
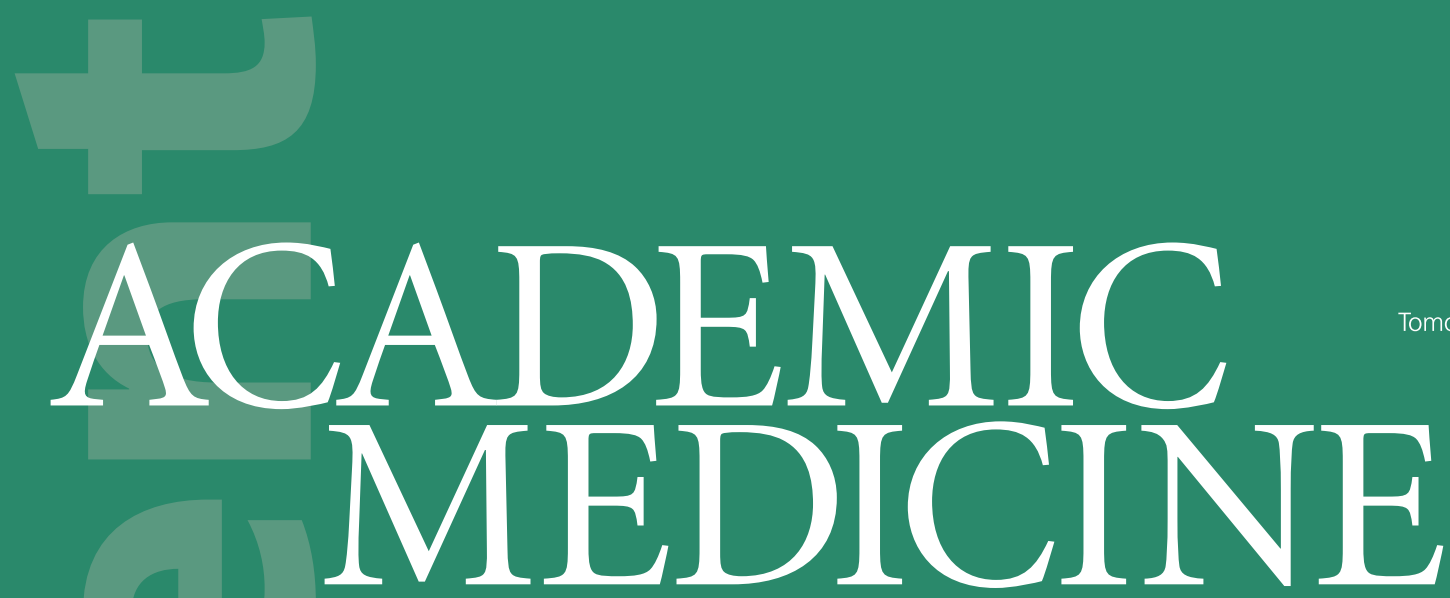

Tomorrow's Doctors, Tomorrow's Cures ${ }^{\circledR}$

Journal of the Association of American Medical Colleges

A Snapshot of Medical Student Education in the United States and Canada: Reports From 145 Schools

Editors: Katherine S. McOwen and Anne L. Farmakidis

Funded by the Association of American Medical Colleges 


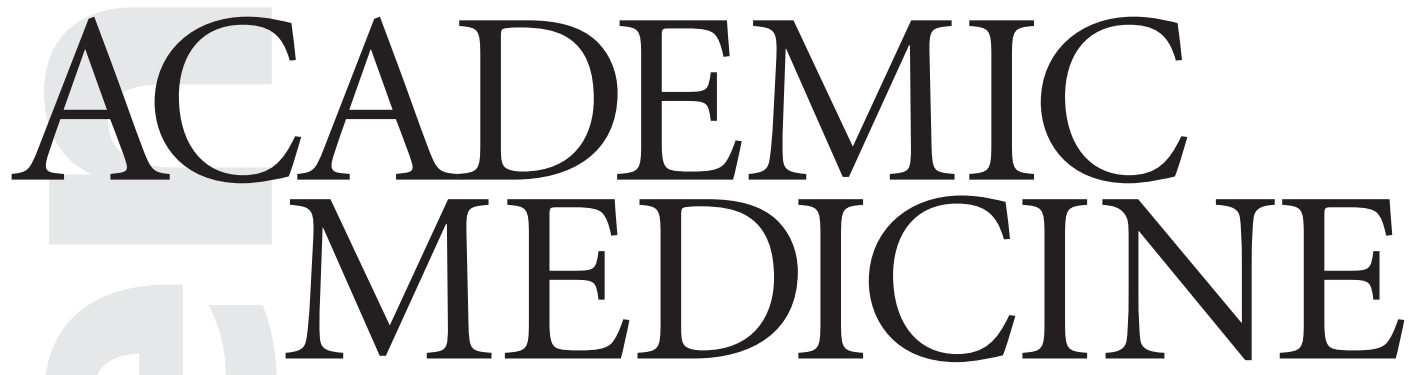

Journal of the Association of American Medical Colleges
September 2020 Supplement

Volume 95

Number 9

A Snapshot of Medical Student Education in the United States and Canada: Reports From 145 Schools

Editors: Katherine S. McOwen and Anne L. Farmakidis

Funded by the Association of American Medical Colleges 


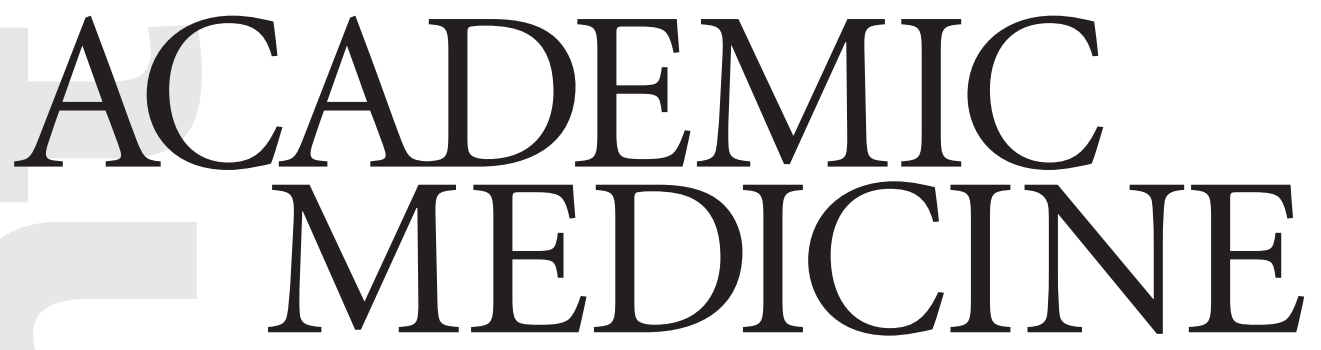

September 2020 Supplement

\section{Journal of the Association of American Medical Colleges}

\section{Editor-in-Chief}

Laura Weiss Roberts, MD, MA

Stanford University

\section{Deputy Editor}

Steven J. Durning, MD, PhD

Uniformed Services University

School of Medicine

\section{Associate Editors}

John Coverdale, MD, MEd

Baylor College of Medicine

Bridget C. O’Brien, $\mathrm{PhD}$

University of California, San Francisco

School of Medicine

Colin P. West, MD, PhD

Mayo Clinic

\section{Assistant Editor for AM Last Pages}

Anthony R. Artino Jr, PhD

The George Washington University

School of Medicine \& Health Sciences

\section{Assistant Editor for Trainee} Engagement

Jesse Burk Rafel, MD, MRes

New York University Langone Health

\section{Assistant Editors}

Irene Alexandraki, MD, MPH

Florida State University

College of Medicine

Dorene F. Balmer, PhD

University of Pennsylvania

Will Bynum, MD

Duke University School of Medicine

Yoon Soo Park, PhD

University of Illinois College of Medicine

at Chicago

Paula T. Ross, PhD

University of Michigan

Michigan Medicine

\section{Assistant to the Editor for}

\section{Social Media}

Ioana Baiu, MD, MPH

Stanford University

\section{Managing Editor}

Mary Beth DeVilbiss

\section{Editorial Staff}

Jennifer Campi

Toni F. Gallo

Elizabeth S. Karlin

Heather Grimm
Laura C. Blyton

Melissa DeCosmo

Charles Rhoads

Whitney A. Staiger

Michael W. Cameron

Lynn Shaull

Publisher's Editor

Suzanne Iler
Academic Medicine (ISSN 1040-2446), is published 12 times per year, monthly, by Wolters Kluwer Health, Inc., at 14700 Citicorp Drive, Bldg 3, Hagerstown, MD 21742. Business offices are located at Two Commerce Square, 2001 Market St., Philadelphia, PA 19103. Periodicals Postage is paid at Hagerstown, MD, and at additional mailing offices. POSTMASTER: Send address changes to Academic Medicine, Wolters Kluwer Health, Inc., PO Box 1610, Hagerstown, MD 21740.
Copyright (C) 2020 by the Association of American Medical Colleges. All rights reserved. Material published in Academic Medicine, in print and online, is covered by copyright. No part of this publication may be reproduced, displayed, or transmitted in any form by any means, electronic or mechanical, without prior written permission.

Published as a supplement to Academic Medicine, September 2020.

academicmedicine.org academicmedicineblog.org @AcadMedJournal 


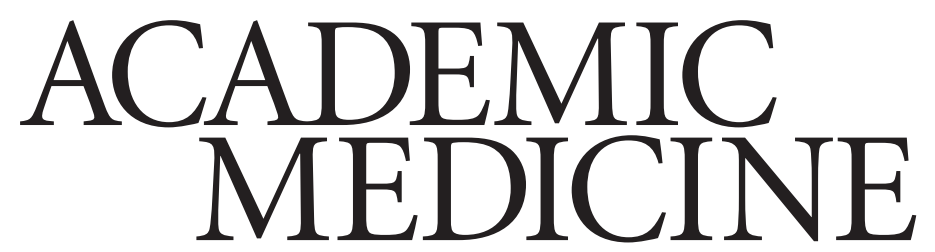

Journal of the Association of American Medical Colleges
September 2020 Supplement

Volume 95

Number 9

\section{A Snapshot of Medical Student Education in the United States and Canada: Reports From 145 Schools}

S1 Foreword: Snapshot 2020

Laura Weiss Roberts, MD, MA

S2 Medical Education in the United States and Canada, 2020

Katherine S. McOwen, MSEd, Alison J. Whelan, MD, and Anne L. Farmakidis, MPS

S5 Curriculum Changes and Trends 2010-2020: A Focused National Review Using the AAMC Curriculum Inventory and the LCME Annual Medical School Questionnaire Part II Angela D. Blood, MPH, MBA, CHSE-A, Jeanne M. Farnan, MD, MHPE, and Walter Fitz-William, MPP

\section{The Reports}

\section{UNITED STATES}

Alabama
S15 University of Alabama School of Medicine Craig Hoesley, MD, and Kevin Leon, MD

S18 University of South Alabama College of Medicine

T.J. Hundley, MD, Anthony Gard, PhD, Elizabeth Minto, MD, and Benjamin Estrada, MD

Arizona

S22 University of Arizona College of Medicine-Tucson

Raquel Hernández Givens, MEd, Sean P. Elliott, MD, Kevin F. Moynahan, MD, and George Fantry, MD

S26 University of Arizona College of Medicine-Phoenix Steven Lieberman, MD, Kathleen Brite Hillis, MD, Glen Fogerty, PhD, Susan Kaib, MD, and Paul R. Standley, PhD

Arkansas

530

University of Arkansas for Medical Sciences College of Medicine James Graham, MD, Sara Tariq, MD, Karina Clemmons, EdD, and Richard Wheeler, MD

\section{California}

S33 California Northstate University College of Medicine Tracy L. Yarbrough, MD, PhD, MAEd, and Catherine Yang, PhD

S38 Kaiser Permanente Bernard J. Tyson School of Medicine Abbas A. Hyderi, MD, MPH, and Mark A. Schuster, MD, PhD

S42 Keck School of Medicine of the University of Southern California Ron Ben-Ari, MD, Kevin Nash, PhD, and Donna Elliott, MD, EdD

S46 Loma Linda University School of Medicine Tamara Shankel, MD, MSHPEd, Lynda Daniel-Underwood, MD, PhD, Daniel Rogstad, MD, PhD, Amy Hayton, MD, and Tamara Thomas, MD 
S50 Stanford University School of Medicine

Daniel Bernstein, MD, Cynthia A. Irvine, MEd, Preetha Basaviah, MD,

James N. Lau, MD, Bahij Austin, Paul J. Utz, MD, and Neil Gesundheit, MD, MPH

S54 University of California, Davis School of Medicine Mark Servis, MD

S57 The University of California, Irvine School of Medicine Khanh-Van Le-Bucklin, MD, MEd, Michael Stamos, MD, and Julie Youm, PhD

S61 University of California, Los Angeles David Geffen School of Medicine Clarence H. Braddock III, MD, MPH, MACP

S63 University of California, Riverside School of Medicine Brigham C. Willis, MD, Christian Lytle, PhD, Maegen Dupper, MD, Rosemary Tyrrell, EdD, Elizabeth H. Morrison, MD, Kendrick Davis, PhD, Kathy Barton, and Deborah Deas, MD

S67 University of California, San Diego School of Medicine Maria C. Savoia, MD

S70 University of California, San Francisco School of Medicine Catherine R. Lucey, MD, Karen Hauer, MD, PhD, Patricia O'Sullivan, EdD, Ann Poncelet, MD, Kevin H. Souza, MS, and John Davis, MD, PhD

\section{Connecticut}

\section{District of Columbia}

S74 The Frank H. Netter MD School of Medicine at Quinnipiac University Lyuba Konopasek, MD, Lisa D. Coplit, MD, and Kim-Thu C. Pham, MD, MPH

S79 University of Connecticut School of Medicine Christine Thatcher, EdD, Ellen Olarsch Nestler, MD, and Melissa R. Held, MD

S83 Yale University School of Medicine Richard Belitsky, MD, Michael L. Schwartz, PhD, Susan E. Larkin, MBA, and Abigail Roth, JD

S87 The George Washington University School of Medicine and Health Sciences

Terry Kind, MD, MPH, Robert Jablonover, MD, and Richard Simons, MD

591 Georgetown University School of Medicine Mary A. Furlong, MD, Racheal M. Ferguson, MHSA, L. Whitman Brown, MBA, and Stephen R. Mitchell, MD, MBA

595 Howard University College of Medicine Debra H. Ford, MD, and Swati Goel, MD

\section{Florida}

S99 Charles E. Schmidt College of Medicine at Florida Atlantic University Sarah K. Wood, MD, and Mark DiCorcia, PhD

S103 Florida International University Herbert Wertheim College of Medicine Vivian Obeso, MD, Janelle M. Fernandez, MS, MBA, Carolyn D. Runowicz, MD, and Robert Sackstein, MD, PhD

S107 Florida State University College of Medicine Alma B. Littles, MD, Nancy L. Hayes, PhD, and Suzanne Leonard Harrison, MD

S111 Nova Southeastern University Dr. Kiran C. Patel College of Allopathic Medicine Paula S. Wales, EdD, Lindsey C. Henson, MD, PhD, and Johannes W. Vieweg, MD 
S115 University of Central Florida College of Medicine

Richard Peppler, PhD, Martin Klapheke, MD, and Jeffrey La Rochelle, MD

5119 University of Florida College of Medicine Joseph Fantone, MD, Maureen Novak, MD, and Adrian Tyndall, MD

S123 University of Miami Leonard M. Miller School of Medicine Alex J. Mechaber, MD, Amar R. Deshpande, MD, Gauri G. Agarwal, MD, Matthew Imm, MD, Rose Maria van Zuilen, PhD, Daniel M. Lichtstein, MD, S. Barry Issenberg, MD, and Laurence B. Gardner, MD

S128 University of South Florida Health Morsani College of Medicine Deborah DeWaay, MD, Bryan Bognar, MD, MPH, and Siwar Bizri, MA

Georgia

Illinois

Indiana

S180 University of lowa Roy J. and Lucille A. Carver College of Medicine Christopher S. Cooper, MD, Susan Lenoch, MA, and David P. Asprey, PhD, PA-C 
Kansas

S184 The University of Kansas School of Medicine

Giulia A. Bonaminio, PhD, Joseph D. Fontes, PhD, Gary Doolittle, MD,

Pam Shaw, MD, Scott Moser, MD, and Michael Robinson, PhD
Kentucky

Louisiana

Maryland
$\mathbf{S 1 8 8}$ University of Kentucky College of Medicine

Charles H. Griffith III, MD, Christopher A. Feddock, MD, and Robert S. DiPaola, MD

S192 University of Louisville School of Medicine

Amy L. Holthouser, MD, Russell W. Farmer, MD, William J. Crump, MD, and Monica A. Shaw, MD, MA

S196 Louisiana State University School of Medicine in New Orleans

Robin English, MD, Joy Sturtevant, PhD, Richard DiCarlo, MD, and Steve Nelson, MD

S199 Louisiana State University Health School of Medicine-Shreveport Christi Rinaudo, EdD, Lucy Robinson, PhD, and Jeff Howells, MS, MBA

S203 Tulane University School of Medicine

N. Kevin Krane, MD, and Jennifer W. Gibson, PhD

S206 The Johns Hopkins University School of Medicine

Nancy A. Hueppchen, MD, MSc, Roy C. Ziegelstein, MD, and Janet D. Record, MD

S211 F. Edward Hébert School of Medicine, Uniformed Services University of the Health Sciences

Arnyce R. Pock, MD, Catherine T. Witkop, MD, MPH, Brian V. Reamy, MD,

Jessica T. Servey, MD, MHPE, Pamela M. Williams, MD, and Jessica L. Bunin, MD

S216 University of Maryland School of Medicine

Donna L. Parker, MD, Joseph P. Martinez, MD, and Nirav G. Shah, MD

Massachusetts

S220 Boston University School of Medicine

Priya S. Garg, MD, Rebecca Halley, and Molly Cohen Osher, MD, MEd

S223 Harvard Medical School

Edward M. Hundert, MD, and Jane M. Neill

S227 Tufts University School of Medicine

Maria A. Blanco, EdD, and Scott K. Epstein, MD

S232 University of Massachusetts Medical School

Anne Campbell Larkin, MD, and Melissa A. Fischer, MD, MEd

Michigan

S236 Central Michigan University College of Medicine

Tina L. Thompson, PhD, Rebecca Molter, MSA, Harold Bell, PhD, and Steven Vance, MD

S240 Michigan State University College of Human Medicine

Dianne P. Wagner, MD, Brian E. Mavis, PhD, and Aron C. Sousa, MD

S245 Oakland University William Beaumont School of Medicine Cynthia H. Ledford, MD, Deirdre G. Pitts, PhD, David M. Thomas, PhD,

Tracey A.H. Taylor, PhD, Robert Noiva, PhD, Robert J. McAuley, PhD, and

Duane G. Mezwa, MD

S249 University of Michigan Medical School

Michelle Daniel, MD, MHPE, Seetha U. Monrad, MD, Sara Weir, MA,

Joseph C. Kolars, MD, and Rajesh S. Mangrulkar, MD

S254 Wayne State University School of Medicine

Jason C. Booza, PhD, Senthil Rajasekaran, MD, MMHPE, and Richard S. Baker, MD

S258 Western Michigan University Homer Stryker M.D. School of Medicine Michael Busha, MD, MBA, Kristine Gibson, MD, Maria Sheakley, PhD, and Jeffrey Greene, PhD 
Minnesota

Mississippi

Missouri

Nebraska

Nevada

New Jersey
S262 Mayo Clinic Alix School of Medicine

Darcy A. Reed, MD, MPH, M. Edwyn Harrison, MD,

Alexandra P. Wolanskyj-Spinner, MD, Debbie A. Dixon, Michele Y. Halyard, MD,

Gerardo Colon-Otero, MD, Marcia L. Andresen-Reid, MS, and Fredric B. Meyer, MD

S266 University of Minnesota Medical School

Austin Calhoun, PhD, Robert Englander, MD, MPH, and Mark Rosenberg, MD

S270 The University of Mississippi School of Medicine

Loretta Jackson-Williams, MD, PhD

S273 Saint Louis University School of Medicine

Debra Schindler, PhD, Lynda Morrison, PhD, Linda Gwinn, Neal Weber, MS, James Duffy, SJ, MD, and Chad Miller, MD

S277 University of Missouri-Columbia School of Medicine Kevin Y. Kane, MD, MSPH, Michael C. Hosokawa, EdD, Kathleen J. Quinn, PhD, and Laine Young-Walker, MD

S282 University of Missouri-Kansas City School of Medicine Paul G. Cuddy, PharmD, MBA, Jennifer Quaintance, PhD, Nurry Pirani, MD, and Mary Anne Jackson, MD

S285 Washington University School of Medicine Eve R. Colson, MD, MHPE, Sabrina Nuñez, PhD, Thomas M. De Fer, MD, Steven J. Lawrence, MD, MSc, Leslie Blaylock, MEd, Amanda Emke, MD, MHPE, Mary E. Klingensmith, MD, Diana L. Gray, MD, Cole Schulte, MHA, and Eva Aagaard, MD

S291 Creighton University School of Medicine Michael Del Core, MD

S294 University of Nebraska Medical Center College of Medicine Regan Taylor, MD, and Amy Cutright, MD

S298 The University of Nevada, Las Vegas School of Medicine Neil G. Haycocks, MD, PhD, and Corrin C. Sullivan, PhD

S302 University of Nevada, Reno School of Medicine Timothy K. Baker, MD, Lisa A. Calvo, MD, Brady J. Janes, PhD, and Gwen S. Shonkwiler, PhD

S305 Dartmouth Geisel School of Medicine Virginia T. Lyons, PhD, John F. Dick III, MD, and Adam R. Weinstein, MD

S309 Cooper Medical School of Rowan University Susan M. Perlis, EdD, Rose Kim, MD, MEd, and Annette C. Reboli, MD

S313 Hackensack Meridian School of Medicine at Seton Hall University Miriam Hoffman, MD, Keith Metzger, PhD, Ofelia Martinez, MD, and Elizabeth Koltz, EdM

S318 Rutgers New Jersey Medical School Maria Soto-Greene, MD, MS-HPEd, Sophia Chen, DO, MPH, Bart Holland, PhD, and Christin Traba, MD, MPH

S322 Rutgers Robert Wood Johnson Medical School Carol A. Terregino, MD, Archana Pradhan, MD, MPH, Joyce Afran, MD, Meigra M. Chin, MD, H. Liesel Copeland, PhD, Robert Lebeau, EdD, Diana Glendinning, PhD, Siobhan A. Corbett, MD, Sarang Kim, MD, and Paul F. Weber, MD, RPh, MBA 
Pablo A. Joo, MD, Todd Cassese, MD, Howard M. Steinman, PhD, Martin N. Penn, MBA, William Burton, PhD, Hai Jung H. Rhim, MD, MPH, and Joshua D. Nosanchuk, MD

S335 Columbia University Roy and Diana Vagelos College of Physicians and Surgeons Jonathan Amiel, MD, Aubrie Swan Sein, PhD, EdM, and Ronald Drusin, MD

S339 CUNY School of Medicine Lisa Auerbach, MD, MS, HPEd, Emine Ercikan Abali, PhD, Rosa Lee, MD, Nicole K. Roberts, PhD, and Erica Friedman, MD

S343 Icahn School of Medicine at Mount Sinai

Robert Fallar, PhD, Valerie Parkas, MD, and Reena Karani, MD, MHPE

S346 Jacobs School of Medicine and Biomedical Sciences at University at Buffalo

Alan J. Lesse, MD, and Michael E. Cain, MD

S349 New York Medical College Jennifer Koestler, MD, Pamela Ludmer, MD, MMEL, and Celia S. Freeman, MBA

S353 New York University Long Island School of Medicine Gladys M. Ayala, MD, MPH, Amy V. Rapkiewicz, MD, Steven E. Carsons, MD, and Steven P. Shelov, MD, MS

S358 New York University Grossman School of Medicine Vicky Harnik, PhD, Steven B. Abramson, MD, Joan Cangiarella, MD, and Mel Rosenfeld, PhD

S362 Renaissance School of Medicine at Stony Brook University Wei-Hsin Lu, PhD, Richard Iuli, PhD, Lisa Strano-Paul, MD, and Latha Chandran, MD, MPH

S367 University of Rochester School of Medicine and Dentistry David R. Lambert, MD, and Christopher J. Mooney, PhD, MPH

S371 Weill Cornell Medicine Yoon Kang, MD, Peter M. Marzuk, MD, Joseph E. Safdieh, MD, Domenick J. Falcone, PhD, and Augustine M. K. Choi, MD

S375 The Brody School of Medicine at East Carolina University Luan Lawson, MD, MAEd, David Gilbert, MD, Robert G. Carroll, PhD, Stephen Charles, PhD, and Kendall M. Campbell, MD

S379 Duke University School of Medicine Colleen O'Connor Grochowski, PhD, and Edward G. Buckley, MD

S383 University of North Carolina School of Medicine Gary L. Beck Dallaghan, PhD, Kurt O. Gilliland, PhD, Beat Steiner, MD, MPH, and Julie S. Byerley, MD, MPH

S387 Wake Forest School of Medicine Kim Askew, MD, E. Shen, PhD, Kimberly Ford, MS, Stefanie Rachis, EdD, Jennifer Mroz, MEd, Nate Warden, MBA, Natalie Barrett, Timothy Peters, MD, and C. Randall Clinch, DO, MS 
S391 University of North Dakota School of Medicine and Health Sciences Marc D. Basson, MD, PhD, MBA, Patrick Carr, PhD, Richard N. Van Eck, PhD,

Susan Zelewski, MD, and Adrienne Salentiny, PhD

Ohio

Oklahoma

Oregon

Pennsylvania

S396 Case Western Reserve University School of Medicine, Including the Cleveland Clinic Lerner College of Medicine Patricia A. Thomas, MD, Amy L. Wilson-Delfosse, PhD, Neil Mehta, MD, Klara K. Papp, PhD, S. Beth Bierer, PhD, and J. Harry Isaacson, MD

S402 The Ohio State University College of Medicine Jennifer W. McCallister, MD, and Daniel M. Clinchot, MD

S406 University of Cincinnati College of Medicine Pamela L. Baker, PhD, and Philip Diller, MD, PhD

S410 Wright State University Boonshoft School of Medicine Brenda Roman, MD, Colleen Hayden, EdD, and Irina Overman, MD

S414 The University of Oklahoma College of Medicine Christopher Candler, MD, EdD, Steve Blevins, MD, Teresa Scordino, MD, LaTasha Craig, MD, and Bryan Touchet, MD

S418 Oregon Health \& Science University School of Medicine Tracy N. Bumsted, MD, MPH, Bart C. Moulton, MD, Tomo Ito, MSEd, George C. Mejicano, MD, MS, and Sharon Anderson, MD, MACP

S422 Drexel University College of Medicine

Amy S. Baranoski, MD, MSc, Donna M. Russo, PhD, and Valerie Weber, MD, MS

S426 Geisinger Commonwealth School of Medicine Andrea K. DiMattia, EdD, John A. Arnott, PhD, Sonia Lobo, PhD, and William B. Jeffries, PhD

S431 Lewis Katz School of Medicine at Temple University Gerald H. Sterling, PhD, David Karras, MD, Larry I. Kaplan, MD, Alisa Peet, MD, Denise Salerno, MD, and Marianne LaRussa

S434 Penn State College of Medicine Britta M. Thompson, PhD, MS, Eileen M. Moser, MD, MHPE, Jed D. Gonzalo, MD, MSc, Daniel R. Wolpaw, MD, Tomi D. Dreibelbis, PhD, MEd, and Terry M. Wolpaw, MD, MHPE

S439 Perelman School of Medicine at the University of Pennsylvania Suzanne Rose, MD, MSEd, Jennifer R. Kogan, MD, Nadia L. Bennett, MD, MSEd, Dennis J. Dlugos, MD, MSCE, Anna Delaney, MBA, Rosalyn Schorr, MSEd, and Judy A. Shea, PhD

S444 Sidney Kimmel Medical College at Thomas Jefferson University Deborah Ziring, MD, Katherine Berg, MD, Nina Mingioni, MD,

Dimitrios Papanagnou, MD, MPH, Urvashi Vaid, MD, and Steven Herrine, MD

Puerto Rico

Rhode Island
S449 Ponce Health Sciences University School of Medicine Georgina Aguirre, MD, and Olga Rodriguez de Arzola, MD

S454 University of Puerto Rico School of Medicine Humberto M. Guiot, MD, Hilton Franqui-Rivera, MD, and Marcel Mesa-Pabón, MD

S457 The Warren Alpert Medical School of Brown University Paul George, MD, Luba Dumenco, MD, Sarita Warrier, MD, Kristina Monteiro, PhD, Steven Rougas, MD, Emily Green, PhD, Michael Mello, MD, Jeffrey Borkan, MD, Thais Mather, PhD, Richard Dollase, EdD, and Allan R. Tunkel, MD, PhD 
S461 Medical University of South Carolina College of Medicine Donna H. Kern, MD, Angela R. Dempsey, MD, MPH, and Debra J. Hazen Martin, PhD

S465 University of South Carolina School of Medicine Greenville April O. Buchanan, MD, Ihsan A. Elkhider, LLB, MSEd, and Angela M. Sharkey, MD

Tennessee

\section{Texas}

$\mathbf{5 4 8 6}$ TCU and UNTHSC School of Medicine Stephen Scott, MD, MPH, Jo Anna Leuck, MD, Jennifer Allie, PhD, Judy Bernas, and Stuart Flynn, MD

S490 Texas A\&M University College of Medicine Jeremy Gibson, MD, Teresa Isbell, PhD, T. Gail Pritchard, PhD, Cayla R. Teal, PhD, MA, Amy Waer, MD, and Emily Wilson, PhD

S495 Paul L. Foster School of Medicine, Texas Tech University Health Sciences Center El Paso

Richard D. Brower, MD, Tanis Hogg, PhD, Maureen D. Francis, MD,

Richard A. Lange, MD, and Paul E. Ogden, MD

S500 Texas Tech University Health Sciences Center School of Medicine, Lubbock

Simon C. Williams, PhD, Michaela Jansen, PharmD, PhD, and

Brian J. Pomeroy, MD, MEHP

S504 The University of Texas Health Science Center at San Antonio, Joe R. and Teresa Lozano Long School of Medicine

Deborah L. Conway, MD, Florence Eddins-Folensbee, MD, Robert M. Esterl, Jr, MD, Joshua T. Hanson, MD, MPH, and Thea K. Lyssy, MA

S508 Dell Medical School at the University of Texas, Austin Elizabeth A. Nelson, MD, LuAnne Wilkerson, EdD, Stephen Smith, PhD, and Susan Cox, MD

S513 University of Texas Medical Branch Galveston

Majka B. Woods, PhD, N. Miles Farr, MD, MPH, and Michael Ainsworth, MD

S517 The University of Texas Rio Grande Valley School of Medicine Andrew Neal Dentino, MD

Utah

S521 University of Utah School of Medicine

Sara M. Lamb, MD, and Wayne Samuelson, MD

Vermont

S525 The University of Vermont Larner College of Medicine Christa Zehle, MD, Kathryn Huggett, PhD, Jesse Moore, MD, and

Cate Nicholas, EdD, MS, PA

\section{Virginia}

S530 Eastern Virginia Medical School

Brooke Hooper, MD, Mily Kannarkat, MD, and Ronald Flenner, MD 
S534 University of Virginia School of Medicine

Megan J. Bray, MD, Maryellen E. Gusic, MD, and Randolph J. Canterbury, MD, MS

S538 Virginia Commonwealth University School of Medicine Michael S. Ryan, MD, MEHP, Diane Biskobing, MD, Lelia Brinegar, EdD, Susan DiGiovanni, MD, and Christopher Woleben, MD

Washington

West Virginia
S542 University of Washington School of Medicine

Suzanne M. Allen, MD, MPH, Michael J. Ryan, MD, Mark Whipple, MD, Kellie Engle, Lynne Robins, PhD, Matthew Cunningham, PhD, and Marjorie D. Wenrich, PhD, MPH

S547 Washington State University Elson S. Floyd College of Medicine James Hupp, MD, DMD, JD, FACS

S548 Marshall University Joan C. Edwards School of Medicine Nitin Puri, MD, PhD, and Bobby Miller, MD

$\mathbf{5 5 5 2}$ West Virginia University School of Medicine Jason S. Hedrick, MA, Scott Cottrell, EdD, Karen Woodfork, PhD, and Norman D. Ferrari III, MD

Wisconsin

5556 Medical College of Wisconsin William J. Hueston, MD

S559 University of Wisconsin School of Medicine and Public Health Elaine M. Pelley, MD, Shobhina G. Chheda, MD, MPH, Christine S. Seibert, MD, Katharina S. Stewart, MD, William Aughenbaugh, MD, and Elizabeth M. Petty, MD

\section{CANADA}

Alberta

British Columbia
S563 University of Alberta Faculty of Medicine and Dentistry

Tracey Hillier, MD, Hollis Lai, PhD, Lyn Sonnenberg, MD, Melanie Lewis, MD, Helly (Rachel) Goez, MD, and Shirley Schipper, MD
S566 University of British Columbia Faculty of Medicine Roger Wong, MD, Sarah Brears, MD, Cheryl Holmes, MD, Amil Shah, MD, Paul Winwood, MB BS, Bruce Wright, MD, Kevin Eva, PhD, and the MD Undergraduate Program Team

\section{Newfoundland}

S570 Memorial University of Newfoundland Faculty of Medicine Tanis Adey, MD, Carla Peddle, MA, and Margaret Steele, MD

\section{Ontario}

S575 Queen's University Faculty of Health Sciences

Leslie Flynn, MD, MEd, MMus, CCFP, FRCPC, and Denise Stockley, PhD

$\mathbf{5 5 7 9}$ University of Toronto Faculty of Medicine Paul Tonin, MEd, Stacey Bernstein, MD, Pier Bryden, MD, Kulamakan Kulasegaram, PhD, Marcus Law, MD, Maria Mylopoulos, PhD, Richard Pittini, MD, Glendon R. Tait, MD, and Patricia Houston, MD

S583 Western University Schulich School of Medicine \& Dentistry Gary Tithecott, MD, MBA, Kyle D. Massey, PhD, Teresa Van Deven, PhD, Shannon L. Venance, MD, PhD, Fred Ross, Lawrence Jacobs, MD, and Jay Rosenfield, MD, MEd

S588 Northern Ontario School of Medicine Brian M. Ross, PhD, MEd, and Catherine Cervin, MD, FCFP, MAEd 
S592 McGill University Faculty of Medicine Beth-Ann Cummings, MD, MSc (HPE), Marion Koch, MD, Melanie Mondou, MD, MSc (HPE), Catherine Millar, MA, and Michelle Elizov, MD, MSc (HPE)

S596 Université de Montréal Faculty of Medicine Margaret Henri, MD, and Geneviève Grégoire, MD

S601 College of Medicine, University of Saskatchewan Greg Malin, MD, PhD, Regina Gjevre, MD, MSc, FRCPC, Patricia Blakley, MD, PhD, FRCPC, and Kent Stobart, MD, MSC, FRCPC

\section{Abbreviations}

S605 Selected Abbreviations Used in This Supplement Jennifer M.G. McDonald 


\section{Foreword: Snapshot 2020}

Laura Weiss Roberts, MD, MA

S American Medical Colleges (AAMC) has published "A Snapshot of Medical Student Education in the United States and Canada" every 10 years as a supplement to Academic Medicine. The undergraduate medical curricula and related features of nearly 150 accredited medical schools are outlined in the current issue. These synopses, taken together, provide a panoramic perspective on the state of undergraduate medical education in 2 North American countries just prior to the coronavirus pandemic in 2020.

Individual entries in this supplement are brief but rich. Authored by education leaders at each school, the descriptions focus on curricular structure, governance, highlights, and innovations, as well as changes over the prior decade. Features such as class size, facilities, and staff support are detailed. The collection allows the reader to compare across schools and also to discern the unique configuration of mission, resources, geography, and educational approaches of each institution.

The snapshot supplement represents a resource for leaders in academic medicine. Documenting core features and trends, the collection illustrates the current context of medical student education and signals where the health professions are headed. Creative new approaches to teaching, mentoring, assessment, inclusion and diversity, professional development, and wellbeing, along with traditional curricular elements, are shared throughout the snapshot supplement. The array of examples should stimulate careful consideration of the intentions and effectiveness of both accepted and novel educational practices.

The idea for collecting curriculum updates from medical schools was originally brought to life years ago by
Brownie Anderson, ${ }^{1}$ and the continued production of this supplement is the direct result of great efforts by Kate McOwen, Anne Farmakidis, and colleagues in Medical Education at the AAMC. As in the past, the snapshot supplement has value because it is comprehensive and accessible, it allows for comparisons across schools, and it reveals patterns and trends in undergraduate medical education. ${ }^{2}$ In our unrelenting efforts to strengthen the educational experiences of future physicians, the collection also provides an opportunity for leaders to reflect on institutional values and, very importantly, how they are expressed in culture and in curriculum.

\section{References}

1 Anderson MB. A guide to the 130 reports in this snapshot supplement to Academic Medicine. Acad Med. 2000;75:Sx-Sxiv.

2 Kanter SL. Foreword. Acad Med. 2010;85(9 suppl):S1. 


\section{Medical Education in the United States and Canada, 2020}

Katherine S. McOwen, MSEd, Alison J. Whelan, MD, and Anne L. Farmakidis, MPS

\begin{abstract}
For the third time this century, the Association of American Medical Colleges has coordinated a collection of reports from their member medical schools that collectively reflect the state of medical education in the United States and Canada. This introduction to the September 2020 supplement to Academic Medicine provides an overview of the collection, with 145 out of 171 eligible medical schools participating in the project. The authors observe trends
\end{abstract}

and similarities across the reports from participating schools, structuring the introduction to mirror the main questions posed to the schools: highlights of each school's medical education program, curriculum description, curricular governance, education staff, faculty development and support in medical education, regional medical campuses, and initiatives in progress. Key findings from the authors include expansion of student enrichment tracks, early clinical encounters, focus on wellness, expansion in competency-based medical education, and continued evolution of approaches to assessment. The authors note that this supplement was produced before COVID-19, and although it robustly chronicles the prepandemic state of medical education, medical education has already evolved and will continue to do so. This view offers important opportunities to observe and study changes in the curricula. n September 2000 and again in September 2010, a supplement to Academic Medicine was published presenting snapshots of medical education. The supplements served as 2 brief windows into undergraduate medical education (UME) at the turn of the century and a decade later, highlighting the wide variety of models of medical education in the United States and Canada to, as Anderson and Kanter ${ }^{1}$ put it in their introduction to the 2010 supplement, "refute the claim that little has changed since the initial reforms that followed the publication of the Flexner Report." We present here a new supplement with the same goal, offering a third brief window into UME, yet highlighting a vastly different landscape for new and existing medical schools alike encountering and reacting to the challenges and opportunities of the last decade. With the support of the AAMC and Dr. Laura Roberts, the editor-inchief of Academic Medicine, we offer this glimpse into the complex and evolving world of medical education in 2020. Even as we write this introduction, reflecting

Please see the end of this article for information about the authors.

Correspondence should be addressed to Katherine S. McOwen; email: kmcowen@aamc.org.

Acad Med. 2020;95:S2-S4.

doi: 10.1097/ACM.0000000000003497

Copyright @ 2020 by the Association of American

Medical Colleges

Supplemental digital content for this article is available at http://links.Iww.com/ACADMED/A977. on submissions that all arrived before the COVID-19 pandemic, UME is rapidly changing again, reminding all of us that education does not hold still long enough to neatly capture its complexities. We hope this glimpse of what has been will offer important lessons to our readers, especially compared with the previous editions of this project. As the pandemic will surely leave its mark on UME, we hope to note in future works the effects of COVID-19 on how we teach and how we learn.

The snapshots contained in this supplement present the story of medical schools in their own words, highlighting that which they deem most important to share with readers in the limited space of approximately 2,000 words and with limited writing time to prepare. Institutions present highlights of their medical education programs, curricular and assessment changes since 2010, descriptions of the structure supporting their educational programs, and their regional campuses, when appropriate. In 2010, 128 schools participated in the supplement, and this year, with a proliferation of new schools, we are pleased to share 145 snapshots, a wonderful representation of the 171 accredited medical schools at the time.

\section{The Process of Creating This Supplement}

In September 2019, medical education senior leaders at each medical school accredited by the Liaison Committee on
Medical Education in the United States and Canada were sent an invitation to participate in the 2020 supplement. A template suggesting the order and composition of topics provided authors with a framework for guidance in deciding how to describe their educational program. (The template is available as Supplemental Digital Appendix 1, at http://links.lww.com/ ACADMED/A977.) Authors were given the leeway to write their snapshot using a narrative or a bulleted format and were asked to share the most detail in the areas they determined important to relate while orienting their writing around the suggested framework. The resulting snapshots allow readers the opportunity to navigate easily between schools with some expectation of similar organization.

\section{Overview of the School Reports}

Highlights of each school's medical education program

We asked authors to make decisions about inclusion based on what they feel is most important to share with a broad audience of readers. Schools have responded with a wide variety of curriculum-, student-, and faculty-related points. Many schools share descriptions of student enrichment tracks in the form of global programs, $\mathrm{PhD}$ studies, and service learning projects. Mentoring is also a widely shared focus, often in longitudinal models and sometimes in learning communities or house systems. Many schools describe their commitment to their states or local communities 
through mission-aligned admissions projects and socially accountable service programs. Integrated curricula are regularly mentioned. Less common but still mentioned in several instances is the intent to serve underserved or rural communities.

\section{Curriculum description}

Readers will note that authors have chosen to share details of their curricula in a variety of formats, including detailed charts, narrative descriptions, and bulleted lists. While the diversity of curricular models is apparent, there are several notable ways in which many or even most schools describe their curricula, pedagogical models, and assessment frameworks.

- Earlier clinical encounters: Schools describe a move toward shorter preclinical time incorporating more integrated basic science and clinical content. Students often experience clinical encounters early or even much earlier in their medical school education.

- New content areas focusing on professionalism, preparedness, and wellness: Schools describe new content areas focused on social and behavioral health, wellness, professional identity formation, population health, exam preparation, and preparation for residency.

- Continued expansion of competencybased medical education: Schools describe their learning objectives in 1 of 3 frameworks: the Accreditation Council for Graduate Medical Education core competencies, ${ }^{2}$ the CanMEDS framework, ${ }^{3}$ or the Physician Competency Reference Set framework. ${ }^{4}$

- Altered assessment landscape: Assessment has changed in format and often in assessor, with schools incorporating peer assessors and other members of the health care team. Schools describe more opportunities for students to be assessed in low-stakes formative assessment models as well as high-stakes summative models. Schools also describe competency-based assessment models that are criterion referenced.

A common challenge expressed by schools focuses on the dispersion of learning to many clinical sites and the difficulty of assessing the quality of learning in a wide variety of sites by faculty. Some schools also share the challenge of finding faculty with the time and experience necessary to teach students in an increasingly competitive environment for clinical learning sites.

\section{Curricular governance}

As expected, most, if not all, schools describe a centralized shared curricular governance model providing consistent oversight of education, although curriculum committees or councils are often composed of a different set of faculty members with different roles on the committees. While at times this centralized structure includes representation from graduate medical education, the committees are always structured in such a way as to support the design and implementation of the undergraduate medical curriculum. Typically, basic sciences and clinical sciences are handled by different subcommittees, and other subcommittees may address assessment or other priorities of importance to the institution. The curriculum committee is always ultimately responsible for the educational program at all educational sites, although what is supported on regional campuses and what is supported on the main campus vary significantly from school to school. We strongly recommend reviewing the curricular governance figures, when provided, for a richer understanding of the variety of models displayed.

\section{Education staff}

At times organized as an office and at times structured as a department, the education staff of schools typically are responsible for all aspects of the educational program from medical school entry to graduation, including curricular oversight, student assessment, and program evaluation. The offices consist of a mix of staff and administrative faculty who often have departmental affiliations elsewhere in the medical school as well. Some schools incorporate into their office or department of medical education all aspects of student support, such as admissions, student affairs, registration/records, and advising; other schools incorporate faculty development and faculty affairs under the umbrella of the office or department of medical education. Also included in some but not all schools are technical support services such as the information technology staff who support curriculum, registration, assessment, and advising. A few schools include the grants office, library, diversity and inclusion office, combined degree programs, and medical education research. The leaders of these organizational units have a variety of titles, but they are typically vice deans or senior associate deans.

\section{Faculty development and support in education}

Faculty development takes many forms, although most schools report both face-to-face and virtual learning opportunities for a variety of teachers including faculty and others. Schools with regional campuses often have regionally hosted programs or traveling programs to support distant teachers. Some schools describe learning academies to support their educators, as well as lunch and learns, journal clubs, medical education fellowships, and certificate programs. Topics tend to be focused on education such as teaching, mentoring, curriculum, assessment, and question writing for assessments; however, other areas are often described as well, such as leadership and diversity and inclusion, including bias training. For schools choosing to describe their promotion and tenure process, teaching evaluations are almost always included in promotion and tenure portfolios along with data about clinical and administrative service and research.

\section{Regional medical campuses}

Schools report a number of models of distributed medical education, including many multicampus schools encompassing from 1 to as many as 15 regional medical campuses (RMCs). The schools offer descriptions of their campuses and, sometimes, the reasons for their model, which often is focused on service to the state's medically underserved areas. RMCs might offer anywhere from just 1 year of education up to the full 4 years, with many schools' RMC models consisting of 2 years of basic sciences and introduction to clinical medicine at the main campus followed by up to 2 years of clinical education at an RMC. Ensuring consistency between main campus and RMC educational programs is a challenge for each school, but they turn to datadriven methods to provide evidence of their effective models. Ultimately, the 
schools describe communication between leadership, faculty, and students at all of the institutions' educational sites as the key to success for RMCs.

\section{Initiatives in progress}

When introducing the suggested format to school authors, we received feedback that many schools would like to include some new initiatives currently ramping up but not fully implemented yet. Schools have been enthusiastic about describing their new initiatives. Some interesting examples involve artificial intelligence and big data. Many schools describe wellness initiatives, sometimes for students and sometimes for the entire medical school. Other schools share plans to implement some model of competency-based medical education including entrustable professional activities frameworks. A few schools describe transition to residency support in the form of data handovers after match and residency preparation boot camps.

\section{Conclusion}

While much remains the same as in previous years, these school reports depict clear trends including almost ubiquitous curricular renewal and redesign descriptions, challenges with finding adequate clinical teaching faculty, and recognition of the educational service of faculty in promotion and tenure. At the same time, the medical schools in this supplement describe with clarity and purpose diverse programs with an array of missions focusing at times on clinical excellence, community service, diversifying the composition of physicians, or scholarly pursuit. As Anderson and Kanter described in 2010 , the stories of the schools in this supplement about their continued efforts to improve their educational programs are testaments to the faculty, administrators, residents, and students of all of the medical schools in the United States and Canada. We think Abraham Flexner would have been pleased, perhaps even amazed, at the substantive changes that have occurred not only in the past 100 years, but in the past decade. ${ }^{1}$

At the dawn of a new decade, we anticipate that this supplement will serve as a reminder of the time before the COVID-19 pandemic, which is testing every aspect of medicine and medical education in the United States and Canada and, quite frankly, around the globe. Throughout the last months, we have experienced the commitment of countless educational leaders dedicated to their patients, students, faculties, and communities in an inspiring show of unity in the face of uncertainty. It is truly a privilege to present their work to you, as we remember what was and look forward to what will be.

\section{Thank You!}

We offer our most sincere thanks to all the authors who described their educational programs with clarity and enthusiasm. The deadlines were short, and they managed to submit their work on time.

Thanks also to the many dedicated AAMC medical education staff members who stepped in to support this work, including Sarah Brown, Michael Cameron, Melissa DeCosmo, Mary Beth DeVilbiss, Toni Gallo, Sara Hunt, Ethan Kendrick, Mallory Lee, Matthew Matarozza, Jonathan O'Loughlin, Charles Rhoads, Whitney Staiger, and Hannah Turner.

Thanks as well to Jennifer Campi, who managed the production of this supplement, and Jennifer McDonald, who copyedited every single snapshot over the course of 2 months. Without them, there would be no supplement and no way to share the wonderful work of our medical schools.

Finally, thanks to the AAMC for supporting this work in print and virtual editions, allowing us to present the programs of education of our member institutions far and wide, and providing this documentation of the wonderful community of medical education that we know and serve.

K.S. McOwen is senior director, Educational Affairs, Association of American Medical Colleges, Washington, DC

A.J. Whelan is chief medical education officer Association of American Medical Colleges, Washington, DC.

A.L. Farmakidis is senior director, Education Resources and Scholarship, Association of American Medical Colleges, Washington, DC.

\section{References}

1 Anderson MB, Kanter SL. Medical education in the United States and Canada, 2010. Acad Med. 2010;85(9 suppl):S2-S18.

2 Holmboe ES, Edgar L, Hamstra S. The Milestones Guidebook: Version 2016. Accreditation Council for Graduate Medical Education. https://www.acgme.org/Portals/0/ MilestonesGuidebook.pdf. Accessed April 20, 2020.

3 Royal College of Physicians and Surgeons of Canada. CanMEDS: Better standards, better physicians, better care. http://www. royalcollege.ca/rcsite/canmeds/canmedsframework-e. Accessed April 20, 2020.

4 Association of American Medical Colleges. CI Physician Competency Reference Set (PCRS). https://www.aamc.org/what-wedo/mission-areas/medical-education/ curriculum-inventory/establish-your-ci/ physician-competency-reference-set. Accessed April 20, 2020. 


\title{
Curriculum Changes and Trends 2010-2020: A Focused National Review Using the AAMC Curriculum Inventory and the LCME Annual Medical School Questionnaire Part II
}

\author{
Angela D. Blood, MPH, MBA, CHSE-A, Jeanne M. Farnan, MD, MHPE, and Walter Fitz-William, MPP
}

\begin{abstract}
Medical school curricula have evolved from 2010 to 2020. Numerous pressures and influences affect medical school curricula, including those from external sources, academic medical institutions, clinical teaching faculty, and undergraduate medical students. Using data from the AAMC Curriculum Inventory and the LCME Annual Medical School Questionnaire Part II, the nature of curriculum change is illuminated. Most medical schools are undertaking curriculum change, both in small cycles of continuous quality improvement and through significant change to curricular structure and content. Four topic areas are explored: cost consciousness, guns
\end{abstract}

and firearms, nutrition, and opioids and addiction medicine. The authors examine how these topic areas are taught and assessed, where in the curriculum they are located, and how much time is dedicated to them in relation to the curriculum as a whole. When examining instructional methods overall, notable findings include (1) the decrease of lecture, although lecture remains the most used instructional method, (2) the increase of collaborative instructional methods, (3) the decrease of laboratory, and (4) the prevalence of clinical instructional methods in academic levels 3 and 4. Regarding assessment methods overall, notable findings include (1) the recent change of the USMLE Step 1 examination to a pass/fail reporting system, (2) a modest increase in narrative assessment, (3) the decline of practical labs, and (4) the predominance of institutionally developed written/computer-based examinations and participation. Among instructional and assessment methods, the most used methods tend to cluster by academic level. It is critical that faculty development evolves alongside curricula. Continued diversity in the use of instructional and assessment methods is necessary to adequately prepare tomorrow's physicians. Future research into the life cycle of a curriculum, as well optional curriculum content, is warranted.

\section{Introduction}

A medical school's ultimate responsibility is to train its students to be compassionate, competent physicians who ensure the health of the public. How this mission is accomplished is as different as the 159 fully accredited U.S. and Canadian medical schools that aim to fulfill this charge. A medical school's curriculum is influenced by numerous external and internal pressures. In this article, using AAMC Curriculum Inventory (CI) and LCME Annual Medical School Questionnaire Part II data, we note curricular changes and trends in the last 10 years and reflect on the range of pressures and influences resulting in the state of undergraduate medical education (UME) today.

\section{Pressures and Influences}

External sources

Aside from the social contract that medical schools maintain with the

\section{Please see the end of this article for information about the authors. \\ Acad Med. 2020;95:S5-S14. \\ doi: 10.1097/ACM.0000000000003484 \\ Copyright (C) 2020 by the Association of American}

Medical Colleges

Supplemental digital content for this article is available at http://links.Iww.com/ACADMED/A963. general public, accreditation heavily influences decisions made by medical schools. The last decade has seen significant change, with the LCME implementing a redesigned system affecting curricular design, delivery, and content. For example, the addition of LCME Element 7.9, Interprofessional Collaborative Skills, correlates with a spike in the number of schools offering interprofessional and team-focused activities. ${ }^{1}$ The accreditation effect impacts not only curricular content but also governance and process, with creation of offices of accreditation, continuous quality improvement, and full-time staff. ${ }^{2,3}$ Forces such as changes in the MCAT and USMLE ${ }^{4}$ have impacted curricular offerings, with the USMLE changes also spurring significant discussion around structure and timing. ${ }^{5}$

\section{Academic medical institutions}

In a nod to accreditation trends, schools have moved from a decentralized, department- and discipline-based structure toward more integrated curricula with centralized administrative oversight. Instructional and assessment strategies have migrated toward a centralized approach, using competency frameworks for instruction and assessment ${ }^{6}$ and embracing outcomes that transcend institutions. Indeed, AAMC CI data show that coverage of Entrustable Professional Activities within curricula has been steadily increasing. ${ }^{7}$ These strategies aim to ensure an outcomefocused product, using time-variable strategies to allow for individualized feedback and development. ${ }^{8}$

With this tremendous sea change comes a tidal wave of data: frequent formative assessments, ongoing narrative feedback, attainment of milestones toward competence-all of these measures create mountains of curricular data. Along the continuum of medical education, big data including predictive analytics and user-created data from electronic medical records are tapped as additional data points in student development. ${ }^{9,10}$ Schools are investing in data visualization systems to use data and share information with stakeholders, including students. ${ }^{11,12}$

In the midst of these pressures, curricular content continues to expand, with more skills and areas of knowledge deemed necessary for graduating medical students. Medical educators make tough decisions with the 130 weeks of instruction minimally required for an educational program's duration (LCME Element 6.8). ${ }^{13}$ These decisions include 
condensing and integrating basic science content ${ }^{14}$ and accelerating entry into clinical clerkships. ${ }^{1}$ Finally, students need to learn how to learn for the rest of their careers, ${ }^{15,16}$ including strategies to manage the influx of information in their postgraduate training. ${ }^{17}$

\section{Clinical teaching faculty}

The changing environment has heightened the need for system-aligned continuing professional development programs. ${ }^{18}$ A systematic review of faculty development initiatives in medical education found that changes in teaching behavior not only were consistently reported by participants but also were detected by students. ${ }^{19}$ While the number of graduate programs in medical education has grown, ${ }^{20,21}$ it remains challenging for faculty to dedicate time to their own professional development as educators, among competing priorities.

Being a physician in an academic medical setting can be fulfilling, but it also brings a large amount of stress. ${ }^{22}$ Pressures for clinical and scholarly activity have grown, while increasing attention is being paid to physician burnout. The measures needed to address burnout are complex; however, a review of faculty vitality suggests that "making sure [clinical] services are adequate and efficient ... would allow more time for faculty to balance their activities while still generating the necessary clinical revenue." ${ }^{23}$ It may be premature to state that faculty engagement with students mitigates burnout, but allowing teaching time for faculty who enjoy their educator role may contribute to faculty vitality.

\section{Undergraduate medical students}

Student priorities may run parallel to, and at times compete with, the planned curriculum. USMLE Step examination preparedness grew increasingly important as test results became a primary component of residency selection. Recently, the USMLE announced policy changes, including a change of the Step 1 to a Pass/ Fail decision. ${ }^{24}$ Before this change, the focus on USMLE performance had led to some unintended consequences, including the development of a stressful Step 1 climate, ${ }^{25}$ with schools under pressure to align curricular content with USMLE blueprints. With the USMLE policy change announcements, there is potential for impact on curricula, medical school policies and practices, and student behaviors.

Residency preparation has deep effects on curricular structure and content. From graduating medical students' perspective, the fourth year of medical school is primarily for preparing for residency. ${ }^{26}$ The number of applications submitted, interviews accepted, and interviews attended by each student has increased, ${ }^{27}$ making finding time for learning challenging. There may be pressure to take away-electives in students' preferred specialty to increase their likelihood of a match in the desired specialty, although away-electives may have little impact on residency match outcomes. ${ }^{28}$ While away-electives have educational benefits, students may be motivated to enroll by other factors related to residency application. ${ }^{29}$ The advent of 3-year medical school programs with direct pathways to residency entry is a recent development. ${ }^{30}$ With limited residency spots available in competitive specialites, ${ }^{31}$ medical school curricula are likely to continue to be affected by the residency application process for the foreseeable future.

\section{Emerging Trends}

Through the prism of these pressures and influences, we note that curricular change is happening at the majority of U.S. medical schools, with $84 \%(\mathrm{~N}=124)$ of schools indicating that they either recently completed, planned, or were in the midst of curriculum change. ${ }^{32}$ Small cycles of curricular change in the form of continuous quality improvement (LCME Element 1.1), ${ }^{13}$ as well as significant changes to the structure and content of curricula, are occurring. ${ }^{1}$

\section{Curricular structure}

LCME Part II data reveal that while overall instructional weeks remain consistent, with the 2018-2019 average at 156.0 weeks, ${ }^{33}$ there has been a shift from years in the curriculum to preclerkship, clerkship, and other (Table 1). In some instances, this structural change has been accompanied by a pedagogical change in basic science instruction. Over $88 \%$ of participating schools reported to the LCME Part II that some or all of their preclerkship instruction is organ based, ${ }^{34}$ with a small decrease in a discipline-focused approach. In addition, longitudinal data from 2010 to 2018 demonstrate that an increasing number of traditionally discipline- or topic-focused courses are more likely to be a component of an integrated rather than an independent course..$^{35} \mathrm{UME}$ programs are generally shifting toward

Table 1

LCME Annual Medical School Questionnaire Part II: Average Required Weeks of Instruction for Participating Schools ${ }^{a}$

\begin{tabular}{|c|c|c|c|c|c|c|c|c|c|}
\hline Data collection period & $\begin{array}{r}2010- \\
2011\end{array}$ & $\begin{array}{r}2011- \\
2012\end{array}$ & $\begin{array}{r}2012- \\
2013\end{array}$ & $\begin{array}{r}2013- \\
2014\end{array}$ & $\begin{array}{r}2014- \\
2015\end{array}$ & $\begin{array}{r}2015- \\
2016\end{array}$ & $\begin{array}{r}2016- \\
2017\end{array}$ & $\begin{array}{r}2017- \\
2018\end{array}$ & $\begin{array}{r}2018- \\
2019\end{array}$ \\
\hline Year 1 & 38.2 & 38.3 & 38.4 & 38.9 & 38.7 & 38.7 & 39.5 & 39.8 & - \\
\hline Year 2 & 35.5 & 35.9 & 35.4 & 34.5 & 35.2 & 35.3 & 34.7 & 35.2 & - \\
\hline Year 3 & 47.3 & 47.1 & 47.1 & 46.8 & 47.0 & 46.9 & 46.7 & 46.6 & - \\
\hline Year 4 & 38.4 & 35.6 & 36.5 & 35.4 & 35.8 & 35.5 & 35.5 & 35.8 & - \\
\hline Average weeks in preclerkship & - & - & - & - & - & - & - & - & 70.3 \\
\hline Average weeks in clinical clerkships & - & - & - & - & - & - & - & - & 64.4 \\
\hline Average weeks in other & - & - & - & - & - & - & - & - & 21.3 \\
\hline
\end{tabular}

aBefore 2018-2019, data were collected according to years in the curriculum (e.g., year 1, year 2, etc.).

In 2018-2019, data were restructured according to preclerkship, clinical clerkship, and other. The data chart is

available at: https://www.aamc.org/data-reports/curriculum-reports/interactive-data/weeks-instruction-and-contact-

hours-required-us-medical-schools. 


\section{Table 2}

\section{AAMC Curriculum Inventory: Participating Schools With Emerging Curricular Content ${ }^{a}$}

\begin{tabular}{|c|c|c|c|c|}
\hline Content area & $\begin{array}{r}\text { Participating schools } \\
\text { with content in } \\
\text { academic year } \\
2013-2014\end{array}$ & $\begin{array}{r}\% \text { Participating } \\
\text { schools with content } \\
\text { in academic year } \\
2013-2014\end{array}$ & $\begin{array}{r}\text { Participating } \\
\text { schools with content } \\
\text { in academic year } \\
2018-2019\end{array}$ & $\begin{array}{r}\% \text { Participating } \\
\text { schools with content } \\
\text { in academic year } \\
2018-2019\end{array}$ \\
\hline Guns and firearms & 21 of 115 schools & 18 & 35 of 131 schools & 27 \\
\hline Nutrition (e.g., food deserts) & 44 of 115 schools & 38 & 64 of 131 schools & 49 \\
\hline Opioids and addiction medicine & 48 of 115 schools & 42 & 86 of 131 schools & 66 \\
\hline
\end{tabular}

aThe search strategy for each of the content areas can be found in the Supplemental Digital Appendices, at http:// links.Iww.com/ACADMED/A963. Please note that for the purposes of this article, nutrition here does not refer to foundational nutrition content (e.g., metabolism). Instead, this report searched for nutrition content in new emerging areas related to social determinants of health (e.g., food access, food insecurity).

integrated course structures, in part due to the desire to improve clinical relevance of curriculum content and the desire to improve efficiency in curriculum delivery.

\section{Curricular content}

There are numerous curricular content areas emerging over the last 10 years that should be explored. This article focuses on the following topics due to their relevance to social determinants of health and health systems science:

- Cost consciousness

- Guns and firearms,

- Nutrition, and

- Opioids and addiction medicine.

The search strategy for each of the content areas can be found in the Supplemental Digital Appendices, available at http:// links.lww.com/ACADMED/A963.

Cost consciousness. According to the AAMC CI, curricular content for cost consciousness has been on the rise, with $80 \%$ of schools reporting content in this area (Table 2). The majority of the curricular content related to cost consciousness occurs in the first 3 academic levels (Table 6 ), and the number of events has risen from 2013 to 2019 (Table 3). Instructional methods are variable for teaching cost consciousness; while lecture continues to be the most common instructional method (Table 4), case-based learning, small-group discussion, and team-based learning are also used with relative frequency. Participation is the most common assessment method (Table 4), and there has been a $50 \%$ increase in schools using a standardized exam, perhaps given the recent implementation of the NBME shelf examination in health care delivery science and innovation. Written or visual media are the primary resource used by schools (Table 4), with some documenting the use of clinical cases. The proportion of cost-consciousness curriculum compared with the entire curriculum continues to rise, most notably reaching $0.83 \%$ in $2018-2019$ academic level 3 (Table 5), and a dramatic $175 \%$ increase in the number of events, with 267 events in 2013-2014 and 733 events in 2018-2019 (Table 6).

Guns and firearms. According to the AAMC CI, curricular content related

\section{Table 3}

\section{AAMC Curriculum Inventory: Timing of Emerging Curricular Content}

\begin{tabular}{|c|c|c|}
\hline Content area & $\begin{array}{l}\text { Timing of content in } \\
\text { academic year } 2013-2014 \\
\text { for participating schools }\end{array}$ & $\begin{array}{l}\text { Timing of content in } \\
\text { academic year } 2018-2019 \\
\text { for participating schools }\end{array}$ \\
\hline \multirow[t]{4}{*}{ Cost consciousness } & Academic level 1 = 37 schools & Academic level $1=78$ schools \\
\hline & Academic level $2=44$ schools & Academic level $2=63$ schools \\
\hline & Academic level $3=50$ schools & Academic level $3=64$ schools \\
\hline & Academic level $4=24$ schools & Academic level $4=32$ school \\
\hline \multirow[t]{4}{*}{ Guns and firearms } & Academic level $1=6$ schools & Academic level $1=17$ schools \\
\hline & Academic level 2 = 10 schools & Academic level 2 = 11 schools \\
\hline & Academic level $3=5$ schools & Academic level $3=10$ schools \\
\hline & Academic level 4 = 1 school & Academic level $4=1$ school \\
\hline \multirow[t]{4}{*}{ Nutrition } & Academic level $1=23$ schools & Academic level $1=30$ schools \\
\hline & Academic level 2 = 15 schools & Academic level 2 = 30 schools \\
\hline & Academic level $3=11$ schools & Academic level $3=21$ schools \\
\hline & Academic level $4=3$ schools & Academic level $4=5$ schools \\
\hline \multirow{4}{*}{$\begin{array}{l}\text { Opioids and } \\
\text { addiction medicine }\end{array}$} & Academic level $1=18$ schools & Academic level $1=57$ schools \\
\hline & Academic level 2 = 28 schools & Academic level $2=44$ schools \\
\hline & Academic level $3=16$ schools & Academic level $3=34$ schools \\
\hline & Academic level $4=5$ schools & Academic level $4=15$ schools \\
\hline
\end{tabular}

${ }^{a}$ A school may have curricular content in multiple academic levels. For the table, each school was counted only once per academic level for which it had relevant curricular content. The search strategy for each of the content areas can be found in the Supplemental Digital Appendices, at http://links.Iww.com/ACADMED/A963. Please note that for the purposes of this article, nutrition here does not refer to foundational nutrition content (e.g., metabolism). Instead, this report searched for nutrition content in new emerging areas related to social determinants of health (e.g., food access, food insecurity). 
Table 4

AAMC Curriculum Inventory: Emerging Curriculum Content Methods ${ }^{a}$

\begin{tabular}{|c|c|c|c|c|c|c|}
\hline $\begin{array}{l}\text { Content } \\
\text { area }\end{array}$ & $\begin{array}{l}\text { Instructional } \\
\text { method used } \\
\text { most often in } \\
2013-2014 \text { by } \\
\text { participating } \\
\text { schools, no. }\end{array}$ & $\begin{array}{l}\text { Instructional } \\
\text { method used } \\
\text { most often in } \\
2018-2019 \text { by } \\
\text { participating } \\
\text { schools, no. }\end{array}$ & $\begin{array}{l}\text { Assessment } \\
\text { method used } \\
\text { most often in } \\
2013-2014 \text { by } \\
\text { participating } \\
\text { schools, no. }\end{array}$ & $\begin{array}{l}\text { Assessment } \\
\text { method used } \\
\text { most often in } \\
2018-2019 \text { by } \\
\text { participating } \\
\text { schools, no. }\end{array}$ & $\begin{array}{l}\text { Resource } \\
\text { type used } \\
\text { most often in } \\
2013-2014 \text { by } \\
\text { participating } \\
\text { schools, no. }\end{array}$ & $\begin{array}{l}\text { Resource } \\
\text { type used } \\
\text { most often in } \\
2018-2019 \text { by } \\
\text { participating } \\
\text { schools, no. }\end{array}$ \\
\hline $\begin{array}{l}\text { Cost } \\
\text { consciousness }\end{array}$ & Lecture (45) & Lecture (68) & Participation (17) & Participation (33) & $\begin{array}{l}\text { Written or visual } \\
\text { media (or digital } \\
\text { equivalent) (17) }\end{array}$ & $\begin{array}{l}\text { Written or visual } \\
\text { media (or digital } \\
\text { equivalent) (19) }\end{array}$ \\
\hline Nutrition & Lecture (29) & Lecture (31) & $\begin{array}{l}\text { Exam-institutionally } \\
\text { developed, written/ } \\
\text { computer based (8) }\end{array}$ & Participation (15) & $\begin{array}{l}\text { Written or visual } \\
\text { media (or digital } \\
\text { equivalent) (9) }\end{array}$ & $\begin{array}{l}\text { Written or visual } \\
\text { media (or digital } \\
\text { equivalent) (10) }\end{array}$ \\
\hline
\end{tabular}

aRegarding how curriculum topics are taught, there are many instructional methods, assessment methods, and resource types available (see AAMC CI Standardized Vocabulary for Instructional Methods, Assessment Methods, Resources, https://www.aamc.org/system/files/2019-08/meded-curriculum-inventory-standardizedvocabulary-082119.pdf). We examined the most often used method or type per curricular topic by number of schools using the given method or resource, which was a selection from the total list, unique per topic. For instructional method, we examined the primary method documented; for assessment methods and resources, we examined each instance of the method or type documented. The difference in these approaches is due to the structure of the AAMC Curriculum Inventory. The search strategy for each of the content areas can be found in the Supplemental Digital Appendices, at http://links.Iww.com/ACADMED/A963. Please note that for the purposes of this article, nutrition here does not refer to foundational nutrition content (e.g., metabolism). Instead, this report searched for nutrition content in new emerging areas related to social determinants of health (e.g., food access, food insecurity).

often used from 2013 to 2019 (Table 4). A small number of schools, 1 to 2 per instructional method, reported using different instructional methods over the most recent years (2017-2019), including clinical experience-ambulatory, clinical experience-inpatient, preceptorship, and problem-based learning. With assessment methods, participation is the most often used (Table 4); however, in 2018-2019, participation was closely followed by exam-nationally normed/standardized, subject (used by 6 schools). This may be due to the increased availability of online educational modules and assessments. Regarding resources, written or visual media have been consistently reported in use from 2013 to 2019; this may be due to the presence of gun violence in the media, making news stories available for medical school curriculum use (Table 4).

Nutrition. For the purposes of this article, we differentiate between foundational nutrition (e.g., metabolism, macroand micronutrients) and emerging nutrition content (e.g., food deserts, food insecurity). According to the AAMC
CI, most schools had foundational nutrition content in their curricula in 2018-2019 (116 of 131 participants, $89 \%)$. While emerging nutrition content occupies a relatively small footprint in the curriculum (Table 8) and fewer schools document emerging nutrition content, it is becoming increasingly represented in UME curricula (Table 2), growing from $38 \%$ of schools reporting such content in 2013-2014 to 49\% of schools reporting it in 2018-2019, with the majority of curricular content related to emerging nutrition occurring in academic levels

\section{Table 5}

AAMC Curriculum Inventory: Proportion of Cost-Consciousness Curriculum Events as a Percentage of All Curricular Events ${ }^{\mathrm{a}}$

\begin{tabular}{|c|c|c|c|c|c|c|}
\hline \multirow{2}{*}{$\begin{array}{l}\text { Academic } \\
\text { level }\end{array}$} & \multicolumn{6}{|c|}{$\%$} \\
\hline & $2013-2014$ & $2014-2015$ & $2015-2016$ & $2016-2017$ & $2017-2018$ & 2018-2019 \\
\hline 1 & 0.11 & 0.15 & 0.18 & 0.17 & 0.28 & 0.33 \\
\hline 2 & 0.21 & 0.21 & 0.22 & 0.17 & 0.31 & 0.31 \\
\hline 3 & 0.25 & 0.29 & 0.40 & 0.45 & 0.55 & 0.83 \\
\hline 4 & 0.23 & 0.28 & 0.31 & 0.22 & 0.31 & 0.38 \\
\hline
\end{tabular}

aThe table compares the number of events for the curricular content area with the overall number of curricular events by academic level for each academic year. 


\section{Table 6}

AAMC Curriculum Inventory: Count of Events With Relevant Curricular Content From Participating Schools ${ }^{\mathrm{a}}$

\begin{tabular}{|c|c|c|}
\hline Content area & $\begin{array}{l}\text { No. events with relevant } \\
\text { content in academic year } \\
2013-2014\end{array}$ & $\begin{array}{l}\text { No. events with relevant } \\
\text { content in academic } \\
\text { year 2018-2019 }\end{array}$ \\
\hline \multirow{4}{*}{$\begin{array}{l}\text { Cost } \\
\text { consciousness }\end{array}$} & Academic level 1 = 71 & Academic level $1=270$ \\
\hline & Academic level 2 = 99 & Academic level $2=162$ \\
\hline & Academic level $3=75$ & Academic level $3=248$ \\
\hline & Academic level $4=22$ & Academic level $4=53$ \\
\hline \multirow{4}{*}{$\begin{array}{l}\text { Guns and } \\
\text { firearms }\end{array}$} & Academic level $1=6$ & Academic level $1=20$ \\
\hline & Academic level $2=10$ & Academic level $2=9$ \\
\hline & Academic level $3=4$ & Academic level $3=11$ \\
\hline & Academic level 4 = 1 & Academic level $4=1$ \\
\hline \multirow[t]{4}{*}{ Nutrition } & Academic level $1=20$ & Academic level $1=44$ \\
\hline & Academic level $2=30$ & Academic level $2=53$ \\
\hline & Academic level $3=33$ & Academic level $3=33$ \\
\hline & Academic level $4=2$ & Academic level $4=3$ \\
\hline \multirow{4}{*}{$\begin{array}{l}\text { Opioids and } \\
\text { addiction } \\
\text { medicine }\end{array}$} & Academic level $1=24$ & Academic level $1=113$ \\
\hline & Academic level $2=53$ & Academic level $2=114$ \\
\hline & Academic level $3=17$ & Academic level $3=47$ \\
\hline & Academic level $4=5$ & Academic level $4=22$ \\
\hline
\end{tabular}

The search strategy for each of the content areas can be found in the Supplemental Digital Appendices, at http://links.Iww.com/ACADMED/A963. Please note that for the purposes of this article, nutrition here does not refer to foundational nutrition content (e.g., metabolism). Instead, this report searched for nutrition content in new emerging areas related to social determinants of health (e.g., food access, food insecurity).

1 and 2 (Table 3 ). In addition, emerging nutrition content has increased by $57 \%$, from 85 total events across all schools in 2013-2014, to 133 total events across all schools from 2018 to 2019 (Table 6). Instructional methods used to teach emerging nutrition content rely heavily on lecture (Table 4) and have increasingly incorporated small- and large-group discussion, as well as casebased learning. The most documented assessment method is participation (Table 4), with more schools making use of institutionally developed written examinations; self-assessment and clinical performance assessment with increasing frequency. Again, written or visual media are the primary resource that the majority of schools have relied upon (Table 4).

Opioids and addiction medicine. According to the AAMC CI, the number of events dedicated to opioids and addiction medicine tripled in some academic levels with a dramatic increase of $199 \%$, from 99 total events across all schools in 2013-2014, to 296 total events across all schools from 2018 to 2019 (Table 6). Most content related to opioids and addiction medicine is taught in academic levels 1, 2, and 3 (Table 3 ) with an increasing number of schools covering this content in 2018-2019 (86 of 131 schools, $66 \%$; Table 2). Consistent with other content areas discussed in this article, the primary methods for instruction are lecture (Table 4), small-group discussion, and case-based learning. Student assessment continues to rely upon institutionally developed written examinations (Table 4) as well as participation, and the most used resource continues to be written or visual media (Table 4). The footprint of opioids and addiction medicine within the overall curriculum has grown, going from a range of $0.04 \%-0.11 \%$ in $2013-2014$ across academic levels $1-4$ to a range of $0.14 \%-0.22 \%$ in $2018-2019$ across academic levels 1-4 (Table 9).

\section{Instructional methods}

Instructional methods continue to evolve as curricula adapt to new realities of health care and health care delivery. As the focus of health care delivery shifts to patient-centered, team-based care, many instructional methods acknowledge approaching medicine as a team sport. When using AAMC CI data to examine the proportion of a given instructional method compared with all other instructional methods, we find that some inherently more collaborative instructional methods are steadily growing in usage, including case-based learning, large-group discussion, and team-based learning, ${ }^{36}$ although these methods collectively make up less than $15 \%$ of overall instructional method use. Perhaps related to the growth of methods that ask students to study and prepare ahead of time such as the flipped classroom method, the use of independent learning, while still a relatively small component of the curriculum (in 2018-2019, 5.88\%), has steadily grown. ${ }^{36}$ Interprofessional education (IPE) - learning from and with other providers of health care or students of health professions - has shown a

\section{Table 7}

AAMC Curriculum Inventory: Proportion of Guns and Firearms Curriculum Events as a Percentage of All Curricular Events ${ }^{\mathrm{a}}$

\begin{tabular}{|c|c|c|c|c|c|c|}
\hline \multirow{2}{*}{$\begin{array}{l}\text { Academic } \\
\text { level }\end{array}$} & \multicolumn{6}{|c|}{$\%$} \\
\hline & $2013-2014$ & $2014-2015$ & $2015-2016$ & $2016-2017$ & $2017-2018$ & 2018-2019 \\
\hline 1 & 0.01 & 0.01 & 0.01 & 0.01 & 0.01 & 0.02 \\
\hline 2 & 0.02 & 0.02 & 0.03 & 0.02 & 0.01 & 0.02 \\
\hline 3 & 0.01 & 0.02 & 0.02 & 0.02 & 0.05 & 0.04 \\
\hline 4 & 0.01 & 0.00 & 0.02 & 0.01 & 0.01 & 0.01 \\
\hline
\end{tabular}

The table compares the number of events for the curricular content area with the overall number of curricular events by academic level for each academic year. 


\section{Table 8}

\section{AAMC Curriculum Inventory: Proportion of Emerging Nutrition Curriculum Events as a} Percentage of All Curricular Events ${ }^{\mathrm{a}}$

\begin{tabular}{|c|c|c|c|c|c|c|}
\hline \multirow{2}{*}{$\begin{array}{l}\text { Academic } \\
\text { level }\end{array}$} & \multicolumn{6}{|c|}{$\%$} \\
\hline & $2013-2014$ & 2014-2015 & 2015-2016 & $2016-2017$ & 2017-2018 & 2018-2019 \\
\hline 1 & 0.03 & 0.03 & 0.02 & 0.03 & 0.03 & 0.05 \\
\hline 3 & 0.11 & 0.08 & 0.08 & 0.10 & 0.08 & 0.11 \\
\hline$\dddot{4}$ & 0.02 & 0.00 & 0.02 & 0.00 & 0.01 & 0.02 \\
\hline
\end{tabular}

aThe table compares the number of events for the curricular content area with the overall number of curricular events by academic level for each academic year. Please note that for the purposes of this article, nutrition here does not refer to foundational nutrition content (e.g., metabolism). Instead, this report searched for nutrition content in new emerging areas related to social determinants of health (e.g., food access, food insecurity).

substantial increase since its inclusion as an LCME Element (7.9) in 2012. Since then, schools have responded with dedicated instruction on, and alongside, interprofessional health care providers.

Based on LCME Part II data, we find that most schools require IPE content as part of their curricula (in 2018-2019, 146 of 151 schools, 97\%), required IPE content has grown steadily over the last 10 years (in 2009-2010, 81 of 130 schools, 62\%), and that although classroom/seminar settings continue to be most often used (in 2018-2019, 87\%), the use of clinical (in 2018-2019, 71\%) and simulation (in 2018-2019, 69\%) settings is steadily increasing also. ${ }^{37}$

When examining the AAMC CI for average number of hours in which students experience an instructional method, we note a steady decline in the amount of lecture. ${ }^{36}$ When considering lecture as a proportion compared with all other possible instructional methods, we find that while lecture has decreased overall since 2012-2013 (60.66\%), it remains the instructional method most often used and retains a relatively large footprint in the curriculum in 2018-2019
(51.10\%). ${ }^{36}$ Also notable, when examining a given instructional method's proportion of the curriculum, the use of laboratory has decreased (in 2012-2013, 6.15\%; in 2018-2019, 5.26\%), with noticeable decline in academic levels 1 and 2.

Clinical experiences continue to be a significant instructional method used in medical schools across the country. Despite the perception that students' clinical experiences are happening earlier, overall AAMC CI data show that while both ambulatory and inpatient clinical experiences are part of academic levels 1 and 2, there is still far more clinical experience present in academic levels 3 and $4 .{ }^{36}$ Meanwhile, LCME Part II data demonstrate a trend toward a decrease in the total number of weeks in required clinical clerkships. Major clerkship experiences such as internal medicine have demonstrated a 1 - to 2 -week decrease in the past 10 years, while others such as radiology have grown. ${ }^{38}$

Simulation continues to be an important instructional method in UME, with an increase in the number of hours across all academic levels. ${ }^{36}$ Service learning demonstrates variability as an instructional method; hours dedicated to service learning have remained stable in academic levels 1 and 2, with recent increases in academic level 3, from an average of 11 hours in academic year 2013-2014 to over 27 hours in academic year 2018-2019 according to AAMC CI data.

\section{Assessment methods}

The most recent and major change in student assessment has been the transition of the USMLE Step 1 to a Pass/Fail reporting system, which is anticipated to begin no earlier than January 2022. This decision includes other changes, such as limiting the number of testing attempts and requiring a passing score on USMLE Step 1 to sit for USMLE Step 2 CS. ${ }^{24}$ Based on LCME Part II data, nearly $100 \%$ of all medical schools require all 3 steps of the USMLE, ${ }^{39}$ with 136 of 136 participating schools (100\%) requiring passing Step 1 for advancement/promotion and 137 of $144(95 \%)$ requiring a Step 1 passing grade for graduation..$^{40}$ Also, an increasing number of schools require Step 2 CK (in 2018-2019, 129 of 144 , or 90\%) and Step 2 CS (in 2018-2019, 119 of 144, or 83\%)

\section{Table 9}

\section{AAMC Curriculum Inventory: Proportion of Opioids and Addiction Medicine} Curriculum Events as a Percentage of All Curricular Events ${ }^{a}$

\begin{tabular}{|c|c|c|c|c|c|c|}
\hline \multirow{2}{*}{$\begin{array}{l}\text { Academic } \\
\text { level }\end{array}$} & \multicolumn{6}{|c|}{$\%$} \\
\hline & $2013-2014$ & $2014-2015$ & 2015-2016 & $2016-2017$ & 2017-2018 & 2018-2019 \\
\hline 1 & 0.04 & 0.04 & 0.06 & 0.07 & 0.09 & 0.14 \\
\hline 3 & 0.06 & 0.07 & 0.06 & 0.11 & 0.15 & 0.16 \\
\hline 4 & 0.05 & 0.06 & 0.10 & 0.08 & 0.09 & 0.16 \\
\hline
\end{tabular}

aThe table compares the number of events for the curricular content area with the overall number of curricular events by academic level for each academic year. 
for graduation. Despite recent trends, the majority of medical schools continue to offer Step 1 at the conclusion of the preclerkship phase (124 of 151 , or $82 \%){ }^{41}$

With regard to grading scales, using LCME Part II data, we find that a Pass/Fail system is increasingly used in preclerkship (from 87 of 141 schools, 62\%, in 20142015 to 116 of $150,77 \%$, in 2018-2019) and physical diagnosis/clinical skills courses (from 91 of 141 schools, 65\%, in $2014-2015$ to 110 of $150,73 \%$, in 2018-2019), whereas required clinical clerkships and fourth-year subinternships and selectives more often use an honors/ high pass/pass/fail system. ${ }^{42}$

Looking to AAMC CI data, we find a diverse group of assessment methods are used across all academic levels, with specific methods used more commonly earlier versus later in the curriculum. Clinical documentation, for example, appears to be used primarily in academic level 3 (the traditional clerkship year), whereas institutionally developed written/ computer-based examinations are more commonly used in academic levels 1 and $2 .{ }^{43}$ Perhaps secondary to accreditation pressures discussed above, data from 2013-2014 to 2018-2019 demonstrate a modest increase in the use of narrative assessment (from $4.87 \%$ in 2012-2013 to $6.81 \%$ in 2018-2019). ${ }^{43}$ Along with the reduction in laboratory as an instructional method, so too do we find that practical labs as an assessment have also declined, dropping by more than half over the last 5 academic years (from 5.96\% in 2012-2013 to $2.83 \%$ in $2018-2019) .{ }^{43}$

Institutionally developed written/ computer-based examinations represent nearly one-third of all assessments (32.78\% in 2018-2019). ${ }^{43}$ Participation is noted as the second most common assessment method, accounting for nearly $20 \%$ of all assessment methods in 2018-2019. With participation measured by student attendance at a given event, it may be that the use of participation and required events is an answer to lapsing student attendance.

\section{Conclusions}

Curricular data show that emerging content areas are added gradually to UME programs. As curricular content is added, one wonders what content, if any, is removed? With a limited number of hours, not to mention stress and burnout as influencing factors among faculty and students, how do UME programs choose what content is added, how much is enough, and what can be removed? We find that among emerging curricular content areas, schools use a likely formula: Lecture is the predominant instructional method, participation is the predominant assessment method, and written and visual media are the predominant resource. Future research into how this formula compares with other more established curricular content areas, as well as the timeline for how these methods and resources may diversify over time as the curriculum content matures, would further illuminate the life cycle of a curriculum.

In this analysis we have focused on required curricular content. Further research on the use of optional content may illuminate how schools are able to respond to an emerging content need and whether approaches vary depending on the need for speedy delivery (e.g., COVID-19); approaches may include optional grand rounds presentations, brown-bag lunches with faculty-facilitated discussions, or other potentially extracurricular activities. As the breadth and depth of content continue to expand, curricularizing content by documenting its footprint on calendars can help schools visualize the amount of content planned. Awareness of the many influencing factors on the UME curriculum, described in detail above, illustrates why thoughtfully inserting even a small amount of new content in an emerging area can be so challenging.

Several trends are notable in the evolution of instructional methods in the last 10 years. Instructional methods remain clustered by academic level, with lecture far and away dominating the instructional methods landscape of the early curriculum. Laboratory time in the early curriculum continues to decrease, as small increases in clinical experiences and case-based learning are evident in academic levels 1 and 2. Despite recent trends of accelerated entry into the clinical clerkships noted nationally, there does not appear to be a substantial change in clinical experiences by academic level. Although modest increases are noted in academic level 2 , inpatient and ambulatory clinical experiences continue to dominate the landscape of the later (academic levels 3 and 4) curriculum.

Despite the goal of training a health care workforce to meet the needs of a diverse patient population, assessment in UME continues to rely upon institutionally developed tests of knowledge. Clinical reasoning, a skill critical to delivering safe and effective patient care, depends on bringing together medical knowledge and clinical assessment, as well as a relative tolerance for ambiguity. These skills cannot be adequately assessed, or encouraged, in an environment with heavy reliance on standardized, multiplechoice-question, computer-based examinations alone. In addition, the second most common assessment method used nationally is participation, which implies student attendance. Educators have for years lamented the state of attendance in UME. ${ }^{44}$ Whether USMLE Step 1, lecture recordings, or generational differences are the cause, how many learners are actually present to participate? Perhaps the lack of attendance says less about students and more about UME.

As schools diversify their instructional and assessment methods and add emerging curricular content, faculty development ensures that medical educators are given the tools to be successful. An example of a curricular content area is the advent of evidencebased medicine curricula: Faculty themselves first needed to be aware of the latest literature and science related to this topic, such that not all faculty may have been initially qualified to teach evidencebased medicine until they themselves had time to learn. An example of an instruction and assessment approach is simulation. Simulation can be extremely valuable when applied correctly; however, when applied incorrectly, simulation can be, at best, inefficient and ineffective and, at worst, traumatic to students. In short, when discussing curriculum change, whether it be related to curricular content, instruction, or assessment, continuing professional development for faculty must be part of the conversation.

As we look ahead to the next 10 years, we must consider the ever-evolving landscape of medical education, including the expansion of clinical knowledge, rapid developments in technology, changes in the transition from UME to graduate medical 
education, licensing and accreditation pressures, and national and emerging health crises. As these forces continue to shape and change the future of our students' experience and their preparation for their role in transforming health care, it is comforting to see both similarities and differences among medical schools, recognizing our common struggles and our common goals.

A.D. Blood is director of curricular resources, Association of American Medical Colleges, Washington, DC

J.M. Farnan is professor of medicine and associate dean, evaluation and continuous quality improvement, University of Chicago Pritzker School of Medicine, Chicago, Illinois.

W. Fitz-William is senior data specialist, Association of American Medical Colleges, Washington, DC

\section{References}

1 Mavis B, Farnan JM. Curriculum change: Reflections on what and how we teach. Curric Context. 2019;6:1-4.

2 Blouin D, Tekian A, Kamin C, Harris IB. The impact of accreditation on medical schools' processes. Med Educ. 2018;52:182-191.

3 Chandran L, Fleit HB, Shroyer AL. Academic medicine change management: The power of the Liaison Committee on Medical Education accreditation process. Acad Med. 2013;88:1225-1231.

4 Gliatto P, Leitman IM, Muller D. Scylla and Charybdis: The MCAT, USMLE, and degrees of freedom in undergraduate medical education. Acad Med. 2016;91:1498-1500.

5 Daniel M, Fleming A, Grochowski CO, et al. Why not wait? Eight institutions share their experiences moving United States Medical Licensing Examination Step 1 after core clinical clerkships. Acad Med. 2017;92:15151524.

6 Goldman E, Schroth WS. Perspective: Deconstructing integration: A framework for the rational application of integration as a guiding curricular strategy. Acad Med 2012;87:729-734.

7 Association of American Medical Colleges. US medical school CI participants including EPA terminology. https://www.aamc.org/ data-reports/curriculum-reports/interactivedata/us-medical-school-ci-participantsincluding-epa-terminology. Accessed March $11,2020$.

8 Mejicano GC, Bumsted TN. Describing the journey and lessons learned implementing a competency-based, time-variable undergraduate medical education curriculum. Acad Med. 2018;93(3 suppl):S42-S48.

9 Arora VM. Harnessing the power of big data to improve graduate medical education: Big idea or bust? Acad Med. 2018;93:833-834.

10 Ellaway RH, Pusic MV, Galbraith RM, Cameron T. Developing the role of big data and analytics in health professional education. Med Teach. 2014;36:216-222.

11 Vaitsis C, Nilsson G, Zary N. Visual analytics in healthcare education: Exploring novel ways to analyze and represent big data in undergraduate medical education. PeerJ. 2014;2:e683.

12 Triola MM, Pusic MV. The education data warehouse: A transformative tool for health education research. J Grad Med Educ. 2012;4:113-115.

13 Liaison Committee on Medical Education. Functions and structure of a medical school: Standards for accreditation of medical education programs leading to the MD degree. https://lcme.org/wp-content/uploads/ filebase/standards/2020-21_Functionsand-Structure_2019-10-04.docx. Published March 2019. Accessed March 5, 2020.

14 Hopkins R, Pratt D, Bowen JL, Regehr G. Integrating basic science without integrating basic scientists: Reconsidering the place of individual teachers in curriculum reform. Acad Med. 2015;90:149-153.

15 Murdoch-Eaton D, Whittle S. Generic skills in medical education: Developing the tools for successful lifelong learning. Med Educ. 2012;46:120-128.

16 Schumacher DJ, Englander R, Carraccio C. Developing the master learner: Applying learning theory to the learner, the teacher, and the learning environment. Acad Med. 2013;88:1635-1645

17 Cutrer WB, Miller B, Pusic MV, et al. Fostering the development of master adaptive learners: A conceptual model to guide skill acquisition in medical education. Acad Med. 2017;92:70-75.

18 Davis DA, Rayburn WF, Smith GA. Continuing professional development for faculty: An elephant in the house of academic medicine or the key to future success? Acad Med. 2017;92:1078-1081.

19 Steinert Y, Mann K, Centeno A, et al. A systematic review of faculty development initiatives designed to improve teaching effectiveness in medical education: BEME guide no. 8. Med Teach. 2006;28:497-526.

20 Artino AR Jr, Cervero RM, DeZee KJ, Holmboe E, Durning SJ. Graduate programs in health professions education: Preparing academic leaders for future challenges. J Grad Med Educ. 2018;10:119-122.

21 Tekian A. Doctoral programs in health professions education. Med Teach. 2014;36:73-81.

22 Arvandi Z, Emami A, Zarghi N, Alavinia SM, Shirazi M, Parikh SV. Linking medical faculty stress/burnout to willingness to implement medical school curriculum change: A preliminary investigation. J Eval Clin Pract. 2016;22:86-92.

23 Shah DT, Williams VN, Thorndyke LE, et al. Restoring faculty vitality in academic medicine when burnout threatens. Acad Med. 2018;93:979-984

24 United States Medical Licensing Examination. Change to pass/fail score reporting for Step 1. https://www.usmle.org/ incus/\#decision. Accessed March 3, 2020.

25 Chen DR, Priest KC, Batten JN, Fragoso LE, Reinfeld BI, Laitman BM. Student perspectives on the "Step 1 Climate" in preclinical medical education. Acad Med. 2019;94:302-304.

26 Benson NM, Stickle TR, Raszka WV Jr. Going "fourth" from medical school: Fourthyear medical students' perspectives on the fourth year of medical school. Acad Med. 2015;90:1386-1393.
27 Gruppuso PA, Adashi EY. Residency placement fever: Is it time for a reevaluation? Acad Med. 2017;92:923-926.

28 Higgins E, Newman L, Halligan K, Miller M, Schwab S, Kosowicz L. Do audition electives impact match success? Med Educ Online. 2016;21:31325.

29 Griffith M, DeMasi SC, McGrath AJ, Love JN, Moll J, Santen SA. Time to reevaluate the away rotation: Improving return on investment for students and schools. Acad Med. 2019;94:496-500.

30 Cangiarella J, Fancher T, Jones B, et al. Three-year MD programs: Perspectives from the Consortium of Accelerated Medical Pathway Programs (CAMPP). Acad Med. 2017;92:483-490.

31 Hayek S, Lane S, Fluck M, Hunsinger M, Blansfield J, Shabahang M. Ten year projections for US residency positions: Will there be enough positions to accommodate the growing number of US medical school graduates? J Surg Educ. 2018;75:546-551.

32 Association of American Medical Colleges. Curriculum change in US medical schools. https://www.aamc.org/data-reports/ curriculum-reports/interactive-data/ curriculum-change-us-medical-schools. Accessed March 5, 2020.

33 Association of American Medical Colleges. Weeks of instruction and contact hours required at US medical schools. https://www. aamc.org/data-reports/curriculum-reports/ interactive-data/weeks-instruction-andcontact-hours-required-us-medical-schools. Accessed March 10, 2020.

34 Association of American Medical Colleges. Curriculum structure during pre-clerkship years. https://www.aamc.org/data-reports/ curriculum-reports/interactive-data/ curriculum-structure-during-pre-clerkshipyears. Accessed March 10, 2020

35 Association of American Medical Colleges. Content documentation as independent course or part of an integrated course. https://www.aamc.org/data-reports/ curriculum-reports/interactive-data/ content-documentation-independentcourse-or-part-integrated-course. Accessed March 10, 2020.

36 Association of American Medical Colleges. Instructional methods used by US and Canadian medical schools. https://www. aamc.org/data-reports/curriculum-reports/ interactive-data/instructional-methods-usedus-and-canadian-medical-schools. Accessed March 11, 2020

37 Association of American Medical Colleges. Interprofessional education requirements at US medical schools. https://www.aamc. org/data-reports/curriculum-reports/ interactive-data/interprofessional-educationrequirements-us-medical-schools. Accessed March 11, 2020.

38 Association of American Medical Colleges. Clinical course required weeks by discipline. https://www.aamc.org/data-reports/ curriculum-reports/interactive-data/clinicalcourse-required-weeks-discipline. Accessed March 11, 2020.

39 Association of American Medical Colleges. USMLE requirements by component. https:// www.aamc.org/data-reports/curriculumreports/interactive-data/usmle-requirementscomponent. Accessed March 11, 2020. 
40 Association of American Medical Colleges. USMLE requirements for advancement/ promotion or graduation. https://www. aamc.org/data-reports/curriculum-reports/ interactive-data/usmle-requirementsadvancement/promotion-or-graduation. Accessed March 11, 2020.

41 Association of American Medical Colleges. USMLE timing requirements at US medical schools. https://www.aamc.org/data- reports/curriculum-reports/interactivedata/usmle-timing-requirements-usmedical-schools. Accessed March 11, 2020.

42 Association of American Medical Colleges. Grading systems use by US medical schools. https://www.aamc.org/data-reports/ curriculum-reports/interactive-data/gradingsystems-use-us-medical-schools. Accessed March 11, 2020.
43 Association of American Medical Colleges. Assessment methods at US and Canadian medical schools. https://www.aamc. org/data-reports/curriculum-reports/ interactive-data/assessment-methods-usand-canadian-medical-schools. Accessed March 11, 2020.

44 Kanter SL. To be there or not to be there: Is attendance really the question? Acad Med. 2012;87:679. 


\section{The Reports}




\title{
University of Alabama School of Medicine
}

\author{
Craig Hoesley, MD, and Kevin Leon, MD
}

\section{Medical Education Program Highlights}

- Rural medicine programs: The Tuscaloosa and Huntsville regional medical campuses oversee special programs designed to promote rural medicine in Alabama.

- Each rural program is a 5-year program including a prematriculation year spent on the undergraduate campus of each program.

- Scholarly Activity: The Scholarly Activity program builds on the research strengths of the university's academic medical center.

- The Scholarly Activity program is a 4-year longitudinal program where students conduct a research project under the supervision of a faculty mentor.

- Upon completion of their projects, students are required to prepare a manuscript.

- Scholarly projects can be undertaken in the following areas of investigations: laboratory-based research; patient-based research; medical humanities; rural and community health; global health and health policy; medical education; and outcome-based research, quality improvement, and medical informatics.

- Service learning: In 2014, the Office of Service Learning was founded to provide students with learning experiences addressing the needs of the community.

- The goal of service learning is to train students about the impact of social factors on health and model the delivery of culturally competent health care.

- The Health Equity Scholars Program is a 4-year program that promotes training in health equity for medically underserved communities.

- University of Alabama School of Medicine (UASOM) is a founding member of the Alabama chapter of the Albert Schweitzer Fellowship training program. Selected students undergo leadership training and conduct a service project in the community.

- Interprofessional education: Interprofessional simulation is a well-established teaching modality. These experiences are coordinated through the University of Alabama at Birmingham (UAB) Office of Interprofessional Simulation for Innovative Clinical Practice.

- Medical and nursing students participate in interprofessional simulations facilitated by faculty members from both

Acad Med. 2020;95:S15-S17.

doi: 10.1097/ACM.0000000000003384

Copyright (C) 2020 by the Association of American Medical Colleges

Supplemental digital content for this article is available at http://links.Iww.com/ ACADMED/A894.

Year school was established: 1969.

School URL: uab.edu/medicine/home. schools. These simulations are a required component during the organ-based modules in the preclinical curriculum and included in some core clerkships.

\section{Curriculum}

\section{Curriculum description}

See Supplemental Digital Appendix 1-Curriculum Overviewat http://links.lww.com/ACADMED/A894.

\section{Curriculum changes since 2010}

- Learning communities_ethics: In 2014, the School of Medicine established learning communities for our student population.

- The primary aim of learning communities is to promote personal well-being and professional development. A longitudinal ethics curriculum is also embedded in this program.

- Clinical Skills Scholars program: This program was established in /2013 to serve as preceptors for the Introduction to Clinical Medicine (ICM) course.

- The ICM course is a longitudinal course spanning the first 2 years of medical school.

- Clinical Skills Scholars are selected through a competitive application process. Each scholar is responsible for a group of first- and second-year medical students and is paired with 2 clinical skills teaching associates who are fourth-year medical students.

- Entrustable Professional Activities (EPAs): In 2018, we piloted a program incorporating the EPAs into the clinical curriculum.

- Students are required to be directly observed by faculty and signed off as competent to perform the skill independently (at or above the level of an intern/PGY-1) on at least 2 consecutive occasions by 2 independent observers.

- The Clinical Skills Scholars are responsible for assuring that the students they taught during the first- and second-year ICM course have successfully completed the required EPAs.

- Evidence-Based Medicine: In 2018, the 1-week-long required Evidence-Based Medicine course was added to the preclinical curriculum.

- Preparation for Residency: Beginning in 2018, a 2-week Preparation for Residency course is now a graduation requirement.

- Components of the course include didactics applicable across all medical specialties, procedural training, simulated case studies, and ACLS certification.

- The course has 2 tracks that students select based on their chosen residency discipline (surgical/procedural or nonprocedural).

\section{Assessment}

The UASOM education program objectives are based on the core competencies outlined by the ACGME. 
See Supplemental Digital Appendix 2-Program Objectives and Assessment Methods - at http://links.lww.com/ACADMED/A894.

The UASOM uses the Customized Assessment Services test item question bank from the NBME during the preclinical curriculum organ-based modules.

- Course directors compile exams for each course using this question bank, which provides faculty with high-quality test items with known statistical performance.

- Students have the opportunity to become familiar with NBME style test questions before taking the USMLE Step 1 examination.

- The school of medicine also uses the item analyses to assist with curricular mapping of the preclinical courses.

- Since 2018, 10\%-15\% of all final exams in each of the organ modules are made up of questions related to prior courses including the foundational sciences course and prior organ modules.

- The Comprehensive Basic Science Examination is offered to all medical students at the end of the preclinical curriculum, before taking USMLE Step 1. This examination is meant to be formative to allow students to focus their Step 1 preparation and assist the Academic Support Team with developing study plans.

\section{Parallel curriculum or track}

Primary care track: In 2018, UASOM established a 4-year MD program for students interested in careers in primary care fields and community-based practice

- The primary care track is based at the Tuscaloosa Regional Campus.

- Students spend their first 2 years of preclinical education in Birmingham before transitioning to their clinical experiences in Tuscaloosa.

- Primary care track students spend their third year in a longitudinal integrated clerkship curriculum based on the Tuscaloosa campus or at an approved community site.

- In addition, students participate in the Leadership in Community and Population Medicine longitudinal course during their third year of medical training.

- During their fourth year, students must fulfill the acting internship requirements (inpatient, acute care, community medicine). Students must also complete 18 weeks of elective credits, which are largely based in community medicine.

UASOM does not offer a 3-year MD degree program.

\section{Pedagogy}

Both the traditional MD program and the primary care track use the following pedagogical approaches to achieve the medical education program objectives.

- Case-based learning

- Clinical experience: ambulatory

- Clinical experience: inpatient

- Discussion: large group $(>12)$

- Discussion: small group $(\leq 12)$

- Laboratory
- Lecture

- Peer teaching

- Self-directed learning/tutorial

- Simulation

- Standardized/simulated patients

- Team-based learning

- Video/podcast

- Workshop

\section{Clinical experiences}

Traditional MD track: Medical students are active in a variety of clinical settings, including both inpatient and outpatient settings, and are exposed to a wide spectrum of medical diseases.

- In Birmingham, students perform clinical rotations at University Hospital (a 1,100+-bed tertiary care facility), the Birmingham VA Hospital, Children's of Alabama Hospital, and ambulatory clinics.

- In Huntsville, students perform clinical rotations at Huntsville Hospital (a 941-bed tertiary care facility) and its associated ambulatory clinics, including rural medicine clinics in north Alabama.

- In Montgomery, students rotate through the Baptist Medical Center South (a 454-bed, acute care regional referral center), Baptist Medical Center East, Vaughan Regional Medical Center, and ambulatory clinics across the surrounding region.

\section{Required longitudinal clinical experiences}

- There are currently no required longitudinal clinical experiences required of all students in the traditional MD track. However, in 2017, the Huntsville regional campus started the Family Medicine Integrated Residency Program, which was primarily developed to foster interest in rural medical practice in Alabama. As part of the program, these students have a longitudinal family medicine ambulatory clinic experience throughout their clinical years.

- Primary care track on the Tuscaloosa regional campus: Students in this track spend their third-year core clerkships in a longitudinal integrated curriculum.

- All medical students first encounter clinical experiences beginning in the first week of medical school in their longitudinal Introduction to Clinical Medicine course.

All students are required to complete an ambulatory medicine acting internship in their third or fourth year of medical school. Those students in the rural programs fulfill this requirement in a rural community.

Challenges in designing and implementing clinical experiences for medical students

- A major challenge of our clinical program is to recruit and onboard community-based physicians as preceptors for our students. UASOM is currently participating in a pilot project through the Society for Teachers of Family Medicine, Building Better Clinical Training Experiences. The goal of this project is to improve student ambulatory experiences and provide 
resources for our community preceptors to enhance their teaching performance.

\section{Curricular Governance}

The Medical Education Committee (MEC) comprises deanappointed faculty, students, residents, and administrative ex officio members. The MEC governs the UME program.

The primary responsibilities of the MEC include:

- Overall curriculum design

- Course and clerkship oversight

- Monitoring program implementation

- Assessment of students and faculty

- Evaluation of programs

There are 3 working subcommittees of the MEC:

- Preclinical Subcommittee (responsible for years 1 and 2)

- Clinical Subcommittee (responsible for years 3 and 4)

- Special Programs Committee (responsible for the dual-degree and rural programs)

In addition to the MEC, the Module Directors Committee and the Clerkship Directors Committee meet at least monthly to implement programs approved by the MEC.

\section{Education Staff}

- The senior associate dean for medical education oversees UME and graduate medical education, medical student services, admissions and enrollment management, the Office of Diversity and Inclusion, and continuing medical education.

- The Office of Undergraduate Medical Education is led by the associate dean for UME. The assistant dean for clinical education assists with the oversight of the third and fourth years of medical education. Furthermore, the Office of UME comprises 12 additional individuals, including 2 with doctoral degrees in education.

- The Office of UME is responsible for the cohesiveness and continuity of educational programs on all 4 campuses. In addition, the office assists with curriculum development, faculty development, and evaluation and assessment of all courses and educational programs.

\section{Faculty Development and Support in Education}

- The UAB Center for Teaching and Learning offers several teaching certificate programs, including a certificate in medical education. In addition, the Office of Interprofessional Simulation for Innovative Clinical Practice offers several faculty development courses in simulation.

- UASOM hosts an annual weeklong faculty development event focusing on medical education, Research and Innovation in Medical Education. This is an annual conference for medical educators to participate in teaching seminars, network with other health care educators, and present their scholarly work in medical education.
- The UAB Healthcare Educators Academy was established in 2019 to provide mentorship, peer support, and faculty development opportunities for current and future health care educators to assist with career advancement in health care education. The Healthcare Academy is a collaborative effort among 5 health professions schools at UAB: the School of Medicine, School of Nursing, School of Public Health, School of Health Professions, and School of Dentistry.

\section{Regional Medical Campuses}

- Consistent medical experiences are maintained across all of our regional campuses. Students are required to keep patient logs, which are reviewed by the clerkship directors. Online educational clinical case presentations are provided to supplement student training if he/she has not participated in the care of a patient with a specific disease during their clerkship rotation. The Office of UME monitors the clinical experiences of students on each of the 4 campuses to ensure comparability across training sites.

- The clerkship directors meet monthly with the Office of UME to review campus-specific data, share innovative teaching modalities, and collaborate to develop new initiatives that can be incorporated across all campuses.

- The clerkship directors from all 4 regional campuses are currently working together to develop a standardized, online repository of didactics, vodcasts, and supplemental resource material that will be used by students in each clerkship discipline.

See Table 1-Regional Medical Campuses.

\section{Initiatives in Progress}

- Expansion of objective structured clinical examinations into the required third-year core clinical clerkships, allowing for longitudinal standardized assessment of students' skills throughout the clinical years.

- Expansion of additional EPAs into the third and fourth years of medical school.

\section{Table 1}

\section{Regional Medical Campuses}

\begin{tabular}{|c|c|c|}
\hline $\begin{array}{l}\text { Regional campus } \\
\text { name }\end{array}$ & Type & $\begin{array}{l}\text { Student } \\
\text { enrollment }\end{array}$ \\
\hline Birmingham & Entire MD program & 100 \\
\hline Huntsville & Clinical & $30-35$ \\
\hline Montgomery & Clinical & 20 \\
\hline Tuscaloosa & Clinical & $30-35$ \\
\hline
\end{tabular}




\title{
University of South Alabama College of Medicine
}

\author{
T.J. Hundley, MD, Anthony Gard, PhD, Elizabeth Minto, MD, and Benjamin Estrada, MD
}

\section{Medical Education Program Highlights}

- The school has a small class size (74 students per class), allowing students to form close bonds with peers and faculty during all phases of the curriculum.

- Preclerkship courses deliver content primarily through small-group learning and simulation exercises, allowing students opportunities to apply basic science concepts to clinical cases.

- Preclerkship students rotate through ambulatory care clinics (year 1) and specialty-based programs of their choice (year 2) synchronously with the organ system-based modules.

- In the clerkship and postclerkship phases of the curriculum, students have the opportunity for extensive hands-on experience and patient interactions, developing a wide breadth of clinical and procedural skills.

\section{Curriculum}

\section{Curriculum description}

- We began planning for curriculum renewal in 2010. The new curriculum, implemented in 2012, is aligned with the 6 ACGME core competencies and possesses a 2-year preclerkship phase composed of integrated, organ system-based modules. This is followed by a clerkship phase and postclerkship phase that integrate a robust clinical curriculum with the basic science concepts introduced in the preclerkship phase. Clinical skills instruction is integrated throughout the curriculum.

See Supplemental Digital Appendix 1-Curriculum Overview and Sequence-at http://links.lww.com/ACADMED/A938.

- The preclerkship phase begins with foundational content, critical to all organ system-based modules. Starting in 2019, basic content in both public health and epidemiology was repositioned and integrated into the first week of this course. An initiative using virtual patient families was developed to facilitate teaching of this material.

- Clinical Skills is a longitudinal course spanning the first 2 years of the educational program. Instruction integrates simulation, standardized patient encounters, and real-life experiences. The course also delivers education related to interprofessional collaboration and culturally responsive medicine.

Acad Med. 2020;95:S18-S21.

doi: 10.1097/ACM.0000000000003444

Copyright (C) 2020 by the Association of American Medical Colleges

Supplemental digital content for this article is available at http://links.Iww.com/ ACADMED/A938.

Year school was established: 1972.

School URL: https://www.southalabama.edu/colleges/com.
- During the Clinically Integrated Introductory course (CLINIC) offered within the clinical skills curriculum, all preclerkship students encounter real patients. Students work with their assigned clinical preceptor for required sessions each semester to practice history gathering, physical exam techniques, and communication skills. During the first year, our students work predominantly in primary care settings. In the second year, students choose a different specialty area each semester to promote professional development, clinical skills, and career exploration.

- We identified key curricular content to be delivered longitudinally, referred to as threads. These threads are integrated horizontally and vertically over a single academic year or multiple years as appropriate. Current threads include bioethics, evidence-based medicine, microbiology, nutrition, scientific method, physician wellness, and population health/ epidemiology/biostatistics.

- We offer students the option to take a selective in their third year and defer their required neurology clerkship to the fourth year. Selectives allow students to explore additional experiences for professional development; options include emergency medicine, neurosurgery, orthopedic surgery, pathology, radiology, and research.

- During the fourth year, we offer a variety of electives. Students are required to take an acting subinternship, a course fulfilling basic science integration, a subspecialty course, and our Transitions to Residency course.

- The Transition to Residency course occurs in spring of the fourth year. It provides education on topics including graduate medical education requirements, health care organization and financing, health care law and malpractice, effective teaching, and leadership skills.

\section{Curriculum changes since 2010}

- The curriculum renewal transitioned our educational program from a discipline-based curriculum to the current structure. A Division of Medical Education was created to facilitate the transition and maintain the current program.

- The new curriculum reduced lecture content by more than half and expanded small-group learning, self-directed learning, and clinical skills instruction.

- Our clinical skills program has expanded significantly. We have appointed a director of clinical skills, introduced a standardized patient program, constructed a clinical skills center recreating an ambulatory clinic, expanded mannequin and procedure simulation, and implemented regularly scheduled weekly clinical skills instruction.

- We developed a standardized list of required clinical experiences and skills for all clerkships. Centralized electronic oversight is provided to assure student achievement of these activities. 
- Our Curriculum Committee, recognizing a need to enhance interprofessional skills, has expanded interprofessional longitudinal experiences. We partner with other institutional health professions training programs to host joint educational sessions throughout the entire curriculum.

- Beginning in 2014, we phased in an integrated quality improvement and patient safety curriculum in the clerkships. Instructional methods include flipped classroom, small-group discussion, facilitated workshops, simulation, standardized patients, and interactive games. Students learn about improvement cycles, adverse event analysis, medication reconciliation, disclosing medical error, reducing health care waste, and clinical decision-making tools.

- In 2019, we implemented a longitudinal physician wellness curriculum spanning the third year. This initiative was developed jointly with our Office of Student Affairs and Student Wellness Council. Sessions include preventing burnout, reducing cynicism, recognizing substance abuse and suicide risk, mitigating stress, promoting wellness, addressing mental health needs, and coping with death and dying.

- We developed a competency coaching program to support students in the new curriculum. This activity provides each first- and second-year medical student with a faculty competency coach whose role is to assist the student longitudinally with the achievement of educational objectives.

\section{Assessment}

- Our education program objectives are based upon the 6 ACGME core competencies and the Physician Competency Reference Set.

See Supplemental Digital Appendix 2-Program Objectives and Assessment Methods—at http://links.lww.com/ACADMED/A938.

- A 3-tier honors/pass/fail grading system and a minimum pass scoring threshold were adopted in the first 3 years. We recently began using only a pass/fail system for the fourth year.

- Preclerkship modules adopted a standardized set of summative assessments administered by the Division of Medical Education, including biweekly exams and a customized NBME assessment as the final exam for each module.

- In the preclerkship curriculum, students maintain their small group for the entire academic year. This allows for peer evaluations to be completed by students on each of their smallgroup team members.

- Numerous formative and 4 summative OSCEs are delivered in the clinical skills curriculum and are required for student promotion. A fifth summative OSCE occurs at the conclusion of the third year.

- During the curriculum renewal process, we identified many opportunities to add assessment methods not previously used.

- Rubrics are now used frequently. Our Curriculum Committee and course directors have found that these types of tools allow for more robust assessment of small-group learning, simulation, and independent learning activities. Examples include:

- Our standardized patient and simulation exercises are assessed using rubrics.
- Self-directed learning activities are assessed using rubrics developed from national accreditation frameworks with emphasis on the quality of evidence-based resources.

- A competency-based assessment rubric is used to measure student progress achieving core clinical skills. The format uses milestones similar to those used in ACGME-accredited residency programs. The tool provides formative feedback during the clinical skills curriculum and summative grading for clerkships.

- All modules and courses undergo an annual comprehensive review by the Assessment and Evaluation Subcommittee of the Curriculum Committee to ensure compliance with accreditation standards, achievement of educational program objectives, and continuous quality improvement.

- The Student Subcommittee of the Curriculum Committee conducts an independent student analysis of each module and course in the preclinical years. This information complements feedback received on course evaluations and is incorporated into the annual review.

\section{Pedagogy}

- Pedagogical approaches used in our curriculum include case-based learning, clinical experiences in the ambulatory and inpatient settings, large- and small-group discussions, laboratory, lecture, preceptorship, reflection, self-directed learning, simulation, standardized patients, team-based learning, virtual patients, and workshops.

- We use a variety of small-group learning strategies. Examples include team-based learning, structured self-directed learning activities, games, and independent small-group projects.

- "Case constructs" are a small-group learning strategy that involve the development of sets of individual cards with specific elements linked to distinct clinical diseases. The elements on cards can be related to epidemiology, pathophysiology, clinical history, physical exam, diagnostic tests, or treatment methods. Card sets are grouped together into a session theme and distributed to student groups. Teams then work to arrange and appropriately match them.

- Team-based learning was uniformly adopted as the preferred small-group learning strategy in the required clinical clerkships. In addition, our team-based learning activities primarily use clinical vignette scenarios.

- Reflective writing is integrated into the CLINIC program, clerkships, and multiple fourth-year electives.

\section{Clinical experiences}

- Clinical sites for our students include our 2 hospitals, University of South Alabama (USA) University Hospital and USA Children's and Women's Hospital, for inpatient experiences. Ambulatory clinical sites include both universityand community-based clinical sites.

- Students begin their exposure to clinical skills instruction in the first weeks of their first year using facilitated demonstrations by faculty as well as application of content delivered in didactic or small groups using standardized patients and mannequins.

- Clinical experience begins in the first semester with the CLINIC program as previously described. 


\section{Curricular Governance}

- The Curriculum Committee provides central oversight for the development, implementation, assessment, and improvement of the educational program.

- There are 7 working subcommittees of the Curriculum Committee:

- Assessment and Evaluation Subcommittee

- Preclerkship Subcommittee

- Clerkship Subcommittee

- Clinical Pathways Subcommittee

- Clinical Skills Subcommittee

- Integration and Educational Strategies Subcommittee

- Student Subcommittee

- The Curriculum Committee membership includes basic science and clinical faculty, students, and educational support personnel from across the institution, all with 3-year term limits.

- Our preclerkship course directors have their primary faculty appointment either in basic science departments or in the Division of Medical Education. Our clinical course directors have their appointments within their respective clinical departments.

\section{Education Staff}

- The Division of Medical Education was formed in 2010 at the onset of our curriculum renewal. This division is overseen by the associate dean of medical education and is composed of administrative leadership, 6 educational support personnel, and 2 full-time faculty.

See Figure 1-Administrative organizational chart.

- Educational support personnel manage educational materials and our learning management system; distribute evaluations to students and faculty; organize clinical skills sessions; coordinate clinical experiences; and prepare, deliver, and analyze assessment data.

- In year 3, there is a full-time coordinator for each of the clerkships, and we also have a selective coordinator. The fourth-year courses are coordinated by 2 full-time personnel.

- The Office of Educational Technologies and Services assists course directors and the Division of Medical Education with multimedia tools, video capturing, and information technology support.

\section{Faculty Development and Support in Education}

- Professional development is offered to faculty through internally conducted workshops, retreats, departmental grand rounds, and faculty meetings in partnership with university faculty development centers. In addition, professional development opportunities at educational meetings are used by faculty with support from the institution.

- Our institution has created the position of associate dean for faculty development and faculty affairs.

- Teaching is a component of the promotion and tenure process at our institution. We offer a clinician-educator track for those faculty interested in this career path. Educational scholarship and excellence in teaching are critical components of this track. Educator portfolios are encouraged to demonstrate excellence in teaching.

\section{Initiatives in Progress}

- We are currently developing a longitudinal 4-year parallel track focusing on primary care. This track will expose selected students to the skills necessary to be collaborative primary care leaders of interprofessional teams delivering high-value,

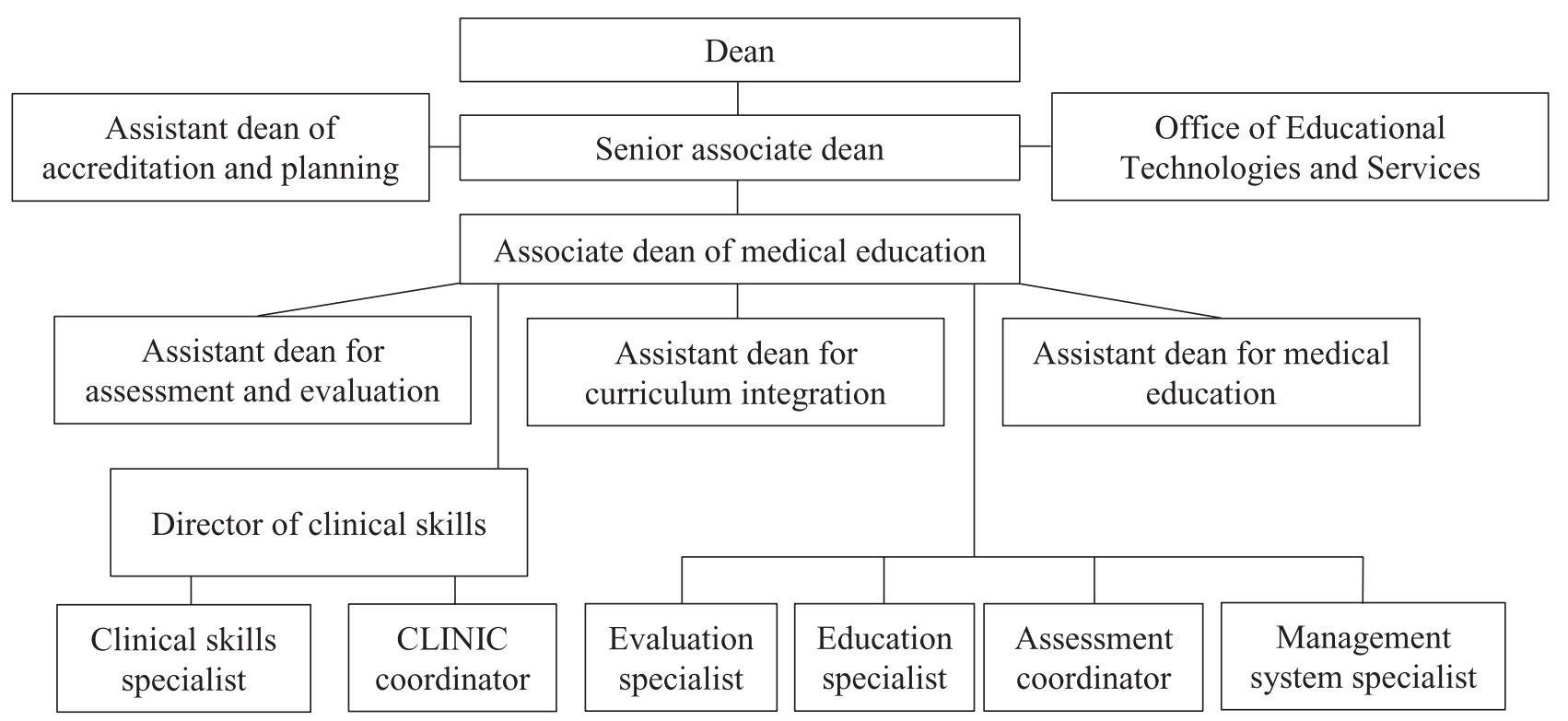

Figure 1 Administrative organizational chart. 
culturally responsive care to patients with complex, chronic diseases. This curriculum will integrate health systems science objectives longitudinally.

- As part of our continuous quality improvement efforts, we have started a review of our Foundations course in the first year. The purpose of this review is to optimize the curricular location of foundational content necessary for each of the organ system modules. We anticipate this review will also enhance the effectiveness of content delivery and time utilization during each of the organ system modules.
-We are introducing point-of-care ultrasound training into the curriculum. Ultrasound workshops have been introduced into 3 of our 10 preclerkship courses and 1 clerkship. Our goal is a 4-year longitudinal ultrasound curriculum beginning in 2020.

- We plan next academic year to institute an Oncology course at the conclusion of the second year, to replace the Multisystem Disorder course.

- Different initiatives related to enhance our students' global health experience are underway. These will complement current educational activities already implemented in Africa and Latin America. 


\title{
University of Arizona College of Medicine-Tucson
}

\author{
Raquel Hernández Givens, MEd, Sean P. Elliott, MD, Kevin F. Moynahan, MD, and George Fantry, MD
}

\section{Medical Education Program Highlights}

The University of Arizona College of Medicine-Tucson (COM-T) offers an integrative 4-year MD program in the newly constructed, state-of-the-art Health Sciences and Innovations Building (HSIB).

- The curriculum features a 3-semester-long preclerkship phase to allow more time for clinical electives and making a specialty career choice.

- The Commitment to Underserved People (CUP) program is a student-directed, cocurricular program that provides students with clinical learning opportunities in 38 program clinics to serve medically underserved populations statewide.

- The state-funded Rural Health Professions Program (RHPP) matches 26 students per class with physicians working in rural areas throughout Arizona to nurture students' interest in rural health care and primary care careers.

- The Societies Program is a 4-year longitudinal mentoring program designed to assure that students acquire robust clinical skills, develop clinical thinking strategies, and begin professional identity formation. The program encourages meaningful, longitudinal relationships among the groups and their mentors; promotes student well-being; and establishes a sense of community.

- The House Advisory System is made up of 4 Societies Houses, each with 20 Societies groups (mentor and students) from each class. Each of the 4 houses is led by a student affairs dean (house dean), who serves as students' points of contact addressing immediate needs and providing individualized career counseling. The house deans work collaboratively with Societies mentors to ensure student success.

- The COM-T offers 8 distinction tracks. Students engage in additional coursework, mentorship, and a thesis or project to satisfy track requirements; successful completion results in students graduating with distinction in that track. Track offerings are bilingual medical Spanish, community service, global health, integrative medicine, leadership and innovation in health care, medical education, research, and rural health. The college also offers several dual-degree programs: MD$\mathrm{MPH}, \mathrm{MD}-\mathrm{PhD}, \mathrm{MD}-\mathrm{MBA}$, and soon, the MD-MEd program.

- The premedical admissions pathway (PMAP) is a 13-month postbaccalaureate program offering conditional acceptance to medical school for approximately 10 students annually who have had greater than average challenges in becoming competitive medical school applicants.

Acad Med. 2020;95:S22-S25.

doi: 10.1097/ACM.00000000000003258

Copyright $(2020$ by the Association of American Medical Colleges

Supplemental digital content for this article is available at http://links.Iww.com/ ACADMED/A816.

Year school was established: 1965.

School URL: https://medicine.arizona.edu.

\section{Curriculum}

\section{Curriculum description}

The curriculum is organized into 3 phases: preclerkship phase, clerkship phase, and transition to residency (T2R) phase.

- Preclerkship: Students proceed through a series of 7 sequenced blocks. Longitudinal themes via Pathways to Health and Medicine (PHM) and the spiraling curricula are integrated throughout this phase. Concurrently with the blocks, students complete the Clinical Reasoning course and Doctor and Patient course. A primary focus of the phase is to expose students to basic sciences translated to clinical medicine framed in social determinants of health, clinical reasoning, and exposure to patients.

- Clerkship: Begins with the Transition to Clerkship course, followed by the required rotations. Students also complete intersessions within the phase building upon longitudinal curricula in the preclerkship phase, covering such topics as health law, physician wellness, and medical ethics.

- T2R: Includes required rotations in emergency medicinecritical care, a surgical subspecialty, and a subinternship. Students may individualize their T2R curriculum to support their medical specialty choice. Transition to Residency Bootcamp and Basic Sciences in Clinical Medicine are 2 new courses. The bootcamp provides education and training in skills necessary for postgraduate training and includes separate tracks in surgery and medicine. The Basic Sciences in Clinical Medicine course provides a return to evidence-based medicine and opportunities to revisit the basic sciences as they inform clinical decision-making.

See Supplemental Digital Appendix 1-Curriculum Descriptionat http://links.lww.com/ACADMED/A816.

\section{Curriculum changes since 2010}

The most significant curricular change has been the full accreditation of the Phoenix regional campus program separate from COM-T's program, affording the opportunity to undergo a curriculum modification. After extensive preparation and faculty engagement, in academic year 2017-2018, COM-T's curriculum transitioned from a 24 -month to a 20 -month preclerkship phase, including a new 6-week basic sciences capstone course designed to support student preparation for the USMLE Step 1 examination. Areas of overlap and redundancy were streamlined to underscore the developmental and spiral curricular design. The clerkship phase underwent similar rigorous review. In this process, the internal medicine clerkship was divided into inpatient and ambulatory components, the surgery clerkship was increased from 6 to 8 weeks, and the neurology clerkship was increased from 3 to 4 weeks. 
Preclerkship block directors emphasized more clinical application and social and behavioral science content, and made greater use of learning technology, including online sources of content, study, and exam preparation. COM-T also formalized USMLE Step 1 preparation into the curriculum. In the clerkship phase, with the successful development of additional clinical sites necessary to accommodate the overlap between students in the legacy and the modified clerkship curricula, COM-T has expanded its faculty resources for clinical instruction. In the T2R phase, the Transition to Residency bootcamp and the Basic Sciences in Clinical Medicine courses were developed based on student feedback, national trends in graduate medical education, and results of the AAMC Graduate Questionnaires preceding the curricular transition.

\section{Assessment}

The medical education program objectives were designed based on the ACGME domains of competence.

See Supplemental Digital Appendix 2-Medical Educational Program Objectives and Assessment Methods-at http://links. lww.com/ACADMED/A816.

Since 2010, more opportunities are available for formative and summative assessment, which include:

- Block self-assessments through interactive home-grown and vended, online modules and practice exams

- More diagnostic practices exams for USMLE Step 1 preparation

- OSCEs administered in the required clerkships

- Formative narrative assessment of clinical skills sessions in the Bootcamp and Basic Sciences in Clinical Medicine courses in the $\mathrm{T} 2 \mathrm{R}$ phase

The year 2 OSCE and end-of-clerkship OSCE have been maintained. Additional improvement changes have resulted in increased uniformity in student assessment strategies among the clerkships. Also, COM-T has piloted the introduction of Entrustable Professional Activities into its student assessment and curricular evaluation approach while expanding the use of OSCEs in the clerkships.

\section{Pedagogy}

The recent curriculum modification provided an opportunity to integrate more active learning strategies since 2010 to achieve the program objectives. Pedagogical approaches used throughout the curriculum include flipped classroom, case-based learning, independent and self-directed learning, reflective writing and journaling, portfolio review, simulations, and standardized/ simulated patients. In addition, the preclerkship phase content is also delivered through team-based learning, virtual labs, and in small- and large-group discussions. Lectures are podcasted for students as additional reference material. The learning in the clerkship phase is based on preceptorship and clinical experience in ambulatory and inpatient settings throughout the rotations. The new simulation center and teaching clinic in the HSIB provides more space to expand patient care-based interprofessional education.

\section{Clinical experiences}

COM-T has a long-standing and productive relationship with a university-based teaching hospital system, community hospitals, community and rural clinics, the Southern Arizona Veteran's Affairs Health Care System (SAVAHCS), and multiple Indian Health Services sites. All are components of the medical students' required and elective clinical experiences, and align with the college's mission and vision goals of exposure to broad diversity of patient care and engagement. Medical students have their first clinical encounter on the first day of medical school. They meet their Societies mentor and interview a patient selected through the program. This first encounter is designed for students to understand their patient's experience. Subsequently, students have clinical experiences weekly in the curriculum, as well as in the student-run CUP clinics.

\section{Required and elective community-based rotations}

As noted, a significant component of medical students' clinical experiences occurs with community partners in both urban and rural settings. Clerkships maintain an active roster of participating sites and preceptors and evaluate them annually for comparability in student experiences, patient exposure, education, and assessment. Instructional development is offered to sites that have either new teaching faculty or opportunities

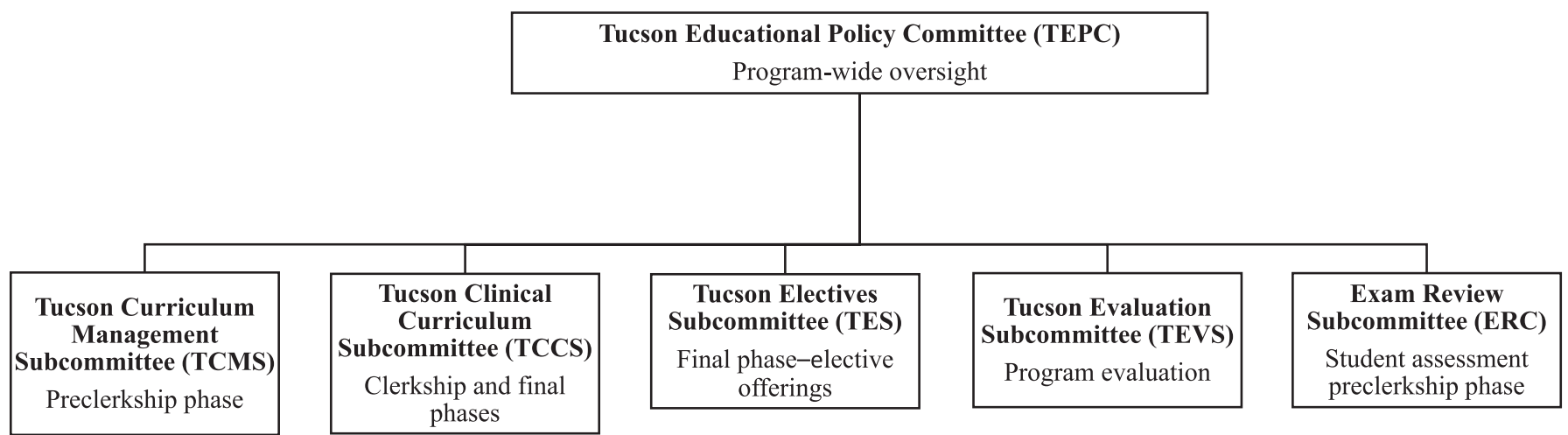

Figure 1 Curricular governance. 


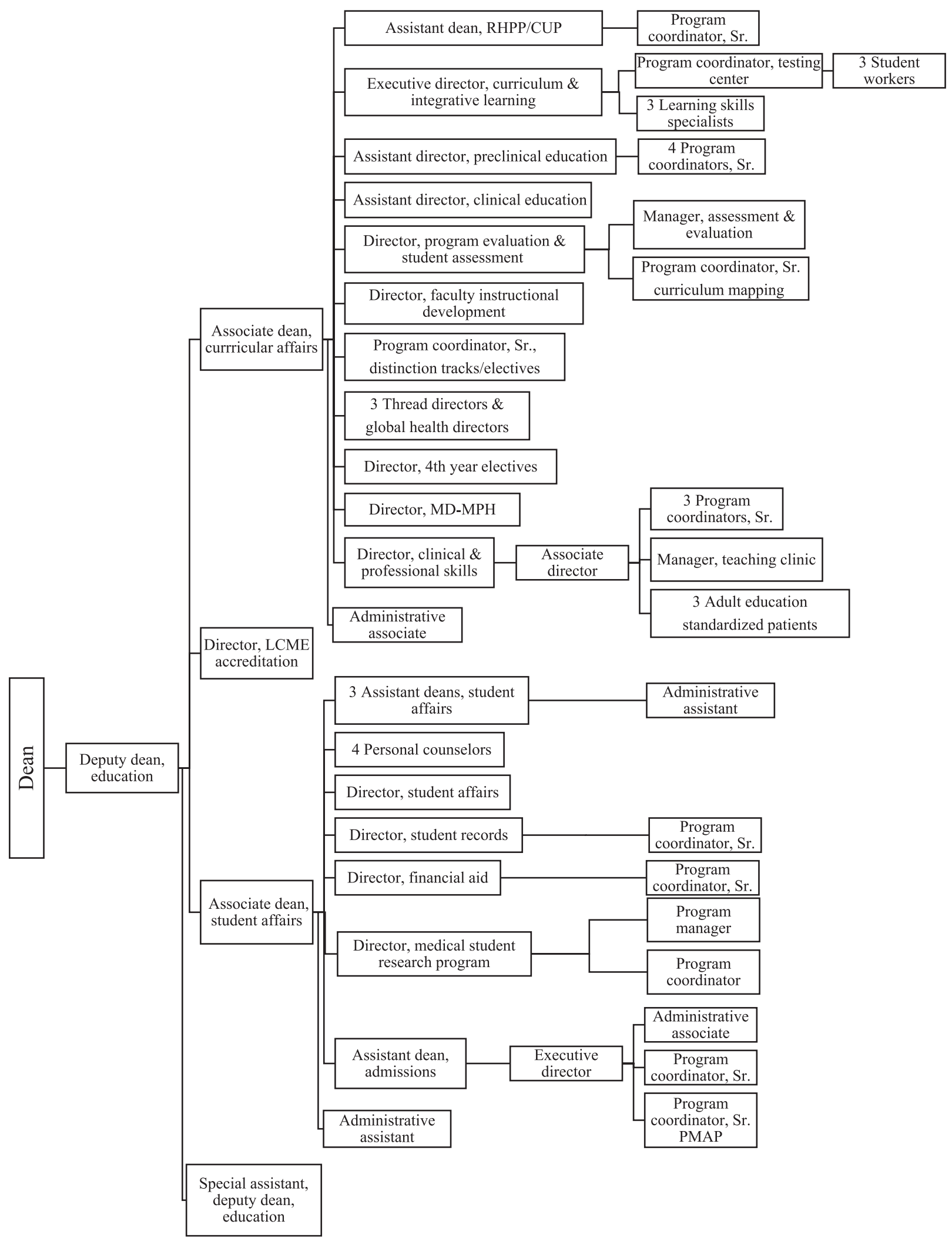

Figure 2 Medical education leadership. 
for improvement in the current staff. To align with the mission of training and retaining students interested in providing community-based health care, all clerkships provide communitybased experiences as part of their rotations. Similarly, the SAVAHCS is a vital component of clerkship training in veterans' health care for the medicine, psychiatry, neurology, and surgery clerkships. Through their active engagement with veterans in these rotations, students are able to build on the instruction in veterans' health care they received as part of the PHM curriculum.

\section{Challenges in designing and implementing clinical experiences for medical students}

An ongoing challenge is monitoring clinical experiences for their impact, benefit, and safety for students' experiences; however, the curriculum committee's monitoring system using annual site reviews has been successful in this regard. Another emerging challenge is competing with 2 osteopathic schools of medicine in Arizona that are able to offer financial support to clinical sites. Not unique to the COM-T, there is also the pressure for teaching faculty to generate significant clinical income to retain their practice and/or position.

\section{Curricular Governance}

The curriculum management and governance structure is designed to oversee the medical education program. The Tucson Educational Policy Committee (TEPC) provides program-wide oversight and is made up of faculty elected by the general faculty and students elected by their peers. The TEPC has several subcommittees that oversee major curricular components, as shown in Figure 1.

See Figure 1-Curricular governance.

\section{Education Staff}

The Office of Curricular Affairs is made up of 26 full- and part-time educational faculty and professional staff who provide leadership, planning, evaluation, and support for the undergraduate medical education program and the TEPC and its subcommittees at COM-T. The office provides organizational responsibility for the CUP community outreach programs, the RHPP, the student development program, and the dual-degree programs. Central to the role of the Office of Curricular Affairs is providing administrative support for all 3 curricular phases. This role includes rigorous assessment and evaluation strategies and support, with cross-linking of educational design and feedback to grow the educational mission further.

See Figure 2-Medical education leadership.

\section{Faculty Development and Support in Education}

Through the Office of Curricular Affairs, COM-T provides professional development to faculty through the services of the director of faculty instructional development. In this role, the director supports faculty in improving their classroom-based and clinical teaching by offering both in-person and online modules. This support extends to all house staff, adjunct faculty, and affiliate clinical teaching faculty at clinical teaching sites through online training. Such development is provided routinely and codified in TEPC policy. Additionally, the Academy of Medical Educators and Scholars (AMES) provides peer-based instructional assessment and instruction.

The role of educational scholarship has grown rapidly in COMT's promotion and tenure process over the last 10 years. Today, all new faculty candidates and faculty candidates for promotion must demonstrate effective teaching in their respective departments and provide peer assessment as demonstration of that educational contribution. Further, the college has created faculty promotion tracks, for example, an educator Scholar track, that require evidence of increasing impact and recognition of the candidate's educational efforts, which are documented in an educational portfolio.

\section{Academy for educators and scholars}

The mission for AMES is to develop and retain outstanding educators and promote excellence in teaching and educational scholarship. AMES recruits its membership from COM-T faculty in all departments, including senior, highly experienced faculty and junior or midlevel faculty who show great promise in education and educational scholarship. AMES offers robust faculty development and mentorship and supports COM-T's success in maintaining a pool of strong educators to support its mission and vision goals. AMES has an annual budget to fund educationbased research, educational scholarship travel grants, and outreach efforts (education-based journal club, speakers, research forums).

\section{Initiatives in Progress}

The COM-T has launched an early acceptance program in partnership with the University of Arizona Honors College. Approximately 10 students enrolled in the Honors College will be accepted during their junior year. Students will experience an individualized curriculum as well as participate in an immersive summer research experience at the COM-T between their junior and senior years before starting medical school. The COM-T is currently also exploring a 7-year combined University of Arizona undergraduate and medical school program, in conjunction with the recently approved Bachelor of Medicine degree that the college plans to offer. Finally, the COM-T is launching an MD-MEd dualdegree program in collaboration with the University of Arizona College of Education. 


\title{
University of Arizona College of Medicine-Phoenix
}

\author{
Steven Lieberman, MD, Kathleen Brite Hillis, MD, Glen Fogerty, PhD, Susan Kaib, MD, and Paul R. Standley, PhD
}

\section{Medical Education Program Highlights}

The University of Arizona College of Medicine-Phoenix (COM-P) evolved substantially in the past decade, from a 48-student-per-year branch campus of the College of MedicineTucson (COM-T) to an independently accredited medical school in 2017. Its educational program emphasizes early, robust clinical skills training and patient experiences, active learning modalities, and personalization of medical education according to each student's career aspirations and preferences.

- Early robust clinical training and patient exposure begins during the first week of medical school in the Doctoring course and Introduction to Medicine block. Standards are high and consistently enforced, enabling students to contribute increasingly to patient care while honing their skills in the community clinical experience, which alternates weeks with Doctoring.

- Active learning modalities include small-group case-based instruction, team-based learning, and various interactive exercises and formats generally requiring preparatory work by students. Independent learning modules-typically 10 - to 20-minute topic-related video presentations - are developed by faculty and the instructional design team to support interactive sessions. In 2017-2018, the college embarked on a 5-year plan to virtually eliminate traditional lectures. Three years in, approximately $65 \%$ of preclerkship basic science sessions are built on active learning modalities.

- Personalized medical education is supported by several major curriculum features in addition to electives and selectives. Additional curriculum modifications further enabling personalization are in development.

- Scholarly projects: All students are required to complete a hypothesis-driven, faculty-mentored research project as a graduation requirement. Choice of project is individually determined by students and spans a wide range of subjects and methods, including traditional laboratory research; retrospective or prospective patient-based research; and population-based, public health, health care system, and education-related topics. Projects span 4 years, and nearly half of students opt to work on a second scholarly project during their enrollment. Each project culminates in a thesis and poster presentation at the annual Student Research Symposium. One-fourth are presented at external conferences and 1 in 6 is published in a peer-reviewed journal.

Acad Med. 2020;95:S26-S29.

doi: 10.1097/ACM.0000000000003463

Copyright (C) 2020 by the Association of American Medical Colleges

Supplemental digital content for this article is available at http://links.Iww.com/ ACADMED/A945.

Year school was established: 2012.

School URL: https://phoenixmed.arizona.edu.
- Certificates of distinction: Students have the option to participate in 1 of 5 certificates of distinction: global health; health humanities; primary care, rural health, or service; and community health. One-third of students currently participate in one such program.

- Dual-degree programs: Current offerings include MD-PhD, MD-MS (clinical translational science), and 4-year combined MD-MPH program. An MD-MBA is underdevelopment. Approximately $18 \%$ of students currently participate a dualdegree program.

- Personalized active learning block: Between years 1 and 2, students design or select from an extensive list a 1-credit learning experience to further their medical education in line with interests and career goals. Experiences have included service learning trips to the Dominican Republic, medical Spanish, scholarly projects, and working with a local clinician to gain exposure to a specialty.

\section{Curriculum}

\section{Curriculum description}

See Supplemental Digital Appendix 1-Curriculum Overviewat http://links.lww.com/ACADMED/A945.

\section{Curriculum changes since 2010}

The curriculum in place in 2010 - an expanded version of Tucson's faculty-developed curriculum-was replaced in 2013 with the current curriculum structure. The preclerkship curriculum features organ system blocks interspersed with 1 - to 2-week capstone experiences in which students apply newly gained basic sciences knowledge in clinical settings while learning about the health care system. Capstone weeks also provide time to work on scholarly projects. The 6-week Transition to Clerkships course uses simulation, standardized patients, and a variety of learning modalities to prepare students for effective clerkship functioning. Required clerkships include the 6 traditional core clerkships in year 3, neurology in year 3 and emergency medicine in year 4. The COM-P strategic plan includes development of a 3-year curriculum with competencybased time-variable transition to residency.

The COM-P facilities on the downtown Phoenix Biomedical Campus were built for a class size of 120. From a branch campus with 24 students per class, the program expanded to 80 in 2013 and to 100 in 2020. The clinical teaching model is distributed, with hospitals in the Banner Health system-the college's primary clinical affiliate-hosting the most student rotations. Students rotate at additional clinical affiliates: Veterans Affairs Medical Center of Phoenix, Phoenix Children's Hospital, Valleywise Medical Center (formerly Maricopa Integrated Health System), Barrow Neurological Institute, Hospice of the Valley, and others. A rural longitudinal integrated clerkship will begin in Payson, Arizona, in summer 2020. 


\section{Medical education program objectives}

COM-P's educational program objectives (EPOs) are based on the 6 ACGME competencies, replacing practice-based learning and improvement with critical appraisal and quality improvement, and systems-based practice with societal awareness and responsiveness, to clarify these domains' focus. EPOs are based on compilation of the AAMC's Medical School Objectives Project, general competencies of physicians developed by the ACGME and American Board of Medical Specialties, and the physician roles of the CanMEDS 2005 report of the Royal College of Physicians and Surgeons of Canada.

\section{Assessment}

During development of the new curriculum and pursuit of independent accreditation, the assessment system was redesigned to evaluate student performance in the new EPOs. The assessment system combines formative and summative assessment using a variety of assessment modalities.

See Supplemental Digital Appendix 2-Competencies, Objectives, and Outcome Measures - at http://links.lww.com/ ACADMED/A945.

\section{Parallel curriculum or tracks}

COM-P is launching a rural longitudinal integrated clerkship in Payson, Arizona (90 miles from downtown Phoenix), for a subset of students enrolled in the rural health certificate of distinction. This program will span 8 months of year 3 and include training in emergency medicine, family medicine, outpatient internal medicine, obstetrics-gynecology, pediatrics, and surgery. Clinical experiences will emphasize continuity with patients and preceptors. Preceding this experience, students spend the personalized active learning block between years 1 and 2 in Payson or another rural setting.

As part of the COM-P strategic plan, a 3-year core curriculum with competency-based, time-variable progression to residency is in the early stages of development.

\section{Pedagogy}

Pedagogical approaches at COM-P have changed extensively since 2010 with the changes in curriculum and independent accreditation. The following constitute the vast majority of learning sessions:

- Case-based instruction is used in all preclerkship curriculum blocks. Cases are usually explored on 2 days, with self-directed study between opening and closing sessions. Each group comprises 1 faculty member and 8 students.

- Clinical experiences include both ambulatory and inpatient rotations at a variety of clinical sites (below).

- Discussion sessions in small and large groups occur throughout the curriculum on a wide range of topics including basic science disciplines, communication skills, and professionalism.

- Laboratory sessions are prominent in the Clinical Anatomy course and neuroscience blocks.
- Lectures are diminishing as part of a 5-year plan to replace passive with active learning approaches.

- Preceptorships comprise the Community Clinical Experience course in the first 2 years and the personalized active learning block for many students.

- Simulation exercises with formative feedback occur in almost all blocks and clerkships.

- Simulated physiology laboratories permit students to gather data from each other to test hypotheses and apply foundational science knowledge.

- Standardized patients are used extensively to train and assess basic clinical skills development in the Doctoring course (years 1-2), as assessments in clerkship OSCEs, and in the summative OSCE at the beginning of year 4 .

- Team-based learning is used to variable degrees in most of preclerkship blocks.

- Online videos, locally produced by COM-P faculty members, are used extensively in preclerkship blocks as independent learning modules for viewing before active learning sessions.

\section{Clinical experiences}

Most students complete the standard block clerkship curriculum.

In addition to rotations in the Banner Health system (COMP's primary affiliate), the college's distributed model of clinical education provides a wide variety of settings and patient populations for required, selective, and elective rotations. Inpatient opportunities include public, children's, veterans, and community hospitals, while ambulatory experiences include private practice, group practice, and federally qualified health center settings. Special opportunities regularly afforded to students include rural and American Indian reservation rotations and a volunteer street medicine program in Phoenix. An educational experience in rural or underserved communities is a graduation requirement.

In the 2019-2020 academic year, the initial pilot of a longitudinal clinical experience was implemented to provide students continuity with patients, faculty, and clinic teams over 3-4 years.

Following the intensive Doctoring curriculum in the summer and fall of year 1, and after demonstrating a required level of competence in history and physical exam skills, students begin authentic clinical experiences in January of year 1.

Students receive extensive experience in community-based locations, as most inpatient and outpatient affiliates, including Banner University Medical Center-Phoenix, are community based. These experiences provide students insight into modern medical practice.

COM-P has adequate clinical training sites for its students and continues to add more in the Banner Health system in preparation for class size expansion. Identification of additional sites is ongoing, with faculty development being a key activity as new sites and faculty members are recruited. 


\section{Curricular Governance}

Virtually all aspects of UME are centralized under the Office of Academic Affairs, including oversight and implementation of curriculum units (blocks, courses, clerkships, electives, selectives) by the Office of Curricular Affairs. In the distributed model of clinical education, educational time for clerkship directors and other leadership positions are "purchased" by the college from clinical affiliates or compensated directly to independent clinicians. Staff support is provided by the college. Some heavily used clinical sites have an appointed site director who reports to the clerkship director. Student assessment, program evaluation, and all other functions are managed centrally. Academic support and career counseling are housed in the Office of Student Affairs.

See Figure 1-Committee structure.

\section{Education Staff}

The Office of Academic Affairs and its units are responsible for virtually all curricular aspects, from design and implementation to program evaluation and CQI. Instructional faculty development, instructional technology, the simulation center, the clinical skills center, and other supporting functions are also housed within academic affairs. The Research Office for Medical Education (ROME) promotes scholarship among faculty and staff and hosts an annual ROME Forum for showcasing these efforts.

See Figure 2-Medical education leadership.

In addition to the UME program and its supporting offices and functions, the Office of Academic Affairs oversees pipeline recruitment programs, admissions, and student affairs.

\section{Faculty Development and Support in Education}

Numerous and varied faculty educational development opportunities are provided. Workshops and small-group sessions on case-based instruction, instructional design, educational technology, and NBME-style item writing are offered annually or more often as needed. The director of faculty development offers a yearlong faculty fellowship for clinical faculty members with a strong interest in teaching. Online modules (with CME credit) include "Tips for effective feedback," "Teaching motivational interviewing," "Communication skills for team-based learning," "Tailoring your teaching to learner needs," "Theory and practice of teaching medicine," and "Optimizing teaching when time is limited."

Tenure-track faculty members are expected to teach, which is an essential part of evaluation for promotion and tenure. Criteria include the quality and quantity of teaching activities, creation and innovativeness of teaching methods and materials, extramural recognition, and educational scholarship. Many education-intensive faculty members are on the education scholar track (non-tenure-eligible). Promotion in this track requires scholarship, much of which is based on scholarship in teaching.

\section{Regional Medical Campuses}

COM-P is launching an 8-month longitudinal integrated clerkship in Payson, Arizona, in the summer of 2020 for a subset of students in the rural health certificate of distinction. Assessing comparability of educational experiences at this site, as at all distributed sites, is part of the regular clerkship review process and includes comparison of student evaluations, grade distributions, NBME subject exam performance, student competency assessment, and required clinical experiences across sites.

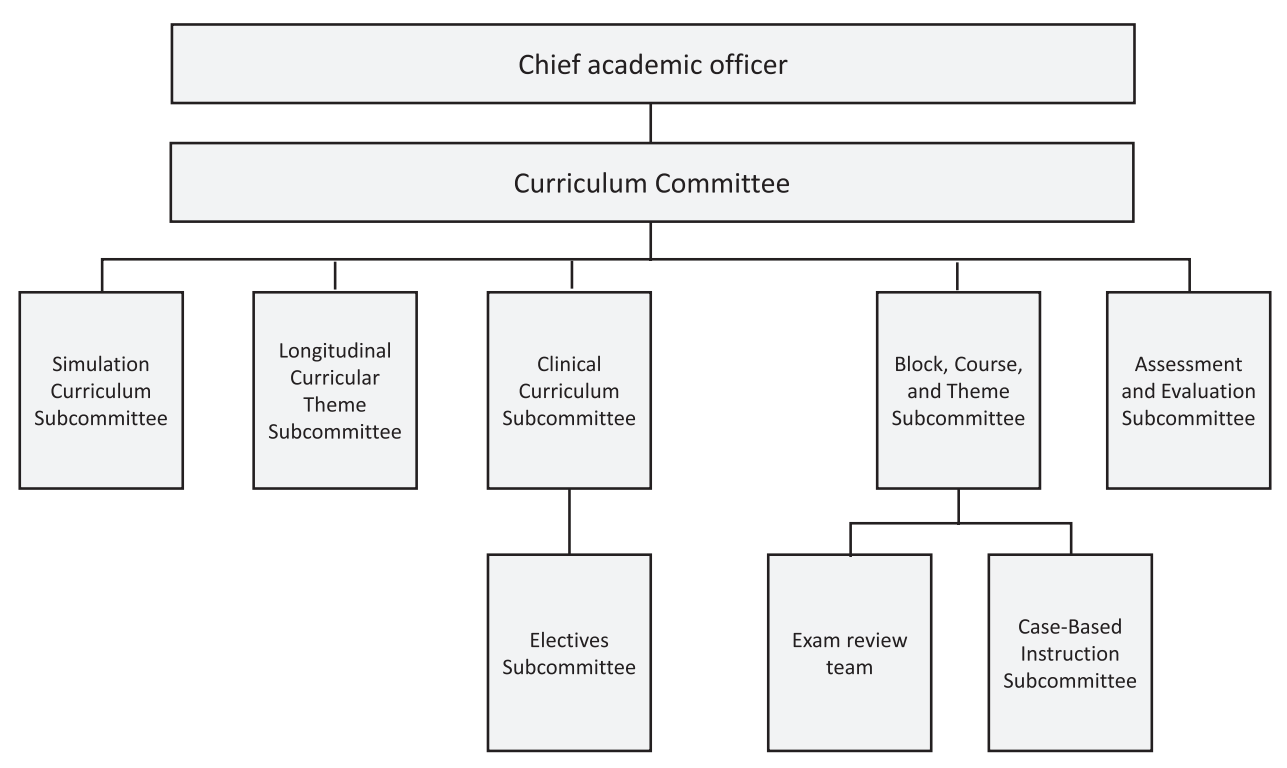

Figure 1 Committee structure. 


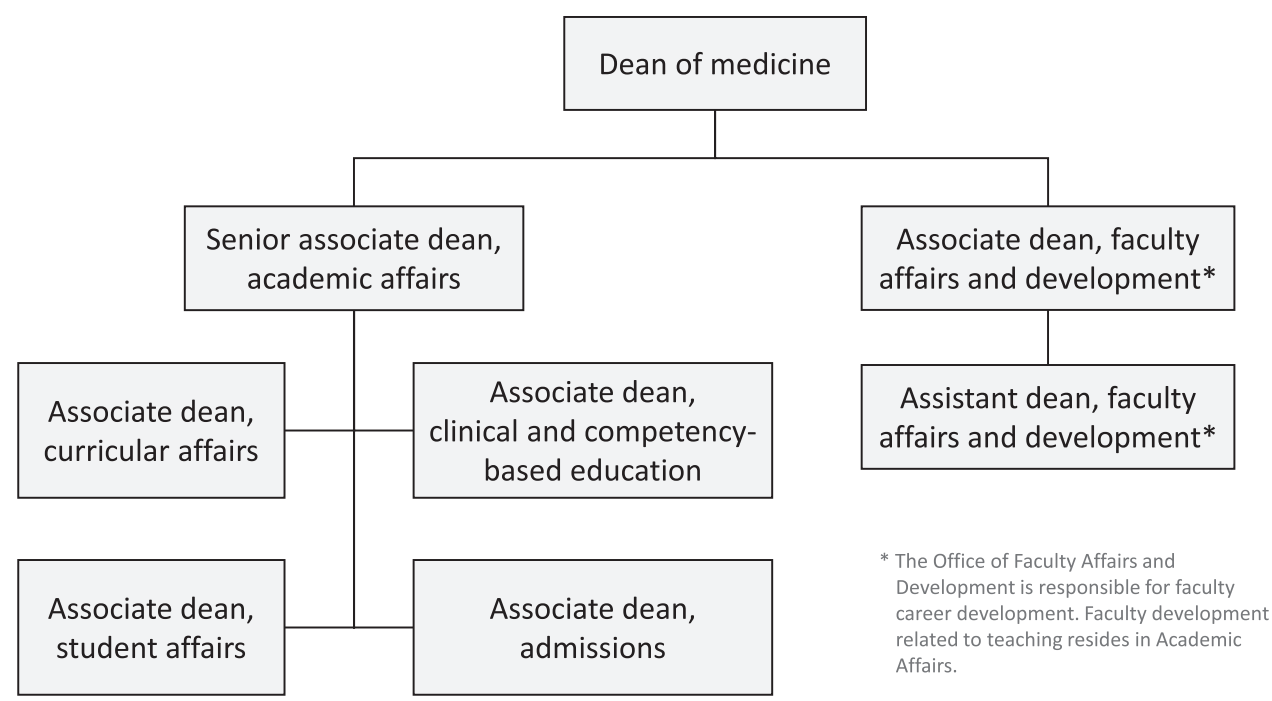

Figure 2 Medical education leadership.

\section{Initiatives in Progress}

Two major interrelated curriculum initiatives are included in the COM-P strategic plan: developing a 3-year curriculum and implementing competency-based, timevariable progression to residency. Pilot projects to develop enabling aspects for these initiatives are currently underway, including:

- A longitudinal ambulatory clinical experience beginning in year 1 and continuing throughout medical school
- Implementation of Entrustable Professional Activity-based assessment in the longitudinal clinic, community clinical experience (years 1-2), longitudinal integrated clerkship (year 3), and several clerkships (year 3)

- Identification of GME programs interested in partnering to create linkages

COM-P envisions creating a 3-year core curriculum with competency assessment that will either confirm student preparedness for GME training or identify a need for additional time for competency development before transition to GME. 


\section{University of Arkansas for Medical Sciences College of Medicine}

James Graham, MD, Sara Tariq, MD, Karina Clemmons, EdD, and Richard Wheeler, MD

\section{Medical Education Program Highlights}

- There is a strong sense of service to the state of Arkansas. As the only MD-granting medical education program in the state, the purpose of the University of Arkansas for Medical Sciences (UAMS) College of Medicine is to produce excellent physicians to serve our citizens. This is accomplished in many ways, but notably in strong primary care emphasis and high retention of graduates who practice in Arkansas.

- The school is moving its curriculum substantially toward active learning pedagogies and away from traditional lecture.

- The school has a series of optional enrichment tracks that students can choose from, including research, global health and service, child health, business/finance, and soon to be a new digital health track. In addition, dual degrees (MD-MPH and $\mathrm{MD}-\mathrm{PhD}$ ) are offered.

- The College of Medicine supports the academic and professional success of medical students through Academic House small-group learning communities, which help medical students develop supportive relationships with faculty and other students that span their course of study and beyond.

\section{Curriculum}

\section{Curriculum description}

The preclinical phase of the curriculum begins with a series of foundational science courses. This is followed by a set of integrated organ system-based modules. There is a longitudinal clinical skills course across the preclinical phase. The third year is composed of traditional clerkships, organized in blocks on the Little Rock campus and in a longitudinal integrated schedule at the Fayetteville/Northwest campus. The fourth year consists of an acting internship, a residency preparation course, the geriatrics clerkship, and a broad choice of electives.

\section{Curriculum changes since 2010}

A major curriculum revision was done in 2012-2013 involving restructuring courses into integrated organ system-based modules and substantially replacing traditional lecture with active pedagogy. In the junior year, clerkships were reorganized to accommodate junior-year selectives. The senior year was expanded by 1 month to better accommodate senior electives and the residency application/Match process.

Acad Med. 2020;95:S30-S32.

doi: 10.1097/ACM.0000000000003303

Copyright (C) 2020 by the Association of American Medical Colleges

Year school was established: 1879.

School URL: https://medicine.uams.edu.
In the 2020-2021 academic year, courses and clerkships will convert at least $30 \%$ of existing lectures to sessions using active learning, primarily peer instruction.

\section{Medical education program objectives}

The medical education program objectives are modified from the ACGME domains of competence.

See Table 1-Medical Education Program Objectives and Assessment Methods.

As the school has moved toward more active learning pedagogy, the assessment of performance has changed as well. Teambased learning is used extensively and incorporates both quiz-based knowledge assessment and peer assessment of team functioning. Peer instruction also uses more low-stakes formative assessment.

\section{Parallel curriculum or tracks}

The school does not currently have a parallel track as defined by the LCME. The school does not currently plan to offer a 3-year $\mathrm{MD}$ degree program in the future.

\section{Pedagogy}

In the preclinical phase of the curriculum, lecture currently comprises about $50 \%$ of contact hours, with the remainder of contact hours coming from team-based learning, laboratory, problem-based learning, and peer instruction sessions. There are also a variety of clinical experiences and standardized patient experiences throughout the first 2 years. In the clinical phase, most of the educational time is spent in ambulatory and inpatient clinical experiences, with an accompanying didactic portion consisting mainly of active pedagogies.

Since 2010, the school has substantially reduced the use of lecture and increased the use of a variety of active/engaged learning pedagogies, including team-based learning, problem-based learning, and peer instruction. In the next academic year, the school will reduce lectures by an additional $30 \%$.

\section{Clinical experiences}

At the Little Rock campus, students rotate in the UAMS hospital, the Arkansas Children's Hospital, the Central Arkansas Veteran's Administration hospitals, and Baptist Health hospitals and clinics. At the Fayetteville campus, students rotate in a variety of private practice clinics, at Washington Regional Medical Center, at Mercy Hospital, at the Fayetteville Veterans Administration Hospital, and at Northwest Hospital.

In the preclinical phase, all students have longitudinal clinical skills courses. In the clerkship year, all students participate in an additional Practice of Medicine 3 longitudinal course. 


\begin{tabular}{|c|c|}
\hline \multicolumn{2}{|c|}{$\begin{array}{l}\text { Medical Education Program Objectives and Assessment } \\
\text { Methods }\end{array}$} \\
\hline $\begin{array}{l}\text { Medical education } \\
\text { program objective }\end{array}$ & Assessment method \\
\hline Medical knowledge & $\begin{array}{l}\text { Multiple-choice examination (MCE), student } \\
\text { performance evaluation, narrative evaluation, } \\
\text { OSCE }\end{array}$ \\
\hline Clinical care & $\begin{array}{l}\text { Student performance evaluation (SPE), narrative } \\
\text { evaluation, OSCE, MCE }\end{array}$ \\
\hline Professionalism & SPE, OSCE, noncognitive academic assessment \\
\hline $\begin{array}{l}\text { Interpersonal and } \\
\text { communication skills }\end{array}$ & $\begin{array}{l}\text { SPE, narrative evaluation, team-based learning } \\
\text { peer assessment, OSCE, peer instruction }\end{array}$ \\
\hline $\begin{array}{l}\text { Lifelong learning and } \\
\text { medical informatics }\end{array}$ & $\begin{array}{l}\text { Problem-based learning assessment, narrative } \\
\text { assessment, SPE }\end{array}$ \\
\hline $\begin{array}{l}\text { Population health and } \\
\text { preventive medicine }\end{array}$ & MCE, SPE, narrative assessment, OSCE \\
\hline $\begin{array}{l}\text { Practice- and } \\
\text { systems-based care }\end{array}$ & MCE, SPE, narrative assessment, IPE assessment \\
\hline
\end{tabular}

In the freshman year, students participate in a required Practice of Medicine 1 clinical skills course. In that course, all students have both standardized and real patient experiences within the first few weeks of medical school.

All students in the first year have clinical experiences in a student-run free clinic near the main campus. In the junior year, Little Rock students rotate on the family medicine clerkship and most spend that time at a variety of regional program sites across the state that are community based. On the Fayetteville/ Northwest campus, all students spend at least some of their required clerkships in community-based private practice settings with adjunct faculty.

Recent challenges include:

- Securing enough clinical sites/faculty/preceptors to accommodate the number of learners. With the expansion of medical schools (new DO-granting schools nearby) and with expansion of other program using clinical teaching sites (physician-assistant and APN programs), having enough sites with adequate patient volume and faculty teaching/ supervision has been challenging. We have secured a number of new teaching sites over the past decade, which maintains appropriate patient and faculty-to-student ratios, but this is a challenge.

\section{Curricular Governance}

The school's curriculum is governed and managed by the College of Medicine Curriculum Committee. The committee is elected from across the departments of the college. The main committee has 21 voting members. It has 4 standing subcommittees: basic science education, clinical education, quality improvement/evaluation, and academic standards.
The departments in the College of Medicine all have important parts to play in the medical education program, but the overall governance of the curriculum is done in a centralized manner by the curriculum committee.

\section{Education Staff}

The executive associate dean for academic affairs oversees the MD degree program as a whole; additional associate and assistant deans serve student affairs and medical education. The college curriculum office has a number of staff who provide direct support to the curriculum and its delivery, including administrative support to course directors, support for educational material development, IT and technical support, and so forth. In addition, the university provides the Student Success Center for academic coaching and tutorial services for medical students. The university also provides the Office of Educational Development to assist faculty in their development in educational work.

The dean of the College of Medicine is the chief academic officer. The executive associate dean for academic affairs oversees the MD degree program in whole. The associate dean for student affairs oversees all student support functions. The associate dean for faculty affairs oversees the faculty development programs of the college. The assistant dean for medical education provides education, assessment, and evaluation support as well as expertise to the program.

The College of Medicine Curriculum Office, led by the executive associate dean for academic affairs, is responsible for the MD degree program, including student affairs. GME programs are led by the associate dean for GME/designated institutional official, who reports to the dean of the college, but has a close working relationship with the undergraduate medical education program. The CPD functions are done at a university-level office.

\section{Faculty Development and Support in Education}

The Office of Faculty Affairs conducts a regular program of faculty development activities, which include, but are not limited to, activities to develop faculty educational skills. In addition, the university-level Office of Educational Development and its Educator's Academy provide additional faculty development offerings focused on education.

Education is included in promotion and tenure decisions; the extent to which it plays a role depends on the specific track in which the faculty member resides. A variety of supporting documentations are considered, including teaching evaluations either by students or peers, educational scholarship, development of innovative teaching materials, and so forth.

The Educator's Academy is a university organization for all health professions schools at our academic health center, including medicine. It serves as a venue for sharing educational expertise across the campus, supporting educational improvement, and providing faculty development in teaching. 


\section{Regional Medical Campuses}

The school established a new regional campus in Fayetteville/ Northwest in 2010. The campus continues to serve third- and fourth-year students. The total student enrollment is 40 students.

All required clerkships have a primary clerkship director (on the Little Rock campus) and a site director at the Fayetteville campus. The educational learning objectives and student assessments are the same at both campuses. Faculty and house staff on both campuses receive orientation to the learning objectives of the program. The College Curriculum Committee regularly reviews a variety of outcome measures by campus to help ensure that educational outcomes are comparable at both campuses. 


\title{
California Northstate University College of Medicine
}

\author{
Tracy L. Yarbrough, MD, PhD, MAEd, and Catherine Yang, PhD
}

\section{Medical Education Program Highlights}

Located in Elk Grove, California, the California Northstate University College of Medicine (CNUCOM) opened its doors in July 2015. Founded as a private medical college by community philanthropists, CNUCOM is envisaged as an educational home for students in one of the nation's most culturally, economically, and ethnically diverse regions. In fulfilling its mission to advance the art and science of health care through innovative education and health care delivery systems, CNCUOM sets itself apart:

- Embedded in the community, with hundreds of community physicians participating in preclerkship training; within our distributed clerkship model, engaged with over 20 hospitals and medical centers and a host of clinics throughout California

- Committed to innovation, with a clinical presentation-led organ systems preclerkship course structure, rich opportunities for student self-directedness, scholarship, team-focused learning, and assessment, and CQI through outcomes-based evaluation

- Integrated within the health professions landscape, as 1 of 3 health professions colleges at $\mathrm{CNU}$; in cooperation with regional health professions programs, participating in an ongoing series of interprofessional collaborations for students and faculty

- Engaged with the population we serve, with students and faculty leading and serving others through local and global institutional partnerships, outreach, volunteering, mentorship, tutoring, and education

\section{Curriculum}

\section{Curriculum description}

The medical curriculum at CNUCOM is divided into 3 phases. Phase A consists of preclerkship, organ systems-based courses, following a clinical presentation-based format. All Phase A students also enroll in longitudinal coursework related to clinical skills, professionalism, public health, and research/scholarship. Phase A students are divided into academic "Colleges," within which many professionalism sessions and aspects of the courseembedded clinical case series take place. Phase B constitutes the core clinical clerkship period, and Phase $\mathrm{C}$ is made up of advanced and elective clinical and nonclinical experiences.

See Supplemental Digital Appendix 1-Curriculum Map-at http://links.lww.com/ACADMED/A973.

Acad Med. 2020;95:S33-S37.

doi: 10.1097/ACM.0000000000003491

Copyright (C) 2020 by the Association of American Medical Colleges

Supplemental digital content for this article is available at http://links.Iww.com/ ACADMED/A973.

Year school was established: 2015.

School URL: https://medicine.cnsu.edu.

\section{Curriculum changes since 2010}

Evolution of the CNUCOM curriculum: Planning began as early as 2010. In 2011, it was determined that CNUCOM would employ a vertically and horizontally integrated organ systems preclerkship curriculum followed by clinical clerkship rotations throughout northern and central California. Led initially by Dr. Joseph Silva, interim dean, and then by Dr. Robert Suskind, founding dean, faculty experts in basic biomedical and clinical sciences joined the team in 2012-2014 and, after preliminary LCME accreditation status in June 2015, the CNUCOM opened its doors in 2015.

Since its first year, a number of quality improvement-driven changes have been implemented across the 3 phases. Highlighted changes to content or other curricular components include:

- Enhancement of curricular content related to scientific inquiry and translational science, and student scholarship and research: The Phase A curriculum includes an experiential and didactic longitudinal thread related to the scientific method, scientific reasoning and research, translational science, and evidence-based medicine. The Self-Directed Student Scholarly Project (SDSSP) 2-course series engages all Phase A students in research and/or scholarship, culminating in presentation of and reflection on the critical thinking process and their personal scientific work at COM research day. Student engagement in faculty research projects and publication has steadily increased in parallel with SDSSP course improvements.

- Restructuring and refocusing of coursework related to professionalism, ethics, and advocacy/public health: Masters Colloquium courses, which longitudinally span Phase A, have been continually revamped. Originally focusing on professionalism and medical ethics, the courses now additionally address health care systems, alternative medicine, physician and student wellness and resilience, and physiciansas-advocates.

- Expansion of core (Phase B) and elective (Phase C) clinical clerkship sites: CNUCOM is a stand-alone medical school without an affiliated hospital or clinical site. This has allowed us to build synergistic relationships with clinical partners regionally and throughout California. Clerkship experiences provide exposure to small/rural clinics, single- and multiprovider clinics and practices, teaching (residency and fellowship program) sites, community and urban inpatient hospital sites, and prison-based practice.

- Development of nonclinical courses/experiences for students across all phases: We continue to enrich available opportunities for participation in nonclinical elective experiences such as courses on leadership, wellness, advanced research experiences, and educational teaching assistantships.

- Evolution of CNUCOM classes: CNUCOM opened its doors to students for the first time in fall 2015, with 60 matriculating students. Since then, enrollment has increased 
to approximately 90 students in each subsequent class, concurrently expanding the educational spaces and necessary infrastructure for team-based and technology-enhanced learning and assessment. As class size has grown, so have the use of active and interactive teaching strategies in the classroom and the breadth of interprofessional educational activities.

\section{Assessment}

Framework for program learning objectives: The CNUCOM program learning outcomes (PLOs) reflect the domains of competence outlined by the ACGME but are largely built on the framework of the AAMC Physician Competency Reference Set (PCRS) and mapped thereto. In general, CNUCOM PLOs map to specific aspects of the following PCRS domains:

- PLO1 (patient care) to PCRS domain 1 (patient care)

- PLO2 (medical and scientific knowledge) to PCRS domains 1, 2 (knowledge for practice), 3 (practice-based learning and improvement), 4 (interpersonal and communication skills), and 6 (systems-based practice)

- PLO3 (communication and interpersonal skills) to PCRS domains 3, 4, and 7 (interprofessional collaboration)

- PLO4 (professionalism) to PCRS domains 1, 5 (professionalism), 6, 7, and 8 (personal and professional development)

- PLO5 (health care systems) to PCRS domains 3, 6, and 7

- PLO6 (reflective practice and personal development) to PCRS domains 1,4 , and 8

Student assessment: Assessment at CNUCOM includes multiplechoice exams, performance-based assessments, and narrative assessments across all phases. Most recently, hybrid exams have been implemented in Phase A.

See Supplemental Digital Appendix 2-Program Objectives and Assessment Methods—at http://links.lww.com/ACADMED/A973.

\section{Parallel curriculum or tracks}

CNUCOM does not currently have parallel or alternative tracks. Through collaboration with our sister undergraduate college, the College of Health Sciences, CNUCOM participates in a directentry pathway (BS-MD program) allowing highly motivated and qualified undergraduate students to matriculate into our 4-year MD program following an accelerated undergraduate course of study.

\section{Pedagogy}

A variety of teaching methods and pedagogies are employed to achieve PLOs. Students are engaged in learning through both large- and small-group activities, independent investigations, and other methods, which are tailored to desired cognitive process domains and content.

See Table 1-Pedagogical Approaches.

Since its initial offerings in AY 2015-2016, CNUCOM has employed continuous improvement of pedagogy and teaching methods. Pedagogy changes in the preclerkship phase include:

\section{Table 1 \\ Pedagogical Approaches}

\begin{tabular}{|c|c|}
\hline Pedagogy & $\begin{array}{l}\text { Examples: phase/course/description } \\
\text { (as needed) }\end{array}$ \\
\hline $\begin{array}{l}\text { Clinical experience: } \\
\text { ambulatory }\end{array}$ & $\begin{array}{l}\text { Phases B \& C/core and elective clinical } \\
\text { clerkships }\end{array}$ \\
\hline $\begin{array}{l}\text { Clinical experience: } \\
\text { inpatient }\end{array}$ & $\begin{array}{l}\text { Phases B \& C/core and elective clinical } \\
\text { clerkships }\end{array}$ \\
\hline $\begin{array}{l}\text { Discussion: } \\
\text { large group (> 12) }\end{array}$ & $\begin{array}{l}\text { Phase A/master's colloquium and organ } \\
\text { systems-based courses/discussions within } \\
\text { Colleges, for example, related to medical } \\
\text { ethics and professionalism }\end{array}$ \\
\hline $\begin{array}{l}\text { Discussion: } \\
\text { small group }(\leq 12)\end{array}$ & $\begin{array}{l}\text { Phases A \& B/master's colloquium and } \\
\text { organ systems-based courses; core } \\
\text { clerkship weekly didactic days/discussions } \\
\text { related to scientific method, biostatistics } \\
\text { and }\end{array}$ \\
\hline Laboratory & $\begin{array}{l}\text { Phase AVorgan systems-based courses/ } \\
\text { gross anatomy cadaver lab }\end{array}$ \\
\hline Lecture & $\begin{array}{l}\text { Phases A \& B/master's colloquium and } \\
\text { organ systems-based courses; weekly core } \\
\text { clerkship didactic days }\end{array}$ \\
\hline Peer teaching & $\begin{array}{l}\text { Phases A, B, \& C/organ systems-based } \\
\text { courses; weekly core clerkship didactic days } \\
\text { and subinternship didactic days, elective } \\
\text { teaching assistantships }\end{array}$ \\
\hline Problem-based learning & $\begin{array}{l}\text { Phase A / organ systems-based courses/ } \\
\text { CCBL series }\end{array}$ \\
\hline Role play/dramatization & $\begin{array}{l}\text { Phase A/masters colloquium/students role- } \\
\text { playing physician or patient in challenging } \\
\text { encounters to develop communication and } \\
\text { interpersonal skills }\end{array}$ \\
\hline Self-directed learning & $\begin{array}{l}\text { Phases A \& B/organ systems-based courses } \\
\text { and core clinical clerkships/clinical case } \\
\text { series, student self-directedness (including } \\
\text { oral presentations) regarding learning } \\
\text { needs during clinical clerkships }\end{array}$ \\
\hline Simulation & $\begin{array}{l}\text { Phase Armedical skills courses/ } \\
\text { standardized-patient encounters to develop } \\
\text { communication, history and physical-exam } \\
\text { taking skills }\end{array}$ \\
\hline Team-based learning & $\begin{array}{l}\text { Phase Alorgan systems-based courses/ } \\
\text { various sessions using TBL across courses } \\
\text { including, for example, sessions on exercise } \\
\text { physiology, electrocardiograms (ECG), } \\
\text { neurophysiology }\end{array}$ \\
\hline Workshop & $\begin{array}{l}\text { Phase AVorgan systems-based courses/ } \\
\text { workshops, including, for example, } \\
\text { diagnostic evaluation of renal insufficiency } \\
\text { with calculations of GFR (glomerular } \\
\text { filtration rate) and other clinical parameters }\end{array}$ \\
\hline
\end{tabular}

- Enrichment of virtual clinical case-based self-directed learning: In years 1 and 2, students participate in self-directed learning through the team-based clinical case series embedded within the organ systems courses. In this method, student teams independently work through a virtual clinical case over the course of each week-developing differential diagnoses, prioritizing clinical workup, identifying their learning 
needs, and investigating the literature, ultimately leading to discussions at the end of each week followed by a faculty-led debrief and discussion. Changes have focused on implementing level-specific goals and emphasizing student skills for lifelong learning, including a transition to just-in-time formative feedback on student case discussions and increased emphasis on feedback regarding student use of learning resources. In AY 2018-2019, CNUCOM implemented problem-based learning into Phase A courses through its clinical case-based learning series.

- Incorporation of peer teaching into high-stakes assessment through hybrid exams: Web-based multiple-choice exams from the NBME have been used routinely for summative assessment since 2015. Select courses in Phase A now also employ hybrid (individual + collaborative) testing for mid- or end-of-course high-stakes exams. In this format, students first take an exam individually and then retake the exam in preformed student teams; the team administration allows clarifying confusing concepts and timely reinforcement of conclusions reached by consensus. These hybrid exams allow students to discuss testing approaches that may differ from their own.

\section{Clinical experiences}

Sites for clerkship experiences: Students rotate through core clinical clerkships in cohorts, and advanced clinical clerkship experiences are tailored to each students' career interests, with a required core specialty subinternship in year 4 . Because CNUCOM does not have its own affiliated teaching hospital, we use a distributed clerkship model for clinical experiences. Students rotate at hospitals, clinics, and medical centers throughout California including large public, private, and $\mathrm{HMO}-$ affiliated hospitals as well as multi- and single-specialty clinics and single- and multiprovider practices. No longitudinal clinical experiences (i.e., longitudinal clerkships) are currently offered.

Student exposure to patients: Students at CNUCOM first encounter patients through the longitudinal Medical Skills courses spanning the first 2 years (Phase A), which include interactions with and evaluation by standardized patients. Students' first experiences with real patients occurs as they embark on their clinical clerkships.

Community-based rotations: Required community-based rotations that include outpatient clinics are pediatrics, family medicine, obstetrics-gynecology, psychiatry, neurology, and surgery. While CNUCOM does not have its own teaching hospital, we offer over 160 community-based "home" electives from which students can choose, including experiences in allergy, dermatology, cardiology, endocrinology, family medicine, family medicine/ psychiatry, integrative medicine, outpatient internal medicine, gastroenterology, hematology, sports medicine, and others.

Clinical clerkship challenges: The challenges in designing and implementing clinical experiences for our medical students have included identifying community physicians (preceptors) in each specialty who are committed and enthusiastic about teaching medical students, comprehensive and flexible preceptor training in teaching and assessment, and developing sustainable clinical experiences and teaching in each specialty rotation at various locations.

\section{Curricular Governance}

The CNUCOM Curriculum Committee (CC) is central to curricular governance. Committees reporting to the $\mathrm{CC}$ are the Phase A, Phase B, and Phase C Subcommittees, as well as the Assessment Committee. The CC is advisory to the dean

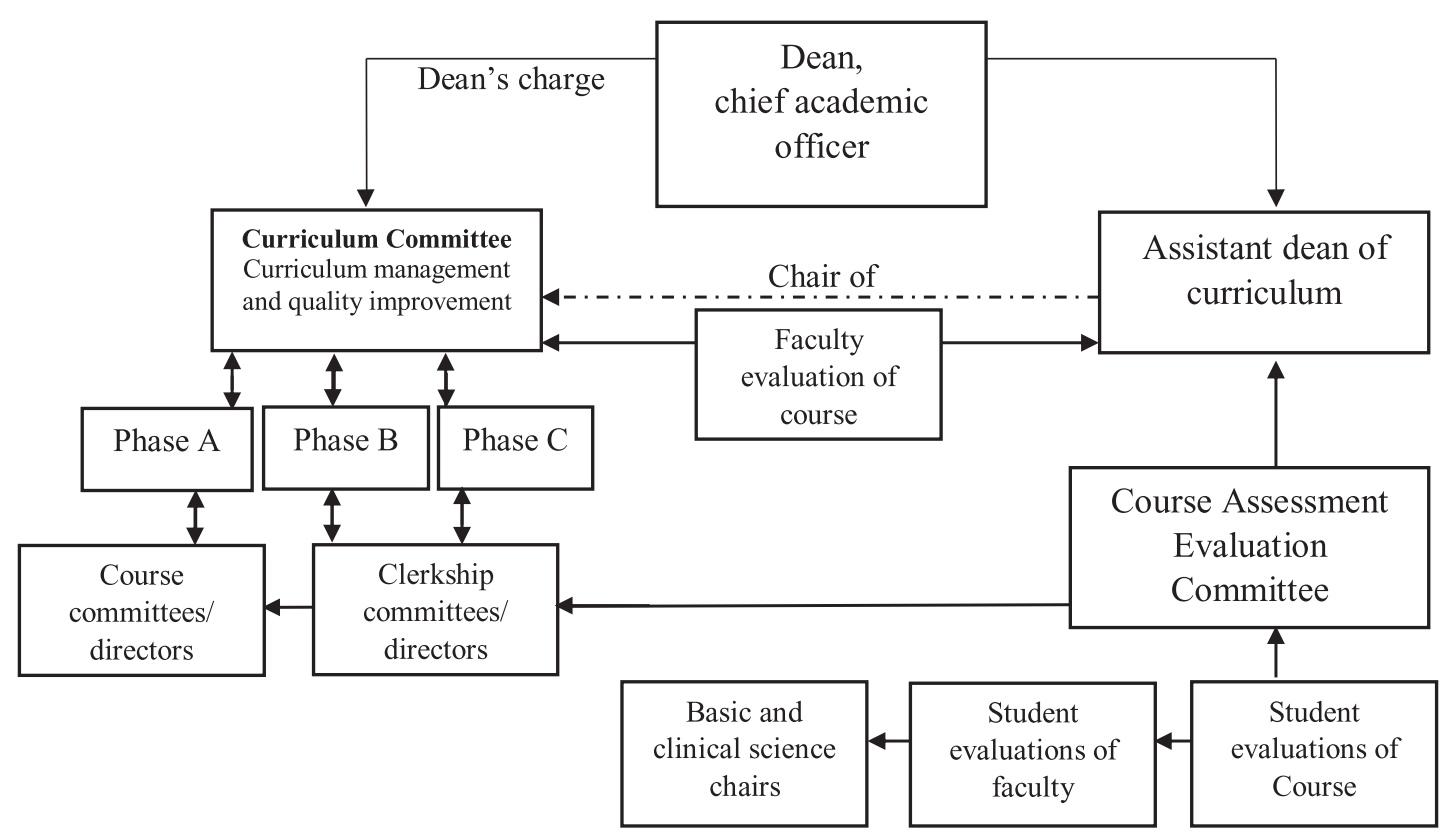

Figure 1 Curriculum management. 


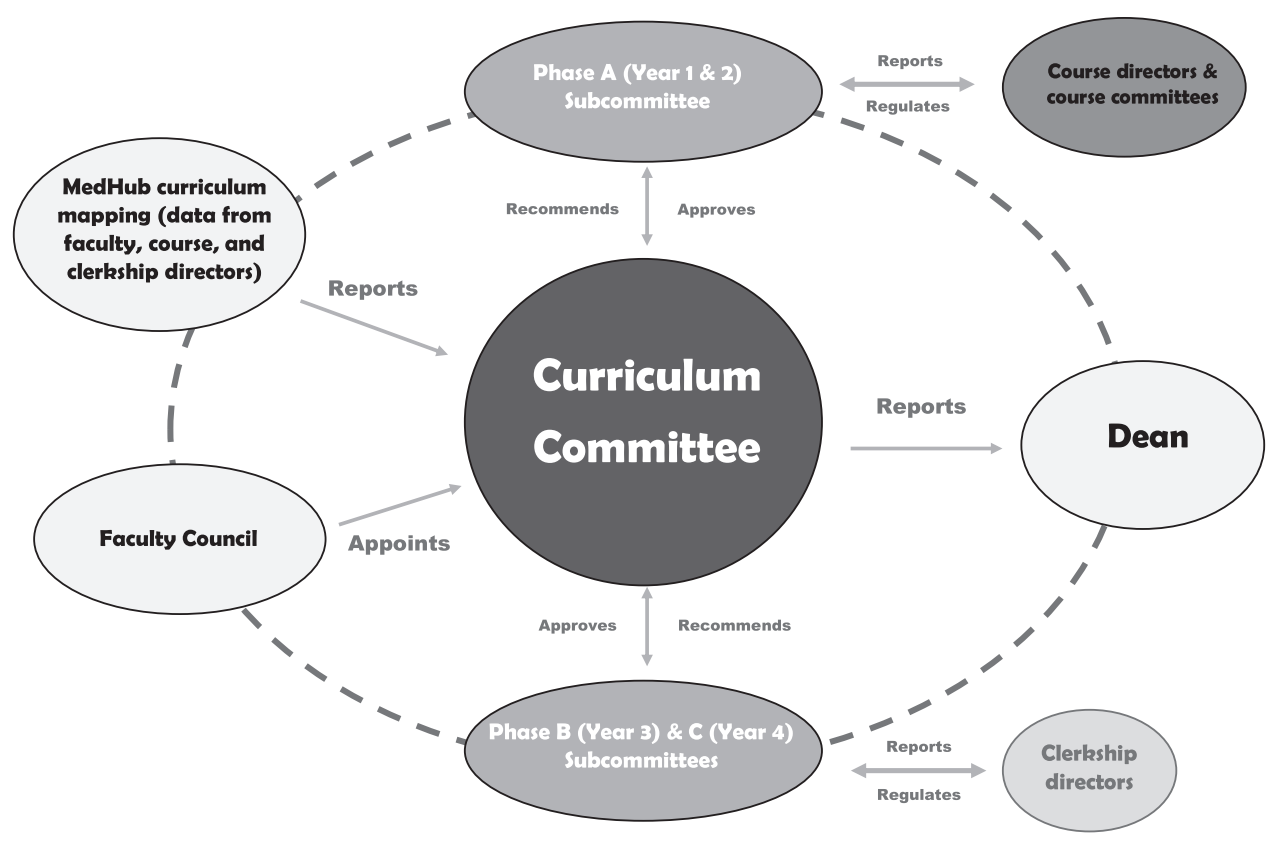

Figure 2 Curriculum review process.

and, in addition to review by the dean, significant changes in the curriculum are reviewed by CNUCOM's Faculty Council. Satellite concerns, such as those related to teaching budget, academic support, etc., are under the purview of the department chairs and dean of the COM.

See Figure 1-Curriculum management.

See Figure 2-Curriculum review process.

\section{Education Staff}

Office of Medical Education: The Office of Medical Education (OME) advances CNUCOM's educational mission by providing core functions through management and oversight of the required medical school curriculum, including courses and clerkships. In addition, OME manages academic support, assessment and evaluation, program accreditation to ensure continuous quality improvement of the curriculum, and

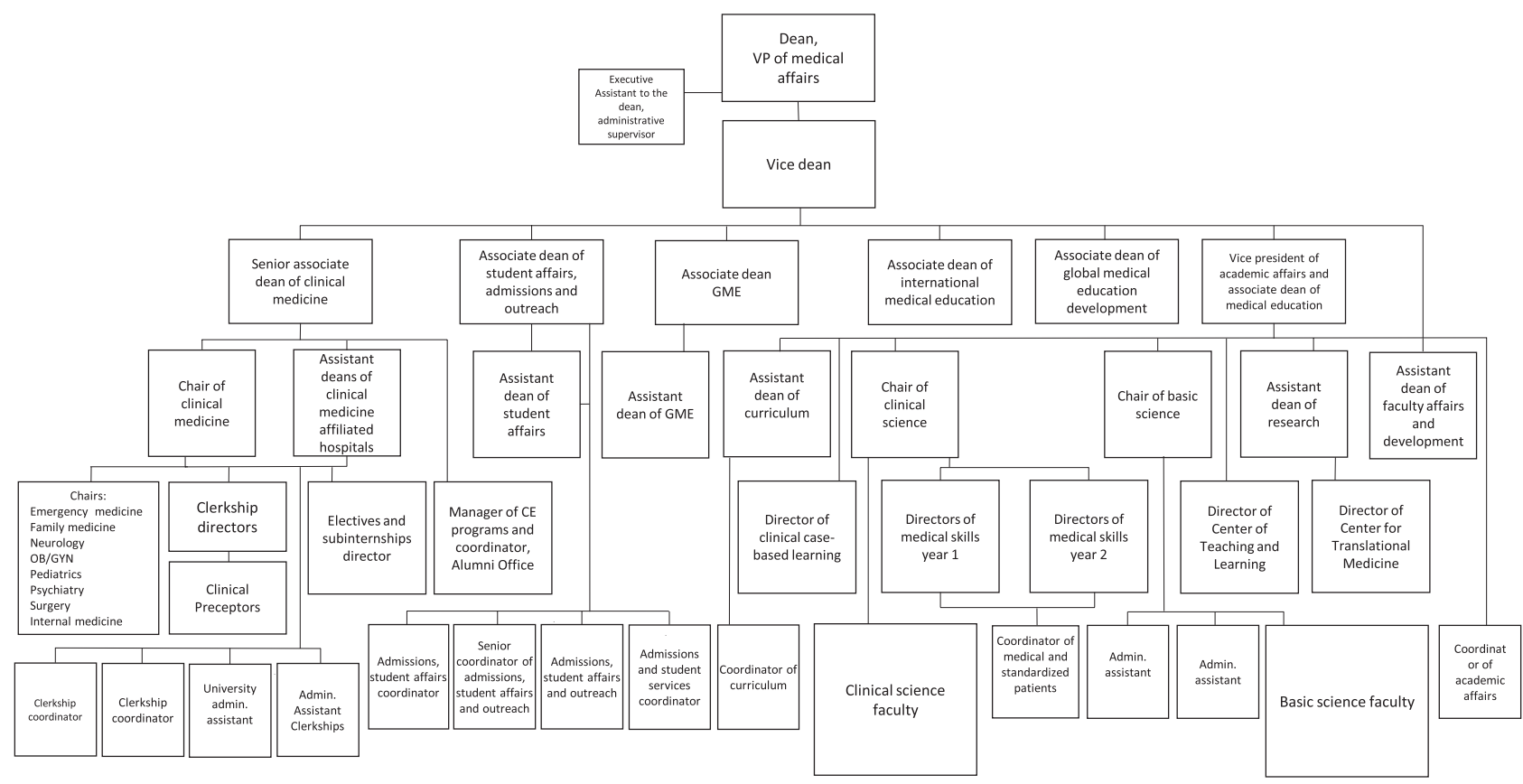

Figure 3 Medical education leadership. 
educational scholarship. Two CNUCOM centers for excellence and development fall within the purview of OME, the Centers for Teaching and Learning (CTL) and Translational Medicine, as well as many other COM committees.

Organizational support and structure: The COM has a full complement of administrative support staff involved in the delivery of our educational program. At the level of Student Affairs, staff support student counseling and academic advising, student wellness, residency applications, and academic/ tutoring support. Faculty in the COM have appointments in 1 of 3 departments-Basic Science, Clinical Science, or Clinical Medicine. The CNUCOM does not have an academic department of medical education nor GME programs.

See Figure 3-Medical education leadership.

\section{Faculty Development and Support in Education}

Faculty professional development: Through collaborations between CNUCOM Faculty Affairs and the university's Office of Continuing Education, regular faculty development seminars are offered on topics such as assessment and pedagogy/andragogy. In addition, CNUCOM's CTL has implemented focused medical education faculty workshops to provide hands-on practice related to assessment implementation. The CNU Institute of
Teaching and Learning Excellence (ITLE) provides a number of university-wide forums for faculty educational enhancement. These include immersive and experiential faculty learning communities and educational topic-specific seminar series.

Faculty promotion and tenure: CNUCOM prioritizes teaching. At each rank, faculty excellence in teaching as reflected on teaching evaluations is factored into promotion decisions, as are faculty participation in educational development opportunities, engagement with curricular improvement, and scholarly productivity (including faculty education-related publications and mentoring student scholarly projects). CNUCOM does not currently have an academy for educators, though the ITLE is developing an academy of faculty educators across all colleges (Medicine, Pharmacy, Clinical Psychology, Master of Pharmaceutical Sciences, and Health Science).

\section{Initiatives in Progress}

As CNUCOM looks toward the future, its commitment to our community and to innovation continues, including planned construction of a teaching hospital, increasing educational collaboration and interweaving with our sister health professions colleges, and expansion of our faculty research infrastructure and portfolio. 


\section{Kaiser Permanente Bernard J. Tyson School of Medicine}

Abbas A. Hyderi, MD, MPH, and Mark A. Schuster, MD, PhD

\section{Medical Education Program Highlights}

The Kaiser Permanente Bernard J. Tyson School of Medicine aspires to train physicians who are outstanding clinicians, committed patient advocates, and innovative leaders. The school leverages the values and capabilities of Kaiser Permanente (KP), one of the nation's largest and highest performing integrated health care systems, which has a focus on patient-centered care, team-based care, population health, technological innovation, and continuous quality improvement, to prepare students for future-facing clinical practice, scholarship, and health system leadership. The school takes a competency-based approach with a curriculum that integrates biomedical science, clinical science, and health systems science (HSS) across all 4 years of training in a spiral fashion, allowing content to be revisited in increasing complexity. The didactic curriculum across the first 2 years emphasizes small-group, collaborative, interactive learning in a case-based format. All core clerkships (other than emergency medicine) use the longitudinal integrated clerkship (LIC) model, starting with family medicine/internal medicine early in year 1 and adding in obstetrics-gynecology, pediatrics, psychiatry, and surgery in year 2. Students stay at their respective KP clinical site ( 1 of 6 sites) for the 2 years, maintaining 1-on-1 relationships with their preceptor and interprofessional team for each specialty, learning clinical skills by following panels of patients over time, and applying what they learn in HSS in the clinical setting (e.g., quality improvement, population health). The school also encourages exploration of population and community health through a service-learning curriculum in which students participate in a monthly longitudinal experience during the first 2 years at 1 of 6 federally qualified health centers. Finally, the school offers a 4 -year course that provides coaching to support personal and professional development, including professional identity formation and resilience skills training.

\section{Curriculum}

\section{Curriculum description}

- The school's curriculum is named INSPIRE (Integration, Nurturance, Scholarship, Population Health, Innovation, Resilience, Equity).

- Early Immersive Experience (EIE) course: This course covers orientation activities; an introduction to the school's mission, vision, and values; and pedagogical approaches to

Acad Med. 2020;95:S38-S41.

doi: 10.1097/ACM.0000000000003471

Copyright (C) 2020 by the Association of American Medical Colleges

Supplemental digital content for this article is available at http://links.Iww.com/ ACADMED/A962.

Year school was established: 2019.

School URL: https://medschool.kp.org/homepage. learning, which include small-group, case-based learning, and team-based learning (TBL). It also allows students to begin developing core clinical skills that will be used in the LIC, which starts in the third week of EIE, and to start building connections with classmates and the larger school community.

- Integrated Sciences courses: These courses are provided across all 4 years of medical school, integrating biomedical science, clinical science, and HSS content in a case-based approach with content revisited in increasing complexity in a spiral fashion.

- LICs: Clerkships begin in year 1 with family medicine/internal medicine using an LIC model with students based at 1 of $6 \mathrm{KP}$ clinical sites. Four additional core clerkships in year 2 also use the LIC model (plus an additional core clerkship in emergency medicine). Students stay at their respective sites, allowing them to learn clinical skills by following panels of patients over time and seeing patients through all phases of diagnosis and treatment, while maintaining 1-on-1 relationships with their preceptors for each field. The LIC experience provides an opportunity to apply what is covered in the classroom to the KP clinical setting, which is organized around interprofessional, team-based care.

- Inpatient immersion blocks: In year 2, there are four 1-week inpatient immersion blocks in internal medicine, obstetricsgynecology, pediatrics, and surgery.

- Service-learning course: Students participate in a monthly longitudinal service-learning experience during years 1 and 2, which takes place at 1 of 6 federally qualified health centers. Within this curriculum, students learn how to recognize social, economic, and environmental factors that influence health; advocate for disease prevention and public health promotion; embrace population care; and collaborate with community partners to help address their needs.

- Reflection, Education, Assessment, Coaching, and Health and well-being (REACH) course: The REACH course takes place every 3-4 months in 1-week blocks and periodically on Monday afternoons. Physician coaches meet with students at regular intervals both 1-on-1 and in small groups throughout their 4 years of medical school to review students' academic portfolios and help them identify strengths and gaps, set goals, and track their progress. The course emphasizes personal and professional development, and includes time for learning about health and well-being and exploring ways to maximize resilience skills.

- Year 3 and 4 experiences: Years 3 and 4 have 20 weeks of required clinical experiences: subinternship (4 weeks; family medicine or internal medicine), subinternship (4; student's preference), advanced clinical rotation (4), rotation in an under-resourced setting (4), and academic medical center rotation (4), as well as required HSS electives (8).

- Scholarly project: Students complete a longitudinal scholarly project through which they meaningfully explore an area of individual interest under faculty mentorship across years 
$1-3$, including 4 weeks of dedicated time in year 3. Advanced scholarly work that involves additional rigor and depth is also available.

- Residency immersive: This course covers both general skills for transitioning to internship and specialty-specific workshops.

- Other degrees: Opportunities have been arranged with nearby universities for other degrees, including Doctor of Philosophy, Master of Health Administration, Master of Healthcare Systems Engineering, and Master of Public Health.

See Supplemental Digital Appendix 1-Curriculum Schematicat http://links.lww.com/ACADMED/A962.

\section{Assessment}

- The school's educational program outcomes (EPOs) are derived from the AAMC's Physician Competency Reference Set
(PCRS) and Core Entrustable Professional Activities (EPAs). The EPOs are competencies, EPAs, or experiences/projects. There are 8 outcome domains, with 5 from the PCRS and 3 additional ones that reflect the school's mission, vision, and values (lifelong learning, interprofessional collaboration and teamwork, and population and community health).

- A Competency Committee evaluates all assessment data in a student's portfolio and makes recommendations to the Student Progress and Promotion Committee, which determines whether the student is qualified to advance to the next year and ultimately graduate. Each of the 8 domains are evaluated using a variety of assessment methods.

See Table 1-Educational Program Outcomes and Assessment Methods.

\section{Table 1}

Educational Program Outcomes and Assessment Methods

\section{Educational program outcomes Assessment methods}

1. Patient care

- Gathers essential information

- Formulates diagnosis and plan

- Demonstrates timely, accurate, and concise documentation

- Manages as part of a team

- Collaborates with patients

- Performs common procedures

- Initiates urgent/emergent care

- Performs safe transitions of care

\section{Lifelong learning}

- Recognizes and manages uncertainty

- Engages in self-directed learning for professional development

- Furthers personal health and well-being

\section{Clinical documentation}

Multisource assessment

Narrative assessment

Oral patient presentation

Peer assessment

Self-assessment

Simulation

Clinical documentation

Multisource assessment

Narrative assessment

Oral patient presentation

Peer assessment
Clinical performance (licensure exams)

Portfolio-based assessment

Workplace-based assessment (with supervisory and/or coactivity scales)

Written/computer-based assessment (institutional exams)

Written/computer-based assessment (licensure exams)

Portfolio-based assessment

Research or project assessment

Self-assessment

Simulation

Workplace-based assessment (with supervisory and/or coactivity scales)

\section{System-based practice}

- Demonstrates appropriate sensitivity to patient, health care, and societal resources

- Applies concepts of quality and performance improvement

- Initiates safety interventions aimed at reducing patient harm

- Advances innovation and systems-level change

Clinical documentation

Multisource assessment

Narrative assessment

Oral patient presentation

Peer assessment

Portfolio-based assessment

Research or project assessment

Self-assessment

Simulation

Workplace-based assessment (with supervisory and/or coactivity scales)

Written/computer-based assessment (institutional exams)

4. Population and community health Multisource assessment

- Advocates with community partners for implementation of Narrative assessment community-level health interventions

- Participates in projects to help reduce health-related inequities of a population
Portfolio-based assessment

Research or project assessment

Self-assessment

Written/computer-based assessment (institutional exams) 


\section{Table 1}

(Continued)

\section{Educational program outcomes}

5. Interprofessional collaboration and teamwork

- Uses knowledge of one's own role and the roles of other health professionals to assess and address the health care needs of patients and populations

- Communicates and interacts with colleagues and team members to create a climate of mutual respect, inclusion, and integrity

\section{Assessment methods}

Clinical documentation

Multisource assessment

Narrative assessment

Oral patient presentation

Peer assessment

Portfolio-based assessment

Self-assessment

Simulation

Workplace-based assessment (with supervisory and/or coactivity scales)

Written/computer-based assessment (institutional exams)

\section{Interpersonal and communication skills}

- Demonstrates person-centered communication that incorporates humility, recognition of uncertainty, honesty, and empathy

- Employs digital technologies to communicate and optimize clinical decision-making and treatment with patients, families, and health care teams

- Demonstrates effective teaching skills

\section{Professionalism}

- Demonstrates accountability, conscientiousness, truthfulness, and discernment

- Engages in behaviors that exemplify humility, value diversity, and foster an inclusive and equitable environment

- Recognizes, analyzes, and proposes solutions to ethical issues and challenges

- Demonstrates a commitment to ethical, legal, and professional standards

8. Medical knowledge

- Demonstrates deep knowledge of the biomedical, clinical, and health systems sciences and applies this knowledge to advance the health of patients and communities

- Engages in a mentored scholarly project to deepen curiosity and foster participation in a community of scholars
Clinical documentation

Clinical performance (licensure exams)

Multisource assessment

Narrative assessment

Oral patient presentation

Peer assessment

Portfolio-based assessment

Self-assessment

Simulation

Workplace-based assessment (with supervisory and/or coactivity scales)

Clinical documentation

Multisource assessment

Oral patient presentation

Portfolio-based assessment

Research or project assessment

Self-assessment

Simulation

Workplace-based assessment (with supervisory and/or coactivity scales)

Written/computer-based assessment (institutional exams)

Clinical documentation

Clinical performance (licensure exams)

Narrative assessment

Oral patient presentation

Peer assessment

Portfolio-based assessment

Research or project assessment

Self-assessment

Simulation

Workplace-based assessment (with supervisory and/or coactivity scales)

Written/computer-based assessment (institutional exams)

Written/computer-based assessment (licensure exams)
Narrative assessment

Peer assessment

Multisource assessment

\section{Pedagogy}

The pedagogical approaches used to achieve the EPOs include the following by course:

- Integrated Sciences courses: Sessions are classroombased, using a hybrid of interactive and flipped classroom approaches. Each week is structured around a primary patient presentation (or case) that unfolds across the week, along with supplemental clinical vignettes. These clinical scenarios serve as context for integrated curriculum delivery with sessions throughout the week covering biomedical science, clinical science, and HSS.
- Three days a week, sessions organized around biomedical science and its application to clinical science and HSS include 8 students in groups, each cofacilitated by a biomedical scientist and a physician-educator. Cofacilitators use various small-group, active learning approaches to engage students, including providing self-directed learning opportunities. Anatomy instruction uses a multimodal approach, incorporating plastinates (human specimens preserved through a process that replaces water and fat with polymers), virtual dissection, augmented/virtual reality, diagnostic imaging technology, and point-of-care ultrasound. 
- Once a week, doctoring sessions emphasize clinical and ethical reasoning and development of clinical skills. Sessions use small-group, interactive teaching and learning methods, such as simulation with standardized patients and procedural-skills training incorporating augmented/virtual reality technology.

- Once a week, HSS sessions are primarily taught using techniques such as TBL. Small teams meet in a large shared space that allows cross-team interaction. Students engage with topics related to community and population health, ethics, health care and social systems, health information technology, inquiry, and quality and safety.

- The service-learning course has monthly opportunities leveraging experiential learning and reflection.

\section{Curricular Governance}

- The Curriculum and Education Policy (CEP) committee oversees the medical education program as a whole and has responsibility for the overall design, management, integration, evaluation, and enhancement of a coherent and coordinated medical curriculum to ensure that it meets the mission, vision, and values of the school and is in compliance with licensing and accreditation standards. The CEP has 3 subcommittees: the Integrated Sciences Subcommittee, the Clinical Experience Subcommittee, and the Longitudinal Coordination Subcommittee. It is responsible for the review of educational policies, standards, and subcommittee reports and recommendations, and votes on what to recommend to the dean regarding the curriculum.

- The Program Evaluation, Assessment, and Improvement (PEAI) Committee is a separate standing committee for educational and noneducational program evaluation and institutional continuous quality improvement.

- Working groups for each major course develop instructional materials and assessment tools. The courses undergo a formal course review, approval, and modification process through PEAI, CEP, and CEP's relevant subcommittees.

- There are 3 departments-biomedical science, clinical science, and health systems science-and the department chairs are responsible for communicating with department faculty and staff regarding expectations, development, and changes that affect the delivery of education. Each chair collaborates with department faculty and staff, the other department chairs, and the Office of Medical Education to develop and deliver the education program.

\section{Education Staff}

- The dean oversees the senior associate deans, who are described below.

- The senior associate dean for medical education (SADME) has primary responsibility for instruction, assessment, program evaluation, accreditation, and support of faculty educator development.

- The SADME leads the Office of Medical Education, which is focused on UME and has its own budget for staff resources to support, manage, and coordinate teaching, assessment, and program evaluation efforts.

- The SADME oversees the associate dean for assessment and evaluation, assistant dean for medical education, and director of faculty educator development.

- The associate dean for assessment and evaluation oversees the senior director for assessment and evaluation and the administrative and faculty directors of simulation.

- Instructional designers, curriculum coordinators, and other staff develop and maintain the tools for curriculum delivery, monitoring, and management, and help implement the curriculum.

- The senior associate dean for academic and community affairs has primary responsibility for community engagement and faculty services, including faculty appointments and career support. The office includes the associate dean for equity, inclusion, and diversity, who oversees the school's efforts to create an equitable, inclusive, and diverse learning environment, and the director of global health.

- The senior associate dean for student affairs has primary responsibility for leading staff and programs that support students and enhance their educational experience, including 4 learning communities each with 48 students ( 12 students per class) in which a variety of student leadership opportunities are embedded. The Office of Student Affairs also monitors the learning environment, oversees the academic support and coaching programs, and includes learning specialists and psychologists.

- The senior associate dean for research and scholarship has primary responsibility for creating an engaging and supportive scholarly environment for students and faculty, including ensuring appropriate mentorship, consultation, data access, and tools to enable successful completion of the scholarly project.

\section{Faculty Development and Support in Education}

- The Office of Academic and Community Affairs provides orientation and resources for faculty academic and career development.

- The Office of Medical Education offers resources for faculty educator development, including intensive training in teaching methods, curriculum development, small-group facilitation, and assessment.

- Faculty rank is determined by a faculty member's performance in educational activities, service to the institution, scholarly achievements, and professional reputation. Participation in educational activities may include direct teaching of students, curriculum development, mentorship, research training, faculty development, administrative leadership of educational programs, evaluations of teaching through student assessment or peer observation, contributions to pipeline programs, or other activities.

Acknowledgments: The authors thank Sophia Harvey, Caryssa Lim, and Caroline Miao for help with preparing this manuscript and also acknowledge the faculty and staff of the Kaiser Permanente Bernard J. Tyson School of Medicine. 


\section{Keck School of Medicine of the University of Southern California}

Ron Ben-Ari, MD, Kevin Nash, PhD, and Donna Elliott, MD, EdD

\section{Medical Education Program Highlights}

The Keck School of Medicine (KSOM) medical education curriculum is integrated and system based with substantial emphasis on the early and progressive development of clinical skills; the clinical relevance of basic science; direct experience with patients or simulations representing a set of common and/or important diseases and conditions; gaining skills in hypothesis-driven research; well-being; professional identity formation; and the roles of the humanities, ethics, art, and law in medicine.

The following courses and programs are highlights within the KSOM curriculum and illustrate how we address select areas of emphasis listed above:

- Clinical skills development: The Introduction to Clinical Medicine (ICM) course is over 50 years old and ensures that students develop clinical skills early in medical school. It is a longitudinal course during years 1-2 with a faculty-to-student ratio of 1:6. Students systematically acquire clinical skills and competency in interviewing, history taking, and physical examination through workshops with standardized patients and hone their skills through encounters with actual patients every other week beginning the first few weeks of medical school. ICM also introduces students to clinical problem solving and documentation in the medical record.

- Mentoring and professional identity formation: The Professionalism and the Practice of Medicine (PPM) course pairs 2 faculty mentors with a group of 24 students in years 1-2 to gain skills and competence in communication, the social and community contexts of health care, ethical judgment, self-awareness and reflection, self-care and personal growth, professionalism, cultural competence, and lifelong learning. Mentoring is extended into year 3 with the Track Mentor Program (a faculty member meets regularly with students on each clerkship track) to continue student professional identity formation and to support a positive learning environment.

- Student well-being: The KSOM hired a director of medical student wellness and has integrated 34 hours of required well-being curriculum throughout years $1-3$ and additional optional activities to facilitate student wellness. The content is not tested/graded and focuses on the lived experience of the medical students, the link between physician well-being and

Acad Med. 2020;95:S42-S45.

doi: 10.1097/ACM.0000000000003294

Copyright (C) 2020 by the Association of American Medical Colleges

Supplemental digital content for this article is available at http://links.Iww.com/ ACADMED/A831.

Year school was established: 1949.

School URL: https://keck.usc.edu. quality/patient safety outcomes, incorporation of student selfreflection, and promotion of appropriate self-disclosure/role modeling by instructors.

- Humanities, ethics, art, and law curriculum: This 4-year curricular thread is integrated into courses including ICM, required clerkships, and year 3 intersessions. In years 1 and 2 , the focus is on collaborative discourse to help students learn to identify, analyze, and resolve clinical ethical problems. Years 3 and 4 include ethics education by clinical role models.

\section{Curriculum}

\section{Curriculum description}

The KSOM is currently a 4-year curriculum. Years 1-2 are delivered as integrated systems and longitudinal courses (ICM, PPM, and the Scholarly Project course). USMLE Step 1 is taken at the end of year 2. Years 3-4 are designed as a continuum and include the core required clerkships (family medicine, internal medicine, internal medicine subinternship, neurology, obstetrics-gynecology, pediatrics, surgery, and surgery subspecialty) and individualized selective and elective rotations, enabling senior students to pursue independent learning plans in pursuit of their specific career goals.

See Supplemental Digital Appendix 1-Curriculum

Description—at http://links.lww.com/ACADMED/A831.

\section{Curriculum changes since 2010}

Changes made to the curriculum since 2010 are highlighted below:

- Learning by cadaveric dissection was better integrated with system content.

- A Transition to Clinical Practice course was established to precede start of core clerkships in year 3 .

- Two single-week intersession courses during year 3 were added for students to pause, reflect, and consolidate clinical/ educational experiences of year 3 .

- The requirement of a scholarly project was implemented through the Scholarly Project course to engage all students in hypothesis-driven research.

- The longitudinal year 3 Track Mentor Program to continue to promote professional identity formation and a positive learning environment was established.

- A number of year 4 electives were made flexible by selfdirected asynchronous learning, distance learning, and/ or flexible clinical shifts to enable students to participate in residency interviews while achieving the objectives of the electives. 


\section{Assessment}

The KSOM medical education program objectives are adapted from the ACGME domains of competence to meet the needs of the KSOM curriculum.

See Supplemental Digital Appendix 2-Program Objectives and Assessment Methods—at http://links.lww.com/ACADMED/A831.

Changes in student assessment implemented since 2010 are highlighted below:

- An end-of-year 2 cumulative exam was discontinued because it did not provide additional student performance information.

- An end-of-year 1 cumulative exam was changed from summative to formative.

- A clinical assessment tool based on observable behaviors was implemented in all required clerkships.

- Narrative assessments by research mentors and some smallgroup facilitators were added.

- OSCEs in required clerkships were supplanted by twice yearly multistation mini-clinical performance examinations with OSCEs reflective of each student's prior clerkship experiences.

\section{Pedagogy}

The KSOM uses a variety of pedagogical approaches within courses and across all 4 years to achieve medical education program objectives.

See Table 1 -Pedagogy.

Changes in pedagogy implemented since 2010 are highlighted below:

- The use of audience response systems in lectures has been promoted and increased.

- Approximately 50\% of the 6-week hematology and clinical immunology system and $100 \%$ of the obstetrics-gynecology

\section{Table 1 \\ Pedagogy}

\section{Course or}

course type

Year 1-2: Systems

Year 1-2:

Longitudinal courses (ICM, PPM, Scholarly Project course)

Year 3-4: Clinical clerkships

\section{Pedagogical approaches}

Case-based learning, large-group discussions, laboratories, lectures, self-directed learning Case-based learning, ambulatory and inpatient clinical experiences (ICM only), small- and large-group discussions, lecture, peer teaching, preceptorship, self-directed learning, standardized patients, team-based learning

\section{Case-based learning, ambulatory and} inpatient clinical experiences, small- and large-group discussions, lecture, peer teaching, preceptorship, self-directed learning, simulation, standardized patients, team-based learning, workshop

Year 3: Transition to Clinical Practice course and/or intersessions
Small- and large-group discussions, lecture, peer teaching, self-directed learning, standardized patients, workshop clerkship curriculum were converted to flipped classroom teaching models.

- The internal medicine subinternship curriculum was converted to a predominantly workshop-based learning approach.

- A year 4 elective, Teaching and Leading in Residency, made up of web-based meetings and distance learning was created.

\section{Clinical experiences}

LAC+USC Medical Center is one of the largest public health care hospital and ambulatory networks in the country and serves as the primary inpatient and ambulatory teaching site for KSOM students. Other health care settings include Keck Hospital and the Norris Comprehensive Cancer Center (USC-owned private healthcare facilities), community-based clinics, private physician offices, and Children's Hospital Los Angeles. The principal challenge in designing and implementing clinical experiences is in identifying new clinical sites for required clerkships, especially in psychiatry and obstetrics-gynecology.

\section{Required longitudinal experiences}

- ICM is delivered in years 1-2 and enables students to achieve competency in basic clinical skills through workshops and encounters with real patients. Students learn from real patients and are exposed to patient care activities in the ICM course in the first few weeks of medical school and every other week in years $1-2$.

- PPM is delivered in years 1-2 as a mentoring and professional identity formation program.

- The Track Mentor Program in year 3 promotes the continued development of professional identity formation and a positive learning environment.

\section{Required and elective community-based rotations}

The family medicine clerkship provides all students with individualized opportunities to experience community-based practices and to understand the role of a primary care physician. A variety of elective community-based experiences are also offered in general internal medicine, neurology, and integrative medicine.

\section{Curricular Governance}

See Figure 1-Medical Education Curriculum Committee governance.

The KSOM provides stipends for the roles of system chairs and clerkship directors and funding for salaries of medical student educators (MSEs). Departments may provide additional funds for MSEs and they support all other faculty time and activities. Departments that oversee required clerkships fund clerkship coordinator positions. Each department receives funding allocations from the Office of the Dean to assist with faculty and staff compensation, which are based on department undergraduate education effort. 


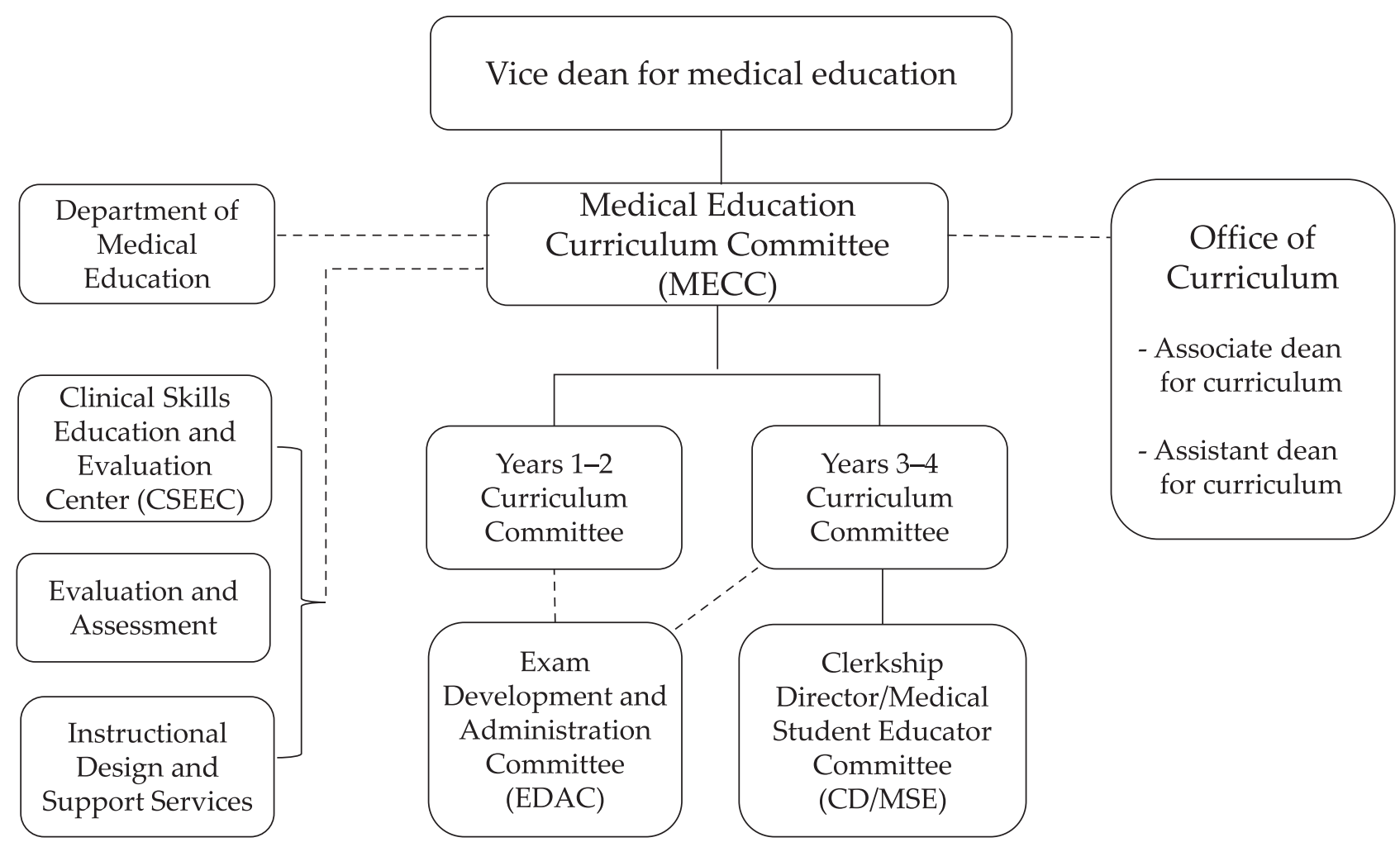

Figure 1 Medical Education Curriculum Committee governance.

\section{Education Staff}

The Office of Curriculum is responsible for overseeing and ensuring the development, implementation, and delivery of the 4-year MD curriculum. The Office of Curriculum provides direct support to faculty course directors, instructors, and medical students. It oversees the scheduling and coordination of courses and faculty and the development and distribution of supporting materials. The Office of Curriculum organizes and administers the Medical Education Curriculum Committee and its subcommittees to ensure continuous quality improvement efforts in all aspects of the MD curriculum.

The Evaluation, Assessment, and Reporting unit within the Department of Medical Education manages the entire evaluation process of all core learning and teaching events throughout the 4-year MD program. It oversees the development of tools (examinations, questionnaires, focus groups, etc.) and produces relevant reports for assessment of outcomes and continuous quality improvement activities.

The role of primary medical education staff and administrative faculty in the Office of Medical Education is to support all aspects of the MD program. The office is responsible for multiple areas outside of educational programming relevant to medical students, that is, admissions, student affairs, diversity, and inclusion, and the undergraduate minor in health care studies. The Office of Curriculum is responsible for overseeing and administering curricular aspects of the MD program or the MD component of dual-degree options.
See Figure 2-Medical education leadership.

The Department of Medical Education is composed primarily of $\mathrm{PhD} / \mathrm{EdD}$ educator faculty members and part-time or voluntary faculty instructors in required longitudinal courses. The department is composed of multiple units, including clinical skills education and evaluation, evaluation and assessment, educational technologies, faculty development, academic support, and instructional design. The department has offered a Masters of Academic Medicine program since 2009 and graduated 103 individuals to date, including many KSOM faculty. The department also sponsors an annual Innovations in Medical Education conference.

\section{Faculty Development and Support in Education}

The Faculty Development Unit in the Department of Medical Education provides direct support for Keck faculty and residents who wish to improve teaching skills and educational scholarship. The unit provides a wide range of services and offerings including customizable faculty development sessions on assessment and feedback, curriculum design, instructional design and strategies, program evaluation, and educational scholarship and research. Interactive workshops are targeted to specific teaching needs including, but not limited to, creating a positive learning environment, identifying learners in difficulty, assessing learners, providing effective feedback, small-group teaching, and teaching in the clinical setting. Small-group or 1-on-1 consultation sessions addressing the specific needs of individual 


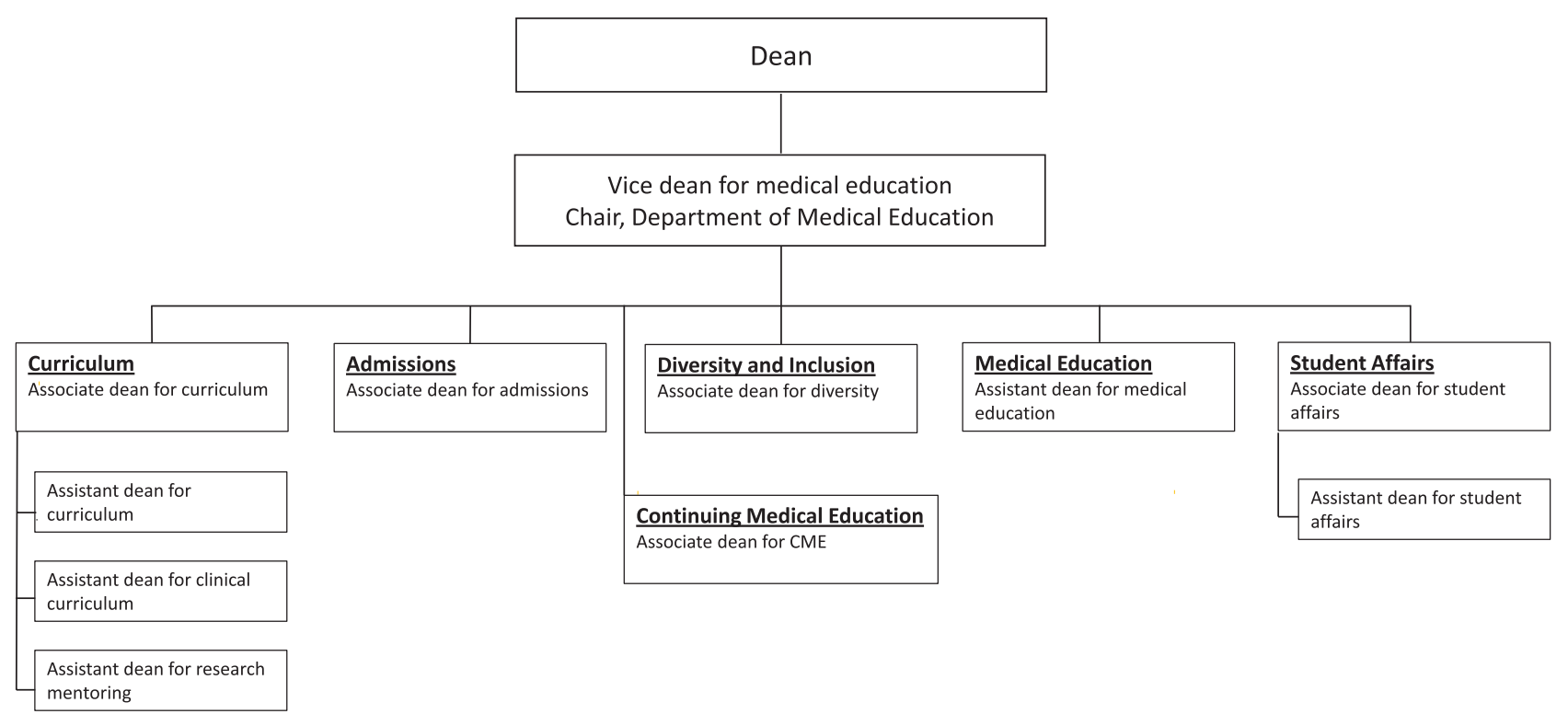

Figure 2 Medical education leadership.

faculty or small groups (disciplines, systems, clerkships, etc.) are available upon request. The department also oversees residents-as-teachers training by directly delivering content or by coordinating with departmental training programs.

The KSOM has established 7 promotion tracks that reflect the different profiles of activities and contributions of faculty. Each track includes expectations for contributions in scholarship, education, and service and, in the case of the clinician-educator and practitioner series, clinical practice. The amount of time devoted to individual areas and expectations in each area differ among the promotion series. Aggregate evaluation reports from students and residents of teaching/education activities are included in the promotion dossiers. Letters from mentees are also solicited and, in the case of the clinician-educator series, evaluation of teaching effectiveness by residency and/or fellowship directors may be requested. Teaching evaluation data are provided annually to each department chair at the time of faculty merit review.

\section{Initiatives in Progress}

The KSOM curriculum will transition to a renewed 4-year physician-citizen-scholar curriculum effective 2021 with the class of 2025. The renewed curriculum will be delivered in 3 phases: preclerkship (18 months), clerkship (12 months), advanced clinical experiences (18 months).

Planned features of the renewed physician-citizen-scholar curriculum are:

- Four hours of active case-based learning each week of the preclerkship phase.
- Enhanced clinical skills and clinical reasoning development in the preclerkship phase.

- Emphasis on active learning strategies throughout the curriculum, early clinical immersion, and a learning environment that fosters the well-being and professional development of students.

- Engagement of students with surrounding communities.

- Enhanced offerings in humanities, ethics, art, and law.

- Provision of a certificate in Social Justice in Medicine.

- A new longitudinal coaching program to guide professional identity formation.

- Integration of health systems science instruction and related clinical experiences throughout the preclerkship and clerkship phases.

- Immersive clinical training in the clerkship phase through a series of core clerkships organized into two 26-week blocks of grouped clinical rotations: 1 block of internal medicine/family medicine/ psychiatry/neurology and 1 block of surgery/anesthesia/ obstetrics-gynecology/pediatrics. The blocks will be enhanced by "just in time" training and by longitudinal cross-cutting themes covering geriatric medicine, chronic disease prevention and management, and mental health during the internal medicine/ family medicine/psychiatry/neurology block; and health systems science, social justice, and quality and patient safety during the surgery/anesthesia/obstetrics-gynecology/pediatrics block.

- Individualized training in the advanced clinical experiences phase to enable the determination and pursuit of one's areas of interest and ultimate career goals.

- An end-of-year 4 Transition to Residency course.

- Step 1 will be taken after the clerkship phase. 


\section{Loma Linda University School of Medicine}

Tamara Shankel, MD, MSHPEd, Lynda Daniel-Underwood, MD, PhD, Daniel Rogstad, MD, PhD, Amy Hayton, MD, and Tamara Thomas, MD

\section{Medical Education Program Highlights}

Loma Linda University School of Medicine has educated medical students for over 100 years. Our unique mission focuses on training students to deliver culturally appropriate wholistic care to individuals, families, and communities. This mission is embedded throughout our educational program. Our admissions process emphasizes selection of students who are mission aligned and committed to service. Our program includes patient care, small-group, and classroom experiences that provide students with the knowledge and skills to care for patients' physical, psychological, social, and spiritual needs. In addition to the 6 ACGME competencies, we have added a seventh, whole person care, to assess mission achievement.

A second strength is our longitudinal, integrated clinical skills curriculum. A centralized clinical skills education center (CSEC) provides consistent reinforcement of clinical skills throughout medical school.

- First-year students practice history, physical examination, and differential diagnosis skills in small-group laboratory sessions.

- Second-year students progress to integrated basic science OSCEs, where they obtain a history, examine a standardized patient, review related basic science, present, and discuss the patient in faculty-led small groups. Workshops focus on oral presentations and note writing.

- First- and second-year students participate in continuity clinic and 5 weeks of inpatient experiences, complementing CSEC learning.

- Third-year students rotate through traditional clerkships, returning to the CSEC for OSCEs and a formative junior clinical skills assessment, including peer, standardized patient, and faculty feedback. A clinical skills enhancement track supports students identified as needing additional support.

- Fourth-year students return to the CSEC for a subinternship OSCE focusing on assessing clinical reasoning, handoff skills, and cross-coverage questions from a nurse.

- The simulation center also enhances students' clinical skills and interprofessional experiences, culminating in assessment of several Entrustable Professional Activities (EPAs).

- An ultrasound curriculum is integrated throughout medical school.

Acad Med. 2020;95:S46-S49.

doi: 10.1097/ACM.0000000000003330

Copyright (C) 2020 by the Association of American Medical Colleges

Supplemental digital content for this article is available at http://links.Iww.com/ ACADMED/A854.

Year school was established: 1909.

School URL: https://medicine.llu.edu.
We deliberately created systems to promote development of students' professional identity, resilience, and wellness. The Christian Physician Formation longitudinal course is taught by clinicians mentoring small groups of students across all 4 years. The course teaches content through small-group discussion, including learning skills, professionalism, resilience, bias, social determinants of health, whole person care, and lifestyle medicine. It allows for mentoring students by faculty, enhances community among students, and creates opportunities for students from different classes to share experiences. For example, junior and senior students meet with sophomore students preparing for the USMLE Step 1 examination to share strategies and provide encouragement.

Students' commitment to service is demonstrated by their development of and participation in local and international service projects. Students provide weekly tutoring and mentorship for disadvantaged elementary through high school students, mentor and educate pregnant and parenting teens at local high schools, provide volunteer care in local free clinics and shelters for the homeless, and provide free music and swimming lessons to children from disadvantaged backgrounds. Many students participate in global health projects and international electives in numerous countries.

\section{Curriculum}

\section{Curriculum description}

See Supplemental Digital Appendix 1-Organ Systems Block Curriculum—at http://links.lww.com/ACADMED/A854.

\section{Curriculum changes since 2010}

The school has been undertaking curricular redesign over the past 3 years, with full implementation of the new curriculum planned for the entering class in July 2020. Specific goals for the new curriculum include:

- Emphasizing topics relating to our unique mission and heritage

- Increasing learning integration horizontally and vertically

- Supporting the development of professional identity, resilience, and wellness

- Transitioning pedagogy to include more active learning

- Using technology to support today's learners

- Allocating resources to support innovation

Aspects of the new curriculum implemented over the past 3 years include increased use of active-learning pedagogies; planned resilience activities; and development of communities to provide mentorship, create community, and support wellness.

New aspects of our curriculum include:

- Organ system blocks, integrating traditional basic science disciplines into one individual course. Content taught 
previously has been reviewed, prioritized, and integrated. Faculty have implemented evidence-based learning strategies such as planned repetition within the curriculum.

- Increased use of active-learning pedagogies. Our problem-based learning (PBL) curriculum has been redesigned as patient inquiry (PIQ). Each block will include 2 to 6 cases, designed to teach and reinforce content and develop lifelong and self-directed learning skills. Other active pedagogies include team-based learning (TBL), case-based learning (CBL), and flipped classrooms.

- Integration of basic and clinical sciences through the use of student-developed integrated illness scripts. These scripts focus on the basic science behind patients' symptoms, create a framework for review before boards, and integrate basic science into the third-year clerkships.

- Creation of integrated examination questions that cross disciplines and are mapped to learning objectives and Bloom's taxonomy. Weekly quizzes will be based on integrated learning objectives. These will count toward the course grade and also serve as formative assessment for learning.

- Two longitudinal courses will continue throughout medical school. The Christian Physician Formation course will be taught by clinical faculty paired with small groups of students for mentoring and nurturing community. Content will include professional identity formation, resilience, social determinants of health, community engagement, lifestyle medicine, and lifelong learning. The Clinical Skills longitudinal course is centered in the CSEC and provides consistent teaching by core faculty.

- Threads including traditional basic science disciplines, social determinants, social advocacy, service learning, academic medicine, pain and palliative care, patient safety and quality improvement, spiritual and whole person care, lifestyle medicine, interprofessional education, and telemedicine are woven throughout the curriculum. Thread directors ensure content is appropriate and updated regularly.

- Intersession weeks in the first and third years allow students to gain resilience and technical skills, explore topics of personal interest, and grow in their professional identity and sense of community.

Class size has not increased since 2010.

\section{Assessment}

See Supplemental Digital Appendix 2-Program Objectives and Assessment Methods—at http://links.lww.com/ACADMED/A854.

Initial development of program objectives was based on the AAMC's Medical School Objectives Project and CanMEDs. With the transition to competency assessment, the Curriculum Committee chose ACGME domains of competence, adding our mission-related whole person care competency. Competencybased assessments were implemented with the class of 2018. The competency subdomains are mapped to EPAs, ensuring students are prepared to transition to residency. Competencies were revised and approved in 2019 to match our curricular change, using the same domains. Our competencies are mapped to the Physician Competence Reference Set via the AAMC Curriculum Inventory Report and uploaded annually.
Evaluations and assessments were revised during our transition to competency-based program outcomes. We created new assessments to better demonstrate student growth, which are completed by each clerkship in our curriculum management system, with data downloaded to a dashboard for Competency Review Committee.

Grading is criterion-referenced for all 4 years to allow for individual student assessment.

We have created an electronic database for our skills log, which is uploaded to student iPads at the start of the third year. Once a skill is completed and assessed using this application, it uploads to our in-house database.

Clinical students receive formative (midyear) and summative feedback from the Competency Review Committee. Preclinical students receive formative and summative feedback at the end of the academic year. When a noncognitive concern (area of strength, praise, or growth) is recognized, faculty send an electronic form to the associate dean for curriculum evaluation and learner assessment.

We completed a review of all learner assessments in 2019 and have centralized the download to a single system, allowing for deeper evaluation and tracking of student growth.

\section{Pedagogy}

A variety of approaches are used to achieve program objectives. During the basic science years, students participate in TBL, CBL, small- and large-group discussion, PBL, self-directed learning, and are provided video/podcasts. Students complete 5 weeks of inpatient medicine and multiple outpatient clinic encounters. Lectures are limited to 8-10 hours per week and engage students through think-pair-share, interactive questions, and large-group discussions. PBL is used to teach content and lifelong learning skills. Students experience laboratory sessions in multiple disciplines, peer teaching, and use standardized patients and simulation. In the clinical phase, clerkship didactics also include CBL, small- and large-group discussions, standardized patients and simulation, and lectures.

We are implementing PIQ as a form of modified PBL. Previously, PBL was used to teach students effective literature search strategies to answer questions; it will now be used to teach actual content. Through patient cases, students will develop their own learning objectives, independently answer questions, and report on what they learned. During PIQ cases, students write integrated illness scripts as a team, deepening understanding of basic science mechanisms behind the symptoms.

Flipped-classroom modalities are increasingly used to replace lecture, including TBL, CBL, and peer teaching. In 2020, standardized TBL activities will increase to approximately 2 sessions per week. CBL activities will also occur directly after the weekly integrated quiz to reinforce learning. Lectures are increasingly employing active-learning techniques, inserting cases, think-pair-share activities, and polling questions to reinforce concepts. 


\section{Clinical experiences}

Clinical sites for required educational experiences include:

- Tertiary care medical center

- Veterans Administration hospital

- 2 county hospitals

- Psychiatric hospital

- Inner-city hospital

- Rotations at affiliated sites in Ohio and Florida

Students also rotate through outpatient sites including a federally qualified health center (FQHC) clinic (San Bernardino, California) and multiple hospital-based and private clinic sites. Students participate in a longitudinal continuity clinic during their sophomore year.

Students are assigned to inpatient services on the first day of school to observe medical care delivery and instill early professionalism. Students are asked to note health care provider communications, listen to a patient's story, and interview a health care professional from another field, then debrief in small groups.

Students may be assigned to community-based outpatient rotations as part of family medicine and pediatric clerkships. We identify clinical preceptors and sites where students see a variety of patients and can meet learning objectives and required patient experiences. Students may also rotate at university, VA, county, or FQHC clinics and have elective rotations options at communitybased sites.

Challenges in designing and implementing clinical experiences include:

- Ensuring that patient experiences are of adequate volume and variety to meet clerkship learning objectives and required patient experiences

- Ensuring that faculty and residents know the learning objectives, required patient experiences, and supervision and evaluation standards

- Evaluating the impact of other learners at the site

- Ensuring that students at each site meet equivalency standards in educational experience and outcomes

- Planning for future needs, based upon projected impact of additional medical schools' student rotations

\section{Curricular Governance}

Courses and clerkships have education committees that work with course/clerkship directors to recommend content and required experiences (based upon faculty expertise and input from national organizations) to the Curriculum Committee. That committee makes final decisions on content and required experiences. Responsibility and expenses for clerkship administrative support is shared between the school and clinical departments. Departments are provided funds to help with educational expenses. Funds are available for clinical clerkships to apply for annual innovation grants, encouraging continual develop of educational advances.
The associate and assistant deans are provided the administrative support and technology necessary to implement and evaluate the curriculum as it is planned and overseen by the Curriculum Committee.

See Figure 1-Curriculum management chart.

The Office of Medical Education is responsible for implementation and evaluation of the curriculum as designed by the Curriculum Committee. Associate deans for graduate medical education and continuing medical education work with this office and are members of the Curriculum Committee. The Office of Medical Student Education works with the Office of Student Affairs in designing programs to promote student success. The Office of Medical Education is responsible for creating the MSPE for each graduating student.

In the Department of Medical Education, leaders in education hold secondary appointments. Faculty who teach, but are not part of a traditional basic science or clinical department, may hold primary appointments in this department.

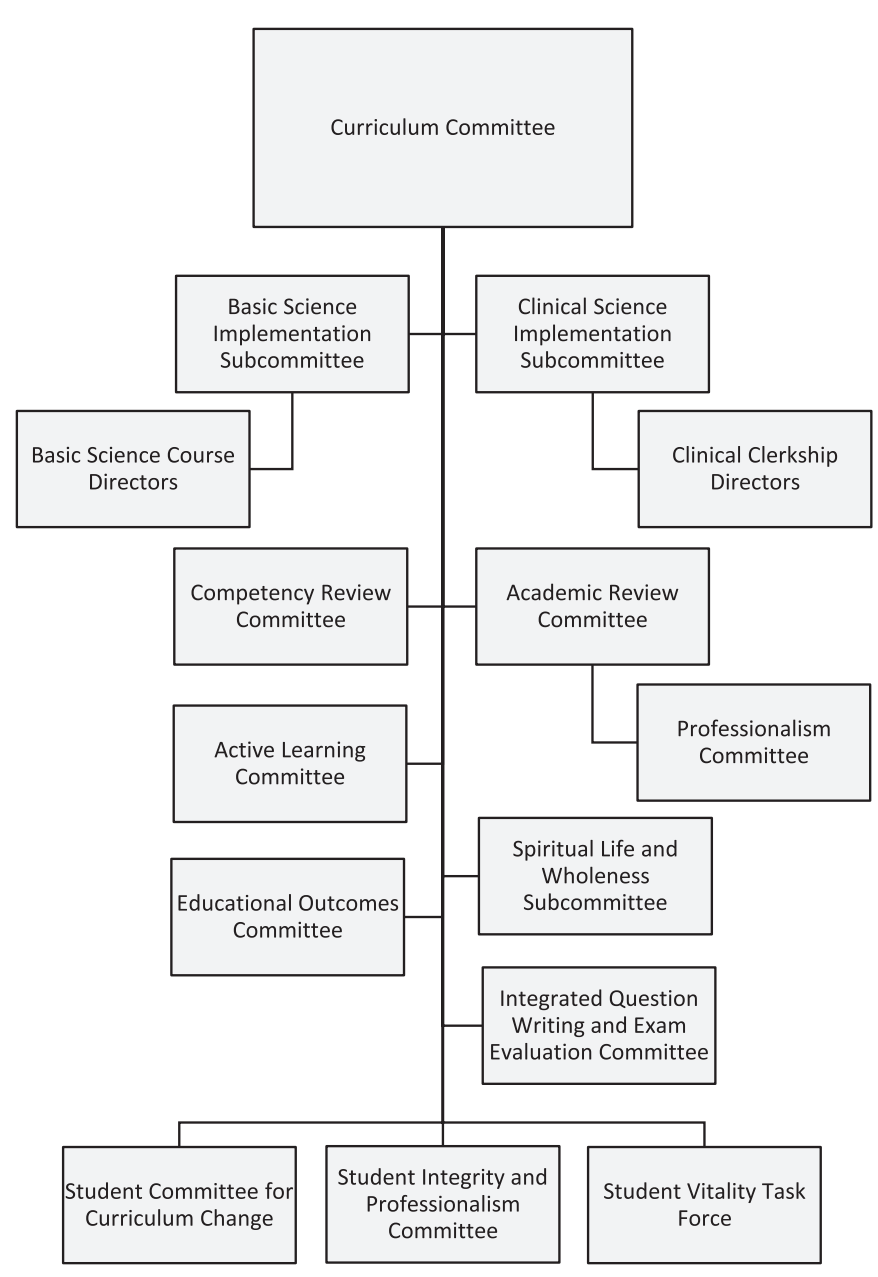

Figure 1 Curriculum management chart. 


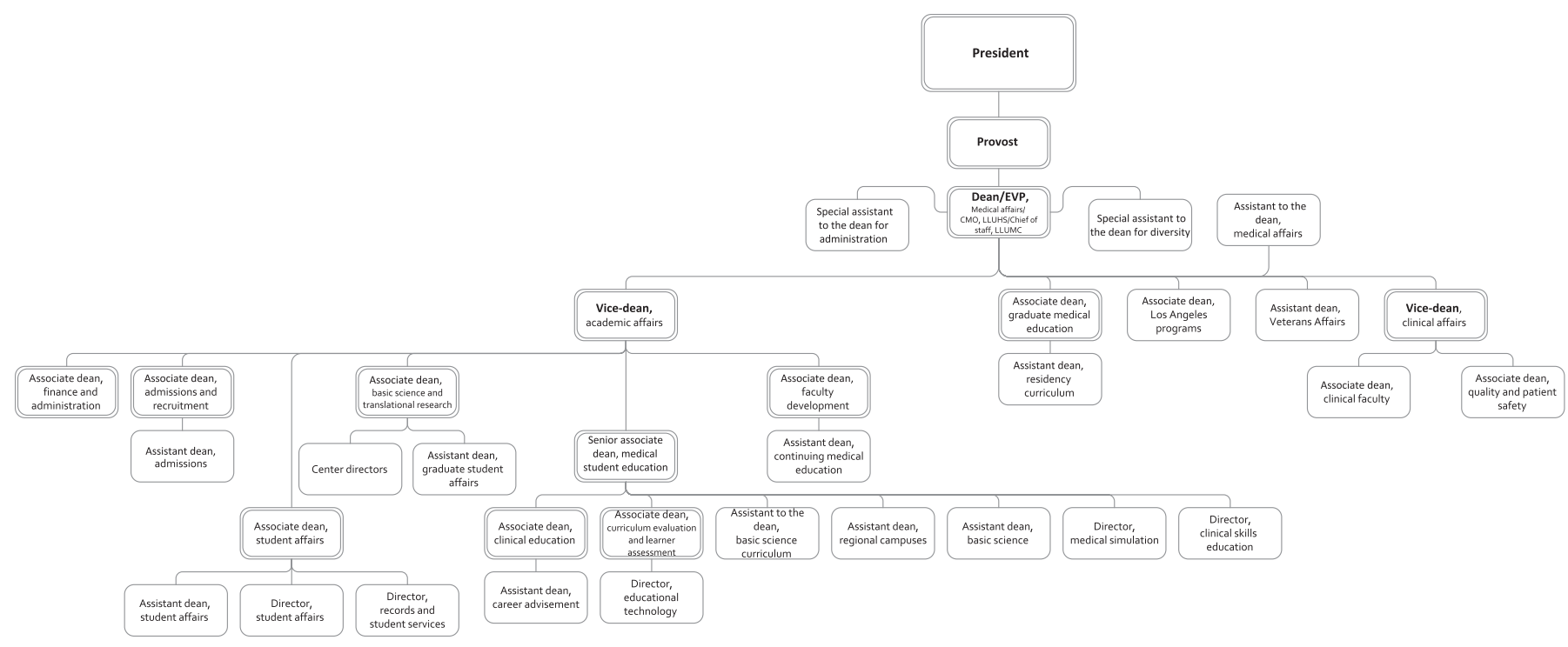

Figure 2 Organizational chart.

See Figure 2-Organizational chart.

\section{Faculty Development and Support in Education}

Faculty are provided professional development focused on education monthly through the dean's office, in addition to multiple departmental faculty educational offerings. The dean's office also offers faculty development at distant clinical sites. An annual faculty development course for junior faculty is offered.

Basic and clinical science educator tracks are available for faculty promotion pathways and include educator portfolios. Promotion requires significant teaching with outstanding evaluations, education-related CME, or training and a publication record related to medical education. 


\section{Stanford University School of Medicine}

Daniel Bernstein, MD, Cynthia A. Irvine, MEd, Preetha Basaviah, MD, James N. Lau, MD, Bahij Austin, Paul J. Utz, MD, and Neil Gesundheit, MD, MPH

\section{Medical Education Program Highlights}

The mission of medical education at the Stanford University School of Medicine is to educate and inspire leaders in medicine and science who will improve human health through discovery, innovation, scholarship, education, and delivery of outstanding patient-centered care. What makes Stanford unique is our Discovery Curriculum, initiated in 2017, expanding Stanford's long-standing commitment to train academic leaders. The Discovery Curriculum provides diverse pathways for exploring scholarly pursuits and developing skills to prepare for careers as thought leaders in medicine. Although students can complete their MD degree in 4 years, the majority spend at least 1 extra year pursuing research or obtaining a master's or $\mathrm{PhD}$.

Unique features:

- Scholarly Concentration Program: Promotes in-depth learning and scholarship in diverse areas that complement medical training, including bioengineering, molecular medicine, informatics, clinical research, community health, health policy, medical education, biomedical ethics, and medical humanities, with additional application areas wherein students can apply knowledge in these core areas to subspecialties. Students work directly with a faculty mentor and complete structured coursework to develop critical thinking skills, skills in evaluating new data, and handson experience using methods of generating new scholarly information. Completion of a Scholarly Concentration project is a graduation requirement. To support student research and ensure that students who spend a fifth research year do not graduate with additional debt, Stanford provides more than $\$ 3,000,000$ annually in competitive funding through our Medical Scholars grants programs, in addition to funding provided to $\mathrm{MD}-\mathrm{PhD}$ students in our $\mathrm{NIH}$-funded Medical Scientist Training Program (MSTP).

- Advanced degrees: In addition to the MSTP, students have obtained master's degrees in diverse fields complementing medicine including clinical research and epidemiology, bioengineering, public health, public policy, health policy, medical anthropology, education, and business. Students benefit from the medical school's location on the main university campus, enabling rich opportunities for scholarship in multiple disciplines.

Acad Med. 2020;95:S50-S53.

doi: 10.1097/ACM.0000000000003493

Copyright (C) 2020 by the Association of American Medical Colleges

Supplemental digital content for this article is available at http://links.Iww.com/ ACADMED/A975.

Year school was established: 1908.

School URL: http://med.stanford.edu.
- Berg Scholars Program: In 2018, Stanford launched a unique 6-year program combining MD-MS training in biomedical investigation. This program provides 5-6 students per year training as physician investigators. Berg Scholars work closely with a faculty mentor and split the second year of medical school over 2 years to pursue a basic science or informatics research project and spend the fourth year of medical school in full-time research, culminating in a master's-level thesis, followed by clinical training in years 5 and 6 .

- Cultural competence, population health, and health disparities: Practicing medicine requires training in implicit bias; cross-cultural interviewing; effects of socio-economics and racism on health and health disparities; immigration issues and health care; gun violence and public health; and sex, gender, and LGBTQ+ issues in health care. Stanford's curriculum provides related instruction, discussion, simulation, and group activities to foster cultural competence and awareness of social justice issues.

- A community of support: In our Educators 4 CARE program, faculty from various medical fields recognized for compassion, advocacy, responsibility, empathy, and teaching excellence serve as small-group teachers and mentors in clinical skills, clinical reasoning, and professionalism for 5-6 students per class, from matriculation until graduation. Additional components of our support community include advising deans, student life dean, Student Wellness Office, learning specialist, onsite psychiatrist and counseling staff, program directors and mentors, and the Stanford Center of Excellence for Diversity in Medical Education.

\section{Curriculum}

\section{Curriculum description}

The Stanford curriculum includes 6 quarters of basic science and clinical preparatory instruction, Scholarly Concentration coursework and a research project, and completion of at least 64 weeks of clinical clerkship instruction. Basic science courses are organized by discipline during the first 2 quarters (Foundations of Medicine), then by organ system in quarters 3-5 (Science of Medicine). In year 1, in tandem with basic science courses, students participate in an early clinical experience (ECE), engaging in patient care either from the patient or physician perspective. The Practice of Medicine spans the first 6 quarters, covering the approach to the patient, physical exam, principles of the psychiatric interview, clinical reasoning, and advanced clinical skills. Specialized threads include nutrition, population health, psychiatry, cultural competence, quantitative medicine, health economics, pain management, addiction medicine, and LGBTQ+ health. In quarter 6, students take a multidisciplinary pathophysiology course synthesizing basic science and early 
clinical learning as well as the Transition to Clerkships course. All students, including those obtaining additional degrees, complete the full curriculum as described.

See Supplemental Digital Appendix 1-Curriculum Map-at http://links.lww.com/ACADMED/A975.

\section{Curriculum changes since 2010}

The Discovery Curriculum strengthens our focus on scientific discovery, innovation, scholarship, and delivery of outstanding patient care. Curricular changes were threefold:

- New courses: In 2017-2019, we launched 4 new courses: Cells and Signaling in Regenerative Medicine, Pharmacologic Treatment of Disease, Pathophysiology Capstone, and Clinical Problem-Solving Capstone. Additionally, Human Anatomy, Embryology, Histology, and Neurobiology courses were reorganized.

- Early clinical experience: Launched in 2019, the ECE includes integration into the clinical setting with a chosen preceptor, development of concrete skills, and introduction to different career paths.

- Longitudinal scholarship via an extended curriculum: Beginning in the fall of 2017, students can apply to take the preclerkship curriculum over 3 years (rather than 2) to enable additional time for in-depth, longitudinal scholarship. This option was created in conjunction with the development of a Master of Science degree in Biomedical Investigation as part of the Berg Scholars program.

Flexibility extends throughout the curriculum, allowing for career exploration, research opportunities and scholarly pursuit, and pursuit of dual degrees.

\section{Class size changes}

We do not anticipate significant expansion of Stanford's 90-student class.

\section{Assessment}

Stanford has a detailed set of program objectives describing expected competencies. For each, a series of assessments have been developed to assure that trainees achieve the objectives (or receive remediation as needed).

See Supplemental Digital Appendix 2-Program Objectives and Assessment Methods-at http://links.lww.com/ACAD MED/A975.

Program objectives were originally based on ACGME domains of competence. We adopted the learning outcomes-oriented language of the Physician Competency Reference Set program objectives and added the institutionally developed domain of competence, Discovery, with its own set of objectives.

We are currently working to incorporate the Entrustable Professional Activities (EPAs) framework into existing clinical assessments. In AY 2020-2021, we will begin implementing workplace-based assessments in selected core clerkships as part of the EPA approach.
There are currently no parallel curricula or tracks offered to meet core educational objectives. Dual degrees, for instance, are considered to be supplemental and not part of the core curriculum.

\section{Pedagogy}

Stanford uses a variety of approaches to achieve program objectives in addition to traditional lecture:

- Case-based and problem-based learning

- Clinical experiences

- Discussion: large group (>12)

- Discussion: small group $(\leq 12)$

- Laboratory sessions

- Peer teaching

- Preceptorship

- Role play/dramatization

- Self-directed learning/tutorials

- Simulation/standardized patients

- Team-based learning

- Video/podcast/flipped classroom

- Virtual patients using 3-dimensional simulation

- Workshops

\section{Changes in pedagogy since 2010}

Compared with 2010, the current curriculum emphasizes active participation and interaction by learners and less lecture time. We estimate that about $30 \%$ of basic science teaching is by lecture, with the remainder in interactive small groups, simulation labs, flipped-classroom sessions, and team-based learning. Students experience direct patient care during their first year through the ECE and Practice of Medicine courses.

\section{Required longitudinal experiences}

In the preclinical curriculum, students follow patients longitudinally in the required ECE. When students enter clinical training, they may enroll in a continuity of care clerkship, wherein during weekly (half-day) clinics students follow a patient panel with an outpatient physician preceptor over a 9to 12-month period. Students also have extensive longitudinal research experiences, described above.

\section{Clinical experience first encounter}

As part of the Discovery Curriculum, students are required to participate in the ECE in their first year, where they are exposed to patients, and their stories in mentored clinical settings.

\section{Required and elective community-based rotations}

In the preclerkship curriculum students may provide health care at local community clinics. Additionally, there are 2 required clinical clerkships providing community-based clinical experiences.

- Preclerkship students lead an annual influenza vaccination campaign, named "Flu Crew," which vaccinates thousands of local students as well as uninsured residents through drives at local and regional churches, community centers, and farms. 
Students also support 2 community clinics that provide free medical services for indigent and uninsured patients. These experiences are part of the Practice of Medicine course, volunteer based, or for academic credit as elective coursework.

- In our ambulatory and emergency medicine core clerkships, students spend 3 weeks serving in a variety of outpatient clinics at Stanford Hospital and Clinics, the Palo Alto Veterans Administration Medical Center, or several community clinics. Students spend an additional 3 weeks in the Emergency Department primarily at Stanford Hospital, a Level 1 trauma center.

- In our family medicine core clerkship (3 weeks), students are distributed to outpatient family medicine clinics throughout our community, in San Francisco, and in Humboldt County.

\section{Challenges in designing and implementing clinical experiences for medical students}

Stanford has ample high-quality clinical training facilities in diverse clinical settings. The cost of providing that training, however, has risen significantly since 2010 as training sites seek more financial support for faculty preceptors involved in teaching. It is a challenge at Stanford, as at many medical schools, to find an appropriate yet affordable model for faculty compensation at affiliated sites.

\section{Curricular Governance}

The Faculty Senate is advisory to the dean of the School of Medicine. Under the dean's direction, the Faculty Senate has curricular oversight. There are 4 committees that report to the Faculty Senate that manage admissions; student scholarship; the curriculum; and student progress, professionalism, and promotion. Each committee is made up of 12-15 faculty members approved by the Faculty Senate's Executive Committee. Stanford has a collaborative model of curriculum oversight. Whereas these committees are part of centralized governance, courses and clerkships are organized by departments with input from education consultants in the Office of Medical Education (OME).

See Figure 1-Curricular governance.

\section{Education Staff}

The OME has primary responsibility for the MD program, including oversight of the curriculum and its associated committees, course and clerkship management, evaluation and continuous quality improvement, curriculum data analytics, and medical student research and scholarship. The office reports to the senior associate dean for medical education, who also has responsibility for GME and CME. OME is led by the associate dean for curriculum and scholarship, who has direct responsibility for the curriculum and its core faculty leaders; the assistant dean for curricular affairs, who manages the curriculum and its associated staff; and the associate dean for the OME, who provides oversight of operations and finances and manages additional staff supporting the curriculum. Twentythree administrative staff support the curriculum and its faculty leaders.

See Figure 2-Medical education leadership.

The primary medical education staff in OME support UME only and are not responsible for other areas of the medical education continuum. Student affairs are handled by the Office of Medical Student Affairs; faculty leaders and staff members collaborate closely in the OME.

Stanford does not have a department of medical education. The majority of faculty appointments, including adjunct appointments, are based in clinical and academic departments. The OME appoints a limited number of adjunct faculty each year who are involved in clinical teaching at affiliated sites.

\section{Faculty Development and Support in Education}

\section{Professional development for faculty as educators}

Stanford's innovative Teaching and Mentoring Academy (TMA) is involved in promoting teaching and mentoring excellence. The TMA offers courses and lectures with the goal of improving basic science and clinical teaching. The TMA has begun 1-on-1 peer feedback sessions and tutorials using skilled faculty to provide input and suggestions to improve teaching quality. Stanford offers a clinical teaching seminar series covering various topics in medical education that culminates in an honors certificate. In addition, faculty are encouraged to participate in courses and workshops offered by the main campus Center for Teaching and Learning.

\section{Role of teaching in promotion and tenure}

Teaching performance is emphasized as a criterion for promotion by the School of Medicine's various appointments and promotions committees. Further underscoring its commitment to teaching, Stanford has a dedicated clinician educator line for faculty. For promotion on this line, faculty must receive outstanding evaluations for their personal teaching performance and demonstrate scholarship in medical education,

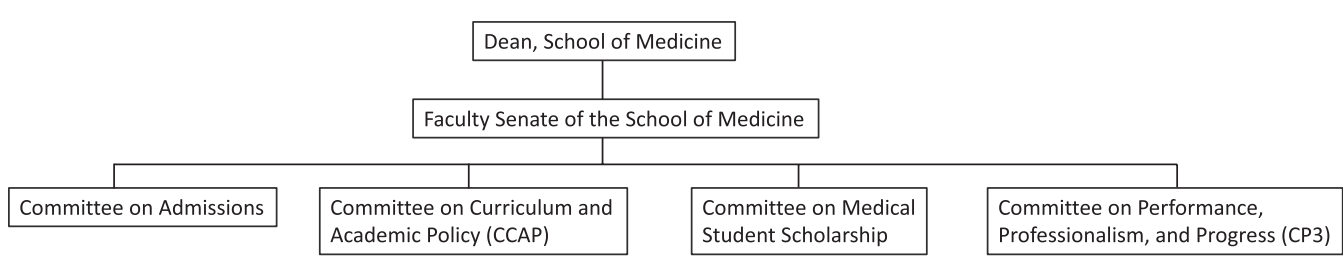

Figure 1 Curricular governance. 


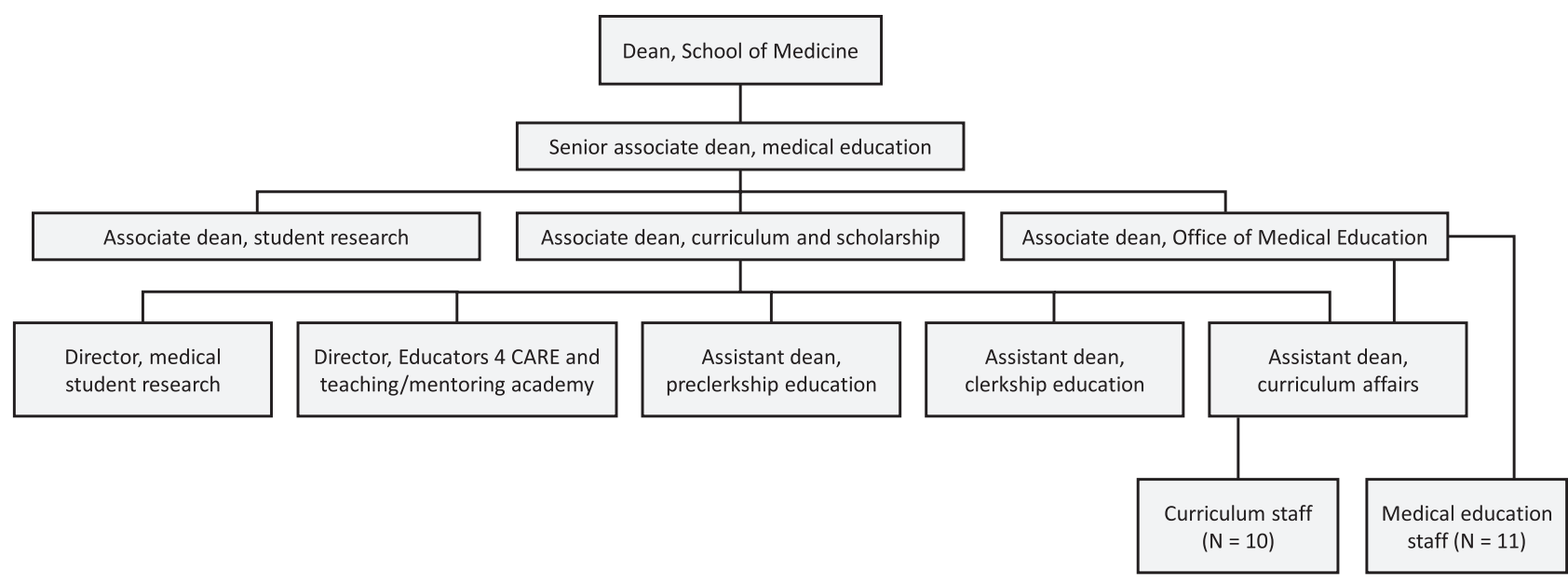

Figure 2 Medical education leadership.

through peer-reviewed publications and/or presentations at national education meetings.

\section{Initiatives in Progress}

- Refinement of the Discovery Curriculum, first introduced in AY 2017-2018
- Innovations in medical education emphasizing interactive learning and enhanced learner engagement

- Development of a curriculum on social justice, diversity and tolerance, and societal citizenship

- Improvement to the student wellness curriculum

- Further development of dual-degree training, including funding opportunities 


\section{University of California, Davis School of Medicine}

Mark Servis, MD

\section{Medical Education Program Highlights}

The UC Davis School of Medicine (UCD SOM) is committed to student-centered education with patient-centered outcomes. Our goal is to train the next generation of compassionate physicians to meet the health care needs of California and the demands of an increasingly complex health care system, a diverse patient population, and a rapidly expanding knowledge base in medicine. We emphasize collaboration and interprofessional education, the acquisition of sophisticated communication and interpersonal skills, the development of intellectual curiosity and critical analytic skills, a commitment to quality improvement and patient safety, and the use of information technology to advance health care delivery and medical education.

- To address the health care workforce needs of our region, we have several tailored clinical pathway programs with specialized admission criteria and curricula including an accelerated primary care pathway, a rural medicine pathway, an urban underserved pathway, and a pathway focused on meeting the health care needs of the northern Central Valley of California.

- To train the next generation of physician investigators, we have research pathways including an 8-year MD-PhD program, a 5 -year research pathway with a master's in Clinical Research, and a Medical Research Fellowship Program to support student research projects.

- We admit a student body matching the incredible diversity of our state and our region. An average of $33 \%$ of our students have come from underrepresented groups in medicine for the past 5 years.

- True to our students' commitment to underserved communities, we are affiliated with 7 student-run clinics and 5 satellite clinics that provide free, culturally attuned services that serve as the "safety net for the safety net" for our region. These clinics are specialized to serve the Asian and Pacific Islander community, Latino community, African American community, South Asian and Muslim community, Filipino community, IV drug users and sex workers, homeless patients, Vietnamese community, Hmong community, Russian-speaking community, LGBTQQI community, and rural underserved and undocumented immigrant patients.

\section{Curriculum}

\section{Curriculum description}

- The preclerkship curriculum is organized in thematic blocks to promote integration of basic sciences and clinical medicine.

Acad Med. 2020;95:S54-S56.

doi: 10.1097/ACM.0000000000003452

Copyright (C) 2020 by the Association of American Medical Colleges

Year school was established: 1967.

School URL: https://health.ucdavis.edu/medschool.
Four blocks of foundational science and pathophysiology courses are grouped by complementary objectives, disciplines, and themes. The longitudinal Doctoring curriculum focuses on clinical skills, clinical reasoning, and behavioral and social medicine, including health equity, and is woven throughout the first 2 years and integrated with concurrent courses. The longitudinal pathology and pharmacology curricula are similarly integrated with concurrent courses.

- The third year begins with a 1-week Transition to Clerkships course, followed by required clerkships in surgery, internal medicine, obstetrics-gynecology, pediatrics, psychiatry, and family medicine, and a longitudinal clinical experience in primary care. A 4-week selective block is included. The longitudinal Doctoring curriculum runs concurrently with the clerkships and focuses on advanced interviewing techniques, clinical reasoning, clinical epidemiology, evidence-based medicine, medical humanities, and ethics/jurisprudence.

- The fourth-year curriculum is designed to provide breadth and depth of learning experiences while helping students attain competencies at an advanced level (subinternship). Special study modules focus on advanced applications of basic sciences and clinical medicine. Students may also pursue a scholarly project. There is flexibility within the schedule to provide diverse opportunities for in-depth exploration of areas of interest while helping students to select and prepare for the residency, ending with a 2-week Transition to Residency course before graduation.

\section{Curriculum changes since 2010}

- We have grown our class size from 100 to 128 students largely through the development of tailored clinical pathway programs to address the health care workforce needs of our region including an accelerated primary care pathway, a rural medicine pathway, an urban underserved pathway, and a pathway focused on meeting the health care needs of the northern Central Valley of California. We anticipate continued growth to an eventual class size of 144 as we develop additional clinical training sites.

- We added a 5-year research pathway with an option for a master's in Clinical Research to expand the number of diverse students pursuing careers in academic medicine.

- We added both a Transition to Clerkship course and a Transition to Residency course to aid students as they transit from one phase of training to another.

- We are currently adopting a transformative new curriculum that is using a learner-centered and patient-centered framework as the twin guiding principles for the new curriculum plan. The new curriculum is anticipated to begin in 2021 and will be a "living" curriculum designed to evolve to meet the needs of physicians and patients in the years to come.

\section{Assessment}

- Medical education program objectives for the UCD SOM mirror the ACGME competency domain framework with 
6 core competencies: patient care, medical knowledge, interpersonal and communication skills, professionalism, system-based practice, and lifelong learning. These graduation competencies have associated subdomains and milestones used to guide curriculum content, pedagogy, and assessment.

- Assessment methods in preclerkship blocks include the use of integrated, case-based questions in end-of-block examinations and customized NBME assessments. The preclerkship blocks also provide biweekly, integrated quizzes for students to monitor their progress and learning. Narrative assessment from faculty is provided in many courses and peer assessment is a part of team-based learning.

- Clinical skills assessment in preclerkship blocks includes the use of standardized patients and other simulations, facultyobserved OSCEs, and practice-based learning exercises. These standardized assessments continue during the clinical clerkships, and oral examinations and OSCEs are used by several clerkships. All required clerkships use an NBME shelf examination.

- End-of-year assessments that involve clinical simulations with standardized patients are used to test interviewing and physical exam skills in Doctoring at the end of years 1 and 2 . An 8 -station clinical performance examination modeled after the USMLE Step 2 CS examination is conducted at the beginning of the fourth year.

\section{Parallel curriculum or tracks}

- The Accelerated Competency-based Education in Primary Care program is a partnership between UCD SOM and Kaiser Permanente-Northern California. Students who are confident that they want to pursue a career in adult internal medicineprimary care or family medicine have the opportunity to complete their undergraduate medical education in an intensive 3-year curriculum, instead of 4 , and are offered a conditional acceptance into a UC Davis or Kaiser primary care residency upon entry into the program.

- Rural Program in Medical Education focuses on meeting the needs of rural communities through unique activities that include specialized coursework, core clerkships at rural affiliated sites, and mentoring by rural physicians. Medical students become skilled physicians and gain the experience necessary to become community leaders and patient advocates in rural communities.

- Reimagining Education to Advance central California Health is for students who are committed to returning to central California to practice medicine and serve their communities. Students have specialized coursework and complete their clinical training at partner sites in northern, central California.

- Transforming Education and Community Health for Medical Students is for students who have a strong desire to work in urban, underserved primary care (internal medicine, family medicine, or pediatrics). Students have unique clinical experiences in community health centers in the Greater Sacramento area, including a longitudinal integrated clerkship, and mentorship from physicians doing community-based health care.
- California Oregon Medical Partnership to Address Disparities in Rural Education and Health is a joint partnership with Oregon Health \& Sciences University, supported by the American Medical Association as part of their Reimagining Residency initiative to address health care workforce shortages in rural, tribal, urban, and other underserved communities between Sacramento and Portland. Students have a specialized curriculum, regional clinical rotations, and are offered conditional acceptance into residency programs in 7 specialties throughout the region.

\section{Pedagogy}

- The regular curriculum and specialized curricula found in the tailored clinical tracks use a variety of pedagogies with an emphasis on active learning. These include problem-based learning, team-based learning, peer instruction, simulation, standardized patients, case-based learning, interactive video and podcasts, laboratory, lecture, preceptorships, and clinical experiences in ambulatory and inpatient settings.

- Major changes in pedagogy since 2010 include an overall increase in active learning pedagogies throughout the curriculum, a decrease in lecture time, and a conversion of content delivery to web-based platforms.

\section{Clinical experiences}

- The tailored clinical pathways use specialized clinical experiences including early exposure to clinical sites from the first day of medical school, the use of longitudinal integrated clerkships in 3 of the tailored clinical pathways, and the development of affiliated clinical rotation sites at Kaiser Permanente-Northern California, Sacramento County, San Joaquin County, and a number of rural health systems and preceptors in northern California.

- Other key community partners for clinical rotations include the VA Northern California Health Care System, Dignity Health, Sutter Health, and Adventist Health.

\section{Curricular Governance}

- The University of California has a tradition of shared governance whereby the faculty and the administration partner with our students to achieve excellence in education. Institutional responsibility for oversight of the curriculum by the faculty rests with the Committee on Educational Policy (CEP), a standing committee of the Faculty Executive Committee. The vice dean for medical education and the associate dean for curriculum serve as an ex officio members of the CEP. Medical student representatives from all 4 years are elected by their colleagues and serve as nonvoting members of the CEP.

- The CEP is charged with design, management, and evaluation of the curriculum and is responsible for ensuring that objectives and teaching methods are linked to graduation competencies.

- The CEP has 4 subcommittees:

- Block Council: Promotes horizontal and vertical integration across the 4-year curriculum 
- Evaluation Subcommittee: Conducts periodic, in-depth, peer evaluation of courses and clerkships

- Curriculum Review Subcommittee: Provides overall assessment and coordination of the curriculum as a whole

- Fourth-Year Oversight Committee: Recommends fourthyear curriculum requirements and approves fourth-year programs of individual students to ensure breadth, depth, and vigor

- The CEP and the associate dean for curriculum appoint a "curriculum liaison" for each major block of curriculum (4 preclerkship blocks, Doctoring for years 1-3, and year 3 core clerkships). The 7 curriculum liaisons provide a linkage between the CEP and the individual courses or clerkships within their blocks to advance integration and encourage collaboration between educational leaders.

\section{Education Staff}

- The Office of Medical Education (OME) partners with the faculty to create a supportive and stimulating learning environment that nurtures the requisite and enduring attitudes, skills, and knowledge required of excellent physicians.

- OME is directed by the vice dean for medical education, who has administrative responsibility for undergraduate and graduate medical education and reports to the dean of the School of Medicine. Associate deans in OME include admissions, curriculum, student affairs, and workforce innovation and community engagement, and an OME manager who is the assistant dean for medical education. The associate deans for student and resident diversity have a dotted line reporting relationship to the vice dean for medical education and a direct reporting relationship to the associate vice chancellor for health equity, diversity, and inclusion.

- OME consists of an interdisciplinary group of academic, technical, and administrative staff who deliver centralized expertise, services, and instructional resources to faculty and students. The services include admissions and outreach, financial aid, student records, information technology, facilities management, and staffing of faculty committees. In addition, OME provides centralized support to all course directors and faculty in the planning, delivery, evaluation, and enhancement of courses and clerkships.

- Academic support functions in OME include the appointment of directors and master educators with formal expertise in academic services, student and career development, professionalism, diversity, wellness, assessment and evaluation, educational technology, research, clinical skills teaching and assessment, and rural health.

- Additional OME units include the Body Donation Program, the Center for a Diverse Healthcare Workforce, the Office of Student Learning and Educational Resources, and the Office of Student and Resident Wellness.

\section{Faculty Development and Support in Education}

- Teaching is essential for faculty advancement in the promotion and tenure process in all faculty series at the University of California. In person and online faculty development offerings in education are provided to faculty, and a 2-year longitudinal Interprofessional Teaching Scholars Program jointly run with the nursing and veterinary schools is offered for those whose academic focus is in education. 


\title{
The University of California, Irvine School of Medicine
}

\author{
Khanh-Van Le-Bucklin, MD, MEd, Michael Stamos, MD, and Julie Youm, PhD
}

\section{Medical Education Program Highlights}

A 5-time Apple Distinguished School honoree, the University of California, Irvine School of Medicine (UCI) has embraced technology as a core component of its medical education program for nearly a decade. In 2010, UCI made a strategic commitment to reinvent the traditional medical school curriculum for the 21st century. The iMedEd Initiative was launched, with UCI leading as one of the early adopters of innovative technologies to promote active, student-centered learning in medical education. 2020 marks the 10-year anniversary of the iMedEd Initiative, and highlights from this period include:

- A 1-to-1 iPad program for every incoming class of students since 2010

- A mature 4-year point-of-care ultrasound curriculum, most recently distinguished by the gifting of Butterfly iQ handheld ultrasound devices to each member of the class of 2023

- Use of Google Glass, virtual reality, and 3D printing to enhance knowledge acquisition and clinical skills development

- Substantial curricular redesign to support a systems-organized, clinically anchored, active learning approach

UCI's mission-based programs constitute another curricular initiative of high priority for the school. The state-supported Program in Medical Education for the Latino Community (PRIME-LC) is the school's signature mission-based program, and was designed to develop leaders, advocates, and culturally competent health care providers for California's under-resourced Latino communities. This 5-year dual-degree program includes educational sessions taught in Spanish, immersive clinical experiences locally and internationally, and a master's degree requirement. The first cohort of PRIME-LC students enrolled in 2004, and the program has continued to consistently graduate students dedicated to serving Latino communities for the past 15 years.

Following the success of PRIME-LC, 2 additional mission-based programs have been established at UCI. Leadership Education to Advance Diversity-African, Black, and Caribbean (LEAD-ABC) is a new, first of its kind, mission-based program aimed at producing future physicians who are committed to serving African, black, and Caribbean communities. Health Education to Advance Leaders in Integrative Medicine (HEAL-IM) is a

Acad Med. 2020;95:S57-S60

doi: 10.1097/ACM.0000000000003312

Copyright (C) 2020 by the Association of American Medical Colleges

Supplemental digital content for this article is available at http://links.Iww.com/ ACADMED/A840.

Year school was established: 1961.

School URL: https://www.som.uci.edu. mission-based program designed to provide a future generation of physicians with additional training and skills in the tools and philosophies of integrative medicine. Both of these 4-year longitudinal programs accepted their first cohort of students in 2019.

\section{Curriculum}

\section{Curriculum description}

See Supplemental Digital Appendix 1-iTEACH Curriculum Map—at http://links.lww.com/ACADMED/A840.

\section{Curriculum changes since 2010}

The UCI curriculum has evolved over the last 10 years from a traditional, discipline-based structure to a systems-organized approach known as iTEACH (Technology-Enhanced, Activity-Coordinated, Humanistic). iTEACH represents a temporal alignment of content organized into thematic blocks, and is a significant departure from the former traditional, discipline-based courses that previously defined the preclerkship years. By maintaining a "systems-organized" perspective, a priority on the integration of basic science and clinical content is explicitly defined, with improved student outcomes as a result. In addition to realignment of the content, iTEACH includes critical opportunities for students to engage in coordinated simulation, ultrasound, and clinical skills modules with the aim of further increasing clinical correlation with the basic sciences by enabling hands-on opportunities to situate student learning.

\section{Assessment}

The current UCI program objectives and Knowledgeable, Skillful, Altruistic, and Dutiful competency framework were developed by a taskforce consisting of faculty, residents, students, and senior administrators. In addition to the school's defined competencies, program objectives are also mapped to the ACGME domains of competence, the Physician Competency Reference Set competencies, and the AAMC Core Entrustable Professional Activities for broader comparability. A varied and comprehensive set of assessment methods are associated with each program objective and used to determine medical students' progress in developing the competencies required for advancement towards the MD degree.

See Supplemental Digital Appendix 2-Program Objective Assessment Mapping — at http://links.lww.com/ACADMED/ A840.

\section{Pedagogy}

The iTEACH curricular redesign featured a renewed reliance on active learning strategies with clinical correlation at its core. Common pedagogical approaches found in the curriculum are team-based learning, small-group discussion, 
and coordinated clinical skills modules that align with systems-organized content utilizing standardized patients, ultrasound, and simulation as foundational resource methods. Successful implementation of these changes was dependent on faculty preceptors who could provide consistent small-group instruction, feedback, and modeling. To address this need, the Dean's Scholars program was initiated in 2018, enabled by the funding and centralization of clinical faculty resources in the Office of Medical Education. For the first cohort of this program, 12 Dean's Scholars from diverse backgrounds and specialties were recruited, interviewed, and selected through a competitive process. Dean's Scholars commit 2 half days a week to serving as content developers, small-group leaders, and clinical preceptors.

This investment in a centralized team of dedicated clinical faculty has provided the following benefits for the medical education program:

- Standardized clinical skills training and assessment

- Coordinated curriculum development and clinical integration opportunities

- Consistent faculty support at small-group activities

- Longitudinal mentorship for students

\section{Clinical experiences}

Students begin clinical experiences during their first year of medical school in the Patient and Community Engagement (PACE) clerkship. PACE is a longitudinal clinical rotation with a community preceptor, and was designed to provide students with consistent workplace-based opportunities to improve medical knowledge while practicing patient care, professionalism, and interpersonal and communication skills.

The community setting exposes students to the unique culture of medical systems and the social determinants impacting the health of patients. During this early clinical experience, students perform a community assessment, as well as develop and implement a quality improvement project within a practice.

In the clinical years, students complete required experiences at the UCI Medical Center, 3 affiliated hospitals (Children's Hospital of Orange County, the Veterans Affairs Long Beach Healthcare System, and Long Beach Memorial Medical Center/ Miller Children's Hospital), and community-based outpatient clinics. UCI does not have any regional campuses.

Annual monitoring of clinical experiences demonstrates a desired balance of inpatient and outpatient experiences across the clinical years, and minimal use of alternative methods to meet core clerkship requirements. As UCI considers an increase in class size, one challenge will be the need to expand capacity in the area of obstetrics-gynecology to accommodate more learners.

\section{Curricular Governance}

Curricular governance is provided by the Curriculum and Educational Policy (CEP) Committee, whose voting members are faculty appointed by the School of Medicine Academic Senate. The CEP has 2 subcommittees: the Basic Science Course Directors Committee and the Clinical Clerkship Directors Committee. Subcommittee membership is made up of course/ clerkship directors, course/clerkship coordinators, and student representatives. The dean and medical education administrators serve as ad hoc committee members. The CEP and its subcommittees meet monthly and are responsible for reviewing all courses on a biennial schedule. The CEP Committee also reviews the phases of the curriculum, and the curriculum as a whole.

See Figure 1-Curricular governance.

\section{Education Staff}

The administrative and academic support for the planning, implementation, evaluation, and oversight of the curriculum is the responsibility of the Office of Medical Education. The Office of Medical Education also oversees the development and maintenance of tools to support curriculum delivery, monitoring, and management through an ongoing, collaborative effort by the divisions of Curricular Affairs and Educational Technology.

The Division of Education Compliance and Quality (ECQ) in the Office of Medical Education oversees the accreditation, compliance, and quality improvement activities of the medical education program, in collaboration with the CEP committee and the divisions of Student Support, Curricular Affairs, and Educational Technology. ECQ is charged by CEP to ensure the school's compliance with the CEP's policy on continuous quality improvement.

The Office of Medical Education's responsibilities reach beyond educational programming to encompass admissions, student affairs, and diversity and inclusion. It also serves as the administrative home for educational activities across the continuum, including pathway programs, graduate medical education, and continuing medical education.

Reporting to the dean, the office is led by a vice dean for medical education, 5 associate deans, 6 assistant deans, 11 faculty directors, a chief administrative officer, and 2 executive staff directors.

See Figure 2-Medical education leadership.

\section{Faculty Development and Support in Education}

Faculty development is conducted across the clinical, research, and educational enterprises of the school. To support the professional development of faculty as educators, the Office of Medical Education conducts a faculty development seminar series covering courses that include:

- Engaging Learners with Effective Didactic Teaching

- Asynchronous Learning + Audience Response

- Effective Clinical Teaching

- Podcasting, PowerPoint and Keynote Tips for Better Presentations 


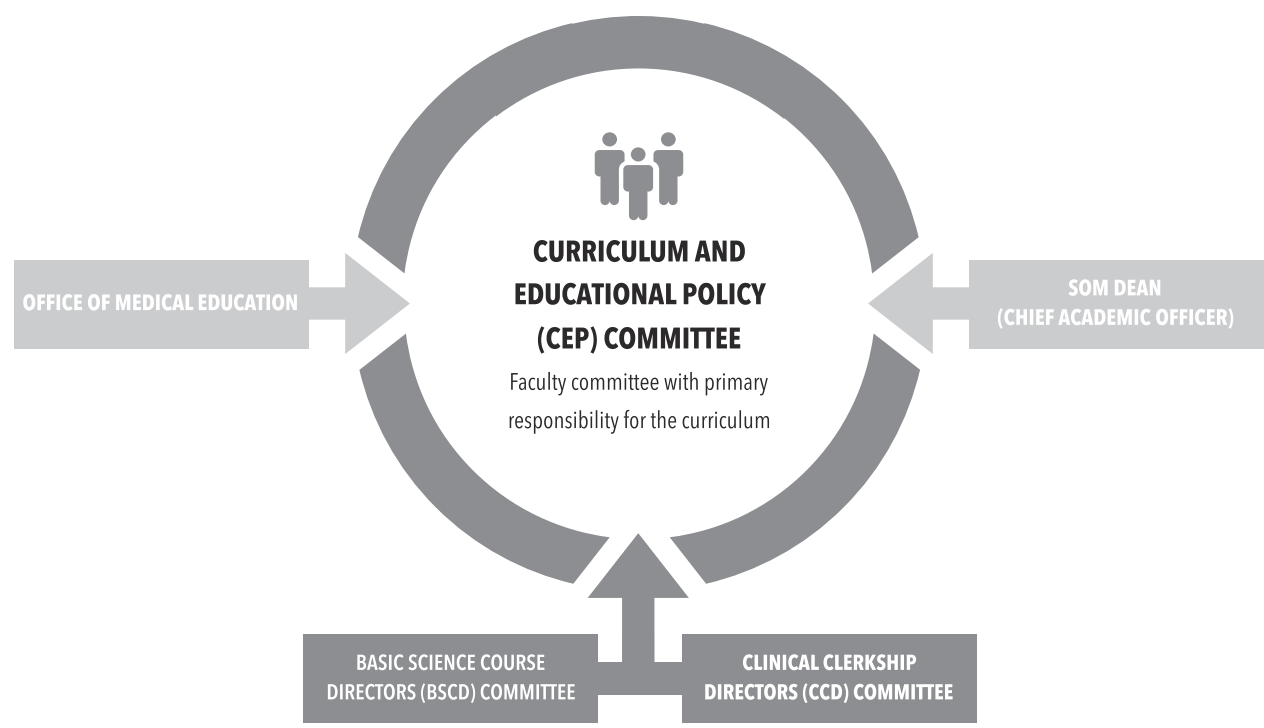

Figure 1. Curricular governance.

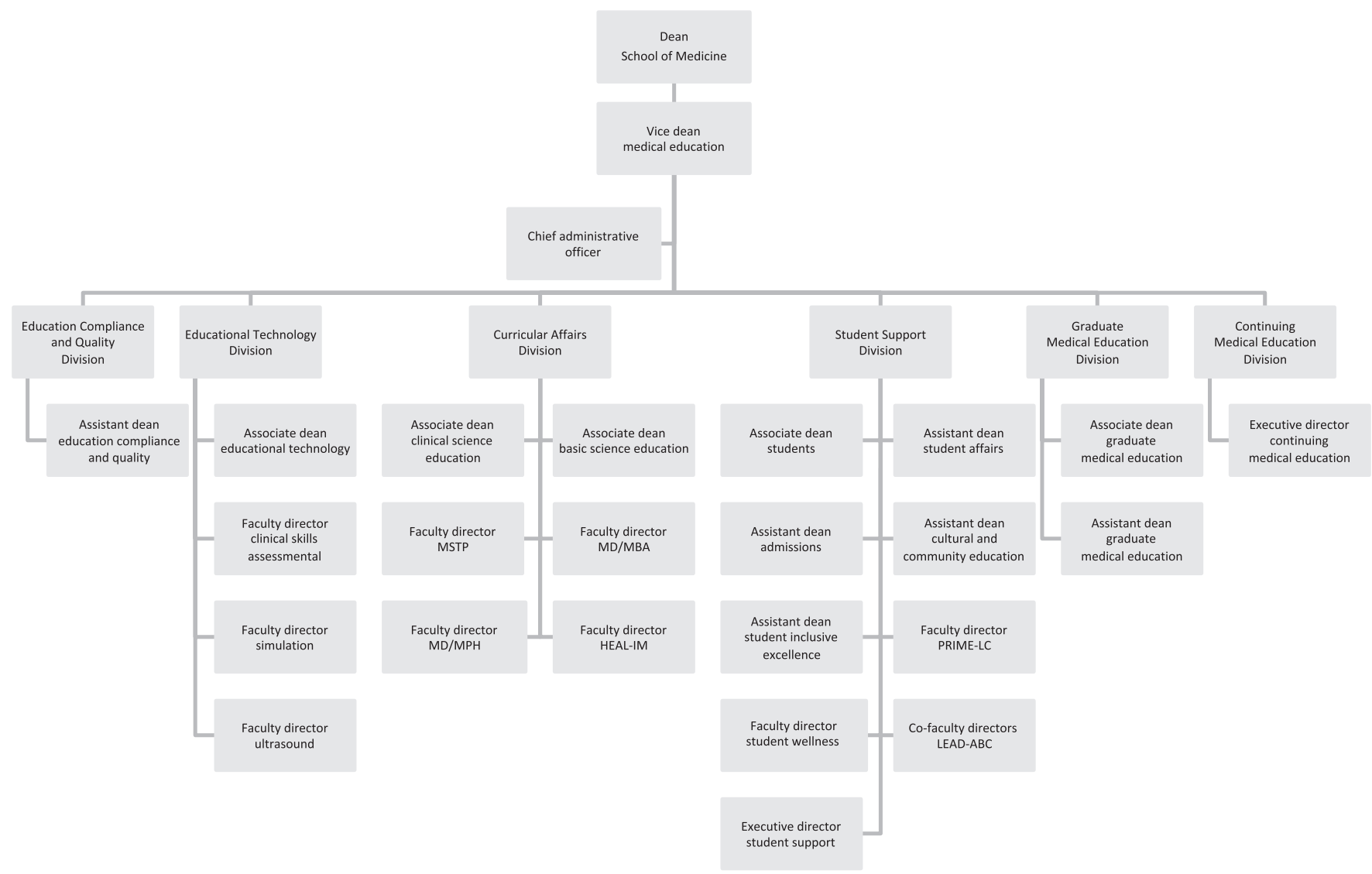

Figure 2. Medical education leadership. 
- Role of the Educator with Medical Students and Policy Review

- Effective Feedback

Additional faculty development opportunities are available through the Division of Teaching Excellence and Innovation on the UCI main campus.
Evidence of teaching is not only considered, but required, in academic promotion and tenure decisions at UCI. Faculty must provide student evaluations of their teaching, and at least one other artifact that demonstrates their support of the university's teaching mission, such as a teaching statement, peer evaluations, or teaching awards. 


\section{University of California, Los Angeles David Geffen School of Medicine}

Clarence H. Braddock III, MD, MPH, MACP

\section{Medical Education Program Highlights}

The David Geffen School of Medicine seeks to "create world leaders in health and science." The mission of the medical education program of the David Geffen School of Medicine at the University of California, Los Angeles (UCLA) is to prepare graduates for distinguished careers in clinical practice, teaching, research, and public service. Recognizing that medical school is but one phase in a physician's education, the faculty has worked to create an environment where students prepare for a future in which scientific knowledge, societal values, and human needs are ever changing. To meet this challenge, our graduates have the skills to provide quality care for their patients, to contribute to the growth of scientific knowledge, and to continue learning throughout their careers. They are involved in service to the community and demonstrate a firm commitment to the humanistic, ethical, legal, and cultural aspects of medicine. We believe that a diverse student body is an integral part of medical education and is essential to the development of patient-centered care.

Our medical education program has several distinct tracks. In addition to our traditional MD program, we also have an MSTP program with 2 subtracks, 1 at UCLA and 1 in collaboration with Caltech. In addition, we have a long, over 40-year collaboration with Charles R. Drew University of Medicine and Science, for students training for the MD degree with special interest in care of the urban underserved. Finally, we have the PRIME-LA program, a 5-year dual-degree program for students training as physician-leaders in addressing health care disparities preventable populations.

Another unique feature of our program has been an increasing emphasis on encouraging students to pursue passions related to our mission and complementary to their pursuit of a career in medicine. This includes actively encouraging students to pursue master's degree, an additional year for research, or other enrichment activities in pursuit of them becoming "an outstanding physician and...."

The Colleges is a program for fourth-year students that provides academic advising, career mentoring, and advanced clinical skill development. The Colleges program provides a venue for structured learning activities that complement electives taken during the fourth year.

Acad Med. 2020;95:S61-S62.

doi: 10.1097/ACM.0000000000003475

Copyright (C) 2020 by the Association of American Medical Colleges

Supplemental digital content for this article is available at http://links.Iww.com/ ACADMED/A958.

Year school was established: 1951.

School URL: https://medschool.ucla.edu.
Finally, we have a very active student body with over 100 student organizations, interest groups, and affinity groups, many with a high level of commitment to the community and social justice issues. This translates into a variety of campus activities, such as sponsoring meetings both regionally and nationally, as well as engagement across the campus with medical students and other health professions programs. A recent development has been the creation of the Health Equity Forum, a group of students from across the health professions who meet and plan activities in solidarity around addressing health equity and social justice issues. We also have a very active clinical outreach program, including the student-run homeless clinic, a mobile clinic program, and a variety of health fairs and other clinical outreach activities.

\section{Curriculum}

\section{Curriculum description}

See Supplemental Digital Appendix 1-Legacy Curriculum Schematic —at http://links.lww.com/ACADMED/A958.

See Supplemental Digital Appendix 2-Redesigned Curriculum Schematic_at http://links.lww.com/ACADMED/A958.

\section{Curriculum changes since 2010}

We are actively involved in curriculum redesign, which will launch in full in August 2021. Among the key features will be early authentic clinical experiences, required clerkships in the second year, and a third year of discovery, in which students will have the opportunity to pursue research, additional degrees, or other enrichment activities.

\section{Assessment}

Our medical school competencies and objectives are comprehensive and organized into the ACGME domains of competence. Details are available from our website: https:// medschool.ucla.edu/current-graduation-competencies.

We use a variety of assessment methods, including regular weekly formative assessments, performance-based assessments, and traditional knowledge assessments. For many years, we have conducted a multistation clinical performance exam, in collaboration with other schools in California. We have recently modified the standard setting methodology, migrating to a criterion-based standard setting method, using the Angoff approach.

\section{Pedagogy}

At our institution, we use a variety of pedagogic methods. We have only a relatively modest amount of traditional lecture and have for many years used problem-based learning as a major instructional approach. In recent years, we have introduced 
newer methods of case-based learning, including team-based learning and flipped classroom approaches.

\section{Clinical experiences}

We have clinical experiences at our university teaching hospital, a VA hospital, 2 county safety net hospitals, a private hospital, and a Kaiser Permanente facility. This provides a wonderful and diverse set of clinical experiences, with diverse patient populations, and also provides learners with experiences of very different health care delivery systems.

Our learners begin a preceptorship in the second half of the first year, which continues through the second year. They begin their required clinical rotations in the third year.

Students complete their clinical preceptorships, core clerkships, continuity clerkships, and clinical electives at Ronald Reagan UCLA Medical Center and affiliated sites, including 2 county hospitals, the Greater Los Angeles VA System of Clinics, an HMO, a private tertiary care medical center, a charity hospital, and dozens of public and private ambulatory clinics associated with these medical centers or based in the community.

\section{Curricular Governance}

We have a curriculum committee that oversees all aspects of the MD program, the Medical Education Committee. That committee has 4 subcommittees, 1 for our basic science curriculum (human biology and disease), 1 for clerkship the curriculum, 1 for our fourth-year program, and 1 for curriculum CQI.
The vice dean for education oversees all aspects of UME, GME, $\mathrm{CME}$, and graduate and postdoctoral education. This individual is supported by associate deans for curricular affairs, student affairs, admissions, medical student research and scholarship, GME, graduate bioscience education (senior associate dean), and CME (assistant dean). In addition, the following offices support the entire educational affairs enterprise: faculty development, educational measurement unit, simulation center, education technology service, and alumni affairs.

\section{Faculty Development and Support in Education}

In the faculty development realm, we have a longstanding medical education fellowship, which recently has evolved to 2 specific tracks. First is a 1-year program for early-career clinician-educators, which focuses on helping them to become scholarly teachers. In the program, the participants work on an education innovation through the lens of education theory, such as developing high-quality learning objectives and more. The second half of this program is a series of 7 seminars that originated in the internationally acclaimed Stanford Faculty Development Program for Clinical Teachers. Through these seminars, these first-year fellows develop high increase capacity as highly effective teachers. The second-year fellowship is for a select group of faculty seeking to become teaching scholars, and as a research training program done in collaboration with the UCLA Graduate School of Education and Information Studies. Fellows in this program engage in research methods seminars and develop a publication-ready research project over the course of this year and beyond. 


\section{University of California, Riverside School of Medicine}

Brigham C. Willis, MD, Christian Lytle, PhD, Maegen Dupper, MD, Rosemary Tyrrell, EdD, Elizabeth H. Morrison, MD, Kendrick Davis, PhD, Kathy Barton, and Deborah Deas, MD

\section{Medical Education Program Highlights}

The University of California, Riverside School of Medicine (UCR SOM) is a unique, community-based medical school for the 21st century. With our initial class having graduated in 2017, UCR SOM is a newly established medical school, yet has a long-standing history of educating medical students. In a shared degree program that began in 1974, students completed the first 2 years of their education at UCR, then transferred to UCLA. With this program as a foundation, and the need for improved access to medical care in the "Inland Empire," a full 4-year program was built. Committed to its local community, UCR SOM focuses on preparing students for careers working with underserved and rural populations; recruiting, training, and retaining students from the area; embracing innovation; and refining a distributed training model.

- The Longitudinal Ambulatory Care Experience (LACE) bridges the gap between classroom and clinical application by providing students hands-on clinical experience throughout inland Southern California. It replaces the traditional "shadowing" preceptorship with a program emphasizing continuity and progressive learning. Participating medical practices are primary care focused and expose students to ambulatory care within the local community.

- Multiple Designated Emphasis (DE) programs:

- The DE in medical Spanish, also known as HABLAMoS (Hispanic And Bilingual Ambulatory Medical Studies), is a 4-year language learning program that includes classroom instruction and experiential learning opportunities in Spanish-speaking clinics, communities, and family homes in Southern California and Spanish-speaking countries.

- The DE in medical and health humanities allows medical students to examine the individual, social, and historical experience of health, pathology, medicine, and clinical encounters.

- The DE in health care leadership prepares medical students to become future health care system leaders, including deans, CEOs, and CMOs.

- The Early Assurance Program is a unique portal for admission to UCR SOM, providing exceptional, "mission-fit" UCR undergraduate students with a guaranteed seat in a future UCR SOM class. The program benefits applicants by reducing financial and psychological stress of applying to medical school.

Acad Med. 2020;95:S63-S66

doi: 10.1097/ACM.0000000000003321

Copyright (C) 2020 by the Association of American Medical Colleges

Supplemental digital content for this article is available at http://links.lww.com/ ACADMED/A848.

Year school was established: 2012.

School URL: https://medschool.ucr.edu.
- The MD/Master of Public Policy Concurrent Degree Program, offered by UCR SOM and the School of Public Policy, develops leaders who will continue their careers shaping health care policy in the United States.

- The Bringing Education \& Service Together Program is an innovative, longitudinal residents-as-teachers curriculum that has been validated in a randomized, controlled trial.

See Supplemental Digital Appendix 1-Distributed Training Model—at http://links.lww.com/ACADMED/A848.

\section{Curriculum}

\section{Curriculum description}

The first-year curriculum integrates human biology and disease, focusing on an integrated approach to human biological systems. Instruction is driven by cases explored in small groups, laboratories, conferences, clinical skills workshops, and independent study. During this time, students begin their 3-year LACE, where they begin to care for patients under the supervision of a mentor. The second-year curriculum focuses on disease processes, followed by an integrative assessment module. Third-year students gain clinical experience through a longitudinal clinical curriculum in core areas of medicine, surgery, family medicine, pediatrics, obstetrics-gynecology, psychiatry, neurology, and emergency medicine at local medical centers and hospitals. In the fourth year, students complete selectives, electives, and a community-based scholarly project.

\section{Curriculum changes since 2010}

Our 4-year curriculum, like the school, is new. Key changes planned in the near future:

- We are continuing to convert instruction to a more active format while progressively reducing number of lectures, using a flipped classroom model and online content delivery.

- With planned construction of a new medical education building expected to be completed in 2023, we anticipate a cutting-edge design that will facilitate the most innovative educational techniques.

- We plan to increase curricular content focused on leadership, medical humanities, humanism, health care disparities, professional ethics, and the science of health care delivery.

\section{Class size changes since 2010}

Class size is now 77, increased from an initial class size of 50. This modest increase has not had any significant effects, but we plan further increases over several years, continuing our mission to increase the physician workforce in our community. The final class size is not determined yet, but increases will require expansions of partnerships with our clinical affiliates. 


\section{Medical education program objectives}

Our outcomes-based educational program objectives are closely aligned with ACGME delineated physician competencies and the AAMC Physician Competency Reference Set. They are also linked to the learning outcomes and assessment strategies of each instructional session in the curriculum.

See Supplemental Digital Appendix 2-Medical Education Program Objectives—at http://links.lww.com/ACADMED/A848.

\section{Assessment changes since 2010}

- Weekly self-assessments: Each week, students complete selfassessments covering learning content from the past week. All assessments were originally formative. To incentivize attendance in the second year, however, we changed the selfassessments to graded quizzes.

- NBME subject examinations: We have substituted these in lieu of traditional examinations in core clerkships.

- LACE: Quality improvement research is a required part of the LACE curriculum as a practice improvement project. Mandatory graded checkpoints throughout the projects, including written submissions and conference presentations, anchor students' learning experience.

- OSCEs: We use OSCEs across the first 3 years. Standardized patients are now trained and employed as OSCE raters.

\section{Parallel curriculum or tracks}

Given the critical shortage of primary care physicians in the area, we plan to implement a 3-year accelerated curriculum leading to conditional acceptance in our affiliated family medicine residency program, followed by a requirement for 3 years of service in the area.

\section{Pedagogy}

- The preclerkship curriculum integrates and correlates normal human biology with disease processes and clinical skills. We use a blend of formats, including lectures, podcasts, casebased sessions, and small-group discussions, with time for self-directed study outside of class. Woven into each block are skill-building threads (doctoring, clinical skills, problembased learning) that serve to integrate scientific concepts into a clinical context. The Doctoring course includes standardized and simulated patient experiences for practice in history taking, as well as adding challenges in the clinical setting. Operating in parallel, the Clinical Skills course provides students with physical exam practice. Anatomy laboratory instruction (in years 1 and 2) integrates cadaver dissection with peer instruction in ultrasound techniques.

- In addition to direct clinical experience, the LACE program integrates a public health lecture series presenting topics on ethics, medical humanities, and more. Every student is required to complete a LACE practice improvement project, which exposes students to the process of literature review, internal review board mechanisms, and the iterative nature of quality improvement. Students present the results of their projects at the school's annual Conference on Teaching, Learning, and Discovery.
- We supplement clinical experiences in the integrated longitudinal curriculum with weekly case-based readings and required written assignments.

\section{Changes in pedagogy since 2010}

- We are transitioning from faculty-centered to student-centered learning approaches. Our aims were to improve student engagement, curricular alignment, and clinical integration in the second year. We replaced poorly attended lectures with self-study assignments comprising readings, videos, and self-assessments, devoting in-class time to small-group problem-solving sessions led by clinical faculty using a scripted "morning report" format. This revision called on second-year students to acquire a larger amount of information outside of class, and student-teacher interactions now emphasize clinical application. This revision also allowed us to achieve better alignment with our program objectives and USMLE Step 1 examination content outline.

- We eliminated instructor-written exams at the end of each block in year 2 and shifted weighting of block summative scores to the NBME subject examination (65\%) and weekly quizzes (35\%), with the goal of reducing stress and incentivizing students to keep abreast of weekly learning goals.

- We incorporated a radiology thread into each of the 9 preclerkship blocks.

\section{Clinical experiences}

As a community-based medical school, UCR SOM partners with many sites to provide experiences that prepare students for practice within different settings and systems. Within the LACE curriculum in years 1-3, students work 1-on-1 with preceptors at ambulatory primary care sites ranging from single-physician private practices to large medical groups. Third-year longitudinal clerkship experiences occur throughout the community at Riverside University Health System (our county hospital), other regional medical centers, federally qualified health centers, physicians' private practices, and university-based UCR Health outpatient and inpatient settings.

\section{Required longitudinal experiences}

Students begin LACE assigned to a community-based clinic and continue this experience for their first 3 years of medical school. This experience allows students to build continuity with the attending preceptor, patients, and staff. During this time, they also complete a quality improvement project to improve health care in our region.

The third-year curriculum includes 3 required clinically integrated longitudinal clerkship blocks:

- Block A: Internal medicine and surgery

- Block B: Family medicine, obstetrics-gynecology, and pediatrics

- Block C (longitudinally integrated throughout the third year): Emergency medicine, psychiatry, and neurology

This longitudinal structure allows us to give students regular feedback throughout the rotation. 


\section{Clinical experience first encounter}

Students engage in clinical experiences after the first month of medical school through the LACE program, which continues throughout the third-year curriculum.

\section{Required and elective community-based rotations}

As a community-based school of medicine, we provide all required and elective clinical rotations in collaboration with community clinical partners based throughout inland Southern California.

Challenges in designing and implementing clinical experiences for medical students

We have designed UCR SOM's clinical experiences to support our mission to improve the health of medically underserved communities in the region. As we prioritize our community's needs within our training program, we face the same clinician shortages that the rest of our region does. We strive to meet these challenges by continually growing and diversifying a complex array of community partnerships.

\section{Curricular Governance}

See Figure 1-Curricular governance.

\section{Education Staff}

\section{Medical education leadership}

- The Office of Medical Education, led by the senior associate dean for medical education, oversees and manages the undergraduate medical education curriculum. Providing additional leadership are associate deans in 4 areas: preclerkship curriculum, clerkship curriculum, quality improvement, and assessment and evaluation.

- Under the University of California's shared governance structure, the school's 36-member Medical Education Committee designs, approves, and oversees the curriculum.

- Professional staff members support scheduling and logistics, coordination of activities at community-based clinical sites, medical simulation instruction, anatomy, information and instructional technology, and curriculum evaluation and assessment.

See Figure 2-Medical education leadership.

Administrative faculty and staff in the Office of Medical Education have sole responsibility for undergraduate medical education. Premedical and graduate medical education programs are administered by the Office of Student Affairs and Office of Graduate Medical Education, respectively.

\section{Faculty Development and Support in Education}

Professional development for faculty as educators

The Office of Faculty Development (OFD) provides many programs, including the Teaching Excellence Academy, a professional certificate in medical education, workshops, webinars, online support, and new faculty orientation. Many are offered as longitudinal programs, including the Clinical Teaching Series, the Teaching Essentials Series, and the Quality Improvement and Patient Safety Series. Special events offered to faculty include the annual Conference on Teaching, Learning, and Discovery, and Women in Medicine and Science events. The

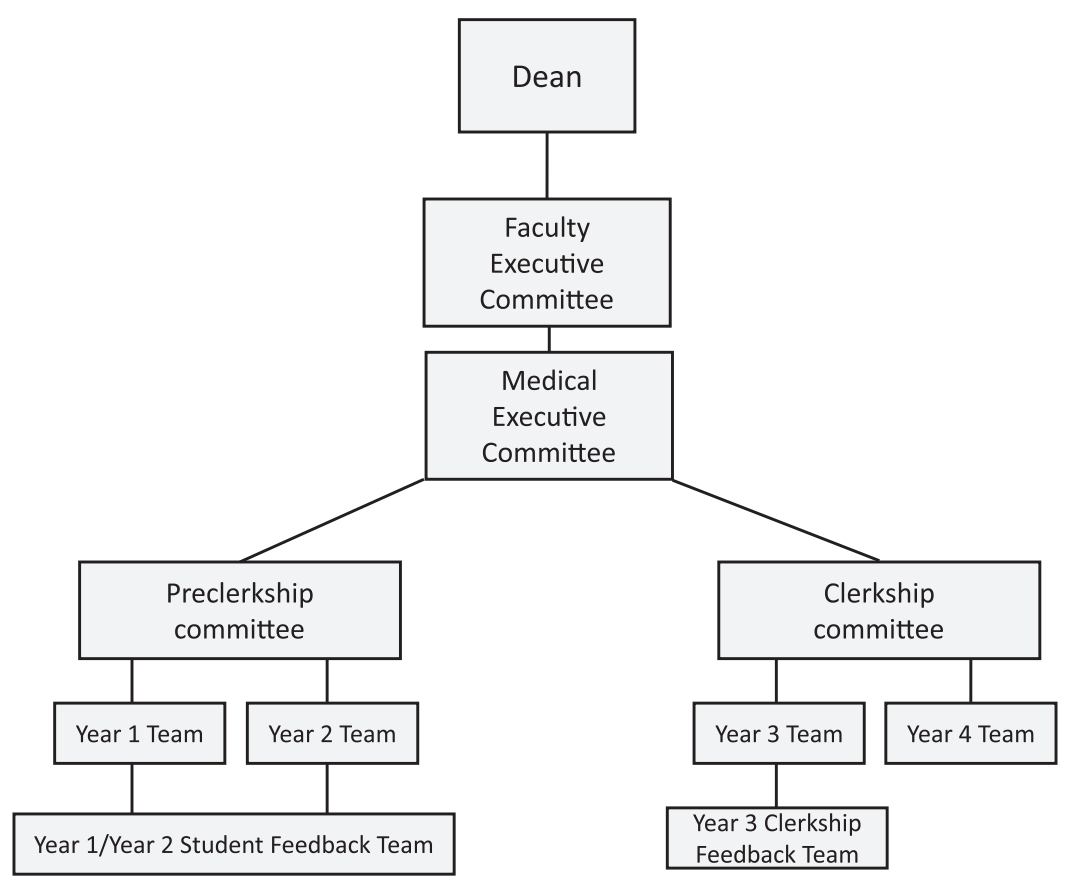

Figure 1 Curricular governance. 


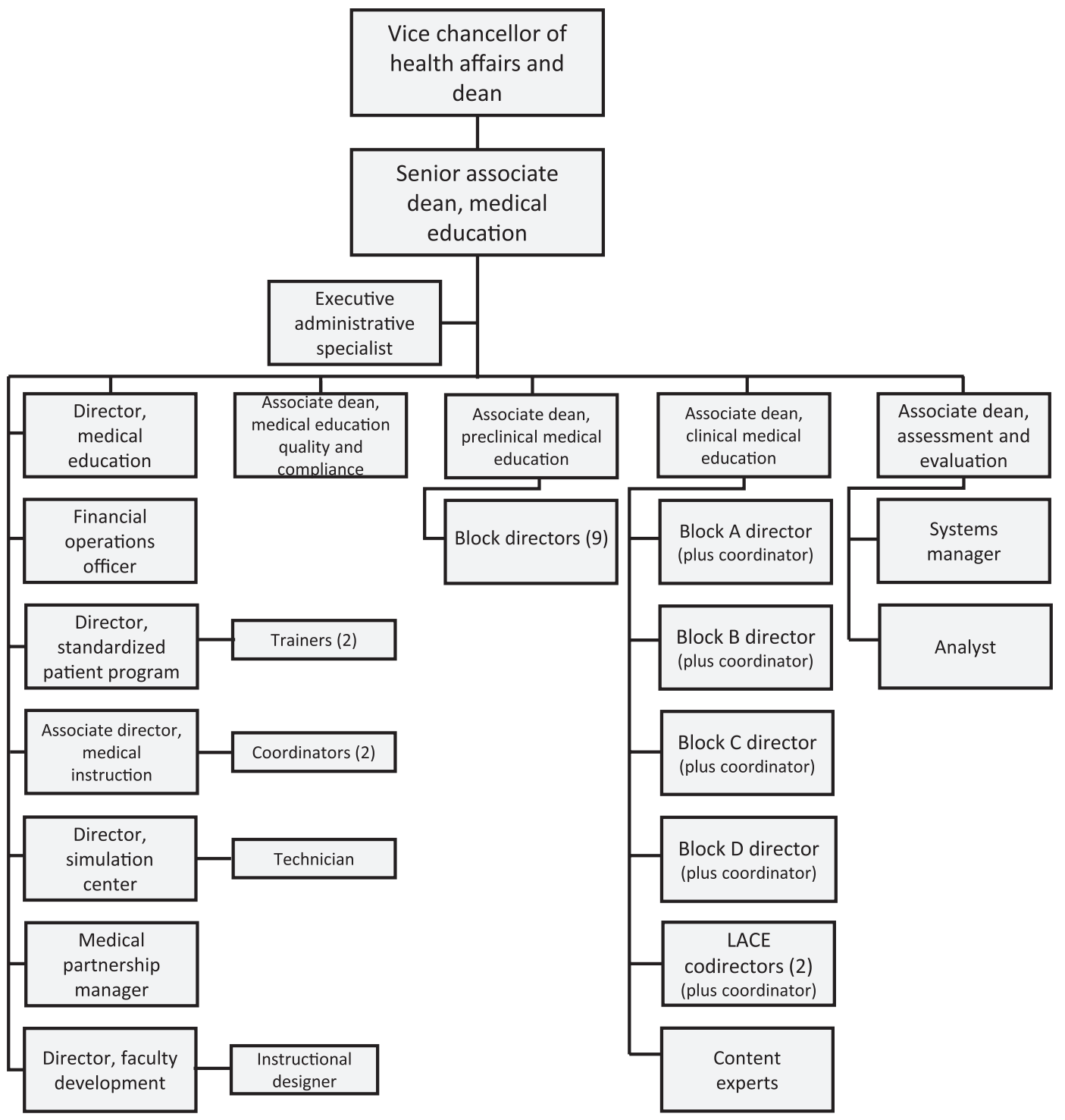

Figure 2 Medical education leadership.

OFD also offers 1-on-1 consultation, classroom observation, and instructional design services.

\section{Role of teaching in promotion and tenure}

Evidence of quality in teaching is an essential criterion for appointment, advancement, or promotion. In judging the effectiveness of a candidate's teaching, the committee considers the candidate's command of the subject; continuous growth in the subject field; ability to organize material and to present it with command of the subject matter and logic; capacity to awaken in students an awareness of the relationship of the subject to other fields of knowledge; fostering of student independence; spirit and enthusiasm; ability to arouse curiosity and encourage high standards; personal attributes as they affect teaching and students; participation in the general guidance, mentoring, and advising of students; and effectiveness in creating an academic environment that is open and encouraging to all students, including development of strategies for the advancement of students in various underrepresented groups.

\section{Teaching academy}

The Teaching Excellence Academy is the flagship program of the OFD. Each year, 10 faculty members are selected to participate in a community of practice learning experience. In addition to attending six 3-hour sessions, participants engage in a scholarly teaching project and present their project at the Conference on Teaching, Learning, and Discovery. The academy is open to anyone with a UCR SOM faculty appointment. Participants must be nominated by either the chair of their department, a member of the leadership team, or graduates from the academy. 


\title{
University of California, San Diego School of Medicine
}

\author{
Maria C. Savoia, MD
}

\section{Medical Education Program Highlights}

- UC San Diego School of Medicine (UCSD SOM) trains students to become physicians who are scientifically astute, clinically skilled, empathetic, and dedicated to the welfare of patients and communities.

- A major revision of the preclinical curriculum took place in 2010. Called the Integrated Scientific Curriculum, it has been highly successful in increasing student satisfaction while maintaining high standards. Clinical immersion begins soon after students matriculate and scientific concepts are reintroduced in the fourth year.

- Recent additions to the curriculum include a longitudinal ultrasound course beginning in year 1 and a fourth-year boot camp to prepare students for the transition to residency.

- The addition of a new 100,000-square-foot medical education building that contains an 18-room professional development center, a large simulation center, an operating suite with 22 fully functioning operating tables, 2 DaVinci robots, a 15 -station microsurgery laboratory, and a hybrid OR has enriched the student experience and allowed students to learn alongside residents and practicing physicians.

- UCSD SOM is one of the few medical schools with an extensive elective curriculum in the first 2 years, which results in diverse educational opportunities. More than 50 elective courses are offered each quarter. Through the elective curriculum, students can participate in our student-run free clinics, lobby the California state legislature, learn to be more observant through drawing the human body, do self-directed, in-depth reading about a scientific topic, or work alongside faculty doing basic or clinical research. An independent study project has always been a graduation requirement.

\section{Curriculum}

\section{Curriculum description}

See Supplemental Digital Appendix 1-Curriculum Map-at http://links.lww.com/ACADMED/A934.

\section{Curriculum changes since 2010}

- Major curricular revision began with faculty retreats in 2008. First-year students entered the new curriculum in 2010. In response to student and faculty feedback, the curriculum has continued to evolve each year.

Acad Med. 2020;95:S67-S69.

doi: 10.1097/ACM.0000000000003446

Copyright (C) 2020 by the Association of American Medical Colleges

Supplemental digital content for this article is available at http://links.Iww.com/ ACADMED/A934.

Year school was established: 1968.

School URL: https://medschool.ucsd.edu.
- Greater emphasis on an organ systems approach has led to an integration of instruction in anatomy and pharmacology across year 1, and the curriculum now consists of both courses and threads.

- Lecture time has been substantially reduced, and small-group active learning sessions have increased.

- First-year students are divided into 6 learning communities, and they remain in the same community throughout 4 years. Students remain within their Communities for the Clinical Foundations course, where they learn about the doctor-patient relationship and clinical skills, but they interact with other community students throughout the rest of the curriculum.

- Core clerkships begin in mid-May of year 2. Pilot projects to improve continuity and clinical skills in the third-year curriculum have been successfully introduced and are expanding.

- Both the fourth-year Principles to Practice course and a new Transitions to Residency boot camp course prepare students for graduate medical education.

- Class size has recently increased from 122 to 134 without a need for any major structural changes.

\section{Medical education program objectives}

The SOM's curricular objectives are defined by the faculty and are reassessed each year.

See Supplemental Digital Appendix 2-Competencies, Objectives, and Outcome Measures_at http://links.lww.com/ACADMED/A934.

In addition to standard course evaluation, a small group of students meets with course instructors and facilitators from Medical Education at the end of each course to provide feedback, a technique that has been highly successful in promoting curricular change.

\section{Parallel curriculum or tracks}

UCSD SOM has 2 separate curricular tracks:

- The Program in Medical Education-Health Equity is part of a system-wide effort at the University of California to train physicians to be better able to meet the needs of the diverse Californian populations who are traditionally underserved by the medical system. Students have required electives in the first 2 years and are supported for an additional year to complete a master's degree to augment their skills. Numerous opportunities to work in underserved communities exist throughout all 4 years.

- The Global Health Academic Concentration exposes students to global health topics throughout all 4 years of medical school. Students are paired with a research mentor based on their academic interests and participate in global health research during the summer between years 1 and 2. Additionally, students participate in a quarter long global health clinical rotation during the fourth year. These international rotations are financially supported by the SOM. 


\section{Pedagogy}

- A wide variety of pedagogical approaches are used throughout the curriculum, including lectures; laboratories; small- and large-group activities; case- and team-based learning; selfdirected learning; and simulation using standardized patients, mannequins, and partial task trainers. All lectures are podcast. Clinical immersion begins early in year 1 . None of these approaches are new, but emphasis has shifted from lecturebased to more active learning modalities.

\section{Clinical experiences}

- During the clinical curriculum, students are exposed to a wide variety of patients in different clinical settings, both inpatient and outpatient. Students rotate through the 2 UCSD Healthcare major hospitals (UCSD Hillcrest and Jacobs Medical Center), the San Diego Veterans Affairs Medical Center, Balboa Naval Hospital, and Rady Children's Hospital. Kaiser, Sharp, and Scripps health care systems also are options for some rotations.

- In 2016, the model for clinical faculty compensation changed to one based on RVU generation, with discretion of allocation of funds to support teaching based on individual divisional/ department plans. This has generally resulted in faculty feeling they have less time to teach and students feeling they have less exposure to faculty.

- A new funding model designed to make faculty "whole" and provide for any lost revenue because of time spent teaching is currently being designed and should be in place during the next academic year.

\section{Curricular Governance}

- In the University of California system, the faculty are in charge of the curriculum. Deans and other administrators serve ex officio and facilitate the work of the faculty.

- The governance structure overseeing education has been in place since the school's inception and functions well.

- A Committee on Educational Policy (CEP) oversees all education in the SOM, which includes undergraduate medical education, graduate medical education, graduate education, and allied health professional education. The chairs and vice chairs of the Core Curriculum Committee (CCC) and Electives Committee (EC), as well as representatives of the Admissions Committee, the Student Affairs Committee, the Standings and Promotions Committee, Skaggs School of Pharmacy and Pharmaceutical Sciences, and a student elected by each of the 4 medical school classes, are members. It meets monthly.

- The CCC oversees all required coursework in years 1 to 4 and meets twice a month. Courses are reviewed in-depth every 3 years using a very structured format.

- The EC, which meets monthly, approves and reviews the electives offerings in the first 2 years and all fourth-year electives, in addition to approving all independent study project proposals.

- The CEP is a subcommittee of the University's Academic Senate, the body that approves changes in grading policy and graduation requirements. The chair and vice chair of the CEP are members of the Health Sciences Faculty Council, which consists of elected representatives of the faculty and other major committee chairs.

\section{Education Staff}

- The support for undergraduate, graduate, and continuing physician education in the SOM resides in the Dean's Office Division of Medical Education (DME), which is led by the dean for medical education.

- Within the DME, units supporting undergraduate medical education include the Office of Educational Support Services (OESS), headed by an assistant dean, and the Office of Educational Technology, Innovation, and Assessment (OETIA), led by an associate dean. OESS provides support to faculty in the delivery of the curriculum and assistance to students as they strive to understand it. Staff in OETIA support the technologic and programming needs of students and teaching faculty, and design and implement course and individual student and faculty evaluation while working with faculty to use state-of-the-art technology in instruction. Staff in OETIA do all the conference and classroom room scheduling for the SOM and UCSD hospitals.

- The professional development center and simulation center (headed by an associate dean) also are part of the DME and are heavily used in the education of medical students, residents, fellows, and practicing physicians.

- Additional resources for undergraduate medical education are centralized in the Office of Undergraduate Medical Education, headed by an associate dean. All committee, teaching laboratory, and administrative curricular support emanates from this office.

- The DME also contains the support for admissions and student affairs, financial aid, community outreach and diversity (headed by an associate dean and assistant dean), graduate medical education (led by an associate dean and ACGME designated institutional official), continuing medical education and educational faculty development (headed by an associate and assistant dean), alumni affairs, and the willed body program.

- The DME has a central Administrative and Business Office, headed by an assistant dean. Centralizing resources in the DME has allowed them to be used efficiently. Whenever possible, funds and equipment are leveraged, and personnel are crosstrained in support of the continuum of education.

See Figure 1-Medical education leadership.

\section{Faculty Development and Support in Education}

- Both the Office of Faculty Affairs and the DME provide support to faculty to help them in their roles as educators.

- The National Center for Leadership in Academic Medicine trains 25-30 junior faculty each year to become successful academicians. A project is required; several faculty each year choose educational projects that they complete with the help of a faculty mentor. 


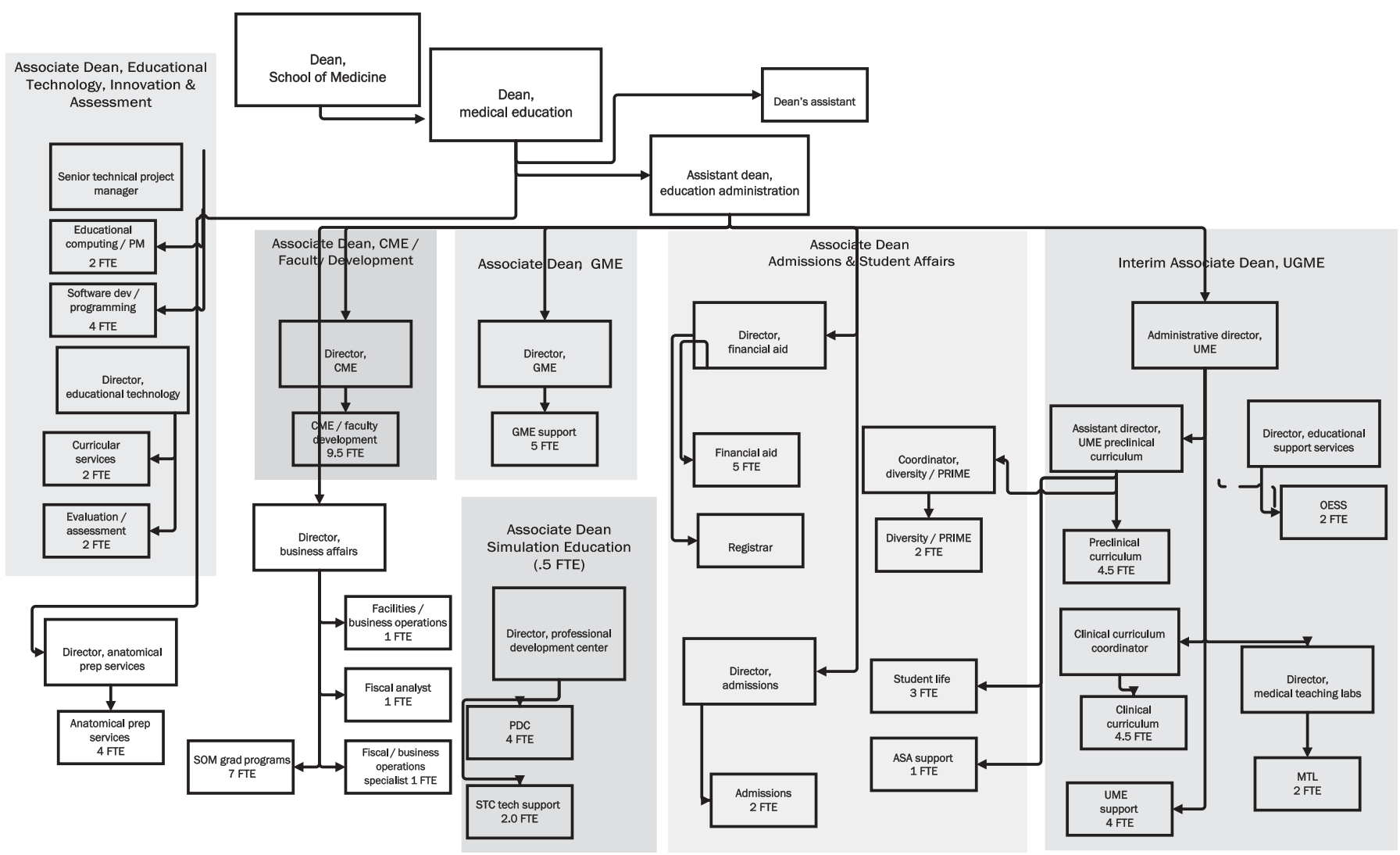

Figure 1 Medical education leadership.

- The Continuing Medical Education and Educational Faculty Development Office supports medical education grand rounds, multiple faculty workshops throughout the academic year, and a yearly faculty educational retreat.

- The SOM has the Academy of Clinician Scholars, which is primarily intended to support excellent academic clinicians, including clinician-educators. Funds are available to support educational pilot projects and educational research.

- Teaching is an important component of academic advancement in all faculty series in the SOM. Although strong teaching evaluations are not sufficient for promotion in any series, poor teaching evaluations have held back faculty from advancement. 


\section{University of California, San Francisco School of Medicine}

Catherine R. Lucey, MD, Karen Hauer, MD, PhD, Patricia O'Sullivan, EdD, Ann Poncelet, MD, Kevin H. Souza, MS, and John Davis, MD, PhD

\section{Medical Education Program Highlights}

In 2016, the University of California, San Francisco (UCSF) School of Medicine launched the Bridges Curriculum, developed to ensure that graduates are prepared to tackle the most complex problems of 21 st-century patients and communities. The curriculum has an enduring focus on providing evidence-based, compassionate care while leveraging emerging skills in systems science and new methods of discovery. The Bridges Curriculum seeks to instill the 4 UCSF physician habits of mind: inquiry, continuous improvement, adaptive leadership, and social justice.

To teach these habits of mind, the curriculum has an inquiry thread focusing on deep exploration into a scholarly area. Each step advances the learner, first to a sophisticated consumer of biomedical science, then to a producer of new knowledge.

Continuous improvement skills are instilled through the clinical microsystem clerkship (CMC), a longitudinal clinical skills curriculum integrating skills in direct patient care, health systems improvement, and interprofessional collaboration. The CMC engages students in systems improvement work directly affecting the quality and safety of care.

During the core clerkship phase, we offer a series of clinical immersion experiences (CIExes) electives, which provide opportunities for deeper exploration of a specialty or subspecialty.

Trained and dedicated faculty "coaches" support students' progression. The coaching program is designed to provide academic guidance for students and support professional/ personal development throughout the curriculum.

\section{Curriculum}

\section{Curriculum description}

The Bridges Curriculum is a 4-year, 3-phase curriculum:

- Foundations 1 (F1) is the preclerkship phase, including foundational sciences coursework and early, robust instruction and practice of health systems sciences (CMC).

Acad Med. 2020;95:S70-S73.

doi: 10.1097/ACM.0000000000003469

Copyright $\odot 2020$ by the Association of American Medical Colleges

Supplemental digital content for this article is available at http://links.Iww.com/ ACADMED/A959.

Year school was established: Founded as Toland Medical College in 1864. Affiliated with the University of California in 1873.

School URL: https://medschool.ucsf.edu.
- Foundations 2 (F2) starts in December, year 2, with six 8-week blocks for 8 core clerkships and 8 weeks of CIExes.

- Career launch starts in March of year 3, including a longitudinal ambulatory subinternship, dedicated time for completing scholarly projects, and advanced clinical training preparing students for their chosen career paths.

Other curricular characteristics:

- Early, robust instruction and practice of health systems science (CMC)

- Incorporation of a dedicated coaching program, integrated with the CMC during the preclerkship phase, embedded in a longitudinal curriculum in assessment, reflection, coaching, and health $(\mathrm{ARCH})$ across all 3 phases

- Deliberate instruction with dedicated time in concepts and application of inquiry in scientific domains

- Intentional incorporation of elements of social justice into all curricular aspects, including a planned required rotation demonstrating these principles in the community-engaged context

- Collaborative integration of foundational science content into clerkships

\section{Curriculum changes since 2010}

The rollout of the Bridges Curriculum will be complete in May 2020, a significant evolution of our curricular content and pedagogy.

Structural changes within the 4-year timeline include:

- A shortened preclerkship phase (ends December, year 2)

- Earlier start of clerkship phase (January, year 2)

- Longitudinal structure of the family and community medicine clerkship

- Shift in placement of USMLE Step 1 examination to after core clerkships (January, year 3)

- Earlier start of postclerkship phase (March, year 4)

Over the coming year, we will also adjust delivery of foundational science content.

\section{Class size changes since 2010}

In AY 2019-2020, UCSF became the sponsoring institution for the San Joaquin Valley Program in Medical Education (SJV PRIME), serving central California communities. The class size increased by 6 students for the first academic year's entering class and will subsequently rise to 12 . These students have been incorporated into class structures with minimal change required in structure or function, primarily because their clerkship and postclerkship phases take place at UCSF Fresno, where they had been placed previously for clerkships while managed by a different institution. 


\section{Assessment}

Medical education program objectives are based on ACGME domains of competence, with the addition of interprofessional collaboration as a separate domain.

See Supplemental Digital Appendix 1-Program Objectives and Assessment Methods__ at http://links.lww.com/ACADMED/A959.

\section{Assessment changes since 2010}

UCSF has implemented programmatic assessment in the F1 phase of the curriculum and is in the process of implementing programmatic assessment in core clerkships, F2. Implementation of programmatic assessment aims to support students' learning across the curriculum and ensure achievement of expected competence. The assessment system emphasizes students' development of reflection, learning, and planning skills in close coordination with their coaches throughout the 4-year curriculum. Students and coaches have ready access to performance data in an individual electronic student dashboard containing visual displays of student progress compared with expected benchmarks and class averages. The dashboard includes score reports from summative assessments and students' reflections and learning goals.

In the core clerkships, the school eliminated tiered (honors) grades in January 2019. This decision was made after a multiyear effort to explore and address concerns about accuracy, fairness, and equity of clerkship grades and the impact of grading on students' learning and well-being. Clerkships are now graded Pass/Fail, with a new requirement for 2 weekly work-based assessments for formative feedback, completed collaboratively by a faculty/resident supervisor working with the student.

\section{Parallel curriculum or tracks}

UCSF has 5 parallel tracks:

- Joint Medical Program is a 5-year integrated MS-MD program for 16 students who begin their education at UC Berkeley and transfer to the UCSF campus for clerkships and senior year.

- Program in Medical Education for the Urban Underserved is a 5-year track accepting 12 students annually. Students take a year off to pursue a master's degree in a field enhancing their leadership ability in the care of vulnerable populations.

- SJV PRIME is a 4-year track recruiting 12 students from the Central Valley of California committed to addressing health care disparities in this underserved area. Students participate in their preclerkship years at UCSF in San Francisco, then relocate to Fresno to complete clinical studies.

- The Medical Scientist Training Program is the UCSF MDPhD program, accepting 12 students yearly into a T32 and institutionally supported combined program.

- The Oral Maxillofacial Surgery (OMFS) program accepts 4 students with DDS degrees into an integrated MD-OMFS residency program.

\section{Pedagogy}

The core curriculum and 5 parallel tracks use these pedagogical approaches:

- Case-based learning

- Clinical experience: ambulatory

- Clinical experience: inpatient

- Discussion: large group (>12)

- Discussion: small group $(\leq 12)$

- Laboratory

- Lecture

- Peer teaching

- Preceptorship

- Problem-based learning

\section{Changes in pedagogy since 2010}

Our work since 2010 has been in identifying the balance between instructional strategies and content to best help learners with cognitive integration, particularly in the contentdense, preclerkship phase.

\section{Clinical experiences}

Clinical sites represent the spectrum of communities for which we provide care, including urban and rural, primary and tertiary/ quaternary, ambulatory, and inpatient sites.

\section{Required longitudinal experiences}

The Bridges Curriculum has numerous longitudinal experiences:

- Longitudinal small groups to support learning for each key element in the preclerkship phase

- Longitudinal delivery of the family and community medicine core clerkship

- 3 longitudinal integrated clerkships

- Longitudinal, ambulatory experience during the postclerkship phase (specialty practice ambulatory subinternship)

- A longitudinal curriculum $(\mathrm{ARCH})$ delivered at key touchpoints across all 3 phases

\section{Clinical experience first encounter}

Students first enter the clinical environment in the third week of the preclerkship phase, during the CMC. This experience starts with a focus on health systems and systems improvement, gradually introducing patient care skills.

\section{Required and elective community-based rotations}

All core clerkship rotations have community-based sites; many clinical electives are also offered at community-based sites. The San Francisco Veterans Affairs Health System is a key affiliate, and over $75 \%$ of students rotate there at some point during medical school.

\section{Challenges in designing and implementing clinical experiences for medical students}

The primary limitation on students' clinical experiences comes from increased demands placed on limited clinical sites by learners from other domestic and international institutions, and across the health care training spectrum. 


\section{Curricular Governance}

The faculty committee with primary curricular responsibility is the Committee on Curriculum and Educational Policy (CCEP), a standing committee of, and deriving its authority from, the Council of the Faculty. The CCEP accomplishes its work through a group of subcommittees: an executive committee, subcommittees focused on operations during the 3 phases, Mapping and Integration Committee, and Student Governance Committee. Each committee has LCME standards of primary responsibility and meets monthly. The executive committee is responsible for operational management of and reporting on CQI through reviewing operations reports and program evaluation data.

\section{Decentralized curricular governance}

None of our governance is managed at the department level. Clerkship directors and staff are funded through a centrally managed budget for curriculum leadership.

\section{Education Staff}

The Medical Education Unit oversees the continuum of medical education, under the vice dean for education and associate dean for medical education (ADME). The ADME oversees all staff, including the medical student programs. The structure includes a central office under the chief of staff, which oversees accreditation, financing, staff engagement, affiliations, communications, and overall strategic support. The ADME oversees educational technology, data and analytics services, and simulation and anatomy centers.

Medical student programs are organized under the associate deans for admissions, students, curriculum, and assessment. Student services provides daily support for students and career advising. The Assessment, Curriculum, and Evaluation Unit oversees curriculum support and clinical phase coordination. It also supports required scholarly activities (inquiry curriculum), student coaching program, and CQI of the curriculum under the director of program evaluation.

\section{Medical education leadership}

The dean provides overall leadership for the School of Medicine. Reporting to the dean is the executive vice dean and vice dean for education, responsible for the medical education continuum. There are 6 associate deans reporting to the vice dean for education:

- Associate dean for medical education: Responsible for staff, program development and support, communications, physical space, technology, finance

- Associate dean for admissions: Responsible for admissions process, student scholarships, financial aid

- Associate dean for students: Responsible for the medical student experience and assisting students and faculty with issues with student activities, supports, career planning, professional development
- Associate dean for curriculum: Responsible for the medical student curriculum, including foundational sciences, clinical and systems sciences, the inquiry curriculum, and curriculum governance and CQI

- Associate dean for competency assessment and professional standards: Responsible for student assessment, including competencies and milestones, and the MSPE; also oversees the coaching program, which provides nonevaluative longitudinal student support

\section{Department of Medical Education}

Medical education staff support the medical education continuum: outreach and postbaccalaureate preparation for medical school, admissions, medical student curriculum, GME, continuous professional development, educational technology, and simulation and anatomy-based instruction. Central staff also support multiple student support services.

The Office of Medical Education includes the Center for Faculty Educators (CFE), which houses our Academy of Medical Education (AME), faculty develop programs, and educational research. The center includes faculty directors of the academy and of faculty development and educational research.

\section{Faculty Development and Support in Education}

\section{Professional development for faculty as educators}

The CFE hosts the AME and an award-winning faculty development program, recipient of an ASPIRE to Excellence Award. Faculty can view online orientation resources for the Bridges Curriculum and coaches receive tailored faculty development. We are initiating workplace-based faculty development through the Learning and Caring Ecosystem program. Faculty can participate in "Teach for UCSF" certificates in general, clinical, simulation, interprofessional, quality improvement and patient safety, and equity and inclusion teaching as well as educational leadership. Education-focused faculty can apply to the longitudinal Teaching Scholars Program and advance to master's and doctoral degrees in health professions education through external partners.

\section{Role of teaching in promotion and tenure}

Faculty must demonstrate quality teaching. Peer observation of teaching can be submitted as data for promotion and tenure application. Clinical educators must show engagement in creative activity and/or dissemination of educational scholarship and/or substantial educational leadership and can be promoted through the University of California "clinical X" series. Educators can embed a teaching portfolio into their $\mathrm{CV}$.

\section{Academy for Medical Educators}

Since 2000, the AME has supported the educators who carryout and advance UCSF's education mission through community, diversity, advocacy, service, and innovation. The AME currently has 163 members from medicine, dentistry, nursing, and pharmacy, selected by a rigorous application process. Programs include education grants, endowed chairs (24), teaching awards, 
quarterly meetings/workshops, an education showcase, teacher observation program, and UCSF-wide initiatives to address diversity/equity/inclusion, wellness, learning climate, and education finance.

\section{Regional Medical Campuses}

Directors of both campuses are ex officio members of the CCEP, which oversees educational programming.

See Table 1-Regional Medical Campuses.

\section{Educational experiences across sites}

Directors of each program meet quarterly with main campus associate education deans to review curriculum, assessment,

\section{Table 1}

Regional Medical Campuses

\begin{tabular}{llr} 
Regional campus name & Type & $\begin{array}{r}\text { Student } \\
\text { enrollment }\end{array}$ \\
UC Berkeley School of Public Health & Preclerkship & 16 \\
ÜCSF San Joaquin Valley & Clinical & $6-12$ \\
\hline
\end{tabular}

student experience, and progress. Each regional campus submits an annual report to the CCEP demonstrating equivalency in student satisfaction, outcomes, and competency. Memorandums of understanding between main and regional campuses are reevaluated and renewed every 5 years. 


\section{The Frank H. Netter MD School of Medicine at Quinnipiac University}

Lyuba Konopasek, MD, Lisa D. Coplit, MD, and Kim-Thu C. Pham, MD, MPH

\section{Medical Education Program Highlights}

Named for the well-known surgeon and medical illustrator, the Frank H. Netter MD School of Medicine at Quinnipiac University (Netter) first matriculated students in 2013 and graduated its inaugural class in 2017. Netter's focus on student education drives all strategic decisions including budgetary allocations and faculty hires. Faculty members in the Department of Medical Sciences are supported to dedicate the majority of their time to teaching, mentoring, and curriculum development. The school is located on an interprofessional campus in North Haven, Connecticut, with the Quinnipiac University (QU) schools of education, health sciences, nursing, and law. Netter's state-of-the-art facilities are custom designed to promote active, collaborative learning and include small-group learning settings and extensive clinical skills training facilities adaptable to evolving learning modalities and technologies.

Netter's commitment to active learning is aligned with its vision of a vibrant health care community to educate and nurture diverse, compassionate physicians who promote wellness and patient-centered care for all of society. Faculty foster the students' growth mindset and responsibility to patients, peers, and colearners. The school shares QU's commitment to the values of academic excellence, a student-oriented environment, and a strong sense of community. These shared values are evident in congruent, linked strategic priorities.

See Figure 1-Mission, vision, and values.

At Netter, basic and clinical science courses are designed to shift the focus from "what is taught" to "what, where, and how students learn." Courses are integrated horizontally and vertically through multiple pedagogies to promote learning, retention, and retrieval. The Scholarly Reflection and Concentration/Capstone (SRCC) course requires every student to select an area of concentration and to complete a capstone project. Students select concentrations in the following:

- Global, public, and community health

- Health policy and advocacy

- Health management and leadership

- Health communications

- Medical education

Acad Med. 2020;95:S74-S78.

doi: 10.1097/ACM.0000000000003276

Copyright (C) 2020 by the Association of American Medical Colleges

Supplemental digital content for this article is available at http://links.Iww.com/ ACADMED/A825.

Year school was established: 2012.

School URL: https://www.qu.edu/schools/medicine.html
- Translational, clinical, and basic sciences

- Medical humanities

- Interprofessional education and practice

The SRCC course extends over 4 years and allows learners to advance a personal interest and develop professionally through exposure to narrative medicine and research mentorship.

Another important element of Netter's student-centered and growth-oriented education model is a comprehensive, multistep student advising program that spans all 4 years. The structure for advising builds on Netter's student-driven "Big Sib" program for entering students and encompasses learning communities, academic coaches, career advisors, and specialty-specific mentors. An academic coach is assigned to each "sibling" cohort and supports students through early transitions, reflection, and goal setting. In the third year, cohorts are transferred to career advisors who support each student through specialty selection, development of a fourth-year schedule, and residency application. Students are encouraged to consult and work with specialty-specific mentors early in the advising process.

The majority of Netter's clinical experiences, including the Medical Student Home (MeSH) longitudinal curriculum in the first and second years, and clerkships and electives in the third and fourth, occur in community settings, made possible through an extensive network of affiliated hospitals and physician offices. Exposure to diverse settings and practice styles enrich student learning, and the clinical affiliates are strengthened by the presence of Netter students and engagement with the school of medicine. A rural longitudinal integrated clerkship at the Northern Maine Medical Center in Fort Kent, Maine, was initiated in academic year 2019-2020 and hosts 4 students annually.

In 2018, Netter expanded its educational footprint as an ACGME-accredited sponsoring institution for GME. Incorporating residency programs has provided benefits for UME and GME programs by expanding the clinical network for students, engaging more residents in teaching medical students, and enhancing the quality of both clerkships and residency programs through the provision of additional learning resources and professional development activities.

The Office of Faculty Development has been critical to successful implementation of new pedagogies, assessment strategies, and the professional growth of faculty members as educators in a new medical school.

Netter's Institutes of Primary Care, Global Public Health, and Rehabilitation Medicine broaden the school's capacity for addressing critical health care issues. Global public and community health themes are integrated throughout the 

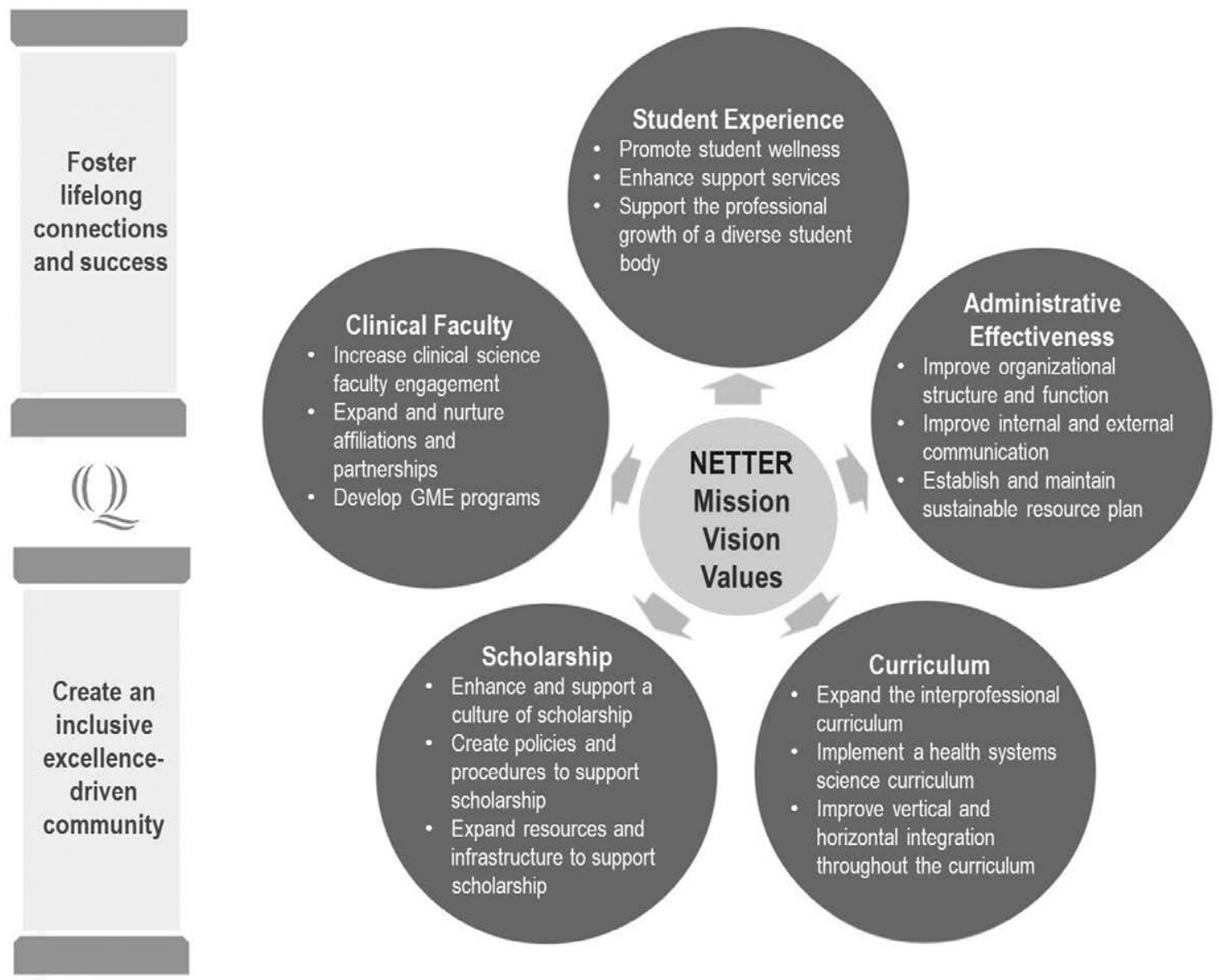
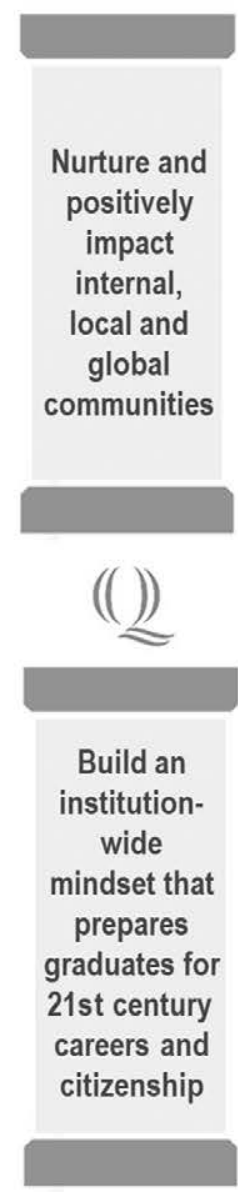

Figure 1 Mission, vision, and values.

curriculum. Collectively, the 3 institutes are designed to improve care, generate new knowledge, and promote student learning in interprofessional fields with high relevance to medical practice in the 21 st century.

\section{Curriculum}

\section{Curriculum description}

The Netter curriculum is holistic in scope, with content including prevention, population health, complementary medicine, and health systems science incorporated intentionally into discussions of patient care. Longitudinal themes encompassing medical humanities, pharmacology, epidemiology, ethics, nutrition, and socio-behavioral science anchor content throughout the curriculum.

The school's curriculum and educational program objectives (EPOs) are based on ACGME and CanMeds competencies. EPOs are framed in outcomes-based terms that facilitate assessment of student progress in developing the knowledge, skills, and attitudes expected of physicians.

See Table 1-Competencies, Sample Educational Program Objectives, and Assessment Methods.
The preclerkship curriculum consists of 3 courses: Foundations of Medicine (FOM), Clinical Arts and Sciences (CAS), and SRCC. Basic and clinical science block content is integrated within and across the 3 core courses and organized around organ systems. The clerkship years include clerkships, electives, and continuation of SRCC.

See Supplemental Digital Appendix 1-Curriculum Map—at http://links.lww.com/ACADMED/A825.

Multiple pedagogies throughout the 4 years focus on active learning. FOM features numerous small-group sessions, including weekly 2-hour case-based discussions embedding basic science in clinical reasoning. A novel social pedagogybased pharmacology curriculum allows first-year students to acquire and apply an interpretive conceptual framework for comprehension, analysis, and synthesis of drug information. CAS instructional methodologies include extensive experiential learning in weekly small-group sessions and examination of standardized patients, a longitudinal 2-year clinical immersion experience $(\mathrm{MeSH})$, and ultrasonography workshops taught in an interprofessional education format with ultrasonography technician students. The pediatric and surgery clerkship 
Table 1

Competencies, Sample Educational Program Objectives, and Assessment Methods

\begin{tabular}{|c|c|c|}
\hline Competency & Sample EPO & Assessment methods \\
\hline Care of individual patients & $\begin{array}{l}\text { Gather medical histories from patients/families; attend } \\
\text { to patient symptoms, concerns, expectations, and illness } \\
\text { experience }\end{array}$ & $\begin{array}{l}\text { Global performance evaluations, multiple-choice } \\
\text { questions (MCQs), OSCEs/FOSCEs, patient write-ups, } \\
\text { patient/procedure logs, self-assessments, small-group } \\
\text { evaluations, WBAs }\end{array}$ \\
\hline Professionalism & $\begin{array}{l}\text { Demonstrate honesty, integrity, and respect in } \\
\text { interactions with patients, colleagues, and faculty }\end{array}$ & $\begin{array}{l}\text { Global performance evaluations, critical incident } \\
\text { reports, narrative medicine evaluations, self- } \\
\text { assessments, small-group evaluations }\end{array}$ \\
\hline Knowledge and scholarship & $\begin{array}{l}\text { Describe essential concepts in human biology, including } \\
\text { molecular, biochemical, genetic, immunologic, and } \\
\text { cellular mechanisms }\end{array}$ & $\begin{array}{l}\text { Global performance evaluations, MCQS, self- } \\
\text { assessments }\end{array}$ \\
\hline $\begin{array}{l}\text { Interpersonal and } \\
\text { communication skills }\end{array}$ & $\begin{array}{l}\text { Communicate patient data to other health professionals } \\
\text { through oral presentations and written and electronic } \\
\text { medical records }\end{array}$ & $\begin{array}{l}\text { Global performance evaluations, OSCE/FOSCES, self- } \\
\text { assessments, small-group evaluations }\end{array}$ \\
\hline $\begin{array}{l}\text { Practice-based learning and } \\
\text { improvement }\end{array}$ & $\begin{array}{l}\text { Use information technology effectively, including } \\
\text { acquiring, storing, retrieving, and analyzing medical data }\end{array}$ & $\begin{array}{l}\text { Evaluation of projects, global performance evaluations, } \\
\text { MCQs, self-assessments, small-group evaluations }\end{array}$ \\
\hline Systems-based practice & Identify factors that contribute to health care disparities & $\begin{array}{l}\text { Evaluation of projects, global performance evaluations, } \\
\text { MCQs, small-group evaluations }\end{array}$ \\
\hline Interprofessional collaboration & $\begin{array}{l}\text { Engage in real and simulated patient experiences with } \\
\text { health professionals from other disciplines }\end{array}$ & Gilobal performance evaluations, self-assessments \\
\hline Citizenship and service & $\begin{array}{l}\text { Identify resources and barriers to health of local } \\
\text { and regional communities, identify vulnerable and } \\
\text { marginalized populations }\end{array}$ & $\begin{array}{l}\text { Evaluation of projects, self-assessments, small-group } \\
\text { evaluations }\end{array}$ \\
\hline Medical practice management & $\begin{array}{l}\text { List the business principles underlying successful health } \\
\text { care delivery models }\end{array}$ & $\begin{array}{l}\text { Global performance evaluations, MCQs, patient/ } \\
\text { procedure logs }\end{array}$ \\
\hline $\begin{array}{l}\text { Concentrated and independent } \\
\text { learning }\end{array}$ & $\begin{array}{l}\text { Demonstrate effective presentation of capstone project } \\
\text { in a self-selected area of concentration }\end{array}$ & $\begin{array}{l}\text { Glassick-informed rubric for project implementation } \\
\text { and scholarly product, MCQs, self-assessments, small- } \\
\text { group evaluations }\end{array}$ \\
\hline
\end{tabular}

curricula include workshops that integrate simulation activities to reinforce basic science learning.

\section{Curriculum changes since 2010}

Netter engages in small-scale tests-of-change as part of its continuous educational quality improvement activities. Changes since matriculating the first cohort include:

- Adding small-group problem-based learning to the secondyear curriculum using clinical cases and introducing concepts of health care disparity, social determinants of health, and stewardship of medical resources

- Shortening preclerkship calendars, allowing earlier start and completion of the third year

- Moving Netter's comprehensive narrative medicine sessions to the second and third years, when students are ready to understand the concepts and clinical contexts

- Increasing vertical curricular integration to reinforce content and highlight connections

Three ongoing efforts to advance the curriculum are formal components of the "Better Netter '23" strategic plan:

- Vertical and horizontal integration throughout the 4 years of medical education with concurrent mapping of the enhanced curriculum
- Expansion of current curricular interprofessional education activities

- Implementation of a health systems science curriculum across the 4 years of medical education

Currently, Netter's assessment system includes multiple assessments and frequent formative and summative feedback to document students' attainment of the EPOs. Assessment modalities include summative and formative OSCEs (FOSCEs), feedback from standardized patients, and workplace-based assessments (WBAs). Recently, formative WBAs have been integrated into the preclerkship clinical curriculum. Important changes in summative assessment have included giving students a choice in the timing of the USMLE Step 1 examination, which can occur at 8 time slots beginning at the end of the second year and spanning the third year, to accommodate students' study and preparation styles. In 2020, the medical school will begin to redesign its assessment system to balance "assessment of learning" with "assessment for learning." The new system will offer enhanced integrated assessments with the goal of further broadening information on students' progress and professional development and preparing them for assessment in GME.

Growth in class size from 60 to 95 students has presented opportunities to adapt and refine the curriculum to ensure an optimal learning experience for every student. This has also 
necessitated recruitment of additional faculty and clinical sites, expanding Netter collaborations and reaching deeper into the community.

\section{Curricular Governance}

The Committee on Curriculum Oversight (CCO) is chaired by the senior associate dean for education and co-chaired by the associate dean for faculty development. It has representation from UME and GME faculty and administrators, students, and QU's health sciences and nursing schools. The CCO oversees all aspects of the curriculum. Subcommittees include the Curriculum Evaluation Committee and course and clerkship committees.

\section{Education Staff}

The Office of Medical Education is housed within the Office of the Senior Associate Dean for Education, providing centralized and local support to both the UME and GME programs at Netter through staff, educational committees, the Standardized Patient and Assessment Center, and the Office of Educational Assessment and Program Effectiveness. The Office of Medical Education works in close collaboration with the Office of Student Affairs, which provides learning support through a combination of in-house coaches (clinical skills remediation, learning strategies, and test-prep) and external consultants (testprep and professionalism coaching).

See Figure 2-Educational leadership.

\section{Faculty Development and Support in Education}

Netter's multimodal faculty development program enhances educators' skills in teaching, mentoring, curriculum development, assessment, inclusion/diversity, leadership, and scholarship and promotes their career advancement. Strong, sustained institutional support has facilitated growth and innovation of a centralized faculty development program that provides comprehensive learning opportunities for medical science educators, clinical faculty, residents, and students who teach. A major focus of the program is engagement of all clinical faculty including those at geographically distant sites. The Teaching for Educators in Clinical Health care traveling workshop series rotates to different affiliate sites to provide core content. An annual clinical faculty retreat provides immersive learning, often with nationally recognized leaders in medical education.

Monthly lunch-and-learn sessions, a medical education journal club, and a facilitated peer-mentored education scholarship group (Scholaris) address the needs of medical sciences faculty. All faculty and residents have access to faculty development offerings within the school's learning management system, which serves as an active repository of resources such as online courses, an interactive tool to create a customized menu of learning opportunities, and a dashboard to track participation. In 2019, webinar options were added, and a new podcast series will be released in early 2020, enhancing access to professional development material.

Netter's faculty members are evaluated for promotion based on teaching, scholarship, service, clinical activity, and professionalism. Faculty describe their contributions using an educator portfolio model. Demonstration of excellence as an educator is required for promotion.

\section{Initiatives in Progress}

Building on the student-focused learning environment, current efforts to innovate in teaching and learning aim for a relationship-

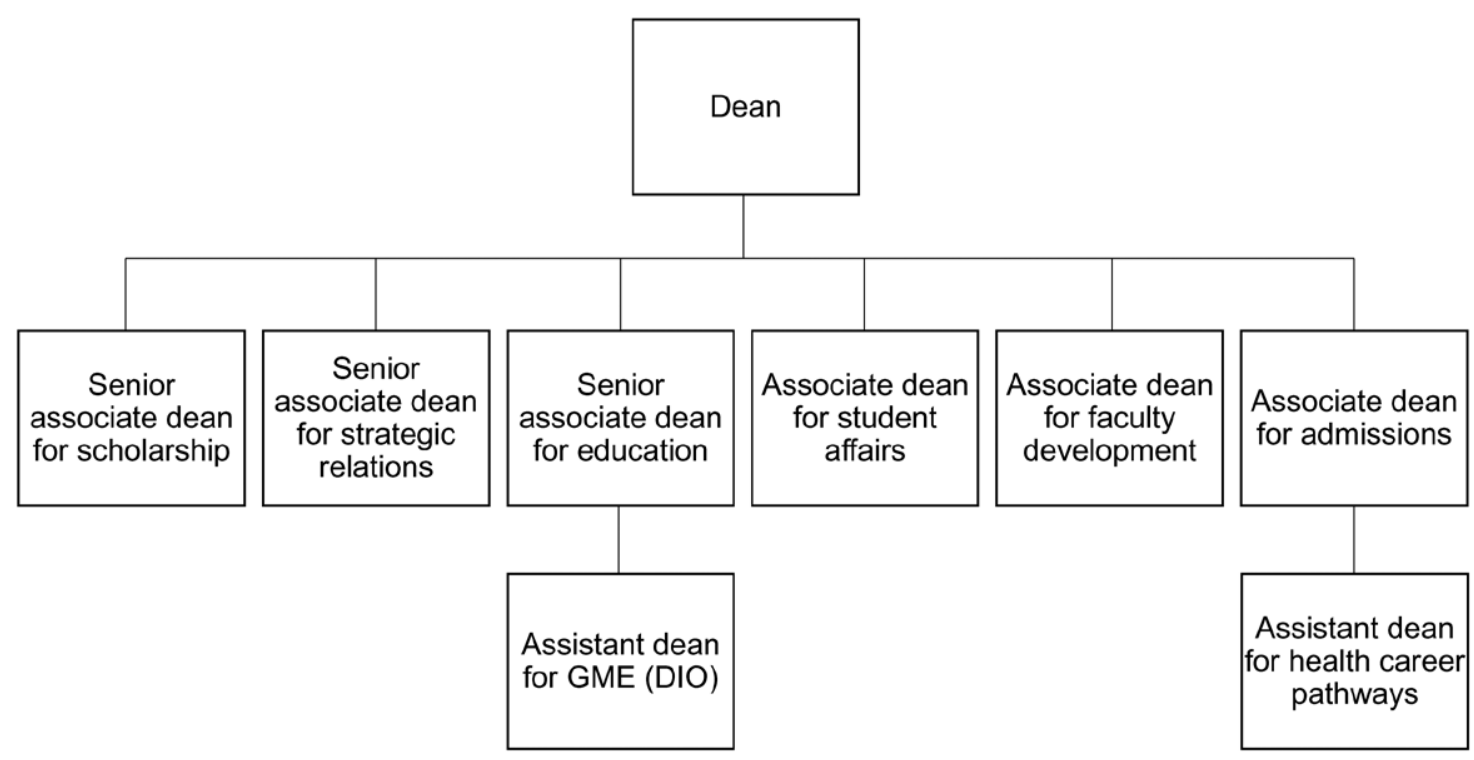

Figure 2 Educational leadership. 


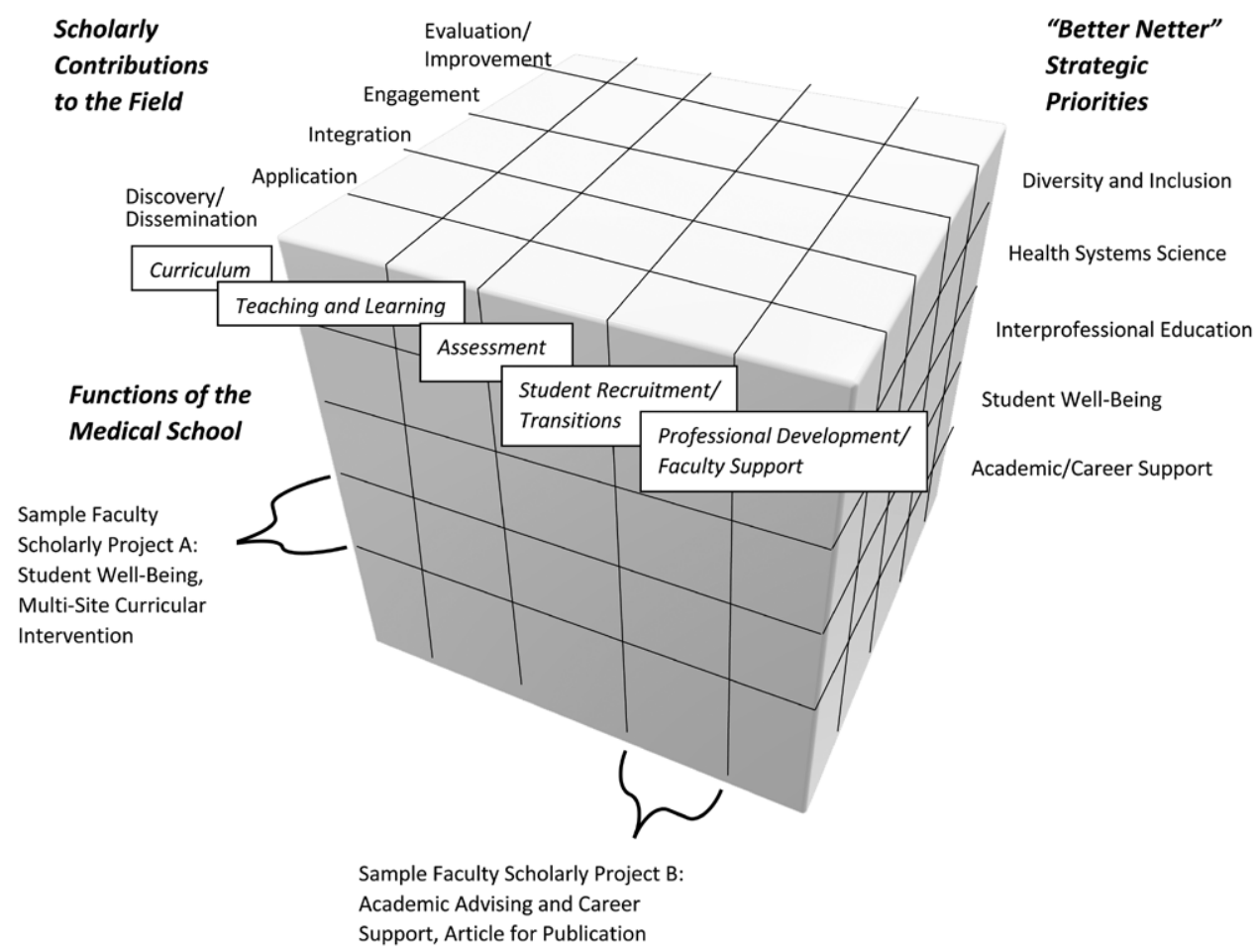

Figure 3 Cube conceptual model.

centered model of medical education and to enhance interactions among learners, faculty, staff, and patients across the QU community and clinical affiliates. The programmatic focus will be on mentor-mentee relationships, coaching, collegiality and inclusion, and interprofessional communication. Relationshipcentered education is also essential to Netter's strategic priorities in diversity and inclusion and student well-being.

Plans call for expanding Netter's efforts to promote student well-being with coordination across the continuum of medical education and the Netter and QU communities. Initiatives bridging student affairs and medical education will focus on well-being skills for learners and how the learning environment, curricular structure, and assessment system affect wellbeing. Netter's GME programs offer sessions on recognizing and responding to distressed learners and clinicians and titrating stress to promote optimal learning. The well-being of Netter's faculty and staff is also addressed by individual- and organizational-focused initiatives. Netter is working with the
Connecticut health care community to design and implement strategies to promote well-being for the region's clinicians.

Efforts to promote educational scholarly activities are represented by the "Netter Cube"-a 3-dimensional conceptual model that links domains for scholarly work that relate to core medical school functions, contributions to the field, "Better Netter" strategic plan priorities.

See Figure 3-Cube conceptual model.

This conceptual model enables mapping of individuals' scholarly work within a local and national context. It also promotes collaboration among faculty with a focus on discovery, dissemination, application, integration, and strategic recourse allocation to support educational scholarship.

Netter's strategic planning is rooted in its mission, vision, and values to move this new school from its launch into a thriving next decade. 


\title{
University of Connecticut School of Medicine
}

\author{
Christine Thatcher, EdD, Ellen Olarsch Nestler, MD, and Melissa R. Held, MD
}

\section{Medical Education Program Highlights}

During the 2016-2017 academic year, a new curriculum, called MDelta (Making a Difference in Education, Learning, Teaching, and Assessment), was implemented. Highlights of this innovative curriculum include:

- A lecture-free Stage 1 curriculum is case based, patient centered, and uses team-based learning (TBL) as the predominant pedagogy via an innovative and integrated approach to assessment.

- Medical students receive a certificate of public health in social determinants of health and disparities.

- Laboratory teaching occurs in the human anatomy lab (cadaveric dissection), the virtual anatomy lab (radiology workstations, Anatomage tables, ultrasound), and the virtual microscopy lab.

- Stage 2 incorporates longitudinal courses, including radiology and geriatrics. In addition, topics such as ethics, patient safety, and basic science content are threaded throughout clerkship conferences.

\section{Curriculum}

\section{Curriculum description}

Stage 1-Exploration-is approximately 18 months and begins with a $2 \frac{1}{2}$-week "launch" period that consists of orientation to the curriculum and to medical school, along with a minicourse in history and physical examination. Patient instructors (standardized patients), along with faculty, provide formative feedback. Students are assigned to a longitudinal continuity practice (Clinical Longitudinal Immersion in the Community, CLIC), which will continue for 3 years. The experiential Patient Advocacy in Communities, Teams, and Health Systems (PACTS) course is part of the clinical suite of courses, which also includes the doctoring course (Delivery of Clinical Care, DoCC). Courses titled Case Oriented Essentials (COrE), Vertically Integrated Teams Aligned in Learning (VITAL), and Laboratory (including cadaveric dissection as well as technology-enhanced virtual laboratory experiences) provide the basic science curriculum and biopsychosocial components so essential to medical training. $\mathrm{TBL}$, along with problem-based learning $(\mathrm{PBL})$ in the clinical reasoning and VITAL courses in the second year comprise the main pedagogical underpinnings. Scholarship \& Discovery is

Acad Med. 2020;95:S79-S82.

doi: 10.1097/ACM.0000000000003283

Copyright (C) 2020 by the Association of American Medical Colleges

Supplemental digital content for this article is available at http://links.Iww.com/ ACADMED/A826.

Year school was established: 1968.

School URL: https://medicine.uconn.edu. introduced with required sessions on topics such as research design, formulating research questions, and data acquisition and analysis, and culminates in a capstone project in Stage 3.

Stage 1, the preclerkship curriculum, is made up of five 10week blocks. Each block is followed by a 2-week Learning Enhancement and Assessment Period (LEAP) intersession. LEAP begins with an integrated examination across courses in Stage 1. For students who have demonstrated a gap in knowledge or who require additional skill development, LEAP provides time and faculty support, and in some cases a reassessment focused on the particular gap.

The summer between the third and fourth blocks is 12 weeks in length, allowing ample time for research or other opportunities for career or scholarly exploration.

Stage 2-Clinical Immersion-begins with the clinical transition period in March/April of the second year. This flexible time is mostly used for board study and may include electives, scholarly work, and/or vacation. Required clerkships include ambulatory medicine, inpatient medicine, ambulatory pediatrics, inpatient pediatrics, neurology, family medicine, psychology, surgery, and obstetrics-gynecology, as well as longitudinal geriatrics and longitudinal radiology elements. Radiology is introduced during the Stage 2 kickoff along with skills training, orientation, and other activities to prepare students for the clinical learning environment. Clerkships are 4 or 6 weeks in length and organized into four 12-week blocks. There are 2 mandatory "homeweeks" (fall and spring). An additional 8-week block at the end of Stage 2, the advanced clinical block, provides time for Step 2 board study, electives, scholarship, vacation, and an option to complete a required Stage 3 clerkship if prerequisites are met. VITAL and radiology content are threaded within the clerkships and homeweeks.

Stage 3-Transformation-includes required clerkships: 4 weeks each of emergency medicine, critical care, and an advanced inpatient experience in either medicine, family medicine, pediatrics, or surgery, as well as 2 weeks of a Transition to Residency (TTR) course. Students complete their scholarly capstone in Stage 3, as well as 20 weeks of electives.

See Supplemental Digital Appendix 1-Curriculum Schematic —at http://links.lww.com/ACADMED/A826.

\section{Curriculum changes since 2010}

There have been a number of changes leading up to curricular reform in the past 10 years, with the culmination leading to the new MDelta curriculum. There was a 5-year planning process and implementation in the fall of 2016 (class of 2020) with these highlights:

- The educational program objectives are based on the ACGME domains of competence. Additionally, the new domains, 
interprofessional collaboration and personal and professional development, were added as part of the curricular reform process through the recommendation of the AAMC.

- The radiology clerkship was first developed as a required 2-week experience in year 4 and then transformed into a longitudinal clerkship during year 3 (Stage 2).

- Moved from a traditional $2+2$ curriculum to an 18-month Stage 1.

- Reduced contact hours per week from an average of 32 hours to 22 required hours in Stage 1.

- Removed all lectures and replaced with primarily TBL pedagogy. Modified PBL and small-group conferences remain. The Clinical Reasoning course was added back after the first year of implementation.

- Lab modifications include adding virtual anatomy and a handoff system, as well as point-of-care ultrasound and radiology workstations, to traditional cadaveric dissection.

- The VITAL course was developed to provide the biopsychosocial aspects of medicine across the 4 years of medical school, incorporating interprofessional education and providing content that contributes to each medical student earning a certificate in Public Health in Social Determinants of Health and Disparities.

- The PACTS course (previously known as Clinical Home) is experiential and complements DoCC and VITAL by providing systems- and team-based content with a focus on health systems science.

- Implementation of LEAP intersessions during Stage 1, which provide integrated examinations and, when needed, individualized knowledge or skill development after the assessment period. This innovation has led to a reduction in summer remediation and a reduction in the number of students needing to repeat an academic year.
- Change in clerkship structure to four 12-week pods.

- Changes in student assessment have included the implementation of shelf exams in family medicine and neurology, joining surgery, obstetrics-gynecology, and psychiatry.

- TTR, piloted as an elective, is now a required course in year 4 in MDelta with skill and experiential training to prepare for internship.

- Incremental increase in class size from 85 students to 110 incoming medical students in the fall of 2019. The increased class size has required additional resources and is especially impactful on clinical capacity.

\section{Assessment}

See Table 1-Medical Education Program Objectives and Assessment Methods.

\section{Pedagogy}

TBL constitutes the main pedagogical approach in Stage 1. Students are required to be prepared through the self-directed learning process, which includes review of curated and homemade online videos, readings, and/or tutorials. There are no lectures. Peer-to-peer feedback is a critical aspect of the pedagogy. The laboratory course uses a modified TBL format and is an integrated experience, including gross anatomy, virtual anatomy, histopathology, radiology, physiology labs, and an introduction to ultrasound. The doctoring course (DoCC) is delivered in small groups with a physician, allied health professional, and fourth-year student co-facilitating; it builds upon and augments the experience of students at their CLIC sites. CLIC is a 3-year curriculum that provides students with a longitudinal immersion and mentorship experience in a community-based (primarily)

\section{Table 1}

\section{Medical Education Program Objectives and Assessment Methods}

\begin{tabular}{|c|c|}
\hline $\begin{array}{l}\text { Medical education program } \\
\text { objective(s) }\end{array}$ & Assessment methods \\
\hline Competency 1: Patient care & $\begin{array}{l}\text { Preclerkship examinations; clinical skills assessments all stages; clerkship required observations; clerkship } \\
\text { evaluations; advocacy project; handoff in lab; Transition to Residency (TTR) course observations }\end{array}$ \\
\hline Competency 2: Medical knowledge & Preclerkship examinations; clerkship examinations; VITAL assignment Stage $2 / 3$ \\
\hline $\begin{array}{l}\text { Competency } 3 \text { : Practice-based learning } \\
\text { and improvement }\end{array}$ & $\begin{array}{l}\text { Clerkship orientation and training for sites; preclerkship examinations; Stage } 2 / 3 \text { VITAL course assignment; } \\
\text { Stage } 2 \text { required curriculum; examinations Stage } 1 \text { and } 2 / 3 \text {; scholarly capstone; lab assessments related to } \\
\text { self-directed learning; clerkship evaluations and observation }\end{array}$ \\
\hline $\begin{array}{l}\text { Competency } 4 \text { : Interpersonal and } \\
\text { communication skills }\end{array}$ & $\begin{array}{l}\text { Preclerkship examinations, clerkship evaluations and observations, clinical skills assessment; VITAL Stage } 1 \\
\text { and } 2 / 3 \text { assignments; human anatomy and lab handoff process; course evaluations in lab and delivery of } \\
\text { clinical care (DoCC) }\end{array}$ \\
\hline Competency 5: Professionalism & $\begin{array}{l}\text { TBL peer evaluations; Clinical Longitudinal Immersion in the Community (CLIC) evaluations; TBL peer } \\
\text { evaluations; Stage } 2 \text { clerkship evaluation; DoCC examinations; HIPAA training and assessment; VITAL/ } \\
\text { Certificate Social Determinants of Health \& Disparities (SDH\&D) projects }\end{array}$ \\
\hline Competency 6: Systems-based practice & $\begin{array}{l}\text { Preclerkship examinations; DoCC examination; launch assessment; PACTS examinations; VITAL homework } \\
\text { assignment; clerkship evaluations; CLIC evaluations; advocacy project }\end{array}$ \\
\hline $\begin{array}{l}\text { Competency } 7: \text { Interprofessional } \\
\text { collaboration }\end{array}$ & $\begin{array}{l}\text { TBL peer evaluations; clerkship evaluations; preclerkship examinations, critical care observation; PACTS } \\
\text { examination; lab evaluation, TBL peer assessments; TTR }\end{array}$ \\
\hline $\begin{array}{l}\text { Competency 8: Personal and } \\
\text { professional development }\end{array}$ & $\begin{array}{l}\text { DoCC journal reflections and assessment; clerkship evaluations; TBL peer evaluations; clinical skills and } \\
\text { CLIC assessments; coach goal setting }\end{array}$ \\
\hline
\end{tabular}


generalist practice for a half day each week. The Clinical Skills Assessment Program provides teaching and assessment activities for medical students during each of their 4 years of school. Using standardized patients called patient instructors, the program assists students as they learn the skills of taking a medical history, doing a physical examination, and communicating with patients. Simulation is included in each of the 4 years of the curriculum. PBL is used in 2 courses in year 2 .

Stages 2 and 3 are clerkship based and consist of both ambulatory and inpatient experiences along with centralized case-based interactive conferences administered via small-group discussions. Workshops, such as in sports medicine, as well as practice in simulation and hands-on experiences are also used. Students in their fourth year may become preceptors/teaching assistants (near-peer teaching) through a developed program that provides principles of adult learning and reflection.

\section{Clinical experiences}

There are several types of clinical sites used for required educational experiences. In addition to the university hospital, we partner with 4 major academic teaching hospitals, which are geographically close to the school of medicine, in addition to a number of community teaching hospitals. Many outpatient practices are used for the required ambulatory clerkships in family medicine, internal medicine, and pediatrics, along with CLIC and other clerkship experiences.

- CLIC is a 3-year required half day per week experience in an outpatient office (predominantly primary care) that provides a longitudinal experience for learners. In addition, through an application process, students may opt to attend another type of practice or specialty, alternating weeks with their usual CLIC, for the final 6 months of the required period.

- Early introduction into the clinical environment has been a philosophical mainstay of our curriculum for decades.
Students' first clinical experiences occur when they begin at their CLIC sites (see above) in mid- to late September of year 1.

- There are always challenges faced in the design and implementation of clinical experiences for medical students. This past decade introduced a new medical school in Connecticut, as well as a regional campus of an out-of-state institution. That fact, along with the state-mandated increase in class size at our institution, has required additional sites to assure excellent quality clinical experiences. Other types of health professions learners (i.e., APRN, PA) also lead to competition in the clinical environment.

\section{Curricular Governance}

The CUME (Committee on Undergraduate Medical Education) serves as the school's curriculum committee for the educational program leading to the MD degree. It approves policies for all aspects of the undergraduate curriculum and determines the structure of the curriculum, educational philosophy, and overall curriculum objectives, as well as criteria for student evaluation, promotion, and graduation. This committee oversees management of the curriculum as well as the ongoing review of individual courses, segments of the curriculum, and the curriculum as a whole (via the Curriculum Evaluation Committee). The Curriculum Advisory Committee uses our mapping tool and other resources to identify gaps and redundancies in the curriculum.

See Figure 1-Governance and operational support.

See Figure 2-UME leadership structure.

The Office of Curricular Affairs has central oversight and coordination of each stage of the curriculum, as well as assessment and accreditation for undergraduate medical education. Graduate medical education and continuing medical education are housed in separate offices responsible for their respective areas.

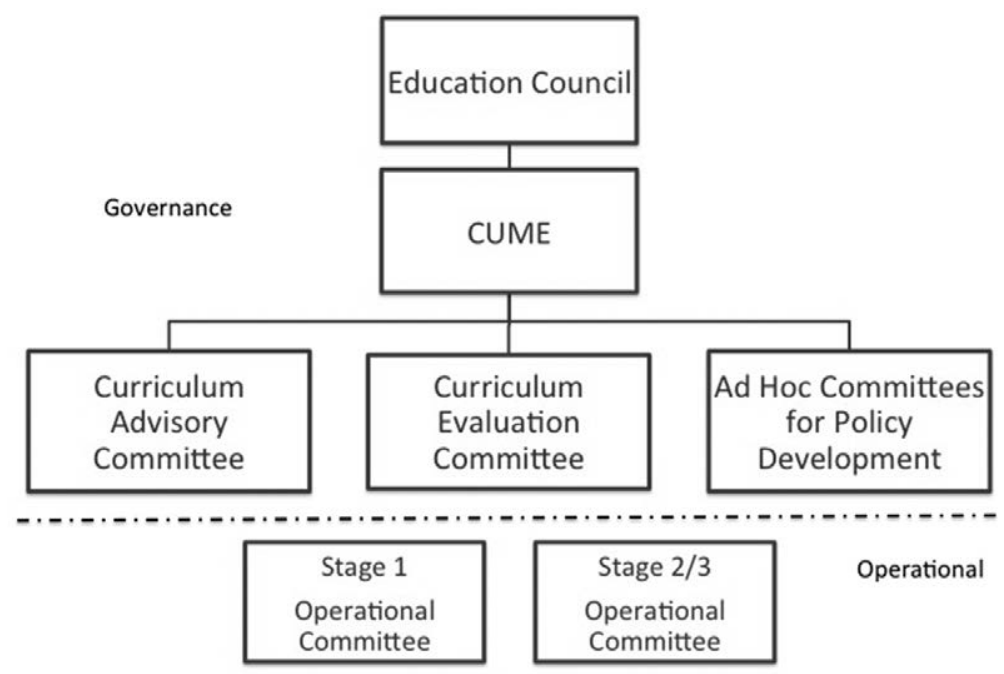

Figure 1 Governance and operational support. 


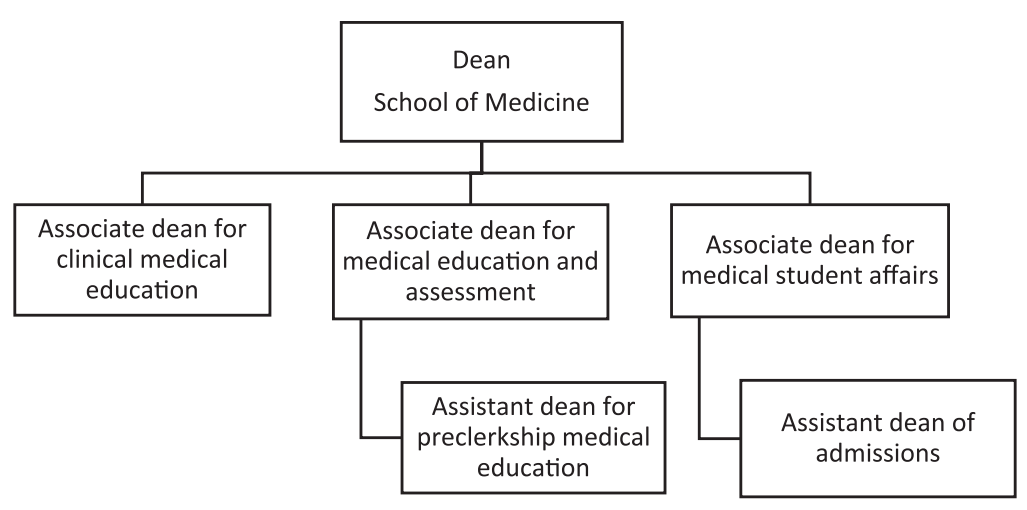

Figure 2 UME leadership structure.

The Office of Curricular Affairs works closely with departmental chairs on distribution of educational FTE and oversight of educational efforts for teaching, assessment, and coaching. Faculty-level directors are provided protected time in the following areas: Stage 2, clinical skills, SIM, student affairs, academic educational affairs, admissions, social and behavioral sciences, basic sciences including laboratory, course directors, clerkship directors, global health, coaching, and wellness.

The student affairs team includes the associate dean for medical student affairs, 2 part-time faculty co-directors, and the assistant dean of admissions, along with support staff. Curricular affairs faculty and staff work closely with student affairs personnel, but have a discrete role in operationalizing the curriculum.

There are several professional categories including medical educator. Faculty are engaged in teaching, research/scholarship, patient care, and other professional activities. Teaching is expected and teaching excellence is required for senior rank promotion. The nature and extent of the other criteria will vary among faculty and professional categories. Teaching excellence can be achieved by participating in educational activities of medical students, residents/fellows, graduate students, and postdoctoral students. 


\section{Yale University School of Medicine}

Richard Belitsky, MD, Michael L. Schwartz, PhD, Susan E. Larkin, MBA, and Abigail Roth, JD

\section{Medical Education Program Highlights}

Yale School of Medicine (YSM) is committed to the scholarly training of physicians and other health care providers with the goal that they will be the leaders of their generation. Our educational mission is "to educate and inspire scholars and future leaders who will advance the practice of medicine and the biomedical sciences." Consistent with our commitment to educating scholars and future leaders, we have a class size of 104 students, with $20 \%$ in our MD-PhD program.

The Yale System creates a highly collaborative learning environment that gives our school its distinct identity and character and makes us unique among medical schools.

- In place since 1931, it is considered the school's most powerful educational strength and is a major factor in our ability to consistently attract extraordinarily talented students.

- In the Yale System, medical students are viewed as mature, highly motivated, and self-directed learners.

- There is a reduced emphasis on grades and no class rank to limit competition. Instead, self-assessment and collaborative learning are emphasized.

- There is a flexible curriculum to accommodate individual learning styles and allow students to pursue individual interests.

- Since 1839, Yale has required a dissertation based on original research.

- The MD thesis is an essential part of the Yale System, and time for this scholarly activity is built into the curriculum. It is designed to promote scientific inquiry, foster critical thinking, and prepare students for lifelong learning.

- Importantly, the thesis requirement gives students the opportunity to work closely with faculty in a mentoring relationship.

\section{Curriculum}

In 2015, following 5 years of extensive planning, a new curriculum was implemented. Eight overarching goals form its foundation, focusing on areas essential for successful future practice:

- Health promotion and disease prevention

- Mechanisms and treatment of disease

- Clinical reasoning

Acad Med. 2020;95:S83-S86.

doi: 10.1097/ACM.0000000000003458

Copyright (C) 2020 by the Association of American Medical Colleges

Supplemental digital content for this article is available at http://links.Iww.com/ ACADMED/A951.

Year school was established: 1810.

School URL: https://medicine.yale.edu.
- Patient care

- Professionalism and communication

- Responsibility to society

- Creation and dissemination of knowledge

- Physician as scientist

See Supplemental Digital Appendix 1-Curriculum Overviewat http://links.lww.com/ACADMED/A951.

\section{Curriculum changes since 2010}

- Integrated course curriculum: Basic and clinical science content is organized and integrated across 8 sequential themebased master courses and 3 longitudinal courses.

- Interprofessional longitudinal clinical experience: Medical students work with physician associate and nursing students as a team and are directly involved in patient care from the beginning of medical school, with an emphasis on interprofessional education, team-based care, and knowledge of the health care system.

- Clinical skills: Teaching and assessment of clinical skills is coordinated and integrated across the 4 years of the curriculum, and emphasize competencies in medical history and communications, physical examination, medical procedures, clinical reasoning, and professionalism.

- Clinical clerkships: A remodeled clerkship year begins earlier in the curriculum; includes integration across disciplines; provides opportunities for greater continuity with patients, teams, and mentorship; and allows students the flexibility to explore career interests.

- Enhanced flexibility: The final 17 months are an advanced training period where students create schedules based on their individual needs and career choices. This includes time for their research, electives and subinternships, residency interviews, and required USMLE Step examinations.

\section{Assessment}

YSM adopted and incorporated the AAMC's Physician Competency Reference Set as the expected competencies for our medical students. These competencies map to our overarching goals through the individual learning objectives represented in the courses, clerkships, and electives throughout our curriculum.

See Table 1-Program Objectives and Assessment Methods.

YSM hired an associate director of student assessment to focus on assessment "for learning" while ensuring assessment "of learning." This approach relies on guided self-assessment, reflection, and self-regulation in learning and professional development, and uses an integrated, developmental, and competency-based approach. In addition, faculty are assisted and supported in the development of various formative and summative assessments used in the curriculum. 


\section{Table 1 \\ Program Objectives and Assessment Methods}

\section{General competengy}

Patient care: Provide patient-centered care that is compassionate, appropriate, and effective for the treatment of health problems and the promotion of health

\section{Medical education} program objective

Health promotion and disease prevention

Patient care

\section{Outcome measure}

Two-station OSCE at the end of the first year (formative)

Seven-station OSCE in the third year (summative)

Advanced Communications Skills OSCE in fourth-year capstone course (formative)

USMLE Step 2 (clinical skills) examination (summative)

Clerkship assessment form based on direct observation (summative)

Students take history and perform physical examination (including sensitive exams) with standardized patients and receive feedback (formative)

Breaking bad news and shared decision-making encounters with standardized patients during the clerkship year (formative)

Knowledge for practice: Demonstrate knowledge of established and evolving biomedical, clinical, epidemiological, and social-behavioral sciences, as well as the application of this knowledge to patient care
Mechanisms and treatment of disease

Patient care

Creation and dissemination of knowledge

Physician as scientist

Qualifying examination in each of the integrated master and longitudinal courses (summative)

Weekly voluntary quizzes and self-assessment examinations (formative)

USMLE Step 1 and Step 2 (clinical knowledge)

Clerkship assessment form based on direct observation (summative)

Self-assessment examinations at the end of each clerkship (formative)

Two-station OSCE at the end of the first year (formative)

Seven-station OSCE in the third year (summative)

Yale Thesis

Practice-based learning and improvement: Demonstrate the ability to investigate and evaluate one's care of patients, to appraise and assimilate scientific evidence, and to continuously improve patient care based on constant self-evaluation and lifelong learning

Interpersonal and communication skills: Demonstrate interpersonal and communication skills that result in the effective exchange of information and collaboration with patients, their families, and health professionals

Professionalism: Demonstrate a commitment to carrying out professional responsibilities and an adherence to ethical principles

Systems-based practice: Demonstrate an awareness of and responsiveness to the larger context and system of health care, as well as the ability to call effectively on other resources in the system to provide optimal health care

Interprofessional collaboration: Demonstrate the ability to engage in an interprofessional team in a manner that optimizes safe, effective patient- and population-centered care

\section{Clinical reasoning}

Patient care

Creation and dissemination of knowledge

Physician as scientist

Patient care

Professionalism and communication

Responsibility to society

Professionalism and communication

Responsibility to society

Patient care

Professionalism and communication

Responsibility to society

Patient care

Professionalism and communication
Clerkship assessment form based on direct observation (summative)

Qualifying examination in biostatistics and Epidemiology and Public Health courses (summative)

Self-assessment and reflection exercises in association with the OSCES

Students complete self-assessments in the integrated courses (formative)

Yale Thesis

Two-station OSCE at the end of the first year (formative)

Seven-station OSCE in the third year (summative)

Advanced Communications Skills OSCE in fourth-year capstone course (formative)

Breaking bad news and shared decision-making encounters with standardized patients during the clerkship year (formative)

Clerkship assessment form based on direct observation (summative)

Essay examination in Professional and Ethical Responsibility Course (summative)

Clerkship assessment form based on direct observation (summative)

Essay examination in Professional Responsibility Course (summative)

Clerkship assessment form based on direct observation (summative) 


\section{Pedagogy}

YSM uses a broad range of pedagogical approaches, including teambased learning, simulation, case-based workshops, standardized patients, iBooks, and augmented and virtual reality, along with more traditional learning approaches using lectures and laboratories. We recently completed a major renovation of education space to support the emphasis on small-group and team-based learning.

\section{Clinical experiences}

- Throughout the first year and a half of medical school, students participate in longitudinal clinical experiences focused on communication, professionalism, and the skills of taking a patient-centered history and physical examination. Direct patient contact and small-group tutorials with faculty are highlights of these early clinical experiences.

- Core clerkships and electives occur at Yale New Haven Hospital and Health System, the VA Connecticut Health Care System, Connecticut Mental Health Center, and Yale University Health Services.

- In addition, global health electives are available in a variety of international and domestic locations including Appalachia Grundy County, Tennessee; Argentina; Chinle, Arizona Indian Health Service; China; San Francisco Free Clinic; South Africa; and Uganda.

\section{Curricular Governance}

The deputy dean for education is responsible for oversight and coordination of the education programs and services.

- The Educational Policy and Curriculum Committee is chaired by the associate dean for curriculum. This committee oversees the policies that govern the medical education program, provides centralized oversight and review of the curriculum, and is responsible for ensuring that the curriculum addresses the school's educational objectives.

- The curriculum directors coordinate the development, implementation, and review of the curriculum. The integration of content throughout the 4 years is fundamental to their roles. In addition, they chair the Curriculum Review Committees that assesses each component of the curriculum and provides feedback to each course, clerkship, and elective director.

- The Thesis Committee is chaired by the associate deans for student research and is responsible for the oversight, coordination, and management of the thesis required of all medical students.

See Figure 1-Organizational chart of deans.

See Figure 2-Curriculum management and governance.

\section{Education Staff}

Multiple offices collaborate to support undergraduate medical education, including admissions; curriculum; diversity, inclusion, community engagement, and equity; financial aid; global health education; $\mathrm{MD}-\mathrm{PhD}$ program; student affairs; and student research.

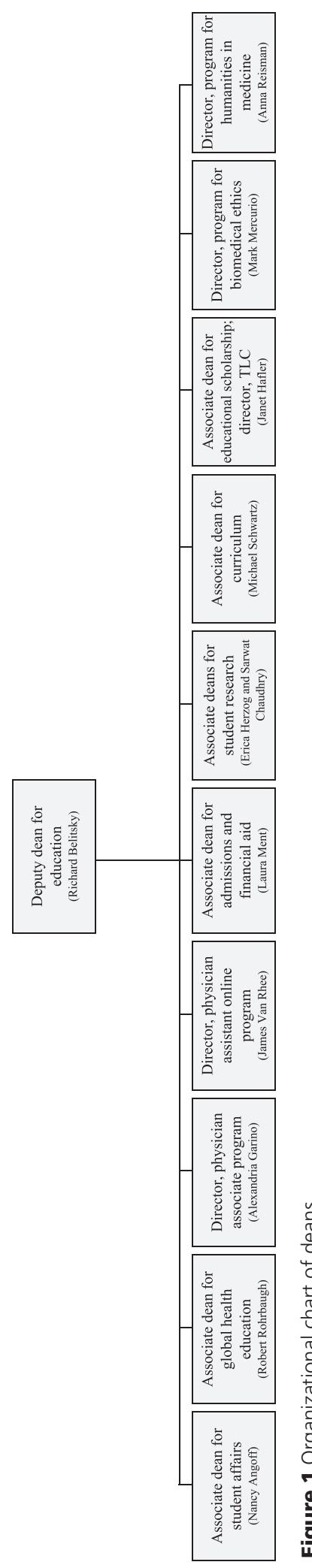




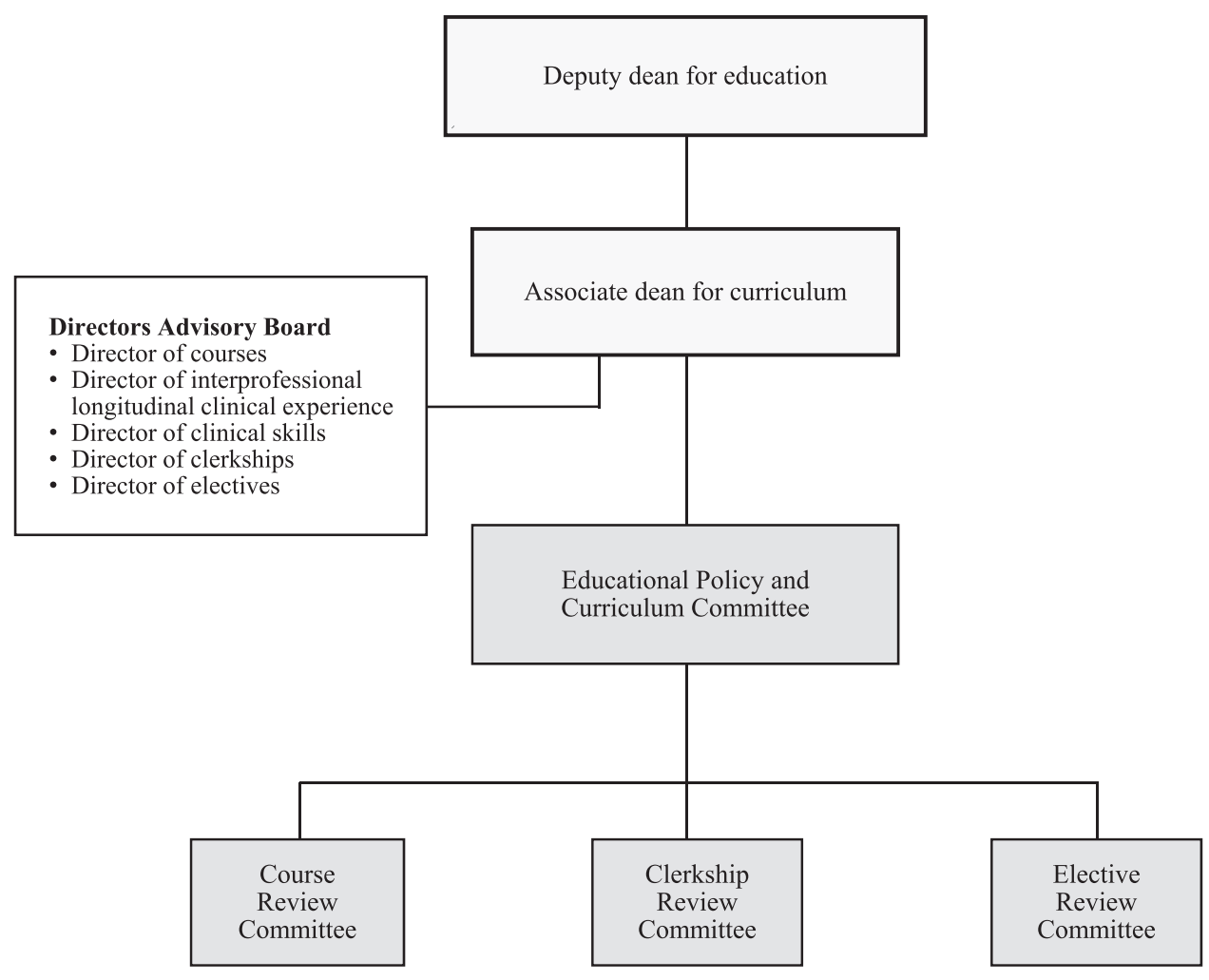

Figure 2 Curriculum management and governance.

\section{Faculty Development and Support in Education}

The YSM Teaching and Learning Center (TLC) provides expertise in curriculum development, pedagogy, assessment, and use of learning technologies. The TLC also provides a variety of professional development programs and services to support educators, including:

- Individual consultations

- Direct observation of faculty teaching with peer feedback

- Seminars and workshops on teaching methods

- Support/mentorship for educational research, grants, and scholarship
- Letters to the appointments and promotions committees summarizing the educational contributions, leadership, and impact of faculty educators in support of promotion/tenure decisions

\section{Initiatives in Progress}

Since its implementation, the curriculum has been continuously and rigorously evaluated. In response, initiatives currently underway to improve the curriculum include:

- Health equity and social justice in medicine

- Sex, gender, and minority health

- Addiction medicine 


\section{The George Washington University School of Medicine and Health Sciences}

Terry Kind, MD, MPH, Robert Jablonover, MD, and Richard Simons, MD

The George Washington University School of Medicine and Health Sciences (GW SMHS) seeks to graduate "physician citizens" committed to local, national, and global public service through excellent patient care, leadership, advocacy for change and innovation, and scholarly inquiry. The MD program is dedicated to admitting a diverse group of students who will be prepared to care for patients with broad and disparate needs. GW has a robust, flexible curriculum that leverages our unique position in the nation's capital. We aim to graduate physicians who exhibit excellent clinical skills; embody respect for the ideals of equity, diversity, and cultural sensitivity; advocate for improvements in health care systems and community health; embody high standards of professional conduct; and embrace lifelong learning. The following guiding principles are reflected in the MD program curriculum:

- Integrating teaching basic sciences, clinical sciences, public health, and population health to build critical thinking skills

- Offering of a range of learning strategies to maximize student engagement and knowledge retention

- Offering early and longitudinal clinical exposure to ground learning in practice and provide meaningful engagement with patients

- Offering opportunities for reflective self-assessment and mentoring to support professional development and wellness

- Individualizing student experiences to take advantage of the unique opportunities in the nation's capital

\section{Medical Education Program Highlights}

- Coursework for all students in patient, populations, and systems, emphasizing clinical public health

- Early exposure to clinical settings

- Close and longitudinal interactions with faculty, advisors, and mentors

- Elective opportunities in the third year and senior year

- Longitudinal primary care experiences

- Experiences unique to Washington, DC, such as curricula at the Holocaust Museum and Capitol Hill legislative offices

- Option to join a scholarly concentration in one of the following areas:

Acad Med. 2020;95:S87-S90

doi: 10.1097/ACM.0000000000003267

Copyright $\odot 2020$ by the Association of American Medical Colleges

Supplemental digital content for this article is available at http://links.Iww.com/ ACADMED/A822.

Year school was established: 1825 .

School URL: https://smhs.gwu.edu.
- Clinical practice innovation and entrepreneurship

- Clinical and translational research

- Community/urban health

- Disaster medicine

- Global health/international medical programs

- Health policy

- Integrative medicine

- Medical education and leadership

- Medical humanities

\section{Curriculum}

\section{Curriculum description}

GW SMHS undertook curricular revisions for the class of 2018, predominantly emphasizing active learning, the integration of basic science and clinical content, clinical public health, an earlier start to the clinical years, third- and fourth-year electives, and a longitudinal primary care experience.

See Supplemental Digital Appendix 1-Curriculum Description—at http://links.lww.com/ACADMED/A822.

There are 3 curricular phases. The Fundamentals of Medicine phase runs from August of year 1 through January of year 2. This phase includes the introductory Foundations course followed by organ system blocks plus a concurrent 3-semester longitudinal Practice of Medicine course (that includes clinical skills and reasoning, professional development, a longitudinal clinical apprenticeship program, clinical integration case sessions, physical diagnosis, formative observed simulated clinical experiences, and informatics sessions) and a 3-semester longitudinal course series on patients, populations, and systems. During summer vacation between the first and second years, students who have opted into a scholarly concentration complete relevant experiential projects. In February through mid-April of year 2, students complete the USMLE Step 1 examination.

The second phase is Fundamentals of Clinical Practice, which begins mid-April with a 2 -week Foundations course followed by clinical clerkships and electives. Most core clerkships are 6- or 8-week blocks; the primary care clerkship has a special format with a 4-week block plus an every other week longitudinal continuity clinic experience throughout this curricular phase. During 2 intersession weeks, students meet to discuss in large and small groups content that spans multiple fields (quality and safety, high-value care, teamwork, wellness, conflict of interest, business of medicine, and clinical public health topics such as obesity and the opioid epidemic).

The third phase is Transition to Advanced Clinical Practice, beginning in May of year 4, culminating in a month-long Transitions to Residency capstone course around Match Day. 
Students complete an acting internship, emergency medicine, neurology (year 3 or 4), anesthesiology (year 3 or 4), and 18 weeks of electives, along with USMLE Step 2 and residency applications. All students complete a longitudinal scholarly project either as part of their scholarly concentration or related to advocacy or research in their area of interest.

\section{Curriculum and assessment}

Program objectives align with ACGME domains of competence: medical knowledge, patient care, interpersonal and communication skills, practice-based learning and improvement, systems-based practice, and professionalism.

In the past decade, we have implemented new assessments as we revised our preclinical curriculum to include organ system block exams; customized NBME examinations; formative OSCEs; reflection essays; peer feedback; and assessments in the patient, populations, and systems course such as group proposal presentations. In addition, we recently introduced pass/fail grading for every course in the preclinical curriculum. Clinical performance-based examinations are used from the first semester on. There is a comprehensive clinical performancebased exam at the end of the preclinical phase and the end of the clinical clerkship year; the latter exam is similar to the USMLE clinical skills licensing exam. Entrustable Professional Activity (EPA) self-assessments begin at the start of the clinical years, with a small EPA pilot in the fourth year.

See Supplemental Digital Appendix 2-Program Objectives and Assessment Methods - at http://links.lww.com/ACADMED/ A822.

\section{Pedagogy}

Pedagogical approaches emphasize active learning as an essential component of every teaching session. We employ several different instructional methods, including a comprehensive standardized patient and simulation program, large- and small-group lecture and discussion groups, smallgroup reflection, laboratories, self-directed learning, casebased learning, video prework, and team-based learning exercises. The Practice of Medicine uses small groups, fostering longitudinal relationships between students and professional development and clinical skills and reasoning mentors ( 8 students to 3 instructors). Throughout the preclinical curriculum, there are clinical integration sessions, with integrated case-based sessions at the end of each week. GW has early preceptorship experiences via clinical apprenticeships with specialists starting in the fall of year 1 .

In the clinical curriculum, instructional methods largely occur in clinical settings (both ambulatory and inpatient) plus weekly didactics that include small- and large-group discussions, casebased learning, standardized patients and simulation, team-based learning, lectures, and online cases.

\section{Clinical experiences}

GW uses a variety of sites for required and elective educational experiences including academic and community-based hospitals and outpatient practices. Our main health systems are The George Washington University Hospital, Children's National Hospital, The Washington DC Veterans Affairs Medical Center, Holy Cross Hospital, Anne Arundel Medical Center, United Medical Center, Lifebridge Health's Sinai Hospital, Northern Virginia Mental Health Institute, Saint Elizabeths Hospital, Psychiatric Institute of Washington, INOVA, The GW Medical Faculty Associates (faculty practice), plus numerous community health centers, federally qualified health centers, patient-centered medical homes, and private physicians' offices in the DC/ Virginia/Maryland metropolitan region.

A diverse array of electives supports the mission to graduate "physician citizens." Some clinical electives include:

- Introduction to Correctional Medicine, where students learn in DC correctional facilities

- Culinary Medicine, where students learn in community-based food kitchens

- Community health electives in low-resource settings to foster socially minded, culturally competent, and cost-conscious practice

- Exploring Implicit Bias in Medicine, Child Health Advocacy, Disaster Medicine, Health Policy, Narratives in and of Medicine, Quality Improvement and Patient Safety, and other electives

Senior-year students can opt to complete electives through the International Medical Programs office as well. For global health scholarly concentration students, these electives are designed to increase awareness about international health systems, global diseases, and assessment techniques for the specific health needs of countries at various stages of development. Other international clinical electives provide students with an opportunity to enrich and diversify their medical education in a different physical and social setting.

Longitudinal clinical learning experiences begin at the start of year 1 and at the start of the clinical clerkship year as follows:

- The Clinical Apprenticeship Program starts in the fall of year 1 and continues throughout the preclinical curriculum (the first 3 semesters of years 1-2). Students are placed with the same preceptor for a longitudinal learning experience focused on interviewing and examining patients in a specialty medicine clinical setting, where students apply interview and physical exam skills they are learning in the classroom.

- The Longitudinal Primary Care Clinic is a component of the primary care clerkship where students are placed with a general internist, pediatrician, geriatrician, or family practitioner for 1 half day every other week throughout the year. This combined block plus longitudinal continuity clinic allows students to appreciate the breadth of primary care diagnoses and the role of care coordination and is anchored in the yearlong teaching relationship between student and preceptor, and possibly several patients.

- During year 4, all students complete mentored longitudinal projects taking a scholarly and/or clinical public health advocacy approach. 
Despite an extensive set of clinical sites and robust electives, challenges in implementing clinical experiences relate to the saturated region, with several other medical schools placing students in the area, as well as traffic and travel around greater metropolitan DC/Virginia/Maryland. Students in the longitudinal primary care clinic travel from morning clerkship responsibilities to afternoon continuity clinic, which can present a challenge depending on location.

\section{Curricular Governance}

Funds to cover teaching throughout the preclinical and clinical curricula are allocated from the medical school to the basic science and clinical departments. The Office of Medical Education (OME) coordinates the preclinical courses, and clinical courses are directed and coordinated by the clinical departments. The school provides funds to the department chairs, who determine their use.

See Figure 1-Curricular governance.

\section{Education Staff}

The senior associate dean for MD programs is responsible for curricular oversight, with an associate dean for clinical education and an assistant dean for preclinical education. The Clinical Learning and Simulation Skills Center, international medical programs, library, and student affairs also report to the senior associate dean for MD programs. The OME develops, delivers, and evaluates both preclinical and clinical curricula, and supports faculty, students, and curricular committees. The office includes a director, decanal faculty, and 8 staff members who, along with departmental coordinators for the clinical clerkships, process and post required coursework and maintain the curricular database. In addition, an Office of Evaluation director and staff support all aspects of program evaluation such as course, clerkship, and site evaluations by students and reviews of courses, clerkships, and curricular phases.

The curriculum committee (chaired by and composed of faculty) coordinates and manages the curriculum. GW uses an internally developed, online curriculum database, searchable and available to all faculty, for mapping all objectives and for tagging keywords and instructional methods.

\section{Faculty Development and Support in Education}

The Center for Faculty Excellence supports the school and its faculty as a leader in innovative education and training by advancing the scholarship and practice of teaching and learning. The center:

- Supports faculty in delivering high-quality, innovative teaching and learning experiences

- Develops areas of inquiry that build education theory and advance education practice

- Promotes the career development of individual faculty and students as education leaders

- Offers workshops, educational modules/videos, a teaching consult service, peer assessments, leadership programs, career development and mentorship, and medical education grants

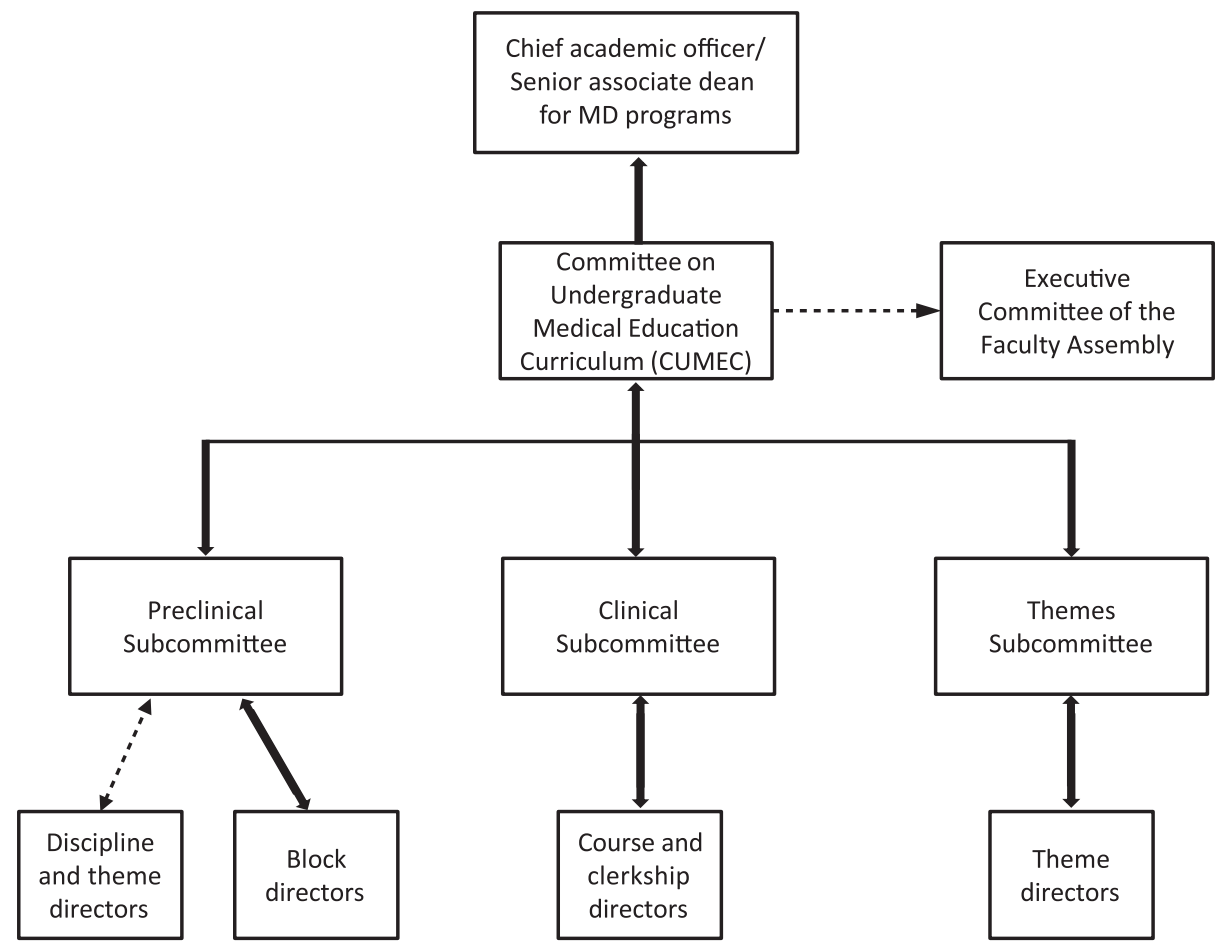

Figure 1 Curricular governance. 
In promotion and tenure decisions, all candidates are evaluated in teaching, scholarship, and service. Tenure-track faculty must be evaluated as very good to excellent in all areas, demonstrate "future promise," and compare favorably to similar faculty at other research institutions. Non-tenure-track faculty must receive very good to excellent evaluations in 2 of the 3 areas, with some achievements in the remaining area.

All faculty are expected to teach. Demonstrating excellence in teaching requires effort beyond the minimum teaching expectations associated with day-to-day work. Faculty document teaching achievements in their CV and teaching portfolio as a systematic collection of materials to show excellence as an educator (teaching narrative statement, teaching and mentoring activities, teaching effectiveness, teaching impact, and professional development as an educator).
The Center for Faculty Excellence's Academy of Education Scholars develops and recognizes excellence in education scholarship and research that advances the practice of medical and health sciences education and improves the quality of educational programs and patient care.

Academy members receive developmental assistance and feedback on their annual education research plan; quarterly individual coaching on research design, execution, and dissemination; access to resources; membership in a collaborative, supportive community of education scholars; and recognition of excellence in education scholarship. Eligibility includes all full-time, regular faculty, across all disciplines, specialties, and teaching levels. Membership is active during the time a faculty member has an approved annual research plan and is the PI on a supported education research study or related project. 


\section{Georgetown University School of Medicine}

Mary A. Furlong, MD, Racheal M. Ferguson, MHSA, L. Whitman Brown, MBA, and Stephen R. Mitchell, MD, MBA

\section{Medical Education Program Highlights}

In 2017, Georgetown University School of Medicine (GUSOM) began implementation of the Journeys curriculum: a dynamic, adaptive curriculum incorporating elements of curiosity, choice, reflection, and, in keeping with our Jesuit mission of cura personalis, care for the whole person.

There are 3 curricular phases: foundational, core clerkship, and advanced clinical. Interactive boot camp sessions precede the foundational and clerkship phases. Between each phase are Journeys periods; these 1-2 months are innovative, flexible, and centered on student choice. The curriculum includes early exposure to doctoring with instruction on the normal history and physical examination within the first 3 months. Students experience a 15 -month preclinical curriculum organized by organ system modules and based on longitudinal basic science disciplines. The clerkship year is 48 weeks, including 6 weeks for students to select among non-core clerkship specialties. Within Journeys II, deep dive seminars reinforce academic curiosity within each students' interests. The advanced clinical phase encourages individualized schedules. The focus of Journeys is to integrate scientific knowledge with clinical work throughout the 4 years and develop empathic, engaged, lifelong learners who care for the patient and for the community.

\section{Curriculum}

\section{Curriculum description}

A highlight is the 1-week intersessions. These occur between regularly scheduled coursework in both the foundational and clerkship phases and include topics such as social determinants of health and the business of medicine. Intersessions are adaptive and topics may change with current health issues or the health climate. A diverse set of pedagogies are implemented as students are asked to discuss today's tough health care issues.

During Journeys workshops, students choose among several 2-hour seminars from 3 categories: physician wellness, cultural competency, and research. Topics include mindfulness and meditation and cross-cultural communication. Many students will have engaged in varying levels of research and our research workshops reflect this variation.

Acad Med. 2020;95:S91-S94.

doi: 10.1097/ACM.0000000000003453

Copyright (C) 2020 by the Association of American Medical Colleges

Supplemental digital content for this article is available at http://links.Iww.com/ ACADMED/A943.

Year school was established: 1851

School URL: https://som.georgetown.edu.
Recognizing that an understanding of basic science and foundational concepts is enhanced with clinical experience, students return to these following the clerkship year as they participate in two 2-week deep dive seminars. These classroom activities elucidate topics of interest related to a student's chosen field. Ranging from specialized neurosurgery tenets to fertility awareness, there are ample subjects that complement students' medical education. To promote creativity and bring arts into practice, several deep dives focus on humanities, spirituality, and culinary medicine.

Our curriculum offers students a forward-thinking journey as they apply knowledge, discernment, and humanity to the care of the whole person.

See Supplemental Digital Appendix 1-Curriculum Framework—at http://links.lww.com/ACADMED/A943.

\section{Curriculum changes since 2010}

Developing Journeys included a review of the attributes of our graduates; guiding factors included curiosity, individualization, and reflective practice.

Examples of how these values are incorporated include longitudinal scholarly tracks, summer research opportunities, professional identity formation program, and Journeys workshops. The longitudinal scholarly tracks are extracurricular experiences in 6 areas from which students may choose: humanities, social justice, primary care, population health, health care leadership, and medical education research. The tracks generate significant interest and provide a mechanism to pursue specific interests. All students engage in an independent scholarly project; the curriculum provides time for activities and supports research with Journeys I workshops. Both the school and MedStar Health provide summer research opportunities and stipends for nearly 100 students. Reflective practice has been enhanced. Students are paired with a professional identity coach for regular meetings to share personal reflections meaningful in their growth as physicians. With curricular prompts, students journal and share thoughts with their coach, building a relationship that enriches their professional advancement.

Embracing cura personalis, we emphasize care of the whole patient and care of the whole physician and have improved the clinical working environment to support programs centered on issues at the heart of stress and burnout. The school has an innovative elective mind-body medicine program for students, now a core part of graduate programs within MedStar. GUSOM offers physician wellness workshops; a nutrition intersession; and humanities-focused deep dive seminars on literature, dance, and music as they relate to medicine. 
Essential to our mission is service to the community. The school's long tradition of providing care to the underserved in Washington, DC, continues with the student-run HOYA Clinic. The Health Justice Alliance is a new partnership with Georgetown University Law Center, and together students advocate for the patient in unprecedented ways. We continue to explore the Jesuit mission within our curriculum and anticipate an even stronger commitment to students, the patient, and the community.

\section{Medical education program objectives}

GUSOM competencies are centered around knowledge, skills, and attitudes necessary to become dedicated, compassionate physicians caring for patients and committed to the health needs of our society. Acquiring and applying knowledge is only part of our goal; developing a curious mind and integrating the psychosocial, spiritual, and cultural dimensions of health care are equally important. We value students as learners and also as teachers and strive to impart accountability, respect, and humility. While framed around KSAs, our objectives are tied to the ACGME core competencies and to AAMC Entrustable Professional Activities.

See Supplemental Digital Appendix 2-Assurances of Learning_at http://links.lww.com/ACADMED/A943.

\section{Assessment}

Our dynamic assessment process provides appropriate, practical formative, and summative assessments in all phases of the curriculum. We broadened the scope of formative feedback in the foundational phase by allowing time each week for students to participate in a practice quiz. The exercise enhances understanding of the scope of students' knowledge and ability to apply material. For skills-associated competencies, the curriculum offers quarterly CURA sessions (clinical unified reasoning activities) and OSCEs as part of the longitudinal cura personalis course, a hypothesis-driven, organ system-based doctoring course. Students practice skills and receive direct feedback from faculty. OSCEs continue throughout the clerkship year. Students take the USMLE Step 1 examination in the second year, before clerkships. A nontiered grading system has been implemented for the foundational phase; grading in the clinical years remains tiered.

\section{Pedagogy}

In the large-group didactics, case-based and problem-based workshops take place routinely. We also engage in interactive approaches that support adult-type learning. Small-group discussions and simulations are implemented across all years to teach individual and team-based skills.

With the support of Dahlgren Memorial Library, we have space for an entire class to participate in team-based learning. In several courses, directors have used podcasts and e-prescribing assignments. Novel in the curriculum is our first virtual patient that students come to know, diagnose, and treat. With exercises in the longitudinal disciplines, students have the opportunity to work directly in our electronic health record system in a training environment created specifically for them.

Enhancing clinical skills was an intentional commitment of Journeys. Our first-year medical students are brought into the hospital on day 2 to interview their first patients and work with physical examination teaching assistants to practice clinical skills. Students are assigned to ambulatory sites for the remainder of their first year, and then in the hospital setting in year 2, reinforcing these skills. Standardized patients reinforce practical skills throughout the curriculum with consistent formative feedback during regular OSCE exercises. We anticipate incorporating ultrasound and virtual reality technologies in the next year, continuing a modern approach to medical education.

\section{Clinical experiences}

As students enter medical school, we aim to impart a sense of excitement about medicine and have included basic life support training and first aid. The cura personalis: Foundations of Patient Care module includes a first patient interview as students meet their patient at the bedside and learn history-taking skills. Clinical exposure continues for the first 8 weeks of the curriculum and provides comprehensive communications instruction. Following block 1, students are competent in performing a normal history and physical examination and prepared for upcoming ambulatory experiences.

The school uses various university-affiliated hospital, community hospitals, community health centers, and private physician offices/clinics to ensure students meet required educational experiences. Students participate in communitybased rotations in family medicine ( 4 weeks), internal medicine ( 2 weeks), and pediatrics ( 3 weeks) during their clerkships and several elective opportunities exist in the advanced clinical phase at Virginia Hospital Center, Inova Fairfax, Walter Reed National Military Medical Center, and the D.C. Veterans Affairs Medical Center.

\section{Longitudinal experiences}

GUSOM began a longitudinal integrated clerkship (LIC) in 2016 at MedStar Franklin Square Medical Center. This clinical experience fulfills the requirements for family medicine, pediatrics, obstetrics-gynecology, and internal medicine. The LIC offers a unique clinical experience as students work with their own patient panel and provide care in all areas over the 6-month period. The LIC integrates clinical disciplines through both outpatient and inpatient care.

With implementation of Journeys and early entry for students into the clerkship phase, we faced a 3-month overlap period. Challenges included identifying new clerkship sites, facilitating faculty development and appointments, and working transparently to engage faculty and staff. We faced the challenge of ensuring adequate inpatient volume and have creatively considered clinical experiences outside of the typical inpatient ward experience, including simulated and case-based scenarios. Ongoing discussion of required clinical experiences 


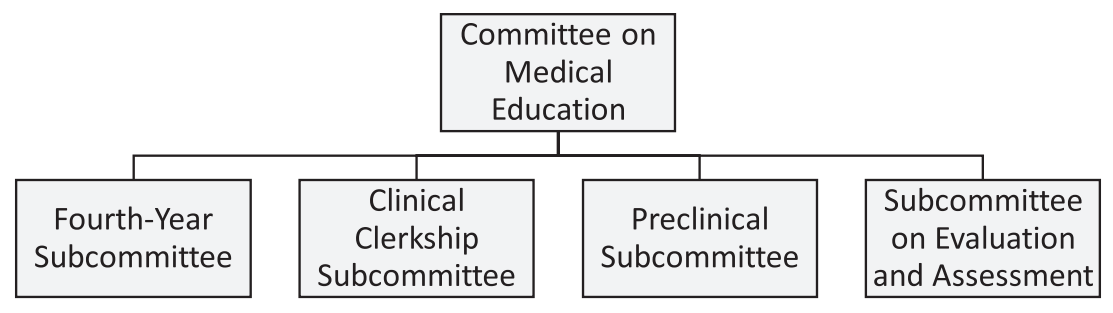

Figure 1 Committees and curricular governance.

has enhanced a longitudinal, integrative approach to common medical problems across disciplines.

We are cognizant of the challenges for faculty development, central monitoring of student instruction, and oversight of resident teaching. The clinical learning environment is critical to optimal learning, patient safety, and care. With MedStar Health, we have invested significant effort to improving the clinical learning environment and enhancing professional behaviors and respect.

\section{Curricular Governance}

The curriculum is overseen by the Committee on Medical Education (COME), composed of a diverse group of basic science and clinical faculty. Subcommittees pertain to each phase of the curriculum and membership is inclusive of all directors. The school's Office of Medical Education (OME) is responsible for the curriculum; oversight occurs with the COME.

With the support of educational coordinators, the OME assists directors in scheduling, gathering resources, preparing assessments, managing student schedules, and providing resources. Direct coordination of clinical rotations is the responsibility of clinical departments. The following tools are used for curriculum management: Canvas, Exam Soft, New Innovations, One45, and VSLO.

The OME is overseen by the senior associate dean for curriculum. Reporting to senior associate dean are 6 academic

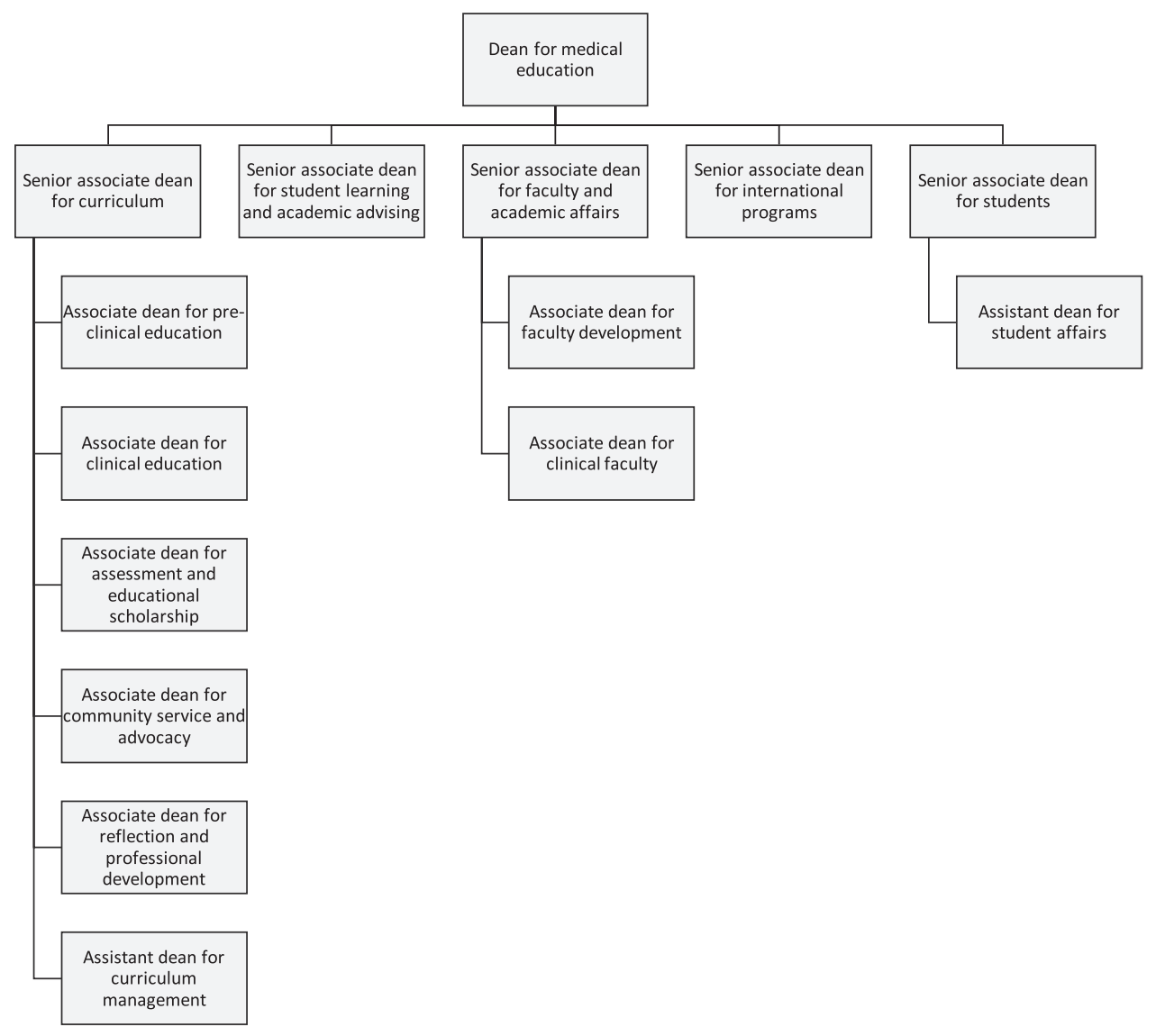

Figure 2 Organizational chart. 
deans, the medical director of the Integrated Learning Center (ILC), and a curricular project manager. The deans are responsible for implementation of the curriculum. The ILC administrative director plans the clinical skills and simulation programs. The assistant dean for curriculum management oversees the day-to-day operations and supervises support staff. The OME collaborates with other administrative offices and works in coordination with its clinical affiliates.

See Figure $1-$ Committees and curricular governance.

See Figure 2-Organizational chart.

\section{Faculty Development and Support in Education}

The Office of Faculty and Academic Affairs supports faculty in important career development processes through courses and longitudinal mentoring programs.

The mission of the Center for Innovation and Leadership in Education (CENTILE) is to support an interprofessional community of educators through a colloquium, grand rounds, workshops, and the Teaching Academy. CENTILE assists with professional development through a teaching certificate program, an educational scholarship consultation forum, and peer evaluations.
The MedStar Teaching Scholars program is a longitudinal program leading to Medical Education Research Certification and Leadership Education and Development certification. The aim of the program is teaching educators how to apply research principles to medical education, to be effective collaborators in medical education, and to develop as a leader in academic medicine.

For both university and MedStar employed faculty, a specific track devoted to medical education exists. There is a range of criteria for each rank in the areas of education, scholarship, and service. Faculty share teaching philosophies and educational goals in their teaching portfolio, which summarizes direct teaching hours, numbers of learners, overall course and individual faculty evaluations, and any teaching awards highlighted for the committee review.

The Teaching Academy is open to faculty and residents cultivating a community dedicated to Georgetown University Medical Center's mission. Membership spans 4 levels from protégé to distinguished educator. Applications are centered on educational philosophy and accomplishments in 1 of 5 areas: direct teaching, educational scholarship, mentorship, educational leadership, or instructional design. Distinguished educators must have achievements in 3 areas. Benefits include faculty development and review of teaching quality. 


\section{Howard University College of Medicine}

Debra H. Ford, MD, and Swati Goel, MD

\section{Medical Education Program Highlights}

Howard University College of Medicine (HUCM), founded in 1868 , takes pride in its long and illustrious history of training students to become competent and compassionate physicians who provide health care in medically underserved communities. In keeping with its mission, HUCM stands as a leader in the education of minority students from disadvantaged backgrounds and continues to graduate more minority physicians than any other medical school nationally. HUCM actively recruits, matriculates, and provides support to a diverse student body that includes many students committed to serve in medically underserved and disadvantaged communities. HUCM is committed to providing care, promoting research, and developing leadership qualities in its students in order to eliminate health disparities in populations who are underserved and under-resourced. HUCM is committed to cultural diversity among faculty, staff, and students, to ensure a respectful and ethical academic environment. The college has adopted new and evolving trends in medical education, including active learning, an integrated medical curriculum, and use of a state-of-the-art simulation and clinical skills center. The curriculum emphasizes interprofessional collaboration and teamwork, patient safety, and the importance of preparing its graduates for the transition to graduate medical education and lifelong learning.

\section{Curriculum}

\section{Curriculum description}

The College of Medicine's (COM's) program of instruction provides the forum for students to acquire the essential knowledge, skills, behaviors, and attitudes necessary for the practice of medicine as set forth in HUCM's educational objectives. The curriculum includes a 2-year foundational science phase; followed by the core clerkship phase in year 3; and a final year of preparation for residency training with acting internships, electives (intramural, extramural, international), and the Intern Readiness course.

See Supplemental Digital Appendix 1-Curriculum Schematicat http://links.lww.com/ACADMED/A957.

\section{Curriculum changes since 2010}

In the last decade, the class size at HUCM has remained stable and the curriculum has seen a continued decrease in didactic

Acad Med. 2020;95:S95-S98.

doi: 10.1097/ACM.0000000000003476

Copyright (C) 2020 by the Association of American Medical Colleges

Supplemental digital content for this article is available at http://links.Iww.com/ ACADMED/A957.

Year school was established: 1868.

School URL: https://medicine.howard.edu. instruction with the integration of techniques such as flipped classrooms, increased small-group and team-based learning, increased clinical correlation activities, and increased integration of simulation-based education across all 4 years. Other improvements and changes are as follows:

- Revamped the Physical Diagnosis course and renamed it as the Introduction of Clinical Medicine 1 and 2 courses

- Restructured the Ethics course during the third year to be the Interdisciplinary Health care Ethics course, emphasizing interprofessional collaboration and teamwork

- Added a new Intern Readiness course, a 4-week mandatory fourth-year transition to residency experience, including clinical and procedural skills expected of PGY-1 residents

- Introduced an Essential Clinical and Technical Skills course, given during the first week of the third year

- Integrated neurology/rehabilitation medicine into the 12-week internal medicine Jr. clerkship

- Added a new emergency medicine 2-week rotation during the third year

- Added "Stop the Bleed" training for all new first-year students

- Began curricula reform planning, which is in its early stages

\section{Assessment}

There are 13 outcome-based medical education program objectives leading to the MD degree. HUCM's competencybased educational program objectives provide the framework across the 4 years of medical school. The program objectives provide faculty with a structured listing of knowledge, skills, behaviors, and attitudes to guide the placement of curriculum content within courses/clerkships and curriculum years/ phases. The AAMC Medical School Objectives, ACGME general competencies, USMLE content descriptions, AAMC Entrustable Professional Activities, and Interprofessional Education Collaborative Core Competencies for Interprofessional Collaborative Practice serve as references for the institutional program objectives. Since 2010, more objective structured clinical examinations (OSCEs), standardized patient assessments, and customized NBME examinations during the second year have been added to the list of assessment methods.

See Table 1-Program Objectives and Assessment Methods.

\section{Pedagogy}

The faculty of the COM use the following instructional methods to deliver components of the medical curriculum, to enable students to achieve the medical education program objectives and competencies listed below:

- Clinical experience: ambulatory and inpatient

- Discussion: large $(>12)$ and small group $(<12)$

- Laboratory: cadaver lab 
- Didactic instruction

- Panel discussions

- Peer teaching

- Preceptorship

- Problem-based learning

- Review sessions

- Role play/dramatization
- Self-directed learning/tutorial

- Simulation

- Standardized/simulated patients

- Team-based learning

- Video (Tegrity)

- Virtual patient: computer-based cases

- Workshops

\section{Table 1}

\section{Program Objectives and Assessment Methods}

Medical education program objectives

1. Demonstrate understanding of current core knowledge of basic biomedical science through an interdisciplinary approach to the understanding of the processes that support normal development, structure, and function of the human organism

2. Demonstrate understanding of current core knowledge of normal and altered structure and function of organ systems, to apply that knowledge to the recognition and management of common and complex clinical conditions

3. Demonstrate the ability to evaluate patients and properly manage their medical problems by: (a) completing comprehensive histories and physical examinations, (b) obtaining focused histories and perform relevant physical examinations, (c) correctly identifying patients' medical problems through critical thinking and formulating hypotheses as to etiology and solutions, (d) successful development of diagnostic strategies, and (e) formulation and implementation of a management plan

4. Apply knowledge of the scientific method in medical diagnosis and treatment and in research; the student should be able to evaluate published findings and to apply scientific evidence-based reasoning to the solution of medical problems

5. Demonstrate understanding of the impact of the various stages of life, as well as the effects of gender, lifestyle, socio-economic status, nutritional factors, genetic characteristics, psychosocial and epidemiologic factors, and culture upon the quality of human health and the prevalence of disease, disease prevention, and health maintenance

6. Demonstrate an understanding of the fundamental concepts of continuity of care (preventive, rehabilitative, and end of life) in addition to the diagnosis of acute medical problems, and be able to apply these concepts to clinical practice on a local, regional, national, or international level

7. Demonstrate mastery of a variety of skills, such as effective communication during interviewing patients and educating patients about their diseases and communicating with their families; the student must also demonstrate appropriate skills during physical examinations (proper use of instruments, application of manual techniques) and in utilization of laboratory resources (ordering appropriate tests, interpreting values)

8. Exhibit appropriate professional behavior in interactions with patient and their families, peers, and other health care professionals to adhere to professional standards of ethical behavior, and to function harmoniously and respectfully as a member of an interprofessional health care team

9. Demonstrate mastery of the curriculum essential to enter and pass USMLE Step 1 examination and progress to and pass USMLE Step 2 examinations (CK and CS) along the pathway toward licensure for the practice of medicine

10. Display skills of independent, lifelong, and progressive learning

11. Manifest self-awareness, self-care, self-assessment, and personal growth sufficient to be a role model for others and maintain a state of wellness

12. Show sensitivity toward the need to address and resolve health disparities at all levels

13. Show mastery of the scholarly expectations of the medical faculty of the College of Medicine as determined by appropriately designed and applied assessment methodologies, including but not limited to written examination performance, practical clinical skills, and technical skills
Assessment methods

- MCQ

- Direct observation by faculty

- Laboratory exam

- MCQ

- Direct observation by faculty

- oSCE

- Standardized patients

- MCQ

- Direct observation by faculty

- oSCE

- Standardized patients

- MCQ

- Direct observation by faculty

- MCQ

- Direct observation by faculty

- Standardized patients

- MCQ

- Direct observation by faculty

- Standardized patients

- MCQ

- Direct observation by faculty

- OSCE

- Standardized patients

- Direct observation by faculty

- OSCE

- Standardized patients

- MCQ

- OSCE

- Standardized patients

- Direct observation by faculty

- Direct observation by faculty

- Direct observation by faculty

- MCQ

- Standardized patients

- MCQ

- Direct observation by faculty

- OSCE

- Standardized patients 


\section{Clinical experiences}

Medical students at HUCM have their first clinical experience during the first semester of the first year and continues throughout the subsequent years. During the fourth year, students have enough time for electives to supplement required learning experiences. Fourth-year students have a required 4-week selective in ambulatory primary care that may be satisfied through elective rotations in family medicine, internal medicine, pediatrics, adolescent medicine, and gynecology. The COM uses the following types of clinical sites for required educational experiences: academic medical centers (inpatient/outpatient), community hospital (inpatient/outpatient), VA medical center, community health clinics, and community physician offices. The sites for clinical education are as follows:

- Howard University Hospital

- Children's National Medical Center

- Washington DC Veterans Affairs Medical Center

- St. Elizabeth's Hospital

- Mary Washington Hospital

- Unity Health Care

- MBI Health Services, LLC (a behavioral health agency)

- Community physicians' offices

\section{Curricular Governance}

The COM's Committee for Curriculum and Schedules (Curriculum Committee) is responsible for the curriculum and has responsibility and authority for overseeing and approving its design, management, and evaluation to ensure that it is coherent, coordinated, and integrated horizontally and vertically. The Curriculum Committee has final authority for all decisions and oversight of the educational program and curriculum. The Curriculum Committee is chaired by the senior associate dean for academic affairs. Faculty and students are elected members of the committee. The Curriculum Committee is a standing committee of the COM. There are 5 subcommittees of the Curriculum Committee: Years 1 and 2, Years 3 and 4, Evaluation, Professionalism, and Clinical Skills/ Simulation Subcommittees.

The Dean of the COM serves as the chief administrative officer for the COM. To ensure an outstanding educational program, the dean is responsible for recruitment and supervision of senior administrators; faculty recruitment, evaluation, and development; budgeting and budget control; and general supervision of the research, teaching, and outreach activities of the college. Most of these activities are carried out by the assistant and associate deans, department chairs, dean's support staff, other support units of the college and university, and faculty as appropriate. The dean's authority and responsibility for the medical education program is exercised by his direct oversight of the senior associate dean for academic affairs, associate dean for student affairs and admissions, associate dean for graduate medical education, and assistant dean for faculty development.

\section{Education Staff}

The Office of Academic Affairs, led by the senior associate dean for academic affairs, provides administrative and academic support for the planning, implementation, evaluation, and oversight of the curriculum and for the development and maintenance of the tools to support curriculum delivery, monitoring, and management. The following administrators are involved in the undergraduate medical education, faculty development, and learner support: dean of the COM, senior associate dean for academic affairs, associate dean for student affairs/admissions, and assistant dean for faculty development.

The Office of Academic Affairs is composed of the Office of Medical Education, the Curriculum Office, and the Data Analysis Center. These offices support the curriculum, learning, and assessment. This office oversees undergraduate medical education and preundergraduate (pipeline) medical education. The Dean of Student Affairs and Admissions also supports the preundergraduate medical education programs.

\section{Faculty Development and Support in Education}

The COM has had an established Office of Faculty Development (OFD) since 2009; its infrastructure is composed of an assistant dean of faculty development (who has a Master Teacher in Medical Education certification) and a full-time program administrator. The office works in conjunction with the OFD Advisory Committee, consisting of COM leadership that includes the senior associate dean for academic affairs, assistant dean for continuing medical education, the health sciences library director, and the director of Howard University's Center for Excellence in Teaching, Learning and Assessment (CETLA), to name a few. The OFD has primary responsibility for 2 signature programs annually, the New Faculty Orientation and the Faculty Retreat. In addition, on a monthly basis, the OFD initiates or takes leadership in logistics for offering monthly programming for all faculty in the COM that include improving educational skills and curricular reform efforts, and keeping the faculty engaged and supporting their professional development in teaching. Programming is determined by the OFD and its advisory committee based on needs assessment surveys, or as requested by other offices with teaching and administrative responsibilities such as the Office of Medical Education, Simulation and Clinical Skills Center, and dean. Specifically, for the provision of teaching and assessment skills, OFD collaborates with CETLA for speakers, distribution lists, and other resources focused on their intended audiences and with the Office of Institutional Assessment and Evaluation, both at Howard University. Specific examples of programming related to supporting faculty as educators include effective active learning facilitation, use of ExamSoft, curriculum mapping workshop, flipping the classroom, how to run an effective journal club, demystifying the library databases, and related topics.

\section{Role of teaching in promotion and tenure}

With regard to promotions and tenure decisions, the faculty of the university is divided into the following 5 ranks: professor, 
associate professor, assistant professor, instructor, and lecturer. The appointed faculty members are required to engage in effective teaching; make scholarly contributions in research and/or creative works; engage in professional development; and render service to the university, the profession, and the community. Faculty on the tenure track must demonstrate a teaching/educational portfolio along with their scholarly works. The health sciences clinical educator track is intended for individuals whose primary interests and responsibilities are patient care and teaching students and residents through clinical instruction within the university's health care delivery sites or at a community health care delivery site. These faculty members are not eligible for tenure. Individuals in this track must have leadership potential and comprehensive knowledge of her or his specialty as indicated by, but not limited to, outstanding achievement in specialty board certification, teaching experience and proficiency, scholarly productivity, leadership roles, membership in societies, and national/ international peer recognition. 


\section{Charles E. Schmidt College of Medicine at Florida Atlantic University}

Sarah K. Wood, MD, and Mark DiCorcia, PhD

\section{Medical Education Programs and Highlights}

The mission of the Charles E. Schmidt College of Medicine at Florida Atlantic University (FAU) as a community-based medical school is to advance the health and well-being of our community by training future generations of humanistic clinicians and scientists and translating discovery to patient-centered care.

Highlights:

- The launch of FAU's new medical school in 2011 provided the opportunity to reimagine medical education with innovative practices in a community-based model.

- Small class size of 64 students provides a close-knit and collegial learning environment.

- Adaptive and responsive classrooms support a technologyinfused curriculum and encourage collaboration and teamwork.

- Early longitudinal exposure to patients, health care systems, and the community as students begin working 1:1 with community-based physicians in their first year.

- Over 1,000 affiliate physicians in a wide variety of clinical practice settings in South Florida.

- Highly successful residency matches for our first 5 graduating classes.

- The recent launch of 5 consortium-based residency programs and 4 fellowships.

Unique features:

- The integrated patient-centered curriculum features a developmental approach to problem-based learning (PBL) that promotes clinical reasoning, problem solving, teamwork, and self-directed learning. Student autonomy is promoted by reducing the full-time presence of faculty facilitators. Students progress from PBL, to inquiry (IQ) cases, to self-led inquiry (SLIQ) cases, to diagnosis and reasoning (DR) rounds across the first 3 years of the curriculum.

- Our Healthcare Careers Outreach Program (HCOP) works toward increasing diversity in the health care workforce. FAU medical students, residents, fellows, faculty, and staff lead immersive hands-on educational activities with local K-12 public school students. Over 2,000 students have been involved in HCOP, and graduates of the program pursuing higher education in the health care field have collectively

Acad Med. 2020;95:S99-S102.

doi: 10.1097/ACM.0000000000003288

Copyright (C) 2020 by the Association of American Medical Colleges

Supplemental digital content for this article is available at http://links.Iww.com/ ACADMED/A829.

Year school was established: 2011

School URL: http://med.fau.edu. received over half a million dollars in need- and merit-based scholarships.

- Research in the college is focused on 4 major areas: healthy aging and geriatrics, neuroscience (including dementia prevention), chronic pain management and opioid use disorder, and genomics and precision medicine.

\section{Curriculum description}

- The preclerkship curriculum includes these courses: Fundamentals of Biomedical Science; Neuroscience and Behavior; Pathophysiology and Therapeutics (PT 1-4): PT1, Hematology, GI and Nutrition; PT2, Cardiovascular and Respiratory; PT, Renal, Endocrine, and Reproduction; PT4, Immunologic and Infectious Disease; and Foundations of Medicine (FOM) 1-4.

- Clerkship curriculum: two 22-week sequential blended longitudinal integrated clerkships (LICs). The Medical and Surgical Sciences LIC integrates the core clerkships of medicine and surgery and includes experiences in geriatrics, radiology, emergency medicine, and other related subspecialties; the Family and Community Health Sciences LIC integrates the core clerkships of pediatrics, obstetrics-gynecology, and psychiatry, and includes experiences in related specialties. Students spend 1 half-day per week paired one-on-one with a community-based primary care physician in a 44-week integrated Community and Preventive Medicine clerkship.

- Postclerkship curriculum: Requires all students to complete at least 1 acting internship, 3 selectives, and 3 electives as well as a Transition to Residency course.

See Supplemental Digital Appendix 1-Curriculum Map-at http://links.lww.com/ACADMED/A829.

See Supplemental Digital Appendix 2-Course and Curricular Thread Descriptions_ at http://links.lww.com/ACADMED/A829.

Curriculum changes since 2010

- Overall curricular structure, sequence, and class size has remained unchanged since 2011.

- We are planning a curricular renewal for academic year 20212022 that would transition from a $2+2$ model to a 3-phase model (preclerkship, clerkship, postclerkship).

\section{Assessment}

- The development of a comprehensive competency-based grading system across all 4 years of the curriculum allows each course and clerkship to assess designated PCRS competencies.

- A new grade designation, Satisfactory with Concern (SWC), was approved to help identify students early who are meeting the minimum expectations for a passing competency grade but 
could be "at risk" in the future if performance and/or behavior is not corrected.

- Students who receive an SWC competency grade are referred to the Student Competency Review Committee for a formative support plan. Neither the SWC nor "at risk" status is entered into the student's permanent transcript or Medical Student Performance Evaluation.

- A pattern of receiving SWC final grades or failure to improve in any competencies of concern may result in a recommendation to the student affairs dean for potential referral to the Promotions Committee.

See Supplemental Digital Appendix 3-Program Goals and Assessment Methods_at http://links.lww.com/ACADMED/ A829.

\section{Parallel curriculum or tracks}

- Students may apply into an MD-MBA and MD-MHA with FAU's College of Business or into an MD-PHD program with the Scripps Research Institute in Jupiter, Florida.

- As GME programs mature, we may develop competency-based education programs that would allow students to move directly from UME to GME within certain specialties.

\section{Pedagogy}

- Substantial time spent in small-group, case-based sessions such as PBL, team-based learning, labs, workshops, and small-group clinical skills sessions with standardized patients and highfidelity simulation manikins.

- Students spend at least 10 hours a week in small groups and are provided with a minimum of 2-3 afternoons per week of independent study to prepare for these sessions.

- A hallmark of our model is the developmental progression over the first 2 years from traditional, small-group case-based learning in PBL groups, to IQ case groups, and finally to SLIQ cases, which uses half the number of faculty because 1 facilitator covers 2 groups and is only present for $50 \%$ of the case discussions. The SLIQ facilitator's role is primarily limited to providing feedback on group process.

\section{Clinical experiences}

- Training at affiliated clinical sites within the first 6 months of the curriculum in weekly hands-on patient care experiences with community-based physician practices, local clinics for the underserved, and in our Clinical Skills Simulation Center.

- In the third year, students rotate through two 6-month blended LICs and a yearlong community-based preventive medicine preceptorship including exposures in a rural health setting, skilled nursing facilities, hospice, an interprofessional experience in a memory and wellness center, and a simulation workshop focused on the care of LGBTQ+ patients.

- A longitudinal pediatric preceptor program was launched in 2017 that allows select students with a strong interest in pediatrics to work with a community-based pediatric preceptor for their entire third year.
- As a community-based medical school, we depend on our partner hospitals and more than 1,000 practicing physicians. Like most medical schools, we are in a constant state of recruitment for community-based primary care physicians to serve as preceptors.

- FAU's partner hospital CMOs serve as associate deans for academic affairs and help identify community faculty to teach in specific sites or disciplines as they credential new physicians to their hospital staff.

See Supplemental Digital Appendix 4-Clinical Curriculum Descriptions—at http://links.lww.com/ACADMED/A829.

\section{Curricular Governance}

- The College of Medicine Curriculum Committee (COMCC) is charged with the responsibility for the curricular continuous quality improvement (CQI) process and reviewing, evaluating, and making policy recommendations for the medical education program as a whole. This includes oversight of curricular design and development, methods of pedagogy, methods of student assessment, and setting standards for student academic and professional achievement, based on the college's medical education mission. There are 2 subcommittees and 3 workgroups of COMCC.

- The Curricular Integration and Program Evaluation Committee (CIPEC) facilitates the ongoing review of the curriculum as a whole, including monitoring curriculum content to identify gaps, redundancies, and appropriate sequencing; ensuring integration of content within periods of study (horizontal integration) and across years (vertical integration); monitoring student learning outcomes and approaches to student assessment; and monitoring program evaluation data.

- The Learning Resources Committee provides direct input and feedback on library collections, services, and policies and facilitates the development and implementation of a vision for effective utilization of technology in teaching, learning, and scholarship among the faculty, students, administration, and staff.

- Ad hoc Curriculum Workgroups meet as needed to review and provide plans for specific courses, years, or phases of the curriculum to the CIPEC and/or COMCC.

- The Clerkship Director Workgroup meets monthly to review all components of year 3 clerkships and year 4 curriculum and make recommendations to COMCC.

- The UME/GME Clinical Integration Workgroup meets quarterly to collaborate, review resources, and coordinate learner placement for year 3, year 4, and visiting students.

\section{Education Staff}

The Office of Medical Education (OME) is responsible for overseeing UME curricular operations and is not responsible for pre-UME, GME, or CME educational programming.

- The senior associate dean for medical education serves as the chief academic officer with responsibility for oversight of the UME/GME medical education program. 
- The assistant dean for medical education/accreditation is responsible for CQI and ensuring UME compliance with LCME standards.

- The assistant dean for medical education/curriculum is responsible for curricular management of UME, OME staff leaders, and course-/year-specific support.

- The first- and second-year basic science curriculum director oversees the foundational science curriculum and supervises all the course directors during the preclerkship phase of the curriculum..

- The first- and second-year clinical science curriculum director oversees the FOM curriculum, the annual institutional competency assessments, and supervises the course directors for the following components of the 4 FOM courses: clinical learning groups, clinical experiences, science of clinical practice, clinical skills/simulation, and IPE/service learning.
- The third-year director oversees the year 3 LIC curriculum and supervises the clerkship and cross-discipline directors in surgery, pediatrics, obstetrics-gynecology, psychiatry, medicine, geriatrics/palliative care, critical care/anesthesia, emergency medicine, and community and preventive medicine.

- The fourth-year directors oversee the year 4 curriculum including acting internships, electives, selectives, the Transition to Residency course, and the Visiting Student Program.

- The director of program evaluation and assessment designs, implements, and monitors program outcomes, oversees the Data Repository for Medical Education Research, and is supported by 2 full-time evaluation coordinators.

- The director and associate director of the OME oversee the course/year-specific support staff for the UME curriculum.

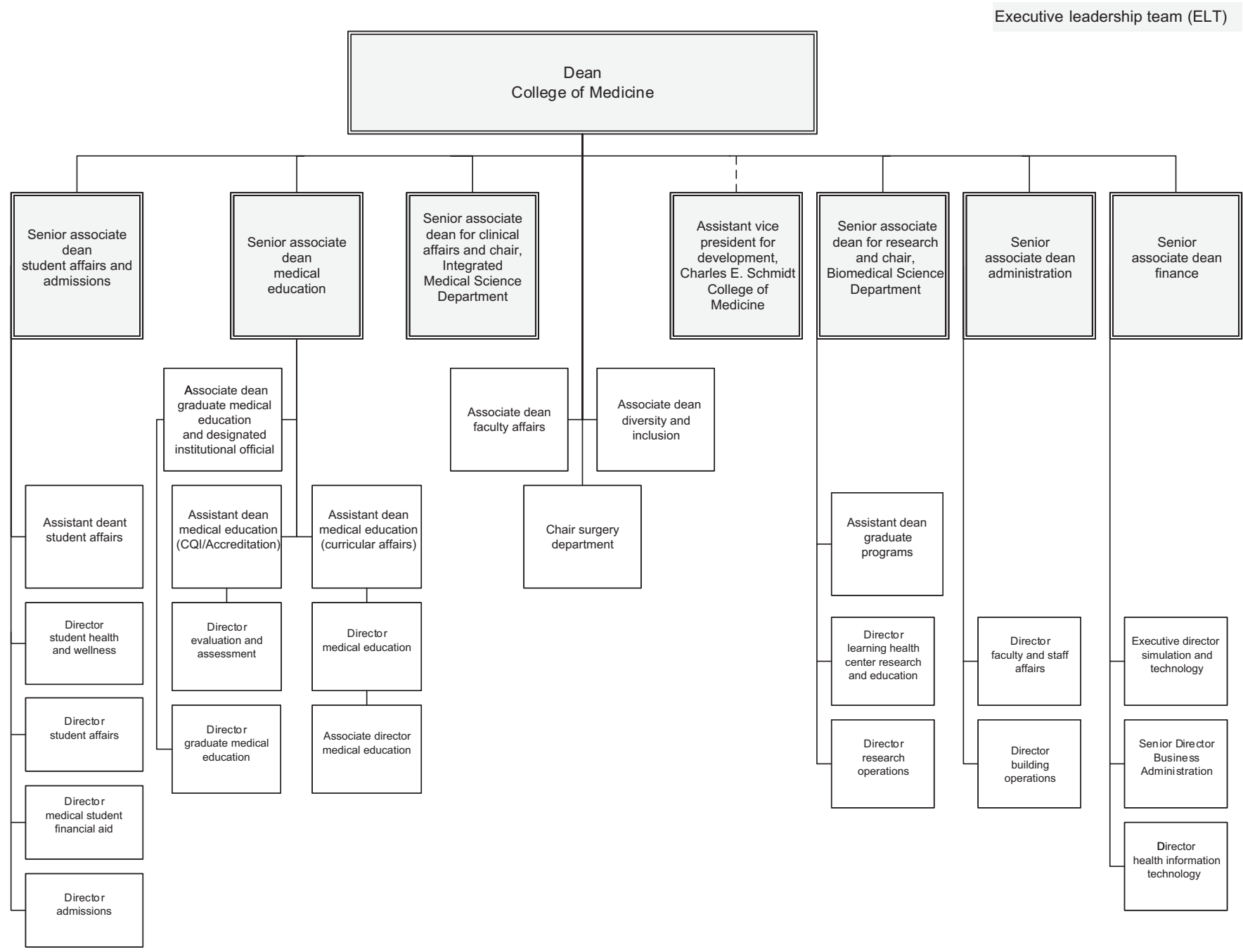

Figure 1 Organizational chart. 
- Education technology coordinators oversee the curricular/ technology initiatives, platforms, and resources that support education and provide ongoing faculty development.

See Figure 1-Organizational chart.

\section{Faculty Development and Support in Education}

- All faculty have opportunities for professional development to enhance skills in teaching, assessment/evaluation, curriculum development, and research/scholarship and are provided resources for career planning, promotion, and tenure.

- The Office of Faculty Affairs provides live and online faculty development and CME offerings, as well as academic enrichment funds to support attendance at professional development conferences.

- Educational Technology holds regular sessions to promote active learning, interactive assessment, and infusing technology thoughtfully into the classroom.

- A peer evaluation of teaching program provides feedback from experienced educators on large- or small-group teaching.

- A wide variety of lecture series are offered on campus, as well as grand rounds at the hospitals.

- All core faculty receive funding annually from their department for attendance at professional meetings, professional membership fees, CME, and resources as needed.

- FAU's Division of Research and the College of Medicine's departments provide support and mentoring for faculty engaging in research and scholarship.

- A Medical Education Scholarship Workgroup meets regularly to improve skills and provide opportunities for educators to engage in educational research activities.
- The FAU College of Medicine plans to launch its Teaching Academy in 2020 to support educators and educational scholarship. It will serve the medical school community by providing enhanced access to professional development, skillbuilding resources, and opportunities for collaboration and advancement.

\section{Initiatives in Progress}

The following initiatives are planned to enhance the strength and success of the medical education program in the coming years:

- The medical college is deeply committed to diversity and inclusion; a Diversity Action Plan is launching in 2020 as part of the school's refresh of its 2017 Strategic Plan.

- Significant efforts to raise more scholarship funds through a large-scale university fundraising campaign to support recruiting a diverse and exceptional student body.

- Continued expansion of our HealthFirst health and wellness program that will focus on student, resident, faculty, and staff well-being and vitality.

- Ongoing investment in community partnerships with affiliate hospitals and community organizations as these valuable, synergistic relationships are critical to support our mission.

- Lastly, plans to proceed with a curriculum renewal that will aim to build on our strengths while incorporating innovative curricular and pedagogical components and fully embracing a 3-phase curricular structure.

Acknowledgments: The authors wish to thank the staff and faculty of the College of Medicine for their hard work, innovation, and commitment to excellence in FAU's medical education program. 


\section{Florida International University Herbert Wertheim College of Medicine}

Vivian Obeso, MD, Janelle M. Fernandez, MS, MBA, Carolyn D. Runowicz, MD, and Robert Sackstein, MD, PhD

\section{Medical Education Program Highlights}

Florida International University (FIU) Herbert Wertheim College of Medicine (HWCOM) provides students with a unique and innovative 4-year MD program. The basic sciences and clinical medicine are well integrated throughout the 4 years by incorporating innovative methods of teaching, learning, and assessment. The curriculum emphasizes patient-centered care and fosters an in-depth understanding of the social determinants of health. The first 3 periods of study were designed using a spiral curricular approach to improve retention and build in complexity. Clinical training starts early in the first year and is augmented by a unique, longitudinal, interdisciplinary experience in underserved community households. Students are immersed early on in various hands-on experiences using simulation-based teaching and assessment. Strong community affiliations offer the advantage of training with a highly diverse patient population in a variety of public and private inpatient and outpatient clinical settings.

The Green Family Foundation Neighborhood Health Education Learning Program (NeighborhoodHELP) is the platform for the community-engaged mission of the college. Emphasizing social accountability and interprofessional education,

NeighborhoodHELP trains future physicians and other health professions students to deliver health care that addresses the social determinants of health to improve the health outcomes of underserved populations. Interprofessional teams of FIU students, faculty, and outreach workers are assigned to and visit underserved households in Miami-Dade County's racially and ethnically diverse neighborhoods. FIU HWCOM has received 2 Association for Medical Education in Europe ASPIREto-Excellence awards based on this signature program, the 2016 award for Social Accountability, and the 2019 award for Inspirational Approaches to Health Professions Education.

Professional development (PD) is one of 5 major strands of the curriculum that runs throughout the 4-year program. The PD strand was the recipient of the 2018 Alpha Omega Alpha Honor Medical Society Edward D. Harris Professionalism Award, recognizing best practices in medical professionalism education. The Professional Behavior course within the strand uses a case-based approach addressing real-world issues. The teaching format offers students the opportunity for thoughtful discussions and reflection, and embraces a humanistic approach. Other

Acad Med. 2020;95:S103-S106.

doi: 10.1097/ACM.00000000000003282

Copyright ( $\odot 2020$ by the Association of American Medical Colleges

Year school was established: 2007.

School URL: https://medicine.fiu.edu. courses within the strand integrate content regarding medical ethics, the doctor-patient relationship, and population-based medicine. The strand prepares students for the professional practice of medicine.

The Research Scholarship course is designed to help students develop the theory and methodological competencies needed to be a lead researcher. Using active learning techniques, students apply the skills needed by clinicians to understand and interpret research studies and learn how to conduct research. Students are assigned a content mentor and complete an original research project by the fourth year.

In 2014, HWCOM was selected by the Association of American Medical Colleges as 1 of 10 medical schools to participate in a pilot to test the implementation of the Core Entrustable Professional Activities (EPAs) for Entering Residency. A program of assessment using the EPA framework has been implemented for the classes of 2019, 2020, and 2021.

Eloquor is the student-published medical arts journal. The mission of Eloquor is to serve as a forum for students to share their art in the form of short stories, essays, poems, and images. Through their art, students share their personal experiences as they encounter the beauty as well as the hardships of becoming a physician. The Florida Medial Student Research Journal (FMSRJ) is a student-run, peer-reviewed research journal. FMSRJ provides opportunities for Florida medical students to develop and publish their research and to participate in the peer review process.

\section{Curriculum}

\section{Curriculum description}

The curriculum is divided into 4 periods: foundations of medicine (Period 1), organ systems-based pathophysiology (Period 2), core clinical clerkships (Period 3), and advanced clinical rotations (Period 4).

Courses and clinical experiences are organized into 5 longitudinal strands that run concurrently throughout the medical education program: human biology; disease, illness, and injury; clinical medicine; PD; and medicine and society.

Clinical experiences in primary care and emergency care settings begin during Period 1. Students participate in the longitudinal service-learning NeighborhoodHELP program, during which medical students collaborate with FIU students from the law school and other health professions (nursing, physician assistants, public health, social work, occupational health) to provide both medical and social care for their assigned family/ household. 
During Period 3 clerkships, students return to campus on select days to participate in the Core Concepts of Clinical Medicine course, a problem-based learning course designed to continue to integrate basic sciences and clinical medicine. The course emphasizes evidence-based medicine and clinical decision making.

During Period 4, students are required to complete a subinternship, a geriatrics rotation, and a 4-week emergency medicine rotation. Broad elective opportunities encourage students to explore personal interests and build competency in preparation for residency. Students participate in the Professional Development and Clinical Medicine Simulation-Based Capstone before graduation. Students also present their original research project completed through the Research Scholarship course at a formal research symposium at the end of the fourth year.

\section{Curriculum changes since 2010}

HWCOM matriculated its first class in the fall of 2009. Curriculum planning and identification of resources were done thoughtfully, anticipating an expanding class size within 4 years. The first cohort consisted of 40 students. Class size gradually increased to a goal of 120 per class in 2016.

See Table 1-Cohort Size, 2013-2019.

Over the last several years, the curriculum has evolved in the following ways:

- Replaced ACGME competencies with the Physician Competency Reference Set (PCRS)

- Added a "social accountability" domain to the PCRS competencies consistent with the college's mission and values

- Enhanced and integrated several key topics throughout curriculum using a spiral approach, for example, evidencebased medicine, nutrition, ethics/advanced care planning, informed consent, pedigrees/genetic counseling, sexual health, LGBTQ+ health

- Expansion of active learning methods: additional resources for faculty development (director of preclerkship faculty development) as well as an instructional design team helped engage faculty and facilitate the transition from passive learning to more active learning methods

\section{Table 1}

\section{Cohort Size, 2013-2019}

\section{Cohort}

Class of 2013

Class of 2014

Class of 2015

Class of 2016

Class of 2017

Class of 2018

Class of 2019
Number of students
- Implementation of the RAPID Simulation Program (Responding Appropriately to a Patient in Distress): practice of basic steps in responding to a patient in distress and learning how to communicate as a team

- Enhanced the student experience in each clerkship by incorporating simulation-based teaching and assessment

- Incorporated innovative elective opportunities, for example, medical education teaching elective, narrative medicine elective

- Expansion of clinical affiliates throughout the community to continue to provide a rich learning environment and a broad range of clinical experiences

- Implementation of EPAs and Entrustment Committees

\section{Assessment}

In the spring of 2015, HWCOM replaced the ACGME domains of competence with the PCRS. This allowed for the inclusion of new competency domains, "interprofessional" and "personal and professional development," which are aligned with HWCOM educational priorities. At the same time, the medicine and society strand revised the additional HWCOM "social responsibility" domain, proposing a "social accountability" domain comprising competencies that parallel the PCRS.

See Table 2-Program Objectives and Assessment Methods.

\section{Pedagogy}

All courses include active learning. Pedagogies used in the curriculum include:

- Large- and small-group case-based learning sessions

- Problem-based learning

- Team-based learning

- Roleplay

- Simulation/task trainers/high-fidelity mannequins

- Standardized patients

- Video

- Virtual patients

- Brief interactive active learning teaching methods in the classroom

- Technology-based classroom games/resources, for example, Kahoot, Jeopardy, Poll Everywhere, Turning Point

Recent changes in student assessment include:

- Incorporating case-based diagnostic examinations in several basic science and organ system courses. Multidisciplinary faculty work groups develop short-answer case-based examinations to promote the application and integration of basic science concepts to clinical cases and assess clinical reasoning.

- Implementation of an exam review process. All exams developed in-house are reviewed by an exam review committee before exam delivery to determine the quality and effectiveness of each question. The committee consists of a multidisciplinary team of faculty, the respective course director, the director of assessment, and an editor.

- Mapping all exam questions to course learning objectives and educational program objectives (EPOs). Student performance in each EPO is tracked and reviewed within and across courses. 


\section{Table 2}

\section{Program Objectives and Assessment Methods}

\section{Medical education program objectives}

Identify the major principles of the sciences foundational to medicine

Explain how the body responds to change, and how it adjusts the functions of cells, organs, and organ systems to maintain homeostasis

Apply the principles of foundational sciences to propose and interpret diagnostic tests, and to determine the etiology pathophysiology, prevention, and treatment of human disease/disorders

Communicate effectively with patients, families, and health care providers, including in situations involving cultural and linguistic differences (and including the effective use of professional interpreters)

Conduct and document complete patient histories and physical examinations using the requisite fundamental clinical skills, and incorporating relevant social, behavioral, and medical factors

Identify and propose initial therapy for acute life-threatening situations

Identify, propose, and coordinate prevention and management plans for acute and chronic disease while working effectively in various health care settings

Apply medical knowledge and critical thinking to develop differential diagnoses

Correctly perform basic procedural skills with attention to patient comfort

Obtain and manage patient information from the medical record

Use evidence-based medicine to provide quality health care to individuals and populations

Apply scientific methods to answer research questions

Identify core principles of health system science including the impact of finance, laws, organizational structures, and health policy on patients and populations

Identify the personal skills and system-level processes that support continuous quality improvement and patient safety, and use standard precautions in the health care setting

Apply knowledge of complementary and integrative medicine to patient care

Identify end-of-life care issues from the physician's and patient's perspectives

Demonstrate skills and habits to sustain lifelong personal and professional growth

Integrate epidemiologic, socioeconomic, behavioral, cultural, and community factors into patient care

Advocate for improved health outcomes at the community level through analysis of social determinants of health, engagement, and reflection

Demonstrate patient-centered counseling techniques and engage patients in collaborative planning to improve health behaviors

Collaborate as a member of an interprofessional team

Anticipate ethical issues encountered in clinical care and research, explain ethically justifiable options and consequences from multiple perspectives, and manage ethical challenges.
Assessment methods

ESA, MCQ, NBME

ESA, MCQ, NBME

ESA, MCQ, NBME

OSCE, PE

OSCE

ACLS/BLS, OSCE

MCQ, NBME, PE

CE, OSCE, PE (oral exam)

$\mathrm{PE}$

OSCE

CE, ESA, MCQ, PE

CE, SGR

CE

\section{CE, OSCE, PE}

CE

CE

$\mathrm{CE}, \mathrm{PE}$

ESA, OSCE, PE

CAPSTONE, PE, SGR

OSCE, PE

CE, PE

CE, OSCE, SGR

Abbreviations: ACLS/BLS, advanced cardiac life support/basic life support; CAPSTONE, project leading to scientific and/or lay presentation; CE, course exam; ESA, essay/short-answer exams; $M C Q$, multiple-choice question exams; NBME, National Board of Medical Examiners subject examinations (including United States Medical Licensing Exam

Part 1); OSCE, objective structured clinical examination; PE, preceptor evaluation; SGR, student-generated reports.

- Implemented a program of assessment using the EPA framework. The program takes a holistic, longitudinal view of student performance in most of the 13 EPAs. Using a student-driven approach and multiple assessment data points, including workplace-based assessment, data have been gathered and reviewed in a newly implemented entrustment committee process for the classes of 2019,2020, and 2021. The process is formative.

\section{Clinical experiences}

Students are assigned a community preceptor during Periods 1 and 2 . Once per month, students spend a half day with their preceptor. Students can participate in emergency medicine shifts at affiliate emergency departments during their first 2 years.
FIU HWCOM employs a community-based model for clinical education. The college does not own or operate its own hospital, rather it forges affiliations with clinical community-based partners who can best serve its mission, goals, and objectives for clinical medical education. Students have the advantage of training with a highly diverse patient population in a variety of public and private inpatient and outpatient settings. Clinical sites include public and private hospitals, academic teaching hospitals, and private clinics. Recently, we have expanded outpatient and inpatient opportunities at the Miami Veterans Administration Medical Center.

One of the challenges of a having a broad community-based model is the variability of faculty expertise in teaching across 
clinical sites. To address this challenge, the assistant dean for faculty development has developed a comprehensive clinical faculty development program. Clerkship reviews occur twice a year. Student evaluations are closely monitored for opportunities to improve teaching. Clinical faculty can have 1:1 consultation, attend workshops, and complete online teaching modules for continuing medical education credits.

\section{Curricular Governance}

The FIU HWCOM curriculum is centrally managed by the Curriculum Committee. The Curriculum Committee and its subcommittees develop and review EPOs, ensure horizontal and vertical curricular integration, monitor the overall quality of courses and clerkships, monitor outcomes of the entire curriculum, and monitor the MD degree program curriculum for achievement of program goals and compliance with accreditation standards.

Subcommittees include:

- Strand Leaders Committee

- Foundational Education Committee

- Clinical Education Committee

- Assessment Committee

- Program Evaluation and Quality Improvement Committee

- Educational Technology Committee

\section{Education Staff}

The Office of Academic Affairs provides oversight of the education curriculum, academic advising, student promotions, and advancement and faculty affairs.

The Office of Medical Education (OME), under the auspices of the Office of Academic Affairs, is responsible for the MD degree program. The OME implements HWCOM's innovative MD curriculum at the direction of the Curriculum Committee and led by the associate dean for curriculum and medical education
In addition, the OME oversees the following:

- Office of Assessment and Evaluation

- Clinical and Classroom Teaching-Faculty Development

- Academic Success Services

- Simulation Center

Faculty within OME have dual reporting structure.

See Figure 1-Deans and directors organizational chart.

\section{Faculty Development and Support in Education}

The OME provides a comprehensive program of faculty development that ensures faculty members have opportunities to enhance their skills in teaching and assessment.

The assistant dean for faculty development oversees a cohesive, comprehensive clinical faculty development program in the framework of the HWCOM's distributed community model of medical education.

The director of preclerkship faculty development provides 1-on-1 consultation as well as workshops that help preclerkship faculty acquire the necessary skills to fulfill their roles as educators.

Funding provided by the OME allows faculty members to participate in programs offered in-house at FIU and to attend regional or national meetings.

\section{Initiatives in Progress}

Since 2009, FIU HWCOM has reported numeric grades for most courses and clerkships. Starting with the class of 2024, the college will implement a new pass/fail system. Clerkships will incorporate a tiered pass/near honors/honors system. Students will have the opportunity to earn an Honorable Mention in a strand. We anticipate that this change will enhance student well-being, encourage self-directed learning, and be the next step toward achieving a true competency-based system.

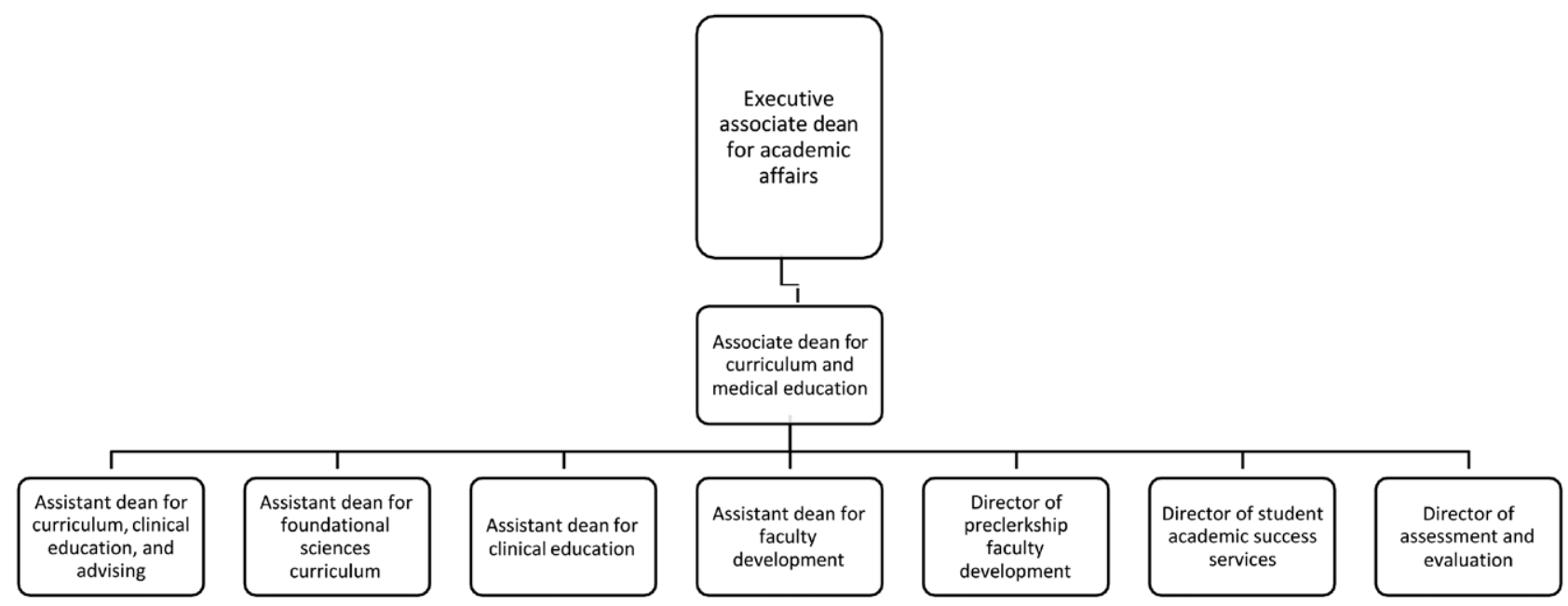

Figure 1 Deans and directors organizational chart. 


\title{
Florida State University College of Medicine
}

\author{
Alma B. Littles, MD, Nancy L. Hayes, PhD, and Suzanne Leonard Harrison, MD
}

\section{Medical Education Program Highlights}

Florida State University College of Medicine is a communitybased medical school with a distributed, regional campus model and a class size of 120. Our 4-year medical education program has a preclerkship phase that takes place at the central campus of Florida State University in Tallahassee and a clerkship phase that takes place at 6 regional campuses and 2 rural sites. Twenty students are assigned to each regional campus (Pensacola, Tallahassee, Orlando, Sarasota, Daytona Beach, Ft. Pierce) for years 3 and 4 . Four students complete their entire third year in rural Marianna, just west of Tallahassee, and 5 students train in nearby Thomasville, Georgia. Students from the regional campuses can complete some of their clerkship training in rural Immokalee, Florida. Highlights of our program include:

- Pairing students with community faculty in an apprenticeshiplike, 1-on-1 relationship for clinical training remains the most unique feature of our educational program.

- Extensive student support and resources, including full-time clinical psychologists and educators who provide academic and personal support, learning communities for study space and relationship formation, fully electronic library with 24-hour access, and regional campus facilities with a campus dean and student support staff.

- Rural Learning Experience: an immersion experience that provides a first exposure to rural communities for many of our incoming students and increases awareness of and commitment to our rural mission.

- Integration of clinical and foundational sciences in all preclerkship courses emphasizes the application of knowledge in clinical context.

\section{Curriculum}

\section{Curriculum description}

- The medical education program begins the Tuesday after Memorial Day.

- Short courses and block sequencing in the preclerkship phase allow flexibility for students who may need "catch up/make up" time.

- Year 3 includes the 6 core clerkships, a 2-week Community Medicine course, the Doctoring 3 course, which includes a longitudinal experience focused on caring for patients with chronic disease, and two 4-week flex blocks.

Acad Med. 2020;95:S107-S1 10.

doi: 10.1097/ACM.0000000000003308

Copyright (C) 2020 by the Association of American Medical Colleges

Supplemental digital content for this article is available at http://links.Iww.com/ ACADMED/A837.

Year school was established: 2001.

School URL: https://med.fsu.edu.
- Year 4 includes 3 required 4-week clerkships (emergency medicine, geriatrics, and a subinternship in either family medicine or internal medicine), a 3-week residency preparation bootcamp, and a minimum of 16 elective weeks.

See Supplemental Digital Appendix 1-Curriculum Map—at http://links.lww.com/ACADMED/A837.

\section{Curriculum changes since 2010}

In AY 2014-2015, we began implementation of our redesigned curriculum, which included changes across all 4 years. While the overall length of the curricular phases did not change, the redesign included shifts in the academic calendar, providing greater flexibility in scheduling. The most significant changes were:

- Preclerkship phase changed from a discipline-based, stacked curriculum to a fully integrated, systems-based, block curriculum.

- New activities for interprofessional learning. We added a Physician Assistant Program in AY 2017-2018, which provides opportunities to bring MD and PA students together in a variety of curricular and extracurricular activities.

- Sequential use of the NBME Comprehensive Basic Science Exam (CBSE).

- Enhanced dedicated USMLE Step 1 examination preparation time as a "course" to provide structured monitoring of readiness to take Step 1 and to identify and intercede with learners as needed.

- Converted all year 3 core clerkships to 6-week duration.

- Added two 4-week flex time blocks in year 3 to be used for clerkship makeups or remediation and to provide early elective opportunities and career exploration.

- Added preclerkship and residency preparation boot camps.

- Added new third-party learning tools for preclerkship and clerkship support.

- Developed a longitudinal integrated clerkship (LIC) for students at the Marianna site. The LIC has the same learning objectives, requirements, and assessments as clerkships at regional campuses.

\section{Strategies for overcoming challenges in curricular changes}

We will continue to address similar challenges as most medical schools, including those associated with the increasing competitiveness of the Match, students' focus on USMLE Step 1 , and the proliferation of third-party learning platforms that respond to student preferences. Our strategies include:

- Emphasis on active learning

- Partnership with a third-party platform as an integral component (not add-on) of our curriculum by:

- Aligning with our learning objectives

- Allowing faculty to "vet" external resources, respond to, and build on them 
- Providing faculty with resources that prepare students for classroom activities and allow time to develop new classroom application activities

- Providing spaced learning tools and formative assessments

- Using NBME customized assessments in all preclerkship courses

Anticipated changes include incorporation of basic ultrasound in the preclerkship phase and training/experience in telehealth across the 4-year continuum.

\section{Assessment}

In 2017, the Curriculum Committee adopted the AAMC Physician Competency Reference Set as our education program objectives, adding a mission-based domain.

- All preclerkship system-based courses include a PICO (Patient/ Problem, Intervention, Comparison, and Outcome) assignment. Each student self-identifies a learning need (based on a patient or case from class or preceptorship), researches, and reports the results via Canvas. Faculty and peers provide individual written feedback during small-group sessions or using Canvas.

- All preclerkship exams are comprised completely or in part of NBME customized assessments.

- We use 3 administrations of the CBSE to guide student selfdirected study and assess readiness for USMLE Step 1.

- The end-of-year 2 OSCE was converted to a high-stakes assessment of readiness for the clerkship year. The midyear-3 formative OSCE conducted at the regional campuses is now used to document any needed remediation as well as preparedness for Step 2 CS.

- We added a quality improvement project in year 3. Groups of 3-5 students research the quality improvement process in a hospital or practice; select an evidence-based, measurable problem; present results in the format of a medical journal article; and conclude by giving presentations to their peers.

See Supplemental Digital Appendix 2-Education Program Objectives and Assessment Methods—at http://links.lww.com/ ACADMED/A837.

\section{Pedagogy}

We continue to use multiple pedagogic strategies in preclerkships and clerkship phases and particularly encourage those that are evidence based for adult learners and are student focused.

\section{Clinical experiences}

- Standardized patient encounters throughout the preclerkship phase

- Preclerkship preceptorships in the offices of community physicians

- Summer Clinical Practicum, a 3-week rotation at the endof-year 1 practicing history and physical exam and clinical reasoning skills with a community faculty member in the office setting

While inpatient training in community hospitals is an integral part of our program, the core clerkship year is, by intent, predominantly an outpatient experience with students working 1-on-1 with faculty in their offices and wherever they provide care. Our mission emphasis on primary care and underserved, rural, elderly, and minority populations is consistent with a significant proportion (60\%-70\%) of clinical training taking place in ambulatory settings that include private physician offices, skilled nursing facilities, managed care organizations, emergency departments, rehabilitation facilities, hospice, and home visits. Instead of a traditional "teaching hospital," our regional campuses are affiliated with over 60 hospitals. Given that patients admitted to hospitals today are typically more complex and spend less time in inpatient areas, our model allows our students to see patients where they receive most of their care. The subinternships may be completed with a residency team or a hospitalist.

\section{Challenges in designing and implementing clinical experiences for medical students}

- Increased competition for community clinical faculty as new medical schools open across the state and region. We have a well-established and loyal group of over 2,300 clerkship faculty.

- Ensuring comparability of educational and student experiences at multiple campuses is an ongoing challenge to which we pay continuous attention.

- Maintaining revenues to compensate community-based faculty in an environment of decreasing revenues is a continuing challenge.

\section{Curricular Governance}

- The Curriculum Committee is charged with central monitoring of the educational program with responsibility for curricular design and development, implementation, and evaluation consistent with the mission of the college.

- The Year 1 and 2 Subcommittee and the Year 3 and 4 Subcommittee of the Curriculum Committee continuously review their years of the curriculum, address implementation issues, and recommend changes to the Curriculum Committee for improvement of content, integration, and evaluation as necessary.

See Figure 1-Curriculum management.

The senior associate dean for medical education and academic affairs (SADMEAA) is the chief academic officer, providing oversight and support for faculty teaching assignments and performance and overall management of the budget for the educational program. The regional campus deans are the principal academic officers at each campus and report to the SADMEAA via the senior associate dean for regional campuses.

See Figure 2-Curriculum governance.

\section{Department of Medical Education}

Units under the direction of the SADMEAA manage and support all aspects of the medical education curriculum. The Office of 
Office of Medical Education - granular data collection and analysis, reported on a defined schedule to

Year 1\&2 and 3\&4 Subcommittees of the Curriculum
Committee - contemporaneous review of student
performance by course or clerkship; regularly scheduled
course and clerkship reviews. Reviews, recommendations, and
alerts to any issues are reported to

Figure 1 Curriculum management.

Medical Education (OME) includes the directors of clinical foundations (preclerkship) and clinical programs (clerkships and electives) and their support staff. Additional curricular support comes from the directors of the medical library, instructional design, the clinical learning center, preceptorships, student counseling services, informatics and information technology (IT), and the associate dean for faculty development. Each regional campus has a regional dean and a support staff of 6 or 7 full-time employees. The education directors work with the campus deans to recruit and train the clerkship directors in their discipline, one per campus. Each clerkship director works with the campus dean to recruit and train local physicians, who are then assigned students to teach and observe.

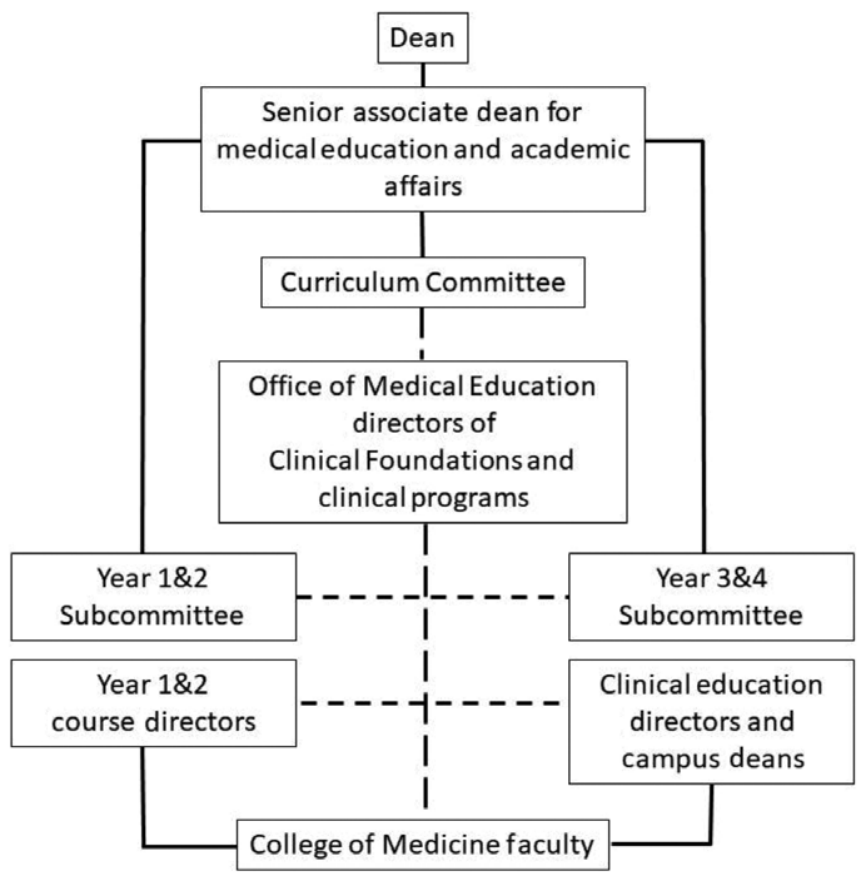

Figure 2 Curriculum governance.
In addition to developing calendars and Canvas sites and assisting faculty in developing materials and assessments, and implementing all courses and clerkships, OME populates the curriculum management database; prepares, administers, and analyzes course and clerkship evaluations and other surveys related to the educational program; and works directly with IT to collect and verify student performance data.

IT also consolidates statistics and presents those data to the faculty, assists in the development of technology for teaching innovations, provides classroom support and videoconferencing, and maintains a secure web-based testing environment. An IT coordinator at each regional campus supports the faculty and students.

\section{Faculty Development and Support in Education}

- The associate dean for faculty development oversees a system to assist the development of faculty as educators including programming offered at the central and regional campuses.

- Faculty receive mentoring and support through their departments, and each full-time faculty member receives an annual stipend to support participation in professional development activities.

- All regional campus faculty complete a core of 4 hours of faculty development before being assigned a student and must participate in ongoing faculty development to maintain their appointment/reappointment status.

- Regional campus deans, clerkship directors, education directors, and central campus faculty and administration meet for 2 days twice a year to provide and receive updates on student performance, curriculum, and current topics in medical education.

\section{Role of teaching in promotion and tenure}

Teaching excellence is a major factor in promotion and tenure. Many of our teaching faculty are not tenure earning, which allows intense participation in teaching and educational 
activities. For faculty members on the teaching faculty and instructional support faculty track, university policies do not require evidence of scholarly productivity for promotion. Community clinical faculty play a major teaching role for the clerkships. Their initial appointment and promotion procedures are overseen by the Committee on Clinical Faculty.

\section{Regional Medical Campuses}

Processes are in place to monitor and assure comparability at all sites:

- Collaboration exists between the director of clinical programs, OME staff, and education directors and campus deans, and regular meetings of clerkship directors, education directors, campus deans, and central campus staff ensure ongoing comparability of the educational program among the campuses.

- Learning objectives, required clinical encounters/skills, assessment methods, and grading policy for each required clerkship are the same for every site.

- Patient encounters, exam scores, and clerkship projects are reviewed by the education director, who assigns the final

\section{Table 1}

\section{Regional Medical Campuses}

\begin{tabular}{lcc} 
Regional campus name & $\begin{array}{c}\text { Type } \\
\text { Clinical }\end{array}$ & $\begin{array}{c}\text { Student enrollment } \\
\text { Daytona }\end{array}$ \\
Ft. Pierce & Clinical & 40 \\
\hdashline Orlando & Clinical & 40 \\
\hdashline Pensacola & Clinical & 40 \\
\hdashline Sarasota & Clinical & 40 \\
\hdashline Tallahassee & Clinical & 40 \\
\hline
\end{tabular}

grade for all students on their respective clerkship at every campus.

- Students at all campuses complete the MedEdIQ evaluation of the clinical faculty and their clerkship experiences. Results are compared by campus and across campuses.

- The Year 3 and 4 Subcommittee and the Curriculum Committee review data from student performance and the learning environment at the regional campuses.

See Table 1—Regional Medical Campuses. 


\section{Nova Southeastern University Dr. Kiran C. Patel College of Allopathic Medicine}

Paula S. Wales, EdD, Lindsey C. Henson, MD, PhD, and Johannes W. Vieweg, MD

\section{Medical Education Program Highlights}

The Dr. Kiran C. Patel College of Allopathic Medicine (NSU MD) is a new medical school at Nova Southeastern University, a private, not-for-profit university with 6 other health professions colleges, including an osteopathic medical school. The colleges share teaching faculty for portions of their curricula, and students from all the colleges take classes in core buildings on the university's main campus. The MD curriculum is integrated, case based, and system sequenced, with a focus on developing physician leaders. Unique aspects of the program include:

- Small class size (50 students/year), with 2 learning communities and physician learning community mentors, who advise students from matriculation to graduation.

- A 16-month integrated preclerkship curriculum that emphasizes active and student-directed learning, introduces clinical experiences in the first course, and includes a research thread and a required research project.

- An affiliation agreement with the Healthcare Corporation of America (HCA)-East Florida Division to provide all required inpatient clerkships at HCA hospitals in south Florida. HCA is currently building a new mixed inpatient ( 250 beds) and outpatient facility on the NSU campus, with capacity for future expansion, which will become a site for faculty clinical practice and training along the educational continuum.

\section{Curriculum}

\section{Curriculum description}

- In the preclerkship curriculum, foundational science blocks are integrated across disciplines and use a hybrid problem-based learning (PBL) model. The first block focuses on core principles and is followed by a series of blocks designed around organ systems.

- The concurrent Practice of Medicine courses include clinical skills training with standardized patients; simulation exercises; patient care experiences in the offices of preceptors; and smallgroup discussions and large-group interactive sessions focusing on clinical reasoning, case presentations, longitudinal topics in the curriculum (e.g., cultural competency, health disparities, societal problems, patient safety), and curricular threads (e.g., ethics and humanities, interprofessional collaboration, research).

Acad Med. 2020;95:S111-S114.

doi: 10.1097/ACM.0000000000003257

Copyright (C) 2020 by the Association of American Medical Colleges

Supplemental digital content for this article is available at http://links.lww.com/ ACADMED/A814.

Year school was established: 2017.

School URL: https://md.nova.edu/index.html.
- After each foundational science block, there are 1 or 2 weeks for reflection, integration, and assessment (RIA). These include formative and summative assessments addressing a range of competencies, curricular activities, student affairs activities, and scheduled one-on-one meetings of students with their assigned learning community mentor.

- The clerkship curriculum begins in March of year 2, after students take the USMLE Step 1 examination. Students take the Clinical Skills and Reasoning course and complete the diagnostic medicine clerkship, both of which integrate material from the preclerkship curriculum and are designed to hone the knowledge and skills students need for the clinical clerkships that follow.

- There are seven 1- or 2-month discipline-specific clinical clerkships, which are organized into 3 clerkship blocks. Each block includes 2 or more required clerkships and is followed by 2 RIA weeks, which include summative assessments for the clerkships students completed during that block and curricular and student affairs activities.

- After taking USMLE Step 2 CK and CS, students will complete a required subinternship, elective experiences, and required research if not completed in year 2 .

See Supplemental Digital Appendix 1-Curriculum Map—at http://links.lww.com/ACADMED/A814.

\section{Assessment}

- The medical education program objectives are based on the competencies in the Physician Competency Reference Set, with the addition of objectives for scholarly inquiry.

- In 2019, after the first iteration of the year 1 curriculum, the program objectives and assessments were revised to eliminate redundancies, simplify language, and ensure that objectives are measurable and aligned with appropriate assessment methods.

See Supplemental Digital Appendix 2-Medical Education Program Objectives—at http://links.lww.com/ACADMED/A814.

\section{Pedagogy}

- NSU MD's preclerkship curriculum employs a variety of active learning and case-based formats that require preparation outside of class. Students have 3 or 4 half days each week protected for independent study.

- Student-directed small-group learning includes sequential disclosure PBL cases for the first 3 preclerkship blocks, followed by inquiry cases (IQs) in the last 2 blocks. Explicit assignments and coaching in self-directed learning skills are incorporated into the PBL and IQ sessions.

- Teacher-directed active learning formats include laboratories, case-based learning (large-group interactive discussions), teambased learning, simulations, and weekly sessions with standardized patients, which employ the hypothesis-driven history and physical examination from the beginning of medical school. 
- Students are required to attend all active learning and clinical sessions ( $\sim 70 \%$ of curricular time in the preclerkship curriculum). Attendance at lectures, where active learning strategies are expected to be incorporated, is not required. All lectures are recorded and archived electronically.

- The clerkship curriculum begins with a course (Clinical Skills and Reasoning) and clerkship (diagnostic medicine) that use many of the same active learning formats as the preclerkship curriculum. The same formats are also used during the weekly half-day core curriculum that runs throughout the clinical clerkships.

\section{Clinical experiences}

- Clinical sites used for required experiences include physicians' offices/clinics for ambulatory preceptorships in the preclerkship curriculum and a combination of hospital inpatient settings and outpatient clinics for the required clerkships. At present, there are ACGME-accredited residencies in all core clerkship disciplines, with the exception of obstetrics-gynecology.

- The outpatient primary care preceptorship in the preclerkship curriculum is a required longitudinal experience.
- Students learn competency in basic clinical skills (history, physical exam, and documentation) with standardized patients during the first semester of medical school. Students interact with real patients during longitudinal preceptorships in the Practice of Medicine course in the second semester of the first year.

- Challenges in designing the clinical experiences have been related to clinical site capacity, location of residency programs, and scheduling constraints due to varying university and hospital partner policies. Cumulatively, these constraints influenced the length of some clerkships, student selective offerings, and the sequencing of some clerkships. A systemic process management approach to learning and assessment in the clerkships is one goal of the weekly on-campus clerkship sessions.

- To ensure educational equivalence in the required clerkships, we limited the number of hospitals for a given discipline and added a weekly core curriculum for all students.

- To adhere to our principle that assessments should reinforce/ reward long-term retention of knowledge and skills rather than short-term recall, we clustered clerkships in blocks, with summative assessments (subject exams, OSCEs, etc.) for all clerkships in a given block during the subsequent RIA weeks.

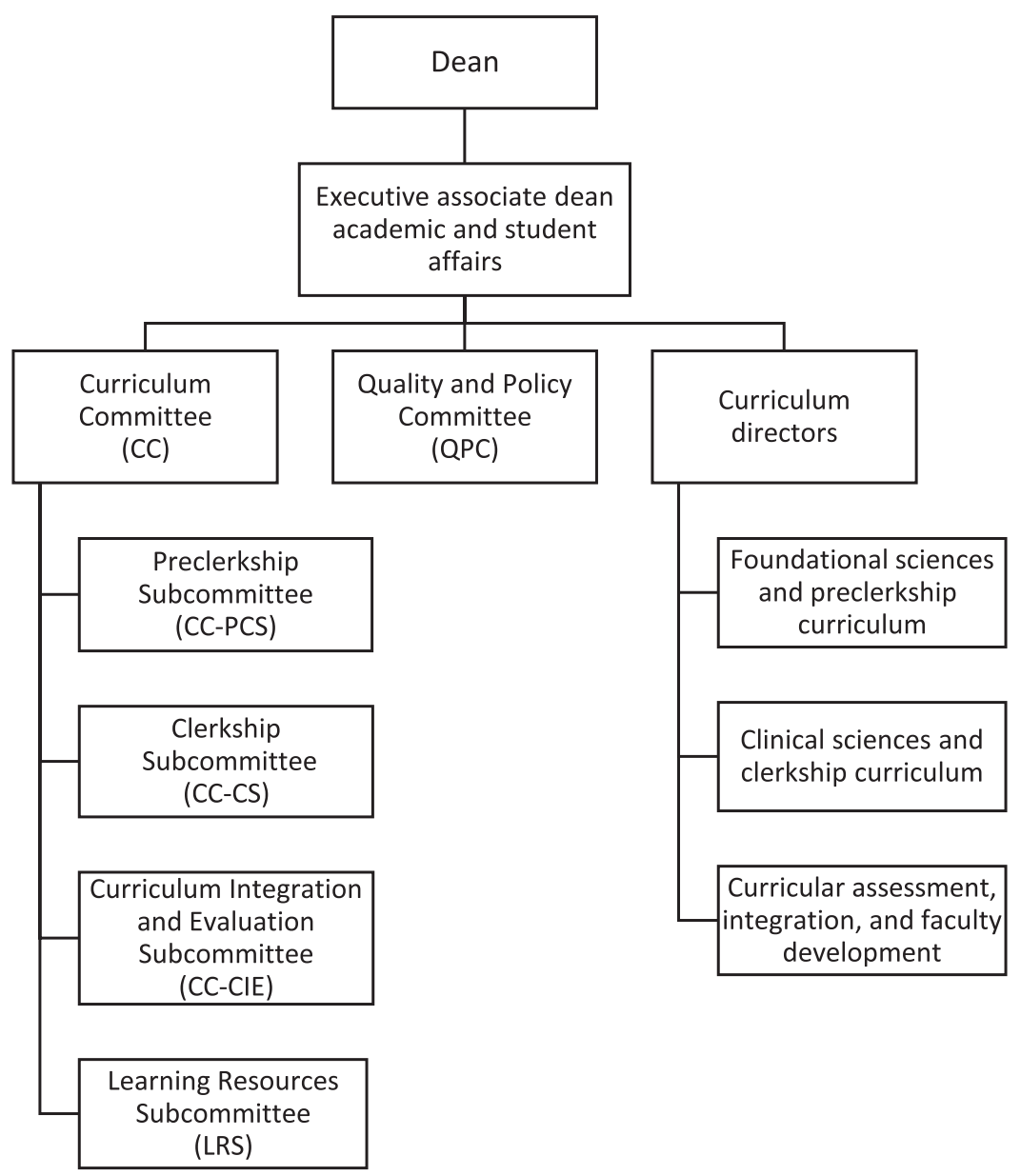

Figure 1 Organizational chart for management of the curriculum. 


\section{Curricular Governance}

- The faculty committees that manage the curriculum include the Curriculum Committee, its 4 subcommittees, and the Quality and Policy Committee, which is responsible for monitoring and evaluating the effectiveness of all programs as well as outcomes of the medical school's strategic plan.

- Day-to-day management and strategic planning for implementation of the curriculum resides with the faculty curriculum directors, who serve on the executive associate dean's curriculum cabinet and also direct or codirect courses.

- All aspects of curricular governance are centralized and based in the Office of Medical Education and ultimately reported to the dean.

See Figure 1-Organizational chart for management of the curriculum.

See Figure 2-Educational program leadership and Office of Medical Education.

\section{Education Staff}

- The Office of Medical Education is responsible for: recruitment and admission of students, including developing diversity pipelines; student advising and other aspects of student affairs; faculty affairs, including recruitment, appointment, annual reviews, and promotions; faculty development; implementation and evaluation of the curriculum, including IT resources for curriculum delivery, program evaluation, and student assessment; administrative support for all medical school committees, including the student promotions committee; grants related to medical education; and all aspects of LCME accreditation. The executive associate dean also prepares and manages budgets for the MD program.

- At present, NSU MD has 3 academic departments: Clinical Sciences, Population Health Sciences, and Medical Education. The Department of Medical Education includes all full-time faculty whose primary responsibility is implementation of the MD program and education of the medical students, with the exception of the clerkship directors and clinical volunteer/affiliate faculty, who are appointed in Clinical Sciences.

\section{Faculty Development and Support in Education}

- Professional development for faculty as educators in provided centrally by the Office of Medical Education. The director of curricular assessment, integration, and faculty development plans the formal faculty development sessions, based on needs identified by the executive associate dean and faculty needs assessment surveys.

- A major focus of faculty development continues to be skills building for design and implementation of the curriculum as it is developed, with additional sessions dedicated to LCME accreditation.

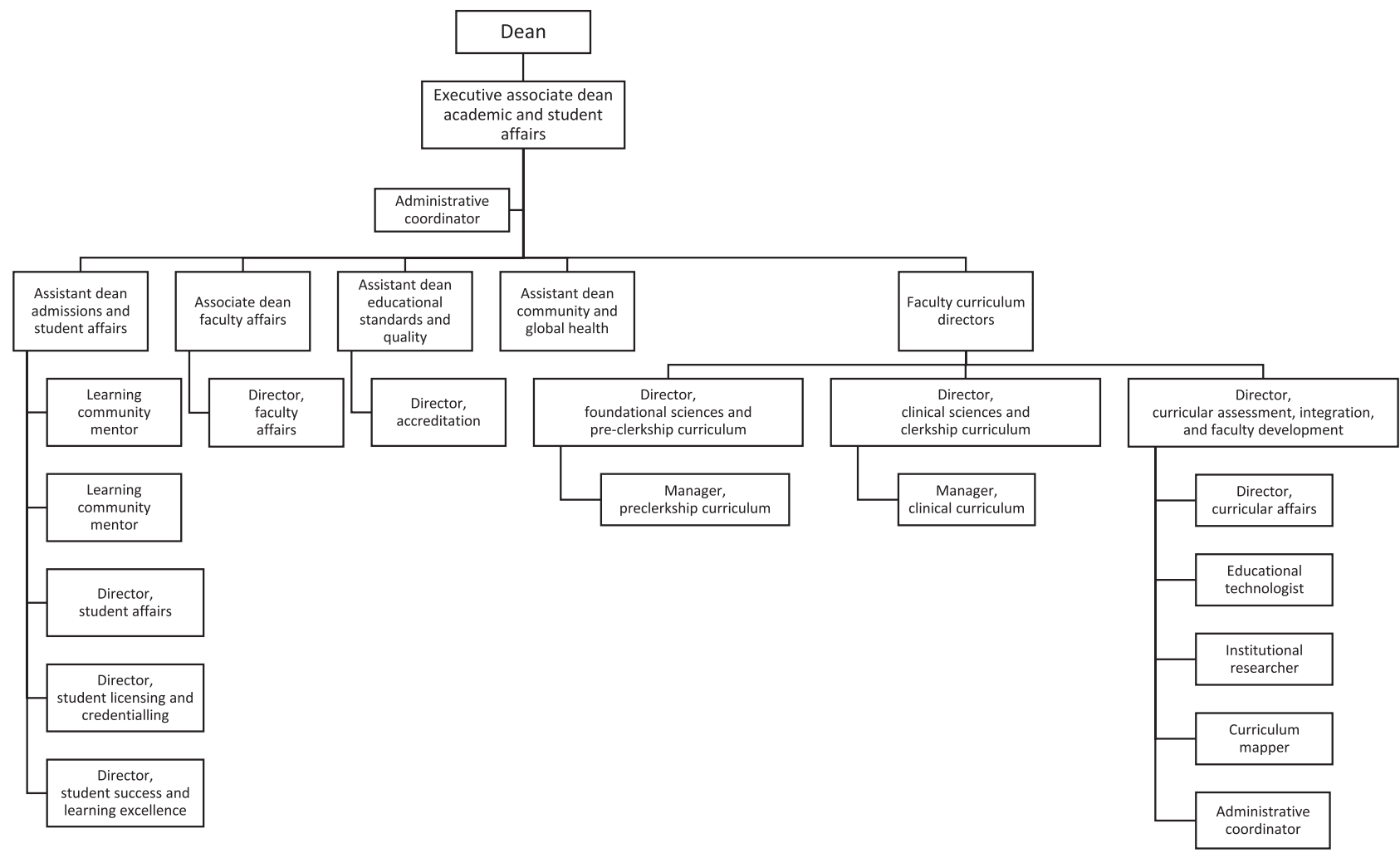

Figure 2 Educational program leadership and Office of Medical Education. 
- There are also frequent, regular sessions for specific teaching roles (e.g., PBL facilitators, clinical skills, clinical skills teachers, OSCE raters) that are built into teaching assignments and schedules and provided by faculty curriculum directors and course directors.

- NSU MD considers 4 categories of academic responsibility for faculty evaluation and promotion: education, research and scholarship, patient care, and service. All full-time faculty are expected to contribute to the education mission.

- Achievements in education, research and scholarship, patient care, and service are emphasized proportionally, based on the individual candidate's percent of effort in these areas over time during his/her progress toward promotion. Recommendations for promotion are based on evidence that the candidate has achieved excellence in the major area of effort, while demonstrating proficiency in all other assigned areas.
- NSU MD uses the AAMC's 5 categories of educational activities in assignment of responsibilities, tracking faculty effort, and consideration for promotion: teaching, defined as direct teaching and creation of associated instructional materials; curriculum development or revision; advising and mentoring; educational leadership and administration; and learner assessment.

- Guidelines and categories of educational effort have been applied to determining rank for initial appointment and for faculty work assignments, tracking of effort, and annual reviews. The first NSU MD faculty candidates for promotion in rank will be considered during the 2020-2021 academic year.

\section{Reference}

1 Armstrong EG, Mackey M, Spear SJ. Medical education as a process management problem. Acad Med. 2004;79:721-728. 


\section{University of Central Florida College of Medicine}

Richard Peppler, PhD, Martin Klapheke, MD, and Jeffrey La Rochelle, MD

\section{Medical Education Program Highlights}

- Students complete a research project during the first 2 years of medical school as part of the Focused Inquiry and Research Experience course. This allows each student to develop skills of intellectual inquiry applicable to the practice of medicine.

- Community interprofessional education experiences in student-run clinics for uninsured patients, farm workers, and homeless, along with global health experiences, occur.

- Longitudinal curricular themes (LCTs) on culture, ethics, geriatrics, medical informatics, nutrition, and patient safety are presented throughout the curriculum.

- A 4-week elective in the third year allows students to experience clinical/research areas to assist in career planning.

\section{Curriculum}

See Supplemental Digital Appendix 1-Curriculum Description —at http://links.lww.com/ACADMED/A827.

\section{Curriculum changes since 2010}

Changes made for the first and second years:

- Increased content in biostatistics and epidemiology

- Decreased lectures with concomitant increase in small-group learning

- Students assigned a personal librarian upon matriculation

- Increased ultrasound skill instruction to complement learning in organ systems

Changes made for the third and fourth years:

- Increased number of clinical placement sites across central Florida

- Implemented 8 LCTs throughout the third year

- Implemented OSCEs in all core clerkships except surgery

- Increased monitoring of standard portions of clerkship OSCE performance over the entire third year to identify areas for individual student focus/remediation

- Added a 4-week elective in the third year

- Decreased the length of the surgery core clerkship from 12 to 8 weeks

- Increased content/simulation in the fourth-year "boot camps" for transition to residencies

- Increased number of "away" rotations allowed in the fourth year

Acad Med. 2020;95:S115-S1 18.

doi: 10.1097/ACM.0000000000003273

Copyright (C) 2020 by the Association of American Medical Colleges

Supplemental digital content for this article is available at http://links.Iww.com/ ACADMED/A827.

Year school was established: 2008.

School URL: https://med.ucf.edu.
- Fourth-year rotations evolved to require an acting internship and emergency medicine or critical care selective plus 6 electives

Changes planned in the third and fourth years:

- Addition of a Practice of Medicine III course in the third year for increased longitudinal focus on clinical skills

- Increase in standardization of third-year core clerkship grading rubrics and narratives

- Addition of intersessions during the third year focused on clinical skills and LCTs

\section{Class size changes since 2010}

The class size has grown from 40 students per year in the charter class to 120 students per year; this has not changed the schools' structure.

\section{Assessment \\ Our medical education program objectives are based on the ACGME domains of competence.}

See Supplemental Digital Appendix 2-Medical Education Program Objectives and Assessment Methods-at http://links. lww.com/ACADMED/A827.

\section{Assessment changes since 2010}

- The mid-third-year OSCE was deleted, and OSCEs were designed and occur in all of the 6 core clerkships except 1 . The end-of-third-year OSCE is a high-stakes assessment for transition to the M4 year.

- The administration of comprehensive basic science examinations was reduced from 5 to 1 in preclerkship years.

\section{Pedagogy}

The curriculum has been very dynamic in terms of changes to module and session levels, such as altering the length and timing of modules and adjusting the portfolio of required clinical experiences. The Offices of Faculty Development, Assessment, and Education Technology collectively assist faculty members with the use of technology, assessment techniques, and different formats of instruction (e.g., team-based learning and self-learning modules). The trend has been to decrease the didactics that occur in a class setting and increase small groups, particularly using clinical cases. When lectures are used, interactive tools and learner response systems are used. Students are exposed to clinical experiences in the first 2 years in the Practice of Medicine modules, which include a Community of Practice clinical preceptor component and experiences in the clinical skills and simulation center with standardized patients or simulation. Cadaver laboratory experiences are used concomitant with medical imaging and ultrasound exposure. 
Faculty continue to improve vertical and horizontal integration, complete item bank annotations, and reduce the proportion of curriculum delivered as didactic lectures.

\section{Changes in pedagogy since 2010}

For the second through fourth years: decreased lectures in core clerkship didactics, with increase in "flipped classroom" and increased use of simulation, small groups, and case-based learning.

\section{Clinical experiences}

Home visits, outpatient, residential, inpatient; Veterans Health Administration; private, public, community-based hospitals.

\section{Required longitudinal experiences}

LCTs are presented throughout the medical school curriculum on 8 topics: culture, health, and society; patient safety; medical nutrition; ethics and humanities; gender-based medicine; geriatrics and principles of palliative care; interprofessional education; and medical informatics.

\section{Clinical experience first encounter}

In the first year, students have experiences in the Community of Practice component of the Practice of Medicine module. This is continued in the second year as well.

\section{Required and elective community-based rotations}

During core clerkships and the third-year elective, third-year students rotate at outpatient clinics at: Orlando VA Bay Pines VA, Nemours Children's Hospital, University Behavioral Center, Park Place Behavioral Health Care, UCF Health, Pasadena Villa, HCA-Ocala Regional, Osceola Sheriff's Employee Clinic, HCANorth Florida Regional, Naples Healthcare System, True Health, Osceola Community Health Services, WellMed, Express Clinic, and private medical offices.

\section{Challenges in designing and implementing clinical experiences for medical students}

- Our school relies heavily on affiliated/volunteer faculty; hence, the most significant challenge has been the increasing requests by third-year clinical preceptors to be paid for teaching medical students.

- Technological and pedagogical problems associated with teleconferencing of third-year didactic sessions is a recurring challenge.

\section{Curricular Governance}

See Figure 1-Curricular governance.

\section{Decentralized curricular governance}

Budgets are developed at the user level and presented by the associate dean for faculty and academic affairs (ADFAA) to the budget committee of the college. Ultimately, the entire college budget is approved by the enterprise and the dean. The medical education program is a high priority, and these funds are provided through the ADFAA. Each module and clerkship have created a budget to handle the specifics of their respective programs. The operating budgets that support the medical education program are centralized in the Office of Faculty and Academic Affairs, which includes the Office of Planning and Knowledge Management, the health sciences library, the Clinical Skills and Simulation Center, the anatomy laboratory, and faculty development and continuous professional development. The Clinical Skills and Simulation Center budget, through the Office of Faculty and Academic Affairs, provides for equipping the facilities and for its operation. This includes funds for the hiring, training, and use of standardized patients within modules and for OSCEs conducted within the core clerkships and at the end of the third year. The support for the anatomy laboratory includes personnel, supplies, equipment, and the purchase of cadavers from the Anatomical Board. Funding for student research projects and funds to present at meetings are available for students. Faculty salary lines reside in the respective departments in which the faculty appointment is based, even though faculty are involved in the delivery of the MD program.

\section{Education Staff}

The responsibility for the medical education program has been delegated to the ADFAA, who reports directly to the dean. The ADFAA is supported by 2 assistant deans of medical education who provide administrative and academic support for the curriculum with a focus on preclinical and clinical sciences. Other individuals who report to the ADFAA include:

- Assistant dean of planning and knowledge management, who oversees the functions of strategic planning, assessment, educational technology, data and knowledge management, and LCME interface

- Assistant dean of clinical skills and simulation, who provides support to the educational program for teaching and assessing clinical skills within the modules and core clerkships (OSCEs) and for high-stakes OSCEs at the end of the second and third academic years

- Director of the health sciences library, whose librarians and staff members assist faculty and students with information resources and mobile technologies

- Director of the anatomy laboratory, a licensed funeral director/ embalmer who supervises 2 individuals responsible for cleaning the laboratory

- Director for faculty development, a coordinator of continuous professional development and staff responsible for the appointment/credentialing and reappointment of the 2,400 nonsalaried core and affiliated/volunteer faculty

- Academic assistant deans at each of the major affiliate hospitals (Flagler; HCA Regional Medical Centers at Osceola, Ocala, and North Florida; NCH Healthcare Systems; Nemours Children's Hospital; Orlando VAMC; and Bay Pines VA Healthcare System) 


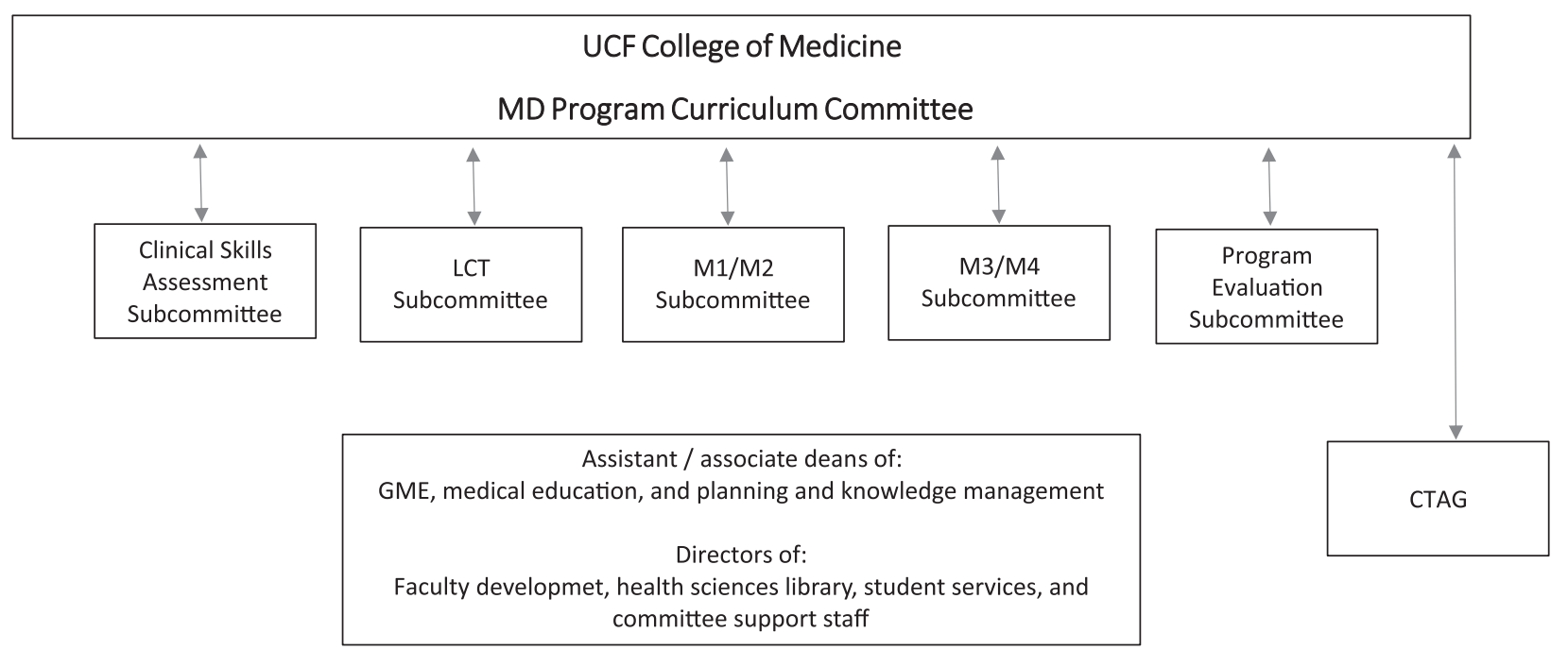

Fig 1. Curricular governance.

The Office of Faculty and Academic Affairs is managed by a director and associate director along with 14 full-time coordinators and administrators who support the instructional modules and clerkships, respectively. Coordinators assist faculty members with the administration of the educational program. There are 2 coordinators for the first-year and secondyear programs, respectively. Two coordinators handle the administration and coordination of the Practice of Medicine
1 and 2 modules, and 2 coordinators and a staff member support the Focused Inquiry and Research Experience 1 and 2 modules. There are 7 clerkship administrators and an associate director for respective clerkships in the third and fourth years. These individuals are responsible for the administrative details associated with the clerkships: orientation, educational activities, student schedules, examinations, OSCEs, and final evaluation.

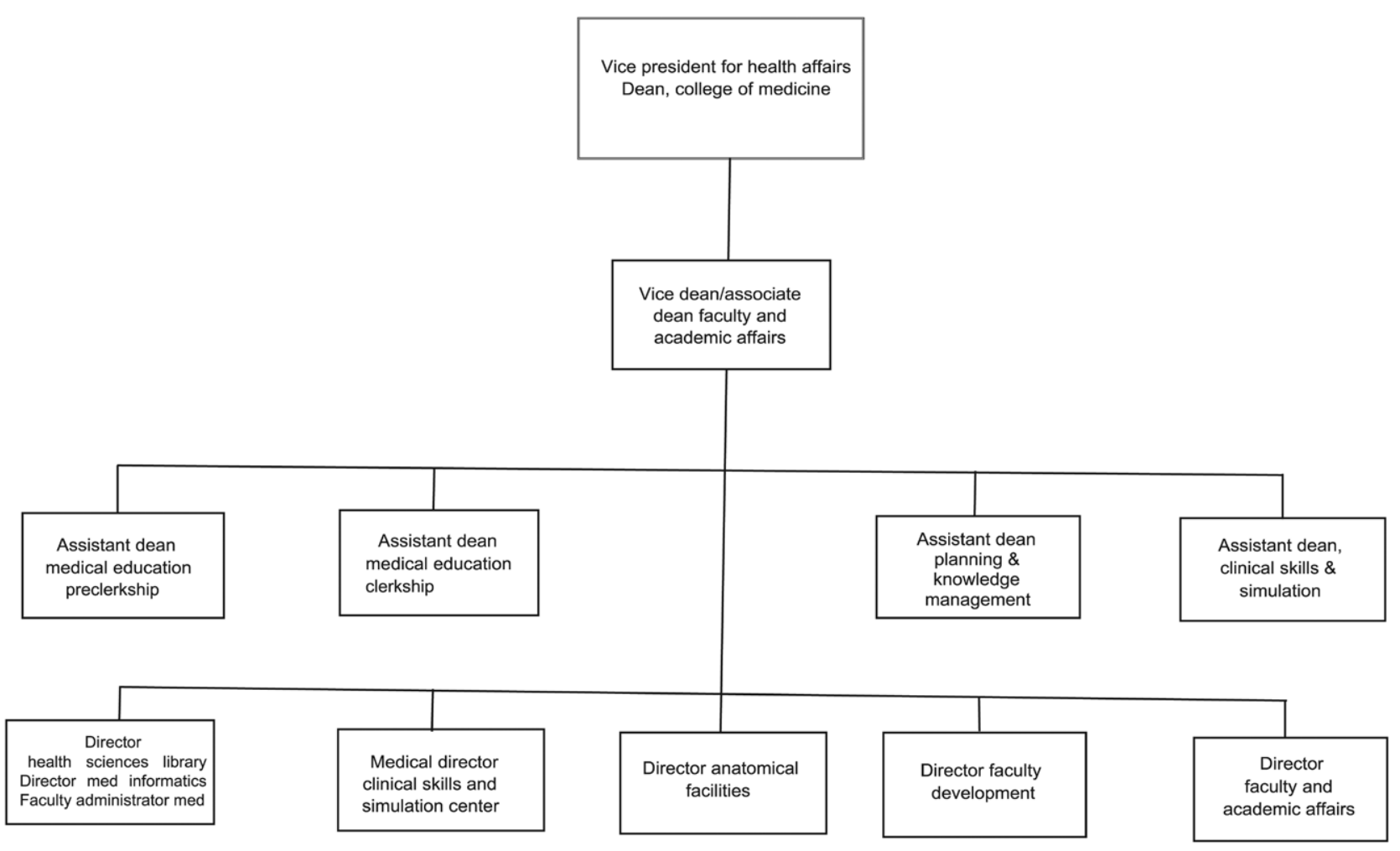

Fig 2. Medical education leadership. 
The Office of Faculty and Academic Affairs is responsible for undergraduate medical education, continuous professional development, nonsalaried faculty appointment, faculty development, planning and knowledge management (to include assessment, educational technology, and knowledge management), the clinical skills and simulation center, and the anatomy laboratory.

See Figure 2-Medical education leadership.

\section{Department of Medical Education}

The Department of Medical Education is 1 of the 5 academic units of the college, where $20 \%$ of the faculty, including librarians, are based. It is composed of faculty who have either a $\mathrm{PhD}$ or MD degree and are responsible for most of the content in the preclerkship curriculum.

\section{Faculty Development and Support in Education}

Professional development for faculty as educators

Faculty are supported by the college's Office of Faculty Development (OFD) and multiple college and university department programs to advance their teaching and assessment skills. Programming within this office occurs on an ad hoc basis, through a series of workshops and/or webbased resources. The ad hoc or personalized approach allows the faculty development professionals (and other medical education faculty members) to work with small groups of faculty members on devising learning objectives, developing appropriate content, identifying appropriate teaching methods, developing formative and summative assessments, and linking assessments to objectives. Workshops are scheduled to facilitate faculty members' understanding of innovative pedagogical techniques, learning theory, and educational technology resources. The OFD collaborates with the Office of Planning and Knowledge Management to present programs related to educational technology and assessment services. There is significant support provided to assist faculty in developing and delivering PowerPoint presentations, case-based materials, selflearning modules, web-based materials, and student assessments. The Office of Educational Technology operates the Faculty Collaboration Center, where faculty obtain individualized instruction related to educational technology, as well as collaborate with other faculty and instructional designers to improve preparation and delivery of content.

\section{Role of teaching in promotion and tenure}

The academic mission requires all faculty members to engage in scholarship and scholarly activity. This requires the dissemination and acceptance of new knowledge by one's peers. For core faculty, their efforts in these areas are assessed during the annual evaluation process and are considered for promotion. Each of the academic units in the college has established criteria for faculty who are in a tenure track and aspire to be awarded tenure and/or be considered for promotion. The tenure probationary period is 9 years. Criteria for promotion of nontenure track faculty have also been established by departments. Clinical departments have established criteria (clinical track) for promotion for physicians (core faculty) who are in practice $50 \%$ of their time or more. Separate criteria have also been developed for those nontenure track faculty (research or teaching) who function as part of a scientific team of scientists working with a primary investigator. Promotion and tenure (P\&T) assistance is provided for faculty at the departmental, college, and university levels. Several departments have a formal mentorship program whereby mentors provide guidance on P\&T timelines, review P\&T packets, and explore scholarly interests and opportunities. 


\section{University of Florida College of Medicine}

Joseph Fantone, MD, Maureen Novak, MD, and Adrian Tyndall, MD

\section{Medical Education Program Highlights}

The University of Florida College of Medicine (UFCOM) strives to provide a highly supportive learning environment that fosters the education and professional development of future physicians, scientists, and scholars who are committed to excellence, humanistic patient-centered care, and service to society. UFCOM engages in continuous curriculum renewal across the spectrum of undergraduate, graduate, and continuing medical ducation, while fostering philanthropic initiatives that support students, faculty, and state-of-the-art infrastructure aligned with UFCOM's vision to be a top 10 public medical school. Medical curricular highlights include longitudinal collaborative learning groups across the curriculum and innovative intensive learning in the preclinical years in societal subjects that affect health: nutrition, pain and addiction, and health policy. Our college continues to support and grow interdisciplinary and interprofessional experiences and service learning through the student-led Equal Access Clinic (https://equalaccess.med.ufl.edu) and through the Free Center for Primary Care (https://freecenter. med.ufl.edu).

\section{Curriculum}

\section{Curriculum description}

Curriculum renewal initiated in 2012 is continuous and has resulted in creation of 3 phases of competency-based curriculum: foundations of medical practice (Phase 1, 68 weeks: biomedical, social-behavioral, and clinical sciences), principles of medical practice (Phase 2, 48 weeks: core clerkships and electives), and advanced medical practice (Phase 3, 40 weeks: advanced-level clinical experiences and electives). Learning outcomes are linked to 6 core competencies.

See Supplemental Digital Appendix 1-Curriculum Map-at http://links.lww.com/ACADMED/A862.

\section{Curriculum changes since 2010}

Current curricular realignment includes integration of health systems science across the 4 years and adoption of the AAMC's Entrustable Professional Activities (EPAs) for entering residency within the 6 core competencies for graduation. Recent changes in clerkship experiences include integration of geriatrics within the family medicine clerkship, providing more intentional

Acad Med. 2020;95:S1 19-S122.

doi: 10.1097/ACM.0000000000003337

Copyright (C) 2020 by the Association of American Medical Colleges

Supplemental digital content for this article is available at http://links.Iww.com/ ACADMED/A862.

Year school was established: 1958.

School URL: https://med.ufl.edu. experiences across the life span. Augmentation of the UF Health Jacksonville Health Science Center as a clinical regional medical campus to attract future physicians dedicated to caring for the medically underserved is currently being developed, reflecting our ongoing commitment to serving the health care needs of the community.

\section{Assessment}

Medical education program objectives are described in outcomebased terms that allow the assessment of medical students' progress in developing the competencies that the profession and the public expect of a physician. ${ }^{1,2}$

The UFCOM has framed its medical education program within the 6 core ACGME competencies for graduation. The 13 EPAs have been integrated within the competency framework. Each of the program objectives are evaluated via several modalities including multiple-choice examinations (both internally and externally developed), direct observation, standardized patient encounters, simulations, portfolio review, USMLE Step examinations, 360-degree assessments, peer assessments, selfassessment, and narrative feedback.

See List 1-Medical Education Program Objectives.

\section{Pedagogy}

The University of Florida utilizes the following pedagogical approaches:

- Role play/dramatization

- Self-directed learning/tutorial

- Simulation

- Standardized/simulated patients

- Team-based learning

- Video/podcast

- Virtual patients

- Workshop

- Supervised medical practice

\section{Changes in pedagogy since 2010}

- Satisfactory/unsatisfactory grading adopted in Phase 1

- 2 additional clinical preceptorships in Phase 1

- Intensive educational immersions in the following topics: research and discovery, nutrition and health, pain and addiction in medicine, population health, and health policy

- Videotaping of didactic sessions for student asynchronous review

- Integration of patient voices into discussions of health and disease in Phase 1

- Integration of geriatrics into the family medicine clerkship

- Integration of health system science across the curriculum

- Development of collaborative learning groups: faculty-led teaching, coaching, and mentoring throughout the curriculum with 8 students per group 


\section{List 1}

\section{Medical Education Program Objectives}

\section{Professionalism}

- Discuss and apply ethical standards of practice

- Demonstrate humanistic and patient-centered care including respect, empathy, and compassion in their role as the patient's advocate

- Demonstrate strength of character and integrity including honesty, altruism, accountability, humility, and moral courage

- Employ measures to balance clinical responsibilities with personal and societal responsibilities

- Strive for excellence in all professional endeavors

- Consistently demonstrate the attitudes, values, and behaviors expected of one who thinks, acts, and will be a physician

\section{Systems-based practice}

- Identify and describe components of health care systems

- Demonstrate a patient-centered approach to diagnosis and treatment that promotes the delivery of safe, high-value, and high-quality patient care

- Participate in and, where appropriate, lead teams including other health care professionals to deliver comprehensive health care that includes facilitating continuity and coordination of ongoing care needs

- Apply patient safety and quality improvement initiatives within health care systems

- Compare and contrast health care policies, financing, and delivery systems, both as they affect populations and to assist patients in navigating these systems

\section{Interpersonal and communication skills}

- Demonstrate knowledge of how cultural issues impact response to illness and interactions with the health care system

- Apply doctor-patient communication strategies appropriate for clinical situations

- Recognize how personal beliefs and biases impact communication

- Present patient information clearly and effectively to all members of an interprofessional health care team

- Use communication technology effectively

- Disclose a medical error

- Deliver bad news compassionately

\section{Medical knowledge}

- Demonstrate understanding of the scientific basis for health and disease including foundational and emerging sciences

- Apply and integrate established and evolving biomedical knowledge and concepts with principles of clinical science to provide optimal clinical care

\section{Patient care}

- Demonstrate the ability to perform an appropriate focused and comprehensive medical history and physical exam

- Critically evaluate clinical findings and initial diagnostic test results to develop appropriate management plans with consideration of patient preferences, ethical principles, and cost effectiveness

- Synthesize new information to refine and reprioritize differential diagnoses, adhering to clinical reasoning best practices

- Critically examine previous management plans when patients were dissatisfied or the desired outcome was not achieved

- Perform common procedures safely

\section{Practice-based learning and improvement}

- Identify clinical questions, search evidence-based databases, critically appraise scientific literature, and apply to patient care keeping with patients' values and preferences

- Apply principles of deliberate practice to self-improvement and professional practice

\section{Clinical experiences}

The types of clinical site the University of Florida uses for required educational experiences are:

- General inpatient hospital wards

- Subspecialty inpatient hospital wards

- The Veterans Administration Health System

- Children's Hospital: freestanding and within hospital

- Emergency departments

- Outpatient clinics in urban, rural, and international settings

- Assisted living and hospice care facilities

- Rehabilitation facilities

\section{Required longitudinal experiences}

- Students on the 12-week family medicine clerkship spend 1 half day per week at a primary care clinic site.

- Students meet 1 half day per week in collaborative learning groups during Phase 1, once a month during Phase 2, and once a quarter during Phase 3 (fourth year). These groups are facilitated by the same faculty member throughout the 4 years.

\section{Clinical experience first encounter}

- Initial clinical experiences occur during the interview day and during orientation with an interactive physician-patient 
interview. Subsequent patient/family presentations occur throughout Phase 1 courses.

- Beginning first semester and continuing through Phase 1, students perform history and physical examinations on patients as part of the Introduction to Clinical Medicine course.

- There are 3 immersive clinical preceptorships in Phase 1 . The first 2-week preceptorship is in December of the first year.

\section{Required and elective community-based rotations}

Although the majority of clinical training occurs in the clinics and hospitals at the 2 health science centers in Gainesville and Jacksonville, additional clinical sites are in the community. These include hospices, assisted living facilities, rehabilitation centers, health departments, Wolfson Children's Hospital, and the Gainesville VA Medical Center. Some students attend private practice sites during the preceptorships in Phase 1.

\section{Challenges in designing and implementing clinical experiences for medical students}

Challenges have included development of appropriate assessment tools and electronic platforms for formative and summative feedback, along with continued faculty development.

\section{Curricular Governance}

The UFCOM Curriculum Committee serves as the faculty decision-making body regarding content, methods, timing, and structure of the medical education program. The Curriculum Committee works with the associate deans for medical education and for student affairs, as well as course/ clerkship directors who will implement changes in curriculum, learning environment, and/or student support services. Major curriculum changes require approval of the Executive Committee of the medical school and dean and the University Curriculum Committee. Membership of the Curriculum Committee consists of:

- Associate dean for medical education (chair)

- Chair of the Course Directors' Committee

- Chair of the Clerkship Directors' Committee

- 6 members from the faculty

- Student academic chair from the fourth-year class

- Faculty council representative

- 2 members from Jacksonville faculty

The Phase 1 Course Committee, Phase 2/3 Clerkship Committee, and Program Evaluation Committee all report to the UFCOM Curriculum Committee on a regular basis. A new position, the director of education quality improvement and accreditation, has recently been created and reports to the senior associate dean for medical education.

\section{Decentralized curricular governance}

Departmental faculty work in tandem with the Office of Medical Education (OME) to provide the curriculum. Budget, assessment, and Phase 1 curricular support is centralized. Direct salary support is provided to all required clerkship directors, course directors, and other faculty discipline leaders. Administrative support for the clinical clerkships is provided through the departments.

\section{Education Staff}

The OME provides centralized support of development and maintenance of the curriculum. This includes instructional technology, online programs, student assessment, program evaluation, simulation, and standardized patient programs. The OME works with the Office of Faculty Affairs and Professional Development to provide faculty development.

See Figure 1-Organizational chart.

The OME is responsible for undergraduate medical education delivery, working in tandem with the Office of Student Affairs. The designated institutional official reports to the dean of the medical school. The graduate programs and admissions report to the senior associate dean for educational affairs. Continuing medical education is coordinated through the offices of Educational and Clinical Affairs.

\section{Faculty Development and Support in Education}

Faculty development is through the Office of Faculty Affairs and Professional Development (https://facultyaffairs.med.ufl. edu). Faculty members of the Society of Teaching Scholars provide additional faculty development programs. The OME oversees a funds distribution model to departments based on educational contributions of faculty as well as direct support of faculty with leadership roles (e.g., course/clerkship directors).

\section{Role of teaching in promotion and tenure}

Teaching/educational efforts are required of all full-time faculty with an academic title. An educational portfolio is required for all faculty members recommended for promotion based on distinction in teaching/education. In addition, the department chair's annual evaluation and letter supporting promotion/tenure will describe the faculty member's teaching contributions and position the candidate's accomplishments within the teaching duties and expectations of the department.

The UFCOM Society of Teaching Scholars honors and fosters teaching and educational scholarship at the UFCOM. The objectives include:

- To provide a forum to recognize and reward UFCOM faculty who have demonstrated excellence in teaching and commitment to the formation of physicians and scientists

- To provide an incentive for the faculty member's professional development as an educational scholar and role model

- To identify a cadre of recognized experts to advise the college of medicine on ways to enhance its educational mission, as well as to serve as mentors/role models to junior faculty, residents, and students 


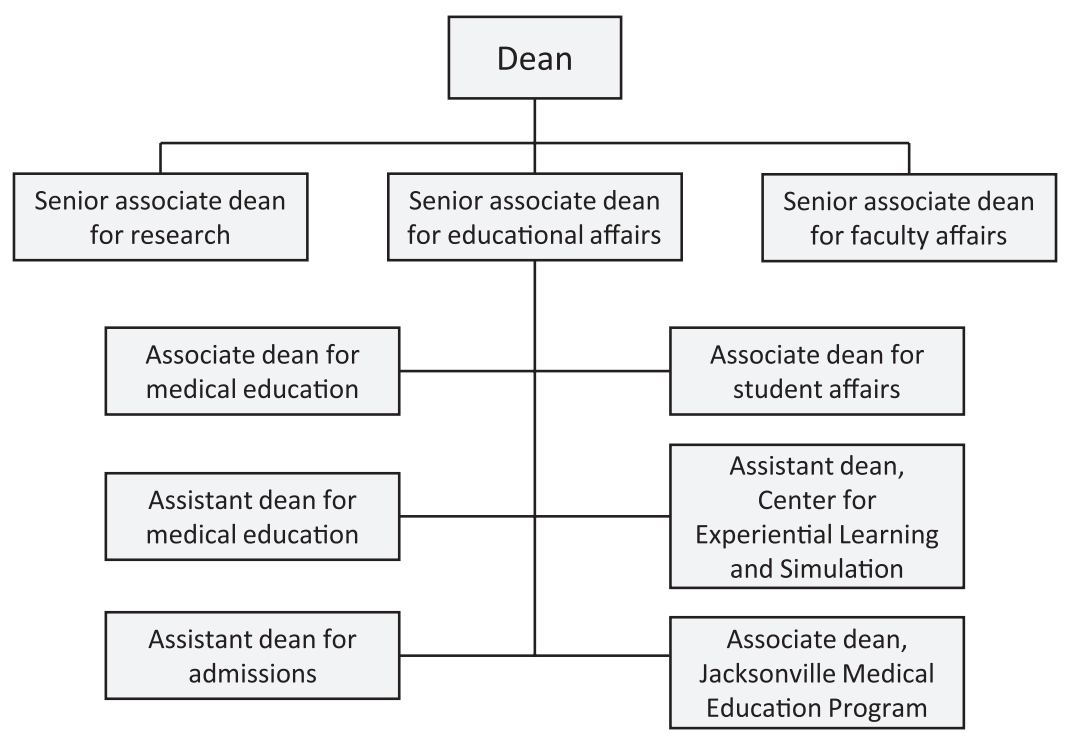

Figure 1 Organizational chart.

- To demonstrate UFCOM's commitment to high-quality teaching and learning, plus continuous quality improvement process of the educational program

\section{Regional Medical Campus}

See Table 1-Regional Medical Campus.

\section{Table 1}

\section{Regional Medical Campus}

\section{Regional}

\section{campus name}

UF Health

Jacksonville

\section{Student enrollment}

Clinical Core clerkships occur at the regional campus with approximately $1 / 5$ of third- and fourthyear students at this site at any one time

\section{Educational experiences across sites}

The core clerkship directors in Gainesville provide educational oversight, including final grading. There is excellent cooperation and constant communication with leadership at both sites regarding delivery of the curricula.

\section{References}

1 Liaison Committee on Medical Education. Functions and structure of a medical school. Standards for accreditation of medical education programs leading to the MD degree. Academic year 2020-2021. Standard 6.1. https://lcme.org/wp-content/uploads/filebase/ standards/2020-21_Functions-and-Structure_2019-10-04.docx. Accessed March 13, 2020.

2 Committee on Accreditation of Canadian Medical Schools. CACMS standards and elements, academic year 2020-2021. Standard 6.1. https:// cacms-cafmc.ca/accreditation-documents/2020-2021/standards-andelements. Accessed March 13, 2020. 


\title{
University of Miami Leonard M. Miller School of Medicine
}

\author{
Alex J. Mechaber, MD, Amar R. Deshpande, MD, Gauri G. Agarwal, MD, Matthew Imm, MD, Rose Maria van Zuilen, PhD,
} Daniel M. Lichtstein, MD, S. Barry Issenberg, MD, and Laurence B. Gardner, MD

\section{Medical Education Program Highlights}

The NextGenMD curriculum at University of Miami Miller School of Medicine (UMMSM) strives to empower learners to transform lives and inspire them to serve the global community.

The NextGenMD curriculum emphasizes:

- Active, case-based, collaborative learning with early clinical experiences

- Integration of basic sciences, clinical medicine, and health systems sciences throughout all curricular phases

- Personalized areas of scholarly concentration including options for a 4-year dual degree or accelerated transition to residency

- Opportunities to study social and cultural determinants of health in South Florida

Graduates will demonstrate competence in 4 core domains: professionalism and interpersonal skills, health systems sciences, biomedical knowledge and clinical care, and practice-based learning.

\section{Curriculum}

The NextGenMD curriculum has 3 phases:

- Phase 1 is grounded in foundational, translational and health systems sciences, learned in a case-based format. Phase 1 is 14 months, including 2 months of summer capstone work.

- Phase 2 consists of integrated clinical clerkships, with continued embedded foundational, translational, and health systems sciences themes. It is divided into four 12week integrated clerkships that begin with introductory bootcamps covering core themes, reinforced throughout the phase, including social determinants of health, patient safety, leadership and health advocacy, wellness promotion, and quality improvement.

- Between Phases 2 and 3, students have dedicated time to prepare for and take USMLE Steps 1 and 2 CK examinations.

- Phase 3 is 17 months and allows students to develop a personalized pathway in a specialized area of interest and pursue scholarly work (including dual-degree pursuits or early transition to residency within our institution).

See Supplemental Digital Appendix 1-Curriculum Overviewat http://links.lww.com/ACADMED/A948.

Acad Med. 2020;95:S123-S127.

doi: 10.1097/ACM.0000000000003459

Copyright (C) 2020 by the Association of American Medical Colleges

Supplemental digital content for this article is available at http://links.Iww.com/ ACADMED/A948.

Year school was established: 1952.

School URL: http://www.med.miami.edu.

\section{Curriculum changes since 2010}

In 2011, UMMSM launched a 4-year MD-MPH dual-degree program designed to produce physician leaders with expanded career opportunities in medicine and public health. It is the largest 4-year dual MD-MPH program nationwide, with approximately 50 students annually. The MD and MPH curricula are fully integrated, and MPH coursework was designed to offer practicum experiences with communitybased clinical partners and capstone projects that integrate public health interventions with clinical practice. Public health immersions are provided in partnership with public health agencies such as the local health department. The MD coursework uses small-group and problem-based learning, along with learning communities involving intensive faculty mentorship. All basic science courses use problem-based learning, with case objectives reflecting integration of multiple core disciplines. Students in this cohort also experience their clinical curriculum on the regional medical campus (RMC), which features a preceptor-based curriculum, focusing on continuity of care and treatment of chronic disease. On the RMC, there are 2 integrated clerkships. The 12 -week integrated medicine clerkship integrates internal medicine with palliative care, geriatrics, and radiology. The 12-week integrated surgery clerkship combines surgery with of anesthesia and radiology. The MD-MPH program used and piloted several pedagogical approaches that became key components of the curriculum.

The NextGenMD curriculum originated from a 2017 taskforce and launched in August 2020.

There have been no changes in class size since 2010 (200 students per class) and none are planned in the future.

\section{Assessment}

The medical education program objectives support our outcomes-based education mission and incorporate and expand upon the AAMC's Entrustable Professional Activities while building a link to GME via ACGME core competencies. Students and faculty track individual progress through e-portfolios using frequent, low-stakes assessments, and students are mentored to create individual learning plans based on interests, demonstrated strengths, and areas for improvement.

See Table 1-Program Objectives and Assessment Methods.

See Supplemental Digital Appendix 2-NextGenMD Domainsat http://links.lww.com/ACADMED/A948.

Students in Phase 1 are assessed primarily with probing shortanswer and essay questions to demonstrate deeper knowledge and mastery learning. Limited use of multiple-choice questions prepares students to meet external progress measures such as USMLE Step examination performance. Leadership and 


\section{Table 1}

\section{Program Objectives and Assessment Methods}

Medical education program objectives

\section{Professionalism and interpersonal skills}

Develops and maintains a professional identity

Maintains emotional, physical, and mental health in the pursuit of continual personal and professional growth

Collaborates as a member of an interprofessional team (includes patients and caregivers)

\section{Assessment methods}

- Narrative medicine-reflections on medicine as a profession

- EPA-based clerkship evaluation form-professionalism section

- Course/clerkship attendance records

- Physicianship incident reports

- Physicianship commendation reports

- Portfolio entries of ongoing professional development

- Faculty mentor/coach portfolio assessment

- Student well-being assessments (e.g., depression, anxiety, stress, burnout, resiliency, imposter syndrome, empathy, work hours)

- Personal well-being plan

- Student journaling

- $360^{\circ}$ and other multisource evaluations

- EPA-based clerkship evaluation form-performance on health care team

- Narrative medicine-reflections on the roles of other disciplines

- IPE competency self-assessment

- Interprofessional team exercises

\section{Health systems science}

Works as a leader in the health care delivery system - Team-focused assessments — evaluation of team function and individual team contributions

- Self and peer assessment of leadership style

- Portfolio entries of leadership roles and institutional service

- Participation in QI projects

- Patient safety exercises

- Resource allocation exercises

Recognizes and addresses social and environmenta determinants of health for patients and populations

- Group community health engagement projects

- Individual public health capstone projects

- Narrative medicine-the patient story

- SP exercises, OSCES

- Portfolio entries of community-based activities (e.g., health fairs, free clinics)

\section{Biomedical knowledge and clinical care}

Obtains, organizes, and communicates clinical data

- EPA-based clerkship evaluation form-reporter section (histories and physical examinations, oral case presentations, written notes)

- Patient write-ups

- SP exercises, OSCEs, mini-CEX evaluations

- Technology-enhanced simulation exercises

Applies foundational science to analyze and prioritize clinical data

- EPA-based clerkship evaluation form-interpreter section

- Patient write-ups/written notes

- NBME subject examinations

- MCQ style progress assessments

- Student generated questions for self-assessment

- Individual and team readiness assessment tests

- Oral examinations

- Clinical reasoning assessments

- Mastery testing: SP exercises, OSCES

- Mini-CEX evaluations

Recommends management for core clinical experiences

- EPA-based clerkship evaluation form - management section

- Patient write-ups/written notes

- Mini-CEX evaluations

- Oral examinations

- NBME subject examinations

- Mastery testing: SP exercises, OSCES

- Direct observation of procedural skills

- Technology-enhanced simulation exercises

\section{Practice-based learning}

Constructs, pursues, and revises an individualized learning plan
- Student self-assessment of strengths, weaknesses, and learning gaps

- Narrative medicine-journaling of personal learning and growth

- Faculty mentor review of the ILP 


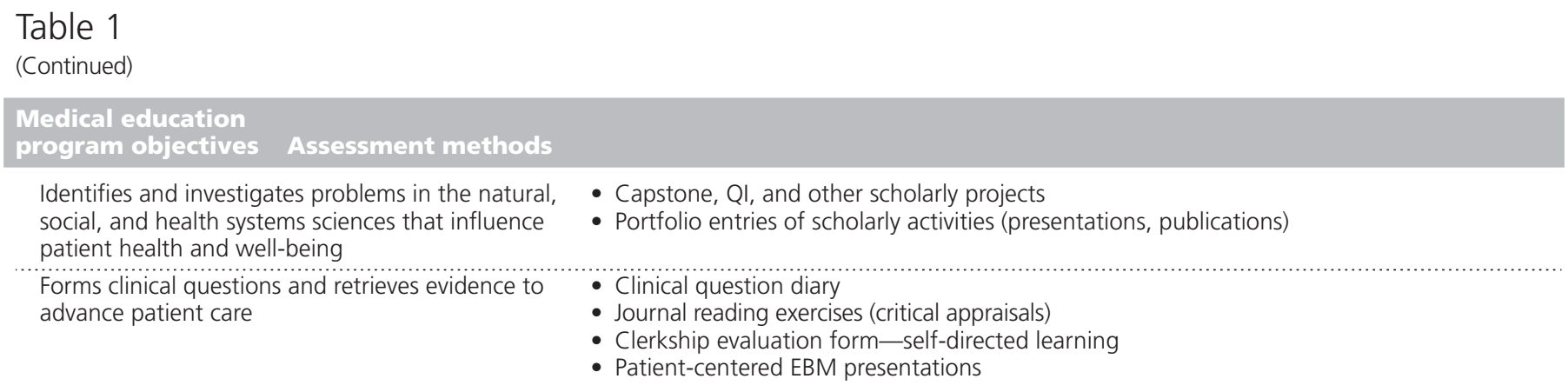

Abbreviations: EPA, Entrustable Professional Activity; IPE, interprofessional education; QI, quality improvement; SP, standardized patient; OSCEs, objective structured clinical examinations; CEX, clinical examination; NBME, National Board of Medical Examiners; MCQ, multiple-choice questions; ILP, individualized learning plan; EBM, evidence-based medicine.

communication skills are evaluated using direct observation, personal reflection, standardized patient assessments, and peer feedback.

In Phase 2, an increasing share of students' evaluations are derived from performance on objective structured clinical examinations and other assessments of real-world applications of knowledge focusing on nuanced areas of patient evaluation and care, leadership, and communication skills. Peer evaluation, personal reflection, and assessment by allied health professionals prepare students to recognize strengths and areas for improvement pertaining to teamwork, professionalism, and personal wellness.

Assessments during Phase 3 confirm readiness for residency and progress toward a scholarly concentration or dual degree. Students are evaluated in patient care settings performing supervised tasks. Scholarly productivity is assessed by mentors who track students' progress developing practical expertise in their chosen pathway and help inform decisions regarding performance and degree eligibility.

\section{Parallel curriculum or tracks}

All students are afforded opportunities to develop a personalized pathway in a specialized area of interest. Students are required to select an emphasis for their scholarly work, which for many culminates in a dual degree, or consideration for an accelerated path to residency.

\section{Pedagogy}

Phase 1 uses a combination of guided, self-directed, case-based collaborative learning (in a team-based learning model), and lab case correlates. Additionally, ongoing clinical skills training linked to cases use standardized patients and longitudinal ambulatory patient experiences. These methods are continued in Phase 2 during the four 12-week integrated clerkships.

\section{Clinical experiences}

In Phase 1, students engage in biweekly early longitudinal clinical experiences in the offices of community preceptors, at the bedside with clinical preceptors, in the health systems as patient navigators, and in the community through an Emergency Medical Service course and ride-along shifts with paramedics to develop skills in emergency response.

Medical students receive clinical instruction at a constellation of primary teaching sites: Jackson Memorial Hospital (thirdlargest public teaching hospital nationwide), University of Miami Hospitals and Clinics, the Miami VA Medical Center, and community physician practices. At the RMC, teaching facilities include 10 community hospital affiliations, health department clinics, and community physician offices.

\section{Required longitudinal experiences}

There are 2 longitudinal themes across all 3 phases of the curriculum:

- Medicine as a Profession covers clinical skills, professionalism, communication skills, population health, health systems sciences, nutrition and wellness, and personal development.

- Scholarly concentration integrates scholarly activities into the curriculum, including research design, epidemiology/biostatistics, quality improvement, and collaborative working environments.

\section{Required and elective community-based rotations}

In addition to the hospitals listed above, students train at community preceptor offices as well as private, state, and federally funded health systems and health centers. The core clerkships in the MD-MPH program occur at the RMC in Broward and Palm Beach. The RMC is affiliated with several partner hospitals, community practices, and the Palm Beach County Department of Health.

\section{Challenges in designing and implementing clinical experiences for medical students}

The expanded role of early clinical experiences and varied ambulatory rotations in NextGenMD increase the challenge of designing and implementing comparable experiences. The curriculum committees closely monitor required clinical experiences to ensure similar exposure for all students. We increased our pool of community preceptors and provide faculty development to ensure comparable experiences and assessments. 
We also expanded ambulatory sites to include satellite offices of our health systems and adjusted faculty compensation to ensure appropriate commitment to the teaching mission.

\section{Curriculum Governance}

The UMMSM Faculty Council charges the Executive Faculty Curriculum Steering Committee (EFCSC) with overall design, management, integration, and evaluation of a coherent and coordinated curriculum. The EFCSC consists of 21 voting members; two-thirds of its members are nominated by the Faculty Council's Committee on Committees and one-third are nominated by the Dean's Office. Two standing committees meet monthly and are responsible for advising the EFCSC on respective curricular components:

- The Basic Science Curriculum Advisory Committee consists of all course directors who lead preclerkship courses, in addition to elected student curriculum representatives from the preclerkship years. This committee includes designated associate and assistant medical education deans who serve in a non-voting ex officio capacity.

- The Clinical Curriculum Advisory Committee makes recommendations regarding the clerkship and postclerkship curricula, consisting of all core, required clerkship directors and elected student curriculum representatives. This committee includes designated associate and assistant medical education deans who serve in a non-voting ex officio capacity.

\section{Decentralized curricular governance}

The clinical clerkships are managed at the department level with central oversight from the Office of Medical Curriculum (by the associate dean for clinical curriculum). Departmental funding for clerkship administration and education comes from an annual allocation from the Dean's Office. The Office of Medical Education sets specific guidelines outlining level of support required for clerkship directors and administration.

\section{Education Staff}

The Office of Medical Education provides administrative and academic support for curriculum delivery, monitoring, and management under the senior associate dean for undergraduate medical education and regional dean for medical education

- Office of Medical Curriculum on the Miami campus and RMC is responsible for curricular operation, and prepares syllabi, distributes handouts, administers examinations, manages the website, and provides administrative support to course and clerkship directors.

- Registrar's Office distributes evaluations, collects results, and summarizes data.

- Office of Program Evaluation (OPE) works with course directors to design course evaluations that are analyzed and distributed to curriculum committees, course directors, administration, students, and department chairs. The OPE director assists course directors in reviewing examination item analyses and determining exam score cutoffs and in creating the assessment instruments used in competency assessment exercises.

- Office of Information Technology (IT) provides IT technical support to medical education programs. The office is responsible for audiovisual support for the curriculum and upkeep of teaching facilities, including the computer lab.

- Medical Education Administration handles all budgetary, purchasing, and human resource needs of the Office of Medical Education.

\section{Medical education leadership}

See Figure 1-Miami campus organizational chart.

See Figure 2-Regional medical campus organizational chart.

\section{Department of Medical Education}

A new Department of Medical Education was established in 2019 to provide primary, secondary, and voluntary appointments to faculty in medical education; it equips teachers with the skills necessary to become more effective in their educator roles.

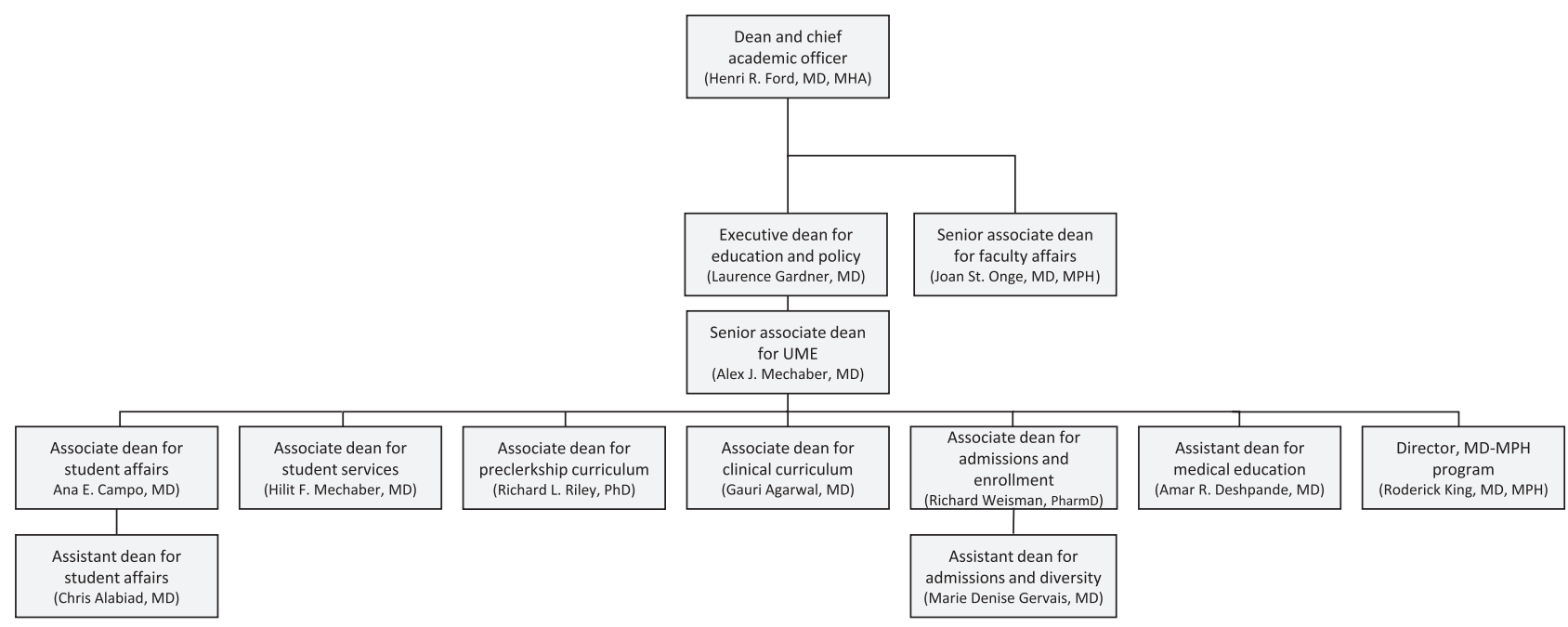

Figure 1 Miami campus organizational chart. 


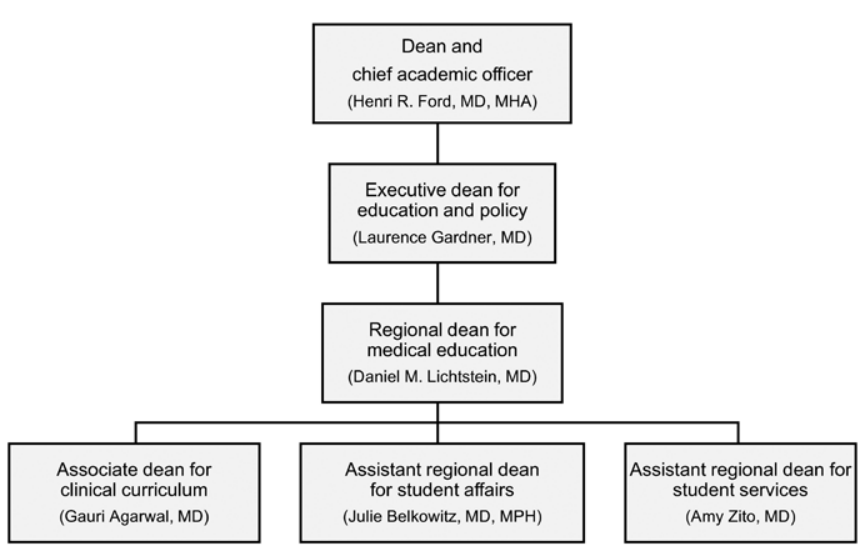

Figure 2 Regional medical campus organizational chart.

Additionally, the department helps create and support a culture of educational research, scholarship, and innovation. The department also provides an academic "home" for faculty whose primary focus is education and allows for development of new and creative approaches to educating learners.

\section{Faculty Development and Support in Education}

The Educational Development Office (EDO) provides consultative and programmatic support to faculty and departments in educational design, instruction, evaluation, and research in medical education. The EDO monitors faculty teaching efforts and generates reports for department chairs and medical finance. Additionally, a redesigned Office of Faculty Affairs and Professional Development offers ongoing faculty development sessions to continue training faculty for their NextGenMD roles.

\section{Role of teaching in promotion and tenure}

In August 2019, the UMMSM adopted new bylaws and guidelines regarding promotion and tenure in 4 faculty tracks: educator, clinical educator, tenure, and research.
Educational contributions are required for appointment and promotion in all tracks, and applicants for promotion are strongly encouraged/required to submit a detailed educator portfolio as a critical part of the process. Publications and other forms of scholarship are also universal requirements for promotion. An important distinction among the educator and clinical educator tracks is the requirement for extramural funding; no significant amount is required in either of the tracks emphasizing education.

See Table 2-Weighted Contributions and Achievements by Track.

\section{Educational experiences across sites}

The UMMSM RMC is the clinical campus for approximately 50 students in the clerkship phase of our 4-year MD-MPH program. An associate dean for clinical curriculum oversees clinical clerkships on both the Miami campus and RMCs. The regional dean for medical education, associate dean for clinical curriculum, and Clinical Curriculum Advisory Committee review the shared objectives, curricular content, required clinical experiences, assessment methods, and all evaluations of all clerkships across sites throughout the year to ensure comparability. Ongoing faculty development efforts, including meetings with clinical faculty and residents, ensure consistency of educational experiences. The Executive Faculty Curriculum Steering Committee, the curriculum's governing committee, also reviews these reports annually.

Our curriculum has evolved over the years to adapt to changing societal needs and evolving pedagogical landscape; to include more student-centered, self-directed, and collaborative learning; increased early clinical experiences; to enhanced focus on social determinants of health; and to place stronger emphasis on leadership skills training-so that we can shape our learners to become change agents who transform lives and are inspired to serve the global community - the mission of the UMMSM.

\section{Table 2}

Weighted Contributions and Achievements by Track

\begin{tabular}{|c|c|c|c|c|c|c|}
\hline Track & Scholarship & Funding & Education & Clinical impact & Reputation & Service \\
\hline Educator & $20 \%$ & $0 \%$ & $60 \%$ & NA & $10 \%$ & $10 \%$ \\
\hline Clinical educator & $10 \%$ & $0 \%$ & $30 \%$ & $30 \%$ & $20 \%$ & $10 \%$ \\
\hline Tenure-earning clinical & $20 \%$ & $20 \%$ & $20 \%$ & $10 \%$ & $20 \%$ & $10 \%$ \\
\hline Research & $30 \%$ & $30 \%$ & $10 \%$ & NA & $20 \%$ & $10 \%$ \\
\hline
\end{tabular}




\title{
University of South Florida Health Morsani College of Medicine
}

\author{
Deborah DeWaay, MD, Bryan Bognar, MD, MPH, and Siwar Bizri, MA
}

\section{Medical Education Program Highlights}

The Morsani College of Medicine (MCOM) moved into its new downtown Tampa location effective January 2020. The college has partnered with Microsoft to create the nation's first Medical School of Innovation, which will allow a more integrated approach to educational pedagogy and technology.

University of South Florida MCOM has 2 distinct MD parallel curricula:

- The Core program is an integrated curriculum offering the ability to personalize the student's experience via an extensive 4-year scholarly concentrations program.

- The SELECT (scholarly excellence, leadership experience, collaborative training) program offers expanded training in leadership, health systems, and values-based patient-centered care.

There are 9 Collegia (learning communities), made up of students at all stages of training, that provide mentoring and personal support throughout the educational journey. The new building has dedicated space for the Collegia program.

The Collegia provide medical students:

- Professional role models and long-term personal relationships

- A sense of community

- Career planning guidance

- Robust strategies for taking care of physical and emotional health

Continuous quality improvement through curriculum mapping:

- By program, course, and session objectives.

- By national discipline-specific objectives, such as pathology and physiology.

- Data analytics enabled by business intelligence software provides actionable reports. Within minutes the number of sessions, location within the curriculum by year and course of a particular topic, can be described, easing the review of gaps and redundancies.

- By 2022, it will be possible to analyze the curriculum by cohort instead of by academic year, increasing the precision of review.

\section{Curriculum}

See Supplemental Digital Appendix 1-Curriculum Schematicat http://links.lww.com/ACADMED/A924.

Acad Med. 2020;95:S128-S131.

doi: $10.1097 / A C M .0000000000003426$

Copyright (C) 2020 by the Association of American Medical Colleges

Supplemental digital content for this article is available at http://links.Iww.com/ ACADMED/A924.

Year school was established: 1971.

School URL: https://health.usf.edu/medicine.
See Supplemental Digital Appendix 2-MD SELECT Curriculum Schematic_at http://links.lww.com/ACADMED/A924.

\section{Curriculum changes since 2010}

Preclinical years:

- Discipline-based courses were replaced with organ system block courses. This change promoted integrated teaching by different disciplines.

- The physical diagnosis course was replaced with a 3-year longitudinal doctoring course addressing history taking, physical diagnosis, patient presentations, and caring for patients from special populations.

- Anatomy is delivered using ultrasound, cross-sectional radiological imaging, and cadavers so that within each lab students can appreciate the regional and systems-based anatomy from clinically relevant perspectives, bridging the gap between the basic and clinical sciences.

Clinical years:

- The third-year clerkships were transformed into integrated clerkships in contrast to discipline-specific clerkships.

- Medical improv techniques are used to enhance student clinical presentation skills.

- Fourth-year students choose from a series of selective courses linked to their area of residency interest. All tracks include a Return to Basic Science selective relevant to the residency field.

- Required short courses were added to the fourth year, including the Patient Safety course and a residency preparation capstone course, to address national calls for improved patient safety training, interprofessional education, and enhancement of internship readiness.

Planned curricular changes:

- An Entrustable Professional Activity (EPA) curriculum, where students master the EPAs through the lens of their future specialty. The goal of this initiative is to provide a disciplinespecific context to the AAMC's EPAs. For example, students pursuing internal medicine will be trained to be entrustable in consenting for blood products, whereas students going into obstetrics-gynecology will be entrustable for consenting for dilatation and curettage, cesarean delivery, and IUD insertion. At graduation, all students will be competent in performing informed consent on a patient in general.

- Continued expansion of the ultrasound curriculum to be a robust, 4-year continuum.

- A longitudinal fourth-year course:

- Core program students will master a Resident as Teacher EPA.

- Students in both programs will receive intense training in addiction and pain management.

- Short courses will allow integration of all curricular components and allow better tracking. 
Changes in pedagogy include:

- Increased use of augmented and virtual reality to enhance understanding of crucial medical concepts.

- Students will be given a case that integrates the didactics for the organ system block and work through the case over several weeks with classmates. The use of technology will enable synchronous and asynchronous case-based work.

- Use of plastinated specimens in anatomy.

\section{Assessment}

The MCOM Curriculum Committee adopted the AAMC's Physician Competency Reference Set as a framework for its program objectives. The SELECT program has additional objectives focused on leadership, values-based patient-centered care, and health systems.

See Supplemental Digital Appendix 3-Program Objectives and Assessment Methods—at http://links.lww.com/ACADMED/A924.

\section{Assessment changes since 2010}

MCOM has put efforts into improving the quality of assessments but has not made major changes to the types of assessments used. An annual 3-part assessment has been added in the SELECT program to better evaluate the areas of health systems, values-based patient-centered care, and leadership. This assessment includes multiple-choice questions, an oral board style section, and standardized patient encounters.

\section{Parallel curriculum or tracks}

- The SELECT program is a parallel curriculum that cultivates leadership competencies with a focus on the development of emotional intelligence. There are 56 students per class.

- Students complete their preclinical years at the Tampa campus alongside the core program students. They complete the clinical years at the Lehigh Valley Health Network campus in Allentown, Pennsylvania, where clerkships have distinct SELECT program objectives focused on leadership, valuesbased patient-centered care, and health systems.

- SELECT program students participate in the following additional curriculum:

- Minicourses at the start of school focusing on emotional intelligence and leadership

- Small-group, intensive SELECT professional development courses in years 1 through 4

- Professional development coaching from trained MD coaches throughout the 4 years

- Summer immersion experience where students complete mentored scholarly work between years 1 and 2 focused on a SELECT program domain

- Students complete a capstone research project relevant to the SELECT domains in year 4.

- Educational comparability to the core program is maintained via:

- Cross-campus videoconferencing of educational committee meetings as well as representation on all committees from both campuses

- Frequent retreats that include both campuses

\section{Clinical experiences}

- Principal clinical sites include Tampa General Hospital, Lehigh Valley Health Network, James A. Haley Veterans' Hospital, Moffitt Cancer Center \& Research Institute, All Children's Hospital, and Bay Pines VA Healthcare System.

- Outpatient sites are a mix of on-campus clinics and multiple MCOM affiliated and community sites throughout the Tampa Bay and Allentown, Pennsylvania, regions.

\section{Required longitudinal experiences}

The SELECT students have a longitudinal primary care rotation, originally 1.5 days per week outpatient experience. It will be transitioned to a 1-week block every 6 weeks with the goal of increasing continuity with patients. The students will continue to be consistently assigned to a particular practice.

\section{Clinical experience first encounter}

This first clinical experience occurs during the first week of school. The students evaluate 3 patients over a 2-week orientation course (Professions of Health). Two of the patients are standardized and one is a real patient. In addition, students interview interprofessional practitioners who care for the type of patients being evaluated.

\section{Challenges in design and implementation of clinical experiences for medical students}

- Maintaining consistent faculty development across campuses and clinical sites

- Maintaining the necessary focus on the education mission in an environment of heightened emphasis on faculty clinical productivity and the generation of patient service revenue

- Stagnant state support for the college over the past several years, including no increases in tuition and/or fees for 5 consecutive years

\section{Curricular Governance}

Governance of the phases of the curriculum: The Committee on Curriculum (CC) is an open meeting enabling anyone to bring a perceived gap or redundancy to the committee to consider whether a rectifying action is necessary. The preclinical and clinical committees present annually to the CC. This presentation represents the work completed in the following areas:

- Plan and develop operational standards across all the required courses

- Consider new content and pedagogical approaches

- Promote horizontal and vertical curricular integration

- Recommend policy changes to the CC

- The Committee of Preclerkship Directors (CPCD) considers feedback from students, teaching faculty, and the individual course directors

- Administrative support for the committee provided by the Department of Medical Education

The presentation includes outcomes data, a SWOT analysis, and recommendations for the upcoming academic year. This review 
allows the CC to oversee the changes and ensure that the changes are congruent with the CC's vision of the program and that program objectives are met. In addition, the CC holds quarterly phases of the curriculum review meetings to take a deep dive into how each phase is working as a part of the entire curriculum. Each course is reviewed by the CC at least once every 2 years. The Course Review Subcommittee evaluates the course first and makes recommendations to the CC. The CC will then determine the recommended changes, request an update before the next full review, and set the next full review date as the CC approves or rejects the components of the course subcommittee review.

The integration director committee maps the content in the curriculum to national objectives for each of the following areas: physiology, pathology, anatomical sciences, behavioral science, biochemistry, biostatistics and epidemiology, cultural competency, embryology, ethics, evidence-based medicine, genetics, histology, human sexuality, immunology/microbiology, introduction to clinical medicine/introduction to patients, neuroscience, pharmacology, and special topics. The integration directors present their findings to the Content Review Subcommittee. This subcommittee reviews and acknowledges perceived strengths, weaknesses, opportunities, and threats identified by the integration directors and completes a content subcommittee summary with recommendations then considered and acted on by the CC.

See Figure 1-Committee on Curriculum chart.

Educational budget for teaching, assessment, and academic support: The educational value unit (EVU) system is overseen and administered by the vice dean for educational affairs and compensates faculty for their teaching and related activities using specific metrics. Educational effort is reported by course directors, then translated into EVUs via approved formulae (incorporating prep time and item writing often associated with teaching sessions). EVUs are then converted to dollar amounts using AAMC salary tables (by rank/discipline) for each faculty member. These funds are transferred to the faculty's academic department for salary support. Course directors and key roles (e.g., Admissions Committee, Curriculum Committee) are provided predetermined EVU allocations and credited in the same way.

See Figure 2-Organizational chart.

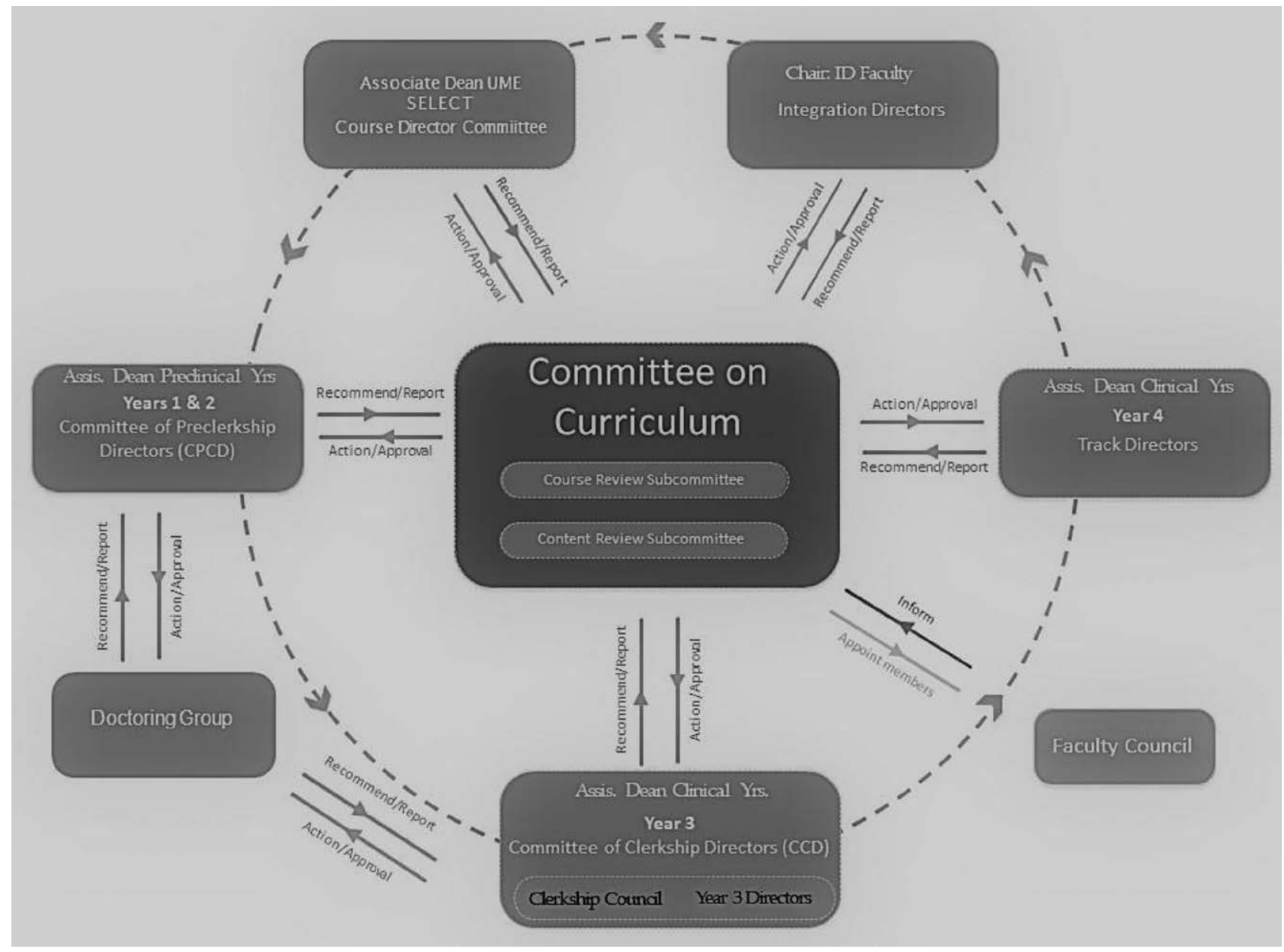

Figure 1 Committee on Curriculum chart. 


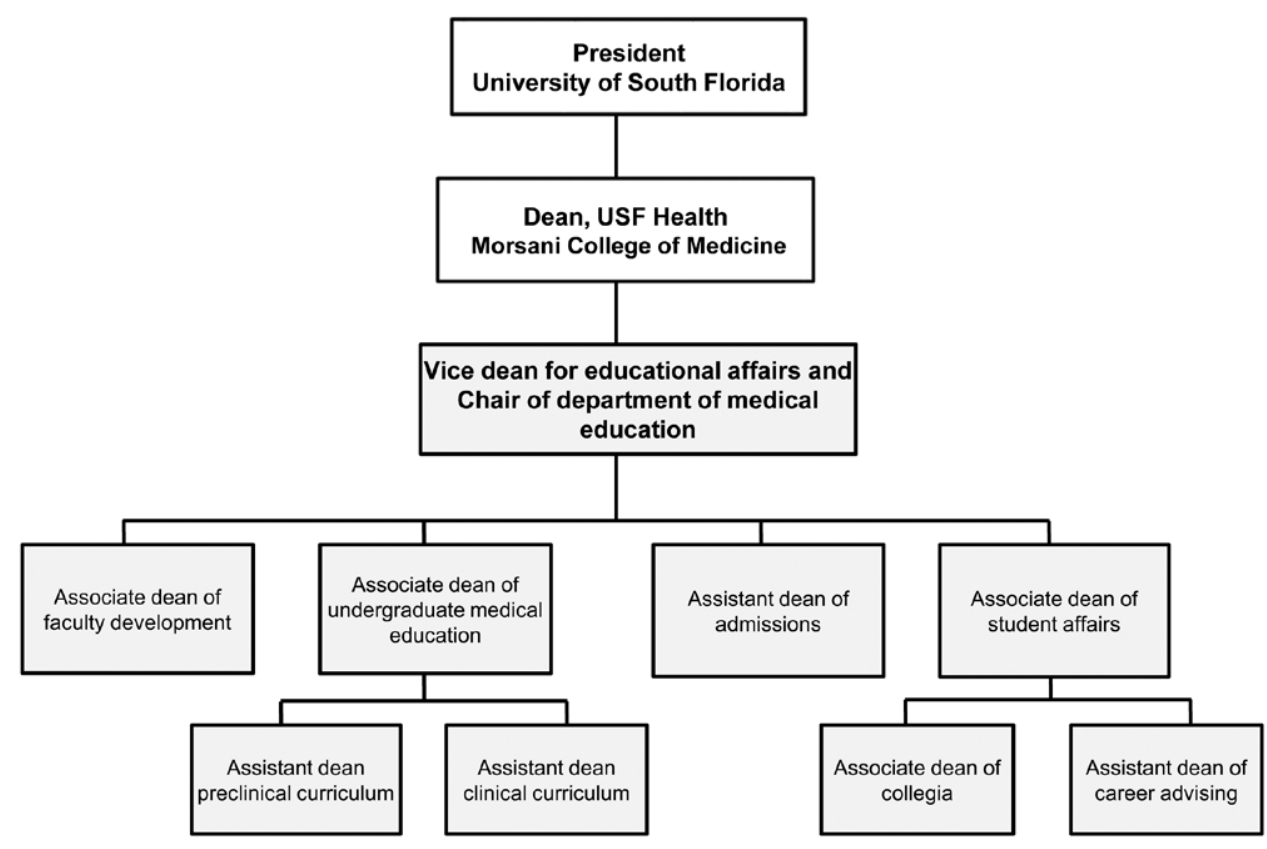

Figure 2 Organizational chart.

\section{Faculty Development and Support in Education}

- Faculty development for education is overseen by the associate dean for faculty development within the Office of Faculty Affairs.

- Multiple centrally planned workshops are available on both campuses including feedback, exam item writing, as well as active learning, lecture based, and clinical teaching skills. Regular webinars by the International Association of Medical Science Educators are offered through the Department of Medical Education.

- There are annual retreats for all phases of the curriculum where faculty development for course and clerkship directors is offered.

\section{Professional development for faculty as educators}

MCOM has an expectation of scholarship from all ranked faculty on both campuses, regardless of pathway for academic progression. The requirements of scholarship vary relative to pathway and assignment. The traditional tenure-earning pathway has the most rigorous expectation of scholarship. A lesser magnitude of scholarship is required for faculty on the clinicianeducator, research scientist, scientist educator, and collaborative research scientist pathways. It is required for promotion to higher rank, although not necessarily for retention. For those faculty on the clinician-educator and scientist educator pathways, the expectations of faculty for educational activities for promotion are substantial. 


\title{
Emory University School of Medicine
}

\author{
J. William Eley, MD, MPH, Erica Brownfield, MD, MBA, and Hugh A. Stoddard, MEd, PhD
}

\section{Medical Education Program Highlights}

The educational program at Emory University School of Medicine (EUSOM) is designed to produce generalist and specialist physicians who are adept at scholarly investigation and are committed to caring for all people and populations, especially those underserved in medicine. Key distinguishing features are:

- Students complete the required longitudinal course, Community Learning and Social Medicine, during which students engage with and learn about the greater Atlanta community, and initiate efforts to promote health and healthy behaviors.

- All students complete an in-depth, mentored research project of 5 months or more, which leads to one or more scholarly products.

- Students participate in a learning community, including faculty-facilitated small groups, from matriculation through graduation.

- Being in Atlanta provides a broad variety of clinical training contexts, including Grady Memorial Hospital, the fifth largest public hospital in the United States, with incomparable commitment to care for underserved patients.

\section{Curriculum}

\section{Curriculum description}

The EUSOM curriculum consists of 4 phases sequenced to inspire learners toward increasing integration of knowledge and independent practice.

- Foundations of Medicine (18 months): Both block and longitudinal courses in biomedical sciences, social and behavioral sciences, health systems science, and clinical practice.

- Application of Medicine (12 months): Blocks of 11 required clinical clerkships.

- Discovery: A 5-month intensive research block during which students design, conduct, and present or publish an individual scholarly project under the direction of a faculty mentor.

- Translation of Medicine: A variety of experiences over 13 months that prepare students for residency. This includes a subinternship, required clerkships in emergency medicine and critical care, a capstone course, and electives.

Several topics are presented as longitudinal courses at developmentally appropriate times across all multiple phases. These courses include: Essentials of Patient Care (which

Acad Med. 2020;95:S132-S135.

doi: 10.1097/ACM.0000000000003331

Copyright (C) 2020 by the Association of American Medical Colleges

Year school was established: 1915.

School URL: https://www.med.emory.edu. includes clinical practice), Ethics, Evidence-Based Medicine, and Community Learning and Social Medicine.

\section{Curriculum changes since 2010}

EUSOM adopted a new curriculum in 2007, fully implemented in 2011. Since then, curricular CQI efforts have focused on improving the alignment and integration across all courses and clerkships. To that end, EUSOM adopted a list of 28 studentphysician activities (SPAs) in 2013 as the program learning outcomes for the MD program.

The SPAs provide a common focus and language to align content, instruction, and assessment across all phases, courses, and clerkships and have unified all components of the program to work toward a common purpose. The courses and clerkships used to prepare students for those outcomes have not been altered substantially since 2007, with the notable exception of creating the required longitudinal Community Learning and Social Medicine course.

In a similar vein, EUSOM adopted a standardized language for categorizing the content being taught and assessed throughout the curriculum. ${ }^{2}$ The Emory Curriculum Alignment Taxonomy (ECAT) was instituted in 2016 to gather data about curricular content and student performance. ECAT data are used regularly by the Executive Curriculum Committee (ECC) to promote alignment and integration across phases, courses, and clerkships.

In 2019, EUSOM began a strategic visioning and comprehensive planning process to renew medical education across the continuum of EUSOM programs, with actualization anticipated for 2023. The upcoming EUSOM curriculum, titled IRIS (inspire, reimagine, innovate, serve), expresses an education vision that will:

- Create coordinated and forward-looking curricula across the medical education continuum to ensure that students, trainees, and faculty thrive in the future of medicine

- Develop distinctive programs for the individual and collective benefit of local, regional, and national education and noneducation communities

- Inspire students and trainees to make medicine better through discovery, health systems sciences, and the incorporation of new knowledge

\section{Assessment}

EUSOM program learning outcomes are defined by the 28 SPAs, ${ }^{1}$ which are mapped to the ACGME core competencies.

See Chart 1-Student-Physician Activities and Assessment Methods.

Major internal (local) assessments for all courses and clerkships have been "tagged" using the SPAs and the ECAT categories described. Thus, a student's growth and 


\section{Chart 1}

\section{Student-Physician Activities and Assessment Methods}

\section{Student-physician activity (SPA)}

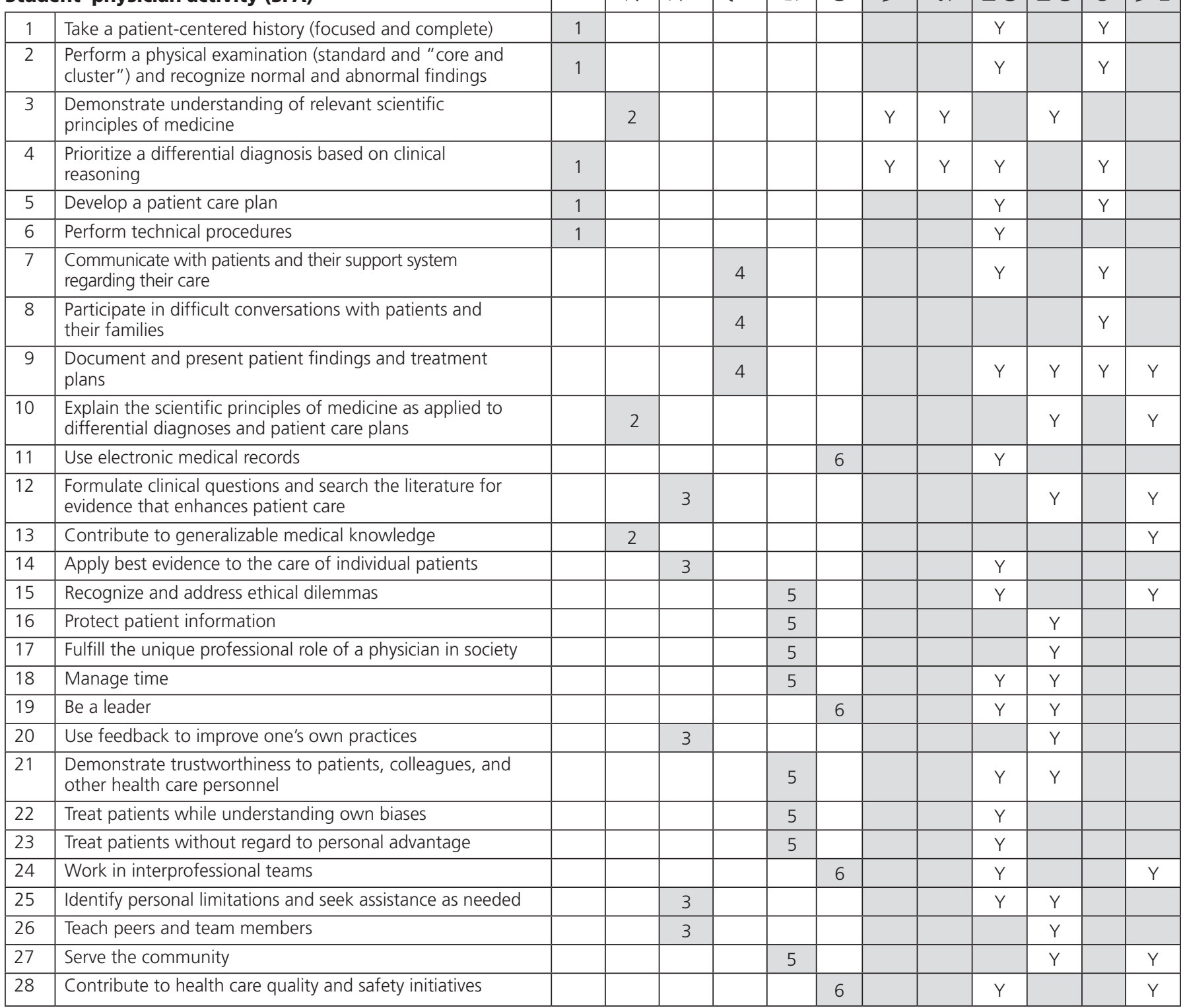


achievement on each of the SPAs can be aggregated across all 4 phases of the program. This tagging is done for written exams, faculty ratings of workplace performance, and summative OSCEs.

- Each item on internally developed written exams is categorized according to SPA and content. Students are informed of their individual results by topic to help guide future learning and pertinent faculty receive reports on the performance of class cohorts to inform curriculum evaluation.

- All clinical assessment forms that are used to record faculty observations of students during required clerkship are tied to SPA criteria. This encourages consistency across clerkships in the criteria for which students are graded and allows for building a profile of student performance on the specific activities of a student-physician in a variety of clinical contexts.

- Summative OSCEs are scored based on a student's performance of a specific activity across all simulated cases. This approach supports the evidence collected from workplace assessments and avoids psychometric issues of case specificity.

\section{Parallel curriculum or tracks}

EUSOM does not have parallel tracks within the MD curriculum, but offers several dual-degree programs. These dual degrees are available: MD-PhD, MD-MSCR (clinical research), MD-MPH, MD-MA (ethics), and MD-MBA. There is not currently a 3-year $\mathrm{MD}$ degree at EUSOM, and no plan is currently in place to offer such.

\section{Pedagogy}

Pedagogy used in the EUSOM program emphasizes clinical practice and experiential learning.

The Foundations phase of the curriculum makes extensive use of actual patients in classroom presentations. These presentations emphasize patient-centered care by offering a first-hand perspective of the condition being discussed and by modeling compassionate care along with clinical reasoning.

The Discovery phase is an experiential scholarly opportunity with the expectation that students will design, conduct, and report on a research question in their area of interest. Students are mentored, but function independently. Similarly, the Community Learning and Social Medicine course is predominantly experiential, with students spending extended time with a community organization.

Throughout the program, students spend time with a faculty facilitator in small-group learning sessions to build their professional identify as physicians and to reinforce the communal and collaborative components of modern health care practice.

\section{Clinical experiences}

The Atlanta metropolitan region presents EUSOM students with a plethora of rich clinical learning environments. Medical students participate in educational activities in 4 different hospital systems:

- Emory Healthcare, the most comprehensive health system in Georgia, which offers training opportunities in practically all medical specialties for a variety of patient types, ranging from ambulatory to quaternary care services

- Atlanta VA Medical Center

- Children's Healthcare of Atlanta

- Grady Memorial Hospital, the largest hospital in Georgia and the fifth largest public hospital in the United States

EUSOM students first experience clinical medicine in the second week of medical school as observers during "Week on the Wards." Participation in patient care commences during the fifth month of their educational program when students see patients in ambulatory settings biweekly for a full year.

In addition to the 4 inpatient facilities, EUSOM students have opportunities in a multitude of outpatient venues, including community-based psychiatric rotations at DeKalb Crisis Center and Skyland Trail.

Despite the extensive hospital networks available to EUSOM students, 2 challenges face the clinical education program for students:

- There is limited availability of outpatient sites for students, particularly for general pediatrics and adult medicine.

- Increasing numbers of learners from other health care programs, other medical schools, and other countries seek clinical training at Emory-affiliated health care systems and hospitals. These outside demands on the resources and facilities create challenges for EUSOM and our health care partners.

\section{Curricular Governance}

A unique structure and function for the EUSOM curriculum committee was instituted in 2013. All final decisions are made by the ECC, with input and support from 10 subcommittees, including a subcommittee of student representatives. ${ }^{3}$ The ECC has embraced CQI processes to manage the myriad details and decisions that need to be made and monitored.

Several members of the ECC are selected by a direct vote of the EUSOM faculty. The remaining members are chairs of the 10 subcommittees who are selected through a process of vacancy notifications, followed by review of applications by curriculum deans and appointment by the dean of EUSOM.

The ECC and its subcommittees collaborate closely with several associate and assistant deans on matters of student academic support, career development, and similar issues of student affairs.

Governance of the EUSOM curriculum and program has become highly centralized over the past 7 years. Funds are transferred from the Dean's Office to various departments to provide salary support for key faculty involved in leading and teaching the MD program. Departments retain primary oversight 
of clinical electives and personnel to lead and coordinate the required clerkships.

\section{Education Staff}

All functions related to the operation of the MD program at EUSOM are under the auspices of the Office of Medical Education and Student Affairs (OMESA). These functions include medical school admissions, curriculum organization and delivery, and medical/clinical simulation activities.

OMESA curricular support includes the coordination of all classroom-based educational events such as scheduling activities, administering course and faculty evaluations, assembling and delivering written exams, producing OSCEs, managing the curriculum inventory, and overseeing data gathering and management for LCME requirements. OMESA student affairs support incorporates scheduling students for required clinical clerkships (this does not include assigning students to the specific services during each clerkship) and arranging months for elective rotations and the Discovery course.

All OMESA units work in collaboration with Information Technology Services (SOM-ITS) to operate the infrastructure needed for program functions and data collection and reporting.

OMESA personnel in several areas also serve other health care education programs of EUSOM. These include the simulation and clinical skills units, the financial aid office, and the registrar.

Additional OMESA personnel fulfill curricular and student affairs needs for several nondegree programs. These include the graduate medical education office and the leadership of various prematriculation "pipeline" and recruitment programs for potential applicants who are demographically underrepresented in medicine. The $\mathrm{MD} / \mathrm{PhD}$ program coordinators also function under the OMESA umbrella.

Dean-level positions in support of medical student education are:

- Executive associate dean for medical education and student affairs

- Associate dean for admissions and student affairs

- Associate dean for clinical education

- Associate dean for medical education, curriculum

- Associate dean for medical education, business and finance
- Assistant dean for clinical education

- Assistant dean for medical education

- Assistant dean for medical education and student affairs

- Assistant dean for medical education research

- Associate dean for education and professional development

- Associate dean for faculty affairs and professional development

- Assistant dean for information technology

- Assistant dean for medical education, Grady

\section{Faculty Development and Support in Education}

School-wide professional development in education opportunities exist for faculty. Examples include Medical Education Day and Learning to Be Better Teachers day. Most opportunities are housed in individual departments or are designed to fulfill specific course or program needs.

The EUSOM promotion and tenure guidelines, adopted in 2017, state that all faculty are expected to "show academic excellence and to participate in all 3 missions: scholarship, teaching, and professional service." Faculty members select 1 of these 3 as an area of distinction appropriate to their intended career path. Criteria and guidelines for promotion, particularly to senior ranks, are established such that individuals can be promoted on the strength of their performance and reputation in teaching.

The Woodruff Health Educators Academy (WHEA) was established in 2017 to promote scholarship of teaching and learning in all schools and programs of Emory University's Woodruff Health Science Center. To that end, WHEA sponsors programs to build capacity among faculty to advance educational pursuits. WHEA programs include an interprofessional journal club and yearlong fellowships in educational scholarship and in health professions teaching.

\section{References}

1 Stoddard HA, Brownfield ED, Churchward G, Eley JW. A process to design program outcomes for health care education: Making sense out of the alphabet soup. MedEdPORTAL. 2015;11:10299. https://doi.org/10.15766/mep_2374-8265.10299.

2 Stoddard HA, Brownfield ED. Creation and implementation of a taxonomy for educational activities: A common vocabulary to guide curriculum mapping. Acad Med. 2018;93:1486-1490.

3 Stoddard HA, Brownfield ED, Churchward G, Eley JW. Interweaving curriculum committees: A new structure to facilitate oversight and sustain innovation. Acad Med. 2016;91:48-53. 


\section{Medical College of Georgia at Augusta University}

Andria M. Thomas, PhD, Renee Page, MD, Ralph Gillies, PhD, Kimberly Loomer, EdD,

Kelli Braun, MD, Lee Merchen, MD, Carol Nichols, PhD, Barbara Russell, EdD, Chris Fly, MD,

Andrew Morris, MD, Kathryn Martin, PhD, Matthew Tews, DO, and D. Douglas Miller, MD, CM

\section{Medical Education Program Highlights}

Established in 1828, the Medical College of Georgia (MCG) is the nation's 13th oldest medical school, with a renowned commitment to medical education, as epitomized by vice dean emeritus Dr. Rhee Fincher. As Georgia’s only public medical school, MCG receives robust state support, now fused with remarkable philanthropic funding highlighted by the transformative $\$ 66 \mathrm{M} \mathrm{J}$. Harold Harrison MD gift that contributed to building a state-of-the-art medical education commons and to funding student scholarships and endowed chairs.

In response to a shortage of physicians in Georgia and with LCME approval, MCG increased class size from 180 to 230 in 2010 and created a series of 4 regional campuses to implement a statewide MD educational program ("One MCG"). Forty of the 230 students matriculate into the 4-year Medical Partnership (MP) created with the University of Georgia in Athens. Three clinical regional campuses each serve as a hub for third- and fourth-year students in Rome, Albany, and Savannah/Brunswick.

See Figure 1-Map of campuses.

MCG educates medical students at more than 350 statewide clinical venues, and over 500 residents/fellows in 53 unique residency/fellowship programs at AU Medical Center in Augusta. Some 25 students are enrolled in the University System of Georgia MD-PhD program.

MCG matriculates a talented, skilled, and diverse class with the attributes that we are seeking for future physicians. The program prepares graduates for the contemporary and future practice of medicine, capitalizing on rich statewide clinical experiences, leading to great success in their residency programs.

\section{Curriculum}

\section{Curriculum description and curriculum changes since $\mathbf{2 0 1 0}$}

To meet the evolving educational needs and professional career imperatives of its students, MCG is undergoing LCMEapproved development of a novel curriculum (MCG 3+). MCG 3+ creates individualized medical education options for

Acad Med. 2020;95:S136-S139.

doi: 10.1097/ACM.0000000000003408

Copyright (C) 2020 by the Association of American Medical Colleges

Supplemental digital content for this article is available at http://links.Iww.com/ ACADMED/A915.

Year school was established: 1828.

School URL: https://www.augusta.edu/mcg. students, while ensuring continued achievement of MCG's established MD program competencies. The redesigned curriculum will provide students completing the 3 -year core MD requirements with a choice from 3 subsequent fourth-year career pathways:

- Accelerated entry into postgraduate year 1 of primary care residency programs in Georgia

- Completion of a dual-degree program (MBA, MPH, MEd, MS)

- Advanced clinical skills and research training in future specialty

To jump-start the planning for MCG's new curriculum, the MCG Office of Academic Affairs employed a unique design strategy. Sprint is well known in the business and technology world as a method for quickly generating solutions to large challenges. MCG's educational leadership participated in two 5-day "sprints," curriculum redesign, and career advising. Both yielded significant program design results and feedback from students, residents, faculty, and content experts. The MCG 3+ program will contribute significantly to MCG's healthier Georgia mission, by graduating physicians who are career confident and practice ready. MCG 3+ is a logical and mission-based extension of prior LCME-approved curriculum enhancements.

Upon completion, MCG 3+ curricular redesign will achieve 3 important goals:

- Guide more students toward primary care careers in Georgia to improve the health of Georgians

- Allow the MD program to translate the lessons and successes from its case-based learning (CBL) track at our MP campus in Athens, and from its longitudinal integrated clerkship (LIC) track at our Rome campus

- Provide students with multiple fourth-year options designed to enhance their careers and/or transition to residency

Scheduling modifications for MCG 3+ beginning in year 1 allow students to complete all core graduation requirements in 3 years (132 weeks). Efficiencies gained in AY2019-2020 with preclinical content delivery through online HMX coursework (offered by Harvard Medical School) will be added to by students remaining active through the traditional summer breaks in the 4-year curriculum beginning in AY2020-2021. The clinical phase of the MCG 3+ curriculum also features greater utilization of a continuity-of-care LIC model for primary care pathway students and by adjustment of traditional clerkship length to 6 weeks for students in the other 2 pathways. The 2-phase MCG 3+ curriculum is structured as follows:

- Preclinical (18 months): The curriculum will continue using organ-based modules. The delivery of content will be accelerated and will primarily employ CBL, similar to that 


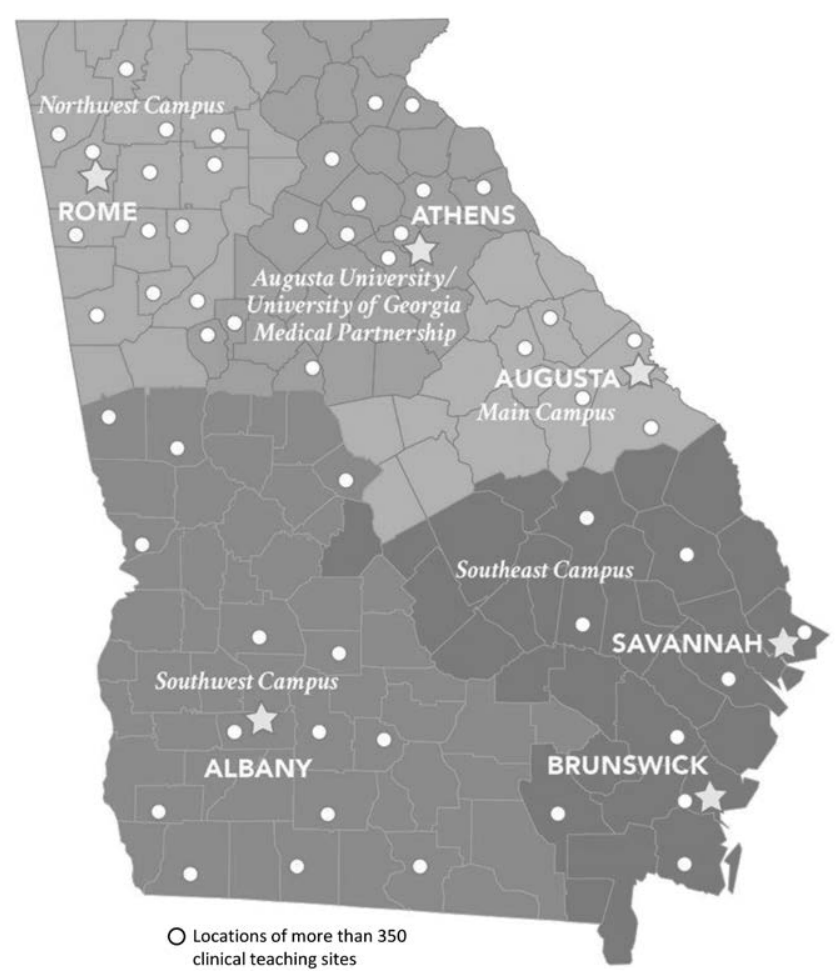

Figure 1 Map of campuses.

being successfully used at our campus in Athens. The current 2-year and 2-cycle (normal then abnormal) preclinical approach will become fully integrated into an 18-month and 1-cycle approach.

- Clinical (18 months): Capitalizing on the success of the LIC at the Rome campus, the curriculum will establish clinical continuity-of-care LIC experiences for MCG 3+ primary care students in all existing clerkship disciplines.

See Supplemental Digital Appendix 1-MCG 3+ Program Schema—at http://links.lww.com/ACADMED/A915.

MCG's Committee on Committees (COC) believes students can achieve all competency-based objectives (CBOs) within these time frames.

MCG 3+ is introducing several educational innovations to the MD program:

- Early preclinical phase patient exposure: student-patient interactions (i.e., home health visits, EMT rides, etc.) in months 1 and 2, and a continuity clinic beginning 3 months after matriculation

- Advanced technology integrated imaging training through a longitudinal "living anatomy" course: the current dissectionbased anatomy course will be modernized to feature more $3 \mathrm{D}$ imaging-enhanced tomography and ultrasound, and virtual reality simulations

See Table 1-Curriculum Design.
Expected outcomes of the MCG 3+ curriculum are:

- Improved career advising designed to better match to individual student interests with aptitudes. This will be measured by carefully tracking student satisfaction and graduate performance data, both internally (graduate survey, program director survey) and externally (AAMC Graduation Questionnaire), to achieve predictive model "fit" at key points in the MD program life cycle.

- Improved student performance metrics as a result of educational enhancements and greater faculty-student engagement in CBL and continuity-of-care clinics. Metrics to be tracked are:

- Module/clerkship grades

- Step scores (with national benchmarks)

- NBME comprehensive basic science and clerkship subject exam performance (with national benchmarks)

- Evaluation data

- Graduation rates (4 years)

- Competency achievement data (e.g., Entrustable Professional Activities [EPAs])

- Reduced medical student debt. The average debt will be reduced by 1 year of MD tuition for primary care pathway

\section{Table 1}

\section{Curriculum Design}

$\begin{array}{lll} & \text { MCG } & \text { MCG } 3+ \\ \text { Curriculum descriptors } & \text { traditional accelerated }\end{array}$

\section{Time phasing}

\begin{tabular}{llll}
\hline Years to MD & 4 & 3 \\
\hline Weeks to MD & 152 & 132 \\
\hline Phases (years) & $2(2)$ & $1(1.5)$ \\
\hdashline Preclinical & $1(2)$ & $1(1.5)$ \\
\hline Clinical & & 1 \\
\hline
\end{tabular}

\section{Design features}

$\begin{aligned} & \text { Competency-based objectives } \\ & (n=31)\end{aligned}$
Clinical integration
System based

\section{Learning cohort size}

\begin{tabular}{lll} 
& $X X$ & $X$ \\
\hline Large group & $X$ & $X X$ \\
\hline Small group & $X$ Xntinuity-of-care clinic "firms" & $X X$
\end{tabular}

\section{(2-3 students each)}

\begin{tabular}{|c|c|c|}
\hline \multicolumn{3}{|c|}{ Teaching methods/pedagogies } \\
\hline Lecture & $x x$ & $x$ \\
\hline Problem-based learning & $\dddot{x} x$ & $x$ \\
\hline Case-based learning & $\dddot{x}$ & $x$ \\
\hline Team-based learning & $x$ & $X X$ \\
\hline Simulation/physical diagnosis & $\dddot{x}$ & $x$ \\
\hline Uiltrasound & $\dddot{x}$ & $x$ \\
\hline Technology (flipped classroom) & $\dddot{x}$ & $x$ \\
\hline
\end{tabular}


students and by conversion of fourth-year students into lower master's-level tuition rates for dual-degree pathway students.

See Supplemental Digital Appendix 2-Medical School "Life Cycle"-at http://links.lww.com/ACADMED/A915.

\section{Assessment \\ MCG faculty define the competencies expected of MD graduates. These competencies are linked to the medical education program objectives, MCG's CBOs. Curricular design, implementation, and evaluation are guided by the CBOs, and these objectives are benchmarks of the knowledge, skills, behaviors, and attitudes MCG expects medical students to achieve by completion of the $\mathrm{MD}$ program. CBOs were developed in COC working groups including faculty, residents, students, and patients, refined by the full committee, stated in outcome-based terms, and linked to 6 general physician competencies outlined by the ACGME Outcome Project.}

The MCG faculty ensures the inclusion and delivery of content with sufficient breadth and depth to prepare students for entry into any residency program and for the subsequent practice of medicine. The COC uses CBOs to outline the content students must acquire and to evaluate student preparedness for residency training. Objective outcome measures confirm student learning and achievement of competency in multiple content domains. The most recent review (October 2019) yielded the addition of a seventh $\mathrm{CBO}$ on personal management and self-care. This new $\mathrm{CBO}$ addresses the need for students to develop an awareness of personal and professional values and needs, the skills to meet those needs, and ability to balance their professional identity with a full life outside of medicine.

MCG has implemented a comprehensive, fair, and uniform system of formative and summative medical student assessments throughout the curriculum to prepare students for promotion and enable their success as future physicians. Summative evaluations use multiple modalities, assess across the CBOs, and confirm satisfactory achievement as defined by the faculty. In the preclerkship years, contribution to summative assessment includes faculty-written exams, NBME-developed examinations, teambased learning activities, objective structured clinical examinations, lab practical exams, oral presentations, problem-based learning assessment, and various exercises with faculty ratings. In the clerkship year, MCG employ: faculty and resident ratings, NBME subject examinations, faculty-developed quizzes, evidence-based medicine assignments, written history and physical examinations, and others. Students are afforded opportunities throughout the curriculum to gauge their level of performance. Whether by lowstake quizzes or personal faculty feedback, formative assessment is provided throughout the curriculum. MCG is partnering with Vagelos College of Physicians and Surgeons at Columbia University to develop a tool to better evaluate medical student narrative feedback to assess EPA attainment.

\section{Curricular Governance}

The MCG faculty senate bylaws specify how the members of the COC are selected, including those who are appointed to the committee as a result of their position in the MD program. Members serve 2-year staggered renewable terms. The members are selected through the process outlined in the bylaws whereby nominations (self, campus leadership, or other) are received and approved by the COC.

The COC is chaired by the vice dean for academic affairs. The vice chair of the COC is the associate dean for curriculum. The chairs of the Phase 1 and 2 and Phase 3 curriculum committees as well as the Phase 1 and 2 and third- and fourth-year directors are members. The Phase 1 and 2 and Phase 3 committee each nominate 3 additional members. The committee includes representation from each campus. There are 3 student representatives from each class (2 Augusta, 1 Athens), with 1 vote per class. Other members include the associate dean for curriculum (Augusta); campus associate dean for curriculum (Athens); senior associate dean for evaluation, accreditation, and CQI; the associate dean for faculty development; and the associate dean for regional campus coordination.

\section{Education Staff}

The medical school's central staff is effective and committed to their administrative roles. Day-to-day leadership of the medical education program is delegated to the vice dean, with the support of regional campus leadership and dean's staff. To enhance support to students, the student affairs office was reorganized in 2015 with the merger of student affairs and diversity functions into the Office of Student and Multicultural Affairs, led by an associate dean and by the appointment of 4 class associate deans. Recently, MCG's medical education programming was further enhanced by restructuring the Office of Academic Affairs to include the graduate medical education and continuing professional development administrative functions. This change creates a continuum that supports learners at all levels.

See Figure 2-Educational staff.

\section{Faculty Development and Support in Education}

Faculty affiliated with any of MCG's campuses have a range of faculty development opportunities, including access to the offerings on the main Augusta campus (live, videoconference, archived, other online programs including MCG Preparing to Teach), as well as digital linkages to MCG's regional campuses, including:

- In-person orientation visits (new faculty) and periodic inperson visits by campus leadership, practice site clerkship directors, or department clerkship directors

- Subscription to teachingphysician.org

- Speakers/workshops

- Office of Faculty Success "Mentoring Minute"

MCG ensures that all faculty are prepared for their role as educators. The approach used includes educational orientation and onboarding, regional clinical faculty meetings, an annual 


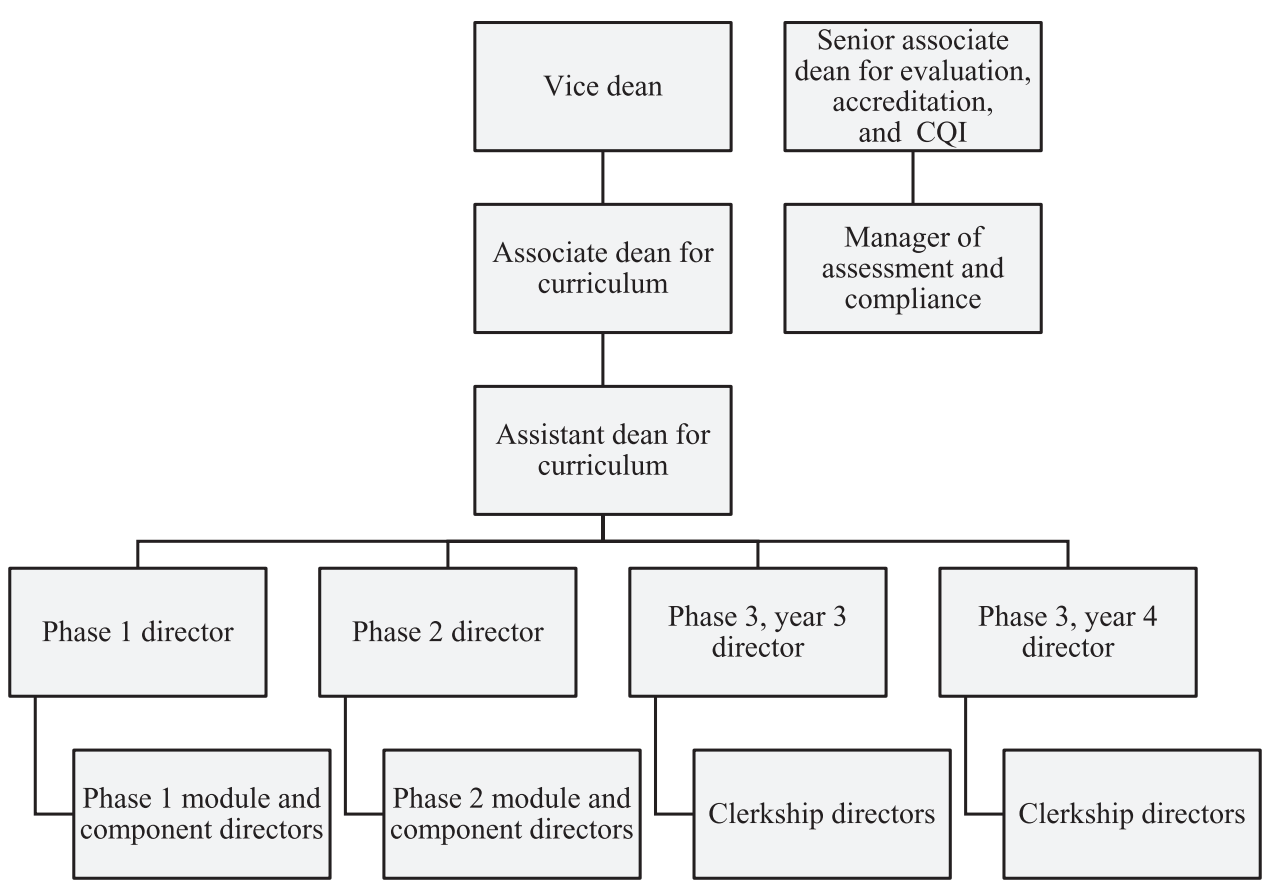

Figure 2 Educational staff.

3-day retreat, and the availability of brief online MedEd Minute presentations.

The Education Innovation Institute offers 2 tracks for faculty development as fellowships: the educational research track (ERT) and the teaching scholars track (TST). The ERT is 2 years in duration and designed to nurture faculty interested in expanding experience in educational research. The 1-year TST is designed to enhance faculty educational skills. In the last 5 years, 44 faculty members have participated in these fellowship programs. Many rich opportunities exist for faculty on all campuses to develop as educators.

The AU Academy of Health Sciences Educators is an organization of distinguished health sciences educators who have demonstrated sustained excellence in educational activities and scholarship.

\section{Reference}

1 Knapp J, Zeratsky J, Kowitz B. Sprint: How to Solve Big Problems and Test New Ideas in Just Five Days. New York, NY: Simon and Schuster; 2016. 


\title{
Morehouse School of Medicine
}

\author{
Martha L. Elks, MD, PhD, Khadeja Johnson, MD, and Aleeia Johnson, MD
}

\section{Medical Education Program Highlights}

The educational program of Morehouse School of Medicine (MSM) is constructed in alignment with MSM's mission priorities of workforce diversity, primary care, and underserved populations. From the start of orientation, strong studentstudent and student-faculty interactions are fostered through learning communities as key elements of the curriculum. Strong academic achievement for the academically diverse student body is assured through high expectations and high support. This support is intrinsic in the curricular structure as well as cocurricular supports including peer tutors and in-course enrichment (ICE). Student engagement with the communities we serve is supported through the service-learning course in community health that occurs longitudinally across the first year. In this course, student-led teams assess a community, develop and implement an intervention, measure and assess the outcomes, and report on the findings. Guided by mission, MSM has been recognized as successful in achieving social mission. ${ }^{1}$

\section{Curriculum}

\section{Curriculum description}

The MSM curriculum is a traditional 2-year preclerkship phase and 2-year clerkship/clinical phase. The first year is integrated into organ systems with 2 longitudinal courses: Community Health, a service-learning course, and Fundamentals of Medicine 1 , a multidisciplinary clinical skills and health systems science course. The second year is a longitudinal discipline-based structure with organ systems-based coordination of topics across courses. There are 6 required clerkships: medicine (with neurology) (12 weeks), psychiatry ( 6 weeks), family medicinerural health ( 6 weeks), pediatrics ( 8 weeks), surgery ( 8 weeks), and obstetrics-gynecology ( 8 weeks). In the fourth year, there is a required selective (subinternship format) and six 4-week electives.

See Supplemental Digital Appendix 1-Curriculum Schematicat http://links.lww.com/ACADMED/A859.

\section{Curriculum changes since 2010}

The integration of the first-year curriculum was done in 2010. To deal with the known phenomenon of some students failing exams early in medical school as they become accustomed to

Acad Med. 2020;95:S140-S141.

doi: 10.1097/ACM.0000000000003332

Copyright (C) 2020 by the Association of American Medical Colleges

Supplemental digital content for this article is available at http://links.Iww.com/ ACADMED/A859.

Year school was established: 1978.

School URL: https://www.msm.edu. the pace and rigor, we developed and implemented an expanded and global program of student exam performance monitoring aligned with a program of ICE. All students have access to nearpeer tutoring and faculty support. Those students who fail more than one exam are supported through ICE, which is a structured program of assessment, academic counseling, and academic skills coaching. This has resulted in a higher student success rate in the first-year curriculum.

Since 2010, there has also been some shifting of rotation time to include neurology content in the medicine rotation. The required subinternship was also introduced.

We are now in a phase of integrating year 2. MSM has a known track record of success for academically diverse students. Often, this involves an extended educational program to allow time for mastery of key content. Before integration of year 1 , this was accomplished by spreading the 2-year curriculum of longitudinal courses across 3 years. Currently, the 5-year track is done by splitting year 2 courses: having students complete Fundamentals of Medicine 2, Microbiology, Nutrition, and Pharmacology courses in the first year (year 2 of matriculation) and Pathology and Pathophysiology courses in the second (year 3 of matriculation). This 5-year pathway is mandated for those with passing averages for the integrated curriculum, of 72 or below. We intend to continue the option of a 3-year preclerkship pathway and are developing the details.

\section{Class size changes since 2010}

Aligned with national priorities and the MSM strategic plan, MSM increased the entering class size from 56 students in 2011-2012 to 100 in 2017-2018. This was achieved through increments of 6-8 students per year over this time frame to allow for sequential expansion of the clinical sites. This increase was supported by a comprehensive plan to increase faculty numbers, staff support, facilities, and practice sites. Over this time frame, community-based practice sites more than doubled and community faculty numbers increased about threefold. We are building toward 2 regional clinical campuses.

\section{Student academic support services}

MSM has a track record of recruiting an academically diverse student body and providing a supportive and nurturing environment that enables the students to have academic success that exceeds that associated with their entering credentials. ${ }^{2}$ This is in part due to curricular content and emphasis, and also due to integrated academic support services. Study strategies and skills are embedded in course work. In addition, the Office of Student Learning and Educational Resources supports learners who face challenges in mastering key concepts. Through the ICE paradigm, students are guided in self-assessment and in using the available supports, including (free) peer tutoring, faculty-led sessions, and other supports. 
Table 1

Learning Objectives and Evaluation Methods

Learning objective

Evaluation methods

\section{A mastery of the concepts necessary for the prevention, diagnosis, treatment, and management of common medical problems, specifically display knowledge of:}

a. The normal development, structure, and physiological function of the body, organ systems, tissues and cells, and their interrelationships, and the molecular, biochemical, cellular, and physiological mechanisms that are important in maintaining the body's homeostasis

b. The biochemical, immunologic, pharmacologic, and microbiologic principles related to issues of disease, laboratory tests, and therapeutics and the various etiologies (genetic, developmental, metabolic, toxic, iatrogenic, microbiologic, autoimmune, neoplastic, degenerative, and traumatic) of diseases (pathogenesis), the associated altered structure and function (pathology and pathophysiology), and characteristic pathologic and laboratory manifestations

c. Common epidemiologic and risk factors for diseases, population health, and the role and impact of psychological, behavioral, social, economic, and cultural factors on health and disease

d. The ethical, legal, gender, psychological, social, interpersonal, sexual health, and economic issues that impact health and medical care

Multiple-choice exams, NBME subject exams, lab exams, quizzes, active learning experiences

Multiple-choice exams, NBME subject exams, lab exams, quizzes, active learning experiences

\section{Basic skills, including the ability to:}

a. Perform and record a complete and accurate history, sensitive to patient needs and the nature of the situation

b. Perform and record an accurate and complete physical examination and mental status examination sensitive to patient needs and the nature of the situation

c. Analyze a patient's clinical presentation, linking to biomedical and health concepts, develop an appropriate diagnostic and therapeutic plan, appropriately using information resources, and laboratory and imaging testing

d. Communicate (in writing and orally) effectively and respectfully with peers, faculty, colleagues, and other members of the health care team, understanding the role of consultations and referrals

e. Communicate and interact with patients in an effective, respectful, and compassionate manner, including counseling them on risks, prevention, lifestyle, and therapy issues

f. Obtain, analyze, and use the medical literature and other information resources to address medical questions and to sustain professional growth and apply techniques of population health, including methods of analysis of the health and health problems of defined populations and development of interventions to improve the health of populations

3. Throughout training, a candidate must demonstrate medical professionalism including ethical behavior, moral reasoning, honesty, integrity, dependability, and commitment to service and health equity

Multiple-choice exams, NBME subject exams, lab exams, quizzes, active learning experiences, community health project

Multiple-choice exams, NBME subject exams, lab exams, quizzes, active learning experiences, learning community assessments, community health project
Clinical evaluations, course and school OSCE

Clinical evaluations, course and school OSCE

Clinical evaluations, course and school OSCE

Clinical evaluations, course and school OSCE, learning community evaluations

Clinical evaluations, course and school OSCE

Clinical evaluations, course and school OSCE, community health project

Clinical evaluations, course and school OSCE, learning community evaluations

\section{Assessment}

The MSM learning objectives are regularly reviewed and are aligned with course learning objectives.

See Table 1-Learning Objectives and Evaluation Methods.

In 2017, we realigned our standard clinical evaluation approach and expanded the utilization of global skills monitoring. We have implemented and expanded parallel programs in the form of shared degree programs with partner institutions. We have an MD-PhD program with the University of Washington (UW) in Seattle (MD with MSM, PhD with UW) and an MD-MBA program with the Scheller School of Business at Georgia Tech (5-year program). We have a long-standing "honors in community service" degree that requires academic excellence, completion of service hours, and completion and presentation of a longitudinal community service project.

We have developed a 3-year track aligned with our family medicine residency. This track is to be implemented in 2021.

\section{References}

1 Mullan F, Chen C, Petterson S, Kolsky G, Spagnola M. The social mission of medical education: Ranking the schools. Ann Intern Med. 2010;152:804-811.

2 Elks ML, Herbert-Carter J, Smith M, Klement B, Knight BB, Anachebe NF. Shifting the curve: Fostering academic success in a diverse student body. Acad Med. 2018;93:66-70. 


\section{Carle Illinois College of Medicine}

Jennifer R. Amos, PhD, William J. Pluta, PhD, Sol Roberts-Lieb, EdD, Judith L. Rowen, MD, Lexi Shurilla, MS, Robert C. Wallon, and Heather Wright

\section{Medical Education Program Highlights}

- Engineering integration: Carle Illinois College of Medicine (CIMED), a public-private partnership between the University of Illinois at Urbana-Champaign and the Carle Foundation, aims to graduate physician-innovators. As the backbone of this reenvisioned medical school, CIMED built engineering into every part of its 4-year, 3-phase curriculum. In Phase 1 preclinical courses, engineering concepts weave through weekly problem-based learning (PBL) cases and Medical Engineering Discovery and Innovation (MEDI) laboratory sessions. In Phase 2 clinical clerkships, an "engineering partner" faculty member rounds with students weekly to identify problems with potential engineering/technology/design solutions, which students then develop and address in the Innovation, Design, Engineering and Analysis (IDEA) course. Capstone and Data Science Project courses are part of Phase 3.

- Early clinical exposure: Clinical exposure begins immediately as students attend weekly sessions with a community-based preceptor for the first year of medical school. A longitudinal family medicine clerkship begins in July to start year 2. During the clerkship, students are the primary providers with faculty oversight in an evening clinic. The students have clinic sessions weekly for 18 months and receive weekly feedback from direct observation of clinical skills via a phone app.

- Innovative admissions process: Quantitative competencies must be evident from transcripts or workplace experiences. No on-campus interview is required for admission. Students produce a portfolio reflecting CIMED's “4Cs": competence, curiosity, compassion, and creativity. A video interview probes other CIMED values including collaboration and diversity. Carle Illinois' first class matriculated in July 2018.

- Frequent and varied assessment: The medical education program at CIMED provides frequent opportunities for student retrieval practice. Throughout Phase 1 of the curriculum, students take weekly quizzes that assess their medical knowledge and receive immediate feedback. Team-based learning (TBL) is used in courses throughout Phase 1, which provides opportunities for individual retrieval and team discussion. Phase 1 assessment also includes 42 OSCE stations, 4 anatomy lab practicals, and a series of 5 progress exams that are designed to support spaced retrieval. In Phase 2 of the curriculum, a progress OSCE program is used to assess student competencies that are linked to Entrustable Professional Activities.

Acad Med. 2020;95:S142-S145.

doi: 10.1097/ACM.0000000000003295

Copyright (C) 2020 by the Association of American Medical Colleges

Supplemental digital content for this article is available at http://links.lww.com/ ACADMED/A831.

Year school was established: 2017.

School URL: https://medicine.illinois.edu.

\section{Curriculum}

\section{Curriculum description}

The CIMED curriculum consists of 3 phases in 4 years: an 18-month preclinical Phase 1; a 12-month clinical clerkship Phase 2; and Capstone and Data Science Projects, clinical electives, and career development in Phase 3.

See Supplemental Digital Appendix 1-Curriculum

Description—at http://links.lww.com/ACADMED/A831.

\section{Assessment}

See Supplemental Digital Appendix 2-Program Objectives—at http://links.lww.com/ACADMED/A831.

\section{Medical education program objectives}

Carle Illinois chose to adopt objectives combining 3 different frameworks: the AAMC Learning Objectives for Medical Student Education, ABET, Inc. Student Learning Outcomes, and KEEN (Kern Entrepreneurial Engineering Network) educational outcomes. It combined these frameworks to adopt objectives that aligned with the college's mission and core competencies.

In particular, the college added objectives that reflected engineering and innovation learning goals for physicianinnovators. ABET is an accreditation agency that accredits engineering, computing, technology, and applied science degrees. ABET student outcomes are required for all engineering degrees and professional licensure as an engineer. KEEN educational outcomes are also widely used in engineering educational programs and help reinforce application of engineering and design skills introduced in the ABET objectives, as well as foster innovation.

\section{Pedagogy}

- PBL: Carle Illinois students participate in three 2-hour PBL sessions per week during Phase 1 . These sessions focus on cases that integrate foundational science, clinical practice, and engineering skills. Unique to CIMED is the addition of an "innovator" role; one student identifies a clinically relevant need from the case and leads a discussion of possible novel solutions. A point of pride at CIMED is having full-time faculty who facilitate the PBL sessions, providing continuity across Phase 1.

- TBL: While the primary pedagogy at CIMED is PBL, additional practices are used to build and solidify content and skill knowledge. These practices take the form of journal clubs, TBL, case review, and lecture. TBL is used often in the first course and then averages 1 to 2 TBL sessions per course for the remainder of Phase 1.

- Laboratory: In addition to traditional cadaver-based anatomy laboratories, students experience MEDI laboratory sessions 
demonstrating the application of engineering principles in medically relevant settings. For example, aerosol particle size and distribution patterns were reviewed in the setting of a lung model replicating asthma physiology.

- Immersive projects: In the latter portion of Phase 1, the MEDI sessions include group projects. In the Phase 2 IDEA Projects course, students identify patient and health care system problems and propose technological solutions. In Phase 3, one of the IDEA projects is selected as the student's capstone project; this is pursued in an interdisciplinary team through the prototyping stage of development. The students also complete a data science project in Phase 3, analyzing large data sets for application to human health concerns.

- Simulation and virtual reality: Standardized patients, task trainers, high-fidelity manikins, and virtual/augmented reality activities are woven into coursework. Ultrasound training includes exploration of the engineering principles as well as the acquisition and interpretation of images. Students are tested to competence in ultrasound and other procedural skills.

\section{Clinical experiences}

Carle Illinois students do most of their training in communitybased care settings. The primary hospital where students train is Carle Foundation Hospital, a 433-bed, Level I Trauma Center. In addition to the 22 outpatient facilities that are associated with Carle Hospital, students rotate through a local physician-owned clinic system, as well as a 210-bed private hospital.

In the first year, students benefit from a yearlong course focused on clinical integration. Through didactics and simulation, students progressively develop the skills necessary to engage in the clinical environment. Students visit a dedicated preceptor in the community who assesses the learner's development of their weekly skill. Carle Illinois students benefit from direct observation and timely, focused feedback.

\section{Required longitudinal experiences}

Longitudinal experiences are woven into 2 of the 3 curricular phases at CIMED. In year 1 of Phase 1 , students are enrolled in the Introduction to Clinical Practice course, a yearlong course that focuses on the development of clinical skills; the course includes weekly sessions in a community physician's practice. Starting in year 2 of Phase 1, students begin a 6-month family medicine clerkship. This clerkship includes ambulatory family medicine rotations, as well as a weekly student clinic. The student clinic continues through year 3 of Phase 2 .

\section{Required and elective community-based rotations}

Phase 1 includes a 6-week elective period titled "Discovery," where students have multiple options, with many selecting global experiences. Carle Illinois students have 7 required clerkships: family medicine, internal medicine, surgery, psychiatry, neurology, obstetrics-gynecology, and pediatrics. Family medicine is community based, and the psychiatry, internal medicine, and obstetrics-gynecology clerkships have portions that may occur in community locations outside of Carle Foundation Hospital. A total of 28 weeks of elective experience is required for graduation; many are community based or have portions that occur at community sites.

\section{Challenges in designing and implementing clinical experiences for medical students}

Similar to other new medical schools, CIMED faced some hurdles in the development of its clinical experiences. With a robust clinical immersion program in the first year, as well as a longitudinal student clinic that runs year-round, recruiting and onboarding new clinical faculty was a challenge. Additionally, Urbana-Champaign is a regional campus for the University of Illinois College of Medicine through 2022 and the needs of learners from both institutions must be balanced.

\section{Curricular Governance}

- Curriculum governance committees: The Curriculum Oversight Committee is true to the CIMED mission with onethird of members from engineering; one-third of members from basic science; and one-third of members with clinical backgrounds, none of which hold an administrative title in the college. The committee sees and participates in review and approval of all courses throughout the 3 phases. To facilitate efficient review, the committee is split into 4 subcommittees: the Preclerkship Subcommittee, Clerkship Subcommittee, Engineering Subcommittee, and Program Evaluation Subcommittee. Each subcommittee does detailed review of proposals and then presents its review for endorsement of the larger committee. In addition, any administrator, faculty, student, or staff member in the college is welcome to submit materials for committee input or attend any of the public meetings. This process ensures that faculty control the curriculum without influence from the administration and that all stakeholders have equal access to the committee to submit proposals.

- Department-led governance: As a public-private partnership, CIMED has a unique faculty model. As in many schools, the clinical faculty are employed by the health system (Carle Foundation or other community partners) and have appointments at CIMED; key personnel such as clerkship directors and course directors have designated FTE contributions to the school. Carle Illinois follows a similar system for its foundational science and engineering faculty; the faculty retain appointments in their home college, and course directors have designated effort in CIMED. The college draws on the wealth of content expertise on the University of Illinois campus. There are two departments within CIMED: Clinical Sciences, and Biomedical and Translational Sciences; the department chairs oversee faculty efforts dedicated to CIMED and report to the dean. Each of the departments maintains an Appointment, Promotions, and Tenure Committee and accesses shared support services at the college level.

See Figure 1-Curriculum Oversight Committee.

See Figure 2-Organizational chart. 


\section{CURRICULUM OVERSIGHT COMMITTEE (COC)}

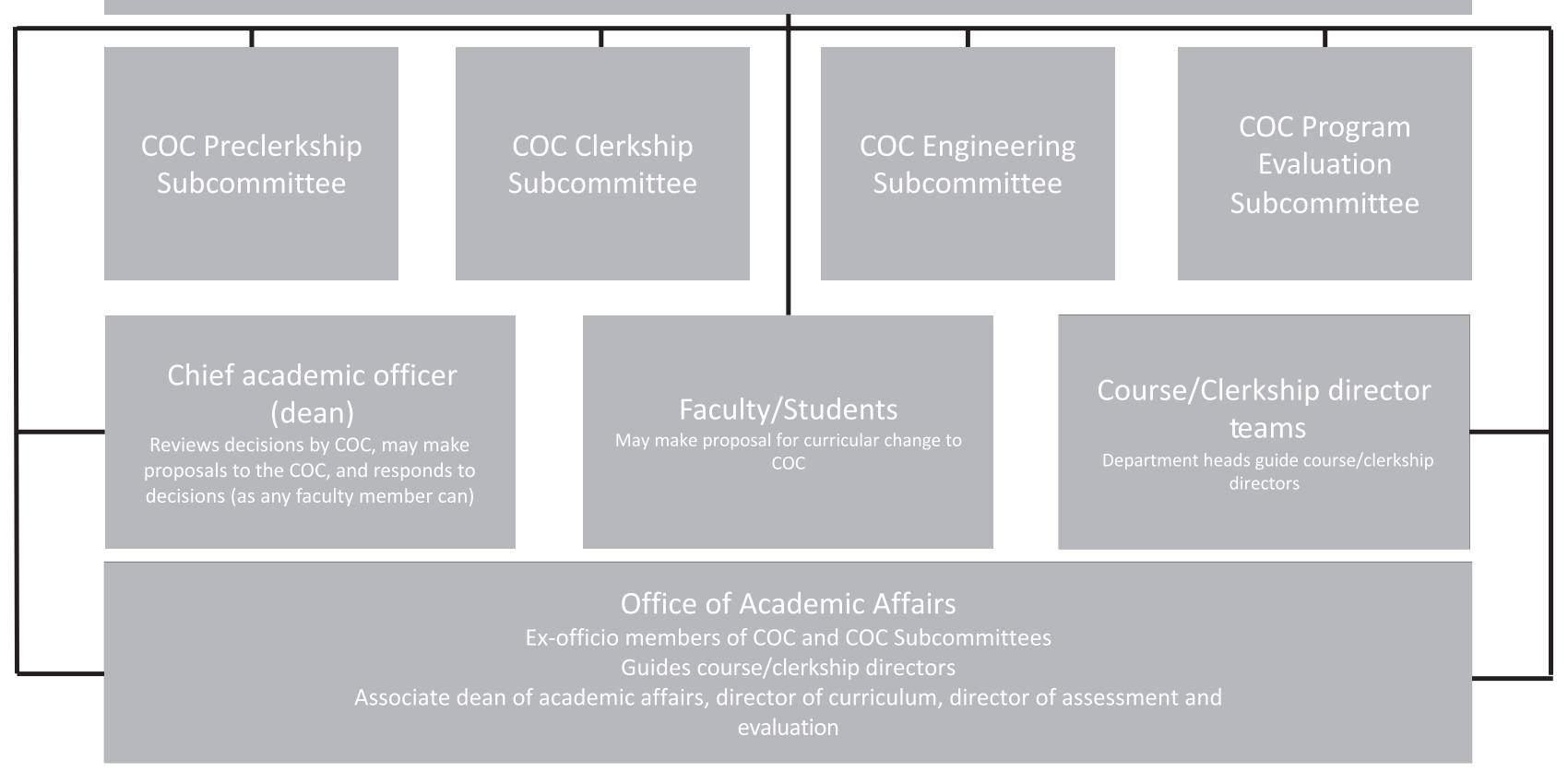

Figure 1 Curriculum Oversight Committee.

\section{Education Staff}

- Course design: All Phase 1 courses are designed by a minimum of 3 faculty: a basic scientist, clinician, and engineer. Academic affairs staff support all facets of curriculum development and delivery, including management of the supporting software, testing programs, and course delivery. An academic affairs faculty or staff member is appointed to each course committee.

- Roles of academic staff: Carle Illinois has various roles to fully support the curriculum. These include: medical education facilitators (PBL); directors for curriculum, faculty development, and assessment and quality improvement; academic affairs coordinator; medical education coordinators (clinical, basic science); medical librarians; simulation center staff (manager, equipment technician, simulated patient coordinator); engineering/IDEA/data science/capstone coordinator; and clerkship coordinators. These staff work with the faculty to ensure the curriculum is created, developed, and delivered according to the values and mission of CIMED.

\section{Dean}
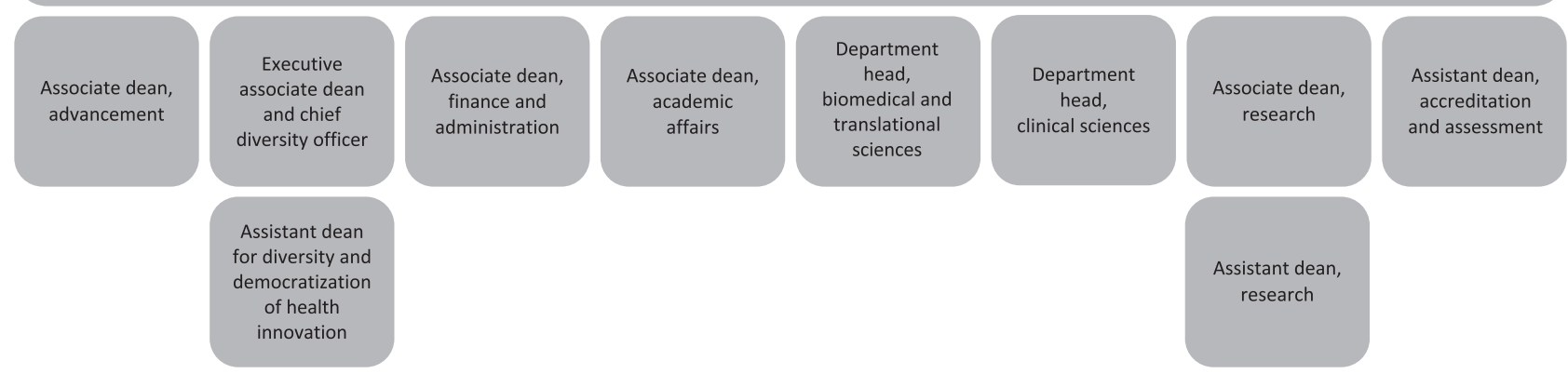

Figure 2 Organizational chart. 


\section{Faculty Development and Support in Education}

- Professional development for faculty as educators: There are a variety of options at CIMED for faculty professional development. Specific training is available for all roles: facilitators, clinical instructors, engineers, and administrative staff. There is an introductory presentation titled "Introduction to Carle Illinois-What Makes CIMED Unique.” An annual curriculum retreat focuses on curricular updates, learning new skills, and network building. It features a mix of presentations, active learning sessions, and workshops. Another annual event, the Problem-Based Learning Workshop, allows all faculty and staff to experience PBL as both a student and facilitator. Faculty may request one-on-one sessions for assistance with tasks such as creating learning objectives, micro-lecture recordings, active learning activities, and so forth. Additionally, the University of Illinois at Urbana-Champaign has extensive faculty development offerings.

- Promotion and tenure: To support both the clinicians and campus faculty, in addition to the faculty with defined FTE roles, there are various tracks for appointment, promotion, and tenure. These include teaching, clinical, and tenure tracks. In each of these tracks, student evaluation and scholarship are included in consideration for promotion. 


\section{Chicago Medical School at Rosalind Franklin University of Medicine and Science}

Jeanette Morrison, MD, Gordon Pullen, PhD, and Sheryl Juliano, MA, MS

\section{Medical Education Program Highlights}

- In 2018, Chicago Medical School (CMS) implemented a revised curriculum delivered in 3 sequential phases. Phase I (first and second years) addresses the foundational knowledge, skills, attitudes, and behaviors of a physician and emphasizes integrated and clinically relevant educational experiences. Phase II (third year) provides 48 weeks of supervised patient care experience in 7 core specialty areas and 1 elective area. Phase III (fourth year) allows students to deepen their understanding of medical specialties and to function at a more advanced level in preparation for residency training.

- CMS is 1 of 5 colleges at Rosalind Franklin University of Medicine and Science (RFUMS), home to over 30 graduate health professions and science programs. This range of disciplines on a single campus allows for team-based, interprofessional learning and practice opportunities that begin in the first quarter with coursework devoted to defining roles and fostering teamwork. This continues throughout the curriculum via service learning, global health, simulation, and patient care experiences.

- As a community-based medical school, CMS has a diverse set of affiliate sites that provide students with rich and varied clinical experiences.

- Students complete the 4-year longitudinal Clinical Reflections course in which they reflect upon their experiences, professional identity, and personal values as they adapt to the culture of medicine.

- Simulation is an integral part of the educational program. A new 30,000 square foot virtual health system opened in 2017 and provides opportunities for learning and assessment in a range of health care environments.

\section{Curriculum}

\section{Curriculum description}

- The 4-year MD-granting curriculum is divided into 3 sequential phases. Phase I is the preclerkship period during the first 2 years of the program. Phase II is the clerkship period during the third year, and Phase III is the period emphasizing residency preparation during the fourth and final year of the program. These latter 2 phases are designed to strengthen the integration of basic and clinical sciences and ensure achievement of core Entrustable Professional Activities (EPAs) for beginning residency.

Acad Med. 2020;95:S146-S149.

doi: 10.1097/ACM.0000000000003347

Copyright (C) 2020 by the Association of American Medical Colleges

Supplemental digital content for this article is available at http://links.Iww.com/ ACADMED/A872.

Year school was established: 1912.

School URL: https://www.rosalindfranklin.edu/academics/chicago-medical-school.
- Woven into the 4-year curriculum are 8 threads that address key topics in health care and enable vertical and horizontal integration: - Health promotion (wellness and promotion)

- Team-based care (interprofessional/interdisciplinary)

- Humanities

- Safety and quality

- High-value care (evidence based)

- Ethics

- Professionalism

- Population health (health disparities/special populations)

See Supplemental Digital Appendix 1-Curriculum Map-at http://links.lww.com/ACADMED/A872.

\section{Curriculum changes since 2010}

- An extensive review of the CMS competencies and objectives in 2013-2014 identified opportunities to strengthen content, methods of delivery, assessment, and integration.

- Following a series of curricular retreats during 2014, 11 guiding principles for teaching and learning were established. These are integration of student-centered, engaging teaching methodologies; diverse learning experiences; team-based learning; systems-organized, clinical conditions-based curriculum that builds on complexity from Phase I to Phase III; early clinical experiences; foundation for success on licensing exams; formative feedback; opportunities for self-study and self-reflection; community engagement; interprofessional learning; and an emphasis on professionalism.

- In early 2015, the Curriculum Committee formed an ad hoc committee to oversee the development of the renewed curriculum. For the first 6 months, this committee focused on overall goals and outcomes, framework, and the impact of curricular change on other colleges at the university. From July 2015 until implementation of the revised curriculum in August 2018, several task forces, comprising faculty and students, were responsible for content development, integration, faculty development, outcome measures, and assessment.

- In late 2015, the Executive University Advisory Task Force was created to support a university perspective on required architecture and logistical changes for the renewed curriculum. Comprising representatives from across the university, this task force continues to meet every 2 months to discuss issues of space, calendar, student financial services, admissions, accreditation, and assessment.

- Assessment of curricular outcomes and effectiveness is ongoing and a comprehensive review of Phase I will occur in the fall of 2020 .

\section{Assessment}

Enhancements to student assessment have been made in all 3 phases. 
Phase I:

- NBME Customized Assessment Services exams are used to provide summative assessment of student learning in several systems-based courses.

- Weekly formative assessments measure student comprehension and academic progress within systems-based courses.

- Improved narrative feedback from clinical case faculty facilitators provides students with formative assessment and supplemental information on academic progress.

Phase II:

- Formative and summative simulation exercises assess student learning on general topics such as teamwork, patient safety, and communication as well as clerkship-specific topics of labor and delivery, ultrasound, and palliative care.

- Increased assessment of student learning via standardized patient encounters measure appropriate clerkship-specific clinical skills.

- An additional midyear, formative objective structured clinical examination provides an opportunity for students to gather a history and perform a physical exam; prioritize an initial differential diagnosis; and seek, synthesize, and evaluate clinical and scholarly evidence.

Phase III:

- Required high-fidelity simulation, interactive small-group discussion, role play, and standardized patients teach and assess the skills necessary to transition from fourth-year student to first-year resident.

\section{Medical education program objectives}

A variety of assessment methods have been implemented during the medical education program, which are used when appropriate to assess each objective.

See Chart 1-Medical education program objectives.

The CMS medical education program objectives are based on the AAMC Physician Competency Reference Set, modified to include competencies required of the AAMC-sponsored Interprofessional Education Collaborative and the AAMC core EPAs.

\section{Pedagogy}

- Pedagogical approaches are aligned with the 11 guiding principles.

- Lectures are still used as a major component to deliver curricular content; however, all lectures must include opportunities for active learning.

- Approximately 25\%-30\% of the classroom hours are now delivered through case-based discussions, laboratories, and workshops.

- First-year medical students learn to use information resources to support self-directed learning during the Essentials of Clinical Reasoning course and Epidemiology course. During Epidemiology, students engage in independent and group work to investigate a clinical research question using the Population, Intervention, Comparison, Outcome method. Students decide upon a research question, evaluate the literature, and independently prepare an oral presentation.

\section{Chart 1}

\section{Medical Education Program Objectives}

\section{Practice-based learning and} to investigate and evaluate one's care of patients, to appraise and assimilate scientific evidence, and to continuously improve patient care based on constant self-evaluation and lifelong learning.

\section{Interpersonal and communication} skills: Demonstrate interpersonal and communication skills that result in the effective exchange of information and collaboration with patients, their families, and health professionals.

\section{Professionalism: Demonstrate a} commitment to carrying out professional responsibilities and an adherence to ethical principles

Systems-based practice: Demonstrate an awareness of and responsiveness to the larger context and system of health care, as well as the ability to call effectively on other resources in the system to provide optimal health care.

\section{Interprofessional collaboration:}

Demonstrate the ability to engage in an interprofessional team in a manner that optimizes safe, effective patient- and population-centered care.

\section{Personal and professional}

development: Demonstrate the qualities required to sustain lifelong personal and professional growth.

\section{Medical education program objectives \\ Patient care: Provide patient-centered care that is compassionate, appropriate, and effective for the treatment of health problems and the promotion of health. \\ Knowledge for practice: Demonstrate knowledge of established and evolving biomedical, clinical, epidemiological, and social-behavioral sciences, as well as the application of this knowledge to patient care.}

improvement: Demonstrate the ability
Assessment methods

\section{- Clinical} documentation review

- Clinical performance rating/checklist

- Exam: institutionally developed, written/ computer based

- Exam: institutionally developed, clinical performance

- Exam: institutionally developed laboratory, practica

- Exam: nationally normed/ standardized, subject

- Multisource assessment

- Narrative assessment

- Oral patient presentation

- Participation

- Peer assessment

- Portfolio-based assessment

- Research or project assessment

- Self-assessment

- Stimulated recall

- Transition to Internship assessment
- Small-group case-based discussions are integrated throughout the Phase I curriculum, providing students with the chance to apply basic science concepts from the week to a patient with a common clinical presentation. The 8 threads are integrated into these cases to enrich the discussion.

- Simulation exercises are embedded in many aspects of the curriculum. The Essentials of Clinical Reasoning course (Phase I) uses multiple simulation opportunities to teach students how to effectively communicate with patients, take a complete history, and perform a physical examination. These skills are reinforced with standardized patient experiences in the second year, during the Phase II clerkships, and in the fourth-year Transition to 
Internship course. The addition of a new, virtual health system in 2017 expanded the simulation capabilities to include a variety of health care settings, such as the operating room, emergency department, and labor and delivery suite.

\section{Clinical experiences}

- During the first-year Clinical Foundations of Medicine course, students are required to spend time in the emergency department, hospice care, and 2 other clinical settings.

- During the second year, students attend a minimum of 4 sessions with a clinical preceptor in either the hospital or outpatient setting.

- Students complete all of their required clerkships, fourth-year subinternship, and various fourth-year electives at affiliate clinical sites, including Advocate Christ Medical Center, Advocate Condell Medical Center, Advocate Good Shepherd Hospital, Advocate Illinois Masonic Hospital, Advocate Lutheran General Hospital, AMITA Health Adventist Health Center, AMITA Health Saint Joseph Hospital Chicago, AMITA Health Saints Mary and Elizabeth Hospital, AMITA Health Resurrection Medical Center Chicago, Billings Clinic, Captain James A. Lovell Federal Health Care Center, Edward Hospital, Elgin Mental Health Center, Elmhurst Hospital, John H. Stroger, Jr., Hospital of Cook County, Little Company of Mary Hospital and Health Care Centers, Louis A. Weiss Memorial Hospital, MacNeal Hospital, Mercy Hospital and Medical Center, Midwestern Regional Medical Center, Mount Sinai Hospital Medical Center, Northwestern Medicine-Huntley Hospital, Saint Anthony Hospital, Waukegan Illinois Hospital Company_-Vista Medical Center East, and Rosalind Franklin University Health Clinics.

- Many students volunteer at the RFUMS Interprofessional Community Clinic. Established in 2013, this clinic allows students from allopathic medicine, pharmacy, physical therapy, physicianassistant, podiatric medicine, and psychology to work together on interprofessional teams to provide supervised health care to underserved and underinsured local community residents.

- The medical school faces increasing challenges from other schools, both local and offshore, for clinical sites and preceptors. In addition, while multiple clinical sites greatly improve the diversity of students' clinical experiences, ensuring comparability of the educational experience within a given clerkship requires sophisticated central monitoring and coordination between faculty, staff, and administration.

\section{Curricular Governance}

- The Curriculum Committee has full responsibility and authority for the design, management, and oversight of the curriculum.

- This committee comprises faculty from the Clinical Sciences Department and the Department of Foundational Sciences and Humanities, with student representation from each year, and administrative (nonvoting) members.

- Four subcommittees (Year 1/Year 2, Year 3/Year 4, Evaluation and Assessment, Vertical and Horizontal Integration) review and provide recommendations to the Curriculum Committee on content, learning activities, alignment with objectives, vertical and horizontal integration, and outcomes measures.

\section{Education Staff}

- The Office of Student Affairs and Education (OSAE) is committed to providing a high-quality educational experience that will produce exemplary physicians. This office supports student success by providing services that optimize academic, personal, and professional growth.

- Working with the Curriculum Committee of the medical school, the OSAE facilitates scheduling classes and clerkships; assists with exam administration; oversees second- and fourth-year elective opportunities; gathers student and faculty evaluations; tracks and analyzes student performance; collects curricular data for the purposes of curricular management, assessment, and enhancement; and acts as a liaison between faculty and administration.

- In addition, the OSAE provides an important advisory function for students during all phases of their education. The office assists students in adapting to the medical school environment, provides academic advising, provides a 4-year program of career and specialty advising, writes the Medical Student Performance Evaluation, and oversees the residency match process.

- Nonclinical faculty who have a major role in teaching medical students have an academic appointment in the Foundational Sciences and Humanities Department. The purpose of this department is to provide an organizational framework within the medical school that places an emphasis on teaching and learning.

See Figure 1-Organizational chart.

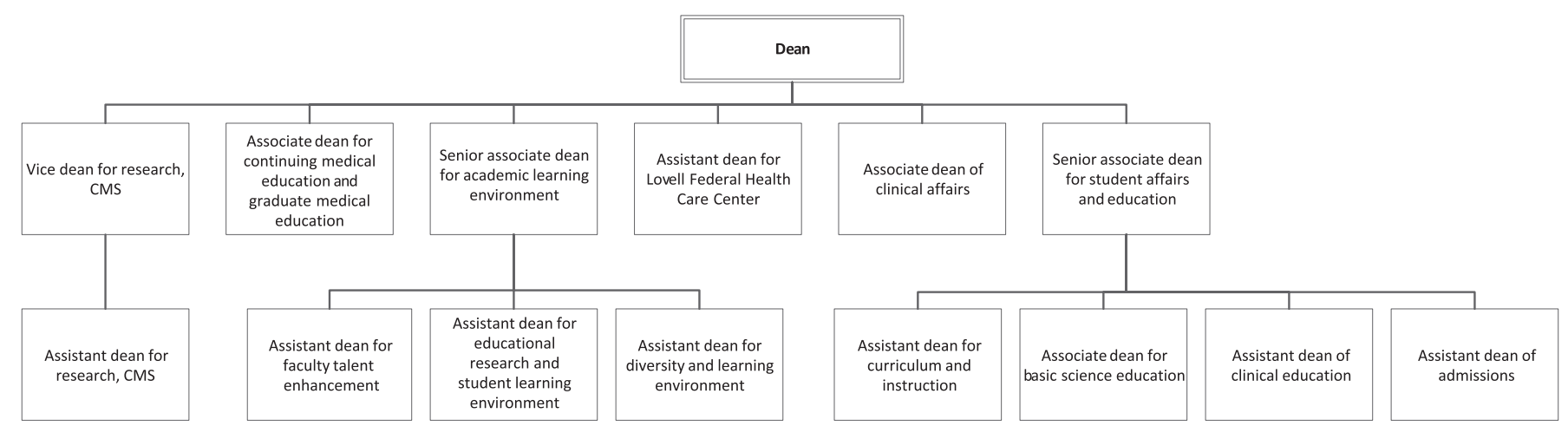

Figure 1 Organizational chart. 


\section{Faculty Development and Support in Education}

- Faculty development is offered by the university through the Office of Faculty Development, Online Learning, and Instructional Design; the Assessment Leadership Council; and the Baldwin Institute for Interprofessional Education. These activities are open to all university faculty and cover a wide range of topics, including mentoring, global health, online teaching, educational technology, educational assessment, and effective teaching strategies.

- In addition, the CMS Office of Academic Learning Environment provides onsite programs at clinical affiliate sites; online modules on teaching, assessment, and student treatment; and bimonthly podcasts on topics and issues related to teaching and learning in higher education.

- Faculty development was an integral part of the curricular revision process, especially for phase I changes in pedagogy and assessment. Faculty-identified topics that needed to be addressed and workshops and seminars were provided on the following topics:
- Small-group facilitation skills

- Small-group assessment skills

- Technology-based teaching

- Clinical case development

- Simulation

\section{Role of teaching in promotion and tenure}

- For promotion in the clinical educator track, faculty must make substantial contributions to the training programs and academic community of the school.

- Promotion to professor in the clinical educator track requires at least 5 peer-reviewed publications as well as recognition at the regional or national level for clinical care, teaching, or research.

- The Master Teacher Guild is an interprofessional association that promotes excellence and innovation in health education. Members are chosen from across the university in recognition of their teaching excellence and provide support and mentoring to other faculty, participate in pedagogical scholarship, and collaborate in assessment of teaching and learning. 


\title{
Loyola University Chicago Stritch School of Medicine
}

\author{
Gregory Gruener, MD, MBA, MHPE, Theresa Kristopaitis, MD, and Neil Clipstone, PhD
}

\section{Medical Education Program Highlights}

At Stritch School of Medicine (SSOM), we facilitate students' professional development as "physicians in training" by providing an educational environment that promotes academic and clinical excellence and also develops lifelong learners and prepares them for a balanced clinical practice. Our primary goal is to graduate a practicing physician who is prepared to assume an academic or research career (participation in research ranges from $70 \%$ to $80 \%$, and approximately $30 \%$ of students pursue our Research Honors program), an outcome that is evident by residency program directors' reviews of SSOM graduates and the leadership roles (e.g., chief residents) they take in those programs.

SSOM is grounded in Jesuit values that embody service and begins with an admissions process that employs mission-based, holistic review. We endeavor to identify the most academically and professionally prepared aspirants who have demonstrated professional growth and reflection, conquered academic challenges, and provided strong contributions to their campus and civic communities. We embodied this commitment as the first medical school to initiate and continue the acceptance of students who identify as Deferred Action for Childhood Arrivals candidates. At SSOM, service that fosters social justice and solidarity with those affected by inequities is evident by graduation: $95 \%$ of the class has engaged in more than one humanitarian, volunteer, or service experience, while 15\%-20\% demonstrate extensive involvement working with underserved populations.

The necessity to actively address the increasing distress experienced by students, residents, and physicians led to integrating existing activities, programs, and groups into a coherent and reinforcing curriculum that develops supportive life skills through professional formation. In addition to current support programs and facilities, this wellness curriculum educates students about the challenges to being well, provides opportunities for reflection on individual experiences within a community of peers, and encourages meaningful discussion about individual and systems-based approaches to these challenges.

The Office of Diversity, Equity, and Inclusion (ODEI) seeks innovative pathways to engage faculty, staff, and students in meaningful learning and professional development experiences that cultivate the capacity to care, teach, learn, and lead in a diverse and global world. A core aspect of our

Acad Med. 2020;95:S150-S154.

doi: 10.1097/ACM.0000000000003289

Copyright (C) 2020 by the Association of American Medical Colleges

Year school was established: 1870.

School URL: https://ssom.luc.edu. process necessitates deep examination of the experiences of underrepresented faculty and senior administrative staff and support for educational initiatives of particular relevance to retention and advancement of a diverse faculty and staff.

\section{Curriculum}

\section{Curriculum description}

The SSOM curriculum comprises 4 phases. The first 2 were developed to prepare students for clinical work and licensing exams; the next 2 ensure robust experiential clinical experiences and assurance of specialty-specific clinical competence. While the total curriculum is 158 weeks, curricular revision is underway to shorten Phase 1 and 2 to allow further innovations in Phase 4.

- Phase 1: Core or foundational clinical sciences, purposefully administered in discipline-specific modules, and a gradually accelerating curriculum to facilitate student transition into development as a physician. This also begins students' introduction to a longitudinal medical practice, bioethics, and our emerging self-care curriculum.

- Phase 2: Integrated curriculum of 3 courses that run in parallel: Mechanisms of Human Disease (pathology and pathophysiology), Pharmacology and Therapeutics, and Patient-Centered Medicine 2 (physical exam and diagnostic skills). After Phase 2, students take the USLME Step 1 licensing exam.

- Phase 3: Seven required clerkships (medicine, surgery, obstetrics-gynecology, pediatrics, psychiatry, neurology, family medicine), an elective, and the Population-Centered Medicine course. At the end of Phase 3, a multistation objective structured clinical examination (OSCE) and end-of-life OSCE are administered.

- Phase 4: Required emergency medicine clerkship, 2 subinternship selectives (SubI-Wards and SubI-ICU), as well as electives, discretionary time, and preparation for residency. During this phase, students take USMLE Step 2 licensing exams.

\section{Curriculum changes since 2010}

A commitment to continuous quality improvement has allowed SSOM to regularly incorporate planned curricular change. Major conceptual changes of how we view and develop our curriculum include:

- Establish a purposeful partnership with clinical affiliates to enhance that learning environment by acknowledging and developing responses to their health system strengths and concerns (e.g., purposeful faculty development/recognition, addressing clinical productivity requirements).

- Align with and ensure graduates meet residency program performance competencies (e.g., EMR familiarity, self-care). 


\section{Table 1}

\section{Medical Education Program Objectives and Assessment Methods}

\section{Medical education \\ program objectives (abbreviated)}

Assessment methods

Medical knowledge

\begin{tabular}{|c|c|}
\hline & $\begin{array}{l}\text { - Clinical documentation review } \\
\text { - Direct observation/narrative assessment } \\
\text { - Oral presentation } \\
\text { - Institutionally developed exam } \\
\text { - Simulation activities } \\
\text { - Standardized patient exercises/OSCES } \\
\text { - USMLE Step } 1 \text { and Step } 2 \text { CK } \\
\text { - Written report/reflection paper }\end{array}$ \\
\hline Patient care & $\begin{array}{l}\text { - Clinical performance rating } \\
\text { - Clinical documentation review } \\
\text { - Direct observation } \\
\text { - Narrative assessment } \\
\text { - Oral presentation } \\
\text { - Institutionally developed exam } \\
\text { - Simulation activities } \\
\text { - Standardized patient exercises/OSCES } \\
\text { - USMLE Step } 2 \text { CS } \\
\text { - Written report/reflection paper }\end{array}$ \\
\hline $\begin{array}{l}\text { Interpersonal and } \\
\text { communication skills }\end{array}$ & $\begin{array}{l}\text { - Direct observation } \\
\text { - } \text { Narrative assessment } \\
\text { - } \text { Reflection activity } \\
\text { - Simulation activities } \\
\text { - Standardized patient exercises/OSCES } \\
\text { - Written report }\end{array}$ \\
\hline $\begin{array}{l}\text { Practice-based learning } \\
\text { and improvement }\end{array}$ & $\begin{array}{l}\text { - Direct observation } \\
\text { - Narrative assessment } \\
\text { - Oral presentation } \\
\text { - Reflection activity } \\
\text { - Self-assessment } \\
\text { - Written report }\end{array}$ \\
\hline Professionalism & $\begin{array}{l}\text { - Narrative assessment (facilitator, faculty, } \\
\text { - Preceptor, resident) } \\
\text { - Participation in required activities } \\
\text { - Reflection paper } \\
\text { - Standardized patient evaluation } \\
\text { - Written ethics paper }\end{array}$ \\
\hline Systems-based practice & $\begin{array}{l}\text { - Institutionally developed exam } \\
\text { - Participation in required activities } \\
\text { - Reflection paper } \\
\text { - Written report }\end{array}$ \\
\hline
\end{tabular}

(Table continues)
Table 1

(Continued)

\begin{tabular}{|c|c|}
\hline $\begin{array}{l}\text { Medical education } \\
\text { program objectives } \\
\text { (abbreviated) }\end{array}$ & Assessment methods \\
\hline $\begin{array}{l}\text { Interprofessional } \\
\text { collaboration }\end{array}$ & $\begin{array}{l}\text { - Direct observation } \\
\text { - Institutionally developed exam } \\
\text { - Narrative assessment } \\
\text { - Participation in required activities } \\
\text { - Simulation activities }\end{array}$ \\
\hline $\begin{array}{l}\text { Personal and professional } \\
\text { development }\end{array}$ & $\begin{array}{l}\text { - Direct observation } \\
\text { - Reflection paper } \\
\text { - Self-assessment }\end{array}$ \\
\hline
\end{tabular}

- Incorporate new skill development or residency expected capabilities (e.g., point-of-care ultrasound curriculum, Institute for Healthcare Improvement Open School).

- Embrace a self-care/wellness mindset throughout, as a core theme equivalent to medical knowledge, skill development, and self-assessment.

- Improve monitoring of experiential clerkship experiences and enhancement of Phase 3 and individualized elective opportunities.

\section{Class size changes}

In recognition of the AAMC's and other professional organizations' predictions of a future physician shortage, SSOM initiated a phased increase in class size to ensure that educational support was aligned with any projected future student class size, currently set at 170 .

\section{Assessment}

Medical education program objectives are described in outcomebased terms that allow assessment of medical students' progress in developing the competencies that the profession and the public expect of a physician.

See Table 1-Medical Education Program Objectives and Assessment Methods.

\section{Medical education program objectives}

The original formation of program objectives used the Medical School Objectives Project, but since that time amalgamated with ACGME domains of competence. We are transitioning to a more relevant hybrid of AAMC Core Entrustable Professional Activities (EPAs) and residency milestones that will facilitate long-term outcome assessments.

\section{Assessment changes since 2010}

- Oversight of all medical students' progression through the curriculum by our Student Promotion Committee and, when necessary, individual remediation plans developed by the Academic Review and Intervention Committee 
- Pass/fail course assessment during Phases 1 and 2

- Standardization of clerkship assessments, weighting, and grade assignments (oversight provided by the Competency Evaluation and Assessment Review Committee)

- End of Phase 3 multistation clinical skills exercise and end-oflife clinical skills exercise

- Monitoring experiential activities in all clerkships, documenting observation of history taking and physical exams, and midclerkship feedback

- Incorporation of interprofessional activities and their formative assessment

- Incorporating EPAs as the final assessment for Phase 4 subinternships

\section{Pedagogy}

We have aligned assessment methods to our pedagogy, but informed by our resources and learners' needs, to support curricular revision and the continued transition from a lecturebased approach to one where student self-assessment and selfdirected learning skills facilitate development of lifelong learning.

Within Phases 1 and 2, we predominantly employ discussion (small- and large-group), lecture, laboratory, role play, selfdirected learning, standardized/simulated patients, team-based learning, video/podcast, and virtual patients.

Within Phases 3 and 4, we predominantly employ clinical experiences (inpatient and outpatient), case-based learning, discussion (small- and large-group), self-directed learning, peer teaching and preceptorship, simulation, and standardized/ simulated patients.

\section{Changes in pedagogy since 2010}

We continue to expand and implement simulation, teambased learning, interprofessional (didactic, experiential, and extracurricular), peer teaching, self-directed learning, and casebased learning.

\section{Clinical experiences}

For required clerkships, we primarily use our clinical affiliate, Loyola University Healthcare System (Loyola University Medical Center [LUMC], Gottlieb Memorial Hospital, MacNeal Hospital) and Hines Veterans Administration Hospital; both Hines and Loyola University Medical Center are on the same campus. This supports a broad range of student experiences, inpatient and outpatient, within an academic medical center's tertiary and quaternary services, Veterans Administration, and a communitybased hospital. Additional clinical training sites include those within Trinity Health system, nearby community hospitals, and affiliated faculty within private practice sites.

\section{Required longitudinal experiences}

We are currently completing development and preparing a pilot of a longitudinal ambulatory experience, eventually for all students in Phases 1 and 2. This will be a progressive curriculum designed to immerse students in the clinical environment along with its faculty and staff. Students will acquire progressive competency with clinical skills, EMR use, screening tools, and patient counseling. This longitudinal exposure will prepare them for their clinical years and opportunities for career exploration.

\section{Clinical experience first encounter}

Student clinical exposure (patient and sites) begins in Phase 1 and extends into Phase 2, as structured curricular activities and as supervised and directed extracurricular experiences.

\section{Required and elective community-based rotations}

Clinical activities occur within our current clinical affiliates (Gottlieb Memorial Hospital, MacNeal Hospital), Hines Veterans Administration Hospital, community hospital affiliates, and affiliated faculty within private practices. Administrative support is also provided to students who seek experiences outside of our current network.

\section{Challenges in designing and implementing clinical experiences for medical students}

The trend for medical schools not to own their primary clinical affiliates, health system mergers, productivity demands for clinicians, increasing numbers of nonmedical student learners, and students from non-LCME-accredited medical schools have placed the same educational burden on all LCME-accredited medical schools. In response, we have focused on developing our students to more effectively contribute to the clinical workplace, support faculty participation and their professional development, strengthen internal clinical affiliations, and strategically prepare for a changing external health care environment. Our greater hope is to collaborate, not compete, with local medical schools by emphasizing our shared commitment to the education of all students.

\section{Curricular Governance}

The Central Curricular Authority (CCA) is a standing committee reporting directly to the dean, who as the chief academic officer exercises authority over curriculum and is responsible for auditing compliance with policies and processes related to its development, delivery, and continuing quality improvement. The CCA oversees curricular management through separate subcommittees; their work informs and is informed by ad hoc participation by other SSOM committees.

See Figure 1-Central Curricular Authority organizational chart.

\section{Decentralized curricular governance}

While collaborative in nature, SSOM provides direction, governance, and support for all faculty (e.g., FTE buybacks, professional development, administrative support, informatics, educational stipends, departmental stipends, clerkship site support)

\section{Education Staff}

SSOM provides both administrative support (e.g., educational coordinators, technology) and support for faculty (e.g., FTE buybacks, professional development, effort capture, educational stipends, departmental stipends). 


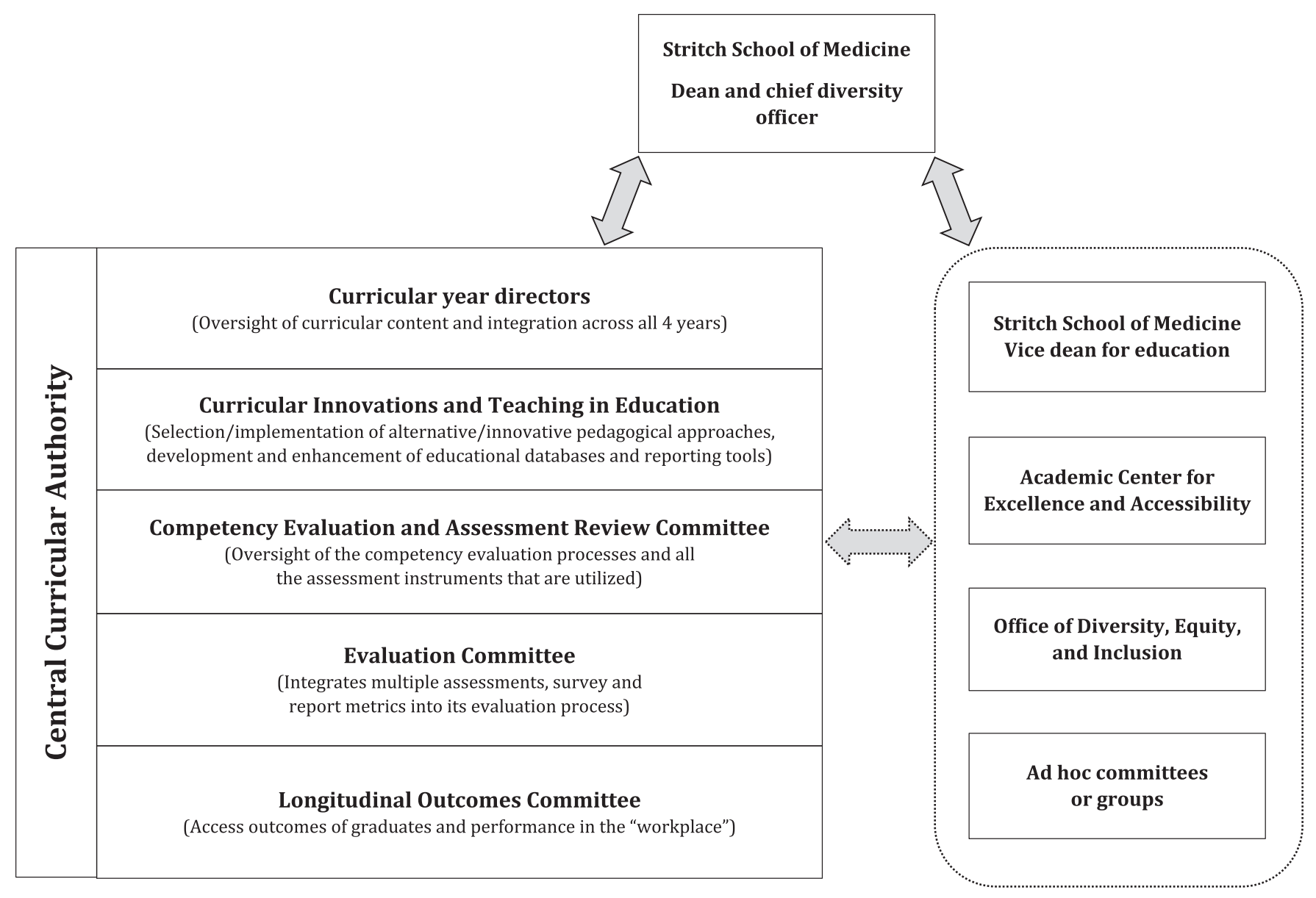

Figure 1 Central Curricular Authority organizational chart.

See Figure 2-Organizational chart.

The Office of Medical Education staff support undergraduate medical education and related committees. Different staff support the Office of Student Affairs, Center for Community and Global Health, and Academic Center for Excellence and Accessibility.

\section{Department of Medical Education}

The Ralph P. Leischner, Jr., MD, Department of Medical Education (DOME) represents the successful amalgamation of the former Leischner Institute for Medical Education professional development program with the support structures of a department. DOME promotes educational innovation, scholarship, and community by developing faculty educators, furthering effective ways of assessment and the broader dissemination of curricular programs. Faculty can receive either a primary or secondary appointment.

\section{Faculty Development and Support in Education}

\section{Professional development for faculty as educators}

DOME provides various faculty development activities, including certificate programs in medical education, Leischner educational grants, and Leischner Institute workshops.
The Division of Continuing Medical facilitates and promotes the process of lifelong learning by providing high-quality, evidencebased educational opportunities designed to advance learner competence, enhance practice performance, promote patient safety, and improve patient outcomes. This division has achieved Accreditation with Commendation status.

The Center for Simulation Education provides innovative, simulation-based educational exercises and performance assessments to promote patient safety and performance improvement. In conjunction with the Institute for Transformative Interprofessional Education (ITIE), it received provisional accreditation by the Society for Simulation in Healthcare.

ITIE was established to educate future health care providers and professionals to work as a team to better care for patients, perform foundational research on interprofessional education/ collaborative practice, and transform health care to incorporate more interprofessional models.

Neiswanger Institute for Bioethics and Healthcare Leadership offers master's degree programs and a hybrid doctoral degree in bioethics and health care mission leadership, as well as online certificate programs for health care professionals. 


\section{ILLINOIS}

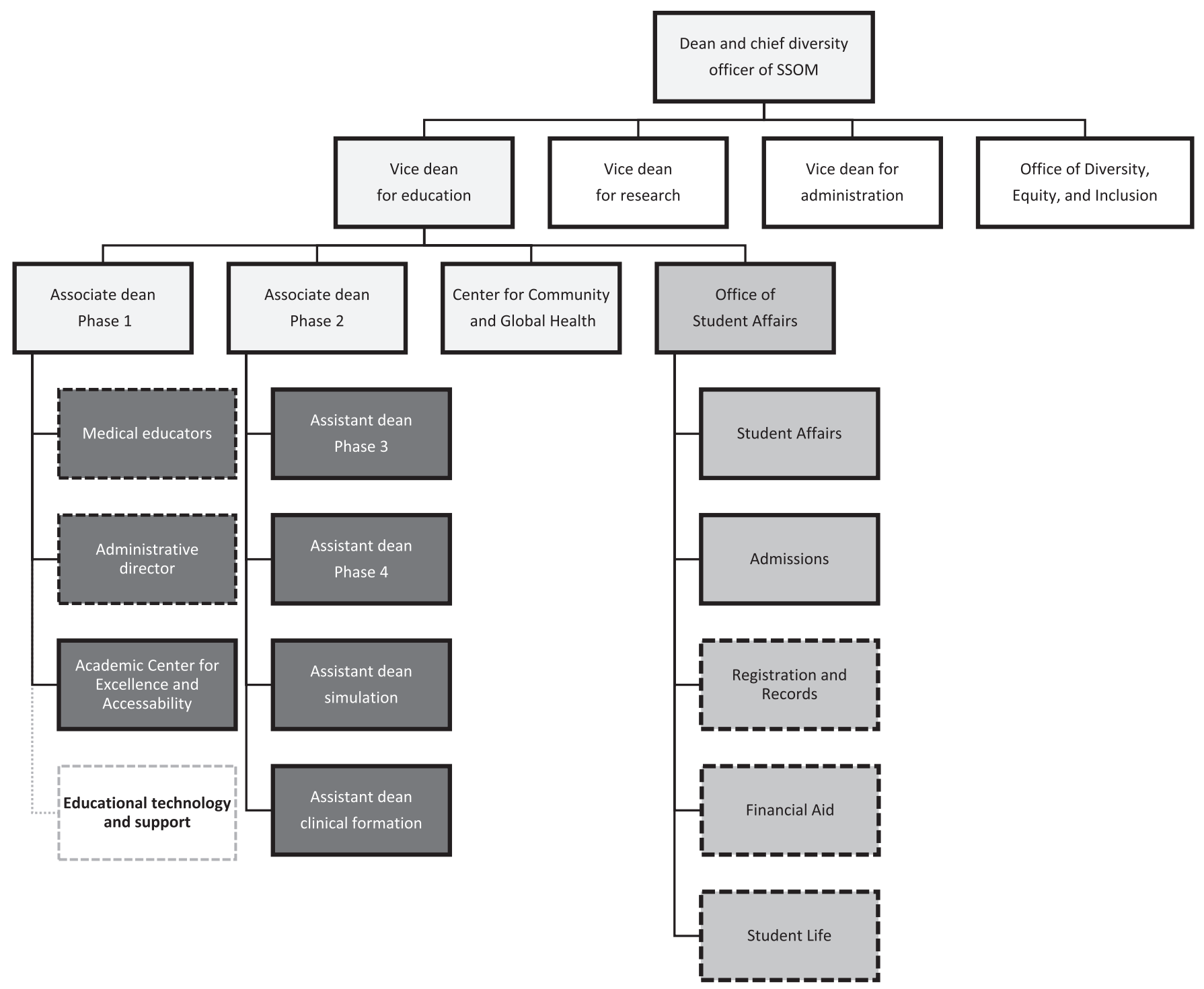

Figure 2 Organizational chart.

ODEI works in partnership with student, faculty, and staff leadership to develop innovative pathways to engage faculty, staff, and students in learning and professional development experiences that cultivate capacity to care, teach, learn, and lead in a diverse global world.

\section{Role of teaching in promotion and tenure}

Our Committee on Academic Rank and Tenure completed a revision of promotion guidelines, instituted in 2020, for all faculty, regardless of their academic track. Evidence of teaching and education were already significant components of each track's promotion guidelines, but these revisions provided clarification and domain-specific examples. Specific attention was paid to the clinical educator track and academic educator track, where evidence of education/teaching efforts and outcomes are more significantly weighted in promotion decisions. Faculty development is supported by a process that assigns all junior faculty to mentors; the outcomes of their meetings and additional performance metrics are captured within an annual faculty performance review that allows faculty to review, comment, and receive feedback on their performance. The formal annual review and a midpromotion review also document faculty progression toward promotion.

DOME is focused on faculty who have a significant commitment to education, including clinical and basic science faculty. This role is being expanded to residents, graduate students, and medical students to capture the continuum of learners on our health sciences campus. 


\title{
Northwestern University Feinberg School of Medicine
}

\author{
Marianne M. Green, MD, Diane B. Wayne, MD, Patricia M. Garcia, MD, MPH, and Sandra M. Sanguino, MD, MPH
}

\section{Medical Education Program Highlights}

- Northwestern University Feinberg School of Medicine (Feinberg) is a large urban medical school with 634 students based in Chicago, Illinois. The curriculum has 3 phases. Phase 1 consists of foundational material and 14 organ-based modules. Phase 2 consists of 6 core clerkships, and Phase 3 is composed of advanced clinical clerkships. Feinberg's curriculum is organized around 8 competencies: patientcentered medical care, communication and interpersonal skills, medical knowledge and scholarship, system awareness and team-based care, personal awareness and self-care, community engagement and service, continuous learning and quality improvement, and professional behavior and moral reasoning. We highlight several components of the educational program below; many of these are also described in a recent publication. ${ }^{1}$

- The Education Centered Medical Home (ECMH) is a longitudinal, immersive primary care clerkship that spans the entire undergraduate medical curriculum. Teams of 16 students, 4 from each class, are embedded into primary care clinics, many of which are in underserved areas. Students care for a panel of medically complex patients under the supervision of a faculty preceptor. Students attend ECMH clinic for 4 hours every other week and see patients in pairs (a third- or fourth-year student paired with a first- or second-year student). Students also serve as peer educators by investigating clinical questions and as health coaches by contacting patients for follow-up on laboratory or radiology studies, consultations, and clinical status. Students act as quality managers by compiling quality-of-care report cards and targeting areas of clinical care for improvement as part of an annual ECMH team quality improvement project. The ECMH serves as a real-world classroom for learning social determinants of health, chronic disease management, and clinical skills. Early reports show students who participate in ECMH report a more positive learning environment, higher quality of primary care training, and greater professional efficacy. ${ }^{2}$

- The Area of Scholarly Concentration (AOSC) is part of Feinberg's mission to mentor and educate students to become inquiry-driven physician-scientists. This longitudinal, mentored scholarly project provides students the opportunity to identify an individualized area of independent study, collect and analyze data, and answer a hypothesis-driven research

Acad Med. 2020;95:S155-S158.

doi: 10.1097/ACM.0000000000003307

Copyright (C) 2020 by the Association of American Medical Colleges

Supplemental digital content for this article is available at http://links.Iww.com/ ACADMED/A838.

Year school was established: 1859.

School URL: https://www.feinberg.northwestern.edu. question. Areas of investigation are broad and may include basic science, clinical and translational medicine, global health, community and family health, and the medical social sciences. The AOSC culminates in writing a thesis before graduation; many students also submit peer-reviewed first-author publications.

- Feinberg fosters habits of self-assessment, personal improvement, and reflective capacity through the use of an electronic assessment portfolio. A faculty committee reviews each student's progress toward Entrustable Professional Activities (EPAs) and the Feinberg competencies at the end of each curricular phase. A well-developed coaching system supports and guides students throughout the process.

- Synthesis and Application Modules (SAMs) are weeklong sessions interspersed throughout the curriculum. SAM sessions are highly interactive and simulation based. These sessions provide students with the opportunity to revisit previously taught concepts and to integrate material across modules using case-based learning and simulation-based education. For example, first-year students review a case of a patient with shortness of breath. The patient's respiratory status worsens and ultimately requires mechanical ventilation for respiratory support. Despite improvement of the respiratory condition, the patient develops multisystem organ dysfunction. Throughout the SAM, students work in small groups within a larger class setting to manage care for this simulated patient. Students work together to answer questions involving pathology, physiology, pharmacology, genetics, and biochemistry that inform the patient's evolving clinical care. This model allows for the synthesis, application, and integration of various elements in the curriculum in a simulated patient care setting.

- In August 2017, Feinberg's Office of Diversity and Inclusion became the first medical school in the country to launch the Sustained Dialogue Program. ${ }^{3}$ This program has been used in college campuses throughout the United States to promote more engaged, cohesive, and diverse communities. Student participants are trained to facilitate longitudinal student dialogue groups that center around challenging topics such as race and privilege, religion, and mental health. This highly rated program provides students the opportunity to understand different perspectives to promote an inclusive campus community.

\section{Curriculum}

\section{Curriculum description}

- The Feinberg curriculum is composed of 4 elements (science in medicine, clinical medicine, health and society, professional development), 5 threads (lifestyle medicine, medical decision-making and diagnostic testing, health equity and advocacy, health care quality and patient safety, teamwork and leadership), and 2 longitudinal experiences (AOSC and 
ECMH). Each element and thread is fully integrated into each phase of the curriculum.

- Phase 1 consists of a Foundations module and 14 organ-based modules. The Foundations module introduces students to the fundamental aspects of basic, clinical, and health system sciences. Each subsequent organ-based module addresses normal and pathological processes in an integrated fashion. Learning in the context of patient problems and clinical care is emphasized. As described above, SAM modules are interspersed throughout Phase 1 to provide an opportunity for spaced repetition and application of prior content to solving new clinical problems.

- Phase 2 is composed of 6 required clerkships (surgery, medicine, pediatrics, obstetrics-gynecology, neurology, psychiatry), continuation of the longitudinal ECMH, and early elective experiences determined by each student's career preferences. Students also spend time in a monthly integrated curriculum (IC2) session incorporating basic and clinical sciences, health and society, and professional development.

- Phase 3 is composed of 4 required clerkships (subinternship, emergency medicine, critical care medicine, physical medicine and rehabilitation), 5 electives, and the longitudinal ECMH. Phase 3 culminates with the Capstone experience including rigorous assessment of EPAs to ensure each Feinberg student is prepared for residency in their chosen field.

\section{Curriculum changes since 2010}

- Feinberg launched a redesigned curriculum in 2012. The traditional structure was replaced with 3 phases as described above. The redesigned curriculum emphasizes 4 curricular elements and 5 threads designed to help students develop into evidence-based clinicians and researchers who provide costeffective, safe, quality care as part of an interprofessional team.

- Formative assessments have been incorporated throughout Phase 1. Students attend weekly synthesis and application sessions in the form of interactive question-based content review. Practice exams are available to allow students to gauge areas of strength and identify areas that need attention before each module exam.

- Phase 2 begins earlier in the curriculum in the redesigned curriculum, in May of the second year. This provides students with additional schedule flexibility, allowing them to participate in electives and research to explore career interests.

- All students are required to complete a 2-week Capstone course before graduation. Students select a track based on their residency choice and have a series of small-group specialty sessions devoted to topics such as first night on call simulations, clinical skills sessions, and didactic content. A multistation clinical skills assessment gives students an opportunity to demonstrate mastery of several EPAs. Opportunities for deliberate practice are included in the course to ensure all students achieve skill mastery before graduation

\section{Assessment}

- Feinberg uses a competency-based educational framework based on the ACGME competencies and the AAMC EPAs.

- The Feinberg assessment system uses a broad array of methods, including clinical performance assessments,
OSCEs, peer feedback, 360-degree assessments, workplace assessments, simulation exercises, and oral and multiple-choice examinations.

- The Feinberg electronic portfolio system serves as a repository of each student's quantitative and qualitative assessment data. As part of the portfolio system, students demonstrate phaseappropriate achievement in 5 competencies: continuous learning and quality improvement, communication and interpersonal skills, patient-centered medical care, professional behavior and moral reasoning, and system awareness and team-based care. EPA assessment is currently being incorporated. Student portfolios are reviewed after each phase by a faculty committee. Students needing additional skills practice receive support through an individualized educational program.

See Supplemental Digital Appendix 1-Educational Program Objectives and Outcome Measures-at http://links.lww.com/ ACADMED/A838.

\section{Pedagogy}

- A variety of pedagogical approaches are used in the curriculum, including but not limited to didactic lectures, problem-based learning, team-based learning, standardized patients, and simulations.

- Over the past 10 years, active learning strategies have been implemented throughout the curriculum. Our new state-ofthe-art active learning classroom allows collaborative learning sessions where students can work together to apply knowledge to solve basic science and clinical problems.

\section{Clinical experiences}

- We are fortunate to have several major sites for clinical education located on the medical campus. These sites include Northwestern Memorial Hospital, the Ann \& Robert H. Lurie Children's Hospital of Chicago, and the Shirley Ryan Ability Lab. Feinberg also has a major affiliation with the Jesse Brown Veterans Affairs Hospital. Required and elective communitybased rotations also occur at the John H. Stroger Hospital of Cook County and several federally qualified health centers.

- The ECMH is a longitudinal clerkship for all students, described above.

- Early clinical training is a cornerstone of the Feinberg curriculum. Students encounter patients during the first week of class and patients are brought to the lecture hall during each module order to provide a clinical context for the science being taught.

\section{Curricular Governance}

In addition to centrally supported activities such as the Feinberg Simulation Center, the Galter Library, and the Clinical Education Center, funds are transferred from the Office of Medical Education to departments to support faculty in leadership roles, resources that are part of the clinical educational program, and administration of the clinical clerkship administration.

See Figure 1-Curriculum governance structure. 


\section{Education Staff}

- The Augusta Webster, MD, Office of Medical Education provides central oversight and support for all aspects of the curriculum. The office encompasses curricular affairs, student affairs, registration and records, and the Honors Program in Medical Education, Northwestern's BA-MD program.

- The senior associate dean for medical education oversees the Office of Medical Education and is responsible for the undergraduate medical education program. The senior associate dean is assisted by the associate dean for curriculum and the associate dean for student affairs. Additional support is provided by the assistant director of student affairs, the director of program evaluation and accreditation, the director of academic success, and the student wellness and support coordinator.

- The senior associate dean for medical education reports to the vice dean for education. Feinberg's vice dean for education oversees the Honors Program in Medical Education, medical student admissions and the medical education program, a Masters in Physician's Assistant program, the doctor of physical therapy program, and a prosthetics/orthotics program. The vice dean for education also has oversight over graduate and continuing medical education. The vice dean for education reports to the dean of the medical school.

See Figure 2-Medical education organizational chart.

\section{Faculty Development and Support in Education}

- The Feinberg Academy of Medical Educators (FAME) was established in 2010. FAME provides school-wide faculty development programming and recognizes faculty contributions to education at the school. FAME currently has 205 members.

- FAME provides monthly lectures and workshops and develops online modules available to all faculty. Recent offerings include topics such as adult learning theory, providing feedback, writing test questions, learner assessment, curriculum development, team-based learning, largegroup teaching, active learning, mentoring, and simulation instructor training.

- FAME offers a 2-year Medical Educator Certificate Program to prepare clinical educators for excellence in teaching practice through participation in a variety of learning and teaching activities. Program participants attend FAME lectures, workshops, and meetings; review online resources; participate in directly observed teaching sessions; create a scholarly project; reflect on program activities; and compile an educator portfolio.

- A clinician-educator career track is available for faculty who contribute to the clinical, educational, and/or research missions of the school but whose major effort is in the areas of clinical practice, practice-related activities, and/or education. This is a non-tenure-eligible track.

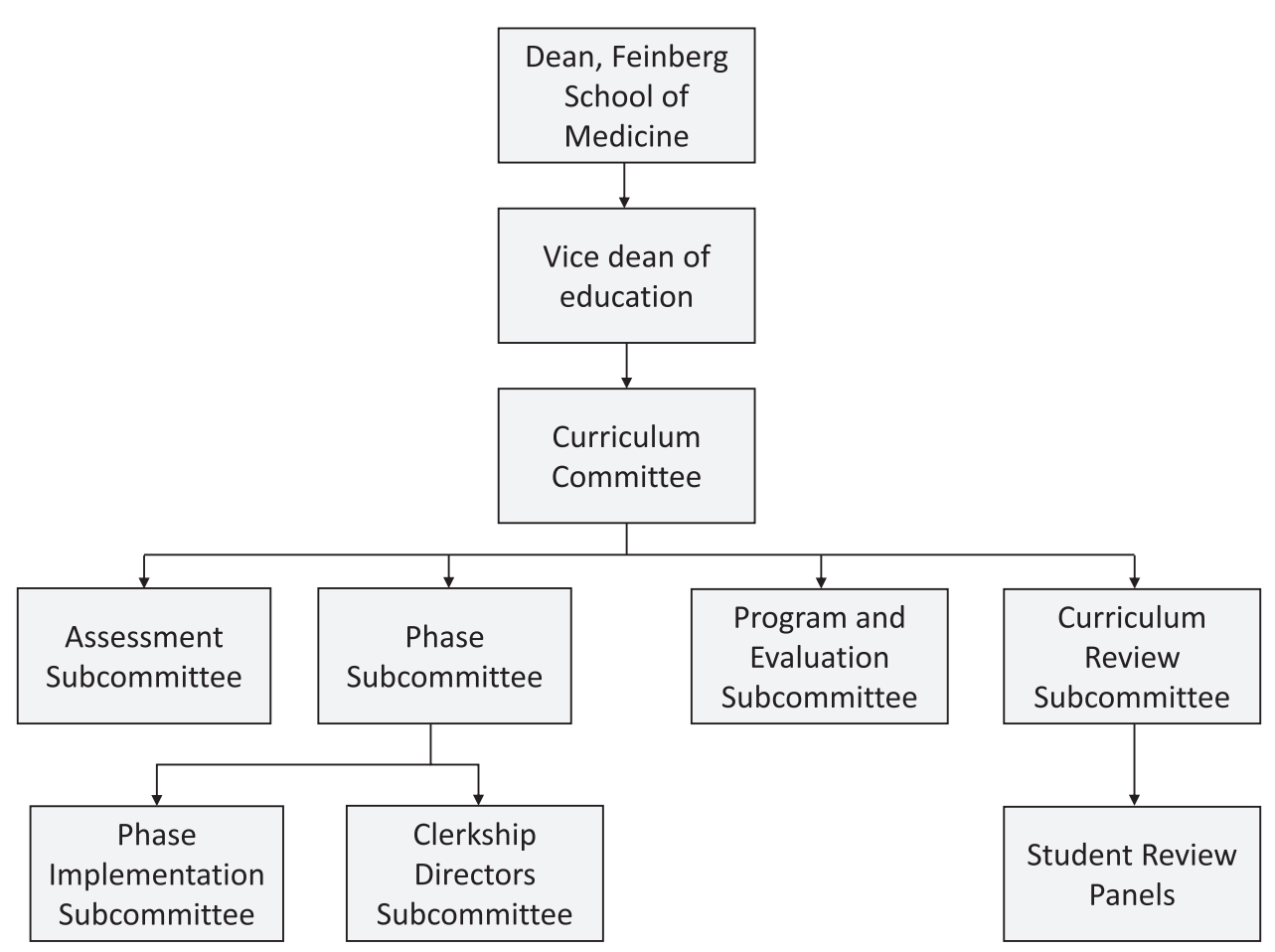

Figure 1 Curriculum governance structure. 


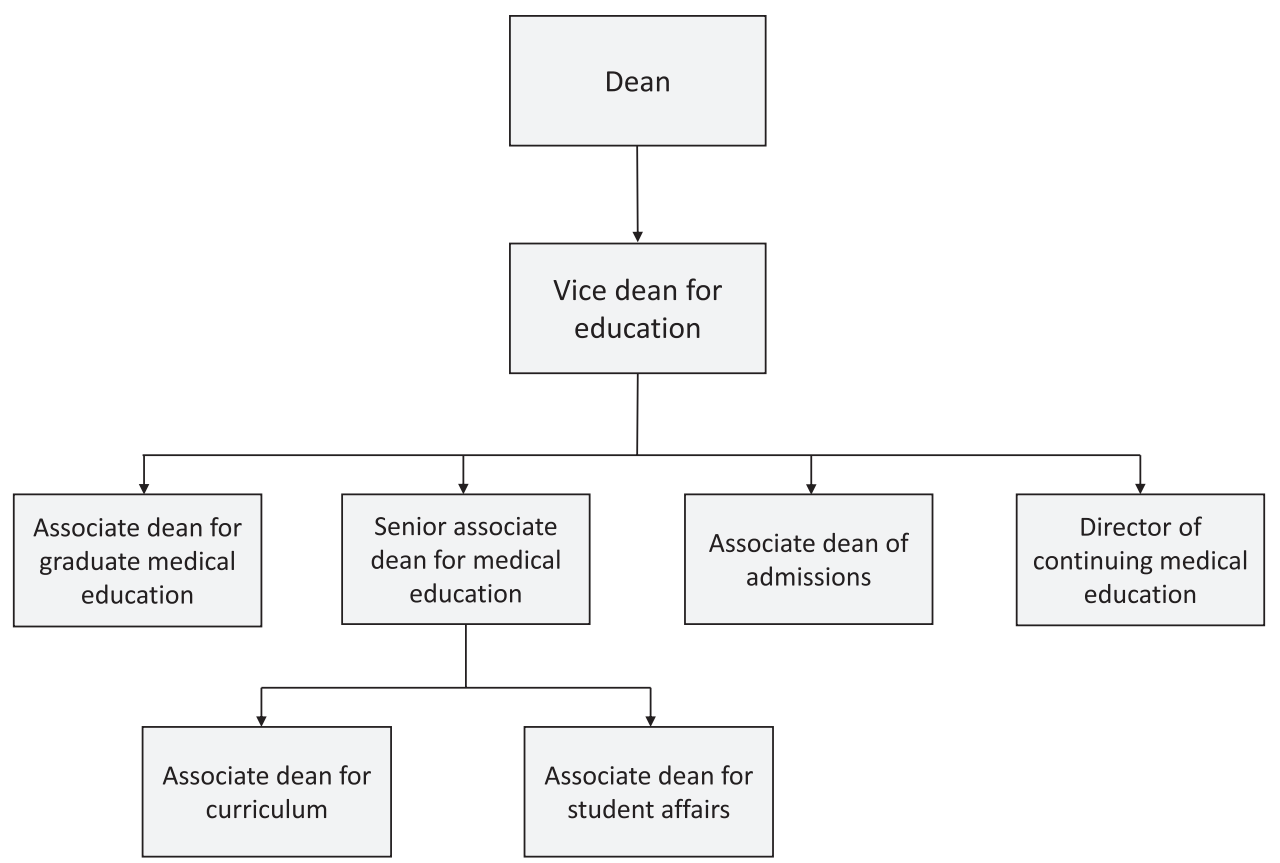

Figure 2 Medical education organizational chart.

- The Department of Medical Education centralizes much of the educational programming, innovation, and education scholarship at Feinberg and its affiliated institutions. Secondary faculty appointments in medical education are awarded to select faculty with significant roles in education.

\section{Initiatives in Progress}

- We are in the process of developing a curriculum that prepares students to use artificial intelligence, digital technology, and data science as clinical tools to practice precision medicine as 21st-century physicians.
- We are aligning each ECMH with a community organization to develop and implement a community health plan aimed at improving a priority health need in a target community.

\section{References}

1 Heiman HL, O'Brien CL, Curry RH, et al. Description and early outcomes of a comprehensive curriculum redesign at the Northwestern University Feinberg School of Medicine. Acad Med. 2018;93:593-599.

2 Henschen BL, Liss DT, Golden BP, et al. Continuity with patients, preceptors, and peers improves primary care training: A randomized medical education trial. Acad Med. 2020;95:425-434.

3 Sustained Dialogue Institute. https://sustaineddialogue.org/our-work/ campus. Accessed March 6, 2020. 


\section{Rush Medical College of Rush University Medical Center}

Elizabeth A. Baker, MD, MHPE, Rahul Patwari, MD, Robert Leven, PhD, Mark Grichanik, PhD, Aimee Szewka, MD, Princess Currence, DSW-C, and Richard Abrams, MD

\section{Medical Education Program Highlights}

\section{Curriculum renewal}

Rush Medical College (RMC) premiered its integrated preclerkship curriculum in 2017-2018. The program roles of practitioner, communicator, leader, advocate, educator, scholar, collaborator, and professional, with a foundation of medical knowledge, guide the curriculum. A single-pass curriculum that combines the normal and abnormal states is anchored around cases from all core clinical disciplines. A flipped classroom format is used, with students preparing independently through readings and video recordings and coming to class to actively participate in case-based collaborative small-group sessions.

The EXPLORE program (EXPeriential Learning Opportunities for preclerkship students at Rush) is a curricular innovation designed to capture the value RMC places on clinical care and personalized, active learning by engaging preclerkship students in meaningful clinical experiences. EXPLORE pairs first- and second-year medical students with authentic clinical work to reinforce and invigorate their classroom learning, as well as to infuse joy and meaning throughout the preclerkship years.

An essential element of our curriculum is an emphasis on formative assessment and early intervention. We are striving to create a true feedback culture, ${ }^{1}$ in which the goal is improvement of performance over time, with liberal and early remediation for all students at risk. We are reforming observation-based assessment in clinical environments to individualize the undergraduate medical education (UME)-GME transition. Additionally, by improving reciprocity between assessment and the medical student affairs processes, we are building a supportive culture reinforcing the idea that wellness and achievement can coexist.

\section{Rush Community Service Initiative Programs}

A hallmark of Rush's community-centered vision is Rush Community Service Initiative Programs (RCSIP). RCSIP has 30 programs in 4 strategic areas:

- Access: 5 clinics and a back-to-school health fair

- Pipeline: colleges, high schools, and grade schools

- Health education: community centers and churches

- Outreach: Cook County Jail, juvenile temporary detention center, and MLK Day of Service

Acad Med. 2020;95:S159-S163.

doi: 10.1097/ACM.0000000000003333

Copyright (C) 2020 by the Association of American Medical Colleges

Supplemental digital content for this article is available at http://links.Iww.com/ ACADMED/A858.

Year school was established: 1837.

School URL: https://www.rushu.rush.edu/rush-medical-college.
Programs are led by teams of student leaders. All students volunteer with RCSIP, which impacts 10,000+ individuals annually, with 280-300 medical students volunteering each year.

\section{Curriculum}

The RMC curriculum is a 4-year program organized around the 8 "roles" physicians embody. These roles are inspired by the CanMEDS roles ${ }^{2}$ and ACGME competency domains. The curriculum begins with 18 months of integrated, one-pass, case-based small-group sessions with clinical and simulation experiences, followed by 2 clinical years composed of clerkships and electives. The preclerkship curriculum is organized into 12 multidisciplinary courses, with an additional research course and the EXPLORE program. The clerkship curriculum is organized into 8 required clerkships, one 4 -week subinternship, a clinical bridge course, and elective experiences.

See Figure 1-Physician roles.

See Supplemental Digital Appendix 1-Curriculum Map—at http://links.lww.com/ACADMED/A858.

\section{Curriculum changes since 2010}

Before the 2010-2011 academic year, the preclerkship curriculum consisted of discipline-based courses with normal body function taught in the first year and disease process and treatment taught in the second year. All curriculum outside the basic sciences was integrated into a single Physicianship course. Multidisciplinary organ system-based basic science courses were introduced in the 2010-2011 (first year) and 2012-2013 (second year) academic years. Pedagogy continued to be focused around live lectures with accompanying labs in anatomy and histology and workshops in physiology.

Our integrated preclerkship curriculum was introduced in 20172018. Multidisciplinary organ system courses continued, with normal and abnormal integrated into single courses for each organ system. The Physicianship course content was distributed based on the roles and integrated into all courses. New courses were introduced to focus on specific populations and complex multiorgan system disease. As the 2017 and 2018 matriculating classes have advanced through the curriculum, the $8 \mathrm{RMC}$ roles have been integrated into required and elective clerkships.

Class size has increased from 128 (2010-2015) to 136 (2016) to 144 (2018). This increase has not resulted in any changes in structure or function.

\section{Assessment}

The overarching goal of the curriculum is to prepare students to practice medicine in the 21 st-century and provide them with the skills necessary to excel in their chosen residency 
programs. Program objectives are based on the CanMEDS roles, describe the roles that define an RMC graduate, and use assessments to monitor student progress toward meeting our objectives.

See Table 1-Roles, Program Objectives, and Assessments.

Assessment activities have been unified under 1 operational unit with 3 primary goals: closely aligning assessments with learning objectives, providing students a consistent experience across courses and assessment modalities, and prioritizing high-volume, low-stakes assessments intended to provide students with frequent feedback. The knowledge-based assessment system in the preclerkship years includes:

- Session assessments

- Formative assessments every 2-3 weeks featuring questions across cases and disciplines

- Summative assessments administered at the end of each course

Knowledge-based assessments, including anatomy and histology practical exams, have been digitized and are administered through Examsoft. Clerkships use NBME Clinical Science Subject Exams for knowledge-based assessment. Performancebased assessments have been expanded significantly with the introduction of clinical skills assessments at the end of each preclerkship course, a gateway OSCE at the end of the preclerkship phase, and 3 OSCEs during the third-year clerkship phase. Finally, assessment systems have been tightly integrated with the Medical Student Affairs processes. Data from each assessment are used just in time to identify students who may benefit from additional support, allowing for timely development and implementation of individualized education plans for success.

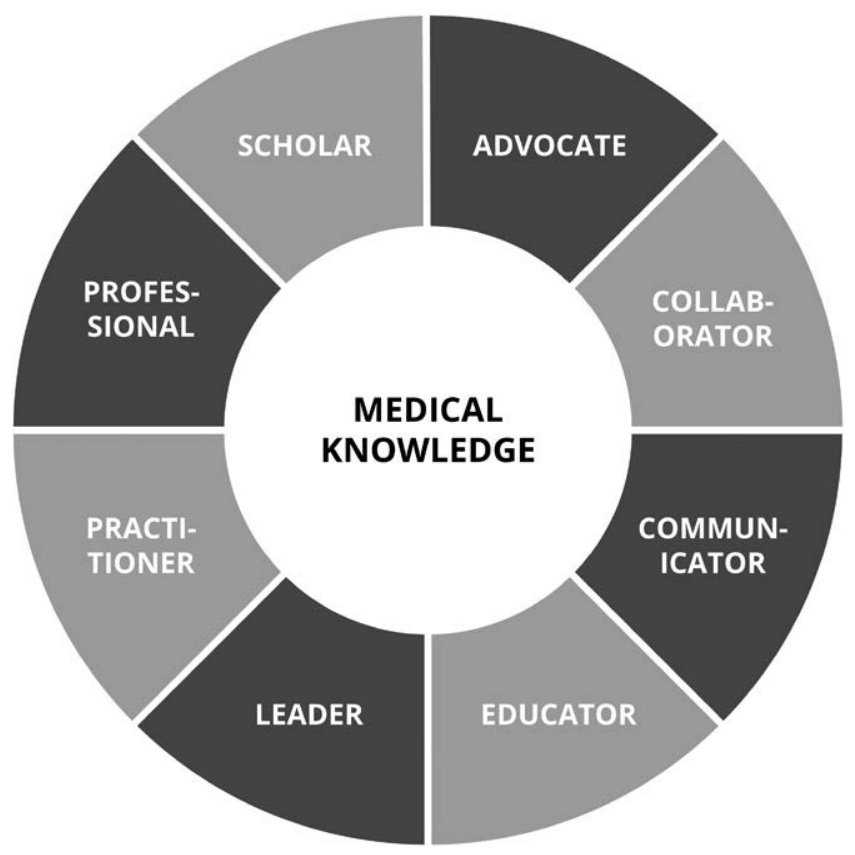

Figure 1 Physician roles.
Assessment changes implemented since 2010 include:

- Preclerkship: Session assessments, formative assessments, and clinical skills assessments

- Clerkship: New workplace-based performance assessments, combined clerkship OSCEs

\section{Pedagogy}

We use a variety of pedagogical approaches.

Preclerkship curriculum:

- Flipped classroom courses use video- and text-based content available online.

- The curriculum is case-based, using both small and large discussion groups, gross and microscopic anatomy laboratory, peer teaching, standardized patients, patient panels, physiology workshops, and authentic patient experiences.

Clerkship curriculum:

- The majority of learning experiences are clinical, in both ambulatory and inpatient settings.

- Virtual patients are occasionally used to complete a student's clinical experience requirements.

- Simulation activities and standardized patients are used in multiple clerkships.

- Standard teaching sessions incorporate lecture, small-group discussion, case-based and team-based learning to deliver a variety of topics.

- Additional activities include self-directed learning (SDL) and peer teaching (EBM/SDL projects).

In the clerkship years, there has been a push to deliver content in a more interactive, flipped classroom format, resulting in a significant increase of case-based or discussion-based sessions.

\section{Clinical experiences}

The majority of required clinical experiences occur at Rush University Medical Center, with a significant component occurring at the John H. Stroger, Jr. Hospital of Cook County, adjacent to Rush University Medical Center. Additionally, we have multiple sites off of our main campus.

We have a required primary care clerkship in family medicine/ internal medicine. While a small percentage of primary care settings are university based, the majority are located in community-based clinics. We have approximately 10 community-based electives in family medicine, dermatology, internal medicine, pediatrics, obstetrics-gynecology, and surgery. In addition, students can develop their own community-based electives based on individual needs.

A major challenge is the ongoing need to recruit and retain outpatient sites. Multiple medical schools compete for community-based clinical experiences in the Chicago metropolitan area. Another significant challenge is around compensation for clinical teachers who are essential for educational experiences in both the academic and community settings. 
Table 1

\section{Roles, Program Objectives, and Assessments}

\begin{tabular}{|c|c|c|}
\hline Role & Program objective & Assessments \\
\hline Advocate & $\begin{array}{l}\text { - Recognize and respond to a patient's health needs by advocating } \\
\text { for the patient within and beyond the clinical environment } \\
\text { - Recognize and respond to societal factors that impact the } \\
\text { health of communities and populations }\end{array}$ & $\begin{array}{l}\text { Rush Medical College (RMC)-developed MCQs and short answer } \\
\text { questions, workplace-based student performance assessment }\end{array}$ \\
\hline Collaborator & $\begin{array}{l}\text { - Work with colleagues to promote mutual understanding, } \\
\text { manage differences, and resolve conflicts } \\
\text { - Coordinate patient care through participation on intra- and } \\
\text { interprofessional teams }\end{array}$ & $\begin{array}{l}\text { Narrative evaluations, workplace-based student performance } \\
\text { assessment }\end{array}$ \\
\hline Communicator & $\begin{array}{l}\text { - Establish professional therapeutic relationships with patients } \\
\text { and their families } \\
\text { - Engage patients and families in developing and implementing } \\
\text { treatment plans that reflect their needs and goals }\end{array}$ & $\begin{array}{l}\text { Communication skills lab, objective structured clinical } \\
\text { examination (OSCE), clinical skills assessment, narrative } \\
\text { assessment, workplace-based student performance assessment, } \\
\text { RMC-developed MCQs and fill-in-the-blank questions, narrative } \\
\text { evaluations }\end{array}$ \\
\hline Educator & $\begin{array}{l}\text { - Establish self-directed learning practices to continually monitor for } \\
\text { and address gaps in skills and knowledge throughout one's career } \\
\text { - Conduct an educational activity }\end{array}$ & $\begin{array}{l}\text { Written reflection and feedback, peer teaching, participation, } \\
\text { experience logs, workplace-based student performance } \\
\text { assessment }\end{array}$ \\
\hline Leader & $\begin{array}{l}\text { Contribute to the improvement of health care delivery in teams, } \\
\text { organizations, and systems } \\
\text { - Organize and lead a team to enhance success }\end{array}$ & $\begin{array}{l}\text { RMC-developed MCQS and fill-in-the-blank questions, } \\
\text { workplace-based student performance assessment }\end{array}$ \\
\hline $\begin{array}{l}\text { Medical } \\
\text { knowledge }\end{array}$ & $\begin{array}{l}\text { - Use medical knowledge foundation skills that are critical to } \\
\text { perform the various roles of a physician }\end{array}$ & $\begin{array}{l}\text { RMC-developed MCQs and fill-in-the-blank questions, anatomy } \\
\text { and histology lab practical, NBME Comprehensive Basic Science } \\
\text { Self-Assessment, NBME Clinical Science Subject Exams, NBME } \\
\text { Mastery Series, externally developed MCQs (i.e., UWorld, } \\
\text { Osmosis), workplace-based student performance assessment }\end{array}$ \\
\hline Practitioner & $\begin{array}{l}\text { - Gather a history and perform a physical exam } \\
\text { - Create and prioritize a differential diagnosis } \\
\text { - Create and implement a treatment plan } \\
\text { - Summarize and share a clinical assessment and management } \\
\text { plan }\end{array}$ & $\begin{array}{l}\text { Physical exam lab, OSCE, clinical skills assessment, observation- } \\
\text { based assessments (e.g., mini-CEX, mental status exam), patient } \\
\text { logs, RMC-developed MCQs and fill-in-the-blank questions, } \\
\text { workplace-based student performance assessment }\end{array}$ \\
\hline Professional & $\begin{array}{l}\text { - Act in accordance with the professional conduct, legal, and } \\
\text { ethical standards expected of the medical professional } \\
\text { - Develop your professional identity as a physician } \\
\text { - Promote the emotional, physical, and spiritual elements needed } \\
\text { to maintain personal well-being in the service of one's self, } \\
\text { colleagues, and practice }\end{array}$ & $\begin{array}{l}\text { Workplace-based student performance assessment, RMC- } \\
\text { developed MCQs and fill-in-the-blank questions, narrative } \\
\text { evaluations }\end{array}$ \\
\hline Scholar & $\begin{array}{l}\text { - Retrieve, appraise, and apply valid evidence to answer a } \\
\text { question about patient care } \\
\text { - Design a research study to address a gap in the medical } \\
\text { literature }\end{array}$ & $\begin{array}{l}\text { Evidence-based medicine case reports, RMC-developed MCOCS } \\
\text { and fill-in-the-blank questions, oral presentations, written } \\
\text { assignments, workplace-based student performance assessment }\end{array}$ \\
\hline
\end{tabular}

\section{Curricular Governance}

The responsibilities of the Committee on Curriculum and Evaluation (CCE) Executive Committee, subcommittees, workgroups, and advisory bodies are outlined below.

- The CCE Executive Committee is charged with developing and executing processes to ensure the curriculum is of the highest programmatic quality, measured by thoughtful design, effective implementation, critical evaluation, and continual revision.

- The Program Design Subcommittee seeks to answer the question "What curriculum do we, the faculty of RMC, want to teach our students?"
- The Implementation and Integration Subcommittee seeks to answer the question, "How do we most effectively and efficiently teach our defined curriculum?"

- The Program Evaluation Subcommittee seeks to answer the question, "Does our program successfully prepare students to be future physicians?”

- The Student Curriculum Advisory Committee advocates for students' well-being and academic success by presenting student perspectives regarding the preclerkship and clerkship curriculum content, implementation, and evaluation.

See Figure 2-Curricular governance. 
To achieve their respective responsibilities, the subcommittees are supported by 3 workgroups-preclerkship workgroup, core clerkship workgroup, and fourth-year elective workgroup-each responsible for conducting comprehensive course reviews, monitoring comparability and distribution of time spent, and implementing action plans.

Curricular governance is centrally managed, in collaboration with clinical and basic science departments. This includes faculty compensation and administrative support for teaching, assessment, and program evaluation. Faculty management occurs primarily at the department level, in collaboration with central administration, with dual reporting for those faculty with teaching FTEs.

\section{Education Staff}

Administrative and academic support for UME is housed within the Office of Integrated Medical Education (OIME). This office integrates support for education across the educational continuum and collaborates closely with Rush University (RU) to assure that systems are integrated across the $4 \mathrm{RU}$ colleges. The OIME is led by the vice dean for education, with the senior associate dean for UME, the senior associate dean for diversity and inclusion, the associate dean for GME, and the associate dean for the learning environment all reporting to the vice dean. UME efforts under the senior associate dean include curriculum, assessment and program evaluation, admissions and recruitment, medical student affairs, and technology. The functions under medical student affairs include academic support, career exploration, and advising. Registrar and financial aid functions fall under RU student affairs.

A distinguishing feature of the OIME is that all facets of medical education are housed in the same physical space, facilitating our ability to truly integrate. A practical example of this is the Early Intervention Committee, which meets weekly to review students' academic progress, achieving integration of curricular, assessment, and student support processes and functions.

See Figure 3-Organizational chart.

\section{Faculty Development and Support in Education}

Professional development is a shared responsibility between RMC and RU. RU offers opportunities for all university faculty, including the Teaching Excellence Bootcamp and the Rush University Teaching Academy Series. Professional development efforts through the OIME are coordinated by the associate dean for the learning environment. The OIME presents monthly grand rounds sessions focusing on issues pertinent to both UME and graduate medical education. Rush Medical College sponsors a variety of specific initiatives, including an annual first- and secondyear clinician-educator boot camp and annual ambulatory preceptor workshop.

RMC has a single track for promotion and tenure, with 4 categories for promotion: research, education, clinical excellence, and service. Superior performance must be demonstrated in at least 2 areas for promotion to associate professor and 3 areas for promotion to professor. All candidates are expected to demonstrate evidence of scholarly productivity regardless of the categories chosen for promotion.

We are currently in the process of reimagining our teaching academy. The Lepper Society of Teachers was initially founded in 1986. Membership is an honor bestowed upon faculty members who have demonstrated significant contributions to medical education. Membership duties are being updated, with

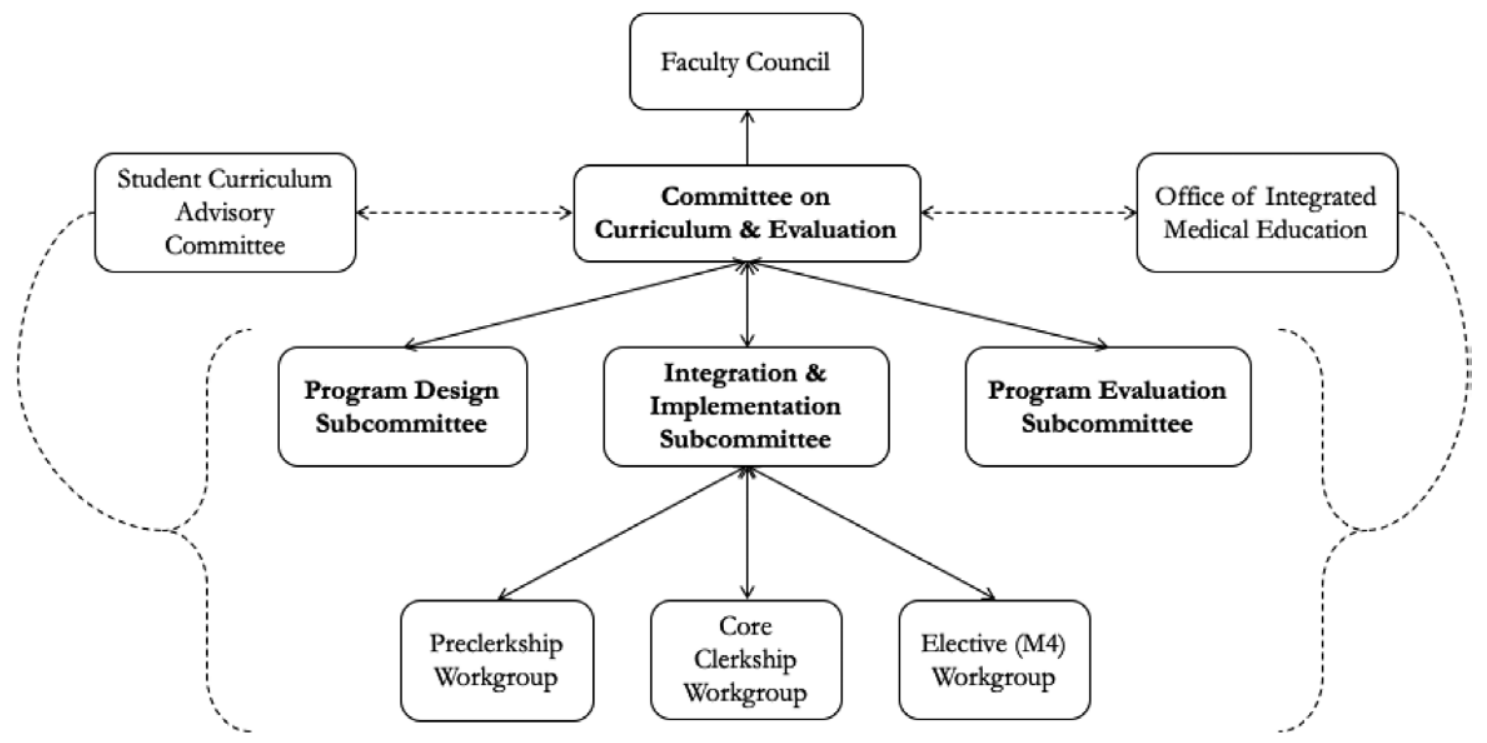

Figure 2 Curricular governance. 


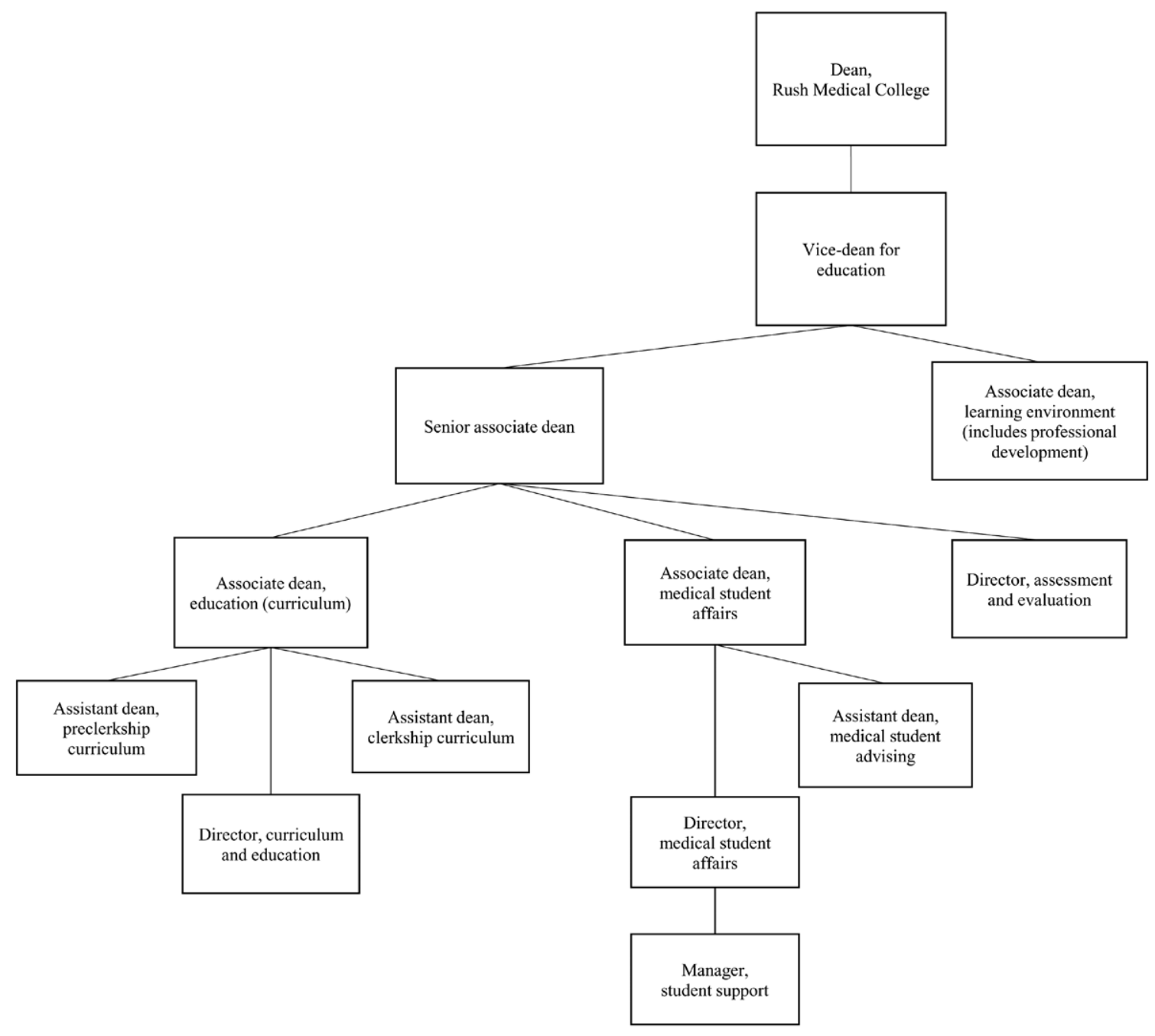

Figure 3 Organizational chart.

the expectation of participation in mentorship and coaching, educational innovation, and faculty development, with stipends allocated to cover the time and effort necessary to fulfill these obligations.

\section{Initiatives in Progress}

\section{Health equity and social justice leadership program}

Students come to Rush passionate about Rush's longstanding commitment to community engagement, service, and health equity. This new 4-year program empowers students to advocate for health equity throughout their careers. Moreover, it will enable RMC to develop future leaders with expertise in health equity and social justice. This program will be used as the model for developing programs specific to each of the 8 roles, with the goal of equipping all students with specialized skills that can be used throughout their careers.

\section{Third- and fourth-year longitudinal courses}

We are creating longitudinal courses to incorporate the role curriculum into the third and fourth years. Clerkship faculty are working with role leaders to craft content that can be applied to discipline-specific clinical scenarios and builds on the leadership, professional, communication, collaborative, clinical, advocacy, educational, and scholarly skills learned in the first 2 years. In addition to delivering the role curriculum, we will bring students together to maintain their class identity. Our overall goal is to have well-defined threads for the role curriculum throughout the 4-year experience.

\section{References}

1 Frank JR, Snell L, Sherbino J, eds. CanMEDS 2015 Physician Competency Framework. Ottawa, ON, Canada: Royal College of Physicians and Surgeons of Canada; 2015. http://www.royalcollege.ca/rcsite/documents/ canmeds/canmeds-full-framework-e.pdf. Accessed March 17, 2020.

2 Konopasek L, Norcini J, Krupat E. Focusing on the formative: Building an assessment system aimed at student growth and development. Acad Med. 2016;91:1492-1497. 


\section{Southern Illinois University School of Medicine}

Debra L. Klamen, MD, MHPE

\section{Medical Education Program Highlights}

Southern Illinois University School of Medicine (SIUSOM) was founded in 1970 and is a small, public, and community-based institution. Campuses are located in Springfield, Illinois, and in Carbondale, Illinois, 180 miles to the south. Since its inception, the school has been known for educational innovation and medical education research. It holds the record in the United States and Canada for the most International Association of Medical Education ASPIRE awards, with 5 in the areas of social accountability, student engagement, simulation, curriculum design/innovation, and assessment.

Highlights include:

- The use of nurse-educators (master's level in educationtrained) to work with medical students years 1-3.

- An innovative third-year clerkship consisting of 8-month-long clerkships with immersion in clinical settings and longitudinal faculty supervisors. There are no lectures or USMLE shelf exams. After the core rotations, students go on to do 15 weeks of a personalized education plan (PEP). They and their advisors choose from a variety of clinical rotations to round out the third year. Critical Clinical Competencies (CCC), an online, video-based, and interactive program, explicitly teaches students clinical reasoning from years 1 through $3 .^{1,2}$

- In 2019, SIUSOM adopted the use of programmatic assessment in the first year of medical school. This assessment system will be spread throughout the rest of the medical school, year-by-year. ${ }^{3}$

- The Center for Human and Organization Potential (cHOP) was launched in 2018. This center has 3 arms: wellness (including culinary and integrative medicine), professional development, and leadership and excellence. cHOP serves all members of SIUSOM, including community members, staff, students, residents, and faculty, in conjunction with other areas of medical education, including the Academy for Scholarship in Education.

\section{Curriculum}

\section{Assessment}

The SIUSOM's campus is split, with the first year occurring in Carbondale, Illinois (at the southern tip of the state), and years 2-4 in Springfield, Illinois (centrally located).

Acad Med. 2020;95:S164-S167.

doi: 10.1097/ACM.0000000000003291

Copyright (C) 2020 by the Association of American Medical Colleges

Supplemental digital content for this article is available at http://links.Iww.com/ ACADMED/A830.

Year school was established: 1972.

School URL: https://www.siumed.edu.
See Supplemental Digital Appendix 1-Curriculum Map—at http://links.lww.com/ACADMED/A830.

There have been several major changes in the curriculum. Several upcoming changes include:

- Expansion of programmatic assessment to include year 2 of the medical school.

- In the fall of 2020, SIUSOM will begin the Lincoln Scholars program. The purpose of this program is to train future physicians who will practice in rural counties of southern Illinois. Students will spend all 4 years in Carbondale. Eight students will be admitted and will train for 3-4 years in a new and innovative curriculum. They will take the first year of medical school with physician assistant (PA) students; rotate through clinical clerkships in year 2; and, if staying in a primary care residency in southern Illinois, may be able to graduate at the end of year 3 .

- Class size will increase in the fall of 2020, from the current 72 to 80 , with the addition of the Lincoln Scholars students.

\section{Medical education program objectives}

See Supplemental Digital Appendix 2-Medical Education Program Objectives and Assessment-at http://links.lww.com/ ACADMED/A830.

The school used a combination of published national and international recommendations to create its goals and objectives. SIUSOM regularly reviews the NBME's content list and LCME standards to assist in a quality improvement process to keep the curriculum up to date.

There have been several student assessment tools and processes that have changed in the last 10 years.

- The school has moved to a programmatic assessment model in year 1 of medical school, and this will be expanded to year 2 the following year.

- SIUSOM has had a senior clinical competency examination (SCCX) (a 14-station, uncued standardized patient examination that senior students must pass to graduate) for over 25 years. Once the new curriculum in year 3 was implemented (2015), the SCCX (now called the summative CCX) has been moved from the early months of year 4 to 8 months into year 3, right after the core clerkship rotations are completed.

- A new clinical reasoning examination, the diagnostic justification examination was implemented to assess clinical reasoning in years $1-3$. This exam asks students to justify their final diagnosis, by narrowing from a listing of their differential diagnosis and arguing through the use of positive and negative signs and symptoms the student collected during a standardized patient examination. ${ }^{4}$

- A clinical reasoning progress test, the longitudinal performance examination, using a diagnostic pattern matching and clinical 
data interpretation multiple choice test, was implemented throughout all 4 years of medical school in $2005 .^{5}$

\section{Parallel curriculum or tracks}

SIUSOM does not currently have parallel tracks, although the new Lincoln Scholars program is in development, which will train 8 medical students with their PA counterparts for a parallel 3- to 4-year curriculum, all conducted in Carbondale, Illinois.

We plan on offering the Lincoln Scholars program as well as our traditional medical school program in a $3+$ format in coming years. This means that students ending their third year would be assessed as ready (or not) for internship. Those deemed ready would graduate. Other students would continue on into their fourth year, but at the time they are deemed ready, they would move on to internship, even if that means at some time before the end of the academic year.

\section{Pedagogy}

SIUSOM uses a wide variety of pedagogy to educate its medical students. The first 2 years of the curriculum are hybrid, with a combination of small-group discussion, large-group discussion, problem-based learning (PBL), an ambulatory clinical experience, laboratory (histology and anatomy), lecture, peer teaching, preceptorships, role play, and self-directed learning video tutorials including virtual patients, simulation and standardized patients. All of these pedagogies continue into the third and fourth years of the curriculum (except for lectures and PBL) with the addition of inpatient and consult/liaison clinical experiences.

CCC online videos were added to the curriculum in 2015. These are interactive, case-based, clinical reasoning educational videos. For an example of the CCCs, please see: http://siucccdemo.org. SIUSOM also requires students to complete the Institute for Healthcare Improvement online curriculum. Please see: http:// www.ihi.org/education/Pages/default.aspx.

\section{Clinical experiences}

We use inpatient settings, ambulatory/outpatient settings (academic and community based), and consult/liaison settings. Most inpatient settings are located in our 2 community-based hospitals, Memorial Medical Center and St. John's Hospital, both located in downtown Springfield, Illinois.

In each of the first 2 years of medical school at SIUSOM, students are assigned one-to-one with a community-based outpatient physician, whom they will meet monthly for 12 months. In the third-year clerkships, students spend 4 weeks each in the following disciplines: emergency medicine, psychiatry, internal medicine, surgery, family and community medicine, obstetrics-gynecology, pediatrics, and neurology. During each of these rotations, a student is assigned a faculty preceptor, who will work with him/her for all 4 weeks. The clerkships are immersion based, so students spend 7.5 hours per day on their service, with no lectures or shelf exams to interfere. During the last 15 weeks of the third year, students design their PEP in conjunction with their clerkship advisors; several options are available for 15-week clinical experiences.
Our students encounter their first patients (both real and standardized) in their first week of year 1 of the medical school curriculum.

Students are assigned community-based preceptors (including those working at the Marion, Illinois, Veterans Administration medical center) during years 1 and 2 (see above). In addition, during the third-year family and community medicine core clerkship, students are assigned to rotate in community-based family and community medicine clinics for 4 weeks. These communities are located across central and southern Illinois.

It is a long-standing challenge to find enough mentoring physicians for students in years 1 and 2 . Even though year 1 is in Carbondale, Illinois, and year 2 is in Springfield, Illinois, it is nevertheless a challenge to find $72-80$ preceptors at each site yearly. It is likewise a challenge to find a preceptor for each student in individual core clerkship rotations (neurology, psychiatry, pediatrics, etc.) since faculty numbers at SIUSOM are small and clinicians rotate frequently. Finally, during the PEP selectives at the latter part of third year, it is difficult sometimes to find enough specialists to satisfy the requests by students for those rotations in the PEP period.

\section{Curricular Governance}

All parts of SIUSOM's curricular governance are centralized. Individual departments do not receive separate budgets for teaching, although an educational component (educational scholarship/teaching) is included in most faculty's job descriptions. The Department of Medical Education (DME) is specifically funded to provide faculty development in education across all disciplines.

\section{Education Staff}

The Office of Education and Curriculum (OEC) is responsible for the support of all undergraduate medical student education. As such, it works with faculty and faculty committees, as they create new curricula, to implement and manage them. Some examples of functions the OEC provides are as follows:

- Recruit, hire, train, and maintain a pool of standardized patients for use throughout the curriculum as a whole

- Maintenance of information technology and software used for educational purposes.

- Support of all curriculum committees

- Classroom scheduling and logistical management of the curriculum

The OEC provides a significant stipend for the years 1-4 directors as well as the Doctoring course directors. The OEC is the home of the nurse-educators, mentioned previously. The OEC is directed by the senior associate dean for education and curriculum, who also serves as the chair of the DME, providing a useful linkage between the two.

Those involved in UME, faculty development, and learner support include: 
- Dean and provost of the college of medicine

- Senior associate dean for education and curriculum

- DME

- Associate deans for equity and diversity, student affairs, and $\mathrm{cHOP}$

The OEC is responsible for the undergraduate medical education area of the educational curriculum only.

The SIUSOM has the DME, which provides ongoing educational development for faculty. Members of the DME sit on every curriculum committee. They work with a wide variety of faculty and staff to assist in curriculum development, student and program evaluation, and medical education research.

See Figure 1-Organizational chart.

\section{Faculty Development and Support in Education}

There are numerous avenues by which faculty can receive professional development in education and educational scholarship. DME faculty meet with those interested in educational research as requested. They provide a wide array of workshops to improve teaching skills. The new cHOP works with DME faculty to provide faculty, staff, community, and learner development in the areas of wellness/resilience, culinary medicine, integrative medicine, educational professional development, and leadership and excellence.

In matters of promotion and tenure, any faculty member cannot be promoted if he/she does not have a ranking of at least "sufficient" in the teaching/educational scholarship arena. Educational scholarship criteria are posted on the school's promotion and tenure webpage. See http://www.siumed.edu/ hop/policies/promotion-tenure-guidelines-2018.html.

SIUSOM is home to the Academy for Teaching and Learning, which is open to faculty and other health care providers. The academy has 2 tiers: the first for those who state their wish to join the academy, and the second for those who have demonstrated excellence in educational scholarship in any one or more of the following categories: development of enduring educational materials, educational leadership, assessment, curriculum development, or teaching.

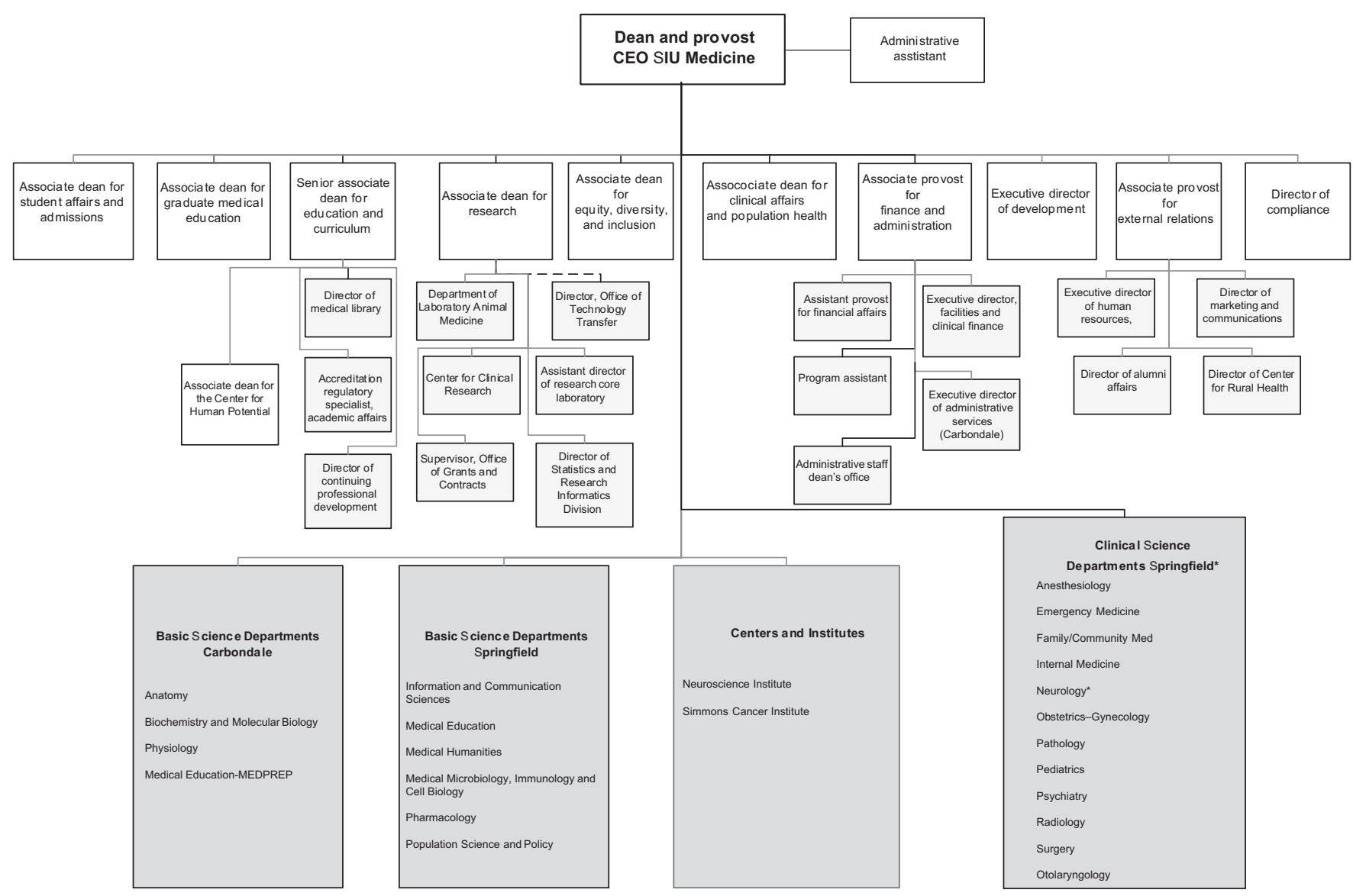

${ }^{*}$ Center for Alzheimer Disease and Related Disorders reports to the Department of Neurology.

Figure 1 Organizational chart. 


\section{Table 1}

\section{Regional Medical Campuses}

\begin{tabular}{|c|c|c|c|}
\hline Regional campus name & Type & Student enrollment & Comments \\
\hline \multirow[t]{2}{*}{ Carbondale, Illinois } & Traditional year 1 & 72 & All first-year students \\
\hline & Lincoln Scholars & $8+$ & To begin fall 2020 \\
\hline Springfield, Illinois & Traditional years $2-4$ & $\sim 226$ & All second-, third-, and fourth-year students \\
\hline
\end{tabular}

\section{Regional Medical Campuses}

See Table 1-Regional Medical Campuses.

\section{References}

1 Klamen DL. Getting real: Embracing the conditions of the third-year clerkship and reimagining the curriculum to enable deliberate practice. Acad Med. 2015;90:1314-1317.
2 Klamen DL, Williams R, Hingle S. Getting real: Aligning the learning needs of clerkship students with the current clinical environment. Acad Med. 2019;94:53-58.

3 van der Vleuten CP, Schuwirth LW, Driessen EW, et al. A model for programmatic assessment fit for purpose. Med Teach. 2012;34:205-214.

4 Williams RG, Klamen DL. Examining the diagnostic justification abilities of fourth-year medical students. Acad Med. 2012;87:1008-1014.

5 Williams RG, Klamen DL, Hoffman RM. Medical student acquisition of clinical working knowledge. Teach Learn Med. 2008;20:5-10. 


\title{
The University of Chicago Pritzker School of Medicine
}

\author{
Jeanne M. Farnan, MD, MHPE, H. Barrett Fromme, MD, MHPE, and Halina Brukner, MD
}

\section{Medical Education Program Highlights}

The University of Chicago matriculated its first class of medical students in 1927 and continues to serve as a leader in training physicians and scientists. The great traditions that underlie our school's history include a full-time teaching faculty, a strong emphasis on research and translating biomedical discoveries to the bedside, and stalwart commitment to outstanding care of individual patients and communities.

The University of Chicago is committed to training future leaders who will contribute broadly to society. This commitment is reflected in the class composition, which is highly diverse in racial and ethnic background, socioeconomic status, geographic origin, age, identity, undergraduate and graduate institution, and field of study. A typical class will represent 35 different home states with approximately $30 \%$ of recent classes consisting of individuals who are underrepresented in medicine. Matriculants will have completed over 40 different undergraduate majors and about half will have taken one or more years off prior to medical school to work, volunteer, or pursue another graduate degree.

The curriculum at the University of Chicago Pritzker School of Medicine (PSOM) includes several unique features. In keeping with the great tradition of scholarly work, the longitudinal Scholarship and Discovery Program teaches students to develop the necessary skills to complete a rigorous mentored scholarly project by the time of graduation, focusing on 1 of 5 tracks: scientific investigation (basic science, clinical research, and social sciences), community health, global health, health care delivery sciences, or medical education. Another highlight includes the personal and professional development thread, a longitudinal program that includes career exploration and advising, student leadership development, practical skills to maintain resilience, and a focus on inclusivity with training in civil discourse. Finally, students participate in early clinical experiences that are anchored in longitudinal, interprofessional teams. Working alongside colleagues from nursing, pharmacy, physical therapy, and other health professions, students learn critical teamwork skills, as well as scope of practice of these disciplines, to provide patient-centered care.

Acad Med. 2020;95:S168-S170.

doi: 10.1097/ACM.0000000000003334

Copyright (C) 2020 by the Association of American Medical Colleges

Supplemental digital content for this article is available at http://links.Iww.com/ ACADMED/A857.

Year school was established: 1927.

School URL: https://pritzker.uchicago.edu.

\section{Curriculum}

Curriculum description

See Supplemental Digital Appendix 1-Curriculum Map-at http://links.lww.com/ACADMED/A857.

\section{Curriculum changes since 2010}

Since the implementation of the Pritzker Initiative Curriculum in 2009, the curriculum has evolved in response to student feedback, continuous quality improvement efforts, a changing medical education landscape, and new opportunities. Through our participation in the American Medical Association ChangeMed Consortium, we implemented the VISTA (Value, Improvement, Safety, Team Training, and Advocacy) curriculum, a longitudinal thread focused on training in health care delivery science. In the preclinical curriculum, instructional methodologies continue to emphasize case-based learning. Other discrete changes include re-sequencing of specific courses to enhance opportunities for self-directed learning and cocurricular activities as well as the implementation of a longitudinal curriculum in interprofessional education. We have increased opportunities for elective time during the clinical years for career exploration and professional development. Finally, there are increased opportunities for students to focus on postclerkship skills and preparedness for transitioning to residency.

\section{Assessment}

The medical education program objectives are derived from the Physician Competency Reference Set and are reviewed and revised annually. Since our most recent curricular renewal, our focus has been to diversify and increase the frequency of student assessment to make informed decisions regarding the attainment of competence in a domain. Preclinical students receive multiple formative assessments in the form of institutionally developed written and laboratory practical examinations, as well as multisource assessment from peers, peer educators, standardized patients, and faculty. The clinical clerkship years include clinical documentation review as well as standardized observed clinical encounters and oral patient presentations.

See Supplemental Digital Appendix 2-Program Objectives and Assessment Methods—at http://links.lww.com/ACADMED/ A857.

\section{Pedagogy}

The University of Chicago PSOM employs multimodal instructional strategies to ensure learners of all types are able to effectively engage with the curriculum. The scientific foundations of medicine use interactive large-group teaching, case-based learning, and laboratory instruction. The longitudinal program and clinical skills rely upon ambulatory and inpatient clinical experiences, simulation, and standardized patients. 
Preceptorships and peer teaching facilitate a smooth transition to the clerkships and postclerkship year, where students use clinical experiences, small-group discussion, and case-based learning to reframe basic science knowledge in the context of bedside care, as well as perform career and scholarly exploration. Finally, time for independent study throughout the curriculum provides the opportunity for self-directed, lifelong learning skills.

\section{Clinical experiences}

The University of Chicago PSOM utilizes 2 main sites for inpatient clinical instruction: the University of Chicago Medicine, a tertiary care academic medical center, and NorthShore University HealthSystem, our primary teaching affiliate, an integrated health system serving patients throughout the Chicago area. Ambulatory experiences take place in various community-based clinics, including federally qualified health centers on the South Side of Chicago.

Students experience significant actual and standardized patient encounters early in their time at PSOM, performing more than a dozen standardized encounters in their first year alone. In addition, students have a 10 -week faculty preceptor experience in which they refine oral case presentation skills in preparation for a seamless transition to the clinical wards. Students participate in their first required clinical experience in the fall of their first year when resident preceptors introduce them to the hospital environment and provide opportunities for history-taking practice, as well as feedback. Students at PSOM also participate in a longitudinal clinical experience throughout their first year entitled the Longitudinal Program and are required to participate in a quarterly longitudinal, interprofessional experience where they are immersed in the clinical experiences alongside physical and occupational therapy, nursing, pharmacy, speech and language pathology, and nutrition. This interprofessional experience continues in the subsequent curricular years, consistent with students' growing clinical capabilities.

Required and elective community-based rotations occur at one of several affiliated family medicine clinics throughout Chicago and the surrounding area. Students may be assigned to any of more than 30 sites that serve diverse populations, from Spanishspeaking only to primarily Asian American communities, as well as other clinical partners such as the Howard Brown Center for Lesbian, Gay, Bisexual, and Transgender Community.

\section{Curricular Governance}

The Education Executive Committee (EEC), chaired by the dean for medical education and reporting to the dean of the division of the biological sciences and PSOM, is responsible for making and endorsing educational policy, oversight of curriculum review committees, integration with graduate medical education, and other aspects of the Biological Sciences Division. The EEC has responsibility for all aspects of the medical educational program, including the medical school, Medical Scientist Training

Program, admissions, and student affairs policies.
The Curriculum Steering Committee (CSC), chaired by the associate dean for evaluation and continuous quality improvement, is the committee responsible for reviewing curricular policy and making recommendations to the curricular subcommittees. The CSC comprises educational leadership, including the chairs of the Preclinical Curriculum Review Committee (PCRC) and the Clinical Curriculum Review Committee (CCRC), educational leadership from our teaching affiliate, and student representation. The CSC performs an annual review of the curriculum, including key process indicators such as pertinent LCME-related elements and national benchmark data from the AAMC. The PCRC is composed of preclinical course leadership, administrative staff, and elected student curriculum representatives. It meets quarterly to perform course reviews and targeted faculty development. The CCRC meets every other month and includes all clerkship directors, as well as administrative staff, student representatives, and the director of fourth-year studies. The CCRC discusses clerkship ratings quarterly, as well as pertinent clerkship metrics such as student mistreatment, midclerkship feedback, and observation of history and physical examination skills. The curriculum at the University of Chicago PSOM is governed centrally through a robust oversight structure, described above.

\section{Education Staff}

The Medical School Education Team consists of the executive director of medical school education and assistant director of medical school education, who oversee operations, including planning, implementation, and oversight of the Curriculum Team staff. These include the registrar, who maintains student records and manages visiting students; the manager of medical education, who supports faculty professional development in medical education; the assistant manager of scholarly activities, who supports the scholarship and discovery curricular thread; 2 curriculum management specialists, who provide technical support and maintain the learning management system as well as evaluation delivery, analysis, and curriculum mapping; the senior programmer analyst and learning technology specialist, who oversee and manage the medical education data warehouse; the clinical performance center and standardized patient program team; and the medical school education administrator, who maintains schedules and provides administrative support to the executive administrative leadership.

The dean for medical education is responsible for the oversight of the medical education continuum that includes undergraduate, graduate, and continuing medical education, as well as simulation. Each part of the continuum is supported by its own faculty leaders and administrative staff. Within the medical school, the medical education staff oversees and supports the curriculum and academic support for students. Separate staff groups support student affairs, admissions, financial aid, and diversity and inclusion.

See Figure 1-Organizational leadership chart. 


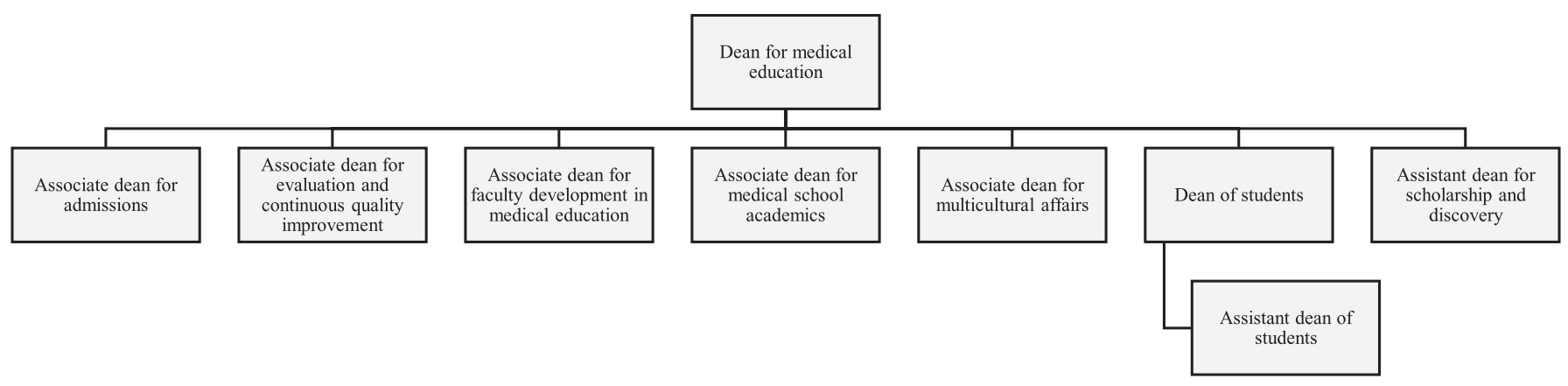

Figure 1 Organizational leadership chart.

\section{Faculty Development and Support in Education}

PSOM has long invested in the development and recognition of educators and teachers. In 2019, PSOM created the inaugural position of associate dean for faculty development in medical education, whose role is to oversee all efforts in this area, which include the programs described below.

The Academy of Distinguished Medical Educators (ADME) is our educator academy, currently in its second decade. The ADME is an honorific academy with a service component, with 2 levels of membership - fellow and master-all of who are faculty. Fellows have a 5-year renewable term, and masters are elected for life. The mission of the ADME is to promote excellence in teaching at PSOM; support scholarship among medical educators; enhance the PSOM curriculum by supporting, recognizing, and rewarding outstanding teachers; build a community among medical educators; and facilitate the creation of an environment that enhances the status of medical educators at the University of Chicago.

The ADME supports numerous programs to fulfill its mission, including the Faculty Advancing Medical Education (FAME) Program and Teaching Consultation Program. In addition, it hosts an annual medical education day event that includes a nationally known keynote speaker, a workshop-based retreat for clerkship and program directors, a poster session of medical education scholarship, plenary presentations, and induction of new fellows and masters into the ADME. The ADME also sponsors 2 grants annually to support medical education scholarship.
FAME is the premier program at PSOM for developing teachers. The program offers workshops 3 times per year to all faculty at Pritzker and NorthShore University Health System. We also offer "targeted FAME" sessions, coordinated with departments and tailored to specific faculty development needs. Most recently, we have offered SNIPPETS, 20-minute skills-focused sessions incorporated into standing departmental meetings.

Individual coaching is as important for personal growth, which is why we offer the Teaching Consultation Service. This model peer observation program is offered to all faculty and offers 1-on-1 observation of teaching in clinical, small-group, and large-group sessions. Consultants are trained members of the academy who pre-brief with participants regarding their goals, observe actual teaching sessions, and debrief performance.

To develop medical education scholars, we offer the Medical Education Research Innovation Teaching and Scholarship (MERITS) fellowship program. This is a 1-year program that consists of didactic sessions on curriculum development, scholarship in medical education, and the theory and practice of teaching as well as mentored projects. A GME-specific MERITS program is also offered to residents across programs who are interested in medical education. We encourage the sharing of scholarship through the monthly Research in Medical Education lecture series that allow faculty, staff, and trainees to present their medical education projects to the larger medical education community. 


\section{The University of Illinois College of Medicine}

Hugh Clements-Jewery, PhD, Jessica R. Hanks, MD, Amy Y. Lin, MD, Sandra Yingling, PhD, and Raymond H. Curry, MD

\section{Medical Education Program Highlights}

The University of Illinois College of Medicine is among the largest and most diverse medical schools in the United States. One of 7 health sciences colleges of the University of Illinois at Chicago (which itself is 1 of 3 universities in the University of Illinois system), its 4 campuses (Chicago, Peoria, Rockford, Urbana) have contributed to training over $40 \%$ of physicians currently licensed to practice in Illinois, including 20\% who are graduates of the MD degree program. The college is among the top 3 LCME-accredited schools in graduating both Latino/ Latina and African American physicians over the last 4 decades. The university's Urban Health Program (established 1978) and the college's Hispanic Center of Excellence (established 1991) are mainstays of this commitment to diversity and inclusion.

The college offers a broad range of career pathways. The Medical Scientist Training Program (Chicago) and the Medical Scholars Program (Urbana) provide MD-PhD training; other dualdegree programs and scholarly concentration programs (elective longitudinal opportunities focusing on global medicine, urban medicine, and several other areas) are also available. The Rural Medicine Program enrolls approximately half the Rockford-based students and has contributed significantly to alleviating physician shortages in rural Illinois, as has the Rural Student Preceptor Program, a longitudinal integrated clerkship option in Peoria.

The MD program has been comprehensively redesigned, with the new curriculum debuting in 2017. This was in conjunction with a major restructuring of the college, expanding the programs in Peoria and Rockford to include all 4 years of the curriculum and phasing out the Urbana campus, which will close in 2022. Concomitant restructuring of the curriculum and the college structure have allowed better assurance of comparable educational experiences; streamlining administrative mechanisms for curricular management; and furtherance of our identity as a single, unified educational endeavor with statewide impact.

\section{Curriculum}

The Illinois Medicine Curriculum is a single, integrated curriculum for the Chicago, Peoria, and Rockford campuses, aligning all major aspects of course and clerkship structure, scheduling, and assessment and emphasizing interdisciplinary integration, active learning, and longitudinal assessment

Acad Med. 2020;95:S171-S174.

doi: 10.1097/ACM.0000000000003367

Copyright (C) 2020 by the Association of American Medical Colleges

Supplemental digital content for this article is available at http://links.Iww.com/ ACADMED/A879.

Year school was established: 1881.

School URL: https://medicine.uic.edu. strategies. All content is organized around 5 themes: foundational knowledge; clinical practice; health, illness, and society; health care systems; and professional development.

The curriculum is presented in 3 phases. Phase 1 consists of 7 consecutive organ-based block courses between 5 and 10 weeks in duration that integrate normal structure and function with disease processes and 3 concurrent longitudinal courses: Doctoring and Clinical Skills (DoCS), Synthesis, and Medical Colloquia. The DoCS course is the primary vehicle for the clinical practice theme. The Synthesis course comprises single weeks that follow each block course, with a capstone segment in the final Phase 1 term. The Synthesis week following a block course provides opportunities for reflection on learning progress and strategies, cumulative integration of prior learning, and engagement in professional development theme sessions with faculty; the capstone segment fosters overall integration and consolidation and a 2-week course, Transition To Clerkships, then completes Phase 1. Medical Colloquia are selective opportunities to explore a variety of presentations, seminars, and workshops extending beyond the scope of the core curriculum and incorporating perspectives from patients, community leaders, and other professions.

See Supplemental Digital Appendix 1-Phase 1 Curriculum-at http://links.lww.com/ACADMED/A879.

Phase 2 begins in May of the second year; 44 weeks of core clerkships (in medicine, surgery, family medicine, pediatrics, obstetrics-gynecology, psychiatry, and neurology) are completed within the first four 16-week clinical terms. Phase 3 requirements include a 4-week subinternship and 12 weeks of selectives in either a medical or surgical "pathway." A third pathway is for students in the Rural Medicine Program. During Phases 2 and 3,24 weeks of elective credits are also required, with flexibility regarding timing. A longitudinal Phase 2/3 course, Clinical Connections and Competencies, consists of a series of small-group sessions and asynchronous online activities that give context and meaning to students' clinical experiences, opportunities to practice and receive feedback on clinical and procedural skills, and a 2 -week transition to residency capstone.

See Figure 1-Phase and term structure.

The college of medicine is a multicampus college and curriculum. The college has encompassed 4 campuses since the early 1970s, when regional sites in Peoria, Rockford, and Urbana were created through an initiative in the state legislature. The Urbana campus ceased enrollment of new students in 2015 to accommodate the formation of the new Carle Illinois College of Medicine by our sister university in Urbana-Champaign. Our last Urbana-based $\mathrm{MD}-\mathrm{PhD}$ students are expected to graduate in 2022. 


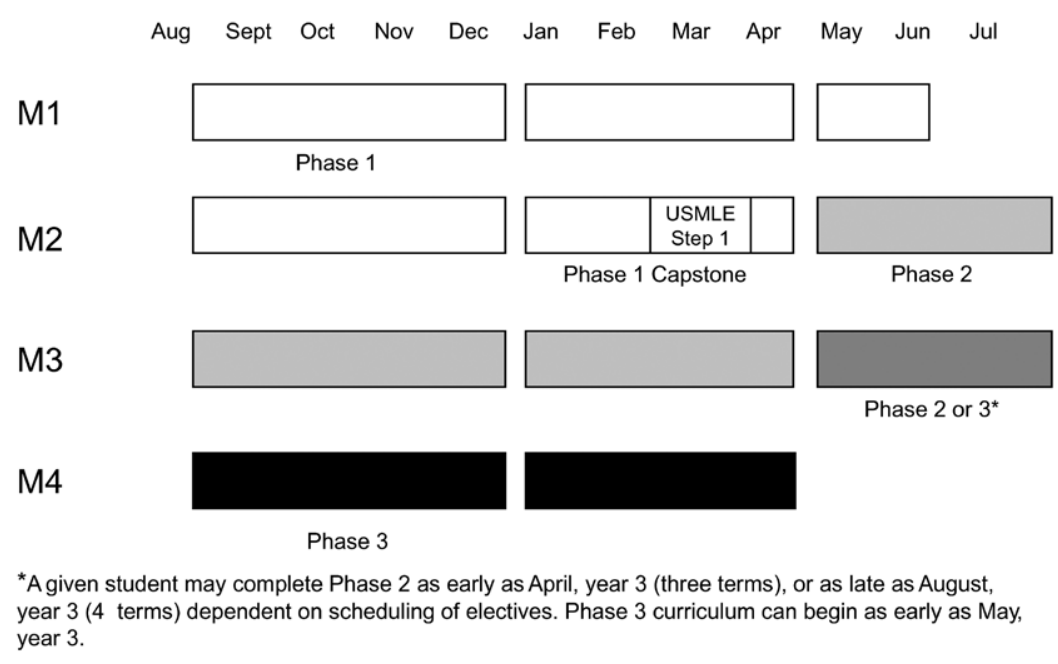

Figure 1 Phase and term structure.

See Table 1-Student Enrollment by Campus.

Our new curriculum's management structure ensures a consistent educational experience across the campuses. The College Committee on Instruction and Appraisal (CCIA) is proportionally representative of all campuses, and oversees subcommittees that provide both campus-specific management of curricular implementation and central authority for policy approval and program evaluation. Each Phase 1 course is led by a team of basic science and clinical faculty from each campus, and clerkships are linked across campuses by Education Coordinating Committees (ECCs) that plan and review students' educational experiences across all affiliates and all campuses. A Phase 1 ECC and Phase 2/3 ECC evaluate the coordination of courses within each phase on behalf of the CCIA.

The Office of Educational Affairs, part of the central dean's administration, evaluates comparability during each block course through weekly assessment performance review and interval evaluations by students. This office also produces course-level and phase-level program evaluation and performance data for review by subcommittees of the CCIA.

\section{Table 1}

\section{Student Enrollment by Campus}

\begin{tabular}{|c|c|c|c|}
\hline Campus & Type & Curriculum & $\begin{array}{l}\text { Enrollment } \\
\text { AY 2019-2020 }\end{array}$ \\
\hline Chicago & $\begin{array}{l}\text { Entire MD } \\
\text { program }\end{array}$ & $\begin{array}{l}\text { Illinois Medicine } \\
\text { curriculum }\end{array}$ & 802 \\
\hline Peoria & $\begin{array}{l}\text { Entire MD } \\
\text { program }\end{array}$ & $\begin{array}{l}\text { illinois Medicine } \\
\text { curriculum }\end{array}$ & 249 \\
\hline Rockford & $\begin{array}{l}\text { Entire MD } \\
\text { program }\end{array}$ & $\begin{array}{l}\text { illinois Medicine } \\
\text { curriculum }\end{array}$ & 229 \\
\hline Ürbana & \multicolumn{2}{|c|}{$\begin{array}{l}\text { A parallel curriculum, the Urbana } \\
\text { Track, is now limited to third- and } \\
\text { fourth-year students as the campus } \\
\text { prepares for closure }\end{array}$} & 37 \\
\hline
\end{tabular}

\section{Medical education program objectives}

The program objectives are based on the Physician Competency Reference Set competency domains. Our curriculum is competency based but not time variable; we do not plan to introduce curricular options spanning fewer than 4 academic years.

In assessing student progress, we emphasize 3 approaches in particular: professional development, learning as a team, and assessment for learning. Our approach to professional development and its assessment has been informed by participation in the AAMC Core Entrustable Professional Activities Pilot Project. Early in the curriculum, we focus on professional identity formation and its role in the development of "entrustability" (discernment, conscientiousness, and truthfulness). In all 3 phases, evaluations of students are based on direct observation, with increasing expectations of professionalism in the clinical environment.

Classroom activities in Phase 1 promote learning as a team. Students work in teams of 6 in team-based learning (TBL) sessions and other case-based learning formats. The block, DoCS, and Synthesis courses maintain stable teams throughout the academic year. Participation in peer evaluation is an element of the grade in block courses and in DoCS.

Many assessment activities are low stakes, designed to instill habits of self-regulated learning and an appreciation of the skills of assessment for learning. These include students' development of a focused learning plan before each block's final exam, customized NBME examinations reflecting block course content, and access to a commercial adaptive learning platform that is integrated with curricular resource materials. Students receive individualized criterion-based and narrative feedback from standardized patients in a sequence of clinical simulations, beginning in Phase 1; they must also demonstrate competence in selected performance-based assessments before beginning the Phase 2 clerkships and again in Phases $2 / 3$ as a graduation requirement. 
All Phase 1 courses are graded on a pass/fail basis; clerkship grades are reported with 3 passing tiers or as fail.

See Table 2-Program Objectives and Assessment Methods.

\section{Pedagogy}

Throughout the curriculum, we have a consistent pedagogical emphasis on active learning; assessment for learning;

knowledge application; and approaches that develop teamwork, communication, and reflective practice skills.

A series of core clinical cases forms the backbone of the Phase 1 block courses, providing clinical context for integrating foundational knowledge and the other curricular themes. Each week, 1 to 3 core case sessions focus on a specific disease or symptom; some sessions are in TBL format. Students work in groups of 6 , with multiple teams led by a faculty facilitator and additional faculty with specific content expertise. Students prepare for sessions with assigned readings, videos, and other resources and work through the case with their team. TBL is also being incorporated into some Phase 2 and 3 clerkships.

These small-group experiences are coupled with lectures and with laboratory work, including cadaver dissection.
Several avenues for reflective practice are also incorporated throughout the curriculum. In Phase 1, these include pre- and postexamination learning plans and narrative writing about students' medical school experiences to further their professional development. Clinical simulation is used in the DoCS course as well as in Phases 2 and 3 for both formative and summative assessment purposes.

\section{Clinical experiences}

The DoCS course introduces students to both ambulatory and hospitalized patients starting in the first term of the first year and integrates these experiences into the work of faculty-facilitated small groups throughout Phase 1. Each Peoria DoCS student is now embedded in a single community-based setting for the entirety of Phase 1; a pilot project in Chicago is providing a 4-year longitudinal team-based experience for selected students.

There is no university hospital in Peoria or Rockford; however, each campus has built strong relationships with the teaching hospitals and physicians in its community over the last halfcentury. In Chicago, the University of Illinois Hospitals and Clinics and the Jesse Brown VA Medical Center are located on campus; a wide variety of other clinical affiliates also participate

\section{Table 2}

\section{Program Objectives and Assessment Methods}

\section{Medical education program objectives}

Patient care: Provide patient-centered care that is compassionate, appropriate, and effective for the treatment of health problems and the promotion of health

Knowledge for practice: Demonstrate knowledge of established and evolving biomedical, clinical, epidemiological, and social-behavioral sciences, as well as the application of this knowledge to patient care

Practice-based learning and improvement: Demonstrate the ability to investigate and evaluate one's care of patients, to appraise and assimilate scientific evidence, and to continuously improve patient care based on constant self-evaluation and lifelong learning

Interpersonal and communication skills: Demonstrate interpersonal and communication skills that result in the effective exchange of information and collaboration with patients, their families, and health professionals Professionalism: Demonstrate a commitment to carrying out professional responsibilities and an adherence to ethical principles

Systems-based practice: Demonstrate an awareness of and responsiveness to the larger context and system of health care, as well as the ability to call effectively on other resources in the system to provide optimal health care

Interprofessional collaboration: Demonstrate the ability to engage in an interprofessional team in a manner that optimizes safe, effective patientand population-centered care

Personal and professional development: Demonstrate the qualities required to sustain lifelong personal and professional growth

\section{Assessment methods}

Formative and summative simulation experiences with feedback from faculty and standardized patients; direct observation of clinical skills in clinical settings; clerkship and subinternship evaluations; assignments related to health care systems and health, illness, and society themes; Step 2 CS

TBL assessments (IRAT and TRAT) of clinical cases; weekly quiz and final exam items mapped to USMLE domains and subdomains; narrative writing assignments; ungraded NBME exams; NBME subject exams in clerkships; Step 1 and Step 2 CK

Evidence-based medicine activities and assessments; weekly quiz and final exam items; preceptor evaluations in longitudinal clinical skills course (DoCS); reflective writing assignments in Medical Colloquia; clerkship and subinternship evaluations

Formative and summative simulation experiences with faculty and standardized patient feedback; direct observation of clinical skills in clinical settings; clerkship and subinternship evaluations; Step 2 CS Ethics activities, weekly quiz and final exam items; faculty assessment of discernment, conscientiousness, honesty in DoCS courses, in clerkships and subinternship; CITI training; narrative writing about ethics in Synthesis and Clinical Connections and Competencies course

Evidence-based medicine and population health activities and assessments; Health Care Systems SAFER projects; clerkship and subinternship evaluations

Health, illness, and society activities and assessments, block 8 capstone community service learning assignment; clerkship and subinternship evaluations

Assessment of professional engagement in course activities, narrative assignments about experiential learning; clerkship and subinternship evaluations; peer feedback; professional development concerns and highlights documentation 
in student education. There is, however, considerable competition for clinical rotation opportunities from area medical schools, an osteopathic school, and schools from other states and countries.

\section{Curricular Governance}

Effective coordination of our multicampus curriculum requires a governance structure that creates a matrix of campus-level and institution-level authorities. The ECC infrastructure for each course, clerkship, and phase was described earlier. The ECCs serve as subcommittees of the CCIA, along with campus curriculum management committees that provide campusspecific oversight.

The administrative leadership of the MD program is also best described by a matrix structure. The regional deans in Peoria, Rockford, and Urbana report to the executive dean, and each regional administration includes an associate dean for academic affairs who directly manages curricular and student affairs. The campus associate deans also work together and in conjunction with the college-wide Office of Educational Affairs, led by the senior associate dean for educational affairs and associate dean for educational planning and quality improvement, and with the senior associate dean of students.

\section{Education Staff}

The Office of Educational Affairs houses the college's resources to support college-wide committees, including the CCIA, ECCs, and Accreditation Monitoring and Quality Improvement Committee. This office houses assessment and evaluation services and supports an enterprise-wide educational database, thus centralizing student assessment, program evaluation, and curriculum-related educational research. The senior associate dean of students oversees the admissions office and centralized student support services, including registration and records, financial aid and debt management, academic support, and an office attending to medical student learning environment. The majority of these areas are also represented on each regional campus by staff reporting primarily to campus leadership and secondarily to the collegewide leadership for that administrative function.

The senior associate dean for educational affairs also oversees continuing medical education and has direct responsibility for graduate medical education at the Chicago campus. The Peoria and Rockford campuses are independent ACGME sponsoring institutions, with primary responsibility borne by the regional dean.

\section{Department of Medical Education}

The Department of Medical Education is the oldest (1959) continuously operating academic unit in the world dedicated to innovation and scholarship in health professions education. Faculty teach and conduct research in the context of master's programs in health professions education and in patient safety leadership and a $\mathrm{PhD}$ program in curriculum studies. Department faculty are also active in the curriculum and, through international consultative services, have assisted in developing over 35 medical schools around the world.

\section{Faculty Development and Support in Education}

Faculty development has been essential to transformation from a primarily lecture-based curriculum to an emphasis on active learning methods. Faculty development at both the campus- and college-wide levels focuses on teaching methods, assessment, and use of educational technologies. Clinical departments receive financial support from central administration for faculty with significant roles in the preclerkship curriculum, particularly college-wide leadership roles. Both the individual and collaborative educational contributions of faculty are recognized for promotion. 


\section{Indiana University School of Medicine}

Bradley L. Allen, MD, PhD, Maureen A. Harrington, PhD, Jennifer Schwartz, MD, Paul Ko, MD, MEd, and Paul Wallach, MD

\section{Medical Education Program Highlights}

Indiana University School of Medicine (IUSM) is the largest MD-granting medical school in the United States, educating over 1,450 medical students across 9 statewide campuses. IUSM, established in 1903, has been a pioneer in regional medical education. Beginning with an expansion from Bloomington to Indianapolis in 1958, by 1971, IUSM expanded with 2-year campuses in South Bend, West Lafayette, Evansville, Fort Wayne, Gary, Muncie, and Terre Haute as directed by Indiana General Assembly legislation. Expansion to the entire 4-year curriculum occurred at all 9 campuses in 2008.

See Figure 1-Map of Indiana University School of Medicine campuses.

The competency-based curriculum, founded on the 6 ACGME competencies, expanded its capacity to address the projected statewide physician shortage by increasing the educational capacity starting in 2008 from a class size of 280 to its current level of 364 in 2016.

In 2013, IUSM received 1 of 11 American Medical Association Accelerating Change in Medical Education grants to develop and implement a teaching electronic medical record in its curriculum and to emphasize the role of health system sciences in improving health care quality.

In 2014, several structural changes occurred at IUSM. An office of continuous quality improvement was created to monitor LCME compliance and progress toward all strategic priorities. Medical student affairs and UME were consolidated into the unified Office of Medical Student Education to serve students' comprehensive needs.

To enhance comparability of the educational program across all 9 campuses, IUSM implemented a single horizontally and vertically integrated curriculum in 2016. The curriculum has students working with patients from the outset of their training and integrates foundational sciences with clinical education throughout the 4 years. Curriculum delivery is supported by a statewide system of course and clerkship directors with representation of faculty from all 9 campuses.

Acad Med. 2020;95:S175-S179.

doi: 10.1097/ACM.0000000000003335

Copyright (C) 2020 by the Association of American Medical Colleges

Supplemental digital content for this article is available at http://links.Iww.com/ ACADMED/A861.

Year school was established: 1903.

School URL: https://medicine.iu.edu.
See Figure 2-Course management structure.

Recognizing the highly interdisciplinary nature of health care, IUSM developed the IU Center for Interprofessional Practice and Education. The center administers IU's longitudinal interprofessional curriculum, Team Education Advancing Collaboration in Healthcare, in conjunction with multiple other health sciences schools statewide.

Along with curricular enhancements, IUSM has greatly expanded its mentoring and advising program. Thirteen full-time masters or doctoral degree educated lead advisors were recruited to work longitudinally with students from their first day of medical school. Lead advisor efforts are supplemented by faculty advisors and career mentors.

The school has invested in promoting diversity, improving wellness programs, and enhancing the teacher-learner environment and other student support services. IUSM and its partner institutions are making major investments to renovate and replace older education and research facilities in Indianapolis and statewide. To create more flexibility for personal self-care and wellness during the busy clinical phase, IUSM created Connection Days. One or 2 days exist between scheduled clerkship start and end dates for students to engage in student-initiated wellness activities, schedule appointments with advisors, or attend to personal needs.

\section{Curriculum changes since 2010}

After an extensive review process, input from faculty and students was used to develop a new curriculum that was launched in the fall of 2016. Our curriculum is divided into 3 phases over 4 years:

- Phase 1: Foundational science curriculum and clinical skill development

- Phase 2: Required clinical clerkships

- Phase 3: Advanced clinical experiences including emergency medicine, critical care, subinternship, and other electives

See Supplemental Digital Appendix 1-Curriculum Schematicat http://links.lww.com/ACADMED/A861.

Phase 1: Our preclerkship curriculum has extensive studentfaculty interactions, increased time for self-directed learning, and early development of clinical skills. Specific changes included:

- Earlier clinical exposure with core history and physical exam skill assessments at multiple points

- Increased use of small-group activities to deliver content

- Expanded integration of clinical sciences into an organ-based system

- Transition to pass/fail grading 


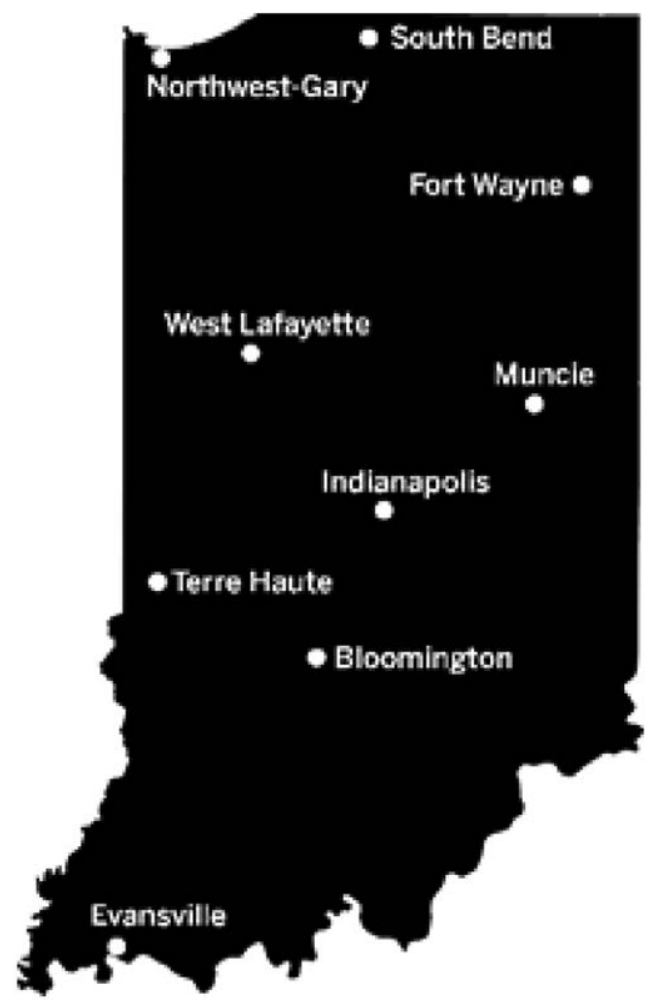

Figure 1 Map of Indiana University School of Medicine campuses.

Phase 2: IUSM has deployed core clerkships throughout all 9 campuses, each with common learning objectives, required clinical experiences, core didactic programs, assessment methods, and grading systems. Statewide clerkship management teams were created, as previously described.

See Chart 1-Phase 2 and 3 required clinical clerkships.

Phase 3: The curricular reform process was critical in the redesign of the final year experience. IUSM was dedicated to several principles:

- Increased responsibility with achievement of competencybased milestones

- Enhanced readiness for residency

- Student-selected experiences aligned with their future career aspirations

The final phase of the educational program offers advanced experiences designed to prepare graduates for the transition to residency. Expectations are more rigorous and students demonstrate enhanced responsibility, knowledge, skills, and abilities. Electives and focused selectives permit students to concentrate on content of their interest, typically related to a specialty choice and professional development needs. Additionally, a transition to residency "bootcamp" (Transitions 3 course) was created to address broad GME preparation topics coupled with specialty-specific requirements.

\section{Horizontally integrated curriculum}

Certain elements of curriculum transcend a specific course or clerkship. Recognizing the importance of preparing our learners to function in a modern health care system, IUSM created intersessions in Phase 2 that emphasize:

- Health systems science and population health

- Enhanced communication skills

- Ethics and student wellness

Participating in the 9-day intersessions course spread across the Phase 2 curricular year, students engage in small-group activities designed to enhance their preparation for patient encounters and to develop and communicate comprehensive plans of care. Intersession activities also allow students to reflect on their clerkship experiences and permit meaningful group sharing and discussion.

The awarding of the American Medical Association grant permitted development of the learning electronic medical record, which is being used to enhance student preparedness in the transition to clerkships course (Transitions 2) as well as to introduce elements of value-based care.

\section{Assessment}

The IUSM medical education program objectives are based upon the ACGME domains of competence. Program objectives are described in outcome-based terms that allow assessment of medical students' progress in developing the competencies that the profession and the public expects of a physician. Intentional alignment of course and clerkship learning objectives to institutional learning objectives, as well as linking specific assessment methods to students' learning outcomes,

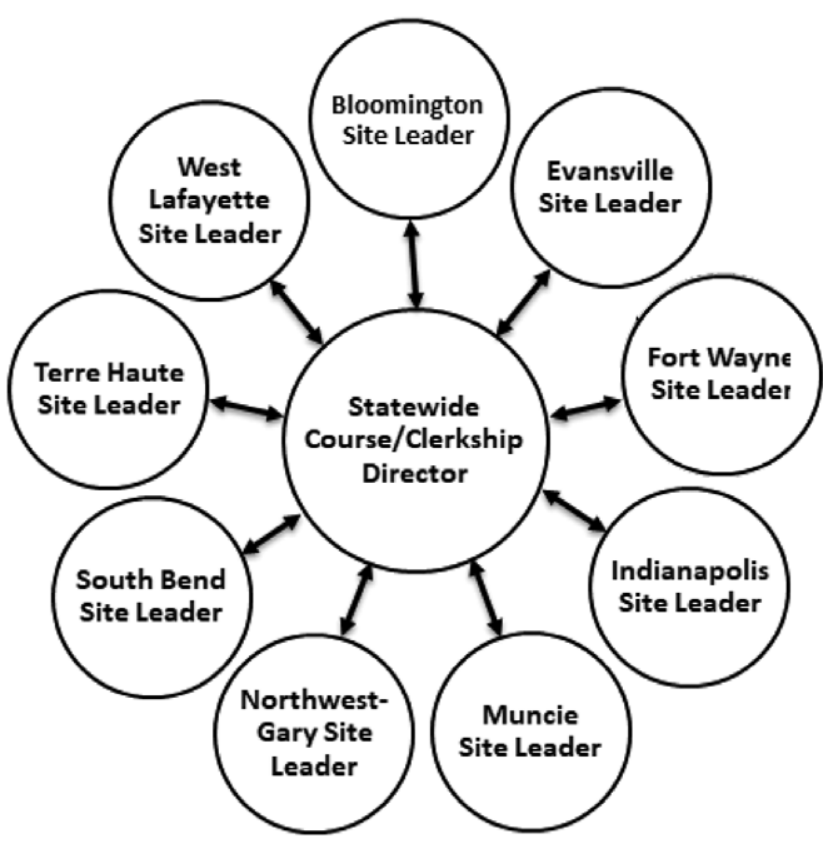

Figure 2 Course management structure. 


\section{Chart 1}

Phase 2 and 3 required clinical clerkships

\begin{tabular}{|l|l|}
\multicolumn{2}{|l|}{$\begin{array}{l}\text { Phase } 3 \text { advanced clinical } \\
\text { experiences }\end{array}$} \\
\hline Anesthesia & Emergency medicine \\
\hline Family medicine & Subinternship \\
\hline Medicine & Critical care selective \\
\hline Neurology & Other electives \\
\hline Obstetrics-gynecology & \\
\hline Pediatrics & \\
\hline Psychiatry & \\
\hline Radiology & \\
\hline Surgery & \\
\hline
\end{tabular}

are tracked in a curriculum inventory map that enables curricular and competency directors to ensure institutional goals are met. Specific initiatives as well as novel curriculum and assessment methods were developed to assess competencies that are traditionally more difficult to evaluate (e.g., systemsbased practice, practice-based learning and improvement, professionalism). Longitudinal tracking of achievement by competency domain has been developed, with the ability to identify learners with areas for improvement and provide them additional focused support.

\section{Parallel curriculum or tracks}

The Bloomington longitudinal integrated clerkship track has been offered at IUSM since 2012. Students in this track participate in all clinical clerkship disciplines by half day experiences per week over the course of the third year, except anesthesiology, which is completed as a block experience. The longitudinal nature of the Bloomington track allows relationships to form within the clinical setting and leads to high faculty and student satisfaction rates.

The Rural Medical Education Program (RMEP) has been offered at the Terre Haute regional campus since 2008. RMEP students complete a 4-year curriculum at this location, which provides longitudinal exposure to health care delivery within rural practice settings. With a particular emphasis on primary care, students learn to appreciate unique aspects of rural health care and are better prepared to practice in a rural environment or engage with those who do.

\section{Pedagogy}

In Phase 1 (preclerkship curriculum), a variety of pedagogical approaches are used, including:

- Case-, team-, and problem-based learning

- Laboratory sessions that involve peer teaching

- In- and outpatient clinical experiences

- Large- and small-group discussion formats
- Lectures

- Self-directed learning

- Simulation/standardized patients

During Phase 2 and 3 (clerkships, electives, and subinternships), pedagogical approaches are based primarily on clinical experiences (inpatient; ambulatory), lecture, preceptorship, and simulation.

Clinical exposures for IUSM students start within the first month of matriculation. In the Foundations of Clinical Practice course, students participate in a longitudinal clinic-based experience in year 1 and again in year 2. Subsequent intensive clinical exposure occurs in various academic, tertiary, and community-based hospitals and clinics in urban and rural areas, which includes partnering with our Veterans Affairs hospitals and clinics statewide. With the IUSM class size expansion, developing new clinical partners at our regional campuses has been an important focus.

\section{Curricular Governance}

Shared governance in the design and implementation of the curriculum is central to the curricular governance structure at IUSM.

The Curriculum Council Steering Committee (CCSC) oversees the design of the school's UME curriculum. The CCSC identifies strategic curricular directions, ensures that the curriculum reflects the broader school educational mission, and is attentive to national trends in medicine and medical education. The CCSC ensures implementation of curricular initiatives, a robust statewide curriculum with appropriate attention to comparability, gaps and unwanted redundancy, and LCME standards. The Foundational Component Committee (FCC) and the Clinical Component Committee (CCC) oversee the creation, implementation, and review of the foundational (FCC) and clinical (CCC) science education. The Academic Standards Committee is charged with systematic evaluation of the curricular components (courses and clerkships) to ensure adherence to the institutional learning objectives and curricular policy, statewide comparability of assessment and outcomes, and equivalency in grading.

See Figure 3-Curricular governance.

\section{Education Staff}

The Medical Student Education Office attends to curricular and student affairs needs, working with the regional campus deans and leadership team at each campus to serve UME needs. IUSM has separate offices of GME, continuing medical education, library services, and a prematriculation program, which also report to the executive associate dean for educational affairs and improvement.

See Figure 4-Organizational chart. 


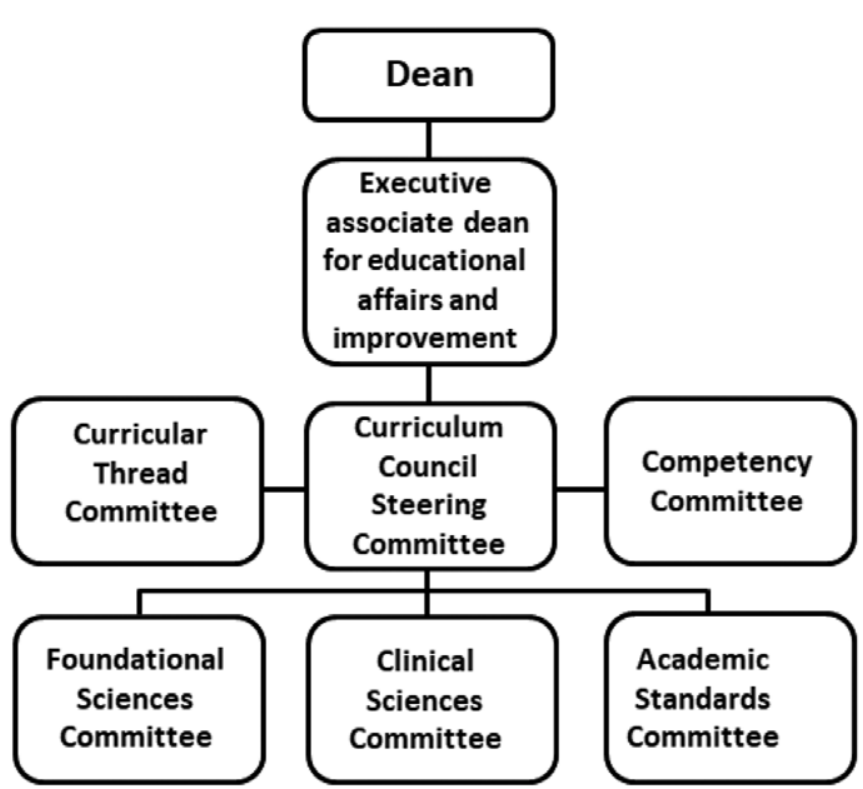

Figure 3 Curricular governance.

\section{Faculty Development and Support in Education}

The IUSM Office of Faculty Affairs, Professional Development, and Diversity directs educational development content and programming that is delivered to all campuses via regionally hosted forums, individual site visits, individual modules, and virtual interactive conferences. All faculty statewide are encouraged to pursue promotion and tenure options through IUSM, which has an enhanced education pathway. IUSM also offers the Academy of Teaching Scholars program for faculty. IUSM Education Day provides an opportunity for faculty, students, residents, and fellows from all departments and campuses to showcase their medical education research through oral presentations, workshops, poster sessions, and small-group discussions. An annual clerkship leaders' summit offers statewide clinical educators

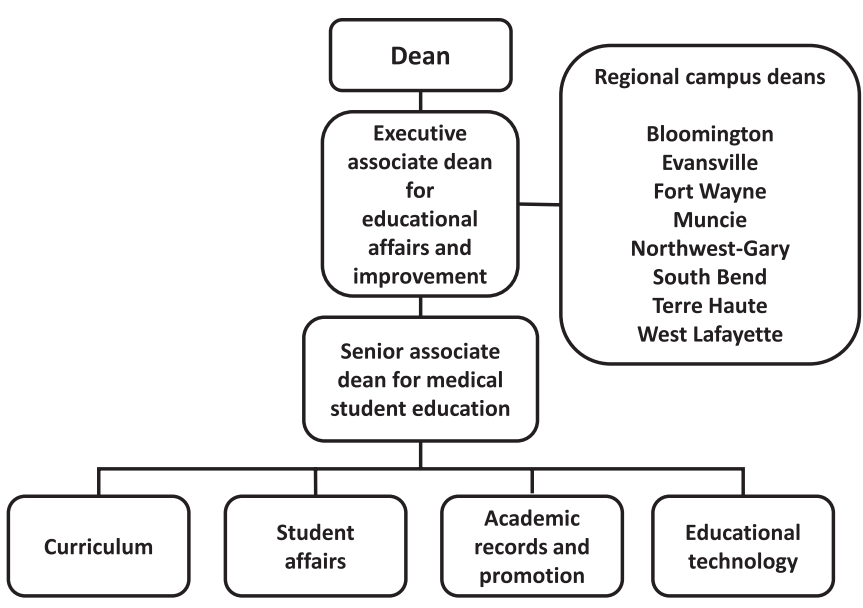

Figure 4 Organizational chart. faculty development content in medical education and facilitates statewide collaboration.

\section{Regional Medical Campuses}

As noted, IUSM features 9 campuses that each offer the full 4-year MD curriculum; 3 regional campuses host MD-PhD students.

See Table 1-Campus distribution.

\section{Initiatives in Progress}

Point-of-care ultrasound

A longitudinal point-of-care ultrasound (POCUS) curriculum specific to medical student education was designed and implemented throughout the entire curriculum in 2019-2020. In addition to its education benefit of cognitive scaffolding, the goal is to introduce learners to the use of POCUS for diagnostic imaging to answer binary clinical questions and to guide procedures. Ultimately, including POCUS in the UME and GME curriculum enhances traditional learning and leads to improved diagnostic and procedural skills.

Sixteen modules were developed and placed into various content-linked components of the curriculum over all phases. Student satisfaction has been extremely high, and there is a phased plan for further enhancement of the POCUS curriculum.

\section{Scholarly concentrations}

The Scholarly Concentration (SC) program is a voluntary track available to all IUSM students that is designed to enhance concepts of the core curriculum while leveraging the reputational focus of our campuses and statewide faculty in novel areas of health care. These topics often highlight areas of health system sciences to round out a learners' understanding of the complex

\section{Table 1}

\section{Campus Distribution}

Campus
Bloomington
Evansville
Fort Wayne
Muncie
Northwest-Gary
South Bend
Terre Haute
West Lafayette
Indianapolis

${ }^{a}$ All campuses are 4-year campuses.

${ }^{b}$ The goal for Phase 2 and 3 (years 3 and 4) enrollment at regional campuses is $50 \%$ of the Phase 1 enrollment.

cMain campus. 


\section{Table 2}

\section{Scholarly Concentrations by Campus}

\begin{tabular}{|c|c|c|}
\hline Scholarly concentration & Campus & Health systems science core domains \\
\hline $\begin{array}{l}\text { Biomedical engineering and applied medical } \\
\text { technology }\end{array}$ & West Lafayette & Value-based care \\
\hline Business of medicine & $\begin{array}{l}\text { Statewide/ } \\
\text { Indianapolis }\end{array}$ & $\begin{array}{l}\text { Health care structures and processes; health care policy, economics, and } \\
\text { management; value-based care }\end{array}$ \\
\hline Care of Hispanic/Latino patients & West Lafayette & Population health; value-based care; health care structures and processes \\
\hline Ethics, equity, and justice & South Bend & $\begin{array}{l}\text { Population health; value-based care; health care policy, economics, and } \\
\text { management }\end{array}$ \\
\hline Health promotion and disease prevention & Muncie & Population health; value-based care; health care structures and processes \\
\hline Health information technology & Indianapolis & Clinical informatics/health informatics technology \\
\hline Aging studies & Fort Wayne & Value-based care; health care structures and processes \\
\hline Human sexuali................. health & Bloomington & Population health; value-based care \\
\hline Public health & $\begin{array}{l}\text { Statewide/ } \\
\text { Indianapolis }\end{array}$ & Population health \\
\hline Medical education & Bloomington & Health care structures and processes \\
\hline Medical humanities & Indianapolis & $\begin{array}{l}\text { Population health; value-based care; health care policy, economics, and } \\
\text { management }\end{array}$ \\
\hline
\end{tabular}

systems in health care. Students in the SC program are expected to develop knowledge, skills, and a mentored scholarly project over the course of their time in the program. Each campus hosts at least 1 SC program, with codirectors managing the delivery of each. Successful completion of the SC in the senior year requires generation of a scholarly product and leads to 2 elective credits. The SC training and projects serve as an additional way for IUSM students to showcase their skills to residency programs.

See Table 2-Scholarly concentrations by campus. 


\section{University of lowa Roy J. and Lucille A. Carver College of Medicine}

Christopher S. Cooper, MD, Susan Lenoch, MA, and David P. Asprey, PhD, PA-C

\section{Medical Education Program Highlights}

The University of Iowa Carver College of Medicine (CCOM) recognizes the importance and contributions of the medical education curriculum and the learning environment in enhancing and maximizing students' professional development. To maintain focus and achieve balance in these critical areas, the educational administration and staff are contained within a single Office of Student Affairs and Curriculum (OSAC).

The mechanisms of health and disease (MOHD) curricular strand comprises 6 multisystem mechanisms that serve as the mechanistic framework for teaching foundational sciences. MOHD 1 and 3 cover the mechanisms of oxygenation, metabolism, and genetics/development. MOHD 2 and 4 cover the mechanisms of immunology/inflammation, locomotion/ integument, and neuropsychiatry. Each MOHD course is codirected by a scientist and a clinician to facilitate integrating fundamental science with clinical relevance.

A unique aspect of our curriculum is the combined curriculum that co-educates physician assistant (PA) and MD students simultaneously. The preclinical phase consists of 13 courses taught to PA and MD students that together use identical coursework, evaluation methods, and grading. This unique feature provides efficiency in training students while helping them learn to work in interprofessional teams throughout the first 18 months of the curriculum.

\section{Curriculum}

\section{Curriculum changes since 2010}

In April 2010, a curriculum renewal committee charged 4 separate design/modeling committees - each containing faculty, staff, and students - to develop a plan to innovate, renovate, and remodel the 4-year medical education program. These committees independently developed 4 new structural models that were presented to each CCOM department and to students. Broad feedback was obtained, and ultimately the most promising and innovative elements of each model were selected and synthesized into a single consensus curriculum.

Acad Med. 2020;95:S180-S183.

doi: 10.1097/ACM.0000000000003447

Copyright (C) 2020 by the Association of American Medical Colleges

Supplemental digital content for this article is available at http://links.Iww.com/ ACADMED/A936.

Year school was established: 1870.

School URL: https://medicine.uiowa.edu.
The preclinical semesters (Phase I) in the new curriculum were reduced from 4 to 3 , and the length of individual courses in the MOHD strand was reduced from an entire semester to 8-10 weeks. Phase I of the curriculum also features longitudinal mentored clinical experiences beginning as early as the first week of medical school.

During Phase II, students complete 9 core clerkships over a 12 -month period. This core clerkship year now begins after 3 semesters of preclinical instruction rather than the 4 weeks in the previous curriculum. The core clerkships comprising Phase II are grouped into four 12-week blocks:

- Ambulatory practice module: community-based primary care, family medicine, and outpatient internal medicine

- Inpatient internal medicine and pediatrics

- Obstetrics-gynecology and surgery

- Neurology and psychiatry

These linked clerkships have shared education days to cover specifically assigned components of the physical examination and mutually agreed upon exemplars, often as part of performance-based assessment activities (e.g., simulated patients, postencounter, computer-based assessments). The recommended timing of the USMLE Step 1 examination was moved to follow completion of core clerkships in Phase II.

In Phase III, an increase from 2 to 3 semesters following completion of Phase II provided increased flexibility for students to individualize their medical education and explore multiple career options. Students engage in an individualized educational experience that is designed to ensure a broad base of medical knowledge and skill set at graduation to prepare for entry into their chosen residency.

See Supplemental Digital Appendix 1-Curriculum Schematicat http://links.lww.com/ACADMED/A936.

\section{Assessment}

The CCOM medical education program objectives are organized around the ACGME framework of 6 domains of competence. To assess program outcomes, the Medical Education Council (MEC) set benchmarks regarding achievement of the objective for each metric in the table. The council reviews outcomes relative to these metrics annually basis. In addition, CCOM developed its own clerkship tracking software, CLERX, that facilitates oversight of required clinical experiences at both clerkship and collegiate levels. This software replaced a commercial product that was more cumbersome for student recording and technically challenging for generating reports for central monitoring of Required Clinical Experience completion. 
A relatively recent enhancement in student assessment was the development of a cadre of physical exam teaching associates (PETAs) to facilitate teaching the physical examination. PETAs are rigorously trained by clinicians to teach the physical exam system according to a validated, clinician-developed checklist delineating the steps of the exam.

See Supplemental Digital Appendix 2-Program Objectives and Assessment Methods - at http://links.lww.com/ACADMED/A936.

\section{Parallel curriculum or tracks}

Distinction track: CCOM's distinction tracks offer students opportunity to pursue scholarship outside of the standard medical curriculum. These voluntary tracks represent ways for students to gain tangible knowledge and skills beyond the basic science and clinical knowledge domains. The pursuit of distinction tracks is documented within a student's medical student performance evaluation and acknowledged during the graduation ceremony. There are 6 tracks: teaching, service, research, global health, humanities, and health care delivery science, management, and policy. Each track includes multiple requirements including didactic content, related educational activities, and a mentored capstone project. Each track includes a council that reviews student work and progress in meeting requirements.

CCOM Rural Iowa Scholars Program (CRISP): This is a comprehensive parallel curricular program focusing on rural medicine throughout medical school by providing supplementing the standard curriculum. Students begin the program the summer before their first semester by shadowing a physician mentor in a rural community. Rural elements are embedded in their medical education through mentorships, seminars, research, and electives. The CRISP clinical experiences include completing continuity of care experiences at a rural site, with specific activities designed to introduce students to community life, health systems, and rural cultures. Additionally, CRISP students complete a rural capstone research project and attend a state or national rural medicine conference. Graduates must practice in an eligible community in Iowa for a minimum of 5 years after completing a qualifying residency to receive loan repayment.

\section{Pedagogy}

A "triple helix" model for the new curriculum was developed with the 3 strands extending throughout all 4 years. The 3 are MOHD, medicine and society, and clinical and professional skills (CAPS). The strands are interwoven in a helical spiral to enhance horizontal and vertical integration of content and to promote deliberate revisiting of material to encourage progressively deeper understanding and mastery. Pedagogical approaches in the new curriculum include case-based learning, problem-based learning, large- and small-group discussion, laboratory and lectures, ambulatory and inpatient clinical experiences, preceptorships, self-directed learning/tutorial, peer teaching, and video/podcasts, as well as an increase in teambased learning, simulation, standardized/simulated patients, and workshops.

\section{Clinical experiences}

Students have their first clinical experience during the first week of medical school. Clinical education occurs primarily at University of Iowa Hospitals \& Clinics, the Veterans Administration Medical Center, the Des Moines Branch Campus, and Regional Medical Education Center (RMEC) sites (for the community-based primary care clerkship). This clerkship maintains an administrative structure in which the orientation of faculty to course objectives and grading is coordinated through 6 RMECs. During this clerkship, students spend 4 weeks with a family practitioner in community practice and 4 weeks at one of the RMECs located statewide.

\section{Curricular Governance}

The MEC is the designated executive body responsible for design, implementation, and evaluation of the CCOM curriculum. The 4 MEC subcommittees are the Medical Arts and Sciences Committee (MASC), the Clinical Experiences Committee (CEC), the Strand Director's Subcommittee, and the Assessment Subcommittee. With the exception of the Strand Director's Subcommittee, the MEC and the other subcommittees are chaired by a UME dean.

See Figure 1-Curriculum committee structure.

The MASC is charged with implementing, managing, and reviewing the preclinical curriculum (Phase I). It reviews and provides feedback on course objectives, sequence of instruction, and integration of material within and across the preclinical curriculum. The MASC reviews preclinical content areas and recommends and implements policies for ensuring quality in courses and fairness in assessments.

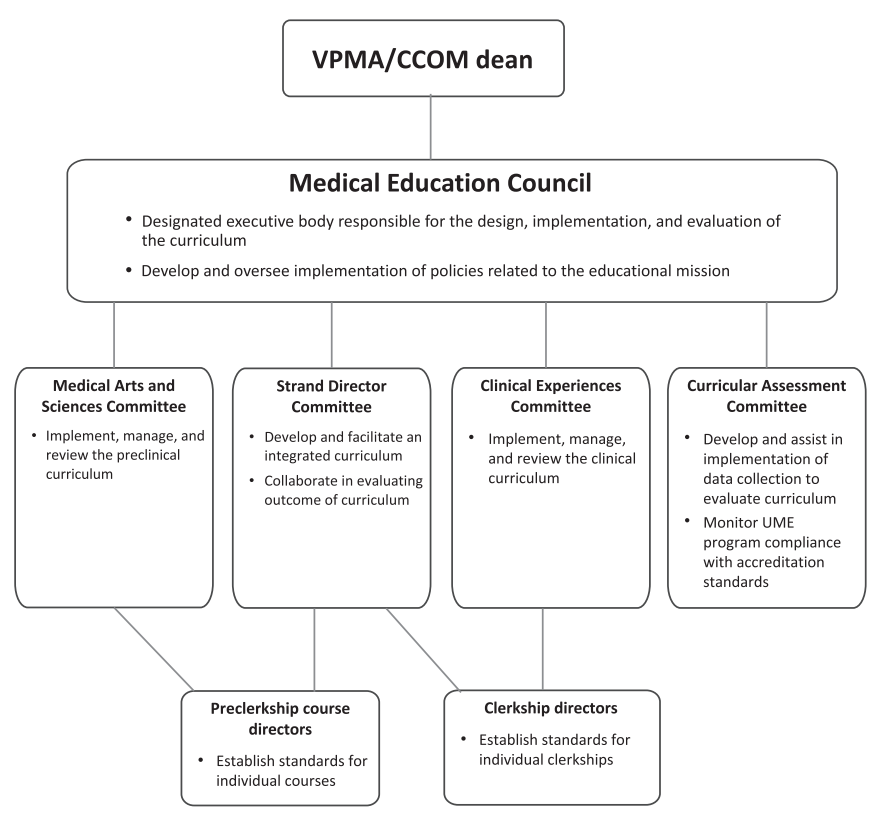

Figure 1 Curriculum committee structure. 
The CEC is charged with the implementation, management, and review of the clinical curriculum (Phase II/III). It reviews and provides input to the MEC regarding clerkship objectives and sequence of instruction within and across the clinical curriculum. The CEC reviews the clinical curriculum to identify and address redundancies and gaps, facilitates integration of foundational science content, and conducts reviews of targeted areas of need specific to the clinical curriculum. The CEC also recommends and implements policies for ensuring quality in courses and fairness in assessments.

The Strand Director Subcommittee is charged with implementing curricular goals, the 3 curricular strands, and the individual required course and clerkship objectives: developing and facilitating the integrated 3-strand curriculum, including coordination and communication across strands, semesters, courses, and clerkships; collaborating and participating in evaluating the overall outcome of the curriculum; and making recommendations for the appropriate utilization/distribution of limited education related resources. It maintains a current map of each strand's goals, objectives, metrics, and integration across the required curriculum. This committee also reviews and provides feedback on course objectives, sequence of instruction, and integration of material within and across the 4 years.

The Assessment Subcommittee is charged with developing and assisting in implementation of a data collection and analysis plan for evaluation of the UME curriculum, monitoring the UME program's compliance with elements of the accreditation standards relevant to the evaluation of the curriculum, and making recommendations regarding policies related to student and program assessment as requested. It reviews and provides feedback on program objectives, sequence of instruction, and integration of material within and across the curriculum. This committee also conducts data gathering and analysis relevant to assessment of the curriculum, develops and implements policies for ensuring quality assessment of the curriculum, and reviews and provides feedback on relevant instruments and methods of data gathering and analysis.

\section{Educational Staff}

The OSAC is the group of 35 professionals who most directly facilitate medical student activities at CCOM. Additionally, each of the 4 learning communities have 2 faculty codirectors. The OSAC mission is to provide an environment of scholarship and humanism in which students can become the well-rounded physicians of tomorrow, fully prepared to enter their professional lives focused on the well-being of their patients and communities. OSAC consists of 10 units, and among its duties are selecting students who will thrive in this academic environment; facilitating the delivery of a medical curriculum providing the best didactic and practical learning experiences possible; providing opportunities for students to experience the world through research, service, travel, and the humanities; and supporting students throughout the learning process. The 5 educational deans for UME are also within OSAC.

See Figure 2-Deans involved in UME.

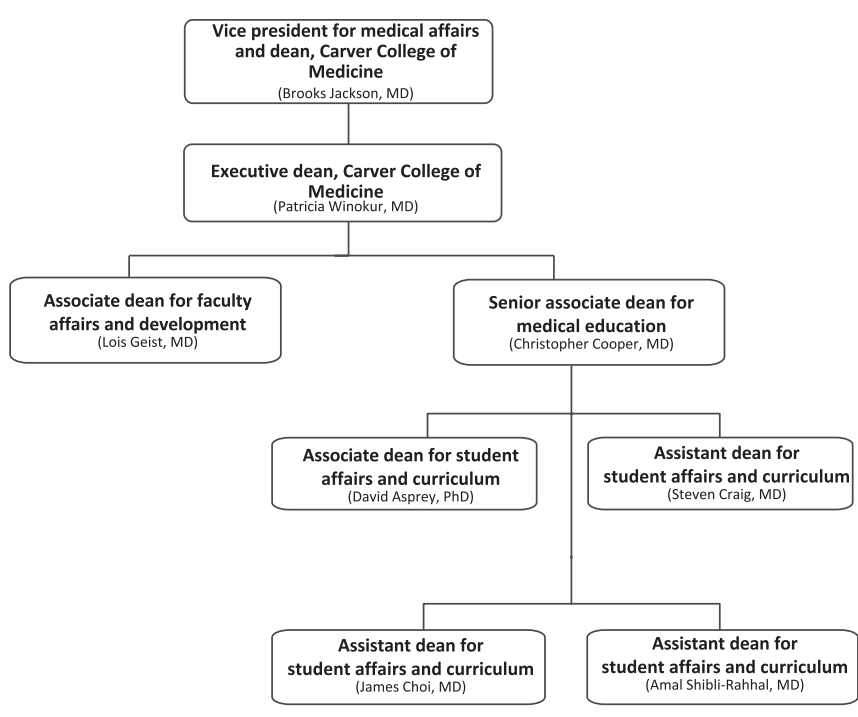

Figure 2 Deans involved in UME.

\section{Faculty Development in Education and Promotion}

The Office of Consultation and Research in Medical Education (OCRME) provides coordination of services and products that support curriculum development, faculty development, measurement, evaluation, and educational research. Departmental seminars and faculty workshops are conducted by OCRME. OCRME also leads the Clinical Teaching Scholars Program for faculty and a master's in medical education program. The OCRME Community Clinical Teaching Scholars Program brings faculty from RMECs to Iowa City for all-day meetings 3-4 times per year to help develop local leaders who can partner with OCRME to conduct faculty development programs at each affiliate site. Each regional site also conducts faculty development programming on site at least twice yearly with guidance and assistance of OCRME staff.

Promotion in the clinical track requires an acknowledged record of continued teaching success, including a record of successful direction of student, fellow, or resident work where applicable. Evaluation of teaching includes review of teaching materials and evaluations in terms of quantity and quality relative to peers. Educational activities including educational scholarship may also contribute to the record of professional productivity. Promotion on the tenure track also includes a similar evaluation of the applicant's teaching record.

\section{Regional Medical Campus}

The assistant dean of the Des Moines branch campus monitors clinical sites, housing, and other facilities and resources. The clerkship directors at each campus work collaboratively to implement the curriculum, including determining identical learning objectives and requirements, formal educational sessions and resources, assessment 
methods, grading policies, and other conditions needed to ensure comparable education and assessment. Clerkships are reviewed regularly through the CEC, and annually the MEC evaluates comparability of education and assessment at the 2 campuses. The MEC determines the comparison measures and directs the medical education monitoring and evaluation team to analyze data and generate a comparability report including measures of student achievement and clerkship quality.

See Table 1-Regional Medical Campus.

\section{Table 1}

\section{Regional Medical Campus}

\section{Regional campus name Type Student enrollment}

University of lowa Carver College of Medicine Des

Clinical 24 students may complete Moines Branch Campus in Des Moines. Some core clerkships have capacity for additional students. Advanced clerkships are also available. 


\title{
The University of Kansas School of Medicine
}

\author{
Giulia A. Bonaminio, PhD, Joseph D. Fontes, PhD, Gary Doolittle, MD, Pam Shaw, MD,
}

Scott Moser, MD, and Michael Robinson, PhD

\section{Medical Education Program Highlights}

Following 3 years of development, we launched a comprehensively revised curriculum in 2017. We designed the revision process to involve all stakeholders in planning, development, and implementation. , $^{1,2}$ Using a "ground-up" evidence-based approach, we developed content, delivery methods, and assessment strategies de novo. The Active-learning, Competency-based, Excellence-driven (ACE) curriculum features:

- Learning communities providing a professional and educational home for every student

- Pervasive, longitudinal clinical engagement, including clinical enrichment weeks ending preclerkship courses

- Diverse active learning strategies promoting cognitive integration of clinical and foundational sciences

- Preclerkship curriculum "bookended" by courses enhancing clinical communication, patient assessment, and clinical reasoning

- Extensive formative assessment coordinated with frequent summative assessments and in-line remediation

- Individual "dashboards" enabling students and faculty to track progress

- Standardized objective measures to assess mastery of clerkship competencies

The educational program is implemented on 3 campuses that share the single vision, mission, and identity of the school of medicine.

Important initial ACE outcomes include:

- Improved student preparation for patient care

- Increased faculty-student interactions and enhanced, close professional relationships initiated early in training

- Enriched faculty collegiality and communication across disciplines and specialties

- Improved longitudinal learner support, including individual coaching and continuity "learner hand-offs" across course/ clerkship transitions

- Improved equity of assessment across campuses

- Enhanced competency-based assessment and expansion of narrative input

- Emphasis on attainment of Entrustable Professional Activities (EPAs) related to graduation competencies

- Development of a cohort of educators prepared in ACE principles and strategies, including coaching and advising

Acad Med. 2020;95:S184-S187.

doi: 10.1097/ACM.0000000000003349

Copyright (C) 2020 by the Association of American Medical Colleges

Supplemental digital content for this article is available at http://links.Iww.com/ ACADMED/A870.

Year school was established: 1905.

School URL: http://www.kumc.edu/school-of-medicine.html.
Encouraged by positive monitoring data and initial internal and external measures of success, we continue to improve the ACE curriculum, maintaining focus on evidence-based innovation, quality enhancement, and involvement of stakeholders. These efforts will ensure our 114-year tradition of excellence in graduating outstanding physicians committed to patients and the public health, support of colleagues, and maintenance of professional vitality and resilience.

\section{Curriculum}

\section{Curriculum description}

Courses (blocks) in the first 2 years are organized around organ systems, "bookended" by clinically intensive initial and final blocks. Year 3 clerkships are aligned with the major medical specialties. The fourth year enables students to individualize experiences within required rotations in critical care, rural preceptorship, subinternship, and electives.

The learning activities of the preclerkship blocks are predominantly case based and focused on problem-solving that integrates diverse scientific and clinical concepts. The blocks incorporate extensive simulation activities to further promote integration and application of didactic material. The culture of the ACE curriculum is centered on patient care. The initial block, Introduction to Doctoring, equips students with interviewing and assessment skills necessary to complete an appropriate medical history and physical examination. This enables our doctors-in-training to participate in health care teams during the enrichment weeks of each block. Students select from a range of enrichment activities, available in diverse medical specialties and clinical environments.

See Supplemental Digital Appendix 1-Curriculum Structureat http://links.lww.com/ACADMED/A870.

\section{Curriculum changes since 2010}

In contrast to previous incremental change with periodic disruptive major revisions, curricular development is now a continuous quality enhancement process using continuous systematic assessment, improvement, and innovation. The ACE curriculum is a learner-centered, patient-focused program integrating foundational and clinical sciences with the goal of appropriate professional identify formation. We intend our students to identify and act as physicians-in-training (with all associated responsibilities) from day 1 . The most significant changes since 2010 include:

- Individual coaching

- Multiple avenues for real-time feedback

- Systematic tracking of mastery of content and achievement of objectives 
- Learning activities prioritizing patient care and enhancing cognitive integration

- Increased interprofessional and small-group experiences

- Expanded human and manikin simulation experiences

- Core EPA framework for the clinical skills curriculum; mapping of EPAs to graduation competencies

\section{Class size changes since 2010}

The entering class has remained at 211 students since 2011. The Kansas City campus matriculates 175 learners, of whom 48 transfer to the Wichita campus for clerkships. The Salina campus, established in 2011, focuses on preparing graduates for rural practice. With 8 students per class, Salina is the nation's smallest 4 -year medical education site. The community-based Wichita campus, established in 1971, expanded to a 4 -year program with 28 students in each entering class in 2011. Wichita graduates 76 students per year and continues to lead the nation in the number of graduates entering family medicine.

\section{Assessment}

Our 35 graduation competencies are based on the AAMC's Physician Competencies Reference Set and the Core EPA frameworks. ${ }^{3,4}$ These graduation competencies provide the framework for objectives in individual courses and clerkships.

See Supplemental Digital Appendix 2-Program Objectives and Assessment Methods-at http://links.lww.com/ACADMED/A870.

A core feature of ACE is robust formative assessment to support learning plus frequent summative assessment to track progress toward competency acquisition. Specific assessments were developed for each objective and competency.

Assessment in the preclerkship phase features:

- Formative assessment as a core educational tool for recallassisted learning and framing of content

- Summative assessment through biweekly multiple-choice examinations and end-of-block clinical skills assessment; inline remediation providing opportunity to remain on track if objectives are met

- Competency-based end-of-year OSCE to evaluate clinical skills

- Small-group performance assessment of students combining checklist and facilitator narrative feedback to track milestone attainment and acquisition of competencies

- Assessment and application of knowledge via 3 spaced NBME Comprehensive Basic Science Examinations

- Pass/fail grading without reported class rank

Assessment in the clerkship phase features:

- Criterion-based grading: students must pass NBME subject exams, a standardized clinical experience, and mandated clerkship experiences

- Committee of clerkship directors responsible for summative grading that integrates narratives on student performance toward EPAs

- Pass-with-distinction/pass/fail grading with reported class rank
Option of obtaining Honors designation by:

- Attaining top quartile of the class on USMLE Step 1 examination and year 3 GPA

- Completing a research/scholarly project

- No history of remediation

- Good standing on all professionalism and personal development competencies

\section{Pedagogy}

Students participate in large- and small-group discussions, lectures, and laboratories. Application of asynchronously acquired knowledge to authentic, clinical problems is a hallmark of ACE, accomplished through diverse learning activities including case-based collaborative learning (CBCL), problembased learning (PBL), flipped classroom, active case sessions, clinical skills and dissection laboratories, simulations, and multiple clinical experiences. Learning strategies involve peerto-peer teaching, interprofessional education, faculty facilitation, preceptorship, self-directed learning, tutorials, video podcasts, and workshops. Teaching is complemented by individual coaching and support from learning communities.

\section{Changes in pedagogy since 2010}

Teaching strategies were changed to promote cognitive integration of foundational and clinical sciences in understanding clinical phenomena. Students are empowered to appreciate how application of scientific knowledge improves the health of patients and the public. Over $60 \%$ of preclerkship contact hours involve active learning activities. The primary active learning modalities are:

- CBCL: This novel activity combines asynchronous knowledge acquisition with small- and large-group problem-solving sessions. The closed-end inquiry of CBCL sessions aims to apply foundational and clinical science principles to understand the promotion of health and the causes, diagnosis, management, and prevention of disease.

- PBL: Expanded and standardized sessions include more cases, improved facilitator preparation, and better integration with course content.

- Flipped classroom: The number of sessions expanded significantly.

- Active case sessions: Novel, large-group discussions involving students, physicians, and foundational scientists aim to consolidate knowledge with clinical reasoning.

- Standardized patients and simulation: Sessions have been expanded, with an additional focus on interprofessional experiences.

\section{Clinical experiences}

- Third-year clerkships occur in family medicine, internal medicine, neurology, obstetrics-gynecology, pediatrics, psychiatry, and surgery.

- Clinical sites for required educational experiences include hospitals, private physician's offices, residency clinics, rehabilitation facilities, long-term care facilities, safety net clinics, and Veterans Administration hospitals. 
- Initial clinical experiences occur in the Introduction to Doctoring course; learning clinical skills (history taking, physical examination) occurs through didactic teaching, standardized patient plus manikin simulation experiences, and personal study of resource materials.

- Enrichment weeks have been placed at the end of each block in the first and second year of the curriculum.

- Community-based learning opportunities occur in thirdyear clerkships. Students may complete part of their family medicine, neurology, pediatrics, psychiatry, and surgery clerkships at a rural Kansas site.

- Required fourth-year rural preceptorship occurs in a Kansas community.

\section{Curricular Governance}

The Education Council is the faculty governance entity responsible for institutional oversight of the educational program. It makes recommendations to the Faculty Council and executive dean regarding program changes. The 16 voting members include elected and appointed faculty, the senior associate dean for medical education, students, and ex-officio representatives from the dean's office. The council has 2 subcommittees: the Phase I Curriculum Oversight Subcommittee (comprising preclerkship block directors) and the Phase II Curriculum Oversight Subcommittee (composed of clerkship directors). Individual campus deans are responsible for managing resources for educational activities on their campus.

See Figure 1-Curriculum governance.

\section{Educational Staff}

The Office of Medical Education (OME) is administratively responsible for the planning, implementation, evaluation, and oversight of the curriculum; the development and maintenance

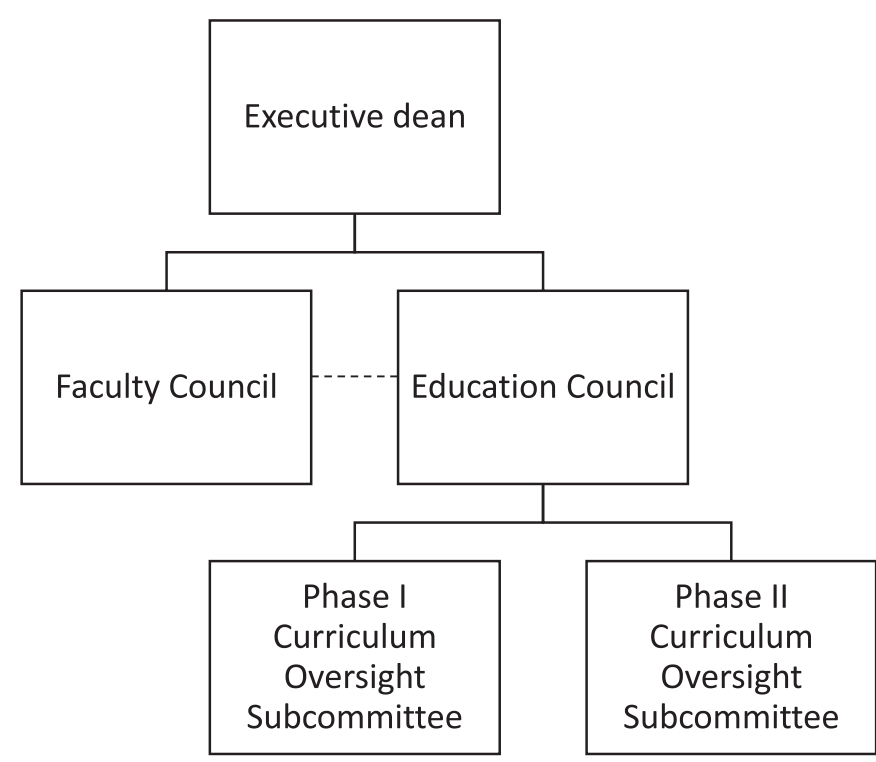

Figure 1 Curriculum governance. of tools that support curricular delivery and management; and support of medical education research and scholarship. Key aspects of the OME include:

- Eighteen staff members and 5 faculty members

- Management of an internal educational grants program supported by the school's medical alumni association ${ }^{5}$

- Support of the Academy of Medical Educators

- Partnerships with educational staff on regional campuses and with offices and units involved in student support, faculty development, and graduate medical education

The executive dean, regional campus deans, senior associate dean, associate deans, and assistant deans on 3 campuses all contribute to managing undergraduate medical education, faculty development, and learner support.

See Figure 2-Undergraduate medical education deans.

\section{Faculty Development and Support in Education}

Faculty Development is provided by departments and the Offices of Faculty Affairs and Development on the Kansas City and Wichita campuses. Schoolwide introductory medical education intensives are offered annually along with longitudinal medical education courses, topical short courses, and a medical education scholarship course. Brief faculty development topics are also provided for departmental faculty meetings.

Intensive faculty development was critical for the success of the ACE curriculum. Specific development workshops were offered on small-group facilitation, flipped classroom, and writing NBME-style exam items. Workshops were provided at the school, campus, and departmental level to prepare for the move to competency-based assessment and integration of narrative comments.

\section{Role of teaching in promotion and tenure}

Educational activities, scholarship, and leadership are central to promotion and tenure considerations. The school has offered a nontenure clinical-scholar track since the 1980s and introduced an educator track in 2014 for educationally focused faculty.

For promotion, education is broadly defined, including teaching and assessment of learners in different educational environments, plus mentoring of trainees and others in research and education. The required documentation summarizes teaching achievements and highlights educational leadership and development of educational materials. The webbased faculty activity database system provides a robust means to document educational accomplishments. The school uses a Boyer-derived approach, recognizing educational scholarship as well as traditional biomedical research as evidence of career progression.

Building and implementing the ACE curriculum expanded leadership and curricular development roles for many faculty educators and provided multiple opportunities for educational scholarship to support academic promotion. 


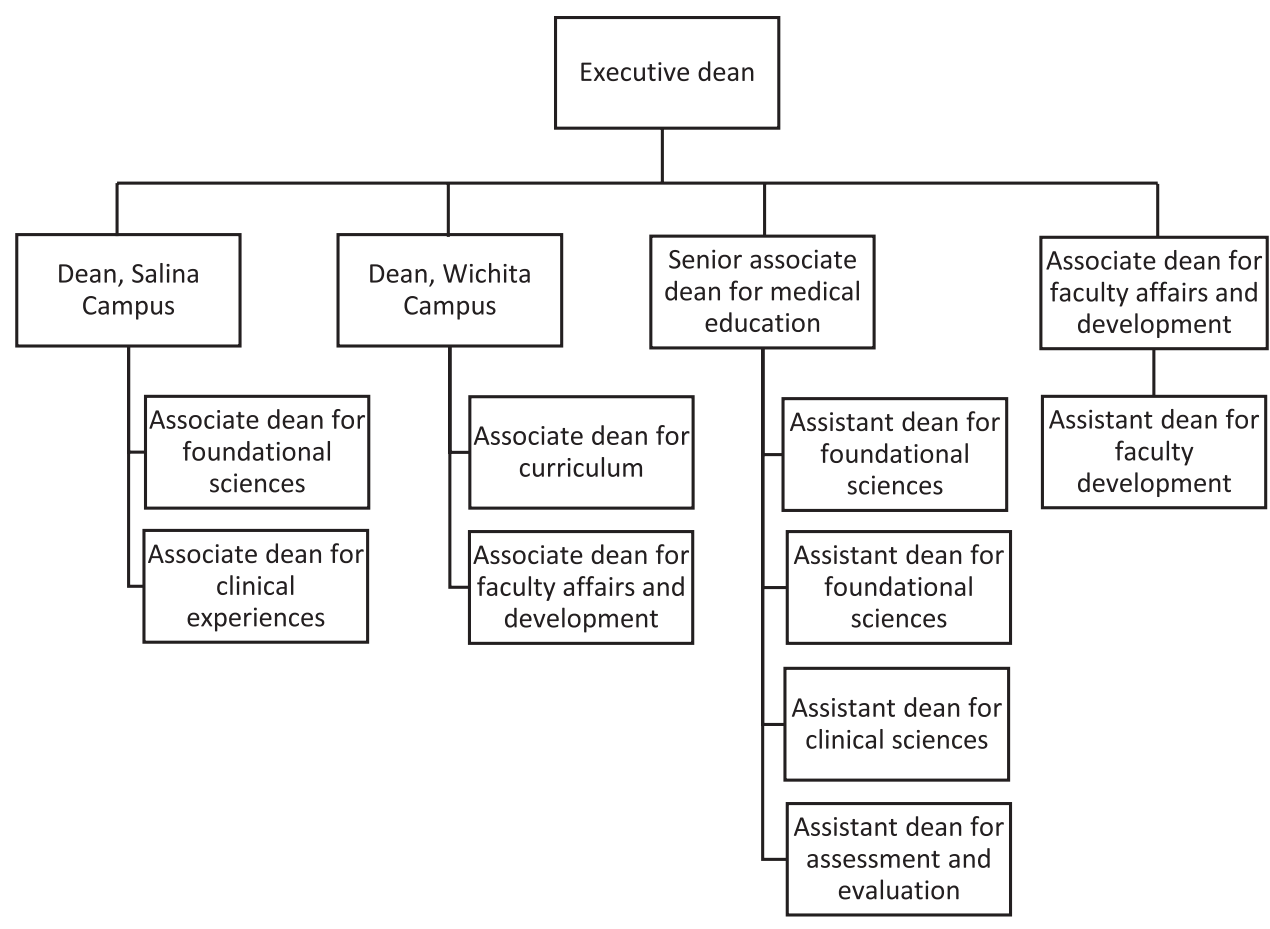

Figure 2 Undergraduate medical education deans.

\section{Academy of Medical Educators}

The Academy of Medical Educators exists to develop, support, mentor, and energize a vibrant faculty to educate the next generation of physicians. The academy is made up of 35 elected medical school faculty and collaborates with the Office of Faculty Affairs and Development to improve teaching and educational scholarship. Academy members focus on educational scholarship and promoting best practices through monthly medical education journal clubs and quarterly research interest group meetings. The academy offers competitive research grants to support innovations in medical education and assessment and sponsors annual travel awards for faculty to present educational research at national and international conferences.

\section{Regional Medical Campuses}

Students complete their undergraduate medical education at 1 of 3 campuses, providing 1 curriculum in different learning environments.

\section{Table 1}

Regional Medical Campuses

\begin{tabular}{|c|c|c|}
\hline $\begin{array}{l}\text { Regional campus } \\
\text { name }\end{array}$ & Type & $\begin{array}{l}\text { Student } \\
\text { enrollment }\end{array}$ \\
\hline Salina & Entire MD program & 32 \\
\hline Wichita & Entire MD program & 208 \\
\hline
\end{tabular}

See Table 1-Regional Medical Campuses.

We employ multiple mechanisms to ensure consistency and quality of educational experiences across campuses. Data from student evaluations plus performance and outcome data across the 3 campuses are analyzed, compared, and reported to educational oversight committees at regular intervals. The school's administrative leaders are responsible for facilitating a comparable educational experience and ensuring that decisions made by the Education Council are enacted at each site. Communication among leadership, faculty, and students on each campus is paramount and maintained through regular electronic and in-person interactions.

\section{References}

1 Quearry M, Bonaminio G, Istas K, Paolo A, Walling A. The impact of communication strategies on faculty members' readiness for curricular change. Med Sci Educ. 2019;29:51-55.

2 Walling A, Bonaminio G, Meyer M, Griebling TL, Paolo T, Nilsen K. Unintended consequences of curricular change: Lessons from legacy students. Med Sci Educ. 2019;29:919-922.

3 Englander R, Cameron T, Ballard AJ, Dodge J, Bull J, Aschenbrener CA. Toward a common taxonomy of competency domains for the health professions and competencies for physicians. Acad Med. 2013;88:10881094.

4 Englander R, Flynn T, Call S, et al. Toward defining the foundation of the MD degree: Core Entrustable Professional Activities for entering residency. Acad Med. 2016;91:1352-1358.

5 Bonaminio GA, Walling A, Beacham TD, Murphy WR, Huyett KM, Simari RD. Impacts of an alumni association-institutional partnership to invest in educational innovation. Med Sci Educ. 2019; doi: 10.1007/s40670-019-00842-x. 


\title{
University of Kentucky College of Medicine
}

\author{
Charles H. Griffith III, MD, Christopher A. Feddock, MD, and Robert S. DiPaola, MD
}

\section{Medical Education Program Highlights}

The University of Kentucky (UK) has grown significantly in both educational capacity and research over the last 3 years. Highlights include our statewide campus expansion, a doubling of our research activity, enhancements in curriculum and programs for student well-being, and leveraging transdisciplinary opportunities of a diverse institution.

- Campus expansion: UK College of Medicine is dedicated to confronting Kentucky's physician shortage and has embarked on a dramatic expansion of class size through the establishment of 4-year regional campuses in Bowling Green in 2018 and northern Kentucky in 2019, augmenting the 2-year clinical campus in Morehead, which focused on training for rural underserved practice via the Rural Physician Leadership Program (RPLP). This expansion initiative has allowed class size to increase from 113 in 2010 to 201 by 2019, with total enrollment expected to increase to 804, achieving the goal of training more physicians for Kentucky in Kentucky, delivering a "first-class medical education close to home."

- Research strategy: To improve overall research activity that fuels opportunities for students multiple initiatives have been launched, leading to a 2 -fold increase in NIH funding over the last 3 years, now totaling more than $\$ 129$ million in annual $\mathrm{NIH}$ awards and over $\$ 200$ million in total external awards. Initiatives include programs such as the Multidisciplinary Value Program that fosters team science across the college.

- Curriculum and student well-being enhancements: Bolstered by a robust continuous quality improvement process, over the last 10 years the College of Medicine has embarked on numerous initiatives to improve the education and wellbeing of our medical students. These include a change from a discipline-based to a systems-based curriculum; the adoption of a competency-based curriculum grounded in the demonstration of Entrustable Professional Activities (EPAs); institution of Pass/Fail grading; a longitudinal third-year course where students are personally coached by faculty as they progress in competency; established "advanced development directors" in the major clinical specialties, who provide career and residency advice for students throughout the clinical years; more infrastructure for wellness, including a studentled "Resilient" initiative and hiring 2 psychologists dedicated to medical student needs; more resources for student success, including an assistant dean and other support staff; greater

Acad Med. 2020;95:S188-S191.

doi: 10.1097/ACM.0000000000003450

Copyright (C) 2020 by the Association of American Medical Colleges

Supplemental digital content for this article is available at http://links.Iww.com/ ACADMED/A942.

Year school was established: 1956.

School URL: https://med.uky.edu. infrastructure and intentionality of pipeline programs to improve diversity and inclusion; and the establishment of a "house" system to allow more cross-class interaction and peer support.

- Transdisciplinary opportunities: As one of only a handful of universities to have 6 health care colleges contiguous on the same medical campus, the UK allows students to tailor their educational experiences and affords many transdisciplinary research opportunities, including those in the $\mathrm{MD}-\mathrm{PhD}$ program, the professional student mentored research fellowship, and the newly enacted scholarly distinction in clinical and translational research.

\section{Curriculum}

\section{Curriculum description}

See Supplemental Digital Appendix 1-Integrated Curriculum Map_at http://links.lww.com/ACADMED/A942.

\section{Curriculum changes since 2010}

In 2012, the College of Medicine modified its existing disciplinebased courses to a hybrid curriculum of integrated organ system-based courses. After this change, the Curriculum Committee noted additional opportunities for integration. Beginning in fall 2016, the original gross anatomy course was modified to a clinical anatomy and radiology course, while biochemistry and genetics were integrated throughout the core principles phase. A new course, Contemporary Practice of Medicine, was then created to teach population health and evidence-based medicine. In June 2018, Entrustment in Clinical Medicine, a new longitudinal course throughout the clerkship year, began teaching content focused on the practice of medicine. Topics included ethics, health care financing, evidence-based medicine, law and medicine, and patient safety. The Curriculum Committee monitors horizontal and vertical integration throughout the curriculum to plan future enhancements.

\section{Class size changes since 2010}

In an effort to uphold the College of Medicine's mission of producing graduates who eventually practice in the Commonwealth and other underserved settings, the college established the RPLP in Morehead, as well as two 4-year regional campuses in Bowling Green and northern Kentucky. All campuses offer comparable educational experiences to the College of Medicine's main campus in Lexington, as well as equivalent methods of assessment. This expansion has increased the class size from 113 to 201 since 2010 .

\section{Assessment}

Medical education program objectives are described in outcomebased terms that allow the assessment of medical students' 
progress in developing the competencies that the profession and the public expect of a physician (2020-2021 LCME Standard 6.1 or 2020-2021 CACMS Standards 6.1.)

UK College of Medicine's medical education program objectives are based upon ACGME and AAMC Core EPA standards.

See Supplemental Digital Appendix 2-Competencies, Objectives, and Outcome Measures—at http://links.lww.com/ACADMED/A942.

Student assessment has become more rigorous and more progressive in objective structured clinical examinations and ensuring that in 4 years of medical school students are competent in Core EPAs.

\section{Pedagogy}

The preclinical phase of the curriculum is an active learning experience. All courses have assignments outside the classroom, including video, podcasts, self-directed learning tutorials, and readings to provide base information. Lectures provide additional information on complex topics. Learning culminates in problem-solving sessions using case-based learning, teambased learning, peer teaching, workshops, and discussions to ensure students have mastered the material. Clinical skills are emphasized using standardized/simulated patients, small-group discussions, peer teaching, and preceptorship.

The College of Medicine has shifted from lecture-based courses to more interactive, flipped classrooms.

\section{Clinical experiences}

The College of Medicine uses both university-based and community-based hospitals and outpatient clinics.

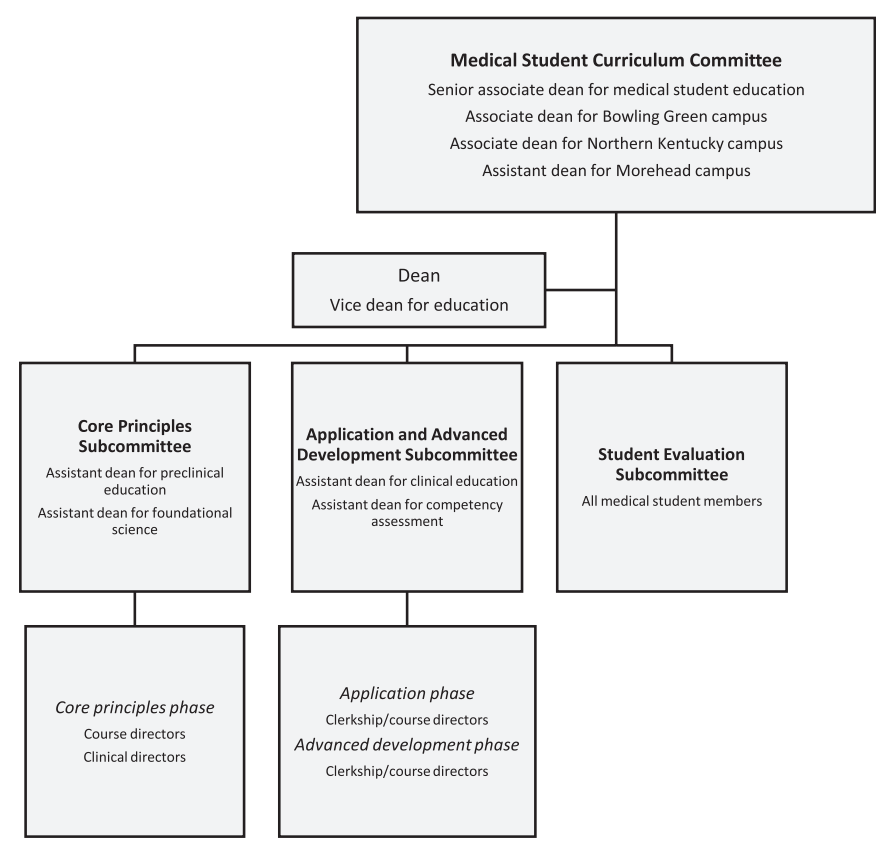

Figure 1 Curricular governance chart.

\section{Required longitudinal experiences}

The College of Medicine requires longitudinal experience in a family practice clerkship as part of the RPLP in Morehead.

\section{Clinical experience first encounter}

During first weeks of their first year, medical students gather clinical experience through training and then demonstrating "entrustable" competency in the basic medical interview and physical examination, initially on standardized patients. In the first semester of medical school, students encounter patients as part of their longitudinal clinical experience ( 4 half-day sessions over several months with a physician preceptor) and at the student-run Salvation Army Clinic.

\section{Required and elective community-based rotations}

Students have a required rotation in an area health education center, as well as at the Lexington VA Medical Center.

\section{Challenges in designing and implementing clinical experiences for medical students}

Students at UK are able to train at some of the best clinical facilities in the state and the country. UK HealthCare, St. Elizabeth Healthcare, Med Center Health, and St. Claire HealthCare provide students with diverse opportunities to experience the spectrum of health care. Therefore, the College of Medicine has not experienced difficulties or challenges in designing or implementing clinical experiences for medical students.

\section{Curricular Governance}

The Kentucky Integrated Curriculum is managed and supported centrally through the Office of Medical Education.

See Figure 1-Curricular governance chart.

\section{Education Staff}

The Office of Medical Education oversees all administrative and academic support for the curriculum. Annual educational faculty development workshops develop new educators, course directors, and small-group preceptors. Course support assists course directors in the planning and implementation of all courses and class sessions. Knowledge and skill assessments are managed centrally using databases to manage both multiple-choice questions and standardized patient cases. Further, the office collects and processes all evaluations and assessments of learning to provide the Curriculum Committee the data necessary for monitoring and managing the curriculum.

See Figure 2-UME organizational chart.

The College of Medicine's graduate medical education programs and faculty report through the vice dean of education, while programs and faculty from undergraduate medical education work under the Office of Medical Education. 


\section{Faculty Development and Support in Education}

\section{Professional development for faculty as educators}

Educational faculty development is provided by the Academy of Medical Educators, which is led by the assistant dean for educational faculty development and medical school senior leadership. The academy has developed 2 workshop series, 1 preparing teachers for the classroom and the other for teaching in clinical settings. The faculty development opportunities are offered via Zoom, a video and web conferencing resource, to accommodate sharing of materials and videos across multiple sites. In addition, leaders from the main campus and all regional campuses meet in Lexington annually for a collaborative faculty development meeting. The Academy of Medical Educators also delivers educational faculty development on each regional campus at least once per academic year.

\section{Role of teaching in promotion and tenure}

Promotion to associate professor entails demonstration of a regional and emerging national reputation in one's specialty, and for professor, an established national and even international reputation. The tenure track of "regular title" is typically attained through demonstrating independence as an investigator through the procurement of extramural funding and a strong publication record. For the tenure track of "special title," promotion occurs through demonstrating scholarship in a wider range of fields, most often in education, while also demonstrating excellence in teaching and course leadership, as evidenced by faculty and course evaluations by learners. Therefore, presenting work at national education meetings, publishing in medical education journals, having educational innovations adopted by others, and/or having leadership positions in educational organizations (NBME, AAMC, etc.) would be hallmarks of faculty who attain successful promotion in this track.

The Academy of Medical Educators, led by the assistant dean for educational faculty development and medical school senior leadership, was founded in 2017 to help educators at the College of Medicine thrive and continuously improve. Its 2 workshop series and utilization of web conferencing allows these resources to be shared across multiple sites, boding well for the college's

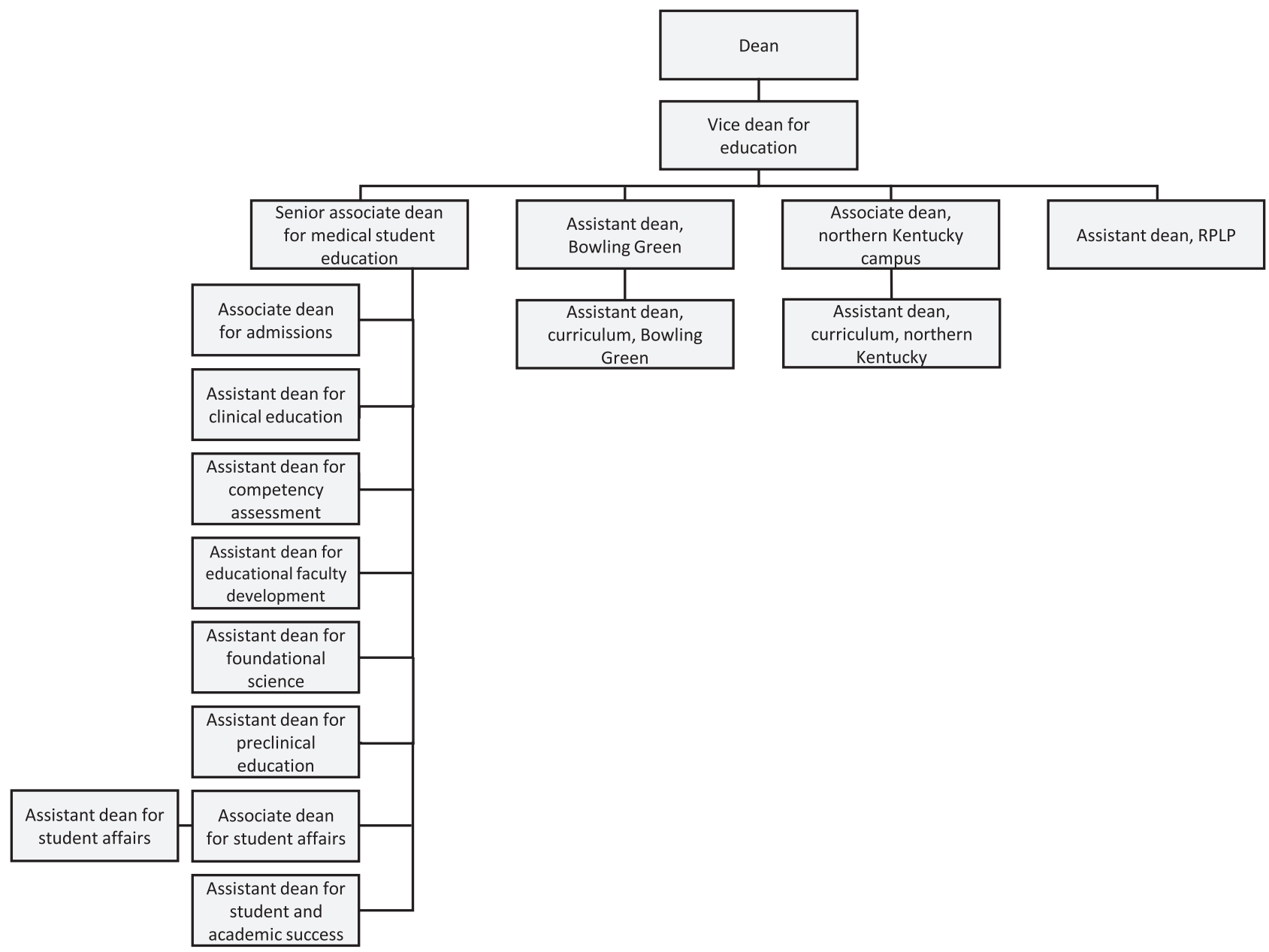

Figure 2 UME organizational chart. 
addition of 2 regional campuses. Leaders from the Academy of Medical Educators also meet in Lexington annually for more collaboration opportunities and deliver educational faculty development to regional sites.

\section{Regional Medical Campuses}

The College of Medicine regularly undergoes its quality assurance process and provides students a survey for feedback on learning environment to maintain consistent educational experiences across the regional campuses.

See Table 1-Regional Medical Campuses.
Table 1

Regional Medical Campuses

\begin{tabular}{|c|c|c|}
\hline Regional campus name & Type & $\begin{array}{l}\text { Student } \\
\text { enrollment }\end{array}$ \\
\hline $\begin{array}{l}\text { UK College of Medicine-Bowling } \\
\text { Green }\end{array}$ & $\begin{array}{l}\text { Entire MD } \\
\text { program }\end{array}$ & $120^{a}$ \\
\hline Rural Physician Leadership Program & Clinical & 24 \\
\hline $\begin{array}{l}\text { ÜK College of Medicine-Northern } \\
\text { Kentucky }\end{array}$ & $\begin{array}{l}\text { Entire MD } \\
\text { program }\end{array}$ & $140^{a}$ \\
\hline
\end{tabular}

Represents full complement of all 4 years. 


\section{University of Louisville School of Medicine}

Amy L. Holthouser, MD, Russell W. Farmer, MD, William J. Crump, MD, and Monica A. Shaw, MD, MA

\section{Medical Education Program Highlights}

The holistic, mission-based admissions process of University of Louisville School of Medicine (ULSOM) selects 162 students for each entering class based on academic ability; desire to remain in Kentucky and serve the needs of its citizens; and evidence of caring, dedication, perseverance, and service to others in need. Students may come from underprivileged backgrounds and communities, and strategic support has been implemented to ensure they succeed and thrive in medical school. Longitudinal coaching is delivered through the Advisory College Program, which assigns incoming students to 1 of 6 colleges. This college serves both as a physical "home" within the school and as a cohort for meetings, socialization, wellness, and advising programming support with faculty and staff. Each college is supported by faculty advisory deans who mentor and coach students individually and in groups longitudinally across all 4 years. Advisory Colleges support development of peer relationships between classmates as well as between classes; the program is a core feature of the healthy, inclusive learning environment of the school. An optional enrichment program providing opportunity for academic exploration and scholarly productivity and increasing the number of students choosing careers in academic medicine is the ULSOM Distinction Track Program. This longitudinal elective experience admits up to $25 \%$ of MD program students into 1 of 4 mentored tracks: business and leadership, global health, medical education, and research. Each track is unique in its requirements, ensures a faculty-mentored longitudinal experience, and culminates in a required scholarly project.

\section{Curriculum}

\section{Curriculum description}

The ULSOM curriculum integrates basic and clinical science topics using didactic, self-directed, and active learning within the $2+2$ hybrid curriculum structure.

- The first 2 years are primarily classroom based, with a 10 -week break between the first and second years for elective, research, or service-learning experiences, including NIH-sponsored Summer Research Scholars Program. Students gain clinical experience through community precepting, standardized patient encounters, and clinical electives during both years.

Acad Med. 2020;95:S192-S195.

doi: 10.1097/ACM.0000000000003304

Copyright (C) 2020 by the Association of American Medical Colleges

Supplemental digital content for this article is available at http://links.Iww.com/ ACADMED/A836.

Year school was established: 1837.

School URL: https://louisville.edu/medicine.
- Following year 2, 10 weeks of flexible time is given for studying and taking USMLE Step 1 as well as vacation.

- The third year consists of 7 core clerkships as well as 6 weeks of elective time to explore additional medical specialties of the students' choice.

- The fourth year requires 11 weeks of selectives in critical, palliative, and ambulatory care as well as an acting internship, with the remainder of the year remaining flexible with elective choice based on the student's desired medical specialty.

See Supplemental Digital Appendix 1-4-Year and 3-Year Curriculum Map—at http://links.lww.com/ACADMED/A836.

Two major programs, the Trover Rural Scholars (TRS) and the Rural Medicine Accelerated Track (RMAT), are targeted to improve the shortage of rurally placed physicians in Kentucky. TRS selects 8 incoming students to complete years 4 and 4 at the Madisonville Trover campus, a distributed clinical campus with a 400-bed hospital and clinics. RMAT is a 3-year path to the MD degree targeted to increase physicians prepared to practice rural primary care. Up to 2 RMAT graduates annually complete all of the required courses and objectives for the 4-year MD degree in 3 years, participating in the Match in their third year. Using vacation and elective time, RMAT students have already completed 12 weeks of family medicine training when they begin other traditional third-year clerkships. TRS and RMAT students complete additional elective experiences, longitudinal weekly primary care clinics, and independent learning assignments that support and assess the additional objectives necessary to function independently in improving patient or community health in a rural or resource-restricted environment. Ensuring a consistent educational experience for students in parallel tracks at a distributed campus requires that they complete all of the same core coursework and are assessed on the same objectives required for the MD degree, as well as continuous monitoring of educational outcomes, work hours, and patient encounters. Technology support, faculty development, and standardized patient encounters are also delivered to support these students and faculty, ensuring that the quality of the learning environment meets or exceeds the level expected for Louisville campus students.

\section{Assessment}

Content and assessment are organized using behaviorally based objectives throughout the 4-year program, requiring evidence of student attainment of required knowledge, skills, and attitudes across AAMC competency domains in each year. The 38 ULSOM program objectives were completely revised in 2017 , and were developed as a novel set after Educational Program Committee (EPC) review of the AAMC Physician Competency Reference Set, ACGME domains of competency, Core EPAs, and the Medical School Objectives Project. The EPC determined 
the course objectives for every course to ensure all competency domains were assessed in every year of the program, and that all required courses include teaching and assessment of student interpersonal communication and professionalism skills and behaviors.

See Supplemental Digital Appendix 2-Program Objectives and Assessment Methods—at http://links.lww.com/ACADMED/ A836.

An area of programmatic strength is the eQuality Curriculum Project, which integrates AAMC physician competencies to better care for LGBTQ+ and DSD patients and populations throughout the 4-year program (https://louisville.edu/ medicine/ume/ume-office/equality). This collaborative, community-based curricular project has resulted in a published toolkit to guide the clinical care of patients, successful applications for educational grant funding, and significant national and international dissemination of associated peer-reviewed scholarly work (https://ir.library.louisville. edu/medicine_ume/1). The eQuality integrated curricular development model is being applied to design curriculum targeting the opioid crisis, gun violence prevention, and health care disparities in medicine.

\section{Pedagogy}

The foundational basic and clinical sciences courses are taught in semester-long interdisciplinary classroom courses, with integration of content led by teams of $\mathrm{PhD}$ and $\mathrm{MD}$ thread and unit directors. These courses require continuous application to the diagnoses and training problems within the curriculum via weekly active learning sessions, including problem-based learning, peer teaching, application exercises, and team-based learning. Semester-long courses are specifically employed because a pilot of shorter modular courses led to an increased rate of student failure and decreased evidence of longitudinal learning. All student small-group learning placements are also longitudinal for at least a semester, up to 2 years, to create more effective opportunities for regular narrative evaluation of professionalism, interpersonal skills, self-directed learning skills, and communication skills by peers and faculty each semester in preparation for working on teams in clerkships. Each of these large classroom courses uses large summative unit examinations over several weeks of material and a cumulative NBME custom shelf exam at the end of each of the first 4 semesters. This layered approach with embedded formative and summative assessment cycles supports development of varied metacognitive strategies for students from varied educational backgrounds.

Basic and clinical science content is organized into units within these semester-long courses, optimizing integration and coordination of content.

- Students start year 1 with the Clinical Anatomy, Development, and Physical Examination courses, relating the developing human to observed anatomy in the dissection laboratory as well as the examination of living patients in the standardized patient clinic.
- In spring of year 1, students examine molecular, biochemical, genetic, immunologic, pharmacologic, microbiologic, and pathologic mechanisms in the Molecular Basis of Life, Defense, and Disease course to prepare them for organ system-based study of health and disease in year 2 .

- The first year concludes with a 2-week immersion in critical examination of the biomedical literature and clinical problem solving in the Biostatistics and Evidence-based Medicine course.

- Second-year students in the Human Systems in Health and Disease courses study the normal microanatomy and physiology of each organ system as well as its primary pathogens, pathology, and therapeutics.

- Integration of core content related to health care disparities, community health, and prevention of disease relevant to the topics under study occurs in each organ system, and each unit is overseen by an $\mathrm{MD}$ and $\mathrm{PhD}$ lead for optimal integration.

\section{Clinical experiences}

Four semester-long Introduction to Clinical Medicine courses run concurrently with the primary science courses, focusing on development of personal and professional identity, navigating health care disparities and cultural humility, an introduction to medical systems science, applied medical ethics, and basic and complex patient communication skills, including health coaching. Students practice emerging clinical skills in our novel longitudinal standardized patient (LSP) program. This program assigns the same LSP to a student for a full year, training students in continuity of care as well as intermittently requiring students to practice caring for a colleague's patients. This placement allows practice developing rapport and building the therapeutic relationship while providing a more consistent setting for monitoring student development and giving feedback about strengths and learning needs. All core basic and complex communication skills are embedded in a naturalistic manner, which includes 12 different characters of varying backgrounds who each have a 2-year biographical storyline. Students also practice using an electronic medical record during their year 1 and 2 LSP experiences, allowing feedback on informatics and clinical documentation skills as well as the assignment of specific evidence-based and clinical reasoning assignments. In addition to written assessments, assigned group work, and satisfactory completion of community preceptorships, students must pass a summative OSCE each semester assessing the clinical skills practiced with their SP over that period.

\section{Required and elective community-based rotations}

Clinical clerkships place students in a variety of inpatient and outpatient sites associated with the health sciences campus as well as community clinical partners and the distributed campus in Madisonville, Kentucky. The majority of students complete a clinical placement in a federally defined medically underserved area via the Kentucky Area Health Education Center grant program, and all Louisville campus students rotate at a Veteran's Administration clinical site, an urban referral specialty and trauma hospital, a freestanding children's hospital, and multiple community clinics and residential care 


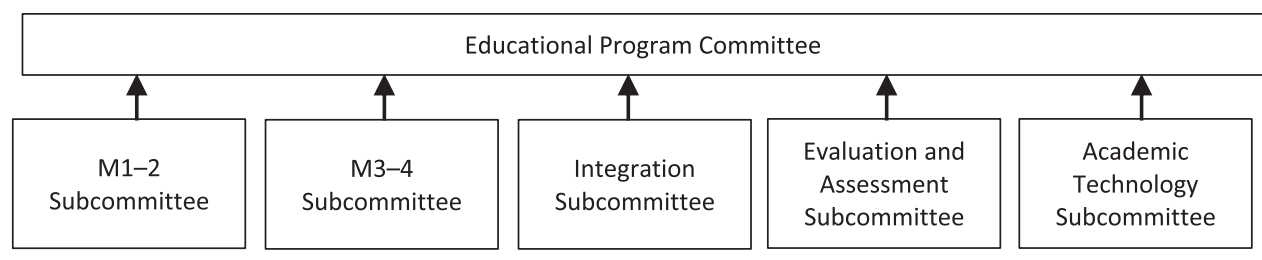

Figure 1 Educational Program Committee governance structure.

facilities. In addition, each clerkship includes independent study modules integrating basic science content as well as societal and health care systems content affecting clinical care. Clerkships use a combination of didactic, case-based, and online assignments to deliver the core curriculum, and each clerkship requires an NBME shelf examination and clinical evaluations assessing attainment of program objectives. Each clerkship requires students to pass a summative Step 2-style OSCE based on required diagnoses for that specialty and observed mini-CEX assessments for core clinical skills. At the end of year 3, students also take a multistation Step 2-style

OSCE assessing core skills for each required clerkship to progress in the program.

Fourth-year students generally position their acting internship early in the year and also use vacation time to study for and take Step 2 CK and CS. The design of this year maximizes flexibility and allows students desiring away rotations or multiple rotations in a subspecialty up to 4 months to explore these options. Students also use this elective time to complete and present original research or work with community and university centers to develop and implement health care-associated service and quality improvement projects. Additionally, many students elect to teach their junior colleagues in a variety of settings in the popular Medical Students as Teachers elective.

\section{Curricular Governance}

The EPC is responsible for the overall design, implementation, management, and evaluation of the medical student curriculum.

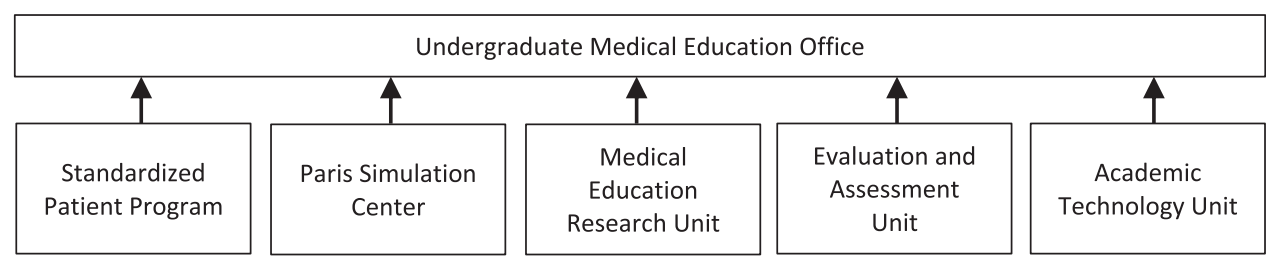

Figure 2 Undergraduate Medical Education Office organization.

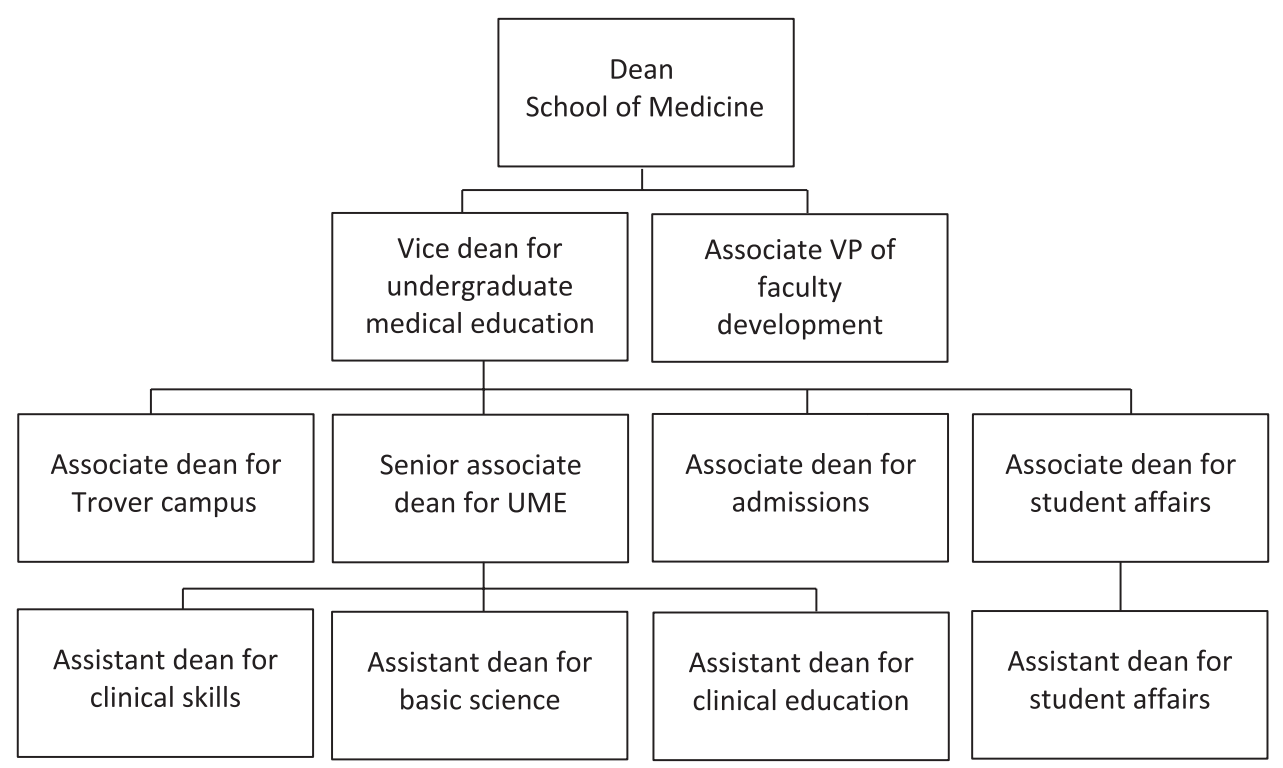

Figure 3 Organizational chart of dean's staff. 
See Figure 1-Educational Program Committee governance structure.

The EPC has the authority to oversee all curriculum-related matters and reports on such matters to the dean and Faculty Forum.

The Undergraduate Medical Education Office coordinates all academic support services for the undergraduate medical education program.

See Figure 2-Undergraduate Medical Education Office organization.

The vice dean for undergraduate medical education oversees admissions, student affairs, and the Undergraduate Medical Education Office.

See Figure 3-Organizational chart of dean's staff.
To support growth in teaching and learning throughout the Health Sciences Campus, the Faculty Development Office, with a new vice president-level position, was created in 2018. Improvements in faculty development as a result of this leadership include creation of a content library for clinical educators to improve the teaching abilities of busy clinical faculty and creation of the yearlong Leadership and Innovation in Academic Medicine program, which has won regional awards for its innovative programming and graduated $>30$ faculty to date. Faculty knowledge of current learning science continues to improve through book clubs and dedicated faculty development programs with a specific focus on diversity and wellness. Faculty with an interest in pursuing education as a career focus also have the ability to enroll in the interdisciplinary Certificate in Health Professions Education, a yearlong, accelerated course of postgraduate study dedicated to education science in the health professions. 


\title{
Louisiana State University School of Medicine in New Orleans
}

\author{
Robin English, MD, Joy Sturtevant, PhD, Richard DiCarlo, MD, and Steve Nelson, MD
}

\section{Medical Education Program Highlights}

Our medical education program is characterized by effective and ongoing collaborations between basic scientists and clinicians and between students and faculty. Unique features of our program include the following:

- We have strong central oversight of the curriculum by clinical and basic science faculty members in the Office of Undergraduate Medical Education (UME), who participate in the development and implementation of class sessions across all courses.

- Each systems course in the preclerkship curriculum is codirected by a clinician and basic scientist.

- We have a robust mechanism to provide feedback through the Aesculapian Society, a student-run organization that fosters ongoing communication between students and faculty. Students meet frequently with course directors to give feedback on individual course sessions and provide formal reports on courses and clerkships. Changes that are made in response to feedback are made public to students.

- Clinical and basic science faculty lead our student learning communities (houses). The houses are integral to both curriculum delivery (small-group seminars) and extracurricular development (mentorship and reinforcement of professional values).

- We enjoy strong support of the educational program from the dean. Resources to enable student learning include a new state-of-the-art team-based learning (TBL) classroom and an AV faculty training suite, in addition to materials for students to use in their courses and in USMLE Step 1 examination preparation. The dean also provides educational stipends for house faculty and has expanded faculty positions and administrative support in the Office of UME.

\section{Curriculum}

\section{Curriculum description}

The School of Medicine curriculum begins in August with a 1-week Introduction to the Profession course, followed by 3 courses: Gross Anatomy, Biochemistry, and Clinical Skills Integration, an introductory clinical skills course, in the fall. The spring semester includes cell biology and anatomy, physiology, medical genetics, medical immunology, foundations of population medicine and health systems, and human behavior and development, as well as continuation of Clinical Skills Integration.

Acad Med. 2020;95:S196-S198.

doi: 10.1097/ACM.0000000000003343

Copyright (C) 2020 by the Association of American Medical Colleges

Supplemental digital content for this article is available at http://links.Iww.com/ ACADMED/A866.

Year school was established: 1931.

School URL: https://www.medschool.Isuhsc.edu.
The second year is made up of the Foundations of Disease and Therapy course, followed by 8 systems courses: Diseases and Therapy of the Neuropsychiatric, Musculoskeletal/Dermatology, Hematologic, Pulmonary, Cardiovascular, Renal, Endocrine/ Reproductive, and Gastrointestinal Systems. A longitudinal Clinical Skills Integration course that focuses on literature evaluation and patient counseling runs throughout the year. Second-year students have opportunities to take a variety of elective courses and to shadow clinicians from a wide variety of specialties. The year ends with a synthesis experience that consists of case-based questions and review. Students take USMLE Step 1 after a period of study time, which is a requirement before they begin their clerkships.

Students begin their third year with a 1-week Clerkship Preparation course. They then rotate through 7 clerkships and an elective:

- Internal medicine: 10 weeks

- Surgery: 9 weeks

- Pediatrics: 8 weeks

- Obstetrics-gynecology: 6 weeks

- Psychiatry: 6 weeks

- Family medicine: 4 weeks

- Neurology: 3 weeks

- Career planning elective: 2 weeks

The fourth year includes 2 acting internships and a Critical Concepts course, which focuses on emergency and intensive care and simulation. Four electives are required, and 3 blocks are reserved for interviews and other activities. The fourth year ends with a 1-week Special Topics course, which includes sessions on internship, financial planning, and opioid prescribing.

\section{Curriculum changes since 2010}

In 2012, we underwent a significant change in the preclerkship curriculum from a completely departmentally based structure to the one described above, which is a hybrid of departmentally based courses and organ system-based courses. Additional changes included enhancement of the curriculum on cultural competency, literature evaluation, health systems, and clinical reasoning. One of the major improvements of the new curriculum was increased integration, both between various basic science disciplines and between basic science and clinical medicine. Another improvement was the expansion of opportunities to take electives in the second, third, and fourth years and the creation of greater scheduling flexibility for senior students.

Planned changes for the future include restructuring some courses to increase active learning opportunities and minor changes in sequencing of some courses. Our core basic science and clinical teaching faculty engage in frequent discussions to continuously improve individual teaching sessions and the curriculum as a whole. 


\section{Assessment}

The 28 medical education program objectives were revised as part of our recent curriculum change and are based on the ACGME competencies of patient care, medical knowledge, practice-based learning and improvement, systems-based practice, professional behavior, interpersonal relationships and communication, interprofessional collaboration, and personal and professional development. Changes in assessment since 2010 include customized NBME examinations in the first and second years, addition of TBL exercises that include individualized and group readiness assessment tests, and development of new graded written exercises.

See Supplemental Digital Appendix 1-Program Objectives and Assessment Methods—at http://links.lww.com/ACADMED/A866.

\section{Parallel curriculum or tracks}

- The Rural Scholars Track trains physicians to practice medicine in rural settings. The preclerkship curriculum for students in this track is identical to the other students' curriculum but with the addition of specific didactic activities relevant to rural medicine. Students in this track complete their third and fourth years at our regional campus at University Medical Center in Lafayette, Louisiana, and spend 1 day per week with a primary care preceptor.

- The Primary Care Track trains physicians interested in primary care. The preclerkship curriculum for students is in this is identical to the other students' curriculum but with the addition of specific didactic activities relevant to primary care. Students spend 1 half day per week with a primary care preceptor during their third and fourth years.

- The MD-PhD Track trains physician-scientists. Students in this track complete the preclerkship curriculum, take USMLE Step 1, and then pursue their $\mathrm{PhD}$. After successfully receiving their $\mathrm{PhD}$, they reenter the clerkships and complete their third and fourth years with other students.

- The MD-MPH Track is either a 4- or 5-year track. Students in the 4-year track begin MPH coursework in the summer before matriculation, and they attend MPH classes during the preclerkship phase of the curriculum. They complete a capstone project in their fourth year. Students in the 5-year track complete their MPH degree between the preclerkship and clerkship phases of the curriculum.

\section{Pedagogy}

We use a variety of pedagogical approaches to achieve our medical education program objectives. We use recorded and live lectures and readings to deliver content. We have implemented approaches to reduce lecture time since 2010, including TBL, use of audience-response systems, and addition of active learning sessions. We use laboratory sessions and simulations for students to apply their knowledge to clinical situations. We use role play for students to practice clinical skills of history taking, clinical reasoning, and communication.

\section{Clinical experiences}

Our students see a very diverse patient population. We place students in ambulatory and inpatient settings in a 1-week preceptorship after their first semester. Second-year students are required to participate in a longitudinal clinical elective and provide a written reflection of their experience. Students complete their clinical clerkships in ambulatory clinics and inpatient units at partner hospitals across the state, including regional campuses in Baton Rouge and Lafayette. Our primary partner hospitals in New Orleans are part of the Louisiana Children's Medical Center corporation and include University Medical Center, Children's Hospital, and Touro Infirmary. Our students also complete rotations at our Veterans Administration medical center.

\section{Curricular Governance}

Three curriculum committees have various roles in the administration of our curriculum. All committees have representation from the general faculty and students. Their main responsibilities are described below.

Curriculum Steering Committee:

- Oversees curriculum changes and approves recommendations from the Curriculum Renewal Committee

- Develops and revises program objectives

- Monitors all educational program outcomes

- Reviews major findings from the Course Evaluation Committee

Curriculum Renewal Committee:

- Develops and revises curriculum structure and content

- Oversees preclerkship and clerkship director committees

- Ensures that course objectives are linked to educational program objectives

- Ensures that pedagogical methods are appropriate to meet course and program objectives

- Approves changes in pedagogy, objectives, and course hours

- Engages in continuous quality improvement of the curriculum

Course Evaluation Committee:

- Reviews all course and clerkship evaluations that are prepared by the Aesculapian Society

- Establishes ad hoc committees to perform in-depth evaluations of courses and clerkships

\section{Education Staff}

All preclerkship courses are centrally administered by the Office of UME, with oversight by the assistant dean for UME and 2 basic science curriculum codirectors. The Office of UME has 3 full-time coordinators who provide administrative support to curriculum management and who assist course directors. Clerkships are administered by departments with central oversight by the assistant dean for UME, the Clerkship Directors' Subcommittee, and 3 clinical curriculum codirectors.

Content directors in the disciplines of pathology, pharmacology, and microbiology work with faculty in the Office of UME and systems-based course directors to ensure adequate content coverage across courses. Faculty members in the Office of UME 


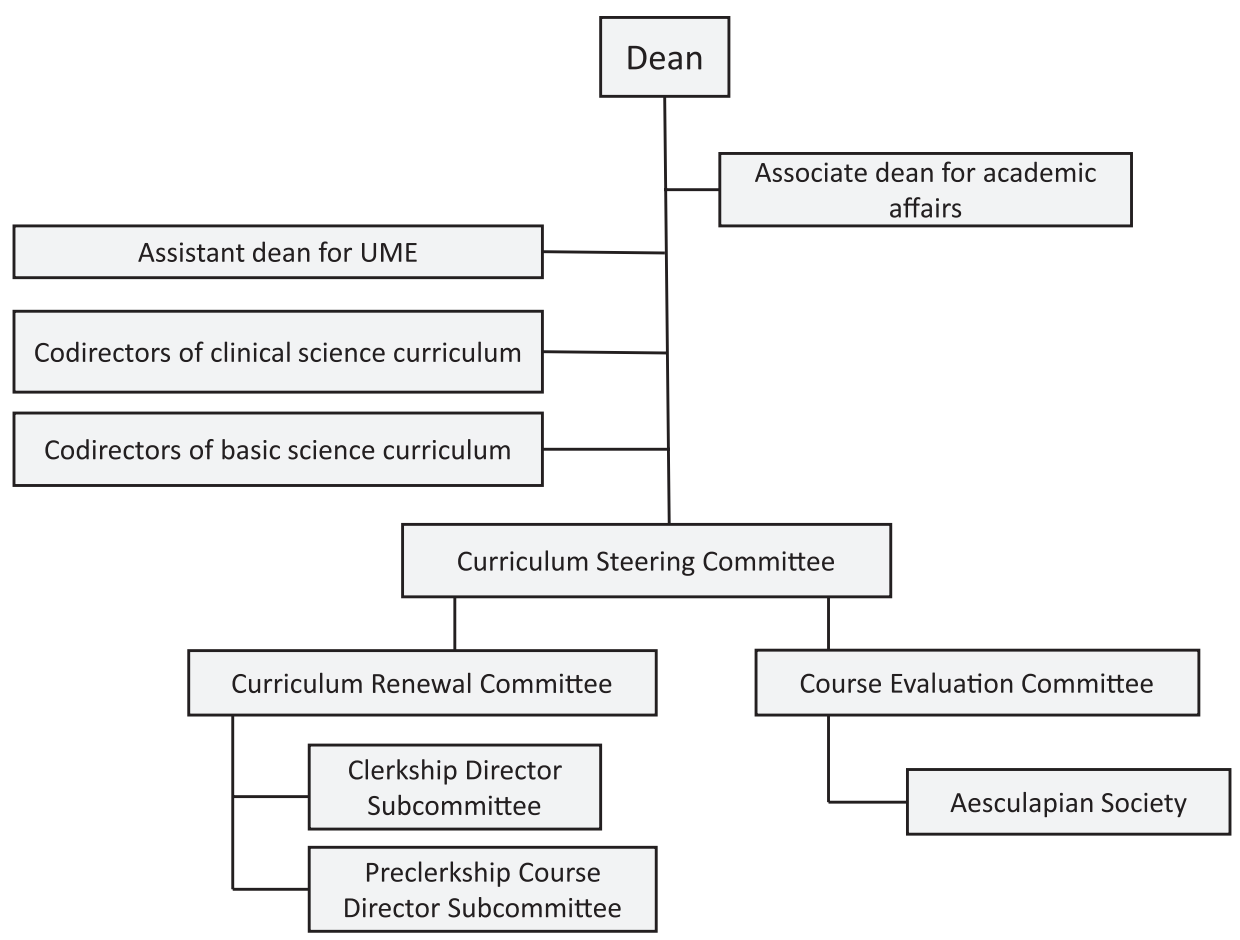

Figure 1 Organizational chart.

also work closely with basic science and clinical faculty in the development of teaching sessions across all courses to optimize integration of basic science with clinical medicine.

Areas outside of educational programming, such as student affairs, are handled by a separate office.

See Figure 1-Organizational chart.

\section{Faculty Development and Support in Education}

We provide professional development for our faculty through internal and external faculty development sessions, including sessions on TBL and innovative teaching techniques. In addition, invited speakers educate our faculty on issues such as resilience

\section{Table 1}

\section{Regional Medical Campuses}

\begin{tabular}{lll} 
Regional campus name & Type & $\begin{array}{l}\text { Student } \\
\text { enrollment }\end{array}$ \\
$\begin{array}{l}\text { Our Lady of the Lake Hospital in } \\
\text { Baton Rouge, Louisiana }\end{array}$ & Clinical & $\begin{array}{l}24 \text { per year for } \\
\text { years } 3 \text { and } 4\end{array}$ \\
$\begin{array}{l}\text { University Medical Center in } \\
\text { Lafayette, Louisiana }\end{array}$ & Clinical & $\begin{array}{l}\text { 6-10 per year for } \\
\text { years } 3 \text { and } 4\end{array}$ \\
\hline
\end{tabular}

and wellness. Teaching contributes significantly to the promotion and tenure process, especially for those on the tenure and full clinical tracks. Educator portfolios are used in the process. There is currently no independent educator track, but discussions regarding this are ongoing.

In an ongoing effort to improve the overall quality of the educational environment for our students, we initiated a pilot program to provide peer-peer feedback for lecturers. The aim was to provide constructive feedback to improve all lecturers' teaching skills. While the pilot program is still in progress, a feedback survey sent to year 1 reviewees resulted in a positive response to a feedback survey questioning confidentiality, bias, and constructiveness of critiques.

\section{Regional Medical Campuses}

See Table 1-Regional Medical Campuses.

The educational experiences are consistent across sites. Site directors for all clerkships are members of our Clerkship Directors' Subcommittee, and they collaborate and communicate with clerkship directors at our main campus in New Orleans. The assistant dean for UME travels to both sites regularly to meet with site directors and provide support as needed. 


\title{
Louisiana State University Health School of Medicine-Shreveport
}

\author{
Christi Rinaudo, EdD, Lucy Robinson, PhD, and Jeff Howells, MS, MBA
}

\section{Medical Education Program Highlights}

- Focus on self-directed learning: First-year students participate in an "immersion" project as a part of the Foundations of Clinical Medicine course, designed to expose students to the clinical and social sides of medicine and focusing on selfdirected learning. Students assess their personal learning needs, select educational activities to meet those needs, and practice skills for continued learning. The medical education elective for fourth-year students also focuses on self-directed learning. It is to involve students in a variety of educational activities to advance their teaching skills. Students create a scholarly project incorporating skills learned in the medical education elective to improve education at LSU Health-Shreveport.

- Early patient contact: First-year students experience their first patient interviews (standardized patient) at the end of orientation week and interact weekly with physicians and patients illustrating the clinical importance of basic sciences throughout Module I, the first semester of preclinical classes. During the remainder of the preclinical curriculum, the Foundations of Clinical Medicine course provides numerous opportunities to interview standardized patients and clinic/ hospital patients.

- Fourth-year online electives: A variety of online electives meet diverse educational needs of these students. The electives allow flexibility in scheduling, particularly during residency interview season, while students further develop medical knowledge and skills. Topics include health system science, nutrition, and a series of reviews on EKGs, genetics, dermatology, and radiology.

- Distinction tracks: In 2014, the Medical Student Research Program (MSRP) was expanded to include the Research Distinction Track (RDT), which offers students an indepth research experience and recognition for their efforts. Expectations for students include participating in dedicated research outside of the regular curriculum, presenting their work on at least 2 occasions, developing and submitting a manuscript for publication, preparing a written grant proposal, and participating in ethical research training. In conjunction with RDT, a student-run peer-reviewed journal (American Medical Student Research Journal) was created as a platform for student publications. LSU Health-Shreveport maintains the MSRP.
Acad Med. 2020;95:S199-S202.

doi: 10.1097/ACM.0000000000003336

Copyright (C) 2020 by the Association of American Medical Colleges

Supplemental digital content for this article is available at http://links.Iww.com/ ACADMED/A860.

Year school was established: 1968.

School URL: https://www.Isuhs.edu/our-schools/school-of-medicine.
- In 2018, a humanism and service learning distinction track was added to develop collaborative leadership skills through meaningful long-term service learning projects. Expectations include the completion of 150 hours of service learning; a community needs assessment; self-reflection essays; and a capstone project, presentation, and manuscript submission. Students who complete all aspects of the Research Distinction or Humanism and Service Learning Distinction tracks receive recognition in medical student performance evaluations and on diplomas.

\section{Curriculum}

\section{Curriculum description}

See Supplemental Digital Appendix 1-Curriculum Description—at http://links.lww.com/ACADMED/A860.

\section{Curriculum changes since 2010}

- Pass/fail grading: In 2016, pass/fail grading was instituted for the preclinical curriculum. The Medical Curriculum Council (MCC) approved this change to improve student well-being and academic outcomes. The pass/fail system was incorporated into all clinical clerkships during the 2019-2020 academic year.

- Use of technology for assessment and formative feedback: Since 2010, technology has been increasingly used and incorporated. In addition to increasing simulation technology to teach and assess clinical skills, ExamSoft permits faculty to provide students with formative feedback on their understanding of course material, including rationale for questions presented and breakdown of assessment performance by topic or learning outcome.

- Enhanced emphasis on critical thinking skills: In 2014-2015, LSU Health-Shreveport's quality improvement committee identified the need for greater emphasis on critical thinking skills. A variety of educational activities were developed to improve students' understanding and use of biostatistics, critical appraisal of research literature, and understanding of research design. In the preclinical years, direct instruction of biostatistics increased, including a problem-solving exercise focused on epidemiology. Journal club discussions were added to each of the required third-year clinical clerkships. In addition, an evidence-based medicine capstone project was incorporated into a required fourth-year course. Initiatives focused on improving students' abilities to incorporate evidence-based medicine into their practice of medicine.

\section{Class size changes}

Class size has increased by approximately 50 students since 2010 . No major changes aside from facility renovation have taken place. A new medical education building is being planned to accommodate future class size increases. Currently, the school 
is recruiting faculty to increase preceptors and lecturers to accommodate class size increases.

\section{Assessment}

See Supplemental Digital Appendix 2-Medical Education Program Objectives—at http://links.lww.com/ACADMED/A860.

LSU Health-Shreveport's program objectives are based on ACGME domains of competence:

- Interpersonal and communication skills

- Professionalism

- Patient care

- Medical knowledge

- Practice-based learning and improvement

- System-based practice

\section{Assessment changes since 2010}

- Pass/fail grading implemented in 2017; among the goals was to decrease student anxiety and improve well-being

- Weekly online quizzes as formative assessment in Module I, with a cumulative quiz preceding Exam I to demonstrate the need for learning instead of memorization

- ExamSoft used for exams and quizzes in all preclinical courses, with postexam reviews of missed questions (formative component)

- A rubric based on ACGME competencies was developed for Module I small-group student assessment. Students complete self-evaluation forms halfway through the semester and meet individually with small-group mentors to discuss selfevaluations.

- An evaluation rubric based on ACGME competencies was developed for all student assessments in third-year clerkships.

\section{Parallel curriculum or tracks}

LSU Health-Shreveport offers an MD-PhD dual-degree program. The medical school curriculum is identical for the dual-degree track, and the $\mathrm{PhD}$ program varies by research area; coursework is limited to advanced courses. Upon completion of preclinical requirements, students matriculate into the $\mathrm{PhD}$ program and complete 5 credit hours of advanced courses in the desired area of study while conducting a research project. Upon successful completion of $\mathrm{PhD}$ research and thesis defense, students review medical knowledge and reenter the MD program, starting third-year clerkships.

LSU Health-Shreveport maintains an RDT for medical students to provide in-depth experience and recognition for carrying out significant research during medical school. The curriculum and duration of coursework/clinical work are identical for these students and all medical students.

LSU Health-Shreveport does not offer a 3-year MD degree program, nor are there plans to develop this for future students.

\section{Pedagogy}

Pedagogical approaches in the preclinical years include lectures (all courses), laboratory sessions (primarily anatomy, also pathology), weekly problem-based learning small-group sessions, case-based learning sessions, self-directed learning, clinical experiences, and standardized patient encounters. Module I also includes weekly patient presentations illustrating the clinical importance of basic sciences throughout the preclinical curriculum. All lectures not involving patients are podcast for real-time use and/or later study.

Pedagogical approaches in the clinical years include both ambulatory and inpatient clinical experiences, lectures, and online learning modules. All required clerkships use combined didactics, small-group learning, and direct patient care (inpatient and outpatient) to enhance student learning. In addition, several clerkships include a standardized patient encounter. Fourth-year courses employ clinical experiences, preceptorships, and selfdirected learning.

The only curricular change peculiar to a parallel track has been to decrease coursework in the $\mathrm{PhD}$ program for $\mathrm{MD}-\mathrm{PhD}$ students. The requirement changed to consider the MD preclinical curriculum comparable to introductory and core graduate school courses, so students matriculating into the $\mathrm{PhD}$ portion of the dual-degree program complete only advanced courses in their chosen department.

\section{Clinical experiences}

Third- and fourth-year clinical experiences are available within the university setting, the Overton Brooks VA Medical Center, Ochsner LSU Health-Monroe, and as community-based medicine outpatient experiences with practicing physicians (designated and approved by the university) in Shreveport and surrounding communities or with affiliated hospitals.

\section{Required longitudinal experiences}

The third-year family medicine clerkship provides a longitudinal patient care experience for students. The clerkship is organized into three 2-week blocks scheduled at specific intervals. During the clerkship, students see and follow patients through the Family Medicine Comprehensive Care Clinic. Every student participates in a minimum of 20 patient encounters. Students demonstrate ownership of their patients by following up with patients on lab results and medical recommendations. Students are responsible for scheduling patients for return clinic visits to ensure continuity of care.

\section{Clinical experience first encounter}

First-year students experience their first patient interviews (standardized patient) at the end of orientation week and interact weekly with physicians and patients illustrating the clinical importance of basic sciences throughout Module I. During the remainder of the preclinical curriculum, Foundations of Clinical Medicine provides numerous opportunities to interview both standardized patients and clinic/hospital patients.

\section{Required and elective community-based rotations}

Some clinical experiences in the third and fourth years are available at Overton Brooks VA Medical Center, Ochsner LSU 
Health-Monroe, community-based physicians in medical practice in Shreveport and surrounding communities, or an LSU Health-Shreveport affiliated hospital outpatient service that is staffed by LSU Health-Shreveport faculty. All third-year students rotate at LSU Health-Monroe, and with community preceptors during clerkships. Fourth-year students participate in a required ambulatory care experience at Overton Brooks VA Medical Center, Ochsner LSU Health-Monroe, Family Practice in Alexandria, or an LSUHSC-S affiliated hospital. Availability of these elective sites varies by medical specialty.

\section{Challenges in designing and implementing clinical experiences} for medical students

The primary challenge in implementing clinical experiences has been in identifying and training faculty preceptors to meet the needs of our increasing student population. Currently, the medical school, in conjunction with our hospital partner, is recruiting faculty to increase numbers of preceptors and lecturers.

\section{Curricular Governance}

The MCC has responsibility and authority to oversee the curriculum for the undergraduate medical students. The MCC is responsible for evaluating programs and course objectives, approving and coordinating course schedules, recommending changes to course scheduling and objectives, and designing experiments in medical education to improve methods of teaching and evaluation of student performance, as necessary.
The MCC represents the faculty and reports directly to the dean of the medical school. The MCC consists of the associate dean for academic affairs, who serves as chair; 2 elected faculty council nominees ( $1 \mathrm{MD}, 1 \mathrm{PhD}) ; 2$ administrative council members (1 MD, $1 \mathrm{PhD}) ; 4$ at-large members selected by the dean; a representative of the Office of Academic Affairs; and the chairs of the major curriculum subcommittees.

See Figure 1-Organizational chart.

\section{Education Staff}

\section{Medical education leadership}

The Office of Academic Affairs is the central source for support and leadership. Led by the senior associate dean for academic affairs, the office is responsible for all curriculum-related areas: program outcomes assessment, curriculum mapping, course evaluations, education and assessment resources for students and faculty, educational facilities oversight, academic computer services, faculty development, and support for the MCC. Currently, office personnel include an associate dean for academic affairs, 2 full-time PhD/EdD educators, 2 technical support analysts, and 2 administrative assistants.

The primary role of the Office of Academic Affairs is developing and implementing the undergraduate medical curriculum. Personnel work closely with representatives from administrative offices, including student affairs and graduate medical education, but are not responsible for services beyond the undergraduate program.

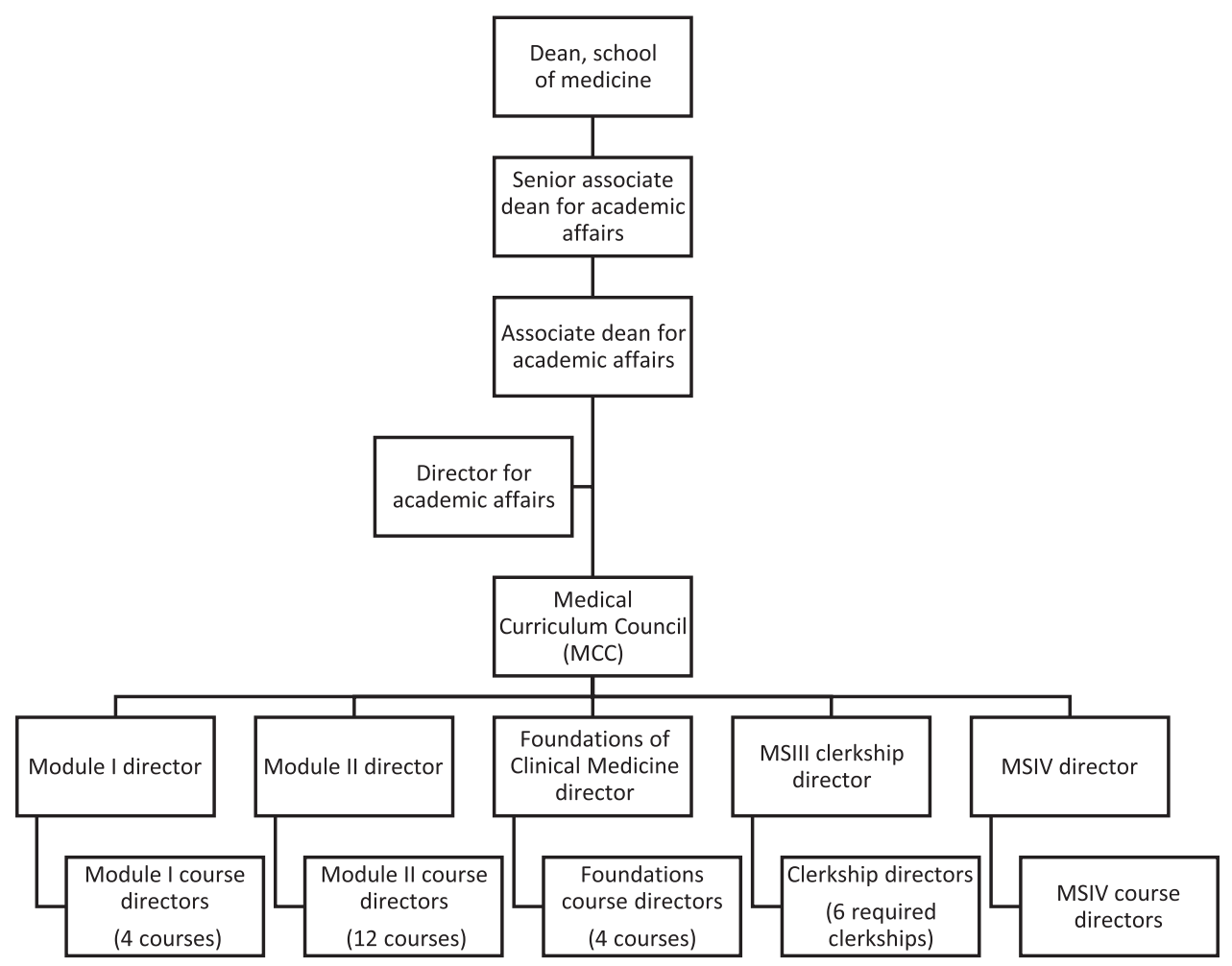

Figure 1 Organizational chart. 


\section{Department of Medical Education}

LSU Health-Shreveport does not have an academic department of medical education. However, planning is underway to create a Department of Medical Education to serve as the academic home of key teaching faculty and offer joint faculty appointments for other medical educators.

\section{Faculty Development and Support in Education}

- Grand rounds sessions cover individual topics in education and curriculum development throughout each year. The Health Sciences Library provides training and resources for faculty in computer programs to assist with lecture presentations.

- Administrative and technical personnel of the Clinical Skills Center are familiar with adult learning theories and apply those principles when demonstrating/teaching faculty how to use simulation equipment. They are involved in designing new scenarios and the ongoing evaluation of existing simulation.
- LSU Health-Shreveport funds faculty with greater-than-average involvement in education to attend educational conferences of their choice, such as the annual Association of American Medical Colleges meeting, so that attendees can bring information on current teaching and evaluation techniques back to the institution.

- Some LSU Health-Shreveport departments maintain mentoring programs to instruct new faculty on teaching basics, especially for didactic lectures.

\section{Role of teaching in promotion and tenure decisions}

Promotion and tenure require participation in education. The educator's portfolio documents types of teaching, student evaluation of teaching quality, and time commitment to teaching over the years of service. LSU Health-Shreveport also recently developed an education track for faculty, having excellence in education as the primary determinant. For this track, excellence in teaching and educational scholarship, demonstrated by leadership in professional education organizations and publication of education-focused manuscripts, are documented in the educator's portfolio. 


\section{Tulane University School of Medicine}

N. Kevin Krane, MD, and Jennifer W. Gibson, PhD

\section{Medical Education Program Highlights}

Tulane University School of Medicine matriculates students from all 50 states, with graduates entering residency programs across the United States in almost every field of medicine. Tulane's curriculum provides students with opportunities that ensure they are well prepared both as applicants and as first-year house officers, regardless of specialty area. The school focuses on community engagement, offering both required and elective programs that emphasize this core value. Unique features of the educational program include:

- 4-year dual-degree programs: For decades, Tulane has graduated 30-40 students per year with MD-MPH degrees and now provides a similar 4-year MD-MBA program.

- Community engagement: All Tulane students participate in community activities through the service-learning requirement (20 hours each in years 1 and 2), the fourth-year community health clerkship focused on cultural competence and social determinants of health, and extensive community clinic opportunities. Under faculty oversight, students at Tulane School of Medicine are also engaged in 27 different volunteer opportunities at 13 locations across greater New Orleans, operating out of homeless shelters, substance abuse rehabilitation centers, and community centers to provide medical care to community members who are particularly vulnerable and have the least access to care.

- Early elective experiences: To provide opportunities for students to consider all aspects of their education and/or participate in a dual-degree program, Tulane offers elective time in the preclinical curriculum 2 afternoons per week beginning in the second semester of year 1. Students use this time to participate in clinical, research, and other healthrelated activities.

- Student engagement in the medical education program is extensive. As a result, there is an atmosphere of cohesiveness and cooperation with both faculty and peers that permeates the student experience and is an essential element of why students choose Tulane.

\section{Curriculum}

\section{Curriculum description}

See Supplemental Digital Appendix 1-Curriculum Overviewat http://links.lww.com/ACADMED/A867.

Acad Med. 2020;95:S203-S205.

doi: 10.1097/ACM.0000000000003340

Copyright (C) 2020 by the Association of American Medical Colleges

Supplemental digital content for this article is available at http://links.Iww.com/ ACADMED/A867.

Year school was established: 1834

School URL: https://medicine.tulane.edu.

\section{Curriculum changes since 2010}

The preclinical curriculum has been significantly reorganized through an evolutionary process whereby independent courses have moved to clearly defined systems-based modules that coordinate and integrate content across basic science disciplines. The overall curriculum is now organized into 2 phases.

- Phase 1 , which is primarily normal structure and function, now begins with the Gross, Developmental, and Radiologic Anatomy course and Foundations in Medicine course. These are followed by systems-based modules, which contain physiology, biochemistry, genetics, and histology.

- Phase 2 now begins in the spring of year 1 and is a series of systems-based modules focused on pathophysiology and disease states, finishing with the recently implemented Brain, Mind, Behavior course that integrates basic and clinical neurological and behavioral science. Students begin clinical rotations in May of year 2.

While the traditional core clerkships in year 3 have not changed, clinical opportunities have dramatically expanded with the recent openings of both large public and Veterans Administration (VA) hospital systems in the medical center. In addition, almost all clerkships now offer community-based sites both locally and regionally that are highly requested by students. Tulane created a community health clerkship focused on social determinants of health and health care disparities required of all students, except those obtaining a dual MD-MPH degree. A transition to residency program will be implemented, initially as an elective, in 2020. Tulane is also planning to completely review its current curriculum in 2020 and anticipates that significant changes to enhance integration, lifelong learning, and clinical training will be forthcoming.

While class size is currently the same as it was in 2010 (190 students), several trial programs, including a new regional campus, were implemented during this time period resulting in a class size that grew to 210 . These programs have been discontinued but significant lessons were learned as a result. While Tulane no longer has a regional campus, the development of the clinical faculty and sites was very successful and continues to provide vital opportunities for students in third-year clerkships.

\section{Assessment}

Tulane's educational program objectives are based on the Physician Competency Reference Set, which includes 8 competency domains and associated outcome-based competency lists. A ninth competency domain, community engagement and service, was added to reflect Tulane's commitment to health equity and public service. 
Significant efforts have been made to enhance student assessment and feedback since 2010. These include:

- Incorporation of an extensive faculty development program, most of which focuses on improved written and verbal feedback to both preclinical and clerkship students

- Newly implemented policies that require written feedback in areas that had not traditionally done so, including narrative assessments by anatomy lab directors and feedback from community preceptors

- Peer evaluation for team-based activities

- Inclusion of formal, self-directed learning (SDL) activities to assess identification, analysis, and synthesis of relevant information, as well as appraisal of the credibility of information resources

- Addition of new full history/physical clinical skills assessments at the end of years 1 and 3

- Administration of all internal and NBME examinations electronically

- Administration of one final exam after each systems-based module in years 1 and 2 that incorporates all disciplines teaching in that module

- Administration of cumulative, customized NBME shelf exams at the end of years 1 and 2

See Supplemental Digital Appendix 2-Program Objectives and Assessment Methods—at http://links.lww.com/ACADMED/A867.

\section{Pedagogy}

As with the curriculum, the pedagogical approaches to achieve educational objectives over the past 10 years have evolved. Lectures are still common, but Tulane has newer educational facilities that are designed for team-based activities including case-based learning (CBL), team-based learning (TBL), SDL, and peer teaching. Clinical experiences begin early in year 1 with preceptorships and are supported by extensive standardized patient and simulation programs, both of which are also heavily used in the clinical years.

Since 2010, the most important change has been an increase in pedagogy that advances active learning. While lectures still constitute just under $50 \%$ of the preclinical curriculum, there have been significant modifications to make them more interactive, including the use of large-group discussion, "clickers," and "just-in-time teaching." Many team-based pedagogical approaches, including CBL, TBL, and SDL, all with peer evaluation, have replaced traditional didactic lectures.

\section{Clinical experiences}

Tulane uses a wide range of public and private inpatient and outpatient clinical sites for educational experiences. These sites include traditional academic settings, community-based hospitals and physician offices, and a network of rural placement sites throughout the Gulf South.

\section{Clinical experience first encounter}

Students encounter clinical experiences within the first month of medical school. This exposure begins with learning medical interviewing with standardized patients in the Foundations in Medicine course. Students are also assigned to community preceptors and can participate in service-learning activities at this time.

\section{Required and elective community-based rotations}

All core clerkships except internal medicine assign limited numbers of students to a variety of required inpatient and outpatient community-based sites. Many of these sites include community hospitals and clinics located in the greater New Orleans area, where students work directly with a communitybased physician. However, there are inpatient sites located across the state of Louisiana and at the Biloxi, Mississippi, VA Hospital, all of which provide housing. In family medicine, the majority of students are assigned to individual rural ambulatory practice locations across the Gulf South. The required community health clerkship also assigns students to local health care agencies; however, there is no direct patient care.

\section{Challenges in designing and implementing clinical experiences for medical students}

The major challenge is ensuring enough meaningful clinical volume and experience for all students, especially in pediatrics, obstetrics, and psychiatry, although this has improved with the opening of new public and VA hospitals. To address this concern, Tulane uses 20 inpatient clinical sites, requiring significant logistical support, including housing for students at distant sites. This requires significant effort to ensure consistency of achieving objectives, comparability of required clinical experiences, effective student feedback, and necessary professional development for all distant faculty. This process is complicated by the realistic demands on both full-time and volunteer faculty for clinical productivity, particularly in the outpatient setting.

\section{Curricular Governance}

\section{Decentralized curricular governance}

While the overall curriculum, including individual courses and the systems-based modules, is overseen by the Curriculum Committee, individual course and clerkship grades remain. Course and clerkship directors are chosen, typically in consultation with academic affairs personnel, by the individual departments. Most funds for educational support are provided directly to each basic and clinical science department from the dean's office, and individual assignments of faculty and support for their efforts are provided at the department level.

See Figure 1-Curricular governance committees.

\section{Education Staff}

The Office of Academic Affairs and the Office of Medical Education (OME) are the organizational loci of administrative and academic support for the planning, implementation, evaluation, and oversight of the curriculum and for the development and maintenance of the tools to support curriculum delivery, monitoring, and management. The OME 
has a $\mathrm{PhD}$ director, assistant director, and learning specialist as well as 7 full-time support staff.

See Figure 2-Organizational chart.

The OME supports undergraduate medical education by providing resources to faculty, students, and administration in the areas of curriculum development, assessment and evaluation, faculty development, educational technology, and educational research. The OME is not responsible for areas outside of educational programming.

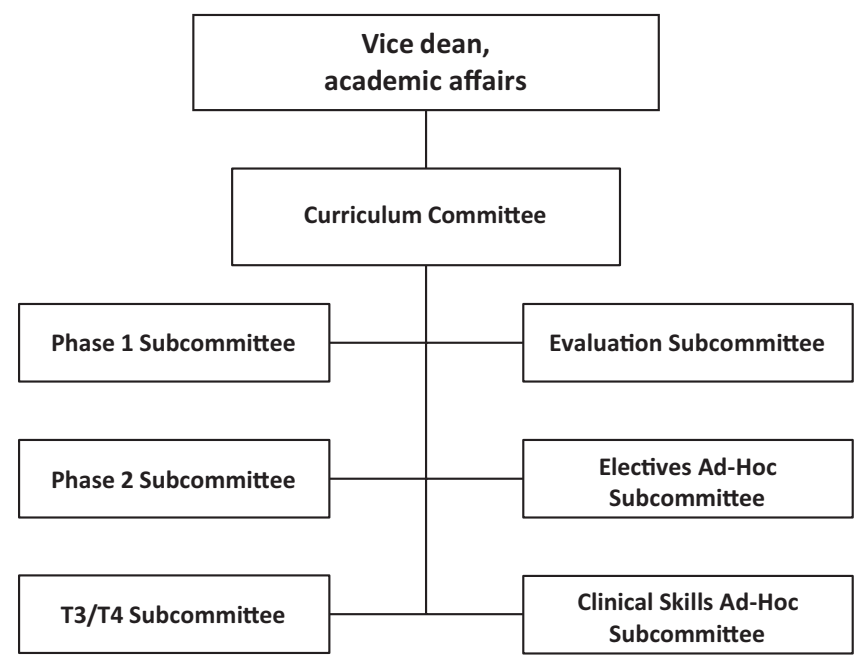

Figure 1 Curricular governance committees.

\section{Faculty Development and Support in Education}

Professional development for faculty as educators

The OME is responsible for creating and organizing faculty development programming in education. Faculty development opportunities are generally provided $1-3$ times per month and include webinars, educational roundtables, innovation seminars, journal club, and half-day workshops. In addition, the OME maintains an extensive faculty development website with teaching resources.

\section{Role of teaching in promotion and tenure}

All faculty are required to participate in teaching and/or education activities; therefore, this is taken into consideration in all tenure and promotion decisions. Teaching and educational

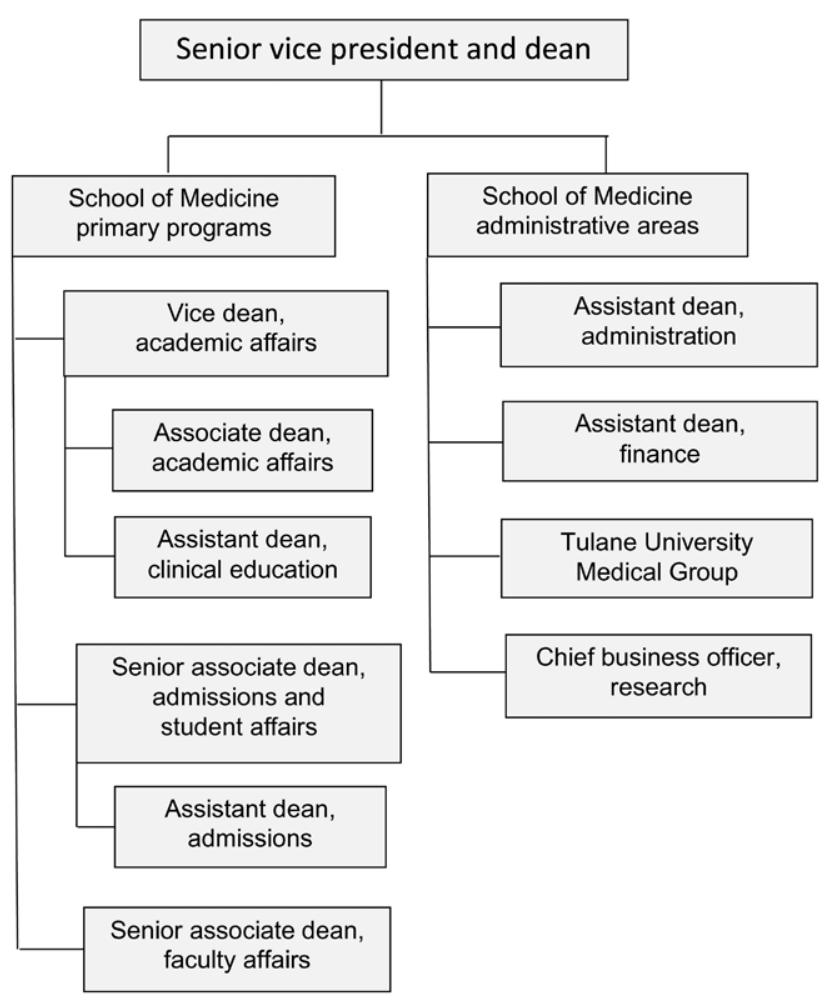

Figure 2 Organizational chart.

activities play a particularly important role in promotion decisions on nontenure tracks. Tulane provides a defined education track for promotion of faculty who spend more than $50 \%$ of their time on educational activities and who view education as their primary academic focus. While quality and quantity of teaching are important, scholarship and service are expected and candidates should have a strong regional reputation as a leader in medical education. Criteria include the development of innovative teaching methods, curricula, educational policy, or educational assessment tools. Candidates are expected to publish influential first or senior author publications related to education or have developed educational materials adopted for use regionally or nationally.

Tulane's Society of Teaching Scholars comprises full-time faculty members who have demonstrated a significant record of educational scholarship and an ongoing commitment to enhancing the quality of teaching and learning at the School of Medicine. Only one award is bestowed each year. 


\title{
The Johns Hopkins University School of Medicine
}

\author{
Nancy A. Hueppchen, MD, MSc, Roy C. Ziegelstein, MD, and Janet D. Record, MD
}

\section{Medical Education Program Highlights}

Johns Hopkins University School of Medicine (JHUSOM) launched the Genes to Society (GTS) curriculum in 2009 to address a paradigm shift in medicine, acknowledging variation in individual health and illness resulting from multiple societal, genetic, and physiologic factors.

See Figure 1-Genes to Society paradigm.

The GTS curriculum includes novel features that have been continued at JHUSOM and have become standard components of medical school curricula elsewhere. These include horizontal strands (scientific, social, or clinical themes covered across the 4 years)and transition courses that assist with matriculation, entry into clinical care, and passage to internship. The integrated curriculum was built on a framework allowing the curriculum to evolve as students, medical knowledge, the practice of medicine, and societal needs evolve.

Specific features include:

- Rich experience in understanding social determinants of health: communication skills, health behaviors, wellness and pathology, and collaboration with interprofessional teams at institutional and community levels. This experience takes advantage of our location in a culturally diverse urban setting that, with the institution's global network, provides opportunities to care for a largely underserved population.

- Personalization and individualization of educational pathways through:

- Primary care leadership track (PCLT) or global health leadership track (GHLT)

- Scholarly concentrations in basic science, clinical research, history of medicine, public health research, and HEART (humanism, ethics, education, the arts of medicine)

- Selectives within Foundations of Public Health and during some core clerkships

- Choice of ambulatory or critical care for advanced clerkship requirement

- Selections among Translational Science courses each quarter

- Multiple local subinternship and electives opportunities (domestic and international)

- Additional degrees (MD-PhD program, and master's degrees offered in public health, medical informatics, biomedical

Acad Med. 2020;95:S206-S210.

doi: 10.1097/ACM.0000000000003480

Copyright (C) 2020 by the Association of American Medical Colleges

Supplemental digital content for this article is available at http://links.Iww.com/ ACADMED/A969.

Year school was established: 1893.

School URL: https://www.hopkinsmedicine.org/som. engineering, business or health care administration, history of medicine, bioethics, and medical education)

- The Colleges Advisory Program (CAP) assigns students to 1 of 4 colleges at matriculation ( 5 students/advisor/class). Advisors teach basic communication and clinical skills, emphasizing patient-centered care. CAP provides longitudinal guidance for academic success and career planning.

- Intentional connections between basic sciences and clinical medicine with Topics in Interdisciplinary Medicine (TIME, 4-day courses interspersed in years 1 and 2) and Translational Science courses (4-day courses following each core clerkship designed to reenergize students in considering clinical applications of basic science research as they reflect on clerkship experiences).

\section{Curriculum}

\section{Curriculum description}

The GTS curriculum is organized around the GTS paradigm, with additional details at https://www.hopkinsmedicine.org/ som/curriculum/genes_to_society/curriculum-overview.html.

See Supplemental Digital Appendix 1-Curriculum Map—at http://links.lww.com/ACADMED/A969.

\section{Curriculum changes since 2010}

On top of our CQI process, a formal curriculum review in AY 2014-2015 identified opportunities to enhance the GTS curriculum: prioritizing actions in competency-based education, content, and further integration; use of content-appropriate pedagogy; inclusion of new topics in medicine; and additional student assessment and program evaluation tools.

Changes since implementation of the GTS curriculum include:

- Identifying emerging topic gaps in interdisciplinary and translational medicine-high-value care, health informatics, regenerative medicine, and genomic medicine-for which new TIME and Translational Science courses were developed

- Identifying a need to enhance clinical experiences in ambulatory medicine, leading to development of a new advanced clerkship in adult ambulatory medicine and increased ambulatory experiences in core clerkships

- Recognition that 8 of 20 original horizontal strands were wellintegrated into the standard curriculum, and reorganization of remaining strands into 3 overlapping content areas: biomedical sciences, health systems sciences, and culture of medicine

\section{Assessment}

- The Physician Competency Reference Set forms the basis for medical education program objectives.

- We have increased use of formative assessments in years 1 and 2 through formative questions embedded in e-lectures and 


\section{Scholarly Concentrations:}

Seminar series and guidance through
mentored scholarly project:
Basic Science
Clinical Research
History of Medicine
Humanism, Ethics, Education, \& the
Arts of Medicine
Public Health Research
Topics in Interdisciplinary Medicine
10 Intersessions in Year 1-2:
Health Care Disparities
Obesity, Nutrition, \& Behavior Change
Global Health
High Value Healthcare
Disaster Medicine
Clinical Informatics
Pain Care
Substance Use Disorders
Patient Safety
End of Life \& Palliative Care
6 Intersessions in Year 3-4:
Translational Science:
Infection
Regenerative Medicine
Genomics
Immunology
Metabolism
Cancer

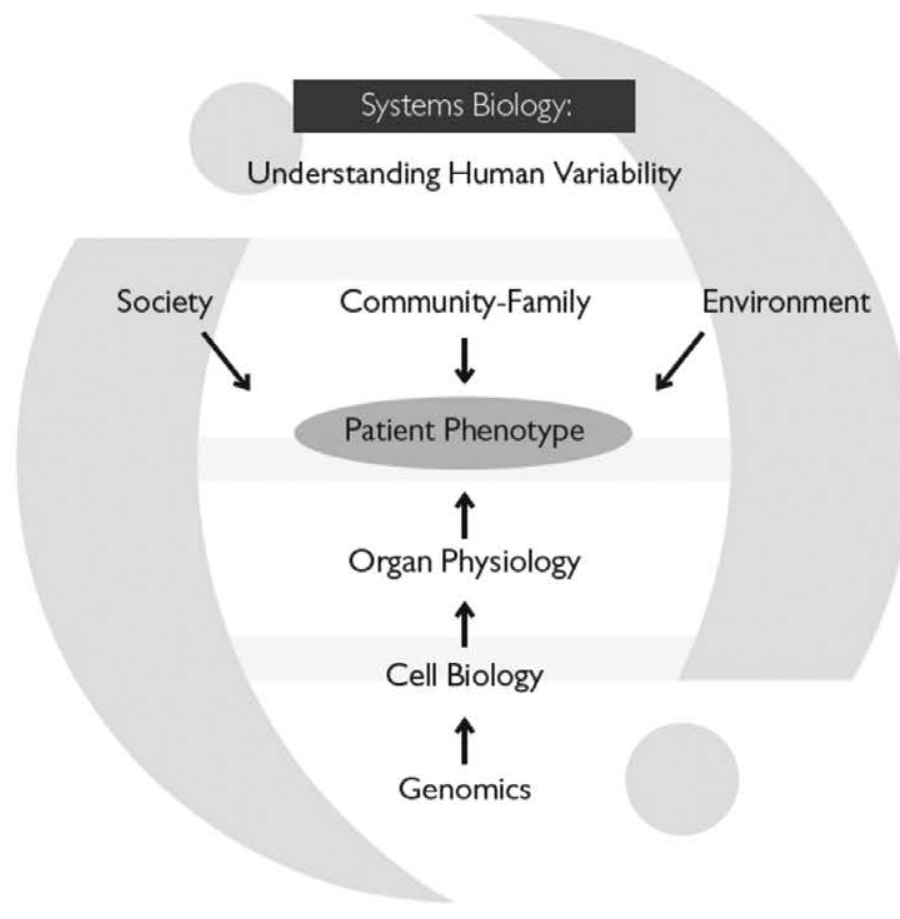

Clinical Skills Development:

YI Clinical Foundations

YI-2 Longitudinal Ambulatory Clerkship

Y2 Transition to the Wards

Y3 Core Clerkships

Y3 Preclerkship Educational Exercises

Y4 Advanced Clerkships

Y4 Transition to Residency

Horizontal Strands:

Biological Mechanisms Aging

Genomics/Proteomics

Neoplasia

Nutrition

Pharmacology

Culture of Medicine

Cultural Competence

Ethics \& Professionalism

Health Care Disparities

Health Systems Science

Biomedical Informatics

Health Policy

Patient Safety

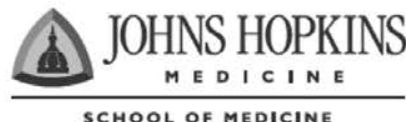

Figure 1 Genes to Society paradigm.

live lectures, pre-small-group-session knowledge checks, and narrative feedback from small-group faculty.

- The Office of Curriculum (OOC) and Office of Assessment and Evaluation (OAE) provide coaching to faculty in item writing to enhance validity and reliability of written and standardized patient assessments.

- All internally written examination items are tagged for multiple domains (Bloom's taxonomy level, USMLE content outline topic, and horizontal strand), to provide each student with an individualized report and provide programmatic reports to educational leaders.

See Supplemental Digital Appendix 2-Program Objectives and Assessment Methods—at http://links.lww.com/ACADMED/A969.

\section{Parallel curriculum or tracks}

- The PCLT and GHLT provide longitudinal educational experiences and mentorship with selected faculty.

- The PCLT prepares selected students to be compassionate, skilled primary care physicians, innovators, team leaders, and advocates for patients and communities.

- The GHLT prepares future leaders in global health by leveraging principles of public health, cultural humility, health equity, health justice, and interdisciplinary teamwork to improve care and advocate for patients and communities, locally and abroad.

- Interested first-year students apply the fall of year 1 and begin longitudinal experiences in January of year 1 .
- Students in the PCLT continue working with longitudinal ambulatory clerkship (LAC) primary care preceptor, spending 1 half day per month during years 3 and 4, allowing a more advanced role in the ambulatory setting with growing clinical experience.

- Some PCLT educational sessions such as workshops are open to all students.

\section{Pedagogy}

Adult learning theory and strategies to promote lifelong learning are employed, with emphasis on interactive learning methods over lecture.

- Active learning modalities in years 1 and 2 build upon new content from lectures, e-lectures, and/or reading, to achieve advanced learning objectives such as application, synthesis, and analysis. Methods include discussion, case-based learning, laboratory, team-based learning, journal club, and virtual microscopy.

- GTS workshops: Students contribute on a rotating basis to weekly workshop presentations in the GTS course, using selfdirected learning to prepare a topic they teach to their peers, which is assessed on the summative examination.

- Clinical courses and clerkships use a variety of content- and skills-appropriate pedagogical methods including role play, simulation, videos, clinical experience, small-group discussion, and peer teaching (Clinical Foundations of Medicine [CFM] 
course); workshops and case-based learning (LAC); and small-group, workshop, role-play, and simulation activities (preclerkship education exercises).

- PCLT: Preceptorship and ambulatory clinical experience are supplemented by workshops, role play, team-based learning, video/podcast, journal club, book club, case-based learning, discussion (large and small group), lecture, and peer teaching.

- GHLT: Students participate in case-based learning, clinical experience, lectures, self-directed learning, role play, and simulation.

\section{Changes in pedagogy since 2010}

- To mitigate unintended differences in outcomes among small groups with different facilitators, the OOC promotes in-person, just-in-time faculty development and provides a template to guide small-group facilitators including learning objectives, student preparation, session timelines, and discussion questions.

- Active learning sessions increasingly use formative or lowstakes knowledge questions at the beginning, to check and reinforce basic concepts from the required student preparation for the session.

- Faculty are shifting from lecture to e-lecture format, where new content is delivered in briefer modules with incorporation of formative knowledge assessments and embedded links for optional foundational or enhancement material.

\section{Clinical experiences}

- Students' first clinical experience occurs in fall of year 1 with CFM, where they learn basic communication and physical exam skills in the simulation center with standardized patients, and in the hospital setting.

- The LAC is a yearlong, weekly experience that provides a longitudinal relationship with a community-based preceptor and their office practice, with opportunities to practice skills learned in CFM, and integrated with the organ systems course in years 1 and 2 .

- TIME courses incorporate simulated patient experiences or patient panels to share patient stories, allowing students to engage in history taking.

- Transition to the Wards is a 3-week course in year 2 where students prepare for core clinical clerkships in sessions including didactics, small-group clinical reasoning, procedural skills and simulations, and brief experiences on inpatient services.

- The core clerkships use Johns Hopkins Medicine affiliates including those in urban and suburban settings, and inpatient and ambulatory facilities in Baltimore and the surrounding community. Students may choose to do a portion of the pediatrics rotation at Johns Hopkins All Children's Hospital in St. Petersburg, Florida.

- The PCLT and GHLT use the clinical practices of Johns Hopkins Community Physicians and other community preceptors, as well as practices specifically serving immigrant populations.

- Several popular electives use specialty practice settings supervised by Johns Hopkins faculty-longitudinal health coaching, rural medicine, austere medicine, year
1 interprofessional practice, Latino health pathway, and subspecialty electives in various disciplines.

Challenges faced in an effort to increase core clerkship capacity and increase ambulatory medicine skills include providing faculty development, recognizing the need to maintain clinical productivity while teaching students, ensuring uniform quality across sites, and identifying new ambulatory settings and implementing faculty and staff development.

\section{Curricular Governance}

The Educational Policy and Curriculum Committee (EPCC) provides central oversight and guidance for the curriculum. Voting members include directors of required courses and clerkships; representatives from horizontal strands, TIME courses, and CAP; 2 department directors; and at-large members selected by student leaders. EPCC subcommittees and their roles include:

- GTS Integration Committee: oversees implementation and evaluation of the 4-year curriculum

- Clerkship Directors and Clinical Skills Committee: oversees and manages clinical portions of the curriculum

- Student Assessment and Program Evaluation Committee: verifies that medical education program objectives are implemented and assessed effectively; facilitates curricular CQI by recommending actions to course leaders via EPCC in response to evaluation data

See Figure 2-Organizational chart.

\section{Education Staff}

The vice dean for education oversees UME, GME, graduate biomedical education, postdoctoral education, and CME. The following offices support medical student education at JHUSOM:

- OOC: The associate and assistant deans for undergraduate medical education, with 3 program administrators and 1 instructional designer, oversee implementation and monitoring of the curriculum and assist course and clerkship leaders in ongoing quality improvement.

- OAE: The assistant dean for assessment and evaluation and the director of the OAE, with a psychometrician, research data analyst, and program evaluation specialist, assist with designing and interpreting results of reliable assessments and program evaluation.

- Office of Academic Computing (OAC): The assistant dean and director of the OAC, an instructional designer, a programmer, and an audiovisual technician facilitate technologic solutions for delivery of educational sessions and online assessments for medical students.

- Office of Information Technology (OIT): The director of OIT leads a team of software engineers and program analysts to provide online applications supporting data collection and reporting in support of medical education broadly at JHUSOM. 


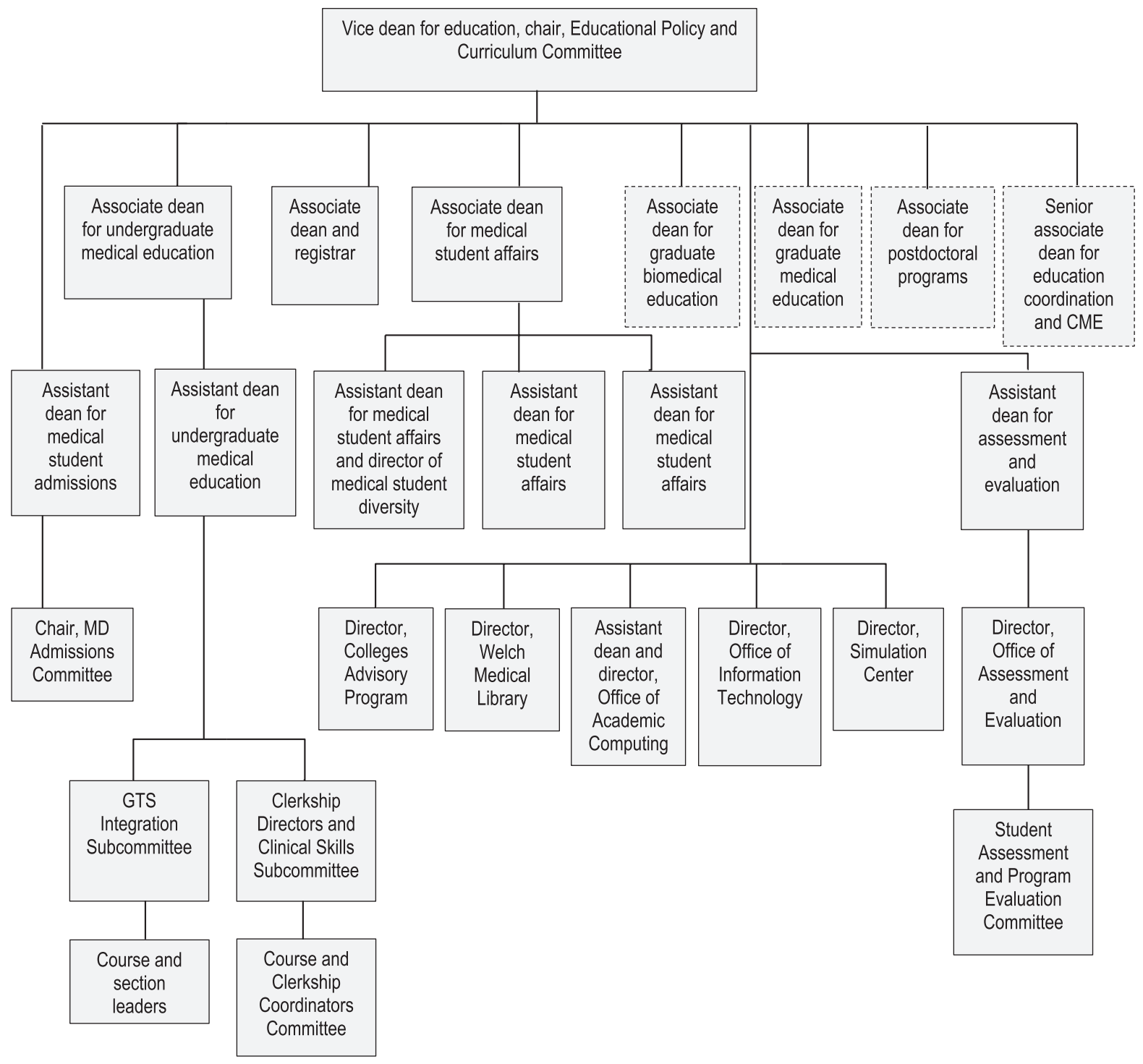

Dotted-line borders indicate leaders of programs outside of undergraduate medical education.

Figure 2 Organizational chart.

- Office of Medical Student Affairs: The associate dean and 3 assistant deans for medical student affairs support students in many aspects of adapting to the medical education environment and navigating ongoing personal and professional matters. One assistant dean also serves as the director of medical student diversity.

- CAP: The director, associate director, academic program administrator, and 22 faculty create a learning community dedicated to supporting medical students' professional growth, career development, and well-being.

\section{Faculty Development and Support in Education}

JHUSOM offers a broad range of faculty development opportunities through the Office of Faculty Development (OFD) and Institute for Excellence in Education (IEE), as well as the Master of Education in the Health Professions degree program, a collaboration of the Johns Hopkins Schools of Business, Education, Medicine, Nursing, and Public Health. The mission of the IEE is to promote, value, and advance the educational mission of JHUSOM, and aligns its programs in 4 pillars: improving teaching; inspiring and supporting 
research, scholarship, and innovation in education; valuing and recognizing teaching and education; and fostering a community of educators.

- Longitudinal programs and workshops are offered multiple times annually in teaching skills and curriculum development.

- Faculty may participate in programs offered by the OFD and in a variety of regularly scheduled educational programs and individual coaching programs offered by the IEE. The IEE also offers online modules on its Improve Your Teaching Skills website (https://www.hopkinsmedicine.org/institute_ excellence_education/Pillar_1/improve_teaching.html). Faculty can apply for small educational project grants through the IEE.

- JHUSOM has a number of annual faculty teaching awards presented each year at our convocation ceremony. Additionally, the IEE offers a number of outstanding educator awards and mentors a student-run Distinguished Teaching Society recognizing faculty and residents who demonstrate the highest standards of excellence in clinical teaching.

\section{Initiatives in Progress}

- Detailed content review of year 1 and 2 courses to determine whether information taught is foundational (prerequisite), core, or enhancement material for interested students

- Implementation of a standard teacher evaluation tool to provide evidence of teaching excellence for faculty annual assessments and promotion

- Implementation of competencies for medical educators

- Piloting new clinical performance assessment tool for the clinical courses based on Entrustable Professional Activities (EPAs), a mobile platform for collection of assessment data in real time, and a dashboard for student and faculty use to monitor individual and class progress

- Creation of UME to GME handoff with EPA data to inform interns' first individualized learning plans 


\section{F. Edward Hébert School of Medicine, Uniformed Services University of the Health Sciences}

Arnyce R. Pock, MD, Catherine T. Witkop, MD, MPH, Brian V. Reamy, MD, Jessica T. Servey, MD, MHPE, Pamela M. Williams, MD, and Jessica L. Bunin, MD

\section{Medical Education Program Highlights}

The F. Edward Hébert School of Medicine, "America's Medical School," is the only medical school owned and operated by the U.S. government. Because Uniformed Services University (USU) medical students are dedicated to national service, they not only graduate debt free but are also paid the salary and benefits of active duty military or Public Health Service officers while they learn to become doctors.

Established to serve as the "leadership academy" for military health and the Public Health Service, USU's curriculum includes 700 hours of military unique training, focusing on areas such as disaster and humanitarian response, tropical medicine, global health, preventive medicine, and health systems science. Students receive extensive training in prehospital trauma life support and have the opportunity to graduate with certifications in advanced life support as well as in advanced trauma life support.

One of the most distinctive features of our 4-year curriculum is our capstone field exercise, called "Operation Bushmaster." During this multiday event, designed to simulate an overseas deployment in support of combat operations, teams of fourthyear medical and graduate nursing students work together to manage a wide array of clinical, logistical, and ethical challenges. There is nothing else like it in American medical education.

\section{Curriculum}

\section{Curriculum description}

See Supplemental Digital Appendix 1-Curriculum Schematicat http://links.lww.com/ACADMED/A946.

\section{Curriculum changes since 2010}

In 2011, with the graduating class of 2015, the School of Medicine began transitioning from a traditional $2 \times 2$ program to an integrated, organ system-based approach called Molecules to Military Medicine. Our revised curriculum includes a 17-month

Acad Med. 2020:95:S211-S215.

doi: 10.1097/ACM.0000000000003460

Written work prepared by employees of the Federal Government as part of their official duties is, under the U.S. Copyright Act, a "work of the United States Government" for which copyright protection under Title 17 of the United States Code is not available. As such, copyright does not extend to the contributions of employees of the Federal Government.

Supplemental digital content for this article is available at http://links.lww.com/ ACADMED/A946.

Year school was established: 1972.

School URL: https://www.usuhs.edu/medschool. preclerkship period comprising 7 organ system-based modules, a 12-month clerkship period, and a 15-month postclerkship period. Key elements of military medical practice and leadership, including 4 field exercises, are threaded throughout the 4-year curriculum.

Also included in the revised program is a 6-week period of advanced didactics in the postclerkship period (bench to bedside and beyond) and opportunities for students to engage in an extended scholarly project of their choice (capstone research).

\section{Assessment}

Our education objectives are based on the ACGME domains of competence. They are:

- Medical and population health knowledge

- Interpersonal and cross-cultural communication skills

- Patient care

- Practice-based learning and improvement

- Professionalism, leadership development, and officership

- Systems-based practice

Each of these overarching competencies is linked to a series of programmatic objectives that are defined in outcome-based terms and assessed using a variety of measures.

See Chart 1-Domains of Competence.

Along with the 2011 curricular revision, several major changes to student assessment were instituted. These included replacing course-/discipline-based examinations with a series of NBME Customized Assessment Services examinations. The traditional letter-based grading framework was changed to honors/pass/fail, and the timing of the USMLE Step 1 examination was shifted to after completion of the core clerkship year.

Additional changes involved use of a core group of teaching faculty who longitudinally follow the development of clinical skills in an assigned group of students through their first 17 months of the curriculum. New assessment methods were introduced, focusing on small-group discussion and exercises. These include assessing pathology case write-ups, concept maps, and Clinical Reasoning Mapping Exercises. Substantially more OSCE assessments were integrated into new curricular structure, including a final, end-of-preclerkship OSCE. In addition, each of the core clerkships use an OSCE as part of their end-of-clerkship assessments.

In addition to covering the same curricular content civilian medical schools teach, our 4-year curriculum includes an additional 700-plus hours of military unique instruction and field exercises that teach team communication, leadership, 


\section{Chart 1}

\section{Domains of Competence}

\begin{tabular}{|c|c|c|c|c|c|c|c|c|c|c|c|c|c|c|c|c|c|c|c|c|c|c|c|c|c|c|c|}
\hline Domains of competence & 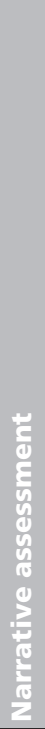 & 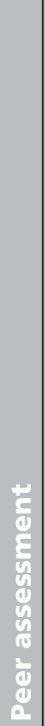 & $\begin{array}{l}4 \\
0 \\
0 \\
0 \\
0 \\
3 \\
0 \\
8 \\
0 \\
0 \\
0 \\
\frac{2}{2} \\
\frac{2}{2} \\
0 \\
0\end{array}$ & 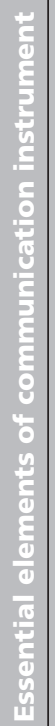 & 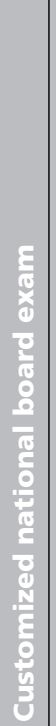 & 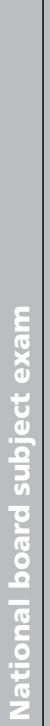 & 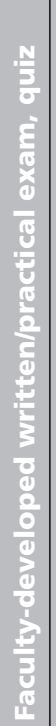 & 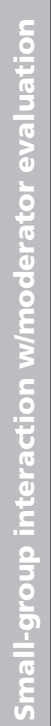 & 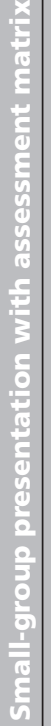 & 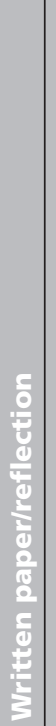 & 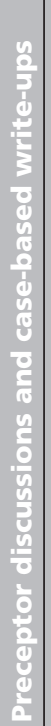 & 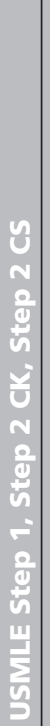 & 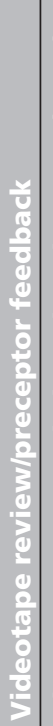 & 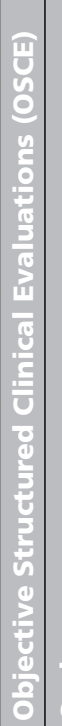 & : & 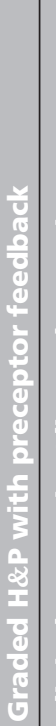 & 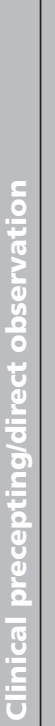 & 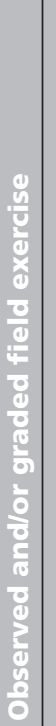 & 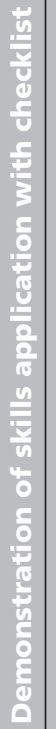 & 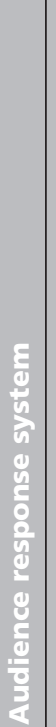 & 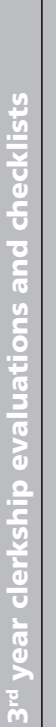 & 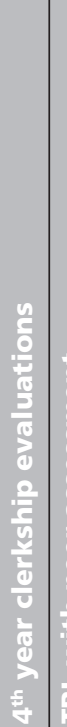 & 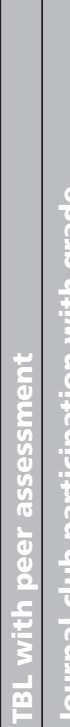 & 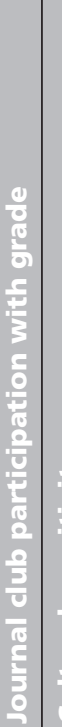 & 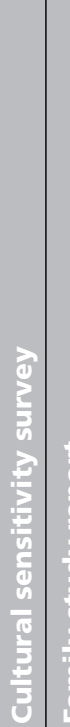 & 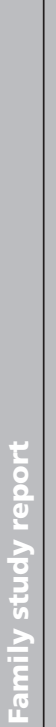 & 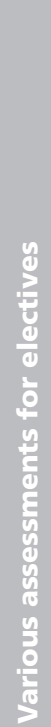 \\
\hline $\begin{array}{l}\text { Medical and population health } \\
\text { knowledge }\end{array}$ & $x$ & $x$ & & & $x$ & $x$ & $x$ & $x$ & $x$ & & $x$ & $x$ & $x$ & $x$ & $x$ & $x$ & $x$ & $x$ & $x$ & $x$ & $x$ & $x$ & $x \mid>$ & $x$ & & $x$ & $x$ \\
\hline $\begin{array}{l}\text { Interpersonal and cross-cultural } \\
\text { communication skills }\end{array}$ & $x$ & & & $x$ & & & & $x$ & & $x$ & $x$ & $x$ & $x$ & $x$ & $x$ & $x$ & $x$ & $x$ & & & $x$ & $x$ & & & $x$ & $x$ & \\
\hline Patient care & $x$ & & & $x$ & $x$ & $x$ & $x$ & $x$ & $x$ & & $x$ & $x$ & $x$ & $x$ & $x$ & $x$ & $x$ & $x$ & $x$ & & $x$ & $x$ & & $x$ & & & $x$ \\
\hline $\begin{array}{l}\text { Practice-based learning and } \\
\text { improvement }\end{array}$ & $x$ & $x$ & $x$ & & $x$ & & $x$ & $x$ & $x$ & $x$ & $x$ & $x$ & $x$ & $x$ & & $x$ & $x$ & $x$ & & & $x$ & $x$ & & $x$ & & & \\
\hline $\begin{array}{l}\text { Professionalism, leadership } \\
\text { development, and officership }\end{array}$ & $x$ & $x$ & $x$ & $x$ & $x$ & & $x$ & $x$ & $x$ & $x$ & $x$ & $x$ & & $x$ & & $x$ & $x$ & $x$ & & & $x$ & $x$ & $x$ & $x$ & $x$ & $x$ & $x$ \\
\hline Systems-based practice & $x$ & & & $x$ & & & $x$ & $x$ & $x$ & $x$ & $x$ & & & & & $x$ & $x$ & $x$ & & & $x$ & $x$ & & & & & $x$ \\
\hline
\end{tabular}

problem solving, and essential principles of military medical practice. This ensures that our graduates are capable, confident, and adaptable in any clinical setting from a tertiary care hospital to a refugee camp.

\section{Pedagogy}

The following pedagogical approaches are currently used:

- Case-based learning

- Clinical experience: ambulatory

- Clinical experience: inpatient

- Discussion: large group

- Discussion: small group

- Laboratory

- Lectures

- Peer teaching

- Preceptorship

- Problem-based learning

- Role play/dramatization

- Self-directed learning/tutorial
- Simulation

- Standardized/simulated patients

- Team-based learning

- Video/podcast

- Workshop

- Military field exercises/training

\section{Clinical experiences}

Since 2010, the School of Medicine has steadily reduced its reliance on the delivery of content via traditional 50-minute lectures and instead is increasing its use of small-group, team, and case-based discussions. We have also incorporated a growing array of interprofessional programs, podcasts, and prerecorded curricular content, and more recently, distance learning to allow for a more interactive and individualized pedagogic approach.

During the preclerkship curriculum, the majority of required clinical experiences are provided at nearby Walter Reed Medical Center as well as at the school's Val Hemming simulation center, where students interact with high-fidelity 
simulators, a cadre of highly trained standardized patients, and a walk-in virtual environment. Required clerkship and postclerkship experiences are delivered at premier military hospitals, clinics, and teaching facilities across the United States as well as hospital and university-based simulation centers.

Students experience their initial clinical encounters during the first week of medical school. These take place during the Foundation in Medicine module and involve working with patients in local military medical centers and our school's simulation/standardized patient center.

\section{Required and elective community-based rotations}

- Required community-based experiences: All students are required to complete 5 weeks of family medicine, with most rotations occurring in ambulatory clinics associated with community hospitals. Requirements for exposure to outpatient pediatrics and to ambulatory neurology may also be completed in clinics associated with community hospitals. Required rotations in internal medicine, neurology, and psychiatry may be completed at one or more VA medical centers.

- Elective experiences: Students have access to a wide variety of clinical electives, both medical and surgical, that can be accomplished in ambulatory clinics, many of which are associated with community hospitals, operationally focused military medical units, and/or a VA medical center.

Challenges in designing and implementing clinical experiences for medical students

One of the major challenges associated with designing and implementing clinical experiences for students relates to the frequency with which military faculty are required to rotate to other duty assignments/duty locations and/or be prepared to deploy to other locations in response to national and/or international contingencies. To meet this challenge, we have put in place an active and ongoing nationwide program of faculty development to ensure consistently skilled and comparable delivery of instruction, feedback, and evaluation. Finally, it should be noted that just as in the civilian sector, the need to balance demands for clinical productivity against time for clinical teaching and mentorship is an ongoing challenge. Given the unpredictability of the military mission, we engage in continuous contingency planning, including identifying alternate training locations.

\section{Curricular Governance}

\section{Decentralized curricular governance}

The only departmentally based components in the curriculum are the department committees that oversee administration of their respective clinical clerkships. While these committees have responsibility for developing and delivering clerkship content and the assessment of individual students, the Executive Curriculum Committee oversees and manages the entire curriculum, including clerkship experiences.

See Figure 1-Curricular governance.

\section{Education Staff}

\section{Medical education leadership}

The Office of Medical Education is staffed by 4 decanal staff: the associate dean for medical education, the associate dean for curriculum, the associate dean for assessment, and the assistant

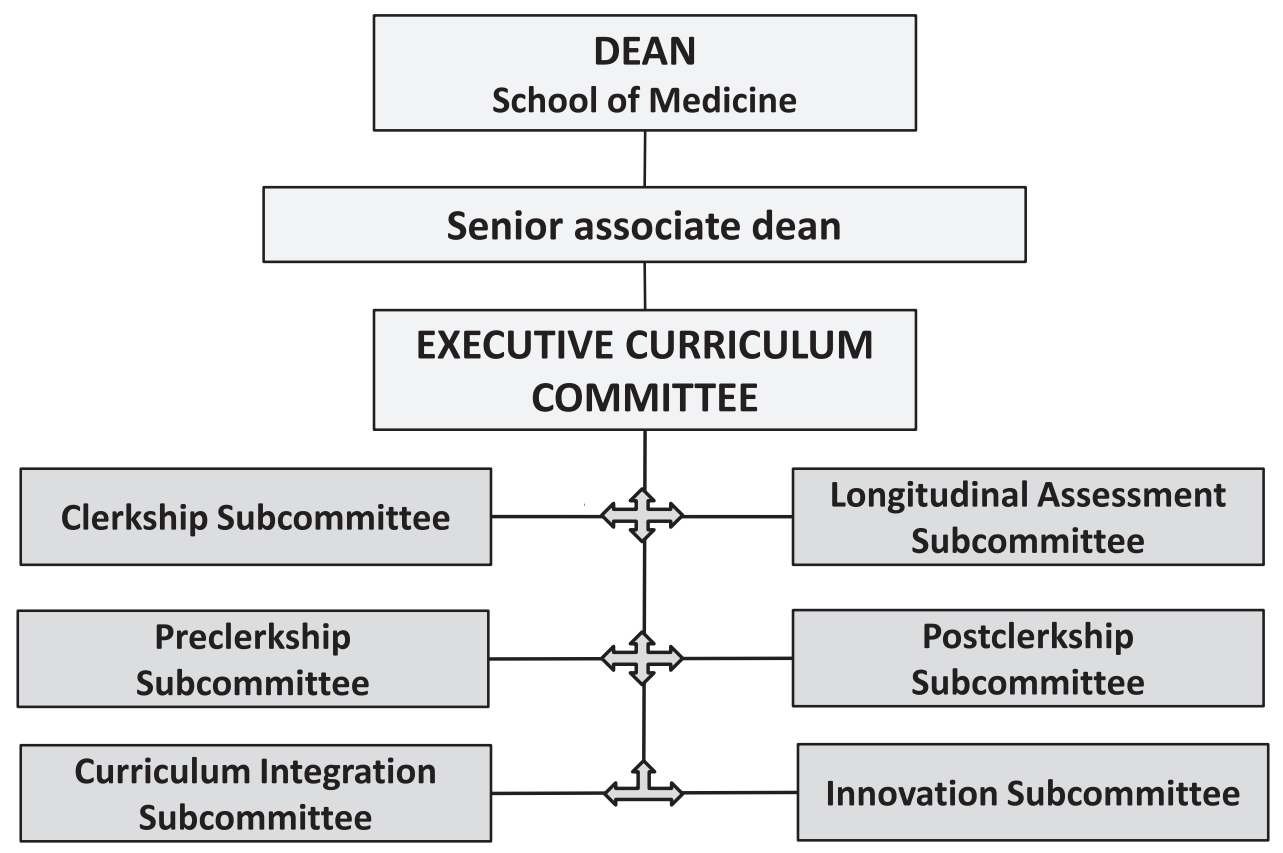

Figure 1 Curricular governance. 
dean for capstone programs. Six full-time staff members provide administrative support to the decanal staff.

\section{Department of Medical Education}

- The primary medical education staff are responsible for the implementation of undergraduate medical education only.

- The Center for Health Professions Education includes health professions graduate education programs granting MHPE and $\mathrm{PhD}$ degrees, a distributed learning office, the newly established Academy for Educators, and a research office focused on ascertaining the long-term career outcomes of our graduates and factors associated with their success.

See Figure 2-Organizational chart.

\section{Faculty Development and Support in Education}

\section{Professional development for faculty as educators}

Since our faculty are dispersed across the United States and periodically move from one teaching hospital to another within the larger organization, our faculty development program has devised a unique approach to standardization. It includes an array of in-person, interprofessional workshops delivered locally and at 25 national teaching hospitals. These workshops address a range of topics, including foundational educational skills and principles, career development, quality improvement, and scholarly endeavors. Educational sessions conducted at USU are recorded and made available via live video teleconference.
We also conduct a semiannual week of structured activities for our key academic leaders. These sessions are tailored to meet the needs of specific cohorts of educators and include preparatory work, assigned readings, and a submitted reflection. Groups are deliberately designed to be interprofessional, and they include leaders of both undergraduate and graduate medical education programs.

To meet the growing faculty development needs of our large health system, we created a robust faculty development outreach program. To date, it has trained 25 interdisciplinary clinicianeducators to support and strengthen our core team. These new faculty developers can each deliver up to 13 standardized presentations on a core set of foundational topics. Because they are embedded in the school's major teaching affiliates and have the flexibility to meet local scheduling requirements, they are an invaluable asset to our national faculty.

Finally, to recognize those who achieve advanced levels of faculty development, we award 4 different certificates.

\section{Role of teaching in promotion and tenure}

Promotion and tenure decisions hinge on achievement in one or more of 4 major areas of scholarship: research, clinical work, institutional service, and teaching.

Teaching is categorized into 3 levels:

- Level 1: direct provision of teaching in the classroom, labs, wards, or clinics

- Level 2: development of teaching materials and curriculum and program or course direction

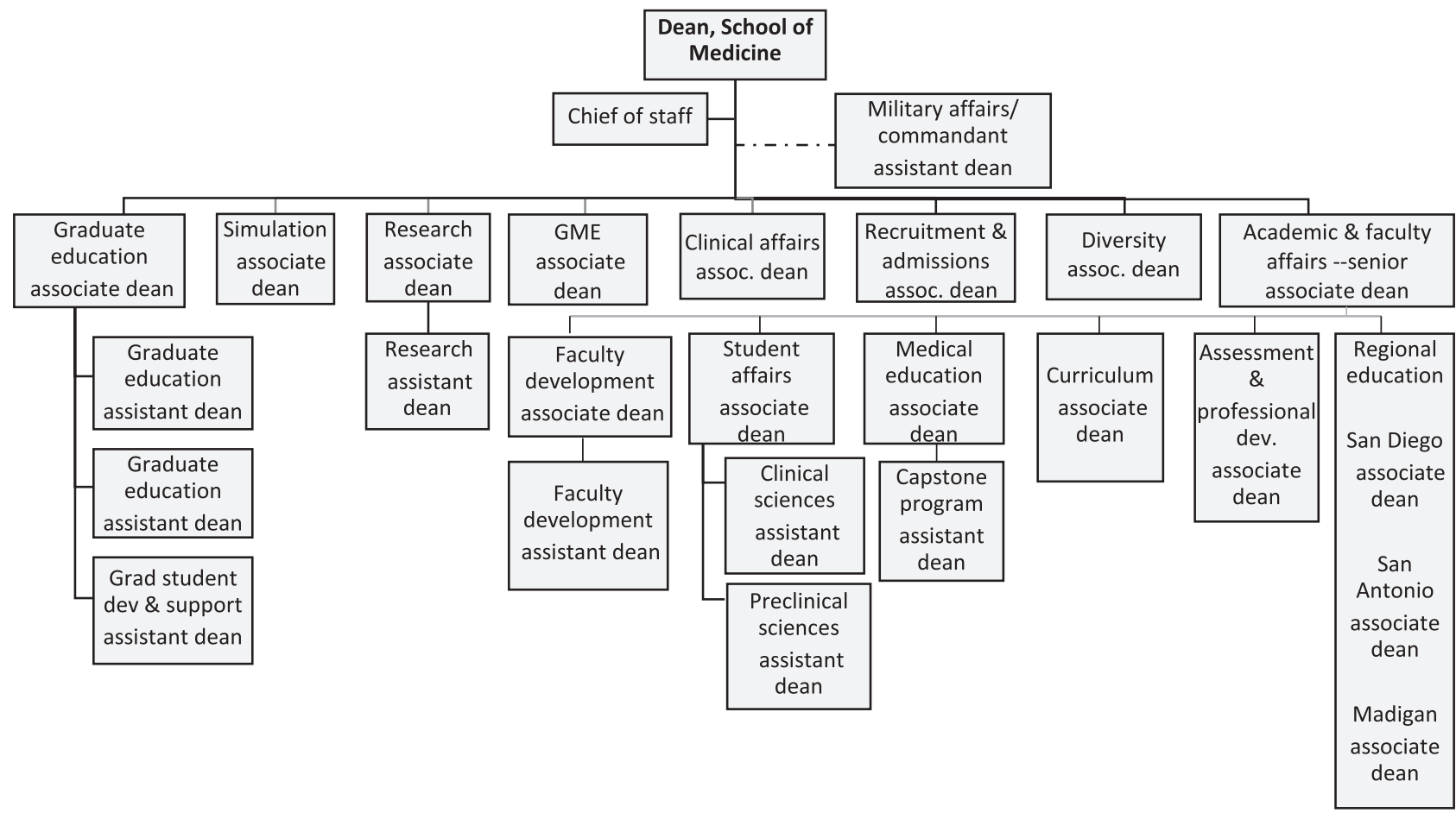

Figure 2 Organizational chart. 
- Level 3: peer-reviewed and disseminated teaching achievements via journals, posters, presentations, digital media, grants, and educational leadership

Educator portfolios are encouraged to document achievements. Promotion to associate professor requires comprehensive level 1 and significant level 2 accomplishment. Promotion to professor requires comprehensive level 1 and 2 and significant level 3 output.

The Academy for Health Professions Educators was officially chartered in September 2019. It will include faculty from USU's
School of Medicine, Graduate School of Nursing, and PostGraduate Dental College. It will serve to assist faculty in their ongoing development as educators and educational researchers.

\section{Regional Medical Campuses}

Although we have a remarkable network of military teaching hospitals, national faculty, and clerkships across the country, and offer elective experiences around the world, all educational activities are coordinated from our home campus in Bethesda. 


\title{
University of Maryland School of Medicine
}

\author{
Donna L. Parker, MD, Joseph P. Martinez, MD, and Nirav G. Shah, MD
}

\section{Medical Education Program Highlights}

The University of Maryland School of Medicine (UMSOM) provides excellence in biomedical education, basic and clinical research, and quality patient care and service. The UMSOM is part of the University of Maryland, Baltimore located on the west side of Baltimore and consisting of 6 other schools: the Graduate School and the professional Schools of Nursing, Pharmacy, Dentistry, Social Work, and Law.

The Office of Admissions recruits and matriculates individuals to enhance the overall health of our communities through development of new knowledge and provision of exemplary patient care. These talented individuals reflect the growing ethnic and cultural diversity of present-day society.

All faculty members participate in the education of medical students on a campus that is home to world-class clinical care and research facilities, including the University of Maryland Medical Center (UMMC), R. Adams Cowley Shock Trauma Center, Institute of Human Virology, Institute for Genome Sciences, Center for Vaccine Development, and the Baltimore Veterans Affairs Medical Center.

Unique features:

- We have maintained and improved an already excellent information technology infrastructure with both personnel and equipment, including an in-house student data management system (MedScope) that provides the Office of Student Affairs (OSA) and Office of Medical Education (OME) with tools to track and analyze student progress.

- Created after the civil unrest in Baltimore in 2015, our 4-year, longitudinal social justice track incorporates an understanding of what social justice means in different populations, evaluates the myriad factors that increase disparities, and discusses ways we can effectively address these issues.

- The Humanism Symposium elective offers preclerkship students, SOM faculty, and Baltimore community members the opportunity to examine what it means to be a humanistic physician. The course is led by third- and fourth-year students with faculty oversight and addresses topics across specialties, from medical ethics to cultural differences, spirituality, and physician self-care.

Acad Med. 2020:95:S216-\$219.

doi: 10.1097/ACM.0000000000003355

Copyright (C) 2020 by the Association of American Medical Colleges

Supplemental digital content for this article is available at http://links.Iww.com/ ACADMED/A874.

Year school was established: 1807

School URL: https://www.medschool.umaryland.edu.

\section{Curriculum}

\section{Curriculum description}

The current curriculum is in the traditional $2+2$ format with normal structure and function taught in year 1 followed by a 12-week summer break, and pathophysiology and therapeutics in year 2. Students spend 1 afternoon a week in the Introduction to Clinical Medicine (ICM) course during years 1 and 2 and complete 40 hours of service learning as a part of the course. Years 3 and 4 consist of ICM III, 2 subinternships, a rural ambulatory experience, and 6 electives required beyond our 7 core clerkships. All students are required to complete a scholarly project as part of the Foundations of Research and Critical Thinking (FRCT) course.

\section{Curriculum changes since 2010}

- Elective time added to third year

- Reduction in the amount of time spent on the core medicine and core surgery clerkship

- Decreased amount of time spent on ICM IV course

- FRCT course designed to stimulate critical thinking, enhance inquisitiveness, and encourage research participation

Education leadership recently embarked on the first major curriculum revision for our school in 25 years. The group convened in June 2018; spoke with other institutions; and studied curricula and organizational charts to understand what structures might work best with our students, our environment, and our values. This group became the Curriculum Council.

In January 2019, the Curriculum Council hosted a retreat attended by nearly 100 faculty members, students, and residents, broadly representing both basic and clinical sciences.

A major result of this retreat was creating a mission statement for our new curriculum. Actualizing the Renaissance Physician: creating lifelong learners who are clinically excellent and possess humanism, professionalism, scholarship, leadership, critical thinking skills, and attention to social justice and diversity. We must cultivate and value our teachers, rethink our assessment and grading methods, and create more space for student wellness.

Following the retreat, the Curriculum Council created 11 working groups with similar broad representation. These groups met throughout the spring of 2019, submitting a substantive report to the Curriculum Council in July 2019. The Curriculum Council synthesized and presented the material to the Executive Committee with subsequent approval at the SOM Council in September 2019. Our required report to the LCME, on major curricular modification, was approved by them in February 2020. 
In the Renaissance Curriculum, the preclerkship phase will be integrated and systems based, and it will be shortened by 2 months. Longitudinal tracks (such as academic medicine, social justice, and humanism) are being developed, which students may choose to pursue during their preclerkship years. The Practice of Medicine course will run longitudinally throughout the curriculum occurring 1 to 2 afternoons per week during the preclerkship phase. In the clinical phase, earlier clerkships and elective experiences will allow students more time to explore career options.

See Supplemental Digital Appendix 1-Preclerkship Curriculum Map—at http://links.lww.com/ACADMED/A874.

See Supplemental Digital Appendix 2-Curriculum Sample Schedule, 2022 — at http://links.lww.com/ACADMED/A874.

In addition to structural changes, we are examining our teaching strategies to improve the quality of lectures and create more active learning opportunities. The renovation of existing education space into active learning spaces for August 2020 will facilitate this latter goal.

As we comprehensively reviewed the curriculum, we identified opportunities for interim improvements in our current (Legacy) curriculum. In the clinical years, we are creating shorter 2-week electives and more ambulatory experiences, expanding our transition to residency offerings, adding emergency medicine as a required rotation, and creating a new back-to-basics selective in the fourth year.

Our educators have been engaged in faculty development activities including item writing, active learning training, and curriculum development. All curricular changes have been conceived with a focus on reducing bias and enhancing student wellness.

\section{Assessment}

We use the AAMC Physician Competency Reference Set as a foundation for program objectives.

See Supplemental Digital Appendix 3-Program Objectives and Assessment Methods - at http://links.lww.com/ACADMED/A874.

\section{Assessment changes since 2010}

- We developed the preclerkship assessment review committee (ARC) to vet all assessments for content, style, and bias at least 1 week before their administration. The ARC is responsible for editing questions for format, fairness, accuracy, and inclusivity; determining length of examinations (i.e., number of questions and time allotted); linking questions to learning objectives and USMLE taxonomies; and reviewing performance of examinations.

- The mandatory scholarly project in FRCT is reviewed by the course director and must meet specific, predefined criteria.

\section{Pedagogy}

The pedagogical approach in the preclerkship phase combines lectures, small-group discussion, laboratory sessions, and clinical correlates, which center around a patient and/or family affected by a disease process. In the clinical phase, a combination of clinical experiences and lectures predominate, supplemented by other modalities such as simulation and standardized patients.

\section{Changes in pedagogy since 2010}

The Renaissance Curriculum will increase the amount of active learning in the preclerkship curriculum and improve the quality of lectures. A working group dedicated to novel teaching methods has been convened to assess educational needs and identify opportunities to improve the delivery of content to learners. This includes new technology, educator development, and integration of parallel curricular resources.

\section{Clinical experiences}

Students rotate through UMMC, Baltimore Veterans Administration Medical Center, academic affiliates within the University of Maryland Medical System (UMMS), local community hospitals, and with individual community preceptors. Students also are required to spend 1 month in an underserved, ambulatory location.

\section{Required longitudinal experiences}

- ICM I, II, and III are all longitudinal experiences in years 1-3 of the curriculum. These courses aim to give students the skills necessary to treat patients and develop meaningful relationships with them through an understanding of the context in which their care is delivered.

- FRCT and completion of a scholarly project are longitudinal over years $1-4$.

\section{Clinical experience first encounter}

Students begin ICM in the first month of their first year. They begin clinical rotations in July of their third year. In the Renaissance Curriculum, this time frame will shift forward with entry into clinical rotations occurring approximately 2 months earlier.

\section{Required and elective community-based rotations}

Students rotate through community-based affiliates within and outside of UMMS during the family medicine clerkship and many elective experiences. The ICM IV course consists almost exclusively of community-based sites.

\section{Challenges in designing and implementing clinical experiences for medical students}

The major challenges in the clinical phase are continued clinical and administrative pressures on faculty members, resident physicians, and community preceptors with resultant worsening in educator:learner ratios. This situation will be particularly challenging during the overlap of the Renaissance and Legacy curricula.

\section{Curricular Governance}

Clinical departments are responsible for providing financial and administrative support for directors of clerkships, subinternships, and electives. Additionally, they bear the cost of NBME shelf examinations for each clerkship. The biomedical 
science departments provide financial support for course directors in the preclerkship years.

See Figure 1-Curricular governance committees.

\section{Education Staff}

\section{Medical education leadership}

The OME is led by the associate dean for medical education and student experience and is responsible for the Office of Academic Support, the learning spaces and teaching technology to support the medical student curriculum, testing services, and IT support. The OME tracks performance of both students and the curriculum. In preparation for the new curriculum, the office added 2 assistant deans (curriculum and assessment) and 3 directors (preclerkship, clinical, and longitudinal). A committee for medical education continuous quality improvement was constituted under the leadership of the vice dean for academic affairs to ascertain medical education goals, implement a systematic process to collect and review data, disseminate outcomes to appropriate leadership and administration, and monitor compliance with the LCME accreditation. The curriculum is organized and managed by the Curriculum Coordinating Committee and made up of course and clerkship directors along with elected faculty, students, and ex officio dean's office personnel; it serves as the main governance body for the medical student educational program. Representation from major medical school departments and students from each year of the program enables a thorough review of existing curricular elements and a forum for decisions about any new curricular initiatives.
The primary medical education staff and administrative faculty in the educational program are responsible for the Offices of UME, OSA, and Admissions under the direction of the senior associate dean for undergraduate medical education.

\section{Faculty Development and Support in Education}

Professional development for faculty as educators

The Office of Faculty Affairs and Professional Development is the central resource for educator faculty development assisting faculty and residents to develop skills as teachers, evaluators, and mentors. The office oversees programs for Women in Medicine and Minority Faculty in Medicine. Their annual new faculty orientation includes information about opportunities to participate in the education mission and breakout sessions for everyday bias training. Promotion and tenure workshops and individual consultations are conducted on designing clinical and teaching portfolios. Educational development workshops on teaching methods and techniques and leadership development are offered throughout the academic year, including a semiannual full-day workshop titled Teaching Skills for Medical Educators.

\section{Role of teaching in promotion and tenure}

Promotion and tenure decisions had previously been focused on researchers with traditional peer-reviewed publications and extramural funding. A clinician-educator path to promotion has recently been established to recognize outstanding and sustained teaching contributions. A wider range of scholarly contributions is now considered that includes video, audio, and web-based activities that are critically assessed and accessible for use by

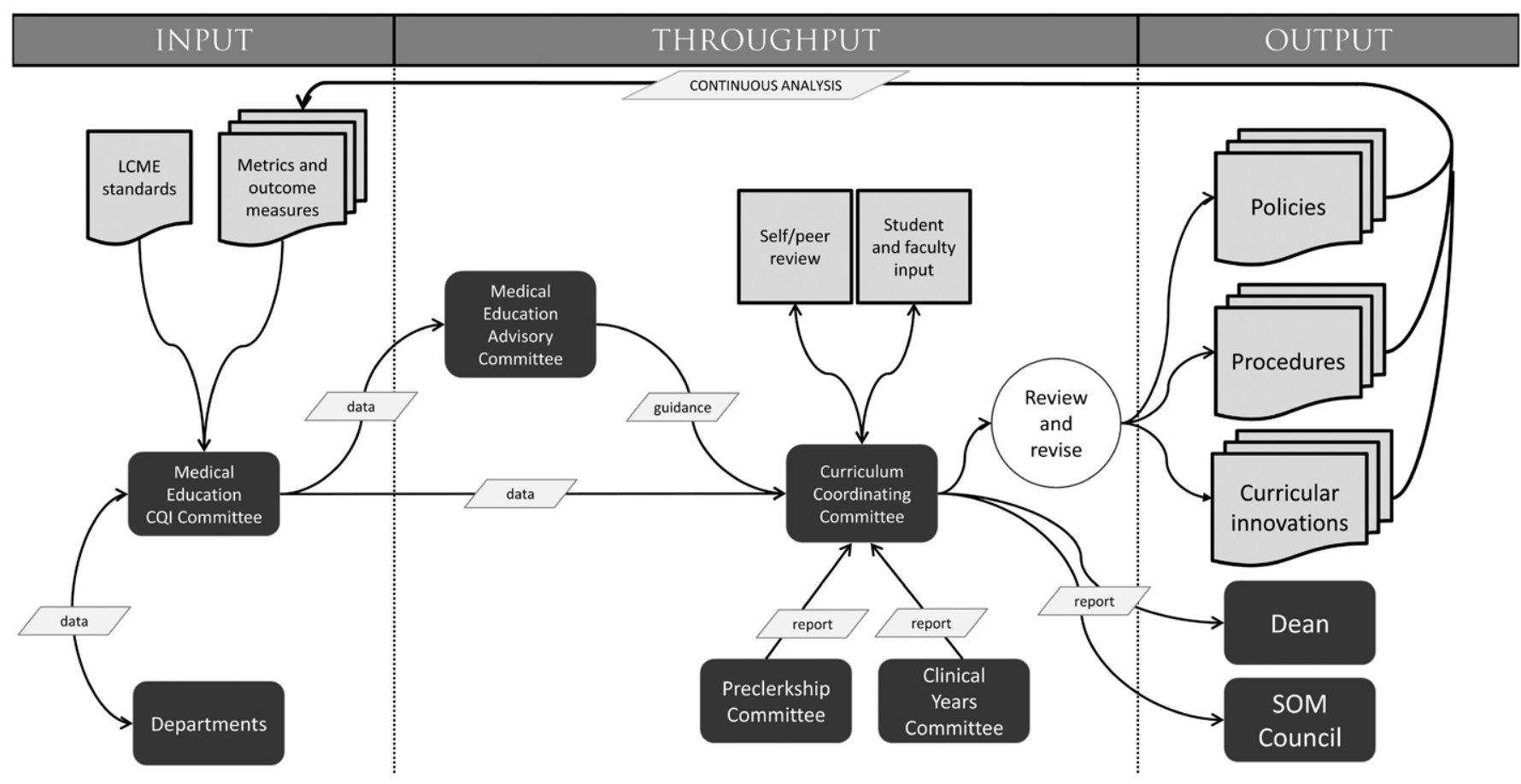

Figure 1 Curricular governance committees. 
members of the broader academic community. Extramural funding is not required for promotion on this path, but promotion to full professor still requires evidence of a national reputation.

\section{Teaching academy}

The Pass and Susel Academy of Educational Excellence, developed in 2007, is an honorific society that recognizes UMSOM faculty members who demonstrate excellence in bedside, classroom, and/or innovative medical education. Nominations are solicited annually, and Faculty Fellows receive an honorarium and are recognized on a plaque in the medical education facilities. In 2014, the academy began inducting a medical student annually as a student teaching fellow honored for their commitment to the medical education mission and demonstrated strength as a teacher. The UMSOM plans to enhance this academy to provide core educators with mentorship, resources, and opportunities for professional growth. 


\title{
Boston University School of Medicine
}

\author{
Priya S. Garg, MD, Rebecca Halley, and Molly Cohen Osher, MD, MEd
}

\section{Medical Education Program Highlights}

Boston University School of Medicine (BUSM) has a long, proud history of integrating the values of our medical school, diversity, commitment to the study and practice of medicine, advocacy, and social justice into the curriculum. In the first week of medical school, students participate in a bus and walking tour that exposes them to Boston neighborhoods where they learn about their patients' demographics, health disparities, and community resources. We continue to address topics of diversity, inclusion, disparities, and the impacts of social determinants of health at an individual behavioral level and at a population health level throughout our curriculum. Since the founding of our program, service and advocacy have been fundamental to our institutional identity. Our students participate in required service learning; a community health assessment project embedded in the Essentials of Public Health (EPH) course; and extracurricular experiences, including numerous service learning/community service organizations and enrichment courses such as the Advocacy Training Program to support our mission and student interests.

Early clinical exposure to patients, longitudinal relationships with faculty, and patient-centered care serve as the backbone of our curriculum and begin with the Doctoring curriculum. During each Doctoring small-group session, groups work through a patient case with their longitudinal faculty facilitator. They role play with actors and expert faculty to practice interviewing and communication skills, and they learn and practice physical exam, diagnostic schema, problem representation development, oral presentation, and notewriting skills to prepare them for the clinical setting. The cases are designed to teach teamwork skills and clinical reasoning, and deliberately develop a framework for building meaningful doctor-patient relationships, understand patients' perspectives and experience of illness, and explore the impact of social determinants on patients' lives.

\section{Curriculum}

\section{Curriculum description}

See Supplemental Digital Appendix 1-Curriculum

Description—at http://links.lww.com/ACADMED/A869.

Acad Med. 2020;95:S220-S222.

doi: 10.1097/ACM.0000000000003352

Copyright (C) 2020 by the Association of American Medical Colleges

Supplemental digital content for this article is available at http://links.Iww.com/ ACADMED/A869.

Year school was established: 1848.

School URL: https://www.bumc.bu.edu/busm

\section{Curriculum changes since 2010}

BUSM has moved toward meaningful integration of content throughout the curriculum. In 2015, the first-year foundational science curriculum was redesigned and became the yearlong Principles Integrating Science in Medicine course, integrating histology, physiology, genetics, and biochemistry throughout the course. In 2019, our Doctoring courses were redesigned to deliberately integrate clinical reasoning, physical diagnosis, and communication skills training into weekly cases. The cases also further reinforce concepts and content taught within the foundational science courses. In addition, anatomy and Doctoring courses have developed cases and sessions together where the physical exam is taught directly after exposure to an anatomic area, highlighting anatomic function and consequences of anatomic lesions that can be illustrated through physical examination. To assist with integration between the foundational science and clinical science phases, we have developed integrated clinical reasoning cases, a series of cases taught longitudinally within foundational science courses to demonstrate how clinical findings (patient signs and symptoms) emerge from anatomic and/or pathophysiologic derangements. For our 4-year integrated curriculum, we have defined the clinical presentations that every graduating BUSM medical student must encounter and adequately demonstrate clinical reasoning skills in by graduation. The BUSM Core, a set of 45 chief complaints and 15 chronic patient presentations, serves as the backbone for curricular integration and ensures spiraling of content as well as defined competence for graduation. In the first 2 years, the core is used in Doctoring cases and for the integrated clinical reasoning cases, and in the clerkship phase, the core informs the required patient encounters that are logged.

The EPH course was redesigned to focus on public health issues faced by the diverse urban population of patients whom students encounter throughout their training. The course includes content in biostatistics, population health, ethics, health care financing, and social determinants of health, and has developed a week of small groups to allow students to more deeply explore emerging topics in public health, engage in service learning, and complete a community project that is presented at the end of the week. Student groups visit local community agencies, learn about the assets and challenges of a Boston neighborhood, and propose an intervention to improve the health of the community based on an identified need.

In response to the state's opioid morbidity and mortality crisis, a vertically integrated substance use and prescription drug misuse curriculum was developed. Core competencies for the prevention and management of prescription drug misuse were defined through a statewide medical education working group convened by the governor. Sessions addressing pain management, substance use screening, neurobiology of substance use disorder, and the opioid epidemic are integrated throughout 
the 4 years. Students learn and practice negotiated interviews with standardized patients and provide screening and counseling for substance use. All students are assessed using standardized patient experiences.

\section{Assessment changes since 2010}

To enhance consistency in student assessment across clerkship sites, we revised our clerkship assessment form, the clinical student evaluation form, during the 2017-2018 academic year to include achievable, observable behaviors expected of a thirdyear clerk in all domains. The behavioral anchor for level 4 (the highest level on the scale) includes language from the AAMC's Entrustable Professional Activities. Since 2010, we have increased the number of objective structured clinical examinations OSCEs in the curriculum. We have added OSCEs at the middle and end of years 1 and 2, and at the end of year 3, to assess communication, physical examination, and clinical reasoning skills as students progress and prepare for the USMLE Step 2 CS examination. The majority of clerkships now also include either a standardized patient-based OSCE or an oral clinical reasoning exam as part of summative assessment at the end of the clerkship. The NBME shelf exam is limited to $30 \%$ of the total grade across all clerkships.

In addition, we have added formative assessments during clerkships to ensure direct observation of core skills. Medical students in each clerkship are given Feedback on Clinical Skills forms that document direct observation experiences in each clerkship and ensure that every component of the physical exam is observed during the third year.

\section{Medical education program objectives}

The curriculum is guided by BUSM's medical education program objectives that are based on the Physician Competency Reference Set. The objectives have been mapped to the 7 BUSM institutional learning objectives, represented by the acronym BU CARES, which are based on ACGME domains of competence.

See Supplemental Digital Appendix 2-Program Objectives and Assessment Methods - at http://links.lww.com/ACADMED/ A869.

\section{Pedagogy}

We use multiple pedagogical approaches in our curriculum. Our foundational science faculty use lecture, videos, largeand small-group discussion, labs, team-based learning, and patient panels to teach curricular content. Human Behavior in Medicine and EPH use lectures, case-based learning, small groups, and workshops to teach. Our Doctoring courses use peer teaching, with fourth-year students teaching the first-year students inpatient interviewing; they also teach second-year students inpatient history and the physical exam portion of the course. The majority of the Doctoring course is taught in small groups facilitated by a longitudinal academy medical educator (AME) who uses techniques such as role play, think-pair-share, deliberate practice, and peer learning. Actors are also used several times in the year when more complex interviews or communication skills are practiced, such as sexual history taking or breaking bad news.

A longitudinal preceptorship occurs throughout the first 2 years of the Doctoring course. The third and fourth years are predominantly clinical experiences in the ambulatory and inpatient settings. Core content is delivered in all clerkships using workshops, simulated patients, lectures, and cases. Self-directed learning is incorporated throughout the program.

\section{Changes in pedagogy since 2010}

The pedagogical changes made since 2010 are numerous including the creation of new videos for flipped content, new application exercises in the classroom to increase active learning, more case-based exercises, and small- and large-group work. We have maintained consistency of faculty and students in small groups in the Doctoring courses to support faculty development and team building. Finally, we have increased the use of simulated patients throughout the 4 years. Currently, we are using virtual reality for the first time to teach first-year students about poverty and social determinants of health.

\section{Clinical experiences}

We immerse students in the clinical setting from the first month of medical school. During the first 2 years, students see hospitalized patients at Boston Medical Center and at the Veterans Administration Boston Healthcare System for approximately 20 hours each year and participate in a longitudinal preceptorship in private group practices and community health centers for approximately 30 hours each year. BUSM is affiliated with over 25 hospitals, multiple community health centers, and private practices, providing our students with a diverse group of patients to learn from during the third and fourth years. Students also have the opportunity to participate in clerkships and electives at our regional campus, Kaiser Permanente Regional Campus-Silicon Valley (KP-SV), located in Santa Clara and San Jose, California. BUSM has successfully addressed the challenge of maintaining consistency of experiences at multiple sites by appointing an assistant dean for affiliated sites (AD-AS), who focuses on faculty and site development.

\section{Curricular Governance}

See Figure 1-Curricular governance.

\section{Education Staff}

The Medical Education Office (MEO) consists of the associate dean of medical education (ADME), 3 assistant deans, 5 faculty, and 13 staff. Each assistant dean oversees a separate area of focus: curriculum and instructional design, affiliated sites, and enrichment (global health, research, and service learning). Our staff provide direct support for administration of the undergraduate medical education program. This includes administrations of all of the preclerkship courses, maintaining and supporting program tools such as the curriculum database and eValue, and collecting 


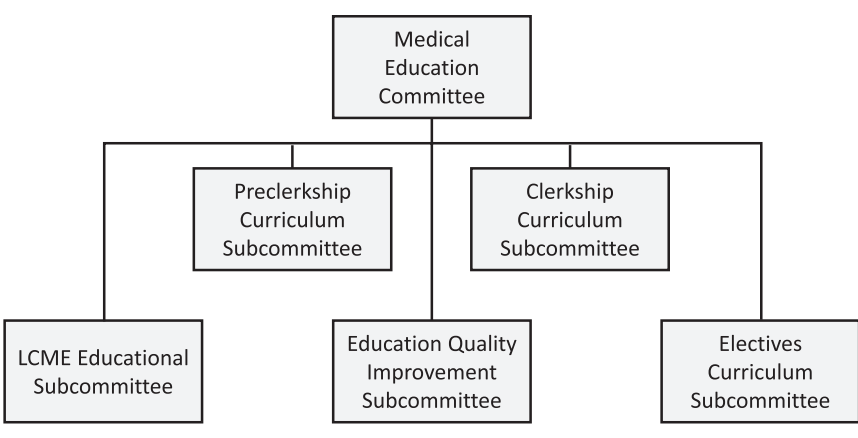

Figure 1 Curricular governance.

and analyzing educational program outcomes and student performance in the curriculum. The MEO works in collaboration with the other deans' offices to support students and faculty. All clerkship coordinators, except one with a dual role in the education office, are located in their respective departments.

Faculty appointments are overseen by the Faculty Affairs Office. Affiliate preceptors are appointed through the faculty's clinical department. Faculty must teach medical students at least 50 hours per year to receive a faculty appointment.

See Supplemental Digital Appendix 3-Organizational Chartat http://links.lww.com/ACADMED/A869.

\section{Faculty Development and Support in Education}

Professional development on the medical campus is overseen by the assistant provost of faculty development for Boston University Medical Campus (BUMC). Five longitudinal faculty development programs and a seminar series are regularly offered: early career, midcareer, women in medicine, underrepresented minorities in medicine, narrative writing, and a longitudinal faculty development seminar series.

The medical campus hosts the annual John McCahan BUMC Education day, a 1-day conference that focuses on teaching and education. The conference includes a keynote lecture; workshops; and oral and poster presentations by medical campus faculty, staff, and students.
Table 1

Regional Medical Campus

\begin{tabular}{lll}
$\begin{array}{l}\text { Regional } \\
\text { campus name }\end{array}$ & Type & $\begin{array}{l}\text { Student enrollment } \\
\text { Kaiser Permanente Branch }\end{array}$ \\
Campus-Silicon Valley & Clinical & $\begin{array}{l}16 \text { full-time equivalent } \\
\text { students }\end{array}$ \\
\hline
\end{tabular}

Within the MEO, the ADME and the assistant dean of medical education for curriculum and instructional design provide faculty development and individual coaching to our faculty educators, including the AMEs who work with students in the year 1 and year 2 Doctoring courses. All AMEs are also provided access to faculty development sessions on medical education scholarship and research held by the MEO. The AD-AS and the manager of affiliated sites provide faculty development and training to affiliated faculty during regular site visits, including visits to KP-SV.

\section{Regional Medical Campus}

To maintain consistency of experience across our clinical sites, we have appointed an AD-AS within the MEO. She and her administrative manager work closely with the $2 \mathrm{KP}-\mathrm{SV}$ assistant deans for BUSM and communicate regularly with them. The KP-SV faculty are also represented in the membership of the curriculum committees.

All Kaiser campus clerkship students participate in the same third-year orientation as students on the Boston campus before leaving for their clerkships at Kaiser. Kaiser students have all of the same learning objectives, requirements, and assessments as the Boston-based students. Kaiser learners use Zoom web conferencing to participate in the required clerkship didactics, which are based in Boston.

See Table 1-Regional Medical Campus.

The operational leadership team monitors the performance of the Kaiser learners to assure that their academic performance and match rates are comparable to classmates. 


\section{Harvard Medical School}

Edward M. Hundert, MD, and Jane M. Neill

\section{Medical Education Program Highlight}

- Harvard Medical School (HMS) offers its students a unique educational environment that includes 15 outstanding affiliated hospitals and the largest medical school biomedical research enterprise in the United States. Harvard enrolls 165 medical students annually who have the opportunity to learn medicine from over 11,000 faculty. A robust advising system helps students connect with mentors as each student's interests develop.

- A 3-phase curriculum offers flexibility in the final phase for all students to choose from hundreds of electives and pursue a scholarly project in areas including basic/clinical research, global health, public policy, or medical humanities. HMS students also have access to degree programs at the other professional schools across Harvard University and at the Massachusetts Institute of Technology (MIT).

- The Pathways curriculum enrolls 135 MD students annually and is highly integrated, weaving basic/social science and clinical experiences weekly and across curricular phases with an innovative form of pedagogy, case-based collaborative learning (CBCL), designed to develop critical thinking and collaboration skills for lifelong learning and high levels of student engagement.

- In collaboration with MIT, the Health Sciences and Technology (HST) program is a unique curriculum track for 30 students admitted annually who are committed to careers as physicianscientists.

\section{Curriculum}

\section{Curriculum description}

The Pathways curriculum is highly integrated across 3 phases:

- The preclerkship phase (14 months) features integrated basic and social science blocks and a weekly longitudinal Practice of Medicine course, introducing students to fundamentals of the physical exam, communication skills, and interprofessional collaboration. Harvard dental students join Pathways students in the phase, and all students begin regular meetings with faculty advisors through the Academic Societies. Pathways students are assigned to 1 of 4 Harvard teaching hospitals for their Practice of Medicine course and remain at that site for the next phase of the curriculum.

Acad Med. 2020;95:S223-S226.

doi: 10.1097/ACM.0000000000003342

Copyright (C) 2020 by the Association of American Medical Colleges

Supplemental digital content for this article is available at http://links.Iww.com/ ACADMED/A865.

Year school was established: 1782.

School URL: https://hms.harvard.edu.
- The principal clinical experience (PCE) phase (12 months) begins in the fall of year 2 and includes immersive clerkships in core clinical fields, a longitudinal primary care clerkship, and longitudinal case conferences and advising at each PCE site.

- The post-PCE phase (19 months) provides flexibility for students to pursue an individualized curriculum, including:

- A required subinternship in medicine.

- A minimum of 3 advanced clinical clerkships at HMSaffiliated hospitals, selected from over 250 offerings.

- A clinical capstone month during the final semester of medical school to prepare students for internship; this features subintern-level clinical responsibilities and special sessions designed to help students identify areas of focus as they start internships.

- Two Advanced Integrated Science Courses (AISCs): innovative courses cotaught by basic and clinical scientists to allow students to explore the boundaries between the known and unknown after completing PCE clerkships.

- A month of advanced topics in social/population sciences and health systems-/value-based care, building on students' clinical experiences during the PCE and advanced clinical experiences; this course is an advanced rejoinder to the firstyear course, Essentials of the Profession, which introduces Pathways and HST students to topics in social medicine, medical ethics and professionalism, health policy, and clinical epidemiology and population health.

- A scholarly project, through which each student is challenged to delve deeply in an area of interest in basic or clinical research, global health, medical humanities, social justice, or another area of interest.

- Opportunities during the post-PCE phase for a majority of Pathways and HST students to extend their medical education by an additional year of enrollment; about half of these complete a project focused on research, international health, advocacy, or entrepreneurship, and another half pursue a $\mathrm{PhD}$ or master's degree.

See Supplemental Digital Appendix 1-Pathways Curriculum Map_at http://links.lww.com/ACADMED/A865.

\section{Curriculum changes since 2010}

- The innovative Pathways curriculum was launched in 2015 after an intensive 3-year planning process involving large numbers of faculty, students, and staff who engaged in a bottom-up curriculum and pedagogical reform initiative. A survey of research on learning theory and educational outcome studies led to a focus on interleaving key content using a flipped classroom model. Implementation required a multiyear effort to create concept videos and other preparatory materials customized for Pathways learners. Pilots were conducted with different forms of pedagogy, resulting in the novel CBCL model. Education spaces in 
the medical education center were renovated to optimize educational form-function correspondence. The new pedagogy also required extensive faculty development and an entirely new assessment system.

- In addition to a complete overhaul of the content, structure, and pedagogy of the preclerkship curriculum, the Pathways reform frameshifted the PCE from year 3 to year 2, requiring a one-time overlap of 2 classes during academic year 2016-2017. This shift required extensive planning; financial resources; and hospital, faculty, staff, and student support.

- The frameshift of the PCE year allowed creation of the new AISCs that are now a highlight of the post-PCE phase. These courses range in subject area from cancer biology, immunology, genetics, pharmacology, and neurobiology to computationally enabled medicine, translational bioengineering, metabolism/nutrition, global health, and health systems science.

- The new clinical capstone course, required for both Pathways and HST students, was designed as a 1-month intensive subinternship with a focus on complex communication skills needed for internship and opportunities for each student to assess their own areas of continued development as they approach residency.

- The HST program introduced 2 new courses for first-year students: Matlab for Medicine, a hands-on introduction to quantitative methods in medicine and health research; and Growth of the Physician Scientist, an integrated, yearlong experience designed to support the personal and professional transformation from student to physician-scientist, building on the themes of patient care, community, mentorship and role modeling, and reflective practice.

\section{Assessment}

- In 2017, the Program in Medical Education (PME) established the new Office of Educational Quality Improvement (OEQI) to manage new student assessment and curriculum evaluation systems. The OEQI tracks curricular and student outcomes through an education data warehouse, which draws data from students' academic records, course exams, and objective structured clinical examinations (OSCEs), and students' evaluations of teachers, courses, and clerkships. The OEQI staff generate reports on student progress that correlate student performance with other key metrics, demonstrate comparability of learning outcomes across clinical sites, and perform many other important assessment and evaluation functions.

- With the launch of Pathways, HMS also implemented the AAMC's Entrustable Professional Activities (EPAs) framework as the basis for all clinical evaluations. Relevant EPAs were mapped to courses and clerkships in each of the 3 curricular phases, and rubrics were created to provide developmentally appropriate feedback to students on all EPAs.

- Students have been graded satisfactory-unsatisfactory (S-U) in the first 2 years of the HMS curriculum for many decades, and this continues. To maintain S-U grading in the first half of the MD program, grading in the core clerkships (now in year 2) was converted to S-U in 2016, when the first class of Pathways students entered the PCE. Students continue to receive quantitative and qualitative feedback in their core courses and clerkships. The required subinternship in medicine and all elective advanced clerkships/subinternships continue to be graded using the honors-with-distinction, honors, pass, unsatisfactory scale, which is also used for departmental summative assessments that grade students by clinical discipline based on their 4-year clinical performance. Formative and summative narratives of student performance are provided in required and elective courses. USMLE Steps 1,2 CK, and 2 CS examinations are taken after the PCE phase; students must pass all 3 and a multistation OSCE administered at the end of year 3 as requirements for graduation.

- In parallel with the above, a broad-based process involving focus groups, benchmarking from other schools and the ACGME, and attention to HMS's mission led to a revision of the MD program objectives.

See Supplemental Digital Appendix 2-Program Objectives and Assessment Methods—at http://links.lww.com/ACADMED/A865.

\section{Parallel track}

- The Harvard-MIT HST program is a unique partnership between HMS and MIT, annually admitting 30 students committed to becoming physician-scientists to a parallel, 18-month preclerkship curriculum. HST students begin the PCE phase in the spring of year 2.

- HST preclerkship courses include MIT graduate students pursuing a $\mathrm{PhD}$ in medical engineering/medical physics and are taught by faculty from both HMS and MIT. Students are given time to do research for a required thesis during this phase.

See Supplemental Digital Appendix 3-HST Curriculum Map_at http://links.lww.com/ACADMED/A865.

\section{Pedagogy}

- HMS's guiding educational philosophy is a belief that meaningful relationships between students and faculty are the most important ingredient in students' personal and professional development. High levels of support and challenge-balanced through the Society Advisor structure in which each student is known well by a supportive, nonevaluative advisor-enable all students to achieve their fullest potential.

- HMS's CBCL pedagogy, developed and tested during the creation of the Pathways curriculum, included a randomized trial with positive outcomes demonstrating a high degree of student engagement and complementarity with other forms of pedagogy employed throughout the curriculum. Students are expected to prepare for each session and work collaboratively in learning studios designed specifically for CBCL.

- The curriculum also includes some large-group, mostly interactive sessions; laboratories; gross dissections; simulations (including interprofessional); standardized patient encounters; and mentored clinical and research experiences. 


\section{Clinical experiences}

- One of HMS's greatest assets is the wealth of clinical experiences available to medical students. Students spend the PCE phase at one of the HMS-affiliated hospitals: Beth Israel Deaconess Medical Center (BIDMC), Brigham and Women's Hospital (BWH), Cambridge Health Alliance, or Massachusetts General Hospital; students at BIDMC and BWH do pediatrics at Boston Children's Hospital. Students also have numerous opportunities for clinical experiences at other HMS-affiliated hospitals during the post-PCE phase.

- All students have a combination of block and longitudinal experiences. Longitudinal experiences include primary care preceptorships in both the Practice of Medicine course and the PCE, along with longitudinal advising and interdisciplinary conferences. The PCE at Cambridge Health Alliance is a longitudinal integrated clerkship.

\section{Curriculum Governance}

- The MD program is governed by the Educational Policy and Curriculum Committee (EPCC), a Standing Committee of HMS, created and charged by the Faculty Council, the elected faculty governing body. The EPCC and its 5 subcommittees (Pathways Preclerkship, HST MD Curriculum, PCE, Post-PCE, and Assessment) include faculty and medical students as voting members, as well as ex officio faculty and staff leaders.

- Other important MD program committees include the Admissions, Financial Aid, and Learning Environment Committees, the Promotion and Review Board, and the Council of Academic Societies, which oversees student advising.

See Figure 1-Curriculum governance.

\section{Education Staff}

- The PME is the organizational structure for the education program that leads to the MD degree. Led by the dean for medical education, with administrative support from the associate dean for medical education planning and administration, the PME includes the Offices of Admissions, Financial Aid, Registrar, Educational Quality Improvement, Curriculum Services, Student Affairs, and Recruitment and Multicultural Affairs; the Academic Societies; and other related functions, including finance, the Scholarship in Medicine Program, disability services, and the Anatomical Gift Program.

- The advising/mentoring function is supported through the Office of Student Affairs, the Academic Societies, and the Office of Recruitment and Multicultural Affairs.

- Curriculum and assessment functions are primarily shared by 2 offices: the Office of Curriculum Services, which supports required courses and clerkships with professional staff, and the OEQI (described above), which supports student assessment as well as course and program evaluation.

See Figure 2-Education administration.

\section{Faculty Development and Support in Education}

- The OEQI also provides faculty development for faculty teachers, mentors, and advisors in the MD program. A faculty director of faculty development devises general and tailored programs for faculty across the MD curriculum, as well as resident-as-teacher initiatives.

- The Academy supports monthly medical education grand rounds; Academy Fellowships in medical education and educational research; annual events such as the Symposium on the Science of Learning and Medical Education Day; and a wide variety of faculty innovation groups in areas ranging from simulation to the hidden curriculum.

\section{Initiatives in Progress}

- The structure of the PCE year is currently under review for innovative new models combining the best of block and longitudinal integrated clerkships.

- In connection with its 50th anniversary, the HST program has launched a major programmatic review, reimagining the best curriculum to prepare physician-scientists for the future.

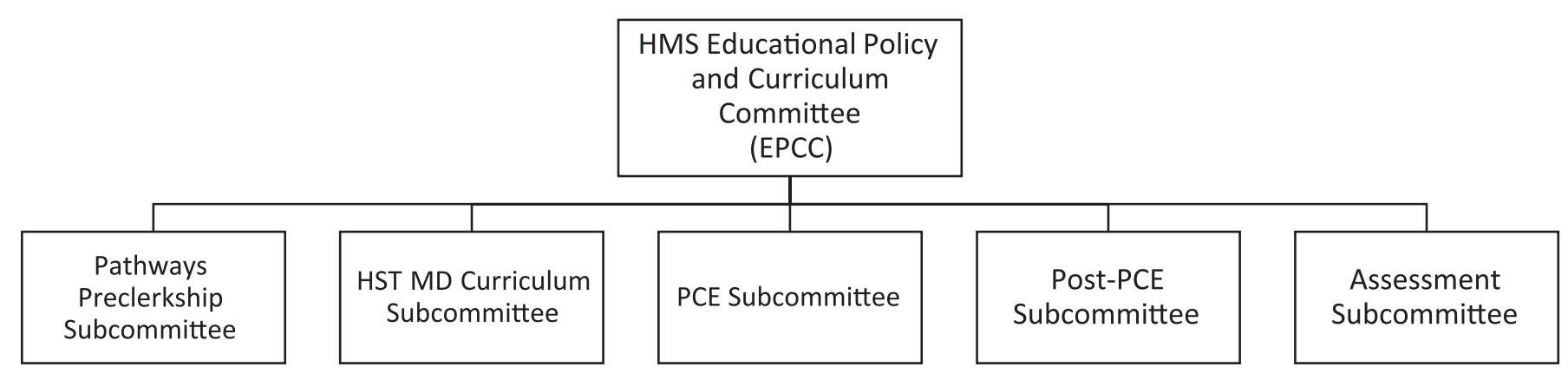

Figure 1 Curriculum governance. 


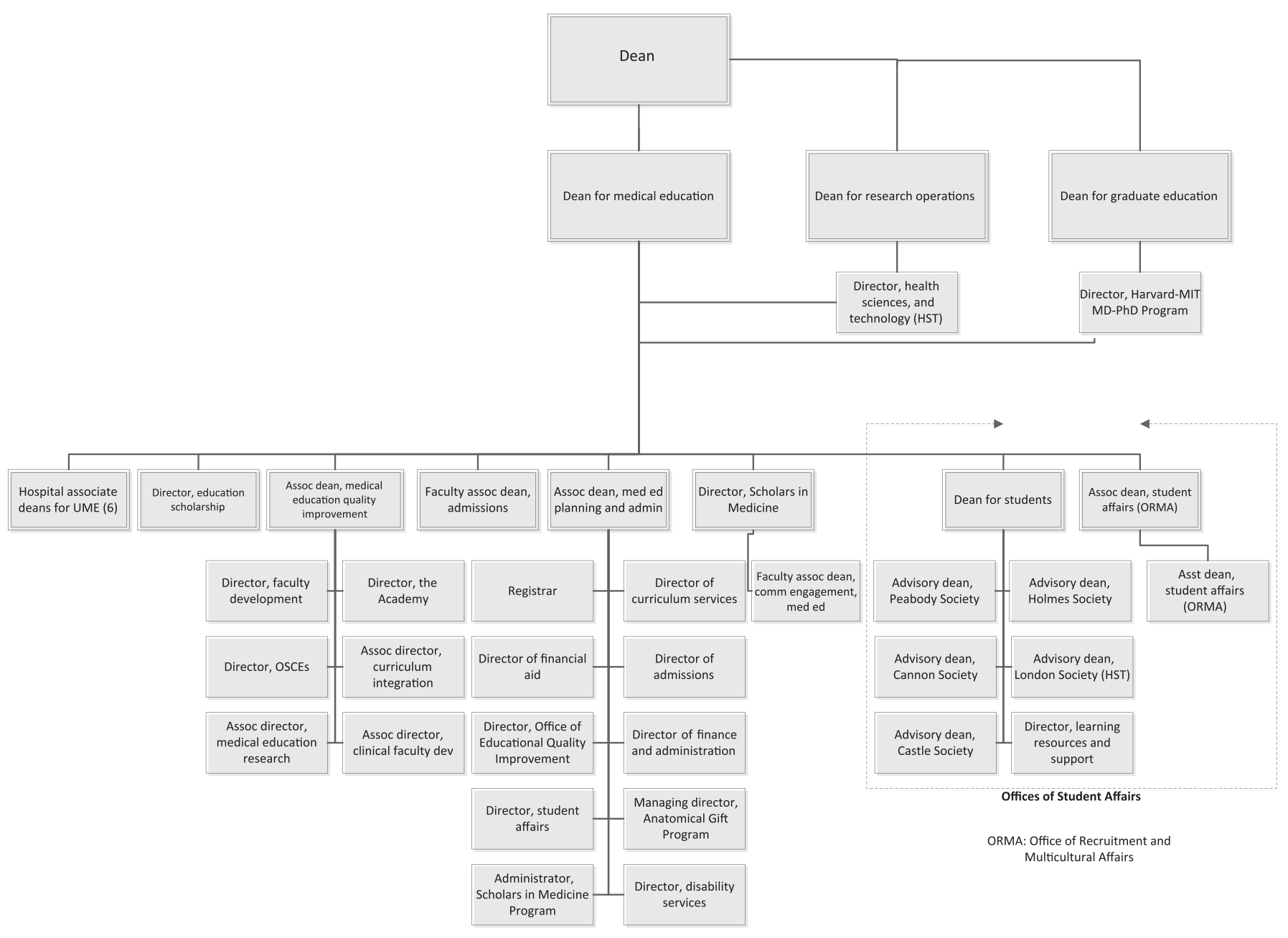

Figure 2 Education administration. 


\section{Tufts University School of Medicine}

Maria A. Blanco, EdD, and Scott K. Epstein, MD

\section{Medical Education Program Highlights}

The new Tufts curriculum (beginning with the class of 2023) seeks to graduate highly skilled and compassionate clinicians imbued with the knowledge, skills, and attitudes to promote the continual improvement of health and health care of individuals and populations. Students develop advanced skills in clinical reasoning and communication, allowing them to improve the patient experience while delivering high-quality and costeffective care. Students cultivate skills in personal reflection, mindfulness, resilience, inquiry, and lifelong learning, essential for adaptation to a constantly changing health care environment. Graduates will be able to apply a detailed understanding of health care delivery systems (and how to navigate those systems), patient-centered outcomes, social determinants of health, and population health to their practice of medicine.

Highlights:

- Curricular threads interwoven throughout the 4-year curriculum

- A new coaching program designed to ensure that a faculty member has a 4-year longitudinal picture of a student's development of clinical skills and professional identity formation

- A Maine Track Program (20\% of the class) that includes a longitudinal integrated clerkship (LIC) with a focus on rural health

- Comprehensive student participation in developing and monitoring the curriculum, including engagement in designing the new curriculum, regular meetings with deans and education staff to discuss all student educational experiences, and full representation on the Curriculum Committee (CC) and its task forces

- Completion of a dual-degree program: MD-PhD, MD-MPH, MD-MBA, or MD-Master of Arts (Fletcher School) by 20\% of students

See Table 1-Curricular Threads.

\section{Curriculum}

\section{Curriculum description}

See Supplemental Digital Appendix 1-Curriculum Overviewat http://links.lww.com/ACADMED/A863.

Acad Med. 2020;95:S227-S231.

doi: 10.1097/ACM.0000000000003338

Copyright (C) 2020 by the Association of American Medical Colleges

Supplemental digital content for this article is available at http://links.Iww.com/ ACADMED/A863.

Year school was established: 1893.

School URL: https://medicine.tufts.edu.

\section{Curriculum changes since 2010}

A new curriculum was launched in August 2019 with the class of 2023 that builds on and further expands on detailed curriculum revisions implemented in 2009. Features of the new curriculum, and other changes since 2010, include:

- Faculty coaching program. Coaches are assigned 10 students (every other class) to support academic performance and personal and professional identity development throughout medical school. The coaching program is nested in a set of learning communities (with 5 coaches per learning community). The coach is responsible for fostering the student's professional identity development (including resilience, ethical practice, self-regulated lifelong learning, navigating uncertainty), providing formative feedback on clinical skills, facilitating self-reflection, and helping students remediate areas of weakness. The coach meets regularly with each student, reviews all formative and summative assessments, participates in the first-year Problem-Based Learning course, and provides formative feedback on clinical skills. A learning portfolio system was launched to facilitate student/coach collaboration.

- Redefined curricular threads woven throughout all 4 years emphasize knowledge, skills, and attitudes related to population health, health care delivery, personal and professional development, and the patient experience.

- A new Population Health and the Profession of Medicine course at the start of the first year introduces students to the health care system, social determinants of health, health care disparities, quality improvement science, ethics and professionalism, and principles of population health. The course includes a community engagement exercise providing early experience in assessing the health needs of populations. This exercise introduces students to the Tufts Community Service Learning Program that is completed during the preclerkship phase.

- New preclerkship thematic blocks (starting in fall of the first year) that promote a more cohesive integration of basic, clinical, and health care systems science and the curricular threads.

- Expanded preclerkship clinical reasoning curriculum that begins in the first year.

- Maine Track students have increased preclerkship time on the Portland, ME campus.

- A preclerkship phase that ends in mid-January with core clerkships beginning in March of the second year.

- New core clerkship integration weeks that return students to campus to more effectively integrate curricular threads.

- A required scholarly project program that includes online modules on research design, translational medicine, quality improvement, and academic writing. Working individually or in teams of up to 4, students collaborate with faculty mentors on a scholarly project in basic science, clinical research, or quality improvement. 
Table 1

\section{Curricular Threads}

$$
\text { Threads }
$$

\section{Subthreads}

\section{Population health}

\begin{tabular}{ll} 
Health determinants & Health disparities \\
Public health & Prevention \\
Culturally competent care & Care of the underserved \\
\hdashline Active citizenship & Global health \\
\hline Social justice &
\end{tabular}

\begin{tabular}{ll}
\hline Health care delivery & Health care systems \\
Cost of health care & Quality improvement \\
Quality and safety & Advocacy \\
\hline Interprofessional collaboration & Clinical informatics \\
Law and reporting obligations & Team-based care \\
\hline Chronic illness care &
\end{tabular}

\section{Personal and professional \\ development}

\begin{tabular}{ll} 
Wellness & Resilience \\
\hline Professionalism & Ethical practice \\
\hline Information mastery & Self-regulated lifelong learning \\
\hline Inquiry/discovery & Navigating uncertainty \\
\hline Professional identity formation & Leadership \\
\hline Teaching & Clinical skills \\
\hline Clinical reasoning & \\
\hline
\end{tabular}

\section{Patient experience}

Advanced communication End-of-life and palliative care Management of substance use disorder

\section{Shared decision-making}

Management of pain Impact of health on patient and family life

- Addition of an end-of-fourth-year boot camp to provide final preparation for residency.

- Significantly expanded interprofessional collaboration experiences (case based, simulated, and clinical) throughout the curriculum with professionals and students including pharmacists, physicianassistants, nurses, social workers, and dieticians.

- Expanded curriculum on end-of-life and palliative care, management of pain, and management of substance use disorders (students now qualify for certification to provide medication-assisted treatment).

- The new Health Justice Scholars Program designed for students committed to serving under-resourced populations. Students work with a faculty mentor, engage in extracurricular programmatic activities, and complete many of their clinical rotations at sites with a large underserved patient population.

- The required Student-As-Teacher Program ${ }^{1}$ was launched and includes online modules and a field teaching experience.

\section{Assessment}

- Tufts has defined 58 overall learning objectives and competencies, addressed in both preclerkship and clerkship phases, grouped using the taxonomy of competency domains proposed by Englander et $\mathrm{al}^{2}$ (https://medicine.tufts.edu/sites/ default/files/education_educational-objectives.pdf).

- Preclerkship course and clerkship objectives and assessments (multiple-choice and short-answer exams, standardized patient and simulated scenario exams, ethical analyses, reflective papers, direct observation of preclerkship small-group work, objective structured clinical examinations, workplace observations) map to the overall learning objectives.

- Since 2010, there has been an expansion of standardized patient assessments, reflection papers, team-based learning assessments, and implementation of integrated cumulative exams.

\section{Pedagogy}

Tufts uses an array of pedagogical approaches to achieve the school's educational program objectives. Of note, the first-year Problem-Based Learning course is delivered exclusively as a self-directed learning experience. Pedagogical changes have been implemented since 2010:

- Reduction in lecture time to promote active and collaborative learning approaches

- Requirement that all lectures include an interactive component

- Requirement that at least $50 \%$ of preclerkship teaching hours use active or engaged learning

- Significant expansion in team-based learning, flipped classroom, and small-group learning

- Expanded simulation education including a comprehensive preclerkship ultrasound curriculum

- Expanded use of standardized patient experiences including for training on advanced communication skills and with interprofessional collaboration

See List 1-Pedagogical Approaches.

\section{List 1}

\section{Pedagogical Approaches}

Case-based teaching

Clinical experience: ambulatory

Clinical experience: inpatient

Discussion: large group (> 12)

Discussion: small group $(\leq 12)$

Flipped classroom

Laboratory

Lecture (interactive)

Patient presentations

Peer teaching

Preceptorship

Problem-based learning

Role play

Self-directed learning

Simulation

Standardized/simulated patients

Team-based learning

Video/podcast

Virtual patient

Workshop 


\section{Clinical experiences}

- Learners encounter clinical experiences during the first month of medical school in the medical interviewing course and through weekly clinical selectives.

- Students subsequently spend a full day each week in a primary care office (the competency-based apprenticeship in primary care) from May of the first year through January of the second year.

- Tufts has a diverse group of affiliated inpatient and outpatient clinical sites for core clerkships and advanced clinical rotations, giving students the opportunity to learn in a range of settings including rural communities, suburban locations, and major urban areas.

- Site directors for each of the core clerkship disciplines meet quarterly to ensure comparability of experience and to refine the curriculum.

- Students in the Maine Track can participate in an LIC (see below).

- Required fourth-year experiences include two 4-week acting internships, a 4-week clinical neuroscience rotation, and a minimum of five 4 -week electives.

- The principal challenge faced in implementing clinical experiences is the competition between medical schools and other health sciences professions programs for core clerkship slots.

See List 2-Teaching Affiliates.

\section{Curricular Governance}

The Tufts curriculum is managed by the faculty-led CC, Office of Educational Affairs (OEA), and Office of Student Affairs (OSA).

- The CC, consisting of elected and appointed members, meets monthly. Members are drawn from basic science and clinical faculty with representation from course and clerkship directors, all major clinical departments, and each major teaching affiliate. Sixteen student members ( 4 from each year) are elected by the student body.

- The CC is responsible for development and implementation of the school's educational policies and the evaluation of the educational program, content, and teaching.

- The CC has responsibility for reviewing all new curricular initiatives.

- The CC oversees the work of task forces and subcommittees, including preclerkship course directors (phase 1), core collaborators (phase 2), the Simulation Curriculum Subcommittee, and the Evaluation Subcommittee.

- The core collaborators, consisting of the overall core clerkship directors and key educational staff, meet monthly and oversee the core clerkships, acting internships, and clinical electives. The group examines all aspects of clinical education including competency-based learning objectives, content within and across clerkships, assessment tools, grading, evaluation of site quality, and best educational practices across sites.

- The CC performs regular peer review of all courses and clerkships (approximately every 3 years) and conducts a yearly review of the entire educational program to ensure the Tufts

\section{List 2}

\section{Teaching Affiliates}

Major hospital affiliates (hosting 3 or more core clerkships)

Tufts Medical Center

Maine Medical Center (Portland, Maine)

Lahey Hospital and Medical Center

Newton-Wellesley Hospital

South Shore Hospital

St. Elizabeth's Medical Center

\section{Other affiliates (hosting 1-2 core clerkships or advanced clinical rotations)}

Atrius Health

Baystate Medical Center

Cambridge Health Alliance

Carney Hospital

Faulkner Hospital

Lawrence General Hospital

Lemuel Shattuck Hospital

Lowell General Hospital

MelroseWakefield Hospital

Metro West Medical Center

Portsmouth Regional Hospital (Portsmouth, New Hampshire)

Salem Hospital/North Shore Medical Center

Winchester Hospital

\section{Longitudinal integrated clerkship sites (all located in Maine)}

Cary Medical Center

Franklin Memorial Hospital

Maine Medical Center

Mid Coast Hospital

Miles Memorial Hospital

Mount Desert Island Hospital

Penobscot Bay Medical Center

Reddington Fairview Hospital

St. Mary's Regional Medical Center

Stephens Memorial Hospital

Togus Veterans Administration Hospital

overall educational learning objectives are achieved in both phases of the curriculum.

- The CC is charged with implementing the educational strategic plan (new curriculum).

- The OEA and OSA work to implement the CC's recommendations and policies.

See Figure 1-Curriculum management.

\section{Education Staff}

The OEA works with the CC, and its subcommittees, to plan, implement, evaluate, and oversee the curriculum while supporting curriculum delivery, monitoring, and management. 
The OEA is exclusively responsible for undergraduate medical education, including:

- Centralized management of the curriculum, including delivery of all preclerkship courses and programs

- Implementation of CC recommendations

- Working with the OSA and the core collaborators to manage the core clerkships and fourth-year required rotations and electives

- Evaluation and assessment of all courses and clinical rotations (overseen by a doctorate-level evaluator)

- Oversight and administration of educational grants

- Faculty development (overseen by a doctorate-level associate dean)

- Conduct and support of educational research

- Administrative support for education-related faculty committees

- Coordination of specialized programs including the standardized patient program and medical education electives

- Oversight of the 9,000 square foot clinical skills and simulation center (a new, expanded facility is scheduled to open in spring of 2022)
- Support of the Accreditation Standards Oversight Committee, charged with continuous monitoring of Tufts' compliance with LCME accreditation standards

The new Department of Medical Education was recently created to provide an academic home for basic science (and some clinician) educators.

\section{Faculty Development and Support in Education}

- Both centralized and hospital-based programs are included.

- A new, comprehensive series of webinars can be watched live or asynchronously.

- In-person centralized and hospital-delivered workshops and sessions, including seminars on teaching, educational research, and educational leadership, and a mutual mentoring program for career advancement. All in-person initiatives focus on promoting the development of communities of practice.

- A longitudinal faculty development program for new coaches has been implemented.

- A faculty medical education journal club consists of regular online exchanges providing summaries of key articles in health sciences education.

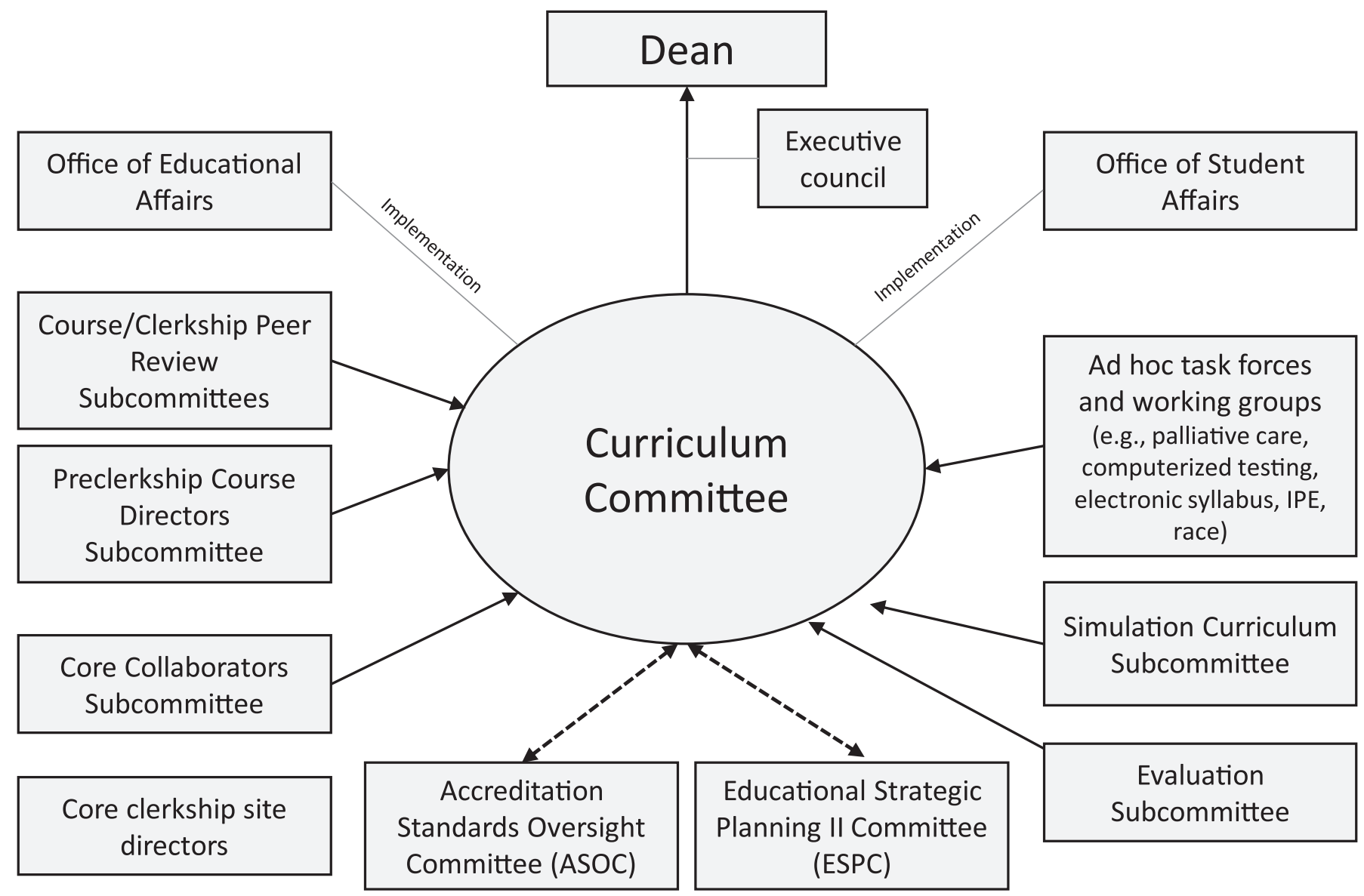

Figure 1 Curriculum management. 
- Faculty receive individualized medical education literature alerts, including monthly email updates from the Hirsh Health Sciences Library.

- Annual Innovations in Medical Education and Innovations in Diversity Education Awards grant programs have provided faculty with approximately $\$ 1,000,000$ in intramural funding since 2005.

\section{Regional Medical Campus}

- Since 2009, Tufts has partnered with Maine Medical Center (MMC) in Portland, Maine, to offer the Maine Track. Forty students (20\% of each class) are annually accepted to the track by a subcommittee of the Admissions Committee.

- Accepted students have close ties to Maine or are otherwise interested in clinical training experiences with exposure to the unique aspects of rural practice and with an interest in returning to Maine to practice.

- Before the class of 2023, Maine Track students spent their first 2 years primarily on the Boston campus, with some specific curriculum components completed in Maine. Students relocated to Maine for core clerkships and portions of the monthly rotations in the fourth year.

- Beginning with the class of 2023, Maine Track students will spend their entire second year in Portland. Preclerkship courses delivered in Maine have identical objectives, content, and assessments as those delivered in Boston. Boston and Maine course codirectors meet regularly and work collaboratively to deliver a consistent educational experience.

- 20-22 Maine Track students annually participate in a 9-month LIC experience either at MMC or at select rural/community sites throughout the state. The remaining Maine Track students complete block core clerkships at MMC.

\section{References}

1 Blanco MA, Maderer A, Oriel A, Epstein SK. How we launched a developmental student-as-teacher (SAT) program for all medical students. Med Teach. 2014;36:385-389.

2 Englander R, Cameron T, Ballard AJ, Dodge J, Bull J, Aschenbrener CA. Toward a common taxonomy of competency domains for the health professions and competencies for physicians. Acad Med. 2013;88: 1088-1094. 


\title{
University of Massachusetts Medical School
}

\author{
Anne Campbell Larkin, MD, and Melissa A. Fischer, MD, MEd
}

\section{Medical Education Program Highlights}

The University of Massachusetts Medical School (UMMS) admitted its first class in 1970 as the only public medical school in Massachusetts, and celebrates its first 50 years in 2020. With a strong emphasis on and dedication to primary care, the school has forged a reputation of excellence through innovative teaching and learning, the early use of standardized patients, and recognized success in all of our missions, including education, research, and clinical care. Now having finished the first decade of the Learner-centered Integrated Curriculum (LInC), we have embraced early clinical experiences, integrated clerkships, and flexible experiences to achieve our core competencies: physician as scientist, problem solver, communicator, professional, advocate, and person. Students participate in interprofessional activities throughout the curriculum, and the spirit of giving back is powerful, with virtually $100 \%$ of students engaging in service to the community. We greatly value the emotional well-being of our students and, as such, have established the robust Student Life Office, emphasizing perhaps our most important competency of physician as person.

\section{Curriculum}

\section{Curriculum description}

Following an extensive planning process, the LInC was initiated in academic year 2010-2011. This innovative curriculum pairs basic and clinical scientists to deliver curriculum integrated across courses, with very early clinical experiences and an increased emphasis on communication skills. Building upon our strong history of simulation and standardized patient experiences, many such experiences were added into the preclinical years with deliberate addition of formative assessment. In the clinical years, clerkships are cohorted into 3 main themes (care of families, care of the adult, perioperative and maternal care), with shared curricula across clerkships and in some cases shared assessments. Longitudinal threads include the capstone Scholarship and Discovery course, in which students engage in using the scientific method to complete a project over the course of 4 years. Additionally, learning communities were established that have longitudinal faculty and peer mentorship, enhanced career advising, and an overall sense of community.

Acad Med. 2020;95:S232-S235.

doi: 10.1097/ACM.0000000000003345

Copyright (C) 2020 by the Association of American Medical Colleges

Supplemental digital content for this article is available at http://links.Iww.com/ ACADMED/A864.

Year school was established: 1971.

School URL: https://www.umassmed.edu.

\section{Curriculum changes since 2010}

The curriculum has continued to evolve, increasing the emphasis on diversity embedded within the curriculum, the expansion of the social determinants of health curriculum, and the addition of a robust interprofessional curriculum and experiences, all of which have greatly enhanced the ability of our students to work in and across teams. Some examples of curricular innovations are:

- An Interstitial Curriculum serves to meet the learning needs of our students across a variety of topics for which there are no discreet experiences within the clerkships, including veteran's care, patient safety, and intimate partner violence, among many others.

- Student-led curricular initiatives are strongly embraced, an example of which is an LGBTQ+ session designed by a secondyear student that was built around the engagement of members of the Worcester community to teach students important culturally competent strategies for caring for this vulnerable population.

- The opioid crisis has not spared Massachusetts, and to meet this public health need, an interprofessional simulationbased curriculum titled Opioid Safe-prescribing Training and Immersion is now required for all students.

- UMMS also now offers pathways for select students who augment their medical school experience through a concentration on global health, clinical and translational research, or rural health, and a recently implemented urban health pathway.

- We are now also proud to offer an MD-MBA degree in collaboration with the UMass Lowell campus.

- Additionally, we have begun an initiative across all learners on the campus to improve and enhance diversity, representation, and inclusion throughout the curriculum.

- In the coming years, we are embarking upon a dramatic change in our curriculum that will work toward increased community engagement, diversity, and interprofessional education. We will be augmenting the use of technology, supporting independent and engaged learning through the use of virtual reality, expanding an academic electronic health record populated with patients designed for learning, and enhancing simulation exercises. Continued amplification of small-group teaching and the codification of a robust health systems science longitudinal thread will be key components of this transformation. An additional critical component of these advancements will be the creation of an academy of teachers, called the Leadership/ Education Academy Program. This curricular transformation is being accomplished under the auspices of our newly developed strategic plan, IMPACT 2025.

\section{Assessment}

- The medical education program objectives for UMMS are framed around the 6 core competencies noted above, which largely parallel with ACGME core competencies. These competencies were initially developed in 2008 and revised in 2017. 
- Student assessment has evolved in the last decade, expanding formative assessment and feedback to help guide self-directed learning.

See Supplemental Digital Appendix 1-Medical Education Program Objectives - at http://links.lww.com/ACADMED/A864.

\section{Parallel curriculum or tracks}

- UMMS recently initiated a parallel track, the populationbased urban and rural community health (PURCH) track. This track is primarily housed and delivered at UMMSBaystate, our new regional campus located in Springfield, Massachusetts. If accepted into this track, students participate in a curriculum with enhanced engagement and exercises related to population-level care, social determinants of health, and disparity. The first class to complete this track will graduate in 2021.

- While UMMS does not currently offer a 3-year track, a task force is actively working toward the adoption of such an option for select students, and we anticipate the initiation of the track within 2 years.

\section{Pedagogy}

- UMMS uses a wide variety of pedagogical techniques to effectively teach medical students. The preclinical years use a combination of self-directed learning, small-group teaching, lab, simulation, and lectures. These experiences are augmented by role play experiences to learn communication skills, standardized patient experiences for learning and assessment, and peer teaching for a variety of topics including physical diagnosis.

- Since 2010, and with the implementation of the LInC, we have integrated our courses across the basic and clinical sciences, enhanced threads across the entire 4-year curriculum, and established a required scholarly project. The learning communities constitute a longitudinal foundational thread that has thrived as an integral component of the curriculum, enhancing peer teaching and promoting the strong sense of community experienced by our students.

\section{Clinical experiences}

- The medical school's primary site for clinical teaching is our clinical partner, UMass Memorial Healthcare, which has 3 campuses in Worcester. We also use more than 80 communitybased hospitals, health centers, and community practices across the region.

- Most recently, UMMS was fortunate to forge an educational alliance with Cape Cod Hospital, which has been an outstanding addition to our robust clinical experiences. Additionally, we have established a regional campus in Springfield, Massachusetts, in collaboration with the Baystate Health System, which oversees our regional campus track.

- Learners engage in their first clinical encounter during the second month of medical school, in which they start the Longitudinal Preceptor Program, which pairs them with a clinician for the purposes of learning communication and physical diagnosis skills.
- Community-based rotations are completed at a variety of practice sites, including federally qualified community health centers, multispecialty groups, and over 200 private practices statewide.

- Challenges in clinical education include limited compensation for community faculty, faculty pressures for clinical productivity, and ensuring the standardization of assessment across diverse sites.

\section{Curricular Governance}

The provision of the curriculum is centralized through our maintained governance. Administration of the courses, however, is maintained through both the Office of Undergraduate Medical Education and the clinical and basic science departments. Support for teaching activities is through the Office of the Dean. In an effort to more effectively meet the needs of clinical faculty, we are moving toward an FTE-based model for support of the educational mission.

See Figure 1-Curriculum governance.

\section{Education Staff}

- The Office of Undergraduate Medical Education is led by the associate dean of undergraduate medical education and oversees a staff of 10 , including her assistant dean of undergraduate medical education and assistant dean of health systems science. The administrators in this office provide support for many of the preclinical courses. The associate dean is responsible for the management and delivery of curriculum management and innovation, working collaboratively with committees and divisions across the institution. The office oversees the OASIS curriculum database, and in collaboration with the Office of Institutional Research, Evaluation, and Assessment continually augments and improves upon the tools and strategies used for student teaching and assessment.

- The Office of Undergraduate Medical Education provides for the oversight of the delivery of the program leading to the MD degree and oversees the interprofessional Center for Experiential Learning and Simulation. The associate dean works closely with the associate dean of student affairs and many others in accomplishing this mission. In addition to the Office of Undergraduate Medical Education, the offices of Admissions, Student Affairs, and Continuing Medical Education are managed through separate offices, but report to the senior associate dean of educational affairs.

See Figure 2-Organizational chart.

\section{Faculty Development and Support in Education}

- Professional development for medical educators is offered through the Office of Faculty Affairs, which provides several programmatic opportunities for faculty across the institution. In addition, the Educational Policy Committee, through its annual education retreat, provides workshops 

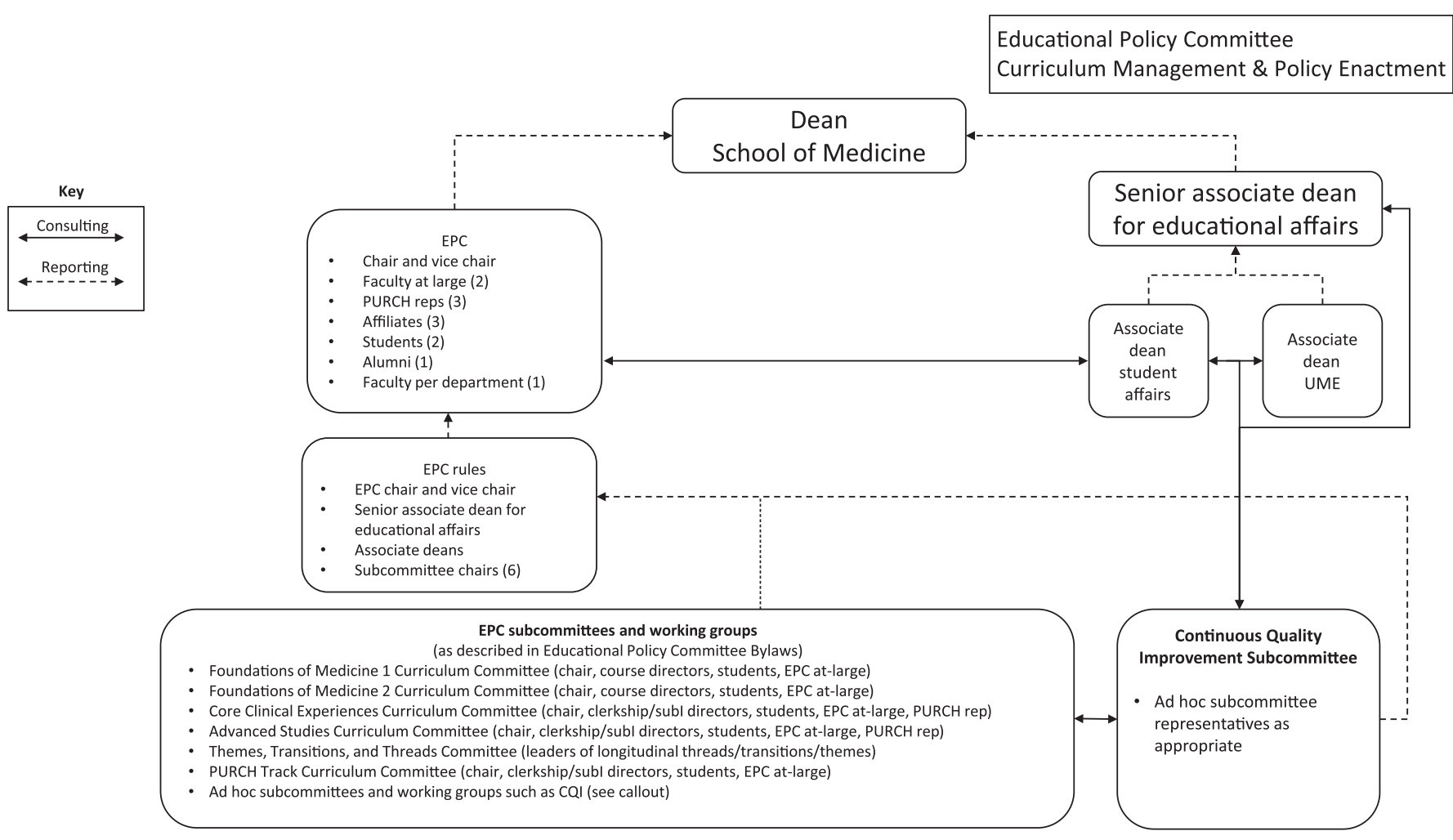

Figure 1 Curriculum governance.

\section{Education Offices}

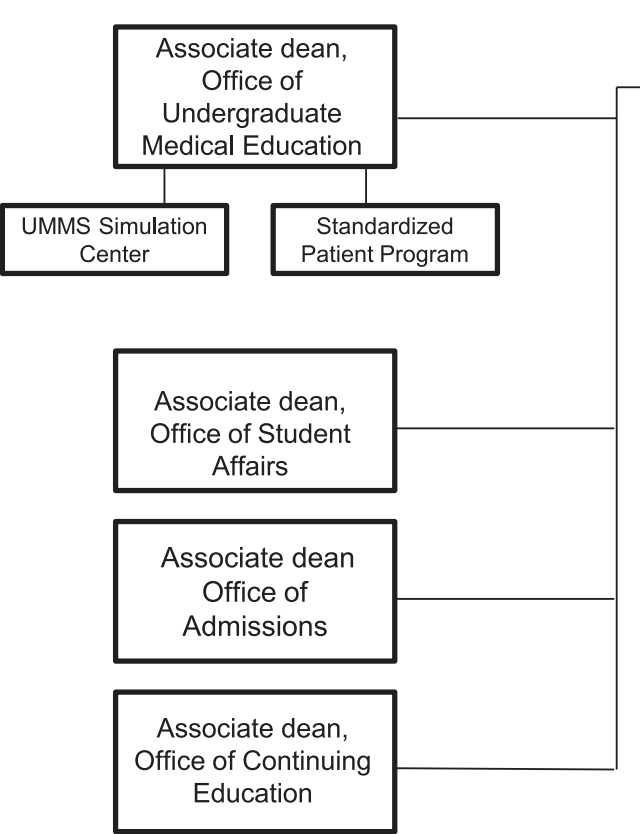

Office of Educational Affairs

\section{Education Resources}

Senior associate

dean,

Office of

Educational Affairs

Academic

Initiatives
Library Services

Center for

Academic

Achievement

Division of Institutional Research,

Evaluation, and

Assessment

Figure 2 Organizational chart. 
and other sessions devoted to the provision of education around teaching skills and techniques. Lastly, the Office of Undergraduate Medical Education, through the associate and assistant deans of undergraduate medical education, provides consultative services for all faculty teaching medical students.

- The UMMS Academic Personnel Policy was recently revised to include for an additional area of distinction in education. It is the expectation that all faculty will engage in education of students, residents, or fellows. However, individuals now may gain promotion based on the quality and quantity of their academic achievements and scholarship as it specifically relates to education. This has been a particularly important enhancement.

\section{Regional Medical Campus}

The UMMS-Baystate regional campus was established with its first class of students in the PURCH track in 2017. Students complete their preclinical courses between the Worcester campus (basic sciences) and Springfield campus (clinical skills and determinants of health). Clinical experiences are completed at the Springfield campus. The 2 campuses use identical grading and assessment tools, and faculty work together closely to provide comparable experiences. Site directors at UMMS-Baystate report to course and clerkship leadership on the main campus, thereby enhancing comparability of the sites. To date, analysis has indicated excellent alignment between the 2 campuses.
See Table 1-Regional Medical Campus.

\section{Table 1}

Regional Medical Campus

$\begin{array}{llr}\text { Campus name } & \text { Type } & \begin{array}{r}\text { Student } \\ \text { enrollment }\end{array} \\ \text { UMMS-Baystate regional campus } & \text { Combination } & 73\end{array}$

\section{Initiatives in Progress}

- In an effort to provide more transparent support of faculty teaching efforts, we are moving toward an FTE-based model.

- A teaching academy will be designed and implemented as part of our strategic plan.

- Student wellness initiatives are being enhanced substantially, and will continue to grow.

- In alignment with the clinical system, a greater focus is being placed on the learning and working environment by adopting common definitions and expectations that will ultimately benefit not only our learners but also all staff and patients.

- Expansion of the academic electronic health record as a tool for teaching and learning across the curriculum is underway.

- Implementation of the Committee on Student Well-being will support innovation in this arena.

- Defined health systems science curricula have been added across all years. 


\section{Central Michigan University College of Medicine}

Tina L. Thompson, PhD, Rebecca Molter, MSA, Harold Bell, PhD, and Steven Vance, MD

\section{Medical Education Program Highlights}

Central Michigan University College of Medicine (CMED) was established with a mission to improve access to high-quality health care in Michigan emphasizing rural and medically underserved regions. The inaugural class was enrolled in 2013 and graduated in the spring of 2017. The CMED curriculum was developed on the framework of a $2+2$ model, with the preclerkship curriculum taking place on the Mount Pleasant, Michigan, campus of Central Michigan University, and clerkship curriculum delivered in our main clinical affiliates, Covenant Healthcare and Ascension St. Mary's Medical Center in Saginaw, Michigan. Since its founding, CMED has expanded from 64 to 104 students per class and grown to include 3 additional branch campuses: Ascension St. John Hospital in Detroit, MidMichigan Health in Midland, and Spectrum Health Lakeland in St. Joseph, Michigan. Most recently, CMED has added an affiliation with University Pediatrics at Children's Hospital of Michigan in Detroit to expand educational opportunities in general and specialty pediatrics.

The CMED curriculum design is mission driven, with an emphasis on development of collaborative problem-solving skills in an active, experiential learning environment. Some key features of the program include:

- Basic sciences delivered using facilitated problem-based learning and nonfacilitated case-based learning/team-based learning (TBL) units.

- A longitudinal Essentials of Clinical Skills (ECS) course that integrates early clinical experiences with community preceptors, a community engagement project, and a formal interprofessional education program.

- A longitudinal Medicine and Society course that promotes appreciation and understanding of topics such as medical ethics, determinants of health outcomes, community needs assessment, epidemiology, and evidence-based medicine.

- An interdisciplinary medication-assisted treatment curriculum for second-year, third-year, and physician-assistant students.

- A 24-week longitudinal integrated clerkship in primary care (comprehensive community clerkship, CCC), with sites focused in rural or urban underserved areas. This curriculum highlights the specific access challenges these patients, communities, and their physicians face in the context of primary medical care.

Acad Med. 2020;95:S236-S239.

doi: 10.1097/ACM.0000000000003356

Copyright (C) 2020 by the Association of American Medical Colleges

Supplemental digital content is available for this article at http://links.Iww.com/ ACADMED/A875.

Year school was established: 2012.

School URL: https://med.cmich.edu.

\section{Curriculum}

\section{Curriculum description}

See Supplemental Digital Appendix 1-Curriculum Schematicat http://links.lww.com/ACADMED/A875.

\section{Assessment}

See Supplemental Digital Appendix 2-General Competencies and Assessment—at http://links.lww.com/ACADMED/A875.

\section{Pedagogy}

- The CMED curriculum is based on adult, self-directed learning methodologies.

- The basic sciences underlying medical practice and patient care are presented to students through a series of integrated, interdisciplinary, systems-based course blocks during the preclerkship curriculum. Each block incorporates a variety of educational methodologies including small-group, case-based discussions; interactive TBL; active lectures; and laboratory experiences.

- The longitudinal ECS course runs concurrently with preclerkship course blocks. In ECS, students learn to perform a clinical examination (history and physical), document patient encounters, and use appropriate communication skills in standardized patient interactions.

- The clinical curriculum consists of 5 separate 4-week hospital-based clerkships and a 24-week longitudinal integrated clerkship, which collectively provide clinical experiences in ambulatory and inpatient settings. A robust videoconference network connects students weekly at distributed sites for small-group discussions and didactic sessions.

- Students are trained using the latest medical simulation technologies at CMEDs-accredited simulation facilities in Saginaw and Mount Pleasant, as well as additional simulation centers at branch campus locations.

\section{Clinical experiences}

- Students first gain experience in controlled, simulated clinical encounters during the ECS course. ECS is an integrated clinical, diagnostic, and therapeutic skill development course using standardized/simulated patients. Emphasis is placed on history taking, physical examination, and diagnostic skills that align with the concurrent organ system being studied. ECS incorporates low- and highfidelity simulation exercises to emphasize clinically relevant basic science concepts covered in the concurrent organ systems courses.

- Years 3 and 4 represent the logical extension of student learning into the clinical environment where students complete required clerkships in both the inpatient and ambulatory settings. 
- The CCC is a unique longitudinal integrated clerkship in the third year of the medical school curriculum. The goal of the CCC is to immerse students in a longitudinal care experience to learn the values of comprehensive patient-centered medical care in the context of a community. Students are placed in primary care practices for a 24-week clinical immersion experience in a rural or urban underserved community, working $2 \frac{1}{2}$ days per week with a primary care provider (a family or internal medicine physician) where they share in the provision of care for both acute and chronic conditions over the course of the clerkship. Students also have longitudinal exposure to community health resources and other medical and surgical specialty experiences, including pediatrics, obstetrics, and emergency medicine. Students learn the course of a patient's illness and appreciate the context in which the patient lives with his or her illness. Students also explore and apply concepts related to population health via completion of a quality improvement project in the primary physician's practice.

- The CCC is a mission-driven experience, offering unique opportunities for students including providing continuity of care, helping patients to navigate access to health care providers within diverse health care systems, and experiencing firsthand what it means to be a community responsive physician. However, such a model presents inherent challenges. The ability to recruit, train, and retain a large cohort of community physicians is a challenge that was magnified when the class size increased from 64 in the inaugural class to 104 students in each subsequent class. Increasing clinical and administrative demands on our community educator faculty, coupled with ever-increasing expectations associated with student assessment and comparability of educational experiences, makes it difficult for them to balance their volunteer educator commitment with clinical demands. Our distributed campuses provide outstanding inpatient educational experiences; however, this clinical training model also comes with unique challenges. These include maintaining comparability of educational experiences and student services across sites, incorporating clinical faculty at branch campuses within the governance structure of the school, implementing effective faculty development activities across sites, and mitigating the financial burden associated with duplication of services.

\section{Education Staff}

- The dean is the designated chief academic officer, but has delegated the day-to-day management of the educational programs to the senior associate dean for academic affairs (SADAA). The Office of Academic Affairs consists of the Office of Medical Education (OME), the Office of Student Affairs (OSA), and Admissions. In addition to the SADAA, OME leadership includes an associate dean for clinical education, an assistant dean for foundational sciences education, 3 clinical assistant deans who oversee regional campuses, a director of assessment and evaluation, and a director of academic success. The OSA includes an associate dean for student affairs, an executive director of OSA, and a senior associate director of financial aid. Faculty development is administered through the Office of Faculty Affairs, and includes an associate dean of faculty affairs and development who reports directly to the dean.

- The OME is responsible for ensuring that the UME curriculum, as developed by the faculty, is delivered and evaluated across all sites in a manner consistent with best practices and policies of the college of medicine and meets accreditation standards. Under the direction of the manager of the OME, curriculum coordinators maintain and update the curriculum database including coordinating content delivery; mapping of learning objectives to educational program objectives; and supporting course/clerkship, faculty, and programmatic evaluation. Staff in the Office of Assessment and Evaluation manage the development, administration, and documentation associated with all student assessments. They ensure that assessments are appropriate for the cognitive domain being assessed and that they are valid and reliable. Administrative faculty (deans, directors) ensure that curricular guidelines, including pedagogy and academic standards, are consistently followed and that outcome data as well as student and faculty feedback inform curricular quality improvement.

- The budget for curriculum management, teaching, assessment, simulation, library services, and academic support is centralized to the Office of Academic Affairs. Each unit within the office develops an annual budget based on prior year expenditures and anticipated curricular needs and is responsible for the management of these funds.

See Figure 1-Curricular organizational chart.

\section{Faculty Development and Support in Education}

- CMED offers a variety of ongoing faculty development opportunities related to instructional methods, assessment and evaluation, educational program leadership, mentoring, and scholarly activity. Programming is available across all sites through workshops, seminars, webinars, and posting of enduring materials online. In addition, the university offers a wide variety of resources and programming through the Office of Curriculum and Instructional Support.

- CMED faculty may be appointed to 1 of 4 tracks: tenure, nontenure, community educator, or visiting faculty. Tenure is only available for those faculty employed by Central Michigan University. Each faculty member receives an appointment letter that outlines their responsibilities associated with clinical, administrative, research, and educational (CARE model) missions of the school. Promotion decisions within each track vary based on the faculty member's achievement across their CARE model; demonstrated evidence of teaching quality is required for promotion and/or tenure for all tracks. While there are no specific promotion requirements involving educational scholarship, faculty in all tracks are expected to show evidence of ongoing research/scholarly activity.

- CMED is currently developing an Academy of Medical Educators that will consist of experienced medical school faculty to serve as a resource for the academic mission of the College of Medicine. The academy will support innovation 


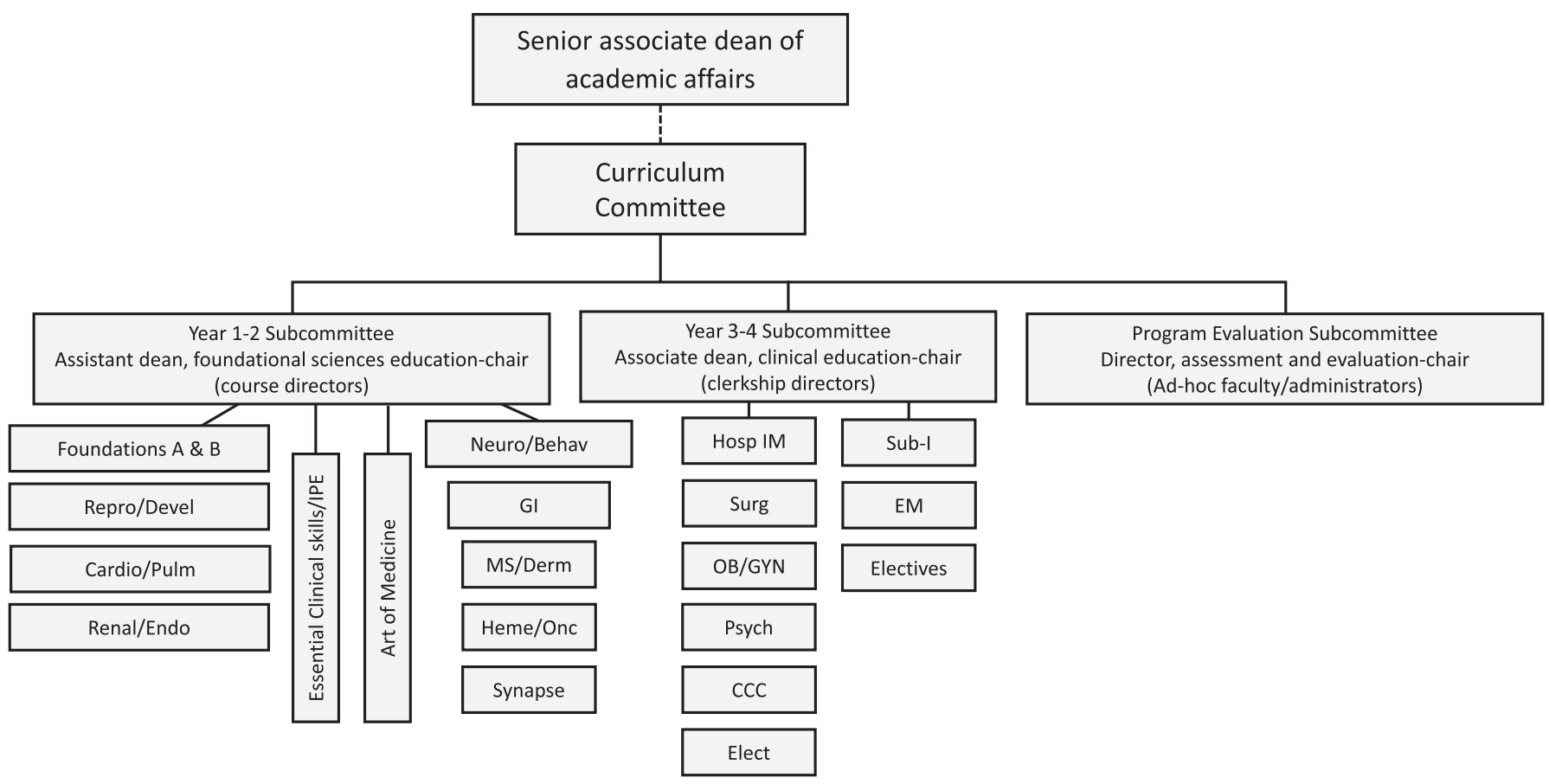

Figure 1 Curricular organizational chart.

and scholarship in medical education, mentoring, leadership, and career development throughout the institution and affiliated clinical sites. Once fully established, the Academy of Medical Educators will provide an opportunity to recognize excellence, innovation, and effectiveness in medical education.

\section{Regional Medical Campuses}

- All students complete their preclerkship curriculum on the Mount Pleasant campus. Clinical students are assigned to either the Saginaw campus or 1 of 3 regional medical campuses to complete years 3 and 4 . A clinical assistant dean is assigned to each regional campus with the responsibility to ensure the delivery of the curriculum, appropriate supervision and timely assessment of students, and the overall learning environment. The clinical assistant deans report directly to the associate dean for clinical education and indirectly to the SADAA. Clinical assistant deans are members of the Year 3-4 Subcommittee of the Curriculum Committee, and participate in monthly meetings of the group. There is a single clerkship director for each discipline, including the director for the CCC. Clerkship directors are responsible for providing faculty at each site with a single course syllabus outlining the clerkship-specific learning objectives. In addition, clerkship directors ensure that faculty involved in teaching are aware of the assessment methodologies and the grading system, the required clinical encounters and procedural skills for each clerkship, and the particular role(s) required of the faculty preceptors to assess student performance in the clerkship. To facilitate comparability, clerkship directors communicate with clinical assistant deans and onsite faculty at least quarterly and more frequently as necessary. Didactics are conducted centrally at the Saginaw campus and are videoconference to each regional campus in real time.

- Outcome data (student performance on NBME shelf exams and clinical evaluations, completion rate of patient encounters/ procedures, distribution of final grades, mistreatment reports, student evaluations, etc.) are tracked and reported to the Year 3-4 Subcommittee semiannually and forwarded to the Curriculum Committee. Any variance across campuses is addressed by a required action plan and resolution monitored by the Curriculum Committee.

- Under the auspices of the Office of Academic Affairs, education and student affairs deans and directors are regularly scheduled to visit each campus to meet with students, coordinators, and faculty to discuss educational and support needs.

See Table 1-Regional Medical Campuses.

\section{Table 1}

\section{Regional Medical Campuses}

\begin{tabular}{lcc} 
Campus name & Type & $\begin{array}{c}\text { Student } \\
\text { enrollment }\end{array}$ \\
Main campus & Preclerkship & 208 \\
\hline Mount Pleasant & Clinical & 144 \\
\hline Saginaw & & \\
\hline Regional campus & Clinical & 24 \\
\hline Ascension St. John Hospital & Clinical & 24 \\
\hline MidMichigan Health & Clinical & 16 \\
\hline Spectrum Health Lakeland & C.m. & \\
\hline
\end{tabular}




\section{Initiatives in Progress}

- The third- and fourth-year curriculum is under review. As part of the review, we are looking at our current clerkship structure including the duration of each required clerkship.
- Clinical performance assessment rubrics are currently under review, with the goal of improved ease of use and alignment with Entrustable Professional Activities.

- CMED will be deploying a new comprehensive learning management system during academic year 2020-2021. 


\title{
Michigan State University College of Human Medicine
}

\author{
Dianne P. Wagner, MD, Brian E. Mavis, PhD, and Aron C. Sousa, MD
}

\section{Medical Education Program Highlights}

The novel Shared Discovery Curriculum (SDC) was implemented in 2016. Its pillars are 3 progressive, longitudinal experiences: the early clinical experience (ECE, year 1), middle clinical experience (MCE, year 2), and late clinical experience (LCE, years 3 and 4). Students learn in authentic patient care environments starting week 9 of medical school. This early interprofessional team engagement enables students to quickly become clinically useful. Clinical experiences require students to integrate their necessary (basic, clinical, and social) science knowledge into the context of authentic patient care.

SDC content is sequenced by what students experience in the clinic or hospital and organized by chief complaints and concerns, rather than disciplines or organ systems, requiring a conceptually advanced framework. Chief Complaints and Concerns (C3) documents define educational end competencies. These are templated using the biopsychosocial model of clinical reasoning, integrating applicable necessary science content. Integration of end competencies with real-world patient care prepares students for how problems are encountered and solved in residency and practice.

The student experience is organized within 1 of 4 learning societies; they provide the structure for small-group learning, faculty coaching, and mentoring. The learning societies have a central role in delivering necessary science content. All learning society faculty convene as an academy, which meets regularly for educator development and support.

Our assessment system is competency based and uses progress testing: all students complete a standardized knowledge and skills assessment suite each semester. Assessments are purposefully aligned with real-world performance requirements to drive relevant continuous improvement of learners and curriculum. This robust assessment strategy includes frequent formative feedback and multimodal summative assessment of each competency. We are the only medical school using high-stakes progress testing to determine graduation competency. Students have consistently performed better than predicted on national certifying examinations, with a significant positive gain since implementation of the SDC.

Just In Time (JIT) Medicine software (https://www. justintimemedicine.com/) powers our curriculum and assessment, providing a transformational link between content

Acad Med. 2020;95:S240-S244.

doi: 10.1097/ACM.0000000000003329

Copyright (C) 2020 by the Association of American Medical Colleges

Supplemental digital content for this article is available at http://links.Iww.com/ ACADMED/A853.

Year school was established: 1964.

School URL: http://humanmedicine.msu.edu. and assessment. It enables the convenient acquisition of realtime, workplace-based assessment. Each student's performance dashboard resides on JIT, readily accessible to students and faculty.

\section{Curriculum}

\section{Curriculum description}

Our 3 clinical experiences reify the philosophy and goals of the SDC. The 24-week ECE begins with learning multiple patient care tasks and their underlying necessary science. Certified safe and useful, students spend 16 weeks in ambulatory clinics caring for patients with their clinic team. The curriculum directly integrates learning about communication and clinical skills, ethics, patient safety science, the social context of clinical decisions, and the biological science behind clinical findings with this foundational patient care experience.

Learning objectives are covered using experiential methodologies in large and small groups and guided study. Weekly simulation sessions form a cornerstone of the curriculum, placing learners in a realistic, safe environment where formative feedback guides improvement. Necessary science is integrated into simulation regularly, providing ongoing opportunities to apply learning to clinical care.

From March to May of year 1, students take intersessions based on individual needs and interests. Some of the intersessions contribute to specialized certificate programs. Certifications exist in leadership for the medically underserved, public health, and 2 rural health leadership options.

The MCE lasts 30 weeks, consisting of ambulatory and inpatient rotations. Each emphasizes integration of clinical work and underlying necessary biological and social sciences required for patient care and work within the health care system. Students learn from residents, attending physicians, and interdisciplinary team members. The MCE includes 5 medical specialty rotations: adult wards, pediatric wards, emergency medicine, newborn nursery, and ambulatory women's health. Students engage in 7 interprofessional rotations: physical therapy, nutrition, pharmacy, respiratory therapy, care management, pain and palliative care, and nursing. They learn the necessary sciences applicable to myriad chief complaints and concerns while garnering robust appreciation for the contributions of health team members.

After the MCE, students have 2 months of intersessions concentrated on areas of need or interest, including a USMLE Step 1 examination preparatory intersession before testing and a summer break.

The LCE engages students in traditional disciplinary clerkships, spending 4-8 weeks on rotations in internal medicine, family 
medicine, pediatrics, obstetrics-gynecology, surgery, psychiatry, a primary care selective, and the ICU. Electives are available early in the LCE, enabling pursuit of interests or completion of research projects or certificate programs.

The SDC's integrative philosophy brings clinical experiences into the first weeks of the curriculum. Similarly, necessary sciences continue to be emphasized in the third- and fourth-year longitudinal advanced skills and knowledge (ASK) sequence. ASK courses require students to continually review and apply necessary science to patient care.

See Supplemental Digital Appendix 1-Curriculum

Description—at http://links.lww.com/ACADMED/A853.

\section{Curriculum changes since 2010}

SDC planning began in 2008. Guiding principles were ratified in 2011 , and the ECE was piloted in 2013. We welcomed our first SDC class in 2016. Guiding principles are:

- Early and ongoing clinical experiences where students are useful

- Necessary science learning integrated with patient care experiences

- Biopsychosocial model of patient care

- Collaborative learning for faculty and students

- Interprofessional training to foster safety and quality

- Technology-enhanced curriculum and assessment

- Alignment of assessment with curricular content and realworld performance

- Assuring competence and striving for excellence

\section{Assessment}

Program objectives are described in outcome-based terms that allow the assessment of medical students' progress in developing the competencies that the profession and public expect of a physician (LCME or CACMS standards).

See Table 1-Program Objectives and Assessment Methods.

Our competency framework is service, care of patients, rationality, integration, professionalism, and transformation. Except for service, our competencies map to ACGME competencies and contain 25 subcompetencies. Each subcompetency has behaviorally specified milestones that describe critical deficiencies or educational "never events," as well as novice, developing, and competent learners. The College Competence Committee determines grade recommendations by reviewing student portfolios of evidence against course competency milestone requirements.

Our assessment progress suite includes NBME Comprehensive Necessary Science Examinations (CBSE, CAS, or CCSE), a progress clinical skills examination (PCSE), workplace-based assessments and Core Entrustable Professional Activities assessments, portfolio artifact review, multisource and peer feedback, and students' individual learning plans.

The PCSE is an 8-station OSCE with 15-minute stations followed by a postencounter task. Competencies assessed are interactional skills, hypothesis-driven history-taking, hypothesis-driven physical examination, counseling, safety, clinical reasoning and documentation, and necessary science applications to patient care.

\section{Three-year curriculum}

We are analyzing data from our progress suite and clerkships to determine how to enable students to complete the curriculum in less than 4 years.

\section{Pedagogy}

We use case-based learning, ambulatory and inpatient clinical experiences, large- and small-group discussions, laboratory, peer teaching, simulation, standardized/simulated patients, team-based learning, videos/podcasts, virtual patients, workshops, and guided problem-based learning methodologies.

We use a small number of traditional lectures, having adopted most of the above methodologies where best suited to specific competencies.

\section{Clinical experiences}

In the ECE, we use primary care offices (internal medicine, family medicine, med-peds, pediatrics) and some urgent care locations. In the MCE, we use hospital system partners for inpatient experiences as well as outpatient offices providing women's health services.

\section{Required longitudinal experiences}

For 15 weeks during the ECE, students spend 2 half days a week in the same primary care office working with the care team. Rural Community Health Program students are longitudinally assigned to a rural community for multiple clerkships.

\section{Clinical experience first encounter}

Our students begin clinics in the ninth week of the ECE.

\section{Required and elective community-based rotations}

We are a community-based medical school with two 4-year campuses and 7 campuses for third- and fourth-year students. In the ECE, students are assigned to communitybased clinics. At each campus, students do required and elective rotations within partner hospital systems, ambulatory sites, and Veteran's Administration facilities, where they exist.

\section{Challenges in designing and implementing clinical experiences for medical students}

Clinical sites are crowded with multiple health care professions learners, implementing new EHR systems, invoking new efficiency and safety processes, and feeling financial productivity pressures. We regularly communicate clinical experience goals for first-, second-, and more traditional third-year clerkship students to our sites; that faculty and site-development work is ongoing and critical for placing 
Table 1

Program Objectives and Assessment Methods

\begin{tabular}{|c|c|c|}
\hline Competency & End-curriculum behavioral outcomes & Longitudinal assessment \\
\hline $\begin{array}{l}\text { Service (no } \\
\text { corresponding ACGME } \\
\text { competency) }\end{array}$ & $\begin{array}{l}\text { - Participates in the provision of beneficial services within the community } \\
\text { - Demonstrates preparation and planning to provide services which } \\
\text { respond to community need } \\
\text { - Demonstrates reflection on their participation in service activities }\end{array}$ & $\begin{array}{l}\text { - Service learning project(s) with planning } \\
\text { documents and reflection }\end{array}$ \\
\hline $\begin{array}{l}\text { Care of patients } \\
\text { (corresponds to } \\
\text { ACGME patient care } \\
\text { and interpersonal and } \\
\text { communication skills) }\end{array}$ & $\begin{array}{l}\text { - Collects complete and accurate patient data } \\
\text { - Synthesizes patient and laboratory data to formulate reasonable } \\
\text { assessments and plans } \\
\text { - Demonstrates the incorporation of patient values into illness } \\
\text { assessment and care plans } \\
\text { - Communicates effectively in writing and orally } \\
\text { - Effectively counsels and educates patients and their families }\end{array}$ & $\begin{array}{l}\text { Direct observations of performance } \\
\text { - Multisource feedback } \\
\text { - Progress Clinical Skills Examination } \\
\text { - Portfolio review }\end{array}$ \\
\hline $\begin{array}{l}\text { Rationality } \\
\text { (corresponds } \\
\text { to ACGME practice- } \\
\text { based learning and } \\
\text { improvement) }\end{array}$ & $\begin{array}{l}\text { - Identifies personal strengths and weaknesses and develops ongoing } \\
\text { individual learning plans } \\
\text { - Demonstrates use of faculty and peer/colleague feedback as a } \\
\text { means of facilitating personal and professional improvement } \\
\text { - Locates, appraises, and assimilates evidence from scientific studies } \\
\text { related to their patients' health problems }\end{array}$ & $\begin{array}{l}\text { - Multisource feedback } \\
\text { - Portfolio review }\end{array}$ \\
\hline $\begin{array}{l}\text { Integration } \\
\text { (corresponds to } \\
\text { ACGME systems- } \\
\text { based practice) }\end{array}$ & $\begin{array}{l}\text { - Demonstrates awareness of cost and access issues in the } \\
\text { - } \text { - Demulation of patient care plans } \\
\text { - Demonstrates respect for all members of the health care team } \\
\text { - Demonstrates knowledge of different types of medical practice and } \\
\text { delivery systems and their implications for controlling health care } \\
\text { allocation and cost } \\
\text { - Demonstrates knowledge of how social and economic systems in } \\
\text { which people live impact health, delivery of health care, and well-being }\end{array}$ & $\begin{array}{l}\text { - Multisource feedback } \\
\text { - Portfolio review } \\
\text { - Progress Clinical Skills Examination }\end{array}$ \\
\hline $\begin{array}{l}\text { Professionalism } \\
\text { (corresponds } \\
\text { to ACGME } \\
\text { professionalism) }\end{array}$ & $\begin{array}{l}\text { - Demonstrates receptiveness to feedback from faculty/peers/ } \\
\text { colleagues/team members } \\
\text { - Contributes actively to group/team process } \\
\text { - Demonstrates respect to patients, colleagues, and team members } \\
\text { - Fulfills responsibilities in courses and on clinical rotations } \\
\text { - Takes responsibility for patient outcomes and is accountable to the } \\
\text { team, the system of delivery, the patient, and the greater public }\end{array}$ & $\begin{array}{l}\text { - Multisource feedback } \\
\text { - Portfolio review } \\
\text { - Progress Clinical Skills Examination }\end{array}$ \\
\hline $\begin{array}{l}\text { Transformation } \\
\text { (corresponds to } \\
\text { ACGME medical } \\
\text { knowledge) }\end{array}$ & $\begin{array}{l}\text { - Applies essential basic, social, clinical science, and systems knowledge } \\
\text { in the care of patients } \\
\text { - Creates new knowledge through research } \\
\text { - Participates in lifelong teaching and learning with peers, trainees, and } \\
\text { patients }\end{array}$ & $\begin{array}{l}\text { - Comprehensive Necessary Science Examination } \\
\text { - Progress Clinical Skills Examination } \\
\text { - Portfolio review } \\
\text { - Multisource feedback }\end{array}$ \\
\hline
\end{tabular}

students earlier and enabling them as useful team members. After the 2013 pilot test, outpatient primary care sites reported unchanged or improved productivity with first-year students, important data as we expanded to almost 100 sites for our entering class.

For the MCE, we meet with multiple stakeholders at each partner institution to communicate rotation goals and enlist participation in these unique placements for second-year students. Multiple learners and changing roles for students comprise the biggest placement challenges.

\section{Curricular Governance}

The ECE, MCE, intersessions, and ASK courses are managed centrally within the associate dean for UME office. Disciplinary clerkships are managed at the department level but supported by Academic Affairs. The centrally managed Office of Academic Achievement supports students across all 4 years. The Office of Assessment is responsible for the progress suite.

See Figure 1-Curricular governance. 


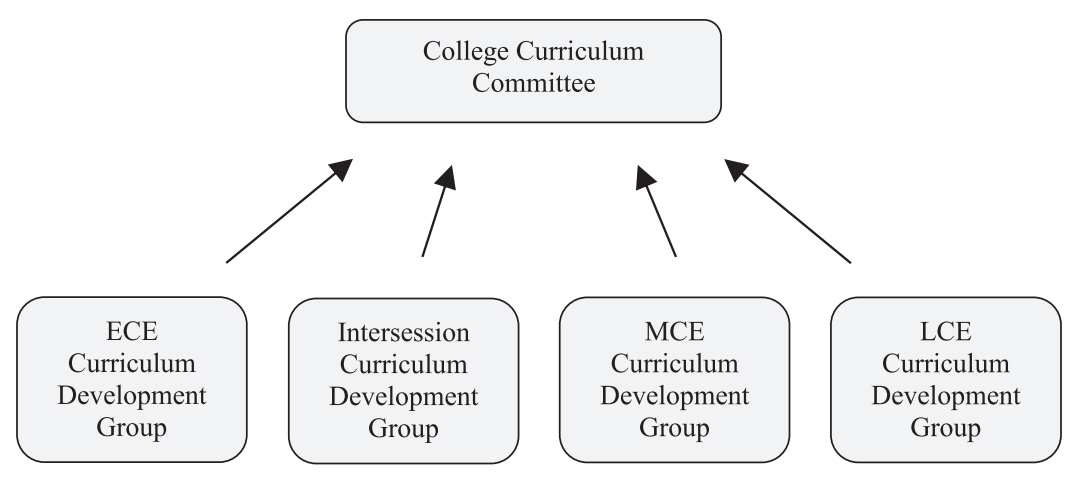

Figure 1 Curricular governance.

\section{Education Staff}

The educational programs of the college exist within the Office of Academic Affairs, led by the senior associate dean for academic affairs. Admissions, student affairs, and continuing and graduate medical education reside within the office of Academic Affairs. The MD curricular program reports to Academic Affairs through the associate dean for undergraduate medical education, who is responsible for undergraduate medical education.

The college benefits from the Office of Medical Education Research and Development (OMERAD), a resource for faculty development, program evaluation, and data analysis.

See Supplemental Digital Appendix 2-Organizational Chart and Administration—at http://links.lww.com/ACADMED/A853.

\section{Faculty Development and Support in Education}

Professional development for faculty as educators

Broad participation from multiple departments formed a platform for the faculty development needed to create the curriculum's innovative clinical, large-group, small-group, and guided study experiences. Participating faculty attended orientations to enable effective delivery of novel offerings. OMERAD offers a clinical educator program for academy fellows and ongoing faculty development occurs under direction of the clinical experience directors.
Small-group teaching in the first 2 years is accomplished within the academy by learning society fellows and by rotation smallgroup leaders who engage in specific orientation to teaching. Academy faculty participate in weekly half day sessions to enable functioning as master teachers and mentors.

\section{Role of teaching in promotion and tenure}

Teaching is 1 of 4 major review categories for promotion and tenure in the tenure track, clinician-educator, or health professions track. All faculty applying for promotion to associate or full professor must fulfill basic criteria in each of the 4 categories, and distinguished criteria in a required number depending on rank sought.

\section{Teaching academy}

The academy is the core of the SDC. Scholar groups of 7-8 students meet with a lead faculty fellow and small cadre of team fellows once or twice weekly during the ECE and MCE. This structure accomplishes multiple curricular goals, including post-clinic debriefing, guided case-based learning and other small-group learning experiences, student portfolio review, and individual learning plan coaching. The academy fosters longitudinal trusting relationships and a rich collegial environment for students and faculty. Lead faculty fellows are clinicians; team faculty include basic and social scientists and bioethicists.

\section{Table 2}

Regional Medical Campuses

\begin{tabular}{|c|c|c|c|c|}
\hline \multirow[b]{2}{*}{ Name of campus } & \multirow[b]{2}{*}{ Location } & \multicolumn{2}{|c|}{ Curriculum phase } & \multirow{2}{*}{$\begin{array}{l}\text { Student } \\
\text { enrollment }\end{array}$} \\
\hline & & Preclerkship & Clerkship & \\
\hline Flint Campus & Flint, MI & & $x$ & 100 \\
\hline Grand Rapids Campus & Grand Rapids, MI & $x$ & $x$ & 340 \\
\hline Lansing Campus & Lansing, Ml & $x$ & $x$ & 240 \\
\hline Midiand Regional Campus & Midland, Mi & & x & 30 \\
\hline Southeast Michigan Campus & Southfield, Ml & & $x$ & 50 \\
\hline Traverse City Campus & Traverse City, MI & & x & 24 \\
\hline Üpper Peninsula Regional Campus & Marquette, Mi & & $\dddot{x}$ & 24 \\
\hline
\end{tabular}




\section{Regional Medical Campuses}

See Table 2-Regional Medical Campuses.

\section{Educational experiences across sites}

The curriculum is managed centrally by the College Curriculum Committee and implemented through Academic Affairs. The associate dean for undergraduate medical education and community assistant deans, who are responsible for curricular implementation in the clinical communities, report to the senior associate dean for academic affairs.

The ECE and MCE are uniquely designed to integrate students' learning with patient care experiences, using 90 C3 documents to define the knowledge, skills, and behaviors required of graduates. End competencies from those documents are programmed into students' learning experiences by week or by ASK course component and delivered to students by core faculty using the same materials and methods. All SDC faculty teachers attend teaching orientation sessions. Academy faculty have weekly educator development sessions aimed at standardizing small-group teaching. Intersessions lead faculty design and orient faculty who deliver those portions of the curriculum. Departmental clerkship committees composed of community clerkship directors design and implement departmental clerkships for comparable experiences and assessment across campuses. The director of the LCE supervises this process across departments and reports to the assistant dean for UME. 


\section{Oakland University William Beaumont School of Medicine}

Cynthia H. Ledford, MD, Deirdre G. Pitts, PhD, David M. Thomas, PhD, Tracey A.H. Taylor, PhD, Robert Noiva, PhD, Robert J. McAuley, PhD, and Duane G. Mezwa, MD

\section{Medical Education Program Highlights}

At Oakland University William Beaumont School of Medicine (OUWB), our notion of the ideal physician is distinctive: an ideal physician is one who, above all else, is kind, compassionate, and provides extraordinary care to patients and communities. To achieve this aim, the medical school merged the approach of a high-achieving liberal arts college with an evidence-based professional medical education.

OUWB achieves its exceptional outcomes through:

- Holistic admissions that broaden the definition of smart, to select candidates with demonstrated empathy, compassion, and a desire to serve others

- Focus on personal development and wellness of students within a student community

- Delivery of unique coursework in ethics and critical thinking

- Providing a hub for community engagement

\section{Curriculum}

\section{Curriculum description}

OUWB's curriculum is cohort based; each class of students forms a community of diverse individuals. Longitudinal courses span multiple years, allowing students to explore medical humanities and clinical bioethics, develop critical thinking through a scholarly project in one's area of passion (Embark), and take a group journey exploring identity and personal wellness with a mentor (PRISM). During the first 2 years, the curriculum facilitates small-group experiential learning in 3 main settings: foundational science laboratories, team-based learning activities, and a clinical skills center where students practice communication and patient care skills with standardized patients. Students traverse the core clerkships via 1 of 6 tracks, as a close cohort of 21 students, gaining experience in 8 clinical disciplines. The senior year allows for individualization of each student's learning plan through 16 weeks of electives coupled with assured readiness for graduate medical education through required clerkships in emergency medicine, anesthesia/pain management, subinternship(s), and diagnostic medicine.

See Supplemental Digital Appendix 1-Curriculum Overviewat http://links.lww.com/ACADMED/A876.

Acad Med. 2020;95:S245-S248.

doi: 10.1097/ACM.0000000000003357

Copyright (C) 2020 by the Association of American Medical Colleges

Supplemental digital content for this article is available at http://links.Iww.com/ ACADMED/A876.

Year school was established: 2008.

School URL: https://oakland.edu/medicine.

\section{Curriculum changes since 2010}

Since OUWB's founding in 2008 and matriculating its first class of 50 students in 2011, the school has focused on delivering highquality yet personally rich learning experiences. Between 2011 and 2015, the class size grew to a full enrollment target of 125 students. As the class size has grown, the curriculum has evolved through:

- Adding more deliberate opportunities for collaborative learning

- Maintaining a strong sense of community amongst students, faculty, staff, and physicians

- Improving the efficiency and effectiveness of curriculum delivery and community outreach

\begin{abstract}
Assessment
The objectives of the medical school curriculum are articulated through measurable knowledge, skills, and attitudes. These are adopted from and include the entirety of the Physician Competency Reference Set (PCRS). ${ }^{1}$ The faculty and leadership designed the curriculum to foster a community of practice ${ }^{2}$ whereby students share a common goal of serving others with kindness and compassion and strive together to master the necessary knowledge, skills, and attitudes. The outcomes-based objectives allow the school to better support each medical student's progress toward acquiring the competencies that the profession and the public expect of a physician. The faculty aligned the assessments with the PCRS competency domains and objectives. Over time, the quality and frequency of assessments have been refined based on student feedback and the trajectory of student learning.
\end{abstract}

See Supplemental Digital Appendix 2-Program Objectives and Primary Assessment Methods—at http://links.lww.com/ ACADMED/A876.

\section{Pedagogy}

Communities of practice ${ }^{2}$ in which students learn together and draw support from each other are integral to the curriculum. For example, students learn and grow as a group over time during anatomy dissection and team-based learning activities. Also, Beaumont Health is the sole clinical site for all required clerkships through which students rotate together within 1 of 6 clerkship tracks. As an example, with the continuity of students within a learning community, the faculty created ethics cases tailored to each clinical clerkship to which groups of students respond, functioning as a mock ethics consult team.

\section{Clinical experiences}

Beaumont Health, specifically the legacy hospitals Royal Oak, Troy, and Grosse Pointe, provides students with 3 clinical 
settings from which to learn about the care of a community of patients. Beaumont Royal Oak provides much of the system's highly specialized care for several diverse ethnic communities in the suburban and urban areas of southeast Michigan. The core surgery, subinternships, and emergency medicine clerkships are in inpatient settings. All other clerkships incorporate substantive experiences in community-based settings, including a psychiatry clerkship in which approximately one-third of the clinical experience is in interprofessional and community-based agencies serving patients with mental health needs.

In addition to required and elective clinical rotations, students choose from several community-based service-learning experiences starting as early as the first year of medical school. Each OUWB student develops patient care and communication skills from the first semester of medical school, initially with standardized patients, and after basic skill mastery, through service-learning opportunities such as health fair and health outreach activities in underserved communities.

Challenges in designing and implementing clinical experiences for medical students

As the class size has grown, the primary challenges were:

- To maintain the strength of the student-student and facultystudent relationships

- To continue to improve the quality of learner engagement

- To provide individualized assessment and feedback to each student

- To foster clinical learning environments where students are active and valued members of the team

\section{Curricular Governance}

The Curriculum Committee provides governance and assumes the primary responsibility for planning, evaluation, and oversight of the curriculum. Membership of the Curriculum Committee and each of its 4 subcommittees include faculty voting members, 1 or 2 nonvoting ex-officio members (associate or assistant deans), and nonvoting student representatives from each of the 4 years of the medical school curriculum. Also, Office of Medical Education and Student Affairs members often participate in discussions as invited guests (nonvoting). The chair of the Curriculum Committee annually reports to the OUWB Executive Committee, a standing committee that includes the dean of the medical school. Per OUWB bylaws, the Curriculum Committee reports its decisions to the associate deans for medical education for operationalization. The budget for teaching, assessment, and academic support is managed centrally by the school.

See Figure 1-Curriculum committee structure.

\section{Education Staff}

The Office of Medical Education (OME) is led by 2 associate deans (of preclinical and clinical medical education) who work closely with the directors of curriculum data management, student assessment, and curriculum evaluation to fulfill the school's educational mission. The director of curriculum data management oversees the curriculum inventory as well as data that assess the quality of the educational program. The director of student assessment is responsible for managing the various

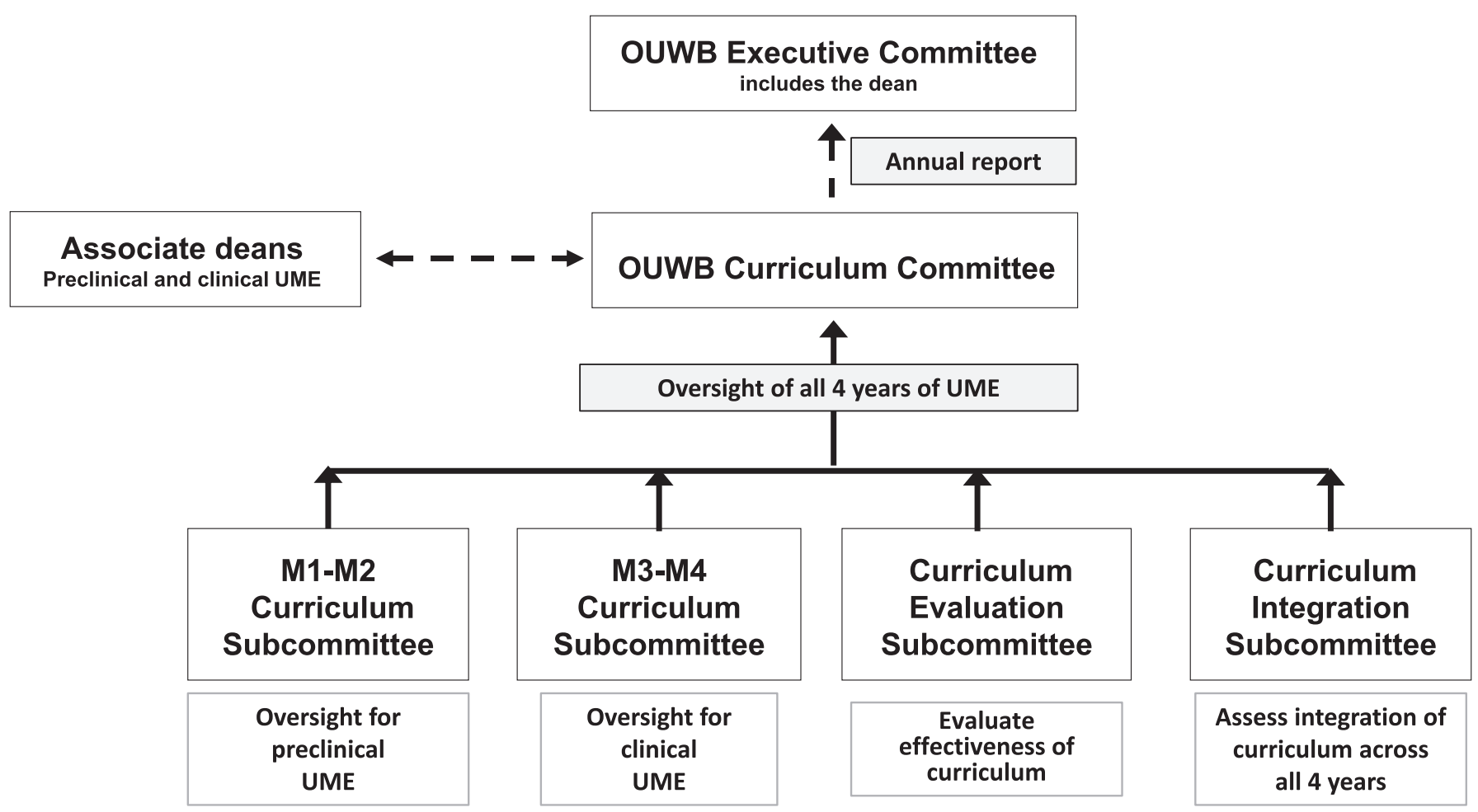

Figure 1 Curriculum committee structure. 
assessment systems used by the school as well as the analysis of outcomes data. The director of curriculum evaluation assists in the design and implementation of curriculum evaluation strategies. Additional members of the OME include the curriculum program administrator and UME operations manager, who directly supervise a team of coordinators who assist their respective course and clerkship directors with the delivery of curricular content. The associate deans and members of the OME work together to support the curriculum committees and faculty they serve to plan, deliver, evaluate, and monitor the curriculum. The OME is responsible for the UME component of the medical education continuum. The office is not responsible for areas outside educational programming. Additional academic services (i.e., the Academic Success Team) reside within the Office of Student Affairs. Opportunities for community engagement and cocurricular service learning are managed by a separate team of professionals in the COMPASS Center for Community Engagement, serving the school's social mission.

\section{Faculty Development and Support in Education}

The OUWB Center for Excellence in Medical Education (CEME) offers a variety of faculty development programs, online webinars, and well-being activities. Teaching improvement workshops and consultations to basic science and clinical faculty, graduate medical residents, postdoctoral scholars, and teaching assistants are offered on an ongoing basis. In addition, CEME has 6 signature faculty development programs:

- Fellowship in Medical Education: An advanced-level program for those interested in building skills for medical education research.

- Medical Education certificate: A program for clinician and scientist educators introducing both the theory and practice of medical education. A range of topics are covered, from the practicalities of running the classroom to the underlying theories that inform and guide educational practices.

- Residents as Teachers: This program gives residents the opportunity to improve their teaching skills through role-play and other activities. Residents attending $100 \%$ of the sessions obtain an Advanced Training in Medical Education certificate.

- Clinical Education Leadership Series: This program is designed to enhance the skills of faculty in clinical education leadership roles.

- Leadership Education and Development (LEAD) certificate ${ }^{3}$ : The LEAD program, developed and delivered by the AAMC, fosters practical knowledge drawn from the best recognized theoretical models and best practices of effective leadership that are relevant to medical education organizations and processes.

- Stanford Faculty Development Program for Clinical Teaching This program for faculty and residents uses a train-the-trainer model based on a 7-component educational framework.

\section{Role of teaching in promotion and tenure}

Teaching, educational activities, scholarly work, and service are important components of promotion and tenure at OUWB.
Each candidate for faculty appointment and promotion must meet expectations as a medical educator if their duties involve education to medical students, residents, fellows, graduate students, or postdoctoral fellows. Achievement in service to the School of Medicine, Oakland University, Beaumont Health, and/or the community is also required. The Office of Faculty Affairs and the Committee on Appointments Promotion and Tenure established the Meaningful Participation Program (MPP) as a means to ensure that faculty members with a clinical focus are active participants in the medical school community. OUWB requires active participation to maintain a faculty appointment; the MPP provides the method by which clinical faculty document their participation. Faculty members are also required to participate in faculty development activities. Only university-employed faculty members are eligible for an award of tenure.

See Table 1-Criteria for Faculty Appointments, Promotion, and Tenure.

\section{Initiatives in Progress}

We aspire to improve continuously and to grow our research and education missions. Though we are privileged to enroll

\section{Table 1}

\section{Criteria for Faculty Appointments, Promotion, and Tenure}

\begin{tabular}{|c|c|}
\hline Rank & Criteria \\
\hline Instructor & $\begin{array}{l}\text { - Entry-level rank } \\
\text { - Appropriate terminal degree } \\
\text { - Appropriate for physicians who have not yet attained } \\
\text { board certification, for chief residents, fellows in } \\
\text { non-ACGME programs, or for individuals who do } \\
\text { not have a terminal degree in their field, and meet } \\
\text { the medical school's criteria for teaching }\end{array}$ \\
\hline $\begin{array}{l}\text { Assistant } \\
\text { professor }\end{array}$ & $\begin{array}{l}\text { - Potential for the development of excellence in } \\
\text { scholarship, teaching, and patient care (if applicable) } \\
\text { - An appropriate terminal degree relevant to the } \\
\text { discipline under consideration } \\
\text { - Clinicians must have completed residency and, if } \\
\text { applicable, fellowship training, and must be board } \\
\text { certified }\end{array}$ \\
\hline $\begin{array}{l}\text { Associate } \\
\text { professor }\end{array}$ & $\begin{array}{l}\text { - } 5 \text { or more years as assistant professor } \\
\text { - Significant contribution to the school of medicine's } \\
\text { programs } \\
\text { - Ongoing teaching and/or service } \\
\text { - Demonstrated expertise and excellence }\end{array}$ \\
\hline Professor & $\begin{array}{l}\text { - } 5 \text { or more years as associate professor } \\
\text { - Exceptionallinternational reputation } \\
\text { accomplishments level of professional } \\
\text { - Significant commitment to school and to one's field } \\
\text { - Sustained commitment to academic programs of the } \\
\text { school } \\
\text { - Demonstrated expertise and excellence }\end{array}$ \\
\hline
\end{tabular}


many students who are first-generation college graduates and physicians, this has contributed to overall student debt. We must strive to reduce this burden if we are to make our program affordable for all. Building on our educational program strengths, we also look forward to further developing research programs in population health informatics, as well as adding graduate programs.

Key initiatives:

- Reduction of student debt

- New research program in population health informatics

- New graduate programs

\section{References}

1 Englander R, Cameron T, Ballard AJ, Dodge J, Bull J, Aschenbrener CA. Toward a common taxonomy of competency domains for the health professions and competencies for physicians. Acad Med. 2013;88:1088-1094.

2 Wenger E, McDermott RA, Snyder W. Cultivating Communities of Practice: A Guide to Managing Knowledge. Boston, MA: Harvard Business School Press; 2002.

3 AAMC. Leadership education and development (LEAD) certificate program. https://www.aamc.org/professional-development/leadershipdevelopment/lead. Accessed March 24, 2020.

4 Stanford Faculty Development Center for Medical Teachers. Stanford faculty development center for medical teachers: Stanford medicine. Stanford Faculty Development Center for Medical Teachers. http://med. stanford.edu/sfdc.html. Accessed March 24, 2020 


\section{University of Michigan Medical School}

Michelle Daniel, MD, MHPE, Seetha U. Monrad, MD, Sara Weir, MA, Joseph C. Kolars, MD, and Rajesh S. Mangrulkar, MD

\section{Medical Education Program Highlights}

The University of Michigan Medical School (UMMS) was founded in 1850. For much of its existence, the curriculum followed a traditional Flexnerian model. Over time, the preclinical curriculum shortened, and the clinical curriculum lengthened. In 2016, the curricular structure inverted. A $17+12+12$-month model transitioned to a $12+12+17$-month model to provide a robust integrated foundation with early clinical immersion, followed by an expanded postclerkship phase for impact-focused work and deliberate professional and leadership development. The new curriculum consists of a "trunk" and "branches," in honor of the oak trees for which Ann Arbor is named.

See List 1-Program Highlights.

\section{Curriculum}

\section{Curriculum description}

The preclinical phase, the scientific trunk, consists of 12 months of foundational learning focused on basic and health system sciences. The core clinical phase, the clinical trunk, consists of the 1-month Transition to Clerkships course and 12 months of departmentally based clinical clerkships. The advanced phase, the professional development branches, is 17 months of required and elective activities. Longitudinal courses are integrated across all 3 phases.

See Supplemental Digital Appendix 1-Curriculum Overviewat http://links.lww.com/ACADMED/A890.

\section{Scientific trunk}

The scientific trunk comprises 6 integrated blocks. Normal functioning is taught concurrently with pathophysiology in a single-pass curriculum. Disciplines (e.g., anatomy, pathology, pharmacology) are threaded throughout all or portions of the year. Basic science content is longitudinally integrated with other courses (e.g., as students learn about the cardiovascular system, they simultaneously learn to perform a cardiac history and physical exam in the Doctoring program). In the scientific trunk, instruction occurs through a combination of lectures ( $\sim 50 \%)$, small groups ( 30\%), labs, standardized patient (SP) interactions, patient presentations, tutorials, and online modules ( 20\% combined).

Acad Med. 2020;95:S249-S253.

doi: 10.1097/ACM.0000000000003373

Copyright (C) 2020 by the Association of American Medical Colleges

Supplemental digital content for this article is available at http://links.Iww.com/ ACADMED/A890.

Year school was established: 1850.

School URL: https://medicine.umich.edu/medschool/home.

\section{Clinical trunk}

Transition to Clerkships prepares learners to think deeply about basic science connected to clinical care through casebased learning and multiple SP assessments. The clerkships include internal medicine, pediatrics, obstetrics-gynecology, family medicine, psychiatry, neurology, and surgery and applied sciences. Learning on clerkships primarily occurs through immersion on inpatient and outpatient care teams. Clerkship didactics supplement clinical learning using a variety of pedagogies (e.g., flipped classroom, simulation, lecture, casebased learning). Three specific initiatives emphasize foundational science in the clinical phase: applied sciences-a 4-week rotation during surgery comprising anatomy, radiology, pathology, and pathophysiology; Science and Practice of Medicine-a longitudinal, case-based learning course on core and cuttingedge science topics; and Health Systems Science-a 1-week course on health disparities, ethics, quality improvement (QI), patient safety (PS), and value-based care.

\section{Professional development branches}

The branches constitute the third and final phase of the UMMS curriculum. Students select 1 of 4 branches:

- Patients and populations (primary care, population health, patient-centered medical homes, longitudinal clinic experience)

- Systems- and hospital-based care (hospitalized patients, health systems, QI, PS, value-based care)

- Procedure-based care (patients requiring diagnostic and therapeutic procedures, technical and nontechnical skills)

- Diagnostics and therapeutics (technologies to diagnose and treat disease; disease-based electives)

The branches aim to ensure clinical excellence, so learners are "ready day one" for residency, while providing flexible, individualized professional development pathways to launch students on impact-focused careers. Students can pursue any elective or residency from any branch. A branch advisor helps learners align their schedule with professional goals. Students use abundant elective time to engage in research or other scholarly pursuits. Branches culminate in specialty-specific residency preparatory courses containing high-yield topics for incoming interns, using a number of pedagogies.

\section{Longitudinal elements}

Doctoring is a 4-year longitudinal clinical skills and coaching program. Small groups are cotaught by 2 physician faculty. One serves as the primary instructor/assessor and the other serves as a coach, helping learners reflect on competency development, work-life integration, professional identity formation, and wellbeing. Chief Concern is a 1-year longitudinal clinical reasoning course teaching students how to think through clinical problems. 


\section{List 1}

\section{Program Highlights}

12-month foundational science curriculum (scientific trunk)

- Normal and abnormal systems taught side by side in single-pass curriculum

12-month core clinical clerkships (clinical trunk)

- Early clinical immersion (September of year 2)

- Basic science emphasis (Science and Practice of Medicine course; surgery and applied science clerkship)

- Postclerkship USMLE Step 1 examination

17-month postclerkship phase (professional development branches)

- Tailored professional development (branch advisor helps design schedule)

- Pursue impact-focused work, Capstone for Impact deliverable

- Residency prep courses (specialty specific)

Longitudinal elements

- 4-year clinical skills and coaching program (Doctoring)

- Health Systems Science course, Leadership Development Program, and interprofessional education

- Clinical reasoning course (Chief Concern in year 1)

- Paths of Excellence (scholarly concentrations), most enter in spring of year 1

- M-Home learning communities

Competency-based medical education

- Learning outcomes dashboard (multisource assessment data)

- Competency committees, holistic review

- Expanding programs of assessment for all competencies

Health Systems Science, leadership, and interprofessional education (IPE) are also 4-year longitudinal threads. The Health Systems Science course involves the study of health policy, disparities, ethics, value-based care, and QI/PS. The Leadership Development Program strives to produce physicians who will become leaders and change agents. Students develop a personal mission, vision, and values statement and attend skill-building sessions, then apply their skills in the classroom, clinic, and extracurricular settings. The IPE thread includes a unique early clinical experience where students observe practicing health professionals caring for patients, as well as classroom and clinicbased experiences with other learners.

The Paths of Excellence are optional scholarly concentrations in which $\sim 80 \%$ of the student body participates. Students choose from ethics, global health and disparities, health policy, innovation and entrepreneurship, medical humanities, PS/ QI/complex systems, scholarship of learning and teaching, or scientific discovery. Learners engage in small-group didactic sessions to deepen knowledge and build community and receive 1:1 mentorship on a capstone.

The Capstone for Impact is a longitudinal experiential project aimed at creating new knowledge and/or delivering a solution, improvement, or innovation to a real-world issue, requiring sustained self-directed learning with guidance from advisors and mentors. Examples of capstones include research on postcholecystectomy opiate prescribing practices, a podcast on the Detroit water shortage, and invention and marketing of a "neovent" (an affordable neonatal bubble continuous positive airway pressure device for resource-poor settings).

\section{Curriculum changes since 2010}

Triggered by a charge from the dean to better prepare learners as leaders and change agents of the future, in 2015 , the faculty voted for a sweeping curricular transformation made up of 8 elements: scientific trunk, clinical trunk, branches, Paths of Excellence Program, Leadership Development Program, IPE, M-Home learning community, and competency-based assessment. The first 6 elements have been described above.

The M-Home emphasizes connections, community, personal authenticity, and meaning and purpose to enhance well-being and work-life integration. The M-Home is organized into 4 houses, serving as the anchor for Doctoring small groups, where personal and professional support is provided by a network of peers, counselors, faculty, and directors.

The approach to assessment transitioned from a higher-stakes, course/clerkship-based model, with a heavy emphasis on medical knowledge, toward a competency-based model, with frequent lower-stakes assessments across multiple competency domains. While students still have graded courses on their academic transcript (exclusively pass/fail in the preclinical phase, mostly honors/high pass/pass/fail in the clinical phase), overall progression through the curriculum is assessed through the lens of competency development.

\section{Assessment}

The medical school identified and reviewed competencies from multiple sources (e.g., ACGME objectives, the Physician Competency Reference Set) and constructed 8 UMMS competency domains, 6 aligned with ACGME objectives. Leadership, teamwork and interprofessionalism, and critical thinking and discovery were added to complete the vision of the new UMMS graduate.

See Supplemental Digital Appendix 2-Program Objectives and Assessment Methods—at http://links.lww.com/ACADMED/A890.

Organizational and structural changes were implemented to support the shift toward competency-based medical education (CBME). A robust electronic learning outcomes dashboard was developed to capture multisource competency assessment data and present them visually, facilitating review and decision making. Data include quantitative measures, Likert-scale assessments of competency development, and narrative descriptions. Competency development is monitored by Competency Committees, with broad representation from faculty, allied health professionals, patient advocates, and student services. These committees follow matriculating cohorts longitudinally and holistically review all students multiple times to ensure appropriate development in all competency domains, and identify support for students. Students have full access to their data, and periodically perform self-assessments, guided by coaches. Identified competency assessment gaps are progressively 
being filled with novel assessments. For example, assessment of the capstone projects focus on harder-to-assess domains such as practice-based learning and improvement, leadership, teamwork and interprofessionalism, and critical thinking and discovery.

Traditional assessments have been reorganized to support learning. Students now take the USMLE Step 1 examination after the clerkships, to foster deep learning of the scientific underpinnings of clinical medicine. Multiple SP-based assessments have been dispersed across the first 2 years to provide frequent, low-stakes assessment and feedback. A postclerkship multistation clinical assessment guides students' branches work and preparation for USMLE Step 2 Clinical Skills.

We are exploring competency-based, time-independent progression through the postclerkship phase, which may facilitate time-variable completion of the MD degree in the future.

\section{Pedagogy}

Overall, we decreased lectures and labs and increased active learning, case-based learning, online modules, simulation, and SP encounters.
Several courses are made up almost entirely of active learning (e.g., Doctoring, Chief Concern, the Leadership Development Program, IPE, Transition to Clerkships, Science and Practice of Medicine).

\section{Clinical experiences}

Students are first exposed to patients in fall of year 1 as part of IPE. Learners are fully immersed in clinical care by September year 2 . The majority $(\sim 85 \%)$ of core clinical experiences occur at Michigan Medicine hospitals. Students also rotate at 2 large urban health care centers, 2 neighboring community hospitals, and the Veterans Administration. The balance of inpatient and ambulatory experiences is roughly 65:35. A 12-month longitudinal clinic is required in the patients and populations branch where students work with the same preceptor, often encountering the same patients.

\section{Curricular Governance}

Curricular governance is overseen centrally by the Curriculum Policy Committee (CPC) and its 5 curricular subcommittees. Competency Committees (CCs) assess competency development and monitor academic progress. CPC and CCs report directly to the Executive Committee (EC). Elected student representatives

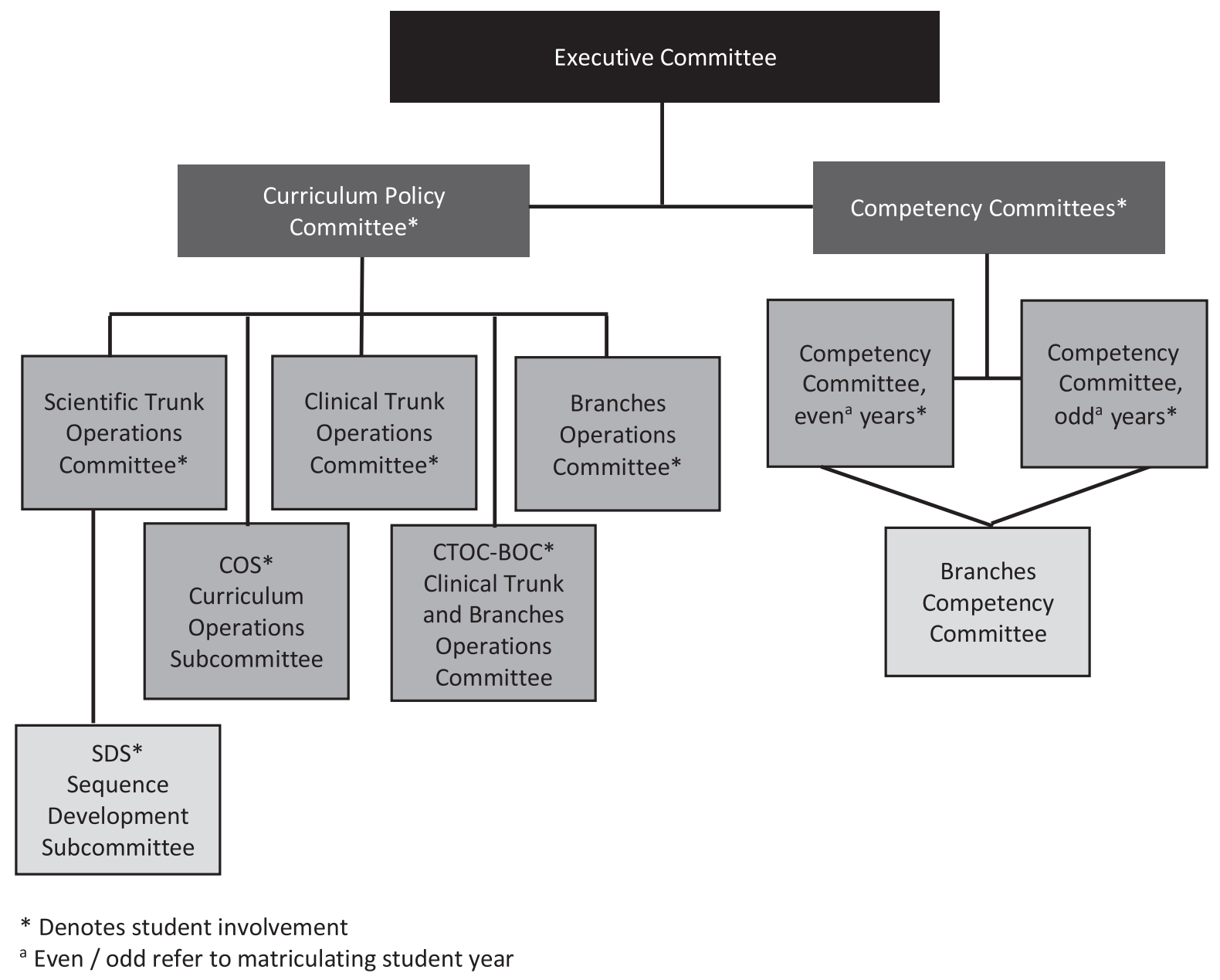

Figure 1 Curricular governance structure. 


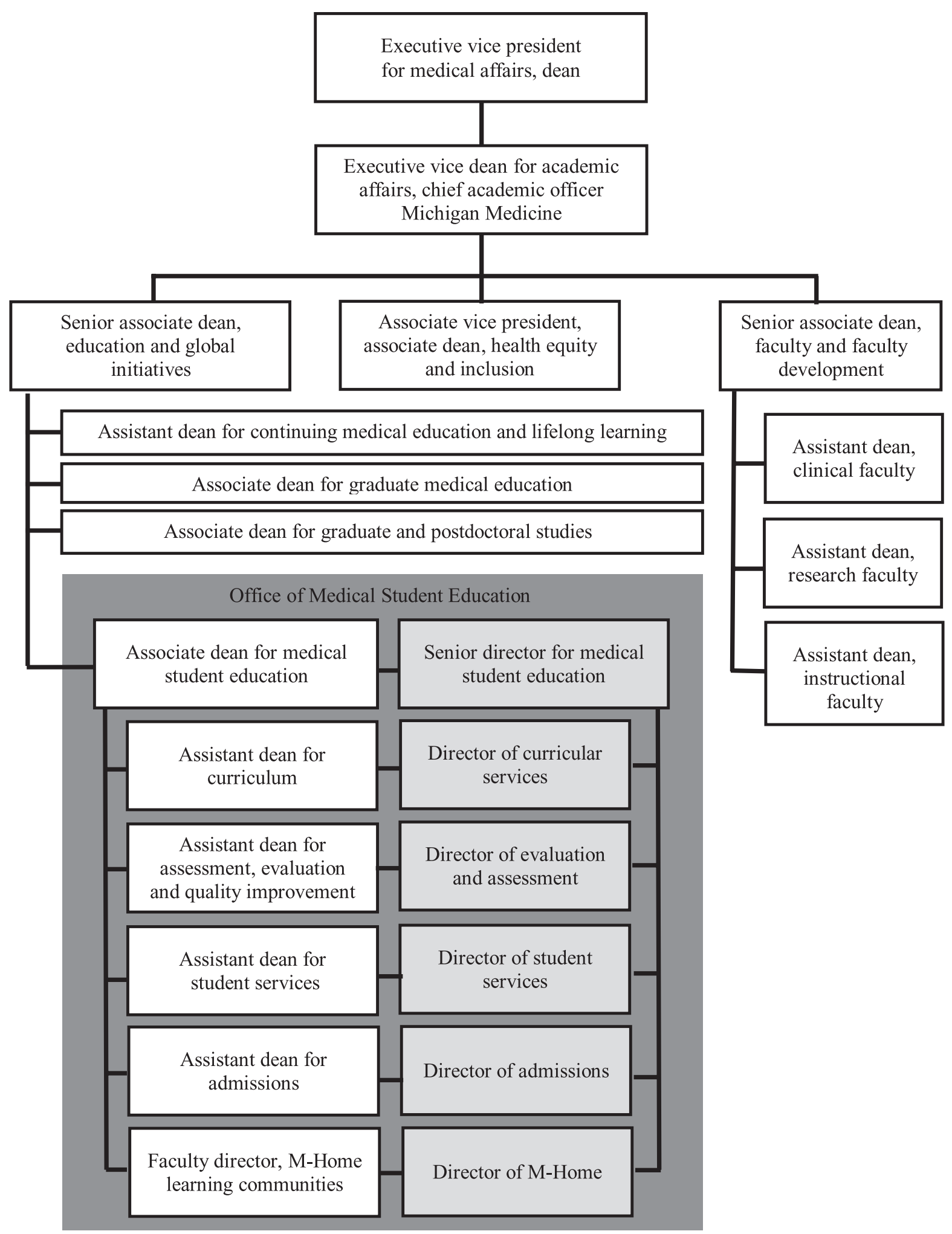

Figure 2 Academic affairs organizational chart. 
serve on all committees, except for the EC, reflecting a core value of partnering with learners.

See Figure 1-Curricular governance structure.

\section{Education Staff}

\section{Medical education leadership}

The Office of Medical Student Education (OMSE) plans, implements, evaluates, and oversees all aspects of the medical student program. The associate dean and senior director for medical student education oversee 4 central units and the M-Home, each collaboratively led by faculty (assistant dean or director) and a staff director. The OMSE sits within Medical School Administration, led by the executive vice president for medical affairs/dean.

See Figure 2-Academic affairs organizational chart.

Curricular leadership and faculty with significant teaching roles are supported by direct salary offsets from the OMSE. Smaller teaching roles are compensated via educational value unitmediated funds flow to departments.

In addition to the OMSE, UMMS has the Department of Learning Health Sciences (DLHS), a first-in-the-nation basic science department focused on the sciences related to learning. DLHS is organized around 4 collaboratives (education, simulation, infrastructure, and implementation) to support academic and research efforts. Key offerings include the Medical Education Scholars Program (that introduces faculty to educational design and scholarship) and a competency-based Master of Health Professions Education.

\section{Faculty Development and Support in Education}

The Office of Faculty Development offers more than 100 unique courses annually to meet the continuing education needs of faculty. Leadership programming and coaching are notable strengths.
Two communities of practice support health sciences education. The first is the Academy for Educational Excellence and Scholarship. With over 400 members, the Academy helps augment the educational rigor and innovation of its faculty by bringing the community together to discuss important topics and engage in peer development and mentoring. The second is RISE (Research. Innovation. Scholarship. Education.), which aims to develop and implement innovative and potentially transformative education ideas. Members receive personal innovation coaching, including instruction on change management.

Faculty educational efforts are recognized in promotion and tenure decisions. The promotion criteria for the clinical track were recently revised, and a clinician-educator pathway was delineated. The definition of scholarship was expanded beyond peer-reviewed manuscripts to include book chapters, innovative teaching practices, educational modules, curriculum development, and patient and community education, among others.

\section{Initiatives in Progress}

As a public institution, UMMS is committed to both innovation and continuous QI to improve the health of patients and society. Three initiatives in progress will continue to guide our work:

- Fully actualizing the CBME vision, by expanding assessments of all 8 competencies and leveraging big data to make judgments. Time variability and effective educational handovers to residency programs are future goals.

- Enhancing well-being and improving the learning environment by adopting system-wide cultural transformation strategies toward kinder, civil, and health-supporting values.

- Growing communities of practice in health sciences education (e.g., RISE) that emphasize both bold innovation and responsible change management, to tackle the big issues facing medical education, and the needs of society. 


\title{
Wayne State University School of Medicine
}

\author{
Jason C. Booza, PhD, Senthil Rajasekaran, MD, MMHPE, and Richard S. Baker, MD
}

\section{Medical Education Program Highlights}

The educational mission of the Wayne State University School of Medicine (WSUSOM) is to develop and graduate a diverse cadre of physician leaders who are comprehensively educated to provide high-quality 21 st-century health care; who are uniquely trained to provide high-quality health care in a complex, highacuity, highly diverse, clinical, and community environment; and who, in alignment with educational goals, understand and demonstrate social accountability in support of the priority health needs of the local, state, and national communities that they serve.

\section{Curriculum}

\section{Curriculum description}

The MD program Highways curriculum is divided into 3 phases and 4 segments:

- Phase 1 , the preclerkship phase, is 18 months and comprises segments 1 and 2 .

- Phase 2, the clerkship phase, is 12 months and comprises segment 3.

- Phase 3, the postclerkship phase, is 18 months and comprises segment 4.

See Supplemental Digital Appendix 1-Curriculum Schema-at http://links.lww.com/ACADMED/A937.

\section{Curriculum changes since 2010}

The newly implemented Highway curriculum for the WSUSOM MD program was launched in 2018 and represents an 18-12-18 model. Specific enhancements include:

- Reorganization of the preclerkship curriculum from a discipline-based to an organ systems-based format in order facilitated horizontal and vertical integration within the curriculum.

- The segment 1 curriculum takes advantage of our gross anatomy full dissection program to ground students' learning of human structure/function; introduces clinical practices including handoffs and certain clinical procedures, basic interpretations of medical imaging, patient safety, and

Acad Med. 2020;95:S254-S257.

doi: 10.1097/ACM.0000000000003445

Copyright (C) 2020 by the Association of American Medical Colleges

Supplemental digital content for this article is available at http://links.Iww.com/ ACADMED/A937.

Year school was established: 1868.

School URL: https://www.med.wayne.edu. team dynamics; and explores humanistic, social science, and public health concepts through a first patient project.

- Early clinical exposure and practice through a redesigned longitudinal clinical skills curriculum begins in segment 1 and progresses through advanced clinical training in segment 4.

- Students begin outpatient clinical training in primary care settings in segment 2 .

- Our newly created Population, Patient, Physician, and Professionalism (P4) longitudinal course provides students a holistic view of clinical practice through the lens of multiple scientific disciplines and includes the social, behavioral, and health systems sciences. The course is built around small-group learning that is facilitated by master clinicians. Classroom learning is supported through service learning opportunities that are designed to integrate concepts taught in P4, clinical skills and foundational science courses.

- Additional changes are occurring in phase 2, segment 3 of our curriculum in which students rotate through their required clerkships. Recent revisions to this phase include:

- Additional curriculum devoted to patient safety and quality improvement

- A focus on student health and well-being to instill best practices early into students' careers

- A move toward improving educational opportunities in an outpatient setting

- The last 18 months of the curriculum (phase 3, segment 4) is devoted to advanced professional development and transition to residency. Revisions to this phase include:

- Residency preparation curriculum

- Expanded elective choices

- Scholarly concentrations

\section{Assessment}

In 2016, WSUSOM adopted new medical education program objectives, which are organized into 8 domains. These new objectives are based on the AAMC's Physician Competency Reference Set and Core Entrustable Professional Activities for Entering Residency.

The WSUSOM competency domains are:

- Domain 1: Knowledge for practice

- Domain 2: Patient care

- Domain 3: Practice-based learning and improvement

- Domain 4: Interpersonal and communication skills

- Domain 5: Professionalism

- Domain 6: Systems-based practice

- Domain 7: Interprofessional collaboration

- Domain 8: Personal and professional development

Along with major curricular changes, multiple forms of student assessments were implemented in addition to the traditional 
single best response (multiple choice question) method of assessment. These include:

- OSCEs

- Essay/written reflection

- Laboratory practicals

- Short-answer examinations

- Oral presentations

- Workplace-based assessments

- Formative assessment

\section{Pedagogy}

WSUSOM currently uses the following pedagogical approaches. As part of an integrated curricular design, case-based and problem-based learning experiences have been added to the first and second segments. Segments 3 and 4 have been redesigned to ensure students have adequate clinical experiences in both an ambulatory and inpatient setting.

- Case-based learning

- Clinical experience: ambulatory

- Clinical experience: inpatient

- Discussion: small group $(\leq 12)$

- Laboratory

- Lecture

- Peer teaching

- Preceptorship

- Problem-based learning

- Self-directed learning/tutorial

- Simulation

- Standardized/simulated patients

- Team-based learning

\section{Clinical experiences}

The required clerkships are offered at 4 separate campuses using 6 hospitals and multiple ambulatory sites. All sites are in the Detroit metro area, the great majority in the city of Detroit with a few in the immediate neighboring suburb. The Detroit Medical Center and Henry Ford Medical Center are large quaternary referral centers in central Detroit that also function as primary, emergency, and critical access centers for the surrounding population. Ascension St. John is a tertiary and referral center, with an active trauma center on the east side of Detroit. The Detroit VA and William Beaumont Dearborn hospitals function in a mixed primary care and tertiary referral role. All of the hospitals (other than the VA) have verified and active trauma centers.

All students rotate at the Children's Hospital of Michigan in downtown Detroit, in addition to ambulatory experiences in pediatrics in the city and immediate surrounding suburbs.

Family medicine and other ambulatory rotations use a network of established primary care practices in and around Detroit, along with the extensive ambulatory network of the Henry Ford Health system.

\section{Required longitudinal experiences}

Beginning with the class of 2022, there is an established clinical skills longitudinal track. This starts with clinical skills simulation and standardized patients in the Kado Clinical Skills Center during segments 1 and 2. Clinical experience is introduced in segment 2 with outpatient assignments. The segment 3 longitudinal course (Clinical Reasoning, Integration, and Skills for Practice) carries the longitudinal curriculum through segment 3. As seniors (segment 4), the students are trained and involved as educators on several levels.

\section{Clinical experience first encounter}

After intensive fundamental clinical training in a simulated environment during segment 1 , the students are assigned to outpatient clinics on a biweekly schedule. They see patients with a structure of increasing expectations as they continue through the year. This is supplemented by continued simulated modules concentrating on advanced skills that can be used in the real clinical environment in a temporally integrated fashion.

\section{Required and elective community-based rotations}

The Clinical Experiential course during segment 2 assigns students to community preceptors, including the VA and federally qualified health centers. During the continuity clerkship (up to 2020) and the internal medicine clerkship (2020 onward), students are assigned to outpatient clinics in Detroit in primary care.

\section{Curricular Governance}

Curricular governance is overseen by the Curriculum Management Committee:

- In accordance with LCME Standard 8 (curricular management), the Curriculum Management Committee (CMC) is the institutional entity that oversees the medical education program as a whole and that has responsibility for the design, management, integration, evaluation, and enhancement of a coherent and coordinated curriculum.

- The CMC authority originates by delegated powers from the School of Medicine Faculty Executive Committee.

- The CMC is by design a predominately faculty committee that has full authority and final approval over curriculum for the medical education program leading to the MD degree at WSUSOM.

- The CMC is specifically organized to incorporate the characteristics of an effective central curriculum authority ("integrated institutional responsibility"); the characteristics of an effective system of curriculum management ("curriculum management"); and the requisite elements to support the design and implementation of a "coherent and coordinated curriculum" as delineated by the LCME.

- The CMC is designed to be a protocol-driven entity that emphasizes data-driven decision-making and CQI philosophy. Through its management and oversight of the undergraduate program leading to the MD degree, the CMC ensures that students learn the requisite knowledge, skills, attitudes, and behaviors consistent with the WSUSOM mission and are well prepared and highly qualified for subsequent medical licensure, postgraduate residency training, and a successful career in the field of medicine.

See Figure 1-Curricular governance organizational chart. 


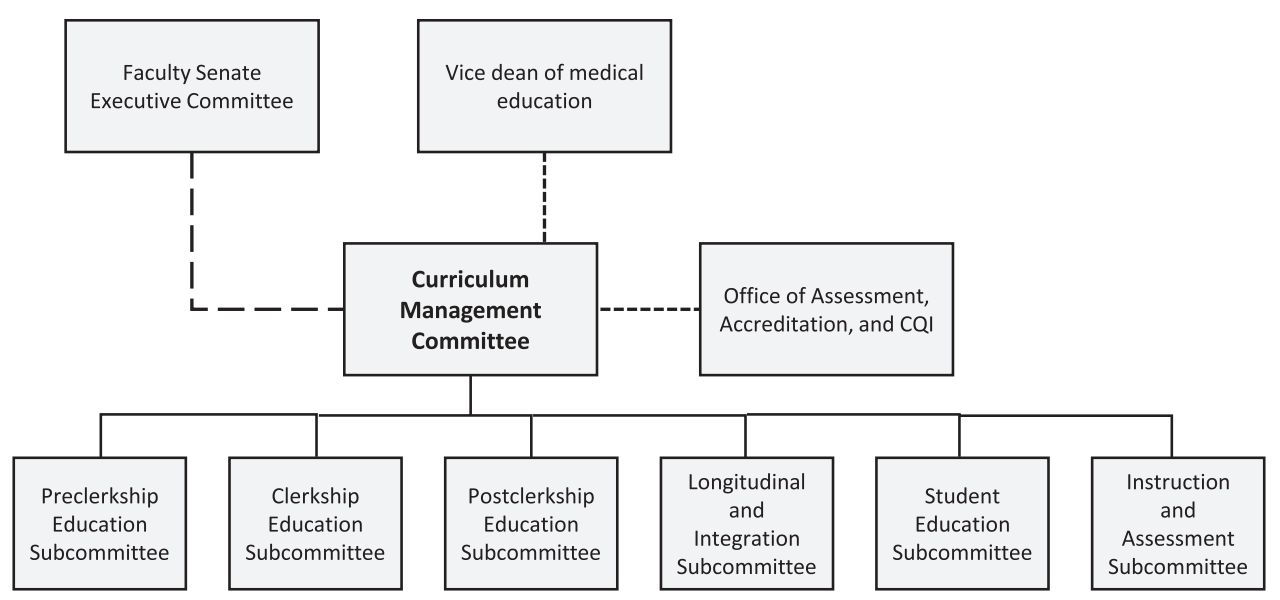

Figure 1 Curricular governance organizational chart.

\section{Education Staff}

- Administrative and academic support for the planning, implementation, evaluation, and oversight of the curriculum and for the development and maintenance of the tools to support curriculum delivery, monitoring, and management is centralized within the Office of Medical Education.

- The vice dean for medical education reports directly to the dean and oversees the Office of Medical Education and all MDrelated educational activities including undergraduate medical education, graduate medical education, and continuing medical education.

- Major administrative units within the Office of Education that provide support to educational programs are the Office of Student Affairs; Office of Admissions and Enrollment
Management; Office of Assessment, Accreditation, and Continuous Quality Improvement; and Office of Learning and Teaching, each of which are led by deans. In addition, medical education support services provide business and logistical support to students, faculty, and administrators under the leadership of a director.

- UME is overseen by the senior associate dean for curriculum and undergraduate medical education, who reports directly to the vice dean for medical education. Lead administrators within UME include the associate dean for preclerkship curriculum and foundational sciences, the associate dean for clerkship curriculum and clinical sciences, the director for community engagement, the director for the simulation and clinical skills center, and the director for medical student research.

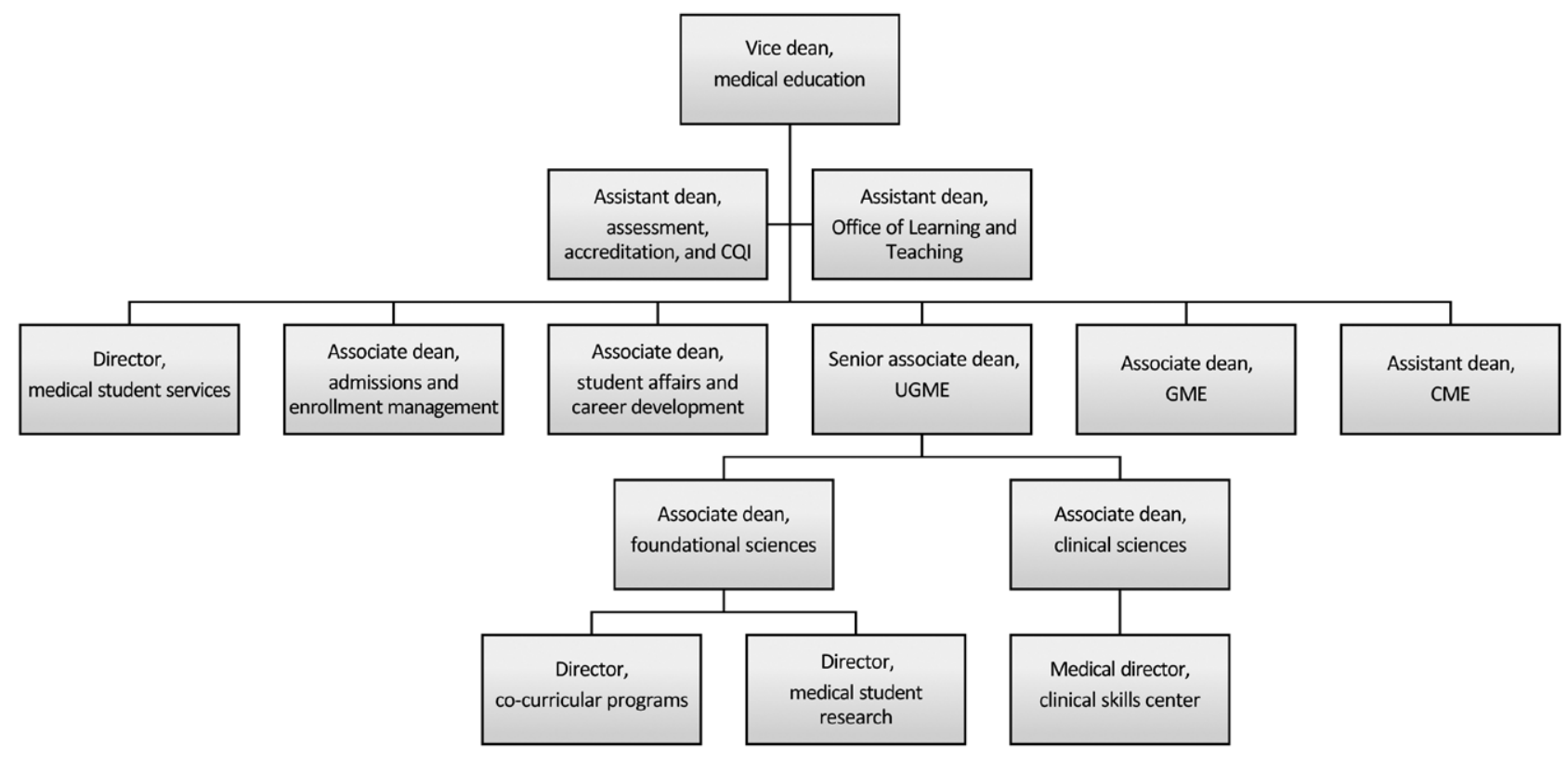

Figure 2 Educational unit organizational chart. 
See Figure 2-Educational unit organizational chart.

\section{Faculty Development and Support in Education}

\section{Professional development for faculty as educators}

Centralized professional development is offered to all faculty and educators. Opportunities include the following:

- Stanford faculty development program

- Curriculum development training

- Education and evaluation training

\section{Role of teaching in promotion and tenure}

WSUSOM values educational activities including teaching, educational scholarship, and other contributions as part of its promotion and tenure process. Documented excellence in medical education is expected. Education may take many forms, including classroom teaching, clinical teaching, development of curricula and educational materials, and mentorship of trainees. Publications that include trainees as coauthors are valued for teaching as well as scholarship. Mentorship of faculty is also an important role for midcareer and senior faculty. Reviews by peers and the recipients of the teaching efforts (i.e., students, residents, fellows, faculty, and staff) demonstrate excellent performance as an educator and/or mentor. 


\title{
Western Michigan University Homer Stryker M.D. School of Medicine
}

\author{
Michael Busha, MD, MBA, Kristine Gibson, MD, Maria Sheakley, PhD, and Jeffrey Greene, PhD
}

\section{Medical Education Program Highlights}

Western Michigan University Homer Stryker M.D. School of Medicine (WMed) enrolled its inaugural class in the fall of 2014 with the mission to educate and inspire lifelong learners to be exceptional clinicians, leaders, educators, advocates, and researchers of tomorrow. The preclinical phase is a teambased learning (TBL) curriculum delivered through systemsbased courses. Clinical education begins in the first year of the curriculum, relying on a robust standardized patient and simulation program and a partnership with the Program of All-Inclusive Care for the Elderly to develop longitudinal relationships with patients and interprofessional faculty. This is combined with a required curricular focus on service learning through community partnerships. The preclinical and clinical phases of the curriculum are designed in a decompressed fashion with a "green" week in between each course/clerkship. The USMLE Step 1 examination occurs after 4 of 6 core clerkships.

\section{Curriculum}

\section{Curriculum description}

See Supplemental Digital Appendix 1-Curriculum Map-at http://links.lww.com/ACADMED/A877.

\section{Curriculum changes since 2010}

Since WMed's inaugural class of 54 students started in 2014, the class size has subsequently increased over 4 years to reach the maximum class cohort size of 84 students. The preclinical curriculum spans 20 months and consists of 13 foundational organ system courses that average 5 weeks in duration, a longitudinal Professions of Medicine (POM) course, and a longitudinal Introduction to Clinical Experiences (ICE) course.

- A TBL format is used in all foundational organ system courses. TBL is an instructional strategy that promotes application of knowledge to clinically relevant scenarios in an active learning setting. TBL holds students accountable for learning independently, promotes the development of high-performing teams, and enhances problem-solving skills. All of this is achieved with less didactic lecture time and more energy in the classroom.

Acad Med. 2020;95:S258-S261.

doi: 10.1097/ACM.0000000000003364

Copyright (C) 2020 by the Association of American Medical Colleges

Supplemental digital content for this article is available at http://links.Iww.com/ ACADMED/A877.

Year school was established: 2012.

School URL: https://med.wmich.edu.
- The POM course includes clinical skills training that heavily uses standardized patients and simulation and is integrated/ aligned with the organ system courses; service learning via the active citizenship component of the course; and sessions on topics such as ethics, epidemiology, and biostatistics in the principles component of the course.

- The ICE course gives students early exposure to clinical medicine and diverse medical teams beginning with Medical First Responder certification during the first term.

- Self-directed learning is accomplished in part through a curricular innovation called Explorations, which runs throughout the preclinical years. Students work in small groups to apply concepts of evidence-based medicine and critical thinking to medically relevant topics of interest.

- The new Multisystem Disorders course is being implemented for the fifth matriculating class as the final course of the preclinical curriculum to serve as a capstone for the preclinical years by integrating knowledge of classic multisystem diseases such as diabetes, lupus, and cystic fibrosis that are difficult to convey in a single organ system course.

- Students are assessed progressively using the NBME Comprehensive Basic Science Exam (CBSE). There are 4 attempts spaced throughout the preclinical years. Students achieving our competency threshold on the third or fourth attempt progress to clerkships, otherwise they progress upon successful remediation of the CBSE.

The clinical applications phase of the curriculum was launched in 2016 for the inaugural class and has undergone significant revisions during the first 3 iterations before hitting a steady state.

- Core clerkships were initially designed with all didactic teaching and assessment in the first and last weeks of the clerkship. Each clerkship was 8 weeks with a dedicated preparatory week that included simulation, facilitated learning sessions, and independent preparation. This pedagogical approach was taken in an effort to allow students to fully immerse themselves in the clinical experience, armed with foundational knowledge and technical skills to be successful as they encounter patients. As a new curriculum, there was also the conscious choice to delay Step 1 until the completion of all core clerkships. While these strategies resulted in successful Step 1 scores, the timing of Step 1 results made career planning difficult.

- For the subsequent class, core clerkships were organized to create four 11-week shared blocks. Family medicine and pediatrics shared a block; women's health and psychiatry shared a block; medicine and neurology shared a block; and the surgery rotation incorporated a midclerkship didactic week with focus on surgical subspecialties, radiology, and anesthesia. We continued to have more concentrated didactics in the first week of each block but added formal once-weekly didactics 
throughout each rotation, as students and faculty both noted improved case discussion and retention of topics that were taught in conjunction with authentic patient interactions. Step 1 was taken three-fourths of the way through core curriculum, which proved to be better timed with career planning needs.

- For subsequent classes, we reverted to the model of independent clerkships, each 7 weeks in length, and have allowed clerkships to balance clinical assignments and didactic teaching as best fits the demands of the clerkship. Students are taking Step 1 in December or January following their fourthcore clerkship experience with an average of between 5.5 and 6 weeks to prepare for the exam.

- Advanced clerkships have evolved over the life of the school in an attempt to provide both rigorous subinternship experiences as well as allow for exploration of individual and career interest. The current requirements include three 4-week rotations in emergency medicine, advanced hospital medicine, and critical care.

- Our curriculum has 4 transition sessions bridging the matriculation to medical school, the move from preclinical to core clerkships, from core to advanced clerkships, and transition into residency. We heavily use our simulation center during these transitions, both for teaching and assessing procedures, and for clinical tasks and certifications (Basic Life Support, Advanced Cardiac Life Support).

\section{Assessment}

The medical education program objectives (EPOs) derive from the Physician Competency Reference Set. The EPOs are classified into 2 groups:
- Knowledge, skills, and abilities for which students must demonstrate competency

- Concepts to which students are exposed or for which they should have an understanding

The Curriculum Committee is responsible for evaluation and movement of EPOs between these 2 groups based on curriculum delivery, developmental appropriateness, and assessment reliability. Structured clinical assessments use the key functions within the AAMC Entrustable Professional Activities framework, which are mapped back to EPOs.

See Supplemental Digital Appendix 2-Program Objectives and Assessment Methods - at http://links.lww.com/ACADMED/A877.

\section{Parallel curriculum or tracks}

While our curriculum does not have separate tracks, we have developed a framework to allow for academic distinctions in an area of curricular focus. This includes a combination of particular electives as well as additional curriculum and scholarship participation within an area of curricular focus. Students apply for an academic distinction and completion is reflected on student transcripts. The first fully implemented academic distinction is in global and public health.

\section{Three-year curriculum}

WMed does not currently offer a 3-year curriculum option but is an exploratory phase for focused specialties in which GME training can be continued at WMed.

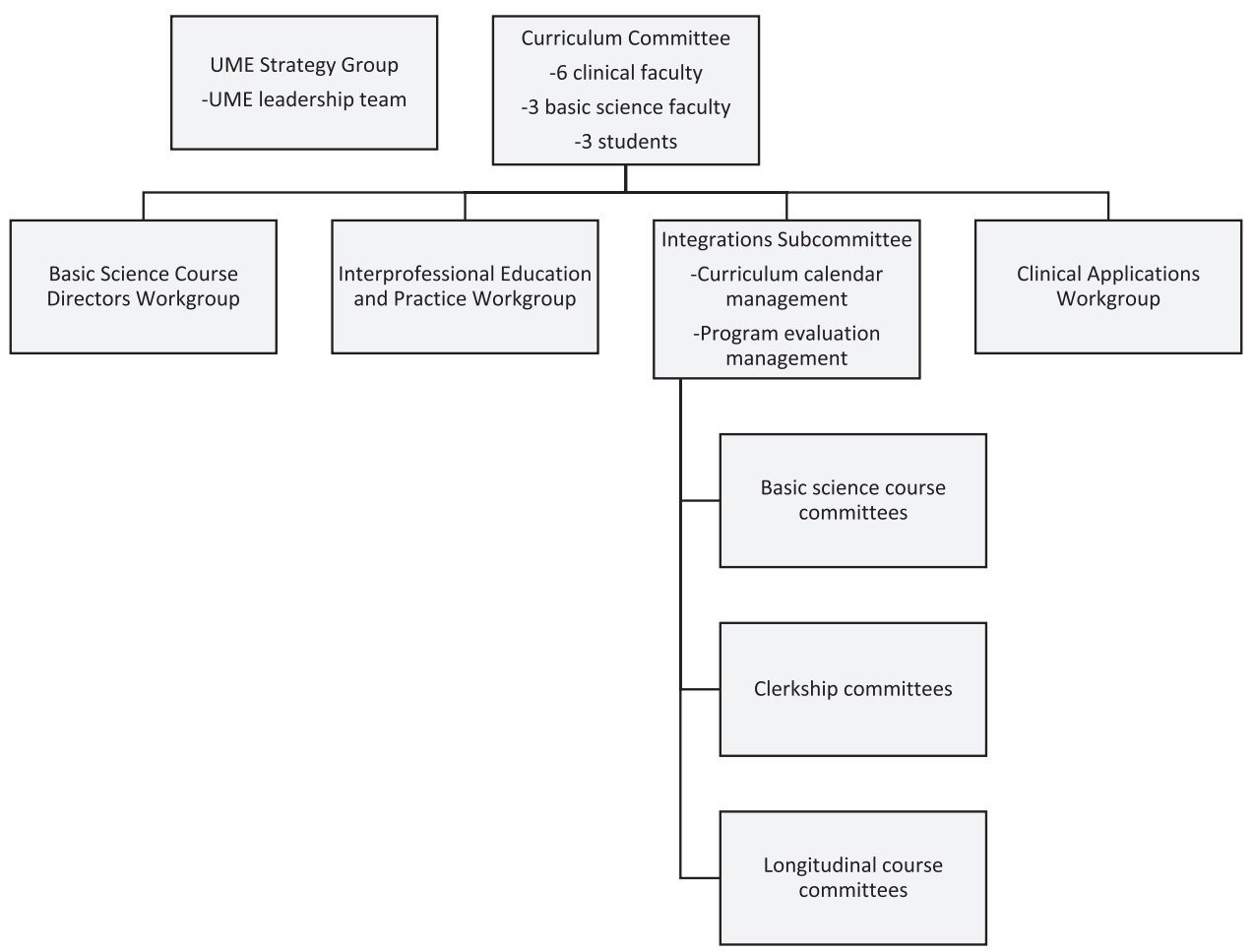

Figure 1 Curricular governance. 


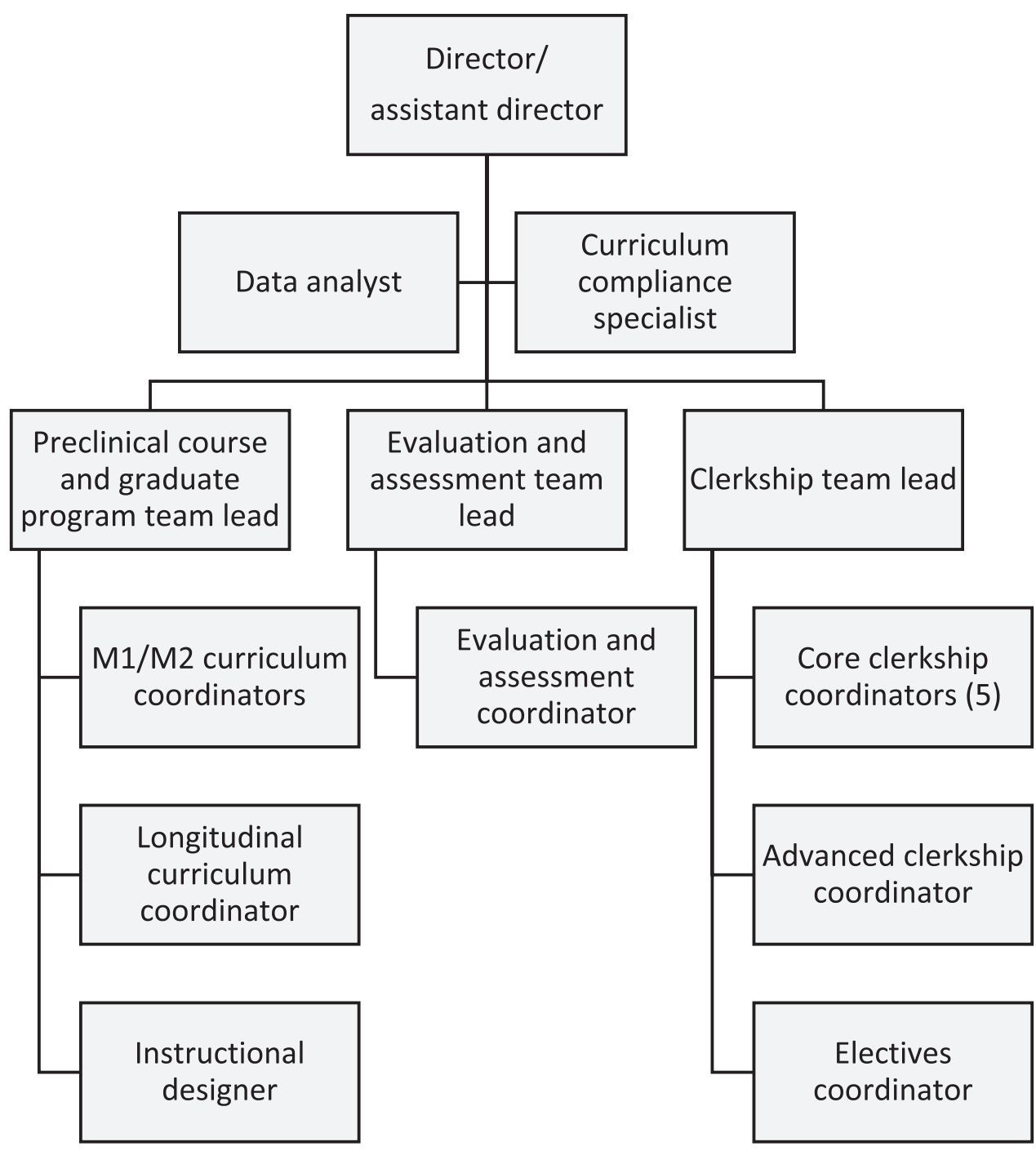

Figure 2 Office of Educational Affairs.

\section{Pedagogy}

The Foundations of Medicine (FOM) curriculum phase for basic science courses is structured in a TBL format. Each week includes a mixture of case-based learning, large-group discussions, lectures, and independent/self-directed learning. This material is then applied to clinical scenarios during a TBL event each Friday. The FOM curriculum for clinical skills development includes large- and small-group discussions, peer teaching, workshops, standardized patient experiences, and simulations, all of which are integrated or aligned with the corresponding basic science course. During the clinical applications phase of the curriculum, case-based learning, large- and small-group discussions, preceptorship, peer teaching, self-directed learning, simulation, standardized patients, virtual patients, and workshops are integrated into clerkship experiences.

\section{Clinical experiences}

Types of clinical sites used for required educational experience:
- Tertiary care community hospitals

- Regional hospitals

- Critical access hospitals

- Level 1 trauma center

- Level 2 trauma centers

- Critical access emergency departments

\section{Required longitudinal experiences}

- Medical First Responder course: Students become certified as a medical first responder as part of their transition to medical school and early clinical skills development. They complete their certification by December of year 1 .

- ICE: Students work with Senior Care Partners throughout their first 2 years of the curriculum, following the same panel of patients along with an interprofessional team of providers.

- POM Clinical Skills: Students focus on doctor-patient communication, physical examination, and documentation skills necessary to complete a patient encounter. 


\section{Required and elective community-based rotations}

WMed Health manages academic physician clinical activity through multiple WMed ambulatory sites and through academic services at its 2 primary affiliate hospitals. All hospital-based rotations occur in community settings, including the Veterans Affairs hospital in Battle Creek, Michigan. Students may be assigned to WMed Health academic services or to communitybased physician groups for inpatient clinical experiences. We also rely heavily on our outpatient community partnerships, including independent private practices, hospital-owned practices, and local federally qualified health centers in Kalamazoo and Battle Creek, Michigan. Some of our core clerkships primarily use our WMed Health clinics for their ambulatory component (surgery, internal medicine) while others employ a mix (pediatric and adolescent medicine; women's health, family, and community medicine; and neurology).

\section{Challenges in designing and implementing clinical experiences for medical students}

- Challenges include securing high-quality preceptors who are able to both teach and complete the required clinical midclerkship and final narrative assessments. We compete with our residents, other health professions students, and the need for clinical productivity. The academic year of our students does not align with resident start dates, causing additional stress in trying to forecast learner capacity within our system.

- We have a strong commitment from system administrators but that does not always translate to ease of acceptance among practice managers and physicians.

- Management of assessment is also a challenge. Busy practitioners can find it challenging to complete and submit assessments at the rapid pace required during many clerkships.

\section{Curricular Governance}

WMed manages the undergraduate medical education curriculum through the centralized Office of Educational Affairs. All internal and external faculty support for teaching, including course and clerkship director roles, are budgeted and managed through the Office of Educational Affairs. The Department of Medical Education, Department of Biomedical Sciences, and the clinical departments interface with the Office of Educational Affairs in delivering curriculum.

See Figure 1-Curricular governance.

See Figure 2-Office of Educational Affairs.

\section{Faculty Development and Support in Education}

The Office of Educational Affairs works closely with the Office of Faculty Affairs to coordinate faculty development events focused on educational delivery needs. These include a combination of embedded "micro" development events within meetings and retreats, larger events including outside speakers, and utilization of course/clerkship directors for faculty development within their individual course/clerkship offerings. The Department of Medical Education also serves a consulting role for individual faculty development in the areas of curriculum management, content delivery, and program evaluation. Teaching data, including degree of engagement, student experience, and student outcomes, are included in promotion decisions. Educational research is included in publication considerations. 


\section{Mayo Clinic Alix School of Medicine}

Darcy A. Reed, MD, MPH, M. Edwyn Harrison, MD, Alexandra P. Wolanskyj-Spinner, MD, Debbie A. Dixon, Michele Y. Halyard, MD, Gerardo Colon-Otero, MD,

Marcia L. Andresen-Reid, MS, and Fredric B. Meyer, MD

\section{Medical Education Program Highlights}

Mayo Clinic established its medical school with an inaugural class of 40 students in Rochester, Minnesota, in 1972. Today, the Mayo Clinic Alix School of Medicine (MCASOM) comprises 3 campuses in Arizona, Florida, and Minnesota with a target enrollment of 424 students. MCASOM employs a single governance structure and delivers a single integrated curriculum, leveraging outstanding educational experiences across the Mayo Clinic enterprise.

Preparing future physicians for unprecedented change in health care requires an adaptable curriculum, a forward-thinking faculty, and a diverse and thriving student body. In 2019, MCASOM began transforming its educational program toward a highly flexible, customizable curriculum that facilitates individualized learning, encourages innovation, and cultivates the myriad talents of the next generation of physicians. This new model emphasizes active learning and authentic clinical experiences that bring value to both education and clinical practice. Student progress is supported with performance analytics and longitudinal coaching. Learning objectives are explicitly values aligned and reinforced within a learning environment and organizational culture that reflects Mayo Clinic's primary value, "the needs of the patient come first" (Dr. William J. Mayo, 1910).

\section{Curriculum}

\section{Curriculum description}

The current MCASOM curriculum culminating in the MD degree is a single 4-year pathway. The first 2 years are made up of integrated blocks in which students simultaneously acquire foundational knowledge in basic sciences and clinical medicine. Students develop clinical competency longitudinally, with progressive independence throughout the 4 years. The third year includes traditional core clerkships, followed by subinternships and clinical electives in the fourth year. A substantial portion of the curriculum is designated for self-directed learning through selectives in the first 2 years and clinical electives in the third and fourth years. There is a longitudinal research curriculum that includes approximately 12 weeks dedicated to a mentored research project. Students are required to submit a first author manuscript and present their research regionally, nationally, or internationally. Just before graduation, students complete the Transition to Internship course and the internship boot camp.

Acad Med. 2020;95:S262-S265.

doi: 10.1097/ACM.0000000000003454

Copyright (C) 2020 by the Association of American Medical Colleges

Year school was established: 1972.

School URL: https://college.mayo.edu/academics/school-of-medicine.

\section{Curriculum changes since 2010}

- New regional campuses: In 2017, MCASOM opened a 4-year regional campus in Phoenix, Arizona, and a 2-year regional campus in Jacksonville, Florida. The Florida campus uses a $2+2$ model in which 12-14 students are chosen to complete their third and fourth years in Florida after completing years 1 and 2 in Arizona or Minnesota. Substantive changes in governance structure, policies and procedures, curriculum management, faculty development, and student services were required to successfully deliver the MCASOM educational program in a comparable fashion across all 3 campuses.

- Science of health care delivery curriculum: In 2017, MCASOM and Arizona State University developed a science of health care delivery curriculum to prepare students for practice within 6 domains: person-centered care; population health; high-value care; teamwork; leadership; and health policy, economics, and technology. All students receive a certificate of completion, and students have the option to pursue a master's degree in science of health care delivery at Arizona State University.

- THRIIVE program: MCASOM implemented a comprehensive program of student support and success at all 3 campuses which includes: academic success coaches, mental health counselors, wellness directors, humanities programming, resilience training, and individualized career specialty advisors and mentors who longitudinally follow students throughout medical school. MCASOM also supports student health and wellness with highly subsidized health insurance and fully subsidized fitness center memberships throughout the 4 years of medical school.

- Integrated MD-PhD program: MCASOM and the Mayo Clinic Graduate School of Biomedical Sciences together redesigned the MD-PhD program from a traditional model comprising separate MD and $\mathrm{PhD}$ curriculum phases delivered in sequence to an integrated model that allows students to progress within the $\mathrm{MD}$ and $\mathrm{PhD}$ components of their education simultaneously. This includes options to intermix clinical clerkships with research activities in ways that best support the educational goals of individual students. This model provides students the opportunity to decrease the total length of their $\mathrm{MD}-\mathrm{PhD}$ training.

\section{Assessment}

Students receive frequent formative and summative assessment throughout medical school. We employ the full breadth of assessment methods, with emphasis on observationbased assessments of clinical skills, communication, and professionalism. Each summative assessment is linked to a learning objective, an ACGME competency, and one or more educational program objectives. The objectives are framed within Mayo Clinic's core values: respect, integrity, compassion, healing, teamwork, excellence, and stewardship. 
See List 1-Educational Program Objectives.

Over the course of 4 years, MCASOM compiles a large body of summative assessments for each student. The Student Promotion and Assessment Review Committee monitors all summative assessment and ensures the satisfactory progression of each student.

Formative assessment is provided to each student at the midpoint and end of each course and clerkship, as well as longitudinally within the curriculum and in the context of coaching or mentoring relationships.

\section{Pedagogy}

In the first 2 years, the predominant pedagogical methods are lecture, small-group case-based learning, and clinical skills practice (with both real and simulated patients). We have implemented online learning and flipped classroom models in select courses. We continue efforts to reduce the number and length of lectures, replacing them with active learning methods and/or independent learning.

\section{Clinical experiences}

The longitudinal clinical skills curriculum begins early in year 1 and spans the first 2 years. Students complete traditional clerkships (block model) in year 3 and choose from a wide range of electives in year 4 . Students provide care to underserved patients in community-based clinics in Rochester and Phoenix. Students may complete clerkships and electives at affiliated hospitals in Arizona and Florida.

\section{Curriculum Governance}

The Curriculum Committee is responsible for ensuring the overall integration, design, management, continuous quality improvement, and evaluation of the MCASOM curriculum. The Medical School Education Committee, chaired by the dean, grants the Curriculum Committee primary responsibility for the curriculum.

See Figure 1-Curriculum Committee.

\section{Education Leadership and Staff}

The medical school is led by the dean and chief academic officer, who is responsible for the conduct and quality of the medical education program for all campuses. Each regional campus has a vice dean who reports to the dean and is responsible for dayto-day operations of the campus. Medical school functions are carried out across the campuses via 4 offices: Academic Affairs, Admissions, Faculty Affairs, and Student Affairs. Each office is led by a senior associate or associate dean, and includes associate deans at both regional campuses.

See Figure 2-Decanal staff.

Operational and administrative support for planning, implementation, evaluation, and management of the curriculum is provided by staff within MCASOM, the

\section{List 1}

\section{Educational Program Objectives}

Consistently demonstrates professional and ethical behavior

Communicates effectively

Demonstrates knowledge of basic human structure

Demonstrates knowledge of normal function

Demonstrates knowledge of pathophysiology of disease

Demonstrates knowledge of pharmaceuticals, therapeutics, and interventions

Applies knowledge of basic sciences to patient care

Contributes to discovery or translation of new medical knowledge

Demonstrates patient-centered approach

Gathers and records relevant patient history systematically

Conducts complete physical examination or selects appropriate components in a systematic and directed fashion

Performs routine clinical procedures and basic lab and diagnostic tests

Accurately interprets data from history, exam, and other findings and formulates a differential diagnosis

Selects and initiates appropriate investigations for the purpose of health screening and promotion, diagnosis, prognosis, or intervention

Makes appropriate clinical decisions and formulates and implements management plans both for treatment of illness, prevention, and relief of symptoms and suffering, and monitors its effectiveness

Participates in health promotion and in prevention of disease and disability

Manages life-threatening conditions

Advocates for patients and the community

Displays effective teamwork

Fosters self-awareness

Demonstrates commitment to personal wellness including physical, emotional, chemical, spiritual, and relationship health

Displays leadership

Promotes patient safety

Demonstrates knowledge of medical economics, health care finance, and health care policy

Understands health care processes and systems

Exhibits behaviors of lifelong learning

Education Technology Center, and the Office of Applied Scholarship and Education Science.

\section{Faculty Development and Support}

Professional development for faculty and residents is provided centrally by MCASOM Office of Faculty Affairs as well as by individual departments. Academic promotion decisions are made by an institutional committee outside the medical school. Excellence in education, including education scholarship, teaching service, teaching awards, curriculum development, and education leadership contribute to academic promotion. The Mayo Clinic College of Medicine and Science has an Academy of Educational Excellence that serves to inspire, develop, recognize, and support Mayo Clinic educators. 


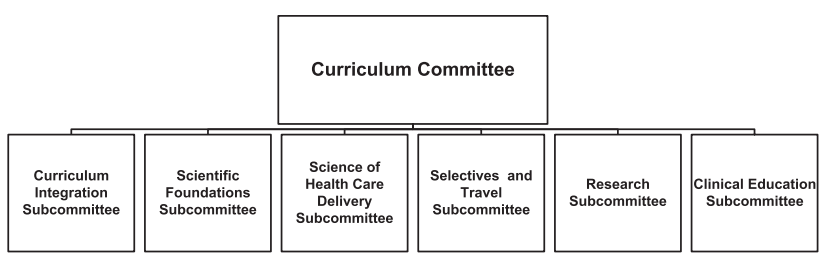

Figure 1 Curriculum Committee.

\section{Regional Medical Campuses}

MCASOM has 2 regional campuses. All students have the opportunity to rotate across all Mayo Clinic locations to maximize the diversity of their educational experience and allow them to benefit from interactions with the many expert clinicians and scientists across the Mayo Clinic enterprise. MCASOM subsidizes students' travel expenses.

See Table 1-Regional Campuses.
The Curriculum Committee, with faculty and student members from all campuses, ensures comparability of the curriculum. The Curriculum Committee reviews course-level and campuslevel dashboards containing aggregate student performance and student satisfaction metrics reflecting comparability. A course-level comparability policy outlines expectations, roles, and responsibilities for course directors and faculty in ensuring comparability across campuses.

\section{Initiatives in Progress}

In recent years, MCASOM has not only expanded to 3 geographically dispersed campuses, but we have also incorporated new content such as health systems science, created online learning resources, and implemented robust student wellness programs. With these foundational elements established, MCASOM is now creating an educational model that will empower students to customize their education, as well as enable faculty coaches to tailor curricula to meet individual

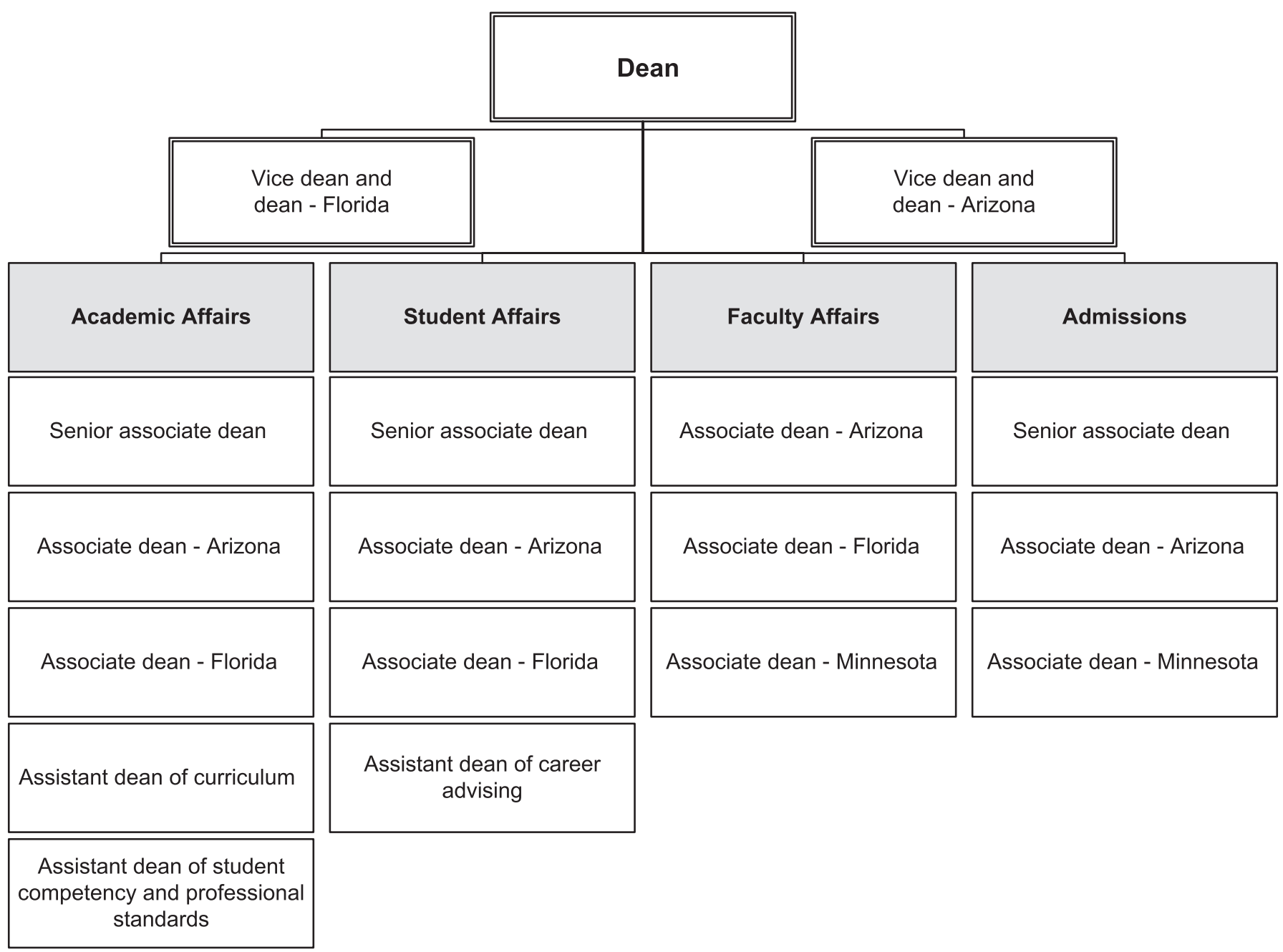

Figure 2 Decanal staff. 
student needs. All students will complete core requirements for the medical degree; however, we will leverage our 3-campus structure to offer flexible course sequencing and numerous opportunities for academic enrichment.

A flexible model should provide students autonomy, alignment with interests, and ability to adapt to change (such as life events, changes in career choice, etc.). These curriculum features may also buffer against student burnout. Longitudinal relationships between students and faculty coaches, supported by performance analytics, will facilitate student reflection, goal setting, curriculum choices, career planning, competency-based advancement, and remediation.

Core components of the new model include:

- Active learning

- Longitudinal clinical experiences with students serving in authentic roles that advance education and add value to clinical practice

- Curriculum phases in variable sequence

- Defined criteria for entry and progress within pathways

\section{Table 1}

\section{Regional Campuses}

\begin{tabular}{|c|c|c|}
\hline Regional campus & Туре & $\begin{array}{r}\text { Student enrollment } \\
\text { (target) }\end{array}$ \\
\hline $\begin{array}{l}\text { Mayo Clinic Alix School of } \\
\text { Medicine-Arizona }\end{array}$ & $\begin{array}{l}\text { Entire MD } \\
\text { program }\end{array}$ & 200 \\
\hline $\begin{array}{l}\text { Mayo Clinic Alix School of } \\
\text { Medicine-Florida }\end{array}$ & Clinical & 28 \\
\hline
\end{tabular}

- Frequent observation-based assessment and feedback

- Technology enabling individualized scheduling, student performance analytics, and monitoring

- Trained and supported faculty coaches

Opportunities for academic enrichment within this new model include existing and newly planned master's degree programs, certificate programs, and research fellowships in emerging areas such as artificial intelligence, innovation and entrepreneurship, regenerative science, and individualized medicine. 


\title{
University of Minnesota Medical School
}

\author{
Austin Calhoun, PhD, Robert Englander, MD, MPH, and Mark Rosenberg, MD
}

\section{Medical Education Program Highlights}

Founded in 1888, the University of Minnesota Medical School (UMMS) is 1 of the 10 largest medical schools in the countrywith 240 students per class across 2 campuses in the Twin Cities and Duluth. As a land-grant institution, we are dedicated to improving the health of the state's citizens and beyond, educating its future physicians, and creating new knowledge through discovery. It is estimated that $70 \%$ of the state's physician workforce is trained at UMMS, either in UME, GME, or both.

Mullan and colleagues defined a social mission score and ranked the nation's $141 \mathrm{MD}$-granting medical schools using that score. ${ }^{1}$ In their 2010 publication, UMMS was 1 of only 2 schools in the country to rank in the top quartile for social mission, NIH funding, and primary care output. Our social mission ranking aligns with our UME vision: "A community, learning together, to prepare exceptional physicians to improve the health and wellbeing of Minnesota and beyond." To achieve that vision, we have delineated 7 guiding principles:

- Build on diversity and inclusion

- Empower students

- Put patients first

- Standardize the outcomes, individualize the learning pathways

- Foster relationships

- Optimize the learning environment

- Provide evidence-based education

These guiding principles are the foundation for UME and are threaded through the following highlights:

- Individualized pathways with the opportunity to focus on rural health, research, earn a dual degree in our FLEX MD program, study abroad, or complete core clerkships in 1 of our 6 unique longitudinal integrated clerkships (LICs).

- We are 1 of 4 schools participating in Education for Pediatrics Across the Continuum. The program allows for competencybased, time-variable progression from medical school to pediatrics residency.

- In 2020, we move into the new Health Sciences Education Center. This state-of-the-art facility is designed to transform health education in Minnesota. The learning environment supports interprofessional education and active learning pedagogies, along with a focus on simulation and emerging technologies including VR/AR. Interprofessional student lounges and study and wellness spaces are also prominent features.

Acad Med. 2020;95:S266-S269.

doi: 10.1097/ACM.0000000000003365

Copyright (C) 2020 by the Association of American Medical Colleges

Supplemental digital content for this article is available at http://links.Iww.com/ ACADMED/A878.

Year school was established: 1888.

School URL: https://med.umn.edu.
- Our regional campus in Duluth has a specific mission in educating physicians dedicated to family medicine to serve the needs of rural Minnesota and Native American communities and is a perennial leader in this area.

- We focus on student, staff, and faculty well-being: engaging our community through innovative programming and tools to incorporate well-being into daily practice.

\section{Curriculum}

\section{Curriculum description}

- Preclerkship years: This phase is organized into 2 academic years and offered in unique formats at the 2 campuses. Each campus provides students with a strong preclinical sequence that ensures essential foundational sciences are covered. Both campuses include additional specialized content areas shaped by the needs of the campus. Content spanning the preclerkship years includes rural medicine education and experiences as well as Essentials of Clinical Medicine and Foundations of Critical Thinking courses. Regional campus students transition to the Twin Cities campus for the final 2 years of clinical training.

- Year 3: Students select to complete their required foundational clerkships in a block or an LIC format during the third year. Students who choose block clerkships rotate in sequenced lanes and with a cohort of students through their required clerkships.

- Year 4: In this time frame, students complete 2 advanced required clerkships (emergency medicine and intensive care subinternship), 2 advanced selective clerkships, and at least 20 weeks of electives.

\section{Curriculum changes since 2010}

The curriculum has evolved over the last decade. Key changes are:

- Intentional integration of basic and clinical knowledge in the foundational science courses

- Greater emphasis on health system science, health policy, and population health

- Expansion of longitudinal clinical experiences, including 4 new LICs

- More individualization of clinical training in year 4 to enhance preparation for residency (advanced care selectives based on future specialty)

- "Return to school" weeks during the clinical phase of training in our new Becoming a Doctor course; this course includes health system science, medical socioeconomics, and pain management and offers an opportunity for students to propose workshop topics

See Table 1-Current Longitudinal Integrated Clerkships.

\section{Class size changes since 2010}

The class size was increased from 165 to 175 on the Twin Cities Campus, and from 60 to 65 on the Duluth regional campus. 
Table 1

\section{Current Longitudinal Integrated Clerkships}

\begin{tabular}{|c|c|c|c|c|c|}
\hline Created & Program & Focus area(s) & Capacity & Setting & Duration \\
\hline 1971 & $\begin{array}{l}\text { Rural Physician Associate } \\
\text { Program (RPAP) }\end{array}$ & $\begin{array}{l}\text { Primary care in rural } \\
\text { Minnesota }\end{array}$ & $36-45$ & $\begin{array}{l}\text { Ambulatory and hospital } \\
\text { settings in outstate towns with } \\
\text { populations } \leq 30,000\end{array}$ & 9 months \\
\hline 2010 & $\begin{array}{l}\text { Metropolitan Physician Associate } \\
\text { Program (MetroPAP) }\end{array}$ & $\begin{array}{l}\text { Primary care for underserved } \\
\text { in the Twin Cities }\end{array}$ & 9 & $\begin{array}{l}\text { Ambulatory and hospital } \\
\text { settings in Minneapolis }\end{array}$ & 9 months \\
\hline 2015 & $\begin{array}{l}\text { Veterans Administration } \\
\text { Longitudinal Undergraduate } \\
\text { Medical Education (VALUE) }\end{array}$ & $\begin{array}{l}\text { Patient safety and } \\
\text { quality improvement; } \\
\text { interprofessional education }\end{array}$ & 10 & $\begin{array}{l}\text { Veterans Administration } \\
\text { Medical Center }\end{array}$ & 10 months \\
\hline 2015 & $\begin{array}{l}\text { Education in Pediatrics across the } \\
\text { Continuum (EPAC) }\end{array}$ & $\begin{array}{l}\text { Competency-based } \\
\text { assessment and progression }\end{array}$ & 4 & $\begin{array}{l}\text { Ambulatory and hospital } \\
\text { settings in Minneapolis }\end{array}$ & 9-12 months \\
\hline 2017 & $\begin{array}{l}\text { Hennepin Longitudinal Clinical } \\
\text { Experience (HeLIX) }\end{array}$ & $\begin{array}{l}\text { Physician advocacy, care of } \\
\text { the underserved }\end{array}$ & 6 & $\begin{array}{l}\text { Hennepin County Medical } \\
\text { Center (HCMC) }\end{array}$ & 9 months \\
\hline 2018 & $\begin{array}{l}\text { Regions Equitable and Affordable } \\
\text { Community Health Program } \\
\text { (REACH) }\end{array}$ & $\begin{array}{l}\text { Population health and the } \\
\text { social determinants of health; } \\
\text { interprofessional education }\end{array}$ & 6 & Regions Hospital/HealthPartners & 9 months \\
\hline
\end{tabular}

aAdvancement in EPAC is competency based and time variable, which accounts for the ranges in length of time.

The rationale was based on projections of future state workforce needs especially for rural and primary care providers and increased classroom and clinical training capacity.

\section{Assessment}

UMMS adopted the AAMC Physician Competency Reference Set (PCRS) as its educational program objectives, adding 2 competencies under a ninth domain, scientific and clinical inquiry. The PCRS is founded in the ACGME and American Board of Medical Specialties Outcome Project competencies, thus providing continuity for students as they head into residency and practice.

\section{Assessment changes since 2010}

Along with curricular changes, we have also created alignment and altered our assessment strategies in the following ways:

- Elimination of grades in the first 2 years moving, to a pass/fail system

- Standardization of assessment based on common course objectives in the preclinical phase, including integration of competency-based testing with robust psychometric analysis

- Development of a framework for assessment for direct observation in the clinical phase, focusing on the Core Entrustable Professional Activities for Entering Residency

- Increased use of peer assessment of students

- Implementation of "Pulse Surveys" in 2018 for real-time tracking of burnout and satisfaction

- Development of the Medical Education Outcomes Center (MEOC) to centralize all educational data, make data-driven decisions, and link educational to clinical outcomes

\section{Parallel curriculum or tracks}

- We have 1 parallel track, comprising the Rural Physician Associate Program (RPAP)/Metropolitan Physician Associate
Program (MetroPAP). These are 9-month, communitybased LICs for third-year medical students. RPAP occurs in rural community training sites, and MetroPAP in medically underserved and diverse urban communities.

- A 3-year MD degree program does not exist at UMMS; however, we have the 7-year BA-MD Joint Admissions Scholars Program, providing an opportunity for exceptional Minnesota residents from broadly diverse backgrounds. Three years are spent taking undergraduate coursework and 4 years are spent at the medical school.

\section{Pedagogy}

The frequency that various instructional methods are employed during years 1 and 2, and years 3 and 4, is varied. A major focus has been to increase the amount of active learning in large-group settings.

See Supplemental Digital Appendix 1-Sessions Using AAMC Instructional Methods in Year 1 and 2 Required Courses-at http://links.lww.com/ACADMED/A878.

See Supplemental Digital Appendix 2-Sessions Using AAMC Instructional Methods in Year 3 and 4 Required Courses-at http://links.lww.com/ACADMED/A878.

\section{Clinical experiences}

- Our inpatient clinical teaching sites at which medical students take 1 or more clinical clerkships include 14 hospitals in Minneapolis and St. Paul, 2 hospitals in Duluth, and 1 hospital in St. Cloud. For our RPAP program, 46 hospitals in Greater Minnesota are used. Multiple ambulatory clinical teaching sites are used, including hospital-associated clinics, community health centers, private physician offices, and rural clinics.

- The first clinical encounter for year 1 students on the Twin Cities campus occurs in the Process of Care clerkships where 
students have the opportunity to see patients in clinic, hospital, and extended care settings. On the Duluth campus, the Rural Medical Scholars Program in the first year places students in rural communities living and working with medical preceptors in a clinical environment.

- The major challenge is capacity for clinical training related to faculty effort, but being the only medical school in the Twin Cities is an advantage. Availability of housing at some sites remains a challenge.

\section{Curricular Governance}

The Education Council (EC) is the curriculum committee of UMMS and is composed of faculty, students, and educational leaders. EC is the approved body for final decisions in curriculum and policy. The committees that report to EC include the Education Steering Committee that serves as an advisory group ("think tank"), Scientific Foundations Committee (Twin Cities year 1 and 2 course directors), Committee on Undergraduate Medical Education Duluth (Duluth course directors), Clinical Education Committee (clerkship directors), and the Assessment Committee that oversees all aspects of assessment in the MD program. All aspects of curricular governance are managed centrally with funds allocated to departments to support faculty teaching.

See Figure 1-Curriculum governance structure.

\section{Education Staff}

The Office of Medical Education (OME), directed by the vice dean for education and academic affairs, administers the medical education program and works with faculty to develop programming and strategy. OME comprises the following components and leaders to oversee the continuum of medical education:

- Admissions and pipeline programs (associate dean of admissions)

- UME (associate dean of UME)

- GME (associate dean and designated institute official)
- Continuing professional development (director of continuing professional development)

- Anatomy bequest program (director of anatomy bequest)

- Program in mortuary science (director)

- Medical educator development and scholarship (MEDS) (director)

- MEOC (vice dean for education and academic affairs)

See Figure 2-Organizational chart.

\section{Faculty Development and Support in Education}

While faculty appointments are in medical school departments and not in OME, the office does provide substantial faculty development and support.

- MEDS is charged with improving the quality and effectiveness of medical education by providing faculty with the skills and tools they need to be effective teachers, educational leaders, and scholars. Programming includes workshops, works-in-progress conference, annual best practice conference, individual consultations, web-based tool kit, and research support through the MEOC.

- The assistant dean for assessment and evaluation provides faculty with workshops and consultations designed to ensure sound teaching practices. These include but are not limited to improving the quality of narrative assessment, creating effective exam questions, and interpreting exam results using effective statistical methods.

- The assistant dean for curriculum and a team that includes 2 directors (director of integrated education in the foundational sciences and director of integrated education in the clinical sciences) work with faculty on all aspects of curriculum development.

- The University of Minnesota Center for Educational Innovation (CEI) supports faculty in identifying sound educational approaches for teaching. Faculty development with a member of the CEI team is a standing item on the monthly course directors meeting. One-on-one consultations with a CEI education specialist are available to provide pedagogical support.

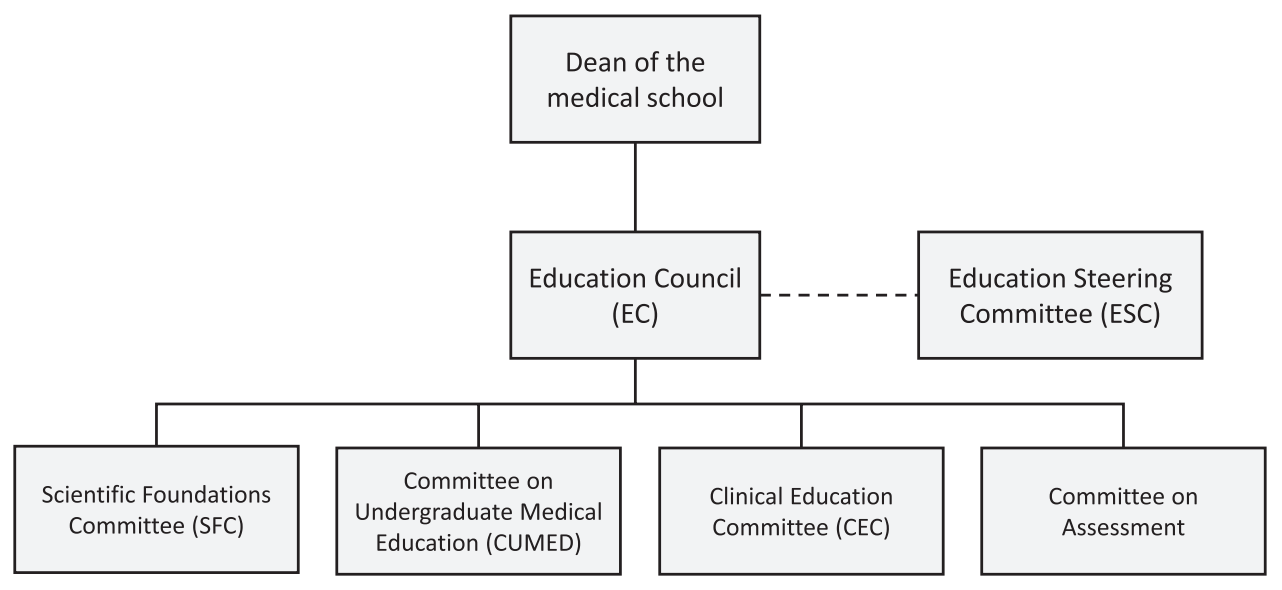

Figure 1 Curriculum governance structure. 


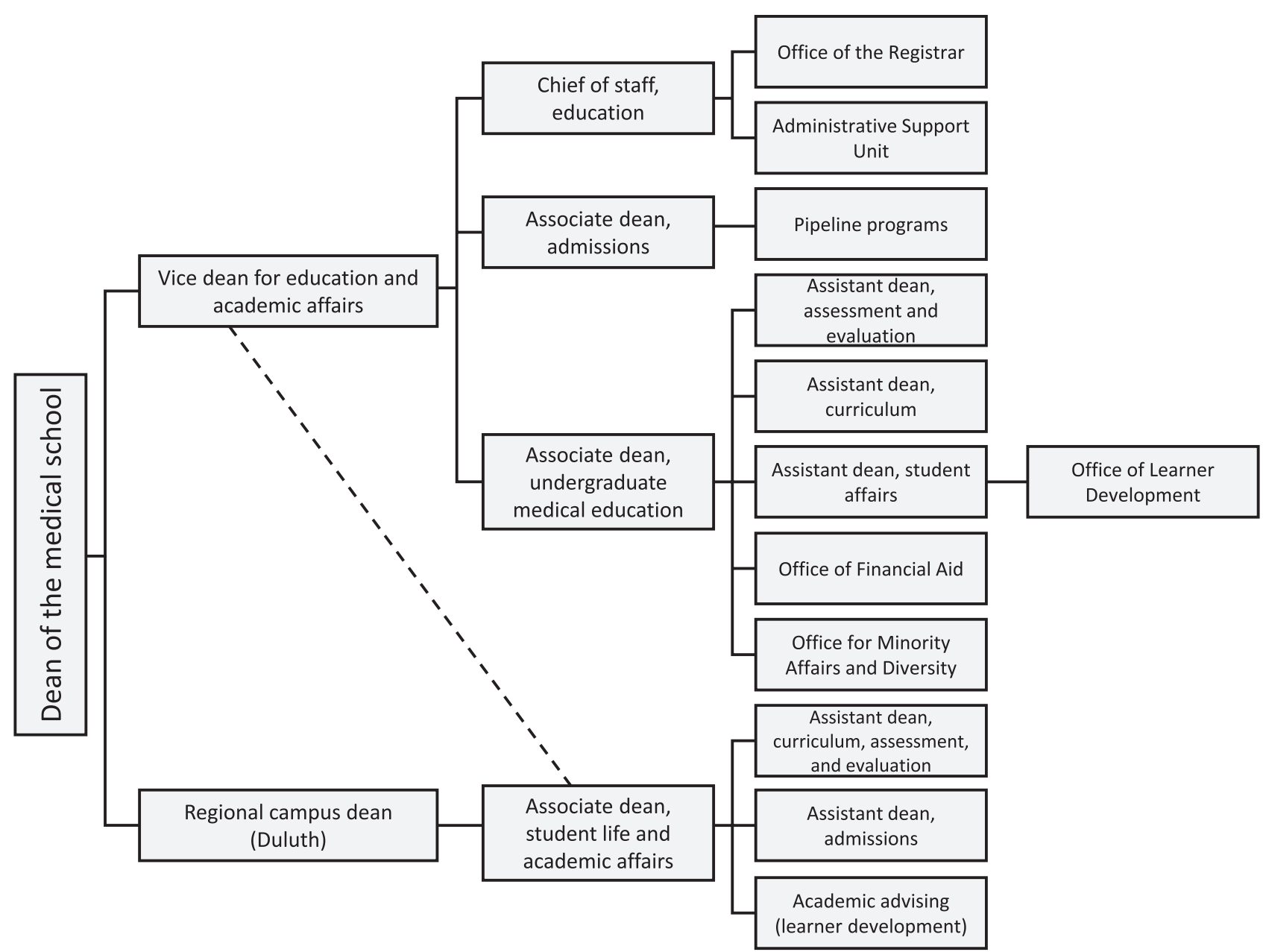

Figure 2 Organizational chart.

\section{Role of teaching in promotion and tenure}

UMMS has 3 tracks for promotion: tenure track, academic track, and master clinician track.

- For promotion on the tenure track, educational scholarship is a requirement.

- For promotion on the academic track, candidates must demonstrate sustained peer-reviewed scholarship in the form of a traditional peer-reviewed publication or other products, including peerreviewed online repositories. Other criteria include development of educational products that have been adopted by others outside of the institution; successful grant funding; instructional, curricular, or assessment innovations; educational policy development; or chapter or book authorship. Candidates must demonstrate excellence in their educational roles demonstrated by learner reviews, teaching awards, and peer evaluations.

- For promotion on the master clinician track, the major focus is clinical excellence, which is defined differently by departments. For most departments, demonstration of teaching excellence is required and must be documented by learner evaluations.

- The University of Minnesota has the Academy for Excellence in the Scholarship of Teaching and Learning, which is open to all health science schools. The academy recognizes faculty who have demonstrated exceptional scholarly contributions to advance learning in their schools and across academic programs.

\section{Regional Medical Campuses}

UMMS Duluth was funded by the legislature in 1969, and the first students matriculated in 1972. Integration of the Twin Cities and Duluth campuses occurred in 2004. The Duluth campus provides the preclinical phase of medical education, after which the students enter the clinical phase of training at the Twin Cities campus. The mission of the Duluth campus is focused on rural medicine and serving Native American communities. A comparable educational experience is demonstrated by common learning objectives and assessments and standardized national examinations. Reporting relationships and regularly scheduled interactions between educational leaders, curriculum committees, and course directors at both campuses facilitate operational issues regarding comparability.

\section{Reference}

1 Mullan F, Chen C, Petterson S, Kolsky G, Spagnola M. The social mission of medical education: Ranking the schools. Ann Intern Med. 2010;152:804-811. 


\section{The University of Mississippi School of Medicine}

Loretta Jackson-Williams, MD, PhD

\section{Medical Education Program Highlights}

- The University of Mississippi School of Medicine is located within the state's only academic medical academic center. The School of Medicine (SOM) began as a 2-year certificate program in 1903 and expanded to a 4-year educational program in 1955. Currently, only Mississippi residents are accepted for the program to encourage the continued growth in the number of physicians practicing within the state.

- For a number of years, the school has focused on the creation of community for students. This concept was extended with the creation of the House Program. This program was established in 2014 as a medical school community designed to ensure that medical students are connected to campus resources and receive adequate social and academic support. First-year students are assigned to 1 of 8 "houses" with mentors and resources including the individual pairing of incoming students with an upper-class "buddy."

- The school provides a proactive academic support program for students that is managed by the assistant dean for academic affairs. The program includes early identification of academic underperformance through the Academic Achievement Program, tutoring and academic counseling through institutional resources, and personal counseling through the Student Counseling and Wellness Center.

\section{Curriculum}

\section{Curriculum description}

- The SOM has a curriculum structure that includes some integrated courses with coordinated sequencing of topics for the remaining courses in the preclerkship phase. There are 7 required clerkships in the third year; a boot camp at the beginning of the third year and in the fourth year at a key transition point; and, finally, required fourth-year rotations for which several selectives are available.

- The curriculum is arranged so that students have an emphasis on "normal" in the first year and "abnormal" in the second year, with an introduction to clinical application and expectations during both years. The first year of the medical education program consists of biochemistry, anatomy, human development, physiology, and histology, and cell biology, along with Integrated Medical Neuroscience

Acad Med. 2020;95:S270-S272.

doi: 10.1097/ACM.0000000000003277

Copyright (C) 2020 by the Association of American Medical Colleges

Supplemental digital content for this article is available at http://links.Iww.com/ ACADMED/A824.

Year school was established: 1903 as a 2-year school; became a 4-year school in 1955 School URL: https://www.umc.edu/som. and Behavior I (MN\&B I) and Introduction to the Medical Profession I (IMP I) courses. The second year consists of microbiology, pathology, and pharmacology, along with MN\&B II and IMP II.

- In the third year of the medical education program, students are required to complete 7 clerkships: surgery, family medicine, pediatrics, internal medicine, psychiatry, obstetrics-gynecology, and clinical neuroscience. In addition, the third year requires 2 electives to allow students to explore different specialties and more ambulatory settings. For academic year 2020-2021, psychiatry, neurology, and neurosurgery have been combined for the third course/clerkship in medical neuroscience and behavior that replaces the clinical neuroscience clerkship.

- The fourth-year requirements include a critical care selective, a procedural selective, an ambulatory selective, and a subinternship. Medical students are also required to take 14 weeks of electives in the fourth year. The fourth year of the medical education program was revised to be on a 2- or 4-week rotation schedule with the noted requirements.

\section{Curriculum changes since 2010}

- The educational program objectives that guide curriculum refinement and development have been expanded to include a deliberate focus on student attainment of skills for physician well-being and interprofessional practice.

- An integrated and longitudinal neuroscience curriculum has been developed and implemented. This includes courses for the first, second, and third years of the curriculum. The first year integrated topics from neurobiology, neurophysiology, psychiatry, and neurology. The second year integrated topics from psychiatry, neurology, neurosurgery, neuropathology, and CNS pharmacology. The third year integrated psychiatry, neurology, and neurosurgery.

- The fourth-year curriculum was redesigned to support the development, growth, and assessment of all students as early medical professionals prepared for graduate medical training.

- Clinical skills assessment and simulation were incorporated into the preclerkship curriculum.

- The focus on interprofessional education was expanded within the curriculum.

- Pain management, service learning, telehealth, and ultrasound were formally added to the curriculum with plans for longitudinal expansion of the topics.

- The class size was expanded from 110 students in 2010 to the current number of 165 . To support the growth of the class size, a new SOM education building was designed and completed in 2017. The building includes 2 large lecture halls, 18 small classrooms, and 2 laboratory spaces. In addition, there is a clinical skills center, dedicated interprofessional training space, and a simulation center within the building. 


\section{Assessment}

The SOM's education program objectives were adapted from Learning Objectives for Medical Student Education, Guidelines for Medical Schools, AAMC, 1998; revised by the School of Medicine Curriculum Committee, July, 2009; updated by the School of Medicine Curriculum Committee, 2011; and reviewed and updated by the School of Medicine Curriculum Committee, January, 2018.

See Supplemental Digital Appendix 1-Medical Education Program Objectives - at http://links.lww.com/ACADMED/A824.

\section{Parallel curriculum or tracks}

There is no current parallel curriculum or track within the educational program. However, the state legislature created the Office of Mississippi Physician Workforce (OMPW) in 2012. The OMPW is charged to monitor and evaluate the composition and distribution of Mississippi's physician workforce. Since the creation of the office, there has been growth of rural graduate education training opportunities in the state. The medical school now has an opportunity to work with these new programs to develop a rural training track that spans undergraduate and graduation medical education programs.

\section{Pedagogy}

- Teaching modalities used in the delivery of the educational programs include the following:

- Case-based learning

- Clinical experience: ambulatory
- Clinical experience: inpatient

- Discussion groups, both large and small

- Laboratories

- Lecture

- Peer teaching

- Preceptorships

- Role play/dramatization

- Simulation

- Standardized patients

- Team-based learning

- Video/podcast

- With the addition of the SOM education building with smaller classrooms, more small-group discussions have been implemented in the delivery of the educational program.

\section{Clinical experiences}

- The university hospitals and clinics are used for required education experiences, with the exception of the family medicine clerkship and a portion of the medicine clerkship at the Veterans Administration medical center. Family medicine uses community preceptors (which would be considered clinical affiliates) for the third-year family medicine clerkship. These preceptors are nonranked faculty members in the SOM with an appointment of clinician-educator.

- Medical students have their first clinical experiences in the first year of the clinical experience in the IMP I course.

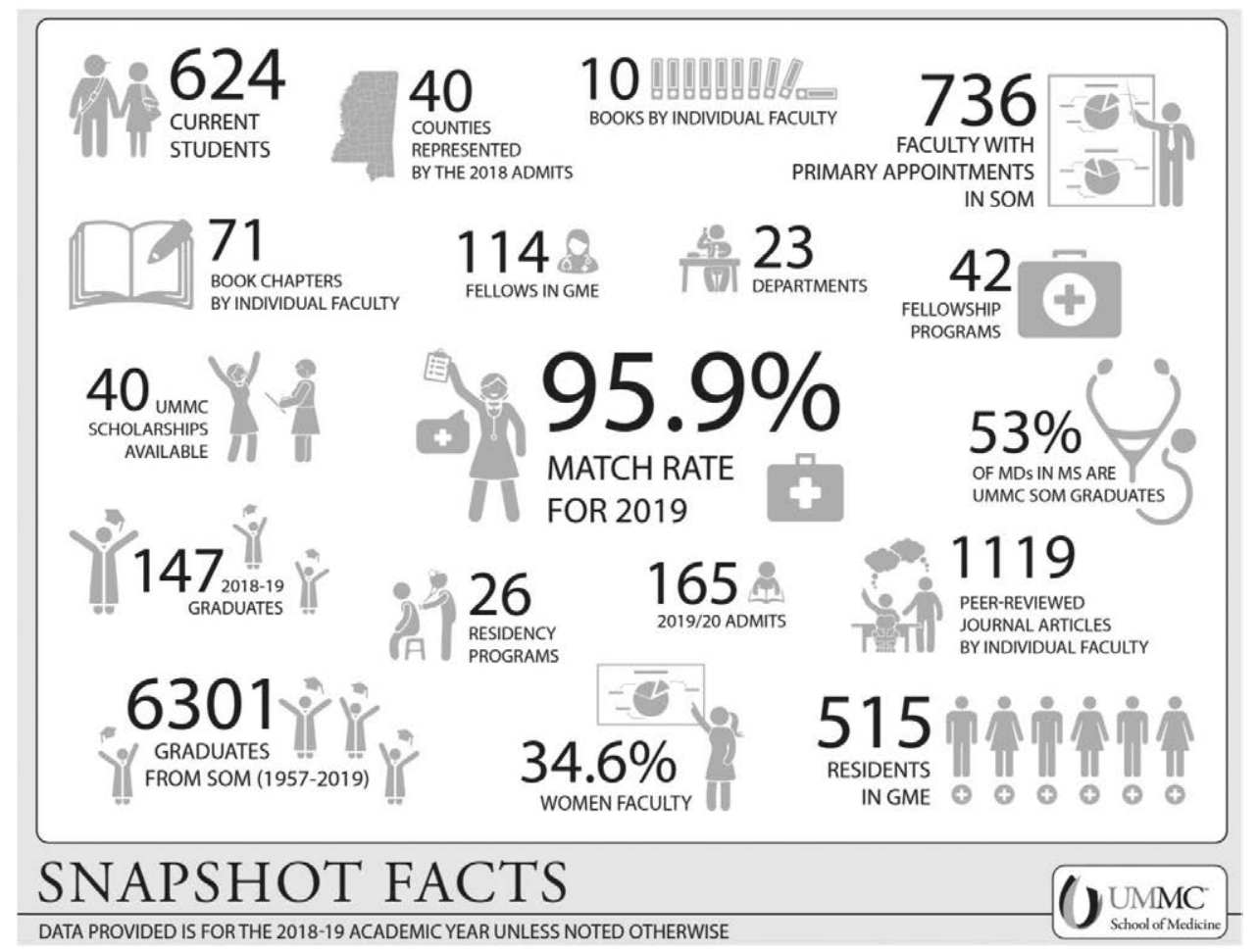

Figure 1 Snapshot facts. 


\section{Curricular Governance}

- The responsibility for curriculum management, design, implementation, and assessment resides with the Curriculum Committee.

- The Curriculum Committee is composed of faculty, school administrators, and students and has 7 subcommittees along with a Steering Committee. The Steering Committee is chaired by the assistant dean for curriculum.

- The Office of Medical Education was established in 2015 and supports the work of the Curriculum

Committee.

\section{Education Staff}

- The growth of the class size and refinements of the curriculum required the addition of several new positions to the administration of the SOM. Those positions include:

- Assistant dean for academic affairs

- Assistant dean for admissions

- Assistant dean for curriculum

- Education quality improvement director

- Director of outreach

- Instructional designer

See Figure 1-Snapshot facts. 


\section{Saint Louis University School of Medicine}

Debra Schindler, PhD, Lynda Morrison, PhD, Linda Gwinn, Neal Weber, MS, James Duffy, SJ, MD, and Chad Miller, MD

\section{Medical Education Program Highlights}

Saint Louis University was founded in 1818 by members of the Society of Jesus (the Jesuits). Founded in 1836, the Saint Louis University School of Medicine (SLU SOM) has, at the heart of its educational mission, the charge to graduate ethically sensitive and clinically outstanding physicians rooted in the Jesuit spiritual attitude of cura personalis (care for the entire person), particularly for those patients on the margins of society.

- SLU SOM has a health care ethics curriculum threaded through the first 3 years of medical school that unites/ integrates the clinical and ethical reasoning of SLU SOM students.

- SLU SOM students gain clinical experience at numerous student-run/student-managed underserved clinics in the St. Louis area.

- SLU SOM students are also extremely active in serving the St. Louis community in nonmedical ways. Service and service learning are integrated into both identity and wellness program initiatives.

\section{Curriculum}

\section{Curriculum description}

See Supplemental Digital Appendix 1-Curriculum Schematicat http://links.lww.com/ACADMED/A880.

\section{Curriculum changes since 2010}

- Recorded all lectures via a lecture recording system

- Moved all course and clerkship syllabi to an electronic platform

- Offered an electronic version of all suggested textbooks

- Moved the start of clinical clerkships to the beginning of May of year 2 from July of year 3

- Implemented a clinical reasoning curriculum in the preclerkship years that includes multiple case-based OSCEs that assess clinical reasoning

- Instituted a new preclerkship Clinical Interviewing course that emphasizes interpersonal skills and history taking

- Instituted a longitudinal course on quality improvement and patient safety that requires all students to complete the Institute for Healthcare Improvement Open School

Acad Med. 2020;95:S273-S276.

doi: 10.1097/ACM.0000000000003366

Copyright (C) 2020 by the Association of American Medical Colleges

Supplemental digital content for this article is available at http://links.Iww.com/ ACADMED/A880.

Year school was established: 1836.

School URL: https://www.slu.edu/medicine/index.php.
- Instituted a longitudinal course on interprofessional education with our nursing and allied health schools

- Expanded the health care ethics curriculum throughout the first 3 years

- Established a 4-week emergency medicine clerkship in year 4

- Introduced a curricular thread on fundamentals of research and research methods

- Expanded the use of simulation throughout the curriculum.

- Moved documentation of required clinical experiences from paper to an electronic platform

- Moved mid-block clerkship feedback from paper to an electronic platform for documentation and tracking

- Implemented required self-assessment as a component of the mid-block clerkship feedback

There are 6 major curriculum changes planned for the next 5 years:

- Beginning with the 2020 academic year, SLU SOM will initiate a process to achieve earlier entry into clinical clerkships after only 18 months of preclerkship curriculum.

- The SLU SOM curriculum will directly address the critical transition points in medical school: entry into a professional school, transition to the clerkship years, and preparation for residency.

- SLU SOM will evolve from a lecture-based preclerkship curriculum to an active learning-based curriculum rooted in instructional methods such as team-based learning, peer instruction, and small-group active learning.

- The preclerkship curriculum will be reorganized to align physiology, embryology, histology, and anatomy in organbased systems so that students receive all normal structure and function before moving onto abnormal structure and function.

- The assessment of clinical performance by students during the clerkship years will be reformed so that students are assessed on the same clinical skills in both the clinical environment and high-fidelity simulation environments. These assessments will align with the AAMC's Core Entrustable Professional Activities (EPAs) for Entering Residency.

- Content threads or courses in the following areas will be added or expanded: health care economics, systems science, leadership, and business ethics.

\section{Assessment}

See Supplemental Digital Appendix 2-Program Objectives and Assessment Methods—at http://links.lww.com/ACADMED/A880.

\section{Medical education program objectives}

Our current medical education program objectives were modeled after the ACGME competencies, Physician Competency Reference Set, and the AAMC Core EPAs for Entering Residency. 


\section{Assessment changes since 2010}

- Required at least one customized NBME exam in each preclerkship course

- Instituted a required minimum passing score for all clerkship NBME subject exams

- Increased the minimum passing score in the preclerkship curriculum to $70 \%$ for all courses

- Implemented 5 separate practice USMLE Step 1-type exams within the preclerkship curriculum

- Implemented a single practice USMLE Step 2 Clinical Knowledge-type exam after the clerkships

- Implemented a practice USMLE Step 2 Clinical Skills-type exam after clerkships

- Implemented the use of formative quizzes from a predetermined question bank throughout the preclerkship curriculum in preparation for summative examinations

- Significantly increased the number of OSCEs in the preclerkship curriculum to assess clinical skills

- Added assessment of clinical reasoning skills in the preclerkship curriculum

- Increased the number of OSCEs in the clerkship curriculum

- Significantly increased the amount of narrative assessment directly from clinicians in the preclerkship years, especially around the assessment of clinical skills

\section{Pedagogy}

Currently, the curriculum relies heavily on lecture and traditional clinical experiences and moderately on simulation and standardized/simulated patients, small-group discussion, and a small number of laboratory activities and videos/podcasts.

Approaches used to achieve program objectives:

- Increased use of videos

- Increased use of simulation and standardized/simulated patients

\section{Clinical experience first encounter}

Students first encounter clinical experiences in the fall of year 1.

\section{Required and elective community-based rotations}

- The family and community medicine clerkship uses community-based resources to provide the appropriate educational experience.
- The internal medicine clerkship and the surgery clerkship use the Veterans Administration Medical Center-John Cochran Division for components of the clerkships.

- Finally, the internal medicine clerkship uses a community hospital for 2-4 students per clerkship rotation.

Challenges in designing and implementing clinical experiences for medical students

- Increasing clinical clerkship capacity, particularly at outpatient sites

- Increasing available faculty time, faculty development, and faculty commitment to assessment and feedback

- Managing tensions between clinical productivity demands for faculty and fulfilling educational requirements

- Managing the increase in need for affiliation agreements for outpatient sites and the increasing legal intervention restricting student-patient interaction to shadowing experiences

\section{Curricular Governance}

See Figure 1-Curricular governance committees.

\section{Decentralized curricular governance}

Student clinical performance assessments and clinical assignments for clerkships and clinical electives are partially decentralized and overseen by the departments. Grade composition has become more centralized regarding standards over the past several years, but it remains department dependent.

\section{Education Staff}

\section{Medical education leadership}

The administrative and academic support for the above is the responsibility of the Office of Curricular Affairs (OCA). The office is led by Chad Miller, MD, the senior associate dean of undergraduate medical education. Reporting to him are the following:

- Associate dean for clinical curriculum (James Duffy, SJ, MD)

- Assistant dean for preclerkship curriculum (Lynda Morrison, $\mathrm{PhD}$ )

- Assistant dean for program evaluation and assessment (Debra Schindler, $\mathrm{PhD}$ )

Dean, school of medicine

\section{Curriculum Committee}

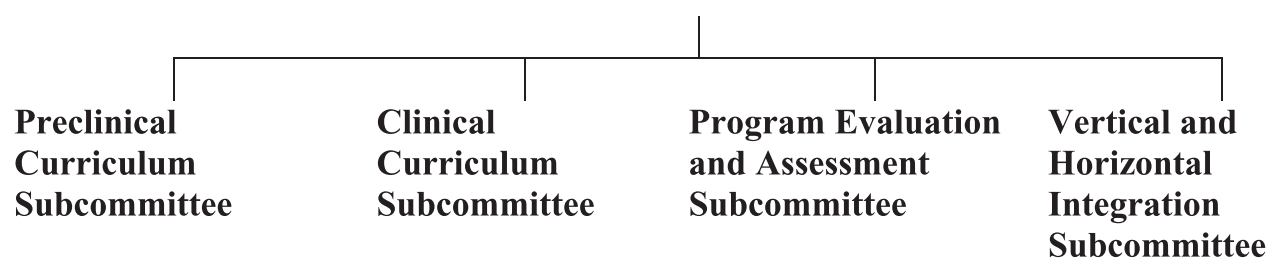

Figure 1 Curricular governance committees. 
- Assistant dean for simulation (Tina Chen, MD)

- Medical director of the clinical skills center (Doug Walden, MD)

- Director of instructional design and assessment (Neal Weber)

- Director of curriculum management (Linda Gwinn)

See Figure 2-Decanal organizational chart.

Office staff includes an office manager; director of the clinical skills center; registrar/coordinator; academic records coordinator; IT data analyst; facilities coordinator; simulation education specialist; and program coordinators for the first-, second-, third-, and fourth-year educational programs.

\section{Department of Medical Education}

The OCA, including all medical education staff and administrative faculty within, is responsible for all aspects of the day-to-day management of the 4-year medical education program. The OCA is not responsible for other areas of the continuum or areas outside of educational programming.

\section{Faculty Development and Support in Education}

\section{Professional development for faculty as educators}

The Office of Faculty Affairs and Professional Development sponsors professional development programs each year. For academic year 2019-2020, the topics included Writing Letters of Recommendation; Faculty Development: Exploring Diversity in Academic Medicine; Examining Our Perspectives on Wellness and Resilience: It's Not Just Yoga, I Promise; and Is It Feedback or Mistreatment? Establishing Expectations and Providing Feedback in Today's Learning Environment. The OCA sponsored the Stanford Faculty Development Program in Clinical Teaching for clinical faculty. Staff from the OCA and the Office of Student Affairs provided workshops for faculty on team-based learning and peer instruction, 2 of our new instructional methods. Finally, the Office of Graduate Medical Education sponsors 2 evening workshops on educational topics each year.

\section{Role of teaching in promotion and tenure}

Faculty on the clinical educator track are expected to submit an academic portfolio for promotion, which includes details regarding scholarly activity, including teaching quantity and quality.

Members of the Academy of Medical Educators are distinguished medical school faculty who are recognized for their sustained teaching excellence and who will provide professional development and mentorship for faculty seeking to enhance their teaching skills. Candidates for the academy must be medical school faculty.

The goals and mission of the Academy of Medical Educators at Saint Louis University include:

- Recognizing medical educators for their teaching activities, their educational scholarship and innovation, and their leadership

- Fostering an environment that promotes the continuous improvement of teaching

- Encouraging educators to link teaching and learning to improved patient outcomes and health care systems performance

- Rewarding educators for their efforts through promotion and public recognition

\section{Initiatives in Progress}

Major initiatives include the following:

- Decreasing the preclerkship curriculum to 18 months

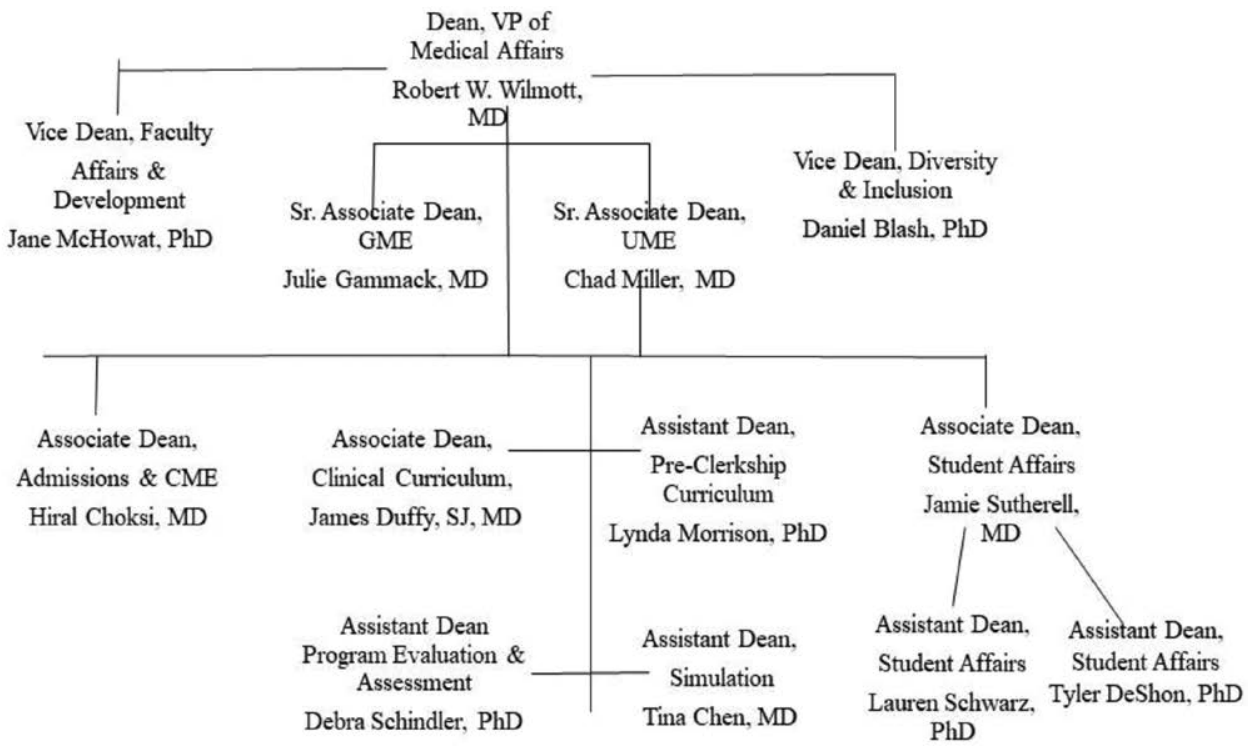

Figure 2 Decanal organizational chart. 
- Transforming from a lecture-based to an active learning and independent study curriculum

- Increasing curricular and professional development during the major medical school transition points: entry into medical school, entry into clerkships, and entry into residency

- Reforming the assessment of clinical performance in the clerkships and alignment with the EPAs
- Increasing the use of technology, including artificial intelligence, for the development and assessment of clinical skills

- Continuing to reform and expand the evaluation and feedback of clinical skills

- Adding a health economics and business curriculum

- Adding a business ethics curriculum

- Expanding the systems science curriculum 


\section{University of Missouri-Columbia School of Medicine}

Kevin Y. Kane, MD, MSPH, Michael C. Hosokawa, EdD, Kathleen J. Quinn, PhD, and Laine Young-Walker, MD

\section{Medical Education Program Highlights}

\section{Patient-based learning curriculum}

The University of Missouri (MU) School of Medicine implemented a hybrid problem-based learning (PBL) curriculum in 1993, which we renamed "patient-based learning." Almost 30 years later, our school is noted for USMLE Step 1 and 2 examination scores consistently above the national means, 96\%$99 \%$ residency match rates, and positive responses to a survey of program directors who supervise our recent graduates (interns).

In 2019, a study summarized variables associated with USMLE scores and identified institutions' curricula that deviated from trend lines by producing higher USMLE scores despite having lower entrance grade point averages and MCAT scores. ${ }^{1}$ Several medical schools outperform or underperform trend line expectations for USMLE, irrespective of entering student qualifications. The University of Missouri School of Medicine was found to significantly outperform in both Step 1 and 2, which may be explained by curriculum and administrative differences. We acknowledge the core faculty dedicated to our PBL model and the many students who choose MU, in part, because of the curriculum.

\section{Integrated simulation activities}

- Simulation activities throughout the 4-year curriculum

- Integrated simulated participant cases and the technological delivery of patient findings added a more realistic dimension to PBL cases

- Established a robust Step 2 Clinical Skills exam preparation and remediation course

- Implemented a fourth-year simulation elective prep-forresidency skills course

\section{The MU Rural Track Pipeline Program}

- Has academic-community partnerships with 9 health systems throughout Missouri.

- Provides students with sequential, ongoing exposure to rural medicine over the course of 4 years. Programs include the Lester R. Bryant Scholars Pre-Admissions Program, the Rural Scholars Program, the Rural Track Summer Community Program, the Rural Track Clerkship Program, and the Rural Track Elective Program. ${ }^{2,3}$

Acad Med. 2020;95:S277-S281.

doi: 10.1097/ACM.0000000000003370

Copyright (C) 2020 by the Association of American Medical Colleges

Supplemental digital content for this article is available at http://links.Iww.com/ ACADMED/A889.

Year school was established: 1872

School URL: https://medicine.missouri.edu.

\section{Diversity and inclusion initiatives}

- Expanded pipeline programs to include the "Called to Academic and Leadership Excellence and Building Character and Confidence" science club, which allows us to attract students as young as fifth grade

- Added inclusion symposiums to applicants' interview day, which improved view of diversity and inclusion

- Added diversity and inclusion primer to prematriculation Med Prep 3 program

- Increased numbers of underrepresented minority matriculants from $4 \%$ in 2016 to $17 \%$ in 2019

- Work with students in implementing diversity and inclusion programs throughout the school year (Diversity Dialogues) with discussions on microaggressions, implicit bias, and inclusion

- Support and encourage faculty and staff to participate in a daylong diversity and inclusion training, Building Inclusive Clinics and Classrooms

\section{Curriculum}

\section{Curriculum description}

- Years 1 and 2 focus on basic science education and early clinical experiences; year 3 focuses on core clerkship experiences; and year 4 offers specialty-specific general electives, advanced clinical selectives (e.g., subinternship), and advanced basic science experiences (e.g., facilitating, tutoring, writing, and research).

- Years 1 and 2 are structured in 10-week blocks ( 8 weeks of content, 1 week of examinations, and 1 week off). Each week includes approximately 10 hours of lecture, 10-12 hours of small-group activities, and time for independent learning. Early outpatient clinical experiences start in the first year. The clerkship phase consists of 7 core clerkships. The elective phase consists of 30 weeks of elective courses. The Contemplating Medicine, Patients, Self, and Society (COMPASS) course runs longitudinally across all 4 years.

See Supplemental Digital Appendix 1-Curriculum Map-at http://links.lww.com/ACADMED/A889.

\section{Curriculum changes since 2010}

- The Next Level of Excellence initiative identified 3 gaps in medical students' educational experience: culturally effective care, population and public health, and professional identity formation. We implemented several strategies to address these gaps. Our key characteristics and overall education goals were updated to include these content areas.

- We implemented COMPASS, a 4-year longitudinal course to enhance students' learning about topics related to professional formation such as transitions, physician roles and vision, stress 
and coping strategies, the impact of culture on health care delivery, and professional/personal choices and boundaries. Learning strategies include group discussion, reflective writing, storytelling, reading, and case problem solving.

- We formed the Incorporating Wellness into the Curriculum Committee, comprising medical students, staff, faculty, and associate deans, to integrate strategies that reduce student burnout and increase resilience.

- The neurology clerkship increased from 2 weeks to 4 weeks in length.

- The Springfield Clinical Campus (SCC) opened in 2016 as part of our class expansion to educate third- and fourth-year medical students.

- We added more active learning strategies including the flipped classroom.

- Block 8 content (at the end of year 2) was shortened to 5 weeks, and more independent study time for USMLE Step 1 exam preparation was added during the block.

\section{Class size changes since 2010}

- We increased our class size to 128 students in AY 2017-2018 and constructed a new medical school building (the PatientCentered Care Learning Center), which includes a high-tech, flat-style classroom, a high-fidelity simulation center, $32 \mathrm{PBL}$ small-group meeting rooms, and an anatomy lab for student learning.

- Up to 64 third- and fourth-year medical students per year complete their clerkship and elective phases at the SCC.

\section{Assessment}

See Supplemental Digital Appendix 2-Educational Goal Statements and Assessment Methods—at http://links.lww.com/ ACADMED/A889.

\section{Assessment changes since 2010}

- Implemented a new computer-based testing platform for exams during the preclerkship phase

- Changed the clinical reasoning exam (CRE) format to reflect a developmental approach of assessing clinical reasoning; changed the CRE grading criteria to satisfactory, marginal, and unsatisfactory and made it a closed resource exam

- Developed a centralized system to track students' completion of required direct observations of the history and physical exams during their clerkship

- Updated the monitoring process to ensure students receive midblock feedback during every clerkship

- Changed our end-of-clerkship faculty evaluation of students' clinical performance from a 9-point scale to a 3-point scale (exemplary, meets expectations, and needs improvement)

- Added a COMPASS capstone assignment during the fourth year to assess students' ability to apply the knowledge and skills learned in COMPASS to a self-described health care encounter where their values were challenged

\section{Pedagogy}

See Table 1—Pedagogical Approaches.

\section{Changes in pedagogy since 2010}

- Implemented the longitudinal, blended, small-group learning experience (COMPASS course), which includes students from each of the 4 classes and 2 faculty guides to address topics related to professional formation

- Incorporated more active learning sessions in lieu of live lectures

- Added a service learning component to IPC in blocks 3 and 4

- Reorganized anatomy content to align with PBL and IPC block content with more focus on the clinical correlation and application

\section{Clinical experiences}

- The main campus primarily uses the University of Missouri Health Care facilities and the Harry S. Truman Memorial Veterans Hospital for outpatient and inpatient clinical training.

- The Springfield regional campus uses CoxHealth System and Mercy Health System facilities for clinical training. Students may also rotate at the local federally qualified health center and the Federal Bureau of Prisons facility.

- The MU Rural Track Pipeline Program has 9 academiccommunity partnerships with hospitals in Missouri, where approximately $25 \%$ of students complete up to 3.5 core clerkships in a rural community.

- The family medicine clerkship uses several community-based practices across Missouri.

\section{Required longitudinal experiences}

The MU AHEC Rural Track Pipeline Program received a grant to develop a longitudinal integrated clerkship at a rural track training site set to begin in June 2021.

\section{Clinical experience first encounter}

- Students are assigned to work with 1 primary care and 1 specialty care preceptor as part of the ACE experience during their first year (required).

- Students may participate in additional clinical experiences during the summer after their first year and during their second year.

\section{Required and elective community-based rotations}

Students have opportunities to train in a variety of communitybased clinical settings, including:

- VA hospital

- Rural and primary care community-based clinics throughout Missouri

- Federal Bureau of Prisons

- Federally qualified health centers

- Psychiatry community-based clinics

Challenges in designing and implementing clinical experiences for medical students

- Recruiting and adequately training community-based faculty to maintain our clinical rotation capacity 
Table 1

Pedagogical Approaches

\begin{tabular}{|c|c|}
\hline Pedagogical approaches & Description of approach \\
\hline Case-based learning & Students work through $64 \mathrm{PBL}$ cases during their preclerkship phase. \\
\hline Clinical experience (ambulatory) & $\begin{array}{l}\text { Students are assigned to work with } 1 \text { primary care and } 1 \text { specialty care preceptor as part of the ACE experience } \\
\text { during their first year (required) and second year (voluntary). Robust clinical learning experiences occur during the } \\
\text { clerkship and elective phases of the curriculum. }\end{array}$ \\
\hline Clinical experience (inpatient) & $\begin{array}{l}\text { Students complete multiple H\&P exams and hospital notes as part of the Advanced Physical Diagnosis (APD) } \\
\text { course during their second year. Robust inpatient clinical learning experiences occur during the clerkship and } \\
\text { elective phases of the curriculum. }\end{array}$ \\
\hline Discussion: large group (> 12) & $\begin{array}{l}\text { Students address topics related to professional formation during COMPASS sessions. Students participate in } \\
\text { interprofessional activities with pharmacy and nursing students. Mix of lecture and flipped classroom learning } \\
\text { experiences. }\end{array}$ \\
\hline Discussion: small group $(\leq 12)$ & PBL and IPC small-group sessions consist of 8 students plus a facilitator. \\
\hline Laboratory & Anatomy lab dissections. \\
\hline Lecture & $\begin{array}{l}\text { We strive to limit lectures during the preclerkship phase to about } 10 \text { hours per week. Students on clerkships and } \\
\text { electives may be required to attend lectures and departmental grand rounds. }\end{array}$ \\
\hline Peer teaching & $\begin{array}{l}\text { Students research and answer learning objectives identified during } \mathrm{PBL} \text { sessions and teach each other what they } \\
\text { learned through mini-presentations. }\end{array}$ \\
\hline Preceptorship & $\begin{array}{l}\text { Students work with faculty at the academic and community-based outpatient and inpatient teaching sites during } \\
\text { ACE, APD, and clerkships. }\end{array}$ \\
\hline Problem-based learning & Renamed "patient-based learning," this is the core of our preclerkship curriculum. \\
\hline Role play/dramatization & $\begin{array}{l}\text { Students role-play to reinforce topics such as use of plain language; teach back; and motivational interviewing } \\
\text { during IPC, interprofessional, and family medicine clerkship experiences. }\end{array}$ \\
\hline Self-directed learning/tutorial & $\begin{array}{l}\text { A large part of our preclerkship curriculum is dedicated to self-directed learning where students research learning } \\
\text { objectives and prepare presentations. }\end{array}$ \\
\hline Simulation & $\begin{array}{l}\text { Students apply knowledge, skills, and behaviors learned during simulated experiences during all } 4 \text { years. We } \\
\text { implemented a skills prep-for-residency elective. }\end{array}$ \\
\hline Standardized/simulated patients & $\begin{array}{l}\text { Students take a history and physical exam to collect the information for the PBL case being discussed that week, } \\
\text { practice male urogenital and female gynecological exams during the second year, and participate in multiple } \\
\text { other sessions for formative feedback and summative assessment. }\end{array}$ \\
\hline Video/podcast & Lectures are recorded and posted immediately afterward for students to access. \\
\hline Other: service learning & $\begin{array}{l}\text { Students complete } 20 \text { hours of service learning with a community organization during their first year for } \\
\text { experiential learning about social determinants of health, health disparities, and population health. The Rural } \\
\text { Track Program offers a } 6 \text {-month service learning Community Integration Program. }{ }^{4}\end{array}$ \\
\hline
\end{tabular}

- Shrinking training opportunities due to significant increases in placement requests for health professions students

- Physician burnout attributed to higher productivity expectations by their health system

\section{Curricular Governance}

- The Curriculum Board is charged with primary policymaking authority for medical student academic programs. Subcommittees of the Curriculum Board include the Preclerkship Steering Committee, which oversees the years 1-2 curriculum, and the Clinical Curriculum Steering Committee, which oversees the years 3-4 curriculum.

- The Curriculum Board membership includes 15 elected members (10 voting faculty and 5 nonvoting students) with representation from both the main campus and the SCC. The Curriculum Board meets monthly and the dean and associate deans attend as ex officio members.

\section{Decentralized curricular governance}

- The simulation center is administratively under the Medical Education Office and the director reports to the senior associate dean. The possibility of the simulation center becoming an independent self-sustaining resource is under study.

- The SCC budget has been separate during its first 5 years of operation, but folding this budget into the overall school of medicine budget for education is being contemplated.

\section{Education Staff}

- The Medical Education Office provides support and resources to the Curriculum Board and its subcommittees to fulfill their charge of curriculum oversight, as well as to faculty in their teaching roles.

- We have a team of dedicated and talented student program coordinators and instructional design specialists with expertise to support our mission: "to educate physicians to provide 


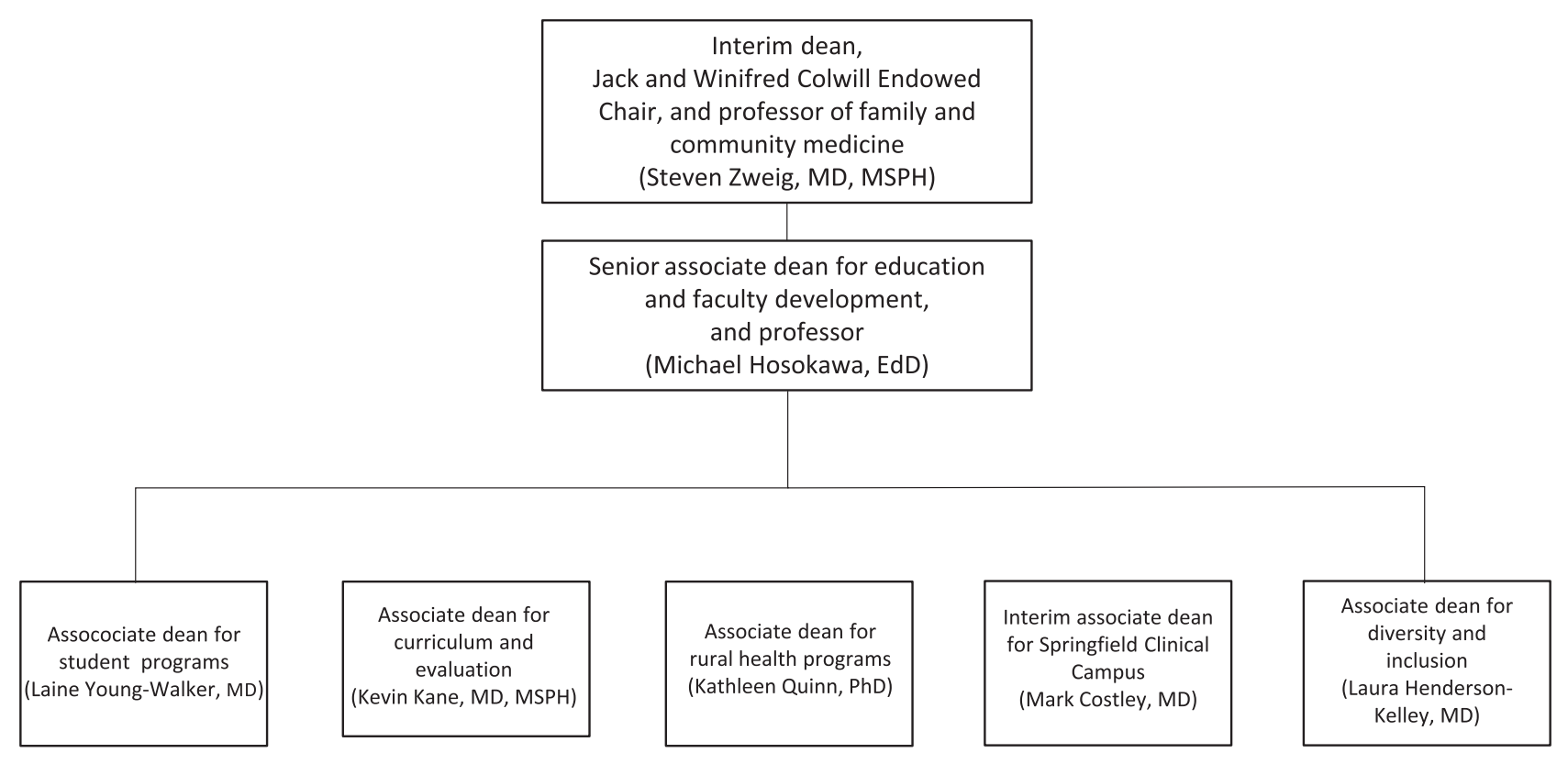

Figure 1 Organizational chart.

effective patient-centered care for the people of Missouri and beyond."

- We have a business technology analyst and a team of programmers who provide support and maintenance of data systems used to support curriculum delivery, monitoring, and management.

- The Medical Education Office provides support to faculty and students for all aspects of medical education, including pipeline programs, preadmission programs, medical school admissions, basic science and clinical curriculum, faculty development, student assessment, rural health experiences, student support, advising, residency match assistance, and graduation.

See Figure 1-Organizational chart.

\section{Faculty Development and Support in Education}

\section{Professional development for faculty as educators}

We support professional development of our faculty educators in several ways:

- Annual Education Day includes guest speakers on medical education topics, a teaching awards ceremony, and poster presentations highlighting curricular innovations.

- Local activities and workshops are hosted by faculty leaders and the Medical Education Office.

- Support is provided to participate in national conferences and faculty development programs.

- A mentoring program has been established.

- We are updating our faculty development resource center, including online modules and articles highlighting our curriculum and best teaching practices, which will be available to all onsite and offsite teaching faculty.

\section{Role of teaching in promotion and tenure}

Faculty can demonstrate excellence in teaching criteria for promotion by presenting evidence of exemplary teaching evaluations by learners, teaching awards, and scholarship such as presentations and publications.

\section{Regional Medical Campuses}

See Table 2-Clinical Regional Campus.

\section{Educational experiences across sites}

- Clerkship leadership and medical school administration track and monitor required patient experiences through our centralized patient log system.

- SCC and main campus clerkship faculty directors and administrative leadership participate in an annual retreat to review annual reports and data and to discuss innovations and challenges.

- The same course learning objectives and assessment methods are used across all learning sites.

- We compare grade distribution and student ratings on end-ofblock evaluations across all learning sites.

- Clerkship directors orient all new faculty to learning objectives and evaluation tools.

\section{Table 2}

\section{Clinical Regional Campus}

\begin{tabular}{lll}
$\begin{array}{l}\text { Regional } \\
\text { campus name }\end{array}$ & Type & Enrollment number \\
$\begin{array}{l}\text { Springfield Clinical } \\
\text { Campus (SCC) }\end{array}$ & Clinical & $\begin{array}{l}\text { Up to } 64 \text { third- and fourth-year } \\
\text { students per year }\end{array}$ \\
\hline
\end{tabular}




\section{References}

1 Ghaffari-Rafi A, Lee RE, Fang R, Miles JD. Multivariable analysis of factors associated with USMLE scores across U.S. medical schools. BMC Med Educ. 2019;19:154.

2 Quinn KJ, Kane KY, Stevermer JJ, et al. Influencing residency choice and practice location through a longitudinal rural pipeline program. Acad Med. 2011;86:1397-1406.

3 Kane KY, Quinn KJ, Stevermer JJ, et al. Summer in the country: Changes in medical students' perceptions following an innovative rural community experience. Acad Med. 2013;88: $1157-1163$.

4 Porter J, Quinn K, Kane K, Stevermer J, Webb W. How we incorporated service learning into a medical student rural clinical training experience. Med Teach. 2016;38:353-357. 


\title{
University of Missouri-Kansas City School of Medicine
}

\author{
Paul G. Cuddy, PharmD, MBA, Jennifer Quaintance, PhD, Nurry Pirani, MD, and Mary Anne Jackson, MD
}

\section{Medical Education Program Highlights}

- The University of Missouri-Kansas City (UMKC) School of Medicine is an accelerated 6-year, combined baccalaureate/MD program, in which students begin integrating undergraduate and medical school coursework with clinical experiences in their first semester. This integration continues through the entire curriculum, with the proportion of coursework and clinical work adjusting across 6 years.

- The docent system is a long-standing hallmark of UMKC medical student education. The docent system comprises a set of longitudinal learning communities consisting of small, multilevel student groups led by an internal medicine physician docent. We embed junior-senior student partnerships in the docent team experience, which provide each student with the opportunity and responsibility to learn from a senior student and to subsequently teach a junior student as the curriculum progresses.

- Since the school's inception, the curriculum has integrated the arts and humanities. During the final 2 years of the curriculum, students are required to select a 4-week arts and humanities course.

\section{Curriculum}

\section{Curriculum description}

- The first 2 years of the 6-year curriculum reflect the emphasis on the baccalaureate degree while providing early clinical exposure.

- The final 2 years of the 6-year curriculum reflect the emphasis on required and elective medical degree coursework while also providing a required arts and medical humanities experience.

See Supplemental Digital Appendix 1-Curriculum Schematicat http://links.lww.com/ACADMED/A974.

\section{Curriculum changes since 2010}

- Greater emphasis has been placed on using standardized patients and simulation in the teaching and assessment of clinical and communication skills.

- An intentional transition from lecture-based to more activelearning teaching modalities including case-based, team-based, and online learning is underway.

- Expanded interprofessional experiences have been introduced into the curriculum longitudinally.

Acad Med. 2020;95:S282-S284.

doi: 10.1097/ACM.0000000000003492

Copyright (C) 2020 by the Association of American Medical Colleges

Supplemental digital content for this article is available at http://links.Iww.com/ ACADMED/A974.

Year school was established: 1971.

School URL: https://med.umkc.edu.

\section{Assessment}

Medical education program objectives are described in outcomebased terms that allow the assessment of medical students' progress in developing the competencies that the profession and the public expect of a physician.

See Supplemental Digital Appendix 2-Program Objectives and Assessment Methods-at http://links.lww.com/ACADMED/A974.

The school's Council on Curriculum adopted a new set of medical education program objectives largely based on the Physician Competency Reference Set used by the AAMC's Curriculum Inventory Report, which in turn is based on the ACGME's competency framework.

\section{Assessment changes since 2010}

- Students sit for a longitudinal set of OSCEs intended to facilitate their development of history-taking, physical exam, clinical decision-making, communication, and professionalism skills.

- Key courses in the preclerkship phase of the curriculum have implemented a remediation process for students who do not meet performance targets.

- The Council on Curriculum requires students who have had academic difficulty or struggle with standardized exams to take and pass, with a minimum score, the Comprehensive Clinical Sciences Examination before sitting for the USMLE Step 2 CK examination.

\section{Pedagogy}

- Clinical experience in the outpatient and inpatient setting has been a signature of the program since its introduction and continues throughout the 6 years.

- Small-group discussion is used extensively in the docent learning communities that span the 6-year program.

- Simulation and standardized patients are used in the final 3 years of the program.

\section{Changes in pedagogy since 2010}

- There has been a substantial increase in the use of standardized patients and simulation-based training and assessment of students.

- Standardized computer-based examinations are now uniformly undertaken in a dedicated onsite testing laboratory.

- Lecture-based instruction has been reduced and replaced with more active-learning focused instruction.

\section{Clinical experiences}

- There is a notable breadth of clinical exposure available to students during their rotations at our hospital affiliates that provides a rich array of patients of all ages, from all socioeconomic groups, and from underserved populations and cultures. 
- These hospital affiliates also provide our students with abundant acute and ambulatory care experiences during all required core clerkships.

\section{Required longitudinal experiences}

- A continuing care ambulatory experience is required of every learner and consists of weekly half-day exposure to a panel of patients throughout the final 4 years.

- An 8-week inpatient internal medicine experience is required for every student in each of the final 3 years. Like the ambulatory care experience, each student experiences this rotation as a member of a docent team that includes 12 students who are supervised by the same physician.

- Longitudinal interprofessional experiences are required for all students across the curriculum.

\section{Clinical experience first encounter}

- Exposure to clinical medicine occurs in the first 2 years of the 6-year curriculum and occurs for 1 half day per week.

- Concurrent with this clinical exposure, students participate in sequential coursework designed to teach communication skills, professionalism, and clinical problem solving and to build, early on, a full understanding of the language and culture of medicine.

\section{Required and elective community-based rotations}

- All students spend 4 weeks interacting with a board-certified family practitioner in a rural setting.

- The intent of this preceptorship is to provide students with an adequate exposure to a rural family medicine practice in a health professional shortage area, provide an introduction to medical practice management, experience the cultural diversity of a rural community, and acquire awareness of the need for primary care physicians in Missouri.

\section{Challenges in designing and implementing clinical experiences} for medical students

- The hospital affiliate institutions that provide required clinical training have experienced increasing requests for student access from osteopathic and offshore medical schools, as well as nurse practitioner and physician assistant programs.

- There is increasing pressure to provide financial incentives to accept students participating in the family medicine preceptorship.

\section{Curricular Governance}

- A curriculum committee consisting of appointed and elected faculty is responsible for the design, implementation, and evaluation of the MD curriculum.

- An associate dean chairs the curriculum committee and reports to the dean.

- Two standing curriculum subcommittees, one led by the vicechair for basic science and the other led by the vice-chair for clerkships, meet monthly and include all basic science course directors and all clerkship directors.

- A steering committee, chaired by the associate dean for curriculum, functions as an executive working committee and includes the 2 vice chairs, an assistant dean for curriculum, and the assistant dean for assessment and quality improvement.

\section{Decentralized curricular governance}

- Purchased teaching-time contracts between the school and the hospital affiliates identify faculty who are course or clerkship directors for required medical education experiences as well as faculty who provide administrative support.

- These contracts specify the amount of financial support provided from the school. Academic department chairs must allocate protected time for these faculty to satisfy their contractual obligations.

\section{Education Staff}

- The Department of Medical Education Support Services is directed by the assistant dean for assessment and quality improvement and staffed by an instructional design specialist, accreditation specialist, database analyst, and administrative assistant.

- The department oversees the medical education program's continuous quality improvement processes; the Clinical Training Facility, housing simulation-based education and standardized patients; and a medical education media center, which has a collection of online and nonprint learning resources.

- The department collects, analyzes, and distributes program evaluation data; monitors compliance with policy and accreditation standards; manages the online student performance and course evaluation system and the curriculum database; offers ongoing education and assessment consultations; and conducts and consults on educational research.

\section{Medical education leadership}

- Faculty holding assistant or associate dean titles provide support at the UGME and GME levels and support faculty employed by major hospital affiliates and/or practice plans.

- Assistant and associate deans supporting student affairs functions report directly to the office of the medical school dean.

See Figure 1-Medical education leadership.

\section{Faculty Development and Support in Education}

- The associate dean for professional development directs faculty development and is supported via a faculty development committee comprising of clinical and basic science faculty.

- A mentorship program is available for early-career, mid-career, and longevity-career faculty.

- Workshops and guidance regarding promotion, mentoring, and career development are provided regularly. Development activities also include workshops/seminars to advance research skills, enhance curricular design, promote active learning, improve instructional methodology and assessment methods, and strengthen team building and leadership skills. 


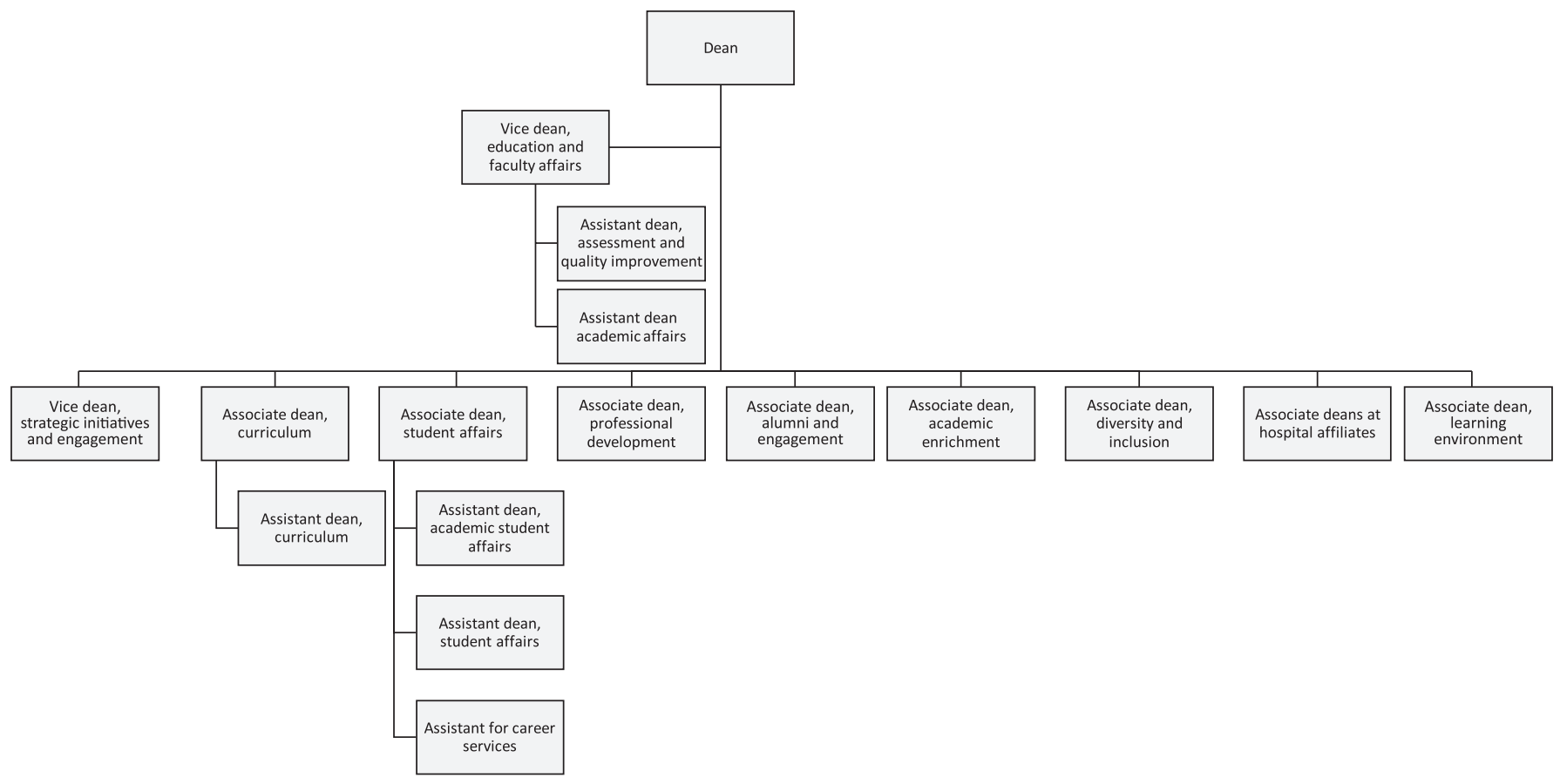

Figure 1 Medical education leadership.

- Funds for faculty travel to present research or for additional professional development opportunities are available.

- A faculty awards review committee annually selects recipients to be recognized for excellence in contributions to innovation and scholarship in medical education; and mentoring for both a senior and junior faculty who have made significant contributions to enhancing and developing the careers of faculty and trainees.

- Teaching awards for medical student preclinical education innovation and a diversity and health equity in medicine award are presented annually.

\section{Role of teaching in promotion and tenure}

- Excellence in teaching, an active commitment to institutional and public service, and acumen of clinical practice are equally weighted for their scholarly merit and importance in the faculty promotion process.

- Promotion guidelines for tenure track and nontenure track faculty reflect the importance that the school places upon teaching based upon the presence of explicit teaching metrics that promotion committees weigh in making academic promotion decisions.

\section{Teaching training}

- The school-based Department of Graduate Health Professions in Medicine offers both a certificate and a master's degree in health professions education.

- Enrollment is open to physicians and physician trainees along with other health professionals, e.g., nurses, pharmacists, physician assistants, etc.

\section{Initiatives in Progress}

- The development of a regional campus is underway.

- The school will launch the Well-Being Index for faculty to encourage them to further their self-care to ensure professional fulfillment throughout the stages of their careers while allowing the school to further monitor the learning environment for improvement opportunities. 


\section{Washington University School of Medicine}

Eve R. Colson, MD, MHPE, Sabrina Nuñez, PhD, Thomas M. De Fer, MD, Steven J. Lawrence, MD, MSc, Leslie Blaylock, MEd, Amanda Emke, MD, MHPE, Mary E. Klingensmith, MD, Diana L. Gray, MD, Cole Schulte, MHA, and Eva Aagaard, MD

\section{Medical Education Program Highlights}

The curriculum at Washington University School of Medicine (WUSM) is in transition. The legacy curriculum is traditional, with an emphasis on inquiry and flexibility. The $\mathrm{MD}-\mathrm{PhD}$ program is one of the largest and most successful in the nation. Over $98 \%$ of students participate in research during medical school. About 50\% spend a year or more in advanced study, often graduating with additional degrees including PhD, MSCI, MPH, MPHS, and MBA. The Office of Medical Student Research and robust grant- and school-based funding support student research. Students have the ability to participate in a large variety of selectives, electives, student groups, and community-engaged experiences.

Planning for curriculum renewal, we committed to these strengths while incorporating best practices in evidence-based medical education. The new curriculum - called the Gateway Curriculum-begins in 2020-2021. It will:

- Be competency based and outcomes oriented

- Enhance horizontal and vertical integration of basic, clinical, social, and health systems sciences

- Ensure early and meaningful clinical exposure

- Incorporate evidence-based active learning methods

- Enhance community engagement and health systems science learning

- Expand opportunities and focused training in academic career development

\section{Curriculum}

See Supplemental Digital Appendix 1-Legacy Curriculum Schematic - at http://links.lww.com/ACADMED/A895.

See Supplemental Digital Appendix 2-Gateway Curriculum Schematic —at http://links.lww.com/ACADMED/A895.

\section{Curriculum changes since 2010}

The Gateway Curriculum intends to inspire learners to create the future of medicine, science, and society by supporting the development of physicians who are leaders in academic medicine including research, education, innovation, and advocacy. The curriculum consists of 3 phases over 4 years.

Acad Med. 2020;95:S285-S290.

doi: 10.1097/ACM.0000000000003385

Copyright (C) 2020 by the Association of American Medical Colleges

Supplemental digital content for this article is available at http://links.Iww.com/ ACADMED/A895.

Year school was established: 1891.

School URL: https://medicine.wustl.edu.
Phase 1 (Gateway to the Foundations) will last 16 months with 7 integrated modules, training students in the foundational basic, social, behavioral, and health systems sciences using a functions (physiological organization) and forms (anatomical organization) framework. Evidence-based active learning and technology will maximize student engagement and learning. Phase 1 will contain three 3-week clinical immersions where students rotate through inpatient, outpatient, and procedural settings. Students will practice clinical skills and develop professional identity while learning about health systems, all framed by patient and interprofessional provider perspectives. Students will also spend 4 weeks exploring academic careers in research, education, innovation, and/or advocacy.

Phase 2 (Gateway to Clinical Medicine) will last 12 months and consist of six 8-week clinical clerkships: internal medicine, surgery, pediatrics, obstetrics-gynecology, neurology, and psychiatry. Each clerkship will begin with foundational science, purposeful reiteration, and expansion of prior and new material (helical learning) taught in a signs and symptoms framework to facilitate knowledge transfer to clinical reasoning. Clerkships will end with 1 week for assessment, reflection, coaching, and communities. Students interested in research/scholarship can delay up to 16 weeks of Phase 2 to pursue this work.

Phase 3 (Gateway to Specialization) will last 20 months with schedules tailored to academic interests and career aspirations. Students will complete a 4 -week internal medicine subinternship and three to four 4 -week advanced clinical rotations. Phase 3 students will complete three 4-week Keystone Integrated Science courses exploring foundational and clinical science in important or emerging areas, including societal implications. A required 4-week capstone course (Gateway to Residency) will occur early in the graduation year focusing on competencies necessary to ensure readiness for internship. The remaining months are elective.

Coaches will support student learning and career development through all 4 years, teaching professional identity formation, reviewing longitudinal performance, and supporting individual student learning goals. Two additional longitudinal elements include students engaging in a Washington University or St. Louis community. Examples of these experiences include student government, research/scholarship, or longitudinal partnership with a St. Louis community organization. Finally, students will have the opportunity to pursue academic interests in research, education, advocacy, or innovation through all phases, including the ability to pursue dual degrees and other yearlong experiences if they desire (e.g., PhD, MSCI, MPH, MPHS, MBA, MA bioengineering, education, health policy fellowship).

\section{Assessment}

See Table 1-Program Objectives and Assessment Methods. 


\section{Table 1}

\section{Program Objectives and Assessment Methods}

\section{Program objective}

Foundational knowledge of practice
Demonstrate knowledge of normal human structure and function at the molecular; genetic; cellular; tissue; organ-system; and whole-body level in growth, development, and health maintenance.

Demonstrate knowledge of the basic mechanisms involved in the pathogenesis of common human diseases and their influence on clinical presentation and therapy.

Demonstrate knowledge of the epidemiology of common and clinically significant diseases.

\section{Assessment method}

MCQ

Short answer

MCQ/Shelf

Short answer

OSCE

MCQ/Shelf

Short answer

OSCE

Mini-CEX

Demonstrate basic knowledge of the impact of ethnicity, culture, socioeconomic status, patient and provider biases, and other social factors on health and disease.

MCQ/Shelf

Short answer

OSCE

Reflections with feedback

Demonstrate basic knowledge of the ethical principles and professional values that underpin the medical profession.

Demonstrate basic knowledge of the common scientific methods used to study health and disease.

Short answer

Reflections with feedback

MCQ

Short answer

EBM rubric

Demonstrate basic knowledge of the methods and principles used for improving quality, safety, and costs of health care delivery for patients and populations

MCQ

Short answer

Reflections with feedback

Patient care $\quad$ Obtain appropriate medical histories that include psychosocial and behavioral factors that influence health.

Direct observation of clinical performance

OSCE

Mini-CEX

Perform accurate physical examinations.

Direct observation of clinical performance

OSCE

Mini-CEX

Discuss the indications, risks, and benefits of common medical procedures: demonstrate proficiency in performing the required procedures of the WUSM graduate.

Direct observation of clinical performance

OSCE/simulation

Mini-CEX

Formulate a prioritized differential diagnosis for the patient's presenting symptoms, discuss expected physical examination findings based on the differential, and identify diagnostic testing required.

MCQ

Chart documentation

Direct observation of clinical performance (rubric/checklist)

OSCE

Mini-CEX

Interpret common physical examination, laboratory, and radiographic studies to inform the differential diagnosis and treatment plan.

MCQ

Short answer/chart documentation

Direct observation of clinical performance (rubric/checklist)

OSCE

Mini-CEX

Develop and carry out with supervision, appropriate, individualized Direct observation of clinical performance

diagnostic and treatment plans for patients across the broad spectrum of acute and chronic conditions.

Assess individual patient risk factors for common clinical conditions.

Direct observation of clinical performance

OSCE

Mini-CEX

Educate patients and families on strategies to reduce risk and promote health.
Direct observation of clinical performance Multisource feedback

OSCE

Mini-CEX 


\section{Table 1}

(Continued)

\section{Program objective}

Interpersonal and communication skills
Demonstrate respectful and effective verbal and nonverbal interpersonal and
communication skills with patients, families, colleagues, and all members of the health care team.

Discuss diagnostic and treatment options in a manner that will facilitate the participation of patients and their families in shared decision-making.

Maintain accurate and thorough medical records.

Maintain a professionally appropriate demeanor.

Professionalism
Demonstrate an awareness of potential conflicts of interest.

Apply legal and ethical principles governing the physician-patient relationship.

Act in the patient's best interest and serve as a patient advocate

Recognize, monitor, and address psychological and physical factors in oneself that may affect professional performance.

Systems-based Work collaborative and effectively in interprofessional teams.

practice

Recognize the roles of various members of the interprofessional health care team and the scope of their practice.

Demonstrate the ability and willingness to adapt to various health care delivery settings (e.g., inpatient, ambulatory, OR, labor and delivery, ED). Recognize barriers to and facilitators of safe, high-quality patient care

Describe individual, team, and system challenges that may contribute to medical errors; demonstrate the ability to identify medical errors when they occur.

Practice-based learning and improvement identify and address personal strengths and weaknesses, to incorporate formative feedback, and to self-assess knowledge and performance to

\section{Assessment method}

Multisource feedback

Direct observation of clinical performance OSCE

Multisource feedback

Direct observation of clinical performance

OSCE

Multisource feedback

Direct observation of clinical performance

Multisource feedback

Direct observation of clinical performance

OSCE

Mini-CEX

Reflection

Multisource feedback

Direct observation of clinical performance

Reflection

Reflection

Multisource feedback

Direct observation of clinical performance

Short answer

Reflection

Multisource feedback

Direct observation of clinical performance Reflection

Reflection

Multisource feedback

Direct observation of clinical performance

OSCE/simulation

Reflection

MCQ

Direct observation of clinical performance

Multisource feedback

OSCE/simulation

Reflection

Reflection

Direct observation of clinical performance

MCQ

Short answer

Direct observation of clinical performance (rubric/checklist)

OSCE/simulation

Reflection

MCQ

Short answer

Reflection

Reflection

Multisource feedback develop a self-improvement plan.

Apply an evidence-based approach to selecting, appraising, and using evidence from scientific studies related to clinical questions and patients' health problems.
MCQ

EBM rubric 
Assessment framework: We created our current program objectives based on the ACGME competencies, adapted through a local Delphi process. The Delphi process (open to all faculty engaged in the current curriculum, program directors from all residency and fellowship programs at Washington University/ Barnes-Jewish Hospital/St. Louis Children's Hospital, and all department chairs or their designees) set measurable, required graduation competencies.

\section{Assessment changes since 2010}

In 2010, we incorporated self assessments and peer assessments and narrative feedback of professional behaviors in the preclerkship courses. Within the Gateway Curriculum, we will implement a variety of assessments. These assessments will appear in dashboards and portfolios for review by learners and coaches.

\section{Parallel curriculum or tracks}

Not applicable.

\section{Pedagogy}

Pedagogical approaches:

- Case-based learning

- Clinical experience: ambulatory

- Clinical experience: inpatient

- Discussion: large group $(>12)$

- Discussion: small group $(\leq 12)$

- Games

- Laboratory

- Lecture

- Mentorship

- Patient presentation

- Peer teaching

- Preceptorship

- Reflection

- Self-directed learning/tutorial

- Service-learning activity

- Simulation

- Standardized/simulated patients

- Team-based learning

- Video/podcast

\section{Changes in pedagogy since 2010}

We emphasize instructional activities requiring students to work in teams, applying concepts, and problem-solving. We did not adopt a single pedagogy, instead relying on a variety of approaches applicable to a given learning activity, supported by technology such as videos, e-learning resources, and audience response systems.

\section{Clinical experiences}

Required clinical experiences occur in inpatient, acute care, and outpatient clinics; operating rooms; and labor and delivery facilities.

\section{Required longitudinal experiences}

None.

\section{Clinical experience first encounter}

Students encounter clinical experiences during the first year as part of the Practice of Medicine curriculum. Beginning in 2020, students will participate in 3 immersion blocks in Phase 1.

\section{Required and elective community-based rotations}

There are no required community-based rotations. Students have multiple options for core rotations and electives including the VA, outpatient clinics, community-based clinics, and hospitals. All students work with patients from underserved backgrounds through federally qualified health centers, Barnes-Jewish Hospital, and St. Louis Children's Hospital.

\section{Challenges in designing and implementing clinical experiences for medical students}

Identifying interested, qualified preceptors is a challenge, although retention has been high. Recently, we created new partnerships with 2 federally qualified health centers in the St. Louis region. We also expanded our engagement with BarnesJewish Hospitals to meet this need.

\section{Curricular Governance}

The curriculum is overseen by the Committee on Medical Student Education (COMSE). COMSE includes representatives from the education leadership team, department chairs, course directors, at large faculty, and students. COMSE's charge includes oversight of curricular content including program and course objectives, content integration, policies and procedures, program evaluation, and continuous quality improvement. COMSE also ensures ongoing compliance with the standards and elements of the LCME. COMSE charges subcommittees, task forces, and work groups for particular purposes such as policy development, curricular subcommittees, focused curricular reviews, specific LCME standards/elements, and the learning environment. COMSE is a subcommittee of the Academic Affairs Committee (AAC), which is the academic subcommittee of the Executive Faculty (EF), the governing body of the school of medicine. COMSE refers matters of broader policy to the AAC and EF.

\section{Decentralized curricular governance}

Course directors and other administrative leaders receive appointments via a cooperative process between the Office of Education (OE) and the departments. Compensation for course directors is shared between the OE and the departments. Operational budgets and administrative support for nonclerkship courses reside in OE. The departments provide operating costs and administrative support for clinical clerkships.

\section{Education Staff}

Currently funded support includes:

- Office of Medical Student Education (15 FTE): OMSE provides oversight of the curriculum for the MD program, including schedule management, evaluations, assessments, course planning, and material support for all course directors. 
- Core teaching faculty (16.9 FTE): We protect and support time for about 50 faculty teaching and administrative roles. This $\$ 4.2$ million effort (\$2.6 million in education support, \$1.6 million to departments) allocates 13 FTE across our curriculum. For the Gateway Curriculum, we will increase to over 85 faculty members with supplemental protected time reaching 16.9 FTE paid through a mix of medical education and department funding.

- Coaching and assessment programs (9.4 FTE): Launching in 2020, our coaching program (7.3 FTE) will train students in professional identity formation, communication, and professional development. A competency committee (2.1 FTE) will review and adjudicate learner progress.

- Educational technology and innovation (4 FTE): This unit collaborates with students, faculty, and staff to support and enhance educational technology integration.

- Instructional design studio (2 FTE): This unit adds internal capacity and a dedicated studio for creation and design of educational materials. Using state-of-the-art technology, the instructional designer and videographer collaborate with faculty on content development, video production, and screencast recording.

- Program evaluation and continuous quality improvement (3.5 FTE): This unit oversees the evaluation of the MD program, synthesizing and presenting data that drives change that aligns with accreditation requirements and institutional strategic planning.

- Medical student research and scholarship (3.4 FTE): This unit supports the design and implementation of the academic career development curriculum and the scholarly work of medical students including alignment with mentors, stipends, and dissemination.

- Immersive learning centers (3.5 FTE): These centers include the standardized patient and high-fidelity simulation centers, which provide comprehensive simulation-based training and assessment for medical students.

- Medical education research unit (2.5 FTE): This unit supports the development and dissemination of education scholarship.

- Academy of Educators (1.7 FTE): The academy provides faculty development, small grants, an awards program, advocacy, and a learning community for educators.

- Center for Interprofessional Practice and Education (CIPE, 2 FTE): CIPE provides coordination and support for interprofessional education and serves as the home for the collaboration between WUSM, Goldfarb School of Nursing, and St. Louis College of Pharmacy.

- Career counseling (2 FTE): This unit provides career, match counseling, and support including creation of the MSPE.

\section{Medical education leadership}

See Figure 1-Organizational chart.

\section{Department of Medical Education}

The primary medical education staff and administrative faculty in the undergraduate medical education (UME) educational

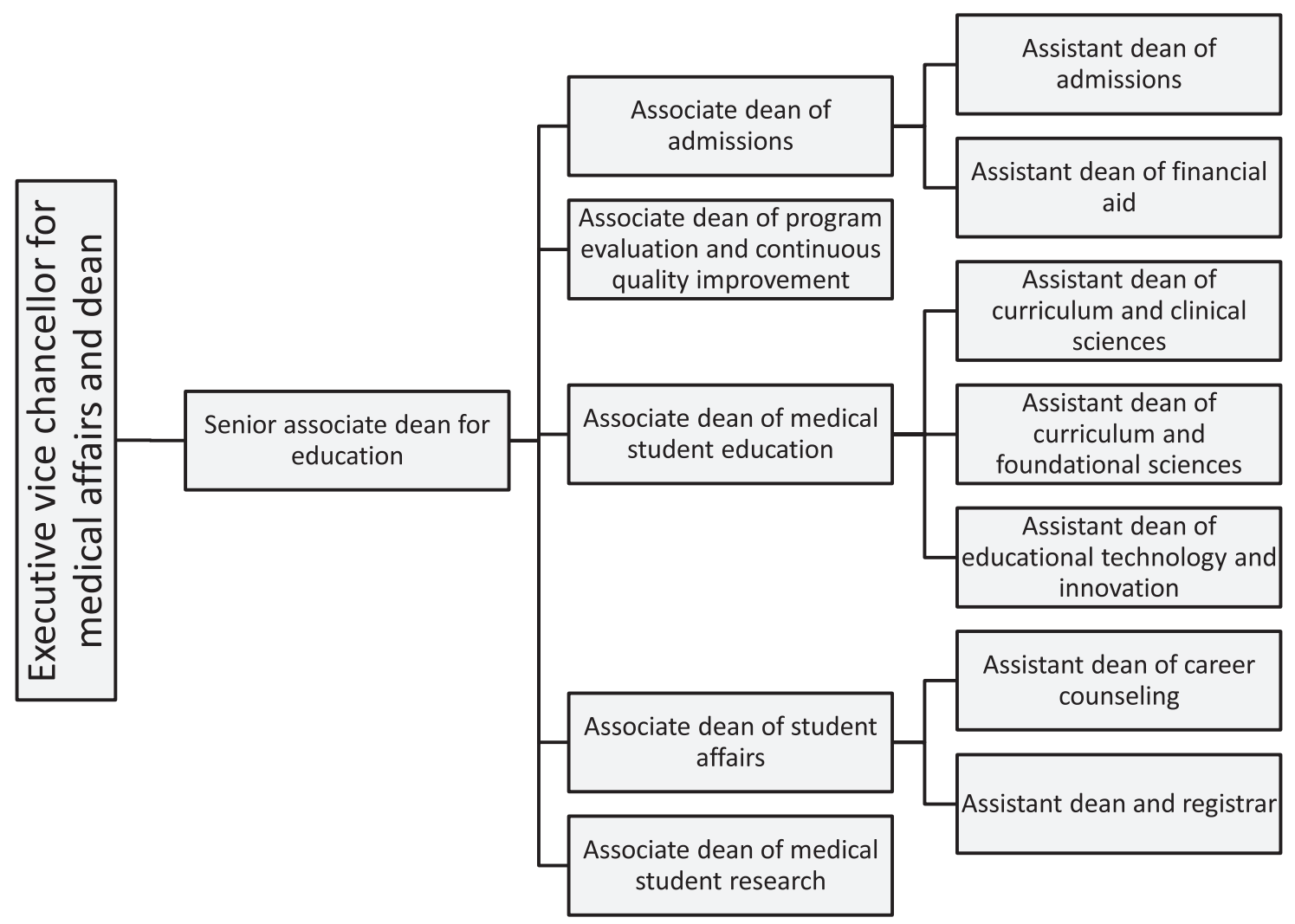

Figure 1 Organizational chart. 
program (OMSE) are responsible for course content and all relevant material support needed to execute the curriculum for UME. There are separate offices with administrative and faculty support for admissions, student affairs, pre-UME, graduate medical education, and continuing professional development, all of which fall under the Office of Education, run by the senior associate dean for education.

\section{Faculty Development and Support in Education}

\section{Professional development for faculty as educators}

The Office of Faculty Affairs and the Academy of Educators host regular workshops for faculty aimed at learning new skills and honing existing ones as academicians and educators.

\section{Role of teaching in promotion and tenure}

Teaching and/or educational contributions are expected and are considered significant components of the promotion dossier for faculty members in the investigator or clinician tracks. Faculty in clinician tracks must submit a clinician-educator portfolio for promotion to the associate and professor ranks.

\section{Teaching academy}

The Academy of Educators:

- Fosters an environment where education is highly valued and conducted at the highest level

- Encourages and recognizes innovative approaches to teaching, learning, and assessment
- Provides advocacy for education in the promotion process

- Organizes mentorship and faculty development programs in medical education

- Supports exceptional educational research and scholarship in the health sciences

Programming includes:

- Faculty development certificate programs in teaching skills, learner assessment, curriculum development, program evaluation, and educational scholarship

- Workshops on teaching, mentoring, and other elements of education and leadership

- Faculty recognition through the Teaching Awards Program that includes competitive 2-year fellowships to fund time to work on innovative teaching projects and curricular development

- Education research and innovation support through a smallgrants program

\section{Regional Medical Campuses}

Not applicable.

\section{Initiatives in Progress}

Our curriculum is undergoing a comprehensive renewal set to launch in fall 2020. Setting the stage for curriculum renewal has included reorganization of the management structure as well as new units and staff, all aimed at ensuring support for and central oversight of the curriculum. 


\section{Creighton University School of Medicine}

Michael Del Core, MD

\section{Medical Education Program Highlights}

Founded in the Jesuit tradition, the Creighton University School of Medicine (CUSOM) strives to improve the human condition with a diverse body of students, faculty, and staff who provide excellence in educating learners, health care professionals, and the public; advancing knowledge; and providing comprehensive patient care. Highlights of our program include:

- An active learning pedagogy that engages students in critical thinking and clinical reasoning skills from the onset

- A horizontally integrated curriculum that reinforces key concepts throughout the 4 years and includes learning opportunities in the medical humanities, patient safety, ethics, health systems science, and other areas

- Highly engaged students and faculty in fulfilling the mission of the school, service to others, in such as activities as the Institute of Latin American Concern in the Dominican Republic, and the Magis Clinic and Project Cura in Omaha

- Faculty who are outstanding leaders and teachers, who are dedicated to improving the human condition through medicine and ensuring the success of our students

\section{Curriculum}

\section{Curriculum description}

- The preclinical curriculum includes 15 required clinically integrated blocks. These blocks are organized by topics/organ system blocks. Each block is designed to integrate related topics in the horizontal integrated disciplines in the blue, green, and gold tracks.

- The gold track includes topics related to personal and professional development, leadership, humanities, ethics, evidence-based medicine, patient safety, science of health care delivery, and service learning.

- The green track includes topics related to physical diagnosis and procedural skills, interviewing, laboratory skills training, ambulatory clinic experience, early hospital experience, interprofessional education and collaborative practice, social medicine, and self-directed learning.

- The blue track includes topics related to anatomy, embryology, histology, physiology, pathophysiology, molecular and cellular biology, genetics, biochemistry, nutrition, microbiology, immunology, pathology, behavioral medicine, diagnostics, and therapeutics.

Acad Med. 2020;95:S291-S293.

doi: 10.1097/ACM.0000000000003377

Copyright (C) 2020 by the Association of American Medical Colleges

Supplemental digital content for this article is available at http://links.Iww.com/ ACADMED/A883.

Year school was established: 1892.

School URL: https://medschool.creighton.edu.
- The clinical curriculum consists of 7 required clerkships during the third year: internal medicine, family medicine, surgery, obstetrics-gynecology, pediatrics, neurology, and psychiatry. Electives, subinternships, and critical care rotations make up the fourth year.

- Horizontal integration of discipline content throughout the required clerkships will result in reinforcement and mastery of basic science and clinical principles introduced during the preclerkship curriculum. With the vertical integration that is planned, each program objective is expected to be covered in each curriculum phase (preclerkship phase and clinical and elective phase).

\section{Curriculum changes since 2010}

- The preclinical curriculum has evolved to include more active/ experiential learning, through a variety of techniques. This includes the use of case-based learning activities, team-based learning activities, self-directed learning, peer teaching, and enhanced clinical skills activities. These changes result in less reliance on passive didactic-based learning.

- Humanities, ethics, patient safety, health delivery science, and service learning, among others, were more intentionally integrated into the curriculum.

- Enhanced clinical skills training, ambulatory clinic experience, and early hospital experiences were added to the curriculum.

- Foundational concepts were integrated into the organ system blocks.

\section{Assessment}

The new curriculum includes a variety of formative assessment methods to ensure students regularly receive feedback to improve their performance. Student mastery of content is assessed at the end of each course, clerkship, and elective. To ensure the program learning objectives are being met, specific assessment metrics have been identified to assess each objective.

\section{Medical education program objectives}

The faculty members have characterized the core competencies of the graduates in domains corresponding to competency domains described by the ACGME and identified other objectives particularly relevant for Creighton graduates.

See Supplemental Digital Appendix 1-Competencies-at http://links.lww.com/ACADMED/A883.

\section{Pedagogy}

- Students participate in 2-3 case-based learning activities per week.

- Students participate in 3-4 team-based learning activities per week.

- There are self-directed learning exercises in each semester of the preclerkship curriculum.

- Didactic lectures average approximately 8 hours per week. 
- Podcasts of heart sounds are used to promote the development of listening and identification skills.

- Podcasts of lectures and other lecture materials are available online.

- Audience response systems (Poll Everywhere) are used for inclass and formative assessment.

- Early hospital experience is provided throughout the preclerkship curriculum.

- Interprofessional education is integrated into the medical school curriculum.

- Student interest selectives in the humanities and special topics, guided research selectives, career exploration selectives, and mission outreach selectives are available throughout the preclinical curriculum.

- Simulation training and interviewing skills including exercises with standardized patients are practiced throughout the curriculum.

- Anatomy, histology, and physiology hands-on laboratories are integrated into the organ system blocks.

\section{Clinical experiences}

Student learning occurs at multiple clinical sites:

- Creighton University Medical Center-Bergan, Omaha, Nebraska

- Omaha VA Medical Center, Omaha, Nebraska

- Children's Hospital and Medical Center, Omaha, Nebraska

- St. Joseph's Hospital and Medical Center, Phoenix, Arizona

- Valleywise Health-Phoenix (formerly Maricopa Integrated Health System)

- Phoenix Children's Hospital, Phoenix, Arizona

- Phoenix VA Health Care System, Phoenix, Arizona

- Rural and urban primary care sites

Ambulatory clinical experiences begin in the fall semester of the first year and continues through the preclerkship curriculum.

A challenge faced in the clinical experience is ensuring comparability of learning and assessment between the regional sites.

\section{Curricular Governance}

- The Educational Program Committee (EPC) oversees the medical education program as a whole and has responsibility for the overall design, management, integration, evaluation, and enhancement of a coherent and coordinated medical curriculum.

- The EPC defines and revises the goals, objectives, structure, and content of the undergraduate medical curriculum. In fulfillment of these responsibilities, it is charged with approving the creation or removal of courses from the educational program, determining the duration and order of courses, appointing course directors, analyzing and distributing data from the Evaluation Committee, and formulating educational policies.

- The EPC consists of the following 18 voting members: 7 faculty members ( 3 basic sciences and 4 clinical with at least 1 clinical faculty member from the Phoenix regional campus) with 4 of these appointed by the dean and 3 elected by the faculty; 5 appointed students ( 1 from each component from the Omaha campus and 1 third-year student from the Phoenix regional campus); 4 component directors; the assistant dean for medical education from the Phoenix regional campus; and the associate dean for medical education, who also serves as chair.

- In addition, the following serve as ex officio, nonvoting members: The medical school dean, the associate dean of the Phoenix regional campus, the associate dean for student affairs (Omaha campus), the assistant dean for student affairs (Phoenix regional campus), the assistant dean for medical education (Omaha campus), the university librarian, and the chair of the Evaluation Committee. Faculty committee members shall be elected by the faculty or appointed by the dean, based on their educational experience and the need for balance among disciplines.

- The committee meets twice per month.

- The EPC has 4 subcommittees, all of which report to the EPC:

o The M1-M2 Component Committee, composed of both basic science and clinical faculty, students, discipline stewards, and administrative staff, is responsible for planning and implementation of the revised integrated curriculum and ongoing review of course performance metrics, component-wide evaluation and grading policies and practices, and content to address omissions or redundancies.

o The M3-M4 Component Committee, composed of clerkship directors, clinical faculty, students, and administrative staff, regularly reviews clerkship/course performance metrics, component-wide evaluation, grading policies and practices, and content to address omissions or redundancies.

o The Evaluation Committee, composed of students, faculty, and the senior director of program assessment and curriculum development, evaluates the medical degree program, courses, and clerkships.

o The Policy Review Committee, composed of faculty and leadership, is charged with reviewing medical education program policies to suggest feedback to the submitting party and to review policies for consistency with other policies.

\section{Education Staff}

The Office of Medical Education (OME) facilitates medical student education by providing educational support and services to faculty and students and by managing and coordinating the curriculum. The office is responsible for the whole spectrum of undergraduate medical education and works closely with the Graduate Medical Education Office in coordinating the educational objectives and learning experiences of our students and residents. The Office of Student Affairs exists to promote the personal, academic, and professional development of students through service, support, and advocacy to assist them in becoming caring, competent health care professionals. 
The OME consists of the associate dean for medical education, who is the chief education officer for the medical education program, and:

- Assistant dean of medical education

- Senior director of program assessment and curriculum development

- A first- and second-year component director, and a third- and fourth-year component director (faculty)

- Medical and education directors for the clinical assessment center

- Instructional designers, and staff to support curriculum, technology, evaluation, and educational research activities.

Areas of responsibility for the OME include management of the medical curriculum, student testing, preparation of student learning materials, curriculum and program evaluation, academic computing, faculty development for teaching, assessment of clinical skills, medical education research and consultation, strategic planning for education, support for medical education committees, preparation and maintenance of educational data and reports, management of medical education space, and oversight of LCME compliance.

\section{Faculty Development and Support in Education}

\section{Professional development for faculty as educators}

- Jesuit Academic Development and Education (JADE) is an online faculty development program to support faculty development activities for the school at both the Omaha and Phoenix campuses. In spring 2019, the school conducted a launch of JADE for school leadership, with launch activities for senior administration and department chairs. The goal of JADE is to provide a range of faculty development activities to meet the faculty's and program's needs.

- The Creighton University Kingfisher Institute was founded with a vision that liberal arts and professional education complement and reinforce one another. In collaboration with Kingfisher, CUSOM instituted a new faculty development series in September 2019.

- The Creighton University Teaching and Learning Center (TLC) promotes a community of reflective and courageous teacherscholars engaged in the personal and professional formation of students. The TLC supports excellence and innovation in teaching and learning through the professional growth of Creighton faculty and staff in the Jesuit tradition.

\section{Role of teaching in promotion and tenure}

- The promotion and tenure process recognizes a clinicianeducator category as an option for career advancement. Evaluation of the candidate's performance by the School of Medicine and subsequently by the University Rank and Tenure Committee specifically includes the evaluation of teaching achievement, which plays a central and critical role in tenure and advancement in rank.

- The Golden Apple award is presented annually by medical students to faculty members and residents for excellence in teaching and dedication to patient care.

\section{Regional Medical Campuses}

Creighton students have participated in clinical rotations in Phoenix since 2007. In 2009, Creighton expanded its partnership with St. Joseph's Hospital and Medical Center in Phoenix by opening a Creighton campus there for third- and fourth-year medical students. Currently, 60 Omaha third- and fourth-year students are selected to complete their clinical training on the Phoenix campus. A 183,000-square-foot Phoenix campus building is expected to open in 2021 at Park Central in midtown Phoenix. When completed, the campus will be home to a 4 -year medical school and will accommodate nearly 900 students, including future physicians, nurses, occupational therapists, physical therapists, pharmacists, and physician assistants.

A single clerkship director works with each site director, to assure that there is comparability between the 2 campuses in terms of required objectives, clinical experiences, and assessment. Twice a year, the Office of Medical Education produces the Clerkship Comparability Report. The report includes the following data divided by campus location:

- Shelf exam performance

- Clinical achievement performance

- Grades

- Data on the timely submission of grades

- Duty hours

- Select evaluations items from the clerkship evaluations 


\section{University of Nebraska Medical Center College of Medicine}

Regan Taylor, MD, and Amy Cutright, MD

\section{Medical Education Program Highlights}

The mission of the College of Medicine (COM) at the University of Nebraska Medical Center (UNMC) is to promote and improve the health of the citizens of Nebraska by advancing health and biomedical sciences through the highest quality education, patient care, research excellence, and community service. We underwent a curriculum redesign implemented in 2017 and anxiously await the graduation of our first class to complete the new curriculum in 2021. We are proud to be one of the leading schools in primary and rural care in the nation and strive to produce physicians to care for the population of Nebraska and the surrounding regions.

\section{Curriculum}

\section{Curriculum description}

Our curriculum is divided into 3 phases: foundations of medicine, clinical applications, and career preparation.

- Foundations of medicine (first 18 months) is organized by organ system to cover basic sciences, pharmacology, and clinical reasoning.

- Clinical applications (12 months) consists of 6 core clerkships (family medicine, internal medicine, obstetrics-gynecology, pediatrics, psychiatry, and surgery).

- Career preparation (13 months) provides students the opportunity to further their clinical skills in a career specialty track with robust advising.

Clinically based topics known as "coils" are woven into the curriculum throughout all phases and include communication skills, physical exam, evidence-based medicine, point-of-care ultrasound, inquiry, interprofessional education, and health systems science.

See Supplemental Digital Appendix 1-Curriculum Map-at http://links.lww.com/ACADMED/A898.

\section{Curriculum changes since 2010}

Our curriculum has undergone a complete redesign, implemented in 2017. We have changed from a traditional 4-year design to 3 phases as described above. Three principles drove our curricular change:

Acad Med. 2020;95:S294-S297.

doi: 10.1097/ACM.0000000000003387

Copyright (C) 2020 by the Association of American Medical Colleges

Supplemental digital content for this article is available at http://links.Iww.com/ ACADMED/A898.

Year school was established: 1880.

School URL: https://www.unmc.edu/com/index.html.
- Enhancing the last year of medical school to best prepare students for residency

- Integrating clinical medicine and basic science throughout the entire 4 years

- Introduction and incorporation of health systems science

In our redesigned curriculum, the final phase of training extended the fourth year of medical school by 3 months (total of 13 months). This allows for further career exploration before submitting residency applications. Once students choose a specialty, they are assigned to a career track so that their last year of training can be more tailored to their future career.

Throughout the entire curriculum, we have integrated clinical topics (coils) so that students get early and consistent exposure to these topics and skills over the course of their education. Content centered around each coil is covered in each block during the first phase of school and revisited during clerkships to allow students to apply what they have learned in a clinical environment. During the third phase, these skills are revisited again in more structured small-group settings to reflect on experiences and explore new opportunities for growth.

The introduction of health systems sciences as a distinct longitudinal curricular thread was an important addition to our curriculum. The domains of social determinants of health, population health, advocacy, quality and patient safety, teamwork, leadership and health care financing are introduced during Phase 1 and reflected on in Phases 2 and 3. Recognizing these as an integral part of training before clerkships better prepares students to navigate the health care landscape and appreciate the challenges that both physicians and patients face in trying to provide the best possible care in our current system.

As we enter the third year of our new curriculum, our goals are to progress from lecture-based learning to more active learning modalities. While our curriculum has changed significantly in the last 10 years, class size has remained fairly steady, last changing in 2012 when we increased from 130 to 132 matriculants.

\section{Assessment}

Our medical education program objectives are based on the ACGME, and specifically follow their domains: medical knowledge, patient care and clinical skills, practice-based learning and improvement, communication and interpersonal skills, professionalism, systems of health care, interprofessional collaboration, and personal and professional development.

With our curricular revision, we transitioned to a pass/fail grading model in Phase 1. The Phase 2 clerkships and Phase 3 career tracks are still graded on an honors/high pass/pass/ marginal/fail system. We have instituted systems to increase formative grading opportunities during Phase 1 to de-emphasize summative multiple-choice tests. 
See Table 1—Program Objectives and Assessment Methods.

\section{Parallel curriculum or tracks}

UNMC does not have any parallel tracks or offer a 3-year MD degree program. There is currently no plan to offer these. Multiple students do independently pursue additional degrees in public health $(\mathrm{MPH})$ or business administration (MBA) at UNMC or affiliated institutions during the course of the MD training.

\section{Pedagogy}

We use a variety of pedagogical methods within our program to facilitate learning:

- During Phase 1, lecture, laboratory, problem-based learning, simulation, standardized patients, small-group discussion, and video modules are used to provide multiple options for students to master the material.

- Phase 2 is experiential (ambulatory and inpatient), with lectures, case-based learning, and procedure labs intermixed.

- Phase 3 is primarily made up of clinical experiences tailored to assist student with choosing a future specialty and then providing a robust clinical experience before residency training. Additionally, there are 2 months of classroom-based education focused on residency preparation. These months are spent with more simulation, standardized patients, smallgroup discussion, and case-based learning.

\section{Clinical experiences}

Students at UNMC are exposed to a variety of clinical sites during their training. Most of their time is spent at Nebraska Medicine, our primary clinical partner located at the UNMC campus in Omaha, Nebraska. These experiences are for both inpatient and ambulatory clinical learning. Students also train at the Omaha Veterans Administration Medical Center, Children's Hospital and Medical Center, and other nonacademic institutions and clinics within Omaha and the surrounding region. Students on their family medicine clerkship are placed at rural clinic sites across the state of Nebraska. Collectively, our students are afforded the opportunity to experience both private and academic settings, as well as urban and rural experiences.

Students have early clinical experience during Phase 1 through the Longitudinal Clinical Experience program. Each student is assigned to a local preceptor in the Omaha metro area. They meet with their preceptor 8 times during Phase 1, and have the opportunity to see and examine patients, as well as spend time with the clinic staff to observe clinic operations and gain appreciation of the many different roles that are required to make a clinic function. These visits start in the second semester of the first year of training. Students also have the opportunity to volunteer at our student-run SHARING clinics during Phase 1.

Currently, our biggest challenge is finding enough clinical sites/ preceptors to accommodate all of our students. As demands on physicians' time continue to increase, the ability to educate an emerging physician, properly evaluate, and provide feedback can be one of the easiest things to cut out of the day. Other expected challenges common to clinical rotations include standardizing

\section{Table 1}

Program Objectives and Assessment Methods

\begin{tabular}{|c|c|}
\hline $\begin{array}{l}\text { Medical education program } \\
\text { objectives }\end{array}$ & $\begin{array}{l}\text { Assessment } \\
\text { method }\end{array}$ \\
\hline $\begin{array}{l}\text { Medical knowledge: Students will apply } \\
\text { and recall knowledge of human structure, } \\
\text { function, development, pathophysiology, and } \\
\text { psychosocial development. }\end{array}$ & $\begin{array}{l}\text { Multiple-choice tests } \\
\text { Step exams } \\
\text { NBME Clinical exam } \\
\text { OSCEs } \\
\text { Clinical evaluations }\end{array}$ \\
\hline $\begin{array}{l}\text { Patient care and clinical skills: Students } \\
\text { will demonstrate and use their knowledge, } \\
\text { procedural and cognitive skills in the diagnosis, } \\
\text { management, and prevention of health } \\
\text { problems encountered in patient care. }\end{array}$ & $\begin{array}{l}\text { OSCES } \\
\text { Clinical evaluations }\end{array}$ \\
\hline $\begin{array}{l}\text { Practice-based learning and } \\
\text { improvement: Student will identify gaps in } \\
\text { understanding, recognize personal limitations, } \\
\text { and demonstrate habits of self-directed learning } \\
\text { to acquire or improve knowledge and skills. }\end{array}$ & $\begin{array}{l}\text { Problem-based } \\
\text { learning small groups } \\
\text { Self-evaluation } \\
\text { Clinical evaluations }\end{array}$ \\
\hline $\begin{array}{l}\text { Interpersonal and communication } \\
\text { skills: Students will employ effective } \\
\text { listening, verbal, and written communication } \\
\text { skills with patients, family members, } \\
\text { colleagues, and other health professionals to } \\
\text { promote optimal health outcomes. }\end{array}$ & $\begin{array}{l}\text { OSCES } \\
\text { Clinical evaluations } \\
\text { Standardized patient } \\
\text { formative feedback }\end{array}$ \\
\hline $\begin{array}{l}\text { Professionalism: Students will employ and } \\
\text { exemplify altruism, accountability, excellence, } \\
\text { duty, service, honor, integrity, and respect for } \\
\text { others into all aspects of their professional lives. }\end{array}$ & Clinical evaluations \\
\hline $\begin{array}{l}\text { Systems of health care: Student will } \\
\text { analyze health care delivery settings and systems } \\
\text { to advocate for patients, identify opportunities } \\
\text { for quality improvement, and optimize patient } \\
\text { safety through open communication of errors. }\end{array}$ & $\begin{array}{l}\text { Multiple-choice tests } \\
\text { Clinical evaluations }\end{array}$ \\
\hline $\begin{array}{l}\text { Interprofessional collaboration: Student } \\
\text { will prepare to function effectively in teams } \\
\text { and within organization, applying community } \\
\text { and other resources to medical problems for } \\
\text { individual patients and groups. }\end{array}$ & Clinical evaluations \\
\hline
\end{tabular}

experiences for all students and providing equivalent evaluation practices among faculty.

\section{Curricular Governance}

The COM Curriculum Committee is the central governing body for our medical school. Each phase has a leadership team including a director who oversees issues pertinent to that level of training. The directors also work closely with the assistant dean for medical education and the senior associate dean for academic affairs. While each of these bodies function somewhat independently, all are ultimately under the jurisdiction of the Curriculum Committee.

Phase 1 organization and governance is centralized within the college with academic support provided by the Office of Medical Education (OME). For Phases 2 and 3, academic support comes from a mixture of clinical departments and the centralized OME. Teaching is provided by basic science and clinical faculty located within each of the academic departments. Financial resources 
for teaching endeavors in all phases are controlled by the senior associate dean for academic affairs.

Assessment is managed at the block, clerkship, and track level. Written examinations are administered and overseen by course leadership. Simulated clinical exams are administered by course leadership with the assistance of the OME and the simulation labs. Clinical evaluations are managed by clerkship and track directors with the support from departmental administrators.

See Figure 1-Curriculum governance structure.

\section{Educational Staff}

The OME consists of 9 staff members who are responsible for the scheduling, coordination of online learning systems (Canvas, Oasis, etc.), website maintenance, curriculum distribution, testing support/administration, and general logistics of the curriculum.

The dean of the COM oversees both the educational and research activities of the college. The senior associate dean for academic affairs is most directly in charge of the educational aspects of the COM. All assistant and associate deans report to the senior associate dean. (See Figure 1 for specific personnel in each role.) The Office of Faculty Affairs is responsible for faculty development and serves all colleges at UNMC.

The role of the OME is primarily limited to undergraduate medical education and oversight of accreditation and other administrative tasks for each phase. The Office of Admission and Student Affairs is separate from the OME and involved primarily in recruitment, admission, and career planning. Graduate medical education has a separate administrative and academic support staff. Both UME and GME report to the senior associate dean for academic affairs.

See Figure 2-Organizational design.

\section{Faculty Development and Support in Education}

The Office of Faculty Affairs offers development opportunities throughout the year on various educational topics. They have single-session, lecture-style events, as well as more longitudinal offerings - short courses that can be enrolled in over 1-2 months and meet multiple times.

Education is highly valued in the promotion and tenure process at UNMC, being 1 of the 4 domains (education, research, service, and patient care) in which promotion criteria are organized. Particular emphasis is placed on the creation of new or innovative teaching methods; mentoring of faculty, staff, or students; evidence of teaching effectiveness (evaluations); and educational scholarly activity.

UNMC also has the Interprofessional Academy of Educators, which aids in faculty development and educational mentorship. Members are selected from the College of Nursing, College of Pharmacy, COM, College of Allied Health, and Graduate Studies. Its function is to promote the use of best practices by all educators, foster and disseminate educational research and innovation, and mentor early career faculty and preceptors in their development to become effective teachers and scholars.

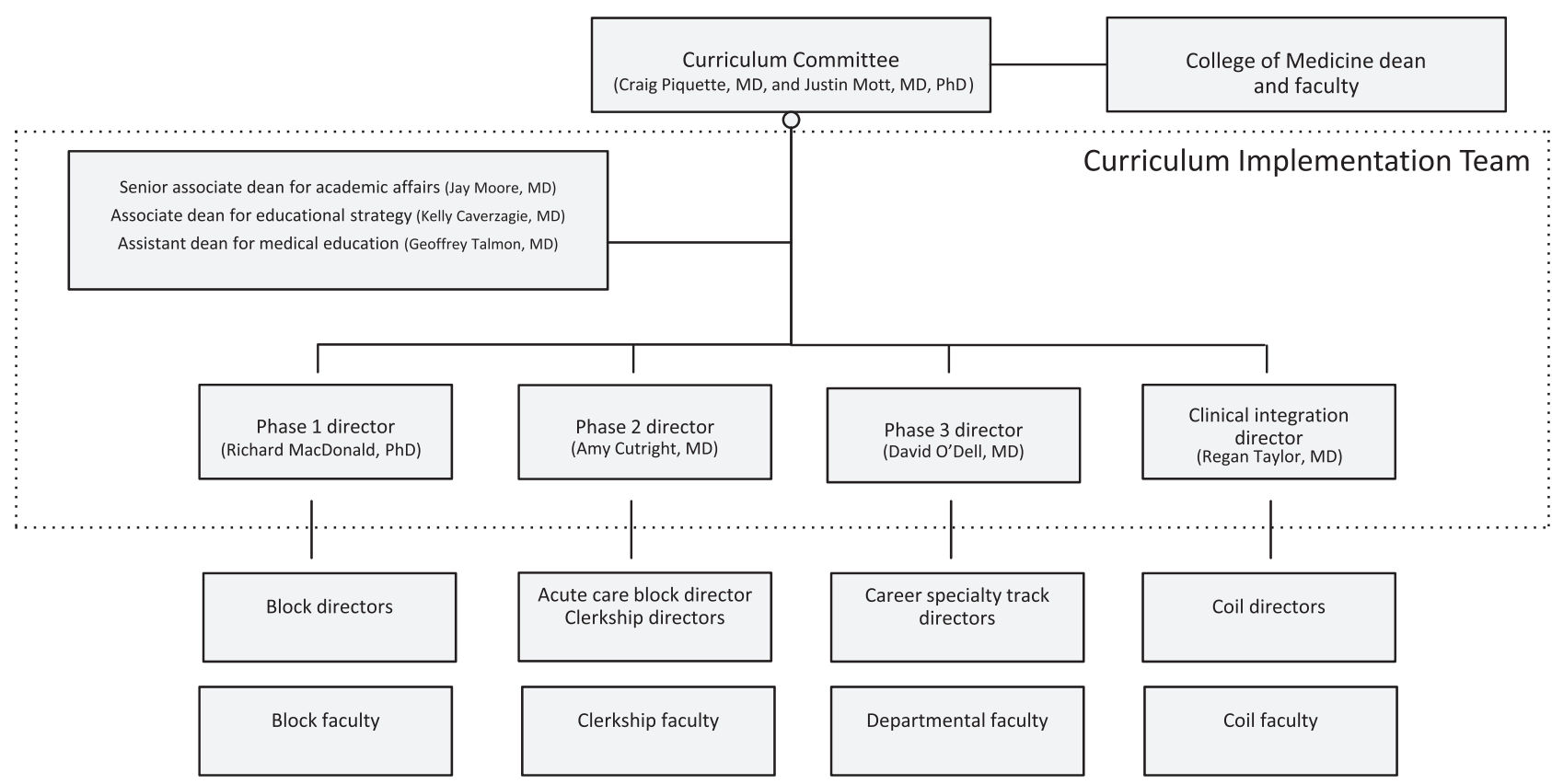

Figure 1 Curriculum governance structure. 


\section{College of Medicine}

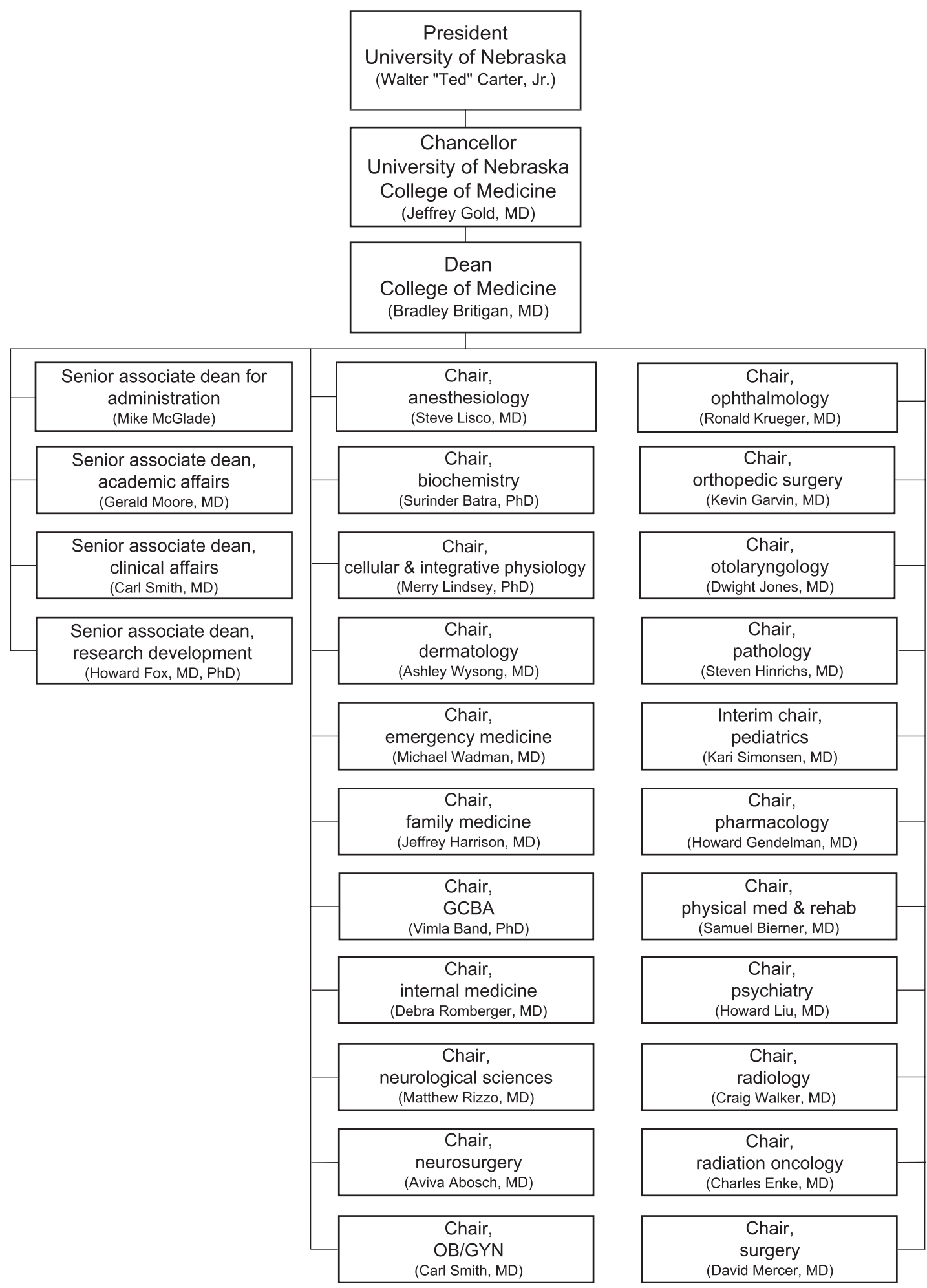

Figure 2 Organizational design. 


\title{
The University of Nevada, Las Vegas School of Medicine
}

\author{
Neil G. Haycocks, MD, PhD, and Corrin C. Sullivan, PhD
}

\section{Medical Education Program Highlights}

The University of Nevada, Las Vegas (UNLV) School of Medicine was founded in 2014 to help address the region's chronic physician shortage. The school's primary mission is to provide a thoughtful, innovative education to students who will practice in Nevada and improve the health of everyone in the state. Major highlights of the school include:

- The relationship between the school and the community. The first 6 weeks of the curriculum include an exposure to population health within Greater Las Vegas. Students are placed in small groups and assigned to underserved neighborhoods in the city. The groups are tasked with performing an assessment of their neighborhood's history, demographics, physical characteristics, and infrastructure, providing social context to the school's patient population. Students also participate in 3 years of longitudinal service learning through the Nevada Community Service (NCS) course, which places them in local community service organizations.

- The diversity of the student body. Understanding the cultural aspects of Nevada's communities is one of UNLV School of Medicine's guiding principles. As such, the medical school has implemented an admissions process that proactively seeks to assemble classes reflecting the state's racial, ethnic, geographic, socio-economic, and educational diversity. The results to date have been successful, with each class possessing significant representation from underrepresented and economically disadvantaged groups, and high proportions of first-generation students.

- Curricular emphasis on analysis and critical thinking. The Analytics in Medicine (AIM) course uses an active learning model and covers foundational principles of bioethics, epidemiology and biostatistics, and evidence-based medicine. Problem-based learning (PBL) likewise promotes self-directed inquiry, synthesis, and teamwork. Both courses seek to equip students with transferable skills that can be applied to any medical decision-making process.

\section{Curriculum}

\section{Curriculum description}

The curriculum is 45 months in duration and has 3 phases: foundations (Phase I, 20 months), clerkship (Phase II, 12 months), and career exploration and scholarship (Phase III, 13 months).

Acad Med. 2020;95:S298-S301.

doi: 10.1097/ACM.0000000000003417

Copyright (C) 2020 by the Association of American Medical Colleges

Year school was established: 2014.

School URL: https://www.unlv.edu/medicine.
- Phase I consists of an emergency response/population health immersion; 7 sequential integrated organ system courses; and 3 concurrent longitudinal courses that encompass basic clinical skills (Foundations of Clinical Practice), analytics (AIM), and service learning (NCS), respectively. Phase I also provides dedicated time for research, which is a graduation requirement.

- Phase II is designed around a longitudinal integrated clerkship (LIC) model, where students complete their core clerkships simultaneously over the course of 12 months.

- Phase III is comprised of 4 -week rotations and provides a breadth of elective experiences and a required communitybased medicine clerkship.

- The curriculum ends with a capstone experience that includes tailored clinical skills training.

\section{Curriculum changes since 2010}

The UNLV School of Medicine welcomed its charter class in 2017 and has not yet undergone substantive curriculum change or renewal. The class size has remained constant at 60 students per cohort.

\section{Assessment}

- The medical educational program objectives were designed using the ACGME domains of competence.

- The majority of Phase I exams are built using the Customized Assessment Service of the NBME. These were initially administered every 2 weeks, but are now progressively spaced out to 3- and 4-week intervals. Cumulative final exams have been instituted at the end of semesters 1 and 2 to promote the longitudinal acquisition of knowledge, and OSCEs are administered to assess student competency in communication and clinical skills through direct observation.

See Table 1-Program Objectives and Assessment Methods.

\section{Pedagogy}

- A wide variety of pedagogies are used. PBL is the cornerstone of the Phase I curriculum and is supplemented with a mixture of interactive lecture, case-based learning, team-based learning (TBL), and large-group discussion. Additionally, in the longitudinal AIM course, students learn how to identify answerable clinical questions as well as ethical issues that arise in patient care, where and how to find information relevant to these questions, and self-direct their study to independently think through these issues to guide practice. AIM uses an active learning format in which students have to prepare individually for sessions and identify learning issues for themselves. The clinical skills course uses standardized patients extensively. Ambulatory clinical exposure begins in the first semester, and the clerkship year contains both ambulatory and inpatient experiences. 


\section{Table 1}

\section{Program Objectives and Assessment Methods}

Medical educational program objective

1. Apply the principles of anatomy, behavioral science, biochemistry, biostatistics, cell biology, epidemiology, genetics, immunology, microbiology, pathology, pharmacology, and physiology to determine the etiology, pathophysiology, diagnosis, treatment, and prevention of significant human diseases.

2. Identify and propose a treatment plan for acute and chronic diseases.

3. Demonstrate personal accountability, altruism, humanism, self-awareness, and humility in the care of patients, self, and others.

4. Communicate effectively with patients, families, and other health care professionals, including in situations involving communication barriers

5. Conduct and document complete and focused medical histories and physical examinations, and recognize confounding factors including age, gender, socio-cultural factors, socio-economic status, family history, and emotional state.

6. Correctly perform basic procedural skills with attention to patient comfort and safety.

7. Integrate epidemiologic, socio-economic, behavioral, socio-cultural, and community factors into patient care.

8. Apply knowledge of wellness, nutrition, hospitality principles, pain management, and integrative medicine into patient care.

9. Anticipate ethical issues encountered in clinical care and research, explain ethically justifiable options and consequences from multiple perspectives, and manage ethical challenges in medical practice and research.

10. Provide appropriate patient-centered counseling techniques to improve outcomes and patient satisfaction and to promote optimal use of health care resources.

11. Identify end-of-life care issues, including palliative care, from the perspectives of patient, family, and health care providers

12. Demonstrate advanced clinical reasoning to develop and narrow a differential diagnosis by merging clinical information with knowledge of mechanisms of disease.

13. Formulate clinical questions and apply evidence-based medicine to provide quality health care to individuals and populations.

14. Practice scholarship based on scientific research methods

15. Identify the personal skills and systems-level processes that support continuous quality improvement, including patient safety.

16. Advocate for the improvement of public health outcomes through community engagement and the analysis of social determinants of health and disease.

17. Discuss the core financial, legal, structural, policy, and regulatory aspects of the U.S. health care system and their impact on delivery of health care.

Abbreviations: ACLS/BLS, advanced cardiac life support/basic life support; Capstone, project leading to scholarly presentation; CD, course exam; NBME, National Board of Medical Examiners examination; OSCE, objective structured clinical exam; PE, preceptor evaluation; SE, self-evaluation; SGF, small-group faculty evaluation.

- Changes have been minimal to the pedagogical approaches. There is a continuous emphasis on replacing passive learning methods with active ones. There has also been more deliberate incorporation of TBL into the basic science courses.

\section{Clinical experiences}

- The school has 10 departments: emergency medicine, family medicine, internal medicine, obstetrics-gynecology, orthopedic surgery, otolaryngology, pediatrics, plastic and reconstructive surgery, psychiatry, and surgery. In addition to ambulatory clinical experiences provided through the faculty practice plan, UNLV Medicine, students are placed in local training sites that include a county hospital with a Level 1 trauma center (University Medical Center), a private hospital (Sunrise Hospital), a federal hospital (VA Southern Nevada), and various private health care organizations (e.g., Optum Care/ Southwest Medical).
Assessment methods

NBME, PE, SGF

ACLS/BLS, OSCE, NBME, PE

$C E, P E$, SGF

OSCE, PE, SGF

OSCE, PE

PE

OSCE, PE, SGF

CE, PE, SGF

CE, OSCE, PE, SE, SGF

OSCE, PE

CE, PE, SGF

CE, OSCE, PE, SGF

$\mathrm{CE}, \mathrm{PE}, \mathrm{SGF}$

$\mathrm{CE}, \mathrm{SE}$

CE, OSCE, PE, SGF

Capstone, SGF

CE, PE
- The clerkship year is organized using a longitudinal integrated clerkship (LIC) model. The required clerkships are interleaved so that students complete them simultaneously across the entire year. Shelf exams are administered at the midpoint and end of the LIC in addition to OSCEs to uniformly assess student competency across a wide range of clinical skills.

- Students have their first required clinical experiences through the Foundations of Clinical Practice course, which pairs them with local physician preceptors, primarily in outpatient primary care offices, through Phase I. These initial clinical experiences are supplemented with extensive standardized patient encounters throughout the longitudinal Foundations of Clinical Practice course to refine students' physical examination, history taking, and oral presentation skills concurrently.

- Elective community-based rotations are offered by the Veterans Affairs Medical Center (VA Southern Nevada Healthcare 
System) and an affiliated hospital, Sunrise Medical Center. At the VA, students are offered required psychiatry rotations with the Behavioral Health Science Department, as well as a wealth of electives opportunities in acute medicine, anesthesia, cardiology, gastrointestinal medicine, general surgery, geriatrics, gynecology, ophthalmology, orthopedics, nephrology, pain medicine, physical medicine and rehabilitation, palliative care, plastic surgery, podiatry, pulmonary medicine, radiology, rheumatology, and thoracic surgery. Sunrise Hospital and University Medical Center provide both required and elective rotations in pediatrics and obstetrics-gynecology, particularly gynecologic oncology, neonatal-perinatal medicine, and pediatric critical care medicine.

- Challenges faced in the design and implementation of clinical experiences are consistent with those of many other institutions: limited clinical education training space for students (preceptor recruitment), lack of shared governance when incorporating community physicians and affiliated entities into the context of medical education within the existing health care system, and physicians' underlying focus and administrative pressure to generate revenue to sustain clinical practices. Hence, particularly with the Phase II LIC, attention was focused on continuity in outpatient and inpatient care settings, developing and modifying technology infrastructures to maintain scheduling accuracy, and allocating resources to support administrative and faculty teaching efforts, including student evaluation and overall program assessment. With the next iteration of clinical training, attention is focused on space due to an overlap between Phase II and Phase III clinical requirements and electives.

\section{Curricular Governance}

- Curricular governance resides with the Curriculum Oversight Committee (COC), which consists of elected and appointed faculty and student representatives. The COC has ultimate authority over curricular matters. The committee contains 4 standing working groups: biomedical sciences, clinical education, community engagement, and LCME compliance. Nonvoting ex officio members are the vice dean for academic affairs and education, the senior associate dean for student affairs and admissions, the associate deans for biomedical science education and community engagement, the assistant deans for curriculum and assessment and clinical education, and the director of educational outcomes and assessment.

- The main budget for instruction, assessment, and academic support is centralized within Academic Affairs and Education. Additional state funds are dispersed to UNLV Medicine departments on a per-faculty basis to subsidize educational activities.

See Figure 1-Curriculum Oversight Committee.

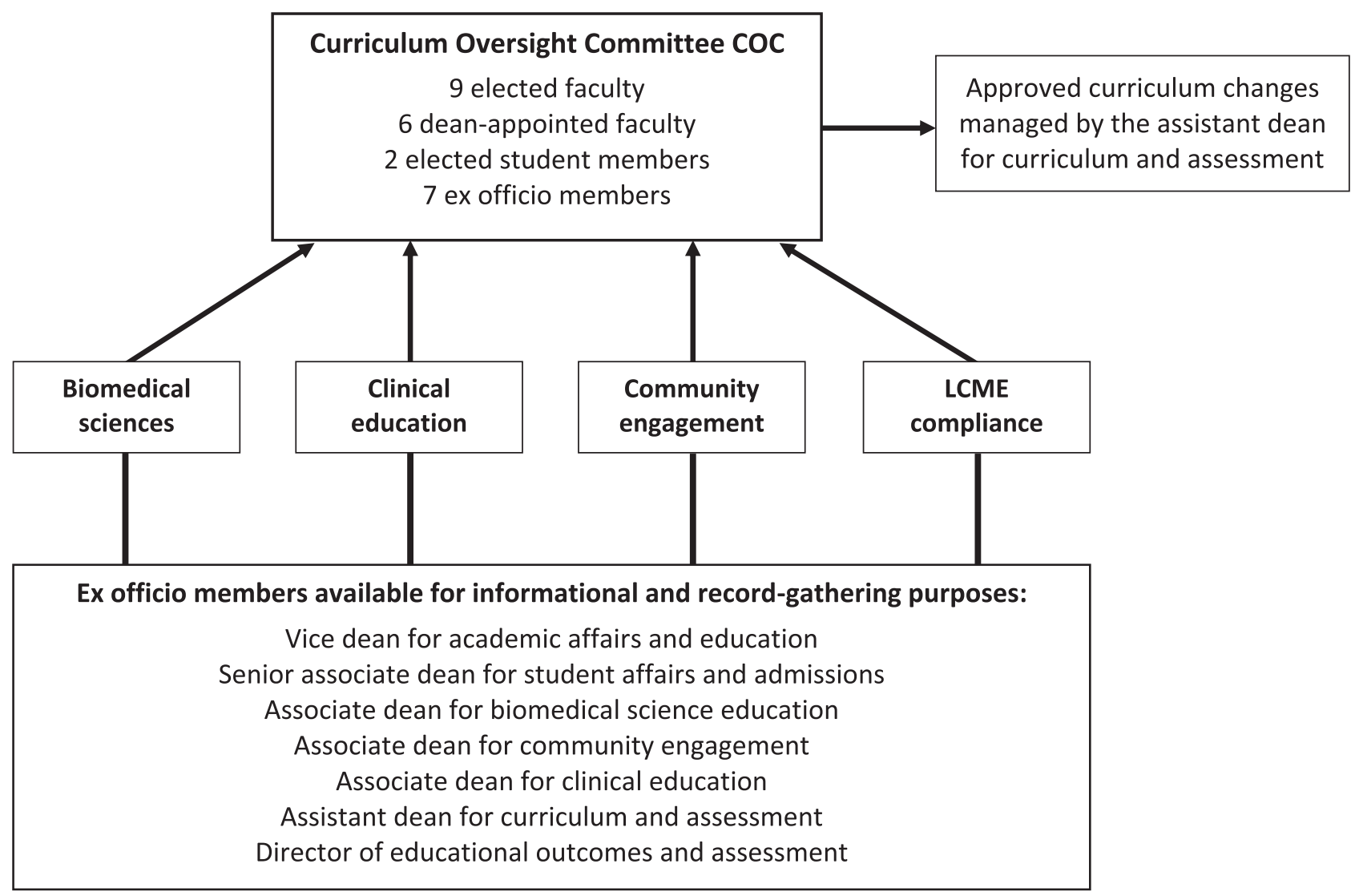

Figure 1 Curriculum Oversight Committee. 


\section{Education Staff}

- Academic Affairs and Education is charged with the planning, implementation, and administrative oversight of the curriculum. The division is led by the vice dean for academic affairs and education, who reports to the dean of the medical school. There are 4 main subdivisions, each led by a dean: student affairs and admissions, biomedical science education, clinical education, and curriculum and assessment. Their respective functions are further supported by directors of case-based learning, anatomy, educational outcomes and assessment, medical student research, community engagement, student affairs, and admissions, as well as the assistant dean of ultrasound education. Academic support is coordinated by a staff member within Student Affairs. Faculty development is coordinated within Faculty Affairs. This is a separate division that is led by the associate dean for faculty affairs, who reports directly to the dean.

- Clerkship directors, the vice dean for academic affairs and education, the assistant dean for clinical education, and the assistant dean of curriculum meet regularly to develop comprehensive plans and assess the implementation of the longitudinal integrated clerkship (Phase II) and advanced clinical electives (Phase III). The UNLV School of Medicine education program objectives and core clinical competencies form the basis for curriculum development, planning, implementation, and evaluation of where and how clinical experiences measure competencies and meet milestones appropriate to the UNLV School of Medicine curriculum and the LCME. Moreover, clerkship directors meet monthly with the assistant dean for clinical education, assistant dean for curriculum and assessment, and director of educational outcomes and assessment to evaluate student performance for entrustment decisions and identify where academic resources or additional support is needed.

- The purview of Academic Affairs and Education is UME. GME is part of Clinical Affairs, which is a separate division. PreUME pipeline programs are coordinated fully or partially by Student Affairs and Admissions.

- The Department of Medical Education provides an academic home for faculty who have substantial roles in education, without primary appointments in other departments, and wish to enhance their academic careers. The key functions of the Department of Medical Education include faculty development, data analysis, assessment, and scholarship.

\section{Faculty Development and Support in Education}

- Programmatic faculty development for teaching has been offered through a joint effort by Academic Affairs and Education and Faculty Affairs. The hiring of a dedicated coordinator for faculty development is in process.

- The large majority of faculty are within clinician-educator tracks, which are nontenure. These tracks define, on an individual basis, the role of teaching and educational decisions in promotion, and these activities are evaluated by the department chairs as part of the annual review. The process is similar for the small number of individuals who are tenured or have tenure-track positions. 


\title{
University of Nevada, Reno School of Medicine
}

\author{
Timothy K. Baker, MD, Lisa A. Calvo, MD, Brady J. Janes, PhD, and Gwen S. Shonkwiler, PhD
}

\section{Medical Education Program Highlights}

The University of Nevada, Reno School of Medicine (UNR Med) combines research-intensive, cutting-edge biomedical sciences with community-based clinical education to create a unique medical school experience for students and faculty. Our mission is to improve the health and well-being of all Nevadans and their communities. Highlights of our program include:

- The required immersive experience in rural health care provides students with an opportunity to engage in the full range of clinical and community health issues experienced by physicians in communities with limited resources and access to specialty care. This provides an opportunity to increase students' clinical skills, knowledge, problem-solving, and decision-making skills while learning firsthand the unique aspects of life and health care in our state's rural and frontier areas.

- UNR Med has a major emphasis on encouraging our future physicians to embrace their role as teachers. Our yearlong Teaching in Medicine elective is taken by nearly the entire class of graduating students, giving them an opportunity to provide education, guidance, and mentorship to their junior colleagues, while developing the skills needed to step into their new teaching role as resident physicians.

- The Student Outreach Clinic is a student-run, voluntary experience founded by students at UNR Med more than 30 years ago. It continues to thrive as a place for students to provide a valuable service to the community, learn from early hands-on experience, and develop the administrative and leadership skills that are increasingly required of physicians. With general medicine, pediatric, women's health, geriatric, and dermatologic clinics, the Student Outreach Clinic has expanded its impact in the underserved Reno health care community.

\section{Curriculum}

\section{Curriculum description}

UNR Med has a traditional 4-year curriculum with 2 years of biomedical sciences education, enhanced by clinical experiences, followed by 2 years of fully immersive clinical education.

- Year 1 includes 40 weeks of instruction across 5 organ systemsbased blocks. The Practice of Medicine (POM) course serves as

Acad Med. 2020;95:S302-\$304.

doi: 10.1097/ACM.0000000000003485

Copyright (C) 2020 by the Association of American Medical Colleges

Supplemental digital content for this article is available at http://links.Iww.com/ ACADMED/A964.

Year school was established: 1971.

School URL: https://med.unr.edu. the introduction to clinical skills course and runs concurrently with the blocks in the fall and spring. POM includes weekly community-based ambulatory care experiences for every student.

- Year 2 includes 31 weeks of instruction across 5 additional blocks in which students revisit the organ systems introduced in Year 1, but with an emphasis on pathophysiology. The Advanced Clinical Skills course runs concurrently with the blocks for both fall and spring and includes a second longitudinal community-based ambulatory care experience.

- The Context of Patient Care course serves as a transition between Years 2 and 3 where students receive exposure to the full spectrum of patient care including health policy, patient safety, public health, and preventative medicine. This course also prepares students for their clerkship experiences with further emphasis on clinical skills and understanding their role as third-year clerks.

- Year 3 marks the transition to the clinical phase of the medical education program. Students rotate through 7 required clerkships over 48 weeks. Additionally, students are provided an opportunity for a 4-week selective experience that is designed for career exploration. Ongoing clinical skills development is done in the longitudinal Clinical Reasoning in Medicine course. The third year also offers an opportunity for select students to have an early experience in rural health by completing their internal medicine and pediatrics clerkships in Elko, Nevada.

- Year 4 includes 32 weeks of electives, in addition to the required 4-week Advanced Clinical Experience in Rural Health course. All graduates of UNR Med are required to have an immersive clinical rural health experience in either the third or fourth year.

See Supplemental Digital Appendix 1-Curriculum Overviewat http://links.lww.com/ACADMED/A964.

\section{Curriculum changes since 2010}

Our preclerkship curriculum underwent a substantial reform in academic year 2012-2013. After an extensive evaluation of the prior curricular structure, the Medical Education Steering Committee adopted a plan to transition from a discipline, coursebased structure to an integrated organ systems-based approach. This included transition to a centralized management system through the Office of Medical Education. This structure continues presently, however, following a recent whole curricular review, discussions have begun regarding the next phase of reform.

The clerkship curriculum underwent a significant change in academic year 2017-2018 with the introduction of 2 new required 4-week experiences each, in neurology and selectives (career exploration). To accommodate these new experiences, 2 existing clerkships (internal medicine and surgery) were shortened from 12 to 8 weeks each. 
For much of our 50-year history, UNR Med was the sole MDgranting institution in the state of Nevada. As public medical education has expanded in our state, it has afforded us an opportunity to focus our resources in Northern Nevada, where the school is based. In academic year 2018-2019, UNR Med ceased the operation of clerkships at the Las Vegas campus and transitioned all student clerkship rotations to the Reno campus.

Since 2010, our class size has increased from 62 to 70 students. This increase in class size did not substantially change our structure but rather was an expansion to fill preexisting available teaching capacity.

\section{Assessment}

Our medical education program objectives were last revised in 2017. With this revision, we transitioned from objectives based on the ACGME domains of competence to objectives based on the Physician Competency Reference Set.

See Supplemental Digital Appendix 2-Program Objectives and Assessment Methods-at http://links.lww.com/ACADMED/ A964.

With the transition to a systems-based curriculum for the preclinical phase of the MD program, several new assessment methodologies were introduced.

- Integration of assessments associated with new pedagogical approaches including team-based learning as well as smallgroup and case-based activities

- Weekly formative self-assessments of foundational biomedical science content

- Assessment strategies to leverage the available technology of a newly constructed high-fidelity simulation center

- Expansion of clinical assessments using standardized patients in Years 1-3 using technology in the newly constructed standardized patient center

\section{Pedagogy}

We use multiple pedagogical approaches to achieve our medical education program objectives, which we have expanded and diversified since 2010. Changes include the expansion of peer teaching in Years 1 and 2 clinical courses, simulations integrated into preclinical courses and clerkships, addition of case-based learning and team-based learning, expansion of the use of standardized patients in Years 1-3, and an emphasis on self-directed learning. Other ongoing pedagogical approaches include:

- Hands-on clinical experiences in ambulatory and inpatient settings

- Small- and large-group discussions

- Laboratory-based experiences for pathology and microbiology as well as traditional cadaver and digital dissection in anatomy

- Lecture, with an increasing emphasis on enhanced, interactive sessions

- Early clinical preceptorship experiences

- Self-directed learning/tutorial

- Simulations

\section{Clinical experiences}

As a community-based medical school, we work closely with our local and regional affiliates to provide a comprehensive clinical education. Students rotate through each of the local hospitals, including the VA hospital. Our UNR Med clinical practices as well as private physician offices and outpatient practices affiliated with the major local health systems serve as clinical training sites for our required and elective experiences.

Students have their first formal clinical experience within weeks of matriculation, when they perform interviews with volunteers who share their personal stories as patients. They work weekly with standardized patients and, beginning in the spring semester of the first year, students participate in weekly community-based ambulatory preceptorships.

While we do have major health system partners, including the local VA medical center, we do not own a hospital or major health system. As a community-based medical school, we rely on our community partners to provide clinical experiences. Nearly all of our required clinical experiences as well as our electives are based in part or entirely at our inpatient and outpatient community sites.

The most significant challenge in designing and implementing clinical experiences for our medical students is the availability of clinical training sites. The community-based nature of our program also brings challenges in ensuring consistent experiences for our students, who learn at a variety of locations with a variety of clinical faculty members.

\section{Curricular Governance}

Our bylaws designate the Medical Education Steering Committee (MESC) as the main curriculum committee with authority for overall design, management, and evaluation of the undergraduate medical education program. Two committees serve under the MESC: the Years 1-2 Curriculum Committee and Years 3-4 Curriculum Committee are responsible for the creation, implementation, coordination, and evaluation of the preclerkship and clerkship/elective phases, respectively.

There is close collaboration with the departments for the management of the curricular content and assessments. Budget management for teaching is divided between the Office of Medical Education, which provides salary support for defined administrative and leadership roles in the curriculum (e.g., clerkship director), and the academic departments, which provide the academic time for faculty to engage in teaching.

\section{Education Staff}

Reporting to the dean, the senior associate dean for academic affairs leads the academic programs of the School of Medicine, including GME, UME, and admissions and student affairs. 
- The UME program is led by the associate dean for medical education, who has broad oversight and responsibility for the curriculum of the MD program. The associate dean does not have direct oversight or responsibility for students before matriculation, nor those in GME programs.

- The associate dean for admissions and student affairs, in collaboration with the associate dean for medical education, is responsible for learner support through the Learning and Wellness Resource Center (LWRC), which includes the learning specialists. The LWRC, in collaboration with the teaching faculty and the staff of the Office of Medical Education, tracks the academic progress of all students from matriculation to graduation and ensures provision of comprehensive support services, as needed.

- Reporting to the dean, the senior associate dean for faculty oversees faculty development activities. Ongoing faculty development needs are determined collaboratively with the Office of Medical Education to ensure alignment with best practices in medical education. The Office for Faculty and the Office of Medical Education partner to deliver effective faculty development across a broad range of topics.

The Office of Medical Education provides administrative support for the implementation and delivery of the curriculum at all levels.

- The director of curriculum development and assessment manages the systems-based blocks that make up the preclinical phase of the curriculum.
- Foundational science content is taught by a combination of basic science and clinical science educators.

\section{Faculty Development and Support in Education}

Our school offers a range of faculty development opportunities to enhance teaching effectiveness, research, and scholarship as well as leadership development. This is accomplished through orientations, workshops, longitudinal faculty development courses, and individual consultations. UNR Med supports programs and departments by facilitating retreats and offering targeted training activities:

- The Association of College and University Educators program provides training in educational best practices.

- The Academy for Development in Academic Medicine program provides education and mentoring for graduate students and residents interested in careers in academic medicine.

- Faculty interest groups such as Women in Medicine are supported.

- The Faculty Development Funding program provides research and professional development funding opportunities to all academic and administrative faculty members.

Teaching and educational activities are included in the evaluation criteria for promotion and tenure decision at UNR Med in all tracks; however, they are particularly emphasized as a metric in the clinical educator track. 


\title{
Dartmouth Geisel School of Medicine
}

\author{
Virginia T. Lyons, PhD, John F. Dick III, MD, and Adam R. Weinstein, MD
}

\section{Medical Education Program Highlights}

The Geisel School of Medicine at Dartmouth aims to graduate the complete physician-one who excels in the basic sciences, delivers outstanding clinical care, discovers and shares new knowledge, and works to improve systems of health care delivery. Program highlights include:

- An innovative health care delivery science curriculum working in collaboration with other graduate institutions at Dartmouth

- Emphasis on pedagogies fostering critical thinking, problemsolving, collaboration, and teamwork

- Thoughtful integration of 18 longitudinal curricular topics across the 4-year curriculum

- Strong sense of community fostered by a small class size and close interactions with faculty

\section{Curriculum}

\section{Curriculum description}

See Supplemental Digital Appendix 1-Curriculum Schematicat http://links.lww.com/ACADMED/A897.

\section{Curriculum changes since 2010}

After several years of planning, Geisel introduced a new highly integrated 4-year curriculum in the fall of 2019. The curriculum provides a strong foundation in the basic sciences, early handson clinical experiences, and innovative and integrated coverage of health care delivery sciences. Learning experiences occur in 3 phases.

Phase I, Foundations of Medicine, consists of an 18-month preclinical systems-based curriculum. Courses are organized to promote integration of topics with natural synergies in the clinical environment, for example, the cardiovascular and respiratory systems. The curriculum has 2 courses spanning the entire preclinical curriculum. On Doctoring introduces students to essential clinical skills through longitudinal learning experiences in small groups, community-based clinics, and simulated environments. The course focuses on patient interviewing, physical diagnosis, clinical reasoning, and communication and counseling skills. The other longitudinal course, Patients and Populations, delves into health care delivery science and exposes students to analytical tools to become

Acad Med. 2020;95:S305-S308.

doi: 10.1097/ACM.0000000000003388

Copyright (C) 2020 by the Association of American Medical Colleges

Supplemental digital content for this article is available at http://links.Iww.com/ ACADMED/A897.

Year school was established: 1797

School URL: https://geiselmed.dartmouth.edu. effective change agents and improve the systems in which they work. Phase I concludes with an innovative case-based course where students work in teams to integrate scientific concepts with evidence-based medicine, clinical reasoning, and communication skills to reflect upcoming clinical environment experiences.

Phase II, Clinical Immersion, provides practice in the core clinical clerkships: inpatient medicine, surgery, pediatrics, obstetrics-gynecology, psychiatry, family medicine, neurology, and advanced ambulatory medicine. These experiences offer a high level of self-directed learning; graded autonomy; and exposure to diverse patients, care venues, and populations across the United States. The clerkships use learning technologies that regularly bring all students together for shared learning experiences and teamwork, even while geographically separated.

Phase III, Exploration and Differentiation, is designed to promote student career exploration. Students decided on a career path can enroll in subinternships and clinical and research electives supporting their path, while students still deciding can select experiences in diverse fields to inform their decision. All students complete a 4 -week subinternship in their chosen field that involves increased patient care responsibility and autonomy. A capstone provides enriched experiences combining the goals of preparation for residency, advanced clinical application of foundation sciences, and the skill of thoughtful self-reflection. Of note, all students, over the course of Phase III, work on a highvalue health care team where they consider, research, develop, plan, and sometimes execute a quality improvement project in their field.

The 3 curriculum phases are linked through integration of 18 longitudinal content areas across the curriculum:

- Biochemistry

- Cells, tissues, and organs

- Child development, health, and illness

- Clinical microbiology and immunity

- Ethics and human values

- Evidenced-based medicine and informatics

- Genetics

- Health care delivery science

- Human anatomy and embryology

- Imaging

- Leadership and professional development

- Nutrition

- Oncology

- Pathology

- Pharmacology

- Physiology

- Race and health equity

- Substance use and pain 


\section{Assessment}

Geisel's medical education program objectives are aligned most closely with the ACGME domains of competence. However, through an iterative process, our curriculum committee has modified these domains to best fit our particular mission and values.

See Supplemental Digital Appendix 2-Program Objectives-at http://links.lww.com/ACADMED/A897.

The purpose of student assessment at Geisel is to ensure that our program contributes to the development of the complete physician by:

- Confirming students achieve our program's learning objectives and competencies

- Providing students with multiple opportunities for selfassessment through assessment design and feedback on performance

- Continually measuring instructional effectiveness through assessment

- Preparing students for USMLE examinations

Geisel uses both formative and summative methods to assess program objectives and individual learning objectives. Methods include written examinations, project assessments, clinical performance examinations (e.g., OSCEs), clinical documentation reviews, narrative assessments, oral patient presentations, and direct observations. Assessments are spaced out to promote reflection and self-assessment of performance. Formative feedback is provided in a timely manner to allow incremental self-improvement. Students also participate in peer assessment to learn how to give constructive feedback and aid their colleagues' growth.

In the clinical phases of the curriculum, midclerkship feedback process is standardized, thus each clerkship follows a similar protocol combining assessment of progress on completing essential skills and conditions, self-assessment on overarching program objectives, and faculty feedback on clerkship learning objectives. Student performance evaluations are standardized and competency based, with descriptive anchors using similar language across clerkships. Recently, we increased the number of peer-to-peer assessments on clerkship assignments such as case write-ups and student presentations. Finally, we have increased the number of OSCEs for each student to better assess integrated clinical skills.

\section{Parallel curriculum or tracks}

The Geisel School of Medicine has a single program of study. We do not offer a 3-year MD degree program, nor do we plan to in the near future.

\section{Pedagogy}

Our choice of pedagogical approaches is based on our commitment to:

- Engage students in a supportive yet challenging environment to ensure they establish a strong knowledge foundation and develop problem-solving and clinical reasoning skills
- Provide numerous opportunities for students to work in teams to practice and develop skills of active listening, effective communication, conflict management, and allocation of tasks

- Facilitate active discussion and debate, enabling students to take advantage of different perspectives to advance knowledge and improve decision-making

Pedagogical approaches used include:

- Case-based learning

- Clinical experience: ambulatory

- Clinical experience: inpatient

- Discussion: large group (>12)

- Discussion: small group $(\leq 12)$

- Laboratory

- Lecture

- Peer teaching

- Preceptorship

- Problem-based learning

- Role play/dramatization

- Self-directed learning/tutorial

- Simulation

- Standardized/simulated patients

- Team-based learning

- Video/podcast

- Virtual patient

Implementation of these principles has led to restructuring class activities, for example, limiting lectures to $\leq 40 \%$ of total contact time for each preclinical course and incorporating more activities using teamwork and peer learning. In Phases II and III, we have increased use of simulation and simulated patients. For example, surgery clerkship uses simulated patients to teach delivery of bad news and task trainers to teach nasogastric tube placement. Several clerkships have either adapted or enhanced use of videoconferencing, which has helped standardize didactic sessions across our geographically diverse sites.

\section{Clinical experiences}

Geisel students begin clinical learning experiences in the first week of Phase I in On Doctoring. This course provides longitudinal clinical experiences in which students practice clinical skills in small groups with 1 or 2 faculty facilitators for the duration of Phase I. A recent innovation is the use of simulated patient encounters in year 1 of the course, allowing a more uniform education of all students and resulting in excellent learning outcomes. In March of year 1, students transition from simulated patient encounters to community primary care practices. Each student is paired with a longitudinal faculty preceptor for clinic visits every other week until the end of Phase I.

During the clinical years, Geisel highly values its ability to engage students in a variety of care venues within and outside of our academic medical center. We use 3 major clinical affiliate health care systems - our local academic medical center, Dartmouth Hitchcock (DH), the nearby White River Junction Veterans Affairs Hospital, and California Pacific Medical Center (CPMC), a large, not-for-profit hospital in San Francisco, California. $\mathrm{CPMC}$ has its own residency and fellowship programs separate 
from DH. The remainder of our sites are mostly located in New England at a mixture of private practices and large and small community hospitals. In addition, we have affiliations with 2 Indian Health Service hospitals in the Southwest, opportunities in rural Alaska and Montana, a county hospital in Santa Clara, California, a private stand-alone children's hospital in Orange County, California, and the state mental health hospital in New Hampshire.

Like many medical education programs, finding a sufficient number of high-quality clinical sites and preceptors has become more challenging for Geisel. Clinical productivity pressures have increased regionally over the past decade. Additionally, the number of health professions education programs such as PA, APRN, and DO programs competing for clinical sites has increased. Once sites are identified and affiliations completed, designing and implementing clinical experiences is less of a challenge. Because we use so many sites, clerkship directors and coordinators are accustomed to providing the necessary outreach and development.

\section{Curricular Governance}

Our curricular governance committee, the Medical Education Committee (MEC), is charged with overseeing our curriculum. The committee consists of clinical and preclinical faculty appointed by the Geisel Faculty Council, student representatives who are elected by their peers, and nonvoting members including key faculty and staff responsible for curriculum implementation. The committee is chaired by one of its voting faculty members. The MEC charges subcommittees to work on various topics including course and clerkship reviews, curriculum revision, and educational program learning objectives. Geisel has both an assessment and an evaluation oversight committee. These committees are composed of key faculty, staff, and students and help to review and set standards for student assessment and evaluation of individual courses and the curriculum as a whole.

\section{Education Staff}

The Office of Medical Education supports coordination, planning, administration, and evaluation of the entire curriculum. This office is led by the senior associate dean for medical education (SADME) and includes directors of administration and finance, student accessibility and support, evaluation and assessment, and biomedical libraries. The Office of Clinical Education and Office of Preclinical Education each are led by an associate dean and director. These offices provide central support to individual course and clerkship directors. The Office of Computing includes an instructional designer and provides curricular support services such as tools for curricular monitoring. The primary medical education staff and faculty are responsible for the UME program alone. Geisel has a separate student affairs team that reports to the SADME and coordinates efforts related to professional development, career advising, and student accessibility and support. Administrative support for UME is provided by the dean and associate deans.

See Figure 1-Administration organizational chart.

In 2016, Geisel established the Department of Medical Education that includes foundational scientists, academic clinicians, and educational staff dedicated to pedagogical innovation and delivery of UME. The department chair is responsible for recruitment, oversight, and professional development of both primary and secondary appointed faculty.

\section{Faculty Development and Support in Education}

Geisel offers several opportunities for the professional development of faculty as educators and teachers. The UME Affairs Office and the Department of Medical Education cohost the Leading Voices in Medical Education lecture and workshop series featuring presentations by distinguished educators from across the country. Central support is provided to identify and send faculty and staff to regional and national educational meetings. Finally, Geisel has an academy of educators that produces a biannual symposium for the community and

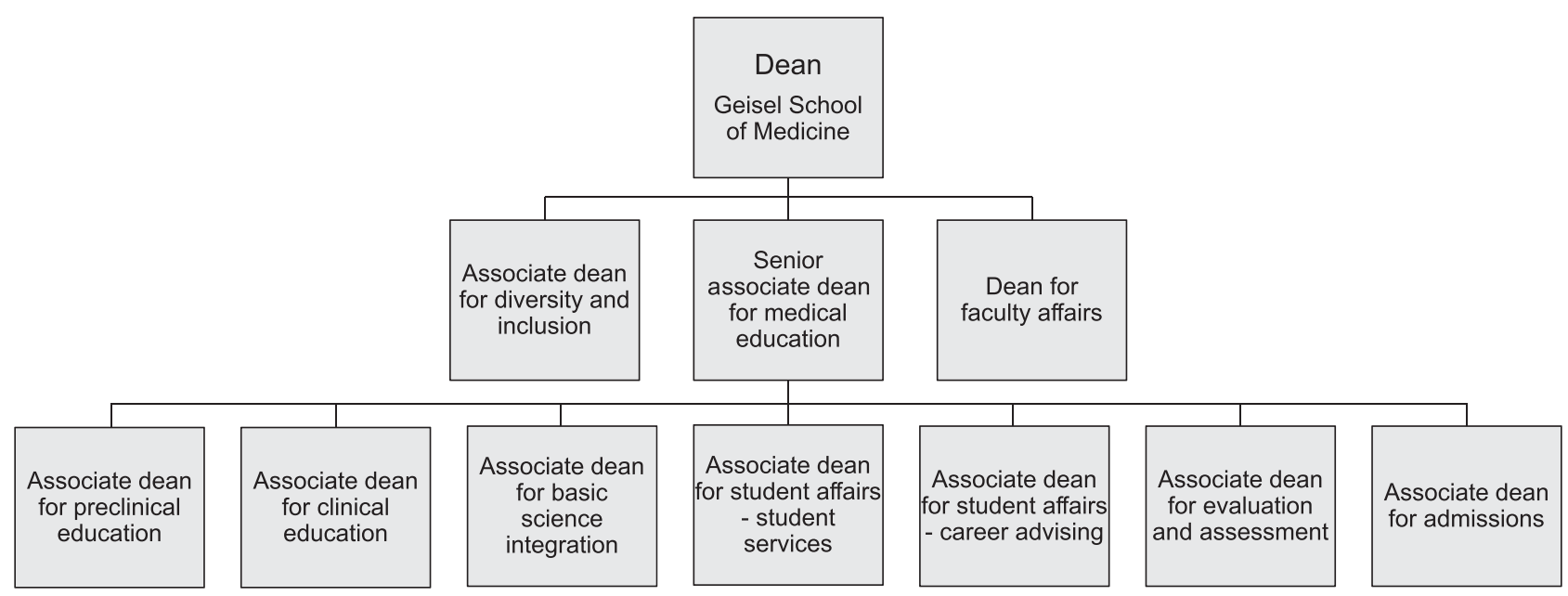

Figure 1 Administration organizational chart. 
maintains a repository of educational resources focusing on medical education best practices. The Geisel Academy of Master Educators is both honorific and service oriented, consisting of distinguished medical school faculty who are peer nominated and peer reviewed for selection.

Teaching and educational activities are very important in promotion and tenure decisions. Faculty must demonstrate evidence of scholarly accomplishment such as publication of content in peer-reviewed journals and excellence in teaching as evidenced by teaching awards, reviews by learners, and peer references. While many of our faculty appointments include educational duties, 2 tracks emphasize educational activities. In the tenure faculty line, individuals in the educator-scholar track are expected to be fully dedicated to innovation and excellence in the delivery of UME. Clinical faculty in the academic medical system faculty line also have a significant role in educational activities and are expected to make substantive contributions to the education of others including medical students, residents, fellows, colleagues, and other health care professionals. 


\section{Cooper Medical School of Rowan University}

Susan M. Perlis, EdD, Rose Kim, MD, MEd, and Annette C. Reboli, MD

\section{Medical Education Program Highlights}

The Cooper Medical School of Rowan University (CMSRU), founded in 2009, accepted our charter class in 2012. Located in Camden, New Jersey, one of our nation's most vulnerable communities, we are deeply committed to our motto "Camden is our classroom, Camden is our home." We have graduated 5 classes in our medical education program, all of whom have exemplified our commitment to provide humanistic education in the art and science of medicine within a scientific and scholarly community. We aspire toward excellence in patient care, innovative teaching, research, and service to our community. We are proud to be the 2019 recipient of the AAMC's Spencer Foreman Award for Outstanding Community Engagement.

CMSRU's medical education program includes the following:

- Clinical experiences that begin in the first year as part of an integrated basic and clinical science educational program that includes didactic, case-based, and self-directed learning.

- The interprofessional ambulatory clerkship experience for first-, second-, and third-year students is delivered in the student-run Cooper Rowan Clinic (CRC) and incorporates medical, pharmacy, physical therapy, and social work students.

- The hybrid third-year experience includes traditional core discipline blocks and a longitudinal integrated clerkship.

- Our systemic 4-year commitment to service learning focuses on the needs of our Camden community.

- A 4-year longitudinal course in health systems science called Scholar's Workshop culminates in a required capstone project.

- A unique primary care 3-year track leads to primary care residency in internal medicine or pediatrics.

\section{Curriculum}

\section{Curriculum description}

The CMSRU curriculum has 2 phases. Phase 1, foundation and integration, prepares students for Phase 2, application, exploration, and advancement. In Phase 1, students develop the essential scientific knowledge along with medical practice skills and behaviors to prepare them for clinical practice. Phase 2 provides for the expansion of basic knowledge in the clinical environment and supports career development. The entire curriculum has an emphasis on evidence-based medicine, critical thinking, problem solving, clinical reasoning, understanding

Acad Med. 2020;95:S309-S312.

doi: 10.1097/ACM.0000000000003423

Copyright (C) 2020 by the Association of American Medical Colleges

Supplemental digital content for this article is available at http://links.lww.com/ ACADMED/A923.

Year school was established: 2012.

School URL: http://cmsru.rowan.edu. the social context of illness, and developing the skills of lifelong learning. Four unique components provide students with opportunities to support their development as physicians:

- An interprofessional ambulatory clerkship experience for first-, second-, and third-year students that is delivered in the student-run, faculty supervised CRC.

- A hybrid third year that incorporates traditional core discipline blocks and the Cooper longitudinal integrated clerkship (CLIC). Students complete 6 -week blocks with 4 weeks devoted to inpatient experiences and 2 weeks completed in an integrated ambulatory experience with assigned preceptors from each of the core disciplines. This permits 14 weeks of continuity experience with their discipline-based preceptors over the course of the third year.

- A 4-year longitudinal Scholars Workshop course that incorporates evidence-based medicine, epidemiology, biostatistics, research methodology, quality improvement and patient safety, and health systems science, culminating in a capstone project with thesis and poster presentation.

- Students have the opportunity to take selectives and electives that connect them to the humanistic side of medicine and encourage development of cultural competence and ethical values.

See Supplemental Digital Appendix 1-Curriculum Schematicat http://links.lww.com/ACADMED/A923.

\section{Assessment}

The CMSRU medical education program objectives correspond to the framework of the ACGME domains of competence. In addition, we identified 3 competencies linked directly to our mission and core values: scholarly inquiry, learning and working in teams, and health partnership. We have disaggregated our competencies into specific objectives that link to national and locally developed assessments.

See Table 1-Sample Program Objectives and Assessment Methods.

\section{Parallel curriculum or tracks}

There are 2 curricular tracks at CMSRU: a traditional 4-year track and a 3-year accelerated track.

- The 3-year accelerated track is for students who are interested in primary care internal medicine or pediatrics and is referred to as PC3.

- Students accepted into the track have a provisional acceptance into our primary care residency in internal medicine or pediatrics, fostering the continuum of medical education across UME to GME.

- The PC3 program includes 2 unique courses (an immersion in communication skills and physical diagnosis and a course 


\section{Table 1}

\section{Sample Program Objectives and Assessment Methods}

\begin{tabular}{|c|c|}
\hline $\begin{array}{l}\text { Medical education } \\
\text { program objectives }\end{array}$ & Assessment methods (select sample) \\
\hline Medical knowledge & USMLE Step examinations, NBME subject examinations, CMSRU developed written and practical examinations \\
\hline Patient care & OSCES, clinical assessments, CLIC preceptor assessments, clinical oral examinations \\
\hline $\begin{array}{l}\text { Interpersonal and } \\
\text { communication skills }\end{array}$ & $\begin{array}{l}\text { OSCES, clinical assessments, weekly active learning group (ALG) assessments, narrative assessments, Scholars } \\
\text { Workshop module assessments }\end{array}$ \\
\hline Systems-based practice & $\begin{array}{l}\text { Clinical assessments, note review, Lean Six Sigma presentation, Scholars Workshop projects, examinations, } \\
\text { Scholars Workshop module assessments }\end{array}$ \\
\hline Scholarly inquiry & Scholars Workshop critical appraisal projects and capstone projects, weekly ALG assessments, OSCES, presentations \\
\hline Learning and working in teams & $\begin{array}{l}\text { TBL scores, weekly ALG assessments, Scholars Workshop module assessments, Life Stages Team Work assessment, } \\
\text { Lean Six Sigma presentation }\end{array}$ \\
\hline Health partnership & $\begin{array}{l}\text { Clinical assessments, service learning reflective essays, Scholars Workshop projects, ambulatory clerkship } \\
\text { behavior checklist }\end{array}$ \\
\hline
\end{tabular}

on transforming health care in urban environments), a patient-centered medical home clerkship that is a venue for interprofessional education, and a hybrid longitudinal integrated clerkship in the third year that is a modification of the CLIC for the 4-year track.

- There is a required subinternship at the end of the third year.

See Supplemental Digital Appendix 2-Primary Care Track Curriculum and Third-Year Details—at http://links.lww.com/ ACADMED/A923.

\section{Pedagogy}

As a new medical school, we have researched a variety of educational pedagogical activities and, in consideration of best practice in adult learning theory, have minimized the amount of time students spend in lectures. We have refocused learning and instructional time to emphasize case-based learning, group discussions, laboratory and application sessions, and simulation/ standardized patients.

- Active learning groups (ALGs) encompass 6 hours weekly for first- and second-year students. The cases are developed by course faculty, reviewed by the ALG Subcommittee of the Curriculum Committee, and cofacilitated by both basic science and clinical faculty teams. There are 8-9 students per group.

- Group discussions are the focus of both the Scholars Workshop and Foundations of Medical Practice courses. Faculty facilitators provide short instructional segments and discussions. The Foundations of Medical Practice course incorporates workshops that develop clinical skills.

- Laboratory and application sessions are part of the core blocks in Phase 1 of the curriculum. The fully integrated nature of the basic sciences within block structures allows for dissectionbased anatomy to occur from the latter part of the first-year curriculum, beginning with the skin and musculoskeletal systems block through the entire second year. In courses that do not have anatomical dissection, application sessions permit deep exploration of virtual microscopy, team-based learning activities, and simulations.

- The Clinical Skills and Simulation Center provides instruction for a variety of courses and clerkships throughout the curriculum. In fall 2019, our simulation center relocated to a new 12,000-squarefoot facility. Our students in all curricular years are able to take advantage of on-demand standardized patient activities. Teams of 4 students can request a 2-hour practice session to review and practice skills being developed within the curriculum, as well as prepare for their OSCEs or the USMLE Step 2 CS examination.

\section{Clinical experiences}

All of our clinical experiences are provided through our affiliation with Cooper University Health Care (CUHC). The expansive network of services and disciplines and wide breadth of patient population associated with CUHC allows students to have a variety of experiences at every level of their training.

- CMSRU students are involved in patient care and clinical reasoning activities from the beginning of the first year. The ambulatory clerkship (years 1-3) adopted an interdisciplinary team-based approach to patient care. Teams are made up of first- through third-year medical and pharmacy students. These comprehensive teams provide care for CRC patients under the supervision of faculty from both disciplines. Both medical and pharmacy students have a specific role on the team relative to their level of training. All patients receive medications via the student-run pharmacy. A social work student intern staffs the clinic and provides support for issues that surface during the patient visit such as housing or food insecurity. Two clinics per month include physical therapy students. Patients may be referred to other services at CUHC. These additional services are provided at no cost to the patient, and 1 or 2 students often accompany patients to their referral visits. This interprofessional team approach provides an invaluable service to the most vulnerable members of our Camden community. 
- All CMSRU students rotate exclusively through CUHC in their third-year clerkships. This permits students continuity of experience with the health system, the EMR, and patient population. The fourth-year students have 4 required clerkships: critical care, emergency medicine, subinternship with student choice of discipline, and interprofessional care of patients with chronic conditions (ICCC). A unique clerkship, ICCC addresses the specific needs of these complicated patients within several pillars of care:

- The Urban Health Institute is made up of interdisciplinary teams that support patients to ameliorate the impact of poverty and chronic stress as social determinants of health.

- ICCC hematology-oncology emphasizes goals of care in treatment and transitions to hospice.

- Geriatric medicine/postacute long-term care emphasizes how aging, compounded with multiple complex medical conditions, affects functional, cognitive, and emotional status.

- Palliative care medicine works with the inpatient palliative care service.

- Collaborative/transitional care provides a combination of experiences in family medicine practice using collaborative care teams, and with inpatient transitional care nurse teams addressing the transition from the acute care unit to homebased care.

- Metabolic and bariatric surgery provides an experience with inter disciplinary teams to support patients to reach their health goals.

- Physical medicine and rehabilitation offers experience with well-coordinated interprofessional teams that improve patient function and quality of life.

\section{Curricular Governance}

The CMSRU Curriculum Committee comprises elected and appointed faculty members with voting privileges and Office of Medical Education ex officio members, without vote. The associate dean for medical education works closely with the Curriculum Committee chair to operationalize the decisions made by the committee. There are 5 permanent subcommittees: Phase 1, Phase 2, Assessment, ALG Case Study Review, and Exam Question Review. Several ad hoc subcommittees assemble as necessary to address curricular enhancements, the biennial review of curricular phases, or the full review of the entire curriculum.

See Figure 1-Curricular governance, support, and implementation.

\section{Education Staff}

CMSRU is responsible for both undergraduate and graduate medical education. The Office of Medical Education is responsible for implementation of the educational program; assessment of students; learning support; and evaluation of courses, clerkships, and faculty. There is a separate Office for Graduate Medical Education that has oversight of GME. The Office of Student Affairs is responsible for nonacademic student support, wellness, activities, career and personal advisement, and student development. The Office of Medical Education has responsibility for the administration, planning, implementation, and evaluation of the curriculum and for the development and maintenance of the tools to support curriculum delivery, monitoring, and management. A variety of software tools are used to enhance delivery of the curriculum including one 45 for our assessment, evaluation, and curricular mapping. Examsoft is used for our examination system, and Progress IQ is used to provide dashboard services of student academic success for our students, advisory college directors, and administrative team.

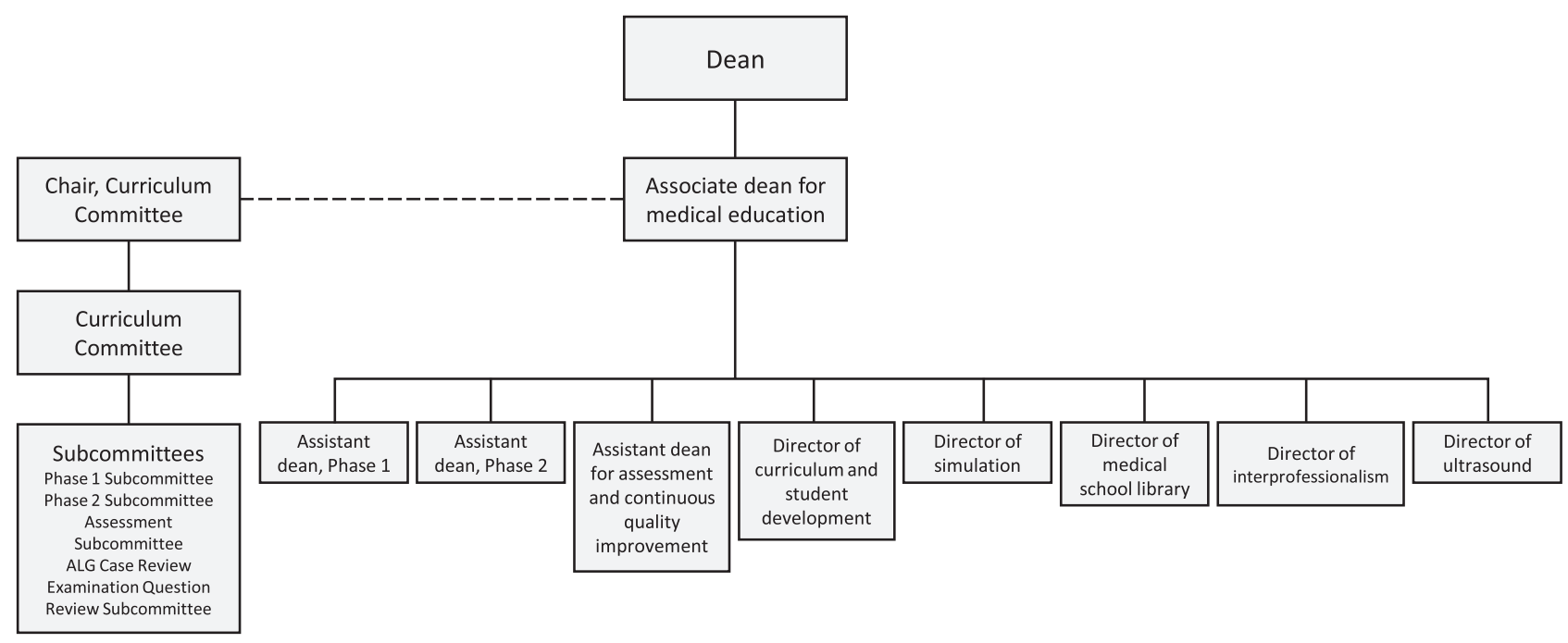

Figure 1 Curricular governance, support, and implementation. 


\section{Faculty Development and Support in Education}

CMSRU faculty development programs assist our faculty to provide exceptional educational experiences for our UME and GME learners, and promote professional vitality and growth. In addition, teaching is highly valued at CMSRU and is 1 of the 4 domains by which faculty are assessed on an annual basis and for promotion and/or tenure.

- Faculty development programs include the CMSRU medical education grand rounds series, targeted medical educationrelated workshops specific to UME and/or GME, and a research seminar series. On a biannual basis, CMSRU hosts a faculty development week; experts in the field of medical education are invited to address specific needs in medical education.

- Two academic tracks, the clinician-educator track and the academic educator track, endorse excellence in teaching as part of the criteria for promotion and/or tenure.

- The Scholarship of Practice and Teaching Pathway for clinical faculty provides an additional means to promotion for clinical faculty whose primary interest is in clinical medicine and teaching. 


\title{
Hackensack Meridian School of Medicine at Seton Hall University
}

\author{
Miriam Hoffman, MD, Keith Metzger, PhD, Ofelia Martinez, MD, and Elizabeth Koltz, EdM
}

\section{Medical Education Program Highlights}

To create the new Hackensack Meridian School of Medicine (SOM) at Seton Hall University, we began with the vision: Each person in New Jersey, and in the United States, regardless of race or socioeconomic status, will enjoy the highest levels of wellness in an economically and behaviorally sustainable fashion. This informed not only our curricular content but also our pedagogies and how we structured the school:

- Community and the determinants of health (DoH): At the center of the vision is the role that community and context have on health and well-being. The framework for the entire SOM curriculum is the DoH: the different types of factors that drive health outcomes, categorized into the biologic/genetic, behavioral, social, environmental, and health care itself. At the heart of the SOM curriculum is the longitudinal Human Dimension course in which medical student pairs are matched with families in the community whom they follow over time. Experiences in the families' homes, communities, and health care settings bring the DoH to life for the students. Students are trained to help and provide benefit to their family and community partners. Students also complete a community assessment project that leads to a community health project in their matched community. All content learned in the Human Dimension course is linked to what they are learning in the rest of the curriculum.

- Individualized educational program: Another central feature of the SOM curriculum is its ability to be individualized to each student's professional goals and developmental needs. Our 3+1 model includes a 3-year core curriculum (Phases 1 and 2) for all students, plus an individualized fourth year (Phase 3 ). Students work with their advisors to plan a Phase 3 that meets their individual needs and goals. They can obtain a second degree or graduate certificate or complete a research year, communitybased project, or clinical immersion. They also have the option of graduating after 3 years and entering residency within the Hackensack Meridian Health (HMH) network.

- Integrated and patient-centered curriculum: Courses in the Phase 1 (preclerkship) curriculum integrate content from the biomedical, behavioral, social, and health system sciences. Each week is framed by a weekly patient presentation in which students generate hypotheses that connect the underlying basic science mechanisms explaining aspects of the case, integrate

Acad Med. 2020;95:S313-S317.

doi: 10.1097/ACM.0000000000003462

Copyright (C) 2020 by the Association of American Medical Colleges

Supplemental digital content for this article is available at http://links.Iww.com/ ACADMED/A950.

Year school was established: 2018.

School URL: https://www.shu.edu/medicine. all content learned within the week, and link the case and the science content to different $\mathrm{DoH}$.

\section{Curriculum}

The $3+1$ curriculum includes the core curriculum: Phase 1 (16-month preclerkship) and Phase 2 (clerkships and advanced clinical rotations), followed by the individualized Phase 3 .

See Supplemental Digital Appendix 1-Curriculum Schematicat http://links.lww.com/ACADMED/A950.

\section{Parallel curriculum or tracks}

One option for Phase 3 is graduation after 3 years with entry into residency within the HMH network. The HMH network has over 190 first-year residency positions in 15 specialties. Students begin the application for this option during the second year and do not make a final commitment until they have completed all clerkships. This is an option for students who have clarity on their career goals and have demonstrated strong academic success.

\section{Assessment}

The SOM educational program objectives (EPOs) were derived from the AAMC's Physician Competency Reference Set. Additional EPOs were created and existing EPOs were modified to more fully reflect the SOM vision and goals for our graduates.

In addition, the SOM's developmental and integrated 3-year core curriculum uses 18 Entrustable Professional Activities (EPAs). These include the 13 AAMC Core EPAs as well as 5 EPAs we developed so that we could fully represent the SOM graduation goals.

Our 18 EPAs form the framework, milestones, and developmental competencies for Phase 1 and Phase 2. All feedback, formative assessment, and summative assessment are structured and delivered using these EPAs.

The 18 EPAs are assessed longitudinally over Phase 2, with increasing expectations over the course of the year. Various methods, both clerkship-specific and longitudinal, are used, including:

- Observation and assessment in the clinical setting, both workplace-based assessment and summative overall assessment; final clinical evaluations are synthesized and created by competency and grading committees

- Assessments in the simulation center, including high-fidelity simulation, standardized patient encounters, and part-task simulation

- Clerkship-based assignments

See List 1-EPAs Taught and Assessed.

See Chart 1-Content of Additional EPAs. 


\section{List 1}

\section{EPAs Taught and Assessed}

1. History and physical examination ${ }^{a}$

2. Prioritize a differential diagnosis ${ }^{a}$

3. Diagnostic and screening tests ${ }^{a}$

4. Orders and prescriptions ${ }^{a}$

5. Documentation ${ }^{a}$

6. Presentation ${ }^{a}$

7. Clinical questions and evidence-based care

8. Handoffs

9. Interprofessional collaboration

10. Acute care

11. Informed consent

12. Procedures

13. Systems failure/patient safety

14. Communication skills ${ }^{\mathrm{a}}$

15. Counseling and education

16. Developing care plans

17. Professionalism and growth ${ }^{a}$

18. Systems, communities, and solutions ${ }^{\mathrm{a}}$

aEPAs taught and assessed by all clerkships. Each clerkship assesses a subset of the other EPAs as well.

See Supplemental Digital Appendix 2-Competencies, Objectives, and Assessment Methods_-available at http://links. lww.com/ACADMED/A950.

\section{Pedagogy}

Each teaching method used in Phase 1 was chosen to build specific skills needed for clinical practice.

During Phase 1, each week is framed by our patient presentation problem-based learning (PBL) curriculum (PPPC). Each week begins and ends with a weekly patient presentation, based on a hybrid of case-based learning and modified PBL. Case-based learning is introduced Monday morning with the patient presentation. Students review the clinical information and develop a differential diagnosis. The case evolves in basic science and clinical skills teaching sessions throughout the week, pushing students to learn underlying mechanisms of disease or treatment. Students also identify questions related to the case and select self-directed learning research topics they present during Friday morning small group, using peer teaching to facilitate a discussion applying information learned to the case. Throughout the week each student creates an individual concept map using the week's patient presentation as a scaffold, connecting and integrating content from all sciences learned throughout the week, and identifying specific DoH at play. During Friday morning small group, the team creates a team concept map integrating the week's content and information from selfdirected research presentations.

Large-group active learning sessions occur 3 to 5 times per week within Phase 1. Flipped classroom methodology is used, with prework assigned and in-class activities focused on knowledge application. Videos and podcasts are used for prework and supplemental learning.

Team-based learning is used regularly in Phase 1, approximately weekly.

Small-group discussions occur in Friday morning PPPC sessions as well as Wednesday morning Human Dimension and Clinical Skills sessions.

Students participate in laboratory sessions approximately once per week during Phase 1, including anatomy (dissection and prosection), pathology, radiology, simulation, and others.

Role play, dramatization, and simulation are used throughout the curriculum to promote active, engaged learning and align with our goal for explicit clinical relevance, maintaining focus on the patient. These experiential sessions include video review, reflection, and development of growth goals.

Simulation and standardized/simulated patient sessions are core components of the 3-year clinical skills curriculum and are also used in basic science teaching sessions.

\section{Chart 1}

\section{Content of Additional EPAs}

\begin{tabular}{|c|c|c|c|c|}
\hline $\begin{array}{l}\text { EPA } 14 \\
\text { Communication } \\
\text { skills }\end{array}$ & $\begin{array}{l}\text { EPA } 15 \\
\text { Counseling and } \\
\text { education }\end{array}$ & $\begin{array}{l}\text { EPA } 16 \\
\text { Developing } \\
\text { care plans }\end{array}$ & $\begin{array}{l}\text { EPA } 17 \\
\text { Professionalism } \\
\text { and growth }\end{array}$ & $\begin{array}{l}\text { EPA } 18 \\
\text { Systems, communities, } \\
\text { and solutions }\end{array}$ \\
\hline Rapport & Health literacy & Health promotion & Conduct & Health inequities \\
\hline $\begin{array}{l}\text { Patient perspective } \\
\text { and empathy }\end{array}$ & Patient education & Chronic disease management & Humanism & $\begin{array}{l}\text { Determinants of health/social } \\
\text { determinants of health }\end{array}$ \\
\hline Cultural humility & Shared decision-making & $\begin{array}{l}\text { Determinants of health } \\
\text { - Broad assessment } \\
\text { - Management plan development } \\
\text { - Interprofessional collaboration }\end{array}$ & Growth & Community \\
\hline Sharing information & Motivational interviewing & & Leadership & Systems \\
\hline
\end{tabular}


The Human Dimension course uses robust experiential learning in families' homes and community settings, as well as workshops on the skills needed for community-based work.

See Figure 1-Sample week: Phase 1.

\section{Clinical experiences}

Throughout the curriculum, students are part of the health care team at a variety of sites across the HMH network, which includes 17 hospitals and over 500 outpatient, community-based, and other care settings. Students complete clinical experiences across a range of settings.

\section{Longitudinal clinical experience}

Beginning in the first year, students are assigned to an ambulatory-based longitudinal clinical preceptorship. The mentoring and role modeling relationships from this longitudinal format build students' professional identity, transition them to the culture and expectations of the clinical workplace, and provide professional development that fosters

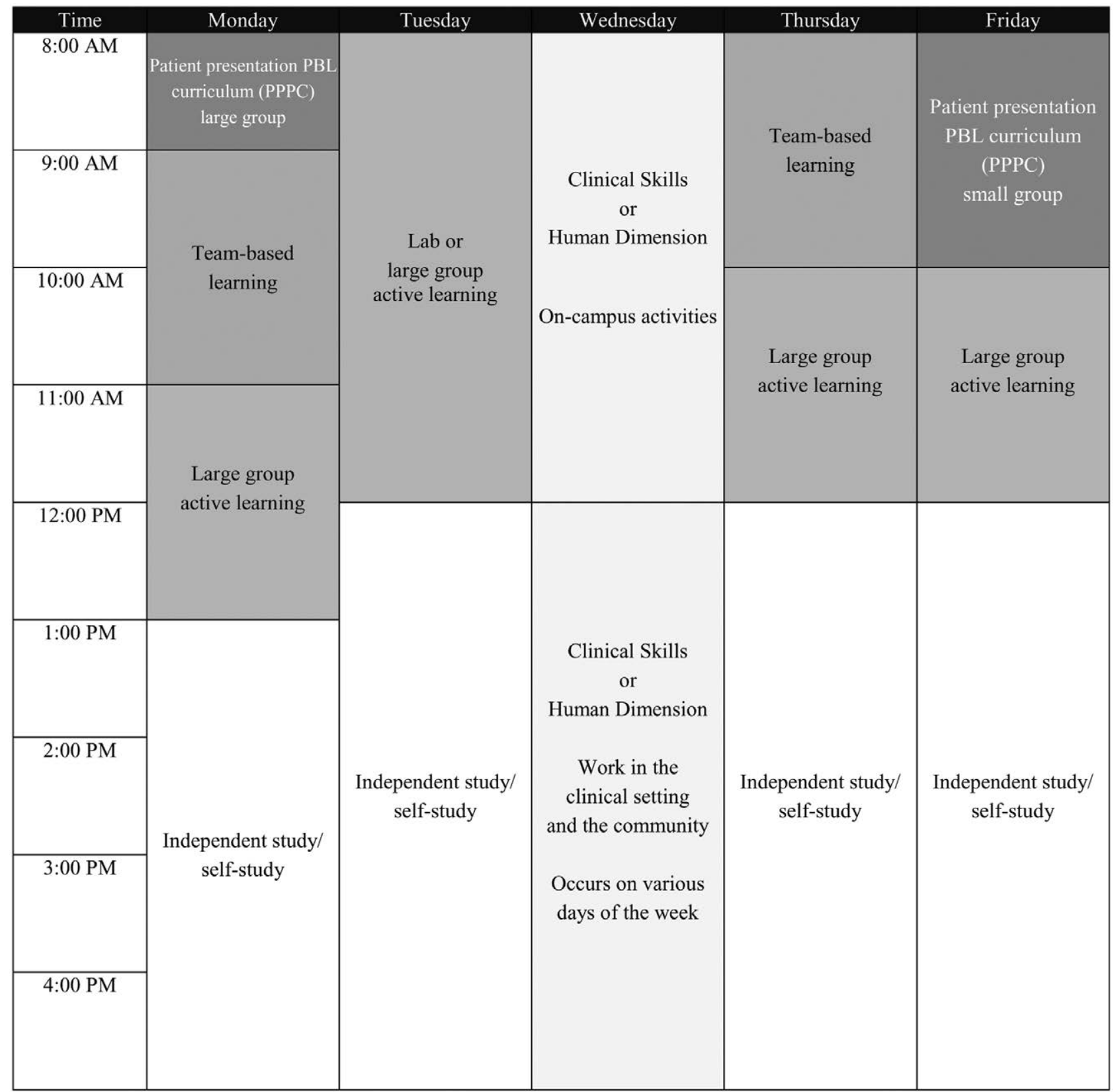

Figure 1 Sample week: Phase 1. 
self-assessment, reflection, and growth. Before beginning, students are trained in patient-centered communication, clinical reasoning, and full history and physical examination so that they can practice and develop these skills in the clinical setting.

\section{Community-based experiences}

Aligned with the SOM vision, many required experiences occur in community and ambulatory sites. All clerkships have an emphasis on the outpatient setting, with each clerkship's outpatient clinical time ranging between $30 \%$ and $80 \%$.

The experiential and service-learning curriculum within the Human Dimension course takes place in community-based settings and families' homes. Students develop communication and patient-centered skills through these experiences.

\section{Challenges in designing and implementing clinical experiences} for medical students

The robust and progressive HMH network is a significant strength of our school. It provides a breadth of learning opportunities, opportunities for integration, and intentional growth of skills across the developmental arc of UME to GME to clinical practice. The cohesive structure of our health network from training to practice provides great opportunities for medical education research.

Challenges encountered in implementation have included:

- Recruitment of clinical faculty

- Onboarding students across multiple sites and settings

- Faculty development

- Logistics and leveraging the strengths of the vast health care network

\section{Curricular Governance}

Our Medical Education Committee has 3 standing subcommittees, one for each phase of the curriculum. Each of the 3 phase subcommittees are responsible for the educational outcomes; the development, review, and enhancement of objectives, content, student workload, student performance, evaluations, and integration of the courses/clerkships; and other curricular components within their phase, as well as of the phase as a whole. The Phase 3 curriculum subcommittee is also responsible for reviewing and monitoring the outcomes of Phase 3 programs, including student completion of degrees and programs, residency match, and internship preparation.

Centralized governance and administration (processes and support) include instructional design, faculty development, administrative support of teaching activities, and compensation for teaching faculty in the preclerkship curriculum. Each curriculum subcommittee, and ultimately the Medical Education Committee, is responsible for the design, implementation, and continuous enhancement of each curricular component and the curriculum as a whole.

Departments are responsible for the quality and outcomes of their courses/clerkships and faculty and are provided data and support for this from central SOM offices. Budgeting of teaching time for clinical faculty is decentralized and varies based on clinical site, faculty employment structure, and responsibilities within the SOM.

\section{Education Staff}

The Office of Medical Education (OME) supports the design, implementation, and continuous enhancement of the educational program. The OME has 5 divisions:

- Educational and curriculum design and delivery

- Curricular administration

- Institutional effectiveness and assessment

- Medical education research and innovation

- Community programs

Administrators and staff support faculty in all phases of the curriculum and collaborate closely with other units of the SOM. The OME administers and supports curriculum committees, the curriculum management plan, and assessment. This includes development, maintenance, and utilization of the learning management system; the curriculum database; and our longitudinal medical education research database (LongMED).

Academic support and advising is a unit within the Office of Student Affairs and Wellbeing, providing academic and learning support to students. Faculty, advisors, and staff within the Office of Student Affairs and Wellbeing and the OME work together closely to support student success.

The following units within the SOM have responsibilities and deans related to UME, faculty development, and learner support:

- Office of the Dean

- OME

- Office of Student Affairs and Wellbeing

- Office of Faculty/faculty development division

- Office of Diversity and Equity

- Office of the Medical Education Continuum

\section{Faculty Development and Support in Education}

Faculty development is a collaboration between the Office of Faculty and the OME, covering a wide range of topics from curriculum development, to classroom and clinical teaching skills, to scholarship and academic development. Faculty development trainers within the Office of Faculty provide training and development for clinical faculty in on-campus, virtual, and clinical settings. Faculty participate in development before beginning a teaching responsibility as well as in development sessions throughout the course/ clerkship.

The vice dean for the medical education continuum and the associate dean of diversity and equity serve as co-chief academic officers of the health network, with GME and CME falling under their scope. Faculty development efforts 
are coordinated and aligned across the UME-GME-CME continuum.

As the next phase of faculty and professional development emerges, a teaching academy may be developed. It is a priority of the SOM to create a culture of learning and growth for students as well as faculty and staff.

\section{Role of teaching and education activities in promotion}

Teaching and education are important factors that are considered for promotion decisions.
The Office of Faculty, Appointment and Promotions Committee, department chairs, and the SOM dean all play a role in the promotions process. The SOM does not offer tenure.

Faculty submit a promotion application to their chair and the Office of Faculty, including an educational portfolio, curriculum vitae, and letters of reference. Criteria for appointment, renewal, and promotion are based on performance in teaching, scholarship (including research), and service (including clinical service). 


\section{Rutgers New Jersey Medical School}

Maria Soto-Greene, MD, MS-HPEd, Sophia Chen, DO, MPH, Bart Holland, PhD, and Christin Traba, MD, MPH

\section{Medical Education Program Highlights}

The strategic vision of Rutgers New Jersey Medical School (NJMS) is to optimize health and social well-being by preparing humanistic leaders in global health care through education of physicians and scientists, building on our strengths of diversity, hands-on clinical training, urban health care programs, and transformative research. In 2015, NJMS's curriculum revision resulted in an organ systems-based curriculum with the following guiding principles:

- Integration of basic and clinical sciences vertically and horizontally

- Longitudinal service learning

- Professional identity development

- Interprofessional and interdisciplinary opportunities

- Health systems sciences

- Active learning activities with innovations in educational technology

- Learner readiness

Furthermore, NJMS's curriculum has unique strengths with a special commitment to the health of underserved communities.

- Health equity and social justice content is integrated throughout all required courses and clerkships to prepare future physicians to be responsive to societal issues.

- A community-engaged service learning requirement teaches students to identify and reflect upon community needs and assets, fostering an enhanced sense of civic responsibility.

- NJMS was one of the first medical schools in the United States to provide training required by the Drug Abuse Treatment Act of 2000 (DATA 2000) for graduating students, which allows them to be eligible for the waiver required by licensing boards.

- A health systems science curriculum was implemented including topics such as patient safety, value-based care, interprofessional collaboration, and clinical informatics.

- Distinction tracks in the areas of entrepreneurship and innovation in medicine, global health, medical education, service, research, and urban health provide enhanced professional development for select students.

- An accelerated 3-year primary care MD track was implemented in July 2019.
Acad Med. 2020;95:S318-S321.

doi: 10.1097/ACM.0000000000003468

Copyright (C) 2020 by the Association of American Medical Colleges

Supplemental digital content for this article is available at http://links.Iww.com/ ACADMED/A956.

Year school was established: 1954.

School URL: http://njms.rutgers.edu.

\section{Curriculum}

\section{Curriculum description}

The curriculum, divided into 3 phases, can be visualized as blocks where each section is taught with an integrated organ systems approach and/or a clinically focused component. These are complemented by threads that cut across the curriculum.

Phase 1 (years 1 and 2), core biomedical content and clinical integration:

- Year 1: Foundations of body systems, followed by an organ systems-based curriculum including musculoskeletal and integumentary, cardiovascular, pulmonary, and renal courses

- Year 2: Digestive, genitourinary, and endocrine systems; and neurology, psychiatry, and biostatistics courses

Phase 2 (year 3), clinical immersion:

- Ambulatory primary care, medicine, pediatrics, surgery, neurology, obstetrics-gynecology, psychiatry, integrative weeks, and electives

Phase 3 (year 4), clinical differentiation:

- Emergency medicine, rehabilitation medicine, acting internship, electives, transition to residency (capstone)

See Supplemental Digital Appendix 1-Curriculum Map-at http://links.lww.com/ACADMED/A956.

Key features of the curriculum:

- Dedicated integration/assessment weeks and capstone courses to enhance critical thinking and problem-solving skills through clinical case-based discussions

- Protected half days in Phase 1 curriculum for individualized learning and wellness

- Ample time for career exploration through electives and selectives

- Specialty-specific skills preparation for transition to residency

\section{Curriculum changes since 2010}

An accelerated 3-year primary care track was implemented while maintaining the same class size.

An organ systems-based curriculum and mappable attainment of student competencies in Entrustable Professional Activities (EPAs) were implemented and linked to the 6 required curriculum competencies. In addition, students have a greater opportunity to track their progress and actively engage in their learning process. We introduced 2 integrative weeks during Phase 2 (year 3 ) focusing on wellness, clinical reasoning, implicit bias, and EPA assessment. A capstone (transition to residency) course was implemented during Phase 3 and includes: 
- Completion of Institute for Healthcare Improvement Basic Certificate in Quality and Safety

- Topics on fatigue, professionalism, teaching medical students, writing orders, transitions of care, informed consent, and preventing burnout

- Basic ultrasound skills workshop

See Table 1-Program Objectives and Assessment Methods.

The medical education program objectives are linked to ACGME competencies and AAMC Core EPAs. In addition to summative assessments and shelf exams, there are formative assessments throughout the curriculum. Competencies in clinical and basic science knowledge are assessed with graphical feedback at regular intervals during Phase 1.

Competencies in EPAs are assessed using integrative OSCEs at strategic points:

- Phase 1: EPAs 1 (history and physical), 2 (prioritize differential diagnosis), 5 (document clinical encounter), and 6 (oral presentation)

- Phase 2: Two integrative weeks with assessments of EPAs 1, 2, 5, 6 with EPA 3 (recommend and interpret common diagnostic tests), 10 (recognize emergent/urgent conditions), and 12 (perform general procedures of a physician)

- Phase 3: Peer-to-peer assessments for EPAs 1, 6, 10 with EPA 4 (enter and discuss orders/discharge a patient), 8 (patient handover to transition care responsibility), and 11 (informed consent)

This allows students to have multiple assessment points and individualized remediation plans in preparation for residency.

\section{Parallel curriculum or tracks}

In July 2019, NJMS enrolled its first cohort into the accelerated 3-year primary care MD program, addressing the national and state shortage of primary care physicians. This parallel curriculum builds upon the strengths of the 4-year curriculum with clinical experiences and community activities. Students commit to a primary care residency (pediatrics, internal medicine, combined internal medicine/pediatrics) and receive a conditional acceptance into the respective residency program.

Highlights of the 3-year curriculum include early clinical immersion, a longitudinal primary care preceptorship starting in year 1, and completion of a population health certificate in conjunction with the Rutgers School of Public Health.

\section{Pedagogy}

NJMS has carefully reviewed its curriculum to increase active as well as independent learning. We have increased small-group sessions, added journal clubs, engaged patients as teachers, and reduced the number of lectures and large-group didactics.

We use flipped classroom sessions, peer and faculty feedback on oral presentations through video submissions, individualized learning plans to identify gaps and weaknesses in clinical skills from the EPA OSCEs, peer teaching, and skills workshops. This is in addition to team-based learning, podcasts, clinical smallgroup sessions using standardized patients and simulation, and e-learning modules.

\section{Clinical experiences}

- NJMS is known for its historic hands-on clinical experiences with a diverse patient population inclusive of vulnerable and underserved communities.

- Clinical sites include the VA New Jersey Healthcare System, university- and community-based hospitals, as well as community- and university-based primary care practices.

- Early exposure to patients is part of the patient-centered medicine program that begins during Phase 1, where students spend 10 weeks in an ambulatory primary care preceptorship

\section{Table 1}

\section{Program Objectives and Assessment Methods}

Medical education program objectives

Mastery and integration of clinical, basic, and health systems sciences

Excellence in clinical skills

Excellence in professionalism and humanism

Commitment to the health of the community and appreciation of social and cultural diversity

Dedication to lifelong learning and personal wellness

Development of effective skills in education and communication

\section{Assessment methods}

Written exams, quizzes, shelf exams, team-based learning (TBL), reports, write-ups, progress notes, lab sessions, presentations, clinical performance, OSCE, Patient Oriented Problem Solving exercise, journal club exercise

Written exams, quizzes, shelf exams, TBL, reports, write-ups, progress notes, lab sessions, presentations, clinical performance, teaching OSCE, OSCE, simulation, case presentations

Written exams, quizzes, TBL, reports, lab sessions, presentations, clinical performance, OSCE, mandatory training-sign-in, HIPAA training

Written exams, quizzes, shelf exams, presentations, write-ups, narrative writing, clinical performance, teaching OSCE/OSCE, integrative OSCE, clerkship reflections, self-test and self-reflection on implicit bias

Written exams, shelf exams, TBLs, write-ups, progress notes, simulation, presentations/reports, clinical performance, OSCE, teaching OSCE, Patient Oriented Problem Solving exercise, integrative OSCE, clerkship reflections, case presentations, interviews/discussions

Written exams, shelf exams, TBLS, lab, write-ups, progress notes, simulation, presentations/reports, clinical performance, OSCE, teaching OSCE, student family health care clinic, Collaborative Approach to Learning Medicine, and other organizations 
setting. During Phase 2, the family medicine clerkship is predominantly situated in community-based practices.

- Electives are offered in community-based health care settings through the departments of Emergency Medicine, Internal Medicine, Pediatrics, and Family Medicine. NJMS also has several core community-based affiliate partners that offer electives in the vast majority of specialties.

- The major challenge in implementing clinical experiences is the placement of learners in available primary care community sites for longitudinal preceptorship experiences.

\section{Curricular Governance}

- The executive vice dean is the chief academic officer. Management of the educational programs is a shared responsibility of the academic departments and the Office of Education, which reports to the executive vice dean.

- The Faculty Organization solicits and provides input to the Committee on Committees, which selects members for the standing committees that are approved by the Faculty Council.

- The Committee on Curriculum, Academic Programs, and Policies (CAP2), a standing committee, is charged with the design, evaluation, revision, approval, and oversight of the curriculum as well as overall vertical and horizontal integration of the education program. It makes recommendations to the dean and Faculty Council regarding implementation of these programs.

- There are 2 standing subcommittees of the CAP2, the Preclerkship Curriculum Committee (PCC) and the Clinical Curriculum Committee (CCC).

- The PCC includes all course directors in the first 2 years of the medical school curriculum.

- The CCC includes all clerkship directors of mandatory thirdand fourth-year clerkships.

- In addition, all committees include student representatives appointed by Student Council and ex officio members from the Office of Education.

- Four task forces are assigned to review our 6 medical education program objectives. They are charged with reviewing program objectives, identifying measurable outcomes used as evidence that competencies are being met, and monitoring selected program outcomes.

- Task forces report their recommendations biannually to CAP2. Subsequently, CAP2 reviews this information, along with annual course and clerkship evaluations, as a means of overall program evaluation of the curriculum.

See Figure 1-Curriculum governance.

The executive vice dean works closely with the academic departmental chairs; course and clerkship directors; and faculty

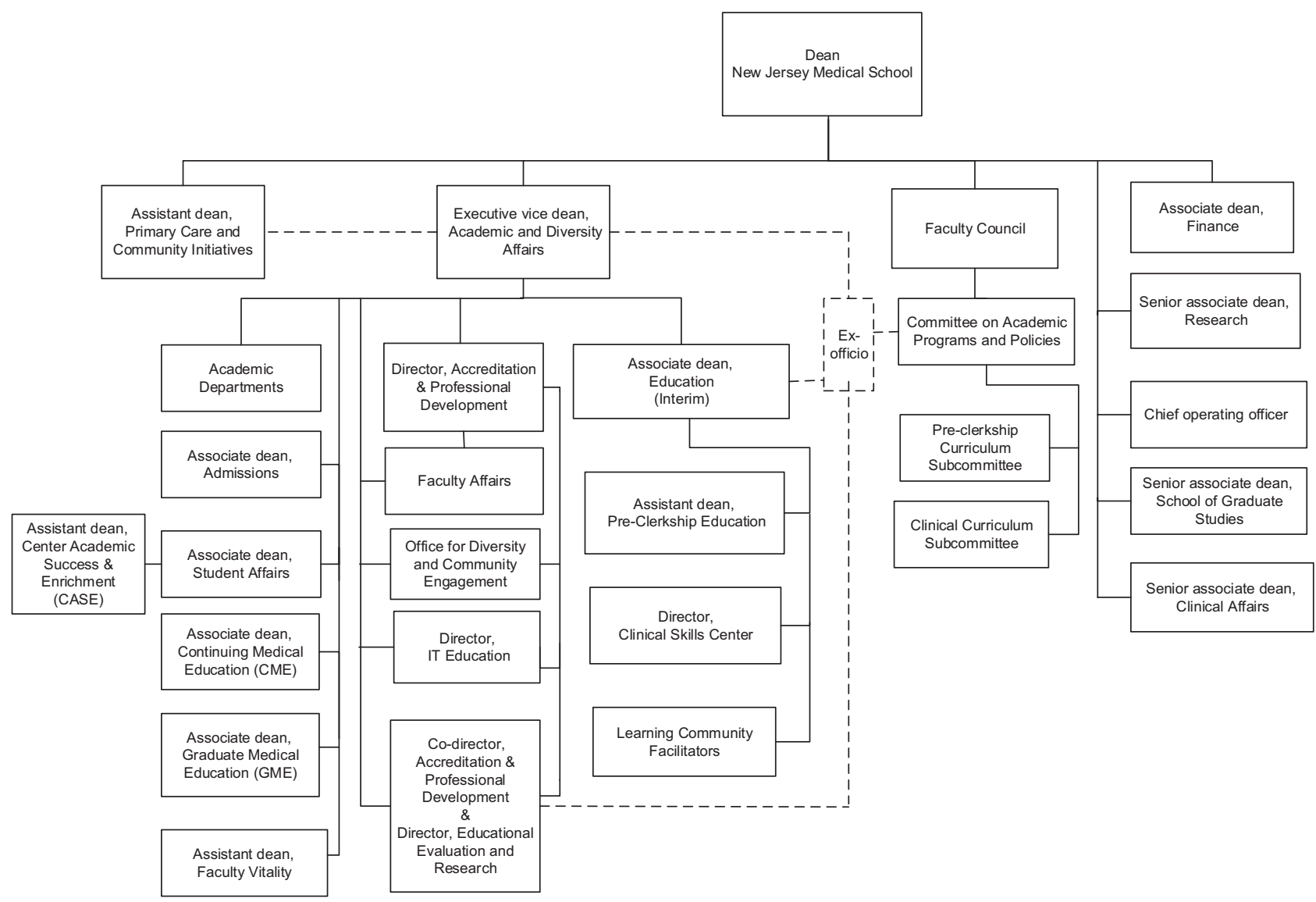

Figure 1 Curriculum governance. 
to implement innovations in education, assessment, and evaluation as they pertain to undergraduate medical education.

In 2015, with the rollout of the new curriculum, all preclerkship courses were managed by the Office of Education with course leadership and teaching faculty financially supported both by the Office of the Dean and departmental mission-based funding. The staff of the Office of Education support the course and clerkship directors in all aspects, including organization, outcome-based measures, and peer review. All assessment and academic support are centralized.

\section{Education Staff}

- The scope and influence of the Office of Education have expanded over the last 10 years.

- The associate dean for education, along with assistant deans for preclerkship and clerkship education as well as a director for evaluation and research, provide monitoring, management and support of content, delivery, and outcomes for all courses and clerkships. The assistant deans contribute to faculty development, which extends to our affiliates.

- The director of clinical skills supervises the standardized patient program as well as simulation for undergraduate medical education.

- The executive vice dean recently recruited a dedicated education technology expert to facilitate innovations in technology within the curriculum.
- The primary medical education staff and administrative faculty are responsible for undergraduate medical education, and in collaboration with the Office of Student Affairs Center for Academic Success and Enrichment, provide learner support.

- The associate deans for GME and CME report directly to the executive vice dean.

\section{Faculty Development and Support in Education}

Faculty development and the recognition of the educational contributions of faculty are an integral part of NJMS.

Professional development for faculty as educators and teachers is held on multiple levels and in multiple venues. Centrally, at Rutgers Biomedical Health Sciences there are workshops on academic promotions and teaching assessments. At NJMS, we provide a number of workshops and seminars, which include a faculty orientation series, faculty mentoring and development, GME grand rounds, annual UME education retreats, resident as teachers, and other faculty teaching development initiatives.

In addition, both the dean's office and individual departments support attendance for faculty at regional and national meetings on educational and faculty development. Teaching and/or education activities are part of promotion criteria in all tracks, including teaching, clinical, research, and professional practice tracks. The clinical educator as well as teaching tracks specifically focus on leadership in teaching and scholarship in the field of education. 


\section{Rutgers Robert Wood Johnson Medical School}

Carol A. Terregino, MD, Archana Pradhan, MD, MPH, Joyce Afran, MD, Meigra M. Chin, MD, H. Liesel Copeland, PhD, Robert Lebeau, EdD, Diana Glendinning, PhD, Siobhan A. Corbett, MD, Sarang Kim, MD, and Paul F. Weber, MD, RPh, MBA

\section{Medical Education Program Highlights}

Rutgers Robert Wood Johnson Medical School has a 4-year curriculum, and for AY 2020-2021, a 2+2 structure. There is an integrated systems-based preclerkship curriculum with normal structure and function, physiology/biochemical/molecular biology principles in year 1, and pathology/pathophysiology/pharmacology in year 2. The curriculum is anchored by the Patient Centered Medicine (PCM) course, teaching skills foundational to becoming a physician. This course also covers humanism, ethics, disparities in health and health care, patient safety, interdisciplinary teamwork, community/population health, and health systems science (HSS). The order of topics and flow of clinical encounters in PCM integrates with the foundational science curriculum, particularly in year 2. Two curricular threads, evidence-based medicine (EBM) and HSS, are integrated over 4 years. There are 8 traditional core clerkships, a critical care selective, 3 weeks of ambulatory experiences, a subinternship, and a 2-week specialty-specific transitions to residency course, focusing on transitions of care, highfidelity simulation, advanced communication, procedures relevant to particular specialties, quality improvement/patient safety, and case-based learning as USMLE Step 3 examination preparation.

\section{Curriculum highlights}

- Development and implementation of the Empathy and Clarity Rating Scale, an inductive developmental tool for formative and summative assessment of clinical skills: This scale with specific attention to empathetic communication was developed interinstitutionally, using the expertise of clinicians, improvisation actors, patients, and communication experts. After commenting on positive and negative observed behaviors, the rater considers the learner's level of training and balance of positive to negative feedback needed, then rates the learner's performance based upon behavioral anchors. The scale allows for rich formative feedback on communication and provides individual longitudinal data regarding approach to gold standard communication skills.

- Implementation of a student-led teamwork training program across the 4 years, anchoring HSS concepts: Our veteran students inspired a movement at the school. Recognizing how health care varies significantly from the synchronized and high-functioning team environment of the military,

Acad Med. 2020;95:S322-S326.

doi: 10.1097/ACM.0000000000003436

Copyright (C) 2020 by the Association of American Medical Colleges

Supplemental digital content for this article is available at http://links.Iww.com/ ACADMED/A930.

Year school was established: 1966.

School URL: http://rwjms.rutgers.edu. students proposed the integration of TeamSTEPPS (Team Strategies to Enhance Performance and Patient Safety) across the curriculum. The student body has embraced the core principles of TeamSTEPPS. Each year reinforces different skills; the movement has led to training in the clinical learning environment, and the skills are foundational to HSS experiential learning activities.

- Frame-of-reference training for clerkship evaluations: With the goal of decreasing the perceived gap between how students and their evaluators assess clinical performance, we built an interactive tool for faculty, residents, and students with the goal to train all stakeholders in using our clerkship evaluation form. Using case presentations developed by core clerkship directors to demonstrate different levels of clinical performance, we designed a web-based, frame-of-reference training tool allowing users to rate a set of case presentations, compare ratings to those determined by the clerkship directors, and receive feedback. We are assessing this program's impact on rating habits and perception of fairness.

- An academic support program that focuses on the alignment of learning, teaching, and assessment: Academic support efforts are framed by the goal of aligning learning, instruction, and assessment. Our longstanding Cognitive Skills Program (CSP) supports student self-directed learning and preparation for future learning through group activities and widely used individual consultation. The CSP faculty support schoolwide educational effectiveness through faculty development in designing and implementing instruction conducive to self-regulated learning and through guidance to faculty and staff. Educators are charged with designing, innovating, and evaluating programmatic assessments that focus on student learning in the near and long term.

\section{Curriculum}

\section{Curriculum description}

See Supplemental Digital Appendix 1-Curriculum Schematicat http://links.lww.com/ACADMED/A930.

\section{Curriculum changes since 2010}

- The ecology of medicine: interprofessional students caring for patients living at home with chronic conditions

- Enhanced patient safety/quality improvement curriculum:

- TeamSTEPPS annually

- Clinical Immersion experience with teams of students

"belonging to" a hospital unit

- Safety Huddle experience with first-year students at primary teaching hospital

- Interprofessional simulations

- Prescription writing workshops with pharmacy students 
- High-fidelity simulation throughout 4 years

- Specialty-specific transition to residency courses

- Workplace-based assessments and additional structure for subinternships

- New primary care track: Primary Ambulatory and Community Clerkship Experience (PACCE)

- 2 new regional medical campuses

- Narrative medicine in the clerkships

- EBM thread

- New topics: digital and connected health, physician wellness, patient- and family-centered care for veterans

- Formative Comprehensive Basic Science Examinations in year 2

- Numerous student-driven and created electives and educational phenomena

- 7 opportunities for distinction: research, medical education, service to the community, bioethics, global health, biomedical innovation and entrepreneurship, and leadership in academic health care

- Expanded communication curriculum with improvisationinformed curriculum and student assessment using the Empathy and Clarity Rating Scale

We are currently planning for an AY 2021-2022 new curriculum rollout. Tenets include enhancing clinical skills/clinical decisionmaking as well as entrustment of core professional activities by increasing the length of the clerkship curriculum and enhancing content in HSS and skills of practice. The curriculum will focus on the quadruple aim for medical education or the Four Cs: curiosity, critical thinking, competence, compassion.

There have been multiple class size changes related to a former regional campus becoming a new medical school. Health systems integrations/consolidations have modified regional campus plans. We anticipate that no more than 30 students will complete clerkships at a regional campus; most students rotate through multiple sites.

\section{Assessment}

We use the ACGME framework as the basis for the medical education program objectives. The Core Entrustable Professional Activities framework is being considered for the new curriculum.

We have implemented the Reporter-Interpreter-ManagerEducator-Professional paradigm since 2010.

See Supplemental Digital Appendix 2-Program Objectives and Assessment Methods—at http://links.lww.com/ACADMED/ A930.

\section{Parallel curriculum or tracks}

The focus for the PACCE track is to provide a clerkship experience that affords the opportunity for continuity of care. The longitudinal relationships between faculty mentors, other health professionals, patients, and peer students are paramount. School-wide and clerkship objectives are identical; however, the student experience in a longitudinal ambulatory setting with a panel of patients over time provides a richer learning environment to internalize many objectives related to primary and ambulatory care in the community. There is enhanced opportunity for transitions of care and interprofessional activities. Total inpatient clerkship time is abbreviated to allow 2 longitudinal blocks of outpatient care. The structure is the same as the traditional curriculum except for the third-year clerkship experience. In AY 2021-2022, we plan to offer a 3-year MD degree program to students pursuing primary care (family medicine).

\section{Pedagogy}

The preclerkship curriculum uses lecture with podcast capture, case-based learning, self-directed learning, laboratory (gross anatomy/neuroanatomy/histology/microbiology), simulation, and team-based learning. The preclerkship clinical curriculum includes lecture and small-group discussions, preceptorship, role play/dramatization, standardized patients, and procedure workshops. The clerkship curriculum includes ambulatory and inpatient clinical experiences, simulation, standardized patients, lecture and small-group discussions, and procedure workshops. The PACCE track has identical pedagogical approaches.

Since 2010, simulation has been incorporated across the 4 years, procedure workshops have been enhanced via specialty-specific transitions to residency courses, self-directed learning activities and team-based learning have been added, and role play and dramatization have been added via improvisation informed curriculum.

\section{Clinical experiences}

Learners first encounter clinical experiences early in the first year. Clinical sites used for required educational experiences:

- Hospitals

- Outpatient offices

- Federally qualified health centers (FQHCs)

- Sites for care of individuals with disabilities

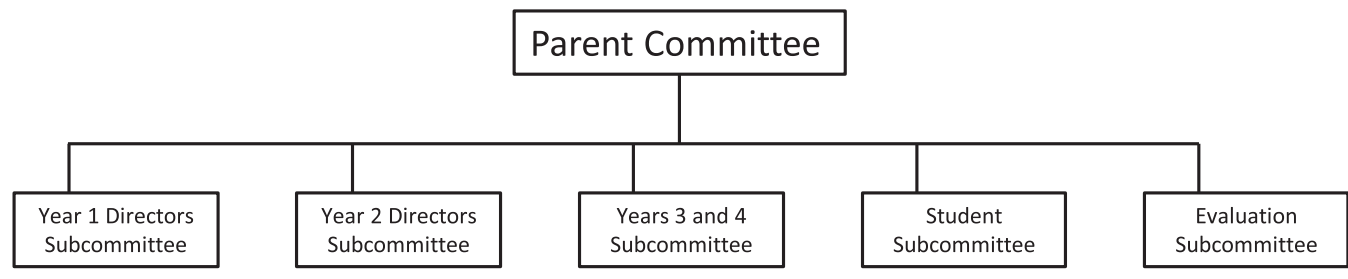

Figure 1 Curriculum governance chart. 


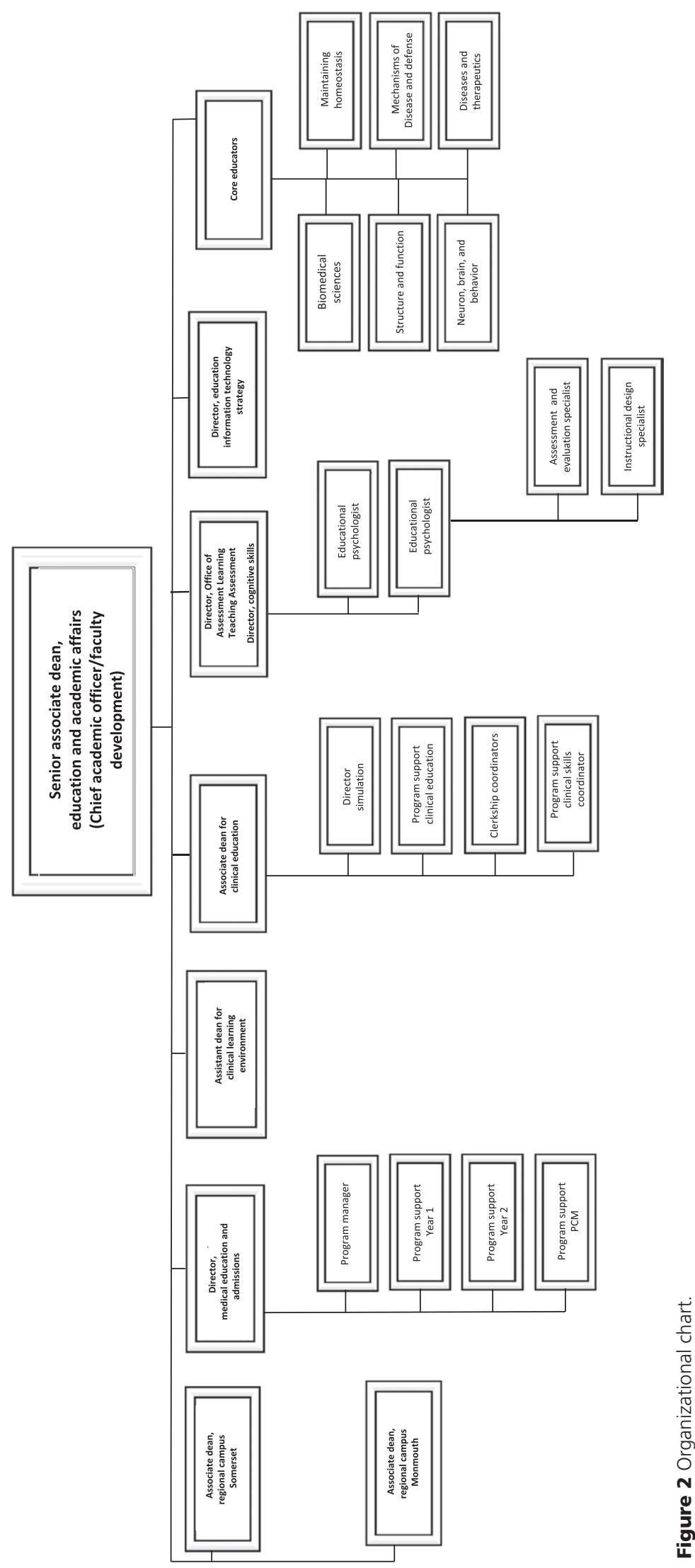


- Sites for care of individuals at end of life

- Home

\section{Required longitudinal experiences}

- All students have required longitudinal experiences as selected experiences

- Continuity experiences with individual practitioners

- Promise clinic (student-run clinic)

- Home

- PACCE track

\section{Required and elective community-based rotations}

All students complete a required 15-hour service-learning experience in the family medicine clerkship. Sites include substance abuse rehabilitation centers, schools, food kitchens, and others. Outpatient sites for required preclerkship and clerkship activities include FQHCs and the student-run clinic. Students in the PACCE track may complete outpatient activities at VA clinics. Electives are offered through the student-run clinic.

\section{Challenges in designing and implementing clinical experiences \\ Considerable oversight is needed to maintain comparability of clinical experiences, bedside/office-based teaching, and student performance assessments. The associate deans for clinical education, the regional medical campuses, and student affairs closely monitor student and faculty experiences.}

\section{Curricular Governance}

The Office of Education supports the chair of the committee, administrative faculty and staff, all assessments, and academic support services.

See Figure 1-Curriculum governance chart.

\section{Education Staff}

The Office of Education is led by the senior associate dean for education and academic affairs (SADEA), the CAO. It comprises faculty administrators (assistant dean for medical education; associate dean for clinical education; director for the Office for Advancing Learning, Teaching, and Assessment), core educational faculty (directors of all of the preclerkship integrated curriculum, director of simulation, and 3 doctorally trained cognitive skills specialists), a director for educational technology, an assessment and evaluation specialist, and support staff (program support specialists for the preclerkship integrated blocks, PCM 1 and 2, clinical courses, and Clinical Skills Center). All faculty administrators directly report to the SADEA. The SADEA collaborates with the Education IT Advisory Committee via the director for education information technology strategy. This collaboration is used to plan and prioritize strategic initiatives and ensure operability of the technological platform for implementing and evaluating the curriculum: course management platform, assessments and evaluations, independent project management, and Medical
Table 1

Regional Medical Campuses

\begin{tabular}{lll}
$\begin{array}{l}\text { Regional campus } \\
\text { name }\end{array}$ & Type & $\begin{array}{r}\text { Student } \\
\text { enrollment }\end{array}$ \\
$\begin{array}{l}\text { Regional medical } \\
\text { campus at Monmouth }\end{array}$ & Clinical & 10 \\
Regional medical & Clinical & \\
campus at Somerset & & \\
\hline
\end{tabular}

Student Performance Evaluation preparation. IT also supports distribution of and attestation to educational policies and learning objectives. The assessment and evaluation specialist performs planning/implementation/evaluation and monitoring of curriculum activities: facilitation of test construction and assessment, providing item-level data, analysis of student performance on exams, guidance for course directors and students on interpreting results, and preparation of curriculum searches.

See Figure 2-Organizational chart.

The role of the medical education staff is to primarily support the UME program. The senior associate dean for education and academic affairs oversees all areas of the continuum, pre-UME, UME, GME, and CPD. Student Affairs, admissions, and registrar faculty and staff are fully integrated in the UME programming.

The Office of Education and Academic Affairs is an equivalent of an academic department. It includes administrative faculty and core educators of the preclerkship curriculum and supports the administrative roles of directors of the PCM courses and thirdand fourth-year curriculum. All faculty maintain a primary appointment within a basic or clinical discipline; however, the salary lines of the administrative faculty and the core educators are under the Office of Education.

\section{Faculty Development and Support in Education}

Professional development is offered through web-based applications and in-person teaching series. The SADEA is responsible for the faculty mentoring program and supporting faculty in developing individualized development plans, which are found to be most helpful for nontenure track faculty who want to develop educational portfolios.

Teaching is mandatory for tenure and nontenure (clinical and research) tracks. Teaching evaluations are reviewed in all tenure decisions. Promotion in the professional practice track requires teaching activity, but not scholarship. Promotion in the clinician-educator and teaching tracks mandate peer-reviewed manuscripts. All faculty develop a teaching portfolio for the promotion process.

The scope of the Institute for Excellence in Education includes faculty from other Rutgers schools beyond health professions, in addition to outside educational experts. The institution serves 3 groups - faculty, learners, and patients: "to engage learners to 
provide high-value health care, communicate respectively in a patient- and family-centered fashion, collaborate and partner with other health care professionals to devise treatment plans and strategies for adherence and self-care, integrate scientific underpinnings of clinical medicine and best evidence into daily practice, and prepare learners to distinguish themselves and prepare teachers for educational scholarship." The institute supports the Medical Education Scholarship Learning Community and the Academy of Medical Educators.

\section{Regional Medical Campuses}

There is close communication between the CAO and principal academic officers at the regional sites. Functional integration of regional campus faculty via standing committees acculturates faculty from all campuses and leads to a shared mental model of the educational program. There is communication between clerkship and clerkship site directors at least every block, the associate dean for clinical education visit the sites at least quarterly, and the student affairs deans meet with all students on a clerkship at least every other block to obtain student feedback from students at all sites. An electronic clerkship evaluation system is monitored each block by the senior associate dean, associate dean for clinical education, and evaluation and assessment specialist. Shelf exam scores and clinical evaluations are reviewed annually for any discrepancies.

See Table 1—Regional Medical Campuses. 


\section{University of New Mexico School of Medicine}

Paul McGuire, PhD, and Craig Timm, MD

\section{Medical Education Program Highlights}

The University of New Mexico School of Medicine was established in 1964 with the mission of advancing the health of all New Mexicans by educating and increasing the diversity of health professionals, leaders, and scientists; providing outstanding and compassionate medical care; advocating for the health of all New Mexicans; and pursuing new knowledge and excellence of practice. Unique features of this medical school include:

- Clinical reasoning curriculum: An integrated curriculum designed to explicitly model and have students actively engage in the clinical reasoning process during relevant case discussions. This curriculum provides structure, guidance, and assessment for self-directed learning and information-seeking skills as well as the skills of critical judgment and medical problem solving.

- Mentored scholarship: Each medical student is required to engage in a faculty-mentored scholarly project. Designed to help students develop practical skills in the scientific method and understanding of the role of research in informing clinical practice, in this initiative students develop and complete a scholarly project in an area of interest related to medical science and/or health care and publicly present or publish their results before graduation.

- Rural and Urban Underserved Program: Designed to support, mentor, and prepare interested medical students in fulfilling their goal of effectively providing health care to underserved groups in New Mexico.

- Early clinical experience: Students begin seeing patients early in their medical education. This includes an emphasis on having students work with community-based physician preceptors, helping them understand the practice environment, and the unique health care challenges faced by New Mexico.

\section{Curriculum}

\section{Curriculum description}

The undergraduate medical curriculum is a 4-year program organized into 3 phases.

See Supplemental Digital Appendix 1-Curriculum Map—at http://links.lww.com/ACADMED/A931.

Acad Med. 2020;95:S327-S330.

doi: 10.1097/ACM.0000000000003437

Copyright (C) 2020 by the Association of American Medical Colleges

Supplemental digital content for this article is available at http://links.Iww.com/ ACADMED/A931.

Year school was established: 1964.

School URL: https://hsc.unm.edu/school-of-medicine.

\section{Curriculum changes since 2010}

The preclerkship curriculum has been revised twice in the past 10 years. The first revision occurred in 2015 with the introduction of new basic science courses and a longitudinal clinical reasoning course replacing the long-standing problembased learning curriculum.

A recent revision beginning in 2019 expanded the length of the preclerkship curriculum from 18 to 20 months to include longitudinal intersessions focusing on wellness, content integration, learning strategies, reflection, and remediation. These intersession weeks (called "WISE" weeks; see Supplemental Digital Appendix 1 at http://links.lww.com/ACADMED/A931) also offer students opportunities to attend workshops focusing on current medical issues in research, clinical practice, and the humanities.

Since 2010, a new education building was also completed, providing a modern facility that promotes the move to a more active learning pedagogy within the curriculum.

The class size increased from 78 to 103 new students in 2010, coincident with the arrival of the first cohort of BA-MD students into the medical school class. Funding was provided for faculty, clinical sites were expanded, curricular administrative support was centralized and expanded, and changes in pedagogy were implemented.

\section{Assessment}

The medical education program objectives are based on the ACGME domains of competence, with additions that reflect the unique nature and mission of our medical school.

See Supplemental Digital Appendix 2-Program Objectives and Assessment Methods—at http://links.lww.com/ACADMED/A931.

Phase I curriculum:

- The Curriculum Committee adopted a pass/fail grading system for the Phase I preclerkship curriculum in 2010.

- The Curriculum Committee adopted the use of low-stakes weekly take-home quizzes during Phase I. This change aims to promote assessment for learning and contribute to the development of professional identity among students.

- The end-of-block summative examinations in Phase I are cumulative and make use of custom NBME examinations created by the teaching faculty.

- Specific clinical reasoning skills are assessed using facultydesigned examinations at the end of each clinical reasoning block.

- Faculty-written questions were transitioned from paper to the use of the ExamSoft program for delivering assessments. 
- With the advent of the Doctoring course, the Phase I performance exams were incorporated into the grades for those blocks, instead of being a separate activity.

- Note-writing activities were introduced in all Phase I performance exams.

Phase II clerkship curriculum:

- SimIQ simulation management software platform was implemented for all performance exams.

- The assessment of clinical competency in the clerkships uses a faculty-developed rubric that has been modified using standard-setting exercises, creating a more transparent process of assessment for students.

- Clerkship-specific skills stations were moved from the objective structured clinical examinations to within the clerkships themselves.

- Several Phase II clerkships developed and implemented endof-block oral examinations for assessment of discipline-specific clinical reasoning.

\section{Pedagogy}

The preclerkship and clinical portions of the curriculum use all of the following pedagogical approaches to achieve the medical education program objectives:

- Ambulatory and inpatient clinical experiences

- Case-based learning

- Laboratory

- Lecture

- Peer teaching

- Preceptorship

- Self-directed and independent learning

- Simulation

- Standardized patients

- Team-based learning

- Video

- Workshops

The School of Medicine Curriculum Committee updated the curricular policies in 2018 to limit the amount of scheduled time and types of pedagogical activities during the preclerkship phase of the curriculum. The committee approved a maximum of 21 hours of scheduled time per week with a maximum of 3 hours of lecture. This has resulted in active learning pedagogies (team-based learning, peer teaching, case-based learning, and laboratories) predominating in the preclerkship curriculum.

\section{Clinical experiences}

We do not have separate tracks. The majority of the required clinical educational experiences take place in the University Hospital and the VA Medical Center Hospital and associated clinics. Students also have substantial opportunities at community-based clinical sites during required experiences in all 4 years of medical school.

Medical students first encounter clinical experiences during the spring semester of the first year as they begin continuity clinic in the Doctoring curriculum. Students are matched with a preceptor in a primary care clinical environment for 1 year and are required to participate in 12 half-day clinical sessions over that period of time.

Medical students have several opportunities in the curriculum to participate in community-based clinical rotations outside of the University Hospital and VA systems. During the continuity clinic experience, about half of the assigned sites are with primary care physicians in the community. The 6-week required practical immersion experience at the beginning of the second year places the majority of students with physicians in community sites, both rural and urban, around the state. All students on their family medicine clerkship are placed in practices outside of the University Hospital system, and a subset of students on other clerkships (surgery, obstetrics-gynecology, pediatrics) also spend time in community-based locations as part of their clerkship experience.

The 2 greatest challenges we face in providing high-quality clinical experiences for our medical students are related to:

- The increases in class size with only modest commensurate increases in numbers of sites resulting in impacts on the number of students on required rotations

- The competing demands that faculty and residents have to provide patient care services while meeting the needs of learners

The School of Medicine continues to address these issues by expanding the number of community-based faculty and sites and by providing faculty and residents with skills to improve their efficiency and quality of teaching while in busy clinical settings.

\section{Curricular Governance}

The Curriculum Committee is a standing committee of the School of Medicine faculty and is responsible for defining the goals and objectives of the undergraduate medical curriculum and for its design, management, and evaluation in accordance with the accreditation requirements of the LCME and the school's mission and vision. This committee is responsible for establishing a process for reviewing, evaluating, and revising the curriculum on a recurring timeline to ensure that the curriculum is coherent, coordinated, current, and effective.

See Figure 1 -Curriculum governance chart.

The assignment of course and clerkship leadership/direction is managed at a departmental level with some financial support from the Office of Undergraduate Medical Education. Otherwise all curricular governance is centralized in the Office of Education.

\section{Education Staff}

- Curriculum Support Center: Staff members focus on providing students and faculty with effective and efficient curricular support in an effort to create an organized and sustainable learning environment. 


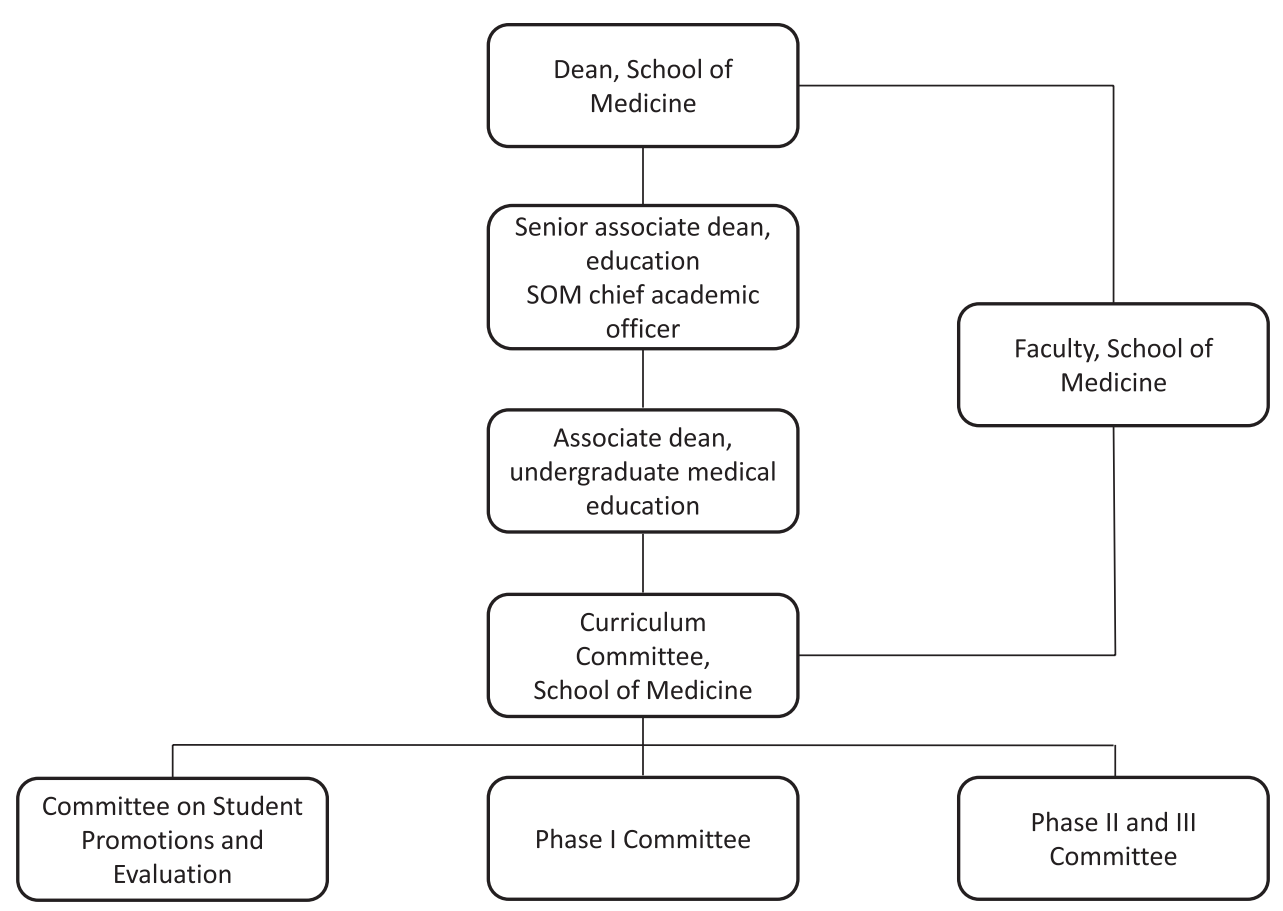

Figure 1 Curriculum governance chart.

- Program Evaluation, Education, and Research: Faculty and staff support the principles of continuous quality improvement and institutional excellence by understanding the educational needs and experiences of students and providing faculty with data and information that can be used to further curricular effectiveness and student learning.

- Academic Multimedia Services: Staff develop and implement academic tools and technologies to enhance student learning and to provide faculty with resources and services that support progressive, efficient educational practices.

- Assessment and Learning: Faculty and staff develop and implement state-of-the-art assessment tools and mentor students in aspects of professional development related to communication skills, clinical skills, and professional behavior.

- Preceptorship Program: Faculty and staff support the recruitment and development of community-based faculty and the identification and matching of preceptors and students participating in the clinical aspects of the curriculum.

- One45: This curriculum management system is used throughout the 4-year medical school curriculum.

- D2L BrightSpace: This learning management system is used for all courses and clerkships.

See Figure 2-Education staff organizational chart.

All SOM education programs are within the Office of Education, headed by the senior associate dean for education. These areas include admissions, medical student affairs, UME, GME, CME, and faculty development. The central office provides support for human resources, financial management, and budgeting, and professional development for all programs.

\section{Faculty Development and Support in Education}

Educational development experiences and resources are provided through the Office for Continuous Professional Learning (CPL). The experiences include 30-40 face-toface and hybrid (combined online and classroom learning) workshops and 3 fully online courses for all faculty and residents as well as community-based volunteer faculty. Consultations on educational development needs are provided to individuals, departments, and programs. Online resources are provided in a variety of formats including for use on smartphones in the flow of work. CPL also facilitates the 1- to 2-year longitudinal Medical Education Scholars program and Residents as Educators courses (in collaboration with the DIO's office) and tracks faculty completion of the Achievement in Medical Education Program certificate (a series of faculty development workshops for improving skills as a medical educator). CPL host the Scholarship in Education Allocations Committee, which annually solicits proposals for education scholarship and selects outstanding proposals in education research that are supported with funds provided by the dean.

\section{Role of teaching in promotion and tenure}

Basic science faculty on the tenure track, the clinician-educator track, and the lecture track are all required to demonstrate excellence in education for promotion. Clinicians on the tenure track have to demonstrate excellence in 2 of 3 areas (clinical service, research/scholarship, and education). Demonstration of excellence is met by consideration of quantity, quality, and evaluation. 


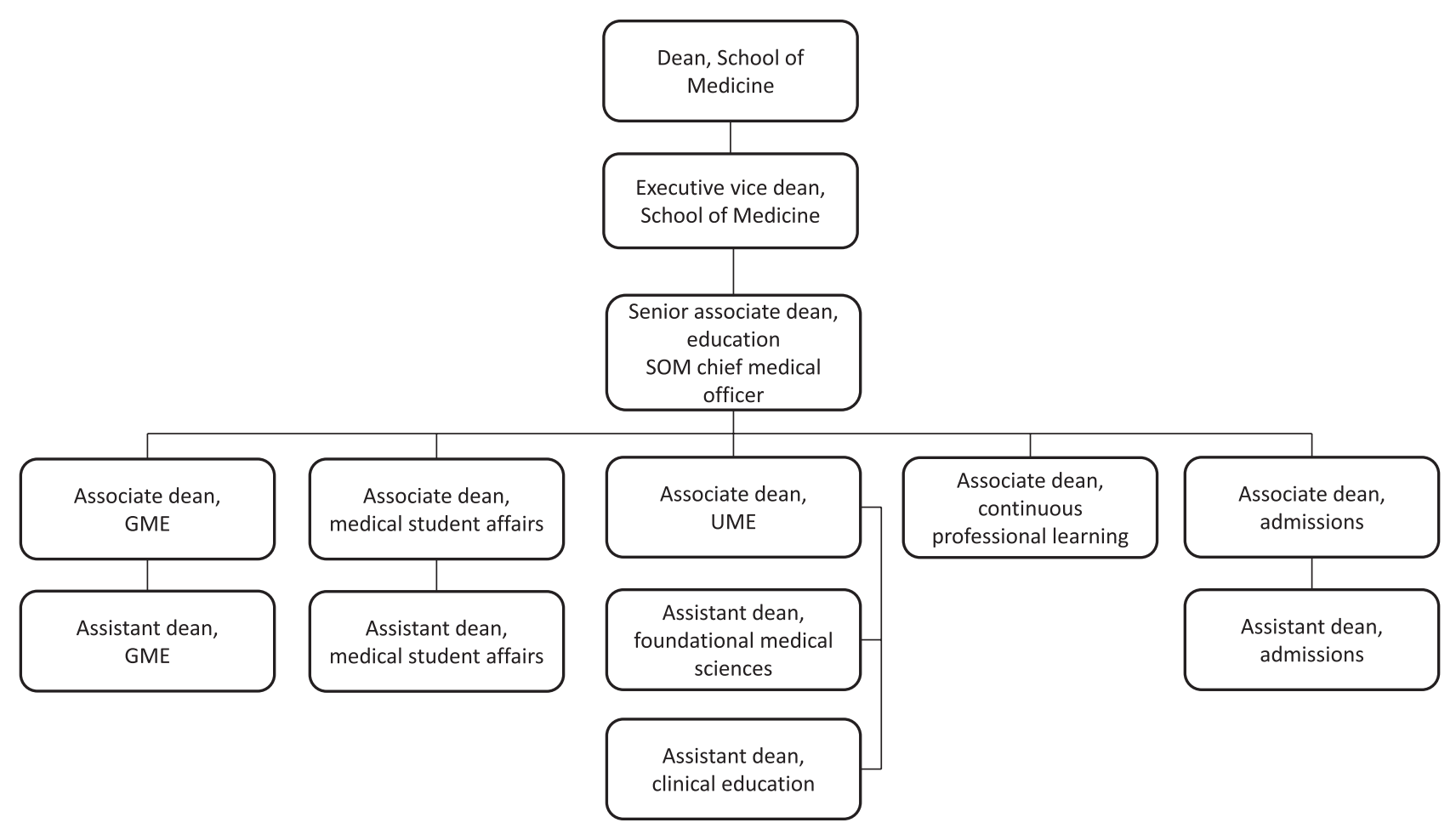

Figure 2 Education staff organizational chart. 


\section{Albert Einstein College of Medicine}

Pablo A. Joo, MD, Todd Cassese, MD, Howard M. Steinman, PhD, Martin N. Penn, MBA, William Burton, PhD, Hai Jung H. Rhim, MD, MPH, and Joshua D. Nosanchuk, MD

\section{Medical Education Program Highlights}

The Albert Einstein College of Medicine (Einstein) curriculum integrates basic, clinical, behavioral, and population health sciences to produce physicians and clinician-scientists that meet the modern, evolving needs of patients and society. Students can pursue electives in the first, second, and fourth years for deeper exploration into areas of interest. A capstone scholarly paper is a graduation requisite.

Recent highlights:

- The Teaching Star Program enhances and supports faculty and nonfaculty instructors' teaching and assessment skills. The program begins with mandatory teaching development seminars and resources for all instructors. Course/clerkship objectives and expected teaching and assessment roles are clarified. Students complete evaluations of instructors' teaching skills after each course/clerkship, which are used as the objective basis for teaching awards and underpin a carefully documented process of improvement plans for any instructor with inadequate teaching skills.

- In 2019, Einstein's Global Health Center and the City University of New York School of Public Health and Health Policy (CUNY SPH) joined together to create an MPH program specifically designed to fit into a gap year within the Einstein MD program. Composed of online and in-person courses, the program will include a summer when students can complete an Einstein global health fellowship that fulfills the CUNY SPH fieldwork requirement. Students will engage in public health research with an Einstein mentor concurrent with MPH coursework during the gap year.

- The peer tutor program is a free service available to all Einstein students, monitored by the Office of Academic Support and Counseling (OASC). Students have the opportunity to work individually or in small groups with upper-class students who have excelled in the curriculum and have excellent interpersonal and communication skills. Peer tutors are available for all basic science courses, USMLE examinations, and subject test preparation. Through this program, students learn more effective study strategies, reinforce course material, and work with others who are facing similar challenges, facilitating their successes.

Acad Med. 2020;95:S331-S334.

doi: 10.1097/ACM.0000000000003422

Copyright (C) 2020 by the Association of American Medical Colleges

Supplemental digital content for this article is available at http://links.Iww.com/ ACADMED/A922.

Year school was established: 1955.

School URL: https://einsteinmed.org.
- In alignment with Einstein's mission of social justice, the curriculum has a robust community-based service learning (CBSL) program. This provides an opportunity for students to partner with members of the Bronx community and local organizations to make a lasting impact on health outcomes and social justice for the Bronx community. The CBSL program combines trainings, workshops, and seminars with experiential education for students to develop the skills to incorporate social medicine in their future practice.

\section{Curriculum}

\section{Curriculum description}

See Supplemental Digital Appendix 1-Curriculum Map—at http://links.lww.com/ACADMED/A922.

See Figure 1-Longitudinal curricular themes.

\section{Curriculum changes since 2010}

In the last decade, the Einstein curriculum has significantly increased integration of foundational science content in the preclerkship years and substantially increased instruction by active learning throughout all 4 years. Einstein has extensively incorporated audience response systems; flipped classrooms; and self-directed, team-based, and case-based learning. Education centers in the former library stacks and a dining hall have created spaces for active learning modes of instruction. Leveraging the Educational Innovations Team, launched in 2019, we plan to continue basic and clinical sciences integration supported by modalities for prelearning that advance active learning sessions. We will be expanding our population health curriculum to more robustly address structural determinants of health faced by communities in the Bronx and developing content to assure that students can harness big data to address their patients' health care needs.

\footnotetext{
Assessment

Einstein established internally developed institutional objectives in 1997, which were refined in 2006. In 2011, an intensive institution-wide process enabled the shift to a competency-based education model. All graduates must demonstrate competency in 7 areas: healer, scientist, advocate, educator, colleague, role model, and lifelong learner. Our competencies are linked to the Physician Competency Reference Set. With this curricular evolution, we initiated a new revision process in mid-2019 with the Medical Education Council committees, to be completed in 2020. In 2018, Einstein implemented policies expanding our assessment system to formally promote assessment for learning. As of 2019, all preclerkship courses have a developmental assessment system (e.g., assessment timelines, mechanisms for formative assessments, assessments that build on previous
} 

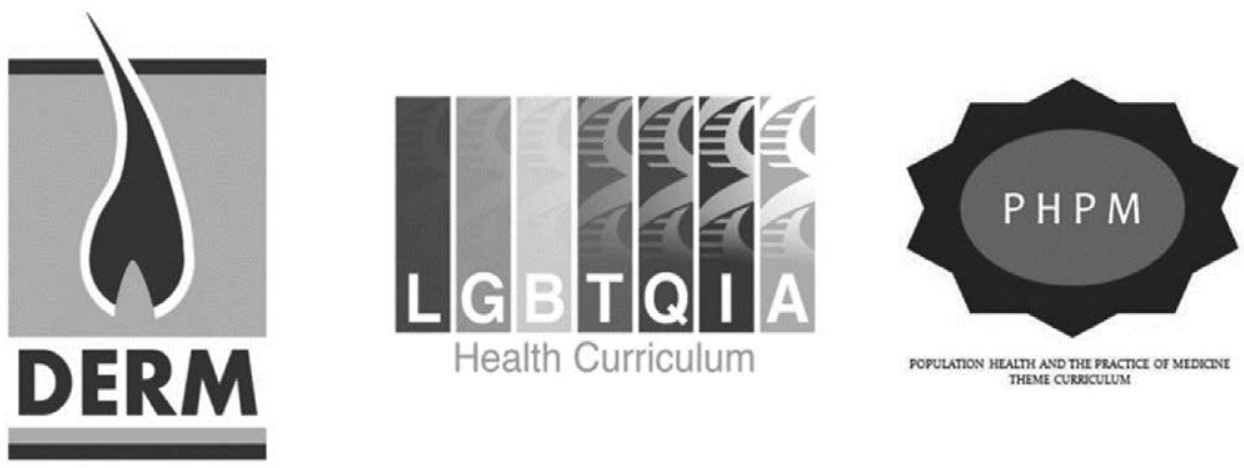

Figure 1 Longitudinal curricular themes.

content) to ensure that students can measure their progress in learning at multiple points during a course. Students can use feedback from no-stakes and low-stakes assessments to reinforce concepts, promote their planning for study, or take corrective steps before final course grades are assigned. Students who do not pass a specified number of assessments must meet for advising during the course with the course director and the OASC. Course directors also receive feedback about students' understanding of material in real time. All students can electronically review their high-stakes multiple-choice question (MCQ) exams and discuss items and content with their instructors.

Also in 2018, a quality improvement policy was introduced for all medium- and high-stakes MCQ exams created by Einstein faculty. Exams are electronically analyzed, and those questions with $>95 \%$ or $<40 \%$ of the class answering correctly, those in which more than one answer was accepted, or eliminated questions are flagged. As a measure of overall test reliability, the Kudar-Richardson Formula 20 (KR20) value for the assessment is also reported. Faculty with training in MCQ standards review the entire exam and mark additional MCQs that do not conform to NBME format guidelines, lack clarity, or are deemed not to be core content. The faculty reviewer prepares an annotated Excel sheet highlighting flagged items and composes a report of the analysis, listing specific questions to be answered by the instructor. The instructor submits a response and meets with the reviewer to discuss the report, with the goal of changing a minimum of $10 \%-20 \%$ of the MCQs on the assessment each year.

See Supplemental Digital Appendix 2-Competencies, Program Objectives, and Assessment Methods—at http://links.lww.com/ ACADMED/A922.

\section{Pedagogy}

We use the following instructional methods:

- Case-based learning

- Clinical experience: ambulatory

- Clinical experience: inpatient

- Discussion: large group $(>12)$

- Discussion: small group $(\leq 12)$
- Laboratory

- Lecture

- Peer teaching

- Preceptorship

- Problem-based learning

- Role play/dramatization

- Self-directed learning/tutorial

- Simulation

- Standardized/simulated patients

- Team-based learning

- Video/podcast

- Virtual patient

- Workshop

As noted, there has been a concerted effort to decrease use of lectures in the foundational sciences curriculum and convert them into interactive sessions using active learning pedagogy. In the clerkship phase, students routinely participate in both ambulatory and inpatient clinical experiences as well as preceptorship in the context of patient care. There is increased use of problem-based learning, peer teaching, role play, and simulations across clerkships. There are both formative and summative assessments through OSCEs using trained standardized patients across the 4-year curriculum.

\section{Clinical experiences}

Einstein students participate in clinical activities in all 4 years of the curriculum. In years 1 and 2, students participate in 1 half day per week preceptorships in ambulatory, emergency medicine, and inpatient services of the Montefiore Health System and Jacobi Hospital Health System, as well as other communitybased practices affiliated with the school. Sites used in years 3 and 4 include inpatient and outpatient services of affiliated municipal, state, voluntary, and academic medical center hospitals and clinics located in the Bronx, Queens, Long Island, and Westchester and Rockland counties.

Einstein students participate in the Clinical Experience Module (CEM) of the Introduction to Clinical Medicine (ICM) course during the preclerkship phase of the curriculum. Assignments to clinical sites are based on student preference and a lottery system, and students visit the same site, typically working with the same preceptor for a full year or even for both years 1 and 2, thus 


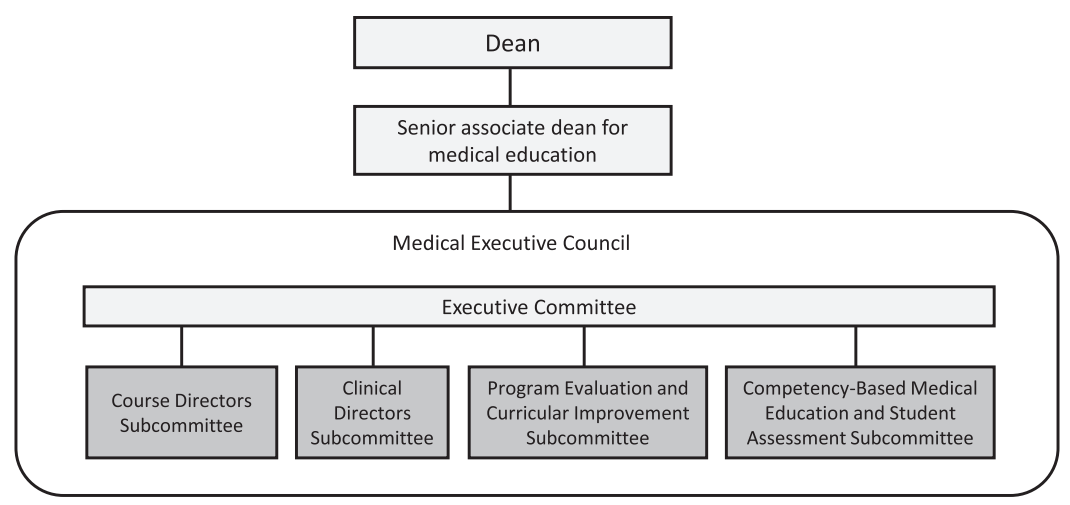

Figure 2 Curricular governance.

having a longitudinal mentoring experience with the provider as well as getting to know the site's patient population and other health professions staff.

In the third year, students spend 1 half day per month away from clerkships, to participate in the Patients, Doctors, and Communities course. This course provides a safe place for reflection and debriefing challenging experiences in the clerkship and serves as a curricular element to teach and assess advanced clinical skills and ethics. It also provides opportunity for students to assess their professional identity formation.

As noted, students start the CEM of the ICM course in the fall of their first year, participating in near weekly experiences throughout the preclerkship curriculum. Additionally, as part of the communication module of ICM, students visit medical and psychiatric wards where they interact with patients under observation.

Einstein students have required community-based rotations as part of the ICM course in years 1 and 2 as well as in components of the family medicine, obstetrics, internal medicine, pediatrics, surgery, psychiatry, neurology, and geriatrics clerkships. There is a required community-based project in the family medicine clerkship. Additionally, required fourth-year rotations in ambulatory medicine have community-based features. Myriad ambulatory opportunities are available to students through fourth-year electives both at the school and away, available through VSAS. Einstein has a formal Office for Community-Based Service Learning that oversees a diverse range of engagement, educational, research, and leadership opportunities for students. Since 1999, students have operated a free clinic serving uninsured adults in the Bronx.

Current challenges to our clinical education include the consolidation of health care systems, which has led to closing or restructuring services offered by historical affiliates. Additionally, the current financial model at our clinical affiliates is based on the relative value unit, which can have a negative impact on the time and interest faculty members have in providing clinical education. The founding of new medical schools in close proximity has also created clinical capacity stress. It is difficult to maintain a fair, reliable system to assess all of our learners' competencies across multiple clinical sites with different types of faculty working. We are working to develop sufficient resources to provide reliable and high-quality clerkship OSCEs.

\section{Curricular Governance}

See Figure 2-Curricular governance.

\section{Education Staff}

Einstein's Office of Medical Education (OME) is responsible for ensuring delivery of the curriculum. In addition to deans for biomedical and clinical sciences, staff in OME support our electronic curriculum by assisting faculty in uploading resources to our learning management system (LMS) and administering exams. The Educational Innovations team of instructional designers assists course and clerkship directors in creating teaching and assessment initiatives that incorporate evidence-based best practices in educational technology and active learning. We also provide support for the educational technologies used at Einstein, including the LMS, a lecture capture system, and an audience response system. We require students to evaluate courses and clerkships, lecturers, and small-group facilitators using an online evaluation tool. We have implemented several programs to monitor the activity of the curriculum and continually drive process improvement.

The primary role of the OME is to expertly deliver and robustly assess Einstein's curriculum to produce physicians who will excel in diverse future activities. Within OME, the Office of Diversity runs a state-funded program for grade 7-12 students that provides preparative opportunities for advancement in science and medicine, which is followed by an extension program for college students. We also run a diversity summer research program for college sophomores and juniors. The Office of Student Affairs is a close partner with OME in actualizing the primary role of medical education staff and faculty.

See Figure 3-Educational staff organizational chart. 


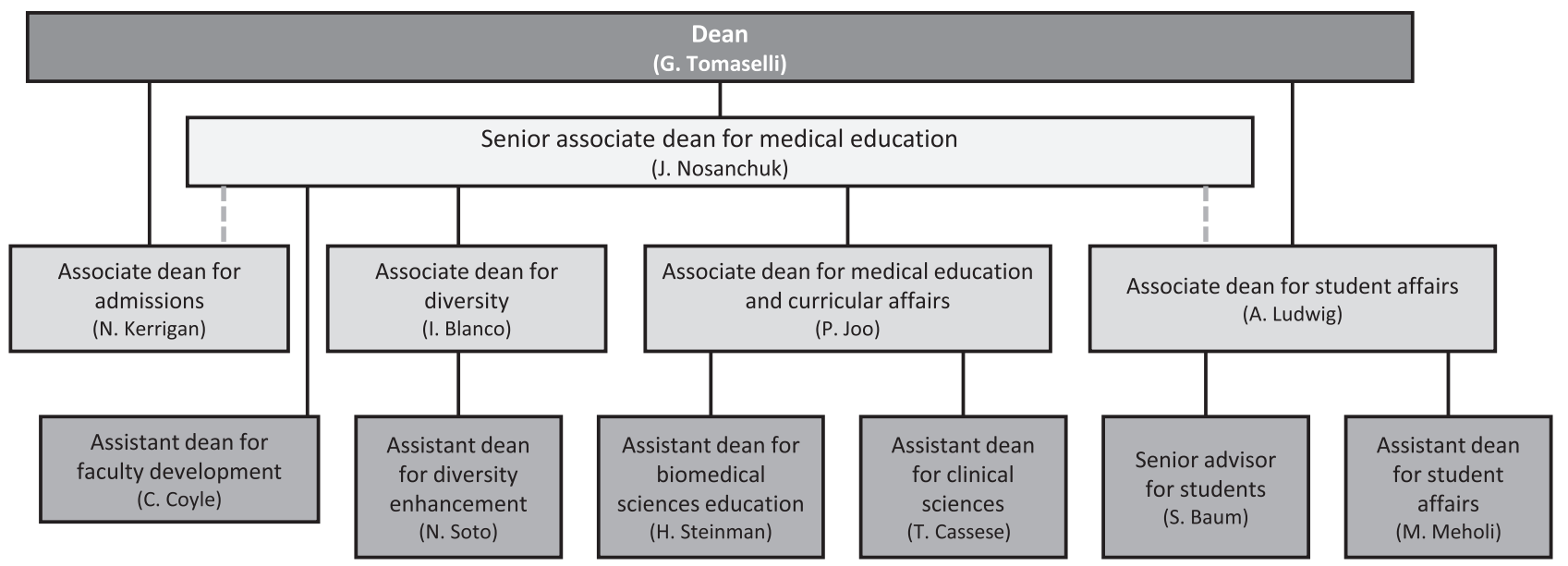

Figure 3 Educational staff organizational chart.

\section{Faculty Development and Support in Education}

The Office of Faculty Development (OFD) offers a comprehensive program of workshops, workshop series, and full-day seminars that promote research seminars and clinical faculty in enhancing their educational and professional skills. The OFD also sponsors major annual events such as Davidoff Education Day, which includes a keynote lecture from a national leader in medical education; the Assessment Summit, which trains course and clerkship leaders in best practices in assessing learners; and the Summer Intensive for New Medical Educators focused, on faculty new to teaching within 5 years.

Teaching is a key component of the promotion process as it is considered a "major leg" in determining promotion. A teaching portfolio is required for faculty in the educator tracks and is strongly encouraged for all faculty undergoing consideration for promotion. Among the various components, the portfolio requires student evaluations provided by OME. To be considered for promotion or tenure, all faculty must document their teaching contributions. 


\section{Columbia University Roy and Diana Vagelos College of Physicians and Surgeons}

Jonathan Amiel, MD, Aubrie Swan Sein, PhD, EdM, and Ronald Drusin, MD

\section{Medical Education Program Highlights}

The Columbia University Roy and Diana Vagelos College of Physicians and Surgeons (VP\&S) was founded in 1767 and was the first medical school in the American colonies to confer the degree of Doctor of Medicine. In 1911, VP\&S entered into its affiliation with Presbyterian Hospital to form the nation's first academic medical center. In addition to the medical school, the medical center includes leading schools of nursing, dentistry, and public health.

Throughout its history, VP\&S has been a research-intensive academic medical center providing high-quality medical care, advancing biomedical knowledge, and training physician leaders. The school's faculty includes 3 Nobel laureates and over 50 members of the Institute of Medicine.

The mission of the school is to discover, educate, care, and lead. Learners' guided exposure and training allow them to exhibit humanism and professionalism in their responsibilities to their patients, to their community, and to society. Graduates' experiences at VP\&S enable them to lead change in medicine in the United States and beyond.

$\mathrm{VP} \& \mathrm{~S}$ is unique in training its students in the world-class Vagelos Education Center, which houses technologically advanced classrooms, collaboration and study spaces, and an advanced simulation center, all located in a bustling urban environment. Students participate in the P\&S Club, an organization of student activities that sponsors over 70 clubs to help students engage with one another, the campus, and the neighborhood community. The school provides a debt-free education to all students with financial need.

Studying in a highly academic environment, all graduates engage in mentored scholarly work teaching them to generate hypotheses, exhibit curiosity, and develop a pattern of lifelong learning, as well as to participate in the process through which new knowledge is generated and to assess the importance of novel ideas. VP\&S learners are enriched by a narrative medicine program aimed at developing their capacity to reflect and observe. ${ }^{1,2}$

\section{Curriculum description}

The Columbia curriculum is in 3 phases. Fundamentals focus on the science of health and disease and on developing clinical

Acad Med. 2020;95:S335-S338.

doi: 10.1097/ACM.0000000000003461

Copyright (C) 2020 by the Association of American Medical Colleges

Supplemental digital content for this article is available at http://links.Iww.com/ ACADMED/A947.

Year school was established: 1767.

School URL: https://www.ps.columbia.edu. skills. The major clinical year (MCY) offers a survey of medical specialties in urban and rural settings. Differentiation and integration (D\&I) provides advanced clinical training and an indepth scholarly project. The curriculum balances scientific rigor with patient-centered care.

See Supplemental Digital Appendix 1-Curriculum Map—at http://links.lww.com/ACADMED/A947.

\section{Curriculum changes since 2010}

The Columbia curriculum changes in response to advances in culture, society, and health care; to changing student needs; and to shifts in available educational resources. Since the last curricular revision in 2010, the school accelerated immersion into the core clinical clerkships into the second year and introduced a scholarly project requirement to promote student research and leadership across disciplines.

In the past 5 years, the school has implemented elements of competency-based medical education including through its participation in the AAMC Core Entrustable Professional Activity (EPA) Pilot. ${ }^{3}$ Throughout the curriculum, students work with coaches to improve knowledge, skills, and attitudes. A longitudinal reflective portfolio ${ }^{4}$ and an advisory dean program ${ }^{5}$ are used for student coaching and advising. An entrustment committee reviews the progress of all students to determine preparedness for residency and offer additional support when needed.

\section{Assessment}

See Chart 1-Medical Education Program Objectives and Assessment Methods.

The school's medical education program objectives are based on the Physician Competency Reference Set (PCRS).

The school has been implementing programmatic assessment over time with a network of low- and high-stakes knowledge and clinical skills assessments. With the adoption of an honor code in 2012, learners take most of their exams unproctored and off-site. Exams are followed by debriefing sessions to promote review and learning from errors. The school uses formative progress exams at the conclusion of each semester of the fundamentals phase to help consolidate learning and promote retention. EPA workplace-based clinical skills assessments are used across the curriculum.

Beginning in 2012, students take the USMLE Step 1 examination after the MCY.6,7

A 3-year MD program for medical school matriculants who have already earned a $\mathrm{PhD}$ was introduced in 2013. 


\section{Chart 1}

\section{Medical Education Program Objectives and Assessment Methods}

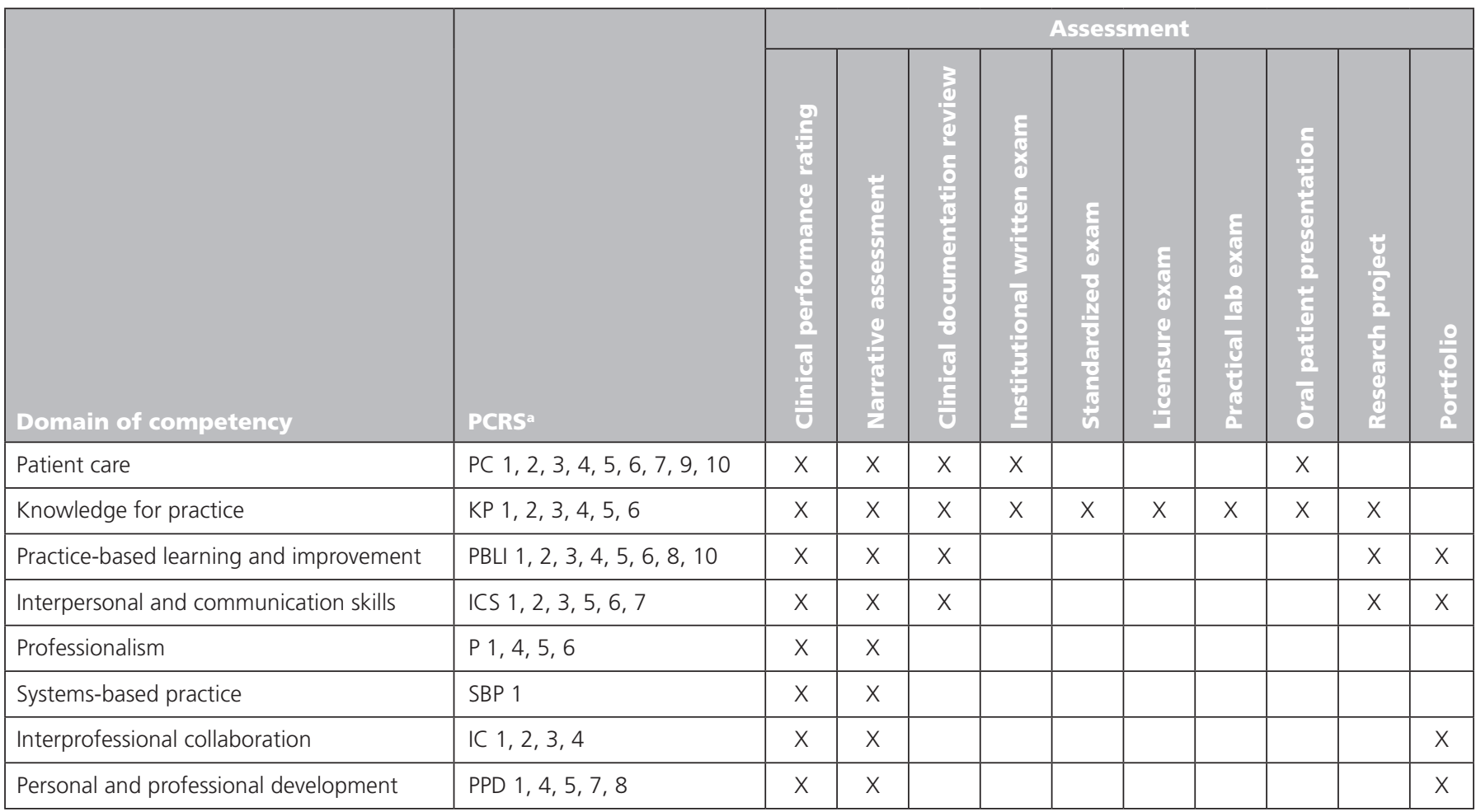

aThe school's medical education program objectives are based on the Physician Competency Reference Set.

\section{Pedagogy}

Students learn in large and small groups in classroom, simulation, and clinical settings. Team-based learning was implemented in small-group sessions in 2010. Flipped classrooms have replaced several lectures with videos and podcasts. Self-directed learning modules have been used to help students learn to identify, analyze, and synthesize information pertinent to learning needs as well as to evaluate the credibility of information sources. Simulationbased learning and assessment has also been increasingly used after the opening of our simulation center.

\section{Clinical experiences}

Clinical experiences begin early in the first semester of medical school, and there is an emphasis on team-based and self-directed learning in the lead-up to clinical rotations. Students rotate through up to 5 clinical affiliates including NewYork-Presbyterian Hospital (a large urban private quaternary care medical center), Harlem Hospital (a public hospital), the John J. Peters Veterans Affairs Medical Center, Stamford Health (a community academic hospital), and Bassett Healthcare (a rural integrated health care system).

\section{Curricular Governance}

The curricular governance structure is centralized and managed through the Office of Medical Education. The Committee on Education Policy and Curriculum (CEPC) oversees the medical education program as a whole and has responsibility for the overall design, management, integration, evaluation, and enhancement of a coherent and coordinated medical curriculum. The CEPC has 8 subcommittees:

- Fundamentals Subcommittee: Oversees the operation of the fundamentals phase to ensure quality and consistency. Manages educational policies in this curriculum phase.

- Major Clinical Year Subcommittee: Oversees the operation of MCY to ensure quality and consistency. Oversight includes the time set aside for NBME exam preparation. Manages educational policies in this curriculum phase.

- Electives Subcommittee: Oversees the operation of D\&I to ensure quality and consistency. Oversight includes the Ready4Residency course. Manages educational policies in this curriculum phase.

- Discover Subcommittee: Oversees the integration of scientific reasoning into the curriculum and student engagement in scholarly work including scholarly elective experiences, the MD-MS program, and the Scholarly Projects Program.

- Educate Subcommittee: Oversees education program evaluation, pedagogical and assessment innovation, and use of educational information technology.

- Care Subcommittee: Oversees clinical skills teaching and assessment across the curriculum, including the Core EPA program and simulation activities. 


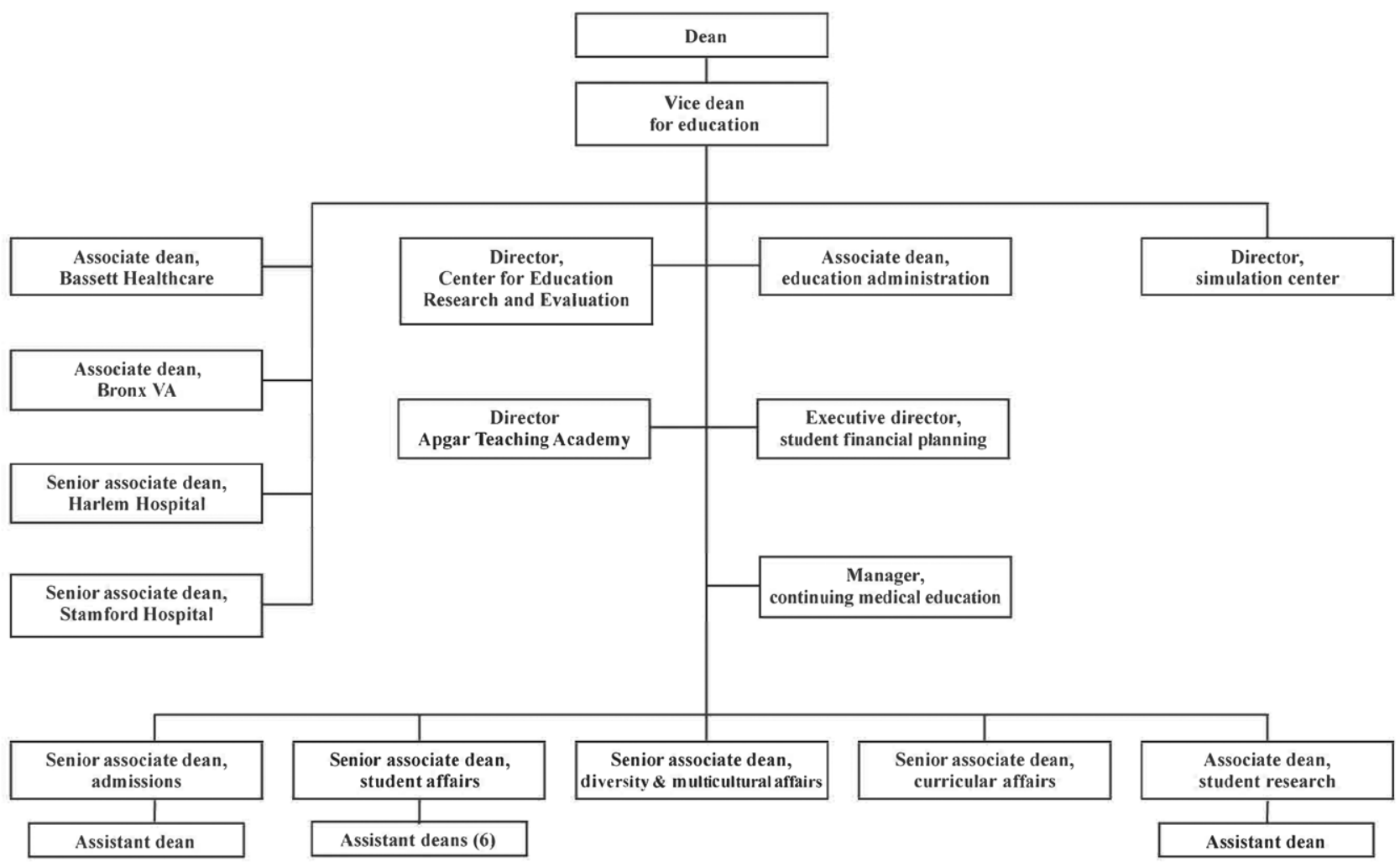

Figure 1 Education staff.

- Lead Subcommittee: Oversees the integration of public health topics into the curriculum and considers the impact of shifts in health policy and the care delivery environment on the UME curriculum.

- Student Curriculum Advisory Board: Acts as the focal point for student participation in curricular initiatives. This includes distributing curricular information to the student body and communicating student perspectives to faculty and administrators; acting as a liaison between students, faculty, and administrators working on curricular projects and the larger student community; incubating long-term curricular ideas; and providing space, resources, and advice to those students and faculty working on curricular improvement.

\section{Education Staff}

The Office of Medical Education, led by the vice dean for education, has responsibility for curricular affairs, student affairs, admissions, multicultural and diversity affairs (including several pipeline programs for students underrepresented in medicine), the Center for Education Research and Evaluation (CERE), student financial planning, the Apgar Academy of Medical Educators, and the Office of Continuing Medical Education. Graduate medical education functions are housed within NewYork-Presbyterian Hospital.

The senior associate dean for curricular affairs and the curricular affairs staff oversee the planning, implementation, evaluation, and oversight of the curriculum. CERE supports this work and uses an extensive continuous quality improvement process. This work is continually presented to the CEPC for oversight and feedback.

See Figure 1-Education staff.

VP\&S does not have an academic department of medical education. All faculty appointments are in clinical or basic science departments.

\section{Faculty Development and Support in Education}

VP\&S supports educator faculty development through a variety of means aimed at promoting educational excellence and innovation and supporting faculty whose scholarly contributions are in education. Teaching and educational requirements are required of all faculty for promotion. The school's promotion track for the educational scholarship and leadership area of focus recognizes direct involvement in the process of promoting learning, support of infrastructure needed for learning, and development of products used by others in learning.

Columbia University (CU), Columbia University Irving Medical Center (CUIMC), and VP\&S itself have developed an array of faculty development resources staffed by experts in teaching, assessment, education technology, and instructional design, including:

- CERE employs 8 full-time educators and data analysts who work with faculty on developing innovative curriculum and 
assessment tools and who support efforts in continuous quality improvement of education programs. Faculty development is a core responsibility of CERE staff, balanced with their work in program evaluation and academic advising.

- The CU Center for Teaching and Learning (CTL) is a university-wide resource supporting pedagogical innovation, implementation of new educational technologies, and development of novel assessment strategies. The CTL operates a branch at CUIMC with 4 full-time and 1 part-time staff working with educators at the medical center to present programs specifically designed to support teaching needs at CUIMC, including preceptorial patient-based teaching, small-group and simulation lab teaching, and lectures. Faculty development is the primary responsibility of CTL staff.

- The CUIMC Office of Professional Development (OPD) offers programs to support educators in developing their careers and demonstrating their impact in support of academic advancement as educators. These programs offer strategies to assess and increase the scope of faculty educational activities, development and communication of educational scholarship, understanding the criteria for promotion in rank in the educational track, and preparation for promotion as an educator. Faculty development is the primary responsibility of OPD staff.

- The VP\&S Virginia Apgar Academy of Medical Educators is a teaching academy that brings together the community of educators at the medical school in workshops to improve teaching skills. The academy cosponsors an annual symposium focused on medical education and also provides grants for curriculum development and education scholarship. The academy's ADEPT program supports senior faculty to coach junior faculty members in lecturing and in small-group teaching. Others support junior faculty in creating multimedia curriculum tools.

- Departments sponsor a variety of faculty development programs including those specifically designed for educators.

Each summer, the Virginia Apgar Academy, the CTL, the OPD, and CERE collaborate on the Summer Institute for Teaching and Learning, an intensive teaching seminar designed for new faculty interested in education. They also have started a program to train a cohort of faculty members on education research techniques, using the AAMC Medical Education Research Certificate sessions.

\section{Regional Medical Campuses}

VP\&S has 1 regional medical campus at Bassett Healthcare that hosts 10 students per year in their MCY for a longitudinal integrated clerkship. There are a variety of complementary mechanisms in place to identify and address inconsistencies across instructional sites in areas such as student satisfaction and grades.

Evaluations are aggregated by clerkship by site and are compared with historic data and cross-sectional data across sites. When quantitative evaluations at particular sites for a clerkship indicate potential site-specific issues, the group reviews student comments and discusses them with the class leaders who prepare for the meeting by querying their classmates for any issues. After grades are submitted, clerkship and site directors are provided access to student evaluations and can identify and address any inconsistencies between sites at that point.

The senior associate dean for curricular affairs, vice dean for education, and CERE meet with each clerkship director twice yearly for continuous quality improvement meetings. At these meetings, the group discusses any changes to the clerkship and issues arising at sites. The group reviews aggregated data for the clerkship by site and develops action plans for correcting any inconsistencies identified. The group also reviews grade distributions on an annual basis to ensure comparability in grades between sites. Finally, the MCY subcommittee reviews comparability data for all clerkships and sites on an annual basis.

\section{References}

1 Miller E, Balmer D, Hermann N, Graham G, Charon R. Sounding narrative medicine: Studying students' professional identity development at Columbia University College of Physicians and Surgeons. Acad Med. 2014;89:335-342.

2 Gowda D, Dubroff R, Willieme A, Swan-Sein A, Capello C. Art as sanctuary: A four-year mixed-methods evaluation of a visual art course addressing uncertainty through reflection. Acad Med. 2018;93(11 suppl):S8-S13.

3 Lomis K, Amiel JM, Ryan MS, et al.; AAMC Core EPAs for Entering Residency Pilot Team. Implementing an entrustable professional activities framework in undergraduate medical education: Early lessons from the AAMC Core Entrustable Professional Activities for Entering Residency Pilot. Acad Med. 2017;92:765-770.

4 Cunningham $\mathrm{H}$, Taylor D, Desai UA, et al. Looking back to move forward: First-year medical students' meta-reflections on their narrative portfolio writings. Acad Med. 2018;93:888-894.

5 Swan-Sein A, Mellman L, Balmer DF, Richards BF. Sustaining an advisory dean program through continuous improvement and evaluation. Acad Med. 2012;87:523-528.

6 Daniel M, Fleming A, Grochowski CO, et al. Why not wait? Eight institutions share their experiences moving United States Medical Licensing Examination Step 1 after core clinical clerkships. Acad Med. 2017;92:1515-1524.

7 Pock A, Daniel M, Santen SA, Swan-Sein A, Fleming A, Harnik V. Challenges associated with moving the United States Medical Licensing Examination (USMLE) Step 1 to after the core clerkships and how to approach them. Acad Med. 2019;94:775-780. 


\section{CUNY School of Medicine}

Lisa Auerbach, MD, MS, HPEd, Emine Ercikan Abali, PhD, Rosa Lee, MD, Nicole K. Roberts, PhD, and

Erica Friedman, MD

\section{Medical Education Program Highlights}

CUNY School of Medicine (CSOM) at City College of New York in New York City is a new 7-year BS-MD program that has been built on the 46-year tradition and success of the Sophie Davis School of Biomedical Education. Students in the prior Sophie Davis School of Biomedical Education program graduated with a BS, which included all required courses from the first 2 years of medical school, and then transferred to one of a number of "cooperating" medical schools to finish their final 2 years of the MD degree. CSOM enrolls students directly from high school and integrates the college and medical school courses over the 7 -year program with a seamless transition in which all students in good academic and professional standing receive their BS after 3 years and continue in the medical school program without taking the MCAT examination.

CSOM is made up of a diverse student body. The majority of its students are from groups underrepresented in medicine, and a significant percentage of its students are first-generation immigrants and/or first-generation college students. Our ideal class size is between 70 and 75 each year, based on the availability of teaching space and the ability of our main clinical partners, St. Barnabas Hospital Health System and Staten Island University Hospital, to accommodate students in clinical rotations. We do not anticipate changing the school size in the near future.

\section{Curriculum}

\section{Curriculum description}

The curriculum of our medical school program is fully integrated across the BS-MD years. The school's Department of Community Health and Social Medicine teaches a robust curriculum in population health and includes coursework in sociomedical sciences; learning the social, ecological, and biomedical aspects of health and illness; population health and community-oriented primary care (COPC); conducting community health assessments; a required 8-week service learning experience completing a community project at a health or social service agency; epidemiology and biostatistics; U.S. health care systems and policy; and population health research.

Our clinical skills curriculum also spans both the BS and $\mathrm{MD}$ programs. These clinical courses begin in the second

Acad Med. 2020;95:S339-S342.

doi: 10.1097/ACM.0000000000003416

Copyright (C) 2020 by the Association of American Medical Colleges

Supplemental digital content for this article is available at http://links.Iww.com/ ACADMED/A920.

Year school was established: 2016.

School URL: https://www.ccny.cuny.edu/csom year of college. In the Practice of Medicine (POM) courses, students learn lifestyle medicine and health coaching skills, and participate in a 3-year longitudinal clinical experience (LCE) at a federally qualified health center (FQHC) beginning in undergraduate year 3. There, they learn health systems science, develop their clinical skills, and identify and address the social determinants of health that affect the health of patients and communities served by these health centers.

See Supplemental Digital Appendix 1-Curriculum Schematicat http://links.lww.com/ACADMED/A920.

\section{Curriculum changes since 2010}

CUNY SOM received preliminary accreditation in 2015 so the curriculum has recently been developed. Aside from minor revisions, there are no plans to change the curriculum. The ACGME core competencies formed the basis for the general competencies along with an additional competency in population health and COPC, which is unique to our academic program and mission. The development of outcome-based educational program objectives in all general competencies focused on the school's mission and vision.

\section{Assessment}

Our 7 core competencies_-patient care, medical knowledge, lifelong learning, interpersonal skills and communication, professionalism, systems-based practice, and population health/ COPC-frame the overall integrated 7-year science and clinical curriculum. The educational program objectives are mapped to the core competencies as well as to the session-level objectives using $\mathrm{LEO}+$, our learning management system.

See Supplemental Digital Appendix 2-Competencies, Program Objectives, and Outcome Measures_at http://links.lww.com/ ACADMED/A920.

\section{Medical education program objectives}

We use a variety of andragogy to achieve our educational program objectives. Although the main instructional method is lecture (54\%), our school provides numerous other teaching methodologies designed to foster application of knowledge, self-reflection, and self-directed learning. Lectures are recorded and available to students within 24 hours after each lecture. Workshops and large-group discussions are used to review and clarify key concepts and use information to analyze and solve problems. Engaged learning occurs in virtually every course through laboratory, small-group, and/or team-based learning sessions. Journal clubs in several courses (e.g., POM, Population Health and Community Health Assessment, selectives in Population Health Research, Introduction to Medical Genetics) expose students to cutting-edge research and promote evidencebased practice and lifelong learning. Self-directed learning starts 
with problem-based learning $(\mathrm{PBL})$ in the third year of the undergraduate program and continues throughout the 16-month integrated organ systems curriculum in the preclerkship years. In each of the 9 organ system modules, students work through 2 PBL cases. PBL groups remain the same for a given module to promote team building and allow facilitators sufficient contact with students to provide each student with meaningful narrative formative and summative feedback. Peer teaching occurs throughout the curriculum, enhancing students' knowledge retention, teaching skills, and self-confidence.

\section{Clinical experiences}

Clinical exposure begins in the summer between the second and third years of the 7-year program in the Evaluation in Healthcare Setting course and continues in the 3-year POM course (undergraduate year 3 and medical school years 1 and 2). Over 30 separate simulation or standardized patient sessions provide numerous opportunities for students to practice their skills in health coaching, history taking, communication and physical examination, clinical reasoning, and oral and written presentations. These experiences, which begin in the third year of the undergraduate program and are completed in the fourth year of the medical school program, are designed to also allow students to identify and resolve ethical issues and to use an evidence-based approach to deliver safe and quality patient care to diverse patients.

Students complete their required clinical educational experiences at clinical sites that include FQHCs for their preclerkship clinical experiences and at our affiliate hospitals for the third- and fourth-year clerkships.

\section{Required longitudinal experiences}

Our students have required primary care opportunities that span 5 years, beginning with a 3 -year LCE for students during their undergraduate and preclerkship years. Students complete these LCE sessions at centers that provide primary care to patients in medically underserved areas. Students also complete their 8 -week required family medicine clerkship at ambulatory family medicine clinics that are either FQHCs or clinics affiliated with hospitals.

\section{Challenges in designing and implementing clinical experiences for medical students}

Our primary challenge has been in creating meaningful clinical experiences at each $\mathrm{FQHC}$ or $\mathrm{CHC}$ site that meet all the course objectives because faculty time and resources for education are often limited. We have been working with the clinical sites to develop value-added activities (such as health coaching, quality improvement, screening, and addressing social determinants of health).

\section{Curricular Governance}

The Curriculum Committee has primary responsibility for the curriculum. Together with the Student Academic Progress Committee, these committees oversee CSOM's educational program and its policies. The Curriculum Committee is responsible for oversight of curriculum development, implementation, and review, and also for development and implementation of policies related to the curriculum. The authority of the Curriculum Committee is provided in the CSOM bylaws. Although the dean has the ultimate responsibility for the educational program, the committee has the authority and responsibility to mandate changes in course and curricular content and in policies and procedures.

See Figure 1-Curriculum governance.

Department chairs have direct responsibility for courses in their department and assign faculty to oversee and teach courses and facilitate PBL sessions. The chairs manage their own budgets for adjunct faculty and provide administrative support for course directors. Otherwise, curricular governance and oversight to ensure compliance with LCME standards and school policies is centralized.

\section{Education Staff}

The Office of Institutional Research and Evaluation, under the Office of Academic Affairs, oversees curriculum management and evaluation. The office's executive director has broad managerial oversight for various aspects of monitoring and evaluating the curriculum. The staff charged with these activities include a learning management system specialist, a mapping specialist, an evaluation manager, and an evaluation specialist. They provide support to the Curriculum Committee and its subcommittees, including the Curriculum Review Subcommittee, Basic Science Subcommittee, and Clinical Subcommittee. Other staff in the office, a data analyst and an academic student support manager, track student performance data. There are also 4 fulltime clerkship administrators with oversight for all required third- and fourth-year clinical clerkships and an administrator with oversight of all affiliation agreements. We also have a full-time administrator to oversee our standardized patient encounters and another administrator to oversee our sites for the preclerkship clinical experiences. The Course Administration office provides audio visual support for courses and oversight of proctoring multiple-choice exams, including those from the NBME. This office consists of a director, an academic testing coordinator, and a technology specialist. We also have administrative staff to support our Curriculum Committee and Student Academic Progress Committee, as well as Basic Science and Clinical Curriculum Subcommittees.

\section{Faculty Development and Support in Education}

The CUNY SOM dean has designated the deputy dean as the chief academic officer of the SOM. The dean, in conjunction with the deputy dean, has the responsibility to develop, implement, and administer the SOM degree programs and report to the president of the City College of New York. The dean appoints the deputy dean, associate deans, and assistant deans to assist with carrying out his/her responsibilities.

See Figure 2-Organizational chart of dean's office. 


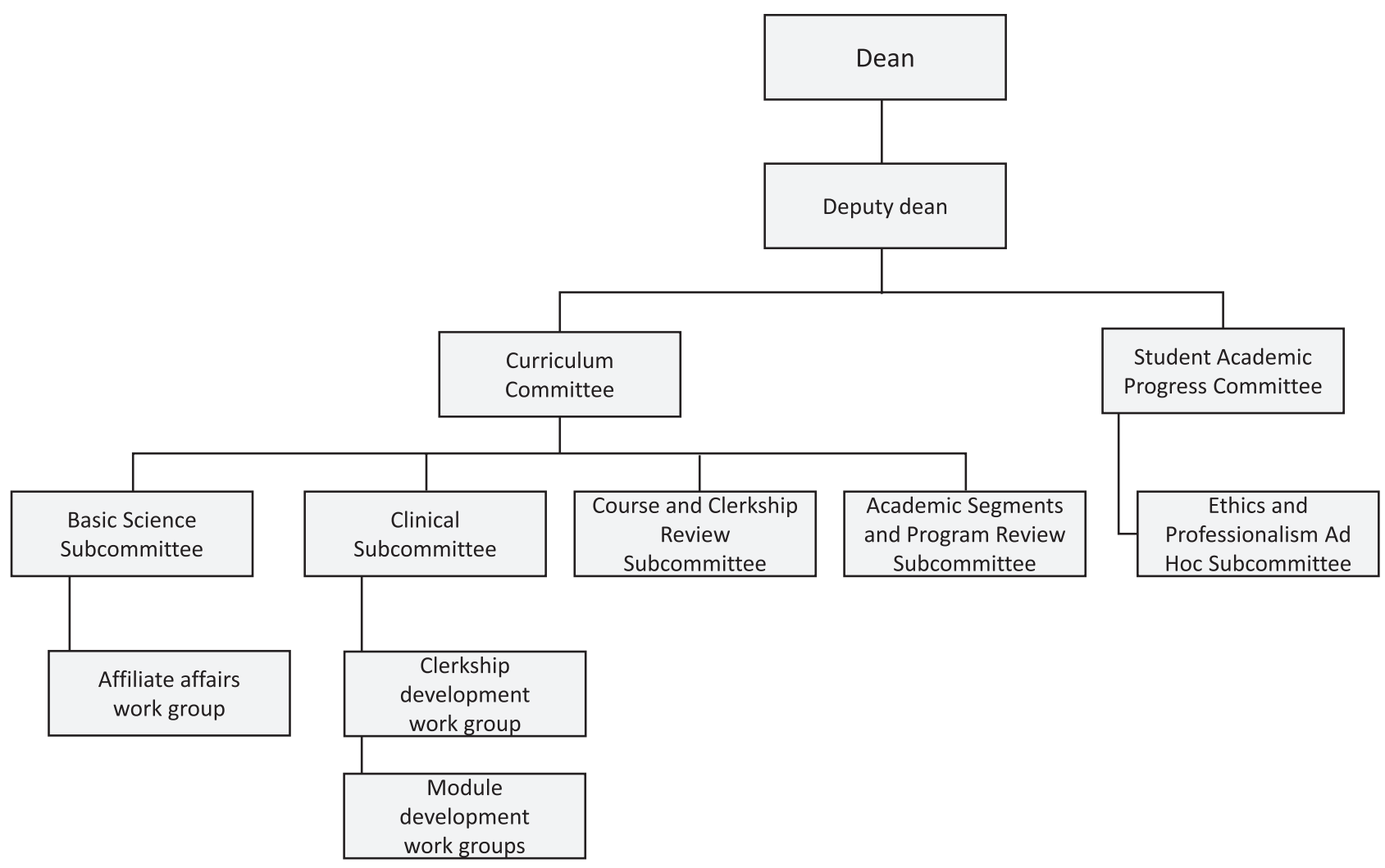

Figure 1 Curriculum governance.

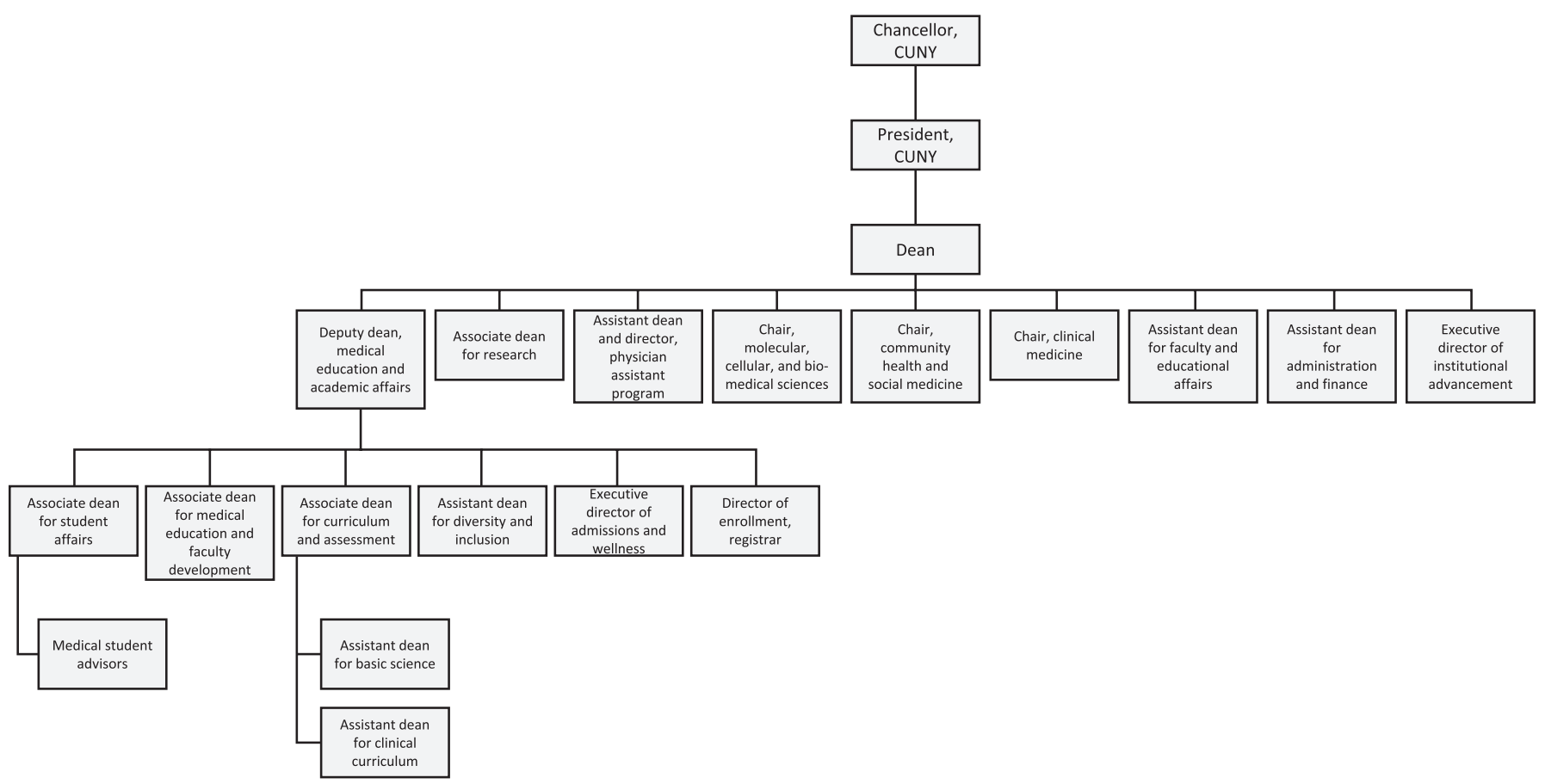

Figure 2 Organizational chart of dean's office. 
The medical education staff and administrative faculty are responsible for the undergraduate (BS) and medical school curriculum. Their role is primarily to support faculty, monitor and assess the curriculum, and oversee student support. The deputy dean or his/her designee also oversees the Office of Student Affairs, which is headed by the associate dean for student affairs, registrar, admissions and financial aid, Office of Course Support, learning resource center, and financial aid. The school does not oversee its affiliate hospitals and is not responsible for graduate medical education or continuing professional development. The Department of Medical Education houses all of the full-time salaried faculty with oversight and teaching of the preclerkship curriculum (excluding population health and basic science) as well as all faculty who are administrators or deans with oversight of faculty development, clerkships, curriculum and assessment, admissions, wellness, and student support (student affairs, learning resource center). 


\section{Icahn School of Medicine at Mount Sinai}

Robert Fallar, PhD, Valerie Parkas, MD, and Reena Karani, MD, MHPE

Icahn School of Medicine at Mount Sinai (ISMMS) celebrated its 50 th year in 2018. Over the years, the school has endeavored to fulfill its missions of education, research, patient care, and service with enthusiasm and distinction. The school was created upon the foundation of clinical and research successes of the Mount Sinai Hospital as well as service to its surrounding communities, and is now embedded in a health system comprising 8 community-based and tertiary care hospitals. The mission of the medical educational program is to produce physicians and scientists who are prepared to enter society as informed advocates and activists, able to advance clinical care and science, and capable of promoting change.

\section{Medical Education Program Highlights}

- As a result of student advocacy and efforts in 2015, the school embarked on addressing biases in all of its functional areas and launched the institution-wide Racism and Bias Initiative in 2017. Educational enhancements that focus on addressing racism and bias were implemented through all 4 years of the curriculum. Currently, the school has a robust, longitudinal curriculum on biases - including sexual orientation, gender identity, culture, disability, race, and others - that is threaded through multiple courses and clerkships. Emphasis is placed on the students' own lived experiences before entering medical school, and on an examination of ways in which their own implicit biases influence their approach to learning medicine and engaging with patients.

- Through shared leadership and accountability in fostering the values of diversity and inclusion, ISMMS continues to enhance diversity and inclusion at all levels in the organization. The school has positioned faculty and senior administrators from diverse backgrounds into leadership, administrative, and/or teaching positions to allow students (including those in pipeline programs) the opportunity to work with and be trained by a diverse array of faculty and administrators.

- Early assurance admissions: ISMMS is the first medical school in the country to create progressive and rigorous admissions pathways for students who seek early assurance of admission. The Donald and Vera Blinken FlexMed Program allows college sophomores in any major to apply for early assurance of acceptance to the school. Once accepted, students are free to pursue their baccalaureate educational plan unencumbered by

Acad Med. 2020;95:S343-S345.

doi: 10.1097/ACM.0000000000003415

Copyright (C) 2020 by the Association of American Medical Colleges

Supplemental digital content for this article is available at http://links.lww.com/ ACADMED/A919.

Year school was established: 1968.

School URL: https://icahn.mssm.edu. the traditional science requirements and the MCAT. Students who are accepted are expected to adhere to all of ISMMS's program requirements before entry. The school also has a small yet innovative early assurance program for active military members.

- ISMMS provides a rich, robust, and supportive environment for faculty and students to engage in scholarly activity. There are also extensive resources to sustain a scholarly environment for faculty. The school is ranked 12th in the nation in $\mathrm{NIH}$ funding. Per the 2018 AAMC Operations Management Survey, the school is in the top $10 \%$ for research dollars per investigator among participating U.S. private medical schools, demonstrating the availability of research and mentorship opportunities for students. ${ }^{1}$

\section{Curriculum}

\section{Curriculum description}

The MD curriculum begins in year 1 with exposure to foundational scientific principles and core patient care competencies. This is followed by the year 2 curriculum, building on these foundational principles with an emphasis on an organ system-based approach to the pathophysiology and treatment of illnesses and diseases. The clerkships in year 3 and year 4 offer exposures to and active participation in patient care across the life span in the core clinical clerkships and prepare students for residency training through experiences designed to promote advanced knowledge and skills development in an individualized manner. There are also a significant number of elective weeks in year 3 (10 weeks) and year 4 (18 weeks) to allow for career exploration.

See Supplemental Digital Appendix 1-Curriculum Schematicat http://links.lww.com/ACADMED/A919.

\section{Curriculum changes since 2010}

- Flextime for all class years provides protected time to use in whatever ways students desire. This includes 1 half day per week in years 1 and 2, scheduled half days off in the required year 3 clerkships, and 1 week in year 4 .

- InFocus weeks throughout the curriculum offer core training in topics critical to medical practice and biomedical research. There are 8 weeks spread over the 4 -year curriculum covering the following themes:

- InFocus 1-4 in years 1 and 2: scholarship and research (basic scientific methodology, study design, research evaluation, statistical methods), East Harlem health, global health, and physician advocacy and human rights

- InFocus 5-8 in years 3 and 4: patient safety and health system science; career planning, teaching skills; leadership; law and economics of medicine 
- All students are required to complete a mentored scholarly project before graduation, and approximately one-third of every class takes an additional scholarly year to pursue these interests or an additional degree. Internal funding covers all ISMMS students doing summer research across the health system. In each of the last 2 academic years, graduating students published over 300 peer-reviewed manuscripts.

- A thoughtful, relevant, and deeply impactful approach is taken to identifying key societal problems in the context of the school's mission and the needs of its community. This focus has led to the development of para-curricular experiences such as the East Harlem Health Outreach partnership, the Human Rights and Social Justice Scholars Program, the NYC Coalition to Dismantle Racism in Health Care, the Human Rights Program, Scrubs Addressing the Firearm Epidemic, and the Racism and Bias Initiative.

- Peer-reviewed graduation distinction is held in 3 categories: global health, medical education, and research.

- PORTAL is an intensively mentored program designed to encourage career development of medical students who seek to become physician-scientists. The program guides students in designing and conducting original research studies addressing the prevention, diagnosis, prognosis, and treatment of disease. Students earn a dual MD-MSCR (Masters of Science in Clinical Research) degree, which prepares them for a future as lifelong learners and for careers in academia, research institutes, industry, or regulatory agencies.

- The Primary Care Scholars Program (PCSP) is a selective program that prepares students to meet the rising health needs of local and global communities. The program provides training, mentorship, and longitudinal experiences in primary care. It creates a forum for collaboration with fellow students, faculty, scientists, and trainees, all of whom are passionate about improving primary care through direct care, research, quality improvement, and advocacy. The PCSP offers students who remain in primary care practice in underserved communities $\$ 80,000$ in loan forgiveness.

- The Ramon Murphy, MD Program for Global Health Education is dedicated to improving the health of underserved communities around the world. The program offers innovative curricula and provides real-world experience in the classroom and in the field at domestic and international partner sites. The Global Health Scholars Program is a 5-year MD-MPH dualdegree scholarship program that offers advanced coursework, mentorship, and field experiences to prepare medical students for careers in global health.

\section{Assessment}

The medical education program objectives are designed to reflect the knowledge, skills, and attitudes expected of all graduating students. These objectives are encompassed in the following 4 competencies, which guide the curriculum. Each is further defined by behaviorally described objectives.
Competency 1, patient care:

- History taking

- Physical examination

- Procedural skills

- Clinical reasoning

- Medical decision making

- Communication skills

Competency 2, scientific and medical knowledge:

- Organ structure and function

- Characteristics and mechanisms of disease

- Healing and therapeutics

- Social determinants of health

- Health care resources and delivery systems

- Ethical principles of medical practice and research

Competency 3, learning, scholarship, and collaboration:

- Self-awareness and commitment to self-improvement

- Methods of investigation, analysis, and dissemination

- Teamwork

Competency 4, professionalism and advocacy:

- Service

- Leadership and accountability

- Honesty and integrity

- Empathy

- Respect

Assessments are linked to every medical education program objective. These assessments incorporate multiple modalities including multiple-choice exams, standardized patients, direct observation with patients, reflections, team projects, oral and written presentations, and self-directed learning activities. All objectives are assessed using both formative and summative assessments to help students monitor their progression.

\section{Assessment changes since 2010}

- Formative assessments to guide learning in all courses and clerkships

- Standardization of the clinical performance assessment form with descriptive anchors for use across all clerkships

- Standardization of NBME subject examination weight in clerkship grades

- Option to take NBME Customized Assessment Services exams throughout years 1 and 2 to guide learning

- Incorporation of self-directed learning activities throughout years 1 and 2 to build skills and receive formative feedback on one's ability to identify and synthesize relevant literature and appraise the credibility of information sources

- Summary statement for the Medical Student Performance Evaluation (MSPE): ISMMS follows closely the recommendations of the AAMC MSPE Task Force for the structure and content of the MSPE; the school stopped ranking students or using ranking qualifiers as part of the MSPE in 2017 
- As of 2018 ISMMS does not participate in the Alpha Omega Alpha Honor Society for medical students, a decision based on the historic racial inequities in the selection of honorees, both at ISMMS and nationally

\section{Clinical experiences}

ISMMS is embedded in a health system comprising 8 community-based and tertiary care hospitals. Students are able to rotate through all of these sites for both inpatient and outpatient experiences. Additional outpatient experiences are provided by affiliated local health centers and primary care providers. Inpatient experiences in several clerkships are also available through rotations at a Veterans Affairs hospital and a public city hospital.

Learners first encounter clinical experiences in the doctoring course Art and Science of Medicine (ASM). During year 1, ASM students meet in small groups with 2 copreceptors, a physician, and a social worker, and visit patients in both inpatient and outpatient settings. In year 2, ASM students advance their clinical exposure with patients, gaining clinical reasoning as well as advanced communication and collaboration skills.

Challenges in designing and implementing clinical experiences include:

- Faculty preceptors faced with challenges regarding time to teach and supervise

- Ensuring comparability of experiences across clinical sites

- Providing continual training regarding teaching skills for house staff and faculty

\section{Curricular Governance}

The Executive Oversight Committee has the authority to set medical education program objectives; establish educational requirements; establish and approve curricular and assessment content; ensure content integration, coordination, and coherence; evaluate educational outcomes; and review/approve all policies related to curricular and student affairs. There are 22 voting members representing faculty, course/clerkship directors, and students from all class years.

\section{Education Staff}

ISMMS's Department of Medical Education oversees the curriculum and program administration. Functional offices within the department include:

- Curricular Affairs

- Student Affairs

- Admissions and Recruitment

- Assessment and Evaluation

- Continuing Medical Education

- Graduate Medical Education

There are 35 faculty with primary appointments in medical education and 105 faculty with secondary appointments.

\section{Faculty Development and Support in Education}

The Institute for Medical Education (IME) resides under the auspices of the Department of Medical Education. There are significant opportunities and resources available for professional development related to teaching, assessment, evaluation, and research skills from the IME. Faculty development is provided by the IME at all sites throughout the health system. Since AY 2018-2019, the IME has 3 faculty who devote $20 \%$ of their effort to providing faculty development at all sites in the health system. Medical Education Grand Rounds Are Broadcast Throughout The Health System, as well as recorded for on-demand viewing.

The Center for Multicultural Affairs (CMCA) also provides research skill development, mentorship, and networking opportunities for faculty who identify as underrepresented in medicine. Funding to support professional development activities comes from a variety of sources, including individual clinical departments, the Department of Medical Education, the CMCA, IME, and the ISMMS dean's office.

Acknowledgments: The authors wish to thank Dr. Peter Gliatto and Dr. Mary Rojas for their assistance in aggregating information for this report. They also thank Dr. David Muller for his assistance in revising the manuscript.

\section{Reference}

1 FY 2018 AAMC Operations Management Tool. Washington, DC: Association of American Medical Colleges; 2020. 


\section{Jacobs School of Medicine and Biomedical Sciences at University at Buffalo}

Alan J. Lesse, MD, and Michael E. Cain, MD

\section{Medical Education Program Highlights}

- A new medical school built in downtown Buffalo as part of the Buffalo Niagara Medical Campus: The educational program at the University at Buffalo Jacobs School of Medicine and Biomedical Sciences (Jacobs School) has undergone significant change over the last 10 years, highlighted by the relocation of the campus to downtown Buffalo. The new education and research building is an 8-story, 628,000-square-foot, \$375-million, state-of-the-art medical school that opened for classes in January 2018. This addition completed the urban downtown medical campus, which also includes the recent openings of the 170,000-square-foot, state-of-the-art Clinical and Translational Research Center (CTRC) in 2012, which is adjacent to Buffalo General Medical Center (Kaleida Health). As a joint venture between the University at Buffalo and Kaleida Health, the CTRC provides a centralized facility for the Clinical and Translational Science Institute. The University at Buffalo and Kaleida Health partnered to build the facility that houses the CTRC (which occupies floors 5 through 8) and the Gates Vascular Institute (floors 1 through 4) and serves as the centerpiece of the NIH-sponsored Clinical and Translational Science Award grant, which concentrates on health disparities and innovative ways to address these issues. Completion of the Conventus Medical Office Building (opened in March 2017) provided additional outpatient clinical and administrative space, which is joined with the Oishei Children's Hospital (opened in November 2017). These new structures joined the Buffalo General Medical Center and the Roswell Park Comprehensive Cancer Center to create a truly urban academic medical center in the heart of Buffalo.

- New facilities allow for greater use of active learning: The new Jacobs School building allows for a greater use of active learning, with designs that facilitate small-group discussion, including a 216-seat, 24-table, active learning room for team-based, case-based, and problem-based learning formats and their hybrid formats. Multiple small-group learning rooms are available, and the building incorporates informal learning spaces called "learning landscapes" on each floor to promote collaboration. The location in the downtown district accentuates service learning projects in the area, including the Lighthouse Free Medical Clinic (a student-run outpatient medical clinic) and the UB Heals program, which provides

Acad Med. 2020;95:S346-S348.

doi: 10.1097/ACM.0000000000003477

Copyright (C) 2020 by the Association of American Medical Colleges

Supplemental digital content for this article is available at http://links.Iww.com/ ACADMED/A954.

Year school was established: 1846.

School URL: http://medicine.buffalo.edu. medical attention and outreach to our homeless populations in Buffalo.

- Campus-wide interprofessional forums: We are especially proud of our 10-school coordinated interprofessional educational forums in the fall and spring that bring together around 600-750 students each semester from the Schools of Public Health and Health Professions (occupational therapy, exercise and nutritional sciences, community health and health behavior, and physical therapy), School of Dental Medicine, School of Social Work, School of Pharmacy and Pharmaceutical Sciences, Jacobs School of Medicine and Biomedical Sciences, School of Nursing, School of Law, and School of Management. Integrated small groups of 6-9 students are brought together to learn about, and from, other specialties in separate forums dealing with the opioid crisis and the management of physical and mental health concerns during the treatment of chronic diseases. Students learn the scope of practice of different professionals and how they can add to the care of a health care team.

- Redesign of the medical curriculum and the establishment of the Medical Education and Educational Research Institute (MEERI): Over the last 3 years, the Jacobs School has produced 5 strategic plans: medical education, clinical practice, research, interprofessional education, and diversity. While all 5 strategic plans are active, the medical education strategic plan has made particular strides. Goal 1 of this plan calls for the redesign of the medical curriculum. To effectively deliver on goal 1, faculty need to be educated on the most effective ways to deliver that goal. Therefore, goal 2 created MEERI, which is geared toward faculty development to best deliver our current and new curricula. We recently recruited Dr. Jennifer Meka, PhD, to direct this institute.

\section{Curriculum}

\section{Curriculum description}

See Supplemental Digital Appendix 1-Curriculum Map—at http://links.lww.com/ACADMED/A954.

\section{Curriculum changes since 2010}

The Jacobs School has changed to a pass/fail grading system in the preclinical years, beginning in 2019. After thorough review and discussion in the Curriculum Committee and dialogue at open school forums, the Curriculum Committee approved the implementation of a pass/fail grading system in Phase 1 of the curriculum. Numeric grades are maintained in the background for use in Alpha Omega Alpha Honor Medical Society selection and letters of commendation for excellence in academics. A tiered grading system (honors/high satisfactory/satisfactory/ unsatisfactory) remains for the clinical years (Phase 2). 
The curriculum of the school has continued to evolve over the last decade with expansion of the Office of Medical Curriculum and the Office of Student and Academic Affairs. A more robust review process has been in place for the last 5 years, with improvement in student evaluations and quality metrics in the Graduation Questionnaire. This work has been greatly assisted through the creation of the new Office of Accreditation and Quality Improvement and continuous monitoring of the LCME Standards and Elements.

\section{Class size changes since 2010}

The Jacobs School increased its class size from 144 to 180 students for the entering class of 2017. The entering class of 2020 will bring the school to its full 720 student enrollment across all 4 years. The new medical school building was crucial in accommodating this increased student population.

\section{Assessment}

Our medical education program objectives are based on the AAMC Physician Competency Reference Set.

See Supplemental Digital Appendix 2-Competencies, Program Objectives, and Assessment - at http://links.lww.com/ ACADMED/A954.

We have increased the use of standardized patients and simulation over the last decade. An integrated curricular thread has been developed for patients with disabilities using real patients and their caretakers. Increased use of OSCEs and a longitudinal OSCE have been implemented across the curriculum.

\section{Pedagogy}

The design of the new Jacobs School enables us to use a full array of teaching pedagogies. We have increased the use of team-based learning, small- and large-group discussion, case-based learning, problem-based learning, and other forms of active learning. Students are required to attend all small-group sessions using active learning techniques. Flipped classroom approaches include the use of short videos and podcasts for preparation. Lecture attendance is highly recommended, but all lecture sessions are recorded and available by streaming and download with speechto-text indexing for rapid retrieval.

Over the last decade, the use of active learning principles has increased and the total number of lecture hours has decreased.

\section{Clinical experiences}

- Students are active participants in clinical activities shortly after the start of their first year. This occurs during the Clinical Practice of Medicine course where they meet weekly with preceptors or in small-group sessions to develop their skills as physicians, both from a scientific understanding of health, disease, and pathophysiology and as empathic physicians in the clinical context. The new 18-station Clinical Competency Center, with audio and visual recording capacity, and the Behling Simulation Center give students experience using standardized patients and human patient simulators to learn the skills necessary for patient encounters in a controlled setting.

- The clinical sites available to the Jacobs School include acute care hospitals (including a National Cancer Institute Comprehensive Cancer Center), a county hospital with a Level 1 trauma center and burn center, and both urban and community hospital locations. A newly constructed pediatric hospital is part of the Buffalo Niagara Medical Campus (BNMC). Clinics are based in both hospital and private settings as well as the Conventus Office complex on the BNMC. The school also has a significant number of required and elective rotations at the VA hospital, which also serves as a site for summer student research projects.

- We have increased our full-time faculty and have also increased our engagement with volunteer faculty to provide additional clinical training sites for our expanded number of students.

\section{Curricular Governance}

The Curriculum Committee manages the integration of the curriculum across all 4 years of medical education. Membership includes both administrative members and members elected by Faculty Council, with the senior associate dean for medical curriculum and a Faculty Council elected member as cochairs of the committee. Student members include 2 representatives of the Organization of Student Representatives (AAMC) and 1 class member from each class year who are elected by the student body. One MD-PhD student is also elected by their peers. The director of MEERI and the medical librarian are also members of the committee.

Permanent subcommittees of the Curriculum Committee are the Phase 1 Committee, composed of course directors of all the Phase 1 courses and student members, and the Phase 2 Committee, composed of course directors for the required clerkships in the clinical years. Students are also an integral part of the Phase 2 Committee as well.

Brief course reviews are completed annually by the Office of Medical Curriculum and presented to the Curriculum Committee, and triennial course reviews are performed by ad hoc review committees composed of Phase 1 and Phase 2 faculty members and a student who has completed the course under review.

\section{Education Staff}

The Offices of Medical Education includes both the Office of Medical Curriculum and the Office of Student and Academic Affairs. The Office of Medical Curriculum consists of a senior associate dean for medical curriculum and 3 associate deans for medical curriculum. A senior curriculum coordinator and 2 curriculum coordinators provide support for delivery, monitoring, and management of the Phase 1 curriculum and also assist with the Phase 2 curriculum. The Office of Student and Academic Affairs has a senior associate dean, 2 associate deans, 1 student services coordinator, and 1 assistant dean of student and academic affairs. This office deals with academic 


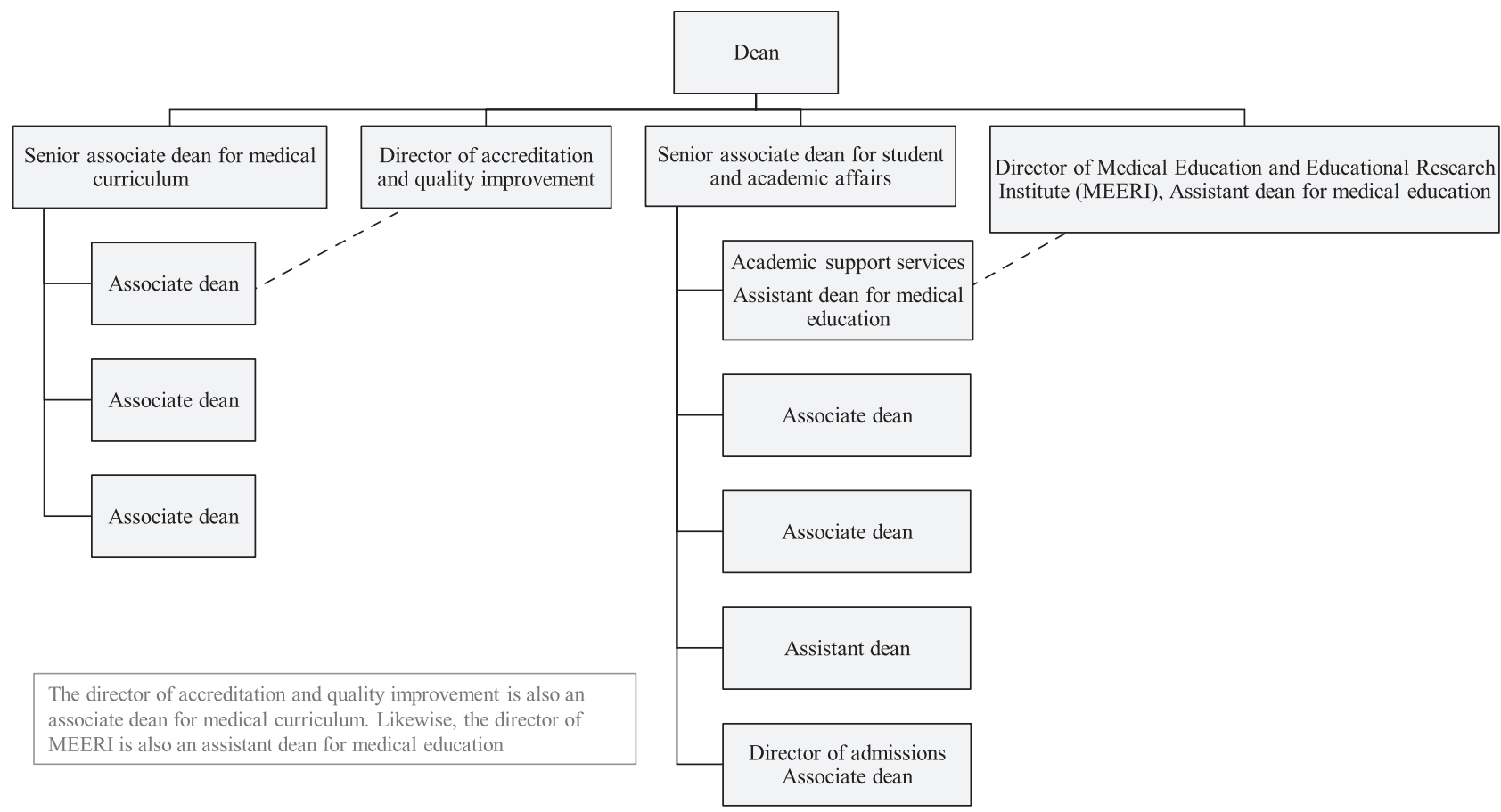

Figure 1 Medical education leadership.

support, career advising, opportunities for research, and school counseling. The recently formed Office of Accreditation and Quality Improvement provides monitoring and support for the curriculum and is staffed by a director of the Office of Accreditation and Quality Improvement and a senior program administrator. The school offers onsite counseling and schoolsponsored psychiatric services at no cost to students.

GME and CME are handled out of separate offices.

See Figure 1-Medical education leadership.

\section{Faculty Development and Support in Education}

The Jacobs School recently established MEERI for the purpose of professional development of faculty. The institute offers personalized sessions for curricular content as well as workshops and conferences for faculty development in the areas of education, developing teaching and instructional design skills, and educational research. The Jacobs School also partners with the Graduate School of Education to enhance the scope of the program and is developing a Masters in Education with specialization in medical education in conjunction with the graduate school.

\section{Teaching academy}

MEERI functions as an academy for our medical educators and oversees the advancement of medical education at the Jacobs School. Faculty from the Jacobs School, both Phase 1 and Phase 2 , have access to the expertise of the institute, as do residents in the graduate medical education program. MEERI also works to promote scholarship in medical education and peer support, as well as career advancement and recognition for medical educators.

\section{Initiatives in Process}

The school has begun a major curricular revision that will be fully implemented with the entering class of 2022. The process began in 2018 with a comprehensive revision of the medical education program objectives, followed by a curriculum retreat involving faculty, students, staff, community representative, hospital partners, and other members of the university community. 


\section{New York Medical College}

Jennifer Koestler, MD, Pamela Ludmer, MD, MMEL, and Celia S. Freeman, MBA

\section{Medical Education Program Highlights}

New York Medical College School of Medicine (NYMC SOM), located in Valhalla, New York, is one of the nation's oldest private health sciences universities (est. 1860). The SOM provides a comprehensive educational program with the goal of developing well-rounded medical students who will become resilient, compassionate, and skilled physicians. The SOM is proud of our strong foundational science education, diverse affiliated clinical training sites, and commitment of its faculty and administration to medical student education. Distinguishing features of the MD program include:

- Longitudinal clinical skills training program that provides early clinical exposure and frequent opportunities for formative and summative feedback

- Highly interactive, asynchronous online elective courses, which allow students the opportunity to explore current issues in medicine (e.g., multiculturalism in medicine, learning to teach, ethical and clinical issues in end-of-life care, practice skills in medical malpractice)

- Areas of Concentration (AOC) program, which allows medical students to engage in a scholarly project in an area of personal interest beyond the formal curriculum. Current offerings include biomedical research, biomedical ethics, medical education, and global and population health

- Comprehensive paracurricular activities (e.g., longitudinal resiliency curriculum, student wellness weeks, NYMedTalks) to support student professional development, well-being, and resiliency

\section{Curriculum Description}

The MD program is organized into 3 vertically and horizontally integrated phases: foundational sciences, clinical immersion, and advanced practice. The program also leverages the state-of-theart Clinical Skills and Simulation Center (CSSC) throughout all 4 years of the curriculum.

The preclerkship curriculum is organized into modular blocks with foundational science and clinical content integrated across disciplines. Longitudinal themes of patient care, interpersonal communication skills, biomedical ethics, interprofessional practice, and evidenced-based medicine are woven into related coursework.

Acad Med. 2020;95:S349-S352.

doi: 10.1097/ACM.0000000000003438

Copyright (C) 2020 by the Association of American Medical Colleges

Supplemental digital content for this article is available at http://links.Iww.com/ ACADMED/A932.

Year school was established: 1860.

School URL: https://www.nymc.edu.
See Supplemental Digital Appendix 1-Curriculum Map—at http://links.lww.com/ACADMED/A932.

Students enter discipline-based clerkships in year 3 of the program with required rotations in internal medicine ( 8 weeks), surgery ( 8 weeks), pediatrics (6 weeks), obstetrics-gynecology ( 6 weeks), psychiatry ( 6 weeks), neurology and rehabilitation medicine ( 4 weeks), and family medicine ( 6 weeks). Students also have one 4 -week elective to assist them in career exploration.

The fourth year is organized into tracks based on specialty interest, thereby allowing students to align their learning experiences with their career trajectories and enhance preparation for residency training. General requirements for fourth-year students include a mandatory subinternship with 2 additional requirements based on specialty track (e.g., emergency medicine, critical care, radiology, diagnostic medicine), 5 elective months based on specialty interest, and 2 transition courses that prepare students for the increased responsibilities associated with entry into the fourth year and residency.

\section{Curriculum changes since 2010}

Since 2010, we have enhanced the integration of our curriculum, increased the use of active learning principles, and incorporated content areas that reflect contemporary issues in health care. Specific areas that have been expanded include women's health, LGBTQ+ health, patient safety, interprofessional collaboration, and pain management. We have also added a required History of Medicine course, providing students with an appreciation of the evolution of modern medicine. In addition, we have increased our curricular focus on research principles to enhance the development of students' analytic and critical thinking skills.

The SOM is currently undergoing a curricular redesign to be launched in academic year 2021-2022. The new curriculum will be organized into 3 integrated longitudinal threads: biomedical sciences, patient-centered care, and health system sciences and will incorporate medical imaging, lifestyle medicine, and "back to basic science" electives in year 4 . Students will have additional time early in the curriculum for career exploration and added elective time in the third year.

\section{Medical education program objectives}

The NYMC SOM curriculum, guided by our medical education program objectives, is designed to offer a breadth of foundational science and clinical training to prepare graduates for entry into competitive residency programs. Faculty of the SOM have defined a core set of competencies and program objectives to outline the expectations that medical students are expected to exhibit as evidence of their achievement by completion of the program. 
See Supplemental Digital Appendix 2-Program Objectives and Assessment Methods-at http://links.lww.com/ACADMED/A932.

The graduation competencies and medical education program objectives were updated and approved by the Education and Curriculum Committee (January 2019) as a component of an overarching curriculum redesign process. The current program objectives were informed by the existing NYMC SOM competencies for graduating physicians, the Physician Competency Reference Set, the AAMC Core Entrustable Professional Activities (EPAs) for entering residency, and the Interprofessional Education Collaborative core competencies.

\section{Assessment changes since 2010}

We have diversified our student assessments throughout the MD program to include more workplace and EPA-based assessments in clerkships, increased the number of objective structured clinical examinations, added new assessments to evaluate self-directed learning and interprofessional teamwork and collaboration, and enhanced our assessment of students' professional competencies with graded reflective exercises and independent learning plans. In 2018, the school modified the grading in preclerkship courses to be fully pass/fail. Grading in core clerkships remains honors/high pass/pass/fail.

\section{Parallel curriculum or tracks}

The SOM introduced its first longitudinal integrated clerkship (LIC) in 2018. The LIC program is 6 months in duration and includes the following disciplines: internal medicine, neurology, pediatrics, and obstetrics-gynecology. Students spend the majority of their time in the ambulatory setting embedded with interdisciplinary teams of health care providers. Regular, unscheduled self-directed learning time is also incorporated into schedules to allow students to follow assigned patients across venues of care (e.g., consultations with subspecialists, scheduled tests, procedures) and assist in maintaining patient-practice continuity.

\section{Pedagogy}

The MD program uses a variety of pedagogical approaches to achieve medical education program objectives, including casebased learning, clinical experiences in both the ambulatory and inpatient settings, large- and small-group discussion formats, cadaveric dissection, laboratory exercises, peer teaching, preceptorship programs, role play, self-directed learning, simulation, standardized patients, and workshops.

The most significant changes in pedagogy over the last decade have been the progressive decrease in the use of traditional large-group lecture and the introduction of a flipped classroom approach; large-group interactive exercises; smallgroup, case-based learning formats; and low- and high-stakes simulation training. The case-based exercises throughout the preclerkship phase of the curriculum use a common architecture; are introduced in a developmental fashion; and are coordinated across courses, enabling students to develop skills that promote self-directed and lifelong learning, critical thinking, and critical appraisal. In addition, the CSSC has
20 fully equipped patient exam rooms and several large simulation rooms, which allow students to practice a broad range of skills such as physical examination, skill training using standardized patients, and team training exercises using mannequins. Multifunctional classroom space allows students to practice procedural skills on a variety of task trainers, complementing their clinical training.

In parallel to these innovations, significant enhancements were made to our instructional technology infrastructure. These include migrating to LEO, a robust health care educationspecific learning management system that provides a single platform for student curricula, assessment, and individualized educational portfolios; the installation of SMART podiums and touchscreen technology; full transition to computer-based examinations; use of video capture; and migration to virtual microscopy.

\section{Clinical experiences}

The SOM uses a variety of clinical training sites including tertiary care centers, academic medical centers, community hospitals and nursing homes, Veterans Affairs medical centers, ambulatory practice sites, and rural practice locations. These facilities provide an extraordinary diversity of patients, medical conditions, and community settings that enrich students' educational experiences.

Clinical training begins during the first week of medical school, where students are trained in cardiopulmonary resuscitation and are introduced to patient-provider communication skills. Over the course of the year, students participate in workshops with standardized patient educators to practice history taking, interpersonal communication, and physical examination skills. Students are then placed in clinical preceptorships where they have the opportunity to participate as members of interprofessional health care teams. Since 2019, first-year students also receive training as health coaches and have opportunities to practice their skills in a variety of community-based settings before being placed with primary care providers in ambulatory practices for longitudinal preceptorships.

The majority of clerkship training is in the form of block specialty clerkships. A subset of third-year students elects to participate in an LIC offered in year 3.

Standardization and coordination of the MD curriculum among our multiple training sites are high priorities and pose particular challenges. In addition, over the last decade, we have recognized increasing competition for clinical training sites from allopathic and osteopathic medical schools, offshore medical schools, and other health professions students.

\section{Curricular Governance}

The Education and Curriculum Committee (ECC) is the faculty committee with primary responsibility for the curriculum. Members are appointed annually by the SOM dean after consultation with the Executive Committee of the Faculty 
Senate, who provide a preferred list of nominated members of the faculty for these service roles. Committee membership is intentionally diverse and includes representatives from basic science disciplines, clinical faculty from major clinical sites, and student representatives.

The ECC and reporting committees oversee all aspects of the curriculum including the development and review of program-level objectives, oversight of the horizontal and vertical integration of the curriculum, monitoring of the overall quality and outcomes of individual courses and clerkships, monitoring of the outcomes of the curriculum as a whole, and monitoring compliance with educational standards of the LCME. The work of this committee is facilitated by outcome data provided by the Office of Data Analytics (ODA) and is supported by staff in the Office of Undergraduate Medical Education (OME).

See Figure 1-Curriculum management.
The budget to fund the MD program is managed by the Office of Academic Affairs, with support from the dean and input from senior leadership and the offices of undergraduate medical education, medical student affairs, and faculty affairs. The annual budget process prioritizes capital requests for educational facilities and technology, faculty development, and student academic support.

\section{Education Staff}

The OME is responsible for providing the administrative support for the planning, implementation, evaluation, and oversight of the MD curriculum. These efforts include support of the ECC and its subcommittees, the CSSC, medical student research, medical education faculty development, education technology, and the AOC program. The ODA provides important support of ongoing continuous quality improvement efforts related to program evaluation.

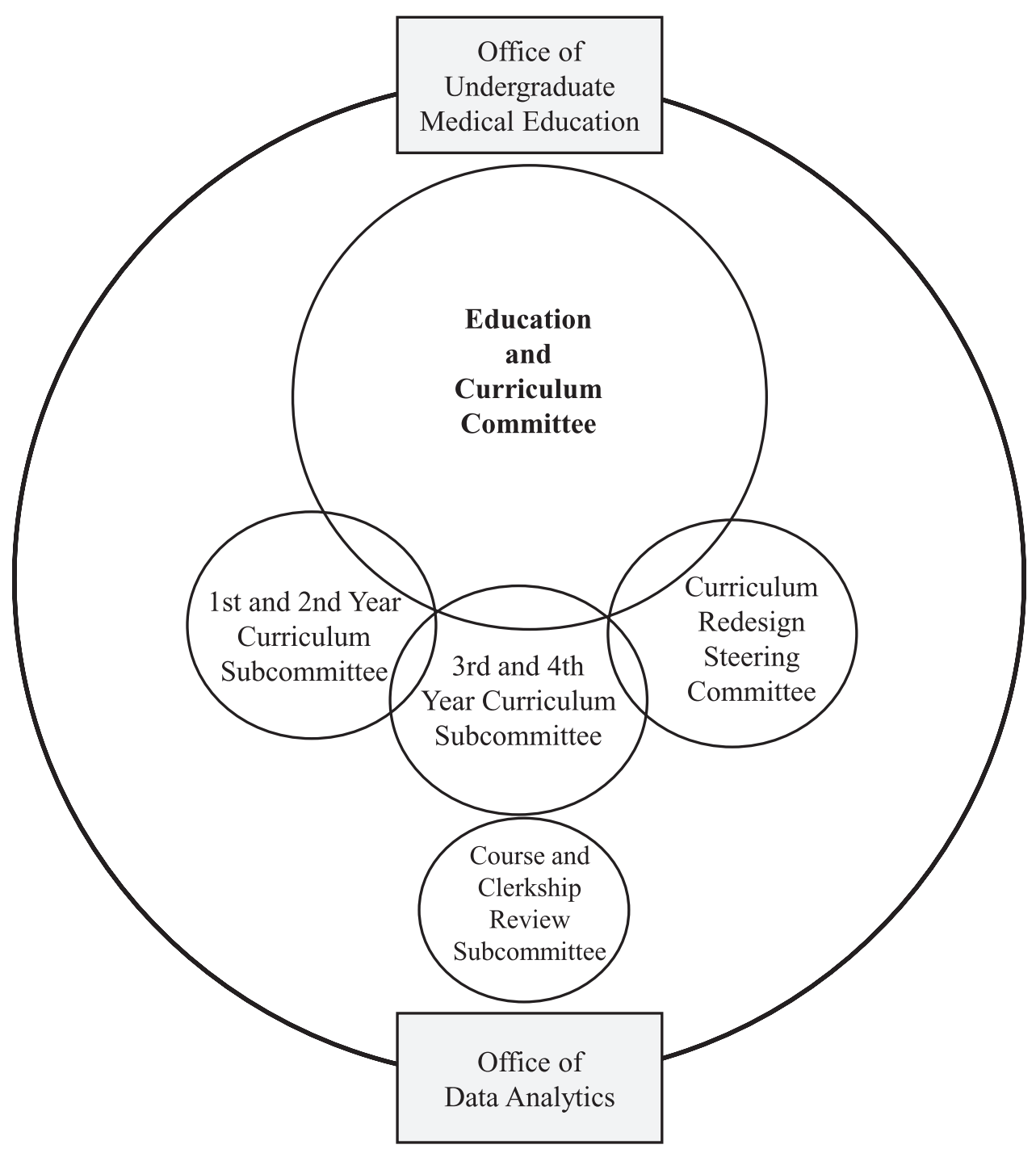

Figure 1 Curriculum management. 


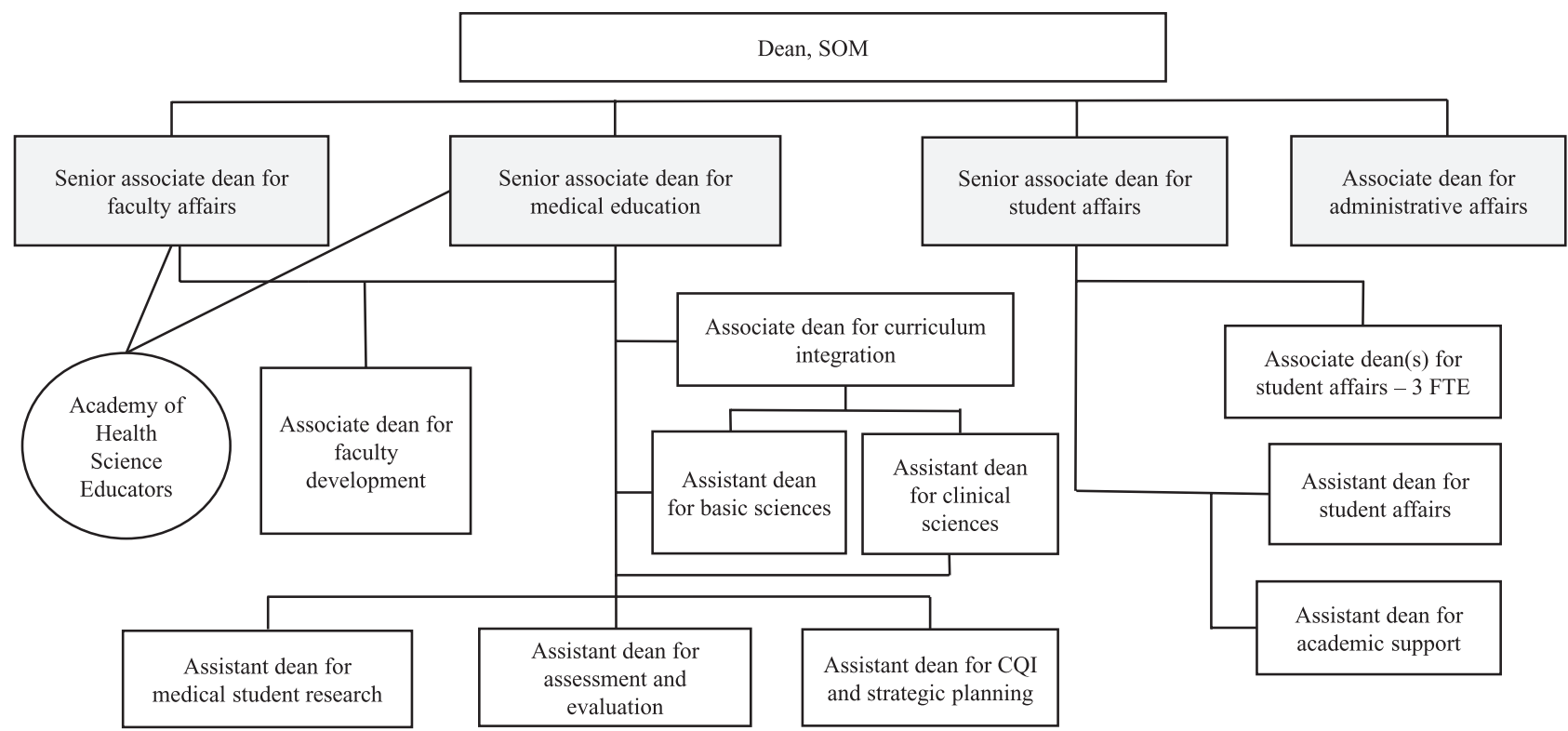

Figure 2 Educational staffing structure.

See Figure 2-Educational staffing structure.

\section{Faculty Development and Support in Education}

The OME and the Office of Faculty Affairs use needs assessment data to design faculty development programs to enhance teaching skills and promote the continuous growth of faculty as teachers, mentors, and educational scholars. These efforts are accomplished through skills development workshops, monthly medical education grand rounds, 1-on-1 consultations, and a faculty mentorship program. The offices additionally support faculty to attend outside programs sponsored by organizations such as the AAMC, Harvard Macy Institute, and International Association for Medical Science Educators.

The college is developing a comprehensive and interprofessional Academy of Health Science Educators, whose mission is to foster a supportive environment for educational innovation, collaboration, and scholarship by establishing a vibrant local community of practice.

The Academy of Health Science Educators aligns with the work of the Tenure, Appointments, and Promotions Committee, which formally established an educator track in 2015. Promotion in this pathway reflects special achievement in the domains of direct teaching, curriculum development, mentoring and advising, and scholarship.

\section{Initiatives in Progress}

The SOM is developing a health systems science curricular thread to include preclerkship modules on research and fundamentals of data-driven decision-making; principles of collaborative practice; population and social determinants of health; health care economics, policy, and structure; patient safety and high-value care; and quality and leadership in teams, with application of relevant concepts in core clerkships. 


\title{
New York University Long Island School of Medicine
}

\author{
Gladys M. Ayala, MD, MPH, Amy V. Rapkiewicz, MD, Steven E. Carsons, MD, and Steven P. Shelov, MD, MS
}

\section{Medical Education Program Highlights}

The New York University Long Island School of Medicine (NYULISOM), located in Mineola, New York, is devoted to educating primary care physicians of the highest quality and who will become leaders of both our community's and nation's health care system. The innovative 3-year curriculum provides students with a scientifically rigorous learning environment founded on the principles of translational science, population health, outstanding clinical science, and application of health systems science.

\section{Notable features:}

- The small class size (40 maximum per class) promotes a collaborative and highly favorable faculty-student learning environment.

- Longitudinally vertical and horizontal integration of basic, clinical, and health systems sciences is implemented across the 3-year curriculum, with problem-based learning (PBL) as a core teaching methodology and use of plastinated cadavers along with autopsy exposure for anatomy instruction.

- Integral to the transformative curriculum that streamlines the flow from medical school into residency, accepted applicants are offered an early, conditionally guaranteed residency opportunity through the National Resident Matching Program in one of our linked primary care residency specialties of internal medicine, obstetricsgynecology, pediatrics, or surgery.

- Longitudinal academic coaching occurs from day 1 . These sessions are designed to assess the student needs, identify areas for improvement and assistance, and plan strategies to achieve academic and career goals. Coaches use our state-of-the-art educational dashboard to review progress and help develop a professional portfolio.

\section{Curriculum}

\section{Curriculum description}

The 3-year curriculum is distributed over 3 phases. Phase I is the preclinical year and Phases II and III are the clinical years.

Phase I (46 weeks) lays a foundation of integrating basic, clinical, and health systems sciences with patient care.

Acad Med. 2020;95:S353-S357.

doi: 10.1097/ACM.0000000000003481

Copyright (C) 2020 by the Association of American Medical Colleges

Supplemental digital content for this article is available at http://links.Iww.com/ ACADMED/A966.

Year school was established: 2019.

School URL: https://medli.nyu.edu.
- It starts with an introduction to core basic science concepts (language acquisition) with case-based clinical learning exercises, along with immersion in integrated clinical skills training that provides the bedside clinical skills needed to become a competent doctor.

- Morning classes in general are dedicated to core principles of medicine, held in small- and large-group formats, which stimulate discussions and critical thinking around the fundamental basic medical sciences through an organ systems approach. The organ systems are cardiology, respiratory, renal, gastroenterology, endocrine-reproductive, musculoskeletalrheumatology-dermatology, brain-mind-behavior, and hematology-oncology. PBL sessions engage students through a self-directed process with facilitators, while providing the clinical relevance of the basic sciences.

- The afternoons are reserved for the longitudinal courses and expose students to the practice of health care delivery and health systems science. Practice of medicine (POM) course sessions convey the essential skills in medical interviewing and bedside examinations. These skills are aligned to the morning morphology of anatomy, histology, pathology, and radiology longitudinal thread, and reinforced through the continuity ambulatory practice experience (CAPE), where students learn the key issues relevant to the actual care of patients. Our high-tech clinical simulation exercises provide further useful feedback to hone the requisite clinical skills for a primary care physician. During the Learning Communities-Social Science Humanities Ethics and Professionalism (LC-SHEP) course, students are exposed via discussion groups to the critical aspects of professionalism, medical ethics, moral reasoning, and medical jurisprudence. In the Health Systems Science (HSS) course, students will develop a fundamental understanding of how health care is delivered; how health care providers work together; and strategies to deliver safer, higher-quality patient care, in addition to learning the essentials of critically appraising and applying the medical literature to patient care. Through these integrated collective learning venues, students will gain insight into the behavioral, moral, and social aspects of medicine and develop the knowledge and skills needed during the clinical curriculum to interact with patients, health care providers, and the health care system.

Phase II ( 49 weeks) is dedicated to clerkships in medicine, neurology and rehabilitation medicine, obstetrics-gynecology, pediatrics, primary care, psychiatry, and surgery. During this phase, students develop the clinical skills necessary to diagnose disease and treat patients.

- On a weekly basis, students continue to take part in one of the longitudinal educational experiences of CAPE, HSS, and LCSHEP. This phase ends with the Capstone 1 course, dedicated to a comprehensive review of basic science concepts and their application to clinical practice, helping students prepare for the USMLE Step 1 examination. 


\section{Table 1}

\section{Program Objectives and Assessment Methods}

Objectives
EPA $1 \mathrm{a}$ and $1 \mathrm{~b}$ :
Obtain a history and
perform a physical
examination both
complete and adapted
to the patient's clinical
situation
situation justify a prioritized differential diagnosis

EPA 3: Recommend and interpret common diagnostic and screening tests

EPA 4: Formulate, communicate, and implement patientcentered plans

\section{Assessments} performance computer based

Multisource assessment

Narrative assessment

Oral patient presentation

Participation

Peer assessment

Self-assessment performance computer based

Narrative assessment

Oral patient presentation

Participation

Self-assessment based

Multisource assessment

Narrative assessment

Oral patient presentation

Stimulated recall
Clinical documentation review

Clinical performance rating/checklist

Exam: institutionally developed, clinical

Exam: institutionally developed, written/

Exam: nationally normed/standardized subject

Clinical documentation review

Clinical performance rating/checklist

Exam: institutionally developed, clinical

Exam: institutionally developed, written/

Nationally normed/standardized subject

Clinical documentation review

Clinical performance rating/checklist, exam: institutionally developed, written/computer

Nationally normed/standardized subject

Clinical documentation review

Clinical performance rating/checklist

Exam: institutionally developed, clinical performance

Exam: licensure, clinical performance

Nationally normed/standardized subject

Multisource assessment

Narrative assessment

EPA 5 and $6:$

Clinical documentation review

Document and present a clinical encounter
Clinical performance rating/checklist

Exam: institutionally developed, written/ computer based

Nationally normed/standardized subject

Narrative assessment

Oral patient presentation
Table 1

(Continued)

\section{Objectives}

EPA 7: Form clinica questions and retrieve evidence to advance patient care

EPA 8: Give and receive the handover in transitions of care as a member of an interprofessional team a patient requiring urgent or emergent care, provide initial assessment, management, and seek help

EPA 11: Obtain informed consent for tests and or/common procedures

EPA 12: Perform general tasks and procedures of a physician
Assessments

Clinical documentation review

Narrative assessment

Research or project assessment

Clinical documentation review

Clinical performance rating/checklist

Multisource assessment

Narrative assessment

Oral patient presentation

Clinical documentation review

Clinical performance rating/checklist

Exam: institutionally developed, clinical performance

Exam: licensure, clinical performance

Multisource assessment

Narrative assessment

Self-assessment

Clinical documentation review

Clinical performance rating/checklist

Exam: institutionally developed, clinical performance

Exam: licensure, clinical performance

Nationally normed/standardized subject

Multisource assessment

Narrative assessment

Oral patient presentation

Self-assessment

Clinical documentation review

Clinical performance rating/checklist

Exam: institutionally developed, clinical performance

Exam: licensure, clinical performance

Multisource assessment

Narrative assessment

Clinical documentation review

Clinical performance rating/checklist

Exam: institutionally developed, clinical performance

Exam: licensure, clinical performance

Multisource assessment

Narrative assessment

Self-assessment

EPA 13: Apply health systems science principles to optimize health outcomes for patients and populations

\section{Clinical documentation review}

Clinical performance rating/checklist

Exam: institutionally developed, written/ computer based

Narrative assessment

Participation

Research or project assessment 
Phase III (37 weeks) begins with a 3-week Capstone 2 course, a structured review of clinical science concepts and their application to the POM.

- Students will also participate in a comprehensive clinical skills exam with series of mock patient encounters that assess skills in communication, taking patient histories, conducting physical examinations, and clinical reasoning - all in preparation for the USMLE Step 2 examinations.

- During this last phase of the curriculum, students will participate in advanced clinical skills development by completing required rotations in emergency medicine, subinternship, critical care, diagnostics and therapeutics ( 2 weeks of advanced applications of clinical and pharmacological management principles in the care of patients), transition to residency (specialty-driven skills development), and a Capstone 3 course whereby students present system science-based projects that arose out of observed opportunities for improvement encountered over the 3 years, along with electives.

- Throughout this phase, students also continue with the longitudinal educational experiences in the HSS, CAPE, and LC-SHEP courses.

See Supplemental Digital Appendix 1-Curriculum Overviewat http://links.lww.com/ACADMED/A966.

\begin{abstract}
Assessment
The medical education program objectives are based primarily on the AAMC Core Entrustable Professional Activities (Core EPAs) with minor revisions incorporating aspects of the CanMEDS Framework to align with the mission and vision of NYULISOM. The critical competencies mapped to our program objectives are driven from the Physician Competency Reference Set competencies using the AAMC recommendations as the benchmark. Developmental milestones using the Dreyfus model of novice to expert were devised to determine the desired behaviors of pre-entrustment vs entrustment. The course objectives are linked to the critical competencies, and course goals act as the framework to develop the learning objectives, which are assessed throughout the curriculum.
\end{abstract}

See Table 1-Program Objectives and Assessment Methods.

\section{Pedagogy}

See Table 2-Major Pedagogical Approaches.

\section{Clinical experiences}

- All of the required student clinical experiences occur on campus in the hospital wards and outpatient clinics at the NYU Winthrop Hospital and its local, community-affiliated clinical training sites or faculty practices.

\section{Table 2}

\section{Major Pedagogical Approaches}

\begin{tabular}{|c|c|}
\hline Pedagogy & Approach \\
\hline $\begin{array}{l}\text { Case-based } \\
\text { learning }\end{array}$ & $\begin{array}{l}\text { Throughout the curriculum patient cases are used to promote discussion, problem solving, and reasoning in clinical and basic } \\
\text { sciences. }\end{array}$ \\
\hline $\begin{array}{l}\text { Discussion: large } \\
\text { group }(>12)\end{array}$ & $\begin{array}{l}\text { Learning Communities and Health Systems Sciences courses use large-group discussions through exchange of ideas, sharing } \\
\text { insights, and knowledge. }\end{array}$ \\
\hline $\begin{array}{l}\text { Discussion: small } \\
\text { group }(\leq 12)\end{array}$ & $\begin{array}{l}\text { Learning Communities and Health Systems Sciences courses break into small-group discussions to further explore ideas and } \\
\text { insights of peers. }\end{array}$ \\
\hline Laboratory & $\begin{array}{l}\text { The laboratory is an integrated unit organized around a systemic approach that uses innovative electronic resources, } \\
\text { plastinations, pathology, radiology, and histology. }\end{array}$ \\
\hline $\begin{array}{l}\text { Problem-based } \\
\text { learning (PBL) }\end{array}$ & $\begin{array}{l}\text { Students are introduced to PBL during the first week of medical school and they have multiple sessions per week throughout } \\
\text { Phase I. Students work in small groups to define clinical questions and through self-directed learning, each student researches } \\
\text { literature and assesses the quality of the evidence. PBL sessions continue within Phase II and are specific to each clerkship. }\end{array}$ \\
\hline Simulation & $\begin{array}{l}\text { Students participate in simulations that mimic or amplify real patient encounters using scenarios based on real life health care- } \\
\text { related situations. We use the highest-fidelity manikins to replicate physiological responses to disease states and administration } \\
\text { of medications and or external lifesaving devices. Students are also exposed to a multitude of low-fidelity task trainers to } \\
\text { develop skills such as suturing, IV placement, and catheterization. }\end{array}$ \\
\hline $\begin{array}{l}\text { Standardized/ } \\
\text { simulated } \\
\text { patients }\end{array}$ & $\begin{array}{l}\text { Learners have a chance to practice interviewing skills or physical exam skills in a safe setting. Standardized patients are trained } \\
\text { to provide an environment where learners can practice a particular interview approach or try a new physical exam for the first } \\
\text { time without risking the comfort, modesty, or safety of a "real" patient. Learners can also have a chance to work in emotionally } \\
\text { charged situations such as domestic violence, angry patients, or a bad-news case without risk. Standardized patient encounters } \\
\text { are learner-centered rather than patient-centered educational experience. }\end{array}$ \\
\hline $\begin{array}{l}\text { Team-based } \\
\text { learning }\end{array}$ & $\begin{array}{l}\text { Team-based learning sessions are incorporated in several courses/clerkships throughout the curriculum. The students prepare } \\
\text { before the in class session then work through exercises in small groups during class time and receive real time feedback from the } \\
\text { facilitator. }\end{array}$ \\
\hline Video/podcast & $\begin{array}{l}\text { Videos and screencasts are assigned as preparation for some class sessions. Students may be assigned faculty vetted videos from } \\
\text { a host on sponsored resources. }\end{array}$ \\
\hline Workshop & $\begin{array}{l}\text { Integrated physiology workshops are strategically placed following Phase } 1 \text { organ systems blocks for the purpose of challenging } \\
\text { students to approach a multifaceted clinical problem. }\end{array}$ \\
\hline
\end{tabular}


- Clinical and nonclinical longitudinally required experiences occur throughout the 3-year curriculum. Once a week, students take part in 1 of the following experiences: CAPE, HSS course, and learning communities.

- The clinical experience in the curriculum starts from the beginning of medical school. Through their CAPE course students join faculty physicians in medical offices or nearby ambulatory care clinics, 1 to 2 afternoons per month. These clinical experiences include geriatrics, internal medicine, family medicine, pediatrics, and obstetrics-gynecology, and after 6 months, students are paired with a preceptor in their chosen primary care specialty for the remainder of the 3 -year curriculum. Students also acquire ambulatory clinical experiences throughout Phases II and III. In addition, oral health education and training is received in collaboration with the Department of Family Dental Medicine.

- Students additionally are exposed to inpatient clinical experiences throughout the curriculum starting in Phase I during their bedside teaching sessions as part of the POM course and recurring in Phases II and III while students are on their clerkships, subinternships, critical care, and electives/selectives.

\section{Curricular Governance}

- The primary responsibility, governance, and management of the curriculum reside with the Evolving Physician Education Committee (EPEC), a faculty policy committee in the School of Medicine. EPEC is advisory to the dean, who has the overall authority for the educational program. The vice dean, appointed by the dean, serves as chair of EPEC. Members to EPEC are both appointed by the dean and elected from the general full-time teaching faculty, as well as student representation, one from each phase of the curriculum.

See Figure 1-Curricular governance.

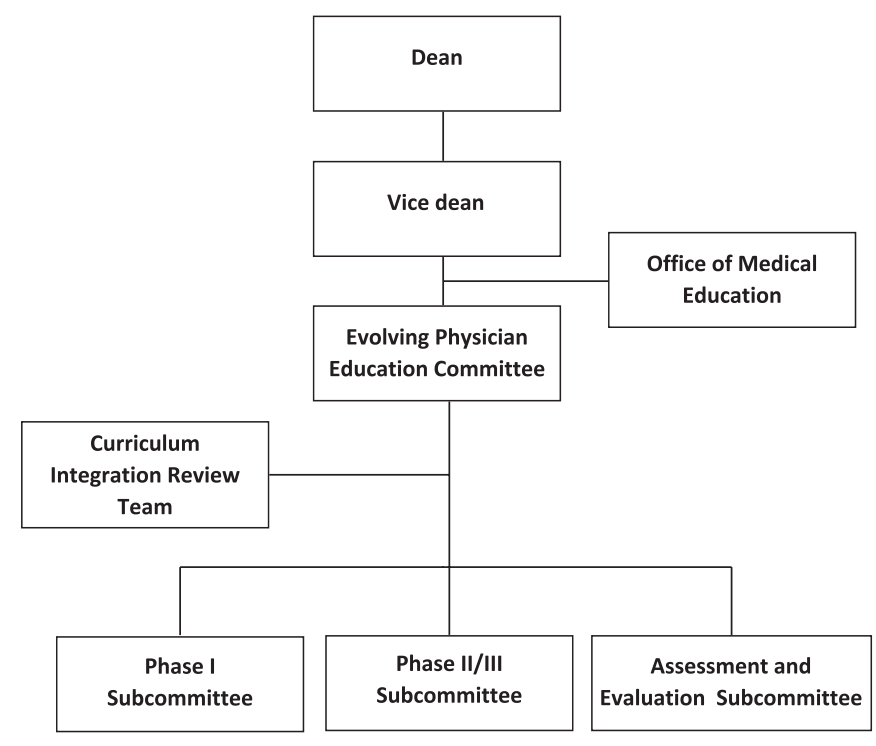

Figure 1 Curricular governance.
The following subcommittees and working group report to EPEC:

- The Curriculum Integration and Review Team (CIRT), a working group chaired by the associate dean for medical education, serves as the intermediate monitor of the curriculum and as such has responsibility for reviewing of the school's educational program, the core curriculum, educational outcomes and delivery, and assessment methods of individual courses before presentation to EPEC. A major task of CIRT is to manage and align the horizontal and vertical integration of the curriculum.

- Phase I and Phase II/III Subcommittees are composed of first-year course directors and clinical/clerkship directors, respectively, as well as longitudinal course and thread directors and are charged to oversee quality and discuss common issues such as policy, pedagogy, and educational technology and course evaluations.

- The Assessment and Evaluation Subcommittee's charge is to ensure a strong alignment between the program, phase, course, and learning objectives with the appropriate assessment rubrics and tools.

All 3 subcommittees direct quality improvement through their recommendations to the EPEC and through their interactions with the faculty.

See Figure 2-Education organizational chart.

\section{Education Staff}

- The Office of Medical Education provides the administrative and academic support for the planning, coordination, implementation, oversight, and assessment and evaluation of the curriculum and its educational committees. It is responsible for supporting the educational deans and core teaching faculty, and for the development and maintenance of the education tools and technology to support curriculum delivery, monitoring, and management. The office consists of a director, a manager for registration and student records, 5 courses/clerkships coordinators, and a full-time educational technology/instructional design professional.

\section{Faculty Development and Support in Education}

- Faculty at NYULISOM have multiple professional development opportunities to enhance their teaching skills and foster career growth. The institution hosts live events such as grand rounds and half-day and full-day workshops and also disseminates upcoming webinars with speakers who are content experts on instructional methods, assessment techniques, and leadership attributes. Core teaching faculty receive structured peer observations with tailored, objective feedback delivered in real time. Opportunities for educational grants are made available to all faculty. Presentation at local and national academic meetings and involvement in local and national medical education societies and organizations are encouraged by the Office of Faculty Affairs. Faculty receive mentorship on manuscript preparation and revisions as needed to increase academic publications in peer-reviewed medical education journals. All early- to mid-level career full-time faculty receive 


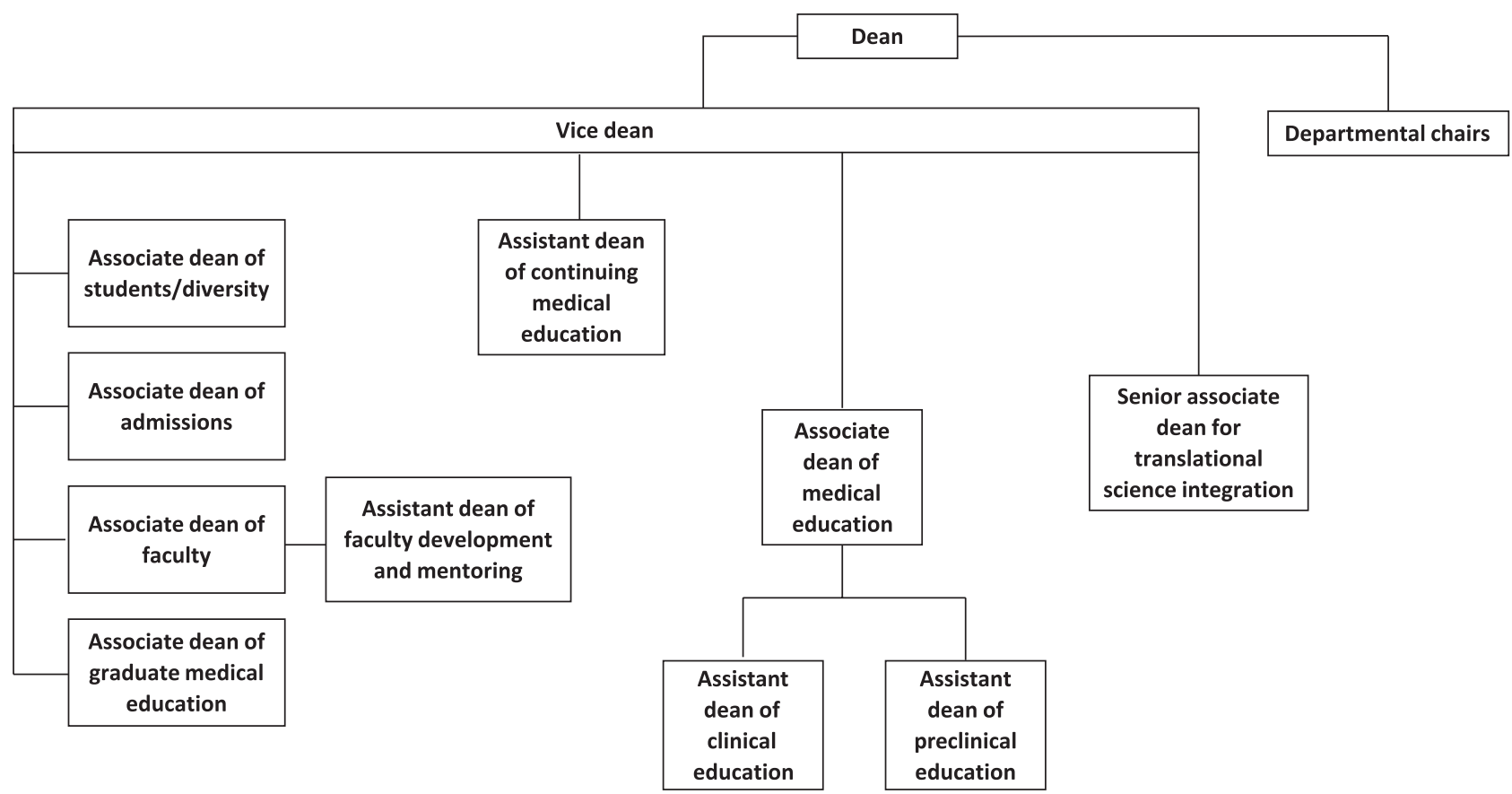

Figure 2 Education organizational chart.

a designated mentor to guide professional development toward academic promotion.

- The faculty educator track recognizes faculty with a high level of commitment to the teaching mission beyond what is expected from the average faculty member. Accepted activities include leadership and administrative roles in educational programs, development of new and innovative curricula, substantive teaching responsibilities, development of unique educational tools and resources, and scholarly or pedagogical work in the field.

- Faculty may qualify for promotion based upon distinguished contributions to the educational mission without publications. Evidence of such contributions should be documented in an academic portfolio and may include distinguished leadership of a medical school course, residency, or fellowship program; number of hours invested per semester; number of courses; or extent of involvement in the educational continuum. Promotion also requires documentation of the effectiveness of teaching as evidence by evaluations, and in the judgment of students, trainees, and peers. Teaching awards (departmental, hospital, school, university, national) and documentation of the effectiveness of teaching programs would be expected.

- The Faculty Scholars Program at NYULISOM offers medical school faculty the opportunity to advance their teaching skills and develop curricula that effectively deliver high-quality, evidence-based medical education. The program offers 2 pathways: an educator pathway (12 months) and a researcher pathway (18 months). Participants are nominated by their departmental leaders, are expected to attend educational sessions, and will complete self-guided assignments on topics such as learning theories, collaborative learning methods, instructional models for designing courses, and how to be an effective leader and mentor in academic environments. Those in the researcher pathway also gain experience searching and appraising literature about medical education, designing research studies, analyzing data, and presenting and publishing research findings. 


\title{
New York University Grossman School of Medicine
}

\author{
Vicky Harnik, PhD, Steven B. Abramson, MD, Joan Cangiarella, MD, and Mel Rosenfeld, PhD
}

\section{Medical Education Program Highlights}

The NYU Grossman School of Medicine (GSOM) and NYU Hospitals Center function as the NYU Langone Medical Center (NYULMC), an integrated academic medical center. It has a unique organizational structure wherein leadership of the hospital and medical school is under a single CEO and dean, crucial to developing and implementing our strategic plan.

The GSOM offers several parallel tracks allowing students a customized education, including 4- and 5-year dual-degree programs.

- MD-MPA, health policy and management

- MD-MPH, global public health

- MD-MS, translational research

- MD-MA, bioethics

- MD-MBA, general management

An accelerated 3-year MD pathway (3YMD) offers conditional acceptance through the NRMP to 1 of NYULMC's 20 residency programs.

Students train at Bellevue Hospital, the country's first public hospital, as well as our flagship Tisch Hospital, new state-of-theart Kimmel Pavilion, Hassenfeld's Children's Hospital, and VA NY Harbor Health Care (VA) system, thus experiencing firsthand a diverse patient population.

All students receive full-tuition scholarships regardless of need or merit.

The Institute for Innovations in Medical Education (IIME), one of the largest medical education innovation groups nationwide, harnesses GSOM's diverse intellectual capital to drive discovery, development, and validation of new information technologies for medical education. We are a leader in use of sophisticated tools to collect, analyze, and display electronic learning data (e.g., dynamic dashboards). The opportunities such data present are potentially transformative: our evaluation of curricular effectiveness can move from anecdotal to epidemiologic, detailed learner-level data can drive the move toward individualized learning and personalized progression, and analyzing performance changes over time can propel us toward competency-driven health education.

Acad Med. 2020;95:S358-S361.

doi: 10.1097/ACM.0000000000003451

Copyright (C) 2020 by the Association of American Medical Colleges

Supplemental digital content for this article is available at http://links.Iww.com/ ACADMED/A941.

Year school was established: 1841.

School URL: https://med.nyu.edu.

\section{Curriculum}

\section{Curriculum description}

Our 145-week curriculum is divided into 4 stages:

- Stage 1: 18 months of preclerkship modules

- Stage 2: 12 months of core clerkships

- Stage 3: a 6-month period between the end of clerkships and beginning of the fourth year

- Stage 4: final 12 months

See Supplemental Digital Appendix 1-Curriculum Schematicat http://links.lww.com/ACADMED/A941.

\section{Curriculum changes since 2010}

Our Curriculum for the 21st century (C21) was implemented in 2010. Driven by our CQI process, we identified curricular gaps and opportunities and instituted a number of major changes (examples below).

MD AWARE Curriculum: The goals of the Medical Students Developing Awareness, Well-Being, And REsilience curriculum are to help students:

- Understand relationships between awareness, well-being, resilience, and burnout

- Foster self-awareness through regular self-assessments

- Recognize signs of stress and respond with healthy coping tools

- Develop and practice evidence-based techniques promoting resilience

- Build strong peer connections

The 7 -session curriculum spans the first 2.5 years. Each session includes a large-group exercise and brief didactic, providing students an opportunity to self-assess their well-being and resilience, and a small-group segment. Topics for each session align with typical challenges faced by students at that point in their training. The small-group segments provide opportunity for sharing experiences in a safe setting with classmates, peer mentors, and nonfaculty-trained staff.

Anatomy curriculum redesign: Our new Living Anatomy module provides students with practical skills that could be further honed throughout medical school. Instead of uncovering structures by dissection, students now examine expertly dissected plastinated specimens and faculty-prosected cadavers. This allows allocation of more time for clinical correlations/clinical reasoning exercises, resulting in more durable retention of anatomical knowledge.

Additionally, we expanded hands-on use of imaging using the same PACS system students will use in clerkships. This allows seamless integration with anatomy content, while acknowledging the reality that most students will "see" inside 
their patients using imaging instead of with a scalpel. We also incorporated AR and VR exercises selected to solve educational challenges the anatomy presents or to gain familiarity with tools currently used to plan surgical procedures. For those who want to experience cadaveric dissection, we will offer clerkship and postclerkship electives.

Academic coaching: Each student is assigned a Violet Society Program (VSP) advisor to provide career advising and guidance on clinical, research, educational, and community-building opportunities. Recently, the VSP was expanded to include academic coaching. Each advisor will partner with their advisee to review academic performance dashboards, identify areas of weakness, and plan strategies for achieving career goals.

Clinical science inquiry (CSI): We designed a mobile platform integrating clinically relevant basic science concepts into core clerkships to help students revisit basic science content. CSI employs just-in-time and situated learning by emailing 2-3 questions a week to students' mobile devices. Questions were curated by a team of clinical and basic science faculty who teach and know the preclerkship content. Completion is mandatory, tracked centrally, but does not contribute to students' final grades.

\section{Assessment}

Our medical education program objectives are based on ACGME competence domains. However, as 1 of 10 AAMCappointed medical schools serving as a pilot cohort for testing implementation of the 13 Core Entrustable Professional Activities (EPAs), we are using EPAs to design assessments of these competencies.

See Supplemental Digital Appendix 2-Competencies, Objectives, and Outcome Measures-at http://links.lww.com/ ACADMED/A941.

In 2016, we appointed a director of integrated clinical skills to oversee assessment of clinical skills, thereby creating a comprehensive, coordinated system of assessments using a common framework. Since January 2019, clinical skills assessments in all curricular stages use behavioral anchors reflecting the Core EPAs. This allows students and their VSP coaches to track, via learner-specific dashboards, trajectories toward increasingly independent practice.

See Supplemental Digital Appendix 3-EPA-Based Clinical Assessment—available at http://links.lww.com/ACADMED/A941.

\section{Parallel curriculum or tracks}

GSOM offers several parallel tracks that allow customized education, including the dual-degree programs previously described and accelerated 3YMD program. The curriculum for the first 2.5 years aligns with our 4-year program, while the last 6 months are devoted to a subinternship, critical care rotation, and elective time to meet the 130 weeks of required education. The 3YMD offers conditional acceptance, through the NRMP, to any of NYULMC's 20 residency programs. Students also have the option of applying to an external residency program.
We offer acceptance into the 3YMD program for prematriculated students as well as 2 opportunities (opt-in) allowing current students program entry at 2 points, April/May of either their first year or clerkship year. Opt-in students are limited to 3YMD residencies available at the time of program application.

\section{Pedagogy}

A major goal of our C21 curriculum was shifting from a largely lecture-based curriculum to a more small-group, active learning environment. Our new structure is approximately $50 \%$ lecture, $25 \%$ small-group conferences, and a 25\% mixture of laboratories, simulation, team-based learning sessions, and workshops. Small-group conferences focus on case-based learning. During the clerkship year, clinical exposure includes both inpatient and outpatient venues.

Additionally, our 18-month Practice of Medicine (POM) module is directed by a full-time faculty member supported by several full-time staff members. Clinical faculty, with time dedicated for weekly teaching, provide sufficient coverage for uniform teaching across the 18-month module. The increased faculty allows more frequent bedside teaching sessions enriched to include interviewing, physical examination, clinical reasoning, oral presentation, and writeups. The number and frequency of POM OSCEs have also increased.

All POM and clerkship OSCEs have direct observation with verbal and written feedback and are assessed using a Core EPA framework. Since all OSCEs use identical checklists, faculty and students can efficiently, effectively track clinical skills acquisition.

Twelve online modules, WISE-On Call, were developed to prepare students for transition to residency through a symptom-based review of conditions they will likely encounter while on call. Modules incorporate didactic review of key concepts and causes associated with presenting symptoms, simulated cases modeling a resident's response to on-call scenarios, case-based practice questions to test students' knowledge, and symptom checklists to direct focus to the larger constellation of symptoms when prioritizing their differential. The modules stress the importance of appropriate interprofessional communication.

We added a mandatory simulation for all graduating students, Night On Call, using WISE-On Call modules. This 4-hour readiness-for-internship multi-instructional method simulation uses standardized patients, nurses, and attending physicians to simulate the experience of a hectic night on call. Launched in 2016, this program has been used to assess graduates' entrustability, through the lens of all 13 EPAs, performing multiple clinical tasks and responsibilities.

\section{Clinical experiences}

The major components of required curricula for the parallel tracks (3YMD; 4- and 5-year dual degree) are identical.

Differences between tracks center around elective requirements. 
All required clerkships use the diversity of patient experiences at various teaching sites. Each clerkship sends students to Bellevue Hospital, Tisch Hospital, Kimmel Pavilion, and Hassenfeld Children's Hospital. Additionally, with the exception of pediatrics and obstetrics-gynecology clerkships, students rotate through the VA. In the 4-week ambulatory care clerkship, students are assigned to various outpatient settings citywide. The pediatrics, surgery, obstetrics-gynecology, and neurology rotations include outpatient clinic experiences. Outpatient clinics are primarily at Bellevue Hospital and the VA. Community-based electives are also offered.

With the opening of Kimmel Pavilion, we added 830,000-squarefeet, including 11 patient floors, to NYULMC's hospital space. Several floors of Kimmel comprise Hassenfeld Children's Hospital, a facility with 68 single-patient rooms, the only pediatric inpatient facility with this feature in Manhattan. Together with our affiliates, our patient census provides rich clinical experiences. While implementing clinical curricular changes can be challenging, the support of clinical chairs and dean is essential for our success.

\section{Curricular Governance}

The Curriculum Committee derives its authority from the dean and oversees the educational program in the broadest sense. Current composition of the committee includes representation from module and clerkship directors, GME Office, selective directors, student body, medical library, assessment experts, and
2 or 3 individuals not directly involved in daily instruction of students. Additionally, we created a Subcommittee on Outcomes and Evaluations to enhance our competency-based curriculum.

See Figure 1-Curriculum oversight structure.

\section{Education Staff}

The Office of Medical Education (OME), under the senior associate dean for medical education, actively supports the curriculum by providing administrative and academic support for planning, implementation, and oversight. Budgeting for teaching, assessment, and academic support is managed centrally by OME.

OME is supported by over 30 individuals. The Division of Education Quality (DEQ), within IIME, provides independent, ongoing curricular evaluation (CQI process) to guide changes in our C21 curriculum.

See Figure 2-Organizational chart.

OME leadership, in consultation with DEQ, is charged with setting policies and standards; however, the Curriculum Committee formally reviews and approves all such policies and standards. Additionally, monthly meetings with clerkship and module directors permit further review of achievement standards, ensuring their alignment with GSOM's broader curricular objectives. While OME focuses exclusively on UME, input from GME and residency program directors helps identify curricular areas needing modification.

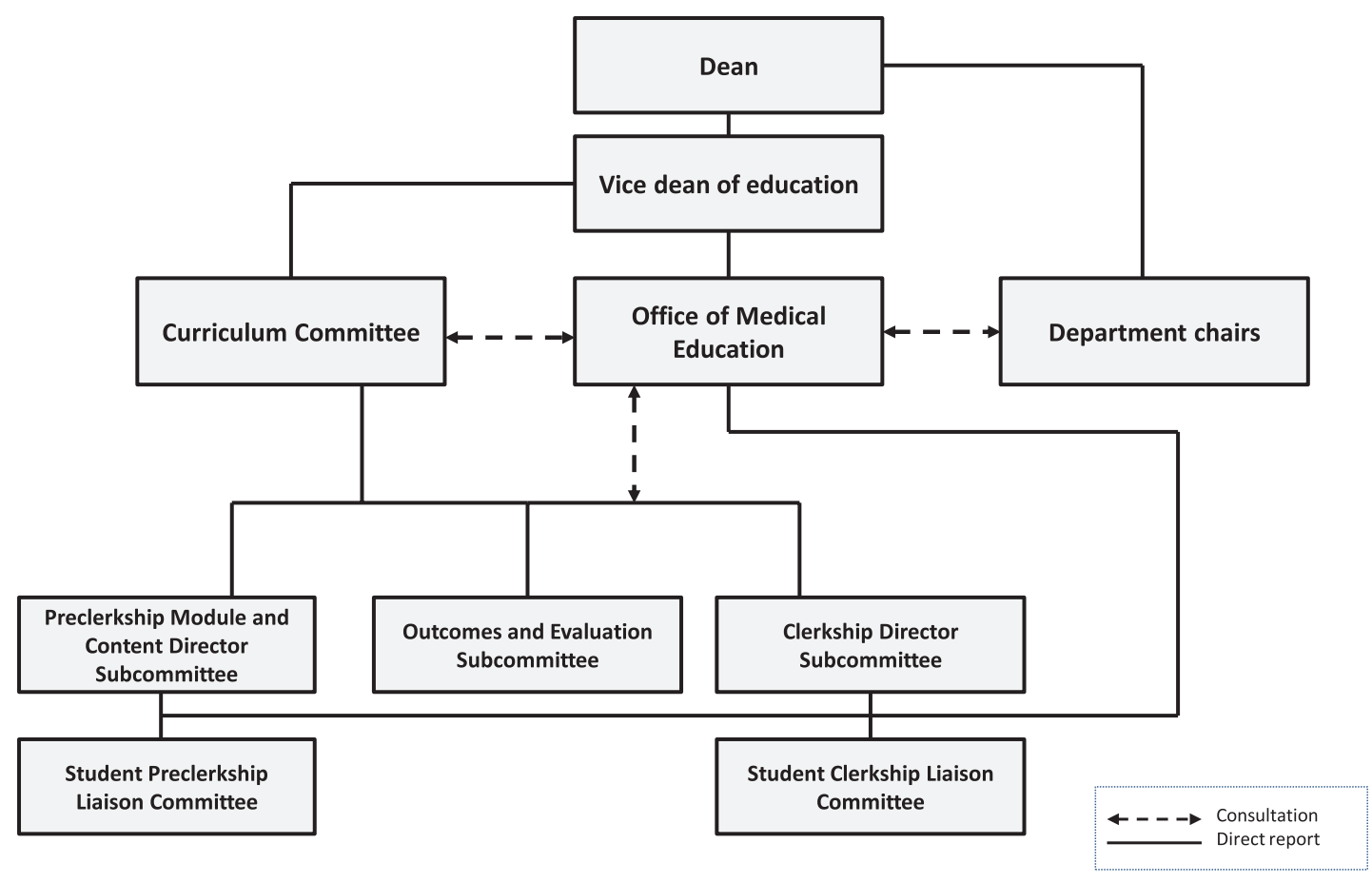

Figure 1 Curriculum oversight structure. 


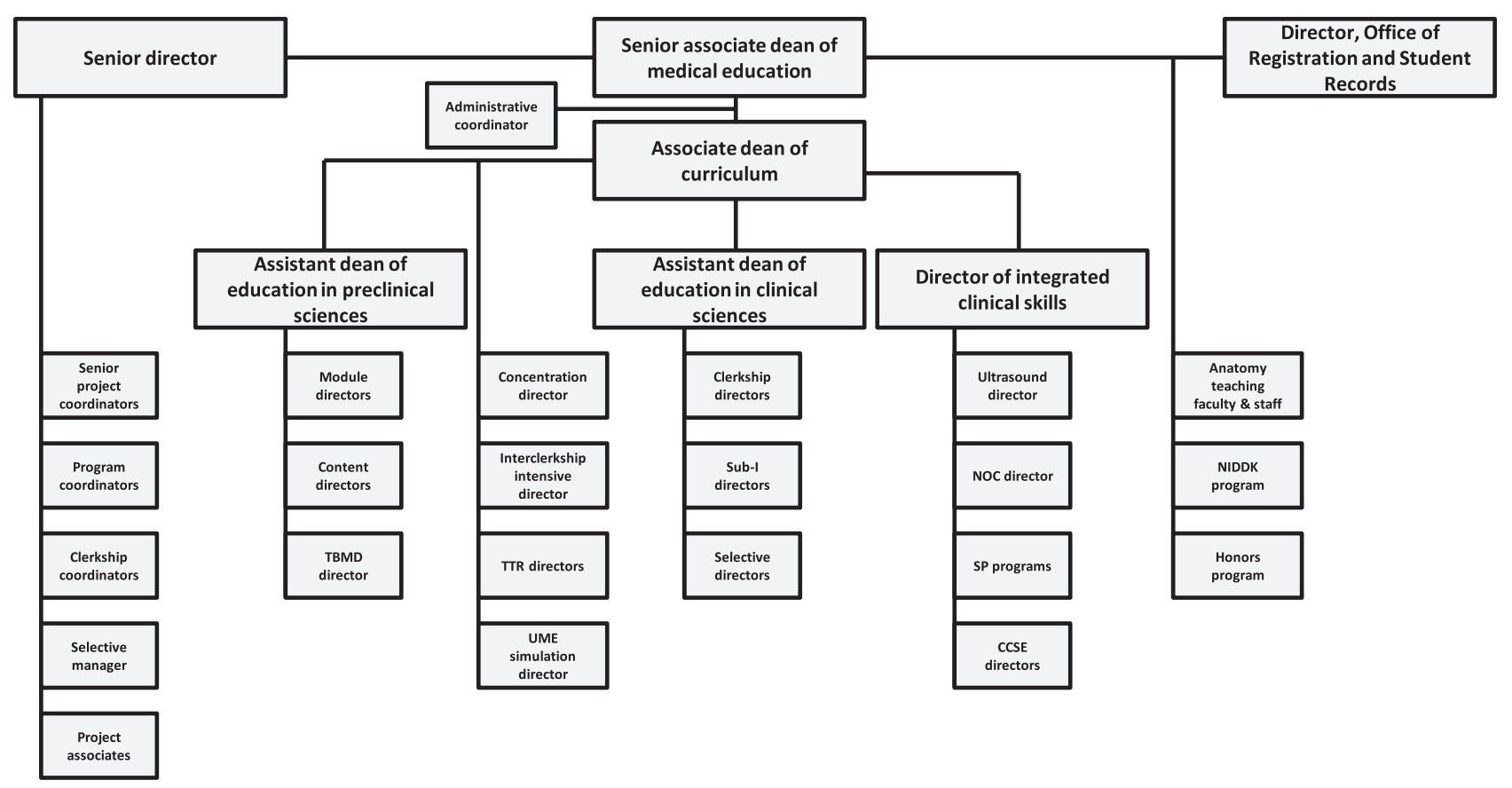

Figure $\mathbf{2}$ Organizational chart.

Beyond these far-reaching goals, clerkship directors are responsible for setting clear expectations for student achievement in clerkships and for establishing and implementing transparent schema for determining grades. The OME reviews these policies to ensure consistency across clerkships, while permitting the flexibility to meet the needs of various clinical disciplines and rotations.

\section{Faculty Development and Support in Education}

OME operates as an educational resource to faculty for developing and assessing effectiveness of course objectives, content resources, and teaching modalities. It conducts or sponsors workshops to improve small-group teaching, assessment strategies, innovative teaching modalities, and evaluation strategies for programs.

IIME provides a resource to assist faculty in developing effective learning modules that optimize learning outcomes by applying individualized, innovative educational approaches. IIME's instructional designer and multimedia teamwork with faculty 1-on-1 to develop, produce, and implement computer-assisted learning experiences supporting learning goals and objectives.

We currently have 8 academic tracks with a common teaching requirement. To be considered for promotion, documenting the scholarship of education requires demonstration of accomplishments via a teaching portfolio. Course leadership and design; the judgment of students, trainees, and peers; and meritorious publications are also considered when assessing a faculty member's teaching.

In 2016, our Educator Community was launched to recognize excellence in teaching and learning, coordinate faculty skill development and peer mentoring in education, strengthen programs supporting medical educator career advancement, and cultivate opportunities for educational innovation and scholarship. It is home to approximately 500 faculty and staff with leadership roles in education and includes faculty across UME, GME, and the College of Nursing.

\section{New Initiatives}

GSOM was 1 of 8 awardees of the AMA's 2019 Reimagining Residency Initiative. We propose developing a new approach to the UME-GME transition, the Transition to Residency Advantage (TRA) program. TRA will be rooted in learnercreated GME training goals, based on insights gained from UME performance data, that drive responsive changes in GME training. Goal setting will be facilitated by existing VSP coaches and a new cadre of GME "bridge coaches." This process will initially focus on graduates who remain for residency and is a "warm" transition with synchronous in-person meetings among learner/UME coach/GME bridge coach, eliminating traditional compartmentalization of education planning, mentoring, and learner support. 


\title{
Renaissance School of Medicine at Stony Brook University
}

\author{
Wei-Hsin Lu, PhD, Richard Iuli, PhD, Lisa Strano-Paul, MD, and Latha Chandran, MD, MPH
}

\section{Medical Education Program Highlights}

In 2012, Renaissance School of Medicine (RSOM) began a curriculum renewal process involving over 150 faculty, educators, and students across the education continuum. This led to the creation of the new LEARN (Learning-centered, Experiential, Adaptive, Rigorous, Novel) curriculum, implemented in $2014 .{ }^{1}$ LEARN provides opportunities for integrated training in basic sciences and clinical disciplines and includes 3 phases: Phase I, foundational phase (18 months); Phase II, primary clinical phase (12 months); and Phase III, advanced clinical phase (16 months).

Curriculum highlights include:

- Transition courses are offered at moments of "educational giant leaps": entering medical school, entering clinical care, and entering residency. Each transition course provides opportunities for students to prepare for the academic and professional rigors of the next phase in training.

- Translational pillars introduce cutting-edge basic science and translational medicine after each 12-week clinical clerkship block in Phase II. Each pillar is organized around a key biomedical concept, such as medical genetics, cancer biology, and metabolic diseases.

- Professional identity formation (PIF): We have integrated evidence-based approaches to enhance PIF and wellness among trainees and faculty. ${ }^{2}$ A standing PIF Working Group provides leadership and support for PIF initiatives.

- WE SMILE (We Can Eradicate Student Mistreatment in the Learning Environment) is a multipronged program to mitigate learning environment concerns. ${ }^{3,4}$ An educational element reviews policies and procedures related to reporting inappropriate behaviors in the learning environment and group discussions of video case scenarios help students identify inappropriate behaviors. WE SMILE training is integrated into each transition course.

- Selective courses focus on current topics in health care. Examples include addiction and pain, telehealth, business of medicine and value-based care, issues in women's health and gender-based medicine, and law and medicine. All students are required to choose at least one selective.

\section{Curriculum}

\section{Curriculum description}

See Supplemental Digital Appendix 1-Curriculum Schematicat http://links.lww.com/ACADMED/A918.

Acad Med. 2020;95:S362-S366.

doi: 10.1097/ACM.0000000000003413

Copyright (C) 2020 by the Association of American Medical Colleges

Supplemental digital content for this article is available at http://links.Iww.com/ ACADMED/A918.

Year school was established: 1971.

School URL: https://renaissance.stonybrookmedicine.edu.

\section{Curriculum changes since 2010}

- Introduced instructional strategies such as team-based learning and provisions for early clinical exposure

- Moved USMLE Step 1 examination requirement to after completing Phase II

- Launched Mobile Medical Education Initiative, with all entering students receiving an iPad

- Established Pathways to Success, a cocurricular program integrating academic and career advising throughout the curriculum

- Initiated 2 academic support programs-Shelf Rx and Clinical $\mathrm{Rx}$ - for students identified as at risk for NBME shelf exam and/or USMLE Step 2 CS failure

- Integrated reflection rounds in all clerkships and Schwartz rounds in several clinical departments

- Increased entering class size from 128 to 136

- Forged new affiliations with Southampton Hospital, Eastern Long Island Hospital, and South Nassau Communities Hospital; in negotiations with Long Island Community Hospital

\section{Assessment}

Our 20 institutional learning objectives (ILOs) are based on the 6 ACGME competencies.

See Supplemental Digital Appendix 2-Program Objectives and Assessment Methods—at http://links.lww.com/ACADMED/A918.

\section{Assessment changes since 2010}

Phase I:

- Criterion-referenced grading system

- Biomedical Building Blocks, Introduction to Clinical Medicine (ICM), Medicine in Contemporary Society (MCS), Themes in Medical Education (TiME) courses changed to pass/fail

- Customized NBME exams in all Integrated Pathophysiology courses

- 16 OSCEs and a summative final OSCE in ICM

Phase II:

- 10 clerkship OSCEs (formative and/or summative), several with a Step 2 CS style postencounter note

- 11-station end-of-Phase II Clinical Performance Examination with postencounter notes and a lab interpretation station

- Competency-based common clerkship evaluation (C3) form used in all core clerkships, providing cumulative competencies mapping for each student

Phase III:

- Standardized subinternship/elective evaluation form

Course, phase, and curriculum:

- Established robust curricular management and program evaluation system using both internal and external quality metrics ${ }^{5}$ 


\section{Parallel curriculum or tracks}

Three-year MD (3YMD):

- Introduced a 3-year curriculum track for a limited number (10-15 per year) of selected students. Acceptance into the $3 Y M D$ track includes provisional acceptance into an RSOM residency.

- 3YMD students take all Phase I and II courses in LEARN, a 4-week Health Systems Performance summer course before the start of Phase I, Teaching in Medicine and Interprofessional Conversations on Cultural Diversity courses, and participate in GME mentored experiences during the second summer.

- 3YMD students complete 135 weeks of credit and must fulfill the same clinical and professional requirements as students in the 4-year LEARN track.

See Table 1-Academic Requirements for LEARN and 3YMD Parallel Tracks.

\section{Pedagogy}

Changes in pedagogy since 2010:

- Increased active learning by additional lab sessions, smallgroup discussions, clinical pathologic conferences, teambased learning, audience response sessions, community site visits, and clinical skills sessions; all Phase I courses use active learning strategies

- Increased the number of standardized patient encounters and formative and/or summative OSCEs in Phase II

\section{Clinical experiences}

Regional clinical campus:

- In 2011, Winthrop University Hospital was designated as a regional clinical campus for 40 clerkship students. All curricular elements, clinical experiences, assessment rubrics, and grading policies were standardized across the 2 campuses. Compliance was closely monitored by the Office of Academic Assessment and Evaluation. However, due to hospital mergers, they are not affiliated with us effective 2020.

\section{Table 1}

Academic Requirements for LEARN and 3YMD Parallel Tracks

\begin{tabular}{|c|c|c|}
\hline \multirow[b]{2}{*}{ Academic requirements } & \multicolumn{2}{|c|}{ No. weeks } \\
\hline & LEARN & 3YMD \\
\hline Phase I & 56 & 56 \\
\hline Phase II & 51 & 51 \\
\hline Phase III & 40 & 12 \\
\hline Summer I and il & 0 & 14 \\
\hline Total & 149 & 135 \\
\hline Achieve 20 ilOS & Yes & Yes \\
\hline Fulfill clinical experiences E-passport & Yes & Yes \\
\hline Pass USMLE Step 1 examination & Yes & $Y_{\epsilon}$ \\
\hline Pass USMMLE Step 2 CK and CS examinations & Yes & $Y$ \\
\hline
\end{tabular}

Clinical sites:

- The primary clinical training site is Stony Brook University Hospital (SBUH), a 628-bed academic medical center. SBUH is the region's only tertiary care center and Level 1 trauma center.

- The Veterans Affairs Medical Center (VAMC) at Northport includes a 508-bed acute care hospital, a 190-bed nursing care home facility, and 5 community-based outpatient clinics.

- Nassau University Medical Center (NUMC), a public teaching hospital, is a 631-bed acute care facility and Level 1 trauma center. NUMC also has an 889-bed long-term care facility.

- Stony Brook Southampton Hospital is a 125-bed facility located on the rural south fork of Long Island. It is a New York State-designated Stroke Center and its emergency department is the sole provider of emergency care on the South Fork.

- South Nassau Communities Hospital is a 455-bed, acute care, not-for-profit teaching hospital in Nassau County.

- Eastern Long Island Hospital is a clinical training site for emergency medicine. Mather Hospital, another local community hospital, is used for internal medicine.

\section{Required longitudinal experiences}

Three required longitudinal courses extend throughout Phase I:

- ICM teaches medical interviewing and physical examination skills, procedural skills, and clinical reasoning skills. Students participate in simulated patient encounters with standardized patients and develop their skills with real patients with their assigned preceptor.

- MCS gives students opportunity to expand their knowledge of ethical, social, cultural, and humanistic issues in medicine. The course focuses on core competencies in the areas of professionalism and ethics, communication, self-awareness, social context of medicine, and health care systems.

- TiME is presented in five 1-week blocks capping each major Phase I course. Each block centers on a different population or life stage.

\section{Required and elective community-based rotations}

Students rotate through community-based outpatient clinics in their primary care clerkship. During this clerkship, all students participate in a home hospice visit and home care visit, done in collaboration with community-based agencies.

\section{Challenges in designing and implementing clinical experiences for medical students}

Recruiting high-quality site directors and educators, increasing clinical demands faced by clinical educators, competition for clinical sites from new regional medical schools, and exclusive training contracts by international medical schools are ongoing challenges to maintaining high-quality clinical sites.

\section{Curricular Governance}

The Curriculum Committee is appointed by the Faculty Senate to develop and supervise the UME curriculum. The committee is chaired by a faculty member voted by the Faculty Senate and an administrative cochair, the vice dean for academic and 
faculty affairs. The committee's functions include specifying the educational mission, goals, and objectives of the medical school; defining the overall content of the curriculum; determining the length and sequence of courses; recommending course directors to RSOM's dean; and reviewing and evaluating courses and the curriculum as a whole. Members include clinical and basic science faculty; representatives from the VAMC, affiliate sites, health sciences library, and medical informatics; and student representatives from each phase. The committee meets monthly.

See Figure 1-Curricular governance.

While course and clerkship directors' salaries are maintained through their respective departments, administration of the curriculum and course and clerkship coordination are managed centrally in the Office of Academic and Faculty Affairs (OAFA). Academic and career advising through the Pathways to Success program is provided centrally along with a full-time learning specialist and specialty advisors recruited from clinical departments. All course and curriculum evaluations, ongoing quality monitoring, management of learner assessments, and curriculum database are coordinated centrally through this office as well.

\section{Education Staff}

The OAFA provides leadership, service, and support to advance RSOM's mission in UME. The vice dean of academic and faculty affairs conducts regular bimonthly meetings with the senior leadership team. The OAFA is supported by 9 administrative assistants and the RSOM registrar. It holds weekly meetings with the medical informatics liaison to discuss ongoing updates to our homegrown curriculum database. The OAFA also maintains regular communications with other offices across the health sciences schools and the University. The OAFA is not responsible for premed, GME, or CME activities.

See Figure 2-Undergraduate medical education administration.

The Office of Admissions, led by an associate dean, is responsible for all aspects of the admissions process. The assistant dean for student diversity reports to the associate dean. Final decisions regarding admissions are made by the Executive Committee of the Committee on Admissions, which reports to the Faculty Senate.

The Office of Curricular Affairs includes 2 assistant deans and the director of Pathways to Success, who report to the vice dean. Each assistant dean is responsible for planning and implementing curricular initiatives in their assigned curricular phase. One assistant dean cochairs the Phase I Subcommittee and one cochairs the Phase II/III Subcommittee.

The Office of Academic Assessment and Evaluation is responsible for ongoing collection/collation of student feedback through end-of-course evaluations, surveys, focus groups, end-ofphase assessments, and OSCE assessments. This office is also responsible for developing evaluation summaries of courses/ clerkships, curricular phases, and overall curricular monitoring. Compliance with LCME requirements is monitored on an ongoing basis. Biannual LCME dashboards are presented at the Phase I and Phase II/III Subcommittee meetings. The director coordinates, conducts, and reports new course proposals, course QI reports, and Level 1 and 2 course reviews in conjunction with faculty to the Learner Assessment and Curricular Evaluation Subcommittee.

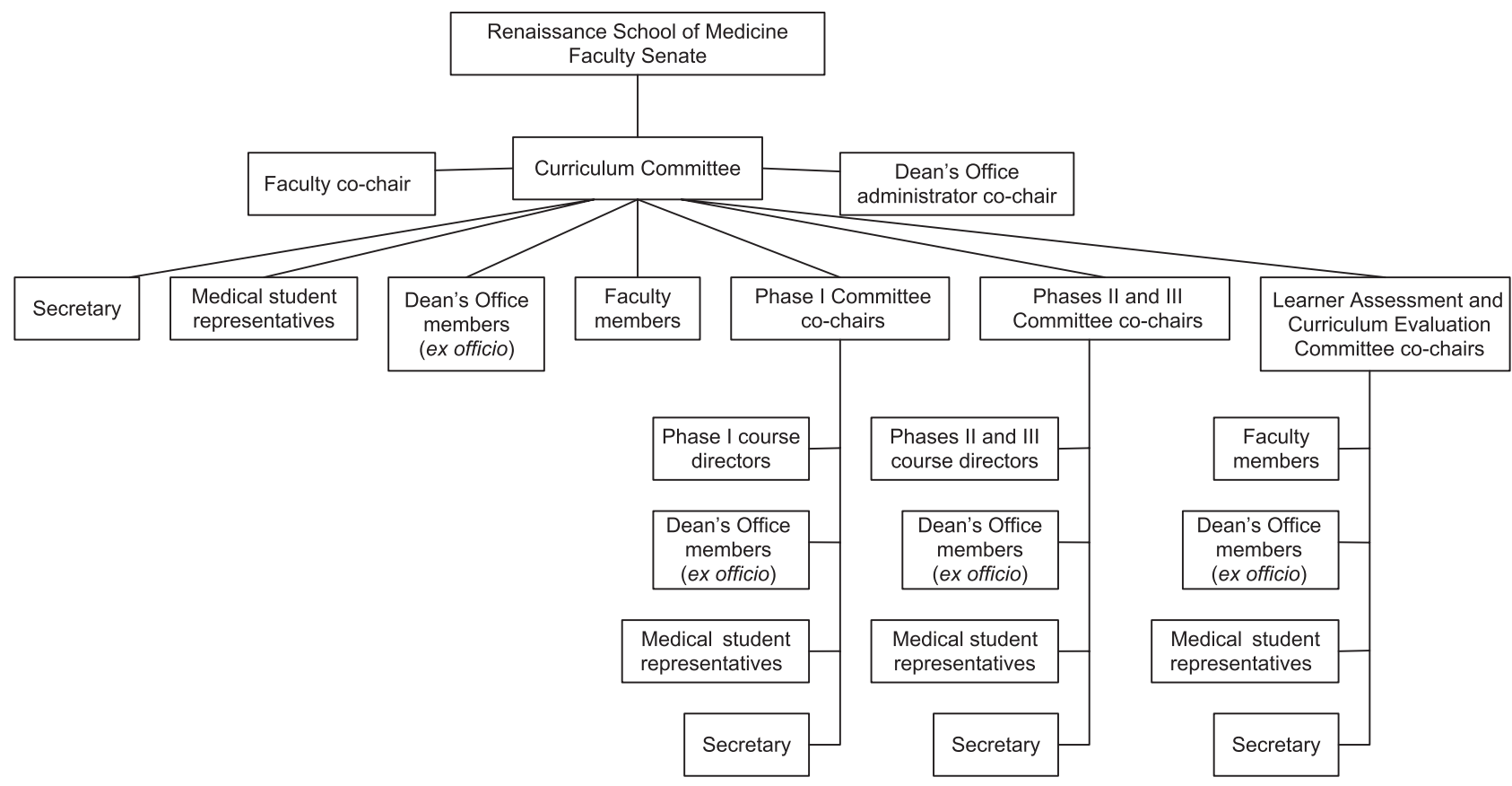

Figure 1 Curricular governance. 


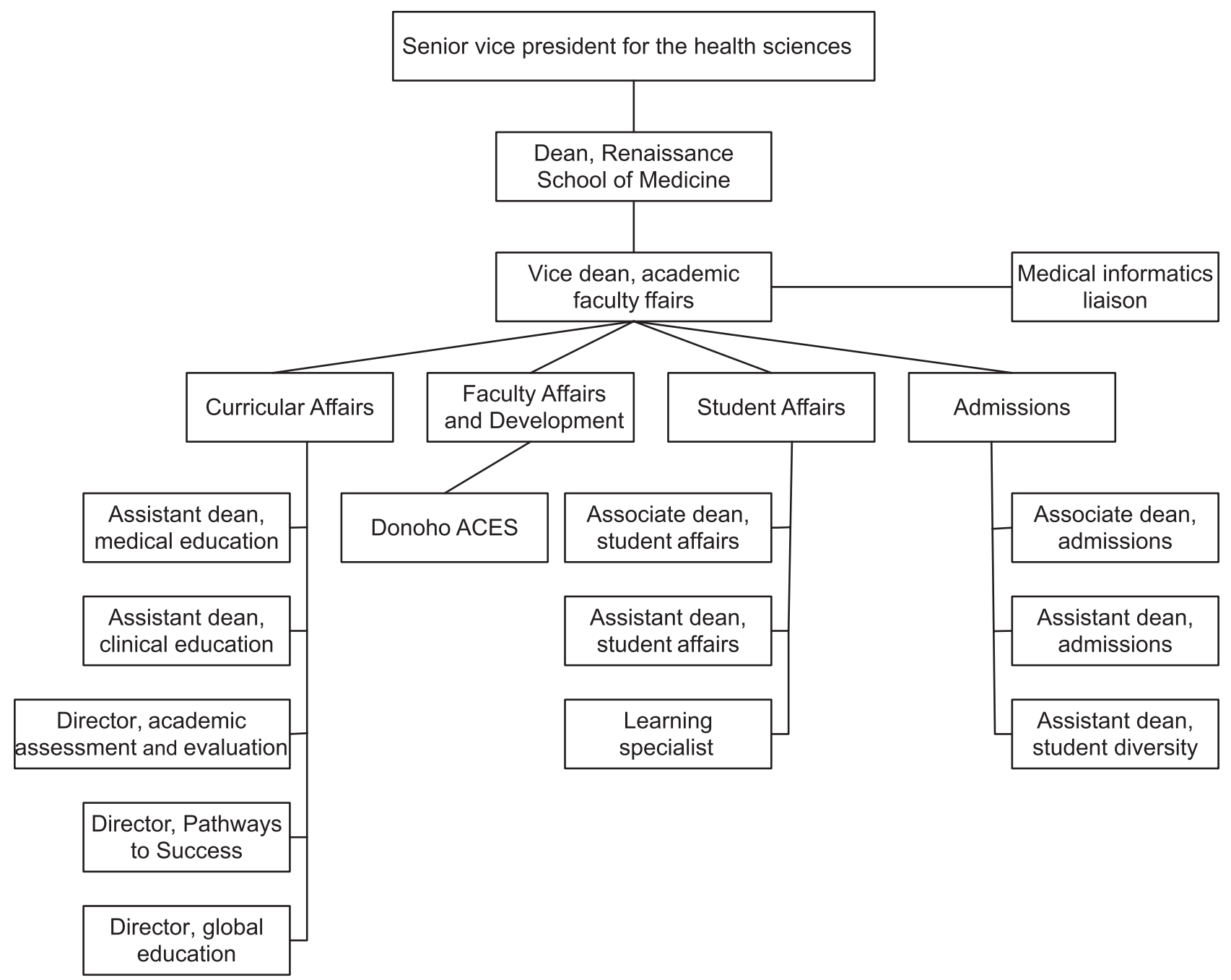

Figure 2 Undergraduate medical education administration.

The Office of Student Affairs is led by an associate dean. The registrar, an assistant dean for student affairs, who is responsible for financial aid support, and a learning specialist report to the associate dean.

The Office of Faculty Affairs (OFA) is led by the vice dean, supported by the director of faculty development. OFA is responsible for all faculty development activities. Annual retreats, workshops, faculty awards, and longitudinal faculty development programs are coordinated through this office. Working in conjunction with the Donoho Academy of Clinical and Educational Scholars, OFA supports junior faculty scholarship through a project implementation and mentoring program.

\section{Faculty Development and Support in Education}

Professional development for faculty as educators

- Through a generous grant, the Donoho Academy for Clinical and Educational Scholars was established in 2014.
A Donoho Distinguished Teaching Professorship was established. One-on-one mentoring to enhance faculty scholarship is provided to junior members over a 3-year period.

- 3 longitudinal faculty development fellowship programs are offered through OFA: Master Educator Fellowship Program, Career Development Fellowship Program, and Leadership in Medicine Fellowship Program. Cohorts of 12-18 faculty are accepted into these programs through a rigorous application process.

- Faculty workshops are offered monthly on various topics. External speakers are regularly invited to present on topics of general interest to faculty.

- An annual medical education retreat with keynote speakers, multiple workshops, and discussions are regular features.

- OAFA coordinates all annual junior and senior faculty awards for teaching, leadership, research, mentoring, and citizenship. 


\section{Role of teaching in promotion and tenure}

- There are tenure-eligible (educator-scholar) and tenurenoneligible (clinician-educator, basic science educator) tracks for promotion of faculty whose primary career focus is education. Promotion on these tracks requires submission of a summative educator portfolio highlighting the faculty member's achievements in any of the 5 educator activity domains. A standard point system is used to determine eligibility for promotion.

\section{Initiatives in Progress}

- Multiple mini-interviews will become an element of the admissions process beginning with recruitment for students entering in 2021.

- The 4-week generic Transition to Residency course at the end of Phase III will be divided into 2 components: a 2-week general training session plus 2-week specialty-specific activities and experiences.

\section{References}

1 Fischel JE, Olvet DM, Iuli RJ, Lu WH, Chandran L. Curriculum reform and evolution: Innovative content and processes at one US medical school. Med Teach. 2019;41:99-106.

2 Chandran L, Iuli RJ, Strano-Paul L, Post SG. Developing "a way of being": Deliberate approaches to professional identity formation in medical education. Acad Psychiatry. 2019;43:521-527.

3 Fleit HB, Iuli RJ, Fischel JE, Lu W-H, Chandran L. A model of influences on the clinical learning environment: The case for change at one U.S medical school. BMC Med Educ. 2017;17:63

4 Fleit HB, Lu WH, Olvet DM, Chandran L. Case studies for recognizing appropriate and inappropriate behaviors in the clinical learning environment. MedEdPORTAL. 2017;13:10638.

5 Shroyer AL, Lu W-H, Chandran L. Drivers of dashboard development (3-D): A curricular continuous quality improvement approach. Acad Med. 2016;91:517-521. 


\section{University of Rochester School of Medicine and Dentistry}

David R. Lambert, MD, and Christopher J. Mooney, PhD, MPH

\section{Medical Education Program Highlights}

Consistent with the University of Rochester School of Medicine and Dentistry's legacy of bridging basic and clinical sciences, our Double Helix Curriculum-Translations and Transitions integrates the scientific foundations of medicine with clinical medicine across a 4-phase curriculum. Home of the Biopsychosocial Model, the school emphasizes a holistic understanding of the patient-the translation of the molecular mechanism of disease and their living environment into strategies for prevention and treatment. A recent curricular addition, Meliora in Medicine, is a 4-year curricular thread that weaves 3 pillars: collaborative practice, technology in medicine, and professional identity formation. Medical humanities remain a school strength and is incorporated as both a dedicated content area and an instructional method. Transitions across the medical education continuum are facilitated with focused activities, curricular elements, self-reflection, and career advising. Specialty-specific competencies assist students in preparing for internship. The medical education program also offers elective pathways in medical humanities and bioethics, medical education, deaf health, Latino health, and global health. Learning in a collegial and collaborative environment, students are guided through the established Advisory Dean Program. The school supports students' intellectual curiosity and passions through funded research opportunities (e.g., academic research track), as well as international and volunteer service work, offering a distinction in research and a distinction in community health. In additional to our MD-PhD MSTP program, the school has multiple combined degree programs (e.g., MD-MBA, master's degrees) and certificate programs.

See Table 1-Meliora in Medicine.

\section{Curriculum}

\section{Curriculum description}

See Supplemental Digital Appendix 1-Curriculum Schematicat http://links.lww.com/ACADMED/A917.

\section{Curriculum changes since 2010}

The medical school's continuous quality improvement process has led to ongoing changes throughout the last decade. Several years ago, we embarked on a curriculum remodel process, from

Acad Med. 2020;95:S367-S370.

doi: 10.1097/ACM.0000000000003412

Copyright (C) 2020 by the Association of American Medical Colleges

Supplemental digital content for this article is available at http://links.Iww.com/ ACADMED/A917.

Year school was established: 1921.

School URL: https://www.urmc.rochester.edu. which emerged the Double Helix Curriculum-Translations and Transitions, which retains core elements of our previous Double Helix Curriculum - the Biopsychosocial Model, including a basic science emphasis throughout the curriculum and early clinical exposure. Curriculum change highlights include streamlined instruction, greater health services science topic presence, and additional clinical requirements. The curriculum is also now defined by phases, a structure that provides a more cohesive and developmentally appropriate clinical curriculum in Phases 1 and 2. The basic science strand in Phases 1 and 2 continues to provide foundational knowledge using a systems-based approach. Streamlining the curriculum now allows students to complete Phase 2 earlier, allowing a longer Phase 3-our clinically immersive period - that now includes a critical care requirement and Family Medicine clerkship. Phase 3 also includes Scientific and Social Foundations of Medicine courses, which include translational basic science and Meliora in Medicine content. The final phase (Phase 4) now includes a course devoted to health systems improvement and an expanded capstone course. Student progress and achievement of objectives are confirmed through 3 end-of-phase assessments.

\section{Assessment}

The medical school has a robust system of student assessment that provides multiple opportunities for direct observation, feedback, and assessment of progress in key knowledge, skills, and behavioral areas that are linked to the school's overall education objectives. Changes in assessment practices in the past decade have included peer assessment across the first 3 phases, Phase 2 Assessment Days (described below), and end-of-phase assessments, which provide students with formative feedback to inform subsequent individual development plans. Current efforts are targeted toward the assessment of professionalism using the institution's IICARE attributes of professionalism (inclusion, integrity, compassion, accountability, respect, excellence) as a guiding theoretical framework.

\section{Medical education program objectives}

See Supplemental Digital Appendix 2-Medical Education Objectives and Assessment Methods-at http://links.lww.com/ ACADMED/A917.

Our curriculum is designed to meet the medical school's overall education program objectives. Each objective is linked to ACGME domains of competence, which help to facilitate the transition to GME. As part of the medical school's curriculum management process, education program objectives are linked to specific session objectives to provide greater specificity with respect to where they are taught and how they are assessed.

\section{Pedagogy}

In addition to lecture, the medical school uses multiple smallgroup learning formats including problem-based learning, 


\section{Table 1}

\begin{tabular}{|c|c|c|}
\hline \multicolumn{3}{|l|}{ Meliora in Medicine } \\
\hline $\begin{array}{l}\text { Collaborative } \\
\text { practice }\end{array}$ & $\begin{array}{l}\text { Technology in } \\
\text { medicine }\end{array}$ & $\begin{array}{l}\text { Professional } \\
\text { identity formation }\end{array}$ \\
\hline $\begin{array}{l}\text { Interprofessional } \\
\text { education }\end{array}$ & Library science & Emotional intelligence \\
\hline Teamwork & $\begin{array}{l}\text { Electronic medical } \\
\text { record }\end{array}$ & Bioethics \\
\hline Leadership & Ultrasound & Wellness and resilience \\
\hline Patient safety/quality & Telehealth" & $\begin{array}{l}\text { Inclusion and } \\
\text { unconscious bias }\end{array}$ \\
\hline Health systems & Informatics & Advocacy \\
\hline
\end{tabular}

team-based learning (TBL), and laboratory experiences for nonclinical courses in Phases 1 and 2 to meet education program objectives. The school uses small-group formats to provide clinical instruction in interviewing and physical examination skills. A robust and integrated simulation program in Phases 1 and 2 facilitates clinical skills development as well as formative and summative assessments in these phases, as well as Phase 3. Simulation activities are also used for teaching in pharmacology, interprofessional education, and team science. Purposeful efforts have resulted in the increased utilization of online learning activities. Humanities-focused pedagogical approaches (e.g., Theater of the Oppressed) are used to provide instruction in specific areas including bias and advocacy. Optional elective pathways (e.g., medical education, deaf health) provide students with focused experiences under faculty supervision.

\section{Changes in pedagogy since 2010}

In 2013, the school transitioned to an electronic curriculum to deliver all content (e.g., TouchBooks, histology and pathology instruction, etc.), which resulted in the school being named an Apple Distinguished Institution in 2019. Another notable change was the addition of TBL activities to Phase 2 of our curriculum and developmentally appropriate interprofessional experiences involving other health professions students. Current medical school initiatives are focused on the incorporation of iPads/mobile devices into an emergent telehealth curriculum.

\section{Clinical experiences}

In the Phase 1 course Foundations of Biopsychosocial Practice, students are placed in a range of settings including ambulatory offices, patient homes, nursing homes, rehabilitation facilities, and a children's museum to provide experiences and instruction in child development, rehabilitation, and refinement of clinical exam skills. Longitudinal ambulatory clinical experiences in Phase 2 are focused in pediatric and adult medicine offices. Required Phase 3 clerkships (medicine, surgery, obstetricsgynecology, pediatrics, neurology, and psychiatry) occur in 4 regional hospitals (community to tertiary referral), with most occurring at the university-owned Strong Memorial Hospital
(SMH) and Golisano Children's Hospital. The family medicine and emergency medicine clerkships are completed in Phases 3 or 4, with the latter occurring at SMH, a Level 1 trauma facility. Clinical electives are offered in a variety of settings spanning regional, national, and international locations.

\section{Required longitudinal experiences}

The school includes a longitudinal ambulatory experience in adult medicine and pediatric settings through the primary care clerkship in Phase 2. Here, students are assigned to an adult care ambulatory office (family medicine or general internal medicine) and a pediatric office. These experiences are completed in conjunction with systems-based courses, which facilitates integration and application of content. Phase 2 also includes 3 assessment days, which require students to perform several standardized patient encounters that present with complaints related to the concurrent systems-based course content. Students conduct a history and examination, complete a patient note, and answer examination questions. Questions are designed to assess and emphasize integration of content and the translation to patients.

\section{Clinical experience first encounter}

Students encounter standardized patients in the first semester of Phase 1 and begin to see patients in a variety of settings in its second half, with the goals of refining history and physical exam skills, oral and written presentations, early clinical reasoning, and developing prevention and counseling skills. Phase 2 includes the longitudinal primary care clerkship.

\section{Required and elective community-based rotations}

To offer a diverse clinical education, the medical school uses the local outpatient VA facility, nursing homes, home care agencies, and other community-based health systems. Elective opportunities exist in these settings and students are able to create their own elective experience with faculty guidance. The family medicine clerkship offers flexibility for completion at distant sites.

\section{Challenges in designing and implementing clinical experiences for medical students}

Consistent with other medical schools, the growing numbers of health professions students have made securing some experiences more challenging than 10 years ago.

\section{Curricular Governance}

All major areas of curriculum design, implementation, assessment, and continuous quality improvement are managed centrally. The majority of the budget for those with administrative and leadership roles is similarly managed centrally. Although clerkship director support is managed centrally and at the department level, allocations to core clinical departments for clerkship instruction is decentralized.

See Figure 1-Curriculum Steering Committee. 


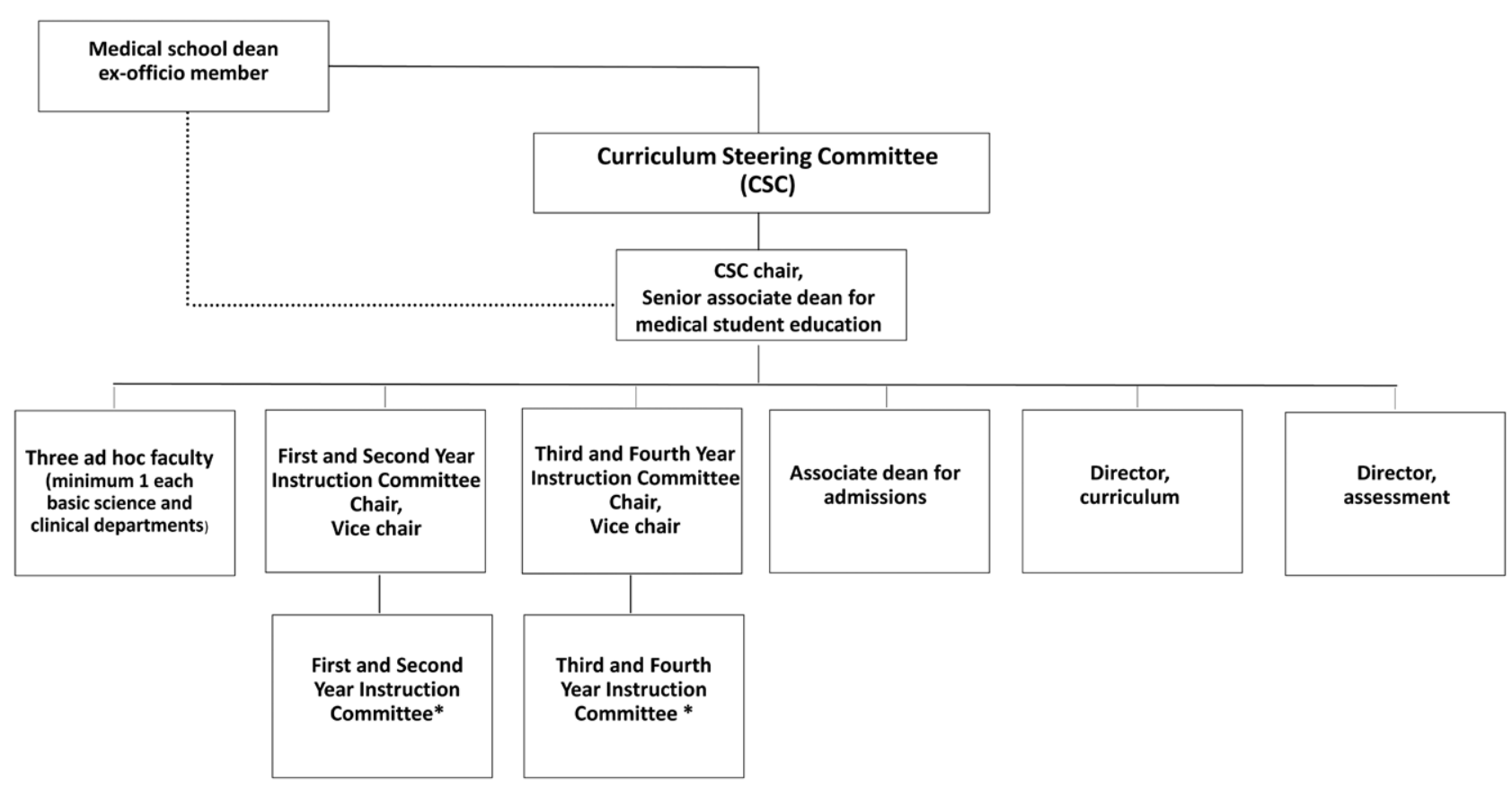

*The instruction committees include course and clerkship directors, theme directors, and library, technology, and education resources staff.

Figure 1 Curriculum Steering Committee.

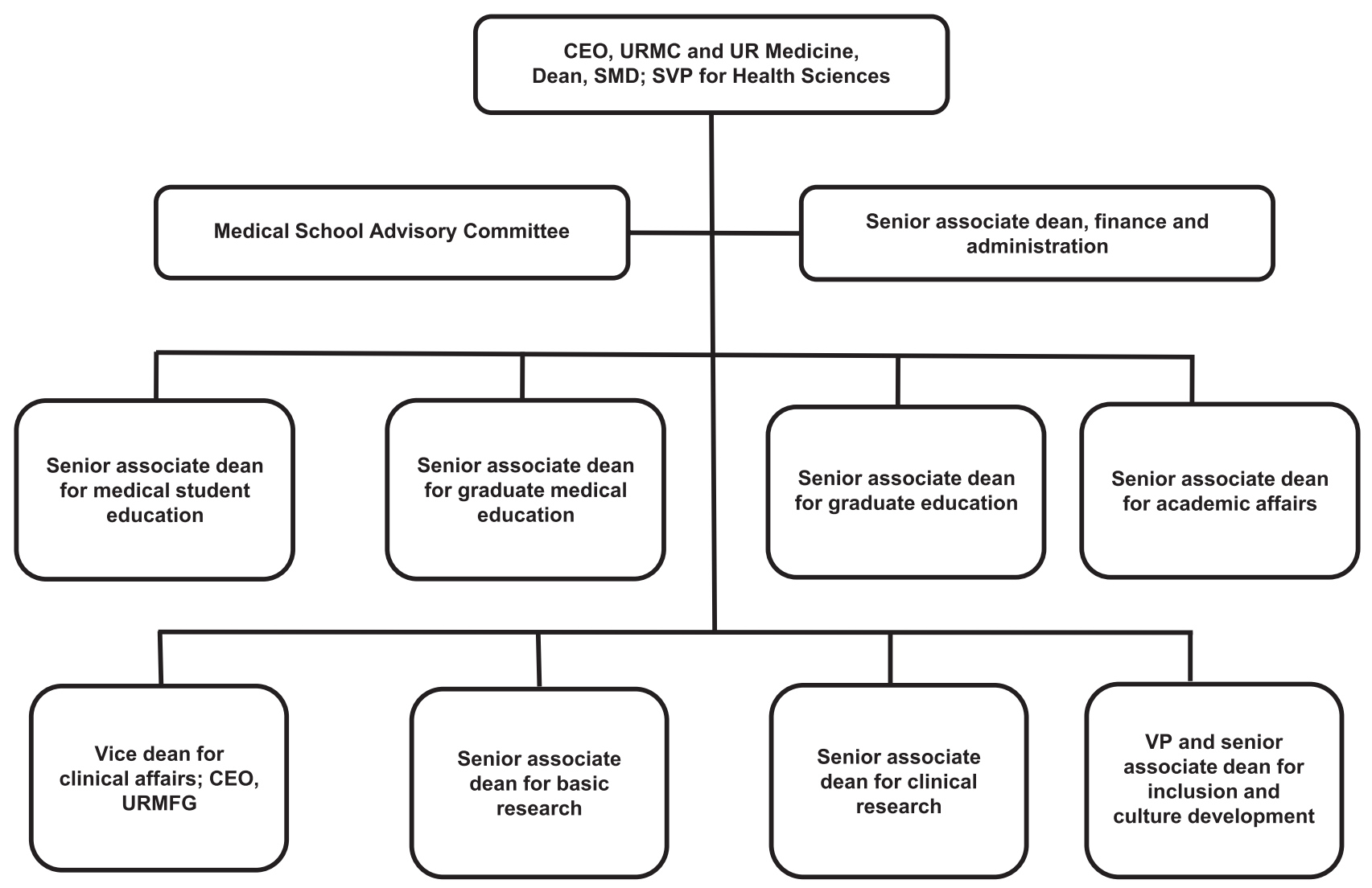

Figure 2 Organizational chart. 


\section{Education Staff}

The school's Office of Curriculum and Assessment (OCA) provides direct support for most courses and pathways in the curriculum, whereas clerkships are coordinated at the department level. Supported by a highly skilled team, the director of assessment and the OCA administrator are integrated into the curriculum governance structure and the medical school's continuous quality improvement processes. The OCA coordinates all student assessment and the evaluation of the curriculum more generally.

Although the OCA only serves UME, the integration of the medical school with a university-owned medical center facilitates regular contact and discussion across the medical education continuum. Our student services office oversees student affairs, working in conjunction with the OCA.

See Figure 2-Organizational chart.

\section{Faculty Development and Support in Education}

Professional development for faculty as educators

Professional development for faculty educators is coordinated through a central office led by a senior associate dean. Numerous onsite offerings address instruction, technology, assessment, and career development. Faculty development topics are defined with input from the school's instruction committees. Medical school resources allow faculty to attend national programs and meetings for professional development.

The university's medical center has the Institute for Innovative Education, which brings together education leaders throughout the medical center to develop and implement shared curricular initiatives. Additionally, the Dean's Teaching Fellowship program supports selected faculty who receive instruction, mentoring, and guidance as they develop an education project over 2 years. To foster creativity and research, there is a collective of medical educators who strategize research initiatives to critically evaluate programs and disseminate best practices.

\section{Role of teaching in promotion and tenure}

All medical school faculty, with the exception of research appointments, must demonstrate excellence in teaching contributions. Teaching contributions and scholarship criteria are defined. At the time of reappointment or promotion, faculty members submit publications, teaching materials, evaluations, and other relevant information regarding educational contributions for review by a committee. 


\section{Weill Cornell Medicine}

Yoon Kang, MD, Peter M. Marzuk, MD, Joseph E. Safdieh, MD, Domenick J. Falcone, PhD, and Augustine M. K. Choi, MD

\section{Medical Education Program Highlights}

Weill Cornell Medicine (WCM) redesigned its curriculum with the class of 2018 as the inaugural graduating class to participate in the new 4-year program:

- One focus was strategic interfaces of foundational and clinical content with patient-centered experiences from day 1.

- WCM has consistently been a leader in the proportion of graduates who enter academic medicine.

- Another hallmark of the new curriculum is the 6-month Area of Concentration (AOC) program. The AOC program provides core research skills and a structured, longitudinal opportunity for mentored scholarly projects in customized areas of interest. To date, there have been over 100 publications associated with the AOC program.

WCM has an institutional commitment to enhancing all aspects of the student learning and living environment.

- Beginning with academic year 2019-2020, WCM provides a unique debt-free education to all students with demonstrated financial need by replacing student loans with scholarships that cover tuition, housing, and other living expenses. WCM also provides support for the academic needs of the entire student body such as study resources and registration for boards exams. The core intent of the WCM program for student financial well-being is to further diversify the breadth of academic choice and opportunity for its students.

- Student mental health is another key WCM area of focus and, as a first step in establishing a national learning community and best practices, WCM hosted the inaugural National Conference on Medical Student Mental Health and Well-Being in 2019 in New York City, in partnership with the Association of American Medical Colleges, the Associated Medical Schools of New York, and the American Foundation for Suicide Prevention. It was the first comprehensive, multidisciplinary forum to examine the specific mental health challenges and needs of medical students.

WCM launched a formal continuous quality improvement (CQI) initiative in 2017 that includes a CQI office, a CQI Steering Committee (chaired by the dean), and a CQI Student Council with student representatives across all 4 classes. WCM

Acad Med. 2020;95:S371-S374.

doi: 10.1097/ACM.0000000000003407

Copyright (C) 2020 by the Association of American Medical Colleges

Supplemental digital content for this article is available at http://links.Iww.com/ ACADMED/A914.

Year school was established: 1898.

School URL: https://weill.cornell.edu. views CQI for the education mission as an iterative process that ensures the outcomes of the medical education program meet programmatic and accreditation goals and that the strategic plan for the education mission aligns with those of the clinical and research missions.

\section{Curriculum}

See Supplemental Digital Appendix 1-Curriculum Schematicat http://links.lww.com/ACADMED/A914.

\section{Curriculum changes since 2010}

The curriculum was reorganized into 3 phases, each designed to integrate basic science and clinical medicine and to prepare the learner for the subsequent phase.

- Phase 1, foundational curriculum: This was shortened from 2 years to 1.5 through the creation of sequential learning units that combine normal and abnormal biology. The longitudinal Patient Care and Physicianship (PCP) unit integrates clinical skills instruction with basic science content.

- Phase 2, clinical clerkships: The medicine and surgery clerkships were both shortened to 8 weeks and a new 2-week clerkship in anesthesiology and critical care medicine was established.

- Phase 3, postclerkship curriculum: A major change in the curriculum was the creation of a 6-month block in years 3 and 4 for the AOC, to allow students to conduct a mentor-guided research project in basic science, clinical investigation, or population-based science. In addition, subinternship offerings expanded to include neurology and emergency medicine. New required courses were created in translational science and advanced clinical ethics.

- Additional curricular enhancements: Transition to Medical School, Transition to Clerkship, and Transition to Residency courses are focused on leaner needs at key transition points. The new curriculum also features enhanced content in cultural competency and health care disparities; student wellness; bioinformatics, patient safety, and telemedicine; interprofessional education; service learning; international elective offerings; and opportunities for accelerated master's programs.

\section{Medical education program objectives}

WCM's 20 program objectives are organized under 7 competencies, 6 of which are the ACGME competencies. The seventh is "scholarship."

\section{Assessment}

See Supplemental Digital Appendix 2-Program Objectives and Assessment Methods—at http://links.lww.com/ACADMED/A914. 


\section{Assessment changes since 2010}

- Since 2010, all courses include additional formative assessments: online self-assessment quizzes, the NBME Comprehensive Basic Science Exam, and midclerkship objective structured clinical examinations.

- Institutionally developed exams emphasize clinical reasoning skills through the use of clinical vignettes.

- Phase 1 faculty and resident rating forms have been standardized to behavioral observational anchors based on preparation, participation, and professionalism and on the ACGME competencies in the clerkships.

- The clerkship grading rubric has been made more transparent and standardized across all clerkships, with a primary focus on clinical performance and Entrustable Professional Activities and a de-emphasis on the clinical subject exams.

\section{Pedagogy}

- Faculty-facilitated peer teaching: case-based and problembased learning, role plays, and self-directed learning sessions

- Critical thinking and analysis of the medical literature, largely through journal clubs and critical appraisal sessions

- Clinical skills: direct supervision in patient care settings, virtual and standardized patients, and interactive workshops and demonstrations

- Lectures and laboratory sessions

\section{Changes in pedagogy since 2010}

- Increased use of blended learning and flipped classroom approaches that involve e-learning modules and video podcasts with self-assessment quizzes

- A shift from problem-based learning to shorter case-based learning

- Increased interactivity in large-group sessions through 1-on-1 pair share exercises and audience participation software

- Additional structured formalized self-directed learning sessions

- E-learning resources

\section{Clinical experiences}

Clinical sites include inpatient, emergency department (ED), and ambulatory outpatient settings at academic tertiary care medical centers; academic specialty hospitals (cancer and orthopedics); and private community hospitals in diverse socio-economic neighborhoods in New York City, Ithaca, New York, and Houston, Texas.

\section{Required longitudinal experiences}

During Phase 1, all students participate in a required longitudinal patient experience, the Longitudinal Educational Experience Advancing Patient Partnerships (LEAP) course. Medical and physician assistant students work in teams led by a faculty mentor. In both an observer and advocacy role, student teams follow a panel of patients with chronic disease by participating in the care of the patient with the patient's physician and health care team through outpatient clinic or ED visits and hospitalizations. The experience is supplemented through monthly seminar sessions conducted by faculty preceptors that include a variety of topics such as interprofessional teamwork, health disparities, insurance access, medication adherence, complementary medicine, and patient safety.

\section{Clinical experience first encounter}

Students first encounter a patient on the first 2 days of classes as part of a large-group session in the PCP unit of the Essential Principles of Medicine course. In addition to weekly in-class patient presentations, students also have clinical encounters as part of LEAP course, described above, and in the first semester of clinical preceptorships.

\section{Required and elective community-based rotations}

The Physician Organization of WCM has a network of satellite primary care and specialty practices to which students may be assigned during the required primary care clerkship or choose in the context of a clinical elective. Some students opt to fulfill primary care requirements at a community-based group in rural upstate New York or a student health center.

\section{Challenges in designing and implementing clinical experiences for medical students}

Challenges include optimizing the clinical environment for multiple learner groups (i.e., medical students, medical residents, physician assistant students) and protecting teaching time for faculty who are under increasing pressures to meet clinical and research productivity metrics.

\section{Curricular Governance}

The Executive Curriculum Committee (ECC) has primary responsibility for the curriculum. Its subcommittees oversee specific courses or phases of the curriculum. The Essential Principles of Medicine Subcommittee and Health, Illness, and Disease Subcommittee oversee these Phase 1 courses. The Clinical Curriculum Subcommittee oversees the Phase 2 clerkships and the subinternships, Transition to Residency course, and clinical electives in Phase 3. The Scholarship, Science, and Ethics Subcommittee oversees the AOC, Translational Science course, Advanced Clinical Ethics course, and science electives in Phase 3. The ECC is the curricular body responsible for reviewing the curriculum as a whole annually. The CQI Curriculum Review Subcommittee also performs a more granular review of the curriculum as a whole by reviewing each required curriculum phase on a regular 3-year cycle.

See Figure 1-Curricular governance committees.

\section{Curricular governance managed at the department level}

Departments work closely with the central curriculum office to ensure that courses are staffed with appropriate teaching faculty. Financial support for teaching activities is managed at the departmental level. Those faculty who hold administrative roles in the curriculum (e.g., course director, unit leader) are provided appropriate central support for their administrative duties. 


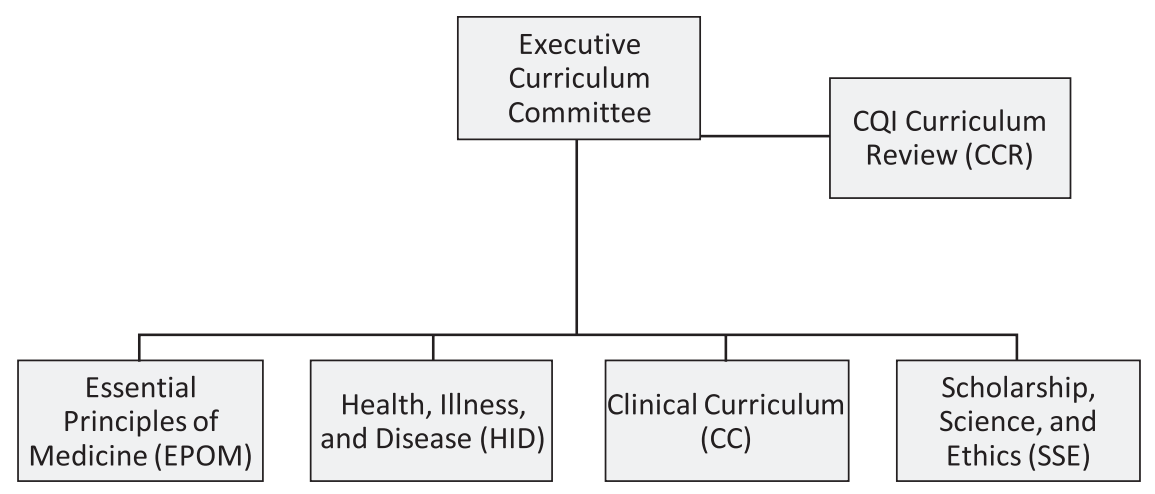

Figure 1 Curricular governance committees.

\section{Education Staff}

\section{Department of Medical Education}

The Office of Medical Education is responsible for the planning, implementation, evaluation, and oversight of the curriculum and for the development and maintenance of the tools to support the curriculum delivery, monitoring, and management.

See Figure 2-Organizational structure.

In addition to oversight of the curriculum, the Office of Medical Education has oversight of the entire medical student experience at Weill Cornell.

- The Office of Admissions recruits and selects the incoming class to the medical college each year.

- The Office of Student Affairs oversees the Office of Student Life and the Office of Student Diversity. The student life office assists students by providing wellness programming and supporting service learning, student organizations, and community service groups. The Office of Student Diversity works closely with all medical students to promote an inclusive atmosphere and oversees pipeline programs.
- The Office of Academic Affairs provides academic and career counseling for medical students, including assisting students with preparing for the residency match. In addition, the Office of Academic Achievement provides robust academic support for all students throughout the curriculum.

- The Office of Operations and Program Development has direct operational oversight over aspects of the clinical curriculum, to include the operation of the Clinical Skills Center. In addition, all aspects of medical education operations, including budget, finance, and human resources, are managed here.

- CQI monitors and reports on curricular institutional elements across the medical education mission and assists with accreditation of the medical education program.

- CME works with the clinical departments to provide continuing professional development opportunities for WCM faculty.

- In addition, the Office of Medical Education works closely with central services such as the Office of the Registrar, Office of Financial Aid, Student Health Services, and Housing Office to ensure medical students receive adequate support.

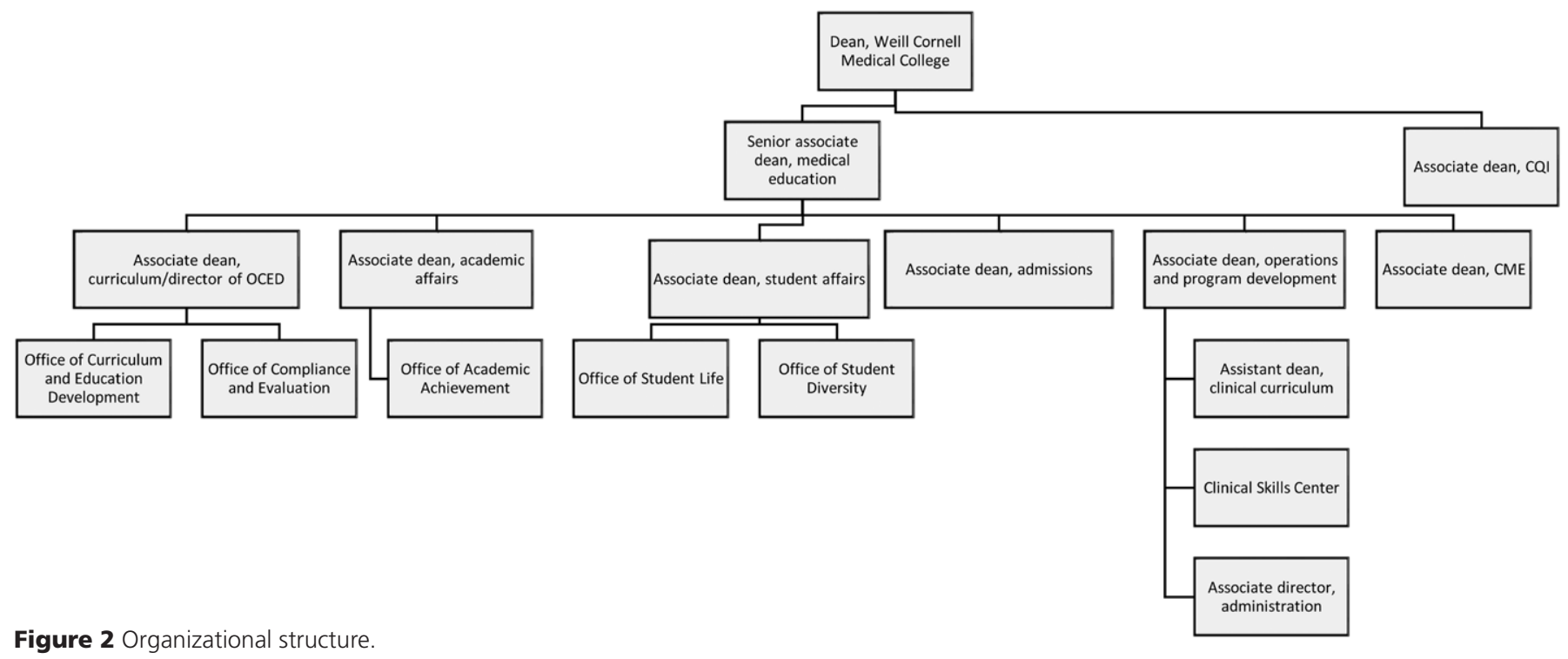




\section{Faculty Development and Support in Education}

\section{Professional development for faculty as educators}

- WCM offers a wealth of professional development programs to improve the skills of faculty as educators, researchers, and clinicians and to contribute to discipline-specific knowledge.

- Central resources include the Office of Faculty Development, the Medical Education Office, eCornell, and the Cornell Center for Teaching Excellence in Ithaca.

- The Office of Faculty Development and the Mentoring Academy hold programs on a range of topics, including the Faculty Enrichment and Educational Development series to provide seminars and workshops to help faculty thrive in their roles.

\section{Role of teaching in promotion and tenure}

- The WCM faculty appointment and promotion system uses 2 types of academic career pathways. One is for faculty members whose primary activity is academic achievement and scholarship. The second is for faculty members whose primary activity is in clinical investigation or teaching excellence.
- In the teaching excellence pathway, excellence in teaching represents the major area of service. Promotion based on teaching quality and quantity alone, in the absence of investigative or clinical care work, is possible.

- Due to the importance of the educational mission at WCM, faculty on all pathways are evaluated for their contributions to teaching and educational activities. Faculty on the pathway recognizing academic achievement in education leadership complete an educator's portfolio and are evaluated in 4 teaching domains.

- WCM recognizes exceptional teaching through annual awards. This includes the Excellence in Medical Education awards dinner where over 20 faculty are recognized for their teaching contributions. Named awards for teaching and mentorship are also presented at Commencement.

- Senior List at convocation recognizes 16 faculty who were nominated as outstanding teachers by the senior class.

- There are 5 Education Scholar Awards, competitive faculty development awards for educators, and multiple endowed professorships in medical education. 


\title{
The Brody School of Medicine at East Carolina University
}

\author{
Luan Lawson, MD, MAEd, David Gilbert, MD, Robert G. Carroll, PhD, Stephen Charles, PhD,
} and Kendall M. Campbell, MD

\section{Medical Education Program Highlights}

The Brody School of Medicine (BSOM) fulfills the threefold mission of increasing the supply of primary care physicians serving the state, enhancing the access of minority and disadvantaged students to a medical education, and improving the health status of citizens in eastern North Carolina. Our commitment to service and mission are highly valued by the BSOM community.

- BSOM is in the top $10 \%$ of U.S. medical schools for graduating physicians who practice primary care, practice in the state, and practice in rural and underserved areas.

- During the past 30 years, BSOM has contributed to improvement in health outcomes and narrowed health care disparities for residents in eastern North Carolina.

- BSOM is in the top $10 \%$ of U.S. medical schools graduating physicians who identify as Black/African American or American Indian/Alaskan Native.

\section{Curriculum}

\section{Curriculum description}

The BSOM curriculum offers early immersion in a variety of clinical settings while laying the foundation of knowledge for the practice of medicine. Education is delivered in a spiral architecture with planned, spaced repetition that ensures students return to key concepts with increasing complexity.

The 20-month foundational phase begins in August and consists of traditional basic sciences content embedded within courses that begin to develop clinical skills, critical thinking, and understanding of social and systemic forces in health systems. This phase provides immersion in clinical care experiences, including a longitudinal primary and community care preceptorship. Activities focused on professional identity formation, wellness, lifelong learning skills, and reflection are woven throughout the reenvisioned Foundations of Doctoring course. The Molecular Basis of Medicine course integrates cellular and biochemical function with its expression in genetic conditions. Newly designed organsystems courses integrate pathophysiology, pharmacology, and clinical applications.

Acad Med. 2020;95:S375-S378.

doi: 10.1097/ACM.0000000000003371

Copyright (C) 2020 by the Association of American Medical Colleges

Supplemental digital content for this article is available at http://links.Iww.com/ ACADMED/A888.

Year school was established: 1969.

School URL: https://medicine.ecu.edu.
The 12-month clinical phase, beginning in April, includes 6 core clerkships and 4 weeks of electives. Electives provide additional time for career exploration and the residency selection process. The radiology clerkship is a longitudinal experience integrated and aligned with each of the core clerkships.

The 13-month differentiation phase begins in April and is the final phase of the curriculum. It includes the required advanced core clerkships and ample elective time for exploration of career interests and individualization of learning experiences. The Transition to Year 4 course prepares students through interprofessional simulation activities, standardized patient encounters, and other advanced workshops. Neurology and physical medicine \& rehabilitation is a new 4-week clerkship focused on both acute and chronic presentations of neurological and musculoskeletal conditions. A newly designed Transition to Residency course with specialty specific integration of foundational sciences refocuses students on foundational concepts needed for success in residency training.

The innovative health systems science (HSS) curriculum is longitudinally interwoven throughout the 4-year curriculum to prepare students to care for patients, families, and communities in a rapidly changing health care environment.

See Supplemental Digital Appendix 1-Curriculum Schematicat http://links.lww.com/ACADMED/A888.

\section{Curriculum changes since 2010}

The BSOM curriculum was revised in 2016 to prepare students to work in the health systems of tomorrow by integrating basic sciences and clinical skills, providing early immersion in patient care experiences, and incorporating HSS as the third pillar of medical education.

Key curricular changes include:

- Longitudinal HSS curriculum

- Increase in problem-based learning, simulation, and selfdirected learning

- Expansion of interprofessional education

- Reorganization of the foundational science curriculum

- Integrated neurology and physical medicine and rehabilitation clerkship

In addition to the formal curriculum, 4 longitudinal cocurricular distinction tracks (research, medical education and teaching, health care transformation and leadership, service learning) allow students to pursue in-depth professional development based on individual interests.

The matriculating class size increased from 82 to 86 in 2017. 


\section{Assessment}

In 2012, BSOM developed new institutional learning objectives through an iterative process anchored on the mission of the school. The ACGME competency domains were selected as an organizing framework. In 2016, the ACGME milestones and the AAMC Core Entrustable Professional Activities were used to identify emerging competencies (HSS and interprofessional education) needed to bridge the continuum from undergraduate medical education to graduate medical education.

Curricular design focused on assessment models that incorporated spaced repetition and retrieval. Practice questions provide formative feedback and evidence to drive self-directed learning. Comprehensive, blended computer assessments are administered for all quizzes and exams. Formative progress testing using the NBME Comprehensive Basic Science Examination occurs in the foundational phase. OSCEs have been expanded throughout the curriculum, and cases have been reformulated to address emerging issues that affect health and society.

See Supplemental Digital Appendix 2-Program Objectives and Assessment Methods—at http://links.lww.com/ACADMED/A888.

\section{Pedagogy}

We use a variety of pedagogical approaches based on institutional learning objectives:

- Case-based learning and discussion, audience response, and problem-solving activities are integrated into lectures to increase student engagement.

- Our longitudinal Foundations of Doctoring course uses small groups, problem-based learning, role play, standardized patients, simulation, preceptorships, and clinical experiences to promote critical thinking and clinical skills.

- Laboratory sessions are used throughout the foundational phase to facilitate active learning.

- Clinical experiences are balanced in the ambulatory and inpatient settings.

- Other techniques include workshops, flipped classroom, case analysis, peer teaching, research presentations, and educational gaming.

Since 2010, learner-centered, experiential, and active learning strategies have been increasingly emphasized. Included are earlier clinical experiences, increased formative feedback, problembased learning, and flipped classrooms. Increased emphasis has been placed on incorporation of simulation, especially in the foundational phase of the curriculum.

\section{Clinical experiences}

- Clinical experiences in the foundational phase occur in community primary care practices that reinforce the patientcentered medical home model of care.

- The majority of required clinical training in the clerkship and differentiation phases of the curriculum is provided on our health science campus and through our teaching hospital, Vidant Medical Center.
- Longitudinal experiences include:

- Foundations of Doctoring course where students participate in a 20-month longitudinal small-group curriculum focused on critical thinking and clinical skills. Students complete a longitudinal primary care preceptorship.

- Radiology clerkship extends over 12 months and is integrated with the core clerkships to emphasize relevant imaging modalities.

- HSS is longitudinally interwoven throughout the entire curriculum.

- Student immersion in clinical settings begin the first week of medical school with shadowing and interprofessional experiences.

- Simulation and clinical skills sessions prepare students for clinical immersions that begin early in the first year.

- Outpatient community-based rotations throughout the state are components for 4 weeks of the family medicine clerkship and 2 weeks of the pediatrics clerkship. On the psychiatry clerkship, students have the opportunity to rotate at a variety of sites including a state psychiatric facility, inpatient and outpatient community sites, substance treatment facilities, and an outpatient Veterans Administration facility. Students have a required ambulatory primary care fourth-year clerkship experience that may be community based.

- Affiliations with community clinical partners have become increasingly challenging as medical schools have expanded. Perceived time constraints and financial impacts, including preceptor payments, are challenges for both institutional and community-based clinical faculty. Appropriate housing for community rotations is a challenge for all our health sciences schools. As we moved to a shortened foundational phase, preserving time for clinical experiences in the foundational phase of the curriculum requires careful attention.

\section{Curriculum Governance}

The Executive Curriculum Committee (ECC) is responsible for institutional oversight and designs, monitors, and evaluates the curriculum. Recommendations for content and structural changes are informed by annual retreats and supported by year curriculum and other committees. The Office of Medical Education (OME) along with the Office of Data Analysis and Strategy offer support through data generation and report creation needed to monitor and evaluate the curriculum. Resource members representing the East Carolina University Health Sciences Library, information technology, faculty development, and diversity affairs ensure appropriate resources for curriculum delivery.

The budget distribution for teaching uses a shared governance model. State teaching dollars are distributed by the Division of Academic Affairs to individual departments based on an educational RVU system to support faculty teaching. Course and clerkship director recommendations are made jointly by the OME, senior associate dean for academic affairs, and department chairs before review and approval by the ECC. The foundational science courses and faculty are supported centrally through 
curriculum coordinators in the OME. The clinical departments employ clerkship coordinators to manage and coordinate medical student activities.

See Figure 1-Curriculum management and governance structure.

\section{Education Staff}

The OME enhances the undergraduate medical education mission through supporting the process of accreditation, leadership in curriculum development, programmatic evaluation, and student assessment. The office is led by the associate dean for curricular innovation in medical education and includes an associate dean for medical education (accreditation), an assistant dean of medical education outcomes and assessment, and a staff of 8 who support the educational program. Administrative support for the foundational science courses and curriculum committees is provided to ensure planning, implementation, evaluation, and oversight of the curriculum. Administration of a systematic course review system-including annual course/clerkship reviews and 3-year course reviews; curriculum mapping; tracking of required clinical experiences and procedures; testing administration; and evaluations of students, faculty, and course/clerkships-is provided through the office. The distinction tracks, longitudinal HSS curriculum, and Teachers of Quality Academy (TQA) are administered through the OME.

The OME is an office in the Division of Academic Affairs and collaborates with the offices of Clinical Simulation, Faculty Development, Student Affairs, Clinical Skills, Diversity Affairs, and Student Development and Academic Counseling to effectively deliver the curriculum and support learners.

\section{Faculty Development and Support in Education}

All faculty receive support from the Office for Faculty Excellence (OFE) that includes education and support for research and teaching methods across the university. Our BSOM Office for Faculty Development (OFD) partners with the OFE to bring programming to the Brody campus. The OFD provides programming tailored to our curricular and faculty needs, a mentoring program for junior faculty, and individual consultations to all faculty. Through the Academic Skills for Medical School Faculty Workshop Series, faculty members can develop and enhance their skills as teachers, clinicians, researchers, and educational leaders.

Every year, the OME hosts Medical Education Day, a day we celebrate innovation and scholarship and advance best practices in education. We also hold a course and clerkship directors bootcamp that supports the growth and development of educational leaders at BSOM. Additionally, we have partnered with East Carolina University's College of Education to offer a graduate-level certificate program, Education in the Health Care Professions, that can be applied to a Master of Arts in Adult Education. The Division of Academic Affairs supports external faculty development for educators and educational coordinators.

Teaching is evaluated and valued, along with service, research, and creative activity, in the promotion and tenure process.

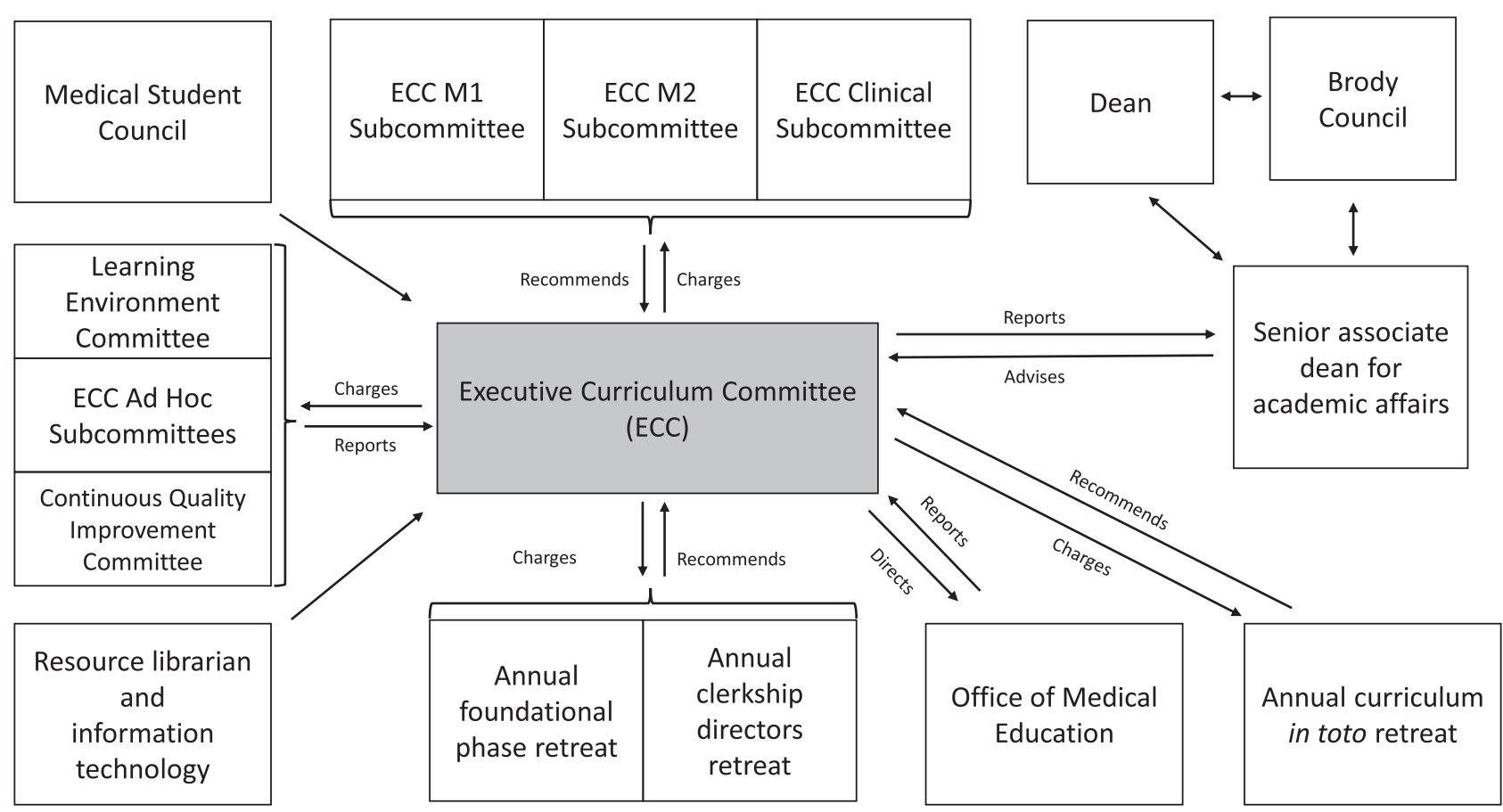

Figure 1 Curriculum management and governance structure. 
Documentation of teaching quality, including peer observation of teaching, is a requirement of the promotion and tenure process for all faculty. Educational grants and manuscripts may be used to fulfill the expectations for scholarly productivity.

We are fortunate to have a cadre of master educators recognized and nominated by their peers and learners. The Master Educator Program recognizes excellence in education in any of the following areas: educational leadership and administration, outstanding teaching or mentorship contribution, educational innovation and curriculum development, educational evaluation and research, faculty development in education, and outstanding educational contribution by a community physician. This program offers a wealth of experience to assist faculty in their teaching roles.

The TQA is a professional development program for health care leaders designed to fundamentally change care delivery through a curriculum focused on building knowledge and skills in HSS. Interprofessional participants engage in advanced learning, apply these skills in projects across the health system, teach others in their environment, and evaluate the outcomes of improvement interventions. Leading by example, TQA participants promote a culture of quality and safety across our enterprise and serve as experts for curricula development for HSS educational experiences. 


\section{Duke University School of Medicine}

Colleen O'Connor Grochowski, PhD, and Edward G. Buckley, MD

\section{Medical Education Program Highlights}

Duke University School of Medicine (DUSOM) offers a unique educational program. The basic sciences are taught in 1 year in 2 half-year integrated courses that use flipped classroom, interactive, team-based, and case-based methods. Core clinical clerkships are completed in the second year either traditionally or longitudinally. A minimum of 10 months of dedicated intensive mentored scholarly investigation is required as a mechanism to facilitate the training of clinician-scientists. We have created a clinical education urgent care clinic experience emphasizing interprofessional clinical care. Our educational program also features longitudinal curricular threads in leadership, cultural determinants of health $\backslash$ health disparities, and ends with a discipline-focused capstone course.

\section{Curriculum}

\section{Curriculum description}

DUSOM's Foundation for Excellence Curriculum consists of 1 year of foundational basic sciences followed by a year of required clinical clerkships in year 2 . The third year is devoted to 10 months or more of scholarly investigation. The fourth year consists of electives and a required subinternship, acute care curriculum, and capstone course.

See Supplemental Digital Appendix 1-Curriculum Map-at http://links.lww.com/ACADMED/A886.

\section{Curriculum changes since 2010}

Our curriculum has evolved over the last 10 years. Highlights of these changes include the following:

Year 1-Preclinical:

- Reduced from 4 to 2 the basic science courses in year 1 and maintained organ-systems approach

- Enhanced the integration of foundational basic science principles

- Implemented a clinical correlations course director to improve the quality, consistency, and increasing complexity of the clinical correlates in the basic science courses

- Aligned physical examinations skills training with organ systems being taught in basic science courses

Acad Med. 2020;95:S379-S382.

doi: 10.1097/ACM.0000000000003374

Copyright (C) 2020 by the Association of American Medical Colleges

Supplemental digital content for this article is available at http://links.Iww.com/ ACADMED/A886.

Year school was established: 1930.

School URL: https://medschool.duke.edu.
- Added a longitudinal 3-year leadership curricular thread beginning in year 1

- Added a longitudinal 2-year course called Cultural Determinants of Health and Health Disparities

Year 2-Required clinical clerkships:

- Eliminated five 1-week classroom-based courses

- Eliminated one 4-week selective but maintained two 2-week selectives

- Added 2 new clerkships, a 4-week clerkship in neurology and a 4-week clerkship in radiology, for a total of 8 required clerkships

Year 3-Scholarly investigation:

- Added several new study program tracks for a new total of 18

- Added 5 approved master's degree programs for a new total of 13

- Transitioned the Medical Statistics course and the EvidenceBased Medicine course from the classroom to online

- Implemented policy guaranteeing students a minimum of 4 weeks of dedicated USMLE Step 1 examination preparation time

Year 4-Electives and capstone:

- Created a standardized acute care curriculum

- Converted the capstone course from a 4-week course to a longitudinal course with required modules beginning in January of the fourth year and culminating in a 3-week classroom-based course in March

- Added specialty-specific boot camps to the capstone course

Multiyear:

- Created an interprofessional education and care (IPEC) clinic supervised by an interprofessional team of faculty; medical students are required to work in the IPEC clinic at least once during their first, third, and fourth years

In 2013, we moved into a state-of-the-art health professions education building that could accommodate more than the 100 students we recruited previously. Since 2013, class size gradually increased to 120 .

\section{Assessment}

Our medical education program objectives are based on the AAMC Physician Competency Reference Set, ${ }^{1}$ with the addition of 2 competency areas reflecting our unique curriculum: scientific foundations of health and disease, and research and scholarly activity.

See Supplemental Digital Appendix 2-Program Objectives and Assessment Methods—at http://links.lww.com/ACADMED/A886.

We have made some changes to our assessment program since 2010. Examinations in the first year now occur on a predictable 
schedule every 2 weeks. We have created a mobile technologyenabled process providing students with actionable formative feedback during each week of each clerkship in the second year and implemented a dashboard for visualizing this data. Lastly, 3 clerkships added mini-OSCEs (obstetrics-gynecology, neurology, and surgery) in addition to the existing mini-OSCE in the medicine clerkship. We also eliminated the week devoted to assessment after the clerkship year, but maintained the summative OSCE, increasing the number of stations from 8 to 10 .

\section{Parallel curriculum or tracks}

We have a primary care leadership track (PCLT) that runs in parallel to our standard curriculum. The goal of the PCLT is to create change agents for the health care system through primary care. The 4-year program offers leadership training, a longitudinal integrated clerkship (LIC) in the second year that includes following pregnant mothers and delivering their babies, time for service with a community health care agency, and research in community-engaged population health. Features of each year are listed below.

Year 1: PCLT students participate in a $21 / 2$-day preorientation in late July at Duke before the general DUSOM orientation. This focuses on leadership skills and team building. Leadership training continues with weekly PCLT team meetings and monthly student-led dinner programs. PCLT students organize a community service project in both the fall and spring. They participate in evaluation of their skills. Students complete the rest of the DUSOM curriculum with students in the traditional MD track.

Year 2: PCLT students participate in a 7-month LIC where they learn medicine alongside faculty in their clinics in family medicine, pediatrics, general internal medicine, and gynecology. In addition, PCLT students serve a community agency working on health issues 1 half day each week during the LIC. Students follow pregnant patients through pregnancy and delivery in a Centering model of group prenatal care. They follow patients to surgery, therapy, and specialty visits. They meet weekly in a primary care seminar to share experiences and learn clinical medicine topics. PCLT students complete an orientation to the Durham community and learn about community engagement.

There are inpatient immersions in internal medicine, neurology, surgery, psychiatry, and pediatrics. PCLT students attend a behavior health seminar as well as population health seminars each designed especially for PCLT with primary care in mind. PCLT students attend the Clinical Skills Foundation and Clinical Skills courses with students in the traditional track.

Year 3: PCLT students develop and implement a population health improvement research project. All projects have a required community- or stakeholder-engaged component. PCLT has a dedicated director of research to guide students' research efforts. PCLT students complete a continuity clinic experience, like students on the traditional track. All students have the option of a 10- or 12-month third year.
Year 4: PCLT students choose from a variety of clinical electives available to all students. Along with traditional students, they complete a subinternship, critical care training, radiology training, and the capstone course.

DUSOM also offers a 3-year pathway in orthopedic surgery. The Accelerated Pathway in Orthopaedics (APO) is a unique opportunity for interested third-year students to pursue a clinical experience in orthopedic surgery and to demonstrate the potential (competency, quality, maturity, etc.) for entering the postgraduate year 1 Duke orthopedic surgery residency class the following year, thereby graduating from DUSOM after their third year of medical school. The goals of the APO are to enhance effectiveness and efficiency of orthopedic surgery training; streamline the number of required years; provide structured preparation for orthopedic surgery residency training; and promote skills in leadership, professionalism, research, ethics, and practice- and systems-based medicine. Features of the APO include combining 3 years of medical school with 5 years of residency training, students earning their MD degree in 3 years, direct admissions into the orthopedic residency program, and providing focused mentorship and coaching.

\section{Pedagogy}

DUSOM uses a variety of pedagogies ${ }^{2}$ to achieve medical education program objectives, including case-based learning, clinical experience in inpatient and ambulatory settings, large- and small-group discussion, laboratories, lectures, peer teaching, preceptorship, role playing, self-directed learning, simulation, standardized patients, team-based learning, video, and workshops. Since 2010, we have more consistently integrated case- and team-based learning, with an increased emphasis on clinical correlations and clinical reasoning.

\section{Clinical experiences}

First-year medical students have their first clinical encounters with real patients in October of their first year as part of the required Clinical Skills Foundation course. Required clerkships on the traditional track occur in Duke-affiliated hospitals and outpatient clinics. LICs are available to up to 12 students in the traditional track and 10 students in the PCLT. LICs occur in Duke-affiliated outpatient clinics and the Durham Health Department. Eighty percent of students will rotate through the Durham Veteran's Administration Medical Center during their clerkship year.

\section{Curricular Governance}

The DUSOM Curriculum Committee (CC) has ultimate authority over the curriculum. The CC has several subcommittees that support different phases of the educational program.

See Figure 1-Curriculum committee organizational chart.

\section{Education Staff}

The Office of Curricular Affairs (OCA), under the leadership of the associate dean for curricular affairs, is responsible for 


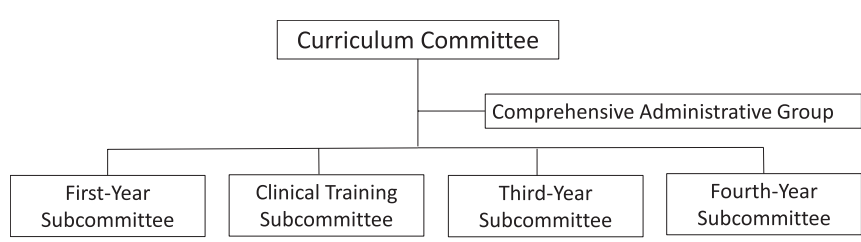

Figure 1 Curriculum committee organizational chart.

the administrative and academic support for planning the implementation and evaluation of the educational program; general oversight of the curriculum; and the development and maintenance of the tools to support curriculum delivery, monitoring, and management. The OCA includes 4 assistant deans (basic science education, clinical education, assessment, and learning environment), a director, and 30 faculty and staff members. The associate dean for curricular affairs reports to the vice dean for education, as do the other associate deans and directors involved in UME, faculty development, and learner support

See Figure 2-Undergraduate medical education organizational chart.

The role of the primary education staff and administrative faculty is support of UME and multiple masters' degree programs housed within DUSOM, and also includes student affairs. The hospital is responsible for GME although there is a dotted line from the designated institutional officer/associate dean of GME to the DUSOM vice dean for education.

\section{Faculty Development in Support of Education}

\section{Professional development for faculty as educators}

Professional development for faculty educators and professional staff in the School of Medicine and the School of Nursing is centrally provided by the Duke Academy for Health Professions Education and Academic Development (Duke AHEAD). The mission of Duke AHEAD is to promote excellence in the education of health professionals by creating a community of education scholars, fostering innovation in health professions education, supporting outstanding teachers, providing faculty development programs, and facilitating quality education research. The mission is accomplished through a variety of annual offerings ranging from education grand rounds to a yearlong certificate program, Education Celebration Day, consultative preparation of dossiers for promotion, grant funding for educational innovations, and teaching awards. Duke AHEAD is interprofessional, supporting educators from the DUSOM's graduate health professions programs (e.g., physician assistant, doctor of physical therapy, occupational therapy) and the School of Nursing.

\section{Role of teaching in promotion and tenure}

To be considered for promotion, faculty must demonstrate effectiveness in clinical or educational practice as well as at least one additional domain (research/original investigation, clinical

\section{Vice dean for education (Edward Buckley, MD)}

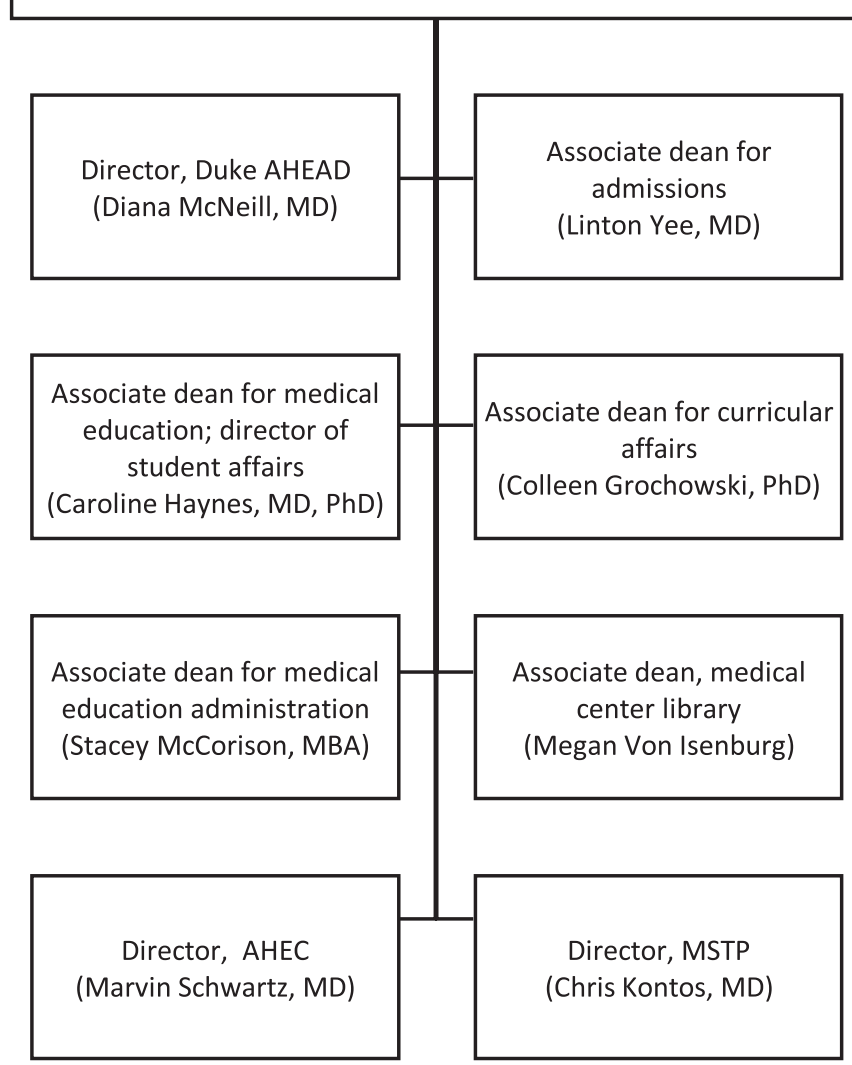

Figure 2 Undergraduate medical education organizational chart.

practice advancement, and administration/service). Effectiveness is demonstrated for the purposes of evaluation for promotion by portfolio development. Contributions are documented and described using one of the following portfolios: clinicianinvestigator, clinician-educator, clinical practice advancement, clinician leader-administrator, or individualized portfolio for other contributions or combination of areas of contribution. An education portfolio provides the framework for the faculty member to submit evidence of impact in education for the purposes of promotion.

\section{Initiatives in Progress}

DUSOM is in the early stages of fleshing out a curriculum innovation initiative. The goals of the innovation are to provide early meaningful clinical experiences for first-year students with level-appropriate clinical skills training immersion the first 2 weeks of medical school and subsequent longitudinal clinical experiences in year 1; to create an explicit clinical framework for teaching the foundational basic sciences; and to develop innovative approaches to clinical education that include leveraging technology, the EHR, and big data. 


\section{References}

1 Englander R, Cameron T, Ballard AJ, Dodge J, Bull J, Aschenbrener CA. Toward a common taxonomy of competency domains for the health professions and competencies for physicians. Acad Med. 2013;88:1088-1094.
2 MedBiquitous Curriculum Inventory Working Group Standardized Vocabulary Subcommittee. Curriculum Inventory Standardized Instructional and Assessment Methods and Resources Types (September 2012 Version). Washington, DC: Association of American Medical Colleges; 2012 


\title{
University of North Carolina School of Medicine
}

\author{
Gary L. Beck Dallaghan, PhD, Kurt O. Gilliland, PhD, Beat Steiner, MD, MPH, and Julie S. Byerley, MD, MPH
}

\section{Medical Education Program Highlights}

The University of North Carolina School of Medicine (UNC SOM) aims to serve North Carolina and beyond as one of the nation's leading public medical schools. We aspire to excellence across the array of careers in medicine, allowing our learners to have access to varied role models and opportunities to inspire them on their way to a career of science and service. Diversity is a core value to our work and community. We admit a very diverse class (currently $21 \%$ of students come from backgrounds underrepresented in medicine) and intend that among our 190 graduates per year, many will specialize in primary care and other needed disciplines. Some will focus on careers geared toward scientific discovery, others focus on public and population health, some in medical education, and all working as leaders to improve health and well-being through their careers as physicians.

In 2014, UNC SOM implemented the Translational Education at Carolina (TEC) curriculum, which was built upon the following principles:

- Be student centered and patient based, while being population, public health, and globally inspired

- Facilitate translation and integration of basic, clinical, and population science to enhance human health and well-being

- Provide a strong foundation for entry into graduate medical education within the broad opportunities of medicine

- Be responsive to the changing health care environment

- Focus on promoting, supporting, and maintaining health, not just treating disease

- Incorporate strengths of the university, including opportunities for interprofessional and cross-disciplinary education

- Provide longitudinal engagement with faculty and robust mentorship

- Instill intellectual curiosity, developing an aptitude for critical thinking and lifelong learning

- Promote the development of leadership skills, professionalism, ethics, humanism, and service to others

The curriculum includes unique features such as:

- Threads to facilitate the translation and integration of basic, clinical, and health systems science to enhance human health and well-being

- Longitudinal outpatient experience incorporating a rigorous quality improvement experience

Acad Med. 2020;95:S383-S386.

doi: 10.1097/ACM.0000000000003375

Copyright (C) 2020 by the Association of American Medical Colleges

Supplemental digital content for this article is available at http://links.Iww.com/ ACADMED/A885.

Year school was established: 1879.

School URL: https://www.med.unc.edu.
- Social and health systems science curriculum that spans all 4 years

- Opportunities to individualize learning such as scholarly concentrations, global health experiences, rural scholar programs, dual-degree programs ( $\mathrm{PhD}, \mathrm{MPH}, \mathrm{MBA}$, and others), and many opportunities for student leadership

\section{Curriculum}

\section{Curriculum description}

The 4-year curricular experience is divided into 3 phases: an 18-month foundation phase, 12-month application phase, and 14-month individualization phase. The foundation phase is divided into 3 courses: Medical Science, Patient-Centered Care, and Social and Health Systems. The application phase provides integrated clerkship experiences to enhance common learning themes. For example, one trimester highlights the vulnerable populations of pediatrics, obstetrics-gynecology, and psychiatry in a streamlined fashion, allowing synergy of perspective. The longer individualization phase allows students time to explore career options before submitting residency applications.

\section{Curriculum changes since 2010}

As of 2011, UNC SOM offered a traditional $2+2$ curriculum with basic science courses in the first 2 years, 1 year of block clinical clerkships, and 1 year of monthlong electives.

In 2011, the faculty and leadership of UNC SOM recognized that the curriculum needed broadening, better integration, and a general rethinking. The newer competencies expected of today's physicians were seen as perfectly congruent with the school's population health mission but were not yet adequately addressed in the curriculum. There was little room for individualization within the medical education program, especially before the selection of residency field was required.

A strategic planning process was initiated. The new curriculum was conceived as 3 large curricular threads, each with identified faculty leadership:

- TEC 1: translation of medical sciences to the care of people (basic science)

- TEC 2: translation of medical sciences to the care of patients (clinical science)

- TEC 3: translation of medical sciences to the care of populations (health system science)

Class sizes have increased to 190 students per year beginning in 2018. UNC SOM has a long history working with the North Carolina Area Health Education Centers to provide training throughout the state and has 3 formal regional campuses.

Asheville and Charlotte have been regional campuses since 2009 
and 2010, respectively, and in 2016, the campus in Wilmington was launched.

See Supplemental Digital Appendix 1-Curriculum Schematicat http://links.lww.com/ACADMED/A885.

\begin{abstract}
Assessment
Assessment is accomplished through direct observation and clinical assessment, objective structured clinical examinations, projects, peer feedback, reflective writing, and multiple forms of knowledge base testing. To better prepare our students for the USMLE Step 1 examination, customized NBME examinations for final block exams were initiated in the foundation phase and are being incorporated for the midterms during the 2019-2020 academic year.
\end{abstract}

See Supplemental Digital Appendix 2-Program Objectives and Assessment Methods—at http://links.lww.com/ACADMED/A885.

\section{Parallel curriculum or tracks}

Even before the implementation of the TEC curriculum, the school offered 2 parallel tracks for the core clinical application phase: the Asheville longitudinal integrated clerkship and Charlotte longitudinal integrated curriculum (CLIC). What varies for students assigned to these programs is the structure of the core clinical application phase. The Asheville longitudinal integrated curriculum, launched in 2009, offers 8 months of integrated ambulatory clinical education and 4 months of inpatient course work for the now 31 students assigned to that campus. The CLIC program was established in 2010 and is offered at the Charlotte regional campus. For 2020-2021, 30 students were assigned to CLIC, which offers 6 months of integrated longitudinal ambulatory education. Another 6 months is dedicated to hospital-based inpatient education. The 2 programs use the same learning objectives, major assessments, and final grade breakdown as the traditional medical degree program.

In 2015, UNC SOM established a 3-year graduation pathway as a parallel track known as Fully Integrated Readiness for Service Training (FIRST). The focus of FIRST is to fast-track selected students into the family medicine residency program local to their campus site and encourage service in a rural area through loan repayment. FIRST students complete the same program objectives and assessments as the traditional medical degree program. The first cohort of students in this track is now completing residency and has been highly successful. The school is now expanding the track to other programs across the state including other disciplines such as psychiatry, general surgery, and pediatrics.

\section{Pedagogy}

In the foundation phase, there are a variety of pedagogical methods deployed, including small-group discussion with case-based learning, large-group lecture (which is now more interactive and case based), video podcasts as preparation for in-class sessions, laboratory sessions including peer teaching, and self-directed learning exercises. In addition, the physical examination course includes preceptorship, simulation, ultrasound skill teaching, standardized patients, and 1 week of clinical experience (either ambulatory or inpatient) per each of 3 semesters. The Social and Health Systems course includes small-group discussion as well as role playing in a health care reform exercise. In the application and individualization phases, pedagogical methods include a balance of ambulatory and inpatient clinical experiences with preceptorship as well as small-group and large-group case-based discussion, simulation, quality improvement project work, reflective writing, and standardized patients.

Additionally, we have an active Office of Academic Excellence that provides 1-on-1 and small-group help for students in need of more attention. Educators in this office assist in enhanced development of standardized test-taking skills, details of clinical performance, and other challenges that individual learners face.

\section{Clinical experiences}

We have worked to replicate longitudinal experiences for each of our students, believing in the benefits of continuity of place, peers, preceptors, and patients as illustrated in evaluation of the LIC model in Asheville ${ }^{1,2}$ and elsewhere. During the application phase, the Central (Chapel Hill, Greensboro, and Raleigh areas) campus and Wilmington have 16-week longitudinal clinical experiences in adult and pediatric ambulatory care. Asheville offers an 8-month longitudinal integrated outpatient experience with discreet inpatient experiences in surgery and adult inpatient care. Charlotte offers a 6-month longitudinal integrated experiences, which includes each clerkship discipline and 6 months of block inpatient experiences.

\section{Challenges in designing and implementing clinical experiences for medical students}

Clinical capacity is a challenge, especially as our class size has expanded. To address clinical capacity in the outpatient setting, we have worked to ensure that students add value. Our students help practices with quality improvement projects, and after the Centers for Medicaid and Medicare Services changes in documentation policy, they have a more active role in documentation. This has helped greatly with preceptor recruitment and retention. We have also invested in administrative support in our large practices to ease the burden of managing students. Finally, we have focused on faculty development through our Academy of Educators. We are also working proactively to address clinical capacity in the inpatient setting by working to develop teaching services in hospitals in our system that have not previously had learners.

\section{Curricular Governance}

The Education Committee has primary responsibility for the curriculum. The senior associate dean for medical student education chairs the committee, which is made up of elected and appointed faculty members, senior administrative staff, and medical students. Regional campus leaders are an integral part of this committee. There are 3 standing curriculum subcommittees 
representing the 3 phases of the TEC curriculum: foundation, application, and individualization phases. The subcommittee cochairs report to the Education Committee.

\section{Education Staff}

The Office of Medical Education provides logistical support for implementation of curriculum delivery. The office works with the director of educational scholarship to design evaluation tools to monitor student perceptions of the curriculum. The director of academic affairs oversees monitoring of the curriculum for quality improvement efforts. In addition to the typical support staff to facilitate student success, we have recently added wellness coaches to help build resilience and improve the mental health of our medical students.

See Figure 1-Organizational leadership chart.

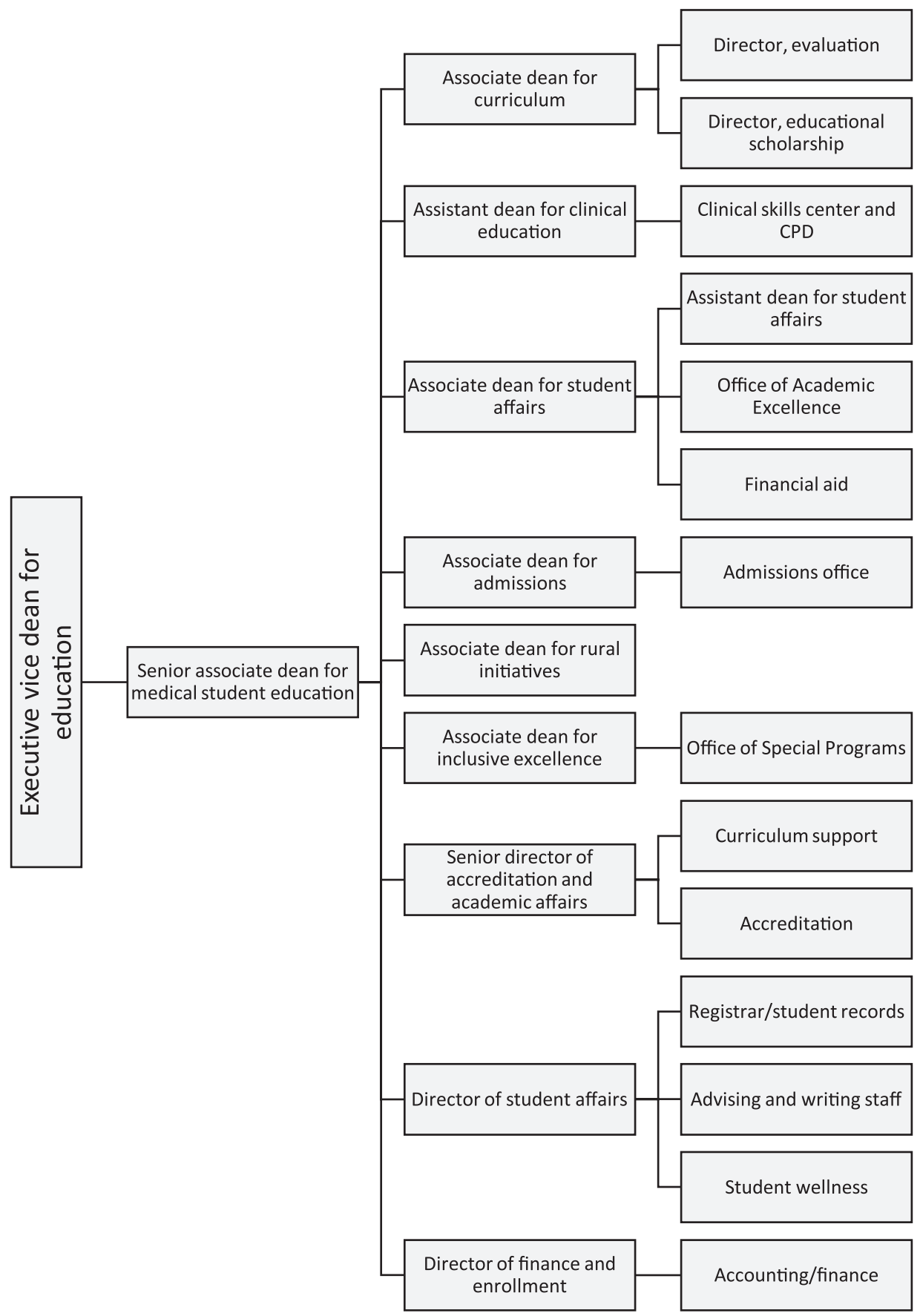

Figure 1 Organizational leadership chart. 


\section{Faculty Development and Support in Education}

The Office of Faculty Affairs and Leadership Development, housed within the Dean's Office, exists to support the academic success of the faculty. The portfolio of programming aims to provide opportunities for personal reflection and growth for all faculty and to support the promotion and tenure process, to ensure individuals hired for leadership positions can develop the skills and ability to lead, and to address the unique needs of faculty subgroups. Program examples include the Academy of Educators, New Faculty Orientation, Coaching Skills for Leaders, Academic Career Leadership Academy in Medicine, and Making the Case.

The Office of Faculty Affairs and Leadership Development has also contracted with an external partner to develop a mobile app that guides faculty through career planning. Though not explicitly linked to promotion, the tool helps faculty develop a career mission and outline SMART goals and objectives to help them achieve that mission.

The Academy of Educators was founded in October 2006 as a part of the school's strategic plan to enhance excellence in teaching through educational research and scholarship. The academy is for medical school faculty only. It offers robust programming at the central campus and at each of the regional campuses. Through online and in-person offerings and an engaged faculty council, the Academy of Educators is a vital component of faculty development for educators.

\section{Regional Medical Campuses}

An instrument we call the Campus Comparability Tool was developed by our Education Committee for tracking measures

\section{Table 1 \\ Regional Medical Campuses}

\begin{tabular}{|c|c|c|}
\hline Regional campus name & Type & $\begin{array}{l}\text { Student } \\
\text { enrollment }\end{array}$ \\
\hline Asheville & Clinical & 26 \\
\hline Charlotte & Clinical & 28 \\
\hline Wilmington & Clinical & 12 \\
\hline $\begin{array}{l}\text { Central campus (which includes } \\
\text { teaching sites in nearby Greensboro and } \\
\text { Raleigh in addition to Chapel Hill) }\end{array}$ & & 124 \\
\hline
\end{tabular}

of comparison. ${ }^{3}$ The measures were derived from the LCME elements addressing comparability of regional campuses. Given the unique attributes of each campus, using these elements allowed us to agree on outcome measures while acknowledging contextual factors at each site.

See Table 1-Regional Medical Campuses.

\section{References}

1 Latessa R, Beaty N, Royal K, Colvin G, Pathman DE, Heck J. Academic outcomes of a community-based longitudinal integrated clerkships program. Med Teach. 2015;37:862-867.

2 Latessa R, Keen S, Byerley J, et al. The North Carolina Community Preceptor Experience: Third study of trends over 12 years. Acad Med. 2019;94:715-722.

3 Foster JH, Byerley J, Tarantino H, et al. Cracking the nut on LCME standard 8.7: Innovations to ensure comparability across geographically distributed campuses. Teach Learn Med. 2019;31:544-551. 


\section{Wake Forest School of Medicine}

Kim Askew, MD, E. Shen, PhD, Kimberly Ford, MS, Stefanie Rachis, EdD, Jennifer Mroz, MEd, Nate Warden, MBA, Natalie Barrett, Timothy Peters, MD, and C. Randall Clinch, DO, MS

\section{Medical Education Program Highlights}

The Wake Forest School of Medicine (WFSM) MD curriculum is called Wake Ready! and provides our learners with the knowledge and skills to be ready for the first day of internship/ residency, while also setting the stage for their future training and areas of interest. Unique aspects of the curriculum include integrated ultrasound, curricular threads (patient safety, social determinants of health, histopathology, dermatology, radiology, pharmacology), and certificate programs.

\section{Curriculum description}

See Supplemental Digital Appendix 1-Curriculum Snapshotat http://links.lww.com/ACADMED/A884.

\section{Curriculum changes since 2010}

Our school has occupied a new state-of-the-art medical education building in July 2016, the Bowman Gray Center for Medical Education. Our class size has incrementally increased since 2010 from around 120 students to its current level of 145 students each year. This facility houses extensive resources in support of active learning; simulation-based instruction; the use of ultrasound in anatomy and physiology instruction; and space for student relaxation, study, and learning communities.

Since 2010, our curriculum has undergone significant revision, including a shortening of the preclinical phase to 18 months. Extensive curricular change of the preclinical phase has included completely new courses focused on clinical skills, clinical reasoning, bioethics and humanities, health systems, ultrasound, and epidemiology. Our clinical curriculum has introduced new clerkship experiences in anesthesiology, geriatrics, and palliative care, as well as integrated instruction in health care disparities. We have partnered extensively with learners during the creation and revision of the curriculum, resulting in practical, experience-based instructional methods. The use of standardized patients in simulated clinical experiences has spread throughout our curriculum, including during basic science blocks of instruction, such as in our teaching of bioethics. Student assessment has been extensively revised, with the creation of experience-focused assessment events, with testing outcomes that correlate with future curricular

Acad Med. 2020;95:S387-S390.

doi: 10.1097/ACM.0000000000003376

Copyright (C) 2020 by the Association of American Medical Colleges

Supplemental digital content for this article is available at http://links.Iww.com/ ACADMED/A884.

Year school was established: 1902.

School URL: https://school.wakehealth.edu. performance. These assessments allow for early identification of students who may require additional instruction before advancing to clinical training. The emphasis on formative assessments is another important feature of the new curriculum. Narrative feedback was given to students during many preclinical courses and throughout the clinical years.

\section{Assessment}

The Undergraduate Medical Education Curriculum Committee (UMECC) reviews and approves the WFSM goals and objectives annually. The framework of WFSM goals and objectives is the Physician Competency Reference Set (PCRS) competencies and the ACGME domains of competence.

WFSM has also implemented an assessment system in clinical years to longitudinally follow growth of student's clinical performance growth. The assessment system not only is based on institutional objectives that aligned with PCRS but also incorporates the AAMC's Entrustable Professional Activities. The assessment methods now include workplace-based assessments, standardized patient encounters, procedure objective structured clinical examinations, clinical subject matter examinations, oral examinations, and professionalism metrics.

See Supplemental Digital Appendix 2-Program Objectives and Assessment Methods—at http://links.lww.com/ACADMED/A884.

\section{Curricular Governance}

The WFSM UMECC, by the authority of the dean, is charged with the oversight of the medical education program leading to the MD degree as a whole. The UMECC is responsible for the overall design, management, integration, evaluation, and enhancement of a coherent and coordinated medical curriculum, in accordance with the mission and vision of the school of medicine, and the accreditation requirements of the LCME. Subcommittees of the UMECC include:

- The Subcommittee on Preclinical Education: responsible for course content, instructional methods, and student assessment for the preclinical curriculum

- The Subcommittee on Clinical Education: responsible for course content; instructional methods; and student assessment for the clinical curriculum, including all clerkship, selective, and elective student experiences

- The Subcommittee on Integration and Transitions in Education: responsible for examining course content, instructional methods, and student assessment to achieve curricular integration needs

- The Subcommittee on Assessment, Learning, and Teaching: responsible for all aspects of learning, teaching, and assessment in the undergraduate medical education program 
- The Medical Education Operations Subcommittee: responsive to UMECC directives and accreditation needs, including component and program review and continuous quality improvement

See Figure 1-UMECC structure.

\section{Education Staff}

The primary role of the Office of Academic Affairs is undergraduate curriculum planning, assessment, program evaluation, curriculum monitoring, and curriculum management. The Office of Medical Education is led by the associate dean for MD program academic affairs, who leads the teams responsible for delivery, planning, evaluation, and oversight of the curriculum. The office is organized into 4 distinct areas, including:

- Preclinical education (1 associate dean, 1 assistant dean, 2 support staff)

- Clinical education (1 associate dean, 2 assistant deans, 3 support staff)

- Longitudinal curriculum (1 associate dean, 1 assistant dean, 2 support staff)

- Evaluations and technology (2 directors, 4 support staff)

See Figure 2-Educational staff.

\section{Faculty Development and Support in Education}

As part of the structure of support for our WFSM faculty, the Office of Faculty Affairs supports the advancement and success of faculty through a variety of institutional opportunities for all faculty to develop themselves as educators, researchers, clinicians, and an amalgamation of these career paths and tracks that represent the faculty at the school of medicine. This support structure includes a number of programs, including the Educator Certificate Program. Cosponsored with academic affairs, the Educator Certificate Program provides practical training for educators aspiring to develop the knowledge and skills needed to advance their teaching and leadership skills in education. Participant selection involves a competitive application process, which includes release time and funding support from their department chair.

Mentoring is a core value and an essential activity at WFSM. One-on-one or team relationships provide an enriching opportunity for both junior faculty (mentee) and senior faculty (mentor). Several departments offer robust mentoring and faculty development opportunities. Institutionally, faculty affairs provide several opportunities in this area, including:

- During a pilot of the Faculty Mentoring Program, participants (mentors and mentees) received training on how to improve relationships, collaborate, and improve confidence in their roles.

- The Faculty Development Committee offers an annual Speed Mentoring event for faculty to network with midcareer and senior faculty.

- The longest-running institutional mentoring program is the WIMS Early Career Women Faculty Mentoring Program. Since 1998, this program has matched early-career women faculty with male and female senior faculty (associate and full professor) mentors.

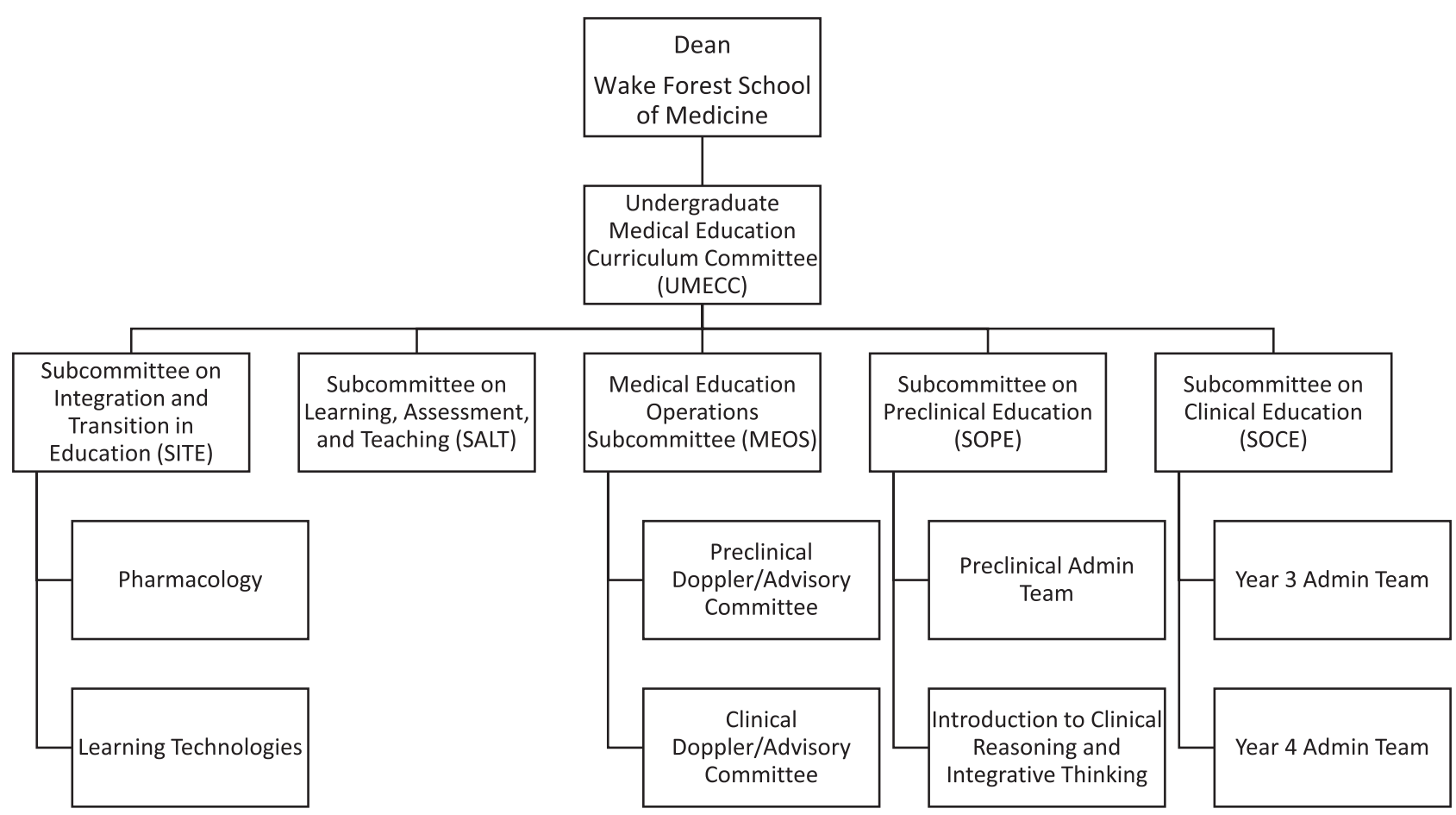

Figure 1 UMECC structure. 


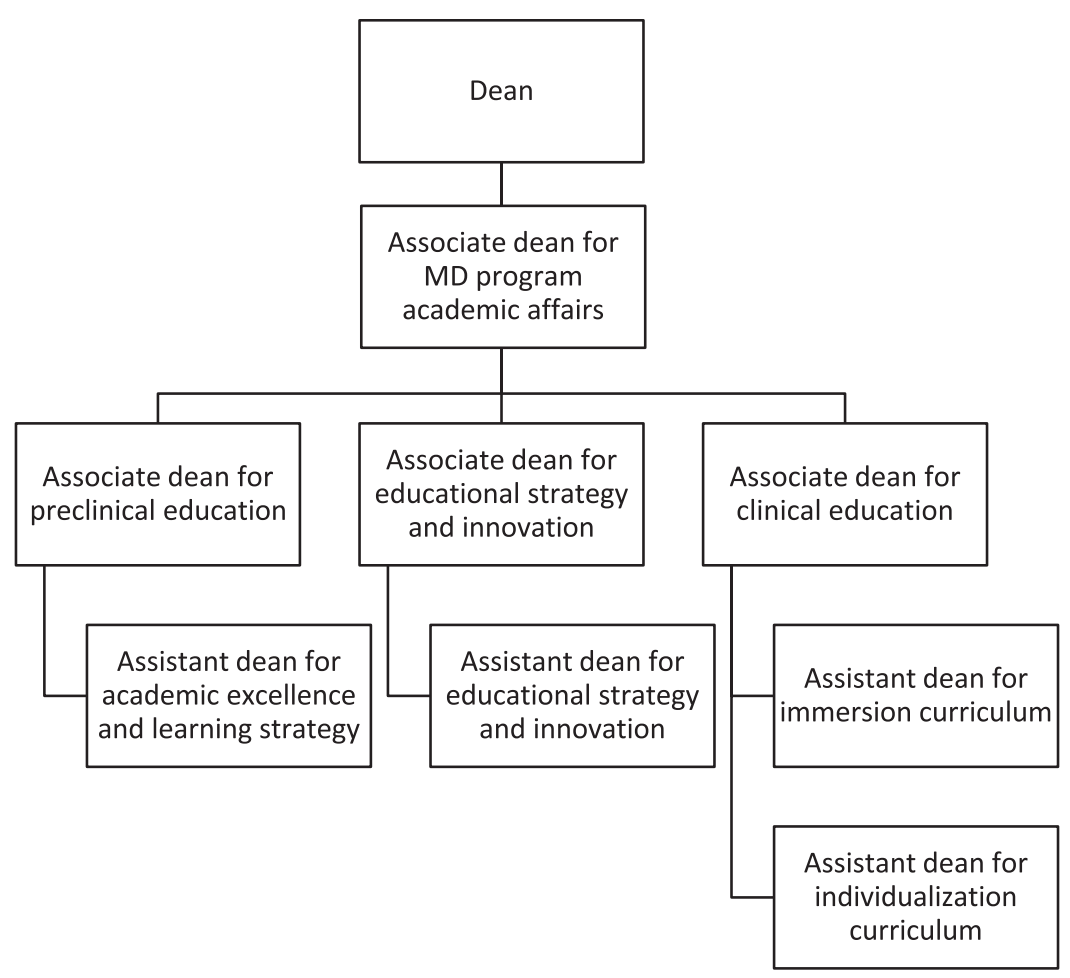

Figure 2 Educational staff.

The "FacDev4me" Podcast Series provides brief interviewstyle episodes on a wide range of topics pertinent to faculty in a health professions education setting. The series is available through RSS feed, on Apple podcasts, or via the podcast web page. A summary outline for each episode is available for download, along with links to resources mentioned. Some of the topics covered include tips for teaching millennials, tips on being a peer reviewer, tips on working with an instructional designer, how to engage multilevel learner groups in the clinical setting, and turning your teaching into scholarship. Four annual scholarships are awarded to attend AAMC conferences: the Early-Career and Mid-Career Women Faculty Leadership Development Seminars, the Mid-Career Minority Faculty Leadership Seminar, and the Minority Faculty Leadership Development Seminar.

WFSM seeks to recruit, retain, and promote faculty of the highest caliber. Faculty may be appointed, reappointed, and/ or promoted in 1 of 2 separate tracks, the tenure-eligible track or the nontenure-eligible (fixed term) track with the following academic tracks: research scholar track, educator scholar track, clinician scholar track, and clinician expert track. We have recently created a clinical faculty pathway. This pathway is a nontenure-eligible faculty classification for physicians who contribute to the mission of our health system through independent medical practice, teaching, and the recurrent direct supervision of patient care provided by medical students, resident physicians, and other health care learners.
The educator scholar track is a nontenure-eligible track for clinical and basic science faculty who spend a majority of their effort and scholarly work in educational activities, including pedagogy, education practice, educational research, and/or education administration. Faculty members on this track are expected to have terminal degrees in their field (e.g., $\mathrm{MD}, \mathrm{PhD}$, EdD). Educational scholarship is valued beyond the activities of high-quality teaching generally required of all faculty members. Scholarly expectations include innovative educational models and practices and a high number of scholarly publications in highly regarded journals.

The clinician scholar track is a nontenure-eligible track for faculty members who contribute to the clinical mission of the institution and whose scholarly activities and publications directly relate to clinical practice or innovation, clinical research, and/or clinical education. Faculty members on this track have clinical responsibilities, may have extramural grant funding, and have substantial evidence of peer-reviewed publications (or equivalent scholarly dissemination). Innovations related to clinical patient care, clinical research, and/or educational scholarship beyond the high-quality teaching that is required of all faculty members are valued.

For promotion, faculty must create a teaching portfolio. This portfolio includes teaching evaluations combined with any supplemental materials they wish to add that highlight their teaching and educational accomplishments. Teaching evaluations or other objective evidence of teaching performance they have 
received for intramural and extramural presentations and activities are included. It is highly recommended to include evaluations from multiple different learner groups (e.g., medical students, graduate students, resident physicians, fellows, practicing physicians, etc.), particularly for faculty on the clinical faculty pathway. Examples of teaching materials they created and used in their educational activities are included. These may include items such as course syllabi, lecture outlines, small-group cases, and other curricular materials.

WFSM also offers the Health Professions Education Institute (HPEI) to support educators, which is a collaborative initiative sponsored by Academic Affairs, Faculty Affairs, and the Continuing Medical Education (CME) Committee. HPEI provides a series of CME activities to help faculty learn contemporary adult education theory and methods to achieve optimal learning outcomes for their trainees. HPEI also contributes to the research mission of the institution. HPEI features a series of programs that provide faculty, staff, and trainees with career development opportunities in medical education.

The Lifelong Learning Series includes education grand rounds and education workshops. These events share best practices in health professions education, provide a forum to disseminate educational scholarship, and promote networking and collaboration across the training programs, including the MD Program, Physician Assistant Program, Pharmacy Program, CRNA Program, and nursing education. The education grand rounds are a CME-approved quarterly lecture series designed to disseminate new health professions education information and results of research; provide a forum for discussion of new techniques and approaches to health professions education; and build networks of educators, clinicians, researchers, and trainees across the health system. The education workshops are often spin-offs of education grand rounds topics and provide an opportunity for grand rounds attendees to "continue the conversation" in a more in-depth development opportunity.

The half-day Annual Medical Education Conference brings together educators from WFSM's health professions training programs. Teaching faculty, graduate educators, and course and program directors are invited to submit abstracts for poster and oral abstract platform presentations that showcase medical education research, curricular innovation, and works in progress. These abstracts are peer reviewed and published on the WakeHealth.edu HPEI page. Excellence in Education Scholarship awards are presented to faculty, staff, student, resident, fellow, or other trainees who demonstrate excellence in dissemination of education scholarship. 


\title{
University of North Dakota School of Medicine and Health Sciences
}

\author{
Marc D. Basson, MD, PhD, MBA, Patrick Carr, PhD, Richard N. Van Eck, PhD, Susan Zelewski, MD, and Adrienne Salentiny, PhD
}

\section{Medical Education Program Highlights}

The University of North Dakota (UND) School of Medicine and Health Sciences (SMHS) is the only medical school in North Dakota. We are a 4-year community-based medical school leading to the MD degree. The preclerkship phase in Grand Forks covers the first 2 years of the program. Third- and fourth-year students are based on 1 of 4 campuses (Grand Forks, Minot, Fargo, Bismarck) and rotate through rural sites. We emphasize skills to provide care for rural and Native American communities. Highlights include:

- The SMHS employs a hybrid problem-based learning (PBL) and team-based learning (TBL) preclerkship curriculum emphasizing small-group, patient-focused learning.

- Preclinical interprofessional education involving psychology, communication sciences, medicine, nursing, dietetics, occupational therapy, physical therapy, and social work is emphasized. In clerkships, students translate interprofessional concepts through experiences such as the Interprofessional Student Community-Based Learning Experiences program.

- Our new (2016) 325,000-square-foot, 4-story SMHS building emphasizes progressive educational infrastructure, including 8 interprofessional learning communities: student-led, noncurricular shared spaces where students from each of the 9 SMHS programs practice clinical skills and interact.

- A state-of-the-art simulation facility is used.

- The Indians Into Medicine Program (INMED) is a national program to help American Indian students meet tribal community needs. INMED provides placements for fully qualified students and offers support, advising, a learning resource center, financial assistance, and tutoring.

- Member of the American Medical Association's Accelerating Change in Medical Education Consortium from 2015 to present. Innovative projects include longitudinal interprofessional continuity of care, rural telemedicine simulations (350+ students in 5 professions), a handbook for designing these simulations, and badging for medical education competencies including interprofessional teamwork and wellness.

See Supplemental Digital Appendix 1-Curriculum Map as of 2019—at http://links.lww.com/ACADMED/A823.

Acad Med. 2020;95:S391-S395.

doi: 10.1097/ACM.0000000000003268

Copyright (C) 2020 by the Association of American Medical Colleges

Supplemental digital content for this article is available at http://links.lww.com/ ACADMED/A823.

Year school was established: 1905

School URL: https://med.und.edu.

\section{Curriculum}

Curriculum changes since 2010

- Program goals and objectives for undergraduate medical education were revised to reflect moves toward competencybased education and expectations of leadership organizations in medical education.

- Developmental, form-based standardized assessment and longitudinal tracking of student leadership behaviors in the firstyear patient-centered learning (PCL) experience were added.

- Weekly "Professor Rounds" convene all students on each clerkship at each campus to practice patient presentation and differential diagnosis.

- Acting internships help students achieve predefined levels of competence in Entrustable Professional Activities (EPAs).

- Integrating USMLE-purchased standardized exams into assessment has increased student exposure to board-style questions and helps gauge student readiness to progress.

- Integrating faculty-selected primary literature into patientcentered TBL cases teaches appraisal of basic, translational, and clinical research.

- Emotional, physical, occupational, and financial wellness activities have been incorporated.

- A second longitudinal clerkship track was added.

A substantially revised curriculum will include earlier clinical electives, compress the preclinical experience, integrate basic and clinical sciences, increase active learning, and strengthen USMLE preparation. Incremental changes are being incorporated with full transition to the new curriculum in 2021.

See Supplemental Digital Appendix 2-Proposed Curriculum to Take Effect in 2021—at http://links.lww.com/ACADMED/A823.

\section{Changes in class size since 2010}

Class size has increased from 66 to 78 , facilitated by a statesupported Healthcare Workforce Initiative (started in 2011) partially aimed at training more practitioners, which also expanded postgraduate residency/fellowship programs. Our new building was constructed to accommodate this increased class. The low student/faculty ratio remains unchanged.

\section{Assessment}

In 2017, we replaced our program goals and objectives with program competencies drawn from national sources and the SMHS mission to "educate physicians ... to enhance the quality of life in North Dakota." Assessments include clinical performance rating/ checklists, institutionally developed clinical performance exams, institutionally developed laboratory practical exams, institutionally developed written/computer-based exams, licensure clinical performance exams, licensure written/computer-based exams, oral presentation, participation, peer assessment, portfolio-based assessment, research or project assessment, and self-assessment. 
See Supplemental Digital Appendix 3-Medical Education Program Competencies and Assessment Methods—at http:// links.lww.com/ACADMED/A823.

The most substantial assessment changes since 2010 are described below. We began using the Comprehensive Basic Science Exam (CBSE) as formative testing after each instructional unit to expose students to NBME-style questions, better track performance, identify early intervention opportunities, and refine curriculum. In the final 2 units of Phase I, the CBSE serves as a "gateway" to Step 1 during the third year.

In 2016, we modified the communication and teamwork skills assessment of interprofessional collaboration to measure telemedicine competency during interprofessional education simulations and to measure interprofessional skills in telemedicine. We use this tool to measure performance during 3 longitudinal continuity-of-care interprofessional simulations every year across 5 disciplines.

In 2017, we identified the need for additional assessment of self-directed learning skills, professionalism, and personal and professional development. The curriculum promotes these outcomes through PCL, a form of PBL in which students rotate case leadership over 64 cases. An observational assessment form ${ }^{1}$ facilitates faculty assessment and student feedback. Students use this feedback to generate future PCL goals. By the 16th case, students are competent to lead future PCL groups.

In 2016, we instituted weekly assessment of students' skills in case presentation, differential diagnosis, and developing therapeutic plans during the clinical phase. ${ }^{2}$ Specially trained faculty use a modified version of a tool developed by Lewin and colleagues. ${ }^{3}$ In 2017, we reconstituted our acting internships to focus on the EPAs, using a modified Ottawa scale ${ }^{4}$ for evaluation.

\section{Parallel curriculum or tracks}

We offer 2 parallel tracks for clerkship completion in addition to our standard curriculum.

MILE (Minot Integrated Longitudinal Experience) consists of 44-week longitudinal clerkships in family medicine, internal medicine, obstetrics-gynecology, pediatrics, psychiatry, and surgery. Students also spend $25 \%$ of their time in a neurology longitudinal curriculum. Students spend 1 week on each clerkship over the first 6 weeks to get to know the clinic, preceptors, and staff in each specialty. The other 4 weeks of the academic year are spent in a rural location to complete 4 weeks of family medicine to fulfill the UND SMHS rural rotation requirement. MILE allows students to experience continuity of care and longer-term preceptor relationships for ongoing formative feedback.

ROME (Rural Opportunities in Medical Education) sends students for 20-28 weeks to a rural site where they complete family medicine and fulfill requirements for half of their internal medicine, obstetrics-gynecology, and pediatrics clerkships. Students on ROME sites for 24-28 weeks have surgical preceptors, and complete half or all of their surgery clerkship depending on experience. ROME students complete neurology and psychiatry and the remainder of internal medicine, obstetrics-gynecology, pediatrics, and surgery at 1 of 3 traditional home campuses. ROME allows students to experience rural medicine, continuity of care, and long-term preceptor relationships.

\section{Pedagogy}

We employ case-based learning, small- and large-group discussion, laboratory, lecture, peer teaching, TBL, PBL, role play/dramatization, self-directed learning/tutorial, video/ podcast, simulation, standardized/simulated patients, and ambulatory and inpatient clinical experience. Since 2010, we have increased self-directed learning and TBL.

\section{Clinical experiences}

Students begin clinical experiences in week 1 via standardized patients and simulations. Additional clinical exposure occurs in weekly case wrap-ups during years 1 and 2 PCL and TBL. Required experiences at clinical sites currently begin in year 3 , but all students will begin clerkships midway through the second year and will participate in clinical electives as early as the end of year 1 starting in fall 2021.

Required clinical educational experiences are completed in a regional campus model. This includes major health systems, the Fargo Veterans Administration, and rural sites in North Dakota and Minnesota. All students complete at least 4 weeks at a rural site. The MILE curriculum is completed in Minot other than a 4 -week period at a rural family medicine site. In ROME, students complete 20-28 weeks at a rural site and the remainder of the year on the Bismarck, Fargo, or Grand Forks regional campus.

Although ROME and MILE represent elective longitudinal tracks, all students undertake 2 formal longitudinal experiences during their clinical years regardless of track: a third-year longitudinal epidemiology course that includes a research project, performed in pairs, and a fourth-year longitudinal patient safety quality improvement curriculum that includes 10 online modules and a safety/QI proposal.

\section{Curricular Governance}

The dean is the chief academic officer of SMHS. Faculty governs the curriculum through the faculty governance structure. Since SMHS offers the MD degree and 7 allied health, public health, and $\mathrm{PhD}$ degrees, the Faculty Advisory Council (FAC) representing all programs has traditionally reviewed medical curricular actions. Through planned streamlining, individual degree-granting programs will be governed by committees limited to that program. Overall issues such as promotion and tenure will continue to be governed by all SMHS through FAC. A proposed new governance structure is targeted for 2020 adoption.

See Figure 1-Current governance structure.

See Figure 2-Proposed new governance structure. 


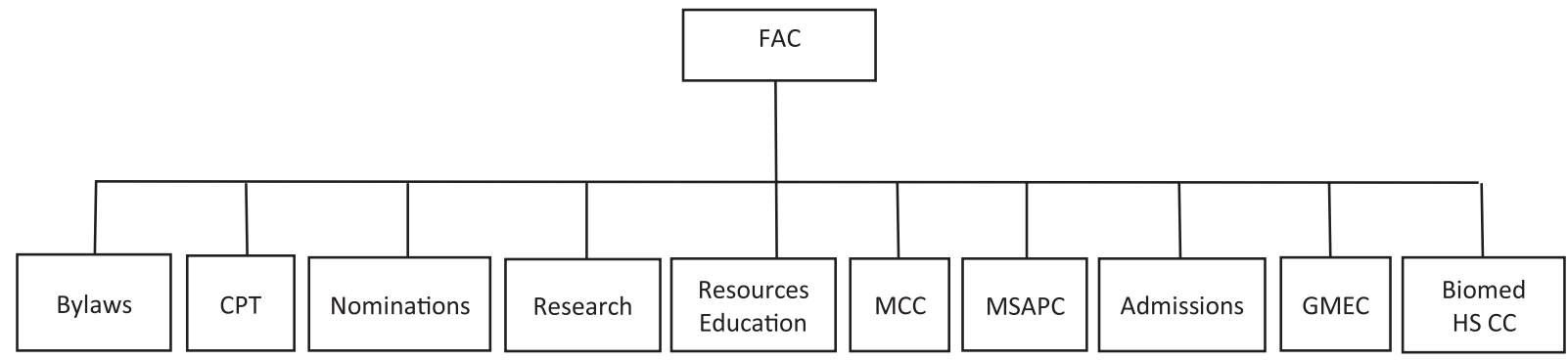

Figure 1 Current governance structure. FAC: Faculty Academic Council. Standing Committees: Bylaws Committee; Committee on Promotion and Tenure (CPT); Nominating Committee (Nominations); Research Committee; Committee for Resources for Education (Resources Education); Medical Curriculum Committee (MCC); Medical Student Academic Performance Committee (MSAPC); Admissions Committee; Graduate Medical Education Committee (GMEC); Biomedical and Health Sciences Curriculum Committee (Biomed HS CC).

Preclinical curricular governance is centralized. Clinical curricular governance is shared between departments and the curriculum committee. Department chairs manage courses in the standard curriculum, overseeing statewide course directors and individual campus-level course directors. MILE and ROME are governed by central boards where each relevant department is represented. All departments report about their courses to the curriculum committee, which has final oversight.

\section{Education Staff}

The dean has ultimate authority over the medical program. The senior associate dean for medicine and research provides direct oversight. Other associate and assistant deans provide additional support and oversight, including undergraduate medical education, faculty development, and learner support. Education Resources, overseen by the associate dean for faculty affairs, provides academic support for the medical program and the school's other degree-granting programs. Nineteen Education Resources faculty and staff assist curriculum committees through administration, implementation, and management of the medical program in addition to providing assessment and instructional design expertise.

See Supplemental Digital Appendix 4-Organizational Chartat http://links.lww.com/ACADMED/A823.

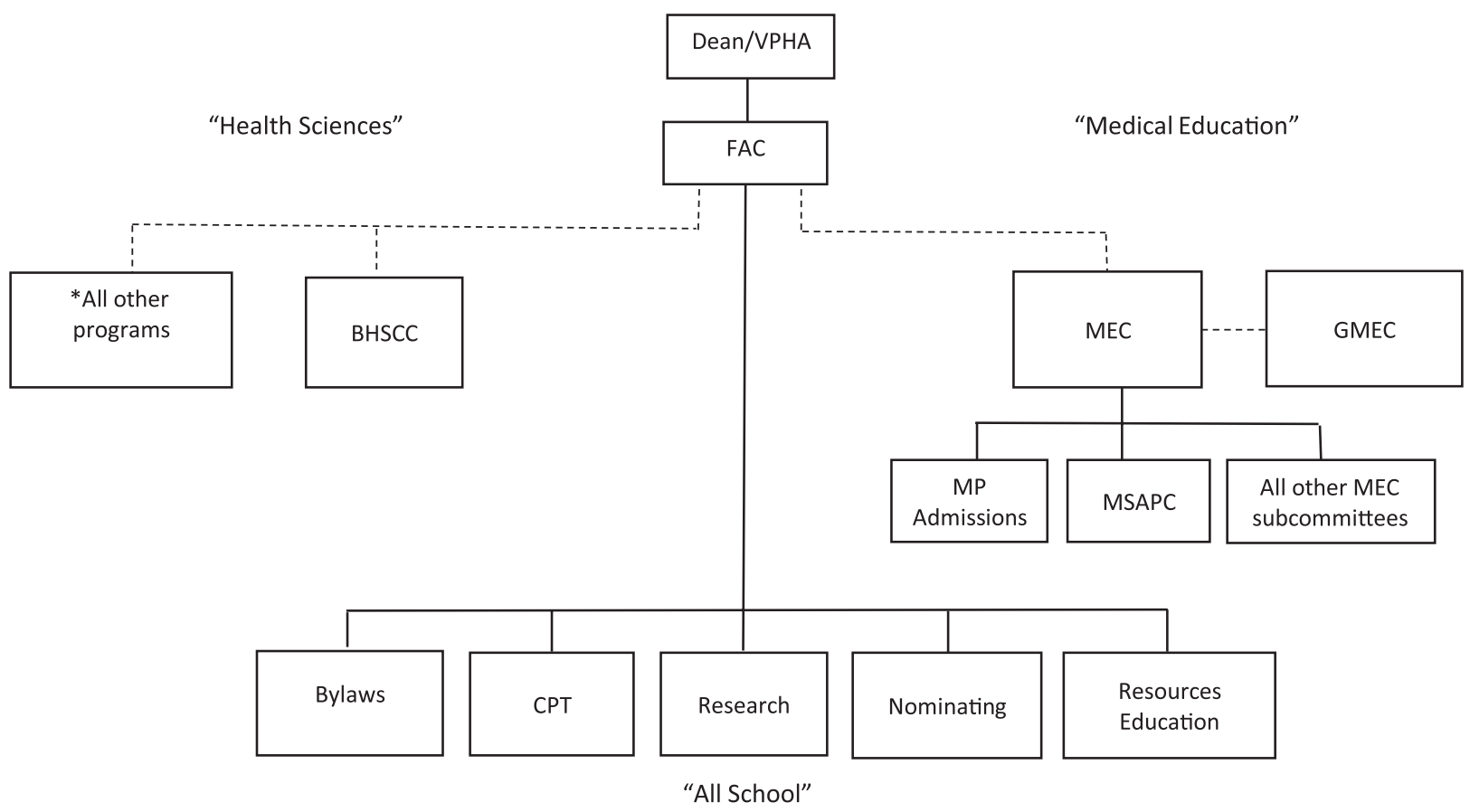

Figure 2 Proposed new governance structure. Faculty Academic Council (FAC); Biomedical and Health Sciences Curriculum Committee (BHSCC); Medical Education Committee (MEC); Graduate Medical Education Committee (GMEC); MP Admissions, Medical Student Academic Performance Committee (MSAPC); Bylaws Committee, Committee on Promotion and Tenure (CPT); Research Committee; Nominating Committee; Committee for Resources for Education (Resources Education). 
The role of the primary medical education staff and administration is educational programming for undergraduate medical education. The senior associate dean for medicine and research manages graduate and continuing medical education, respectively, using different staff.

\section{Faculty Development and Support in Education}

Excellence in teaching is integral to promotion and tenure. SMHS provides a wide range of faculty development and support. The associate dean for faculty affairs oversees SMHS faculty development, emphasizing promotion and tenure. The associate dean for teaching and learning oversees SMHS faculty development initiatives, emphasizing teaching, innovation, education scholarship, and curriculum design and evaluation. An instructional designer provides faculty development and support regarding curriculum design, education scholarship, teaching and assessment, and technology-based learning tools. The senior associate dean for medicine and research is focused particularly on developing the clinical faculty. The clinical department chairs and statewide clerkship directors, under leadership of the senior associate dean for medicine and research, provide more specialized feedback and faculty development for clinical faculty. The assistant dean for medical education focuses on faculty development for the medical curriculum.

Chairs can direct faculty to meet with an instructional designer for remediation, including individual consultation, recommended workshops, teaching observation, and analysis of teaching materials and documentation. Faculty can also seek professional development from Education Resources.

Faculty development programming is advertised by emails, e-newsletter, website, digital signage, and lobby kiosks. Sessions are livestreamed and recorded. Individual consultations are available in person, by phone, or via conferencing software. Key leadership travels to the regional campuses.

See List 1-Formal Faculty Development Sessions at the SMHS Grand Forks Campus Over One Year.

\section{Regional Medical Campuses}

To maintain an equivalent educational experience across the 4 regional campuses, performance by campus is monitored by each department and the curriculum committee. Campus deans meet regularly to standardize solutions across campuses for nonclerkship educational experiences.

See Table 1-Type and Enrollment for Regional Campuses.

Each required clerkship has a statewide clerkship director who coordinates campus clerkship directors to ensure consistent educational experience. The senior associate dean for medicine and research meets regularly with statewide clerkship directors, and separately with campus deans, and with department chairs, to further assure consistency of educational experience across sites and clerkships. Two faculty from the Department

\section{List 1}

Formal Faculty Development Sessions at the SMHS Grand Forks Campus Over One Year

Topic

- Strategies to promote student success

- Measuring development of competencies over time

- Use of concept maps to synthesize knowledge

- Game-based learning in medical education

- Teaching critical thinking

- Research poster design (part 1 of 3)

- Research poster design (part 2 of 3)

- Interprofessional education

- Research poster design (part 3 of 3)

- Universal design and accessibility

- Converting to active learning

- Curricular design: Classifying outcomes

- Curricular design: Writing objectives

- Book study: "Make It Stick: The Science of Successful Learning" (session 1)

- Book study: "Make It Stick: The Science of Successful Learning" (session 2)

- Book study: "Make It Stick: The Science of Successful Learning" (session 3)

- Faculty lecture on improvement of teaching

- How to use asynchronous methods

- Small-Group Peer- and Self-Evaluations Reveal Behaviors Correlated with Learning Outcomes

- Book study: "Telling Ain't Training" (session 1)

- Book study: "Telling Ain't Training" (session 2)

- Book study: "Telling Ain't Training" (session 3)

\section{Table 1}

Type and Enrollment for Regional Campuses

\begin{tabular}{lllr}
$\begin{array}{l}\text { Regional } \\
\text { campus name }\end{array}$ & Type & Year & $\begin{array}{r}\text { Student } \\
\text { enrollment }\end{array}$ \\
Bismarck & Clinical & $\begin{array}{l}\text { 3rd year } \\
\text { \#th year }\end{array}$ & 22 \\
\hline Fargo & Clinical & 3rd year & $19-21$ \\
\hline Grand Forks & 4th year & 33 \\
& $\begin{array}{l}\text { Combination } \\
\text { preclerkship }\end{array}$ & 1st year & $19-21$ \\
\hline and clinical & 2nd year & 78 \\
& & 3rd year & 78 \\
\hline Minot & 4th year & 14 \\
& Clinical & 3rd year & $19-21$ \\
\hline
\end{tabular}


of Family and Community Medicine coordinate rural site education and meet regularly with students and preceptors at each rural site.

The chairs also monitor student experiences at rural sites by meeting with each student either in person onsite or by teleconference. Finally, each clerkship reports annually by campus overall grades, shelf exam performance, preceptor scores, and completion of required clinical encounters to the curriculum committee, which addresses discrepancies. Feedback such as the AAMC Graduation Questionnaire is also reviewed separately by campus.

\section{References}

1 Center for Excellence in Teaching, Learning, and Assessment. Rubric for evaluating small group facilitators. http://www.cetla.howard.edu/ teaching_strategies/facilitating_small_groups/docs/4-26-12\%20 Rubric\%20for\%20Evaluating\%20 Small\%20Group\%20Facilitators.pdf. Accessed February 25, 2020.

2 Lancaster I, Basson MD. What skills do clinical evaluators value most in oral case presentations? Teach Learn Med. 2019;31:129-135.

3 Lewin LO, Beraho L, Dolan S, Millstein L, Bowman D. Interrater reliability of an oral case presentation rating tool in a pediatric clerkship. Teach Learn Med. 2013;25:31-38.

4 Gofton WT, Dudek NL, Wood TJ, Balaa F, Hamstra SJ. The Ottawa Surgical Competency Operating Room Evaluation (O-SCORE): A tool to assess surgical competence. Acad Med. 2012;87:1401-1407. 


\section{Case Western Reserve University School of Medicine, Including the Cleveland Clinic Lerner College of Medicine}

Patricia A. Thomas, MD, Amy L. Wilson-Delfosse, PhD, Neil Mehta, MD, Klara K. Papp, PhD, S. Beth Bierer, PhD, and J. Harry Isaacson, MD

\section{Medical Education Program Highlights}

Case Western Reserve University (CWRU) School of Medicine (SOM) has 2 collaborative MD degree programs: the University Program, established in 1843 as the Western Reserve School of Medicine, and the Cleveland Clinic Lerner College of Medicine (College) program, launched in 2002.

- The University program's 4-year curriculum, Western Reserve2, merges the disciplines of public health and medicine into a single integrated program, with a goal to prepare students to function effectively in collaborative teams and interprofessional settings. A 4-month research experience and MD thesis are required for graduation.

- The 5-year College Physician Investigators program is designed to train graduates to become physician investigators and scientists committed to the advancement of biomedical research and clinical practice. During the fourth year, students complete a master's-level thesis and graduate with an MD degree with special qualification in biomedical research.

- Students can experience 4 unique health systems in Cleveland.

- Five pathways (Wellness and Preventive Medicine, Urban Health, Humanities, Health Care Innovation and Technology, and World Medicine) offer enhanced curricular activities to students with a focused interest.

- Students in both programs experience a longitudinal curriculum in interprofessional education (IPE) and collaborative practice in the newly designed interprofessional Health Education Campus.

\section{Curriculum}

Curriculum description

University program:

- The Western Reserve2 University program curriculum begins with a preclerkship phase of 20 months, the Foundations of Medicine and Health, delivered in 6 blocks, that integrate basic, clinical, and health systems science education. Three longitudinal blocks, Structure (anatomy, histopathology, radiology), Foundations of Clinical Medicine (doctoring), and Systems and Scholarship (research and health systems science skills) run through this phase (http://casemed.case.edu/curriculum).

- The average week has 20 hours of formal instruction to enable self-directed learning.

Acad Med. 2020;95:S396-S401.

doi: 10.1097/ACM.0000000000003411

Copyright (C) 2020 by the Association of American Medical Colleges

Year school was established: 1843.

School URL: https://case.edu/medicine.
- In the 48-week clerkship phase, students elect a disciplinebased block structure or 1 of 2 longitudinal clerkship models.

College program:

- The College program begins in year 1 with a basic and translational research block and consists of the foundational Molecular Medicine course, basic science research project, and basic science journal club, followed by sequential organ-system blocks, with an emphasis on normal.

- Year 2 begins with a clinical research block consisting of a clinical research project and clinical research journal club as well as courses in clinical epidemiology and medical biostatistics, followed by organ systems with emphasis on abnormal.

- A theme is chosen for each week of the curriculum, which forms the organization for problem-based learning groups, seminars, and the Advanced Research in Medicine seminar.

- Foundations of clinical medicine and longitudinal clinic occur in both years. Students are assigned a longitudinal preceptor for clinical skills development with every other week half day clinic sessions beginning in year 1 .

- Advanced Research in Medicine and Art and Practice of Medicine courses are presented longitudinally in all 5 years.

- All College program students are enrolled in the Cleveland Clinic longitudinal clerkship.

- Students participate in a basic/translational research project in the summer before year 1 , and a clinical research project in the summer before year 2, and then a full year of research in year 4 .

- Year 5 includes 2 acting internships and a capstone course to prepare for residency.

\section{Curriculum changes since 2010}

- Students in both programs participate in the Foundation in Interprofessional and Collaborative Practice course using a hybrid online and small-group format over the first year. Participants include CWRU dental medicine, nursing, physician assistant, and graduate social work students.

- Anatomy education in both programs has moved pedagogy from traditional reliance on dissection and prosection to a multimodal approach that incorporates augmented reality, virtual reality, Sectra tables, radiologic imaging, and point-ofcare ultrasound, in addition to cadaveric dissection.

University program:

- Responding to grant initiatives in IPE and collaborative practice, team skills have been incorporated as part of the small-group process and assessment.

- A longitudinal course in the preclerkship phase, Systems and Scholarship, introduces the skills and tools for research, as well as health systems sciences of population health, quality improvement, and community engagement. 
- Core clerkship-year students return to the Health Education Campus once a week for Science and Art of Medicine Integrated, case-based, small-group learning that integrates basic, clinical, and health systems sciences into clinical practice.

College program:

- Year 4 Capstone

- Longitudinal didactics in health systems science

- Increased content in professional identity formation

- IPE in longitudinal clinic

\section{Class size changes}

- In anticipation of the move to a larger campus, the University program increased its incoming class to 185 students per year. This required additional IQ small groups in the preclerkship phase and prompted the development of longitudinal clerkship models to increase capacity in the core clerkship year.

- The College program values and retains a small class size of 32 students per year.

\section{Assessment}

- Our joint medical education program objectives are based upon the ACGME's 6 domains of competence, with the additions of interprofessional collaboration, personal and professional development, and research and scholarship.

- Both programs use frequent formative assessments and rely heavily on portfolio systems for students to demonstrate achievement of competence.

See Table 1-Program Objectives and Assessment Methods.

University program:

- Enhanced assessment of health systems sciences

- Frequent low-stakes formative assessments to assess students' mastery of the material. High-stakes summative assessments are infrequent; essay questions to assess student mastery.

- "Remediation" for lapses in professionalism has shifted to a coaching model.

College program:

- Moved from performance standards to milestones, revised annually

- Enhanced ePortfolio system used to document students' progress in 9 competencies of the portfolio-based, programmatic assessment system

- Revised assessment forms to ensure alignment with milestones; no grades for completed courses or clerkships

- Increased students' curricular experiences and assessments in the areas of patient safety and continuous quality improvement

- Expanded faculty development activities in the areas of direct observation, interactive teaching, coaching, competency-based assessment, and written feedback.

\section{Pedagogy}

Similar pedagogical methods are used in both programs.

See Table 2-Pedagogical Methods.

\section{Clinical experiences}

- Students in both programs have early patient encounters within the first weeks of the curriculum.

The SOM is affiliated with 4 health systems in Cleveland, which provide both inpatient and ambulatory sites:

- University Hospitals-Cleveland Medical Center

- VA Northeast Ohio Healthcare System

- The Cleveland Clinic

- MetroHealth Medical Center

\section{Required longitudinal experiences}

- In the University program, year 1 electives are available to provide students longitudinal experiences working with community patients and populations.

- The College program requires the longitudinal clinic in years 1 and 2, a 12-week longitudinal ambulatory block in the year 3 Longitudinal Clerkship, and a longitudinal weekly half day clinic during year 4 .

- University program students can elect:

- Cleveland Clinic Longitudinal Clerkship or

- MetroHealth Medical Center Longitudinal Integrated Clerkship, designed as a yearlong longitudinal ambulatory experiences with 2-week integrated discipline-based hospital rotations.

\section{Required and elective community-based rotations}

- University program students are required to complete a 10week community-based primary care clerkship (CPCP) in either spring of year 1 or summer or fall of year 2 .

- Community-based electives available to students include semester-long and yearlong experiences as patient navigators in the VA Center of Excellence in Primary Care practice, or at a PCMH refugee clinic in Cleveland; working with interprofessional students and elders in the community in the Aging in Place elective; and working on clinical quality improvement initiatives in interprofessional teams.

\section{Curricular Governance}

The Committee on Medical Education is a committee of the CWRU SOM faculty and provides overall governance of the MD degree. Neither the University nor College program has departmental-level courses in the preclerkship curriculum and all budget and academic support remains centralized at the program level. All faculty with dean titles are funded for educational effort centrally. Clinical departments budget for clinical faculty teaching effort.

See Figure 1-Curricular governance.

\section{Education Staff}

\section{Medical education leadership}

University program:

- The vice dean for medical education oversees all administrative offices for the MD degree program. 


\section{Table 1}

Program Objectives and Assessment Methods

\begin{tabular}{l}
$\begin{array}{l}\text { Competencies and program } \\
\text { objectives }\end{array}$ \\
$\begin{array}{ll}\text { Research and scholarship } & \\
\begin{array}{l}\text { Analyses and effectively critiques a a } \\
\text { broad range of research papers. }\end{array} & \begin{array}{c}\text { Journal club preceptor } \\
\text { assessment } \\
\text { Research preceptor assessment }\end{array} \\
\begin{array}{l}\text { Demonstrates ability to generate a } \\
\text { research hypothesis and formulate }\end{array} & \text { MD thesis evaluation } \\
\text { questions to test the hypothesis. } & \\
\text { Demonstrates ability to initiate, } \\
\text { complete, and explain his/her research. }\end{array}$ \\
\hline
\end{tabular}

\begin{tabular}{|c|c|}
\hline Knowledge for practice & \\
\hline $\begin{array}{l}\text { Demonstrates ability to apply } \\
\text { knowledge base to clinical and } \\
\text { research questions. } \\
\text { Demonstrates appropriate level of } \\
\text { clinical, basic, and health systems } \\
\text { science knowledge to be an } \\
\text { effective starting resident physician. }\end{array}$ & $\begin{array}{l}\text { Synthesis essay question responses } \\
\text { CAPPS question responses } \\
\text { Seminar case narrative preceptor } \\
\text { assessment } \\
\text { Oral presentation assessments } \\
\text { CPCP preceptor assessment } \\
\text { Longitudinal clinic preceptor } \\
\text { assessments } \\
\text { Clerkship clinical assessments } \\
\text { NBME Health Systems } \\
\text { examination }\end{array}$ \\
\hline
\end{tabular}

\section{Interpersonal and communication skills}

\begin{tabular}{|c|c|}
\hline $\begin{array}{l}\text { Uses effective written and oral } \\
\text { communication in clinical, research, } \\
\text { and classroom settings. }\end{array}$ & $\begin{array}{l}\text { Simulated patient checklists } \\
\text { Video reviews of simulated } \\
\text { encounters }\end{array}$ \\
\hline $\begin{array}{l}\text { Effectively communicates } \\
\text { knowledge as well as uncertainties. }\end{array}$ & $\begin{array}{l}\text { Reflective papers } \\
\text { Longitudinal clinic preceptor } \\
\text { assessments }\end{array}$ \\
\hline $\begin{array}{l}\text { Demonstrates effective } \\
\text { communication with patients using } \\
\text { a patient-centered approach. }\end{array}$ & $\begin{array}{l}\text { CPCP preceptor assessments } \\
\text { Clinical skills exams } \\
\text { (years } 2 \text { and } 3 \text { ) } \\
\text { Clinical assessment forms in } \\
\text { clinical rotations }\end{array}$ \\
\hline
\end{tabular}

\section{Professionalism}

Consistently demonstrates compassion, respect, honesty, and ethical practices.

Meets obligations in a reliable and timely manner.

Recognizes and addresses lapses in professional behavior.
Narrative facilitator assessments

Preceptor assessments

Clinical assessment forms

Reflective essays

\section{Personal and professional development \\ Critically reflects on personal values, priorities, and limitations to develop strategies that promote Narrative facilitator assessments Small-group assessments} personal and professional growth.

Identifies challenges between personal and professional responsibilities and develops strategies to address them.

Recognizes when personal views and values differ from those of patients, colleagues, and other caregivers and reflects on how these can affect patient care and research.
Table 1

(Continued)

\begin{tabular}{|c|c|}
\hline $\begin{array}{l}\text { Competencies and program } \\
\text { objectives }\end{array}$ & Assessments \\
\hline \multicolumn{2}{|l|}{ Patient care } \\
\hline $\begin{array}{l}\text { Obtains thorough and accurate } \\
\text { information through an H\&P } \\
\text { adapting to the clinical setting. }\end{array}$ & $\begin{array}{l}\text { Preceptor assessments } \\
\text { Clinical assessment forms } \\
\text { Clinical skills exams }\end{array}$ \\
\hline $\begin{array}{l}\text { Uses evidence from the patient's } \\
\text { history, physical exam, and other } \\
\text { data sources to formulate and } \\
\text { prioritize clinical decisions. }\end{array}$ & \\
\hline \multicolumn{2}{|l|}{$\begin{array}{l}\text { Incorporates diagnostic, therapeutic, } \\
\text { and prognostic uncertainty in clinical } \\
\text { decision-making and patient care } \\
\text { discussions. }\end{array}$} \\
\hline \multicolumn{2}{|l|}{$\begin{array}{l}\text { Incorporates patient perspective, } \\
\text { values, and goals into all aspects of } \\
\text { the clinical encounter. }\end{array}$} \\
\hline $\begin{array}{l}\text { Identifies and critically analyses } \\
\text { relevant literature and practice-based } \\
\text { guidelines to apply best evidence of } \\
\text { patient care and management. }\end{array}$ & \\
\hline
\end{tabular}

\section{Teamwork and interprofessional collaboration}

Performs effectively as a member Clinical skills assessment forms of a team.

Direct observation of team skills

Respects and supports the Self-assessments of team skills

contributions of individuals on an interprofessional health care team to deliver quality care.

\section{Systems-based practice}

Applies knowledge of health care Clinical skills assessment forms systems to patient care discussions. SOAP-V cases in clinical skills

Demonstrates awareness of context of care, patients' values, and health care system resources in clinical decision-making. exams

Quality improvement project NBME Health Systems Science examination

Applies principles of quality improvement and safety to patient care.

\section{Reflective practice}

Demonstrates habits of ongoing reflection using feedback from others as well as self-assessments to both identify learning needs (cognitive and emotional) and practice continuous quality improvement.

- The associate dean for curriculum, with the director of office of curricular affairs, leads faculty and staff tasked with planning, implementation, and coordination of curriculum delivery, monitoring, and management.

- The associate dean for assessment and CQI and 3 staff members design and implement assessment and program evaluation.
Reflective writing plans

Portfolio assignments
Preparation of personal learning 
- The assistant dean for educational technology supervises the administrative and academic computing team.

College program:

- The executive dean of the Cleveland Clinic Lerner College of Medicine oversees all administrative offices of the MD degree.

- The associate dean for curricular affairs chairs the College Program's Curriculum Steering Council, which oversees the development, implementation, and evaluation of the College program curriculum and assessment policies and procedures.

- The director of assessment and evaluation is responsible for maintaining the integrity of the College LL portfolio-based assessment system and for evaluating and continuously improving the program's curriculum.

Role of the primary medical education staff and administrative faculty in the educational program:

- The vice dean for medical education is responsible for all UME MD programming, the MSc in Physician Assistant Studies, and the MSc in Anesthesia degrees, and the Office of Continuing Medical Education.

- The assistant dean for medical education in the Office of Graduate Education has oversight for all pre-UME and graduate-level programs in the SOM.
- GME is directed by designated institutional officials and Offices of GME in the respective clinical affiliates.

- Each of the clinical affiliates also maintains a health systembased office of Continuous Professional Development.

See Figure 2-Organization of education deans.

\section{Department of Medical Education}

The vice dean for medical education is director of the Center for Medical Education, which appoints all part-time teaching faculty who do not otherwise hold departmental appointments, as well as all physician assistant program faculty and other allied health professional teaching faculty. This center resides within the Division of General Medical Sciences in the SOM; the dean serves as department chair for the division.

\section{Faculty Development and Support in Education}

\section{Professional development for faculty as educators}

- The SOM vice dean for faculty development is responsible for faculty development within the medical school. This office supports a weekly faculty development program that includes teaching skills, career, and leadership development.

- The director of the Center for Advancement of Medical Learning is responsible for developing educators' skills in teaching,

\section{Table 2}

\section{Pedagogical Methods}

\begin{tabular}{|c|c|c|}
\hline Content & University program & College program \\
\hline $\begin{array}{l}\text { Preclerkship basic } \\
\text { science, health } \\
\text { systems sciences }\end{array}$ & $\begin{array}{l}\text { - Inquiry-based 9-person small groups } \\
\text { - Self-directed study } \\
\text { - Weekly feedback }\end{array}$ & $\begin{array}{l}\text { - PBL 8-person small groups with faculty facilitator } \\
\text { - Self-directed study }\end{array}$ \\
\hline $\begin{array}{l}\text { Supplemental } \\
\text { methods }\end{array}$ & $\begin{array}{l}\text { - Team-based learning } \\
\text { - Interactive lectures }\end{array}$ & - Small-group seminars \\
\hline Anatomy & $\begin{array}{l}\text { - 2-week cadaveric dissection followed by } \\
\text { longitudinal curriculum in preclerkship phase }\end{array}$ & - Fresh-frozen cadaveric prosections in longitudinal curriculum \\
\hline $\begin{array}{l}\text { Supplemental } \\
\text { anatomy methods }\end{array}$ & $\begin{array}{l}\text { - Augmented reality } \\
\text { - Radiologic imaging } \\
\text { - Point-of-care ultrasound }\end{array}$ & $\begin{array}{l}\text { - Rirtual reality } \\
\text { - Rectra tables } \\
\text { - Point-of-care ultrasound }\end{array}$ \\
\hline Doctoring course & $\begin{array}{l}\text { - Discussions } \\
\text { - Satient panel presentations } \\
\text { - Role play }\end{array}$ & $\begin{array}{l}\text { - Seminars } \\
\text { - Role play/dramatizations }\end{array}$ \\
\hline $\begin{array}{l}\text { Early patient experience } \\
\text { (years } 1 \text { and } 2 \text { ) }\end{array}$ & - Community-based primary care clerkship & - Longitudinal clinic 1 half day every other week \\
\hline Core clinical year & $\begin{array}{l}\text { Block discipline-based rotations or option of } \\
2 \text { longitudinal clerkships }\end{array}$ & $\begin{array}{l}\text { - Longitudinal clerkship year with } 12 \text {-week longitudinal ambulatory block, } \\
\text { integrating pediatrics, internal medicine, family medicine, women's } \\
\text { health, and palliative care } \\
\text { - Additional hospital-based, team-based care blocks in inpatient medicine }\end{array}$ \\
\hline Research & $\begin{array}{l}\text { - Systems and Scholarship course introduces } \\
\text { research methods in years } 1 \text { and } 2 \\
\text { - 4-month block in year } 3 \text { with required thesis }\end{array}$ & $\begin{array}{l}\text { - Advanced Research Methods course presented in all } 5 \text { years } \\
\text { - Basic/translational science laboratory experience in year } 1 \text {, clinical science } \\
\text { research experience in year } 2 \text {, and research year with required thesis }\end{array}$ \\
\hline
\end{tabular}




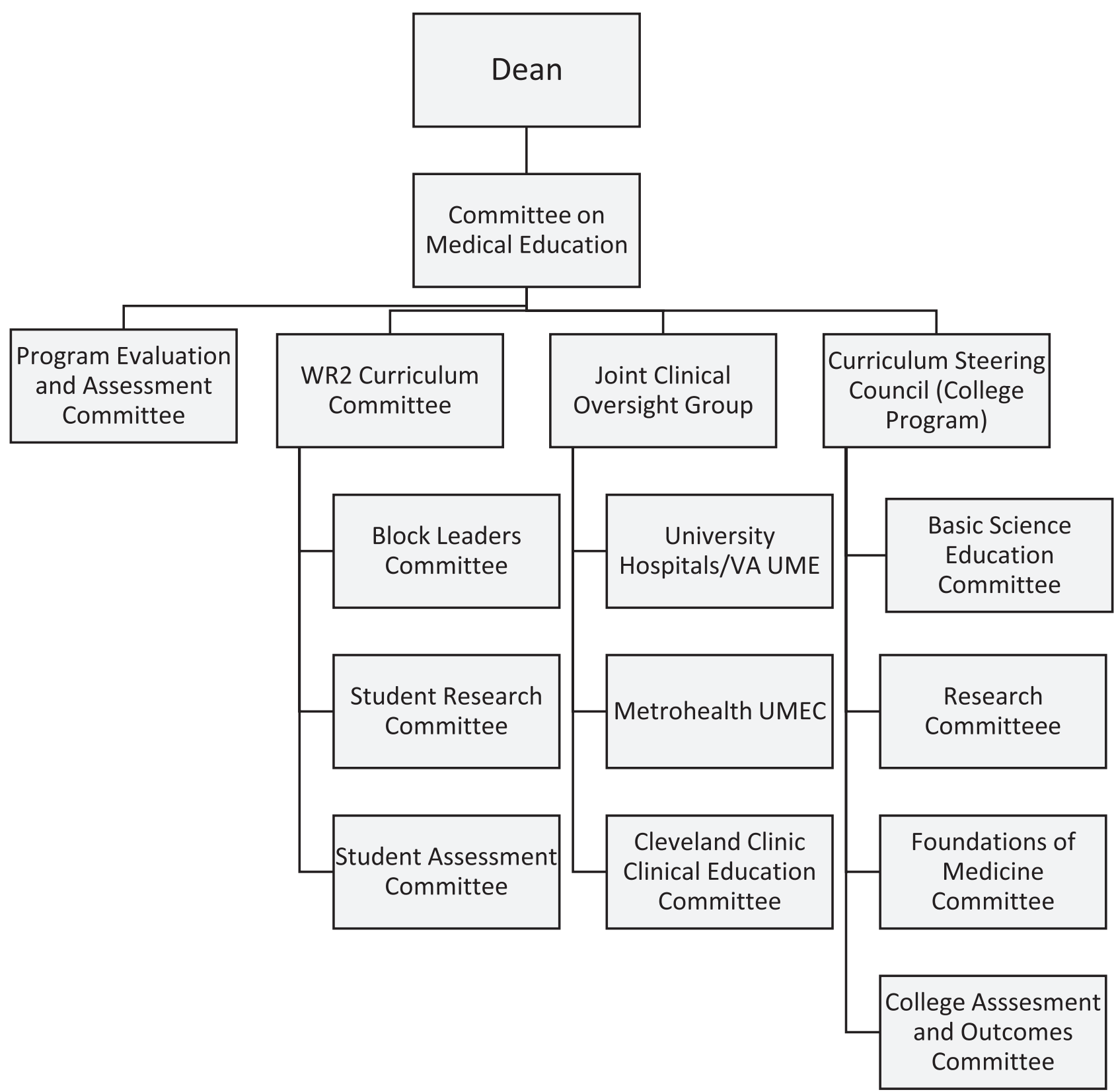

Figure 1 Curricular governance.

curriculum development, assessment, and evaluation, and the professional growth of faculty, resident, and student educators.

- The director of the Center for Educational Resources in the College program and the Cleveland Clinic Education Institute (CCEI) works closely with College program leadership regarding issues pertaining to faculty development.

- The CCEI has partnered with Cleveland State University to offer a Master of Health Professions Education, available to interprofessional teaching faculty.

- The CWRU SOM Academy of Scholar Educators (https:// case.edu/medicine/faculty-staff/academy-scholar-educators) was formally organized in 2017 to recognize educational excellence and build a service community of scholar educators within the SOM. Membership is determined through a selection process that includes an educator's portfolio and nominations.

\section{Role of teaching in promotion and tenure}

- Faculty are awarded promotion in the nontenure track based onevidence of scholarship as well as regional and national reputation in 2 of 3 areas: research, service, and education. Faculty are encouraged to submit an educator's portfolio as evidence of scholarship and impact if seeking promotion based on educational effort. 


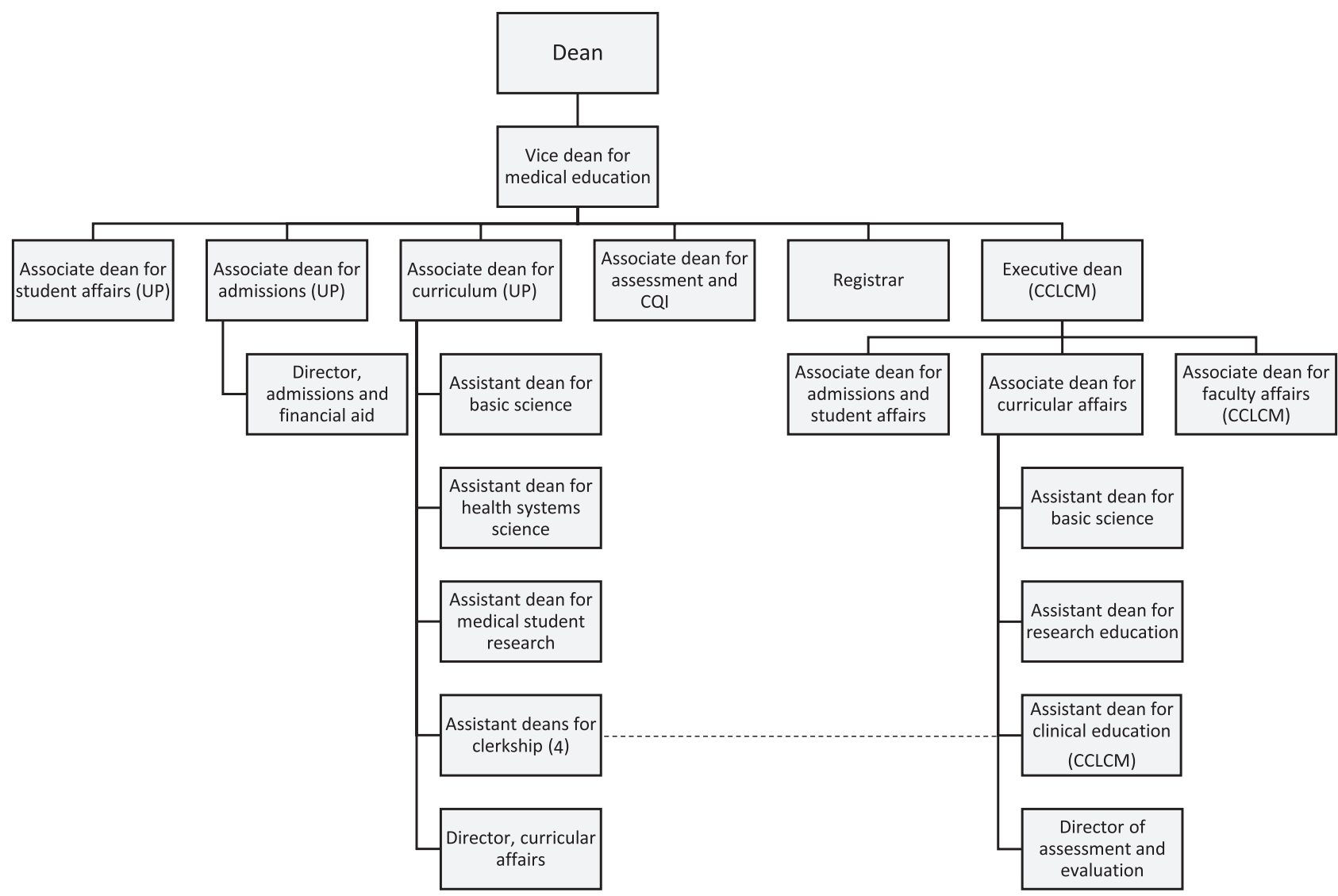

Figure 2 Organization of education deans.

- Tenure at CWRU is granted solely on the basis of research productivity.

\section{Initiatives in Progress}

- New curricular initiatives in IPE and collaborative practice will expand interprofessional teams in community-based activities with our CWRU health professions students.
- Big data and artificial intelligence are being integrated into the curriculum.

- Climate change content will be added in both programs.

- Building on popularity of the pathway, wellness and preventive medicine will be fully incorporated into the curriculum for all students. 


\section{The Ohio State University College of Medicine}

Jennifer W. McCallister, MD, and Daniel M. Clinchot, MD

\section{Medical Education Program Highlights}

- The Lead.Serve.Inspire (LSI) curriculum incorporates innovative longitudinal projects such as the Community Health Education Project to meet the education program objectives. Students work collaboratively in teams to partner with a community health organization to identify a specific need within the population and implement and study an intervention to address it.

- The Ohio State University has developed specific and intentional initiatives to support the well-being of its students. The MINDSTRONG program ${ }^{1}$ provides cognitive behavioral skills-building techniques to enhance resiliency and selfprotection of participants.

- Faculty Development for Medical Educators (FD4ME) is an interactive, online faculty development program that delivers a wide range of peer-reviewed topics in medical education.

\section{Curriculum}

\section{Curriculum description}

LSI is a competency-based curriculum developed to meet the evolving needs of students and educators in a rapidly changing educational environment. By integrating foundational science concepts with early clinical experiences, incorporating flexible instructional methods and targeted assessment strategies, the curriculum fosters critical thinking and prepares students to provide patient-centered care in diverse settings. It includes 3 parts of study over 4 years: Part 1 (Clinical Foundations, 18 months), Part 2 (Clinical Applications, 12 months), and Part 3 (Advanced Clinical Management, 12 months).

Part 1 allows students to build a strong foundation of biomedical, behavioral, and clinical sciences while developing the knowledge, skills, and attitudes needed to function as a physician in clinical settings. Material is delivered in 8 multisystem integrated curricular blocks with unifying content threads running longitudinally throughout (e.g., anatomy, diversity and inclusion, pharmacology, scientific inquiry).

See Supplemental Digital Appendix 1-Part 1, Clinical Foundations_-at http://links.lww.com/ACADMED/A835.

Acad Med. 2020;95:S402-S405.

doi: 10.1097/ACM.0000000000003302

Copyright (C) 2020 by the Association of American Medical Colleges

Supplemental digital content for this article is available at http://links.Iww.com/ ACADMED/A835.

Year school was established: 1914.

School URL: https://medicine.osu.edu.
Students participate in weekly longitudinal group (LG) sessions where they develop skills required for relationshipcentered care. Through longitudinal relationships with faculty, students cultivate skills in history and physical examination, clinical reasoning, and health coaching. They build upon these experiences with biweekly longitudinal practice sessions where they join clinical sites to hone the skills introduced in LG through participation in patient care and integration into the health care team.

Professional development weeks are interspersed throughout Part 1, providing students with a focused career development curriculum and opportunities to explore a wide range of career paths early in training. One-week selectives allow students to explore areas of interest complementary to the learning objectives for Part 1.

Several longitudinal curricular components begin in Part 1. The Community Health Education Project introduces students to concepts of population-based medicine through service learning and provides an opportunity for interprofessional collaboration. The Educational Portfolio and Faculty Coaching Program uses an online portfolio to incorporate self-assessment through reflective writing. Faculty coaches provide feedback and support each student's self-directed learning and professional growth. The applied health systems science curriculum provides a foundation in the key concepts of high-quality care and process improvement.

Part 2 provides core clinical experiences to refine the knowledge, skills, and attitudes needed to interpret and apply knowledge while working with individual patients and patient populations. These core clinical immersions are delivered in three 16-week rings of integrated clinical content: understanding patients within populations (family medicine, pediatrics, geriatrics), understanding patients with reproductive and surgical needs (obstetricsgynecology, perioperative medicine, surgery, radiology), and understanding patients with specialized medical needs (internal medicine, neurology, psychiatry).

Each ring begins with a 1-week ground school that combines foundational knowledge with practical skills development in the clinical skills lab to prepare students for the specific clinical environment.

Students continue work in the Educational Portfolio Coaching Program and in applied health systems science.

Part 3 gives students advanced clinical training across a spectrum of patient care frameworks to acquire the ability to effectively manage patients within diverse health care systems.

- Advanced Management in Hospital Based Care (AMHBC) provides students with advanced experiences in an acute 
care setting. Students spend 4 weeks each in the emergency department and on a mini-internship.

- Advanced Management and Relationship Centered Care (AMRCC) provides 8 weeks of ambulatory setting advanced training at 2 selected sites.

- Students must complete at least four 4-week electives, one of which must be an advanced competency. The advanced competencies offer enhanced skill development in a domain identified as a priority for each student's advancement.

- Students must enroll in at least 1 clinical track in Part 3. These individualized educational plans provide a framework to prepare for internship in their chosen specialty. Students work toward entry-level residency milestones.

- Students complete their capstone project for applied health systems science and present a summative portfolio to a faculty committee during Part 3.

\section{Curriculum changes since 2010}

The LSI competency-based curriculum replaced the previous curriculum in the fall of 2012. Two learning pathways (the Integrated Pathway and the Independent Study Pathway) were replaced by this single curriculum, focusing on integrating foundational sciences with clinical care and longitudinal projects throughout.

- Med 1-2 was reorganized into multiorgan system blocks with horizontal content threads (Part 1, Clinical Foundations).

- The Clinical Analysis and Problem Solving Program for teaching foundational clinical skills was replaced with a combined program using LG and longitudinal practice. Students develop essential clinical skills early in Med 1 and learn to function as part of the health care team in an ambulatory setting.

- New longitudinal programs, including the Community Health Education Project, the Educational Portfolio and Coaching Program, and the Applied Health Systems Science Project, were added.

- Med 3 combined the existing 7 core clinical clerkships into 3 rings with integrated clinical content and educational sessions (Part 2, Clinical Applications). A clinical skills immersion experience (ground school) was introduced at the beginning of each ring.

- Med 4 was revised to include an emphasis on care of the acutely ill patient (AMHBC) and the chronically ill or ambulatory patient (AMRCC) in addition to focusing on individualized career development (Part 3, Advanced Clinical Management). The learning experiences were aligned to help students better prepare for residency, and specialty-specific clinical tracks were created. Advanced competency electives were added to enhance core skills.

- The LSI curriculum was designed to allow continuous content and delivery improvement. For example, with the recent opioid crisis, we were able to modify the curriculum to ensure that students were graduating with required skills.

\section{Assessment}

In February 2018, the Executive Curriculum Committee adopted the AAMC Physician Competency Reference Set as the basis of the medial education program objectives. ${ }^{2}$
See Supplemental Digital Appendix 2-Medical Education Program Objectives and Assessment Methods-at http://links. lww.com/ACADMED/A835.

\section{Assessment changes since 2010}

Since the implementation of LSI in 2012, there has been an increased emphasis on the use of multimodel competency-based assessments. Summative evaluations such as OSCEs, medical knowledge exams, and nationally normed/standardized content exams are administered during designated weeks. Expert faculty raters are trained to perform direct observations of competence and are available as resources for students who need remediation. Evaluations are cumulative and mastery based, providing clear evidence that each student has met competency.

\section{Parallel curriculum or tracks}

- The OSU Primary Care Track is an accelerated 3-year doctor of medicine program intended for students interested in a career in family medicine. The program offers early handson experiences through longitudinal, practice-based clinical encounters in addition to professional development.

- The Medical Scientist Training Program incorporates the LSI curriculum with a partnering graduate program (biomedical sciences, neuroscience, or biomedical engineering) to provide a rigorous curriculum in both medicine and research, facilitating the development of outstanding clinician-scientists.

\section{Pedagogy}

LSI delivers a curriculum that fully integrates foundational sciences with clinical practice.

Part 1 includes a variety of classroom and asynchronous methods that enable mastery of content. In-person experiences including lecture, small- and large-group discussion, case-based learning, team-based learning, and panel discussions are recorded when assessments or protected patient information are not included. Livestreaming and digital recording as well as self-directed learning allow students to prepare for in-classroom sessions and to determine how they learn most effectively. Laboratory sessions, simulation, and incorporation of standardized/ simulated patients supplement these approaches. The use of virtual patients and a longitudinal preceptorship provide an early introduction to patient care and application of foundational science principles in the clinical environment.

In Parts 2 and 3, ambulatory and in-patient clinical experiences integrate with simulation and self-directed learning as the primary instructional methods. Students participate in lectures, small-group discussions, case-based learning, and workshops as well as other activities such as specialty-specific conferences and journal clubs. Reflection, research, and independent learning provide opportunities for self-directed education.

The implementation of LSI provided an opportunity to focus on innovation and efficiency in teaching. The incorporation of high-fidelity simulation, digital classrooms, and virtual reality allows faculty to effectively deliver cutting-edge content. Faculty routinely use a flipped classroom design to incorporate 
team-based learning and small-group discussions, emphasize use of prosection over primary dissection in gross anatomy, and encourage reflection by students to make learning more efficient and engaging. Each area of content is offered in at least 2 formats to allow flexibility for student learning styles and preferences.

\section{Clinical experiences}

Students first encounter nonsimulated clinical experiences after 9 weeks of their first year as part of the Longitudinal Practice curriculum. They train in a variety of clinical environments: the large academic Ohio State University Wexner Medical Center, large metropolitan community hospital partners in the city, small community-based hospitals, and a large children's hospital. Ambulatory experiences are offered in a range of settings, including academic and community practices and the outpatient Veteran's Affairs clinic.

Required longitudinal clinical experiences include the LG and longitudinal practice in Part 1 and a subset of students who select a longitudinal ambulatory experience in AMRCC in Part 3.

\section{Curricular Governance}

The Executive Curriculum Committee is responsible for curriculum planning, implementation, evaluation, and oversight, with the management and coordination of the curriculum vested in the vice dean of education and associate dean of medical education.

See Figure 1-Curricular governance.

\section{Education Staff}

The Office of Medical Education provides academic and administrative support for the implementation and maintenance of the curriculum under the direction of the associate dean of medical education.

- Faculty positions required to administer the curriculum are supported centrally by the College of Medicine with release time for FTE \% based on a standard formula.

- Faculty teaching contributions are tracked via a centralized database so total teaching effort can be factored into the departmental budget allocation process annually.

- Staff support for academic program directors and budget support for implementation of the curriculum are administered through the Office of Medical Education and the Office of Curriculum and Scholarship.

- The offices of medical education, student life, research education, and admissions solely support undergraduate medical education. The offices for diversity and inclusion

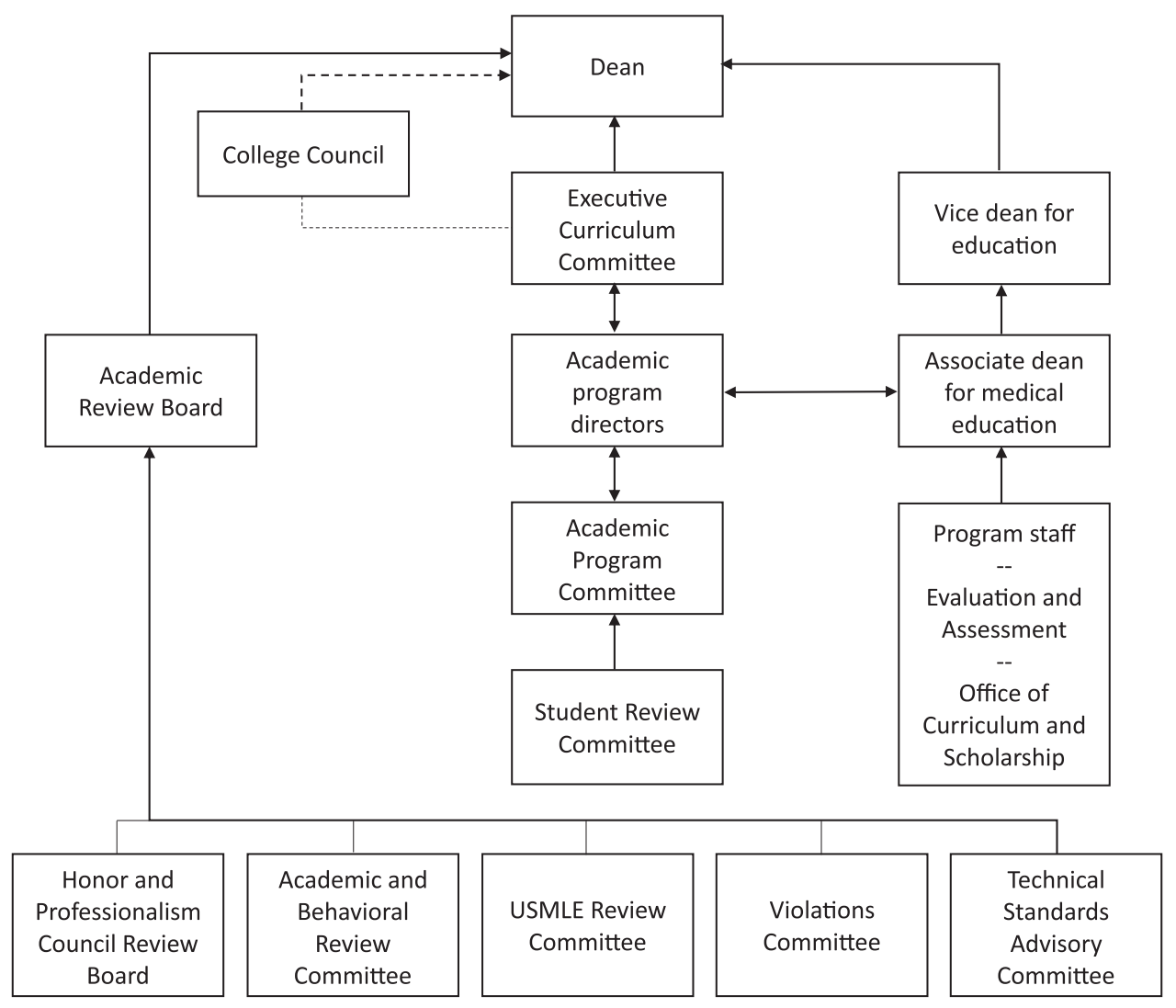

Figure 1 Curricular governance. 


\section{Dean
Vice president for health sciences}
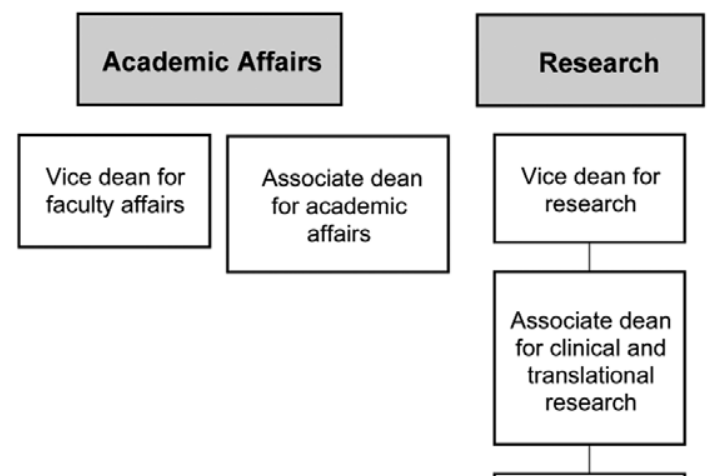

Associate dean for research

Figure $\mathbf{2}$ Organizational chart.

and curriculum and scholarship additionally support undergraduate and graduate medical education.

See Figure 2-Organizational chart.

\section{Faculty Development and Support in Education}

The Center for Faculty Advancement, Mentoring, and Engagement (FAME) provides comprehensive faculty career development for all domains of the academic medical center, including programming to develop faculty to be expert educators, leaders, clinicians, and scholars. The educator development programs in teaching/leadership and educational research support excellence and advancement for an annual cohort. Peer review of teaching is offered through FAME in all teaching venues. FD4ME, developed at Ohio State, is an online faculty development and continuing education platform for health professions educators. The College of Medicine partners with the College of Education and Human Ecology to offer a Master of Arts in Biomedical Education degree.

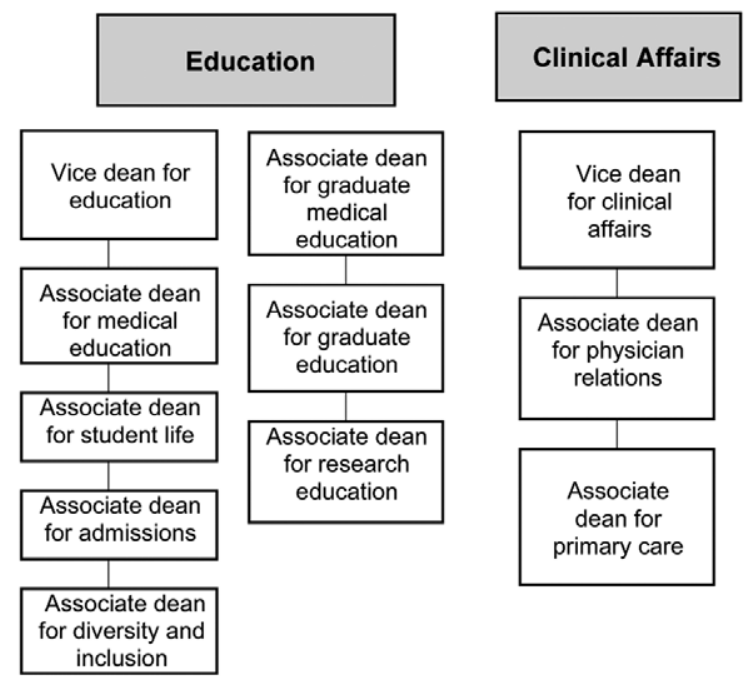

Faculty on the clinical (nontenure) track are eligible for promotion via 1 of 3 pathways: clinician-educator, clinicianscholar, and clinical excellence. Promotion of clinical faculty on the clinician-educator pathway is based upon convincing evidence that the candidate has developed the necessary level of leadership and recognition as a teacher. A distinctive record of teaching and mentoring excellence is required, demonstrated by exemplary learner, colleague, and national peer evaluations. Teaching awards and other honors are evidence of excellence. Candidates should demonstrate curricular innovation, new teaching modalities or evaluation methods, program or course development, and contributions to scholarship through peerreviewed publications or dissemination of work focusing on pedagogy of education or other related content.

\section{References}

1 The Ohio State University College of Nursing. MINDSTRONG. https:// nursing.osu.edu/offices-and-initiatives/mindstrong. Accessed March 5, 2020.

2 Englander R, Cameron T, Ballard AJ, Dodge J, Bull J, Aschenbrener CA. Toward a common taxonomy of competency domains for the health professions and competencies for physicians. Acad Med. 2013;88:1088-1094. 


\section{University of Cincinnati College of Medicine}

Pamela L. Baker, PhD, and Philip Diller, MD, PhD

\section{Medical Education Program Highlights}

- University of Cincinnati College of Medicine's (UCCOM's) location in an urban environment, serving a diverse and expanding patient population, is aligned with the university's strategic initiative Next Lives Here, which includes urban health as a priority.

- UCCOM has partnered with 7 local health care systems and over 150 primary care sites to provide students with a diverse and exceptional clinical learning environment.

- An integrated curriculum with early clinical exposure and enhanced curricular components such as a remodeled interprofessional curriculum in partnership with the College of Pharmacy, augmented self-directed learning (SDL) activities, and innovative use of analytics and dashboards to inform curricular improvements and promote student success. Key features include an ultrasound program, a longitudinal Healthcare Emergency Management (HCEM) course related to natural and man-made disasters, and global health electives.

- An outstanding student body that is increasingly diverse (49.7\% women; 23\% URM; 7\% LGBTQ+) and performs well on metrics such as USMLE examinations and success with the NRMP Match process.

\section{Curriculum}

See Supplemental Digital Appendix 1-Curriculum Map-at http://links.lww.com/ACADMED/A850.

\section{Curriculum changes since 2010}

- Students are provided significant opportunities for SDL experiences and receive feedback on their SDL skills, particularly in their first and second years.

- Beginning in 2018-2019, UCCOM partnered with the College of Pharmacy to create an interprofessional learning experience that includes simulation center activities partnering medical and pharmacy students and includes opportunities for medical students to shadow in a pharmacy setting.

- In 2017-2018, the college implemented an ultrasound curriculum in the first and second years to train students in a technology with increasing utility in point-of-care practice.

- In 2019-2020, the use of QR code technology was implemented to provide real-time formative feedback in the third year.
Acad Med. 2020;95:\$406-\$409.

doi: 10.1097/ACM.0000000000003325

Copyright (C) 2020 by the Association of American Medical Colleges

Supplemental digital content for this article is available at http://links.Iww.com/ ACADMED/A850.

Year school was established: 1819.

School URL: https://www.med.uc.edu.

\section{Class size changes since 2010}

- UCCOM medical student class size has had small incremental increases over the past 10 years, none of which changed the medical school's structure or function. Most recently, in 2018-2019, the class size increased from 175 to 180 .

\section{Assessment}

See Supplemental Digital Appendix 2-Program Objectives and Assessment Methods - at http://links.lww.com/ACADMED/ A850.

\section{Program objectives and competencies}

- In 2014, UCCOM adopted the 8 domains of the Physician Competency Reference Set as the overarching program objectives.

- The UCCOM program objectives are further aligned with the AAMC Core Entrustable Professional Activities (EPAs).

- As part of a 3-year cycle, UCCOM uses continuous quality improvement (CQI) to review the program objectives to ensure that we are appropriately teaching and assessing student learning.

\section{Changes in assessment}

- In 2011-2012, UCCOM implemented biweekly multiplechoice exams in foundational science courses.

- In 2011-2012, UCCOM implemented assessment of SOAP note-writing skills using faculty trained to provide feedback using a standardized rubric as part of the longitudinal primary care clerkship (LPCC) in the first and second years.

- In 2015-2016, UCCOM standardized the assessment strategy for third-year core clerkship rotations.

- In 2016-2017, UCCOM implemented the Assessment of Core Entrustable Professional Activities (ACEPA) OSCE, a comprehensive, simulation-based, EPA-aligned assessment that provides formative feedback to learners before beginning clerkships.

- In 2016-2017, UCCOM implemented assessment of ultrasound skills in years 1 and 2.

- In 2018-2019, UCCOM implemented an assessment of interprofessional skills based on an encounter with a pharmacy student in the simulation center using a self-reflection tool. In 2019-2020, pharmacy residents will provide narrative feedback on the encounter.

\section{Pedagogy}

UCCOM employs numerous pedagogical approaches to achieve our medical education program objectives, including:

- Case-based learning

- Clinical experience: ambulatory

- Clinical experience: inpatient

- Directed observation with checklists 
- Discussion: large group $(>12)$

- Discussion: small group $(\leq 12)$

- Flipped classroom

- Laboratory

- Lecture

- Peer teaching

- Preceptorship

- Problem-based learning

- Role play/dramatization

- SDL/tutorial

- Simulation

- Standardized/simulated patients

- Student self-reflections

- Tabletop exercises (i.e., group discussion around emergency planning, response, preparedness)

- Team-based learning

- Topic debates

- Video/podcast

- Virtual learning modules

- Virtual patient

- Workshop

- Other

UCCOM introduced the following changes to pedagogical approaches since 2010:

- Flipped classroom

- Scaffolded SDL activities

\section{Clinical experiences}

UCCOM uses 2 types of clinical sites for required educational experiences.

Inpatient:

- Community hospitals

- Level 1 Trauma Center

- Level 3 obstetrics-gynecology

- Mental health facilities

- Pediatric care centers

- Short- and long-term rehabilitation centers

- Veterans Administration hospital

Outpatient:

- Federally qualified health centers

- Mental health facilities

- Pediatric care centers

- Primary care facilities

\section{Required longitudinal experiences}

- First- and second-year students participate in the LPCC; gross anatomy and dissection, which is embedded in each organ system course; learning communities, which enroll a cohort of 12 students who meet weekly with a clinical faculty member; the Clinical Skills course; Physician and Society course; and the Principles and Application of Interprofessional Experiences courses.

- The only 4-year required longitudinal experience is HCEM, which engages students in the management of emergency medical situations and their role in emergency and disaster systems-based practice. Components include first responder, disaster preparedness, terrorism preparedness, and emergency medicine preparedness.

\section{Clinical experience first encounter}

- Clinical experiences start immediately following orientation with the First Responder component of the HCEM, which prepares students to handle a medical emergency, within or outside of a health care facility. In the fall term, students begin a clinical skills course where they practice fundamental patient-centered and interprofessional skills in a state-of-the-art simulation center. This is followed by the LPCC in the spring, which pairs a medical student with a community primary care preceptor where students learn patient care in a real-world setting.

\section{Required and elective community-based rotations}

- All students participate in the LPCC during the first and second years, in an ambulatory primary care setting. All required third-year rotations also include an ambulatory component.

- As part of required third-year clerkships, students complete rotations served by our 10 affiliate health care systems (e.g., Cincinnati Children's Hospital Medical Center, Department of Veteran's Affairs, The Christ Hospital).

\section{Challenges in designing and implementing clinical experiences} for medical students

- UCCOM is fortunate to be located in a rich environment with 7 local health care systems with which we have partnered to provide clinical experiences for our students. UCCOM also has the advantage of being part of an academic health center. To better navigate placement of learners, UCCOM hired a program manager in 2017-2018 for this role.

- UCCOM maintains strong relationships with the health care systems as well as our primary care partners and continues to pursue opportunities to expand clinical sites.

\section{Curricular Governance}

- The Cincinnati Medicine Education Committee structure guides and makes decisions concerning medical education in alignment with the UCCOM program objectives and LCME standards.

- Our committee structure provides opportunities for continuous improvement, collaboration, innovation, and scholarly activities. Key strengths include: entrusts faculty, encourages collaboration across all UCCOM medical education programs, encourages interprofessional collaborations, and solicits student input.

- The Education Program Committee (EPC) reviews and approves all UCCOM curricula as well as new and updated educational initiatives, monitors the learning environment, reviews the educational program performance, and identifies opportunities for further innovation. EPC reports to the College of Medicine Council.

- Three standing subcommittees report to EPC: Years 1 and 2 Curriculum Committee, Years 3 and 4 Curriculum Committee, and the Clinical Core Competencies Team, all of which 
comprise course/clerkship/elective directors, faculty-at-large, staff, and medical students.

- The 3 subcommittee chairs serve on the EPC and report at each EPC meeting.

The dean's office provides funding at the department level for administrative time (e.g., clerkship/elective director), teaching effort, and coordinator support.

\section{Education Staff}

\section{Medical education leadership}

The Office of Medical Education (OME) is responsible for the planning, implementation, evaluation, and oversight of the curriculum and for the development and maintenance of the tools to support curriculum delivery, monitoring, and management. Staff to support these efforts include the following:

- Associate deans for medical education (2)

- Assistant dean for medical education, assessment, and evaluation

- Director of medical education

- Director of first- and second-year curriculum

- Director of third- and fourth-year curriculum

- Director of intersessions

- Director of electives

- Director of ACEPA

- Data warehouser/data analyst

- Program director, Leo

- Program manager, evaluation and classroom support

- Program director, third and fourth year
- Program director, chief proctor/performance and advancement committees

- Program manager, interprofessional experiences and learning communities

- Program manager, support to the associate and assistant deans

- Student assistant

See Figure 1-Organizational structure.

OME is solely responsible for management of the curriculum and, in addition to educational programming, has full oversight of the CQI process. OME also oversees the annual Medical Education Retreat, which includes the Offices of Students Affairs; Admissions and Special Programs; and Diversity, Equity, and Inclusion.

\section{Department of Medical Education}

The Department of Medical Education (DME) is the academic home to faculty with select primary or secondary appointments who teach in undergraduate or graduate medical education. DME full-time faculty include individuals with primary teaching responsibilities within the first and second years. DME also includes the Body Donation Program.

\section{Faculty Development and Support in Education}

\section{Professional development for faculty as educators}

- UCCOM provides multiple opportunities for faculty to improve their knowledge, skills, and abilities in the assessment of teaching and learning through centralized resources of the Office of Faculty Affairs and Development (OFAD) and OME.

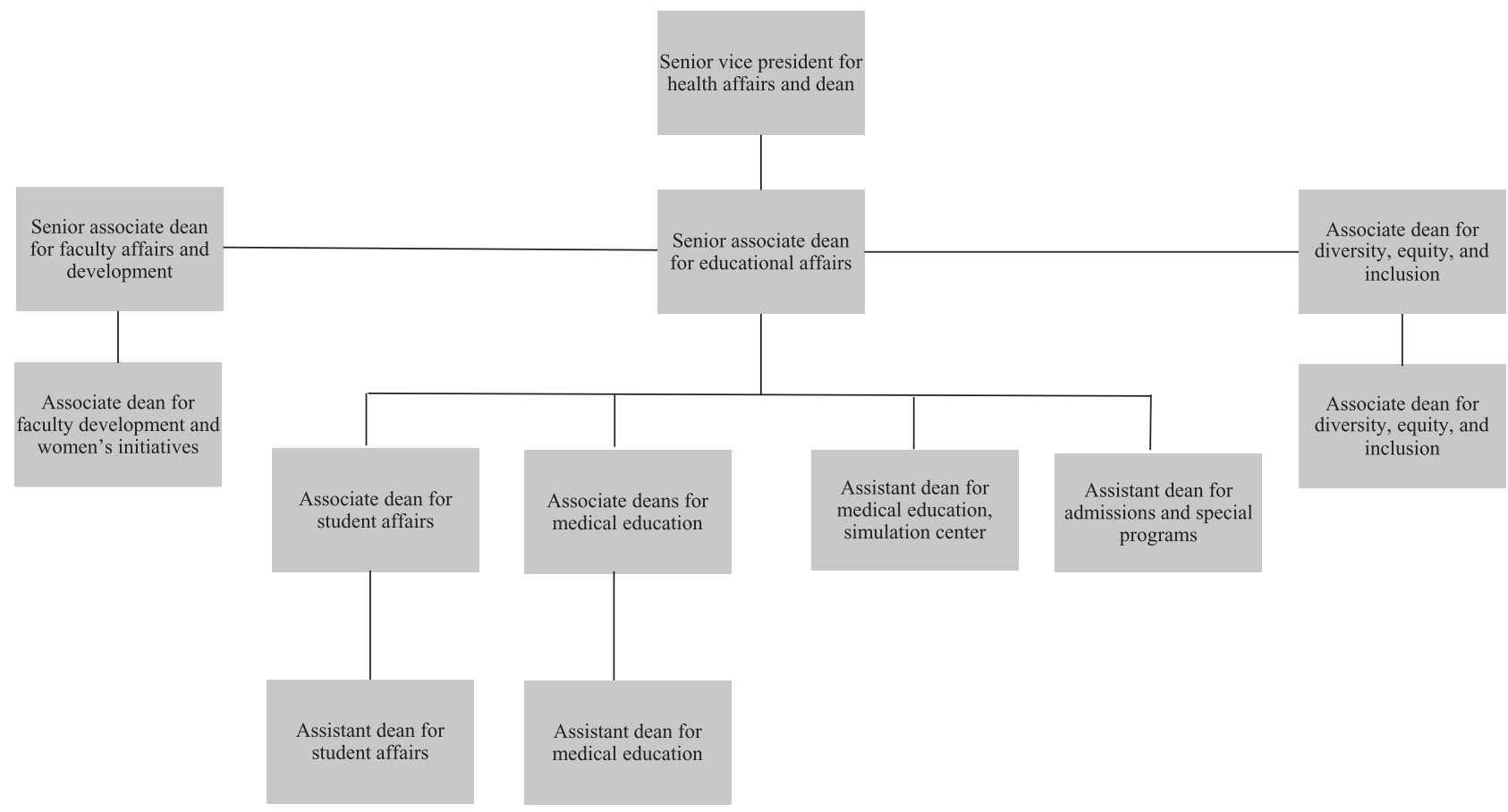

Figure 1 Organizational structure. 
Faculty can also use university resources such as UC's Center for the Enhancement of Teaching and Learning and its Center for Excellence in eLearning.

- OME provides the teaching and learning series offered through OFAD. Monthly workshops are offered on topics ranging from evaluation and assessment to providing constructive feedback to learners in a clinical setting. OME staff also meet with course/clerkship directors to provide individualized support in improving teaching and assessment skills. OME also sponsors the webinar series offered through the International Association of Medical Science Educators.

\section{Role of teaching in promotion and tenure decisions}

- For promotion and tenure decisions, description of teaching/ mentoring activities; evaluations from students, residents, and peers; and/or description of administrative activities regarding teaching (e.g., development and organization of new and innovative teaching or evaluation methods, directorship of a clerkship or residency program, participation in institutional or national education committees) are considered as part of the annual performance evaluation and appointment, reappointment, promotion, and tenure (ARPT) decisions.

- UCCOM also offers an educator track (nontenure) in both the basic science and clinical departments that adheres to the UCCOM guidelines for ARPT.

\section{Initiatives in Progress}

UCCOM is working to:

- Increase use of data analytics and dashboards to inform curricular improvements and monitor individual student performance.

- Explore the revision of the curriculum to a 3-phase model.

- Plan for an Interprofessional Simulation Center.

- Develop an AAMC Project Medical Education event.

- Explore the possibility of creating an institute/academy for educators. 


\title{
Wright State University Boonshoft School of Medicine
}

\author{
Brenda Roman, MD, Colleen Hayden, EdD, and Irina Overman, MD
}

\section{Medical Education Program Highlights}

The WrightCurriculum at Boonshoft School of Medicine (BSOM) is a lecture-free curriculum designed using teaching and learning strategies that are evidence based in the science of learning. Our goal is that students will become self-directed learners with skills in critical thinking and evidence-based practice. In the Foundations of Clinical Practice phase (20 months), students master assigned content outside the classroom so that time with faculty is highly interactive, averaging about 15 hours a week, and is devoted to applying content by answering high-order-thinking questions about biomedical concepts and clinical application. Faculty teaching becomes focused on probing for deeper understanding.

Retrieval-based learning, a highly effective learning strategy, is used in peer instruction (PI) sessions and team-based learning (TBL), while WrightQ, our form of problem-based learning, primarily uses elaborative interrogation and self-explanation. Our curriculum was designed using interleaving and spaced repetition-all of these strategies have evidence for improved learning. Both TBL and WrightQ take place in small collaborative groups, which our students report as being engaging, helpful, and fun.

The clerkships and electives of the Doctoring phase provide the learning for clinical skills while reinforcing the integration of biomedical and clinical sciences. Throughout the 2 phases of the curriculum, which also include the Clinical Medicine and Scholarship in Medicine courses, extracurricular courses such as Healer's Art, and service learning, our students build a set of professional skills and attitudes. The capacity to listen generously to patients and their families, conduct a comprehensive or focused physical examination, work collaboratively with other health care professionals, keep current with the medical literature, and make clinical decisions that are ethical and evidence based are some of the characteristics of humanistic physicians. Besides preparing students for success on national licensing examinations, the WrightCurriculum is delivered in the context of a supportive and collaborative learning environment, and fosters the personal and professional growth of its students.

Acad Med. 2020;95:\$410-\$413.

doi: 10.1097/ACM.0000000000003313

Copyright (C) 2020 by the Association of American Medical Colleges

Supplemental digital content for this article is available at http://links.Iww.com/ ACADMED/A839.

Year school was established: 1973.

School URL: https://medicine.wright.edu.

\section{Curriculum}

\section{Curriculum description}

See Supplemental Digital Appendix 1-4-Year Curriculum Schema-at http://links.lww.com/ACADMED/A839.

\section{Curriculum changes since 2010}

Over the last decade, we increasingly replaced lectures with TBL, PI, and WrightQ. The WrightCurriculum debuted as a lecturefree curriculum in 2017 using those 3 main teaching modalities, as well as increased use of simulation and standardized patient sessions. We are the first medical school in the United States to extensively use PI, adapted from Eric Mazur. In PI, classroom time is devoted to solving problems that are presented as questions about specific case vignettes and/or diagnostic or therapeutic interventions, and out-of-class time is for mastering core content. Students use audience response devices to answer faculty-generated questions, first as individuals, and then again after consulting with a peer nearby who may have a different answer choice. Faculty-led teaching moments clarify student misconceptions.

The "Q" in WrightQ is about learning how to ask the right questions - ones that lead to understanding of the principles of pathophysiology underlying disease and making informed, evidence-based decisions, including developing a differential diagnosis. Working in small groups with a faculty facilitator, students investigate a clinical case; determine what information is needed to understand what is going on; then proceed independently to learn that information to come back; and, with the group, share, explain, and decide on what are the possible diagnoses and best therapeutic approach for the patient.

\section{Class size changes since 2010}

Our medical school size increased from 100 students to 120 students over the past decade, but has not had any impact on our medical school's structure or function.

\section{Assessment}

The overarching dimensions associated with the BSOM medical education program objectives are complementary with the ACGME competency domains:

- Foundations of knowledge-Medical knowledge

- Application-Patient care (clinical skills)

- Integration-Systems-based practice

- Human dimension-Personal and interpersonal development

- Learning about and working with oneself-Personal growth and professional development

- Learning how to interact with others-Interpersonal skills and communication

- Caring/valuing_-Professionalism

- Learning how to learn-Practice-based learning 
See Table 1-Medical Education Program Objectives and Assessment Methods.

BSOM has also implemented changes in student assessment:

- One of the hallmarks of our "flipped and engaged" curriculum is that students receive feedback daily on what they know, but more importantly, on what they do not know so they can better focus their studies. These frequent, low-stakes assessments using TBL and PI provide students with regular, graded feedback to assess how they are performing within the curriculum, and allow opportunities for early intervention of those students who are struggling.

- WrightQ provides an opportunity for peer feedback, as well as narrative feedback from faculty facilitators.

- Wanting to capitalize on the learning that happens from discussion with peers, we changed our multiple-choice examinations to include a group component, similar to a group Readiness Assurance Test in TBL (for all exams aside from the end-of-course exams).

- We use customized NBME examinations for our end-of-course examinations during the Foundations phase.

- The NBME Comprehensive Basic Science Examination is administered twice during the Foundations phase as a progress test and to provide guidance on preparation for the USMLE Step 1 examination.

- In 2016, BSOM implemented a mid-third-year, 6-station OSCE; this has been positively received and therefore we added an additional 6-station OSCE during the Doctoring phase. In 2019, NBME end-of-clerkship "shelf" examinations were eliminated in favor of more OSCEs to provide more focused feedback on clinical skills.

- The NBME Comprehensive Clinical Sciences Examination is administered twice and used as progress testing during the Doctoring phase and also as a guide for preparedness for USMLE Step 2 CK.

- With the implementation of the WrightCurriculum in 2017, all students are required to complete a scholarly project before graduation.

\section{Parallel curriculum or tracks}

- BSOM received approval from the LCME in 2019 to offer a 3-year curriculum track for students interested in pursuing careers in either family medicine or pediatrics, with plans to expand in the future. This parallel curriculum provides an opportunity for students to complete all requirements of the MD degree within 3 years, with the prospect to enter into Wright State University-sponsored residency programs upon successful completion of all MD and residency admission requirements.

- For over a decade, we have offered a Global Health Certificate to students completing additional study in the area of global and/or refugee health.

- In 2019, the Faculty Curriculum Committee (FCC) approved the Wright Rural Health Scholars Certificate, with half of students' core clinical education being at rural sites to promote careers in rural medicine.

\section{Pedagogy}

- In our backward design of the WrightCurriculum, we embraced the theory that "assessment drives learning." Thus, we use a variety of pedagogical approaches that include daily formative, graded feedback to our students, specifically through the use of PI, TBL, and WrightQ.

- We also use simulation in several of the Foundations modules and clerkships, as well as standardized patient encounters in our Clinical Medicine course.

\section{Table 1}

\section{Medical Education Program Objectives and Assessment Methods}

\begin{tabular}{|c|c|c|}
\hline \multirow[b]{2}{*}{$\begin{array}{l}\text { Medical education program } \\
\text { objectives }\end{array}$} & \multicolumn{2}{|c|}{ Assessment methods } \\
\hline & $\begin{array}{l}\text { Foundations of clinical } \\
\text { practice curricular phase }\end{array}$ & Doctoring curricular phase \\
\hline 1.2. Describe the determinants of health & $\begin{array}{ll}- & \text { CBSE } \\
\text { - } & \text { PI } \\
\text { - } & \text { TBL } \\
\text { - WrightQ }\end{array}$ & $\begin{array}{l}\text { - Clerkship summative evaluations } \\
\text { - CCSE }\end{array}$ \\
\hline
\end{tabular}




\section{Table 1}

(Continued)

\section{Medical education program objectives}

\author{
2.1. Conduct patient interviews and physical \\ examinations
}

\subsection{Diagnose patient health problems}

\section{Foundations of clinical}

- Standardized patient exams (SPE)

- Objective structured clinical examinations (OSCEs)

- Simulation

- SPE

- OSCES

- NBME customized final exams

- $\mathrm{PI}$

- $\mathrm{TBL}$

- Formative MCQ exams

- WrightQ

- Simulation

\subsection{Propose evidence-based health maintenance and} therapeutic options

3.1. Connect knowledge of patient populations and health delivery processes in making diagnoses and therapeutic recommendations

- WrightQ

- SPE

- OSCES

- Simulation

- NBME customized final exams

- PI

- $\mathrm{TBL}$

- Formative MCQ exams

- WrightQ sessions

3.2. Advocate for the humane, just, safe, and prudent care of persons

3.3. Adapt to the complex economic and social structure of health care delivery

4.1. Reflect upon one's personal strengths and weaknesses to make positive changes in one's behavior

4.2. Find one's own meaning in medicine

4.3. Take care of oneself

4.4. Deliver effective patient presentations and document accurately in the medical record

4.5. Communicate and work effectively with others

\section{- PI}

- $\mathrm{TBL}$

- PI

- TBL

- Student mission statements

- Self-assessments

- SPE

- OSCES

- WrightQ

- NBME customized final exams
- Self-evaluation based on peer feedback

- PI

- $\mathrm{TBL}$

- WrightQ

- Formative MCQ exams

- Interprofessional Engagement (IPE)

4.6. Demonstrate leadership skills in a variety of settings

5.1. Care deeply about becoming an excellent physician through a life of service

5.2. Care about and support others in the profession

5.3. Value and behave in a manner consistent with the highest ethical standards of the profession

- WrightQ

- Student mission statements

- Peer evaluations

- Professional behavior requirement for advancement

- Student learning and career action plans medical professional

6.2. Stimulate intellectual curiosity to question and advance knowledge through scholarship

6.3. Appropriately use evidence-based resources to address uncertainty in medicine and gaps in knowledge/skills

- Scholarly project

- WrightQ

- TBL

- Scholarly project practice curricular phase

\section{Assessment methods}

\section{Doctoring curricular phase}

- Clerkship summative evaluations

- OSCES

- Clerkship summative evaluations

- OSCES

- Clerkship summative evaluations

- OSCES

- Didactic case applications

- Preceptor evaluations

- Clerkship summative evaluations

- Didactics

- Clerkship summative evaluations

- Clerkship summative evaluations

- Student self-reflections

- Student self-reflections

- Midrotation student evaluations

- Clerkship summative evaluations

- OSCES

- Clerkship summative evaluations

- IPE sessions
- Clerkship summative evaluations

- Learning and career action plan

- Personal statements for residency application

- Preceptor evaluations

- Clerkship summative evaluations

- Professional behavior requirement for advancement

- Personal statement (ERAS)

- CV (ERAS)

- Scholarly project

- OSCES

- Scholarly project 
- In moving to a lecture-free curriculum, we implemented PI in 2014 and WrightQ in 2015.

- With the implementation of the WrightCurriculum in 2017 as a lecture-free curriculum, core teaching and learning modalities are PI, TBL, and WrightQ.

\section{Clinical experiences}

- Clinical experiences at BSOM begin with interprofessional experiences in the community during the Foundations phase. The clinical medicine curriculum includes weekly sessions with preceptors to learn and review physical exam skills and interpretation.

- Students complete their core clerkships and electives during the Doctoring phase. We have 17 affiliated hospitals, including our local Veterans Administration hospital and clinics, and dozens of private practices, as well as rural sites. The diversity of patient experiences produces a graduate who is well informed about the social determinants of health and can adapt to most any clinical setting.

- The primary challenge we have experienced has been in placing our students with enough preceptors, as our students are competing with other allied health professions trainees for preceptor availability. We have had to recruit new sites and hospitals to ensure students maintain a valuable clinical experience.

\section{Curricular Governance}

- The FCC provides oversight and management of the MD curriculum.

- The FCC approves all decisions pertinent to the MD curriculum and student policies, including annual review of the entire MD curriculum, the 2 phases (Foundations and Doctoring), and all of the preclinical modules (courses) and clinical clerkships.

- Three subcommittees report directly back to the FCC: the Foundations Subcommittee, Doctoring Subcommittee, and Assessment and Evaluation Subcommittee.

See Figure 1-Curriculum governance structure.

\section{Education Staff}

The Office of Medical Education at BSOM became the Department of Medical Education (DME) in December 2019, allowing for faculty appointments. The associate dean for medical education serves as the department chair, which has 10 faculty and 24 staff to manage and support the undergraduate medical education program. This includes:

- Working with faculty to design and deliver the curriculum

- Assist with development and administration of assessments

- Collect and interpret data for faculty to evaluate outcomes of teaching innovations, education research, and the curriculum

- Provide in-class technological support of teaching activities

- Lead the development of WrightQ sessions

- Assist in quality improvement processes of questions

- Develop the interprofessional educational program

- Monitor the service-learning requirement

- Oversee all evaluations of faculty and courses/clerkships

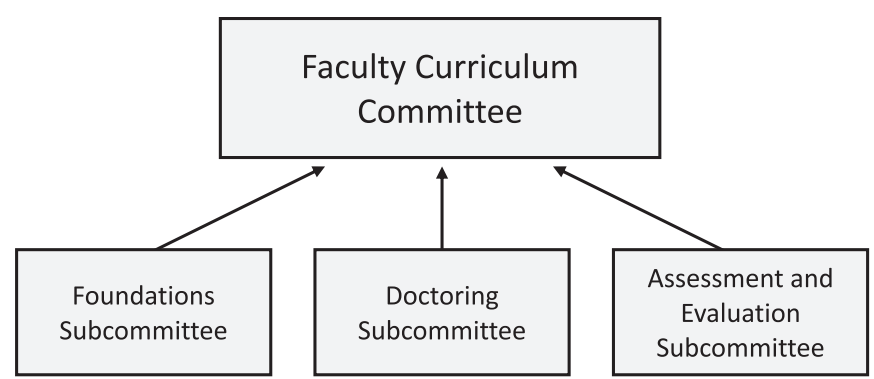

Figure 1 Curriculum governance structure.

The dean of medicine provides the leadership for the BSOM. The associate dean of medical education and the associate dean of student affairs and admissions work in concert with their units' faculty and staff to provide support for our students in their academic, career, and personal needs.

The organizational structure of the DME is led by the associate dean of medical education, with the following faculty and staff leadership positions reporting directly to the associate dean:

- Assistant dean of clinical skills education

- Director of Foundations phase

- Director of Doctoring phase

- Director of medical education and accreditation

- Director of assessment

- Director of therapeutics

- Director of educational scholarship and program development

The DME works closely with the FCC to ensure that the content, format, and evaluation methods of the curriculum meet the standards for accreditation of the LCME, including providing leadership and support of the Continuous Quality Improvement Committee.

\section{Faculty Development and Support in Education}

The DME organizes the Scholarship in Teaching and Research in Medical Education series, focusing innovations in teaching, developing assessments, and honing faculty facilitation skills. Additionally, there are monthly medical education research seminars.

There is an educator track in regard to faculty promotion, where high-level teaching and education activities are required. The majority of publications are to be educationally related. There are teaching excellence awards, innovations in education awards, and faculty development awards for undergraduate medical education to recognize exceptional contributions by faculty.

\section{Initiatives in Progress}

- Continuing to refine our lecture-free curriculum, examining data that support the success of the students in our curriculum. 


\title{
The University of Oklahoma College of Medicine
}

\author{
Christopher Candler, MD, EdD, Steve Blevins, MD, Teresa Scordino, MD, LaTasha Craig, MD, and Bryan Touchet, MD
}

\section{Medical Education Program Highlights}

The systems-based preclinical curriculum at the University of Oklahoma College of Medicine emphasizes the connections between basic and clinical sciences through coursework designed to promote learning and retention, develop early clinical skills, and provide students with enrichment experiences in the medical humanities. Depending on which track they selected during the admissions process, students take all 4 years of the curriculum at either the Oklahoma City academic medical center campus or the Tulsa School of Community Medicine campus where the core clinical curriculum is supplemented with experiences in community medicine. All students participate in lectures, smallgroup activities, team-based learning, and standardized patientbased learning exercises, a type of self-directed learning activity that blends the use of standardized patients with problem-based learning. During the first 2 years, students are assigned to modules of 15-16 students that are used to organize small-group learning sessions and foster student wellness through a social support system.

The clinical curriculum begins with a 1-week preparatory course designed to facilitate students' entry into traditional clerkships. All students must take three 2-week selective career exploration courses from a variety of subspecialty areas. At the end of the third year, students must take and pass an end-of-year OSCE.

\section{Curriculum description}

See Supplemental Digital Appendix 1-Curriculum Schematicat http://links.lww.com/ACADMED/A933.

\section{Curriculum changes since 2010}

Since 2010, the educational program has evolved through incremental changes in both the preclinical and clinical segments of the curriculum. These changes include:

- A required 7-week course, MS2 Capstone, was added at the end of the second year to reinforce and synthesize major basic science concepts.

- A required 4-week course, MS4 Capstone, was added to the fourth-year curriculum to reinforce important clinical topics.

- A required 1-week course, Clinical Transitions, was added at the beginning of the third year to prepare students for the clinical clerkships.

Acad Med. 2020;95:S414-S417.

doi: 10.1097/ACM.0000000000003439

Copyright (C) 2020 by the Association of American Medical Colleges

Supplemental digital content for this article is available at http://links.Iww.com/ ACADMED/A933.

Year school was established: 1900.

School URL: https://www.oumedicine.com/college-of-medicine.
- A required 2-week course, Joint, Skin, and Bone, was added to the second-year curriculum.

- Two first-year courses were combined to form a single course, Foundations of Medicine, where basic molecular and cellular biology, physiology, pathology, and pharmacology are taught.

- The gross anatomy course, Human Structure, was expanded by 2 weeks and moved to the beginning of the first year.

- The Blood, Hematopoiesis, and Lymphatics course, originally taught at the end of the first year, was placed earlier in the semester after the Immunology and Microbiology course.

- A longitudinal course, Lifestyle Medicine and Health Promotion, was created for the School of Community Medicine to address track-specific topics in community and population health.

- New educational program objectives were created to emphasize curricular threads such as cultural sensitivity and societal problems.

The class size has not changed since 2010 .

\section{Assessment}

The medical education program objectives are aligned with and organized under the ACGME general physician competency domains.

See Supplemental Digital Appendix 2-Program Objectives and Assessment Methods — at http://links.lww.com/ACADMED/ A933.

Since 2010, computer-based, multiple-choice examinations remain the principal means of assessing students in the preclinical curriculum. Written evaluations by faculty and residents, OSCEs, NBME subject examinations, and internally developed written and oral examinations remain the principal means of assessing students in the clinical curriculum. All students are required to take an 8-station OSCE at the end of the third year to assess skills in medical history-taking, physical examination, communication, establishment of a differential diagnosis, and development of a diagnostic plan.

The curriculum committees have established guidelines for summative assessment, formative assessment, comparability, remediation, and weighting of assessment.

\section{Parallel curriculum or tracks}

Developed to address the burden of complex chronic diseases among Oklahomans, the 4-year School of Community Medicine track supplements the core curriculum through additional curriculum components regarding social determinants of health, lifestyle medicine and health promotion, health systems science, quality improvement and patient safety, and interprofessional education. The School of Community Medicine (SCM) track delivers its specialized curriculum through SCM-specific 
required courses and through curriculum threads that run through common core courses and clerkships. SCM track students participate in special lectures, projects, cases, activities, simulations, and various community experiences that have been designed to address the SCM educational program objective and subobjectives associated with community medicine.

The College of Medicine recently received a Health Resources and Services Administration grant to fund enhancements in undergraduate medical education with the aim of increasing the number of primary care physicians in the state. As part of this grant, the College of Medicine is exploring the development and implementation of a 3-year medical school curriculum pilot for a small number of students in the SCM track at the Tulsa campus.

\section{Pedagogy}

During the preclinical curriculum, the educational program is delivered chiefly via lectures, self-directed learning, standardized patients, workshops, and preceptorships. The clinical curriculum uses inpatient and ambulatory experiences, lectures, workshops, and simulations.

Since 2010, there have been no significant changes in pedagogical approaches used throughout the curriculum.

\section{Clinical experiences}

The Oklahoma City clinical curriculum is primarily based at the OU Medical Center and Veterans Affairs hospitals. The Tulsa clinical curriculum uses community hospitals such as Saint Francis Hospital, St. John Hospital, and Hillcrest Medical Center.

Students at the School of Community Medicine in Tulsa participate in a longitudinal experience providing supervised patient care at a community-based clinic during the first and second years. During the third year, Tulsa track students work 10 hours per month to provide continuity care for a fixed panel of 15-20 underinsured patients.

Students begin clinical experiences early in the first year. They interview and examine standardized patients during the first week of medical school. They also gain clinical experience in community physicians' offices in the first and second years. At the School of Community Medicine in Tulsa, students visit patients' homes within the first week of school and take a supervised history using the appreciative inquiry model to ascertain social determinants of health. The students also interview the physician to discuss barriers to care and what might improve the patient's outcome.

The third- and fourth-year clinical experiences consist primarily of core clinical clerkships and electives situated within inpatient hospital units as well as outpatient hospitalbased clinics and outpatient private community-based clinics. On the Oklahoma City campus, third-year students rotate in community-based clinics during the family medicine clerkship. They also participate in a number of ambulatory clinics during obstetrics-gynecology, psychiatry, and pediatrics. Most Oklahoma City clerkships include both VA inpatient and outpatient experiences.
The SCM in Tulsa relies on community partners to provide required and elective clinical training opportunities. Students on the Tulsa campus rotate in community-based private hospitals, the Schusterman OU Physicians Clinic, and in community outpatient clinical experiences throughout the third and fourth years of medical school.

Maintaining a sufficient number of primary care clinical preceptors has been a challenge for curriculum planners. Obstacles include competition for training sites, preceptor clinical productivity pressures, preceptors' burden of electronic medical records, and the administrative burden associated with the assessment of learners.

\section{Curriculum Governance}

The College of Medicine has established a robust central curriculum governance model that is described in a curriculum governance charter. While there is no decentralized or department-based component to the curriculum governance system, the departments receive funding to employ course and clerkship directors; these individuals participate in the curriculum committees that collectively design, develop, and implement the components of the medical education program.

See Figure 1-Curricular governance framework.

\section{Education Staff}

A central Office of Medical Education (OME) consists of 3 units that provide distinct areas of support for the undergraduate medical educational program. The first provides general administrative and academic support for the planning, implementation, evaluation, and oversight of the undergraduate medical education program. Support is also provided for faculty development and for maintenance of the curriculum database. This unit comprises the director of OME and 4 administrative assistants. The second unit, the Clinical Skills Education and Testing Center, is the home for instruction and assessment activities involving standardized patients, simulation, and surgical skills. It consists of a medical director, an administrative director, a unit manager, a simulation specialist, and 2 coordinators. The third unit, made up of a unit manager and 3 staff assistants, provides classroom support and oversees the Willed Body program.

See Figure 2-Organizational chart.

The OME is devoted primarily to undergraduate medical education and to faculty development; programs related to student affairs, admissions, and graduate medical education are supported through separate offices.

\section{Faculty Development and Support in Education}

The Oklahoma City campus-based Office of Faculty Development (OFD) develops and delivers faculty development programming that supports faculty knowledge and skill-building 


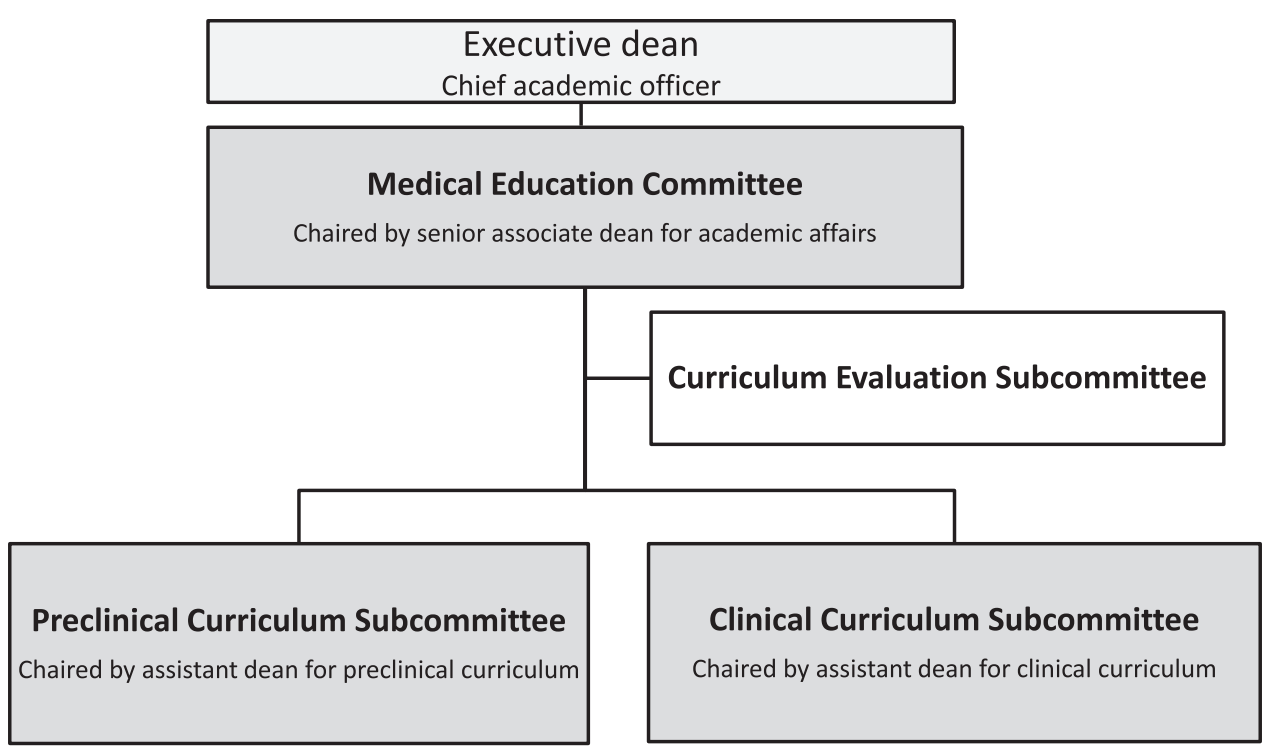

Figure 1 Curricular governance framework.

across the academic career life cycle. OFD offers a variety of programs and workshops for faculty and educators on both campuses. The college-based Academy of Teaching Scholars (ATS) is administered through the College of Medicine Office of the Executive Dean. The ATS offers assistance to both Oklahoma City- and Tulsa-based faculty interested in developing skills related to teaching, assessment, and educational research. On the Tulsa School of Community Medicine campus, the associate dean of undergraduate medical education and faculty affairs is responsible for facilitating many of the Tulsa-based faculty development offerings.
The college employs a faculty advancement system that includes several distinct faculty promotion pathways (basic science faculty, research faculty, clinician-scientist, clinicianeducator, and clinician). If teaching is a major portion of a faculty member's advancement application, evidence must be presented that the faculty member has developed and/ or conducted teaching programs of high quality. Teaching criteria include contributions to professional meetings, scholarly investigation, publication, and innovation. The definition of educational scholarship emanates from the larger context of scholarship; tenure-track scholarship

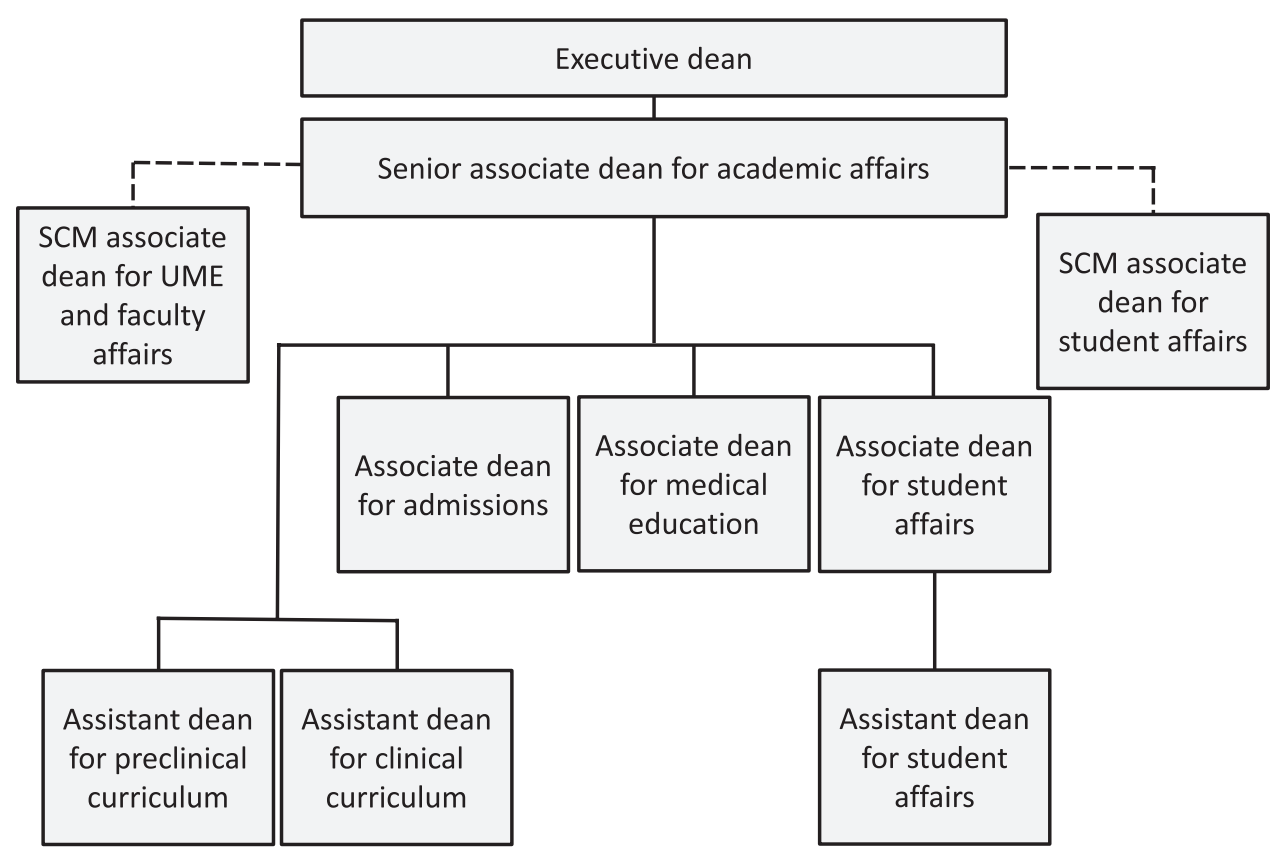

Figure 2 Organizational chart. 
includes publications indexed by the National Library of Medicine or a similarly recognized organization while nontenure-track scholarship considers public presentation and peer review of products or programs, both internal and external to the institution.

The College of Medicine ATS serves college faculty on the Oklahoma City and Tulsa campuses to:

- Recognize/reward teaching excellence and promote the value of teaching

- Develop and advance the skills of College of Medicine educators

- Recognize/reward teaching improvements and accomplishments

- Promote educational research

- Assist with faculty advancement (promotion and tenure; regional, national, and international programs; regional, national, and international awards)

\section{Regional Medical Campuses}

The Tulsa SCM is a regional campus of the University of Oklahoma College of Medicine. The MD program core curriculum is represented, and class enrollment is currently capped at 30 students.

A consistent educational experience is monitored and managed by the central curriculum governance system. The Medical Education Committee (MEC) annually reviews a comparability scorecard containing detailed side-by-side data for all Oklahoma City and Tulsa campus courses and clerkships. The scorecard rates each course and clerkship on a variety of comparability dimensions including learning objectives, outcomes, required clinical encounter compliance, nonfaculty instructor preparation, student satisfaction, assessments, educational experience, and grading system. Courses or clerkships that are flagged for one or more comparability concerns are required to submit a status report to the MEC that details actions taken. The MEC will monitor all comparability concerns and take action if necessary, until the issue is resolved. 


\section{Oregon Health \& Science University School of Medicine}

Tracy N. Bumsted, MD, MPH, Bart C. Moulton, MD, Tomo Ito, MSEd, George C. Mejicano, MD, MS, and Sharon Anderson, MD, MACP

\section{Medical Education Program Highlights}

Oregon Health \& Science University (OHSU), founded in 1887, is the only academic health center in the state of Oregon. OHSU is committed to graduating physicians with demonstrated skills in lifelong learning and improvement-leaders who can adapt to and develop future innovations in the health system, leading to better outcomes for patients and communities. In addition to transferring information and skills, OHSU School of Medicine strives to prepare the student for lifelong learning and scholarship; synthesis of information, critical reasoning, and problem solving; self-assessment and reflection; and collaborative clinical practice. The curriculum explicitly integrates the scientific basis of medicine with relevant clinical experiences within and across each phase of learning. It offers students progressive patient care responsibilities, fosters independent learning, and allows individualization of educational experiences. Students learn in an integrated curricular model, in which scientific principles of normal and abnormal human structure and function are woven throughout and incorporated as threads. The goal of the OHSU School of Medicine curriculum, YourMD, is to effectively prepare the MD graduate for residency training and professional practice to best serve and meet the needs of society in the 21 st century. Key aspects include:

- Competency-based education and assessment: Students are taught and assessed on their progress in attaining competencies linked to courses, clinical experiences, and other curricular elements that span the 6 domains of competence aligned with the ACGME framework. Frequent formative and summative assessments provide students with timely feedback to support their continued learning. Faculty portfolio coaches help students identify areas of academic strengths and challenges, and they offer resources and advice to overcome identified academic problems. Two assistant deans for student affairs who are dedicated to medical students, along with course directors, other UME educational leaders, peer tutors, and facultyled review and tutoring sessions, are all available to support students.

- Integration of curriculum: Integration and spiraling of basic, clinical, and health system sciences promotes comprehension and retention.

- Lifelong learning: Students have opportunities to develop this critical skill through their mentored scholarly project, narrative

Acad Med. 2020;95:S418-S421.

doi: 10.1097/ACM.0000000000003405

Copyright (C) 2020 by the Association of American Medical Colleges

Supplemental digital content for this article is available at http://links.Iww.com/ ACADMED/A913.

Year school was established: 1887.

School URL: https://www.ohsu.edu/school-of-medicine. medicine, written reflections, and meeting with their faculty portfolio coach to develop individualized goals. Learnercentered pedagogical approaches foster development of critical reasoning and independent problem-solving skills.

- Individualization of curriculum: Opportunities for curricular flexibility and customization are provided throughout the curriculum and start in year 1 with clinical preceptorships, enrichment activities, electives, and service learning.

\section{Curriculum}

\section{Curriculum description}

See Supplemental Digital Appendix 1-Curriculum Template (Without Acceleration) — at http://links.lww.com/ACADMED/A913.

See Figure 1-Curriculum threads.

\section{Curriculum changes since 2010}

In 2012, OHSU embarked upon a radical curriculum transformation. The innovative YourMD competency-based, integrated, and learner-centered curriculum replaced the previous traditional $2+2$ discipline-based curriculum and was launched with the entering class of 2014. YourMD includes 2 phases and 3 transition courses that bridge the progression from premedical to the foundations of medicine phase, from the foundations of medicine phase to the clinical experience phase, and from the clinical experience phase to postgraduate residency training. Starting in 2014, the class size gradually phase increased from 139 to 160 per year to address projected workforce shortages. The faculty responsibilities and availability of institutional resources were taken into account before final decisions regarding class expansion. The expansion of class size was successfully accommodated by the opening of a new education facility, hiring of new staff positions, and newly recruited clinical sites.

\section{Assessment \\ OHSU School of Medicine UME program competencies were compiled and devised using the AAMC Physician Competency Reference Set, the ACGME 6 core competency domains, clinical informatics competencies, and OHSU interprofessional core competencies.}

See Supplemental Digital Appendix 2-Program Objectives and Assessment Methods—at http://links.lww.com/ACADMED/A913.

OHSU School of Medicine uses competency-based assessments in both simulated and authentic clinical environments. Using standardized assessment instruments, trained faculty who are qualified assessors determine students' milestone level along each competency. All required courses in the preclerkship curriculum phase have common assessment components, 


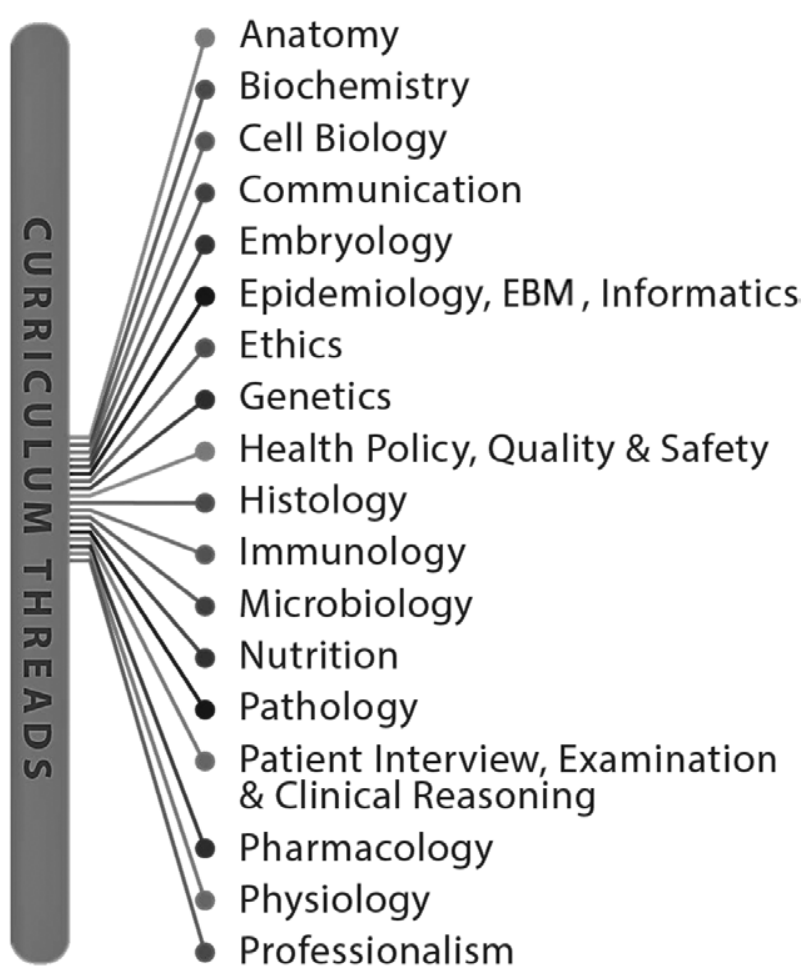

Figure 1 Curriculum threads.

including weekly quizzes on both knowledge and skills attainment to provide frequent and low-stakes feedback. In addition, all required courses in the clerkship phase have a standardized assessment structure. A workplace-based assessment platform provides students a mechanism to receive ongoing, immediate, and formative feedback on the 13 Core Entrustable Professional Activities (Core EPAs) for Entering Residency.

\section{Pedagogy}

OHSU School of Medicine uses a multimodal pedagogical approach. In particular, case-based learning, peer teaching, lecture, preceptorship, simulation, self-directed learning/ tutorial, standardized patients, video, virtual patient, lecture, lab, workshops, group discussions, narrative medicine, and both inpatient and ambulatory clinical experiences are all used to achieve the medical education program objectives.

\section{Clinical experiences}

OHSU students complete clinical rotations at a variety of clinical sites. This includes OHSU Hospital, the Portland Veterans Affairs Medical Center (VAMC), and many community-based hospitals and outpatient facilities throughout Oregon and Southwest Washington, including rural sites across Oregon.

All students are required to complete a continuity clinical experience, which is typically an 8-week full-time core or elective clinical experience offered in a variety of clinical disciplines allowing students to maintain continuity in at least 2 of the following 3 ways: continuity with the same patient population, continuity with the same health system, and continuity with the same preceptor(s).

OHSU students are exposed to clinical experiences through their preceptorship course in year 1 . Students can choose from multiple disciplines with curricular emphasis on systembased practice, practice-based learning and improvement, and interpersonal communication competencies.

OHSU students are required to complete a rural rotation. Students can choose from various disciplines including but not limited to family medicine, internal medicine, pediatrics, surgery, neurology, psychiatry, cardiology, anesthesiology, and otolaryngology. In addition, many students complete required and elective clinical rotations at community sites and the Portland VAMC.

Challenges faced in designing and implementing clinical experiences include:

- Providing clinical experiences across a large geographical area. The large area creates the challenge of ensuring that each site has adequate support such as housing and clinical space as well as ensuring that the rotations provide appropriate instruction across disciplines.

- Creating objective rubrics for assessment of the medical education program objectives, as well as training faculty, residents, and others who assess medical students on the rubrics.

\section{Curricular Governance}

See Figure 2-Curricular governance committees.

\section{Education Staff}

The Office of Undergraduate Medical Education (UME) provides administrative and academic support for all aspects of UME in the School of Medicine. The Office of UME is led by the associate dean for UME and a team of 2 assistant deans for student affairs, an assistant dean for admissions, 4 directors, and 25 additional professional and administrative support staff whose responsibilities include admissions, assessment, evaluation, course coordination, registration and scheduling, curriculum data, technical support, scholarships, wellness, student affairs, outreach, and accreditation/continuous quality improvement. The Office of UME provides administrative support to the UME Curriculum Committee (UMECC) and its 5 subcommittees: Foundations of Medicine, Core Clinical Experience Directors, Curriculum Development, Curriculum Evaluation, and Curriculum Resources. UMECC is responsible for reviewing and revising the overall goals and objectives of the UME curriculum, as well as assuring that these goals and objectives are achieved.

See Figure 3-Dean chart. 


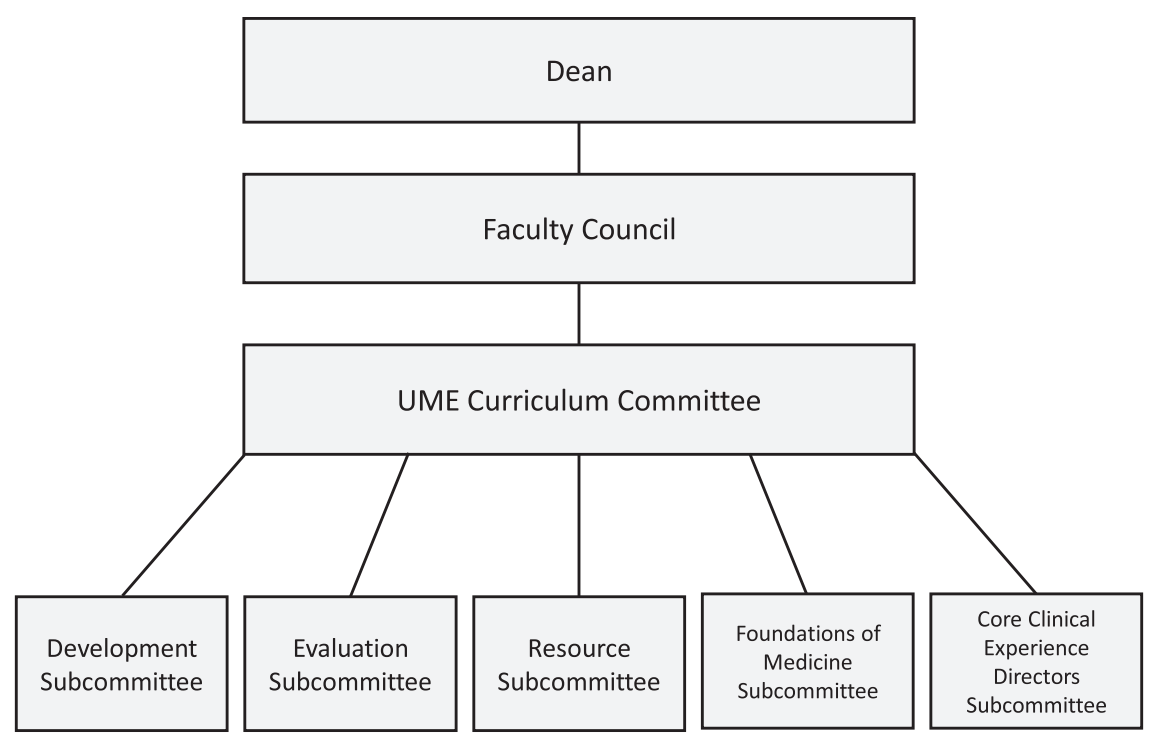

Figure 2 Curricular governance committees.

\section{Faculty Development and Support in Education}

Faculty in the School of Medicine have access to a plethora of knowledgeable individuals and resources to help them improve their skills in teaching and assessment.

- The school's assistant dean for continuing professional development and assistant dean for faculty development help coordinate workshops, seminars, and lectures in a wide variety of education topics for teachers at our institution in both clinical and basic science departments, as well as for teachers at our regional clinical partner sites.
- The Office of UME has a full-time doctoral-level director of UME assessment, and the Office of Graduate Medical Education supports a part-time doctoral-level faculty member, both of whom assist and train faculty members for learner assessment as well as course and program evaluation.

- The School of Medicine supports the faculty-driven Educators' Collaborative, which hosts monthly faculty development and education grand rounds as well as the annual Symposium on Educational Excellence daylong conference that includes small-group discussion on topics of interest such as academic writing, curriculum design, and best practices for improving

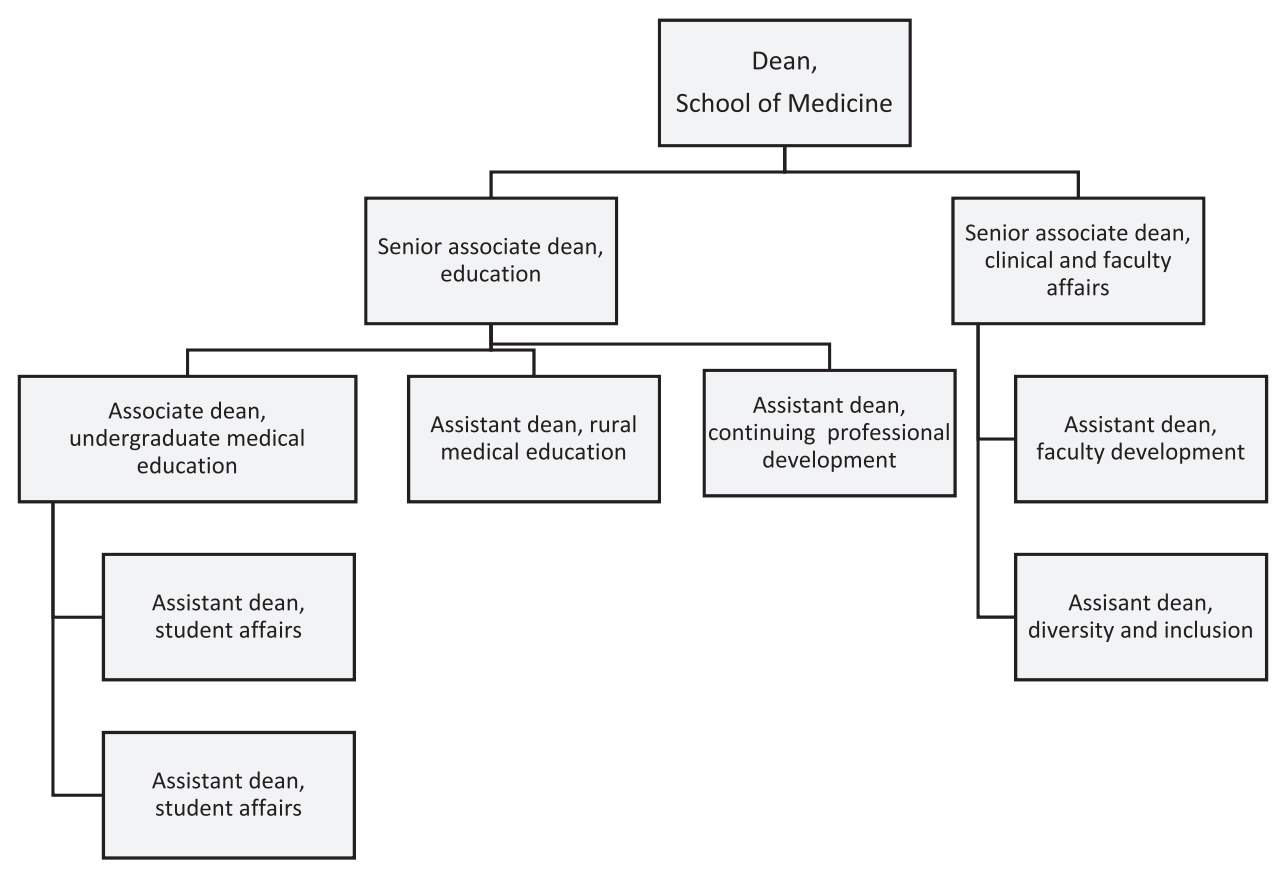

Figure 3 Dean chart. 
teaching and assessment skills for learners across the educational continuum.

- The School of Medicine supports the Education Scholar Program, a 9-month longitudinal cohort program where participants learn both education theory and methods. Program activities include sessions about effective education strategies for small- and large-group teaching, flipped classrooms, asynchronous learning, and mentoring for participants related to their application of educational concepts to current educational projects.

- The Teaching and Learning Center is a centrally located resource accessible to faculty across the institution. Its main focus is to assist faculty in skill development on a variety of topics such as how to perform formative and summative assessments of student learning and how to develop, implement, and improve best practices in teaching and course design.

- The Provost's Office of Educational Improvement and Innovation is tasked with leading institution-level faculty development initiatives such as professional development series workshops aimed at new/junior faculty. Efforts include coordinating faculty leadership and professional development grants, cosponsoring education mini-grant opportunities with School of Medicine, and creating new resources such as the Fostering Respectful and Equitable Education website for faculty to reference as they make their courses more inclusive.

\section{Initiatives in Progress}

- YourMD curriculum transformation initiative: The UME curriculum was radically transformed beginning in 2012, and the first cohort of the new curriculum launched with the entering class of 2014. Since that time, the curriculum has continued to evolve and adapt so that graduates are optimally prepared to practice in the highly complex and everchanging health care delivery environment of today. The School of Medicine has partnered with and received support from national organizations such as the American Medical Association's Accelerating Change in Medical Education and
Reimagining Residency consortia, and the AAMC's Core EPAs for Entering Residency pilot, which has provided rare opportunities to advance innovative ideas that promise to transform medical education on a national and international scale. Being a part of the community of forward-thinking change agents from across the nation has been vital to the success of our transformation initiatives at OHSU. We believe our bold and courageous spirit of continual improvement toward "better"-better processes and enhanced outcomeswill benefit our students, staff, faculty, institution, and most important, our patients.

- In 2019, OHSU, in conjunction with the University of California, Davis, was awarded an American Medical Association grant to establish a robust graduate medical education collaborative known as COMPADRE, short for the California Oregon Medical Partnership to Address Disparities in Rural Education and Health. COMPADRE will place hundreds of medical students and resident physicians to train under faculty and community physicians at 10 health care systems, 16 hospitals, and a network of federally qualified health center partners throughout Northern California and Oregon. COMPADRE's main goals are to address health care workforce shortages in rural, tribal, urban, and other communities that lack resources; increase access to health care providers; and improve the health of patients from ethnic and racial minority groups who are disproportionately affected by certain conditions.

- As part of the Core EPA pilot, OHSU is working toward ensuring every student is entrustable in the 13 Core EPAs. This includes forming an Entrustment Group whose focus is to make summative decisions, based on defined criteria, on whether a student has achieved a level of skill that will result in "badges" symbolizing that they are residency ready. Our goal is to find students who are behind and require extra support, as well as those who may accelerate through the curriculum. In the future, this will allow students to have a time-variable education and advance to residency when they are ready, not when a certain amount of time has passed, thus helping to address the growing physician shortage as well as reduce debt. 


\section{Drexel University College of Medicine}

Amy S. Baranoski, MD, MSc, Donna M. Russo, PhD, and Valerie Weber, MD, MS

\section{Medical Education Program Highlights}

Embracing the legacy from our forebearer medical schools, the Women's Medical College and Hahnemann Medical College, our "college of opportunity" emphasizes self-directed, collaborative, interdisciplinary learning. The Drexel University College of Medicine (DUCOM) curriculum, Foundations and Frontiers, provides early instruction in clinical reasoning based on the principles of problem-based learning (PBL). All in-classroom learning occurs in teams. Our longitudinal community-based health advocacy practicum exposes students to work with vulnerable populations and highlights the importance of social determinants of health. We teach the discipline of health systems science in an innovative immersion format.

\section{Curriculum}

\section{Curriculum description}

See Supplemental Digital Appendix 1-Curriculum Description —at http://links.lww.com/ACADMED/A812.

\section{Curriculum changes since 2010}

In 2014, DUCOM launched a curriculum renewal project that resulted in implementation of the new curriculum, Foundations and Frontiers, in 2017. Drexel's goal is to create physicians who excel at medicine's foundational skills and possess expertise in new and emerging fields such as health care quality and patient safety, population health, informatics, and health economics. The curriculum consists of 3 phases:

Phase 1: Foundations

- Integrated basic science courses use flipped classroom modality. Faculty prepare videos, handouts, and web-based tutorials for student independent learning. Active learning sessions apply this material using methods including casebased learning $(\mathrm{CBL})$, team-based learning, and simulation. Laboratory components support the anatomical threads.

- CBL: Students develop clinical reasoning skills while working through cases in a small group guided by a faculty facilitator. The cases integrate basic, clinical, and social science content using the principles of PBL. Multidisciplinary team-based learning activities expand on topics addressed in CBL.

Acad Med. 2020;95:S422-S425.

doi: 10.1097/ACM.0000000000003256

Copyright (C) 2020 by the Association of American Medical Colleges

Supplemental digital content for this article is available at http://links.Iww.com/ ACADMED/A812.

Year school was established: 1848.

School URL: https://drexel.edu/medicine/.
- Foundations of Patient Care course: Physical exam and communication skills are taught longitudinally with integrated professionalism formation through small groups, standardized and virtual patient encounters, and simulation experiences.

- Health Advocacy Practicum: A community-based longitudinal experience in which students work with vulnerable populations while learning about health disparities, social determinants of health, and patient advocacy.

- Frontiers: An innovative course organized into four 1-week immersion experiences including public health, population health, quality/patient safety, health informatics, and health economics.

Phase 2: Applications

- This phase is organized in a distributed model offering educational experiences in both academic hospital and community settings.

- It begins with a 2-week introduction to the clinical experience.

- Block clerkships in internal medicine, surgery, pediatrics, family medicine, psychiatry, neurology, obstetrics-gynecology, and ambulatory medicine are 5 or 10 weeks long. Most clerkships are a combination of inpatient and ambulatory clinical experiences.

- Ambulatory medicine combines outpatient clinical experiences and self-directed learning focusing on important topics such as addiction, chronic disease management, and high-value care.

- The neurology clerkship incorporates instruction on translational research and includes a short research project.

- All students participate in faculty-facilitated professionalism formation small groups.

Phase 3: Transitions

- Required experiences in emergency medicine and an acute care subinternship are included.

- Most experiences are individualized according to the student's career choice with the oversight of a faculty pathway advisor.

- This phase concludes with a capstone course focused on transition to residency.

\section{Class size changes since 2010}

While the class size has remained constant over the last decade, we are adding a satellite 4-year medical school campus in proximity to our clinical partner, Tower Health, in Wyomissing, Pennsylvania. With the Tower campus, our class size will increase to 300 beginning with the entering class in August 2021.

\section{Assessment}

See Supplemental Digital Appendix 2-Medical Education Program Objectives and Assessment-at http://links.lww.com/ ACADMED/A812.

During the recent curriculum redesign, our medical education program objectives were rewritten. We continue to use the ACGME competencies for our framework. 


\section{Assessment changes since 2010}

- In 2015, after a review by a subcommittee of the curriculum committee, we converted to a pass/fail system from tiered grading in Phase 1.

- Formalized narrative assessment and peer evaluation and self-assessment of group performance were added to multiple courses in Phase 1.

\section{Pedagogy}

As detailed above, our pedagogical approach includes the following:

- Phase 1: Flipped classroom with faculty-prepared materials (videos, handouts, and web-based tutorials), CBL, teambased learning, self-directed learning, laboratory, conferences, simulations, and standardized/virtual patients.

- Phases 2 and 3: Inpatient and ambulatory experiences using a block format structure. Self-directed learning and small-group and standardized/virtual patient activities supplement clinical experiences.

\section{Changes in pedagogy since 2010}

In 2010, DUCOM had 2 parallel tracks, 1 lecture-based and a second smaller track using PBL. As team-based learning gained in popularity, we implemented this format into the lecture-based curriculum and soon both students and faculty saw multiple benefits. In 2017, we moved to a unified curriculum using both $\mathrm{CBL}$ and team-based learning.

\section{Clinical experiences}

- DUCOM has several academic affiliate partners, including hospitals; health systems; and practices in urban, suburban, and rural settings. Partners are sponsors of residency programs and have long histories as academic and community-based teaching institutions.

- Six sites are designated as regional academic campuses where cohorts of students spend the entirety of their required thirdand fourth-year clinical experiences.

- St. Christopher's Hospital for Children, jointly owned by Drexel University and Tower Health, provides inpatient pediatric experiences.

- Students can experience an integrated delivery system through our academic partnership with Kaiser Permanente-Northern California.

\section{Required longitudinal experiences}

The Health Advocacy Practicum provides a service-learning experience in Phase 1 with structured content about the social aspects of medicine. Students gain health advocacy skills to support the well-being and resilience of individuals and their communities, particularly in the face of social adversity. Students are placed in settings working with communitybased populations including the homeless, elders, youth at risk, people contending with chronic medical illness or disability, people returning home from incarceration, or refugees. Over a 10 -month period, students obtain a unique perspective into how people interact with the health care system.

\section{Clinical experience first encounter}

- In Foundations of Patient Care, students begin required clinical experiences in the second week of the curriculum. The initial sessions focus on foundational communication skills working with standardized patients. Early experiences with patients, primarily in chronic care settings, are integrated within the first month of medical school.

- Students work with patients in the Health Advocacy Practicum in year 1 as described above.

- Basic science courses bring patients into the classroom for certain segments of the curriculum early in year 1 .

\section{Required and elective community-based rotations}

- Students spend 10 weeks in community-based practices. In family medicine, students see patients of all ages presenting with both common and more complex medical conditions. In ambulatory medicine, students rotate in internal medicine community-based practices.

- During the required pediatrics clerkship, students have exposure to 2 weeks of ambulatory pediatrics.

- In Phase 3, there are multiple community-based rotations available in various specialties and subspecialties.

\section{Challenges in designing and implementing clinical experiences for medical students}

The continuum of clinical experiences at DUCOM was redesigned during the 2014-2015 curriculum renewal project. This included rewriting the MD program objectives during the visioning stages of curriculum renewal. Implementation considered the realities of the availability of experiences at our clinical sites, including competition from other medical schools at some sites. The closure of Hahnemann University Hospital in September 2019 necessitated expansion of our clinical relationships.

\section{Curricular Governance}

\section{Curricular governance committees}

- The Educational Coordinating Committee (ECC) is responsible for the oversight and management of the curriculum. The ECC includes 13 faculty members: 1 basic science chair; 1 clinical science chair; and 11 members at large, including 1 member from a regional campus. There are 4 student members, 1 from each class. The ECC reports to the dean of the college through the Executive Committee of the faculty, which includes department chairs and elected faculty representatives.

- The Curricular Phase 1 ECC subcommittee consists of all directors of required courses, all content thread leaders, and 4 medical students.

- The Curricular Phases 2 and 3 ECC subcommittee includes all faculty directors of required clerkships/courses taught in the clinical years, all regional campus associate deans, 4 fourth-year class pathway directors, and 2 medical students.

- The ECC subcommittees report directly to the ECC. 


\section{Decentralized curricular governance}

- Basic science departments hire basic science educator faculty who are $100 \%$ dedicated to the education of medical students. Although funding for these positions is in the respective departments, evaluations and hiring decisions are made with the senior associate dean for curriculum's input.

- All departments, both basic science and clinical, receive funds through an allocation that is tied to educational time (including delivery and prep time).

- In clinical departments, funds from the Office of Educational Affairs (OEA) provide dedicated time for teaching and curriculum governance (clerkship directors, etc.).

- Assessment and academic support activities occur centrally from the Office of Curriculum and Assessment which is located within the OEA.

\section{Education Staff}

The OEA, led by the senior vice dean for educational and academic affairs, provides support and direction for the planning, implementation, evaluation, and oversight of the undergraduate medical education program. The Office of Curriculum, led by the senior associate dean for curriculum, provides direct oversight for the Office of Assessment and Evaluation and is located within the OEA. Personnel including the senior associate dean for curriculum; the associate dean for evaluation and assessment; and their respective staff provide support for curriculum delivery, monitoring, management, and assessment.

\section{Medical education leadership}

See Figure 1-Medical education leadership.

The OEA contains all the faculty and administrative staff supporting the MD program, and is responsible for all facets of undergraduate medical education, including admissions, curriculum, student affairs, and all related areas. The head of the OEA reports directly to the dean of the college of medicine.

\section{Faculty Development and Support in Education}

\section{Professional development for faculty as educators}

- The Office of Faculty Development provides administrative support and hosts programs in professional, leadership, and faculty development for College of Medicine (COM) faculty. Faculty development programs are offered in discipline content, curricular design, program evaluation, student assessment methods, instructional methodology, and research methodologies and fundamentals to enhance skills and leadership abilities.

- The Faculty Development Committee, composed of mid- to senior-level faculty representing departments across the COM, provides support to the dean's office in planning programs pertaining to faculty development in teaching, clinical service, research/scholarship, intramural/extramural service, and mentoring and leadership.

- Periodic faculty development events are held at regional campuses.

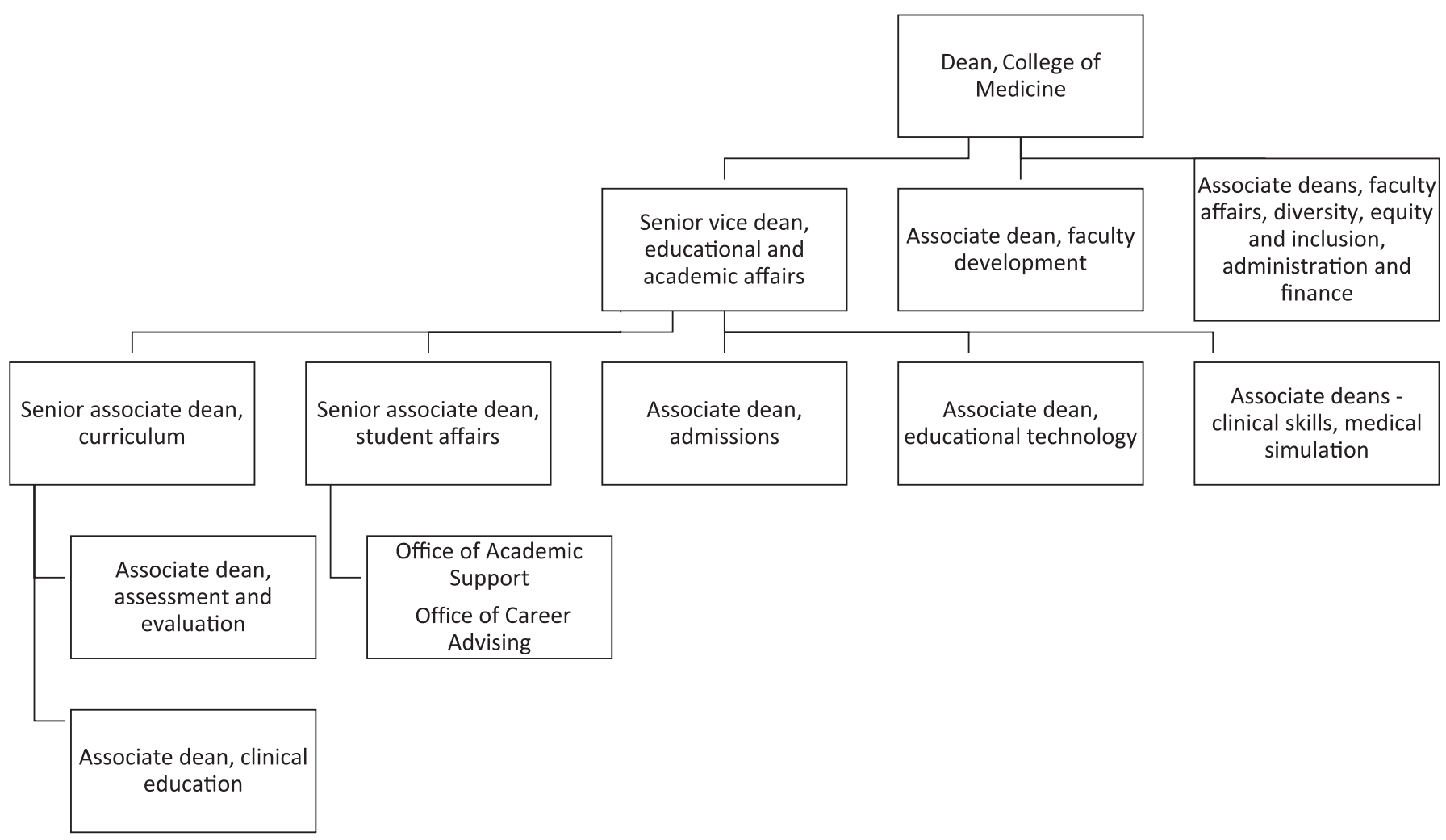

Figure 1 Medical education leadership. 
- An e-newsletter focused on faculty development, called

"I-Teach," is provided to all community faculty 3 times yearly.

\section{Role of teaching in promotion and tenure}

Education is highly valued in promotions and tenure decisions. Scholarly activity in education supports promotion as well as educational success, as measured by course evaluations and educational outcome data, teaching awards and recognition, and service on institutional and national educational committees. In addition, the COM has created a promotional track for clinician educators to recognize clinical faculty with interest and expertise in teaching.

\section{Regional Medical Campuses}

See Table 1-Regional Medical Campuses.

\section{Educational experiences across sites}

- Comparability and consistency across clinical sites is monitored both in process and outcomes by the ECC.

- Learning objectives, required clinical encounters lists, and access to the assessment system are distributed electronically to the faculty at the beginning of each academic year and posted on course websites.

- Clerkship directors/coordinators contact site directors/ coordinators via email at least once per block, reviewing student placements and performance.

- Annual site visits occur at regional campuses and other sites by the educational leadership team.

\section{Table 1}

\section{Regional Medical Campuses}

\begin{tabular}{llr} 
Regional campus name & Type & $\begin{array}{r}\text { Student } \\
\text { enrollment }\end{array}$ \\
Allegheny General Health System & Clinical & 40 \\
\hline Crozer Chester Medical Center & Clinical & 30 \\
Kaiser Permanente-Northern California & Clinical & 20 \\
Bay Area & & \\
\hdashline Pinnacle Health System & Clinical & 20 \\
\hdashline Reading Hospital/Tower Health & Clinical & 20 \\
Wellispan-York Health System & Clinical & 20 \\
\hline
\end{tabular}

- The regional deans and DMEs are members of the ECC Phases 2 and 3 subcommittee and attend monthly meetings either in person or via videoconferencing.

- An annual report is generated of course evaluations and student performance specific to each site and shared with all clerkship site directors and regional deans. This report is presented to the ECC Phases 2 and 3 subcommittee as part of the annual course review for each clerkship. Metrics used to assess comparability across sites include: clinical encounter/ patient volume (data collected from the electronic student patient logs and monitored centrally), grade distribution (final grade, clinical grade, and shelf exam means), and student feedback (generated centrally from the student-completed course evaluations). 


\title{
Geisinger Commonwealth School of Medicine
}

\author{
Andrea K. DiMattia, EdD, John A. Arnott, PhD, Sonia Lobo, PhD, and William B. Jeffries, PhD
}

\section{Medical Education Program Highlights}

Geisinger Commonwealth School of Medicine (GCSOM) was founded as The Commonwealth Medical College (TCMC) in 2009. Established by the community, without a parent university or sponsoring hospital system, the school collaborated with volunteer community faculty and local health care institutions to deliver the curriculum. From the beginning, the school has embraced a mission to serve the community and to replenish its physician workforce. It has been innovative in significant ways:

- The first medical school to use the longitudinal integrated clerkship (LIC) for the entire class

- Using active learning and the flipped classroom extensively, with lectures representing less than $23 \%$ of classroom time in the preclinical years

- Integrating community experiences, community health, and community service fully into the curriculum

On January 1, 2017, the school integrated with Geisinger, a highly functioning, integrated health care delivery system, which immediately provided robust and stable clinical learning venues; access to over 1,600 clinician-educators to serve as faculty; and exposure to cutting-edge research and clinical innovation in informatics, data science, precision health, genomics, implementation science, and outcomes research. Geisinger's existing graduate medical educational programs include over 450 learners in 48 accredited residency programs, providing enhanced learning for GCSOM students in an environment of cutting-edge innovations in education, research, and patient care.

- The Family-Centered Experience (FCE) is an integral part of GCSOM's patient-centered approach to medical education. This program matches a pair of first-year medical students with a volunteer family from the community to teach them about the impact of illness on family life. Students follow their volunteer family for 2 years and participate in scheduled debriefing sessions with peer students and faculty regarding their experiences, observations, and lessons learned in an effort to foster student compassion and understanding.

- All first-year medical students complete longitudinal community health intervention projects (L-CHIPs) in collaboration with community partners to learn about community health research. The L-CHIP experience, anchored in the Physician and Society course, includes presentations

Acad Med. 2020;95:\$426-\$430.

doi: 10.1097/ACM.0000000000003428

Copyright (C) 2020 by the Association of American Medical Colleges

Supplemental digital content for this article is available at http://links.Iww.com/ ACADMED/A929.

Year school was established: 2008.

School URL: https://www.geisinger.edu/education. on community-based participatory research, scientific method, research design, clinical and epidemiological research methods, research ethics, and biostatistics. Students work in small groups, to perform a literature review, interact with community partners, help develop and/or investigate a researchable question and proposal for community health intervention, and present a poster at a spring symposium.

- All third-year medical students are required to complete quality improvement community collaboratives to introduce the core competency of systems-based practice. Under the mentorship of QI staff in GCSOM teaching hospitals, students apply the principles of performance improvement, identify and analyze a health care delivery problem, and help develop and carry out a quality intervention in small groups. Through these projects, students learn to engage stakeholders to make changes in their routines that improve health care delivery and patient safety.

- A professional identity formation curriculum was developed to address explicitly the professionalism competency. It maps longitudinal identity growth with an ultimate goal of becoming a reflective practitioner by providing a system of academic coaching for students. This includes a referral process to the GCSOM Center of Learning Excellence focused on early identification of academic and professionalism issues. The process documents each student's professional identity formation growth throughout medical school through the use of an ePortfolio.

\section{Curriculum}

\section{Curriculum description}

See Supplemental Digital Appendix 1-Curriculum Schematicat http://links.lww.com/ACADMED/A929.

\section{Curriculum changes since 2010}

- Initially, the third year was a 12-month LIC with brief "bursts" of inpatient experiences. Based on feedback from students and preceptors, the third year was redesigned in 2017 into a hybrid LIC/block curriculum that consists of 6 months of inpatient experience and 6 months of outpatient LIC experience. This redesign eliminated interruptions in the continuity experience and provided a more robust inpatient experience, which facilitates full student engagement on clinical teams for a total of 23 weeks as compared with the previous 10 burst weeks.

- In 2019, the Longitudinal Continuity Experience (LCE), with 1 half day per month in year 2, was launched as part of the Art and Practice of Medicine course. The LCE pairs students to outpatient clinic sites where they are expected to practice their clinical skills and learn to navigate the day-to-day operations of an outpatient practice, under the guidance of a GCSOM faculty physician preceptor. 
- In 2017, a new 4-year interprofessional education (IPE) core curricular element was established. The IPE component introduces foundational concepts of IPE in the first 2 years through IPE exercises with nursing and pharmacy students, using a Team STEPPS model. Third-year IPE sessions include multiple health care learners and simulated patient scenarios. In the fourth year, all students complete a required 2-week IPE rotation on a highly functioning interprofessional team to learn about roles, responsibilities, and factors that enhance or hinder team function. All IPE sessions are aligned with Interprofessional Education Collaborative core competencies.

- In 2017, TCMC became GCSOM. This resulted in the realignment of the clinical campus structure around 3 Geisinger sites: North (Scranton), South (Wilkes-Barre), and Central (Danville). A fourth campus site had been established earlier in Sayre, Pennsylvania, in affiliation with the Guthrie Health System. In 2019, a fifth campus was established within AtlantiCare in Atlantic City, New Jersey. All sites follow a similar clinical training curriculum.

\section{Assessment}

The ACGME core competencies inform GCSOM's MD program objectives. Each of the 6 competencies has subcompetencies that are measured using formative and summative feedback, objective structured clinical examinations (OSCEs), team-based learning, exams, essays, peer evaluations, and performance on national exams.

See Supplemental Digital Appendix 2-Medical Education

Program Objectives—at http://links.lww.com/ACADMED/A929.

\section{Pedagogy}

See Figure 1-Pedagogical approaches.

\section{Clinical experiences}

The sites used for the required rotations are primarily Geisinger or Guthrie network locations; however, students also rotate with GCSOM faculty at the Wilkes-Barre VA Medical Centers and at community-based sites for some clinical experiences.

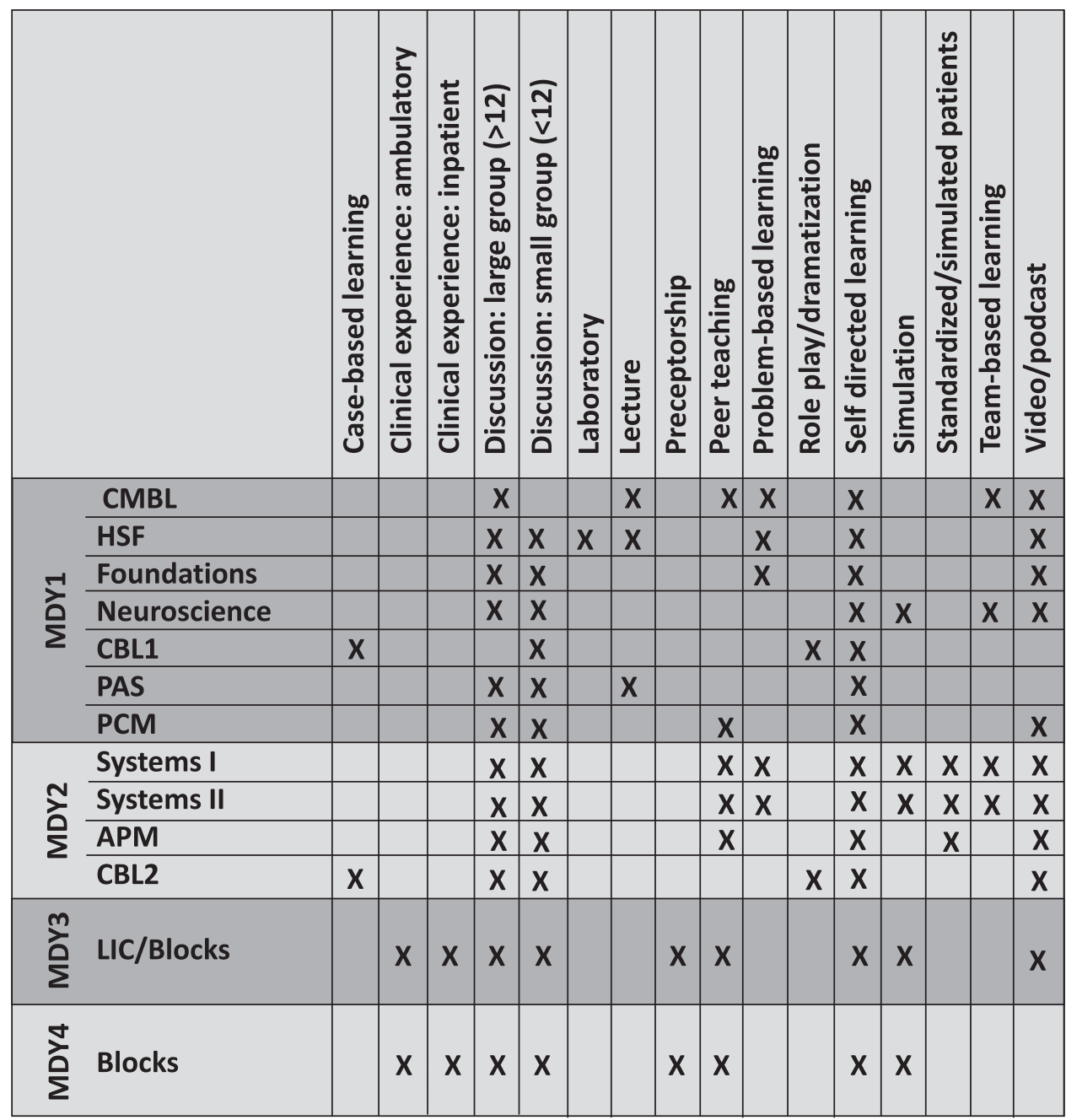

Figure 1 Pedagogical approaches. 
All GCSOM third-year learners complete a yearlong clerkship comprising a 6-month longitudinal integrated experience and discipline-specific block rotations in 6 core specialties. Half of the class begins the third year with longitudinal experience and half begins with block rotations, switching at midyear.

GCSOM students spend a considerable amount of time at community sites during their clinical training, beginning with their first-year L-CHIP project and FCE, and continuing with their second-year LCE longitudinal outpatient experience. During the third-year LIC, students in the outpatient curriculum spend at least 1 half day weekly with an assigned physician in an ambulatory setting in each of the 6 core specialties, followed by intervals of 1-4 weeks in block inpatient rotations. Additional community experience is found in the 100 hours of required community service completed by each GCSOM student.

GCSOM faced significant challenges in maintaining the original clinical campus structure as the health care environment in northeast Pennsylvania underwent structural change. Since the integration with Geisinger, we have established stable campuses and are in the process of renewing our medical education program and governance to match better the integrated health system. This integration has helped GCSOM establish early clinical exposure, enhancements in our required third- and fourth-year required rotations, and expansion of our elective rotation catalog. We are beginning a period of curriculum renewal in which we will further optimize our clinical education program by leveraging the strengths of the Geisinger and Guthrie health care systems.

\section{Curriculum Governance}

See Figure 2-Curricular governance committees.

\section{Education Staff}

The Office of Academic Affairs, led by the vice dean for medical education, comprises 16 staff who support planning, implementation, evaluation, and oversight of the curriculum. The chair of the Department of Medical Education (DME)

\section{Medical Curriculum Committee (MCC)}

Faculty committee that provides central oversight of the curriculum with the responsibility of review, oversight and decision-making authority for curriculum of the MD program

\section{1}

\section{Foundational Education Subcommittee}

The FESC Subcommittee is charged to provide recommendations to the MCC concerning the oversight, review, development, assessment and implementation of the preclinical curriculum of the MD program in the first 2 years

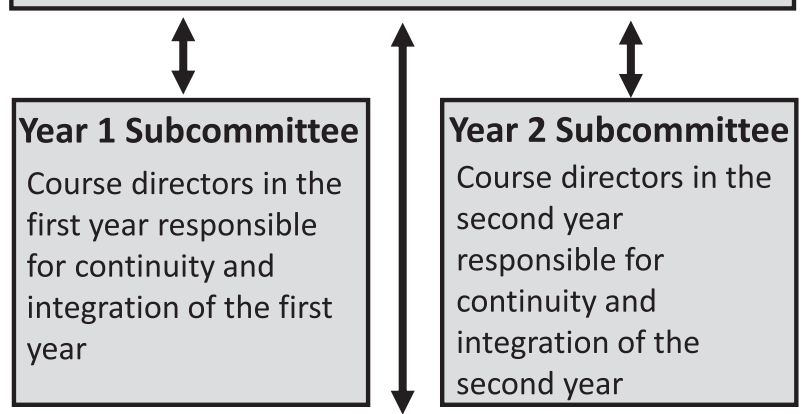

\section{Assessment Subcommittee} To develop uniform assessment standards for assessment during integrated science courses in the preclinical phase; acts in an advisory capacity to faculty on the proper conduct of remediation and examination review

\section{Years 3 and 4 Subcommittee}

The Years 3 and 4 Subcommittee is charged to provide recommendations to the MCC concerning the oversight, review, development, assessment and implementation of the clinical curriculum of the MD program in the second 2 years

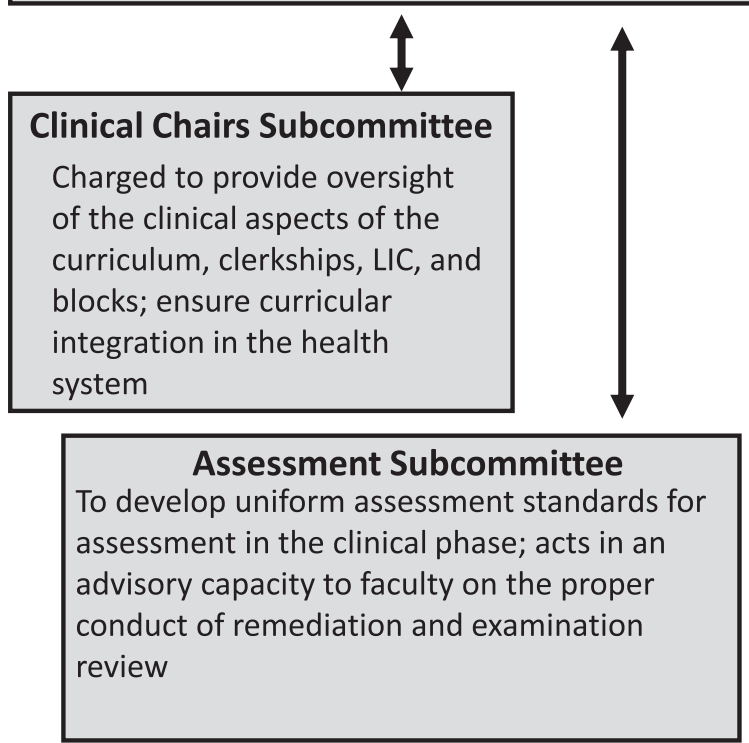

Figure 2 Curricular governance committees. 


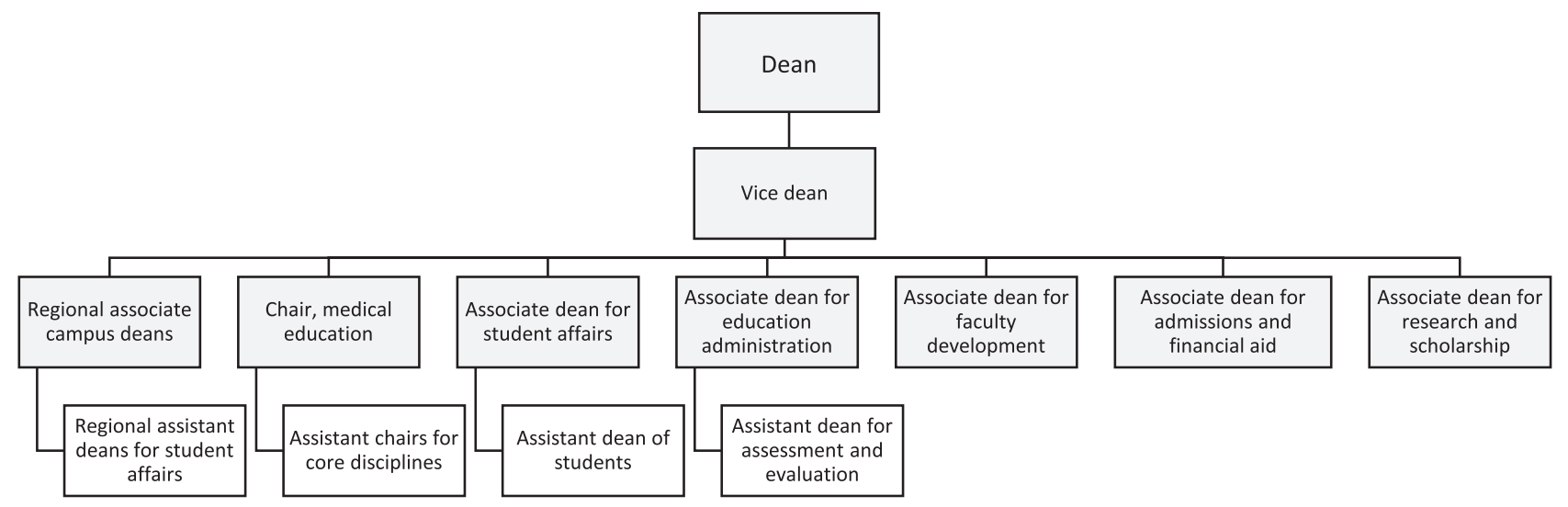

Figure 3 Medical education leadership.

reports to the vice dean. The DME, with 43 faculty, is responsible for curriculum development and delivery in years 1 and 2. In addition, the department has 6 assistant chairs in core clinical disciplines (family medicine, internal medicine, obstetricsgynecology, pediatrics, psychiatry, surgery) who are responsible for curriculum development, assessment, and comparability in the required clinical curriculum. Five regional associate campus deans lead the clinical campuses. Each campus associate dean is responsible for delivery of required clinical curriculum in years 3 and 4. Every campus has an assistant dean for student affairs and core clerkship directors, a regional education specialist (master'slevel educator responsible for monitoring student progress and implementing the curriculum), and support staff.

See Figure 3-Medical education leadership.

Each campus has a robust GME program that is administered by separate staff. Likewise, education for other learners outside of UME is administered through separate individuals. The UME mission at each campus has its own staff, as described above.

In 2018, GCSOM combined its 2 medical school departments (basic and clinical science) into the single DME that represents all clinical and basic science disciplines at the medical school.

\section{Faculty Development and Support in Education}

The school has a robust faculty development program that is administered by the associate dean for faculty development. This includes a quarterly workshop in education topics at each campus, a yearlong medical education certificate program, and additional faculty development programs. Other campusspecific development activities include NBME-style questionwriting workshops, narrative composition workshops, facilitator development, professional identity workshops, implicit bias training, and well-being/resiliency workshops.

All faculty at GCSOM are evaluated based on teaching excellence (quantity and quality) and use of a scholarly approach in education and their level of scholarship (engagement). All faculty submit educational portfolios that include evidence of excellence and documentation of engagement (abstracts, workshops, presentations, seminars, posters, etc.) based on the AAMC's Group on Educational Affairs criteria, including teaching, learner assessment, curriculum development, mentoring/advising, and educational leadership/administration.

\section{Regional Medical Campuses}

See Table 1-Regional Medical Campuses.

\section{Educational experiences across sites}

Consistency of educational experiences across sites is monitored at quarterly intervals. Regional campus leadership and the assistant chairs for the 6 core disciplines are sent aggregated data that include shelf exam performance, preceptor assessments of student performance, OSCE scores, and clinical encounters and skills. This allows student progress and campus comparability to be evaluated and discussed in real time. The regional deans are also responsible for meeting with the hospital and clinical leadership within their campuses to ensure that the learning environment is appropriate for students. This is also monitored using a learning environment survey that is delivered annually to first- and third-year students. An online event-triggered learning environment form can also be submitted by students at any time during their education.

\section{Table 1}

\section{Regional Medical Campuses}

\begin{tabular}{|c|c|c|}
\hline $\begin{array}{l}\text { Regional } \\
\text { campus name }\end{array}$ & Туре & $\begin{array}{r}\text { Total student } \\
\text { enrollment }\end{array}$ \\
\hline \multirow{2}{*}{$\begin{array}{l}\text { North Campus (Scranton, } \\
\text { Pennsylvania) }\end{array}$} & Preclerkship & 226 \\
\hline & Clinical & 45 \\
\hline $\begin{array}{l}\text { South Campus (Wilkes-Barre, } \\
\text { Pennsylvania) }\end{array}$ & Clinical & 41 \\
\hline $\begin{array}{l}\text { Central Campus (Danville, } \\
\text { Pennsylvania) }\end{array}$ & Clinical & 57 \\
\hline $\begin{array}{l}\text { AtlantiCare Campus (Atlantic } \\
\text { City, New Jersey) }\end{array}$ & Clinical & 16 \\
\hline $\begin{array}{l}\text { Guthrie Campus (Sayre, } \\
\text { Pennsylvania) }\end{array}$ & Clinical & 49 \\
\hline
\end{tabular}




\section{Initiatives in Progress}

The Abigail Geisinger Scholars Program awards up to 10 students per class up to 4 years of tuition and fees in the form of a loan, which is forgiven upon completion of a service commitment as a Geisinger physician. The award recipient is obligated to practice as a Geisinger-employed physician upon completion of residency training. One year of service is required for each year of support, with a 2 -year minimum commitment. Thus, participants who complete their obligation will have no tuition debt. This initiative was launched in the spring of 2019.

The Geisinger Primary Care Scholars Program, launching in the spring of 2020, supports up to 40 students per class with full tuition and fees and a $\$ 2,000$-per-month stipend in the form of a forgivable loan. Upon completion of residency training, the student will become a Geisinger-employed primary care physician (family medicine, medicine, medicine-pediatrics), providing 1 year of service for each year of support. The objective of this program is to create new regional primary care physicians who have no educational debt from pursuit of their MD degree.
A 2020 curriculum renewal effort is currently gathering the talent of all stakeholders across our 2 health systems to create a curriculum that will produce practitioners who are ideally suited to practice medicine in a changing health care environment. Thus, the renewed curriculum will include themes that reflect strengths evident in our health care systems based on the following principles and foundational elements:

- Integration of basic and clinical sciences throughout the curriculum

- Establishment of signature, evidence-based pedagogies

- Creation of meaningful roles for students in the clinical environment with progressively increased levels of responsibility throughout training

- Explicit inclusion of professional development formation

- Service to the community

- Leveraging system and regional strengths to ensure curricular excellence

- Ensuring that graduates are prepared to become excellent residents 


\section{Lewis Katz School of Medicine at Temple University}

Gerald H. Sterling, PhD, David Karras, MD, Larry I. Kaplan, MD, Alisa Peet, MD,

Denise Salerno, MD, and Marianne LaRussa

\section{Medical Education Program Highlights}

The educational programs of the Lewis Katz School of Medicine (LKSOM) are designed to train the next generation of physicians, physician-assistants, medical researchers, and bioethicists. The school uses a patient-centered approach in education to train students to meet the needs of a diverse patient population. The educational programs include: MD degree, MS and $\mathrm{PhD}$ degrees in biomedical sciences, an MA degree in urban bioethics, 2 postbaccalaureate programs (BCMS, ACMS), an MS degree in physician assistant studies, and several dual-degree programs (MD-PhD, MD-MPH, MD-MBA, MD-MA).

Unique features of the school include:

- A clinically focused MD program that uses patient scenarios to teach basic science principles, disease management, and health maintenance throughout the curriculum

- An MA degree program in urban bioethics, addressing health disparities in an urban community

- A Doctoring College system that enhances team-building, small-group learning, precepting, and advising

- A commitment to meeting the medical and social needs of our community through service-based learning

- Postbaccalaureate programs with direct admission to the medical school for qualifying students

\section{Curriculum}

\section{Curriculum description}

The medical student education program comprises 2 phases: Phase 1 (preclerkship) and Phase 2 (core and advanced clinical clerkships). Beginning in August 2020, LKSOM will adopt a single-pass organ system-based Phase 1 curriculum, in which normal structure and function of each organ system will be taught in conjunction with the pathology, pathophysiology, pharmacology, diagnosis, and management of diseases. The curriculum begins with foundational Human Structure, Cellular Function, and Host Defenses and Threats courses, followed by courses focusing on organ systems. Doctoring courses, integrating with the basic sciences across Phase 1 , focus on clinical and communication skills and the societal aspects of patient care. Phase 1 ends in February of the second year,

Acad Med. 2020;95:\$431-\$433.

doi: 10.1097/ACM.0000000000003320

Copyright (C) 2020 by the Association of American Medical Colleges

Supplemental digital content for this article is available at http://links.Iww.com/ ACADMED/A846.

Year school was established: 1901.

School URL: https://medicine.temple.edu. followed by a USMLE Step 1 examination study block before the start of Phase 2 .

See Supplemental Digital Appendix 1-Curriculum Map, 2020-2021—at http://links.lww.com/ACADMED/A846.

The expanded Phase 2 program consists of core clerkships in family medicine, internal medicine, neurology, obstetricsgynecology, pediatrics, psychiatry, and surgery; required advanced clerkships in critical care, emergency medicine, and a subinternship (medicine, pediatrics, surgery); a scholarly project; and 24 weeks of elective opportunities.

\section{Curriculum changes since 2010}

In 2020, LKSOM is changing the Phase 1 medical school curriculum to a single-pass organ system model in which normal body structure and function is taught in conjunction with diagnosis and management of disease. The Phase 1 curriculum has been shortened by 6 weeks, allowing for an expanded Phase 2 curriculum that provides more time for clinical electives, a scholarly project, and more robust Transition to Clerkships and Transition to Residency courses.

In preparing for this change, several modifications have been made since 2010:

- Incorporation of ultrasound and other diagnostic imaging to the gross anatomy course

- Identification of curricular threads that are integrated throughout the 4-year curriculum

- These threads include basic science topics such as pharmacology and pathology, and clinical science topics including communication skills, patient safety and quality, interprofessional education, social determinants of health and health care disparities, and bioethics

- Change to a pass/fail grading system for Phase 1

- Incorporation of weekly clinical reasoning conferences that tie basic science principles to clinical medicine

- An expanded Transition to Clerkships course

- Integration of radiology across third-year clerkships

- Incorporation of Academic Friday sessions into the third-year curriculum, for reflection on clinical experiences, review of basic science principles, and an emphasis on student well-being

- A capstone course that serves as a transition to residency, including a scholarly project recognition session that highlights student scholarly achievement, and specific residency "boot camps"

\section{Class size changes since 2010}

Class size increased from 180 to 210 students in 2011. This corresponded to the opening of a regional campus at the St. Luke's Hospital and Health Network in Bethlehem, Pennsylvania. 


\section{Assessment}

The school assesses student performance through a combination of internal and national examinations, including multiple-choice exams, formative and summative clinical skills exams, direct observation, and narrative evaluation in clinical clerkships. Most recently, LKSOM incorporated narrative evaluations in smallgroup activities in the Phase 1 curriculum, to provide feedback on interpersonal and communication skills, clinical problemsolving skills, ability to work in a team, and presentation skills.

See Supplemental Digital Appendix 2-Program Objectives and Assessment Methods_at http://links.lww.com/ACADMED/ A846.

\section{Medical education program objectives}

The medical education program objectives were developed based on the competency model of the ACGME, with modification based on the Physician Competency Reference Set and Core Entrustable Professional Activities. The objectives, which are reviewed annually, are divided into 7 defined areas:

- Medical knowledge

- Patient care

- Interpersonal and communication skills

- Professionalism

- Practice-based learning and improvement

- Systems-based practice

- Interprofessional collaboration

\section{Pedagogy}

Since 2010, lecture hours continue to be reduced, with a goal of no more than $40 \%$ in the revised curriculum. This has allowed for increased use of small-group learning, case-based discussions, simulations, and other active learning modalities. Over the past 4 years, the school has implemented clinical reasoning conferences, in which students work in small groups to solve clinical cases tied to the basic science content. LKSOM has also increased the use of standardized patients for teaching and assessing clinical skills, including patient history taking and physical exam skills, and professionalism. Each student in the preclerkship curriculum is assigned a clinical preceptor who monitors the student's progress.

\section{Clinical experiences}

LKSOM uses a variety of clinical sites for required clinical clerkships. These include inpatient, hospital-based clinics and community practice settings.

Students first encounter clinical experience during their Doctoring courses. Students learn from standardized patients and are provided opportunity for additional clinical experiences with their Doctoring preceptors beginning in their first year. Clinical experience is expanded in the second year, with time spent in the hospital and with community-based physicians.

Currently, each student is required to take a core clerkship in family medicine that includes community-based physician practices. Community-based electives are also offered in medicine, family medicine, and pediatrics as part of the fourthyear advanced clerkships.

Challenges in designing and implementing clinical experiences:

- Assuring comparability of clinical experiences across sites

- Assuring comparability of student evaluations across clinical sites

- Mergers of health systems, which can affect availability of clinical sites

\section{Curricular Governance}

The Curriculum Committee is a standing committee of the school, responsible for establishing and updating the education program objectives; monitoring where students learn and are assessed on each of the objectives; and reviewing and evaluating the design, quality, and effectiveness of the curriculum. Committee membership includes basic science and clinical faculty, affiliate faculty, a student from each class, and the education deans. The committee is co-chaired by a faculty member and the senior associate dean for education (SADE). The committee has several standing subcommittees:

- Phase 1 course directors

- Phase 2 clerkship directors

- Education Strategic Planning

- Academic Standards and Promotions

- Student Feedback

- Student Learning Environment and Appeals

- Technology in Education

- Assessment of Student Performance

Subcommittees are co-chaired by a member of the dean's staff and a faculty member. Subcommittees submit reports and recommendations to the curriculum committee for discussion and approval. The Office of Education, led by the SADE, in consultation with department chairs, assign responsibility for curricular content areas to appropriate faculty. The dean is responsible for the financial resources necessary for the education mission of the school.

\section{Education Staff}

The Office of Education consists of the SADE, associate deans for preclerkship and clinical education, associate dean for interprofessional education, associate dean for clinical skills, and assistant dean for clinical simulation. Staff include director of clinical simulation, director of the standardized patient program, and director of educational IT, all with support staff. Additionally, the SADE and the Office of Education have an administrative assistant and 2 managers with 9 curricular support staff.

The responsibilities of the office include management of the curriculum, continuous quality improvement, initiation of the curriculum review processes, implementation of curriculum revisions and evaluation, coordination of faculty development in education, and coordination of use of simulations and 
standardized patients in the curriculum. The SADE is responsible for all student education (medical, physician assistant, postbaccalaureate, graduate) at LKSOM. He is a member of the Dean's Leadership Committee and assures that each program has the necessary resources and support staff. Each of the programs is headed by an associate dean, who reports to the SADE. The curricular support staff noted above are only responsible for support of the medical student education program. There are additional support staff in each of the other student education programs. There are also separate graduate and continuing medical education offices.

Student support services are managed by the Office of Student Affairs with the associate deans for student affairs reporting to the SADE. To enhance student, resident, and faculty diversity and provide support, LKSOM established the Office of Health Equity, Diversity and Inclusion (OHEDI) headed by a senior associate dean. The staff of the Student Affairs Office and OHEDI work in coordination to assure support for all students.

\section{Faculty Development and Support in Education}

Professional development for faculty as educators

LKSOM established the Richard J. Kozera Teaching Academy to plan and coordinate the school's effort in educational faculty development for LKSOM and affiliate faculty. The academy works closely with the medical school's Office of Faculty Affairs, which sponsors faculty development programs in career development, and with Temple University's Center for the Advancement of Teaching, which is responsible for faculty development in education for all university faculty. The academy sponsors an annual faculty education retreat, with recent programming including compliance with LCME guidelines, teaching skills, student assessment, and feedback. Monthly programs have included an educational journal club, a book review, and a session regarding best practices in teaching skills. Faculty from the university's other health professions schools are invited to attend.

\section{Role of teaching in promotion and tenure}

The school has developed management tools to evaluate faculty contributions to the education mission and reward excellence in student and resident education. Performance is tied to annual compensation and used in tenure and promotional decisions. The school's mission-based management system assures availability of clinical faculty to support the medical education program. This includes doctoring faculty preceptors, core clinical educators in the preclerkship curriculum, and course and clerkship administration. The school has developed a prototypic teaching portfolio for faculty who contribute to the education mission of the school.

\section{Regional Medical Campus}

LKSOM has a regional medical campus at the St. Luke's Hospital and Health Network in Bethlehem, Pennsylvania.

\section{Table 1 \\ Regional Medical Campus

\begin{tabular}{|c|c|c|}
\hline $\begin{array}{l}\text { Regional } \\
\text { campus name }\end{array}$ & Type & $\begin{array}{l}\text { Student } \\
\text { enrollment }\end{array}$ \\
\hline $\begin{array}{l}\text { St. Luke's Hospital } \\
\text { and Health Network } \\
\text { (Bethlehem, } \\
\text { Pennsylvania) }\end{array}$ & $\begin{array}{l}\text { - Preclerkship } \\
\text { - Clinical } \\
\text { - Combination } \\
\text { X Entire MD program }\end{array}$ & $\begin{array}{l}\text { Academic year } \\
\text { 2020-2021: } \\
\text { Planned enrollment }{ }^{\mathrm{a}} \\
40 \text { first year } \\
30 \text { second year } \\
30 \text { third year } \\
30 \text { fourth year }\end{array}$ \\
\hline
\end{tabular}

aBefore August 2020, 30 students per class were accepted to the St. Luke's campus. As of August 2020, the St. Luke's campus class size will increase to 40 students per class

The campus was established in 2011 as a $1+3$ model, where 30 students completed first-year courses at the Philadelphia campus and the last 3 years of the program in Bethlehem. Beginning in August 2020, the campus expands to a 4-year program with class size increasing to 40 students per class. Students are accepted to the campus upon admission to LKSOM. Noncampus students may take individual clinical clerkships in Bethlehem.

See Table 1-Regional Medical Campus.

The St. Luke's education and student affairs deans collaborate with their counterparts at the Philadelphia campus to oversee the curriculum and student support services. Each course/ clerkship site director works closely with the course/clerkship directors in Philadelphia to assure comparability in the education program.

\section{Educational experiences across sites}

The curriculum at both the Philadelphia and Bethlehem campuses follow the same program and course/clerkship objectives and use comparable teaching methodologies. Lectures in the preclerkship curriculum are recorded and made available to students at both campuses. Small-group activities including case-based discussions and conferences are facilitated by faculty at each campus. Faculty facilitator guides for smallgroup sessions are shared amongst faculty from each campus to ensure consistency and comparability. Assessment methods at both campuses are identical. Students are assessed by multiplechoice exams, direct observation, and clinical skills assessments. Performance on school-generated exams, USMLE Step 1, $2 \mathrm{CK}$, and 2CS and residency match rates have demonstrated comparability in student performance at both campuses. The curricular content, teaching methodologies, assessments, and student feedback are regularly reviewed to assure educational comparability between campuses. 


\section{Penn State College of Medicine}

Britta M. Thompson, PhD, MS, Eileen M. Moser, MD, MHPE, Jed D. Gonzalo, MD, MSc, Daniel R. Wolpaw, MD, Tomi D. Dreibelbis, PhD, MEd, and Terry M. Wolpaw, MD, MHPE

\section{Medical Education Program Highlights}

The vision of Penn State College of Medicine (PSCOM) for its MD program is "to guide the development of a humanistic, systems-ready physician who is adaptive, critical thinking, collaborative, and scholarly." The curriculum is built on a 4-pillar framework of biomedical science, clinical science, health systems science (HSS), and health humanities. It is designed to align with a dynamic, evolving health care system.

The curriculum structure has 4 phases: Phase I, foundations; Phase II, clinical core; Phase III, discovery; and Phase IV, residency preparation. Below are several unique aspects that ensure alignment with our vision and the 4 pillars.

Flexibility and individualization include a choice of curricular track: The Hershey central curriculum (HC) or 2 parallel curriculum tracks-a 3-year accelerated option (HC3+) and a 4-year University Park regional campus curriculum track (UPC). During clerkships, choices include block or longitudinal integrated clerkship (LIC) models, rural or urban health centers, and large health systems or community-based settings.

Integration of the 4 pillars across each curricular phase facilitates longitudinal interleaving:

- Biomedical Science courses in Phase I are organized by organ system. Problem-based learning (PBL)/inquiry groups are the core pedagogical method during Phase I to ensure selfdirected learning and cognitive engagement, ${ }^{1}$ complemented by a variety of framing, synthesis, and interactive largegroup activities. During Phase II, students work in small groups to prepare interactive peer teaching sessions focusing on application of biomedical sciences to clinical sciences as well as HSS and health humanities. The USMLE Step 1 examination is scheduled at the end of Phase II/early Phase III to promote longer-term retention of biomedical science principles. ${ }^{1}$

- Clinical science education begins early in Phase I, focusing on developing history and physical exam skills in the context of critical thinking and clinical reasoning. Within the Society framework, 3-5 students work with faculty coaches and draw on a range of simulated and patient-based

Acad Med. 2020;95:\$434-S438.

doi: 10.1097/ACM.0000000000003409

Copyright (C) 2020 by the Association of American Medical Colleges

Supplemental digital content for this article is available at http://links.Iww.com/ ACADMED/A916.

Year school was established: 1967.

School URL: https://med.psu.edu. clinical experiences. Phases II-IV develop and reinforce these skills.

- HSS coursework in Phase I includes weekly sessions and patient navigation. During patient navigation, students participate in interprofessional collaborative care teams to apply HSS concepts in the clinical setting while working with patients to help them achieve better health outcomes. ${ }^{2,3}$ A 2-week course, Translating HSS to the Clinical Setting, occurs postclerkship with electives during Phases III and IV.

- Health humanities courses have dedicated experiences across all years, with weekly sessions in Phase I, longitudinal smallgroup experiences during Phase II, and a required selective in Phases III and IV.

Longitudinal learning is promoted through longitudinal learning relationships, longitudinal clinical courses, and transitions courses. Longitudinal learning relationships are promoted through many activities:

- Society advisor-coaches who provide coaching in clinical skills and across competency domains

- Longitudinal small groups in health humanities and HSS

- Required medical student research project mentoring

- Longitudinal clinical experiences such as LIC options and longitudinal clerkship block experiences

Courses dedicated to helping students transition to medical school, clerkships, and residency are scheduled at each transition period.

Assessment for learning is emphasized through regular weekly practice assessments during Phase I to promote retrieval practice. ${ }^{4,5}$ Collaborative exams administered midcourse provide opportunities for students to solidify learning. ${ }^{6,7}$ Critical thinking and clinical reasoning are promoted through summative constructed response examinations during Phase $\mathrm{I},{ }^{8-10}$ objective structured clinical examinations (OSCEs), and other clinical reasoning assessments during Phases I-III. In addition, students' progress toward PSCOM's 10 competency domains (and 23 unique subcompetencies) is determined through multiple central assessments and/or portfolio entries. ${ }^{11,12}$

\section{Curriculum}

\section{Curriculum description}

PSCOM offers 3 curricula: Hershey (HC), accelerated (HC 3+), and University Park (UPC).

See Supplemental Digital Appendix 1-Curricula Schematicsat http://links.lww.com/ACADMED/A916.

See Table 1-Overview of Curricula. 


\section{Table 1 \\ Overview of Curricula}

\begin{tabular}{|c|c|c|}
\hline Characteristic & $\begin{array}{l}\text { Hershey central } \\
\text { curriculum (HC) }\end{array}$ & $\begin{array}{l}\text { Hershey curriculum } \\
\text { Accelerated 3+ Track (HC3+) }\end{array}$ \\
\hline Brief description & $\begin{array}{l}17 \text { months Phase I (preclerkship) } \\
\text { course work with longitudinal } \\
\text { integration of biomedical sciences, } \\
\text { health humanities, health systems } \\
\text { science, and clinical sciences. } \\
\text { PBL is the core learning. Patient } \\
\text { navigation in year } 1 . \\
45 \text { weeks of core clerkships with } \\
\text { flexibility for individualized choices. } \\
\text { Options for blocks and longitudinal }\end{array}$ & $\begin{array}{l}17 \text { months Phase I (preclerkship) course } \\
\text { work identical to the Hershey central } \\
\text { curriculum. } \\
\text { PGY-1 pathways (early GME residency } \\
\text { entry): Clinical curriculum accelerated } \\
\text { with entry into residency after } 3 \text { years. } \\
\text { Some clerkships/clinical electives done } \\
\text { during summer after 1st year; options } \\
\text { for blocks and longitudinal integrated } \\
\text { clerkships. }\end{array}$ \\
\hline
\end{tabular}

integrated clerkships.

clerkships.
EPD pathways (enhanced professional development):

4th year focused on either research (clinician-scientist path) or master's degree in education (MEd) (clinicianeducator path).

\begin{tabular}{|c|c|}
\hline Average size entering class & 130 \\
\hline Typical duration of study & 4 years \\
\hline \multicolumn{2}{|l|}{ Accelerated pathways } \\
\hline Joint degrees & MPH, MBA MEd, MSTP program \\
\hline Clinical sites & $\begin{array}{l}\text { Milton S. Hershey Medical Center } \\
\text { and Penn State Health practice } \\
\text { sites, community-based affiliate } \\
\text { sites }\end{array}$ \\
\hline Research and scholarship & $\begin{array}{l}\text { Required medical student research } \\
\text { project; options for more extensive } \\
\text { research experiences; resources of } \\
\text { Penn State University College of } \\
\text { Medicine available }\end{array}$ \\
\hline
\end{tabular}

$20 \quad 12$

3 years (PGY-1 paths) 4 years

4 years (EPD paths)

Family medicine, emergency medicine, neurosurgery, orthopedics, internal medicine

MEd MPH, MBA

Milton S. Hershey Medical Center and Penn State Health practice sites, community-based affiliate sites

Mt. Nittany Medical Center, Penn State Health practice sites, surrounding community practices

\section{Required medical student research project \\ Clinician-scientist pathway; final year for research}

University Park

curriculum track (UPC)

Early immersive, longitudinal clinical experiences linked to inquiry-driven small-group learning. Longitudinal integration of biomedical sciences, health humanities, health systems science, and clinical sciences. Patient encounters in clinical settings provide the scenarios for inquiry and discovery in PBL-like facultyfacilitated small groups (Inquiry or "IQ groups").

Longitudinal integrated clerkships in year 2. Advanced biomedical and foundational science immersions following clerkships.

\section{Curriculum changes since 2010}

Substantial curriculum changes have occurred since 2010:

- Phase I was changed from a 2-year, discipline-based period of study relying largely on lectures and occasional PBL sessions to a 17-month, organ-based curriculum emphasizing selfdirected PBL and critical thinking. HSS was incorporated into the required core curriculum through a longitudinal Science of Health Systems course, paired with patient navigation in year 1.

- Phase II was changed from nonintegrated rotationbased clerkships to three 15-week blocks of integrated disciplines: internal medicine and surgery; pediatrics and obstetrics-gynecology; neurosciences, psychiatry, and health equity. Family and community medicine was changed to a longitudinal clerkship across the 3 blocks. Two-week career exploration and synthesis electives and a 4-week elective period were also added.

- Phases III and IV included the development of the Translating HSS to the Clinical Setting course. This course provides an opportunity for clinically experienced students to refocus on the application of HSS with dedicated mentorship and culminates in a capstone project and Yellow Belt Lean Six Sigma certification. Other additions to Phases III and IV include a summative portfolio and a variety of new elective options, including the addition of specialty-specific boot camps.

\section{Class size changes}

While there have been major changes in the curriculum, class size has remained stable since 2010 .

\section{Assessment \\ PSCOM has 10 competency domains (and 23 subcompetencies with milestones) with achievement fostered by society advisor- coaches and tracked by the Competency Progress Committee. These competencies were adapted from the Physician Competency Reference Set. ${ }^{13}$}

See Supplemental Digital Appendix 2-Competencies, Program Objectives, and Outcome Measures-at http://links.lww.com/ ACADMED/A916. 
To ensure a robust competency-based medical education, multiple foundational changes and additions in assessments have been implemented since 2010:

- Phase I changes included collaborative exams, summative end-of-course constructed response exams, formative and summative simulation-based exams/OSCEs to assess clinical reasoning and skills, regular practice assessments such as weekly quizzes, and Pass/Fail grading.

- Phase II changes included integrated simulation-based exams/ OSCEs at the end of each 15-week clerkship block to assess clinical reasoning. In addition, the USMLE Step 1 examination was moved to the end of Phase II.

- Phase III and IV changes included addition of a summative portfolio and competency review for each student.

\section{Parallel curriculum or tracks}

As described above, PSCOM offers the HC curriculum and 2 parallel curriculum tracks: $\mathrm{HC} 3+$ and UPC.

All parallel curriculum tracks are designed around PSCOM's 4-pillar framework with slight design variations for each curriculum during the phases.

See Figure 1-Four-pillar framework.

PSCOM has 2 options within the $\mathrm{HC}+$ curriculum. In the accelerated residency entry pathway, students graduate in 3 years. In the career enhancement pathway, students accelerate their MD coursework and participate in value-added training during their fourth year.

- The accelerated residency entry pathway is offered in family medicine, orthopedics, neurosurgery, emergency medicine, and internal medicine. In addition to participating in all Phase
I courses, students begin some clerkship curricula as early as spring of year 1. They complete clerkships in Phase II and have reduced elective requirements in Phases III and IV.

- The career enhancement pathway is offered for clinicianscientists and clinician-educators. Students in the clinicianscientist pathway use their final year to participate in clinical coursework and intensive mentored research, while those in the clinician-educator pathway participate in clinical coursework along with graduate courses in education, culminating in a joint MD and master's degree in Education within 4 years.

\section{Pedagogy}

Pedagogical approaches for each of PSCOM's curricula are briefly described below. In 2010, neither the UPC nor the HC3+ had been implemented.

- $\mathrm{HC}$ and HC3+ pedagogy: PBL during Phase I is now the central pedagogical approach for medical knowledge competencies and standardized patients. Students first encounter longitudinal clinical experiences during patient navigation and through their society-based course,

Foundations of Patient Centered Care. Many types of clinical sites are used throughout the curriculum, including practices within Penn State Health for a majority of the students and affiliated inpatient and outpatient sites across Pennsylvania. As discussed, longitudinal clinical experiences are offered during Phase II as LICs and a longitudinal family and community medicine clerkship.

- UPC pedagogy: Longitudinal PBL with actual patient cases from clinical immersions are central to Phase I learning. Students first encounter clinical experiences upon entry into the curriculum and immediately begin precepted clinical experiences 2 half days per week for their first year. These experiences become the basis for PBL (inquiry groups)

\section{Patient Centered and Systems Focused MEDICAL EDUCATION}

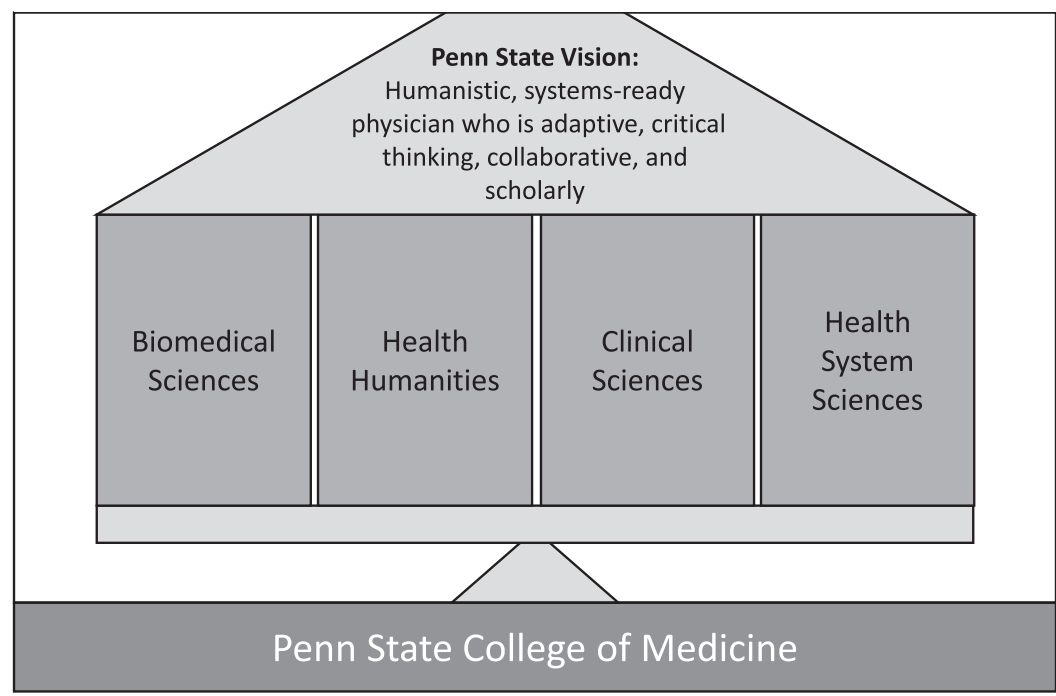

Figure 1 Four-pillar framework. 


\section{Table 2}

\section{Pedagogical Approaches for Each Curriculum}

\begin{tabular}{ll} 
HCHC3+ & UPC \\
- Case-based learning & - Case-based learning \\
- Clinical experience: ambulatory & - Clinical experience: ambulatory \\
- Clinical experience: inpatient & - Clinical experience: inpatient \\
- Discussion: large group (>12) & - Discussion: large group (>12) \\
- Discussion: small group ( $\leq 12)$ & - Discussion: small group $(\leq 12)$ \\
- Laboratory & - Laboratory \\
- Lecture & - Preceptorship \\
- Preceptorship & - Problem-based learning (inquiry \\
- Problem-based learning & groups) \\
- Role play/dramatization & - Self-directed learning/tutorial \\
- Self-directed learning/tutorial & - Simulation \\
- Simulation & - Standardized/simulated patients \\
- Standardized/simulated patients & - Workshop \\
- Team-based learning & - Other: patient navigation \\
- Video/podcast & \\
- Other: case-based collaborative & \\
\hline
\end{tabular}

facilitated by master teaching faculty. Clinical teaching sites in this curriculum include a mixture of Penn State Health and affiliated sites.

- Required community-based rotations: A required clerkship for all students, regardless of curriculum track, is the 4 -week health equity clerkship, which provides the application of HSS principles in community-based primary care practices within physician shortage areas and medically underserved populations. Students experience models of health care and physician adaptation in the care of vulnerable populations.

- Challenges: Similar to medical schools nationwide, clinical capacity is a challenge, along with the changing landscape of health care systems acquisitions and mergers. In addition, the fast-paced, high-productivity clinical learning environment presents challenges to direct observation, assessment of clinical reasoning, provision of high-quality feedback, and timely and valid clinical assessments.

See Table 2-Pedagogical Approaches for Each Curriculum.

\section{Curricular Governance}

The Committee on Undergraduate Medical Education (CUMED) manages all phases and aspects of the curriculum. Teaching in non-patient care settings, assessment, and academic support are budgeted through a central College of Medicine process.

See Figure 2-Curriculum governance committees.

\section{Education Staff}

The vice dean for educational affairs reports to the PSCOM dean and is responsible for the MD and physician assistant programs and associated offices (Student Affairs, Student Admissions, Student Mental Health and Wellness, Health Systems Science, Cognitive Skills Center, Interprofessional Education). The vice dean also leads the graduate medical education and continuing education programs, along with the simulation center, Harrell Health Sciences Library, Education Program on Human Structure, and Woodward Center for Excellence in Health Sciences Education. Associate and assistant deans provide key support to the programs.

The role of the primary medical education staff and administrative faculty is to support the continuum of the medical education and physician assistant programs along with areas outside of educational programming, such as those indicated above. The Office of Medical Education (OME) provides administrative and academic support for the central curriculum and the 2 parallel tracks. Professional staff within OME includes curriculum operations managers and instructional designers. The Office of Evaluation and Assessment supports and oversees evaluation and assessment and the development and maintenance of tools to support the management and data-driven decisions of CUMED.

\section{Faculty Development and Support in Education}

\section{Professional development for faculty as educators}

The Woodward Center for Excellence in Health Sciences Education offers opportunities to enhance teaching, learning, and educational scholarship for faculty, residents, students, and postdoctoral fellows. The Woodward Center offers a variety of resources, from single-topic sessions to multiday workshops, peer review of teaching, and longitudinal mentoring. PSCOM does not have an academy/institute for educators, but does have an honorific award/society for medical school faculty.

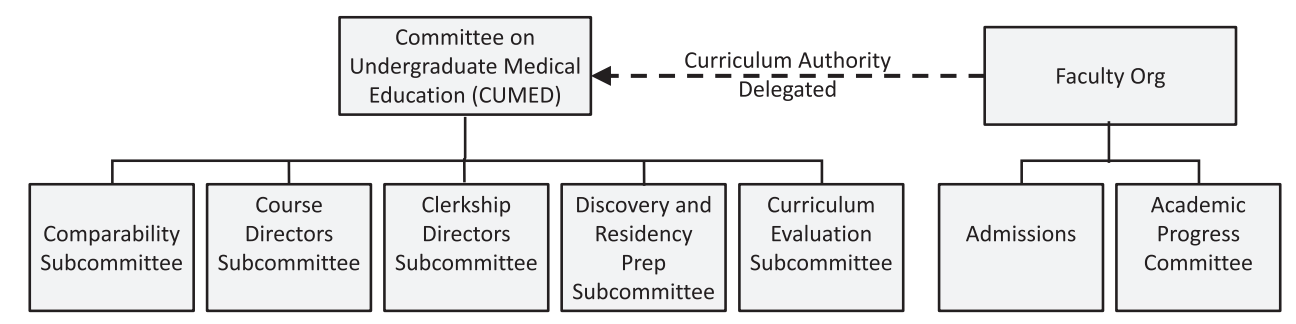

Figure 2 Curriculum governance committees. 


\section{Table 3 \\ Regional Campuses}

\begin{tabular}{|c|c|c|}
\hline $\begin{array}{l}\text { Regional campus } \\
\text { name }\end{array}$ & Туре & $\begin{array}{l}\text { Student } \\
\text { enrollment }\end{array}$ \\
\hline University Park & Parallel 4-year curriculum & $\begin{array}{l}\text { 48/year } \\
\text { (12 per class) }\end{array}$ \\
\hline $\begin{array}{l}\text { Kaiser Permanente } \\
\text { (Washington, DC) }\end{array}$ & $\begin{array}{l}\text { Longitudinal integrated } \\
\text { clerkship curriculum }\end{array}$ & 4/year \\
\hline $\begin{array}{l}\text { Summit Health (WellSpan } \\
\text { Health, Summit) }\end{array}$ & $\begin{array}{l}\text { Longitudinal integrated } \\
\text { clerkship curriculum }\end{array}$ & 2/year \\
\hline
\end{tabular}

The associate dean for faculty professional development supports professional development opportunities to faculty, including promotion and tenure counseling and the Junior Faculty Development Program for early career MD and $\mathrm{PhD}$ faculty.

\section{Role of teaching in promotion and tenure}

Teaching and educational activity is included in promotion/ tenure decisions; faculty must demonstrate excellence in at least 2 of 3 areas (teaching, research, patient care). To show excellence in education, faculty are expected to provide evidence of educational scholarship and national reputation of the academic level for which they are being considered.

\section{Regional Medical Campuses}

See Table 3-Regional Campuses.

We employ several techniques to ensure comparability between sites:

- Clerkship directors on the Hershey campus oversee clerkships in their specialty area at each site.

- Affiliate calls for each clerkship are conducted on a quarterly basis to ensure that all clerkship sites are enacting the clerkship comparably, such as clerkship goals and objectives, faculty orientation, student assessment, and course evaluation.

- In addition to calls, an annual affiliate site meeting (face-to-face) occurs with clerkship directors, site directors, and faculty at all sites. At the meeting, any changes to clerkships are discussed and site-specific data are reviewed to ensure comparability.

- The Curriculum Evaluation Subcommittee reviews each course in-depth. For any courses offered at multiple sites, all data for each site are reviewed and comparability issues are identified for the course directors to address and CUMED to manage.

- The Comparability Subcommittee reviews all site data to ensure comparability and reports to CUMED for management of issues.

\section{References}

1 Jurich D, Daniel M, Paniagua M, et al. Moving the United States Medical Licensing Examination Step 1 after Core Clerkships: An outcomes analysis. Acad Med. 2019;94:371-377.

2 Gonzalo JD, Thompson BM, Haidet P, Mann K, Wolpaw DR. A constructive reframing of student roles and systems learning in medical education using a communities of practice lens. Acad Med. 2017;92:1687-1694.

3 Gonzalo JD, Lucey C, Wolpaw T, Chang A. Value-added clinical systems learning roles for medical students that transform education and health: A guide for building partnerships between medical schools and health systems. Acad Med. 2017;92:602-607.

4 Weinstein Y, Madan CR, Sumeracki MA. Teaching the science of learning. Cogn Res Princ Implic. 2018;3:2.

5 Dunlosky J, Rawson KA, Marsh EJ, Nathan MJ, Willingham DT. Improving students' learning with effective learning techniques: Promising directions from cognitive and educational psychology. Psychol Sci Public Interest. 2013;14:4-58.

6 Levine RE, Borges NJ, Roman BJB, et al. High-stakes collaborative testing: Why not? Teach Learn Med. 2018;30:133-140.

7 Thompson BM, Haidet P, Borges NJ, et al. Team cohesiveness, team size and team performance in team-based learning teams. Med Educ. 2015;49:379-385.

8 Kang SHK, McDermott KB, Roediger III HL. Test format and corrective feedback modify the effect of testing on long-term retention. Eur J Cogn Psychol. 2007;19:528-558.

9 McDaniel MA, Roediger HL $3^{\text {rd }}$, McDermott KB. Generalizing testenhanced learning from the laboratory to the classroom. Psychon Bull Rev. 2007;14:200-206.

10 Stanger-Hall KF. Multiple-choice exams: An obstacle for higher-level thinking in introductory science classes. CBE Life Sci Educ. 2012;11:294306.

11 ten Cate O, Carraccio C. Envisioning a true continuum of competencybased medical education, training, and practice. Acad Med. 2019;94:1283-1288.

12 Murray KE, Lane JL, Carraccio C, et al; Education in Pediatrics Across the Continuum (EPAC) Study Group. Crossing the gap: Using competencybased assessment to determine whether learners are ready for the undergraduate-to-graduate transition. Acad Med. 2019;94:338-345.

13 Englander R, Cameron T, Ballard AJ, Dodge J, Bull J, Aschenbrener CA. Toward a common taxonomy of competency domains for the health professions and competencies for physicians. Acad Med. 2013;88:1088-1094. 


\section{Perelman School of Medicine at the University of Pennsylvania}

Suzanne Rose, MD, MSEd, Jennifer R. Kogan, MD, Nadia L. Bennett, MD, MSEd, Dennis J. Dlugos, MD, MSCE, Anna Delaney, MBA, Rosalyn Schorr, MSEd, and Judy A. Shea, PhD

\section{Medical Education Program Highlights}

The University of Pennsylvania's Perelman School of Medicine (PSOM), established in 1765, is the oldest medical school in the United States, with a long tradition of academic excellence and scientific discovery. Contemporary highlights include:

- A beautiful and purposefully designed new space, the Jordan Medical Education Center, opened in 2015.

- The Center for Student Life, a unique integration of the offices of student affairs, diversity and inclusion, curriculum, and the new position of associate dean for student success and professional development.

- A highly developed, team-facilitated learning environment with students assigned to a learning team of 6-7 students, linked to a House system with advisory deans; supported by a 2-day, business model-inspired team-building retreat informed by work from the Wharton School.

- An Academy of Master Clinicians, which is a distinction related to exemplary patient care: honorees provide mentorship and support to students through the House system.

- A world-class MD-PhD and MD+ program, with nearly $70 \%$ of students graduating with an additional degree or certification (12 master's programs, 10 certificates).

- PennHealthX, a program to support exploration and innovation in health care.

See List $1-\mathrm{MD}+$ Programs.

\section{Curriculum}

Penn's curriculum has been rebranded as "Learning for Life." Curriculum refresh has been iterative, building upon innovations from the last major curriculum revision, which included shortening the preclerkship curriculum (PCC) to 16 months, establishing a team-facilitated learning environment, and advancing individualized education. We are setting a galvanizing vision for UME and redefining competencies required for graduation while considering curricular advances that address the question of how we can best continue to train future-ready physicians to perform in a rapidly changing field.

Class size has remained stable at approximately 150 students per class, with no plans to change class size. Our students represent

Acad Med. 2020;95:S439-S443.

doi: 10.1097/ACM.0000000000003317

Copyright (C) 2020 by the Association of American Medical Colleges

Supplemental digital content for this article is available at http://links.Iww.com/ ACADMED/A845.

Year school was established: 1765.

School URL: https://www.med.upenn.edu/psom/education. a diverse and talented group of socially conscious learners, many with prior experiences that encourage maturity and promote leadership skills. PSOM has developed a culture of inclusion fostering our community as a whole, while directly supporting affinity groups.

\section{Assessment}

Almost all student assessment at PSOM fits into 1 of 4 categories: preclerkship formative, clinical formative, preclerkship summative, and clinical summative. For graduation, students are required to pass a 12-station summative OSCE as well as USMLE Step1, Step 2 CK, and Step 2 CS examinations. Students not pursuing a dual degree complete a required, 3-month Scholarly Pursuit capstone.

See List 2-Description of Assessment Methods.

Assessment methods have evolved continuously since 2010. We now use more secure exam software allowing for more robust and flexible testing. In preclerkship courses, team exams count toward the final course grade. We have transitioned to a Pass/ Fail system in the PCC, while maintaining high performance standards. All clerkships except one have incorporated a multistation OSCE.

\section{Pedagogy}

The PCC at PSOM, spanning 3 semesters, is the first part of a modular, vertically and horizontally integrated curriculum, and it includes content from modules 1, 2, 3, and 6. The first semester covers core principles across module 1 (basic science), module 3 (technology and practice of medicine), and module 6 (professionalism and humanism).

See Supplemental Digital 1-Modular Curriculum—at http:// links.lww.com/ACADMED/A845.

The second and third semesters cover integrative systems and disease across modules 2 (pathophysiology), 3, and 6. Smallgroup sessions, almost always conducted in learning teams, are the core of PCC learning. Near-peer support is provided in small-group facilitation, anatomy sessions, and tutoring opportunities.

- Didactic learning: Faculty provide lecture slides and notes with learning objectives, an organizational road map, and are asked to "chunk" content in 2-4 segments, answering questions after each segment. Attendance is optional, except for live-patient clinical encounters.

- Small-group team-facilitated learning: Small groups are student directed with faculty facilitation. Attendance is required. Learning teams work at their own pace and the House system provides additional academic and wellness support. 


\section{List 1}

\section{MD+ Programs}

\section{Dual degree (generally 5+ years)}

- MD-PhD

- MD-JD

- OMFS

- MD-MTR

- MD-MBA

- MD-MBE

- MD-MPH

- MD-MS

- MD-ML

- MD-HPR

- MD-Non-Penn Masters

\section{Certificate (4 years)}

- Research Year

- Medical Education

- Academic Surgery

- Healthcare Management, Entrepreneurship, and Technology

- Public Health

- Clinical Neuroscience

- Bridging the Gaps

- Global Spirituality

- Self-directed learning: In module 2, selected small-group sessions have a student-organized "teaching point," a 5 -minute presentation on a topic related to, but not covered in concurrent course materials. Each module 2 course also has self-directed learning topics, not formally covered in any lecture or small group, but featured on exams.

- Hands-on learning and simulation: The Introduction to Clinical Medicine (ICM) course provides direct, hands-on, interprofessional and experiential learning via standardized patients (SPs) and simulation. Bedside ultrasound is integrated across the curriculum.

- Longitudinal patient care: The Longitudinal Experience to Appreciate Patient Perspective (LEAPP) and the Elder Home Care Experience (EHCE) are both required. LEAPP is integrated with the Health Care Systems course in the PCC, and EHCE is integrated with the Doctoring course.

\section{Curriculum changes since 2010}

- Paperless course materials: students receive iPads for syllabi, slides, and notes (2011)

- Ultrasound Diagnostics course (2015)

- Cancer Biology 1-week foundations course, followed by oncology content throughout the curriculum (2016)

- "Inside Out": fully integrated with ICM, anatomy, ultrasound, and radiology (2017)

- "Yoganatomy": integration of anatomy and wellness curriculum (2017)
- Culinary medicine, art and medicine, narrative medicine preclerkship electives (2019)

- Preclerkship week to facilitate transition to clerkships (2019)

- The Measey Primary Care Pathway Program for students interested in pursuing careers in family medicine, outpatient general pediatrics, general internal medicine, and/or geriatrics (2019)

\section{Clinical experiences}

PSOM introduces clinical experiences early in the curriculum. During orientation, an accomplished Penn physician-scientist describes a clinical/scientific discovery and then introduces the students to a relevant patient. Students interact with patients during the Doctoring and ICM courses. We continue to introduce earlier patient contact and opportunities for interprofessional team-based learning.

All clerkships have some didactic component in addition to immersion in the clinical environment, and many use selfdirected learning and flipped classroom sessions. Since 2010, we have introduced a high-value care curriculum in the internal medicine clerkship, a simulation bootcamp session for senior students in pediatrics, and an expanded 2-week surgery bootcamp, focusing on simulation experiences and practical skills.

All students rotate through a variety of inpatient and outpatient clinical sites within the University of Pennsylvania Health System, including The Hospital of the University of Pennsylvania, Penn Presbyterian Medical Center, and Pennsylvania Hospital; along with several affiliated hospitals, including Veteran's Affairs Medical Center and Children's Hospital of Philadelphia. Students are exposed to diverse patient populations with general and specialized diseases. There are opportunities to rotate at community hospitals and clinics in the tristate area.

Outpatient experiences are integral within the clerkship year, including a 4-week clerkship in family medicine and a 3-week outpatient pediatrics clerkship, along with outpatient weeks in others. Students have multiple volunteer outpatient experiences. Close to $70 \%$ of students participate at community health clinics; and approximately $30 \%$ of students participate in 4- to 6-weeklong global clinical experiences in locations such as Botswana, Peru, Tanzania, and Guatemala. Students can participate in service-learning opportunities or may complete an externship learning from Penn's Community Health Worker program. In 2019, a group of students participated in a pilot emergency medical training program, with follow-up longitudinal outpatient experiences working alongside city emergency support services.

\section{Challenges in designing and implementing clinical experiences for medical students}

Multiple factors affect a student's medical education experience. PSOM has worked to limit the impact of identified challenges:

- Our structure of student placement and faculty oversight at the clinical sites mitigate the challenges of high-turnover inpatient and rapid-paced outpatient clinical environments.

- Scheduling and selection of highly rated teaching sites help us address the challenges of limited outpatient clinic space. 


\section{List 2}

\section{Description of Assessment Methods}

\section{Competencies}

Science of medicine: Bioscientific basis of disease

- Epidemiologic approach to medicine

- Decision-making/evidence-based medicine

- Information systems

- Health care systems

- Research principles and methodology

Technology and practice of medicine

- Clinical practice of medicine including diagnosis management and treatment

- Prevention and nutrition

- Self-directed learning

- System-based practice

Humanism and professionalism

- Professional skills and attitudes

- Intra- and interprofessional collaboration

\section{Assessments: Formative}

Preclerkship

- Small-group, case-based discussion

- Problem sets

- Journal club discussions in Module 1

- Reflective writing assignments (in preparation for and during smallgroup discussions)

- Teaching point presentations during Doctoring course

- 1-on-1 meetings with Doctoring preceptors

Clinical

- Small-group work

- Patient write-ups

- Simulation

In some clerkships:

- Feedback cards

- History taking/physical exam

- Oral case presentation

- Written documentation

- General skill

- Evidence-based medicine

- Mid-clerkship feedback review

\section{Assessments: Summative}

Preclerkship

- Multiple choice question exam (some with matching and shortanswer, tending to be team based)

- LEAPP self-reflection papers

- Elder home-visit reflection

- Doctoring capstone and disability assignments

- Two standardized patient exams: history/history and physical

- Self-directed learning activities

Clinical

- End-of-clerkship exams (NBME, Aquifer, in-house)

(List continues)
List 2

(Continued)

- OSCES

- Preceptor and residents' ratings and narrative

- All clerkships > 4 weeks have other requirements, for example, an EKG quiz, presentation, ultrasound logs

Other

- Penn Summative Clinical Skills Inventory (12 standardized patient exams)

- USMLE Step 1

- USMLE Step 2 CK

- USMLE Step 2 CS

- Scholarly Pursuit project

- We have created simulation and SP satellites to accommodate just-in-time training needs.

- We have monitored and addressed inconsistencies in clinical education across sites.

- We collaborate with GME and faculty affairs to clarify student roles on teams.

- We prioritize Penn's students' education within our health system.

Opportunities exist to make the expectations, assessment, and evaluation processes more cohesive.

\section{Curricular Governance}

PSOM's curricular governance is evolving to ensure centralized oversight of operational groups. The Education Council is charged by the dean to maintain excellence and improve the efficiency of the many programs that make up the entirety of Penn Medicine, which includes the health system and PSOM. The Medical Faculty Senate (MFS) remains the formal instrument exercising authority with respect to admissions, educational affairs, and governance. MFS has delegated responsibility to the Undergraduate Medical Education Committee (UMEC) to review all matters related to UME including curriculum, assessments, and policy. Two subcommittees report to UMEC: the Program Evaluation Committee (PEC) and the Policy Committee (PC). PEC must annually review all curricular elements of modules, courses, and clerkships, with triennial in-depth review. The PC is tasked with ongoing evaluation and maintenance of educational policy.

See Figure 1-Curricular governance overview.

\section{Education Staff}

The Office of the Senior Vice Dean for Medical Education (SVDME) is the central administrative body responsible for UME and continuing medical education, with an integrated and collaborative relationship with GME. The SVDME and the chief operating officer oversee faculty administrators and staff responsible for admissions, curriculum, student affairs, student success and development, inclusion and diversity, assessment, community engagement, and wellness. 


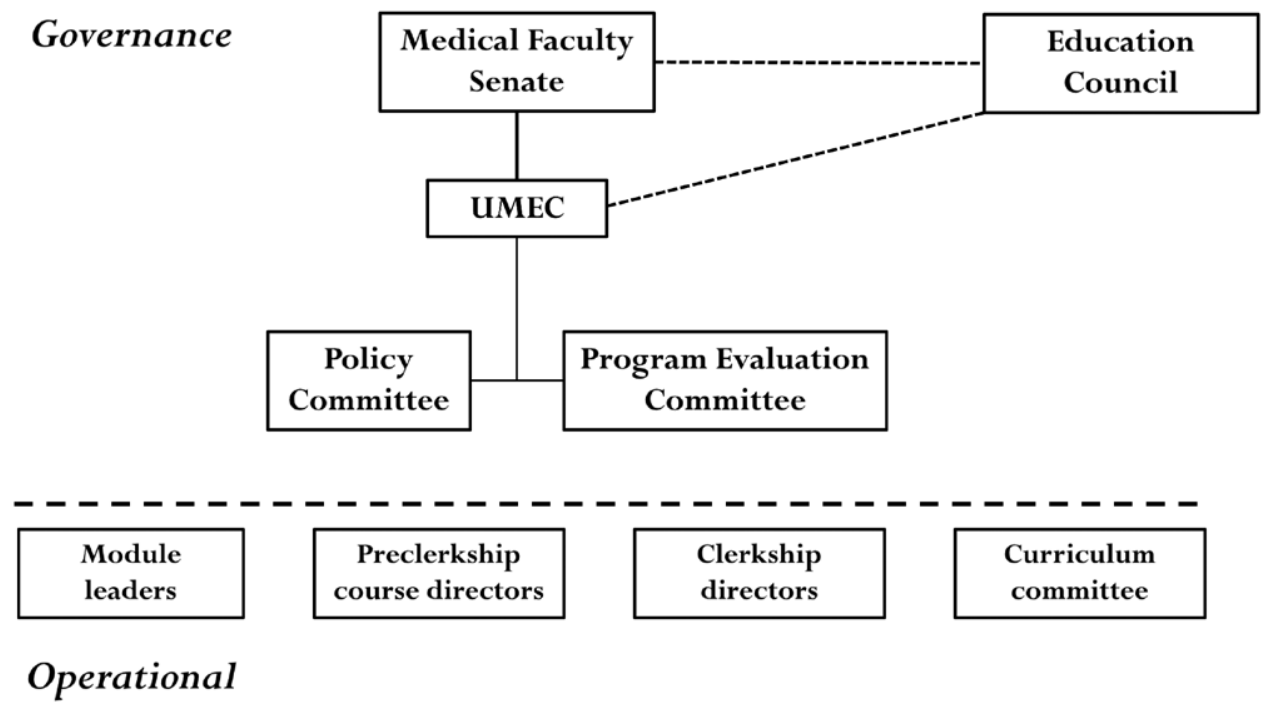

Figure 1 Curricular governance overview.

See Figure 2-Education team organizational chart.

Each associate dean works with staff to perform relevant tasks such as those related to admissions, curriculum, assessment, and student affairs. In total, there are 30 staff working with 7 associate deans and 4 assistant deans. Cross-collaboration is expected and facilitated with weekly UME leadership team meetings. Collectively, the office is responsible for providing support for the course and clerkship directors in the planning, implementation, and oversight of the curriculum.
Strategic direction for clinical education is under the supervision of the associate dean for UME clinical and health systems sciences curriculum and, along with scheduling clinical experiences, is managed centrally. Implementation and day-to-day management of the required clinical clerkships and electives rest with the clinical departments.

\section{Faculty Development and Support in Education}

Teaching is an essential PSOM mission. All faculty on the tenure, clinician-educator, and/or academic clinician tracks must submit

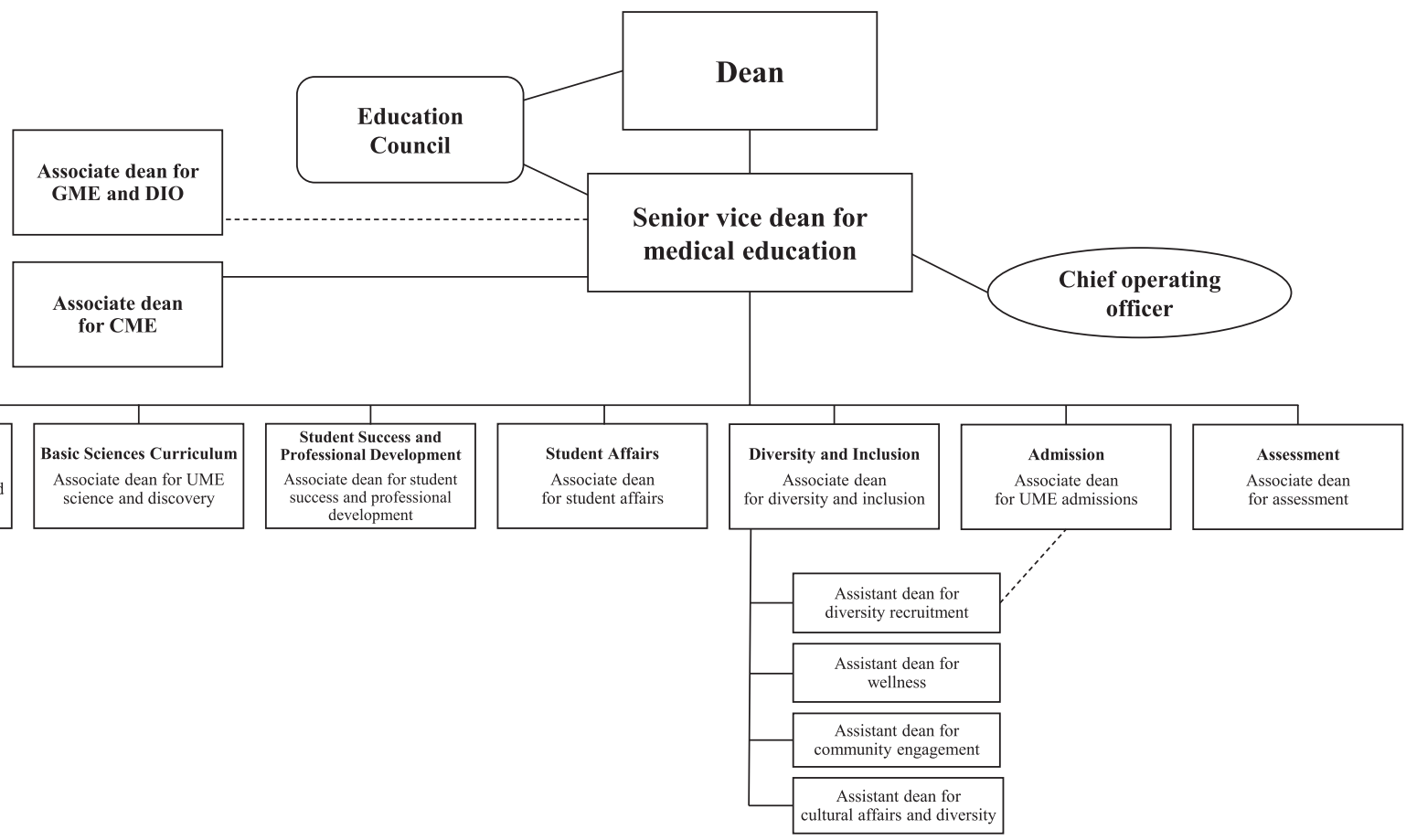

Figure 2 Education team organizational chart. 
teaching evaluations and a descriptive self-assessment during reappointment and promotion opportunities. We have developed an improvement plan for situations where faculty might benefit from enhancement of teaching skills. Our faculty have scored a nearly perfect 4.0 on a scale of $0-4$ in over 10,000 documented student evaluations.

The Faculty Life and Professional Development "Advance" Program is designed to orient faculty to institutional and learning expectations, support pedagogically sound best practices, develop core competencies in teaching, and provide resources and skill-coaching for faculty. All new faculty are required to complete the Onboarding for Teaching Program.

The overriding objective of the university's procedures for academic appointments and promotions is the recruitment and retention of a distinguished faculty. This objective stresses intellectual leadership as the chief criterion for academic advancement. Accordingly, a high degree of excellence is expected at PSOM in both research and teaching for members of standing faculty. Historically, teaching at Penn was judged by student evaluations of faculty teaching. Currently, we are looking to increase the presence of peer faculty evaluations as well as other assessment modalities including student focus groups. Notably, Penn created an academic clinician track in 2004 to recognize the excellent clinician-teachers at Penn. Advancement comes through excellence in clinical work and teaching, along with a recognized area of concentration.

\section{Initiatives in Progress}

Within the past 2 years, our restructured organization in UME has created a collaborative matrix of staff working with an effective faculty leadership team to support our students and curriculum. We are iteratively evaluating our curriculum as we explore additional innovations and will continue our reflective process to drive the future of our educational programs. Working groups of the newly convened Education Council will be charged with improving our curriculum and assessments, educational scholarship productivity, and use of technology, while leveraging partnerships with the Penn community, our interprofessional colleagues, and our regional clinical partners.

PSOM continues to build upon our legacy of excellence in education with outcomes of distinction supported by student and faculty satisfaction, student performance on internal and board examinations, our Match results, and by surveys of residency program directors of our graduates' performance. We are committed to training future-ready physicians who will serve as leading advocates for advancing science, discovery, and health care while furthering all relevant missions for our patients, communities, and society. 


\section{Sidney Kimmel Medical College at Thomas Jefferson University}

Deborah Ziring, MD, Katherine Berg, MD, Nina Mingioni, MD, Dimitrios Papanagnou, MD, MPH, Urvashi Vaid, MD, and Steven Herrine, MD

\section{Medical Education Program Highlights}

Established in 1824, the Sidney Kimmel Medical College (SKMC) of Thomas Jefferson University (originally Jefferson Medical College) is a large, private medical college in Philadelphia. The first U.S. medical school to incorporate bedside teaching, SKMC has long held the reputation for producing superior clinical graduates. The school is distinguished in the quantity and depth of student community engagement.

SKMC recently underwent a significant curricular redesign (JeffMD), transitioning from a $2+2$ paradigm to a 3 -phase program integrating fundamental science, clinical science, and health systems science (HSS) throughout all 4 years.

Highlights:

- Varied pedagogical approaches with tight integration of clinical and scientific concepts in small groups and limited lecture hours

- Utilization of frequent low-stakes assessments

- Robust scholarly inquiry requirement for all students in one of several tracks: clinical and translational research, design, digital health, health policy and systems, humanities, medical education, population health

- Competency-based final curricular phase with pathways to teach and assess general and specialty competencies linked to graduate medical education milestones

- Home of the Jefferson Longitudinal Study of Medical Education

\section{Curriculum}

\section{Curriculum description}

The JeffMD Curriculum consists of a preclerkship phase (Phase 1), core clerkship phase (Phase 2), and specialization phase (Phase 3).

See Supplemental Digital Appendix 1-Curriculum Overviewat http://links.lww.com/ACADMED/A849.

See Supplemental Digital Appendix 2-Curriculum Schematic With Structure and Sequencing-at http://links.lww.com/ ACADMED/A849.

\section{Curriculum changes since 2010}

- JeffMD curriculum successfully launched in 2017 with complete implementation in 2021

Acad Med. 2020;95:S444-\$448.

doi: 10.1097/ACM.0000000000003326

Copyright (C) 2020 by the Association of American Medical Colleges

Supplemental digital content for this article is available at http://links.Iww.com/ ACADMED/A849.

Year school was established: 1824.

School URL: https://www.jefferson.edu/university/skmc.html.
- Increased use of active learning techniques with $>40 \%$ reduction of lectures

- Customization opportunities via scholarly inquiry tracks and specialty pathways

- Centralization of curricular development, administration, assessment, and quality control

Future changes:

- Expand assured admission programs to increase student body diversity

- Optimize application of HSS concepts through early clinical experiences

- Adopt competency-based assessment and learner advancement

Class size changes since 2010

While class size has increased from approximately 260 to 270 students, JeffMD offers more opportunities for small-group active learning compared with our legacy curriculum. SKMC introduced a regional campus in 2017 that includes a 6-student longitudinal integrated clerkship (LIC).

\section{Medical education program objectives}

Developed and adopted in 2000 with reference to AAMC Medical School Objectives Project Guidelines (2000), SKMC graduation competencies and linked medical education program objectives were revised in 2016 referencing AAMC core competencies and Entrustable Professional Activities (EPAs) and ACGME transitional year milestones.

See Table 1-Medical Education Program Objectives and Assessment Methods.

In 2017, the SKMC Student Assessment Office was created to provide oversight of all student assessments. With launch of the JeffMD curriculum, SKMC developed the Jefferson Competency Assessment Tool (JeffCAT), a secure online platform that aggregates student assessment data from multiple modalities, providing an integrated portrait of student performance throughout the curriculum. JeffCAT benchmarks student performance to SKMC graduation competencies, EPAs, and ACGME milestones. Administrators and students can track progress longitudinally to identify areas of strength and opportunities for improvement.

For Phases 2 and 3, SKMC instituted the Jefferson Direct Observation Tool (JeffDOT), a mobile phone-based application for bedside use, to encourage frequent microassessments and track student progress. JeffDOT uses checklists that, in aggregate, are designed to stratify learners' skill level and provide evidence of progress toward workplace competence.

Additional JeffMD assessment changes by phase are described below. 


\section{Table 1}

\section{Medical Education Program Objectives and Assessment Methods}

\begin{tabular}{|c|c|}
\hline $\begin{array}{l}\text { Medical education } \\
\text { program objectives }\end{array}$ & Assessment method \\
\hline $\begin{array}{l}\text { Competency 2: Knowledge for } \\
\text { practice }\end{array}$ & $\begin{array}{l}\text { MCQs, peer assessment and facilitator rating scale, rating scale, student presentations, research reports, critical } \\
\text { reading papers }\end{array}$ \\
\hline $\begin{array}{l}\text { Competency 3: Practice-based } \\
\text { learning and improvement }\end{array}$ & Direct observation, rating scale (end-of-course evaluation form), critical reading papers \\
\hline Competency 5: Professionalism & $\begin{array}{l}\text { Direct observation, rating scale (end-of-course evaluation form), peer assessment and facilitator rating scale, } \\
\text { clinical skills small group, OSCE, standardized patient checklists }\end{array}$ \\
\hline $\begin{array}{l}\text { Competency 6: System-based } \\
\text { practice }\end{array}$ & Rating scale, simulation \\
\hline $\begin{array}{l}\text { Competency } 7 \text { : Interprofessional } \\
\text { collaboration }\end{array}$ & Rating scale (end-of-course evaluation form), rating scale (Jefferson team observation scale), direct observation \\
\hline
\end{tabular}

Phase 1:

- Computer-based administration of assessments (quizzes, exams, case-based learning [CBL] peer assessments) through educational technology

- Weekly low-stakes quizzes to assess mastery of information

- Rubric grading for CBL, including peer and facilitator assessments

- Longitudinal tracking of student performance on the NBME Comprehensive Basic Science Self-Assessment over 3 administrations

- End-of-block, end-of-year 1, and end-of-Phase 1 OSCEs

Phase 2:

- Work-based structured assessment of clinical skills longitudinally

- Project-based assessments in scholarly inquiry

- Additional end-of-clerkship OSCEs

- Integration of NBME subject exams into clerkship assessment structure

- End-of-clerkship evaluation mapped to SKMC competencies

- End-of-Phase 2 OSCE

Phase 3:

- End-of-course evaluations mapped to SKMC competencies

- Project-based assessments in scholarly inquiry and advanced basic science courses

- Group simulation experiences

- Implementation of an end-of-Phase 3 OSCE

- Work-based structured assessment of clinical skills in required Phase 3 courses

\section{Parallel curriculum or tracks}

In 2017, SKMC launched the LIC at the Atlantic Health System, which emphasizes outpatient care, integration between disciplines, and longitudinal relationships with faculty and patients.

SKMC plans to offer a 3-year graduation option for 8-12 students, linked with graduate programs at Thomas Jefferson University Hospital (TJUH), beginning in 2021.

\section{Pedagogy}

Various pedagogical approaches are used throughout the 4-year curriculum. Simulation, standardized patients, lectures, and small- and large-group discussion are employed during all phases. Students have in-patient and ambulatory experiences in all phases. Peer teaching occurs during CBL and teambased learning (TBL) in Phase 1 and continues during patient rounds in Phases 2 and 3. Self-directed learning is specifically incorporated into $\mathrm{CBL}$ during the preclerkship phase and occurs routinely during the clinical curricular phases.

Some pedagogical approaches are limited to specific phases. In Phase 1, the cornerstone of the curriculum is small-group CBL using a guided inquiry approach. TBL is extensively incorporated as well. The Phase 1 communication skills curriculum uses role plays and a preceptorship. Gross anatomy dissection and virtual microscopy labs occur in Phase 1 as well as during advanced basic science courses in Phase 3.

SKMC moved from a predominantly lecture-based curriculum to a longitudinally integrated, case-based organ system curriculum requiring close collaboration between basic and clinical scientists. SKMC now uses more active learning approaches including CBL, TBL, audience response, flipped classrooms, and patient panels. A longitudinal scholarly inquiry track was added to teach and foster research principles; humanities selectives were expanded. 


\section{Clinical experiences}

All required clinical experiences occur at either TJUH or one of several affiliated community-based academic medical centers. Each offers exposure to a diverse patient population and an opportunity to see medicine practiced in a variety of settings. Students can participate in one of our branch campus programs, which focus most clinical rotations at a particular community site. Christiana Care Health System in Delaware serves as a regional medical campus, providing students in the Delaware Branch Campus program with clinical experiences. Students enrolled at Main Line Health Branch Campus do most clinical rotations at the hospitals of the Main Line Health Systems. Students enrolled in the LIC complete their clinical requirements at the Atlantic Health System in New Jersey. However, most students will complete core clerkships at both TJUH and community-based academic affiliates.

\section{Required longitudinal experiences}

Students in the LIC receive longitudinal clinical exposure with a small host of dedicated preceptors in major specialties. All students have a 4-year scholarly inquiry longitudinal experience incorporating research projects and mentoring.

\section{Clinical experience first encounter}

SKMC students' first clinical exposure is 4 months into the first year, when they partner with community health workers at outpatient sites for first-hand exposure to the challenges patients face and ways social determinants affect patient care and health care delivery.

\section{Required and elective community-based rotations}

SKMC does not require any community-based rotations. However, most students complete at least part of their required core clerkships or fourth-year rotations at an affiliated community-based academic medical center.

\section{Challenges in designing and implementing clinical experiences for medical students}

Considerable effort is invested to maintain the number and quality of clinical sites for students to ensure they receive outstanding clinical teaching within the geographic area despite the high demand for clinical placements.

\section{Curricular Governance}

\section{Decentralized curricular governance}

- There is granular departmental involvement in core clerkships through the clerkship directors and coordinators, although there is central oversight of Phase 2.

- The LIC, due to significant administrative need and its location remote from the main campus, has considerable autonomy in its functions.

- Most of the educational budget is administered centrally, although a portion is within control of the departments.
See Figure 1-Office of the Dean administrative organizational chart.

\section{Education Staff}

- The office staff includes 1 vice dean, 1 senior associate dean, 3 associate deans including 1 dean of professionalism, 1 business manager, 2 administrative assistants, and 5 educational coordinators.

- A curriculum technology specialist performs curricular mapping, technologic tools training, and building/problemsolving with the learning management system.

- An assistant dean of assessment, director of student assessment, senior psychometrician, and 2 data analysts comprise the Undergraduate Medical Education Student Assessment Office.

- The Office of Academic Affairs works closely with the SKMC Center for Research in Medical Education and Health Care, particularly on the Jefferson Longitudinal Study of Medical Education.

- A faculty member is supported part time as phase director for each of the 3 phases of the curriculum, ensuring longitudinal integration and spiraling of content.

- The office is responsible for medical student curriculum planning and evaluation, student promotion and remediation processes, accreditation activities, and affiliate collaborations.

- The administrative faculty and staff in the office are responsible for undergraduate and graduate medical education.

- It is not responsible for areas outside of educational programming.

See Figure 2-Curricular governance committees.

\section{Faculty Development and Support in Education}

\section{Professional development for faculty as educators}

The Office of Faculty Affairs and Faculty Development (OFAFD) provides a multifaceted faculty development program offered continuously across the academic year and features content across 3 thematic domains to assist faculty with their career advancement: effective teaching, research skills, and leadership and management. Curricular content is curated by an interdisciplinary, interprofessional cadre of faculty members across the 10 schools of the university. Content represents domains of expressed need for faculty skills, with a particular focus on pedagogy. The program includes a series of in-person workshops, with remote access for affiliate faculty. Sessions are recorded and archived, and remain available for asynchronous access. Faculty are eligible to earn continuing medical education credits for participation. In addition, a series of interactive modules in pedagogy are developed and made available for faculty.

The OFAFD sponsors its annual signature Faculty Days Program, a full-day interprofessional multidisciplinary event consisting of presentations by and for Jefferson faculty who share their 


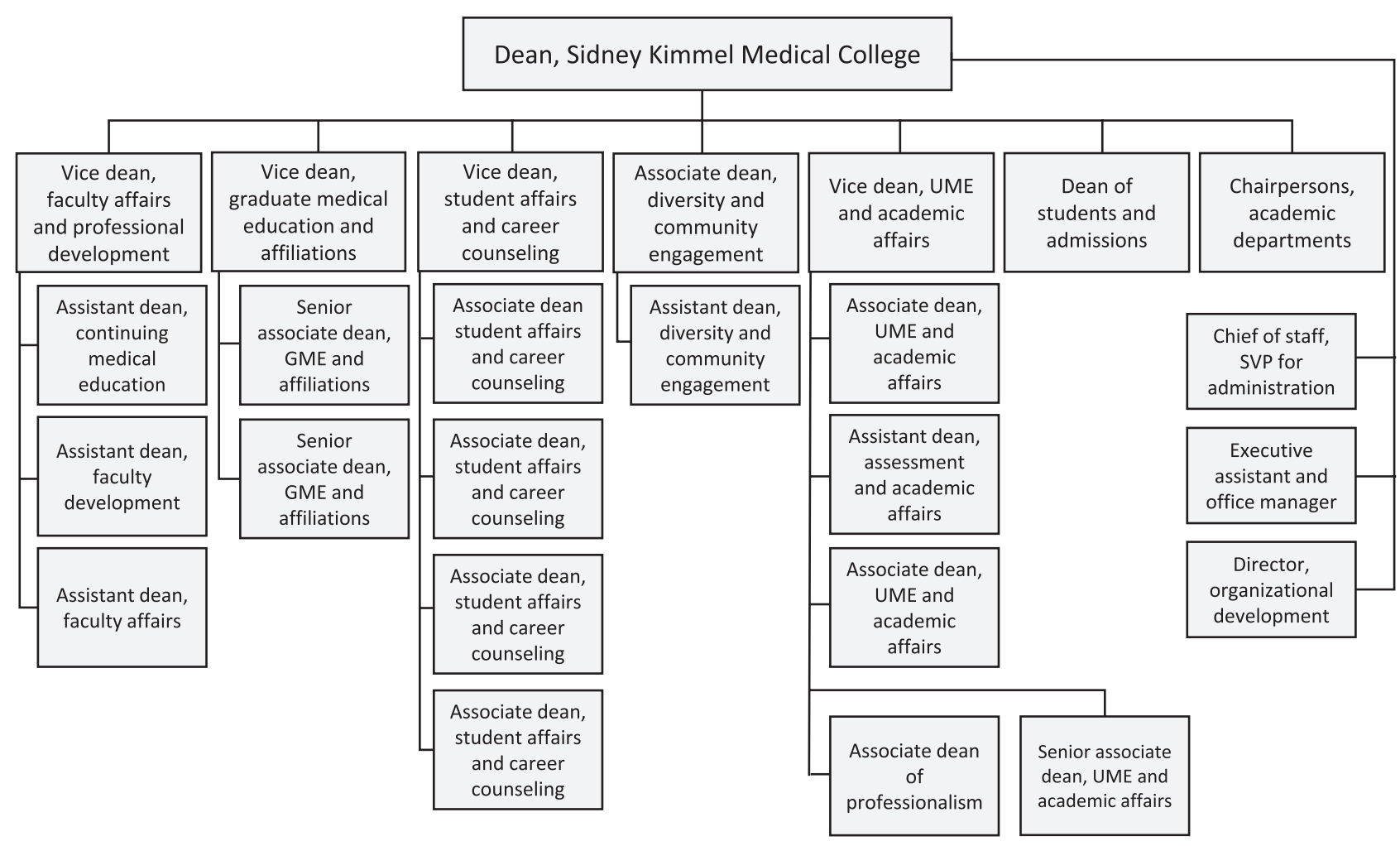

Figure 1 Office of the Dean administrative organizational chart.

knowledge and strategies to improve teaching and learning for learners across the continuum.

Most recently, to equip faculty with skills to facilitate CBL sessions in the setting of the revised curriculum, the OFAFD developed an immersive 4-part multimodal training program. Part 1 offered faculty a series of asynchronous modules covering principles of facilitation, including peer-vetted resources. Part 2 provided faculty with video recordings of actual CBL sessions modeled by seasoned faculty facilitators with medical students. Part 3, an in-

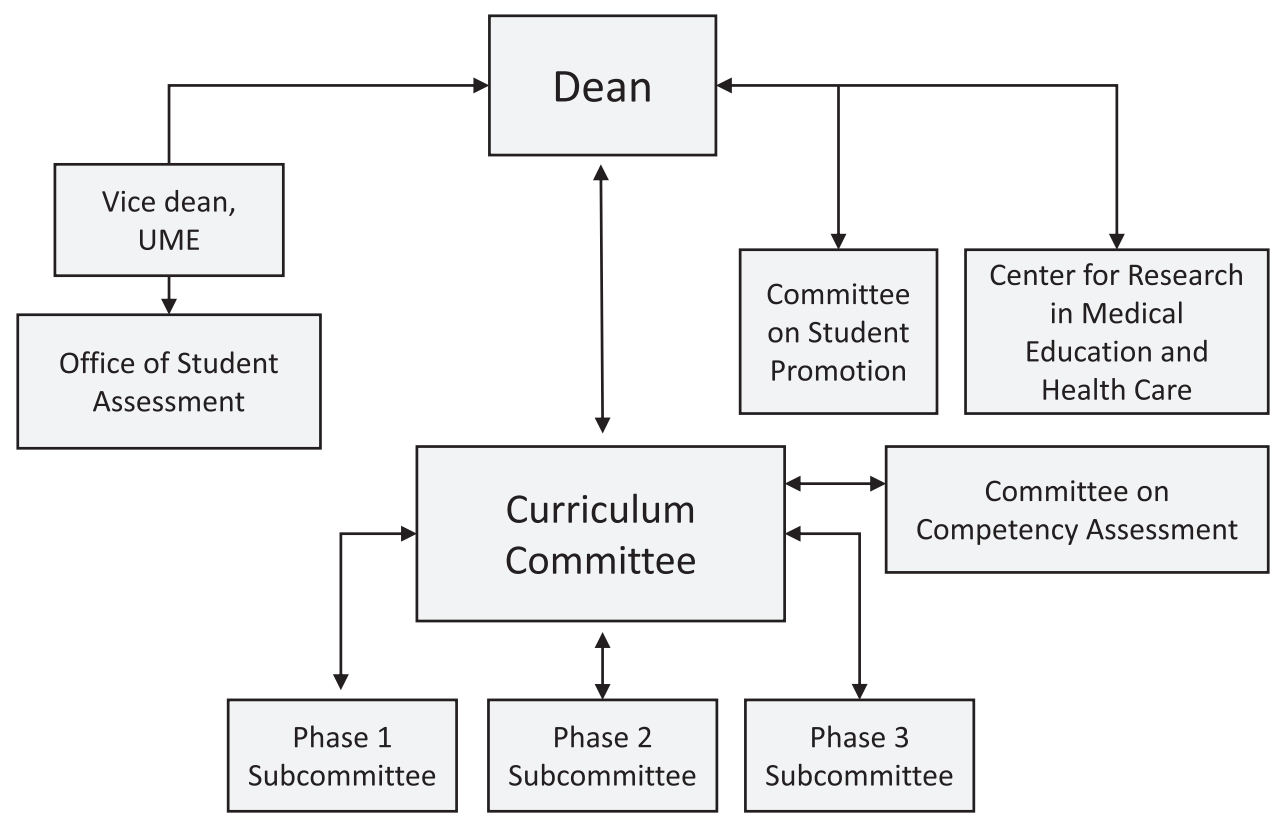

Figure 2 Curricular governance committees. 
person workshop, immersed faculty in a simulated CBL session, followed by a large-group debriefing. An optional Part 4, offered to faculty weeks before their first session (just-in-time), was executed through co-facilitation with expert facilitators.

\section{Role of teaching in promotion and tenure}

Faculty contributions to the educational mission of SKMC are heavily weighted in promotion and tenure decisions. Contributions to teaching are evaluated through faculty members' education and teaching portfolios, which are reviewed during appointment and promotion procedures. Excellence in teaching remains a critical criterion for promotion across all tracks at SKMC. Furthermore, educational scholarship can be leveraged for promotion and tenure decisions in scholarshipbased tracks in the medical school.

\section{Regional Medical Campuses}

The Delaware Branch Campus offers all core clerkships, as well as required fourth-year rotations such as subinternships, to 6-8 students each academic year. The Atlantic Health System
Branch Campus is the site for the LIC, enrolling 6 students each academic year; expansion up to 12 students per year is under consideration.

\section{Educational experiences across sites}

The Delaware Branch Campus Program and the LIC program are relatively local, allowing participating students to commute to the SKMC campus for required educational activities and assessments. Each clerkship has a single set of educational objectives that are reviewed annually with the branch campus clerkship site directors, who are accountable to the main clerkship directors. The LIC Program was designed to meet SKMC educational goals and objectives. Patient logs, with identical targets as those for the rest of the student body, are used to monitor clinical experiences. All students submit standard end-of-clerkship questionnaires after every clerkship and, at each site, the results of which are used to compare student experiences across sites and assure comparability of educational experiences. Finally, a student-run clerkship liaison program regularly queries the student body about their clinical experiences and reports their findings to the core clerkship phase director. 


\section{Ponce Health Sciences University School of Medicine}

Georgina Aguirre, MD, and Olga Rodriguez de Arzola, MD

\section{Medical Education Program Highlights}

The Ponce Health Sciences University School of Medicine (PHSU-SOM) is a 4-year program emphasizing primary care and lasting 166 weeks. It is in Ponce, a city on the southern coast of Puerto Rico, a U.S. territory subject to Puerto Rican and U.S. regulations.

PHSU-SOM was founded in 1977 and has graduated more than 2,100 physicians. The school has successfully educated first-generation college students from low socio-economic status, thereby changing financial outcomes and educational opportunities for generations.

PHSU-SOM offers bilingual (Spanish-English) education, contributing significantly to training the physicians necessary to care for the growing Hispanic population in the United States. All alumni are fully bilingual (English-Spanish).

PHSU-SOM's research enterprise makes a significant contribution in addressing health disparities for the Hispanic population (e.g., developed the first Puerto Rican tumor tissue repository, uncovered breast cancer predisposition factors for Puerto Rican women). The medical program offers various opportunities for participation in service learning activities through the established community medicine curriculum and student interest group organizations, across all years of study.

\section{Curriculum}

\section{Curriculum description}

See Supplemental Digital Appendix 1-Curriculum Schematicat http://links.lww.com/ACADMED/A968.

\section{Curriculum changes since 2010}

PHSU-SOM Curriculum Committee has implemented curricular changes during the past 10 years. The most relevant are:

- Flipped classroom: In 2014, the Medicine Program Curriculum Committee (MPCC) started a pilot project to adopt the flipped classroom model for the preclinical curriculum, to decrease lecture time and incorporate more active learning strategies. Students' satisfaction with this modality increased, which was adopted for most preclinical courses.

Acad Med. 2020;95:S449-S453.

doi: 10.1097/ACM.0000000000003483

Copyright (C) 2020 by the Association of American Medical Colleges

Supplemental digital content for this article is available at http://links.Iww.com/ ACADMED/A968.

Year school was established: 1977.

School URL: https://www.psm.edu/school-of-medicine.
- Professional development course: Designated time for students' exposure to general medical education and humanism topics was converted to a required course in 2013, with 5 major components: history of medicine, cultural traditions of the community, translational research, medical practice, and preparation for residency.

- Introduction to Clinical Practice: Patient and student safety and quality improvement topics were incorporated into this course, including measures to protect students during encounters with patients with aggressive behaviors.

- Simulation laboratory: Faculty incorporated high-fidelity simulators for teaching clinical skills and medical management in the clerkships, including a birth simulator.

- Interprofessional Perspectives in Health Disparities: The MPCC approved a 12-module, 1-credit course for first-year students on social health determinants and health disparities.

- Interprofessional education: Clinical psychiatry and pediatric clerkships incorporated interprofessional collaborative experiences in the outpatient clinic and hospital. Other health professions students or practitioners involved include psychology $\mathrm{PhD}$ and PsyD interns and social work, nursing, or medical record technician students. For example, during the pediatric clerkship, students participate in managing patients during ward rounds with pediatricians and psychologists, then reflect and agree an integrated response to patient and family needs.

- Longitudinal radiology: In 2019, PHSU-SOM initiated a thirdyear course with online modules including short recorded lectures organized by common conditions and radiological studies seen in the 6 third-year clerkship specialties.

\section{Class size changes since 2010}

PHSU-SOM admitted 66 students in 2010 increasing to 100 students in 2019. In 2019, a 20-student regional clinical campus was opened in preparation for increased numbers in the clinical years.

\section{Assessment}

PHSU-SOM medical education objectives are based on ACGME domains of competence.

See Supplemental Digital Appendix 2-Program Objectives and Assessment Methods—at http://links.lww.com/ACADMED/A968.

\section{Assessment changes since 2010}

- Using iClickers audio response system for continuous assessment of student understanding

- Using NBME customized assessment for physiology and infectious disease course examinations

- Administering NBME comprehensive basic sciences examination at least 5 times during the second semester of year 2, to provide progress measurement in preparation for USMLE Step 1 examination 
- Opening a 1-semester independent study course for reviewing basic sciences for students needing additional time for passing USMLE Step 1 examination

- Incorporating the NBME shelf examination in the family medicine clerkship

- Increasing weight of the NBME clinical subject examination for final clerkship grades to $30 \%$, using the equated percent score

- Assigning a weight of 5\%-20\% of final grade to objective structured clinical examinations for every third-year clerkship

- Incorporating a professionalism assessment form for all clerkships and assigning it a 5\% weight for all core clerkships final grade

- Creating an interdisciplinary faculty committee for evaluating and correcting exam questions, including evaluation of content, quality, and organization

\section{Pedagogy}

PHSU-SOM uses various pedagogical approaches to achieve program objectives:

- Case-based learning

- Clinical experience: ambulatory

- Clinical experience: inpatient

- Discussion: large group (>12)

- Discussion: small group $(\leq 12)$

- Laboratory

- Lecture

- Preceptorship

- Problem-based learning

- Role play/dramatization

- Self-directed learning/tutorial

- Simulation

- Standardized/simulated patients

- Structured community service learning

- Video/podcast

- Workshop

\section{Changes in pedagogy since 2010}

With incorporation of the flipped classroom, in-class sessions are devoted to assessing student understanding and discussing difficult topics through cases, think-pair-share, games, and other active learning modalities. More short patient videos have been incorporated in family medicine and psychiatry preclinical classes. Computer cases have been incorporated in all clerkships to complement clinical experiences. Clerkships have begun including exercises in the simulation laboratory to expand teaching advanced skills.

\section{Clinical experiences}

PHSU-SOM is affiliated with several hospitals and health care facilities, most in the southwest of Puerto Rico. The school also owns an outpatient clinic (the PHSU Wellness Center) on its main campus that serves as a teaching site for clinical clerkships and the psychiatry residency program. The affiliation network provides over 2,000 beds and several ambulatory facilities for clinical teaching and training.

\section{Required longitudinal experiences}

Several longitudinal experiences along the 4 years of study have been developed since 2010 .

- Geriatrics: Students are exposed to a longitudinal geriatrics curriculum including didactic and experiential activities to train skilled professionals who can deal competently and humanely with health problems of the elderly population, especially in Hispanic communities. In the first 2 years, through lectures and small-group discussions, basic concepts of geriatrics, gerontology, the aging process, and geriatric pharmacology are introduced. During the first year, students perform a medical history of an elderly person in a community setting. After a lecture on falls in the elderly, students participate in a community activity to assess risk of falls and educate participants on fall prevention. In the third year, they learn the comprehensive geriatric assessment during the family medicine clerkship and perform at least one supervised comprehensive assessment. In the internal medicine clerkship, students participate in small-group discussions about geriatric syndromes: dementia, dizziness, pressure ulcers, and urinary incontinence.

- Research: Basic scientific principles of clinical and translational research are addressed through several courses and clerkships. Through case discussions, literature review, scientific papers discussions, and workshops on evidencebased medicine, students in the preclinical curriculum in medical ethics, behavioral sciences, biochemistry, pharmacology, epidemiology, and biostatistics, are introduced to principles of the scientific method and applying results for prevention and treatment of diseases. In third-year clinical clerkships, students present patient cases and seminars including relevant research studies. In the third-year professional development course, PHSU faculty involved in research present lectures on topics such as the institutional review board process and grant writing skills, among others. Fourth-year students can do elective rotations in their area of research interest.

- Professionalism: Professionalism is taught and evaluated across the curriculum. During the first week of classes, the topic is introduced in the community medicine course, where societal expectations of physicians are discussed followed by a small-group discussion of cases relevant to first-year students. In the anatomy course, faculty instruct students expected laboratory behaviors and evaluate their noncognitive behaviors and professionalism. Evaluation of noncognitive behaviors is included in all courses with smallgroup discussions. In all sessions with standardized patients, community activities, and patient encounters through all 4 years, professional behavior is formally assessed, and feedback is given to every student.

- Radiology: In the first-year gross anatomy, embryology, and imaging course, students are introduced to basic radiology imaging and techniques as well as landmarks of common radiologic studies for each anatomic region. In the second year, in Fundamentals of Pathophysiology and introduction to clinical skills courses, imaging characteristics of common 
diseases are presented. A third-year longitudinal radiology course offers online modules including recorded video lectures organized by common conditions and radiological studies seen in third-year clerkship specialties. Fourth-year students complete a required 4-week radiology rotation.

\section{Clinical experience first encounter}

In the first year, students are introduced to history taking. They practice taking a medical history with a standardized patient and complete the patient note. They are assigned to a community center to perform medical histories for elderly patients and report on the encounter. Twice during the semester, they are assigned to primary care offices with a faculty preceptor to observe and participate in clinical encounters.

\section{Required and elective community-based rotations}

PHSU-SOM has affiliations with multiple communitybased hospitals, clinics, and private physician offices where students do required rotations in family medicine, internal medicine, pediatrics, psychiatry, obstetrics-gynecology, surgery, emergency medicine, and radiology, as well as elective rotations. The SOM is affiliated with the Puerto Rico VA Hospital and several VA ambulatory clinics where students do required and elective rotations in internal medicine and its specialties, physical medicine and rehabilitation, psychiatry, and others.
Challenges in designing and implementing clinical experiences for medical students

The most important challenge to implementing new clinical experiences is conflicting time demands among all the educational experiences, particularly in the preclinical curriculum when faculty and students are focused on teaching and learning foundational knowledge necessary for the next level of training. Students are particularly concerned about passing USMLE Step 1 and matching into their desired specialty.

\section{Curricular Governance}

Each academic department prepares, submits to the dean, and manages a budget once it is approved by the administration. Faculty salaries and stipends, NBME examinations, online computer cases subscriptions, and other teaching material are budgeted departmentally. Faculty may request the department pay for faculty development expenses to improve teaching and assessment skills or knowledge in their fields.

See Figure 1-Curricular governance.

\section{Education Staff}

Planning, implementing, evaluating, and overseeing the curriculum is under the associate dean for medical education.

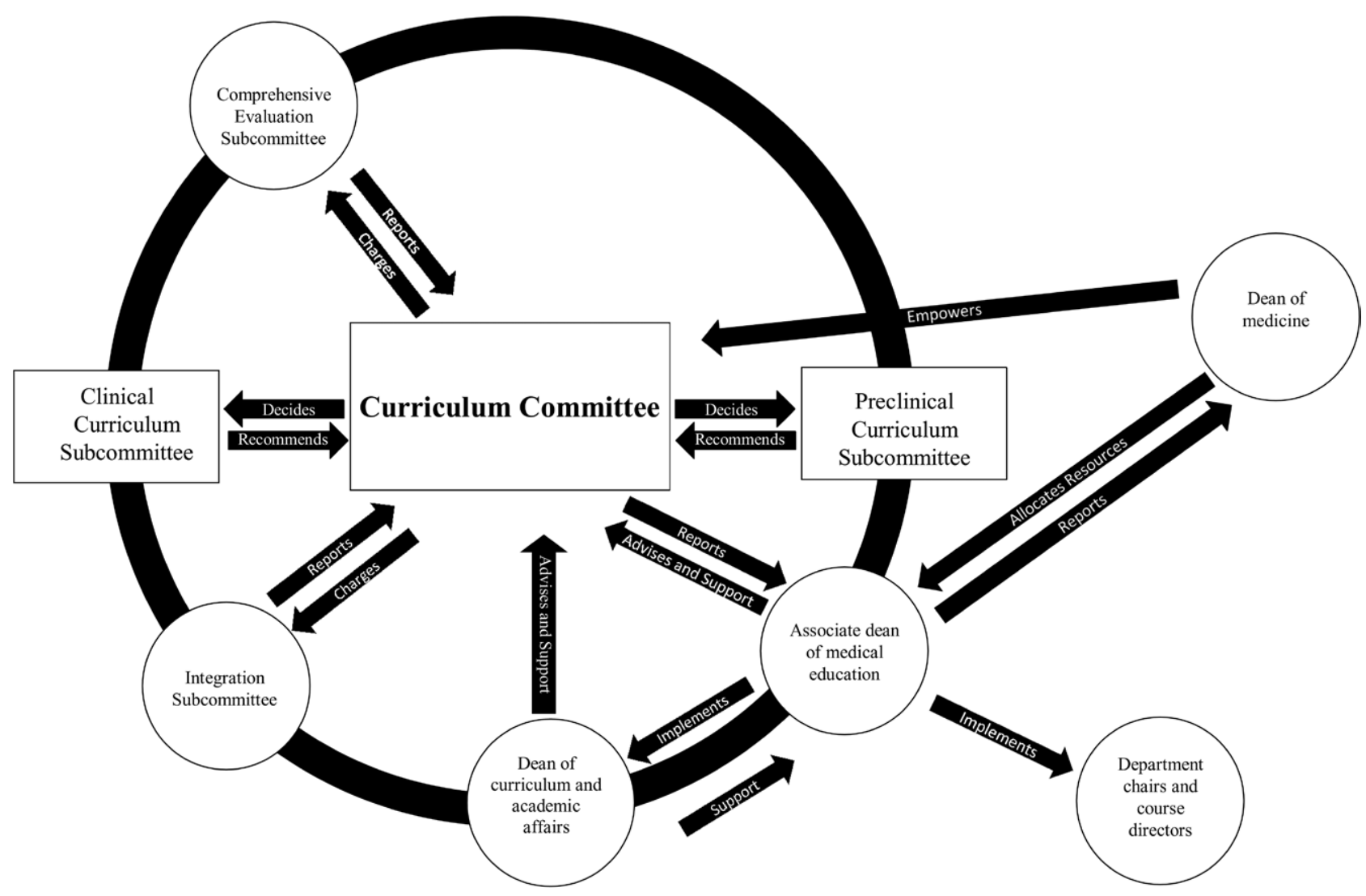

Figure 1 Curricular governance. 
A
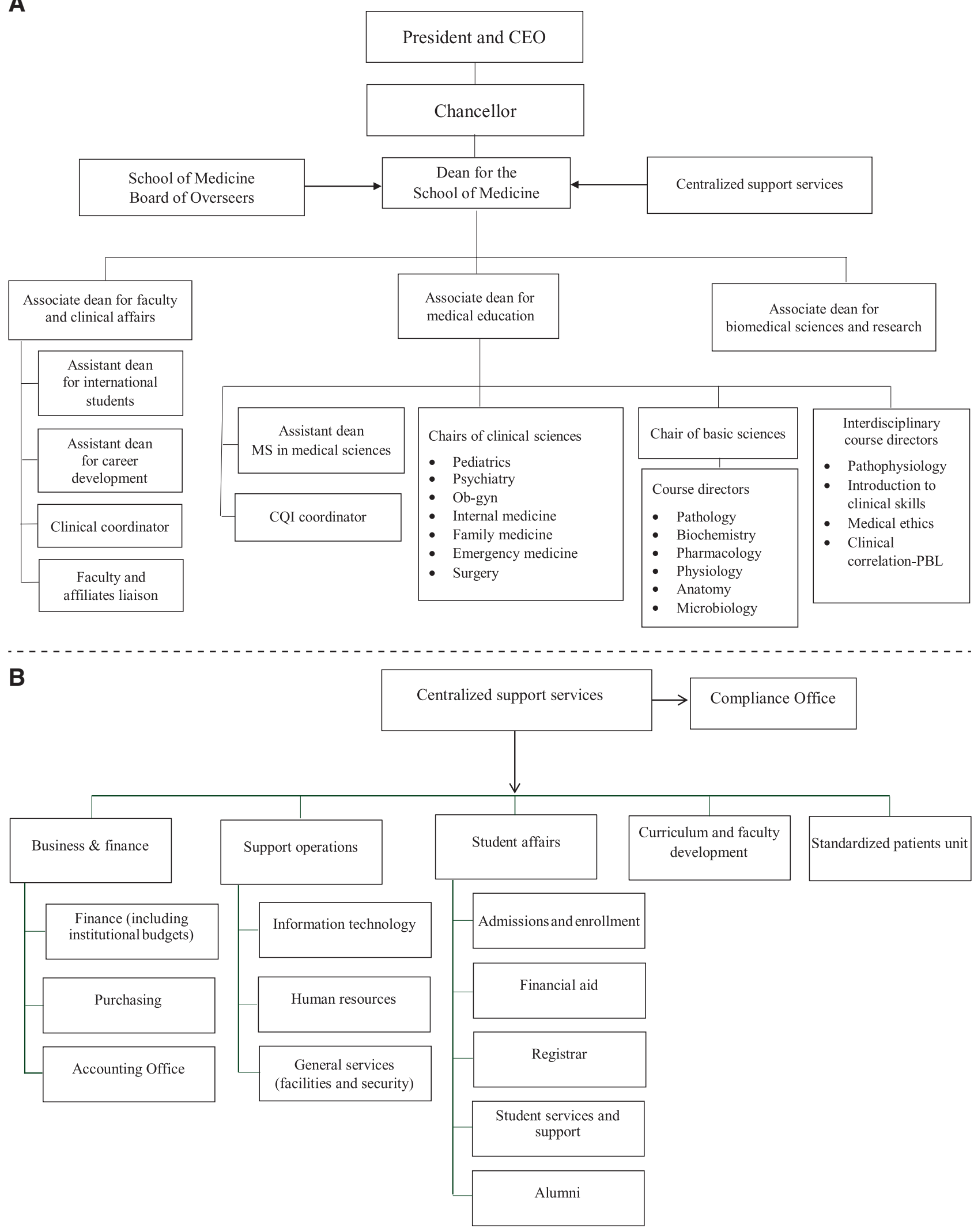

Figure $\mathbf{2}$ Organizational chart. 
The coordinator of quality improvement and an administrative assistant support these activities. The development and maintenance of tools to support curriculum delivery, monitoring, and management are supported by the dean of the SOM, the university's dean for curriculum and academic affairs, academia media director, and academic affairs office staff, which includes 4 educational technologists who manage e-learning services and lecture video recordings; the information technology department, a 16-person division in charge of technological advances; and the standardized patients program, including a program director, assistant director and 4 trainers. Each of the 8 academic departments has an administrative assistant who manages electronic databases and all clerical tasks. The associate dean for faculty and clinical affairs and the clinical coordinators support implementation of the curriculum and its oversight in clinical sites.

See Figure 2-Organizational chart.

The role of primary medical education staff and administrative faculty is implementing the UME curriculum. The affiliated hospitals have offices and administrative structure to support GME; PHSU has a dedicated office for CME that supports continuing professional development. PHSU has a Student Affairs Office that offers services to all students. An assistant dean for career development provides guidance and assistance to students related to career advising and residency application.

\section{Faculty Development and Support in Education}

\section{Professional development for faculty as educators}

The Faculty Development Division is at the university level under the supervision of the PHSU chancellor and coordinates educational activities related to improving PHSU-SOM faculty teaching and assessment skills.

The division is headed by the dean of curriculum and academic affairs, who has a doctoral degree in education. Additionally, the division has the support of 2 experienced educational technology specialists, both with master's-level education-related degrees. PHSU-SOM identifies faculty development needs from faculty directly, department chairs, course directors, residency program directors, and curriculum committee recommendations. It also takes into consideration current educational trends from medical education literature, professional organizations (such as the AAMC and AMA), and professional development meetings. Resources such as student feedback at the end of courses and clerkships, course/clerkship evaluation forms, end-of-year student evaluations of teaching and learning components, and annual departmental faculty evaluations are used to identify needs and provide alternatives for improvement. The specialist in educational technology provides training and continued instruction to faculty on platforms available at PHSU (e.g., Canvas, Moodle, iClickers, ExamSoft).

\section{Role of teaching in promotion and tenure}

The main basis for faculty promotion is performance. There must be evidence of a growing record of excellence in teaching, scholarship, mentoring, service, and performance of duties and responsibilities of a faculty member.

The primary responsibility of a faculty member is teaching. A faculty member must demonstrate thorough knowledge of the subjects taught. Primary focal points for evaluating competence and performance as a teacher include the ability to impart knowledge effectively; develop independent critical thinking and intellectual self-reliance; transfer know-how and incubate skills essential for success in a rigorous, diverse, and changing professional environment; use multiple instructional modalities; bridge differences in learning styles; narrow traditional success gaps among groups; conduct fair, appropriate, timely evaluation of students' work; and contribute to outcomes exceeding projections of traditional academic quality indicators. A faculty member must also demonstrate commitment to best teaching practices. The Faculty Development and Evaluation Committee, composed of 5 senior faculty members, is the institutional body responsible for recommendation of faculty promotion.

\section{Regional Medical Campuses}

PHSU-SOM has 1 regional medical campus, Mayagüez Medical Center Clinical Campus, enrolling 8 students. The learning objectives, assessment methods, required clinical experiences, and evaluation forms used for clerkships at the regional campus are identical to those used at the main campus. Each clerkship coordinator and department chair documents the orientation about objectives, assessment methods, and required clinical experiences that is given to the regional campus faculty who supervise PHSU students. During midclerkship formative evaluations, clerkship coordinators assess student progress in fulfilling experiences needed to attain clerkship objectives. Clinical departments collect and analyze data about learning outcomes, student satisfaction, duty hours, rate of clinical experiences completion, and teaching quality, using forms identical to those used on central campus to address any inconsistency. 


\section{University of Puerto Rico School of Medicine}

Humberto M. Guiot, MD, Hilton Franqui-Rivera, MD, and Marcel Mesa-Pabón, MD

\section{Medical Education Program Highlights}

The University of Puerto Rico (UPR) School of Medicine is the only state-funded school of medicine in Puerto Rico. It was originally founded as a school of tropical medicine and has been accredited by the LCME since 1954. At the time, it was the first and only school of medicine in Puerto Rico. The curriculum includes activities in both English and Spanish, and the vast majority of students and faculty members identify themselves as Latino and as fully bilingual.

\section{Curriculum}

\section{Curriculum description}

See Supplemental Digital Appendix 1-Curriculum Map-at http://links.lww.com/ACADMED/A967.

\section{Curriculum changes since 2010}

Several changes have been implemented over the last 10 years to contribute to the evolution of the curriculum. Some of these changes include:

- In the preclinical years, corequisite courses in the first level have been fully integrated to match the integration of the second level, which has been integrated since 1998.

- In the clinical years, the third and fourth levels are now visualized as a continuum.

- Longitudinal threads about several relevant topics (interprofessionalism and patient safety; health disparities and cultural competency; neurosciences; ethics, humanism, and professionalism; evidence-based medicine; obesity, pain management, and palliative care; and clinical and communication skills) were created and immersed within the 4 levels of training.

- Curricular maps and assessment blueprints have been developed and used in each course.

\section{Assessment}

The frameworks from the ACGME core competencies and the AAMC Core Entrustable Professional Activities are used as reference for the medical education program objectives at UPR School of Medicine, but the curriculum also responds to historical processes, regional/national needs, and current trends.

See Table 1-Program Objectives and Assessment Methods.

Acad Med. 2020;95:S454-\$456.

doi: 10.1097/ACM.0000000000003482

Copyright (C) 2020 by the Association of American Medical Colleges

Supplemental digital content for this article is available at http://links.Iww.com/ ACADMED/A967.

Year school was established: 1954.

School URL: https://md.rcm.upr.edu.

\section{Assessment changes since 2010}

Since 2010, several changes have been implemented in student assessment. Some of the changes are:

- All tests are offered using online platforms, such as ExamSoft, NBME customized assessments, and NBME subject examinations.

- Although passing the USMLE Step 1 examination has been a requirement for graduation for years, more recently the USMLE Step 2 CK and Step 2 CS examinations were added as requirements for graduation as well.

\section{Parallel curriculum or tracks}

Regarding parallel tracks, a structured research program (research track) is provided at the UPR School of Medicine by enrollment in 3 sequential courses. Students who complete all 3 courses receive a Research Track Certificate at the graduation ceremony.

The UPR School of Medicine also offers the possibility of concurrent degrees in addition to the MD degree at the same campus or in collaboration with other campuses of the UPR or with other institutions:

- MD-MS: UPR Medical Sciences Campus solely or in collaboration with Mayo Clinic

- MD-MSc: UPR Medical Sciences Campus

- MD-JD: UPR Medical Sciences Campus and Río Piedras Campus

- MD-PhD: UPR Medical Sciences Campus solely or in collaboration with Mayo Clinic, Yale University, or MD Anderson

\section{Pedagogy}

To achieve the medical educational program objectives, the following pedagogical approaches are employed:

- Case-based learning

- Clinical experience: ambulatory

- Clinical experience: inpatient

- Discussion: large group (>12)

- Discussion: small group $(\leq 12)$

- Flipped classroom

- Laboratory

- Lecture

- Peer teaching

- Preceptorship

- Problem-based learning

- Role play/dramatization

- Self-directed learning

- Simulation

- Standardized patients

- Team-based learning

- Video/podcast

- Workshop 
Table 1

Program Objectives and Assessment Methods

$\begin{array}{ll}\text { Medical education } & \text { Assessment methods } \\ \text { program objective } & \text { Institutional quizzes and exams } \\ \text { Knowledge } & \text { NBME customized assessments } \\ & \text { NBME subject examinations } \\ & \text { Formative assessments } \\ & \text { Case presentations } \\ & \text { USMLE Step 1, Step 2 CK, and Step 2 CS } \\ \text { examinations } & \text { Noncognitive academic factors } \\ & \text { (professionalism) evaluation } \\ & \text { Active learning activities } \\ \text { Small-group discussions } \\ \text { Case presentations } \\ \text { Objective structured clinical examinations } \\ \text { USMLE Step 2 CS }\end{array}$

\section{Changes in pedagogy since 2010}

Since 2010, the tendency has been to continue to decrease passive activities (such as lectures and conferences) while increasing active learning and clinical experiences.

\section{Clinical experiences}

The clinical sites for the required educational experiences include outpatient primary and specialized settings (public, community based, and private), as well as secondary, tertiary, and quaternary public and private inpatient institutions.

\section{Required longitudinal experiences}

Medical students have longitudinal experiences on the following topics: interprofessionalism and patient safety; health disparities and cultural competency; neurosciences; ethics, humanism, and professionalism; evidence-based medicine; obesity, pain management, and palliative care; and clinical and communication skills.

\section{Clinical experience first encounter}

Although clinical clerkships start during the third level of training, all students are exposed to some clinical experiences throughout their preclinical years. Some of the activities include interactions with real patients as part of the Introduction to Clinical Diagnosis, Fundamentals of Clinical Diagnosis, and Integration Seminar courses. The students also have the opportunity to complete a summer elective in emergency medicine or observation/shadowing with our faculty during their preclinical years.

\section{Required and elective community-based rotations}

To guarantee community-based experiences, all students are requested to complete a family medicine clerkship, which is entirely based on outpatient clinics at primary care facilities spread throughout the island. Students also complete a required experience in population health, which requires them to visit and complete a project at a local community. Community hospital-based experiences are included in portions of the third-year required clerkships (i.e., internal medicine, surgery, obstetrics-gynecology, psychiatry, and pediatrics), as well as in most fourth-year elective subinternships. In addition to these required community-based experiences, there are several elective community-based rotations available for students to select.

\section{Challenges in designing and implementing clinical experiences for medical students}

Finding and maintaining enough clinical settings to accommodate all our medical students always requires effort and creativity, but we have succeeded at providing an appropriate number of scenarios for the design and implementation of the required clinical experiences.

\section{Curricular Governance}

In terms of curricular governance, the Curriculum Committee is the institutional body that oversees the medical education program as a whole and has responsibility for the overall design, management, integration, evaluation, and enhancement of a coherent and coordinated medical curriculum. The Curriculum Committee analyzes the curricular program and the evaluation system throughout the 4 levels of the MD degree program. It has the empowerment to endorse changes to the curriculum and evaluation system and its implementation in the MD degree program. This committee is composed of administrative, faculty, and student representatives. The Medicine I, Medicine II, and Medicine III/IV Committees are advisory to the Curriculum Committee and are directly responsible for reviewing the structure and content of the curricular offer within each academic year/level.

Most courses, clerkships, and electives are managed at the department level, although they are overseen by the Medicine Committees and the Curriculum Committee, and they are evaluated by the Department of Evaluation and Medical Research (which falls under supervision of the associate dean of academic affairs). Due to their uniqueness or interdisciplinary approach, a few courses fall directly under the associate dean of academic affairs (such as epidemiology, ethics, fundamentals of clinical diagnosis, integration seminars, mechanisms of disease, introduction to clinical diagnosis, public health, and population health).

\section{Education Staff}

The Curriculum Committee and the Office of Curriculum (under the associate dean of academic affairs) supervise and develop strategies for the planning, implementation, evaluation, and oversight of the curriculum. The Office of Evaluation and Research on Medical Education (also under the associate dean of academic affairs) keeps records of the students and performs 


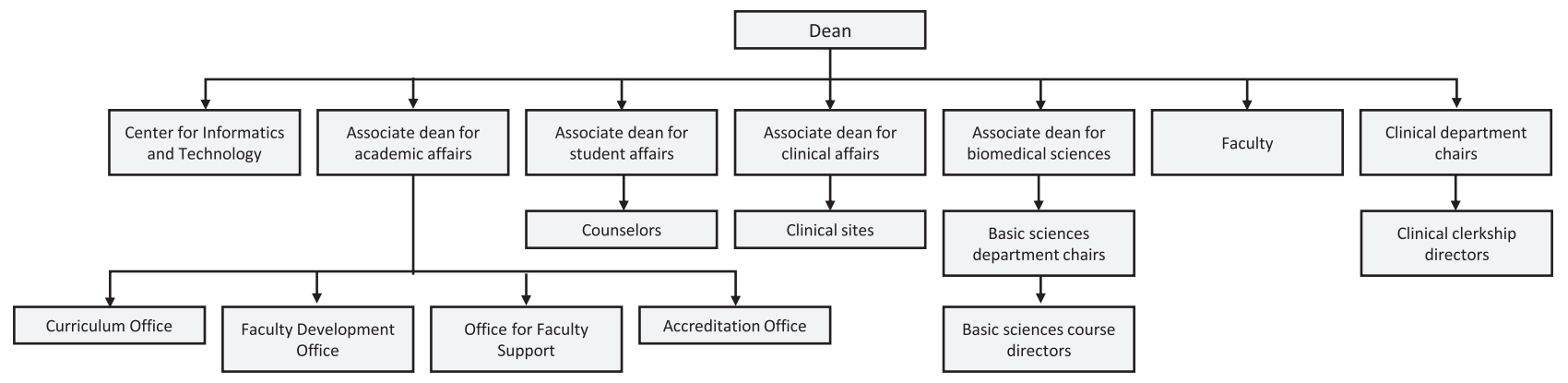

Figure 1 Medical education leadership.

periodic evaluations of courses and faculty. The associate dean for clinical affairs assesses the adequacy of clinical sites, while the Accreditation Office estimates compliance with LCME standards. Administrative and clerical staff in each basic science and clinical department provides support for their corresponding courses, while the staff under the associate dean of academic affairs provides similar support for those unique courses that fall under that deanship (as described above). The Office of Faculty Development and Support assists during the preparation and administration of tests, as well as during the coordination and development of educational activities for the faculty.

See Figure 1-Medical education leadership.

There is active participation of administrative faculty in the Curriculum Committee to identify gaps in the educational program and to guarantee a continuum throughout medical training. Under the associate dean of academic affairs, the Hispanic Center of Excellence is responsible for designing preundergraduate medical education activities as part of our pipeline and outreach programs, particularly geared toward students from disadvantaged backgrounds. Similarly, the Office of Curriculum is responsible for undergraduate medical education through clinical shadowing and research experiences for college students. Jointly, the associate dean for graduate medical education is a member of the Curriculum Committee, so that the clinical years serve as a transition into residency training. Although the associate dean for academic affairs and the associate dean for clinical affairs participate in some academic and career counseling for students, the ultimate responsible for student advising, counseling, wellness is the associate dean for student affairs.

\section{Faculty Development and Support in Education}

\section{Professional development for faculty as educators}

Faculty members are offered workshops and educational activities on creating learning objectives, delivering presentations, student assessment, and question writing, among other topics. These activities are designed and offered by the Faculty Support Office, under the associate dean for academic affairs.

\section{Role of teaching in promotion and tenure}

Time and effort dedicated to teaching activities, committees, and research, as well as student evaluations, are taken into consideration for academic rank promotions.

\section{Initiatives in Progress}

Future goals in our school include to design and implement a longitudinal thread on health systems science throughout the 4 levels of the MD degree program, to increase the time and options for electives in the fourth level, and to perform a full integration in the preclinical years (first and second levels). 


\section{The Warren Alpert Medical School of Brown University}

Paul George, MD, Luba Dumenco, MD, Sarita Warrier, MD, Kristina Monteiro, PhD, Steven Rougas, MD, Emily Green, PhD, Michael Mello, MD, Jeffrey Borkan, MD,

Thais Mather, PhD, Richard Dollase, EdD, and Allan R. Tunkel, MD, PhD

\section{Medical Education Program Highlights}

The educational program at the Warren Alpert Medical School of Brown University (AMS) is an innovative, 4-year medical degree granting program. Unique features of the program include:

- A longitudinal, integrated curriculum on opioids and opioid use disorder that results in the granting of a Drug Addiction

Treatment Act waiver for all medical students

- A longitudinal, multidisciplinary curriculum on diversity, inclusion, and social justice

- A focus on interprofessional education

- The Primary Care-Population Medicine (PC-PM) program

\section{Curriculum Description}

See Supplemental Digital Appendix 1-Curriculum Description —at http://links.lww.com/ACADMED/A828.

There are 3 phases to the AMS curriculum: preclerkship (approximately 17 months, with summer research options of approximately 10 weeks in between the first and second years), clerkship (approximately 12 months), and postclerkship (approximately 12 months).

- In the preclerkship phase, the first semester serves as a foundation for the remainder of the systems-based curriculum, which begins with the second semester of year 1. At the end of year 2, students complete the clinical skills clerkship (CSC), a 2-week course preparatory course for thirdyear clerkships.

- During the clerkship and postclerkship phases of the curriculum (years 3 and 4, respectively), students must complete at least 80 weeks of instruction, including 44 weeks of required clerkships.

- Upon successful completion of the clerkships, postclerkship students complete a minimum of 36 weeks of clinical electives, 24 of which must be at AMS.

Throughout their time at AMS, students are also able to participate in the Scholarly Concentrations program, a scholarship-oriented interdisciplinary elective.

Acad Med. 2020;95:\$457-S460.

doi: 10.1097/ACM.0000000000003274

Copyright (C) 2020 by the Association of American Medical Colleges

Supplemental digital content for this article is available at http://links.Iww.com/ ACADMED/A828.

Year school was established: 1963.

School URL: http://medical.brown.edu.

\section{Curriculum changes since 2010}

Preclerkship changes: As a member of the American Medical Association's Accelerating Change in Medical Education Initiative, AMS developed a new course in 2014 for all first-year medical students titled Health Systems Science. This course focuses on health disparities, health care systems, race and medicine, epidemiology, biostatistics, the scientific method, and patient safety and quality improvement.

Other preclerkship curriculum changes included shortening the preclerkship curriculum by 5 weeks and further integration of anatomy with other courses.

Clerkship changes: Since 2010, 2 major changes included the development of a transition course for students (the CSC) before the start of formal clerkships. In addition, neurology was added as a 4-week clerkship and psychiatry was shortened from 6 to 4 weeks; this was developed into an integrated 8-week clerkship in psychiatry/ neurology that includes longitudinal experiences in both disciplines.

\section{Class size changes since 2010}

Class size was approximately 99 students per class in 2010. The class size increased to 120 in 2012 and 144 in 2015. Approximately 16-24 students per year are part of the PC-PM program, which led to the creation of a longitudinal integrated clerkship (LIC) during their clerkship year for this cohort of students. The increased class size has otherwise not changed AMS's structure or function.

\section{Assessment}

See Supplemental Digital Appendix 2-Medical Education Program Objectives and Assessment Methods-at http://links. lww.com/ACADMED/A828.

The 9 abilities (competencies) and the subabilities (medical education program objectives) were designed and implemented in the early 2000s. The Curricular Refinement, Innovation, and Strategic Planning-Continuous Quality Improvement (CRISPCQI) Committee is responsible for the ongoing review of the 9 abilities and subabilities, including their linkage, by working directly with course/clerkship directors. The Medical Curriculum Committee (MCC) is responsible for reviewing, providing feedback, and approving updates to ensure oversight of the medical education program.

During the 2017-2018 academic year, the MCC, in conjunction with CRISP-CQI, updated the 9 abilities and subabilities to integrate the AAMC Core Entrustable Professional Activities into the existing teaching and assessment framework. In addition, all subabilities are mapped to the Physician Competency Reference Set. 


\section{Assessment changes since 2010}

Student performance evaluations (SPEs) are completed by smallgroup faculty and community mentors (Doctoring only) in the preclerkship phase of the curriculum and by clinical faculty in the clerkship and postclerkship phases. In the 2016-2017 academic year, all SPEs were standardized to include ratings on the abilities linked to the course/clerkship. This ensured a high level of integration between teaching and assessment methodology. Student progress is tracked longitudinally throughout program by the AMS Competency Committee.

Self-directed learning assignments and data problem sets (in which students analyze precollected data using Microsoft Excel) are new methods of assessment in the preclerkship phase of the curriculum.

\section{Parallel curriculum or tracks}

AMS initiated the PC-PM program in 2014. This program is a dual-degree curriculum that prepares students for a career in medicine while providing comprehensive, longitudinal training in population medicine through 9 additional courses and a thesis requirement. Graduates are awarded both an MD degree and a Master of Science in Population Medicine.

\section{Pedagogy}

Preclerkship IMS courses at AMS use a mixture of pedagogical approaches including lectures, case-based learning (CBL), laboratory, large- and small-group discussions, team-based learning (TBL), self-directed learning, and workshops.

Preclerkship Doctoring courses use a mixture of lecture, CBL, large- and small-group discussions, peer teaching, preceptorship (community mentor experience), role play, standardized patients, and video modalities. Year 3 clerkships are primarily clinical experiences in both ambulatory and inpatient settings, as well as lectures, CBL, and large- and small-group discussions. Year 4 encompasses mostly clinical experiences in both ambulatory and inpatient settings, with subinternships and electives using varying additional modalities including CBL and lecture.

\section{Changes in pedagogy since 2010}

There are 3 major changes to pedagogical approaches since 2010:

- TBL and CBL. Both preclerkship IMS courses and clerkships have integrated more active modes of teaching, replacing small-group sessions with required TBL and CBL.

- CBL and standardized patients. Clinical skills content in Doctoring is primarily taught using case-based small-group discussions and weekly access to standardized patients.

- Peer teaching. The CSC at the end of the second year is primarily taught by fourth-year medical students who participate in a 2-week training elective.

\section{Clinical Experiences}

\section{Clinical sites}

AMS uses a variety of clinical sites for required educational experiences. Students begin working at clinical sites during the
Doctoring course in years 1 and 2 (preclerkship phase). Students spend 1 half day per week at these sites over the course of 14 weeks each year.

Students continue training at clinical sites during both the clerkship and postclerkship phases of the curriculum. AMS has 7 required clerkships: family medicine, internal medicine, surgery, psychiatry, neurology, pediatrics, and obstetrics-gynecology. All clerkships use a combination of inpatient and outpatient sites, except for family medicine (which exclusively uses outpatient family medicine practices). Students in the PC-PM participate in an LIC.

The main clinical sites of AMS are located within 3 health care systems in Rhode Island: Lifespan, Care New England, and the Veterans Affairs Medical Center.

\section{Required longitudinal experiences}

Students spend 1 half day per week within the Doctoring course (in the preclerkship phase of the curriculum) at a clinical site. In both years 1 and 2, students spend 14 sessions with a mentor at an assigned site.

Students in the PC-PM program participate in the year 3 LIC, which consists of 31 sessions with a preceptor in each of the 7 core clerkship areas.

While not required, students have the option of doing an advanced clinical mentorship in the clerkship and postclerkship phases of the curriculum. Students can spend 1 half day per week with a mentor of their choice (usually in the specialty area they intend to enter).

\section{Clinical experience first encounter}

As part of the Doctoring course, learners are assigned to a community mentor within the first 2 months of the preclerkship curriculum, which continues into preclerkship year 2 .

\section{Required and elective community-based rotations}

AMS's required 6-week family medicine clerkship is exclusively community based. Students are assigned to a family medicine practice (practice types include federally qualified community health centers, residency teaching practices, and private practices). These practices are located in Rhode Island, southeastern Massachusetts, and eastern Connecticut.

Students on several other required clerkships-internal medicine ( 4 weeks) and pediatrics ( 2 weeks) —also spend time at community-based sites. In addition, a number of elective rotations are community based, ranging from a primary care experience in family medicine to specialty rotations in specialties such as cardiology and HIV care.

\section{Challenges in designing and implementing clinical experiences}

The major challenge in designing and implementing clinical experiences for AMS students is in recruiting clinical sites, especially outpatient sites. Two contributing factors are learners from other health professions (such as physician-assistant and nurse-practitioner students needing similar opportunities) 
and the increasing demand for clinical productivity placed on physicians. In addition, adequate and consistent faculty development of clinical preceptors across settings poses an ongoing challenge.

\section{Curricular Governance}

The MCC serves as the final decision-making body regarding the medical curriculum as stated in the AMS bylaws. The MCC ensures continuous oversight of the medical education program and is charged with oversight, review of curricular content and integration, and evaluation of the medical education program.

There are 4 standing subcommittees of the MCC: CRISP-CQI, the Program in Educational Faculty Development Subcommittee, the Subcommittee on Diversity and Inclusive Teaching and Learning (CDITL), and the Administrative Policy Committee.

CRISP-CQI oversees the Subcommittee on Years 1 and 2, Subcommittee on Years 3 and 4, the Clerkship Directors Committee, and the Subcommittee on Scholarly Concentrations and provides a summary of recommendations to the MCC for final approval to ensure ongoing central oversight of the curriculum.

The Program in Educational Faculty Development Subcommittee provides programming and services to support the instructional practice of faculty and residents who teach medical students in required and elective portions of the 4-year curriculum.

With input from the Office of Medical Education and Continuous Quality Improvement (OME-CQI) and the Office of Student Affairs, CDITL regularly reviews the curriculum with regard to diversity and inclusion. As potential biases and inaccuracies are identified, CDITL provides recommendations on curricular change to correct inaccuracies and suggests faculty development to improve future iterations of the curriculum.

The Administrative Policy Committee regularly reviews AMS policies and procedures. The final authority on whether to change or adopt a particular policy rests with the MCC.

See Figure 1-Curricular governance.

\section{Education Staff}

The OME-CQI is led by the associate dean for medical education, who also oversees the clinical curriculum, with a focus on years 3 and 4 . There are 2 assistant deans for medical education, who oversee years 1 and 2. Other key leaders in the OME-CQI include the director of the Doctoring Program, the director of evaluation and assessment, and the associate director of the preclerkship curriculum. These individuals, with administrative support from coordinators and assistants, plan, implement, evaluate, and oversee the curriculum.

\section{Medical education leadership}

The role of the primary medical education staff and administrative faculty is development, implementation, and evaluation of curriculum at AMS. The office is not responsible for graduate medical education or continuous professional development. A separate Office of Student Affairs reports to the senior associate dean for medical education.

See Figure 2-Medical education leadership.

\section{Department of Medical Education}

There is a Section on Medical Education within AMS, which can appoint faculty, either with primary or secondary appointments, in the area of medical science.

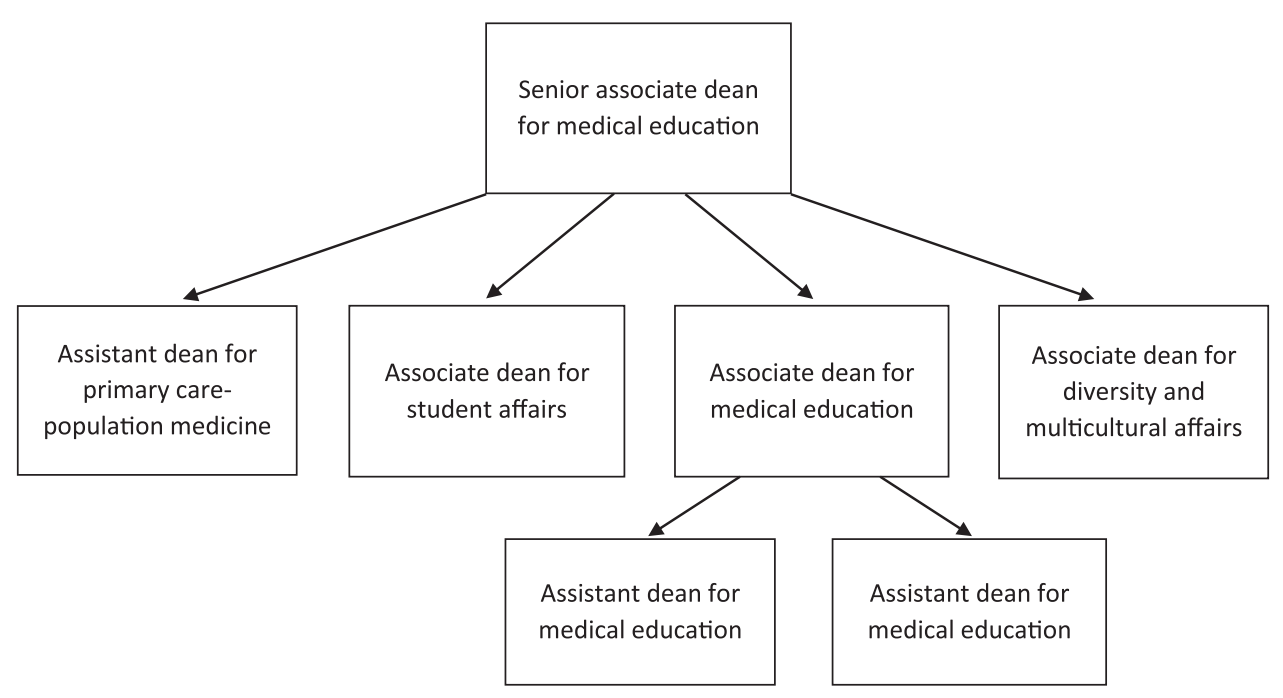

Figure 1 Curricular governance. 


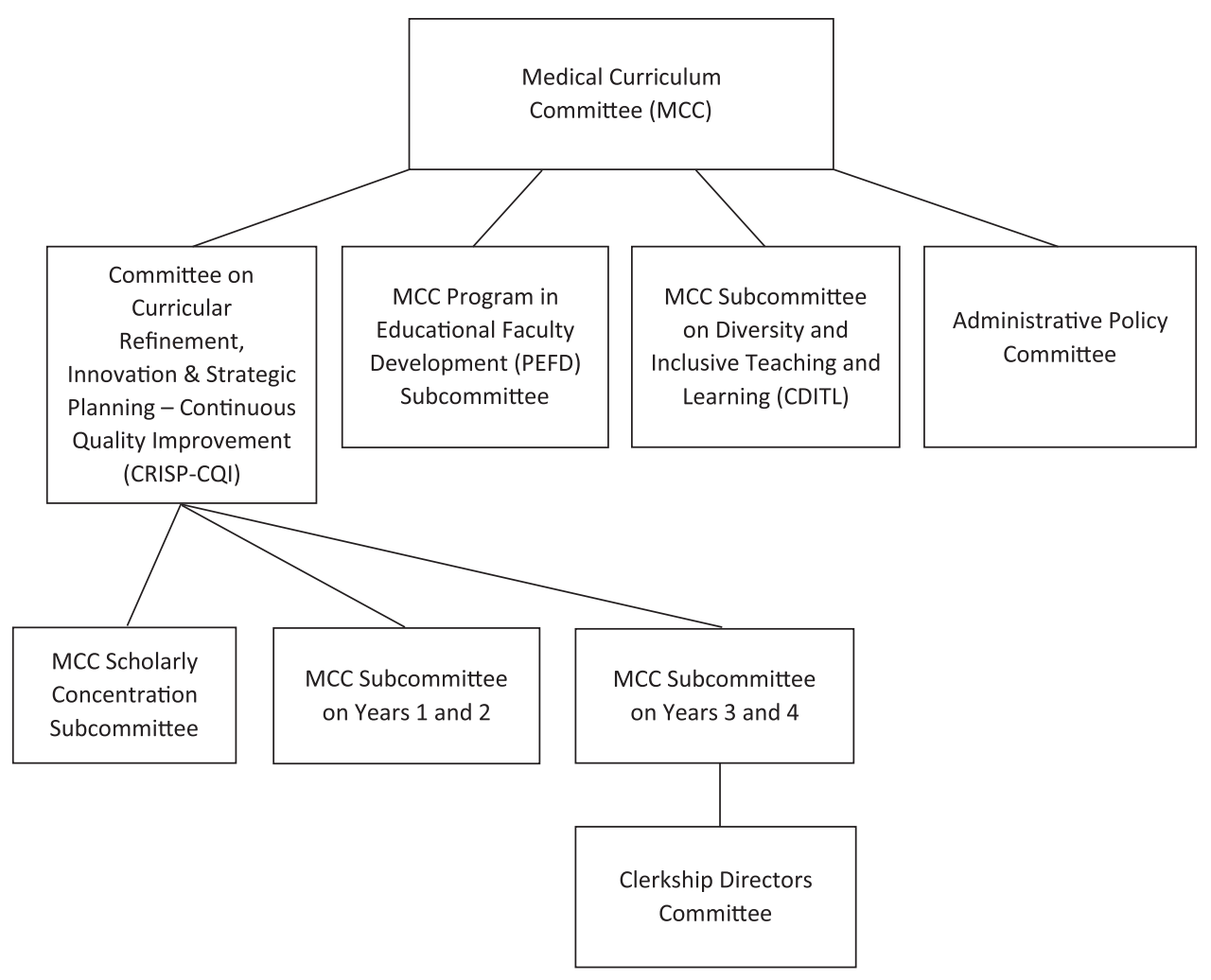

Figure 2 Medical education leadership.

\section{Faculty Development and Support in Education}

Professional development for faculty as educators

The Program in Educational Faculty Development, an arm of the Office of Faculty Professional Development and a subcommittee of the MCC, provides a central source of programming and support for instructional skill building. Annual programming includes workshops in 8 core areas (presentation and lecturing skills, small-group facilitation, using technology in teaching, clinical teaching, teaching the challenging learner, evaluation and effective feedback, mentoring and advising, and inclusive teaching), a TedTalksstyle event, a full-day retreat, and a medical education scholarship poster session.

\section{Role of teaching in promotion and tenure}

Metrics used to account for teaching and/or education activities include major educational roles, excellence and innovation in teaching (learner evaluations, list of mentees, CME course evaluations, conference presentation evaluations, teaching awards), continuous record of scholarship (peer-reviewed publications; book chapters; CQI documents; policy statements; curriculum development; book, article, or curriculum reviews; position pieces), and local/regional/national reputation in area of expertise (leadership roles in national organizations, organization of conference sections, creation of research methodologies, curriculum and assessment tool development, invited presentations, testimonials by outside referees). 


\title{
Medical University of South Carolina College of Medicine
}

\author{
Donna H. Kern, MD, Angela R. Dempsey, MD, MPH, and Debra J. Hazen Martin, PhD
}

\section{Medical Education Program Highlights}

- A longitudinal interprofessional education curriculum includes required interprofessional courses and activities in the preclerkship phase, required interprofessional experiences in each clerkship, a robust offering of electives (e.g., culinary health elective), and increased opportunities to work with underserved populations in various community settings through the CARES student-run free medical clinic.

- The new Flex Curriculum offers students options for pathways: global health with scholarship support for global field experiences, health humanities, physician as teacher, clinical and basic science research, a primary care parallel curriculum, and an accelerated 3-year MD degree program that tracks into a residency position in 1-on-1 residency programs.

- A newly developed wellness program, Student Wellness Council, and learning community model emphasize the importance of personal wellness, resilience, and supportive relationships.

\section{Curriculum}

\section{Curriculum description}

See Supplemental Digital Appendix 1-Curriculum Map—at http://links.lww.com/ACADMED/A912.

See Figure 1-Flex pathways.

\section{Curriculum changes since 2010}

In 2009, a new preclinical integrated curriculum was implemented that replaced traditional courses with a comprehensive, organ system-based curriculum of basic science and clinical science content administered through a coordinated, central governance model.

Building on successes and lessons learned, the new Flex Curriculum preclerkship phase ( 66 weeks) was implemented in AY 2018-2019 as organ system blocks that cohesively blend learning of normal and abnormal to teach the spectrum of human health and disease in engaged learning formats. Learners engage in early clinical experiences in the hospital, the community, and simulation settings that enhance application of basic science concepts.

Acad Med. 2020;95:S461-S464.

doi: 10.1097/ACM.0000000000003404

Copyright (C) 2020 by the Association of American Medical Colleges

Supplemental digital content for this article is available at http://links.Iww.com/ ACADMED/A912.

Year school was established: 1824.

School URL: https://medicine.musc.edu.
In the Flex pathways phase (13 weeks), each learner selects a scholarly concentration pathway (basic science or clinical science research, health humanities, global health, or physician as teacher) that best meets their educational needs, professional interests, and career aspirations. Alternatively, students who are eligible may apply to the accelerated medical pathway (AMP), which leads to graduation after 3 years with entry into a conditional residency position at the Medical University of South Carolina (MUSC).

The clinical immersion phase ( 49 weeks) comprises 7 core clinical clerkships: family medicine, internal medicine, neurology and rehabilitation medicine, obstetrics-gynecology, pediatrics, psychiatry, and surgery. Students choose 2 selectives for additional immersion in specialties of their choice. The clinical enrichment phase (34 weeks) is made up of a new, required critical care elective, advanced clinical electives, and the Internship 101 Capstone course, all of which are designed to prepare learners for the transition to increased levels of responsibility for patient care.

The class size was increased from 174 to 180 when the regional campus was established. The regional campus offers a primary care parallel track that integrates the traditional curriculum with unique opportunities for learners to attain greater breadth and depth of experience in the areas of primary care, community medicine, population health, and service learning.

\section{Assessment}

Medical education program objectives are described in outcomebased terms that allow the assessment of medical students' progress in developing the competencies that the profession and the public expect of a physician.

\section{Medical education program objectives}

The medical education program objectives were informed by the following competency frameworks: ACGME domains of competence, AAMC Core Entrustable Professional Activities, and the Physician Competency Reference Set competencies.

See Supplemental Digital Appendix 2-Program Objectives and Assessment Methods—at http://links.lww.com/ACADMED/A912.

MUSC College of Medicine offers 2 parallel curricula:

- Primary care parallel curriculum at the AnMed Health regional campus (https://medicine.musc.edu/education/medicalstudents/curriculum/anmed-clinical)

- AMP 3-year parallel curriculum (https://medicine.musc.edu/ education/medical-students/curriculum/clinical-curriculum/ accelerated-medical-pathways)

\section{Pedagogy}

The medical education program uses a variety of pedagogical approaches in the preclerkship phase and the clinical immersion 


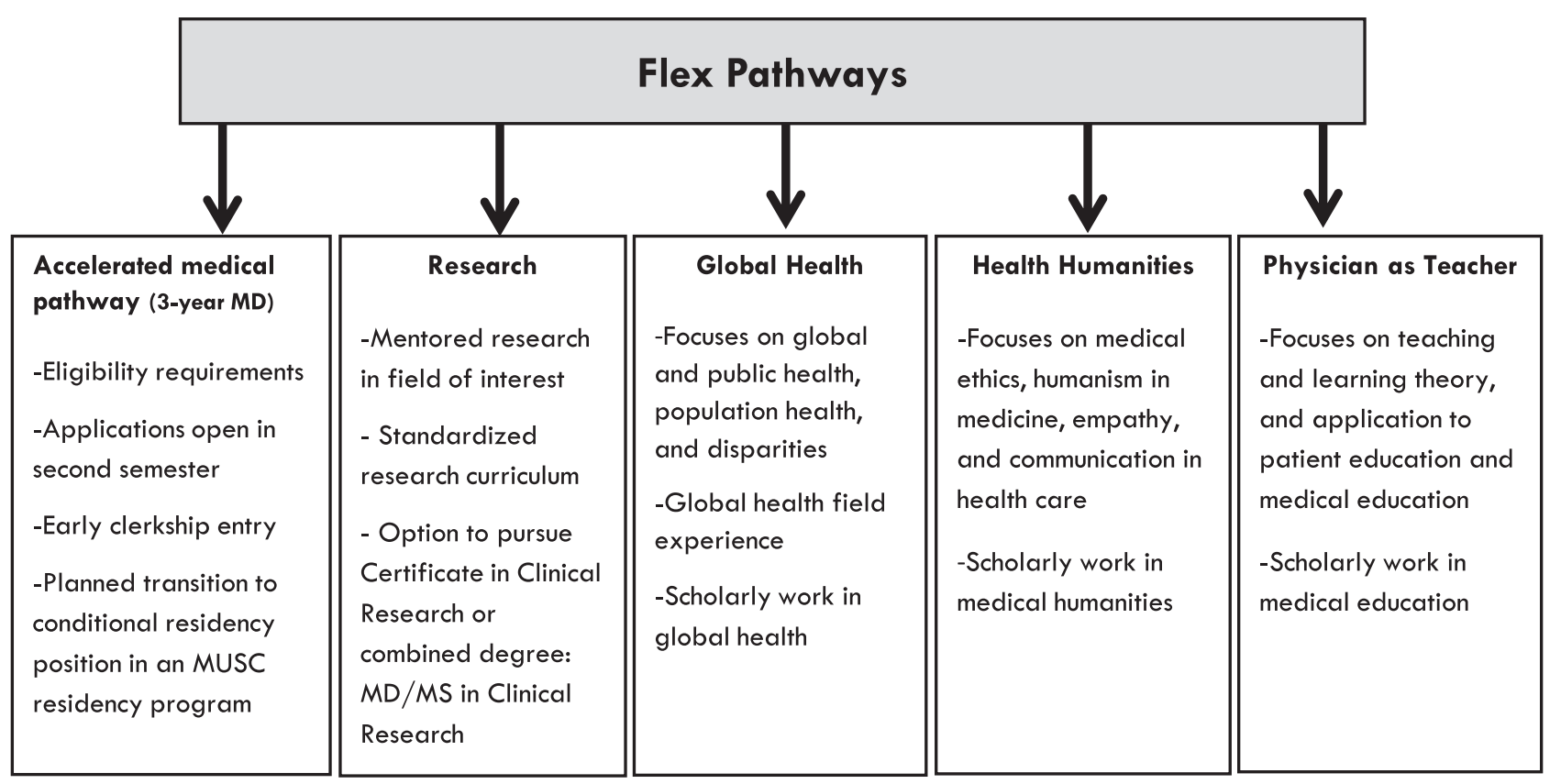

Figure 1 Flex pathways.

and enrichment phases. Pedagogical approaches that are new to the curriculum since 2010 are indicated with $\left(^{*}\right)$.

- Case-based and team-based learning* are used throughout the organ system-based preclerkship blocks.

- Lecture-based didactics are used to prepare learners for applied learning experiences.

- Multiple-patient panels are used to enhance the learner's understanding of the patient experience of disease, the health care system, and disparate treatment. ${ }^{*}$

- The new multisystem integration block ${ }^{\star}$ emphasizes the use of self-directed learning and clinical problem solving.

- Anatomy laboratory teaching is enhanced by a peer teaching model.*

- The longitudinal clinical skills curriculum that spans the preclerkship phase uses a combination of small-group discussion, simulated and standardized patient encounters, inpatient teaching rounds, and clinical reasoning case-based learning strategies.

- The AnMed Health clinical campus uses a longitudinal continuity clinic model.*

- The core clerkship curricula use inpatient care, ambulatory care, skills workshops, small-group discussion, and simulationbased teaching in each clerkship.

\section{Clinical experiences}

Clinical sites for required educational experiences:

- Charleston campus: MUSC hospitals and ambulatory clinics, which include Storm Eye Institute, Shawn Jenkins Children's Hospital, Pearl Tourville Women's Pavilion, Main Hospital, Ashley River Tower, Institute of Psychiatry, Hollings Cancer Center; Ralph H. Johnson VA Medical Center; Roper Rehabilitation Hospital
- AnMed Health Clinical Campus: AnMed Health hospitals and ambulatory clinics, which include the AnMed Health Medical Center and AnMed Health Rehabilitation Hospital; Patrick B. Harris Psychiatric Hospital

\section{Required longitudinal experiences}

- The Longitudinal Clinical Skills course spans the preclerkship curriculum.

- The senior mentor program pairs each student with a community-dwelling senior for the duration of the MD degree program. Students meet with their mentors each semester; the curriculum reflects core geriatric competencies that address topics pertinent to healthy aging (e.g., nutrition assessment, fall risk prevention, advance directives).

- Medical ethics small-group discussion sessions occur throughout the third- and fourth-year curriculum (3 per semester); students discuss issues that arise during their clinical rotations and apply foundational principles first introduced in the preclerkship curriculum.

- The Careers in Medicine program and the financial literacy curriculum are required beginning at prematriculation through the spring semester of the senior year.

\section{Clinical experience first encounter}

Students work with standardized and simulated patients beginning the first week of medical school and continuing in each curriculum block from year 1 through year 3 . Real patient care experiences begin in the first academic year:

- Fall I: Each student is assigned to PARTNERS experiences with 2 different interprofessional clinical teams and submits structured reflections on team member roles, effectiveness, and impact on the quality of the patient care. Students are 
also introduced to the senior mentor they will work with for all 4 years.

- Spring I: Each student is assigned to a master clinical skills teacher and participates in teaching rounds 5 times over 3 semesters with a focus on history-taking, physical examination, clinical reasoning, and medical documentation.

- Summer I: In the clinical immersion phase, each student prerounds/rounds with a clinical team in their preferred specialty for 4 consecutive days and participates in clinical care experiences.

\section{Required and elective community-based rotations}

The medical education program uses community-based, ambulatory clinical sites across the state for the 6-week family medicine rural clerkship. Surgery, medicine, psychiatry, and neurology clerkships use the Ralph H. Johnson VA Medical Center. The rehabilitation medicine portion of the neurology clerkship occurs at the Roper Rehabilitation Hospital.

\section{Curricular Governance}

The Undergraduate Curriculum Committee is responsible for curricular governance and has 4 working committees that carry out planning, implementation, assessment, and evaluation functions:

- Preclerkship Planning and Evaluation Committee

- Clinical Sciences Planning and Evaluation Committee

- Selectives and Electives Committee

- Teaching Effectiveness Assessment Committee

The College of Medicine education funds flow process allocates funding to basic science and clinical science departments based on faculty teaching effort and course direction responsibilities.

\section{Education Staff}

Medical student education is organized centrally through the Office of Curriculum, Office of Student Affairs, Office of Admissions, and an Office of Assessment, Evaluation, and Quality Improvement (OAE-QI). The Office of Curriculum organizes the planning, implementation, and evaluation of the curriculum with the support of the OAE-QI; these offices collaborate to develop and maintain the tools to support curriculum delivery, monitoring, and management. The Office

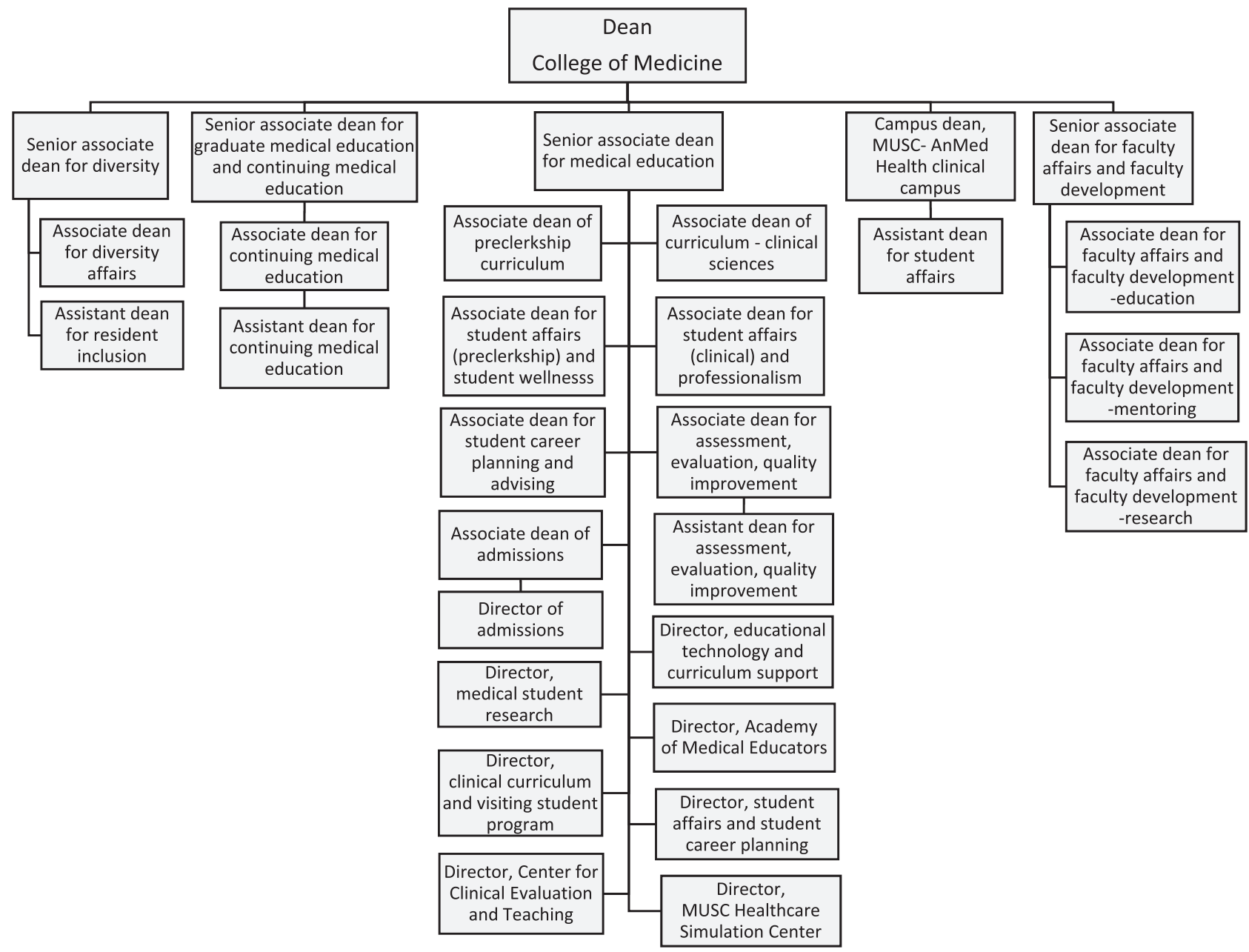

Figure 2 Medical education administration. 
of Medical Education works collaboratively with basic science and clinical science departments to secure faculty appointments and protect faculty effort for teaching, course direction, and education leadership for the medical education program.

See Figure 2-Medical education administration.

\section{Faculty Development and Support in Education}

The College of Medicine develops faculty for their role in teaching, assessment, mentoring, and advising students through the collaborative efforts of the Dean's Office, associate deans for faculty affairs and faculty development, Office of Education, and clinical science and basic science departments. Education grand rounds, round tables, workshops, and special programs offered through the college's Academy of Medical Educators and the university Apple Tree Society provide a wide variety of development opportunities.

\section{Professional development for faculty as educators}

The Academy of Medical Educators (AME) promotes teaching effectiveness and innovation in medical education and recognizes teaching excellence and scholarship, through a variety of faculty development opportunities:

- Provides peer observation of teaching in which AME members mentor faculty to improve teaching skills, educational materials, objectives, and assessments

- Offers workshops for members and nonmembers on topics of teaching and learning

- Recognizes teaching excellence and scholarship; hosts the annual Pearls for Medical Educators event in which distinguished faculty are invited to share their insights and experiences

AME welcomes applications from faculty, fellows, residents, administrators, and staff affiliated with medical education program. Based on defined criteria, individual applicants join AME in 1 of 4 membership categories: distinguished teacher, master teacher, developing teacher, and education specialist. As part of the application process, members are asked to develop an education portfolio in domains that include teaching activity; curriculum; and/or assessment development, advising and mentoring, educational leadership, and educational research. A detailed description can be viewed on the AME website (https://medicine.musc.edu/faculty-affairs/academy-of-medicaleducators).

\section{Table 1}

\section{Regional Medical Campus}

\begin{tabular}{lll}
$\begin{array}{l}\text { Regional } \\
\text { campus name }\end{array}$ & Type & Student enrollment \\
AnMed Health Clinical Campus, & Clinical & $\bullet 12$ third-year students \\
Anderson, South Carolina & & $\bullet 12$ fourth-year students \\
\hline
\end{tabular}

The Apple Tree Society is a university-wide academy that promotes excellence in teaching and learning for the faculty of all 6 colleges of the medical university.

\section{Role of teaching in promotion and tenure}

Excellence in teaching and mentoring of students, curriculum development, innovation in education, scholarship in education, and education leadership and course direction activities are viewed as critical components of the criteria for promotion and tenure consideration in the clinician-educator track. The College of Medicine Appointment, Promotion, and Tenure Committee sets criteria that value teaching and education-related efforts. Scholarly activity, curriculum development, and sustained efforts in teaching and education leadership are documented in a faculty intramural teaching effort report.

\section{Regional Medical Campus}

See Table 1-Regional Medical Campus.

\section{Educational experience across sites}

The clerkship curriculum at the regional campus uses the same objectives, core didactics, required patient care experiences and procedures, assessments, and expectations for feedback and observation of clinical skills as the main campus. Regional campus site directors for each clerkship participate along with clerkship directors in the CSPEC monthly meetings and biannual retreats; one of the biannual retreats for clinical education is held at the regional campus. At-large faculty and associate deans from the regional campus are active members of the Curriculum Committee and subcommittees. Clinical clerkship directors and associate deans/directors for education, student affairs, and assessment and evaluation have regularly scheduled conference calls with regional campus site directors and faculty and make site visits to meet with campus faculty. Regular meetings are conducted to collect feedback from regional campus students. 


\title{
University of South Carolina School of Medicine Greenville
}

\author{
April O. Buchanan, MD, Ihsan A. Elkhider, LLB, MSEd, and Angela M. Sharkey, MD
}

\section{Medical Education Program Highlights}

Through a synergistic partnership between the state's largest public university and the state's largest public hospital, the University of South Carolina School of Medicine (UofSC SOM) Greenville welcomed its charter class in the fall of 2012. Focused on expanding the physician workforce in South Carolina, the curriculum was developed to create a new type of graduate and a different kind of doctor: one responsive to society's medical needs and acquainted with the foundations of the quadruple aim, structured communication, professionalism, and awareness of the socio-economic environment.

- Vision: "Cultivate a culture of curiosity and commitment to others to transform the health and wellness of communities."

- Emergency medical technician (EMT) training ${ }^{1}$ provides students an opportunity to better understand their patients' life circumstances and the community in which they live, acute and subacute care delivery, and the importance of working as part of an interprofessional team.

- The Lifestyle Medicine Curriculum is a pedagogic shift to a need-based curricular approach focused on disease prevention and lifestyle-related noncommunicable chronic diseases currently affecting population health. This curricular thread is developed around the 6 pillars of lifestyle medicine-exercise, nutrition, substance avoidance, sleep, stress management, and relationships ${ }^{2}$ - and creates graduates prepared to influence patient outcomes and lower costs related to chronic disease management. UofSC SOM Greenville is considered a pioneer in designing and implementing a Lifestyle medicine curriculum. ${ }^{3}$

- The fourth-year curriculum includes specialty tracks for students to assure a solid general training and preparation for their chosen specialty. Through a menu of selectives and electives, students are guided through a variety of clinical experiences to prepare for their future career. The fourth year culminates in a 2-week intensification to assure adequate knowledge and skill in core areas through small-group learning and simulation, followed by 2 weeks of intensification in their matched specialty to ensure mastery of entry-level intern skills. Several of these specialty intensifications are based on national boot camp programs. ${ }^{4,5}$

\section{Curriculum}

\section{Curriculum description}

The curriculum is an integration of interactive experiences from classroom to clinical to community, designed to foster

Acad Med. 2020;95:S465-S468.

doi: 10.1097/ACM.0000000000003403

Copyright (C) 2020 by the Association of American Medical Colleges

Supplemental digital content for this article is available at http://links.Iww.com/ ACADMED/A911.

Year school was established: 2011.

School URL: https://www.sc.edu/study/colleges_schools/medicine_greenville. and enhance the acquisition and application of essential knowledge and skills including communication, diagnostic reasoning and problem-solving, critical thinking, and quality patient care.

Curricular themes:

- Integration of biomedical science and core clinical skills

- Early introduction of core clinical skills (EMT and integrated practice of medicine)

- Lifestyle medicine

- Behavioral, social, and population health

- Professionalism

The Integrated Practice of Medicine module (IPM) is a 4-year longitudinal course focused on clinical skills, behavior/social/ population health, and clinical reasoning.

See Supplemental Digital Appendix 1-Curriculum Overviewat http://links.lww.com/ACADMED/A911.

\section{Curriculum changes since 2010}

- Implementation of substance use disorder curriculum (2019): Based on recommendations from vertical integration mapping of behavioral health content and in response to the local and national opioid epidemic, substance use disorder content was further integrated and medicationassisted treatment waiver training (https://www.samhsa.gov/ medication-assisted-treatment) was added to the fourthyear intensification, to provide students with additional knowledge of addiction and recovery processes. Faculty partner with one of our community agencies FAVOR (Faces and Voice of Recovery) Greenville, to deliver additional curricular content.

- Delivery and assessment of biostatistics and epidemiology: The NBME Step 1 report following the 2016-2017 academic year revealed student performance below the national mean in the area of 1-PBLI: Evidence-Based Medicine. This was hypothesized to be a consequence of disjointed content delivery within the first and second years of the curriculum and the absence of summative assessments. In response to these findings, the content and distribution of evidence-based medicine/epidemiology/ biostatistics material was revised. Specifically, content was adjusted to shift from the delivery of broad concepts and foundational knowledge in the first-year IPM module into more specific, granular content that was delivered and assessed in the second year. During the second year, this material was integrated into biomedical science modules where it is was assessed via summative written examinations. This approach helped improve students' performance (NBME) and student satisfaction (AAMC Graduation Questionnaire). 


\section{Assessment}

The school's educational program objectives were initially based on core competency domains described by the ACGME and specific program objectives suggested by the AAMC's Medical School Objectives Project. In 2019, the Curriculum Committee aligned its educational program objectives with the Physician Competency Reference Set, ${ }^{6}$ maintaining some of the original medical knowledge objectives to better reflect the biomedical science module objectives. In addition, a set of lifestyle medicine objectives reflect our unique curriculum and align with the Agency for Healthcare Research and Quality's quadruple aim ${ }^{7}$ to improve health care through modalities outside of or adjunctive with the traditional medical model that focuses on curing diseases.

See Supplemental Digital Appendix 2-Program Objectives and Assessment Methods—at http://links.lww.com/ACADMED/A911.

These educational program objectives are the backbone for curriculum planning. As faculty develop module/clerkship content, session-level objectives are mapped to the module/ clerkship-level objectives, which in turn are mapped to the educational program objectives.

\section{Pedagogy}

Our curriculum focuses on student-centered learning to support lifelong learning skills using different approaches to engage learners, reflecting the diversity of instructional methods used in the preclinical years in a recent curriculum inventory report (2018-2019), including:

- Problem-based, team-based, and self-directed learning: During first- and second-year IPM, weekly clinical reasoning cases provide a basic patient presentation and students individually review the information provided, develop a hypothesis regarding the case, and identify knowledge gaps and resources needed to follow up on their hypothesis, reinforcing the skills of lifelong learning. Small-group and case-based discussions during years 3 and 4 emphasize problem-solving skills in patient care.

- Blended learning: Our blended learning approach offers individualized and small-group computer-based, self-directed experience supported by direct interaction with faculty in a classroom setting.

- Real, standardized/simulated/virtual patients: Throughout the curriculum, students have ample opportunities for patient encounters including real and standardized patients, simulation, and online virtual patients. Practice and application of medical problem-solving skills is integrated into standardized patient encounters and simulation scenarios in the Clinical Skills and Simulation Center across all 4 years. During the clinical years, students apply their medical knowledge and clinical skills (i.e., development of a differential diagnosis, diagnostic testing, interpreting results, analysis of information, and incorporating patient concerns) through daily interactions with patients, morning reports, and rounds.

See Table 1-Preclerkship Phase Instructional Formats.
Table 1

Preclerkship Phase Instructional Formats ${ }^{a}$

\begin{tabular}{|c|c|c|}
\hline Instructional method & $\begin{array}{l}\text { Primary } \\
\text { method: } \\
\text { no. events }\end{array}$ & $\begin{array}{l}\text { Nonprimary } \\
\text { occurrences: } \\
\text { no. events }\end{array}$ \\
\hline Case-based instruction/learning & 196 & 269 \\
\hline Demonstration & 14 & 2 \\
\hline Discussion, large group (> 12) & 148 & 165 \\
\hline Discussion, small group $(=12)$ & 107 & 82 \\
\hline Laboratory & 195 & 32 \\
\hline Lecture & 559 & 73 \\
\hline Role play/dramatization & 39 & 12 \\
\hline Patient contact & 56 & $\ddot{0}$ \\
\hline Self-directed learning & 22 & 229 \\
\hline Simulation & 44 & 26 \\
\hline Team-based learning & 4 & 3 \\
\hline
\end{tabular}

a Used in 2018-2019 academic year.

\section{Changes in pedagogy since 2010}

The instructional goal of the curriculum is to limit use of the traditional lecture-based didactic methods and encourage active learning and student-centered learning. Selection of the instructional methods is guided by the session-level objectives. Thus, various teaching methods are planned to deliver different elements of the curriculum. For example:

- Medical knowledge: Instructional delivery includes lecture, case-based instruction, small-group, large-group discussion, laboratory, and self-directed learning.

- Clinical skills: Case-based instruction, small-group, problembased learning, laboratory, real/standardized/virtual patients, and simulation.

\section{Clinical experiences}

- During third- and fourth-year clerkships, selectives, and electives, students have a broad exposure to the clinical environment including a Level 1 trauma center, intensive care units, and inpatient wards, along with hospital-based clinics and community practices. This variety contributes to students' understanding of all aspects contributing to the comprehensive care of the patient and resources available in various locations. The need for ancillary services is also emphasized. In addition, students have exposure to nursing home and long-term care facilities to better aid in the understanding of transitions of care. Students may elect extramural rotations during their fourth year, including global health electives.

- The distribution of clinical experiences reflects a balance that a physician would encounter in the various core specialties, while considering the overall exposure of students to inpatient and ambulatory settings in their third year.

- Medical students begin their clinical learning in the EMT program, subsequently participating in ambulance shifts and gaining an understanding of prehospital medicine as they follow patients into the clinical setting. In addition, students have sessions in the hospital and clinics as part of IPM during the first and second years. 
Table 2

\section{Ambulatory and Inpatient Experiences During Clerkships ${ }^{a}$}

\begin{tabular}{lcc} 
& \multicolumn{2}{c}{$\%$ Total clerkship time } \\
\cline { 2 - 3 } Clerkship & Ambulatory & Inpatient \\
Emergency medicine & 100 & 0 \\
\hdashline Family medicine & 90 & 10 \\
\hline Internal medicine & $25-37$ & $75-63$ \\
\hdashline Obstetrics-gynecology & 40 & 60 \\
\hdashline Pediatrics & 50 & 50 \\
\hdashline Psychiatry/neurology & 55 & 45 \\
\hline Surgery & 50 & 50 \\
\hline
\end{tabular}

aln 2018-2019 academic year.

- As part of the core clinical curriculum, all students have community-based weeks during their family medicine, internal medicine, obstetrics-gynecology, and pediatrics rotations. In addition, there are outpatient experiences in psychiatry and outpatient surgery and neurology practices; the emergency medicine clerkship places students in more rural emergency settings as well. In addition, there are elective opportunities specifically in rural family medicine and community practices.
- Many students asked for additional hospital experiences, specifically in internal medicine, so we made a deliberate shift to additional time managing acute patients, while maintaining a balance with outpatient family medicine to help students recognize illness and understand the importance of preventive care and lifestyle medicine. One of our biggest challenges has been the desire of students to see patients in the Children's Hospital as well as labor and delivery in the affiliated tertiary care hospital, where student numbers are limited to ensure a robust learning experience. We have been fortunate to engage community physicians with the support of the clinical departments.

See Table 2-Ambulatory and Inpatient Experiences During Clerkships.

\section{Curricular Governance}

The faculty committee with primary responsibility for the oversight and management of the curriculum is the Curriculum Committee. The curriculum is centralized, with support provided to the various departments through administrative staff from the SOM and a transfer of dollars to cover clinical teaching and administrative roles.

See Figure 1-Curricular governance committees.

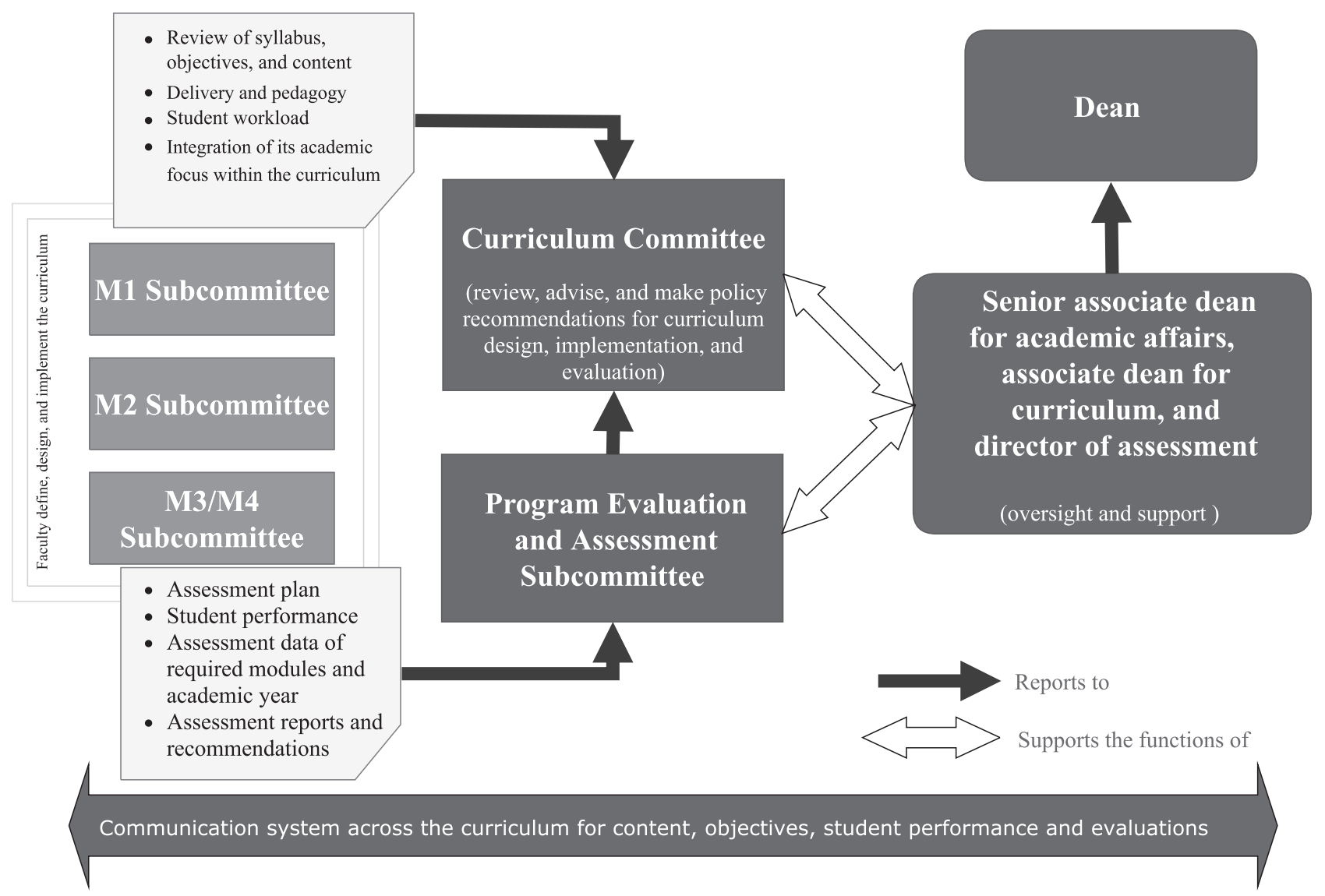

Figure 1 Curricular governance committees. 


\section{Educational Staff}

The Office of Academic Affairs is responsible for the planning, implementation, evaluation, and oversight of the curriculum and for the development and maintenance of the tools to support curriculum delivery, monitoring, and management. The associate dean for curriculum is supported by 2 curriculum managers, 1 focused on preclinical and the other on clinical. Curriculum coordinators provide support for all 4 years. The senior associate dean oversees the Department of Academic Affairs, including the faculty director for assessment, manager of assessment, and assessment coordinator.

The organizational structure includes the dean as the lead for the school and the chief academic officer for the health system. The senior associate dean for academic affairs serves under the dean as the chief operations officer. Other associate deans include curriculum, GME, student affairs, faculty affairs, institutional culture and inclusivity, research, and a director for business operations and finance. The senior associate dean for academic affairs oversees student affairs and supports the educational program across the continuum.

\section{Faculty Development and Support in Education}

The Office for Faculty Affairs and Professional Development supervises the broad set of responsibilities related to faculty development with a full-time associate dean and a full-time director focused entirely on faculty development. In addition, the Office for Academic Affairs employs a director of instructional design who provides faculty development through programs and individual consultation on instructional issues, technologies, and learning theory.

Faculty also have access to professionals in the University's Center for Teaching Excellence. These full-time staff are available for consultation regarding instructional design and general educational questions and concerns (https://www.sc.edu/about/ offices_and_divisions/cte/consultations/index.php).

\section{Role of teaching in promotion and tenure}

The University of South Carolina is a Carnegie Research institution, and as such subscribes to a model where faculty must demonstrate a national or international reputation to progress to the rank of professor. This must be accompanied by tangible evidence of excellence in both scholarship and teaching. Research and scholarship including medical educational research are essential and highly valued elements in the life of the faculty member. In preparation for annual reviews, faculty compile documentation of the evidence of their accomplishments, which are included in their supplementary file for promotion.

\section{Initiatives in Progress}

- The school is currently in the process of developing the Academy for Educators, which will be open to biomedical and clinical science faculty who demonstrate excellence and a promise of growth in field of medical education.

- The Curricular Refresh team will convene in April 2020 to review vertical integration documents and Curriculum Committee recommendations in an effort to integrate valuebased care and additional health system science initiatives, while enhancing commitment to the community.

\section{References}

1 Blackwell TH, Halsey RM, Reinovsky JH. Emergency medical technician training for medical students: A two-year experience. Prehosp Emerg Care. 2016;20:518-523.

2 American College of Lifestyle Medicine. https://lifestylemedicine.org/ What-is-Lifestyle-Medicine. Accessed April 6, 2020.

3 Trilk JL, Elkhider IA, Asif I, et al. Design and implementation of a lifestyle medicine curriculum in undergraduate medical education. Am J Lifestyle Med. 2019;13:574-585

4 Hartke A, Devon EP, Burns R, Rideout M. Building a boot camp: Pediatric residency preparatory course design workshop and tool kit. MedEdPORTAL. 2019;15:10860.

5 American College of Surgeons. Division of Education. ACS/APDS/ASE Resident Prep Curriculum. facs.org/education/program/resident-prep. Accessed April 6, 2020.

6 Englander R, Cameron T, Ballard AJ, Dodge J, Bull J, Aschenbrener CA. Toward a common taxonomy of competency domains for the health professions and competencies for physicians. Acad Med. 2013;88:10881094.

7 Bodenheimer T, Sinsky C. From triple to quadruple aim: Care of the patient requires care of the provider. Ann Fam Med. 2014;12:573-576. 


\section{East Tennessee State University James H. Quillen College of Medicine}

K. Ramsey McGowen, PhD, and Kenneth E. Olive, MD

\section{Medical Education Program Highlights}

The Quillen College of Medicine (QCOM) mission is to educate future physicians, especially those with an interest in primary care, to practice in underserved rural communities. The curriculum consists of a preclerkship phase and a clinical phase. Significant integration is accomplished through coordination among courses and through the Doctoring course sequence, a comprehensive clinical skills course that builds on concepts from other courses and provides the clinical skills foundation required for the practice of medicine.

\section{Rural primary care track}

- Longitudinal community-based parallel curriculum for students interested in rural primary care.

- Learning objectives equivalent to generalist track with alternate pedagogy based on rural community-based experiences.

\section{Interprofessional education}

- Formal component of the curriculum since the introduction of an interprofessional communication course in the late 1990s:

- Medical, nursing, pharmacy, and clinical psychology students work with standardized patients and interprofessional faculty facilitators in small groups.

- Elective 2-year interprofessional education (IPE) pilot program for a subset of students introduced in 2012:

- Based on the IPE collaborative competencies (values and ethics, roles and responsibilities, interprofessional communication, teams and teamwork).

- Additional disciplines include nutrition, speech pathology, audiology, physical therapy, public health, and social work.

- Students participated in a 2 -year sequence of interactive small-group workshops.

- Required IPE sequence for all first-year students from medicine and pharmacy along with smaller numbers of students from the other disciplines began in 2018.

- Interprofessional experiences embedded in third-year clerkships, for example, interprofessional home visit in family medicine clerkship.

\section{Doctoring curriculum}

- Four-year longitudinal clinical skills curriculum (Doctoring courses) created by consolidating existing separate courses plus enhanced content in systems-based practice, patient safety, and pain management.

Acad Med. 2020;95:\$469-S473.

doi: 10.1097/ACM.0000000000003323

Copyright (C) 2020 by the Association of American Medical Colleges

Supplemental digital content for this article is available at http://links.Iww.com/ ACADMED/A852.

Year school was established: 1974.

School URL: https://www.etsu.edu/com/.
- Focus on clinical skills, medical decision-making, professional development, and professional identify formation.

- Pedagogy primarily interactive and experiential and emphasizes simulation with task trainers, high-fidelity mannequins, and standardized patients.

- Includes required IPE experiences.

- Doctoring III includes interactive seminars around themes of preparing to practice medicine in the real world, for example, clinical reasoning, medical jurisprudence, patient safety.

\section{Community medicine clerkship}

- Six-week required clerkship in a rural and underserved county.

- Focuses on population health, prevention, health education, access to care, interprofessional care, multiple roles of the physician in a community, cultural competence, evidencebased medicine, the role of nonclinical components of the health care system, and social determinants of health.

- Service-learning activities include participation in a rural outreach health fair and performing an epidemiologic assessment of the community in which they conduct the health fair.

\section{Curriculum}

\section{Curriculum description}

See Supplemental Digital Appendix 1-Generalist Track Curriculum—at http://links.lww.com/ACADMED/A852.

See Supplemental Digital Appendix 2-Rural Primary Care Track Curriculum—at http://links.lww.com/ACADMED/A852.

\section{Curriculum changes since 2010}

- Initiated required 2-year sequence of IPE for all students.

- Developed Doctoring courses.

- Implemented community medicine clerkship.

- Duration of preclerkship phase reduced to 73 weeks in 2017.

- Integration increased in preclerkship foundational science courses through sequencing and coordination among course directors, although structure remains largely discipline based.

- Incorporated 6 weeks of protected USMLE Step 1 examination study time between end of the second year and beginning of clinical clerkships.

- Advanced start date of fourth year to allow students more time for individualized preparation before completing residency applications.

\section{Changes in class size since 2010}

- Class size increased to 72 students in academic year 2012-2013.

- Class size increase associated with the creation of a required 6-week community medicine clerkship in an additional rural community. 


\section{Assessment}

See Chart 1-Medical Education Program Objectives.

Changes in student assessment since 2010:

- Pass/fail grading implemented in academic year 2019-2020.

- Computerized exam administration across curriculum.

- Increased use of formative assessment through active learning strategies (e.g., flipped classroom, problem-based learning, team-based learning).

- Adopted use of NBME Comprehensive Basic Science Examination for formative assessment.

- Increased use of online modules with embedded assessments as a part of course structures.

\section{Medical education program objectives}

- Program objectives were developed in 2014 based on the general expectations of the Physician Competency Reference Set.

- Current educational objectives include minor revisions resulting from ongoing curricular review.

Parallel curriculum or tracks

- Single parallel track (rural primary care track [RPCT]), up to 16 students per class: optional experiential track to which students apply after admission.

- The goal is to educate physicians to practice in underserved rural communities.

\section{Chart 1}

\section{Medical Education Program Objectives}

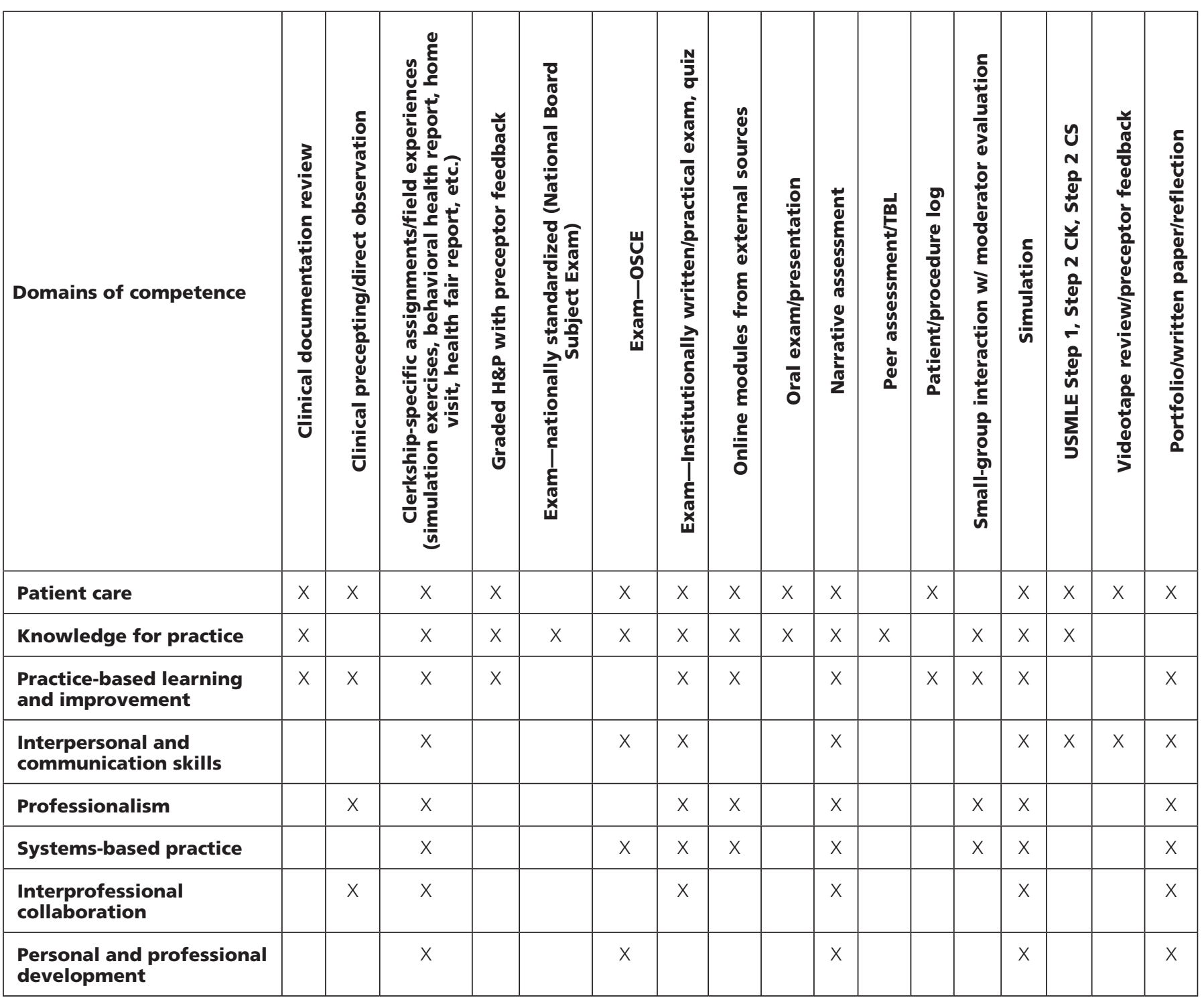


- Emphasizes community immersion, rural culture, and leadership development.

- RPCT experiences begin in year 1 and continue across all 4 years.

- Preclerkship RPCT students spend 1 day each week in a rural setting.

- RPCT students complete components of Doctoring I and II (clinical skills) courses at rural sites.

- Preclerkship RPCT students take 2 courses not taken by generalist track students: Rural Health Research and Practice (first year) and Rural Community-Based Health Projects (second year).

- Third-year RPCT students complete a longitudinal 12-week clerkship in a rural community, replacing 6-week family medicine and community medicine clerkships taken by generalist track students.

- Community-based health intervention projects in both preclerkship and clerkship phases.

- Fourth-year students perform 1 required selective in a rural setting.

- Overall students obtain approximately $25 \%$ of their education through rural experiences.

- RPCT students are more likely to pursue family medicine, primary care, and practice in rural or underserved areas.

\section{Pedagogy}

Preclerkship phase:

- All preclerkship courses use multiple pedagogical methods, including lectures, online modules, laboratories, simulation, and large- and small-group discussions.

- Several courses employ self-directed learning.

- Team-based learning is the primary methodology in 1 course and is a component of others.

- Preclerkship clinical skills courses employ case-based and problem-based learning, standardized patients, ambulatory clinical experiences, workshops, precepting, and large- and small-group discussions.

- Video and podcasting are integral to instruction since all lectures are recorded.

- Several courses employ service learning.

Clinical phase:

- Primary instructional methods in the clinical phase are inpatient and ambulatory clinical experiences.

- Self-directed learning, simulation, standardized patients, lectures, small-group discussions, precepting, and workshops are widely used throughout the clinical phase.

- Service learning is used in multiple clerkships.

Changes in pedagogical approaches since 2010:

- Increased emphasis on service learning in Doctoring I course, community medicine, family medicine, and pediatrics clerkships.

- Required 2-year IPE component of preclerkship curriculum.

- Addition of ultrasound and increased simulation.

- Recorded lectures for asynchronous viewing in all preclerkship courses.
- Team-based learning in selected classes and increased active learning pedagogies in all courses.

- Resequencing and coordination of foundational science content.

- Enhanced use of educational technology.

\section{Clinical experiences}

Generalist track:

- Longitudinal clinical preceptorship experiences occur in community physicians' offices in first and second years.

- Faculty practices are the primary sites for ambulatory experiences in the third and fourth years.

- Inpatient experiences occur at 4 local medical centers: Johnson City Medical Center, Bristol Regional Medical Center, Holston Valley Medical Center, and James H. Quillen Veterans Affairs Medical Center.

- LeConte Medical Center supports the community medicine clerkship.

RCPT:

- Experiences in rural sites use physician offices, health departments, nursing homes, federally qualified health centers, and community hospitals.

\section{Required longitudinal experiences}

- Two semester preceptorship experience in first year at 1 site.

- One semester preceptorship experience in second year at a different site.

- Required 2-year IPE component of preclerkship curriculum.

\section{Clinical experience first encounter}

- First clinical encounters occur during the first month of medical school.

\section{Required and elective community-based rotations}

Required:

- All inpatient experiences are conducted in community-based medical centers.

- Family medicine clerkship: rotation in 1 of 3 university community-based family practice centers.

Elective:

- Third-year 2-week electives use a variety of community-based specialty options.

- Senior electives: multiple specialty options are available, with over one-third in community-based settings.

Challenges in designing and implementing clinical experiences for medical students

- Merger of 2 major health systems into 1 has realigned some services requiring adjusting the location of student clinical experiences.

- Increase in other health professional training programs has increased pressure on community-based clinical resources, including osteopathic schools, physician-assistant programs, and advanced practice nurse programs. 


\section{Curricular Governance}

The QCOM curriculum committee is the Medical Student Education Committee.

\section{Decentralized curricular governance}

- Curricular governance is centralized at the curriculum committee level.

- Overall curriculum content, design, and management and curri culum policies are the responsibility of the curriculum committee.

- Course/clerkship administration such as staff support, faculty assignments, daily schedules, and content on assessments are determined at the department level.

- Course scheduling decisions, such as timing of labs, didactics, small-group assignments, and locations for clinical assignment, are scheduled at the department level within parameters outlined by the Office of Academic and Faculty Affairs.

- Curriculum committee policy (Course/Clerkship Governance) defines actions that are the responsibility of the Office of Academic and Faculty Affairs and the curriculum committee and those which are the responsibility of course directors and faculty members.

\section{Education Staff}

\section{Medical education leadership}

- The Office of Academic and Faculty Affairs is responsible for support of the medical student education program under the oversight of the executive associate dean for academic and faculty affairs.

- The associate dean for curriculum oversees planning, implementation, and evaluation of the curriculum and faculty development.

- The director of rural programs provides oversight for the RPCT.

- Six full-time administrative staff plus 6 support staff members coordinate implementation and evaluation of the curriculum, functioning of the curriculum committee and its subcommittees, management of the curriculum database, and accreditation compliance.
- Simulation and standardized patient programs are managed by the Office of Academic and Faculty Affairs. A senior director of experiential learning, a simulation director, a simulation lab manager, and a standardized patient coordinator administer these programs.

- Classroom technology is supported by 2 staff members within the Office of Academic and Faculty Affairs, with additional support from the university Office of Academic Technology Support.

See Figure 1-Decanal staff.

- The Office of Academic and Faculty Affairs is responsible for the administration of the undergraduate medical education program and for faculty affairs, including faculty development.

- Separate offices and associate deans are responsible for student affairs, graduate medical education, continuing medical education (CME), learning resources (medical library), and clinical affairs.

\section{Department of Medical Education}

- The Section of Medical Education within the Office of Academic and Faculty Affairs is responsible for faculty members and courses not primarily based in other departments.

- Six faculty members who function primarily as educators have their primary academic appointments in the Section of Medical Education. Five part-time faculty and 25 volunteer faculty have appointments in the Section of Medical Education.

- Four staff members support these activities.

\section{Faculty Development and Support in Education}

\section{Professional development for faculty as educators}

- Faculty development related to educational roles is available at both the institutional and departmental levels.

- The associate dean for curriculum organizes monthly faculty development sessions over a broad range of medical education topics relevant to both basic science and clinical faculty. CME credit is generally offered.

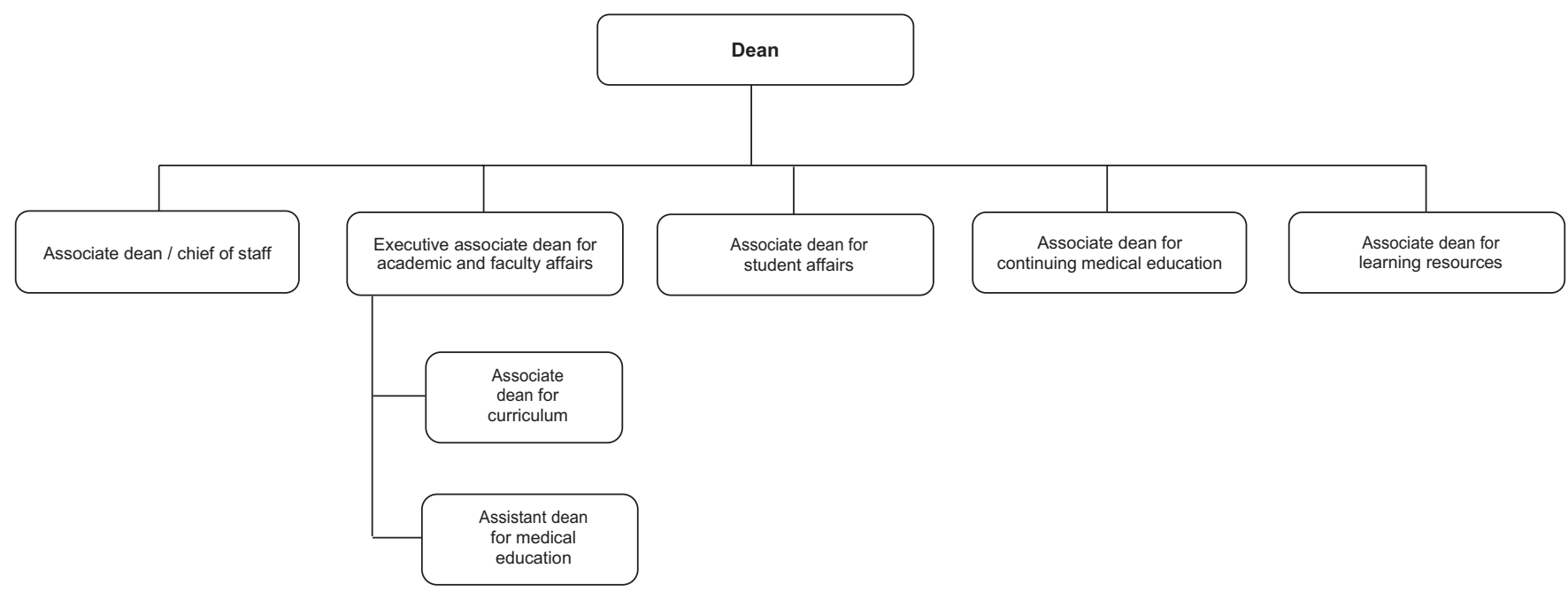

Figure 1 Decanal staff. 
- The Office of Academic and Faculty Affairs maintains a faculty development website with recordings of monthly faculty development programs and links to online resources.

- The vice chair for education in the Department of Biomedical Sciences organizes every-other-month faculty development sessions for preclerkship faculty focusing on educational roles and topics.

- The East Tennessee State University (ETSU) Center for Teaching Excellence organizes regular faculty development conferences and trainings, provides liaison between faculty development presenters and the QCOM faculty, and directly offers workshops and trainings to QCOM faculty.

- The ETSU Office of Academic Technology Services provides training, resources, and support for the use of technology in teaching.

- The Office of the Dean provides financial support for preclerkship course directors attendance at educationally oriented professional meetings.

- Department chairs provide financial support for clinical faculty to attend professional meetings for enhancement of discipline specific knowlege and development in faculty roles as teachers.

\section{Role of teaching in promotion and tenure}

- Teaching and education are central factors in decisions for promotion at all levels of advancement and for tenure.

- Criteria for determining if teaching and education contributions meet criteria for advancement are established at departmental level but generally includes consideration of:

- Teaching evaluations of "excellent" or better by students, positive peer evaluations of effectiveness, and nominations for teaching awards.

- Significant participation in at least 1 course or clerkship or equivalent residency teaching.

- Service as course/clerkship director or active roles in curricular planning or educational program management.

- Mentoring junior faculty in educational roles.

- Evidence of teaching outcomes through such measures as scholarly activity related to teaching published in peerreviewed publications, development of innovative teaching methods, authorship of textbooks, textbook chapters, lab manuals, podcasts; presenting at national/international meetings that focus on medical or biomedical science education; presentation of CME programs; contribution to funded education/training grants. 


\section{Vanderbilt University School of Medicine}

William B. Cutrer, MD, MEd, and Donald Brady, MD

\section{Medical Education Program Highlights}

- Curriculum 2.0: This is a responsive, agile system of lifelong learning designed to prepare students, through highly personalized education, to adapt and respond to a rapidly evolving health care system. Curriculum 2.0 focuses on how to learn and understand concepts and relations, more than memorizing facts.

- Innovative, personalized curriculum: Vanderbilt University School of Medicine (VUSM) offers early clinical exposure, placing students in a weekly continuity clinical experience in year 1 and core clerkships in year 2. In the 2-year postclerkship phase, students personalize learning experiences to facilitate professional growth and development.

- Competency-based assessment with portfolio coaching: Our programmatic assessment system with UME-specific competency milestones allows measurement from day 1 through graduation. The behavioral language guides student learning with explicit, standardized expectations, allowing for monitoring student progress over time. Assessment of Entrustable Professional Activities (EPAs) also informs student development. A comprehensive online learning portfolio captures assessment data, facilitating evidencebased self-assessments, faculty coaching, and personalized learning plans.

- Master clinical teachers: This program uses experienced clinicians to provide individualized attention to clinical skills development through direct observation and customized learning.

- Colleges program: Upon matriculation, students are sorted into 1 of 4 advisory colleges. Two college mentors lead each college and provide students a rich environment of academic/ career mentoring, general support, and encouragement. Students discuss professional development topics, wellness, and career exploration.

\section{Curriculum}

\section{Curriculum description}

Medical school in 3 phases:

- Year 1: Foundations of Medical Knowledge (FMK) provides students with a broad foundation of knowledge and skills focusing on conceptual understanding.

Acad Med. 2020;95:\$474-S477.

doi: 10.1097/ACM.0000000000003399

Copyright (C) 2020 by the Association of American Medical Colleges

Supplemental digital content for this article is available at http://links.Iww.com/ ACADMED/A908.

Year school was established: 1875.

School URL: https://medschool.vanderbilt.edu.
- The 1-week Foundations of the Profession course precedes FMK, providing an ethical and historical framework for understanding the practice and profession of medicine.

- Year 2: Foundations of Clinical Care (FCC) provides core clerkships in medicine, surgery, pediatrics, obstetricsgynecology, psychiatry, and neurology.

- A symptom-based framework of core clinical problems is coupled with a portfolio system tracking individual studentpatient encounters.

- Diagnostics and therapeutics threads throughout the FCC phase, explicitly addressing strengths and limitations of various diagnostic/therapeutic approaches and elucidating clinical reasoning.

- Years 3 and 4: The immersion phase is a highly individualized period using clinical context to enhance prior learning. Students deepen foundational science knowledge in meaningful clinical contexts, solidify clinical skills, and enhance practice-based learning skills, while expanding knowledge and skills related to scholarship, leadership, and professional development.

- 3 longitudinal courses span the curriculum:

- Research program fosters development of research acumen. It includes a tailored 3- to 6-month mentored research experience. During their first 2 years, students participate in research-focused coursework and training. An intensive 1-month project planning course follows, after which students engage in 3 to 6 months of full-time mentored research during years 3 and/or 4 .

- Learning communities use intentionally developed longitudinal groups of faculty and students to enhance students' professional development, specifically addressing metacognition, reasoning, ethics, service, leadership, and knowledge and understanding of the broader health care environment.

- The Foundations of Health Care Delivery (FHD) course allows students longitudinally to learn about health systems science while gaining important skills to function in and eventually modify those systems.

See Figure 1-Curriculum framework.

See Supplemental Digital Appendix 1-Curriculum Schematicat http://links.lww.com/ACADMED/A908.

\section{Curriculum changes since 2010}

- Curriculum 2.0 visioning began in 2008 with recognition that learning based on continuous development and assessment of competencies was needed for effective and compassionate care under challenging circumstances. Graduates should be able to assess practice outcomes, identify learning needs, and engage in continuous learning to provide the highest quality care. Core guiding principles are that learning should be competency based and embedded in the workplace. 


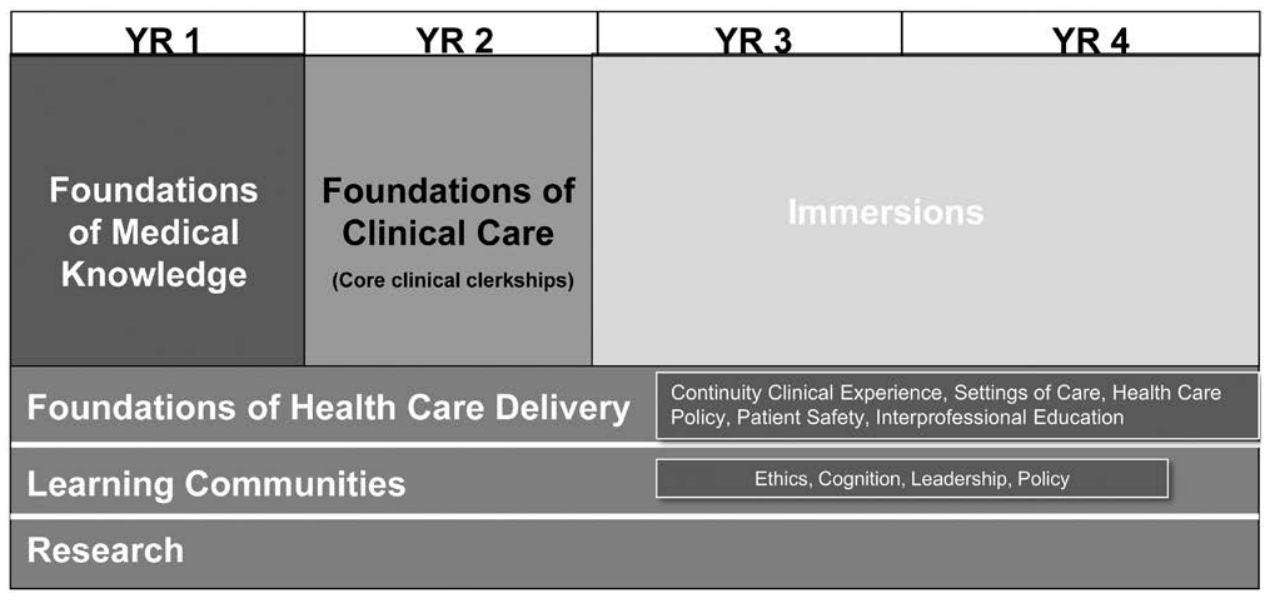

Figure 1 Curriculum framework.

- In 2013, the curriculum transitioned from a $2+2$ model to Curriculum 2.0. A hybrid phase facilitated the transition.

- We introduced a more explicit focus on leadership development, master adaptive lifelong learning, and health systems science.

- In addition to teaching CQI, we model this via our own CQI efforts, including a student curriculum committee and expansive program-wide evaluation system.

- No large-scale curricular changes are planned; ongoing refinement of Curriculum 2.0 will continue.

\section{Assessment}

- Vanderbilt's competency-based assessment system models the 6 ACGME competency domains with UME-specific behaviorally described milestones for each. We added EPAs to our assessment system in 2015.

See Supplemental Digital Appendix 2-Program Objectives and Assessment Methods—at http://links.lww.com/ACADMED/A908.

\section{Assessment changes since 2010}

Grading:

- FMK phase is pass/fail.

- Given the foundational nature of core clerkship experiences, the FCC phase is pass/fail.

- Immersion phase grades are honors/high pass/pass/fail. Grade determination combines focus on the medical knowledge domain and performance across the other 5 domains.

Competency-based programmatic assessment with portfolio coaching:

- By combining UME-specific competency milestones and core EPAs, over time students monitor progress and development with more explicit standardized expectations.

- Guided by a portfolio coach, students have frequent opportunities for formative feedback encouraging identification of areas for improvement.

\section{Pedagogy}

- Various learning modalities, guided by the learning issue being addressed, are used throughout each phase. Self-directed learning is emphasized.

- Within FMK, core science blocks use case-based learning, lecture, laboratory, team-based learning, gross anatomy dissection, virtual patients, and large-/small-group discussions. Physical diagnosis uses role play/dramatization, simulation, standardized/simulated patients, and clinical experiences in various settings.

- Within FCC, core clerkships emphasize clinical experience within the inpatient and ambulatory settings, supplementing with other strategies such as large- and small-group discussions, simulation, standardized/simulated patients, lectures, workshops, peer teaching, and preceptorship.

- In the immersion phase, students experience various different pedagogical approaches depending on the course type.

- Advanced clinical experiences (ACEs) and acting internships primarily use clinical experience supplemented with other strategies (group discussions, simulation, lectures, peer teaching).

- Integrated science courses (ISCs) deepen foundational science understanding within a meaningful clinical context by pairing simulation, team-based learning, case-based learning, gross anatomy dissection, and lecture with targeted clinical experiences.

\section{Changes in pedagogy since 2010}

- During FMK phase, lectures were deemphasized, case-based learning became core to the weekly schedule, and team-based learning was added phase wide.

- During FCC phase, use of flipped classroom strategies and morning report-style case-based discussions have increased, complementing clinical experiences.

- For the immersion phase, Vanderbilt created a new type of course, the ISC.

- ISCs reinforce the foundational sciences that underpin clinical medicine. 
- Foundational science includes traditional preclerkship sciences, social and behavioral sciences, health services and population science, quantitative methods, and informatics.

- Students engage in meaningful clinical experiences related to foundational science topics, promoting workplace learning.

- ISCs ensure students learn to integrate foundational science knowledge into clinical scenarios through exploration of patient evaluation and management plan development.

\section{Clinical experiences}

- Most clinical education takes place in the VUMC campus, including Vanderbilt University Hospital, the Vanderbilt Clinic, Monroe Carell Jr. Children's Hospital at Vanderbilt, Vanderbilt Psychiatric Hospital, and Vanderbilt Stallworth Rehabilitation Hospital.

- Students also learn at the Nashville VA Hospital, on the Vanderbilt campus.

- We work with partners in the Vanderbilt Health Affiliated Network to provide experiences at local community hospitals, for example, obstetrics-gynecology rotations at NorthCrest Medical Center.

- Our pediatric clerkship, as well as other primary care courses, use ambulatory facilities in community settings throughout Nashville.

\section{Required longitudinal experiences}

- In FHD, students have a weekly continuity clinical experience beginning in September of year 1 .

- FHD offers a longitudinal experience to learn about systems of health care as well as foundational skills to better understand how health systems function.

- Topics explored include patient-centered care, team-based care, medication reconciliation, patient education and health coaching, social determinants of health, and advocacy.

\section{Required and elective community-based rotations}

- Students have several opportunities, especially during the postclerkship immersion phase, to work in community settings.

- Students during both the FCC and immersion phases learn at the Nashville VA Hospital.

- Pediatric clerkship students rotate at pediatric clinics throughout Nashville and surrounding areas.

- Immersion phase students must complete a primary care experience, which can be fulfilled via:

- Primary Care Advanced Clinical Experience (ACE) at various clinics around Nashville and surrounding areas

- Shade Tree Clinic (student-run free clinic) ACE, where students deliver care, guided by faculty directors, to underprivileged patients from Nashville

- Community Health ISC, combining primary care clinical experiences in community settings with classroom didactics on population health, social determinants, and health equity.

Challenges in designing and implementing clinical experiences for medical students

- Curriculum 2.0, through addition of a first-year longitudinal clinical experience and transition of core clerkships into year 2, added more than 100 new students into clinical settings.

- Having learners in all 3 phases active in clinical settings challenges us to prepare teachers for engagement with learners of all stages.

- Growing interest in health equity and social determinants of health pushes us to find more clinical experiences where students can integrate clinical skill and patient/communitycentered learnings.

- Our competency-based learning system, because of earlier clinical engagement of students, challenges us to provide students more competency-based role responsibility within current curricular and licensure-based restrictions.

\section{Curricular Governance}

- Curricular governance is a primarily centralized function.

- The senior associate dean for health sciences education (SADHSE) semiannually reviews with each department chair budgets for teaching and all central allocations to departments for teaching, course oversight, and course directorships.

- FMK and ISC course directors are compensated on a percent effort basis, at a level agreed upon by the SADHSE, associate dean for UME, faculty member, and appropriate department chair.

- For interdisciplinary courses, teaching support comes directly from VUSM.

- For core clerkships, course directors are compensated directly by departments on a percent effort basis.

\section{Education Staff}

Dean's Office staffing for medical education:

- The dean delegates to the SADHSE responsibility for oversight of all aspects of physician education, from admissions process to CPD.

See Figure 2-Organizational chart.

Office of Health Sciences Education (OHSE):

- OHSE, overseen by the SADHSE, governs a range of educational activities at VUSM and VUMC, including UME, GME, physician CPD, and advanced degree and certificate programs in related fields.

- OHSE oversees student affairs and the Office of Enrollment Services.

- OHSE provides many services supporting these academic programs, including education technology and informatics, simulation programs, educator development programs, financial and administrative oversight, student services, and education research resources.

- The SADHSE and associate dean for UME sponsor an annual curriculum summit.

- Vanderbilt has a small Division of Medical Education and Administration. Most faculty members, and all new faculty, have primary appointments in a clinical or basic science department. 


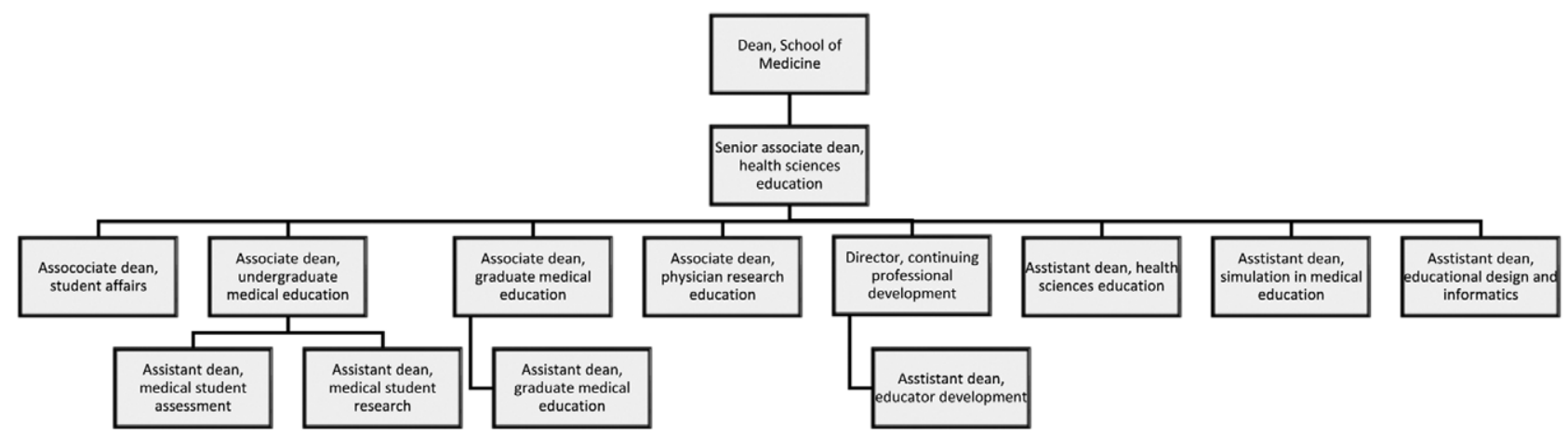

Figure 2 Organizational chart.

Office of Undergraduate Medical Education (OUME):

- OUME has a strongly collaborative culture. The associate dean for UME and administrative director cultivate teamwork and enthusiasm for continuous improvement across a diverse group who oversees provision of appropriate learning materials to students and faculty via the learning management system, secure space for educational sessions, and facilitate collection of accurate student assessment data via an online portfolio platform.

- 4 OUME staff program managers oversee program coordinators/assistants daily work and collaborate with faculty phase/program directors to ensure impactful learning opportunities.

- OUME uses multiple curricular teams (FMK, FCC, and immersion phases; longitudinal elements; evaluation and assessment) and specific program teams (Research, Vanderbilt Program for Interprofessional Learning, Medical Innovators Development Program).

- Overall function and effectiveness are monitored to guide measurement activities, conduct outcomes analysis, and facilitate programmatic improvements.

\section{Faculty Development and Support in Education}

Professional development for faculty as educators

- 2 regularly scheduled lecture series support educators: Flexner dean's lecture series and health sciences education grand rounds.
- Our educator development program within OHSE offers education workshops and provides individual consultations to programs and departments on request.

- Health Professions Education Research Day annually highlights health professions education scholarship.

- The GME office cosponsors with ACGME an annual 3-day faculty development course on assessment and feedback.

\section{Role of teaching in promotion and tenure}

- For both tenure and basic science educator/clinician-educator tracks, teaching is an important appointment and promotion criterion and requires an educator's portfolio.

- The educator track requires demonstration of excellence in teaching.

- The investigator track requires, at minimum, demonstration of a high level of effectiveness.

- For the clinical practice track, any teaching predominantly occurs in the course of clinical duties.

- The Academy for Excellence in Education (AEE) advocates for development, support, and recognition of dedicated educators; sustains a community of educators; and fosters educational excellence, innovation, leadership, and scholarship.

- The AEE organizes health sciences education grand rounds, to discuss recent advances in teaching and learning in medicine, and the annual John E. Chapman Lecture in Medical Education (in cooperation with the Flexner deans' lecture series).

- The AEE annually inducts approximately 5 new members. Candidates are self-nominated or peer nominated. 


\section{Baylor School of Medicine}

Nadia Ismail, MD, MPH, MEd, Jennifer Christner, MD, Nital Appelbaum, PhD, and Alicia Monroe, MD

\section{Medical Education Program Highlights}

- Baylor College of Medicine's School of Medicine (BCM SOM) is a top-ranked, lowest-tuition, private medical school with access to the resources of the world's largest health science center, including diverse faculty and students who support patient-centered and community-engaged health care.

- In 2014, BCM SOM entered a joint venture with Catholic Health Initiatives (CHI) to establish an academic private, adult hospital, Baylor St. Luke's Medical Center. It is jointly owned and governed by Baylor and CHI St. Luke's Health.

- Medical students gain clinical skills and experience caring for diverse populations with common as well as rare and complex conditions in a wide variety of health systems:

- A single payer system (VA Medical Center)

- A private hospital (Baylor St. Luke’s Medical Center)

- Underserved populations in a public health system (Harris Health System-Ben Taub Hospital and community clinics)

- The top 5 ranked children's hospital (Texas Children's Hospital)

- A psychiatric hospital (the Menninger Clinic)

- A rehabilitation hospital (Memorial Hermann Institute for Rehabilitation \& Research, TIRR)

- In an effort to allow more flexibility in scheduling and exploration of interests, required core clerkship rotations were shortened to 12 months.

- BCM was also one of the first medical schools to make its foundational curriculum 18 months in 2001 and was one of the first institutions to move the USMLE Step 1 examination to after clinical rotations. This allows students to have the experience on the NBME subject exams and clinical experiences to better prepare for Step 1.

- In 2019, BCM's Team Launch program developed a required "launch pad" curriculum for all BCM students to foster evidence-based teamwork skills needed to care for our growing and complex patient population. Curriculum is delivered in an interprofessional format with students and facilitators from BCM's 3 health sciences schools. Topics include team training, leadership skills, effective communication, team cognition, cooperation, conflict management, negotiation methods, and strategies for building and optimizing high-performing teams.

Acad Med. 2020;95:S478-S481.

doi: 10.1097/ACM.0000000000003327

Copyright (C) 2020 by the Association of American Medical Colleges

Supplemental digital content for this article is available at http://links.Iww.com/ ACADMED/A856.

Year school was established: 1900.

School URL: https://www.bcm.edu.

\section{Curriculum}

\section{Curriculum description}

The foundational curriculum spans the first 18 months, while the remaining years focus primarily on clinical exposure.

See Supplemental Digital Appendix 1-Curriculum Map-at http://links.lww.com/ACADMED/A856.

\section{Curriculum changes since 2010}

- BCM SOM is in the midst of a curriculum renewal with anticipated implementation by 2022. Currently, the Curriculum Committee (CC) is analyzing the medical school's program outcomes data to ensure SOM students are prepared to practice medicine in 2030 and beyond.

- New courses since 2010 include Determinants, Disparities, and Social Health of Populations; Critical Thinking and Problem Solving; and Translational Research and Population Health. These courses broadened the scope of content from the prior curriculum to address the growing needs to educate our students on the social determinants of health and how to mitigate them.

- Our medical school class size has remained stable at 187 students admitted per year since 2010.

\section{Assessment}

- Program objectives are based on the ACGME domains of competence, AAMC Core Entrustable Professional Activities, and the Physician Competency Reference Set. A blend of these 3 frameworks has resulted in our own framework, called the Core Competency Graduation Goals (CCGG). The CCGGs comprise 7 competency areas, each with at least 3 major objectives.

- The Division of Evaluation, Assessment, and Research works on innovative methods for student assessment and program evaluation (e.g., development of a universal student assessment form for clinical curriculum, clinical skills assessment through simulation).

See Supplemental Digital Appendix 2-Core Competency Graduation Goals and Mapped Assessment Methods—at http:// links.lww.com/ACADMED/A856.

\section{Pedagogy}

Since 2010, our pedagogical approaches have evolved to integrate more active learning (e.g., case-based learning, team-based learning, self-directed learning, standardized patients) into the curriculum. Faculty development opportunities have also been integrated on such pedagogies to foster expertise in adult learning theories.

Pedagogical approaches used to achieve our medical education objectives include:

- Case-based learning

- Clinical experience: ambulatory and inpatient 
- Discussion: large (>12) and small group $(\leq 12)$

- Laboratory

- Lecture

- Peer teaching

- Preceptorship

- Role play/dramatization

- Self-directed learning/tutorial

- Simulation

- Standardized/simulated patients

- Team-based learning

- Video/podcast

- Workshop

\section{Clinical experiences}

Medical students rotate through a diverse set of clinical sites for required educational experiences at BCM SOM. Private and public health systems within a densely located medical center allow our students to gain both ambulatory and inpatient experiences. The medical school also has relationships with physicians in private practice settings across Texas, and over 100 voluntary faculty that enhance nonacademic medicine experiences.

- Current affiliate clinical sites include:

- Baylor St. Luke's Medical Center

- Harris Health System

- MD Anderson Cancer Center

- Menninger

- Texas Children's Hospital

- Children's Hospital of San Antonio

- VA Medical Center

- TIRR Memorial Hermann

- Early exposure to patient interaction and clinical experiences is priority at BCM SOM. Clinical experiences are required starting in August of the first year of medical school and lasts through the fourth year. Students start clinical rotations during their second year through the end of medical school, and the shortening of core clerkships allows BCM SOM students greater flexibility in exploring career paths earlier in the curriculum.

- The Patient, Physician, and Society (PPS) course allows early clinical exposures through a longitudinal preceptorship experience. Additional formative "patient" experiences are provided during the entire first year through the simulation lab and Standardized Patient Program.

- We have multiple opportunities for primary care experiences including community-based rotations through the family medicine clerkship and assignments with community-based preceptors in the PPS 1 and 2 courses.

- Recruiting and retaining voluntary community faculty members remains a challenge for BCM SOM. We are unable to offer any incentives with the exception of a voluntary BCM faculty appointment.

\section{Curricular Governance}

The CC at BCM SOM has sole authority to oversee the entire medical education program to ensure that the school complies with LCME standards and that students fulfill the CCGGs, through the work of several committees.

The CC is responsible for the development and implementation of the medical educational program including program and learning objectives, assessment/evaluation methods, and design of content and its sequencing.

There is a collaborative relationship between departmental chairs and BCM SOM educational leaders, since the clinical curriculum is delivered within the clinical departments. Through departmental chair support, the medical school dean meets with the departmental vice chairs for education quarterly to ensure continued progress on BCM SOM's curricular mission. The vice chairs have a dual report to their departmental chair and to the dean of the school of medicine. Further, all course and clerkship directors have a dual report to their departmental vice chair of education and the associate dean of curriculum. There are well-defined job descriptions for all educational leadership positions.

- The BCM Provost Office supports educational leadership and teaching activities through teaching dollar support. Medical school educational leadership collaborates with the departmental leadership (chairs, vice chairs for education) to identify and support individuals for teaching faculty roles. For example, clerkship directors are supported through teaching dollars and identified by joint decision-making between the departments and the Curriculum Office (CO).

- BCM is also positioned to develop individual faculty and establish leadership goals on a yearly basis so there is fair evaluation of members who are supported by teaching dollars. There is a review of teaching metrics for faculty and an annual renewal process; teaching performance data are also communicated back to the departmental chairs.

See Figure 1-Curricular organizational chart.

\section{Education Staff}

- The CO and curriculum leadership work together to provide administrative support and resources to implement the decisions of the CC.

- Since 2010, BCM SOM administrative and academic support for planning, implementation, evaluation, and oversight of the curriculum has grown to include a new position, the director of accreditation, who maintains oversight of accreditation and continuous quality improvement. The CO, with support of the Division of Evaluation, Assessment, and Research, provides planning, implementation, evaluation, and oversight of the foundational curriculum. The director of administrative operations is responsible for the oversight of 6 coordinators, the curriculum mapping specialist, and a project manager. Course directors and coordinators meet regularly with the director of administrative operations to ensure a shared mental model of curricular activities and establish clear communication within and external to the CO.

- More recently, interdepartmental offerings such as subinternship education days, interprofessional education 


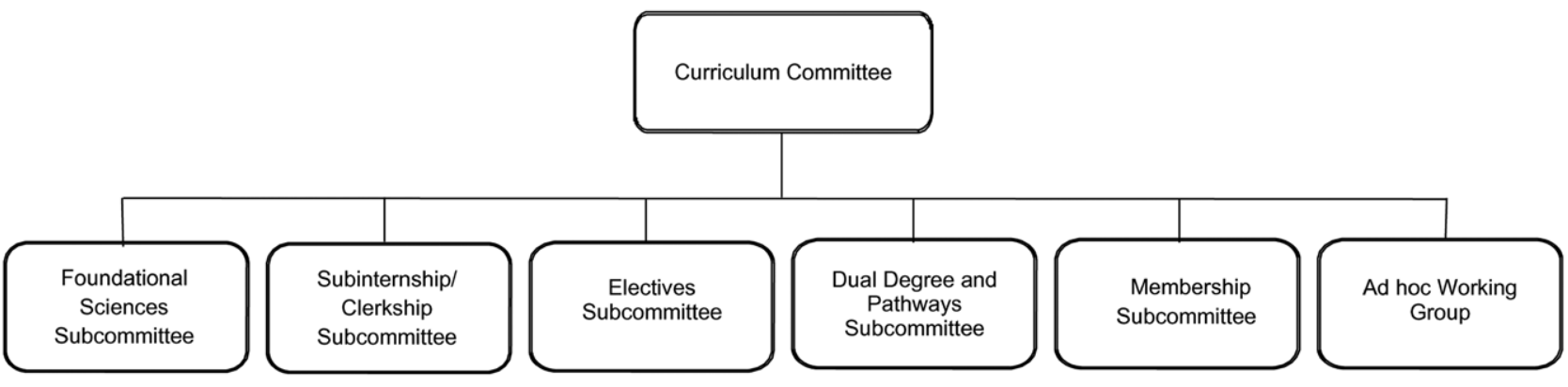

Figure 1 Curricular organizational chart.

activities/curriculum, and curriculum data management (curriculum mapping) are also supported centrally by CO.

- The medical school dean is responsible for the entire continuum of medical education including undergraduate and graduate medical education, and continuing professional development. Within this oversight includes areas outside of educational programing (e.g., admissions; student affairs; evaluation, assessment, and research).

See Figure 2-Dean's organizational chart.

\section{Faculty Development and Support in Education}

BCM's Office of Faculty Development (OFD) offers a wide range of professional development opportunities for faculty at all stages of their career. From initial onboarding sessions to 1-on-1 coaching sessions to provide guidance on career progression, readiness for academic promotion, and development of award portfolios, the OFD invests in the growth of BCM faculty.
Medical school faculty can participate in several development opportunities designed to promote teaching skills:

- Master Teachers Fellowship Program: A 2-year program to develop faculty members' knowledge (i.e., key educational principles and theories), skills (i.e., lecturing, facilitating, designing, evaluating), and ability to serve as an educational leader.

- Peer Coaching for Educators: A program in peer coaching for any BCM faculty member who wants to have his/her educational activities reviewed in various settings including lectures, grand rounds, clinical rounds, small group-based learning, and 1-on-1 interactions. Each faculty is assigned a coach and includes confidential discussions, observation, feedback, and a formal letter of documentation for teaching awards and/or promotion.

- Workshops: Faculty can register for lunchtime and afternoon development workshops that focus on topics such as educator bootcamps, creating an educational portfolio,

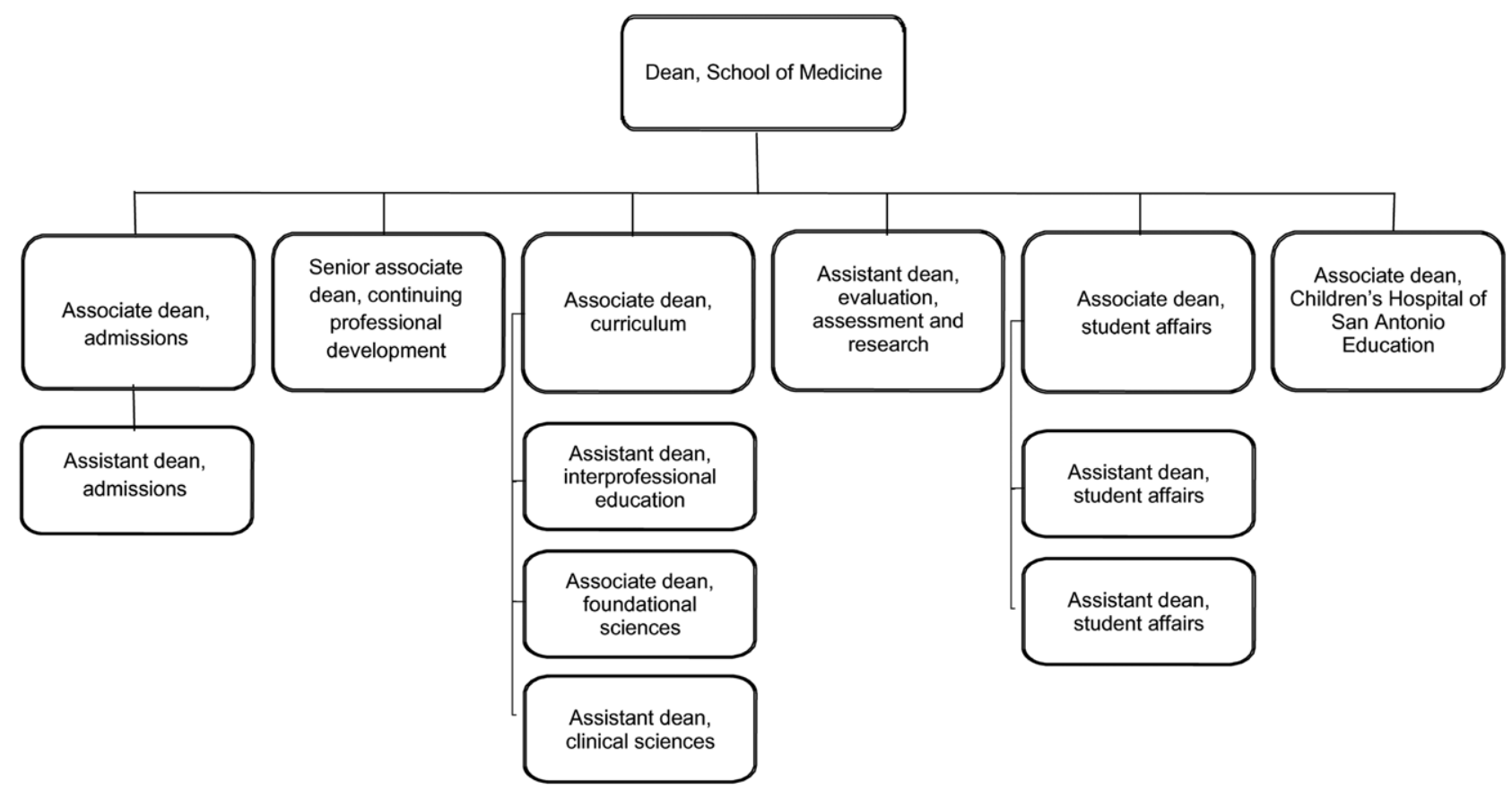

Figure 2 Dean's organizational chart. 
guidance on promotion, refining one's curriculum vitae, and implementation of small-group teaching. An all-day teaching-oriented symposium is also offered annually through partnership with other local medical schools.

A core component of faculty development includes recognition of strong educators at our institution. The OFD oversees 3 awards to promote our educational mission:

- Barbara and Corbin J. Robertson, Jr. Presidential Award for Excellence in Education: BCM's most prestigious, college-wide, competitive award for faculty educational service. Awardees are recognized for shaping the lives of students through innovative teaching methods and mentoring to prepare trainees to adopt the mantle of leadership regardless of the career path they choose.

- Norton Rose Fulbright Faculty Excellence Award: A criterion-based program that recognizes faculty contributions in 4 categories: teaching and evaluation, development of enduring educational materials, educational leadership, and educational research.

- John P. McGovern Outstanding Teacher Award: A studentnominated award that recognizes faculty for outstanding teaching in the medical school.
BCM promotion and tenure decisions require an education portfolio for teaching faculty. The portfolio must state the quantity of contributions (length of service/instruction, number of learners, etc.) and describe the specific role of the faculty member. It must also include, if applicable, a quantitative summary of all teaching critiques and reviews by students and peers. If assessments of the outcomes of the teaching are available, these should be included in the portfolio. The Faculty Appointments and Promotions Committee must judge quantity of teaching, for example, whether the educational activities are a major, moderate, or minor proportion of time and effort and more important, whether or not the quality is below average, average, above average, or outstanding.

BCM faculty are eligible for membership into an academy based on their acceptance of a Norton Rose Fulbright Faculty Excellence Award. The Academy of Distinguished Educators' mission is to recognize educational achievements and teaching contributions of individual faculty; promote professional development, innovation, and excellence in education; and promote a free exchange of ideas regarding educational theory and methods. 


\title{
McGovern Medical School at The University of Texas Health Science Center at Houston
}

\author{
Patricia M. Butler, MD, Christine Ford, EdD, and Allison R. Ownby, PhD, MEd
}

\section{Medical Education Program Highlights}

The curriculum of McGovern Medical School (MMS) integrates basic science and clinical experiences with an emphasis on active learning in small groups and new pedagogical methods. Weekly opportunities for student self-assessment and frequent feedback from faculty engage students in shaping their learning experiences. During the second semester, students begin a yearlong longitudinal patient care experience supervised by community preceptors. Required core clerkships begin in the spring of the second year. The accelerated schedule provides more elective opportunities for students to focus on career interests and residency preparation.

MMS offers a variety of programs that enhance and extend the core curriculum:

- There are 14 interdisciplinary scholarly concentrations available to students to enrich their educational experiences through learning and scholarly activities. Students enrolled in a concentration are required to complete a scholarly project before graduation.

- Career focus tracks (CFTs) provide a baseline structure to the fourth year based on career aspirations, as well as more formal career mentoring, while maintaining the flexibility to pursue additional areas of interest. The 4 CFTs - applied anatomy, primary care, acute care, and academic career-provide guidance and mentorship to students in the specialty to which they plan to apply.

- A poverty simulation occurs at the end of the second year before the students begin the clerkships. The simulation assigns all students to play the role of a family member experiencing varying degrees of poverty. The goal of the simulation is to enhance students' awareness of the realities of their impoverished patients in hopes that they will deliver culturally appropriate and economically sensitive care as well as to identify community resources to address their patients' medical and nonmedical needs.

- During the Transition to Clerkships course, all the secondyear students participate in an interprofessional mass casualty simulation. MMS students work with UTHealth students from the Cizik School of Nursing, the School of Public Health, the School of Dentistry, and the School of Biomedical Informatics

Acad Med. 2020;95:S482-\$485.

doi: 10.1097/ACM.0000000000003430

Copyright (C) 2020 by the Association of American Medical Colleges

Supplemental digital content for this article is available at http://links.Iww.com/ ACADMED/A928.

Year school was established: 1970.

School URL: https://med.uth.edu. in a mass casualty simulation. Students are directly observed by faculty from each school and are provided formative assessment focused on their interactions with the students from the other health care disciplines.

\section{Curriculum}

\section{Curriculum description}

The medical education program consists of 3 major phases: integrated medical science, required clerkships, and career focus tracks.

- The integrated medical science phase consists of a Foundations of Medical Science course including team-based learning, organ system modules, and Doctoring courses, which include problem-based learning sessions during Doctoring 2 and 3.

- The second phase of the medical education program consists of required clerkships.

- The third phase consists of the career focus tracks. During the tracks, the students complete 3 required advanced clinical selectives (ambulatory care, advanced patient care, and critical care) and 7 four-week electives.

See Supplemental Digital Appendix 1-Curriculum Map-at http://links.lww.com/ACADMED/A928.

\section{Curriculum changes since 2010}

Over the last 10 years, the curriculum has evolved to be more fully integrated by transitioning from a curriculum based on basic science disciplines to one based on organ system modules. There has been a significant effort to include clinical faculty teaching within the preclerkship curriculum. The revised curriculum also features 5 longitudinal themes. The themes are clinical skills, population health, ethics and professionalism, evidence-based medicine, and systems of care/health systems science. The revised curriculum was implemented in 2016.

Since 2010, the class size increased from 230 to 240 students per class per year. This increase did not affect the medical school's structure or functions.

\section{Assessment}

Medical education program objectives are described in outcomebased terms that allow the assessment of medical students' progress in developing the competencies that the profession and the public expect of a physician (2020-2021 LCME Standard 6.1).

The Curriculum Committee reviews and approves the educational program objectives yearly. There are educational program objectives related to patient care, clinical procedures 
(routine and specialized), medical knowledge, interpretation of medical data/practice-based learning and improvement, interpersonal and communication skills, professionalism, and systems-based practice. The educational program objectives are based on the ACGME domains of competence as well as selected Core Entrustable Professional Activities.

Since 2010, we have adopted NBME customized examinations for organ system modules in years 1 and 2 .

See Supplemental Digital Appendix 2-Program Objectives and Assessment Methods—at http://links.lww.com/ACADMED/A928.

\section{Parallel curriculum or tracks}

MMS introduced a longitudinal integrated clerkship (LIC) pilot in academic year 2019-2020 for 10 third-year medical students. In the pilot year, the LIC was 24 weeks long and included the internal medicine, pediatrics, obstetrics-gynecology, and geriatrics disciplines. Students complete their clinical learning in an integrated fashion, rotating between the various specialty areas every half day with continuity of preceptors, patients, and locations. For the 2020-2021 academic year, family medicine will be added to the LIC, and the duration of the LIC will increase to 28 weeks.

\section{Pedagogy}

In the preclerkship curriculum, a variety of pedagogical approaches are used including problem-based learning, team-based learning, lectures, laboratories, preceptorships, simulations, standardized patients, and case-based learning to deliver the majority of content. Active and self-directed learning modalities are emphasized.

In the clinical curriculum, we use ambulatory and inpatient clinical experiences, lectures, team-based learning, case-based learning, small-group discussions, simulations, and standardized patients. The majority of content is delivered through direct patient experiences.

The simulation program has grown significantly since 2010. Two preclerkship modules (cardiovascular and gastrointestinal) and 2 clerkships (internal medicine and neurology) incorporate simulation activities. As indicated above, active and self-directed learning modalities are emphasized, and the overall number of lecture hours has decreased with the addition of flipped classroom activities in the preclerkship curriculum.

\section{Clinical experiences}

Clinical education occurs in several sites including outpatient clinics, hospitals, and community preceptor offices.

Students first encounter standardized patients during the Doctoring 1 course in the first semester of the first year. Beginning in the second semester of the first year, students participate in a longitudinal clinical experience where a half day per week is spent working with a preceptor in an outpatient setting.
Students participate in a required ambulatory medicine selective during the fourth year. This is an outpatient clinic-based selective, allowing students to choose a particular area of focus consistent with their career trajectory. Students also revisit the principles of evidence-based medicine and complete a critical review of the literature for a clinical question based on a patient experience.

We are fortunate to be affiliated with 2 large teaching hospitals providing complementary clinical experiences and a large number and diversity of patients. Challenges associated with the clinical education of our students are related to the large class size (240 students per year) and faculty attendings with multiple responsibilities.

\section{Curricular Governance}

The Curriculum Committee is charged by the dean to provide oversight of the medical education program, including the design, management, integration, evaluation, and enhancement of a coherent and coordinated medical curriculum. There are 6 subcommittees of the Curriculum Committee:

- The Policy Review Subcommittee reviews and discusses proposed policies and procedures before presentation to the Curriculum Committee for formal approval and implementation (if approved).

- The Preclerkship Education Subcommittee provides oversight of all preclerkship courses and modules including adherence to the preclerkship contact hours policy; the academic workload policy; review of pedagogical methods used; and the coordination of educational content with other modules, the problem-based learning curriculum, and the longitudinal themes.

- The Clinical Education Subcommittee provides oversight of the medical student required clinical experiences, including adherence to the duty hours policy, review and approval of required clinical and procedural/skills experiences, completion by students of required clinical and procedural experiences, completion of midrotation feedback, submission of grades, and appropriate supervision by full-time faculty.

- The Preclerkship Evaluation Subcommittee reviews the preclerkship courses and modules annually and presents findings to the Curriculum Committee.

- The Clerkship and Advanced Clinical Education Evaluation Subcommittee reviews the clerkships and required advanced clinical selectives annually and presents findings to the Curriculum Committee.

- The Continuous Accreditation Review and Quality Improvement Subcommittee provides ongoing monitoring to assure medical school compliance with LCME accreditation standards and elements and engages in continuous quality improvement activities to enhance medical education programmatic quality.

The dean of the medical school allocates state funds to departments specifically for education. These funds are used to support faculty with major teaching responsibilities. (e.g., 
clerkship directors) as well as clerkship coordinators. The dean also allocates funds to the Office of Educational Programs for general support of the educational program (e.g., core faculty, course/module directors, simulation activities, etc.).

\section{Education Staff}

The Office of Educational Programs (OEP) is responsible for the planning, implementation, evaluation, and oversight of the curriculum. The OEP provides administrative support for all of the curriculum review activities. The OEP also develops and maintains tools to support curriculum delivery, monitoring, and management. The OEP includes a vice dean, 4 associate deans, 3 assistant deans, 1 director of educational development, 1 director of educational technology, 2 educational specialists, 1 medical educator, 2 senior program managers, 3 program managers, 1 instructional designer, and 4 educational coordinators.

See Figure 1-Education staff.

The OEP faculty and staff provide general administrative oversight and management for all aspects of the undergraduate medical education curriculum. This includes chairing the Curriculum Committee and its associated subcommittees; administering interdepartmental courses and fourth-year clinical selectives; managing the standardized patient program; administration of the comprehensive clinical competency examination at the end of the third year; as well as several student programs, including the preentry program, the summer research program, the scholarly concentrations program, and the career focus tracks. The OEP monitors student performance outcomes to measure the efficacy of the curriculum, including student performance on national examinations and performance in residency training programs.

The office is also responsible for managing the Surgical and Clinical Skills Center. The OEP is not responsible for medical school admissions or student affairs.

\section{Faculty Development and Support in Education}

Faculty development activities include educator development sessions, educational retreats, online faculty development through Magna Commons “20-Minute Mentor” videos, the Health Educators Fellowship Program, and the Academy of Master Educators. The OEP provides workshops as requested by the medical school departments, as well as individual consultations with faculty to enhance their teaching skills and understanding of learner evaluation.

The monthly educator development sessions are recorded so that faculty who are not able to attend sessions can access the material. It is intended that these sessions will be live streamed to faculty at other sites in the future.

Faculty must demonstrate ability in 4 domains of achievement to be considered for promotion and tenure: clinical/patient care, research/scholarship, education, and service/administration. Teaching activities, such as didactic teaching, problem-based learning facilitation, and student mentoring, may count toward the education domain. Educational scholarship can also be applied to the research/scholarship domain provided it

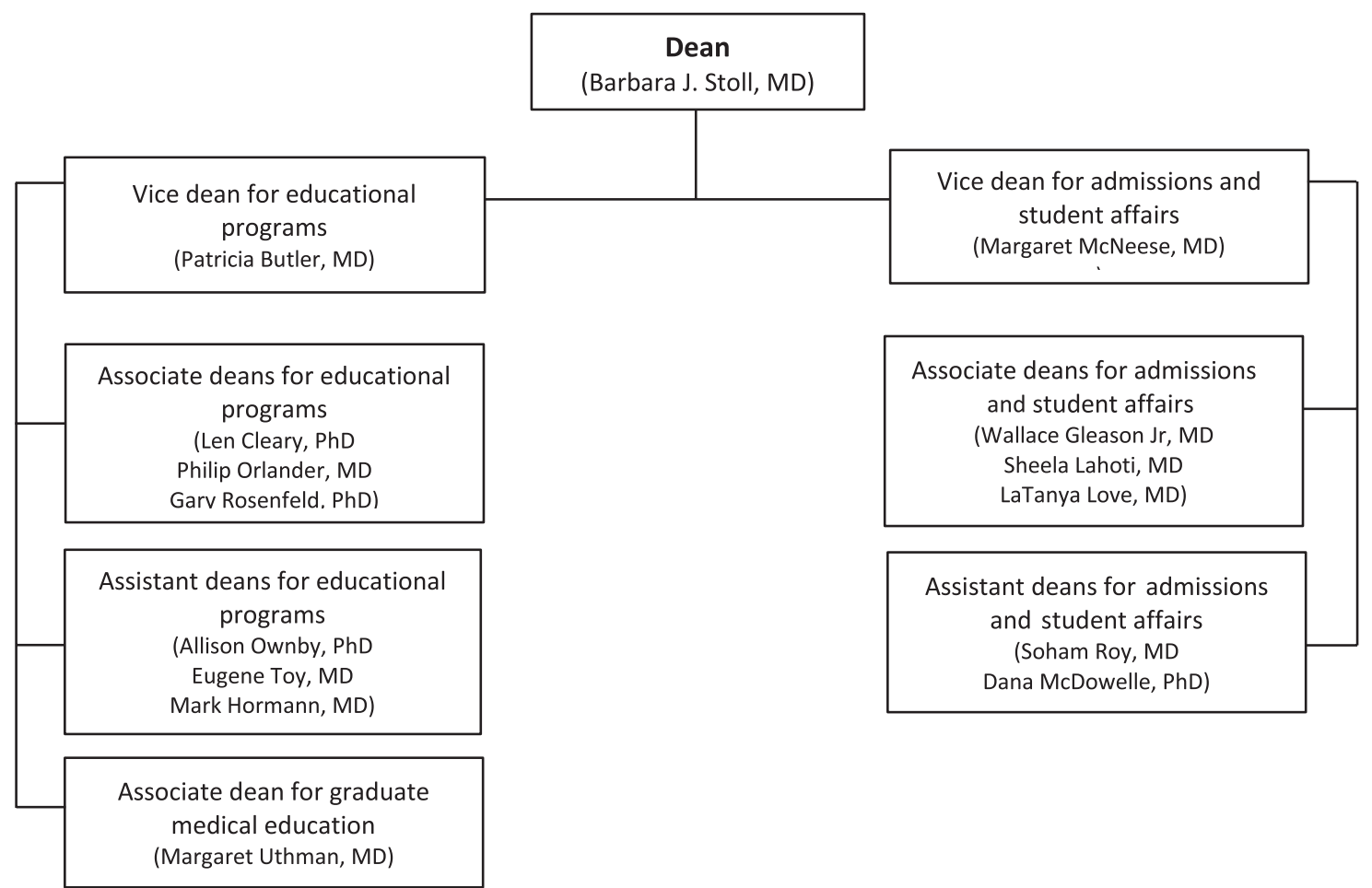

Figure 1 Education staff 
demonstrates impact on the candidate's field. Scholarly activity can be in many forms (e.g., peer-reviewed publications, invited articles, awards and honors, speaking invitations).

MMS Academy of Master Educators (AME) is a formal organization of distinguished scholars recognized for their teaching and educational excellence, and committed to the enhancement of medical education. A goal of the AME is to have diverse membership representing the many disciplines in health science education. AME members encourage faculty who are dedicated to scholarly teaching and to educational scholarship and serve as professional role models and mentors for faculty, residents, fellows, and students. AME membership is open to any medical school faculty member. 


\section{TCU and UNTHSC School of Medicine}

Stephen Scott, MD, MPH, Jo Anna Leuck, MD, Jennifer Allie, PhD, Judy Bernas, and Stuart Flynn, MD

\section{Medical Education Program Highlights}

The Texas Christian University and University of North Texas Health Science Center (TCU and UNTHSC) School of Medicine's vision is to transform health care by inspiring empathetic scholars. Along with the ability to "walk in the patient's shoes," these physicians will excel in the science of medicine. Graduates will be outstanding communicators, compassionate healers, and lifelong learners, prepared to ask and answer the medical questions of the future.

The Compassionate Practice is an interdisciplinary curriculum for building awareness, listening, inquiry, and engagement skills to foster exceptional connections between physicians and their patients, teams, and communities. Using theatre and other communication pedagogy, we train doctors to improvise, build authentic connections, and take responsibility for their audience. We use journalism and narrative medicine techniques to train doctors to speak in a language appropriate to their audience's understanding. Through storytelling and written narratives, students learn to connect, reflect, and build resilience. Incorporating public health, psychology, diversity, communication research, and more, The Compassionate Practice prepares students to become resilient, empathetic physicians and globally conscious leaders.

The School of Medicine (SOM) focuses on maximizing student learning with the patient at the center, most visibly through employing active learning methods and the longitudinal integrated clerkship (LIC) model. Students become part of a physician's practice and see patients from the beginning of medical school and remain in practices over time, enhancing student-patient and teacher-learner relationships, which in turn leads to increased student entrustment, learning, and well-being. Through engagement in a fully distributed, community-based model focusing on ambulatory and team-based care, students care for and learn from patients in diverse settings over extended periods of time.

In its commitment to developing the physician of the future, the SOM has developed the Physician Development Coaching Program, pairing each student with a coach and additional student team members. This cohort is an integrated home within the SOM to foster student professional identity formation while providing an additional layer of support for academic success. Coaches assist in developing resiliency, collaboration, communication, and continuous improvement for personal and professional development.

Acad Med. 2020;95:\$486-S489.

doi: 10.1097/ACM.0000000000003395

Copyright (C) 2020 by the Association of American Medical Colleges

Year school was established: 2018.

School URL: https://mdschool.tcu.edu.

\section{Curriculum}

\section{Curriculum and pedagogy}

Using principles of effective learner-centered pedagogy, the curriculum applies foundational concepts in the breadth of sciences relevant to clinical medicine through progressively integrated, contextual experiences. A combination of individualized, preparatory work and in-person, applied sessions are used throughout the curriculum. Specifically, there are no traditional lectures. Instead, students complete online prework modules (e.g., digital resources including videos, graphics, journal articles, online modules, textbook excerpts, review questions, etc.) before course or clerkship sessions, which then use modalities maximizing the presence of the teacher/facilitator and learner.

The curriculum has 3 sequential phases, with longitudinal courses and interdisciplinary curricular components woven into each.

Phase 1 spans 14 months and focuses on the foundational science and practice of medicine, organized by system and integrated with patient care. Basic and clinical sciences faculty members lead each course, promoting integration and immediate application of concepts. In weekly content framing sessions, students are exposed to the "why" for the week, grounded in a patient story and often with a patient or panel present. This is followed by patient-centered inquiry-based learning, a modified form of faculty-facilitated problem-based learning that challenges students with real-world problems, generates self-directed learning issues, and promotes personal reflection and skill. Throughout the week, students participate in content application sessions, conducted in the format most conducive to achieving learning objectives: case-based learning, teambased learning, laboratory, station, simulation-based activities, small group, peer teaching, or work with standardized patients. Embedded in 4-week-long segments in Phase 1, the Future Accelerators of Medicine course anticipates major changes in health care by 2030 and focuses on 4 topics: genomics, artificial intelligence/machine learning, screening diagnostics, and biotech "wearables" and devices.

Students are simultaneously enrolled in 4 longitudinal courses beginning in Phase 1:

- Phase 1 LIC: Students are paired 1-on-1 with an internal medicine or family medicine preceptor and participate in 4-hour clinic sessions every other week. Students apply and reinforce the communication, patient-centered care, and decision-making skills learned in Clinical Skills as well as broader concepts learned in other courses. Preceptors help students identify several selected patients who students follow throughout the 3 phases of the curriculum, enhancing opportunities for students to demonstrate emerging clinical 
skills and medical knowledge over time, experience various transitions of care, and develop rich physician-patient relationships.

- Clinical Skills: Students learn and practice history taking, physical exam, written and oral case presentations, and basic clinical decision-making, emphasizing communication skills and integrating The Compassionate Practice. In 35 four-hour sessions, integrated and aligned with weekly content of other courses, students connect and apply knowledge through standardized patient encounters, small- and large-group discussions, role play, high-fidelity simulation, and other active learning modalities.

- Scholarly Pursuit and Thesis: Students acquire academic inquiry and skills essential to be lifelong learners, practice evidence-based medicine, critically appraise the literature, and engage in ethical scientific inquiry. Each student works with a mentor to complete a 4-year research project and thesis.

- Preparation for Practice: Concepts essential to being a professional, well-rounded provider in the modern health care system, organized in 10 themes, are integrated into the curriculum and course sessions and taught using multiple modalities:

- Ethics

- Gerontology

- Health care delivery

- Health care policy and advocacy

- Informatics

- Patient safety and continuous quality improvement

- Physician as educator

- Population health (includes global health and social determinants of health)

- Psychological and behavioral sciences

- Team-based care (encompasses traditional interprofessional education)

The Phase 1 curriculum concludes with a 4-week period when students select appropriate electives or a break from their studies.

In Phase 2, students develop the core skills of being a physician through immersive engagement with the health care community of Tarrant County, including inpatient and outpatient settings. The Phase 2 LIC builds on Phase 1 foundational knowledge and skills, beginning with a 2 -week transition course focusing on skills for entering various patient-care settings. Nine weeks of inpatient experience in hospital medicine, pediatrics, surgery, and obstetrics are followed by 10 months of longitudinal clerkships in 8 core disciplines (including the internal or family medicine practice from Phase 1). Students follow a panel of assigned patients across specialty appointments, emergency room visits, labor and delivery, or the operating room. The LIC aligns students with patients and mentors, from whom they receive meaningful formative feedback regularly and frequently. Clerkship directors monitor and assess students' progress and achievement. Students also return for weekly Learning, Application, and Pondering Sessions: applied, active learning sessions using all Phase 1 formats described for content application sessions, integrating foundational and clinical science concepts in patient care.
Phase 3 facilitates transition from medical student to doctor. In the 16-week "Develop" stage, students have continued longitudinal patient care 1 full day per week, with additional sessions focused on Preparation for Practice course themes, including a required group project addressing 1 of 7 societal issues in the surrounding community. Students continue work on their Scholarly Pursuit and Thesis project. The remainder of Phase 3 provides extended time to complete required advanced clinical experiences (subinternship, critical care), individualized learning opportunities, and 4-year research thesis and culminating presentation. Students complete their journey with a residency bootcamp, covering just-in-time topics, and final reflections on the Art of Medicine course, returning to campus to reflect on physician identity, active citizenship, resilience, lifelong learning, and future goals.

\section{Program objectives and assessment}

Educational program objectives reflect the school's emphasis on inspiring empathetic scholars and align with both ACGME competency domains and AAMC core entrustable professional activities.

See Chart 1-Program Objectives and Assessment Methods.

\section{Clinical experiences}

As Fort Worth's medical school, the SOM partners and has affiliation agreements with all the major systems and numerous physician practices and groups throughout Tarrant county and the Dallas-Fort Worth metroplex:

- Major community hospital and system

- 2 large private adult care hospitals

- Children's hospital

- Large and small physician groups

The SOM uses an all-longitudinal model for core clinical learning experiences, emphasizing and promoting essential physician values and skills, including ongoing development of empathy, communication, and clinical reasoning. Clinical experiences begin in Phase 1 (14 months), with biweekly, halfday placement in a primary care internal or family medicine practice. In Phase 2, students continue in the discipline from Phase 1 and are placed in 7 additional practices in core clerkship disciplines (family medicine, internal medicine, obstetricsgynecology, pediatrics, surgery, psychiatry, neurology, emergency medicine). Nine weeks of inpatient immersions (surgery and hospital medicine, 3 weeks; obstetrics-gynecology, 2 weeks; pediatrics, 1 week) are followed by 40 weeks of LICs across all core clerkship disciplines. Students adopt a panel of 1-2 patients per discipline, beginning in Phase 1.

\section{Curricular Governance}

The Curriculum Committee (CC) has primary responsibility and decision-making authority for the curriculum and has 3 subcommittees under its oversight: Assessment and Evaluation Subcommittee, Course and Clerkship Review Subcommittee, and Curriculum Policy and Resource Subcommittee. The CC lays out 
Chart 1

\section{Program Objectives and Assessment Methods}

\section{Medical education program objective(s)}

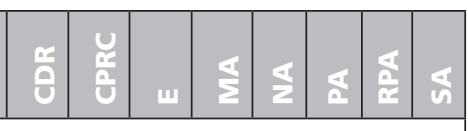

\section{Patient-centered care}

Conduct a comprehensive and problem-focused history and physical exam.

Elicit and synthesize diagnostic data and contextual factors to develop an accurate differential diagnosis.

Develop evidence-based and patient-centered care plans to prevent, treat, and manage acute and chronic diseases.

Perform basic procedural skills with gathering of consent, use of universal precautions, and attention to patient comfort.

\section{Medical knowledge}

Apply established and emerging principles of biomedical and clinical science to diagnostic and therapeutic decision-making, clinical problem-solving, and other aspects of evidence-based health care.

Apply established and emerging principles of social and behavioral science to health maintenance, including the impact of psychosocial-cultural influences on health and disease.

Apply established and emerging principles of epidemiological science to the identification of health problems, risk factors, and disease prevention/health promotion efforts for patients and populations.

\section{Communication and collaboration}

Demonstrate empathy and clarity to develop a therapeutic relationship with patients and their families.

Collaborate as a member of a health care team in shared decision-making.

Provide accurate and relevant written documentation and oral presentation and confirm understanding.

Consider others' perspectives in the context of role, experience, and circumstance.

\begin{tabular}{|c|c|c|c|c|c|c|c|}
\hline$x$ & $x$ & $x$ & $x$ & $x$ & & & $x$ \\
\hline$x$ & $x$ & $x$ & $x$ & $x$ & & & $x$ \\
\hline$x$ & $x$ & $x$ & $x$ & $x$ & & & $x$ \\
\hline & $x$ & $x$ & $x$ & $x$ & & & $x$ \\
\hline
\end{tabular}

\section{Professionalism}

Demonstrate compassion, empathy, altruism, and integrity in all interactions.

Demonstrate ethical judgment and support policies, protocols, and procedures (e.g., privacy, confidentiality) in the care and protection of patients.

Demonstrate respect for the diversity and uniqueness of others and promote a culture of inclusive excellence.

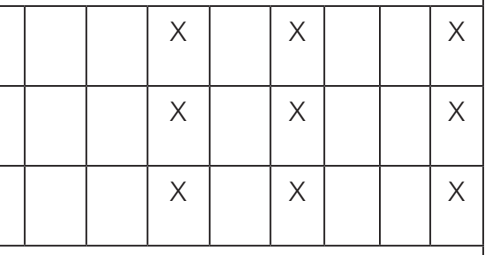

\section{Critical appraisal and lifelong learning}

Critically appraise and evaluate literature to discover and implement new knowledge and skills.

Demonstrate skills associated with lifelong learning to improve the delivery of patient-centered and evidencebased care.

Use technology to advance one's own learning or that of others.

\section{Systems and service}

Apply principles of patient safety, quality improvement, and cost-effective care to prevent adverse events and improve health care outcomes using an integrated approach.

Describe and assess systems in communities and health care settings to identify gaps and support continuous quality improvement.

Describe strategies for advocating and engaging with communities in a leadership role to address factors in population health.

\section{Personal and professional formation}

Accept responsibility, follow through on tasks, and use resources to seek help.

Evaluate one's own progress and wellness through reflection and goal setting.

Provide and respond to constructive feedback in a receptive manner.

Demonstrate self-preservation techniques in routine and stressful encounters.

Abbreviations: CDR. clinical documentation review: CPRC. clinical performance rating/checklist: $E$ : exam (includes institutionally developed, licensure, nationally normed); MA: multisource assessment; NA: narrative assessment; PA: peer assessment; RPA: research or project assessment; SA: self-assessment. 
charges for each subcommittee, which submit minutes, reports, and recommendations for vote directly to the CC. The CC has constituted task forces for the development and monitoring of each course and phase under the Course and Clerkship Review Subcommittee.

\section{Education Staff}

\section{Medical education leadership}

The dean of the medical school serves as the chief academic officer. With the dean, the senior associate dean for educational affairs works with the assistant deans for admission, curriculum, student affairs, and assessment and continuous quality improvement to develop, deliver, and monitor the educational experience. The team also includes the assistant dean for narrative reflection and patient communication, director for clinical skills, and executive director for simulation.

The majority of faculty members with education roles are appointed to the Department of Medical Education, led by a department chair. The department's role is to foster the growth of educational skill, practice, and innovation, partnering with the Office of Faculty Affairs and Development. The department is an academic home and supports its faculty's career development. Faculty with primary educational responsibility within a clinical discipline (such as community preceptors) are appointed in clinical departments, with close collaboration between the clinical department chair and the leader of the associated curricular area.

The educational mission is supported by a centralized support staff, allocated to support the curricular structure by mission and phase. This includes a combination of director and assistant director positions working with designated coordinators for each course (Phase 1 and longitudinal courses: approximately 4-5 total FTE; Phases 2 and 3: 4-5 FTE), along with an instructional designer, academic technologist, and director for assessment. Student support is provided through the school's innovative coaching model, incorporating six 0.5 FTE and six 0.2 FTE physician coaches, a staff director for student affairs, assistant director for student success and student success navigator, assistant director for wellness, physician director for career advising, and coordinators.

\section{Faculty Development and Support in Education}

The TCU and UNTHSC School of Medicine Faculty Development Program recognizes and supports faculty by providing appropriate knowledge, skills, tools, and resources to foster thriving as educators, scholars, and academic professionals. The program emphasizes 4 pillars: medical education and curriculum development, personal and professional development, research and scholarship development, and leadership development.

Faculty development programming is available to all SOM faculty; residents; and graduate student instructors and affiliated TCU, UNTHSC, and community hospital, practice, and other partners. It is offered in a variety of formats, including face-toface and asynchronous programming, retreats, workshops, grand rounds, and online resources.

To ensure that faculty are recognized for their unique and diverse contributions, the SOM has identified 5 areas of accomplishment:

- Education/educational leadership

- Investigation

- Community engagement

- Clinical innovation and improvement

- Service/administration

These areas ensure that faculty academic contributions and scholarship activities reflect a broad definition of scholarship, including the scholarship of teaching and learning, scholarship of discovery, scholarship of application, or scholarship of engagement. Given the broad definition of scholarship and inclusivity of these 5 areas, all faculty are expected to demonstrate sustained contributions to scholarship in at least 1 domain to be considered for promotion. 


\section{Texas A\&M University College of Medicine}

Jeremy Gibson, MD, Teresa Isbell, PhD, T. Gail Pritchard, PhD, Cayla R. Teal, PhD, MA, Amy Waer, MD, and Emily Wilson, PhD

\section{Medical Education Program Highlights}

Since 2010 and its subsequent 2012 LCME accreditation review, Texas A\&M University College of Medicine (TAMU-COM) continues to strive for excellence in rural and population health, military medicine, and innovation.

Institutional restructuring and growth:

- 2011: The Dallas campus opened and is currently the largest regional clinical campus.

- 2013: The Texas A\&M Health Science Center, including TAMU-COM, moved under TAMU administratively and functionally. Notably, TAMU is 1 of only 17 institutions in the nation to hold the triple designation of a land-grant, sea-grant, and space-grant university.

- 2014: The Houston campus was established, bringing the regional campus number throughout Texas to 5 .

- 2016: The Texas A\&M Health Office of Interprofessional Education and Research was established to create and sustain a culture of collaborative learning among the health care professions of the Health Science Center.

- 2017: TAMU-COM partnered with Darnall Army Medical Center to expand military medicine education opportunities.

- 2018: TAMU-COM partnered with the U.S. Air Force to establish the Air Force's 59th Medical Wing as a clinical training site for medical students.

Curriculum development and implementation:

- 2013: The A\&M integrated medicine (AIM) track inaugural cohort began on the Bryan-College Station campus. This was the first longitudinal integrated clerkship program in Texas.

- 2015: The medical education program shifted to a shortened 18-month preclerkship curriculum and an expanded 30-month clinical curriculum.

- 2017: The health systems science (HSS) track inaugural cohort began on the Round Rock campus.

- 2017: The Practice of Medicine course series began and now spans all 4 years. In addition to clinical skills training, the courses provide important training in humanities, ethics, altruism, and leadership.

- 2019: The engineering medicine (EnMed) track inaugural class began on the Houston campus, becoming the nation's

Acad Med. 2020;95:S490-S494.

doi: 10.1097/ACM.0000000000003398

Copyright (C) 2020 by the Association of American Medical Colleges

Supplemental digital content for this article is available at http://links.Iww.com/ ACADMED/A907.

Year school was established: 1977.

School URL: http://medicine.tamu.edu. first fully integrated engineering and medical education curriculum, allowing graduates to receive both the MD degree and a master's in engineering in 4 years.

\section{Curriculum}

\section{Curriculum description}

TAMU-COM has a traditional curriculum and 3 tracks. This curriculum spans 18 months of preclerkship and 30 months of clerkship and electives. The traditional curriculum uses a primarily lecture-based preclerkship approach and a block model clerkship.

See Supplemental Digital Appendix 1-Curriculum Model—at http://links.lww.com/ACADMED/A907.

\section{Curriculum changes since 2010}

- Preclerkship period was redesigned to 18 months, creating expanded elective opportunities in all 4 years, including a period of career exploration during the third year

- Increased utilization of nonlecture-based learning experiences, including faculty-facilitated small groups, simulation, online learning modules, and case-based (traditional curriculum) and team-based learning platforms (EnMed)

- Inclusion of earlier clinical training experiences, for example, the first week of medical school is now devoted to training in taking a basic medical history and measuring vital signs; clinical correlations are highlighted in learning experiences, such as case synthesis and integrations exercises during preclerkship organ-system courses

- Improved horizontal and vertical curricular integration of key curricular content, including clinical skills training, medical ethics, societal problems, communication, and interprofessional education

- Evolving interprofessional education curriculum, including Disaster Day, the nation's largest student-led interprofessional emergency response simulation

- Established the Medical Student Research Scholars Program supported by the Office of Medical Student Research

- With the addition of the Dallas and Houston campuses, increased capacity to accommodate up to 200 students per year; availability of 5 campuses allows TAMU-COM to offer full preclerkship curriculum on 2 campuses, partial on 2 , and 3 parallel curricular tracks

\section{Assessment}

- Medical education program objectives are described in outcome-based terms, allowing the assessment of medical students' progress in developing the competencies the profession and the public expect of a physician. (2020-2021 LCME Standard 6.1 or 2020-2021 CACMS Standards 6.1). 
- Texas A\&M COM does not use a single framework; rather, the program objectives are organized into knowledge-based $(\mathrm{K})$ and skill-based (S) areas, as well as attitudes and behaviors $(\mathrm{AB})$. Each objective is mapped to ACGME competencies and Core EPAs.

- Attitude and behavior objectives reflect the Aggie core values of respect, excellence, leadership, loyalty, integrity, and selfless service.

See Supplemental Digital Appendix 2-Program Objectives and Assessment Methods—at http://links.lww.com/ACADMED/A907.

\section{Assessment changes since 2010}

- There is an increased reliance on objective structured clinical examinations for both formative clerkship and summative clinical skills assessment.

- Physical Examination Teaching Assistant skill competencies were added, as well as increased use of small-group facilitator assessment, implementation of NBME customized examinations, and introduction of a value-based portfolio system for assessment of attitudes and behaviors.

\section{Parallel curriculum or tracks}

A\&M AIM: The integrated medicine track is designed to provide students with a longitudinal patient experience and integrated clerkship content. This track focuses on longitudinal patient care and 1-on-1 relationships with faculty preceptors. Students learn to manage patients in multiple disciplines during the track. There are no additional medical education program objectives for this track.

HSS: The HSS track is designed to produce students with enhanced skills for navigating and leading in an evolving U.S. health care system. This track emphasizes the intersection of science and health care delivery, where systems thinking, innovation, and evaluation can improve the quality, safety, and value of health care. In 2020, TAMU-COM will expand the HSS track from a single campus to multiple regional campuses as a part of planned future integration into the traditional curriculum.

EnMed: The EnMed track is designed to incorporate engineering and medicine within 1 track. Students enter the track with an undergraduate degree in engineering and expand on these skills while completing their medical degree. EnMed focuses on knowledge, skills, and experience to develop new therapies, devices, algorithms, and diagnostic or treatment processes. The track includes concurrent curricula, resulting in 2 degrees: MD and Master of Engineering (MEng). Students have dual enrollment in both TAMU-COM and TAMU College of Engineering. EnMed's goal is to produce a new kind of doctor, the "physicianeer," who is capable of transforming health care through innovation.

Three-year MD degree programs: At this time, Texas A\&M does not offer a 3-year MD degree program, nor is one planned for the future.

\section{Pedagogy}

The following pedagogical approaches are used throughout the curriculum: case-based learning, clinical experiences (ambulatory and inpatient), discussion (large and small group), laboratory, lecture, preceptorship, self-directed learning, simulation, standardized patients, team-based learning, and video/podcast.

AIM track: Clerkship objectives are achieved primarily through longitudinal clinical experiences in each core specialty. Other modalities include small-group discussion, lectures, self-directed learning, and simulation.

HSS track: Track objectives are achieved through core curricular courses, electives, and a capstone course. The foundation course is primarily interactive with limited didactics. For students in clerkships, courses are small groups and case-based discussions. Electives are self-directed learning projects with faculty mentors. The capstone course is a multisemester, longitudinal project with a faculty mentor. Additional modalities include online modules, videos, and journal readings.

EnMed track: Preclerkship learning objectives are achieved primarily through team-based learning with integrated engineering content. Clerkships are completed as traditional block clinical experiences. The bulk of the 30-credit-hour engineering degree is achieved through a prematriculation course (discussions, laboratory) and during the elective time, which is used to complete a capstone design and innovation project.

Changes in pedagogical approaches:

- Increased active learning modalities, including large- and small-group discussion, case-based learning, and clinical and basic science integration experiences

- Specific course for all students to teach self-directed learning skills, with additional self-directed learning integrated into courses

- A fourth-year capstone course that integrates several active learning modalities to ensure residency readiness

- A longitudinal clerkship track that was implemented, as well as team-based learning (primarily in the EnMed track)

\section{Clinical experiences}

Wide geographic distribution of the 5 regional campuses across Texas provides opportunity for students to gain clinical experience across the full spectrum of clinical care from rural to specialty care. One or more major health care affiliates at each site provide inpatient experience with exposure to residents and fellows.

\section{Required longitudinal experiences}

Bryan-College Station regional campus offers students a longitudinal clerkship curriculum.

\section{Clinical experience first encounter}

Nonsimulated clinical experiences for students begin in the third semester, preclerkship, as a preceptorship and are completed at a variety of community-based clinics. 


\section{Required and elective community-based rotations}

TAMU-COM does not have its own teaching hospital; thus, it relies on a variety of clinical affiliates to provide communitybased rotations across regional campuses. These experiences are supplemented by strong relationships with both military and VA medical centers, which provide partial clerkship experiences and electives.

\section{Challenges in designing and implementing clinical experiences for medical students}

There are 2 major challenges to the design and implementation of clinical experiences:

- Maintenance of comparable learning experiences within a complex multicampus and multitrack model

- Regional competition for clinical experiences due to a growing number of medical schools in the state; areas of particular need are inpatient pediatrics and inpatient psychiatry

\section{Curricular Governance}

- The Curriculum Committee (CC) governs the curriculum, sets educational policy, and provides direction.

- 5 standing subcommittees report to the CC: Preclerkship, Clinical, Elective, Interprofessional Education, and Student.

- The CC also receives reports from task forces assigned to address specific issues.

- The executive dean for education and academic programs provides management support for the curriculum through the Office of Medical Education (OME).

- Budget for teaching, assessment, and academic support is managed centrally.

See Table 1-Curriculum Governance and Management.

\section{Education Staff}

\section{Medical education leadership}

The OME leads and supports all aspects of the medical education process at TAMU-COM. The primary medical education staff and administrative faculty are housed under the OME organization umbrella. Administrative and academic support for curriculum oversight, delivery, monitoring, and management is achieved primarily through the offices of Academic Affairs and Evaluation and Assessment.

See Figure 1-Office of Medical Education.

\section{Department of Medical Education}

The Department of Medical Education (DME) serves as the centralized source for development and promotion of faculty medical educators through professional mentoring, career development, and collaborative scholarly research opportunities. The mission of the department is to promote and nurture those key faculty dedicated to the improvement and advancement of both undergraduate and graduate medical education.

The DME is home to the medical college's volunteer faculty and faculty with primarily administrative roles. The organizational structure was designed to support faculty at the 5 campuses and 4 teaching sites. The DME is led by a department head with assistance from an associate department head. The associate campus deans and associate site deans serve as divisional team leads.

\section{Faculty Development and Support in Education}

The Office of Faculty Development provides the primary support for teaching faculty. A variety of live and web-based resources supporting instructional methodology, student assessment,

Table 1

Curriculum Governance and Management

\begin{tabular}{|c|c|c|c|c|c|}
\hline Area of responsibility & $\begin{array}{l}\text { Course/ } \\
\text { clerkship } \\
\text { directors } \\
\text { and faculty }\end{array}$ & $\begin{array}{l}\text { CAO/associate } \\
\text { dean for } \\
\text { medical } \\
\text { education }\end{array}$ & $\begin{array}{l}\text { Office of Medical } \\
\text { Education staff } \\
\text { (OAA, OEA, OSA, } \\
\text { OFD) }\end{array}$ & $\begin{array}{l}\text { Curriculum } \\
\text { Committee }\end{array}$ & $\begin{array}{l}\text { Curriculum } \\
\text { Committee, } \\
\text { Subcommittee(s) }\end{array}$ \\
\hline Medical education program objectives & $\mathrm{I}, \mathrm{R}, \mathrm{Rec}$ & $R, \operatorname{Rec}$ & $C, R, R e c, I$ & $D, R, A$ & $R, \operatorname{Rec}$ \\
\hline Course/clerkship objectives & $C, I, R, R e c$ & $\mathrm{R}$ & $\mathrm{R}, \mathrm{Rec}$ & $D, R, A$ & $\mathrm{R}, \mathrm{Rec}$ \\
\hline $\begin{array}{l}\text { Course/clerkship content, instructional } \\
\text { methods, and assessment methods }\end{array}$ & $C, I, R, \operatorname{Rec}$ & $\mathrm{R}$ & R, Rec & $D, R, A$ & $\mathrm{R}, \mathrm{ReC}$ \\
\hline Course/clerkship quality and outcomes & $C, I, R$ & $\mathrm{R}$ & $R, \operatorname{Rec}$ & $D, R, A$ & \\
\hline Faculty/resident teaching & $C, I, R$ & $R$, Rec & R, Rec, I & $D, R, A$ & \\
\hline $\begin{array}{l}\text { Curriculum content, including horizontal } \\
\text { and vertical integration }\end{array}$ & $\dddot{1}$ & $\mathrm{R}$ & $C, R, \operatorname{Rec}$ & $D, R, A$ & \\
\hline Outcomes of curriculum phases & I, R, Rec & $R$, Rec & R, Rec & $D, R, A$ & \\
\hline Outcomes of the curriculum as a whole & $\because$ & $R, \operatorname{Rec}$ & $R, \operatorname{Rec}$ & $D, R, A$ & \\
\hline
\end{tabular}

Abbreviations: (D) Determines curriculum direction; (C) Create $=$ Create the product or process in response to direction; (I) Implement = Carry out the process or utilize the product; (R) Review = Receive and consider the results of an evaluation of the product or process and/or of its outcomes; (Rec) Recommend = Propose an action related to the process or product based on a review or evaluation; (A) Approve/take action = Have final responsibility for an action related to the product or process; OAA = Office of Academic Affairs; OEA = Office of Evaluation and Assessment; OSA, Office of Student Affairs; OFD = Office of Faculty Development. 


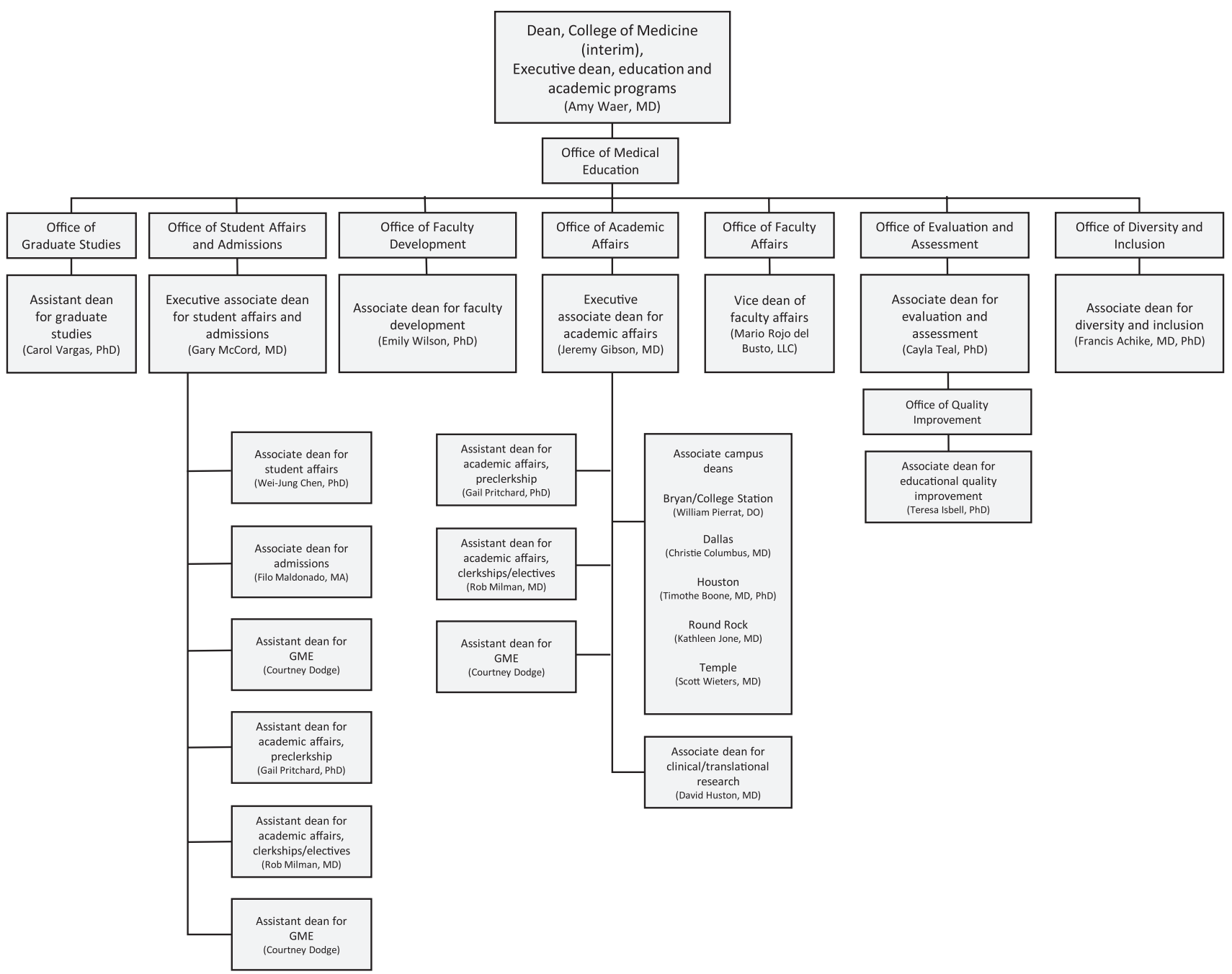

Figure 1 Office of Medical Education.

and curriculum development are delivered to faculty across all campuses and sites. The office also delivers a Medical Educator Program certificate focused on developing learning objectives and linking those objectives to formative and summative assessments as well as the appropriate teaching methods to assist students in meeting those objectives. Individual faculty development is available through an instructional consultation program.

\section{Role of teaching in promotion and tenure}

Both annual faculty reviews and promotion/tenure decisions include consideration of educational activities such as educational scholarship, quantity and quality of teaching, and service on curricular-related committees.

\section{Teaching academy}

The Academy of Distinguished Medical Educators (ADME) includes faculty members who have demonstrated excellence in teaching and scholarship throughout their career. The ADME is committed to promoting excellence in medical education by:
- Supporting teaching excellence through coaching and mentorship

- Developing and delivering faculty development workshops

\section{Table 2}

\section{Regional Clinical Campuses}

\begin{tabular}{|c|c|c|}
\hline $\begin{array}{l}\text { Regional campus } \\
\text { name }\end{array}$ & Type & $\begin{array}{l}\text { Student } \\
\text { enrollment }\end{array}$ \\
\hline Bryan-College Station & - Entire MD program & 156 \\
\hline Dallas & $\begin{array}{l}\text { - Partial preclerkship } \\
\text { - Clinical }\end{array}$ & 137 \\
\hline Houston & - Entire MD program & 134 \\
\hline Round Rock & - Clinical & $50^{\circ}$ \\
\hline Temple & $\begin{array}{l}\text { - Partial preclerkship } \\
\text { - Clinical }\end{array}$ & 121 \\
\hline
\end{tabular}


- Advancing curriculum and instruction through promoting innovative practices

- Facilitating educational research and scholarship

\section{Regional Medical Campuses}

See Table 2-Regional Clinical Campuses.

\section{Educational experiences across sites}

Comparable learning experiences across the regional campuses are ensured through:

- Identical program learning objectives for required clinical experiences, including patient encounters and procedure logs
- Clear organizational reporting lines for faculty course leadership and course coordinators

- Executive clerkship director position with oversight over all teaching sites

- Centrally monitored student feedback, inpatient/outpatient proportions, use of alternate learning experiences, timeliness of grades, and duty hours

Equitable assessment of students across the regional campuses is ensured through:

- Identical written assessment

- Standardized objective structured clinical examination

- Centrally monitored campus performance metrics 


\title{
Paul L. Foster School of Medicine, Texas Tech University Health Sciences Center El Paso
}

\author{
Richard D. Brower, MD, Tanis Hogg, PhD, Maureen D. Francis, MD, Richard A. Lange, MD, and Paul E. Ogden, MD
}

\section{Medical Education Program Highlights}

- The Paul L. Foster School of Medicine (PLFSOM) educational program features a highly integrated preclerkship curriculum in which the foundational sciences are taught in the context of common clinical presentations. The program deliberately incorporates principles of adult education, including learning in the context of intended application, expert-guided deliberate practice, protected unscheduled time, and frequent formative assessment and feedback.

- Preclerkship phase instruction occurs in systems-based units, with each week following a clinically focused experiential learning cycle. ${ }^{1}$

- Each week a motivating context for learning is established via a common clinical presentation and diagnostic scheme. Subsequent content is derived from the foundational concepts required to "work" the diagnostic scheme. The diagnostic scheme is also applied in a medical skills session, including a standardized patient encounter. Each week culminates in case-based small-group discussions that integrate newly acquired knowledge, skills, and early diagnostic reasoning.

- A required 2-year Spanish language curriculum is fully integrated with the clinical presentation framework.

- The traditional core clerkships occur in three 16-week blocks consisting of set clerkship pairs. The pairings facilitate integration of didactic and practice-based experiences designed to emphasize the interdependence of the specialties.

\section{Curriculum}

\section{Curriculum description}

See Supplemental Digital Appendix 1-Curriculum Description —at http://links.lww.com/ACADMED/A821.

Preclerkship phase course explanatory notes:

- Scientific Principles of Medicine (SPM): Integrated biomedical sciences course as described above.

- Medical Skills: Introductory clinical skills instruction fully aligned with the SPM course.

- Society, Community, and the Individual (SCI): Regional health systems, medical social sciences, community-based clinical experiences, and medical Spanish.

Acad Med. 2020;95:S495-S499.

doi: 10.1097/ACM.0000000000003269

Copyright $\odot 2020$ by the Association of American Medical Colleges

Supplemental digital content for this article is available at http://links.Iww.com/ ACADMED/A821.

Year school was established: 2008.

School URL: https://elpaso.ttuhsc.edu/som.
- Masters' Colloquium: A discussion-based course taught in the school's learning communities ("colleges") covering medical ethics; "physicianship"; and the role of the physician in medicine, research, and society.

- Scholarly Activity and Research Program: All students design, implement, and present a scholarly project prior to graduation.

Curriculum changes since 2010

Preclerkship phase:

- Increased utilization of team-based learning and interactive

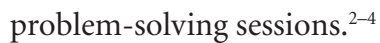

- Systematic integration of session-level self-assessment quizzes. ${ }^{5}$

- Transition of gross anatomy instruction from regional dissections to a blended multimodal approach. ${ }^{6}$

- Enhanced integration of interprofessional education, including activities related to teamwork and communication skills, the roles and responsibilities of health care professions, and the ethical use of opioids.

Clerkship phase:

- Two 1-week third-year intersessions emphasizing common cross-clerkship content and integrating clinical experiences with basic sciences.

- A 2-week simulation-based "boot camp" to prepare the graduating students for residency.

PLFSOM is currently planning its first major curricular update. Modifications will include:

- Shortening the preclerkship phase to 3 semesters.

- An earlier clerkship phase, concluding with a flexible USMLE testing, remediation, early elective, and scholarship block.

- Transition to a longitudinal integrated clerkship model designed to promote transdisciplinary integration.

Class size changes since 2010

See Table 1-Class Size Changes.

- Expansion occurred according to a predetermined development plan and did not involve substantial modification of the school's structure.

\section{Assessment}

See Table 2-Medical Education Program Objectives and Assessment.

A complete copy of the PLFSOM educational program goals and their subsidiary objectives can be accessed via the school's online academic catalog, at https://elpaso.ttuhsc.edu/som/ catalog/default.aspx. The PLFSOM medical education program goals and objectives are based on the Reference List of General 


\section{Table 1}

\section{Class Size Changes}

\section{Year} Number of new matriculants

2009-2010a

2010-2011

2011-2012

2012-2013

2013-2014

2014-2015

2015-2016

2016-2017

2017-2018

2018-2019

2019-2020

alnaugural class.

Physician Competencies as proposed in 2013 by Englander and colleagues. $^{7}$

\section{Changes in assessment during the preclerkship} phase since 2010

- Transition from norm- to criterion-referenced grading for unit summative exams.

- Implementation of periodic progress testing using formative administrations of the NBME Comprehensive Basic Science Examination.

- Addition of regular evidence-based medicine literature reviews and problem sets.

\section{Changes in assessment during the clerkship phase since 2010}

The final assessments in the clerkship phase for all required courses were redesigned for academic year 2015-2016, increasing narrative feedback; adding a final competency grade in each of 8 domains; and including a final overall grade based on clinical work-based assessments, clerkship assignments, objective structured clinical examination performance, and NBME subject test scores.

\section{Clinical experiences}

Inpatient clinical sites:

- County general hospital

- County children's hospital

- State psychiatric center

- Military medical center

- Private hospital systems: 2 local, 1 outlying

Ambulatory clinical sites:

- Multiple Texas Tech University Health Sciences Center El Paso clinics

- Multiple community private practices
- Multiple federally qualified health clinics

- Veterans Health Administration clinics

- Active military medical center clinics

- Prison system clinic

- Multiple freestanding surgical centers

- Clinics of 1 local and 1 outlying private hospital system

\section{Required longitudinal experiences}

There is a longitudinal experience in each of the 16-week thirdyear clerkship blocks:

- Internal medicine/psychiatry block: psychiatry selective

- Obstetrics-gynecology/pediatric block: mother/baby experience

- Family medicine/surgery block: family medicine selective

\section{Clinical experience first encounter}

Learners begin clinical experiences within the first week of medical school:

- The medical skills course introduces students to history taking, physical exam, and diagnostic reasoning skills via standardized patient encounters, simulations, and expertguided sessions.

- Students attend clinic with a community preceptor 12 times across the preclerkship phase.

\section{Required and elective community-based rotations}

Required: preclerkship phase

- SCI course

- Spanish instruction and community assessment and cultural competency activities

- Students work with a primary care preceptor 8 days in year 1 and 4 days in year 2 (including experiences with a dentist, an optometrist or ophthalmologist, and a pharmacist)

Required: clerkship phase

- Family medicine clerkship

- Minimum of 1 day per week for 5 weeks with a community preceptor

- 1 week with community hospice

- Surgery clerkship

- 1 week with a community general surgeon

Elective: clerkship phase

- Psychiatry clerkship

- Several longitudinal selectives are community based

- Internal medicine clerkship

- Students have an option to spend 3 weeks at a community inpatient site

- Surgery clerkship

- Third-year students can choose from a variety of community-based subspecialty 3 -week selectives 


\section{Table 2}

\section{Medical Education Program Objectives and Assessment}

\begin{tabular}{|c|c|}
\hline $\begin{array}{l}\text { Medical education } \\
\text { program objectives }\end{array}$ & Assessment methods a \\
\hline $\begin{array}{l}\text { Goal } 1 \text { : Patient care } \\
\quad \text { (10 subsidiary objectives) }\end{array}$ & $\begin{array}{l}1 \mathrm{~F} / \mathrm{S} ; 2 \mathrm{~F} / \mathrm{S} ; 3 \mathrm{~S} ; 4 \mathrm{~F} / \mathrm{S} ; 6 \mathrm{~S} ; \\
7 \mathrm{~S} ; 8 \mathrm{~F} / \mathrm{S} ; 10 \mathrm{~F} / \mathrm{S} ; 11 \mathrm{~F} ; \\
12 \mathrm{~F} / \mathrm{S} ; 13 \mathrm{~F} ; 16 \mathrm{~F} / \mathrm{S} ; 17 \mathrm{~F}\end{array}$ \\
\hline $\begin{array}{l}\text { Goal } 2 \text { : Knowledge for practice } \\
\text { (6 subsidiary objectives) }\end{array}$ & $\begin{array}{l}1 \mathrm{~F} / \mathrm{S} ; 2 \mathrm{~F} / \mathrm{S} ; 3 \mathrm{~F} / \mathrm{S} ; 4 \mathrm{~F} / \mathrm{S} ; 6 \mathrm{~S} ; \\
7 \mathrm{~S} ; 8 \mathrm{~F} / \mathrm{S} ; 10 \mathrm{~F} / \mathrm{S} ; 11 \mathrm{~F} ; 12 \mathrm{~S} ; \\
16 \mathrm{~S} ; 17 \mathrm{~F}\end{array}$ \\
\hline $\begin{array}{l}\text { Goal 3: Practice-based learning } \\
\text { and improvement } \\
\text { (6 subsidiary objectives) }\end{array}$ & $\begin{array}{l}1 \mathrm{~F} / \mathrm{S} ; 2 \mathrm{~F} / \mathrm{S} ; 3 \mathrm{~F} / \mathrm{S} ; 4 \mathrm{~F} / \mathrm{S} ; 7 \mathrm{~S} ; \\
10 \mathrm{~F} / \mathrm{S} ; 13 \mathrm{~F} / \mathrm{S} ; 16 \mathrm{~F} / \mathrm{S} ; 17 \mathrm{~F}\end{array}$ \\
\hline $\begin{array}{l}\text { Goal 4: Interpersonal and } \\
\text { communication skills } \\
\text { (4 subsidiary objectives) }\end{array}$ & $\begin{array}{l}1 \mathrm{~F} / \mathrm{S} ; 2 \mathrm{~F} / \mathrm{S} ; 3 \mathrm{~F} / \mathrm{S} ; 4 \mathrm{~S} ; 5 \mathrm{~S} \\
6 \mathrm{~S} ; 10 \mathrm{~F} / \mathrm{S} ; 11 \mathrm{~F} / \mathrm{S} ; 13 \mathrm{~F} \\
16 \mathrm{~S} ; 17 \mathrm{~F}\end{array}$ \\
\hline $\begin{array}{l}\text { Goal 5: Professionalism } \\
\text { (7 subsidiary objectives) }\end{array}$ & $\begin{array}{l}1 \mathrm{~F} / \mathrm{S} ; 2 \mathrm{~F} / \mathrm{S} ; 3 \mathrm{~F} / \mathrm{S} ; 4 \mathrm{~F} / \mathrm{S} ; 6 \mathrm{~S} ; \\
7 \mathrm{~S} ; 8 \mathrm{~S} ; 10 \mathrm{~F} / \mathrm{S} ; 11 \mathrm{~F} ; 12 \mathrm{~F} / \mathrm{S} ; \\
15 \mathrm{~F} / \mathrm{S} ; 16 \mathrm{~S} ; 17 \mathrm{~F}\end{array}$ \\
\hline $\begin{array}{l}\text { Goal 6: Systems-based practice } \\
\text { (4 subsidiary objectives) s }\end{array}$ & $\begin{array}{l}1 \mathrm{~S} ; 2 \mathrm{~F} / \mathrm{S} ; 3 \mathrm{~S} ; 4 \mathrm{~S} ; 7 \mathrm{~S} ; 8 \mathrm{~F} / \mathrm{S} ; \\
10 \mathrm{~F} / \mathrm{S} ; 16 \mathrm{~S}\end{array}$ \\
\hline $\begin{array}{l}\text { Goal 7: Interprofessional } \\
\text { collaboration } \\
\quad \text { (4 subsidiary objectives) }\end{array}$ & $\begin{array}{l}1 \mathrm{~S} ; 2 \mathrm{~F} / \mathrm{S} ; 3 \mathrm{~F} / \mathrm{S} ; 4 \mathrm{~F} / \mathrm{S} ; 10 \mathrm{~F} / \mathrm{S} ; \\
12 \mathrm{~F} / \mathrm{S} ; 15 \mathrm{~S} ; 16 \mathrm{~F} / \mathrm{S}\end{array}$ \\
\hline $\begin{array}{l}\text { Goal 8: Personal and } \\
\text { professional development } \\
\text { (5 subsidiary objectives) }\end{array}$ & $\begin{array}{l}1 \mathrm{~F} / \mathrm{S} ; 2 \mathrm{~F} / \mathrm{S} ; 3 \mathrm{~F} / \mathrm{S} ; 4 \mathrm{~F} / \mathrm{S} ; \\
10 \mathrm{~F} / \mathrm{S} ; 12 \mathrm{~F} / \mathrm{S} ; 15 \mathrm{~F} / \mathrm{S} ; 16 \mathrm{~F} / \mathrm{S}\end{array}$ \\
\hline
\end{tabular}

Abbreviations: F, formative assessment; S, summative assessment; F/S, both formative and summative.

assessment type codes using MedBiquitous Terminology:

1. Clinical documentation review

2. Clinical performance rating checklist

3. Exam, institutionally developed, clinical performance

4. Exam, institutionally developed, written/computer based

5. Exam, institutionally developed oral

6. Exam, licensure, clinical performance

7. Exam, licensure, written/computer based

8. Exam, nationally normed/standardized subject

9. Multisource assessment

10. Narrative assessment

11. Oral patient presentation

12. Participation

13. Peer assessment

14. Portfolio-based assessment

15. Practical/lab

16. Research or project assessment

17. Self-assessment

\section{- Fourth-year electives}

- Family medicine: Big Bend rural health care elective, border health Spanish immersion course, public health and community-based approaches for disease prevention

- Internal medicine: multiple subspecialty community-based electives

- Surgery: clinical ophthalmology, orthopedic surgery, otolaryngology/head/neck surgery

- Physical medicine and rehabilitation

Challenges in designing and implementing clinical experiences for medical students

- Development of clinical sites over an expanded geographical area
- Competition for clinical sites with private osteopathic medical schools and midlevel practitioner programs

- Variable credentialing requirements across clinical sites

- Recruitment and retention of community preceptors

\section{Curricular Governance}

See Figure 1-Curricular governance.

Curricular governance is centralized, as is provision of academic information technology, including web-based content and assessment applications. These functions, plus program accreditation, management of the clerkship phase, and comprehensive program coordination, are managed by the school's Office of Medical Education (OME).

\section{Education Staff}

- The OME is led by an associate dean for medical education and 2 assistant deans. Staff include: a director of accreditation and educational program improvement, a finance and administration director (shared with the academic Department of Medical Education), an associate director for assessment and evaluation, a senior analyst, an instructional design specialist, and 3 program coordinators.

- The OME is supported by a dedicated academic information technology team consisting of an associate director and 3 senior analysts.

- The Department of Medical Education is a transdisciplinary, mission-based academic department responsible for the operational management of the preclerkship curriculum (and constituting the school's Preclerkship Phase Committee). It consists of 26 full-time and 6 part-time basic scientist and clinician-medical educators, and 15 program support staff. Faculty appointed to the Department of Medical Education generally commit $80 \%$ effort to undergraduate medical education.

- Operational management of the clerkship phase curriculum is led by an assistant dean for clinical instruction, supported by 29 clinical department faculty with subsidized roles as faculty clerkship/course directors or assistant directors and 15 program support staff.

\section{Medical education leadership}

See Figure 2-Medical education leadership.

\section{Faculty Development and Support in Education}

\section{Professional development}

The institutional Office of Faculty Development centrally manages programs to enhance faculty skills as educators and teachers, including:

- An annual 8-month course for junior and midcareer faculty, focusing on teaching strategies, assessment, clinical simulation, and online learning technologies 


\section{Curriculum and Educational Policy Committee (CEPC)}

A 10-member dean-appointed faculty committee, chaired by the associate dean for medical education. This committee has full authority to plan, coordinate, review, and modify the curriculum (subject to concurrence by the dean and input from the PLFSOM Faculty Council).

\section{Evaluation of Educational Programs Committee}

A dean-appointed faculty committee, advisory to the dean and the CEPC, that reports independently to the Faculty Council. It provides oversight on the design and interpretation of the school's educational program performance evaluations.

\section{Preclerkship Phase Committee}

Consists of the preclerkship phase faculty course directors, other Department of Medical Education faculty, and course coordinators (staff). Chaired by the assistant dean for basic

science instruction. Responsible for the implementation and management of the preclerkship phase curriculum as approved and overseen by the CEPC. Also provides feedback and recommendations to the CEPC.

\section{Clerkship Phase Committee}

Consists of the faculty directors for the required phase components and the associated coordinators (staff). Chaired by the assistant dean for clinical instruction. Responsible for the implementation and management of the clerkship phase curriculum as approved and overseen by the CEPC. Also provides feedback and recommendations to the CEPC.

Figure 1 Curricular governance.

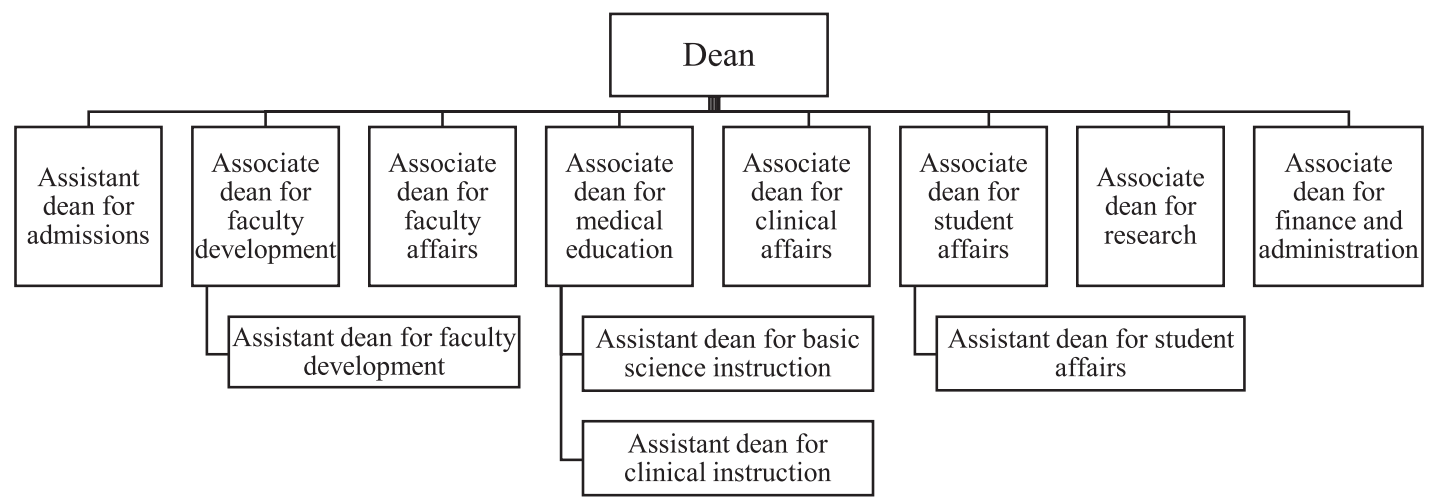

Figure 2 Medical education leadership.

- A comprehensive faculty mentoring program that pairs trained senior faculty mentors with early- to midcareer faculty members

- Quarterly “Need to Know” grand rounds to provide practical teaching skills

- A community preceptors' development program

\section{The role of teaching in promotion and tenure}

Faculty requirements for tenure and promotion are based on specific accomplishments in 4 areas: teaching, scholarship, clinical service, and academically related public service. Faculty may designate teaching as their primary category. Accomplishments are rated per established guidelines to be level 
1 (locally recognized), level 2 (regionally recognized), or level 3 (nationally recognized). Promotion and tenure decisions follow an algorithm based on the level of accomplishment in each of the 4 performance domains.

\section{References}

1 Armstrong E, Parsa-Parsi R. How can physicians' learning styles drive educational planning? Acad Med. 2005;80:680-684.

2 Nesic N, Quest D, Gest T, Bramblett DE, Blunk D. Four clinical concepts: A template for cognitive integration of clinical and basic sciences. Med Sci Educ. 2015;25:483-488.

3 Blunk DI, Tonarelli S, Gardner C, Quest D, Pettit D, Leiner M. Evaluating medical students' clinical reasoning using clinical and basic science concepts presented in session-level integration sessions. Med Sci Educ. 2019;29:819-824.

4 Blunk DI, Brower R, Hogg T, Perry C, Pettit D, Kupesic Plavsic S, Quest DW. A team-based approach compared with two other case study methods. Med Sci Educ. https://doi.org/10.1007/s40670-019-00845-8. Published November 30, 2019.

5 Baatar D, Lacy NL, Mulla ZD, Piskurich JF. The impact of integration of self-tests into a preclerkship medical curriculum. Med Sci Educ. 2017;27:21-27.

6 Lavezo JL, Beale EG, Lacy NL, et al. Cadaver biopsies positively impact the medical student educational experience. Med Sci Educ. 2017;27:543-547.

7 Englander R, Cameron T, Ballard AJ, Dodge J, Bull J, Aschenbrener CA. Toward a common taxonomy of competency domains for the health professions and competencies for physicians. Acad Med. 2013;88: 1088-1094. 


\title{
Texas Tech University Health Sciences Center School of Medicine, Lubbock
}

\author{
Simon C. Williams, PhD, Michaela Jansen, PharmD, PhD, and Brian J. Pomeroy, MD, MEHP
}

\section{Medical Education Program Highlights}

Texas Tech University Health Sciences Center (TTUHSC) School of Medicine was established in 1969 as a multicampus institution with an administrative center in Lubbock and 3 regional campuses across west Texas. While one of these regional campuses has since become an independent medical school in El Paso, TTUHSC has continued to operate a distributed educational program across the region.

Four current campuses are located in Lubbock (2 campuses), Amarillo, and in the Permian Basin (Odessa/Midland). The preclerkship curriculum is completed by all students in Lubbock, and clinical studies are completed on these 4 campuses.

TTUHSC School of Medicine introduced its first accelerated medical program (family medicine accelerated track, FMAT) in 2011. This program allows students interested in a career in family medicine to complete the MD degree in 3 years and complete residency in one of the 3 family medicine programs in the institution.

The school has integrated ultrasound-based diagnosis training and simulation experiences throughout the curriculum, with several opportunities for interprofessional education.

Students can supplement the preclerkship curriculum with elective experiences in culinary medicine, global health, human trafficking, technology in medicine, and many more.

\section{Curriculum description}

The standard 4-year curriculum is made up of 20 months of preclerkship blocks followed by 2 years of clinical immersion training.

The FMAT is an accelerated curriculum that prepares students to enter a family medicine residency after 3 years.

See Supplemental Digital Appendix 1-Curriculum Schematicat http://links.lww.com/ACADMED/A949.

\section{Curriculum changes since 2010}

The overall structure of the curriculum has not changed dramatically in terms of timing of transitions from preclerkship to clerkship phases or in the length of the major courses. Certain

Acad Med. 2020;95:S500-S503.

doi: 10.1097/ACM.0000000000003457

Copyright (C) 2020 by the Association of American Medical Colleges

Supplemental digital content for this article is available at http://links.Iww.com/ ACADMED/A949.

Year school was established: 1969.

School URL: https://www.ttuhsc.edu/medicine/default.aspx. content areas have moved between courses to meet learning needs of the students and to accommodate findings from curriculum audits. The content of individual courses has been altered to accommodate contributions from external sources and changes in pedagogy. The fourth year has been altered with the reassignment of geriatrics and neurology content from required courses to third-year clerkships.

Curriculum revisions in the near future will include accelerated progression of students from the preclerkship to the clerkship phase and potential changes to the overall structure of the clerkship phase to ensure that students have early exposure to specialty areas of practice.

\section{Class size changes since 2010}

The size of the matriculating class has increased from 140 to 180 since 2010. This resulted from 2 major alterations to the curriculum. First, 10 positions were added after establishment of the family medicine accelerated program. Second, the medical school established a second clinical campus in Lubbock, located within the Covenant Medical campus. Thirty students who complete their clinical training at this campus were added to the matriculating class in 2015.

\section{Assessment \\ Methods for summative assessment of student performance have not changed dramatically since 2010. However, there has been significant effort to provide more formative assessment of student progress. In addition, for the summative assessment tools we do use, more focus has been placed on establishing the validity evidence for these tools in our educational program.}

\section{Medical education program objectives}

The medical education program objectives are directly linked to the Physician Competency Reference Set competencies to facilitate mapping of student attainment of objectives.

\section{Parallel curriculum or tracks}

The FMAT program is an accelerated track specifically designed for students with a defined interest in the field of family medicine. Students in this program complete the medical school curriculum in 3 years and proceed into a family medicine residency program on one of the TTUHSC campuses. Thus, students in this program complete medical school and residency in 6 years. Over 50 students have completed this program since its inception in 2011, and the vast majority of graduates who have completed residency training are currently practicing in family medicine in Texas, many in underserved communities. Partly as a result of the FMAT program, TTUHSC is ranked by 
the American Academy of Family Physicians as one of the top $8 \mathrm{MD}$-granting medical schools for contributions to the family medicine workforce.

\section{Pedagogy}

Students in the preclerkship curriculum are educated using a variety of pedagogical approaches including case-based learning, small-group discussion, laboratory, lecture, peer teaching, problem-based learning, team-based learning, and simulation including standardized patient interactions. In addition, significant portions of each class are delivered as videos and self-directed learning modules. The curriculum has changed dramatically from a primarily lecture-based curriculum to one involving enhanced use of active learning methodologies. In addition, the curriculum is supplemented by a variety of external resources, including question banks and online learning systems.

\section{Clinical experiences}

Multiple clinical sites are used for student education throughout the curriculum on each of the campuses.

See Table 1-Sites for Clinical Training.

Learners encounter numerous simulated patient encounters in the first year of the curriculum to begin developing communication and physical examination skills within the Development of Clinical Skills course. Learners interact with patients in inpatient settings in the second-year curriculum as part of the continuation of these areas of training.

\section{Challenges in designing and implementing clinical experiences for medical students}

One challenge related to comparability in clinical experiences on our distributed campuses is ensuring that each learner has the opportunity to encounter a broad variety of patients in both the inpatient and outpatient settings. Each clerkship has established a specific list of required clinical encounters for their learners, and completion of these encounters is carefully monitored.

A second area of focus is in the development of measures to balance the educational and clinical responsibilities of both faculty and community physicians to ensure that we are providing an excellent educational experience to our learners.

\section{Curricular Governance}

Curricular governance is almost entirely centralized within the Offices of Academic Affairs and Student Affairs in Lubbock working closely with education offices on each campus. Clerkship departments provide administrative support for clinical activities on all campuses.

See Figure 1-Educational committee structure.

\section{Education Staff}

The Office of Academic Affairs oversees the administration of the medical school curriculum and includes divisions focused on the separate but overlapping areas of curriculum, student assessment, and program evaluation and educational technology.

See Figure 2-Education staff.

The Office of Academic Affairs has primary responsibility for the undergraduate medical education program, working closely with the Offices of Graduate Medical Education and Faculty Affairs and Development to coordinate with educational activities at the GME and CME levels.

The Department of Medical Education was established in 2013 to bring together faculty who are focused on teaching in the first and second years of our medical school's curriculum. The department includes basic scientists, medical educators, and clinicians, whose activities are focused on the education of preclinical students. The Department of Medical Education brings synergy, collaboration, and a shared mission for education, as well as innovation and expanded communication across courses and educational experiences.

\section{Faculty Development and Support in Education}

The Office of Academic Affairs holds an annual conference for faculty from all clinical campuses that includes faculty development workshops/seminars. In addition, the Office of Academic Affairs works closely with the Office of Faculty Development in developing and delivering faculty development courses offered annually and seasonally.

Faculty can list educational scholarship as 1 of their 2 primary areas of focus and can receive tenure and achieve promotion based on exhibited excellence in education.

\section{Table 1}

\section{Sites for Clinical Training}

\begin{tabular}{|c|c|c|c|c|}
\hline \multirow{2}{*}{$\begin{array}{l}\text { Type of } \\
\text { training }\end{array}$} & \multicolumn{4}{|c|}{ Location } \\
\hline & Lubbock & Lubbock-Covenant & Amarillo & Permian Basin \\
\hline Outpatient & TTUHSC clinics & Covenant Medical Group clinics & TTUHSC clinics & TTUHSC clinics \\
\hline Inpatient & $\begin{array}{l}\text { University } \\
\text { Medical Center }\end{array}$ & $\begin{array}{l}\text { Covenant Medical Center and } \\
\text { Covenant Women's and Children's } \\
\text { Hospital }^{a}\end{array}$ & $\begin{array}{l}\text { Northwest Hospital } \\
\text { and Baptist Saint } \\
\text { Anthony Hospital }\end{array}$ & $\begin{array}{l}\text { Medical Center Hospital } \\
\text { (Odessa) and Midland Memorial } \\
\text { Hospital (Midland) }\end{array}$ \\
\hline
\end{tabular}

astudents on Lubbock-based campuses get experience in both hospital systems, although the majority of their experience is on their home campus. 


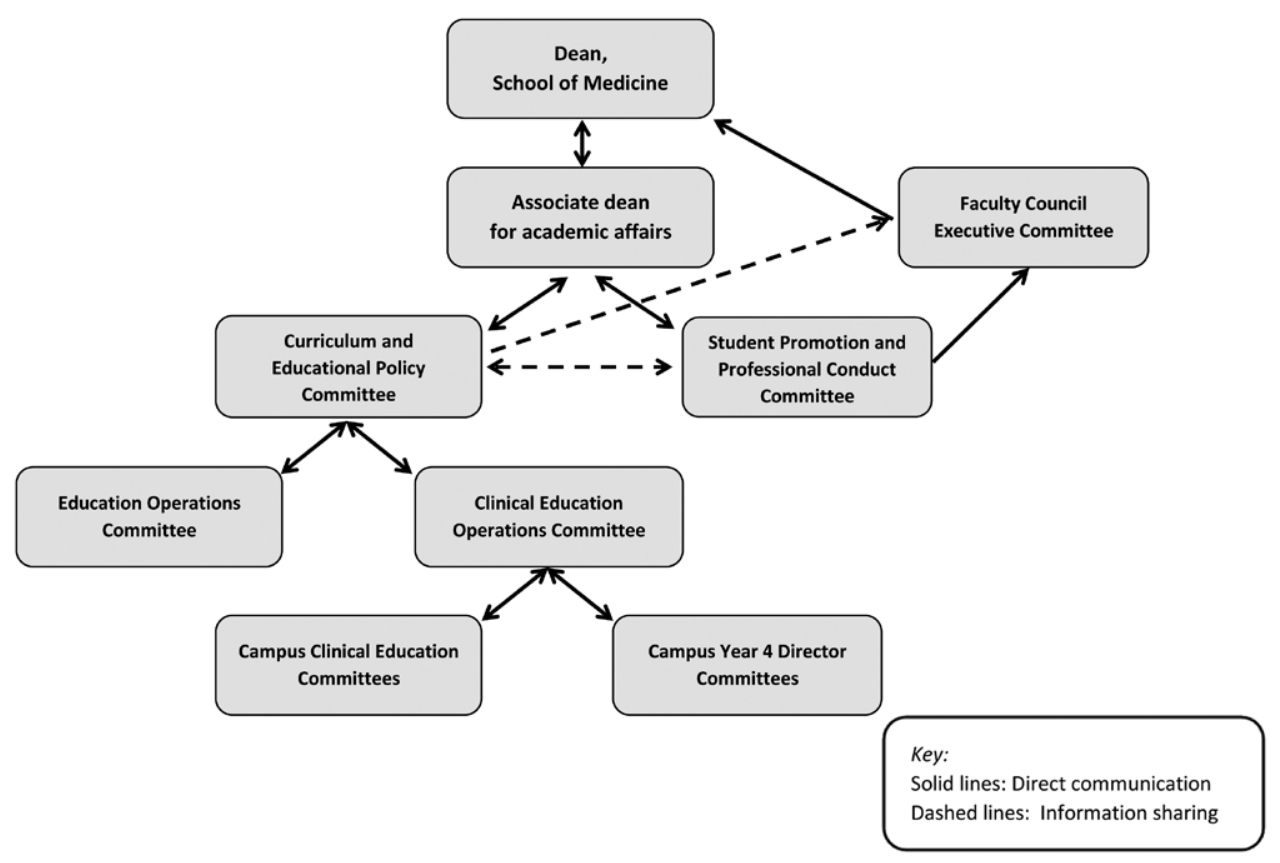

Figure 1 Educational committee structure.

The School of Medicine Teaching Academy was established in 1999 as a community of faculty who had displayed excellence in medical education. The academy offers expertise in faculty development activities and peer support for all educators in the school. In 2019, the Council of Distinguished Educators was established as an interdisciplinary group focused on collaborative practices, particularly in interprofessional education.

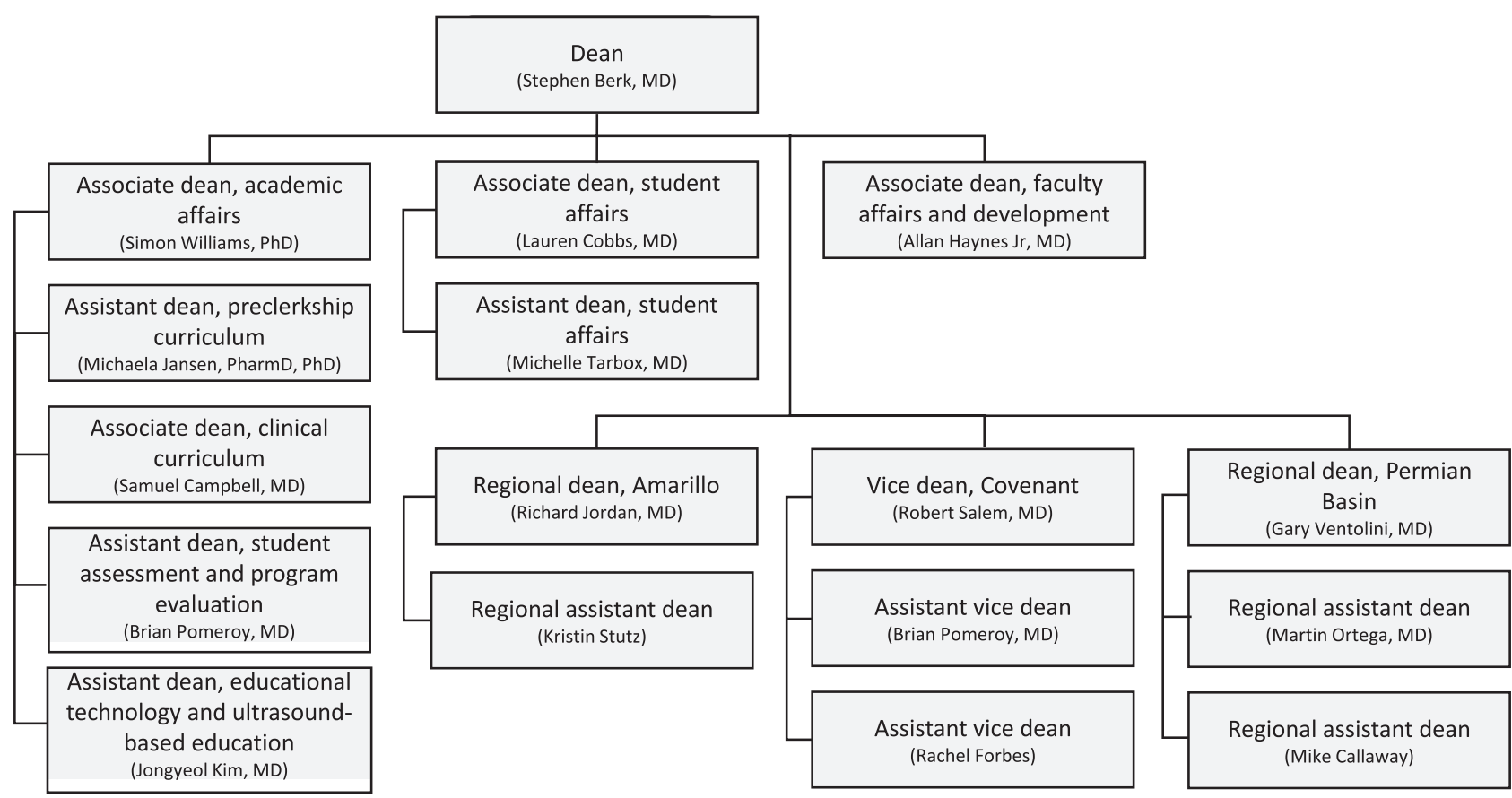

Figure 2 Education staff. 


\section{Regional Medical Campuses}

TTUHSC School of Medicine operates 4 campuses, 2 located in Lubbock and one each in Amarillo and the Permian Basin (Odessa and Midland). All students (180 per year) complete the preclerkship in Lubbock, and 72 remain on the Lubbock campus for clinical training. One hundred and eight students are distributed to regional/branch campuses.

See Table 2-Regional Medical Campuses.

Each campus has a freestanding education office that is in direct communication with the centralized administrative office. Each campus has representation on all curriculum and other committees with responsibility for cross-campus comparability, and the leadership of each clerkship meets every 2 months to review data comparability and to address any concerns.

\section{Table 2}

\section{Regional Medical Campuses}

Regional campus name Type Student enrollment

\begin{tabular}{|c|c|c|}
\hline Amarillo, Texas & Clinical & 54 per year (year 3 and year 4 ) \\
\hline Lubbock-Covenant & Clinical & 30 per year (year 3 and year 4 ) \\
\hline $\begin{array}{l}\text { Permian Basin (Odessa/ } \\
\text { Midland, Texas) }\end{array}$ & Clinical & 24 per year (year 3 and \\
\hline
\end{tabular}




\section{The University of Texas Health Science Center at San Antonio, Joe R. and Teresa Lozano Long School of Medicine}

Deborah L. Conway, MD, Florence Eddins-Folensbee, MD, Robert M. Esterl, Jr, MD, Joshua T. Hanson, MD, MPH, and Thea K. Lyssy, MA

\section{Medical Education Program Highlights}

The Long School of Medicine (Long SOM), a U.S. Department of Education Hispanic-Serving Institution, has a strong and supportive faculty and numerous opportunities for building clinical and research skills. Medical research institutes and nationally recognized cancer treatment programs combine education and research to provide some of the country's most innovative care. The MD degree program offers several unique features:

- Degree distinction programs in research, medical education, and humanities

- Dual-degree program for MD-MPH, whereby a student can earn both degrees in 4 years

- Dual-degree program for MD-PhD, offered by an NIHdesignated Medical Scientist Training Program

- The Center for Clinical Ultrasound Education, integrating bedside ultrasound curriculum throughout all 4 years of medical school

\section{Curriculum}

\section{Curriculum description}

The medical education program is named the CIRCLE curriculum (Curricular Integration: Researchers, Clinicians, Leaders, Educators). The name represents the continuous cycle of learning for physicians, as well as the roles students and graduates are encouraged to embrace. The CIRCLE curriculum has been in place since 2012. The Long SOM class size has remained stable at approximately 220 students per year since 2010 .

The 20-month preclinical curriculum begins with 3 foundational modules (Molecules to Medicine, Attack and Defense, and Language of Medicine) in July of the first year. The foundational modules are followed by 8 sequential organ-system modules, concluding in February of the second year. Two longitudinal modules (Medicine, Behavior, and Society; Clinical Skills) thread throughout the entire preclinical curriculum. Within each module, there is an integration of knowledge in a systematic fashion as follows: normal structure and function;

Acad Med. 2020;95:S504-S507.

doi: 10.1097/ACM.0000000000003397

Copyright (C) 2020 by the Association of American Medical Colleges

Supplemental digital content for this article is available at http://links.Iww.com/ ACADMED/A900.

Year school was established: 1968.

School URL: http://som.uthscsa.edu. pathogenesis/pathophysiology; clinical manifestations; interpretation of diagnostic tests; therapeutic interventions (including pharmacotherapy); relevant clinical/translational research, evidence-based medicine, and value-based care; and epidemiology/prevention. Each module has a weekly theme, culminating in a team-based interactive patient case. In March of the second year, students have the option to begin their clerkships and electives, creating flexibility later in the third and fourth year for career preparation, research, licensing examinations, and service activities.

The clinical curriculum comprises 8 required rotations: emergency medicine (4 weeks), family medicine ( 6 weeks), internal medicine ( 8 weeks), neurology (4 weeks), obstetricsgynecology (6 weeks), pediatrics (6 weeks), psychiatry (6 weeks), and surgery ( 8 weeks). Students assume increasing patient care responsibilities commensurate with achievement of specific milestones and competencies derived from nationally recognized standards, such as the AAMC's Core Entrustable Professional Activities (EPAs) and Physician Competency Reference Set (PCRS).

The elective curriculum provides students flexibility to determine their own developmental needs as they transition to residency. The schedule includes 8 weeks of selectives ( 4 weeks of inpatient subinternship, 4 weeks of ambulatory care), 20 weeks of electives, and 4 weeks of mandatory didactics.

See Supplemental Digital Appendix 1-Curriculum Overviewat http://links.lww.com/ACADMED/A900.

The curriculum has changed substantially since 2010 . Before the launch of the CIRCLE curriculum in 2012, it had a traditional "Flexnerian" format: basic science in the first 2 years followed by clinical science. Classroom sessions previously employed primarily lecture-dense, passive learning experiences.

Preclinical modules are co-led by 2 module directors, a pairing of 1 scientist-educator and 1 clinician-educator. Discipline coordinators serve as subject matter experts who ensure vertical and horizontal integration of their area of expertise across the educational program. Learning objectives drive session design and delivery, student assessment, and course/program evaluation. There has been a net reduction of classroom hours, leveraging best practices in blended learning, an emphasis on application of knowledge over memorization, and team-based learning. Demonstration of accountability, collaboration, and diligence are emphasized as foundations of physician excellence. As such, each preclinical module has designated portions that require in-person student engagement to meet passing requirements. 
The clinical phase of the curriculum also represents an evolution in student experiences, assessment frameworks, and sequencing. All students now take 4-week core clerkships in emergency medicine and neurology during their third year. Unified clinical assessment tools are used across all 8 clerkships and are built on the concept and framework of Core EPAs. Clerkship students now take part in 2 new experiences that transcend any individual clerkship: the cross-clerkship clinical skills (CS) exam, a series of 3 sequentially more complex objective structured clinical examinations that replicate and prepare students for the format of USMLE Step 2 CS examination; and the TeamCare series, large- and small-group interactive half-day sessions where all clerkship students are brought together as a class to cover widely applicable clinical topics, such as patient safety/quality improvement, value-based care, physician resilience, and challenging patient encounters.

\section{Program objectives and assessment methods}

The Long SOM competencies and objectives were initially developed by a working group of the Curriculum Committee (CC) in 2008 in preparation for a comprehensive curriculum renewal. The Medical School Objectives Project served as the primary framework upon which we based our final product. They are the backbone of instructional design, student assessment, and program evaluation of the curriculum. All competencies and objectives are mapped to module, clerkship, and elective objectives; session objectives within modules; assessment tools; and the PCRS.

See Supplemental Digital Appendix 2-Competencies, Program Objectives, and Assessment Methods_at http://links.lww.com/ ACADMED/A900.

In keeping with full integration of foundational science and clinical content in preclinical modules and reduction in classroom contact hours, approaches to assessment have changed since 2010. Daily opportunities for formative feedback on acquisition of key concepts and frequent quizzing have been incorporated into preclinical modules. Items on summative module examinations mimic USMLE Step 1 item format. Before students begin electives and clerkships, they must demonstrate readiness to pass USMLE Step 1 via performance on the NBME Comprehensive Basic Science Exam.

In the clinical phase, assessment of clinical performance is captured using a common tool across clerkships. The domains map to Core EPAs and performance levels are descriptive in nature, permitting students to monitor their own progress within key domains and set developmental goals for ongoing improvement.

\section{Pedagogy}

The Long SOM uses a wide variety of pedagogical approaches to achieve program objectives and competencies. The appropriate approach is selected based on the topic, desired learning outcomes, level of the learner, and educational setting. A pillar of the curriculum renewal in 2012 was an emphasis on applied knowledge and engaged, often collaborative, learning.
Students are provided guided preparation for each session, and face-to-face time targets higher levels of cognitive skills: applying, analyzing, evaluating, and creating. This is primarily accomplished through case-based learning, large- and smallgroup discussions, simulations, role play, standardized patient encounters, and team-based learning.

\section{Clinical experiences}

The CS longitudinal module runs for the duration of the preclinical phase of the medical education program, providing students with opportunities to learn medical history-taking and physical examination skills using standardized and real patients. Reinforcing the learning and development continuum of the module, CS core clinical faculty facilitators shepherd a small cohort of students at the CS simulation center from the first week of medical school through the end of the course, serving as role models and coaches in the development of key skills.

Students have their first clinical experiences in the first semester as part of the CS module's longitudinal preceptor component. Students meet periodically in clinical settings with their assigned preceptor, engaging in basic CS and patient encounters, through the end of the preclinical phase.

Long SOM and its faculty practice plan own and run outpatient sites but currently do not own a teaching hospital. Thus, most clinical rotations for our students occur at community-based locations for both inpatient and outpatient experiences. Most of these clinical sites are within the San Antonio metro area. Inpatient experiences are conducted primarily at 2 locations: University Hospital, which is a Level 1 trauma center, a Level 4 NICU, and a Level 4 maternal care facility, and the Bexar County safety net hospital and a regional referral center for central and south Texas; and at the Audie L. Murphy Memorial Veterans' Hospital. Students also have opportunities to work at several other local hospitals, including a large military hospital located at Brook Army Medical Center, as well as private hospital systems. Outpatient experiences occur primarily at University Health System (Bexar County) and UT Health/Long SOM faculty practice sites, as well as in private practice settings.

As it is with many other medical schools, it can be a challenge to find and maintain enough quality placement sites to host medical students for clinical experiences. There is added competition locally and regionally pursuing placements for students in an already limited pool of potential sites.

\section{Curriculum Governance}

The Long SOM Faculty Assembly bylaws designates the CC as the faculty committee with ultimate authority and responsibility for central oversight of the medical education curriculum, including the overall design, management, integration, evaluation, and enhancement of a coherent and coordinated curriculum.

The CC maintains 2 standing subcommittees to fully carry out its overarching charge: Evaluation Subcommittee and Design and Integration (D\&I) Subcommittee. The Evaluation Subcommittee 
is charged with recurring review of all required modules, clerkships, didactics, and (s)electives within the medical school curriculum. The D\&I Subcommittee is charged with monitoring and influencing design of the curriculum to ensure the opportunity for students to achieve the stated program competencies and objectives.

See Figure 1-Curriculum design, implementation, and evaluation.

\section{Education Staff}

\section{Medical education leadership}

The Office for Undergraduate Medical Education (OUME), under the direction of the vice dean for UME, has primary responsibility for supporting the UME program. It is a comprehensive academic home comprising 3 administrative units: admissions and outreach, curriculum oversight, and student affairs.

The curriculum oversight team, led by the associate dean for curriculum, works closely with the CC and course/module and clerkship directors to provide administrative, operational, and academic support for the development and maintenance of tools (i.e., Canvas LMS, one 45 curriculum management software) to support curriculum delivery, monitoring, evaluation, and management.

The student affairs team sponsors a wide variety of services, programs, and events for students in the Long SOM. Its mission is to help create a supportive and positive environment for students that enhances and supports learning to promote the personal and professional development of students as they progress through the curriculum.

\section{Department of Medical Education}

Separate from the OUME, the Department of Medical Education (DME) supports individuals interested in conducting medical education research and facilitates translation of research findings into educational practice across the continuum of medical education. Faculty with cross appointments in the DME also hold faculty appointments with their academic home department.

See Figure 2-Dean leadership.

\section{Faculty Development and Support in Education}

The OUME has an education development services (EDS) team dedicated to working with faculty members on development; implementation; and enhancement of existing and new courses, modules, initiatives, programs, and services in support of the teaching mission. The EDS staff meet with faculty on an individual basis as well as provide workshops on teaching and assessment of students.

The OUME hosts the AAMC Medical Education Research Certification program locally in conjunction with a faculty development week when invited guests from other institutions and local education experts provide sessions on a variety of

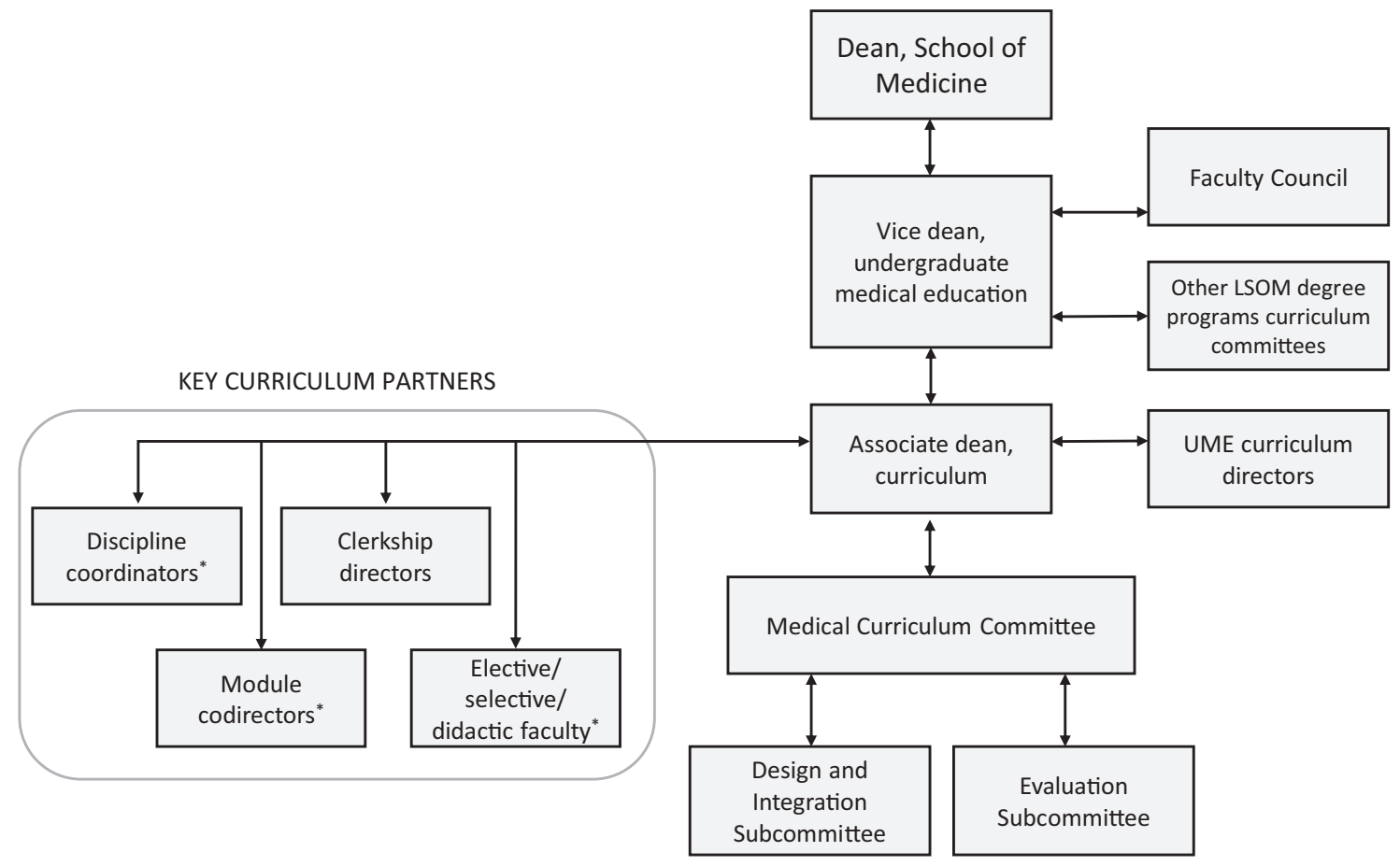

${ }^{*}$ Faculty groups are each represented by a voting member on the Medical Curriculum Committee.

Figure 1 Curriculum design, implementation, and evaluation. 


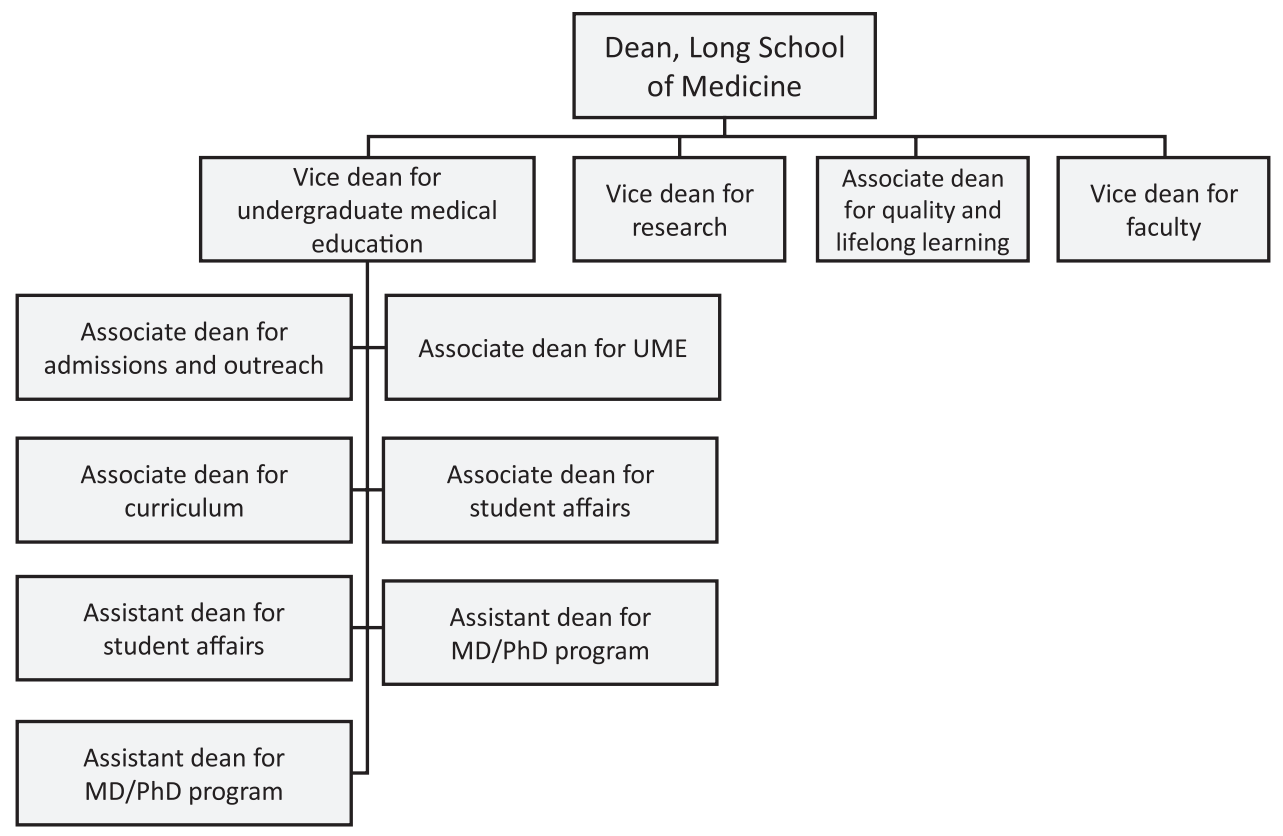

Figure 2 Dean leadership.

topics relevant to educational best practices, teaching, and educational research.

\section{Role of teaching in promotion and tenure}

In promotion and tenure decision, documented excellence (i.e., scholarly achievement) in teaching is considered a fundamental academic activity and is evaluated for advancement in rank based on performance in original or imaginative accomplishments in the conduct of academic responsibilities in teaching and research. Strength and leadership in education is demonstrated when a faculty member engages in sustained scholarly activities such as teaching in the curriculum, serving as a director of a training program, or serving as a module/course director or discipline coordinator.

\section{Initiatives in Progress}

Planned curriculum initiatives include a detailed analysis of the fourth year with resultant changes to its goals and structure (target implementation date 2022) and a coordinated, integrated interprofessional education plan that spans all 4 years of the curriculum (target implementation date 2020). 


\title{
Dell Medical School at the University of Texas, Austin
}

\author{
Elizabeth A. Nelson, MD, LuAnne Wilkerson, EdD, Stephen Smith, PhD, and Susan Cox, MD
}

\section{Medical Education Program Highlights}

Dell Medical School (DMS) at the University of Texas (UT), Austin, enrolled its first class in 2016. The curriculum is designed to train outstanding doctors who are also prepared as physician leaders. They should be as comfortable taking on transformational health care challenges as they are caring for patients. Every curricular component is designed to focus students on the distinct challenges of 21st-century health care and medicine and how to apply creativity in solving those challenges. Students complete a 4-year leadership curriculum and have the opportunity to individualize leadership applications during the year 3 Innovation, Leadership, and Discovery (ILD) 9-month course.

The highly integrated educational program incorporates guided, self-directed learning, interprofessional education, longitudinal coursework, and a health systems science curriculum.

\section{Curriculum}

\section{Curriculum description}

- Year 1 curriculum-Essentials: The preclerkship curriculum includes courses that integrate scientific, social, and clinical knowledge for students to prepare and succeed in the clinical years of medical school. The curriculum consists of 5 integrated block courses and 3 longitudinal courses (Leadership, Interprofessional Education, and Developing Outstanding Clinical Skills) that continue throughout all 4 years.

- Year 2 curriculum-Delivery: There are five 8-week block clerkships, a 4-week emergency medicine clerkship, and time to complete up to 4 weeks of electives. A weekly longitudinal primary care, family, and community medicine clerkship begins in year 2 and continues through year 3.

- Year 3 curriculum-Growth: Students have up to 8 weeks between year 2 and the start of the ILD course to prepare and take the USMLE Step 1 examination. ILD is a 9-month course that allows students the option to complete a UT master's degree, or a distinction in research or population health. There are shared core competencies for the ILD course, which must be met irrespective of the option chosen. Current degrees available include public health, business administration, educational psychology, health care transformation, biomedical engineering, and design in health care.

Acad Med. 2020;95:S508-S512.

doi: 10.1097/ACM.0000000000003392

Copyright (C) 2020 by the Association of American Medical Colleges

Supplemental digital content for this article is available at http://links.Iww.com/ ACADMED/A903.

Year school was established: 2015.

School URL: https://dellmed.utexas.edu.
- Students continue their primary care clerkship with weekly preceptor half-day experiences and participate in a servicelearning community project as part of their interprofessional education course. At the end of year 3 , students have a weeklong clinical skills course to transition into the fourth-year clinical experiences.

- Year 4 curriculum-Exploration: Students are required to complete two 4 -week acting internships, 4 weeks of critical care, a 2-week geriatrics/palliative care clerkship, and an intensive residency preparation experience (Milestone 1). In addition, 22 weeks of electives are required.

See Supplemental Digital Appendix 1-Curriculum Schematicat http://links.lww.com/ACADMED/A903.

\section{Assessment}

The DMS graduation competencies are modeled after the 6 ACGME domains, with the addition of a seventh domain specific to DMS, leadership, and innovation. There are 18 competencies and 68 measurable enabling objectives. These provide the foundation for all curricular components and student assessments. Students also complete an annual competency reflection and describe curricular and noncurricular activities and assessments that promoted those competencies in which they have experienced the most growth over the year.

See Chart 1-Competency Domains, Objectives, and Assessment Methods.

Since the first class began in 2016, all assessments have been newly developed, implemented, and refined. The clinical performance assessment form used in the clerkship and clinical electives includes leadership, curiosity, emotional intelligence, and systems thinking as unique items, each described at 4 levels of accomplishment. The NBME Comprehensive Basic Science Examination is used as a progress test with 4 repetitions across years 1 and 2 of the curriculum. Students complete multiple OSCEs during years 1 and 2 with individual feedback provided on performance and writeups. A multistation clinical performance examination is given in the spring of the third year as students reenter clinical medicine.

\section{Pedagogy}

- Self-directed learning/problem-based learning (PBL): Block courses in the preclerkship curriculum include PBL, called PILLARS, for 3 to 6 hours a week with a faculty facilitator. Students discuss 1 or 2 clinical cases each Monday morning and develop a list of basic, clinical, and social science learning objectives that they will all pursue prior to the cases discussion. At the follow-up discussion, students teach each other what they have learned, assist one another in clarifying and deepening understanding, and apply the results to the patient.

- Simulations/standardized/simulated patients: In the 4-year Developing Outstanding Clinical Skills course, teams of 5 


\section{Chart 1}

\section{Competency Domains, Objectives, and Assessment Methods}

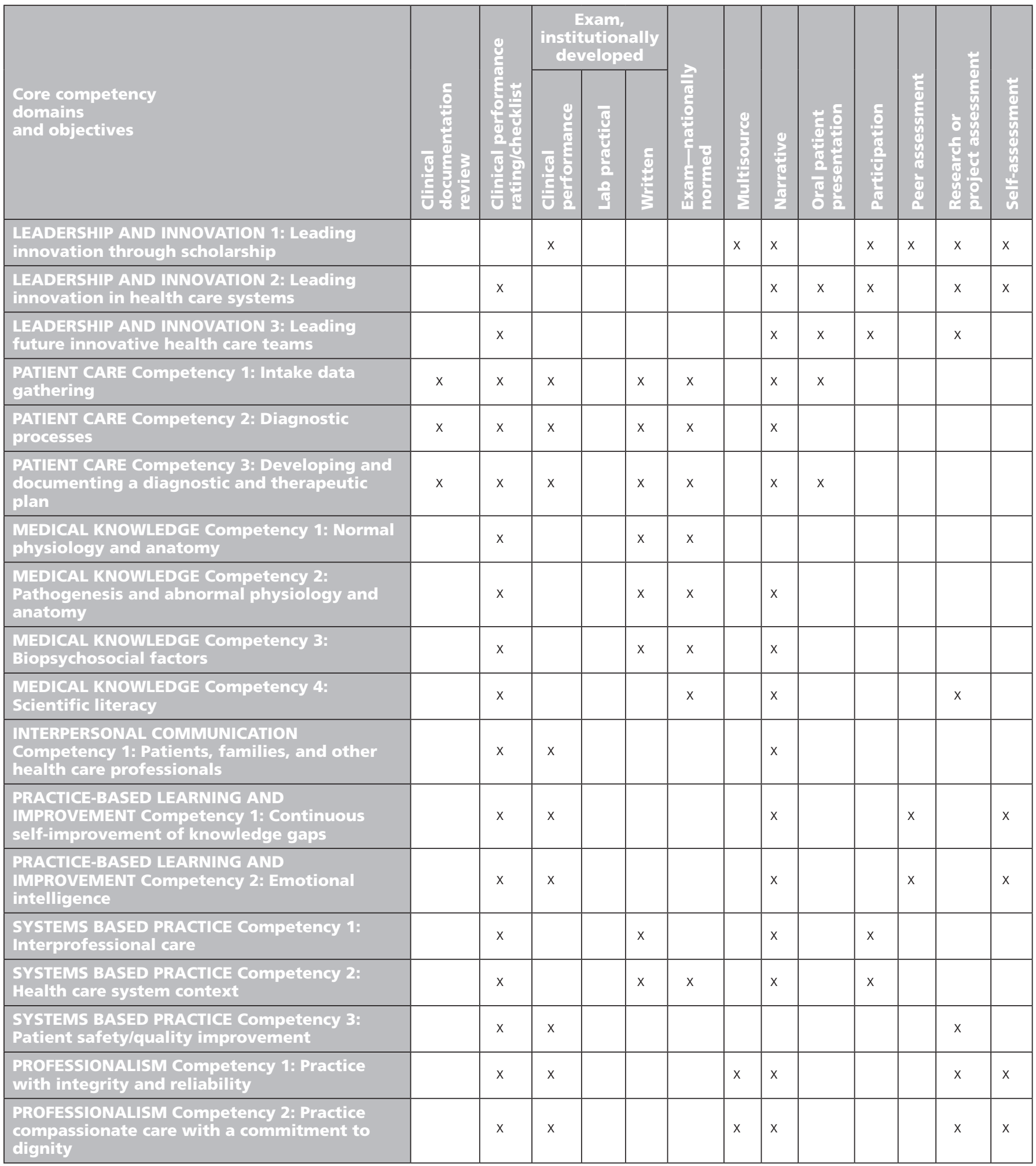

Assessment methods from: MedBiquitous Curriculum Inventory Working Group Standardized Vocabulary Subcommittee. Curriculum Inventory Standardized Instructional and Assessment Methods and Resource Types (March 2016 version). Washington, DC: Association of American Medical Colleges; 2016. 
students work with a single clinical facilitator beginning in year 1 , using role play, simulation, standardized patient exercises, real patient encounters, and OSCEs for both formative feedback and summative assessment. All of the core clerkships use simulation as a part of midpoint feedback. Emergency medicine and surgery include simulation for skill development. The transition-to-clerkship course (Milestone 0) incorporates simulation for skill development and assessment.

\section{Clinical experiences}

The required clerkships have identified the core conditions and clinical skills or procedures that must be completed by each medical student to pass. Each condition and clinical skill is associated with a clinical setting and level(s) of expected responsibility. The conditions/skills logs are structured to assist students and instructors in assuring a consistent clinical experience. Students' feedback on the conditions/skills log has been very positive; students report that it forces them to look for a range of conditions and skills while they are in their clerkships, and discuss incomplete sections of the log with their residents and attending physicians. The Curriculum Committee reviews the logs across clerkships for the inclusion of essential medical conditions with attention to community needs, coverage of skills, level of responsibility, and settings. Of particular note, students complete a 2-year longitudinal clerkship in primary care, family, and community medicine at a single site to promote continuity of care, a population perspective, and consistent clinical supervision.

Ascension Seton hospitals in Austin serve as the inpatient sites for the core clerkships and clinical electives. The major inpatient site is Dell Seton Medical Center at the UT. Other sites include Dell Children's Medical Center (pediatrics), Seton Shoal Creek Hospital (psychiatry), and Seton Main Hospital (women's health, emergency medicine). Austin's Central Health CommunityCare is the primary ambulatory care partner.

\section{Service learning and community service}

DMS is committed to serving its community and has therefore embedded service learning into its required curriculum within the interprofessional courses in years 1, 3, and 4. In the year 1, Foundations of Interprofessional Collaborative Practice course, teams of medical, nursing, social work, and pharmacy students identify and explore a community health-related need and develop a public service announcement. In the year 3 course, teams plan and implement a community service learning project based on the Travis County Public Health Critical Indicators report. Medical student teams work with members of established community organizations to achieve their project aims. They develop and deliver a community project then present the results at a forum to which community stakeholders are invited. Students are also involved in volunteer community service across all years of the curriculum and can develop their own interventions or draw on the UT Austin volunteer opportunities website.

\section{Curricular Governance}

The Undergraduate Medical Education Curriculum (UMEC) committee, a DMS standing committee, "is responsible for the overall design, management, integration, evaluation, and enhancement of a coherent and coordinated curriculum." The UMEC subcommittee structure was designed to foster integration across disciplines, courses, and years of the curriculum. Routine subcommittee reporting at the twice monthly UMEC meeting (Scientific Foundations, Healthcare Delivery, Curricular Innovation, Innovation, Leadership and Discovery, and Evaluation and Assessment Committees) fosters both horizontal and vertical integration. Clinical clerkships and elective education include input from the departments but are ultimately approved and financially supported centrally.

See Figure 1-UMEC and subcommittees.

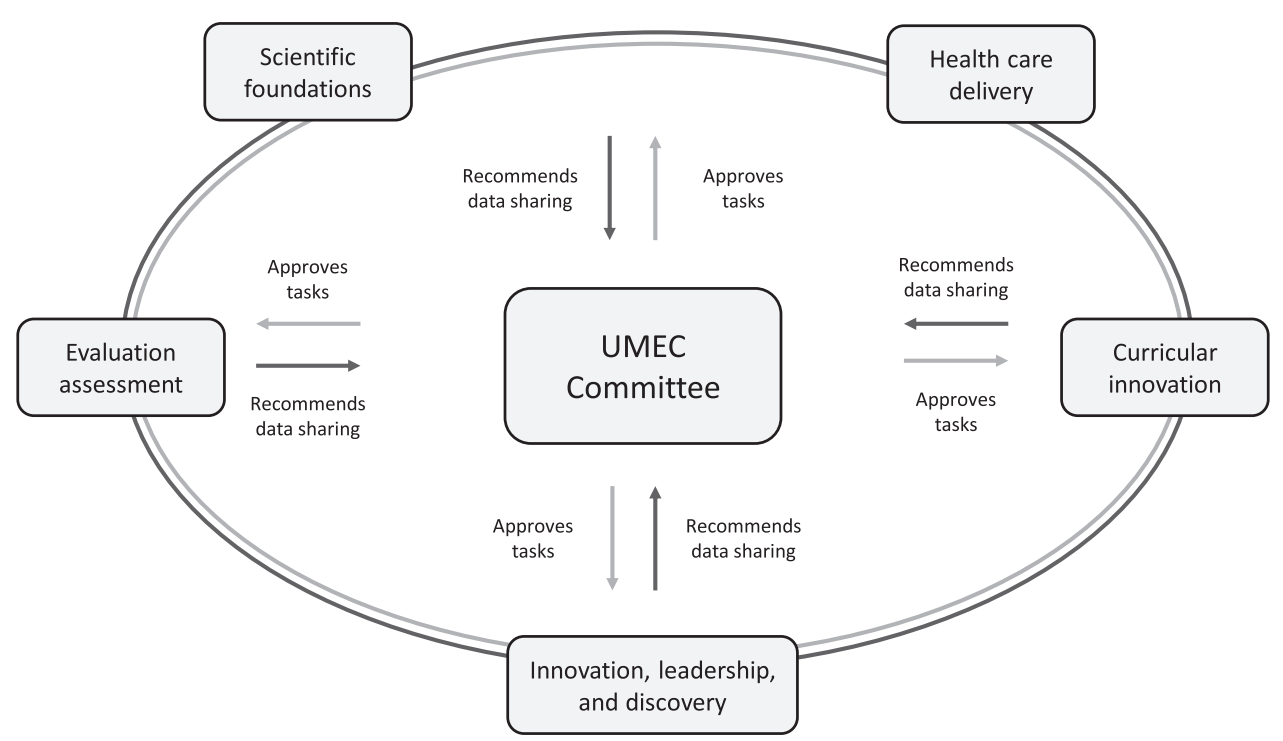

Figure $1 \mathrm{UMEC}$ and subcommittees. 


\section{Education Staff}

\section{Department of Medical Education}

The Department of Medical Education serves as the organizational locus for the planning, implementation, and evaluation of the medical education program across the continuum. The department is chaired by the executive vice dean for academics and includes faculty members who serve as the educational leadership team, consisting of an associate dean for UME, associate dean for evaluation and faculty development, associate dean for student affairs, and associate dean for graduate medical education. Assistant deans for clinical skills integration, interprofessional integration, and value-based care are also faculty members in the department and have extensive curriculum development and teaching responsibilities in their respective domains. There are also 6 full-time core faculty members who serve as course directors and teaching faculty for the scientific foundations courses in year 1. Together these faculty members provide support for all curricular components.

A senior-level curriculum manager and 5 education coordinators staff various curricular components and maintain the tools that support curriculum delivery, monitoring, and management, including Canvas as the learning management system and one that serves as the curriculum database support the UME division. These UME staff also support the Curriculum Committee and its subcommittees.

The evaluation and faculty development division of the department includes the associate dean and the faculty member who directs program evaluation and educational research, who are both doctoral-level educators, the director for the standardized patient program, and an education coordinator.

\section{Faculty Development and Support in Education}

\section{Professional development for faculty as educators}

Professional development opportunities for teaching, assessment, and scholarship are widely available with programs at the school, departmental, and university levels. Faculty members can earn a DMS certificate in clinical teaching by participating in 24 hours of school and external teaching enrichment activities. The centerpiece of the certificate is a 14-hour course offered twice annually, Extraordinary Learning and Teaching in Clinical Settings. Best practices in medical education grand rounds, a medical education journal club, and an educational research-inprogress conference occur monthly. Departmental conferences, workshops, and grand rounds feature professional development topics on a regular basis. A course on leadership is available for junior faculty members, that is, coordinated with the leadership course for first-year medical students.

The Department of Medical Education also supports the whole continuum of medical education through administrative support for student affairs, admissions, graduate medical education, and continuing medical education.

See Figure 2-Organizational chart.

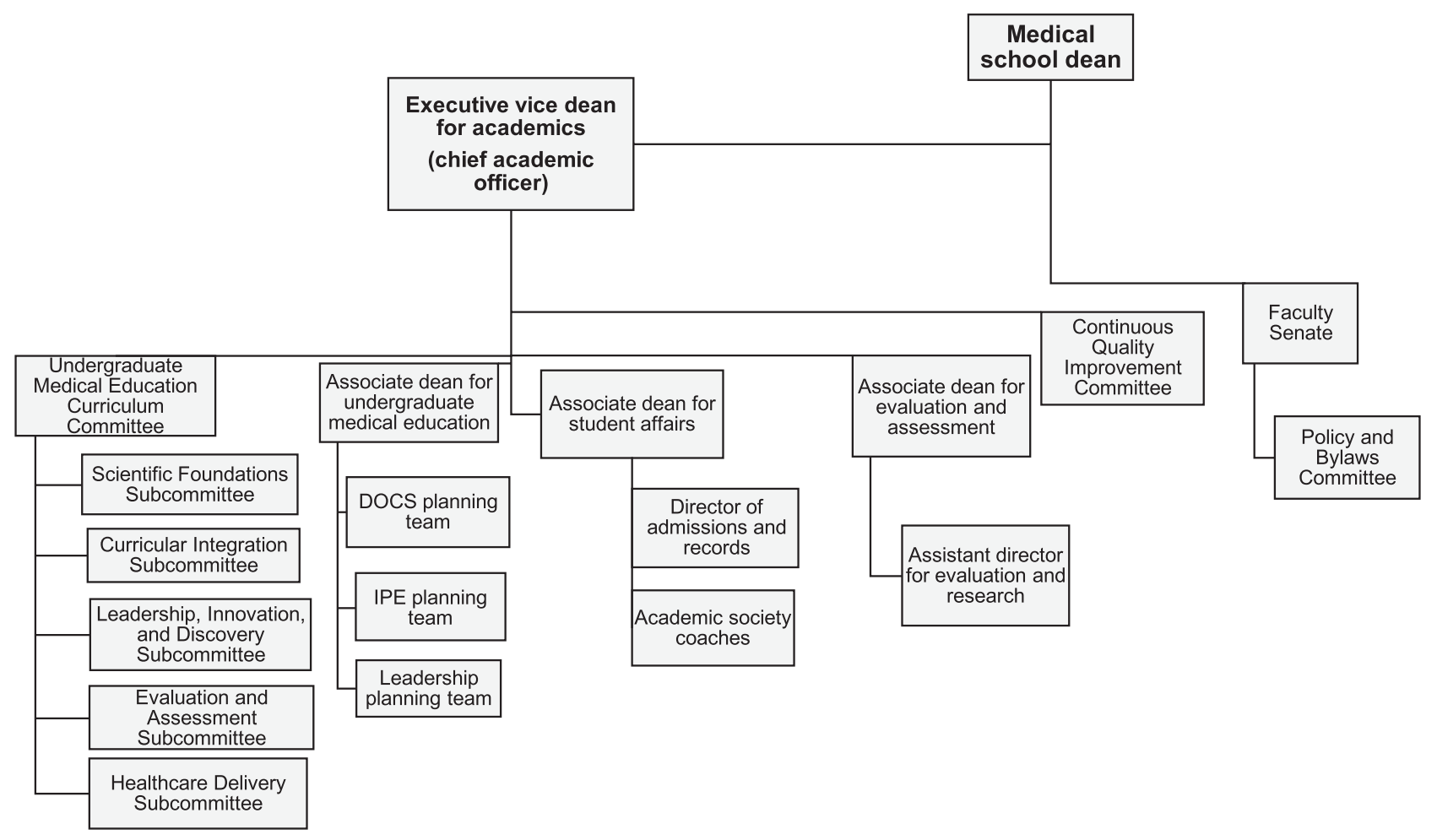

Figure 2 Organizational chart. 


\section{Role of teaching in promotion and tenure}

There are 2 faculty tracks, regular (regardless of funding source) and affiliate (volunteer). The criteria for promotion for faculty in the regular series include the opportunity to select an area of excellence in education, clinical expertise, community engagement, or research, each requiring a different type of scholarly activity.

Regular faculty members are expected to continuously engage in both teaching and scholarship, while expectations for affiliate faculty are less rigorous. Peers define scholarship as the creative application of the intellect that results in development of a product that is available for assessment for quality. Furthermore, this product is made public for others to learn from or build upon. Criteria have been delineated for use in assessing products of scholarship. Scholarship may consist of traditional peer-reviewed manuscripts or new forms of dissemination such as blogs.

\section{Teaching academy}

The DMS Academy of Distinguished Educators recognizes the school's most outstanding educators and educational leaders and fosters the growing community of Dell Med teachers. The academy provides peer-observation opportunities and sponsors an annual educational innovation and scholarship event with poster presentations by faculty, residents, and students. Starting in the fall of 2019, the event also serves as a celebration of faculty members and residents receiving DMS teaching awards. 


\title{
University of Texas Medical Branch Galveston
}

\author{
Majka B. Woods, PhD, N. Miles Farr, MD, MPH, and Michael Ainsworth, MD
}

\section{Medical Education Program Highlights}

Founded in 1891, the University of Texas Medical Branch (UTMB) at Galveston is the state's first medical school. While fostering educational innovation and growth to better train tomorrow's doctors, the school of medicine remains focused on its core mission areas: excellence in education, service, research, and patient care. UTMB is uniquely situated to provide students with varied educational opportunities across the mission areas as well as support and recognition of faculty in pursuit of educational excellence.

Students have the option to pursue a scholarly program track, focusing on an area of interest, giving them an additional defined educational experience, and providing them with real-world applications. These include aerospace medicine, bilingual health, community engagement, geriatric health, global health, medical humanities, physician healer, public health, rural health care, and translational research.

UTMB's John P. McGovern Academy of Oslerian Medicine recognizes and honors the physician faculty who dedicate their careers to teaching in medicine and exemplify the principles of excellence, compassion, integrity, and respect. The academy, supported by the John P. McGovern Foundation of Houston, provides funding that supports outstanding teaching and exemplary student scholarship (Oslerian societies).

Students, faculty, and staff at UTMB serve patients from a wide variety of backgrounds through community service, ongoing clinical experiences, and volunteerism. The UTMB GalvestonTexas Department of Criminal Justice Hospital (UTMB-TDCJ) is the first major university hospital focused on offender care. Students have the opportunity to rotate through the facility multiple times during their training. St. Vincent's Clinic in Galveston is a community-based health facility where students may serve unfunded and underfunded patients. In addition, multiple agencies in Galveston county allow students and faculty to volunteer time and efforts to serve the community and improve health care.

Professionalism coupled with professional identity formation is woven throughout the curriculum and related experiences. Students' first course, Mindfully Evolving, Thriving, and Advocating (META), focuses on student wellness, learning,

Acad Med. 2020;95:S513-S516.

doi: 10.1097/ACM.0000000000003381

Copyright (C) 2020 by the Association of American Medical Colleges

Supplemental digital content for this article is available at http://links.Iww.com/ ACADMED/A891.

Year school was established: 1891.

School URL: https://som.utmb.edu. cultural humility, and clinical skills, while emphasizing integrity, compassion, and respect for everyone. This course is intended to launch the learner into the curriculum and into their lifelong journey to become a compassionate and excellent physician.

\section{Curriculum}

\section{Curriculum description}

Courses in the integrated medical curriculum begin with a focus on scientific principles integral to the practice of medicine: META; Gross Anatomy and Radiology; Molecules, Cells, and Tissues; Pathobiology and Host Defense; and Neuroscience and Human Behavior. In year 2, the curriculum shifts to organ-based blocks and culminating courses: Cardiovascular and Pulmonary; Renal Fluid and Electrolytes; Gastrointestinal and Nutrition; Endocrine and Reproduction; Dermatology, Hematology, and Musculoskeletal; and Great Syndromes. All courses integrate traditional basic science disciplines for each of the major systems of the human body; are interdisciplinary; and are based on self-directed, problem-based learning (PBL), with supplemental large-group lectures and laboratory sessions.

The Practice of Medicine course serves as a focal point in clinical skills development during years 1 and 2. It emphasizes practical development in communication, medical interviewing, and physical examination skills, complemented by principles of medical ethics, professionalism, and evidence-based medicine/ clinical reasoning during weekly small-group sessions with clinical faculty. Basic skills in year 1 are reinforced through regularly scheduled half-day visits to primary care physician practices in the community. Experiences are expanded in year 2 with periodic university-based clinical experiences to practice and develop clinical abilities.

Year 3 consists of 48 weeks of instruction in internal medicine, pediatrics, surgery, obstetrics-gynecology, psychiatry, family medicine, and an elective. Year 4 consists of 40 weeks of instruction, which include neurology selective, emergency medicine selective, acting internship selective, ambulatory community selective, basic science/humanities selective, and electives totaling 20 weeks.

\section{Curriculum changes since 2010}

UTMB has a PBL focus in the first 2 years of medical school. The PBL cases continue to evolve. Every case has a patient focus and is driven by key objectives. New topics are continually assessed for addition into the cases, including professionalism, population health, medical humanities, communication skills, bioinformatics, and clinical reasoning. An increased emphasis on the clinical application of foundational science knowledge, including patient presentations, has been a focus of recent years. In addition, structured peer-to-peer feedback on written work has increased. 
In addition to the PBL-focused Foundational Science courses, students also participate in a Practice of Medicine course during the first and second years. These courses continue to adapt to provide both exemplary clinical skills experiences and focused reflection on the practice of medicine. UTMB continues to use standardized patients in small-group settings to train and reinforce excellence clinical skills, as well increasing the number of clinical site visits that start in year 1 .

In 2015, the Curriculum Committee moved all courses to NBME customized exams for both the midterm and final. Additional assessments include weekly quizzes created in house, tied to objectives covered during PBL cases and structured after NBME style items; lab exams; oral presentations; and objective structured clinical examinations. These exams are built by course directors and are a direct reflection of the defined objectives from the course. Courses are required to review and rebuild the exams and refresh $20 \%-30 \%$ of items annually.

Course and clerkship directors have worked on coordination of the early clinical skills curriculum as a path into the clerkships. This led to many innovations and curricular improvements, including anatomical dissection with direct clinical applications mainly focused on surgical interventions during gross anatomy, further clinical skills development within the Practice of Medicine course, increased use of high-fidelity simulators in small-group and team-based learning activities, and intentional instructional design of clinical skills elements to provide students multiple exposures and opportunities to develop their abilities.

Curriculum revitalization efforts are currently underway to further improve instructional design as well as students' experiences and opportunities related to clinical training. Efforts include designing and providing an increased number of early clinical opportunities; creating more robust peer-to-peer feedback; focusing on professionalism; redesigning the clinical years to create a more robust fourth-year experience; increasing team-based learning opportunities in all years; and analyzing the entire 4-year curriculum to identify gaps, redundancies, and strengths.

\section{Assessment}

The integrated medical curriculum is designed using the 29 curriculum goals of the AAMC Medical School Objectives Project.

See Supplemental Digital Appendix 1-Program Objectives and Assessment Methods—at http://links.lww.com/ACADMED/A891.

\section{Clinical experiences}

A wide array of clinical sites are used for required experiences, including UTMB health system academic inpatient and outpatient sites, UTMB community outpatient clinics, external community outpatient clinics across Texas, and affiliated community and teaching hospitals' inpatient and outpatient sites across the state of Texas.

We have an optional longitudinal clinical experience offered to third-year students.

\section{Clinical experience first encounter}

Students' first clinical experiences begin within the first 2 months of medical school as part of the first-year clinical skills curriculum.

\section{Required and elective community-based rotations}

Between their first and second years, students have an 8-week period where they may participate in a statewide community placement program. They are placed with a community preceptor anywhere in Texas in pediatrics, internal medicine, or family medicine.

As part of the core clerkship curriculum, the majority of students spend a portion of their clerkship in external outpatient community sites. This includes the possibility of placement for 4 weeks of family medicine, 4 weeks of internal medicine, 2 weeks of pediatrics, and 2 weeks of obstetricsgynecology. There are opportunities for students to participate in individual inpatient and outpatient rotations at our regional campuses.

Students may participate in student-created community electives in their field of choice during their fourth year of medical school. UTMB's Office of Clinical Education facilitates adjunct faculty appointments, evaluations, and affiliation agreements for community preceptors.

\section{Challenges in designing and implementing clinical experiences for medical students}

In Texas, the number of medical schools and medical students continues to expand, increasing competition for clinical training. Additionally, the evolution of medical education to include earlier and more frequent clinical exposure has led to increased pressure on the utilization of limited clinical teaching sites. The financial pressures in the current health care environment that emphasizes clinical RVUs and efficiency have increased challenges in implementing quality clinical educational experiences.

\section{Curricular Governance}

Administrative and academic support for the curriculum is organized through the Office of Academic Affairs. Academic Affairs manages the curriculum through its organizational structure of offices and oversight groups.

Offices:

- Educational Affairs: General oversight led by the associate dean for educational affairs

- Instruction Management: Administers years 1 and 2 of the medical school curriculum

- Clinical Education: Administers years 3 and 4 of the medical school curriculum

- Educational Development: Program evaluation, research, and faculty development

- Admissions and Student Affairs: Student admissions, academic and career counseling 
- Physician Assistant Studies: Instruction and assessment of physician assistant students

Curriculum oversight and support:

- Curriculum Committee: Primary policy and curriculum implementation authority

- Operations Committee: Supports Curriculum Committee with policy analysis/recommendations

- Longitudinal Clinical Assessment: Identifies and assists learners with clinical skills deficiencies

- Student Societies: Groups sponsored by John P. McGovern Academy of Oslerian Medicine

See Figure 1-Organizational chart.

\section{Education Staff}

The primary role of the medical education staff and faculty is to serve students and faculty throughout all phases of undergraduate medical education-from recruitment, admissions, curriculum development, instruction, and support to competency assessment, advancement, and remediation. These programs operate under the umbrella of the Office of Academic Affairs, as does the Office of Continuing Education, which sponsors continuing professional development activities across the health professions. Graduate medical education is managed by a separate process administered through an associate dean for graduate medical education.

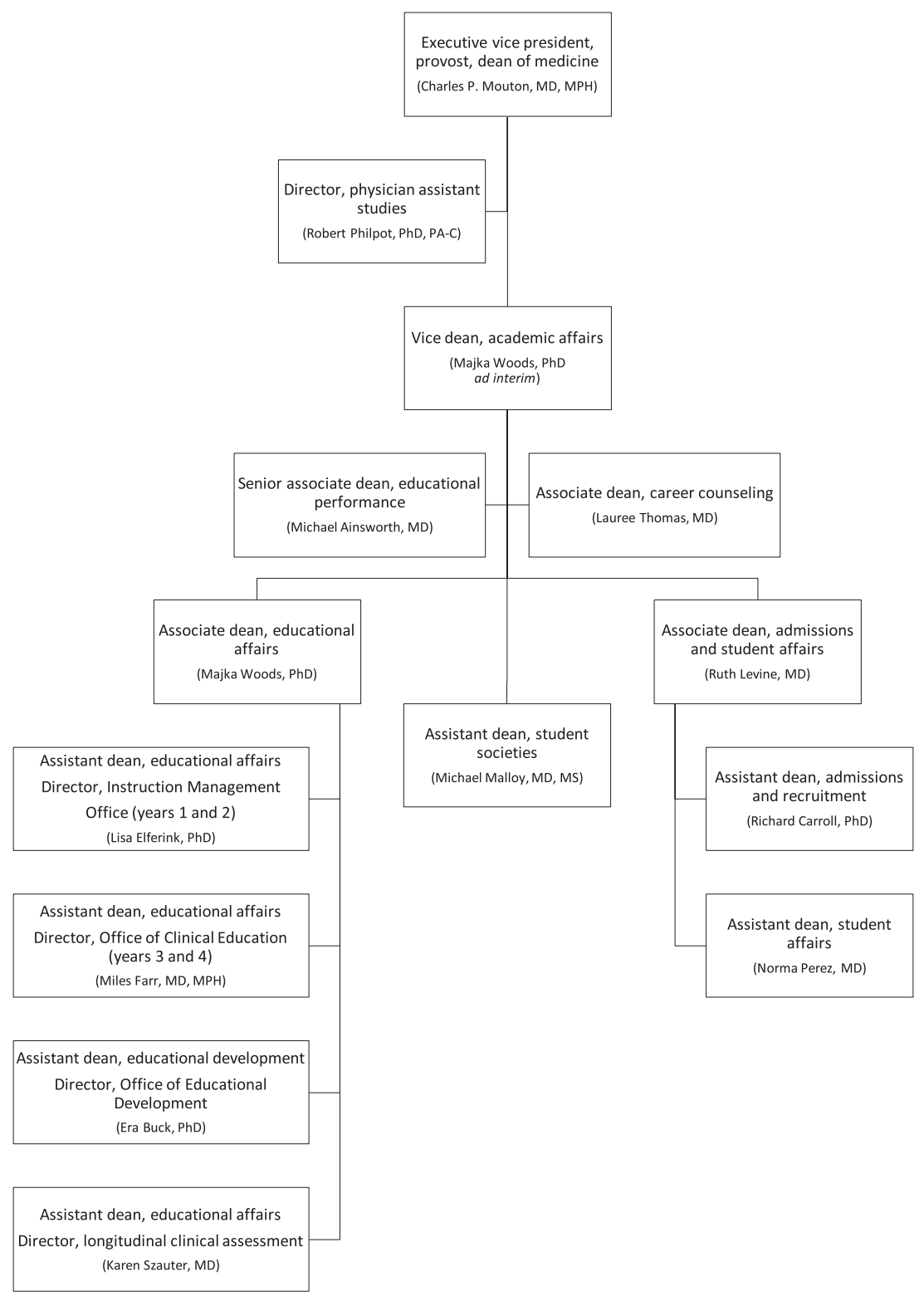

Figure 1 Organizational chart. 


\section{Faculty Development and Support in Education}

\section{Professional development for faculty as educators}

Professional development is offered in a range of categories through the Academy of Master Teachers, Office of Educational Development, Office of Faculty Affairs, and other sources. These include basic and advanced teaching skills, including PBL and team-based learning; feedback and assessment of learners; mistreatment and professionalism; scholarly development of faculty through educational projects; and services for mentor and mentee career advancement in education including educator portfolio development for APT, educational symposia, grand rounds, journal club, and visiting speakers.

\section{Role of teaching in promotion and tenure}

Research, clinical, and educational accomplishments and productivity are all considered important missions in the academic community. Appointment or promotion in the tenure track requires excellence in 2 missions (patient care, education, and/or research). Appointment or promotion in the nontenure track requires excellence in 1 of these missions. Consideration for promotion and/or tenure on the basis of education requires submission of an educational portfolio documenting the faculty member's accomplishments in teaching quantity and quality, educational scholarship, and leadership.

The Academy of Master Teachers is health institution wide in scope and function, incorporating members from the Schools of Medicine, Nursing, and Health Professions and the Graduate School of Biomedical Sciences. It is an interprofessional community of outstanding educators selected by their peers, serving the institution through promotion of educational excellence. The academy sponsors continuing professional development activities serving the needs of all teachers.

\section{Regional Medical Campuses}

The Houston campus is approximately 1 hour from our Galveston, with a Houston-based campus clerkship director
Table 1

Regional Medical Campuses

\begin{tabular}{|c|c|c|}
\hline Location & Type & Student enrollment \\
\hline Houston, Texas & Clinical & $\begin{array}{l}24 \text { for core clerkship year; } \\
\sim 230 \text { for individual clinical rotations }\end{array}$ \\
\hline $\begin{array}{l}\text { University of Texas Health } \\
\text { Science Center at Tyler }\end{array}$ & al al & $\begin{array}{l}12 \text { for core clerkship year; } \\
\sim 85 \text { for individual clinical rotations }\end{array}$ \\
\hline
\end{tabular}

and an onsite Houston campus coordinator. There are many clinical experiences shared by students at both the Galveston campus (main) and Houston campus. The assistant dean of clinical education participates in site visits throughout the year.

The University of Texas Health Science Center (UT-HSC) at Tyler regional campus is supported through direct UTMB funding for administrative and clinical education of students. UT-HSC Tyler has local clerkship directors for every specialty, and UTMB provides funding support for an undergraduate medical education coordinator. We have videoconferencing capability to ensure Tyler students participate in key group learning activities for each clerkship. The assistant dean for clinical education makes several site visits each year to the Tyler campus, and Tyler clerkship directors and coordinators participate in monthly clerkship directors' meetings via conference call.

See Table 1-Regional Medical Campuses.

As part of the annual clerkship review process, a close review of regional campus equivalency is performed including review of shelf exam performance, distribution of grades, and student survey responses for key performance metrics separated out by campus location. Educational Affairs monitors student survey results throughout the year to identify student concerns at a regional campus location. 


\title{
The University of Texas Rio Grande Valley School of Medicine
}

\author{
Andrew Neal Dentino, MD
}

\section{Medical Education Program Highlights}

The University of Texas Rio Grande Valley School of Medicine (UTRGV SOM) uses innovative, competency-based medical education to train the next generation of physicians who are committed to improving the lives and well-being of their communities through innovative research and compassionate, patient-centered care, as well as biomedical scientists who will lead the charge in advancing research on diseases such as diabetes and infectious diseases that affect the Rio Grande Valley (RGV) and beyond. ${ }^{1}$

The mission of the UTRGV SOM is to educate a diverse group of medical students and future biomedical scientists, to develop physicians who will serve across all disciplines of medicine, to bring hope to patients and communities by advancing biomedical knowledge through research, to integrate education and research that advances the quality and accessibility of health care, and to engage with the RGV communities to benefit Texas and the world. ${ }^{2}$

\section{Program objectives}

The UTRGV SOM is committed to providing a forwardthinking medical education experience that graduates physicians dedicated to practicing scientific, evidence-based, and patient-centered medicine in any setting but particularly in underserved communities. The curriculum provides a unique educational experience that integrates basic science disciplines, clinical skills, and professional development throughout the 4-year program by incorporating and promoting:

- Principles of evidence-based practice and scientific research

- Community and population-based health care

- Patient advocacy and cultural awareness

- Interprofessional collaboration and communication

- Ethical and professional behavior

- Lifelong learning and problem-solving

The school's distinctive geographic location at the border of the United States and Mexico offers a rich bicultural experience that will afford students the opportunity to experience and to learn about border medicine and the interplay between the crosscultural, socio-economic, and environmental determinants of health. ${ }^{3}$

\section{Acad Med. 2020;95:S517-S520. \\ doi: 10.1097/ACM.0000000000003396 \\ Copyright (C) 2020 by the Association of American Medical Colleges}

Supplemental digital content for this article is available at http://links.Iww.com/ ACADMED/A901.

Year school was established: 2015.

School URL: https://www.utrgv.edu/school-of-medicine/index.htm.

\section{Curriculum integration}

Woven throughout this 4-year integrated curriculum are:

- Interprofessional education: Students are given the opportunity to experience interprofessional education and team-based care by working and learning alongside an array of disciplines that include nursing, pharmacy, physician assistants, public health, social work, behavioral health, and occupational therapy.

- Early clinical exposure: Each student is assigned to a preceptorship where they are given the opportunity for direct patient interaction during the preclerkship years.

- Underserved medicine: Students are given the opportunity to work in underserved communities, Colonias, and at the student-run free clinic, to learn about health care disparities and population health. ${ }^{3}$

In concert with the strategic plan of our institution, highlights of our educational program include the theme of interprofessional education throughout the curriculum; our utilization of problem-based learning in the preclinical years; our many and varied clinical sites along the border communities of the RGV; and our dual-degree programs: $\mathrm{MD}-\mathrm{MS}$ in Bioethics, MD-MS in Biomedical Informatics, and MD-MPH.

\section{Dual-degree programs}

Bioethics: Students who enroll in the UTRGV MD-MS in Bioethics program will gain advanced learning and develop skills in bioethics and social justice to:

- Address complex bioethical issues of health disparities in multicultural populations

- Transform challenges into opportunities of change that promote health equity

- Shape clinical practice, research, and policy to advance equitable delivery of health care

The program focuses on bioethics, social justice, and health equity, allowing graduates to cultivate specific skills to create opportunities and manage resources effectively while remaining responsive to the diverse challenges within complex health care, social, political, and economic climates.

Biomedical informatics: The UTRGV SOM along with the UTHealth Houston School of Public Health and the School of Biomedical Informatics are offering 2 dual-degree programs. Starting with the class of 2022, medical students can apply to these programs through the School of Public Health and the School of Biomedical Informatics if they are in good academic standing at the School of Medicine.

Students who enroll in the MD-MS in Biomedical Informatics program will learn how to decipher large amounts of data related to patients to: 
- Make the best clinical decisions regarding patient care, including patient safety

- Inform the development of public health and clinical research initiatives

A biomedical informatics degree is useful in clinical and research settings because it allows health care professionals and researchers to take a large amount of data, information, and knowledge to make inferences regarding medical treatment, drug development, and clinical trials. With biomedical informatics, health care providers can develop protocols to improve care such as reducing medication errors or hospital readmissions and improve patient safety. At the population health level, biomedical informatics can help spot trends and assess the burdens of disease, including financial and economic burdens, to make decisions about interventions.

Public health: Student enrolled in the MD-MPH program will learn and develop skills in the core public health disciplines of:

- Biostatistics

- Environmental and occupational health

- Epidemiology and disease control

- Health promotion and behavioral sciences

- Management, policy, and community health

UTRGV SOM students will be able to complete the MD and MPH programs in 4 years because of shared credits between the 2 institutions. ${ }^{4}$

\section{Curriculum}

\section{Curriculum description}

See Supplemental Digital Appendix 1-Curriculum Overviewat http://links.lww.com/ACADMED/A901.

\section{Curriculum changes since 2010}

The UTRGV SOM was originally developed in 2015-2016 to accept its inaugural class, which will graduate in 2020. The launch of the school occurred with multiple educational, clinical, and community-based stakeholders to advance the school's mission of interprofessional education, care for the underserved, and border health. Since that time the Central Curricular Authority Committee (CCAC) has advanced with multiple subcommittees spanning the preclinical and clinical years, and to include evaluation, assessment, and quality improvement. In preparation for our first graduating class, the Offices of Educational Affairs (OEA), Student Affairs (OSA), and Faculty Affairs (OFA), and multiple faculty committees such as the Optimum Learning Environment Committee and SOM Faculty Assembly have all worked together to refine and strengthen the curriculum to its present model.

\section{Assessment}

See Supplemental Digital Appendix 2-Program Objectives and Competencies—at http://links.lww.com/ACADMED/A901.

Medical education program objectives are described in outcomebased terms that allow the assessment of medical students' progress in developing the competencies that the profession and the public expect of a physician (2020-2021 LCME Element Standard 6.1 or 2020-2021 CACMS Standard 6.1).

Our medical education program objectives follow ACGME rubrics for the domains of evaluation of competence for our students in our curriculum.

In the preclerkship years, end-of-module exams were changed to be NBME examinations for all modules except for the final exam for the Medicine, Behavior, and Society course, which is administered via ExamSoft with questions developed by the teaching faculty for the module. In the clerkship years, the clinical performance assessment has replaced the clinical student assessment to better reflect students' overall performance in the areas of medical knowledge, clinical skills, and altruism.

The following methods of assessment are used:

- Clinical document review

- Clinical performance ratings/objective structured clinical examination

- Exam, SOM developed: written/competency-based

- Exam, licensure: written/competency-based

- Exam, SOM developed: oral

- Exam, NBME: norm/standardized, subject

- Exam, SOM developed: clinical performance

- Exam, licensure: clinical performance

- Multisource assessment

- Narrative assessment

- Oral patient presentation

- Participation

- Peer assessment

- Portfolio-based assessment

- Practical (lab)

- Research or project assessment

- Self-assessment

- Stimulated recall

\section{Curricular Governance}

The OEA and the CCAC are responsible for the planning, implementation, and oversight of the curriculum. OEA has the following administrative and academic support:

- Vice dean for academic affairs

- Associate dean for educational affairs

- Assistant dean for educational affairs, preclerkship

- Assistant dean for educational affairs, clerkship

- Program specialist

- Administrative associate

- Education development specialist

- Systems analysts

- Clerkship academic coordinators

- Preclerkship course coordinators

- Instructional development designer

- Medical simulation specialist

- Standardize patient educator 


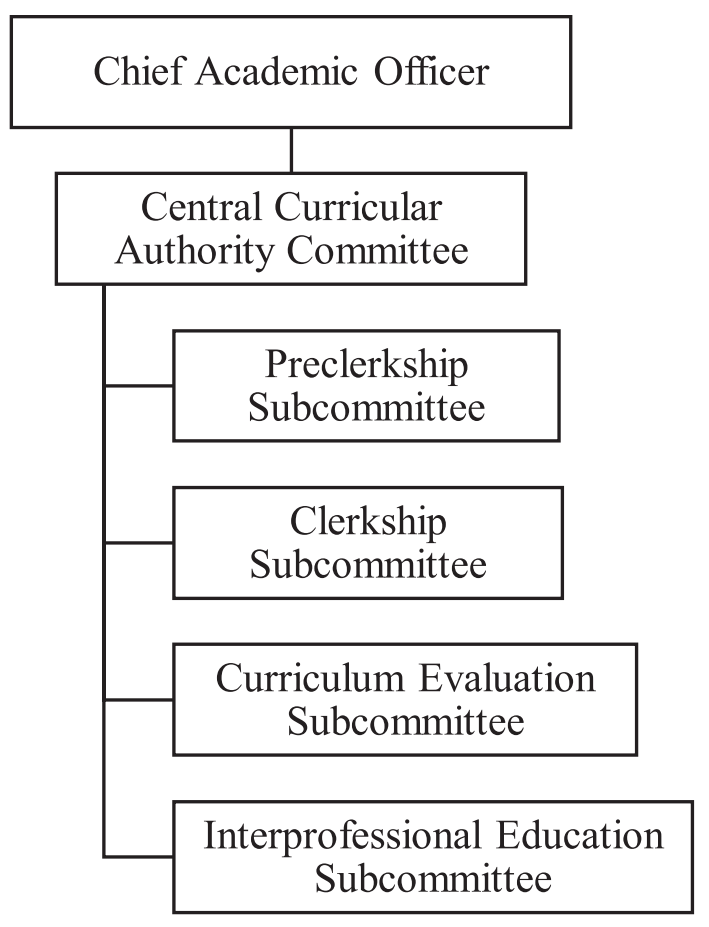

Figure 1 Curricular governance committees.
CCAC is comprised of membership from the preclerkship and clerkship faculty, teaching faculty representatives, and faculty members at large. CCAC has 4 subcommittees: Preclerkship Subcommittee, Clerkship Subcommittee, Curriculum Evaluation Subcommittee, and Interprofessional Education Subcommittee, with membership made up of faculty and assistant/associate deans who directly recommend and support the curriculum.

See Figure 1-Curricular governance committees.

The Office of Assessment, Evaluation, and Quality Improvement (OAE\&QI) is responsible for the development and maintenance of tools to support curriculum delivery, monitoring, and management. OAE\&QI has the following administrative support:

- Associate dean for evaluation and quality

- Assistant dean for assessment, evaluation, and quality improvement

- Continuous quality improvement manager

- Systems analyst

- Testing coordinator and assessment

OEA, CCAC, and OAE\&QI work together to collaboratively manage and evaluate the curriculum on an ongoing basis.

See Figure 2-Organizational chart.

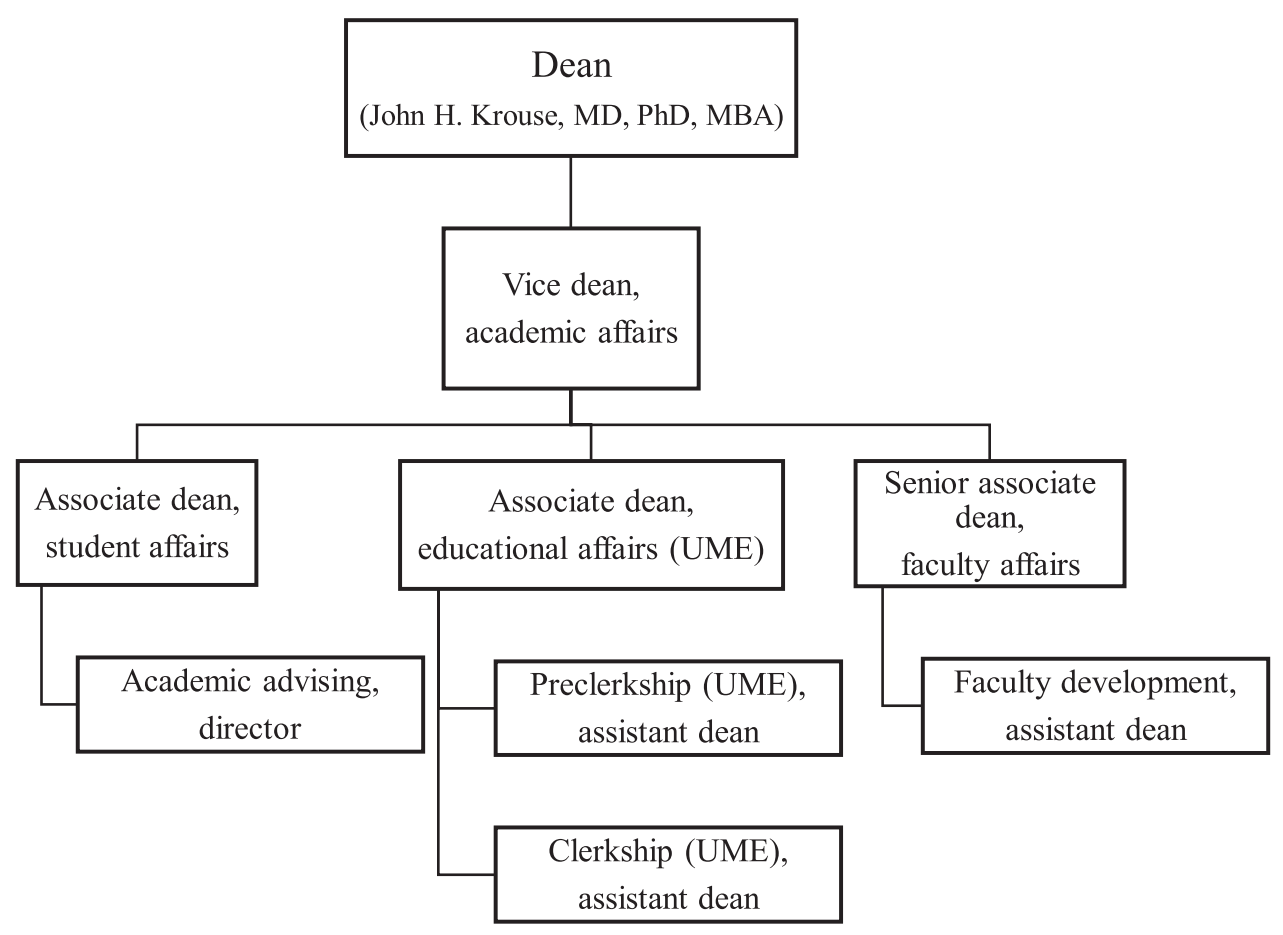

Figure 2 Organizational chart. 
Faculty Educator Series

\begin{tabular}{|l|l|}
\hline Thursday, September 5, 2019 & IAMSE - The Current Landscape of Faculty Development \\
\hline Challenges and Opportunities
\end{tabular}

Figure 3 Faculty educator series.

\section{Education Staff}

The associate dean for educational affairs is responsible for the oversight of the UME curriculum in collaboration with the assistant deans for educational affairs (preclerkship and clerkship). Together they work with staff and faculty in each phase of the curriculum to implement the CCAC-approved curriculum. OEA is responsible for UME education only, but collaborates with areas of the continuum. OEA is not responsible for areas outside of the educational programming.

\section{Faculty Development and Support in Education}

The Office of Faculty Affairs has developed a robust faculty development curriculum for faculty educators. The Faculty
Educator Series was formulated by the UTRGV SOM annual needs assessment and standpoint survey information. We have started a hashtag \#FacultyFriday, which is intended to remind faculty of the faculty educator series planned every first Friday of the month. All sessions are recorded for asynchronous viewing and available on the faculty affairs website via blackboard.

See Figure 3-Faculty educator series.

Our promotion and tenure guidelines each have 3 tracks or pathways: education, research, and clinical service. For tenure track, the criteria require excellence in 2 or more pathways depending on their faculty effort. The standard is demonstration of the ability to secure national funding equivalent to an $\mathrm{NIH} \mathrm{RO1} \mathrm{in} \mathrm{their} \mathrm{area} \mathrm{of} \mathrm{expertise.} \mathrm{For} \mathrm{nontenure} \mathrm{track,}$ demonstration of excellence in education pertinent to the specific type of students or trainees whom the faculty member teaches is required. At present, we do not have clinical educator portfolios but expect to develop educational portfolios for all 3 pathways.

Presently, we do not have an institute for educators. The Faculty Educator Series is open to all health professions schools. We plan to use the Faculty Educator Series program to develop a Master Teacher Certificate in AY 2021 (15 credit hours). Our office works closely with the UTRGV Division of Health Affairs. We communicate our faculty development programs to the assistant vice president for faculty affairs, Division of Health Affairs. The other schools/colleges in the division include Health Professions, Social Work, and Nursing. Schools under development are Podiatry and Pharmacy, with founding deans named for both programs. We will also kick off our faculty mentoring program in mid-January 2020, which has been developed in partnership with AAMC staff.

\section{References}

1 The Strategic Plan for The University of Texas Rio Grande Valley. Edinburg, Texas: The University of Texas Rio Grande Valley School of Medicine; 2017.

2 The University of Texas Rio Grande Valley. Undergraduate Medical Education. Office of Educational Affairs. https://www.utrgv.edu/schoolof-medicine/education/educational-affairs/index.htm. Published January 2020. Accessed March 2, 2020

3 The University of Texas Rio Grande Valley. MD Program. Program Objectives. https://www.utrgv.edu/school-of-medicine/education/ educational-affairs/md-degree-program/index.htm. Published January 2020. Accessed March 2, 2020

4 The University of Texas Rio Grande Valley. Office of Admissions. School of Medicine Programs. https://www.utrgv.edu/som/admissions/somprograms. Published January 2020. Accessed March 2, 2020. 


\section{University of Utah School of Medicine}

Sara M. Lamb, MD, and Wayne Samuelson, MD

\section{Medical Education Program Highlights}

The University of Utah School of Medicine (UUSOM) is the only medical school in Utah affiliated with an academic medical center (University of Utah Health) and is a state-funded, 4-year program. In addition to the MD degree, students can pursue 1 of multiple combined degree opportunities (MD-PhD, MD-MBA, MD-MSPH, MD-MPH, MD-MS of Biomedical Engineering). UUSOM's clinical learning experiences are concentrated within 4 major affiliated teaching hospitals, affording a diversity of exposure to a variety of health systems within the intermountain west.

Unique features of the program include:

- A longitudinal learning community model for teaching clinical skills and medical decision-making (CMC). This engages faculty as coaches and master clinician-observers and augments students' growth and mastery of the Core Entrustable Professional Activities (Core EPAs).

- Focused effort to support the professional identity, leadership, and career development of students. The Real MD (Relationships Excellence Authenticity Leadership) program is a key extracurricular initiative emphasizing the student to physician transformation.

- The core educator model, created in 2017, has invigorated teaching faculty and enhances recognition and support for the growth and development of faculty as educators. The model focuses on the development and support of approximately 190 foundational science and clinical faculty who interface with students in the delivery of the core curriculum.

- Pathways of Excellence offer students additional training in areas of distinction for the University of Utah Health. Students can engage in longitudinal, 4-year elective programs of study to obtain expertise in precision medicine, population health, value-driven health care, students as teachers, wellness and health promotion, or global medicine.

- UUSOM is 1 of 4 schools implementing a competency-based, time-variable parallel track for a small cohort of students pursuing a career in pediatrics (Education in Pediatrics Across the Continuum [EPAC]).

\section{Curriculum}

\section{Curriculum description}

See Supplemental Digital Appendix 1-Core Curriculum Map_at http://links.lww.com/ACADMED/A904.

Acad Med. 2020;95:S521-S524.

doi: 10.1097/ACM.0000000000003391

Copyright (C) 2020 by the Association of American Medical Colleges

Supplemental digital content for this article is available at http://links.Iww.com/ ACADMED/A904.

Year school was established: 1905.

School URL: https://medicine.utah.edu.
See Supplemental Digital Appendix 2-Core Curriculum With EPAC Parallel Track-at http://links.lww.com/ ACADMED/A904.

\section{Curriculum changes since 2010}

A number of modifications have been implemented since 2010:

- Implementation of a longitudinal learning community model to teach clinical skills and medical decision-making, integrating clinical coaches and master clinician-observers

- Major revision to the longitudinal curriculum addressing the medical humanities, ethics, and complexities of a changing health care system; and how culture, race, gender, economic factors, and access to care impact patient outcomes

- Implementation of a competency-based, time-variable parallel curricular offering for students pursuing pediatrics

- Required experiences in the final year of the curriculum to increase students' preparedness for internship and mastery of Core EPAs (advanced internal medicine, critical care, and a required core subinternship).

- Pathways of Excellence offerings (4-year programs of study in precision medicine, population health, value-driven health care, students as teachers, wellness and health promotion, or global medicine) for students to achieve academic excellence beyond the core curriculum

- Core educator initiative, a major modification to funds flow supporting medical student, including significant resource allocation to support programming for faculty professional development, scholarship, and education research

- Strategic planning efforts beginning in 2018 to achieve the "Exceptional Learning Experience," using a framework for organizational excellence and systems thinking to optimize the value, quality, and experience of faculty and students at UUSOM

The UUSOM class size increased from 82 to 102 students in 2012. In 2015, the class size further expanded to 125 as the leadership of the school was able to successfully secure increases in state funding allocation to support the effort. Modest incremental changes to scheduling in the clinical learning environment and modifications to gross anatomy and clinical skills simulation spaces were planned for and implemented to accommodate the class size change.

\section{Assessment}

Medical education program objectives are based on a number of frameworks including the ACGME competencies, AAMC Core EPAs, and Physician Competency Reference Set.

See Supplemental Digital Appendix 3-Program Objectives and Assessment Methods-at http://links.lww.com/ ACADMED/A904. 
Significant changes to student assessment since 2010 include the following:

- Increased emphasis on noncognitive/attitudinal behaviors with narrative assessment through all 4 years of the program

- Implementation of peer feedback/ratings in the setting of team-based learning (TBL) and case-based learning (CBL)

- Clinical curriculum assessment alignment with Core EPAs

- Pilot of competency-based strategy for supporting the mastery of foundational science knowledge in the preclerkship foundational sciences courses (Knowledge for Practice Assessment of Competence Committee)

\section{Parallel curriculum or tracks}

The UUSOM implemented the EPAC pilot in 2013. As 1 of 4 medical schools collaborating with the AAMC, UUSOM's program has been successful in accomplishing time-variable, competencybased advancement from medical school through residency to fellowship/professional practice. UUSOM's students are assessed to a greater extent on the 13 Core EPAs than the traditional students. Once students complete the medical school graduation requirements and show competency in all EPAs, they transition to the pediatric residency program at the University of Utah.

\section{Pedagogy}

A mix of pedagogical approaches are used in the core curriculum, including significant use of active learning methods such as CBL, TBL, interactive labs, small-group discussion, clinical experiences (both ambulatory and inpatient), simulation (high and low fidelity), role play/dramatization, and standardized patient experiences. Learning communities are an important structural characteristic, serving as the foundation for the longitudinal clinical skills program. Self-directed tutorials are used in a number of courses across the 4-year program as is peer teaching. Pure large classroom didactic lectures are becoming much less prevalent in the program.

The parallel track for EPAC requires all EPAC students to have the same experiences as their counterparts in the core curriculum; however, EPAC has an increased amount of clinical experiences, primarily ambulatory, in the form of a pediatric preceptorship as well as a number of workshops and an increased volume of assessment of the Core EPAs.

Significant shifts in the implementation of active learning methods have been underway since 2010 with increased emphasis on consistent use of CBL across the preclerkship phase and the implementation of TBL in 2017. A small percentage of the program is delivered using traditional, didactic, lecture-based methods. The launch of Layers of Medicine and CMC have increased the use of small- and large-group discussion methods, peer teaching, role play/dramatization, and inpatient and ambulatory clinical experiences.

\section{Clinical experiences}

- Clinical sites used in the required clinical curriculum include academic hospitals and clinics, community-based hospitals, and clinics including a number of rural sites (both inpatient and ambulatory) throughout Utah and Idaho. Across the 7 core clinical clerkships, there are 12 different inpatient sites and 9 types of ambulatory teaching sites that are used (including community health centers, rural clinics/Area Health Education Centers, and Veterans Affairs sites).

- The school does not use longitudinal integrated clerkships.

- Learners are introduced to the clinical method beginning the first week of the curriculum and encounter patients in the clinical environment midway through the first year of the program in ambulatory and inpatient settings.

- Students have a required 6-week family medicine clerkship (100\% ambulatory), which is largely set in communitybased clinics. Several sites are located in remote parts of Utah and Idaho. The surgery clerkship preceptorship (2 weeks) additionally places students in community-based clinics including rural sites in Utah. A small number of students complete a portion of their obstetrics-gynecology clerkship in rural Utah community clinics and hospitals.

- The VA system is an important training site for students during the internal medicine, surgery, and neurology clerkships for both inpatient and ambulatory experiences.

- Faculty training to ensure consistent and fair summative assessment proposes a notable challenge given the large number of faculty we engage in the core clinical curriculum. While our students provide us with data indicating that they receive high-quality clinical educational experiences, we continue to have reports of medical student mistreatment, which is counter to our values as an institution. We continue to work to identify opportunities and strategies to address the mistreatment of medical students in the learning environment.

\section{Curricular Governance}

There are no elements of the curricular governance that are decentralized or managed at the department level.

See Figure 1-Curricular governance chart.

\section{Education Staff}

The administrative and academic support for the planning, implementation, evaluation, and oversight of the curriculum is through the Office of Curriculum, which is overseen by the associate dean of curriculum. There are 3 assistant deans who direct the foundational sciences curriculum, clinical curriculum, and operations for curriculum evaluation and quality improvement, respectively. A staff director of curriculum and faculty support assists the Office of Curriculum leadership and teaching faculty in accomplishing the missions of the education program as well as supporting the Curriculum Committee (CC). The Office of Curriculum is responsible for identifying and maintaining the tools that support the delivery, monitoring, and managing the curriculum. The Education Technology Subcommittee of the CC also investigates and proposes solutions to curricular technology needs with membership including faculty and staff from information technology, library sciences, and University of Utah main campus education technology, as well as students. 


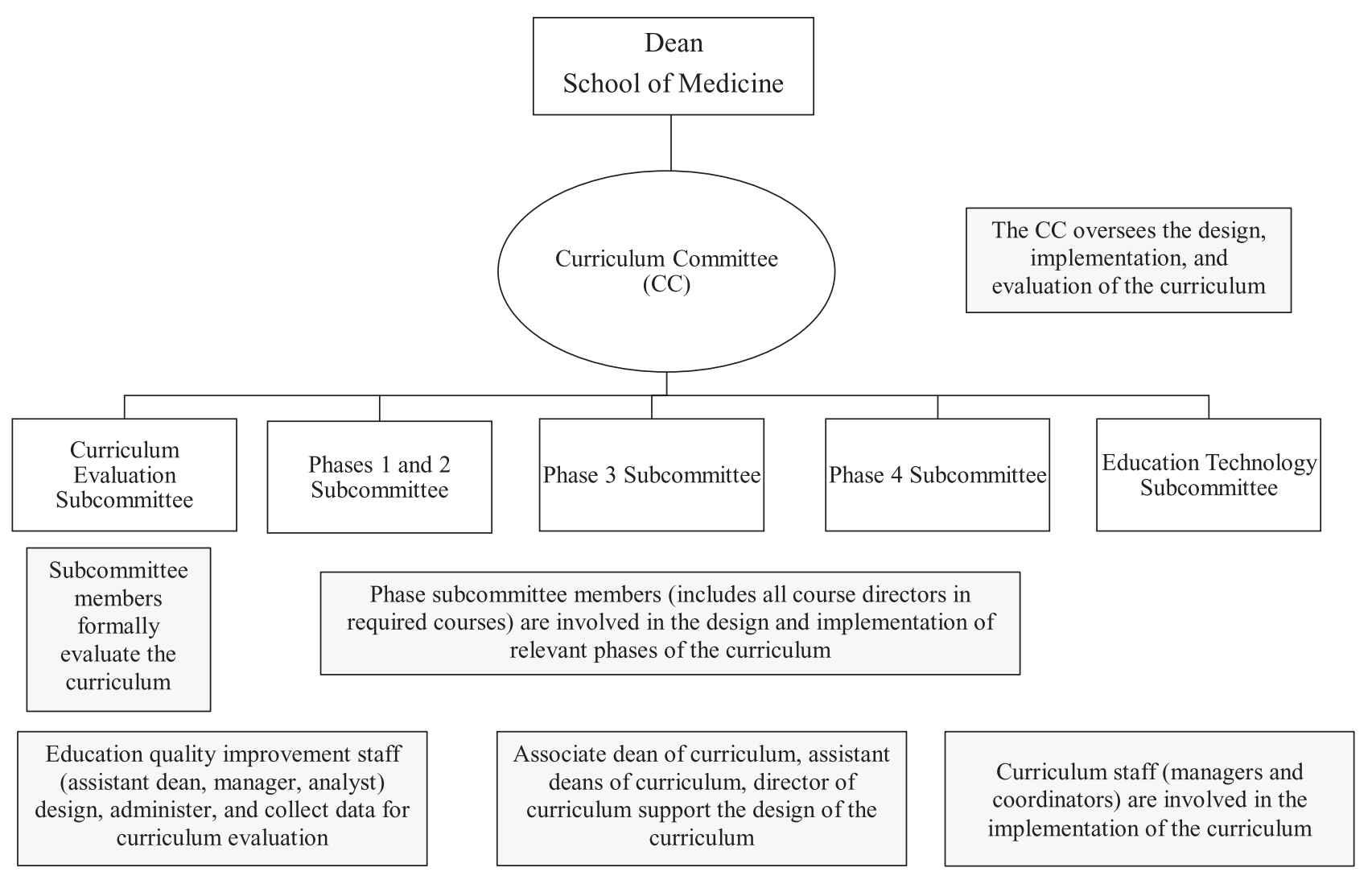

Figure 1 Curricular governance chart.

The medical education staff and administrative faculty ensure the implementation and continual improvement of the medical student program. The administrative leads in admissions, student affairs, health equity and inclusion, curriculum (includes the Office of Education Quality Improvement), continuing medical education, and finance work closely to ensure the delivery of a high-quality program. Faculty development explicit to the needs of the medical student program is directed by the offices of curriculum, student affairs, and admissions. The associate dean for GME closely collaborates with the Dean's Office administrative faculty and staff. However, GME reports to the University of Utah Health Administration. The UUSOM does not have an academic department of medical education.

See Figure 2-Dean's office organizational chart.

\section{Faculty Development and Support in Education}

Professional development for education faculty is central to the mission of the Office of Curriculum. The core educators primarily deliver the curriculum and engage in regular professional development through retreats, skills-specific trainings, workshops, journal clubs, etc. These experiences help build a community of educators and prepare faculty for their roles in the program and to take a scholarly approach to education. The Offices of Student Affairs and Admissions conduct regular trainings for faculty who serve key roles in their operations, such as advisors, mentors, academic coaches, interviewers, and selection committee members, respectively.

In promotion and tenure decisions, education excellence criteria have been developed and are used by the departments and committees responsible for the faculty appointment, review, and advancement procedures. The criteria for educational scholarship and education effectiveness and excellence were recently revised with broad input from key education administrators, faculty, and leadership at the school.

In addition to the professional development programming that has heretofore been described, the UUSOM has an associate dean for faculty development/associate vice president for health sciences education. This individual directs faculty development efforts for the health sciences colleges' faculty, which includes oversight of the Academy of Health Sciences Educators (AHSE). The AHSE includes faculty from all 5 health sciences colleges at the University of Utah (nursing, pharmacy, dentistry, health, medicine).

\section{Initiatives in Progress}

The UUSOM is reaching a final phase of planning a new medical education building. The development of a Pathway of 


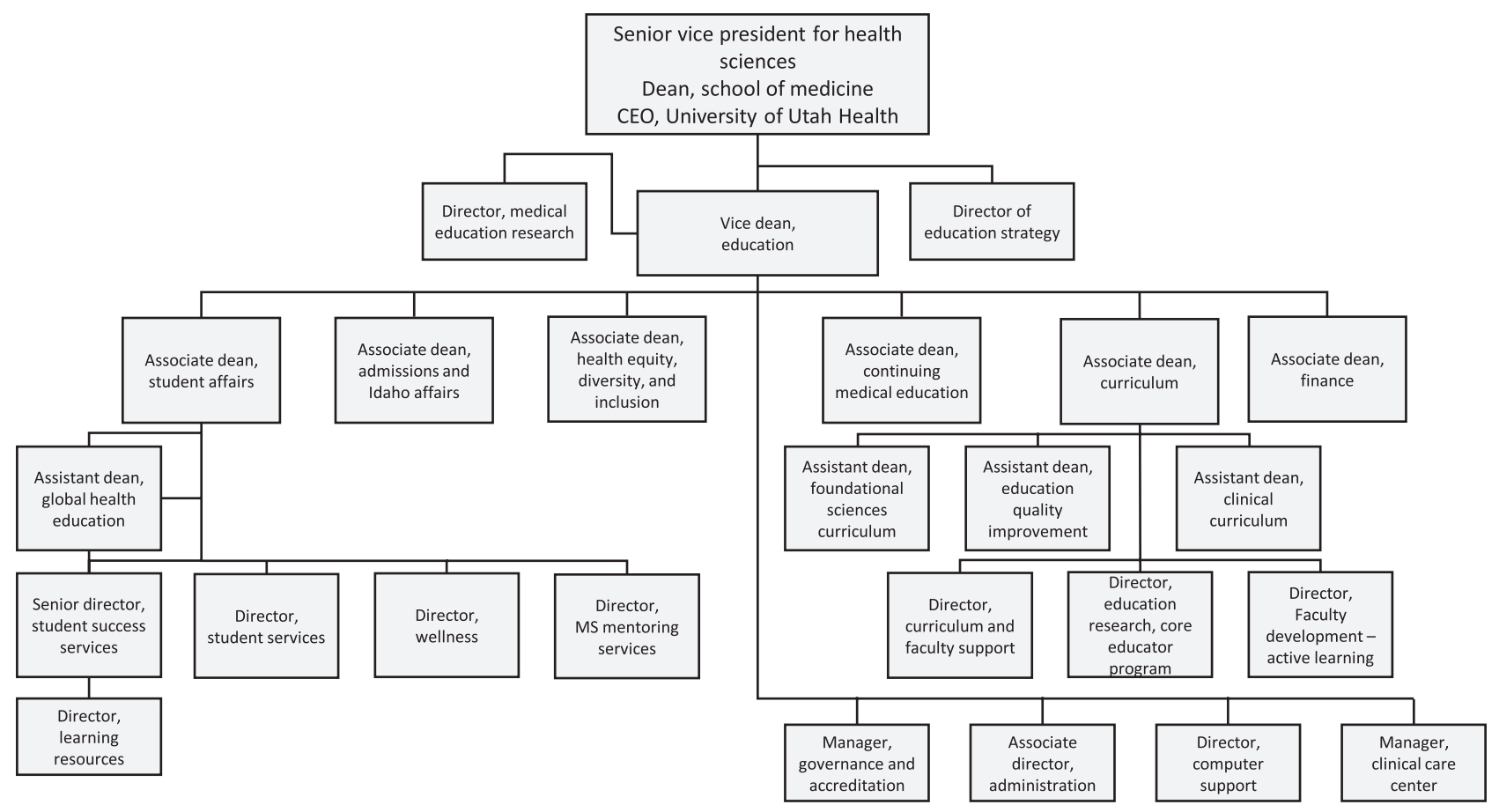

Figure 2 Dean's office organizational chart.

Excellence and a graduate certificate in Rural and Underserved Health are being planned for the coming 1-2 years as part of the Rural and Underserved Utah Training Experience (RUUTE). With significant support from the Utah state legislature, the RUUTE program aims to enhance the training, health care access, and long-term socio-economic benefit for rural and underserved communities of Utah by expanding interest, awareness, and placement of students and physicians. Finally, strategic efforts to redesign the education program and achieve the "Exceptional Learning Experience" with a focus on relationships, community, diversity, professionalism, excellence, learning and continuous improvement, partnerships with students, and student centeredness are mapped out for the upcoming 3 years. 


\title{
The University of Vermont Larner College of Medicine
}

\author{
Christa Zehle, MD, Kathryn Huggett, PhD, Jesse Moore, MD, and Cate Nicholas, EdD, MS, PA
}

\section{Medical Education Program Highlights}

- Larner College of Medicine (LCOM) is a 4-year academic curriculum, the goals of which are to educate a diverse group of dedicated physicians to serve across all disciplines of medicine, be respected for innovative and outstanding teaching, advance medical knowledge through research, integrate education and research to advance the quality and accessibility of patient care, and engage with our communities to benefit Vermont and the world.

- LCOM is recognized for our inclusive approach and evidencebased innovation in medical education.

- Our location on the same campus as the main University and adjacent to a medical center affords tremendous opportunity for collaborative academic programming and research between colleges and disciplines.

- The Vermont Integrated Curriculum (VIC) offers students early access to broad clinical experience, firm grounding in the basic sciences, and opportunities to conduct research and engage in community service.

- Active learning has been shown to provide students superior benefits compared with lecture. We recognize the importance of this newer teaching/learning pedagogy for retention of knowledge, engagement, and collaboration among learners and educators. We strive to deliver $100 \%$ of required teaching sessions with active learning. To effectively do so, Medical Education, Information Technology, and the Teaching Academy collaborated on developing the Larner Learning Commons, a laboratory for teaching designed to support leading-edge medical education.

- Faculty development is integral to the success of our transition to active learning. In 2014, the college established the Teaching Academy, providing resources to support all faculty to become better educators and improve the efficiency and quality of medical education. The Teaching Academy fosters a community of educators that leads educational innovation and scholarship by promoting faculty members' pursuits of education excellence and scholarship.

\section{Curriculum}

\section{Curriculum description}

The educational program comprises 3 levels:

- Level 1: development of fundamental science knowledge in a clinically relevant context and acquisition of clinical skills

Acad Med. 2020;95:S525-S529.

doi: 10.1097/ACM.0000000000003442

Copyright (C) 2020 by the Association of American Medical Colleges

Supplemental digital content for this article is available at http://links.lww.com/ ACADMED/A935.

Year school was established: 1822.

School URL: med.uvm.edu.
- Level 2: core clerkships emphasizing basic principles and practices of clinical medicine

- Level 3: additional opportunities for application of medical knowledge with increased student responsibilities in caring for patients

See Supplemental Digital Appendix 1-Curriculum Diagramat http://links.lww.com/ACADMED/A935.

\section{Curriculum changes since 2010}

- Enacted commitment to active learning

- Updated selection of longitudinal topics: social determinants of health, evidence-based medicine, health systems science, point-of-care ultrasound (POCUS), interprofessional education (IPE), and opioid epidemic management

- Established a branch campus in Connecticut that hosts our global health program, offering global health experiences in countries including Uganda, Russia, Vietnam, and Zimbabwe

- Development of a Longitudinal Integrated Clerkship (LIC) at 2 sites in rural Vermont and New York. LIC students are embedded in family practice offices where they develop their own panels of patients and have "burst" weeks of intensive exposure to inpatient medicine

\section{Class size changes since 2010}

LCOM has made modest incremental changes in class size. Class size increased in 2016 from 115 matriculating students to 120 . The addition of 4 seats in 2019, with 2 seats reserved for students from Vermont, brought our class size to 124 with 30 seats for Vermonters.

\section{Assessment}

The medical education program objectives are based on the Accreditation Council for Graduate Medical Education domains of competence. LCOM has made the following assessment changes since 2010:

- Changed grading in the foundations level in 2016, from a pass/ fail/honors to a pass/fail system

- Completed inclusion of a clinical skills exam (CSE) in all clerkship courses

- Continued to explore use of CSEs at the conclusion of the medicine acting internship to provide a standardized assessment of knowledge, skills, and behaviors required of medical students in the inpatient setting, to add to inpatient observations

\section{Pedagogy}

- Classes are delivered in a variety of active learning modalities. Each modality is different, and expectations vary. Attendance is required for all active learning class sessions. 
- LCOM employs best practices in simulation-based education and assessment in the clinical skills curriculum, which is vertically and horizontally integrated in the VIC. Human (standardized/simulated patients) and nonhuman (manikins, task trainers, virtual reality) simulation modalities are integrated into courses and clerkships and linked to course/ clerkship objectives.

See Chart 1-Teaching Modalities.

The following pedagogical changes have been implemented since 2010:

- Enacted commitment to increase the amount of active learning in the curriculum, striving for $100 \%$ active learning activities

- Developed a prematriculation curriculum delivered online or in person with a goal of improving student success

\section{Clinical experiences}

- Vermont campus: The University of Vermont Health Network serves Vermont and northern New York through 6 hospital locations, large multispecialty outpatient clinics, and satellite facilities with more than a million patient visits each year. All clerkships are offered at the University of Vermont Medical Center.

- Saint Mary's Medical Center, located in West Palm Beach, Florida, offers a broad spectrum of medical care. Clerkship rotations include pediatrics, obstetrics-gynecology, and surgery.

- Connecticut campus: Students at the Connecticut campus complete clerkships at the Danbury and Norwalk locations. Danbury Hospital is a regional medical center and university teaching hospital. Norwalk Hospital is a community teaching hospital with a wide range of clinical programs. The patients served represent a remarkable cross section of economic, ethnic, racial, and linguistic backgrounds. All clerkships are offered at the Connecticut campus.

- LIC sites:

- Central Vermont Medical Center is a hub for regional health care delivery.

- Hudson Headwaters Health Network is a not-for-profit system of community health centers headquartered in Queensbury, New York. The primary care clinics are designated as Level 3 patient centered medical homes.

\section{Required longitudinal experiences}

- Professionalism, Communication, and Reflection (PCR) is a yearlong course that meets in small groups with a faculty preceptor once a week. Important themes of the course include professionalism, self-awareness, communication skills with peers and colleagues about difficult subjects, understanding culture and diversity in medicine, and social and economic factors affecting health.

- Second-year PCR groups partner with local community organizations to apply their leadership, professional, and team skills to a public health project, which results in a required poster presentation at LCOM and optional scholarship at national conferences. These projects begin to develop the background in population-based medicine and prevention a physician needs to fully address a range of health issues.

- Doctoring in Vermont (DIV) is a course that spans the first and second years of the foundations level. Students spend 8 sessions in a primary care office observing direct patient care and practicing interviewing and examination skills.

- The LCOM clinical skills curriculum is horizontally and vertically integrated in the VIC. The curriculum includes professionalism, communication, medical interviewing, physical exam, and clinical reasoning (presentation and

\section{Chart 1}

\section{Teaching Modalities}

\begin{tabular}{|c|c|c|c|c|c|c|}
\hline $\begin{array}{l}\text { Quick Overview of Active } \\
\text { Learning SOPs }\end{array}$ & Team-Based Learning & Case-Based Learning & Workshop & Problem-Based Learning & Integrative Review & eLearning Module \\
\hline $\begin{array}{l}\text { Independent Learning } \\
\text { (Before class) }\end{array}$ & Yes & Yes & Yes & No & Yes & N/A \\
\hline $\begin{array}{l}\text { Time estimate for Independent } \\
\text { Learning (Students) }\end{array}$ & $2-4$ hours to read and master & $1: 1$ ratio for class time, prior to class & $1: 1$ ratio for class time, prior to class & 1:1 ratio for class time, after class & 1:1 ratio for class time, prior to class & N/A \\
\hline Prep Materials Due & $\begin{array}{l}\text { Monday, the week prior to the } \\
\text { session }\end{array}$ & $\begin{array}{l}\text { Monday, the week prior to the } \\
\text { session }\end{array}$ & $\begin{array}{l}\text { Monday, the week prior to the } \\
\text { session }\end{array}$ & N/A & $\begin{array}{l}\text { Materials for review identified for } \\
\text { students a week in advance }\end{array}$ & N/A \\
\hline $\begin{array}{c}\text { \# of Objectives per preparatory } \\
\text { hour }\end{array}$ & $\begin{array}{l}\text { Aprox } 10 \text { for Independent Learning } \\
\text { and } 5 \text { for in-class }\end{array}$ & $\begin{array}{l}\text { Aprox } 10 \text { for Independent Learning } \\
\text { and } 5 \text { for in-class }\end{array}$ & $\begin{array}{l}\text { Aprox } 10 \text { for Independent Learning } \\
\text { and } 5 \text { for in-class }\end{array}$ & Students set objectives. & 5 in-class objectives covered & N/A \\
\hline Readiness Quizzes, IRAT, GRAT & Yes. IRAT \& GRAT in class & $\begin{array}{c}\text { Yes. 7-10 question RQ completed } \\
\text { before class. Annotated answers at } \\
\text { the close of the quiz. }\end{array}$ & $\begin{array}{c}\begin{array}{c}\text { Yes. 7-10 question RQ completed } \\
\text { before class. Annotated answers at } \\
\text { the close of the quiz. }\end{array} \\
\end{array}$ & None & None. & $\begin{array}{l}\text { Yes. An assessment typically } \\
\text { accomanies a module. }\end{array}$ \\
\hline In-class Activities & $\begin{array}{l}\text { Teams of students work on a } \\
\text { Significant problem that is not a } \\
\text { regeneration of the material tested } \\
\text { on the readiness tests }\end{array}$ & $\begin{array}{c}\text { Students solve a case or cases, with } \\
\text { guided questions to facilitate student } \\
\text { application of knowledge }\end{array}$ & $\begin{array}{c}\text { Students work through non-case- } \\
\text { based problems, challenges, puzzles, } \\
\text { or tasks to further the application of } \\
\text { knowledge }\end{array}$ & $\begin{array}{c}\text { Active discussion and analysis of the } \\
\text { problem, hypotheses generation, } \\
\text { investigation of knowledge gaps, and } \\
\text { peer teaching }\end{array}$ & $\begin{array}{c}\text { Students work through problems, } \\
\text { questions, or cases that are designed } \\
\text { to integrate content and concepts } \\
\text { from prior sessions }\end{array}$ & N/A \\
\hline $\begin{array}{l}\text { Answers Provided } \\
\text { After the Session }\end{array}$ & No & No & No & N/A & No & N/A \\
\hline
\end{tabular}


electronic health record), which are taught and assessed with the use of standardized/simulated patient methodology. Students are assessed in these domains with CSEs at the end of courses and clerkships. They are also required to pass an endof-level CSE between foundations and clerkship to proceed to clerkship, as well as to pass a 12-station CSE at the end of clerkship to graduate.

- The 4-year integrated LCOM ultrasound curriculum is a comprehensive longitudinal POCUS course. The curriculum begins in the first weeks of medical school and continues throughout all 4 years of medical training with the intent that students become competent in using POCUS in clinical patient care.

\section{Clinical experience first encounter}

- LCOM students are introduced to their first patient on the first day of orientation, where they learn the importance of patient communication skills.

- Additional clinical experiences occur during their first semester in the PCR course, which includes clinical history taking, spiritual care, and nurse shadowing.

- Clinical skills continue to develop in DIV, which begins in the second semester of the first year.

\section{Required and elective community-based rotations}

LCOM partners with outpatient clinics in Vermont, Connecticut, Florida, and upstate New York to deliver our clinical curriculum.

\section{Challenges in designing and implementing clinical experiences for medical students}

LCOM values diversity, equity, and inclusion and considers these factors when making decisions about clinical affiliations and curricular experiences. With increasing numbers of learners in the clinical environment, communication and collaboration among educators and clinical systems is necessary to ensure a high-quality clinical curriculum for our students.

\section{Curricular Governance}

The Medical Curriculum Committee is the institutional body that oversees the medical education program. This committee provides integrated institutional responsibility for overall design, management, integration, evaluation, and enhancement of a coherent and coordinated medical curriculum. The committee implements needed changes to the curriculum and is cognizant of national medical education priorities and requirements.

See Figure 1-Committee structure.

\section{Education Staff}

The Office of Medical Student Education (OMSE) supports all aspects of the medical education curriculum, consisting of multiple teams involving 2 clinical campuses and additional clinical affiliates:
- The teams within the OMSE comprise faculty and staff, including administrative, admissions, medical student services, preclinical curriculum, clinical curriculum, active learning, Teaching Academy, and clinical simulation lab.

- The Office of Diversity and Inclusion, Technology Services, and Dana Medical Library provide integral support for successful program delivery.

- Clinical simulation facilities and staff are on both Vermont and Connecticut clinical campuses.

OMSE supports high-quality educational programs and services for medical students and faculty development in educational methods and innovation. OMSE, which includes the senior associate dean for medical education, associate/assistant deans, and directors, is directly responsible for UME, continuing professional development, and academic integration across the University of Vermont Health Network.

\section{Faculty Development and Support in Education}

Professional development for faculty as educators is offered centrally through the Teaching Academy, which provides:

- Opportunities for professional development to all faculty in curricular design, program evaluation, learner assessment methods, instructional methodology, and educational scholarship, including educational research

- Programs open to all LCOM faculty including residents, fellows, and postdoctoral fellows

- Faculty development opportunities and webinars available to clinical affiliates and regional campuses by Internet connection, recordings, and remote viewing

- Faculty development activities, including medical education grand rounds and retreats, offered at multiple instructional sites (e.g., regional campus)

- Programs addressing educational skill development, mentoring, diversity and inclusion, IPE, leadership development, career development, academic program evaluation, and educational scholarship

- Programs including an annual intensive teaching course, annual medical education retreat, medical education grand rounds, and mentoring groups

- Access to national webinars and courses from organizations such as the AAMC, AMEE, International Association of Medical Science Educators, and Medical Education Research Certificate program

- Brief online videos on core teaching topics available at the Teaching Academy website

- Peer observation of teaching, a formative evaluation conducted by academy members

\section{Role of teaching in promotion and tenure}

- Teaching and education activities are expected of all faculty.

- Promotion and reappointment decisions include consideration of the 5 AAMC educator activities.

- Membership in the Teaching Academy is considered in promotions decisions. 


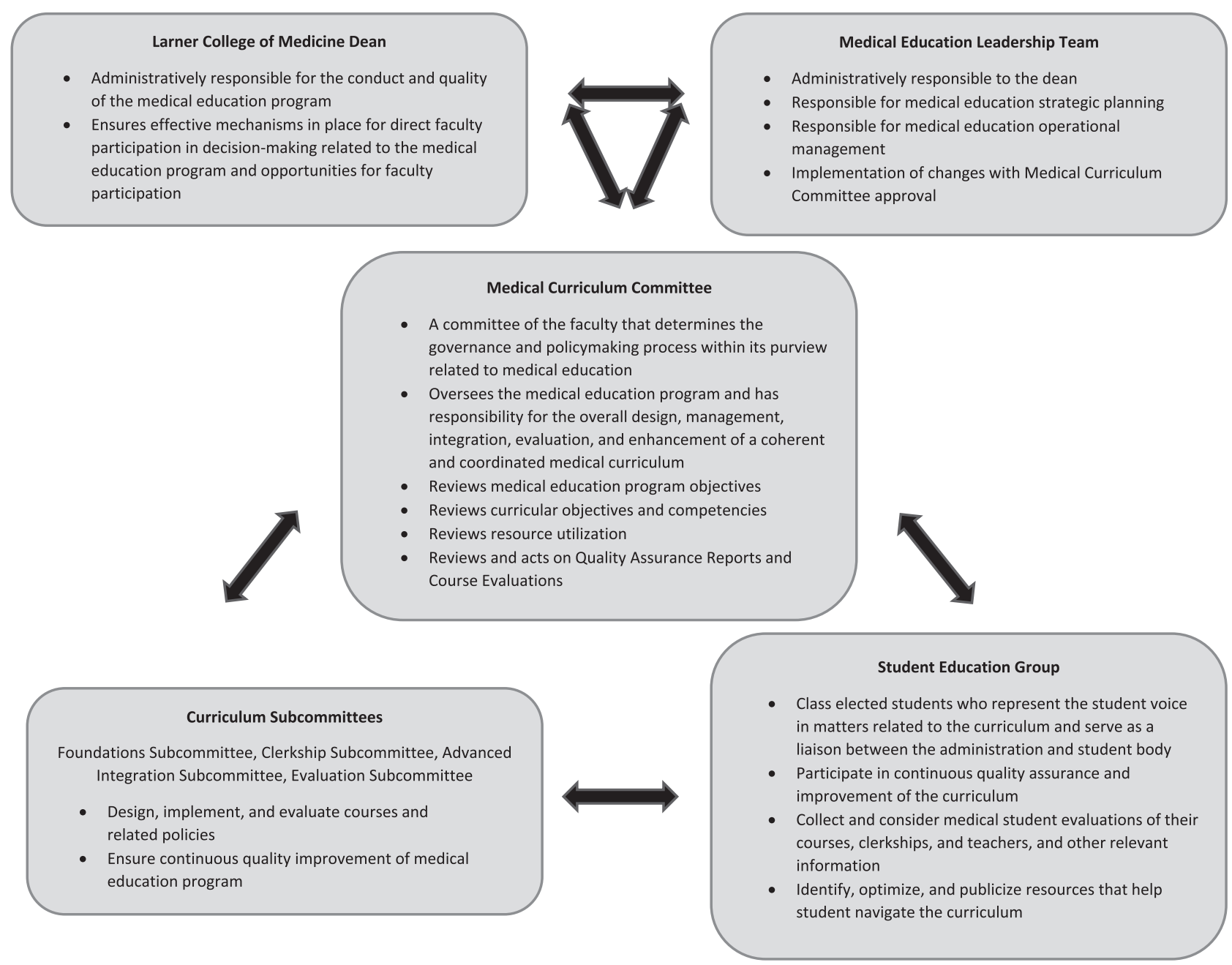

Figure 1 Committee structure.

- The Education Scholar Pathway is for individuals whose predominant effort is dedicated to the overall education of health professionals and individuals in the health sciences.

\section{Teaching Academy}

The LCOM Teaching Academy mission is to become a community of educators that leads educational innovation and scholarship by promoting faculty members' pursuit of education excellence and scholarship:

- Sustains and supports an interdisciplinary community of educators who value the scholarship of teaching and learning while facilitating educator development

- Improves efficiency and quality of medical education through collaboration and scholarship

- Promotes an academic environment that increases value and impact of educators locally, regionally, and nationally

- Provides recognition of excellence through peer-reviewed membership and awards for teaching and educational excellence
Scope of the Teaching Academy:

- Membership is open to all LCOM faculty across all instructional sites.

- The 4 tiers of membership are distinguished educator, master teacher, member, and protégé.

- Protégé membership is for residents, fellows, and postdoctoral students.

- Membership requires applicants to submit a portfolio for peer review.

\section{Regional Medical Campuses}

LCOM has a regional campus focusing on the clinical curriculum located in Connecticut, described above. Student enrollment includes 70 students (35 students/year) from levels 2 and 3.

\section{Educational experience across sites}

The LCOM regional campus in Connecticut has the same programmatic objectives and assessments as the Vermont campus and the LIC. 
- All clerkship courses, assessment activities, and quality improvement processes are managed centrally by OMSE and supported by technology services.

- Monthly clerkship meetings are held that include all course directors for required clinical courses; leadership from the Vermont, Connecticut, and LIC sites; and clinical support staff.
- Information is presented in the course quality assurance reports and reviewed and approved by the Medical Curriculum Committee.

Acknowledgments: The authors wish to thank members of the Medical Education Leadership Team, Medical Communications, and Office of Medical Student Education who provided input for this project. 


\section{Eastern Virginia Medical School}

Brooke Hooper, MD, Mily Kannarkat, MD, and Ronald Flenner, MD

\section{Medical Education Program Highlights}

The mission of Eastern Virginia Medical School (EVMS) is deeply rooted in the education and training of truly caring and competent physicians with a dedication to serving their community. Our curriculum is intentionally designed to be patient-centered, learner-focused, and community-oriented using an innovative and case-based approach.

Highlights include the following themes integrated throughout the 4-year curriculum:

- Health systems science: EVMS uses a virtual community of culturally diverse families to help students learn. Beginning in the first week of preclerkship training, students learn the importance of looking beyond symptoms and diagnoses to include family dynamics, health disparities, social determinants of health, access to community resources, and the complexities of care in specific patient populations. In addition to body donors, a "living donor" also meets with students on a regular basis during the first year. Students review the living donor's medical records, build a relationship, and learn about the patient and family experience. During clinical training, students are excused from clerkships 1 day per block to discuss the experience of various health systems science topics as applied and observed in the clinical setting as part of the CareForward Curriculum Day Series.

- Community-engaged learning: EVMS students participate in a community-engaged learning initiative for 4 years, developing a depth of expertise outside of the classroom in pathways such as first response, nutrition and exercise, underserved and health inequities, elderly and chronically ill, infectious disease, or global health equity.

- Student and physician wellness: Student and physician wellness is a guiding principle throughout the EVMS curriculum. Students participate in a longitudinal curriculum throughout their 4 years, intended to promote wellness, academic success, and professional development in a diverse and collaborative atmosphere. Students are excused from clerkships for 1 half day during each clerkship for personal wellness care and activities.

- Robust ultrasound curriculum: EVMS is proud to be one of a handful of medical schools nationwide to have a robust ultrasound curriculum, giving students an edge in the use of this powerful tool. Students begin using ultrasound equipment in the first week of preclerkship training and have access to handheld ultrasound scanners throughout their clinical years.

Acad Med. 2020;95:S530-S533.

doi: 10.1097/ACM.0000000000003393

Copyright (C) 2020 by the Association of American Medical Colleges

Supplemental digital content for this article is available at http://links.Iww.com/ ACADMED/A902.

Year school was established: 1973.

School URL: https://www.evms.edu.

\section{Curriculum}

\section{Curriculum description}

See Supplemental Digital Appendix 1-Curriculum Overviewat http://links.lww.com/ACADMED/A902.

\section{Curriculum changes since 2010}

In 2016, our CareForward Curriculum was implemented. The overarching goal is to enhance learning through integration and innovation; the core purpose is to educate caring, compassionate, skillful caregivers with the knowledge and understanding to competently navigate the health care system's complexities.

Preclerkship curriculum:

- Integration of basic sciences disciplines into an organ system framework

- Earlier emphasis of clinical reasoning to explicitly teach a hypothesis-driven history and physical exam before entering clerkships

- Principles of health systems sciences introduced, including evidence-based medicine, cost-conscious care, interprofessional teamwork, health care financing, and patient safety

- Increased use of team-based learning, case-based learning, and flipped classroom teaching methods

- Practicing clinicians paired with basic science faculty for each organ system module, facilitating integration of foundational basic sciences with clinical sciences

Clinical curriculum:

- Transitioned from 8-week to 6-week rotations for obstetricsgynecology, surgery, pediatrics, family medicine, and psychiatry. Transitioned from 8-week to 12-week rotations for internal medicine, which now includes neurology and geriatric experiences.

- Added career exploration clerkship in the third year to incorporate additional elective experiences facilitating development of professional identity and immersion with interprofessional teams.

- Increased formal career advising opportunities (both global and individual specific) as part of the curriculum to proactively address the climate of increasing competitiveness for residency.

- Added Transition in Practice Series (TIPS) to augment preparedness for next level of training:

- TIPS Clerkship: transition from preclerkship and clinical education phase.

- TIPS Residency: transition from undergraduate to graduate medical education.

Longitudinal courses:

- Addition of community-engaged learning: This 4-year curriculum integrates meaningful community service 
with structured learning experiences and reflection to enhance student physicians' overall learning. It provides real-world context for social determinants of health, an understanding of our local Hampton Roads community's needs, longitudinal participation in a specific initiative, and culmination in a presentation (including needs assessment, quality improvement recommendations, and research within that serviced population) by fourth-year medical students.

- Addition of student and physician wellness curriculum: This 4-year curriculum is designed to educate and train medical students in knowledge and skills promoting resiliency in the practice of medicine. This course builds upon the progressive development of the medical student over time and thus has a different emphasis each year.

\section{Class size changes since 2010}

Class size has increased by $16 \%$ since 2010 . To accommodate our current number of students in the preclerkship curriculum, we have a new 89,000-square-foot education and administration building under construction, set to open in 2020. Although we do not employ a regional medical campus, we have increased our outreach to clinical sites/preceptors in our community to maintain student clinical experiences in both the preclerkship and the clinical curriculum.

\section{Assessment}

Our medical education program objectives were originally drafted to reflect the 6 ACGME domains of competence: practice-based learning and improvement, patient care, professionalism, medical knowledge, evidence-based practice, and interpersonal and communication skills. As additional frameworks (e.g., Core Entrustable Professional Activities) have emerged, we used these as guidelines to further refine our internal framework for delivering curriculum content and assessing the learner at different time points.

See Supplemental Digital Appendix 2-Program Objectives and Assessment Methods—at http://links.lww.com/ACADMED/ A902.

With the implementation of the CareForward Curriculum, new (or modified) tools of assessment have been implemented.

Preclerkship phase:

- Introduction of rubrics for reflection and self-directed learning

- Use of NBME-based questions for summative examinations

- Addition of institutional requirement to pass Clinical Basic Science Examination prior to being eligible to take USMLE Step 1 examination

- Modification of clinical evaluation form for clinical experiences

- Alignment with medical program objectives and CareForward curriculum objectives

- Inclusion of target performance anchors

- Transition to pass/fail grading in 2019-2020
Clinical phase:

- Introduction of rubrics for reflection and self-directed learning

- Modification of objective structured clinical examinations in third year as preparation for Step 2 Clinical Skills examination

- Modification of clinical evaluation form for clinical experiences

- Alignment with medical program objectives and CareForward curriculum objectives

- Inclusion of target performance anchors

\section{Pedagogy}

We employ a multimodal approach to achieve our medical education program objectives.

Preclerkship phase:

- Team-based learning, small-group discussion, peer teaching, and case-based learning: These modalities are used to integrate knowledge of basic and clinical science content in the organ system modules.

- Standardized/simulated patients: Our longitudinal clinical skills thread (designed to teach the skills of history taking and physical exam) employs standardized patients.

- Simulation (combined human and nonhuman): This modality illustrates principles taught in the basic sciences module and integrates clinical skills.

- Preceptorship: Students work with a clinical preceptor in the preclerkship phase to gain exposure to a specific field and to practice skills initially taught in simulation with standardized patients.

- Virtual family cases: Patient stories simulate real-world clinical scenarios in complexity. The individual patient story is created in the context of a larger virtual family to illustrate important concepts (medical complexity, impact of illness/chronic disease on both the individual and the family, social determinants of health, and patient safety).

- Self-directed learning: Students are asked to explore specific areas of personal interest as relates to a particular organ system module. Students are also given feedback on the quality of the used sources.

- Suturing workshops.

Clinical phase:

- Clinical experiences: Students rotate in inpatient, ambulatory clinics, and subacute care facilities during the third-year clerkship rotations.

- Small-group discussion ( $\leq 12)$ : Clinician-educators guide students through interpretation of diagnostic studies (EKGs, lab interpretation, imaging) in small-group settings.

- Peer teaching: Students present to small groups of their peers in the presence of the clerkship directors in different clerkships.

- Standardized/simulated patients and simulation: Simulated patient encounters are designed for increasing fidelity and alignment with the Step 2 Clinical Skills exam.

Accounting for generational changes in our learners, technological advances, and the sheer increase in the amount of available medical information to the current learner, our pedagogical approach continues to evolve. With the understanding that acquisition of knowledge is no longer 
"restricted" to textbooks or even faculty, the delivery of curriculum continues to shift away from required lectures and toward more active learning sessions. We are using the varied modalities just described to emphasize and more explicitly teach "how" and "why" rather than "what."

\section{Clinical experiences}

EVMS coordinates required clinical educational experiences at a variety of affiliated clinical sites, including a Level 1 trauma medical care facility, a standalone children's hospital, a Veterans Affairs Medical Center, and a military medical facility, as well as several other community hospitals and outpatient clinics, including clinics providing uncompensated care to residents who are low-income, uninsured, or underinsured.

\section{Required longitudinal experiences}

Students continue to participate in community-engaged learning initiatives and the student and physician wellness curriculum throughout the clinical phase. Additionally, students are excused for a day each clerkship to participate in a longitudinal experience incorporating professional development, health systems science, and wellness throughout the clerkship experience.

\section{Clinical experience first encounter}

Beginning the first week of medical school, students work with standardized patients. Within the first 6 months, students begin their preclinical longitudinal mentorship program with physicians in hospitals and clinics.

\section{Required and elective community-based rotations}

All students rotate outside of the primary teaching hospital as part of our community-oriented emphasis, including rotations at the Veterans Affairs Medical Center and military medical facility, as well as several other community hospital partners.

\section{Challenges in designing and implementing clinical experiences for medical students}

The growing size of the medical school class has increased our reliance on volunteer community partners to train students. The resulting challenges include providing regular communication about medical education objectives, required clinical experiences, and procedures; student policies; and student feedback with a broad range of community partners. This has also resulted in needing additional resources to regularly evaluate site comparability measurements in an effort to keep student expectations and assessments standardized and fair. We have also found that our student population is reporting increased difficulties with access to transportation due to the expanding radius of required clinical experiences $(10 \%-15 \%$ of the class from 2018 to present day).

\section{Curricular Governance}

See Figure 1-Curricular governance chart.

\section{Education Staff}

The Medical Education Committee (MEC) has the primary responsibility for oversight and management of the curriculum at EVMS. The Office of Academic Affairs provides administrative support and lends educational expertise (including creation and maintenance of curriculum map) to help the MEC execute its charge from the dean.

See Figure 2-Organizational chart.

The medical education staff and administrative faculty in the Office of Academic Affairs are responsible for the 4-year MD educational program (not pre-UME or GME). The Office of Student Affairs provides services to students in both the school of medicine and school of health professions.

Faculty have academic appointments to basic science departments or clinical departments. EVMS does not currently have an academic department of medical education.

\section{Faculty Development and Support in Education}

\section{Professional development for faculty as educators}

The Office of Faculty Affairs and Professional Development provides support in the following ways:

- Financial support to promote scholarship and national recognition

- Networking events to increase opportunities for collaboration and interdisciplinary networking among faculty members

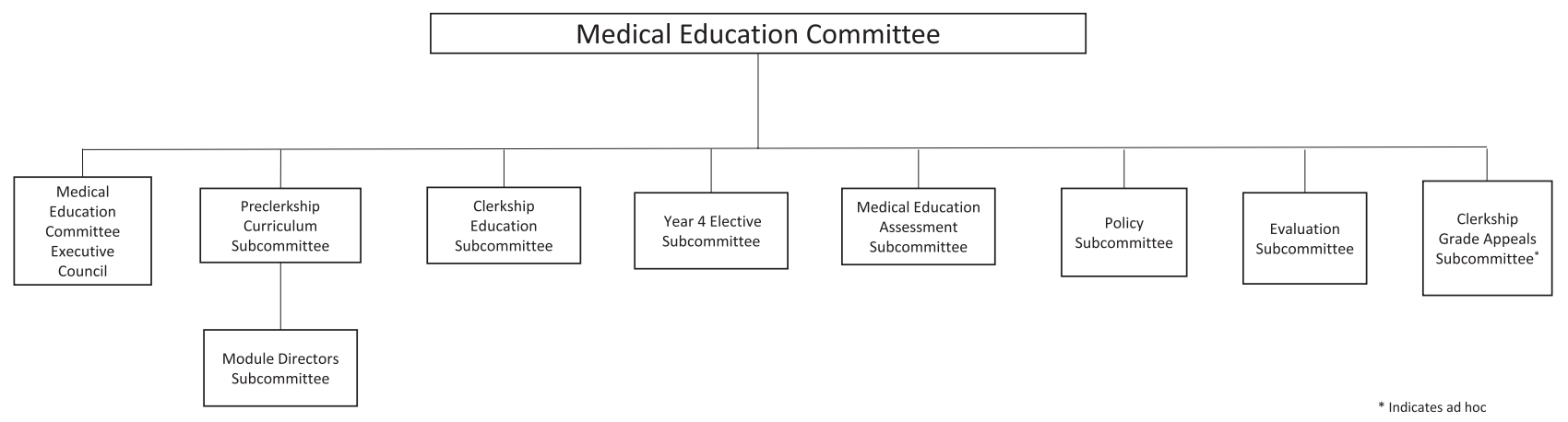

Figure 1 Curricular governance chart. 


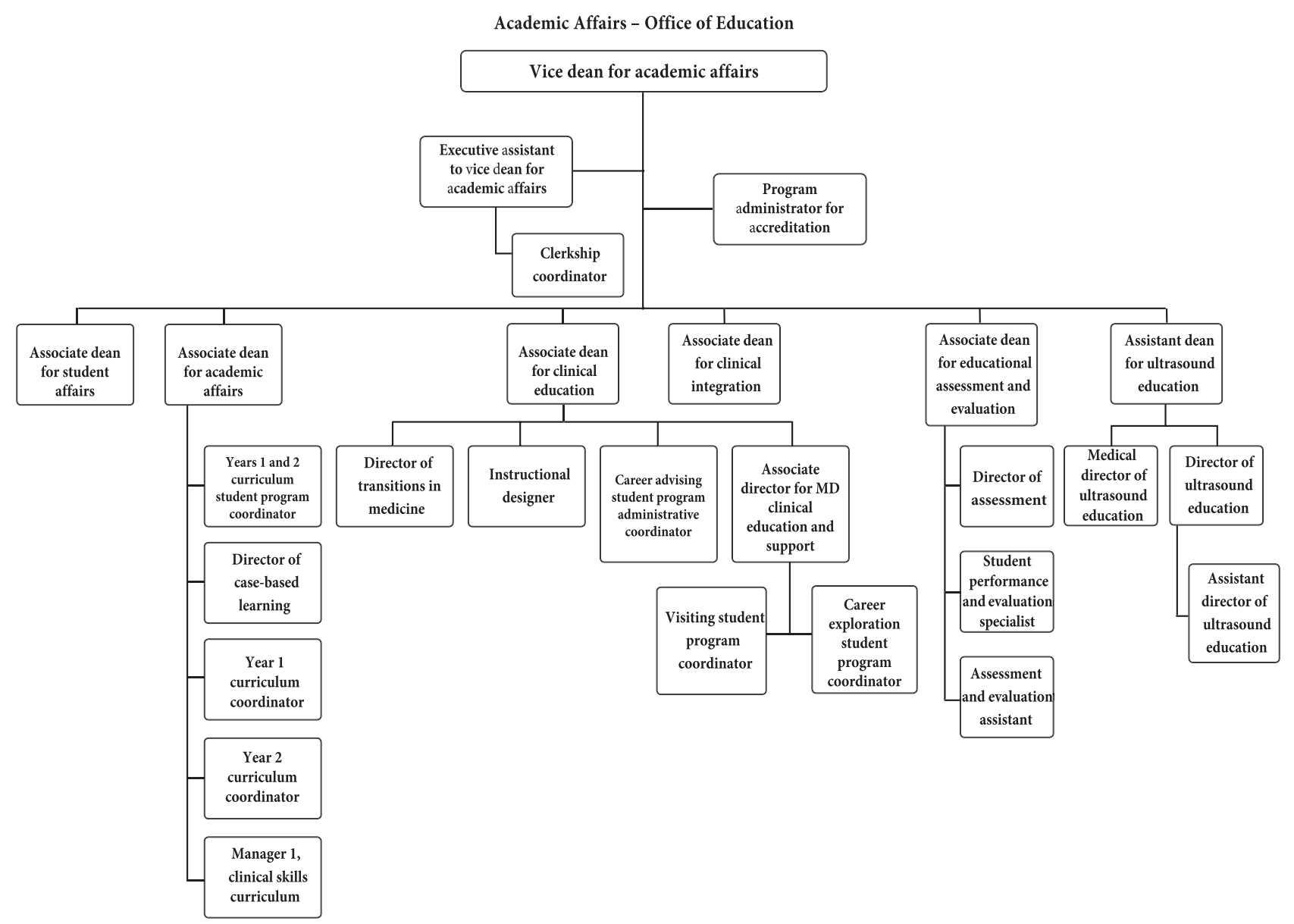

Figure 2 Organizational chart.

- Professional development activities at EVMS (workshops, online "bytes," and education grants)

- Academy of Educators

\section{Role of teaching in promotion and tenure}

In promotion and tenure decisions, teaching is 1 of the 4 domains used for evaluation of a faculty member. The thresholds for promotion are described in 3 levels: "competent," "meritorious," and "excellent." A faculty member must meet set criteria in at least
2 domains to achieve promotion to associate professor and at least 3 domains to achieve promotion to professor.

Current faculty of the medical school and school of health professions are eligible for membership in the Academy of Educators. Its core purpose is to develop educators, educational leaders, and scholars across the career continuum; recognize and celebrate educators' contributions and achievements; nurture and develop educators as a community of practice; and advance educators and education. 


\section{University of Virginia School of Medicine}

Megan J. Bray, MD, Maryellen E. Gusic, MD, and Randolph J. Canterbury, MD, MS

\section{Medical Educational Program Highlights}

The Next Generation (Next Gen) curriculum at the University of Virginia School of Medicine (UVA SOM) is grounded in the Twelve Competencies Required of a Contemporary Physician ${ }^{1}$ and was implemented in 2010 in alignment with these goals:

- Assure all graduates demonstrate mastery of the competencies required of a contemporary physician

- Integrate content around organ systems

- Integrate clinical and basic science within each educational experience and across all phases of the curriculum

- Incorporate experiential and active learning activities

- Provide frequent developmental activities for clinical skills

Unique features of the current program include:

- Integrated program of assessment incorporating competencybased metrics such as the Entrustable Professional Activities (EPAs) program:

- A longitudinal, integrated system of learning and assessment.

- Students are directly observed by residents, fellows, faculty, and master assessors in authentic patient encounters within the workplace.

- Students receive immediate feedback to support their continued development.

- Data are aggregated and analyzed for summative decisionmaking by the Entrustment Committee to grant graduated autonomy as students' skills develop.

- EPA assessments supplement information from other clinical evaluations.

- Cohesive student support system:

- College deans and learning communities: Four student affairs deans each lead a learning community of students and faculty coaches and intentionally engage and support learners as they navigate the curriculum as a cohort.

- Faculty coaches aid students in analyzing and interpreting data from clinical performance evaluations, OSCEs, and EPA assessments to cocreate individualized student learning plans. The college deans work closely with coaches to identify resources to aid students in addressing issues that affect their clinical performance.

- Master assessors are faculty selected and trained to be expert assessors provide immediate feedback to students during real-time assessments of authentic clinical tasks (EPAs). They

Acad Med. 2020;95:S534-S537.

doi: 10.1097/ACM.0000000000003328

Copyright (C) 2020 by the Association of American Medical Colleges

Supplemental digital content for this article is available at http://links.Iww.com/ ACADMED/A855.

Year school was established: 1819.

School URL: https://med.virginia.edu. are empowered to feed forward information about a student to the students' faculty coach and college dean. Coaches address clinical performance issues, and college deans identify resources to address nonacademic issues affecting students' performance. Master assessors additionally provide summative feedback as members of the Entrustment Committee.

- Alpha Omega Alpha (AOA) Peer Mentoring Program: AOA Honor Society student volunteers offer mentoring and academic support for coursework across the curriculum, provide strategies to prepare for national licensing exams, and share advice about how to navigate one's role as a student member of clinical teams.

- Experiential learning designed to promote professional identity formation: The Patient Student Partnership (PSP) program is a longitudinal program that pairs students with a patient at matriculation. The intent is not only to give students a framework for acquiring medical knowledge but also to provide an opportunity to serve as a patient advocate while learning about socio-economic systems that affect patient health and access and utilization of health care.

- Enhanced student participation in the clerkship phase curriculum review process: Student Medical Education Committee representatives meet with clerkship directors quarterly to provide ongoing student feedback and participate in the annual review done by the Curriculum Committee.

\section{Curriculum}

\section{Curriculum description}

The Next Gen curriculum is organized into 3 phases:

- The preclerkship phase is 18 months in length, is system-based, integrates foundational science and clinical medicine, and uses active learning strategies. In parallel, students complete a Foundations of Clinical Medicine (FCM) course that focuses on the development of clinical skills and diagnostic reasoning and allows students to apply learning to patient experiences, both in simulated and inpatient settings, and through the PSP and reflection on experiential learning in the Social Issues in Medicine (SIM) course.

- The clerkship phase is 1 year in length and includes disciplinespecific clinical rotations completed in 12-week blocks with one's learning community.

- The postclerkship phase is 46 weeks long and includes 10 weeks of required courses, allowing for individualization of each student's learning and preparation for residency training.

See Supplemental Digital Appendix 1-Curriculum Map—at http://links.lww.com/ACADMED/A855.

\section{Curriculum changes since 2010}

In addition to the highlights described above, other recent innovations have been implemented. 
- Curricular interventions to ease students' transitions across the phases of the curriculum:

- Classrooms to clinics: End of preclerkship phase course in which students use biomedical knowledge and apply their clinical skills and diagnostic reasoning to undifferentiated patient cases. Learning occurs in laboratory, classroom, and simulated settings.

- Transitions course: Initial course in the clerkship phase focused on learning (knowledge, skills, behaviors) that students require to assume their role as a new member of the patient care team.

- DxRx: Just before the postclerkship phase, this course emphasizes systems-based practice, health disparities, population health, health policy, and advocacy.

- Intern Readiness course: Post-Match, discipline-specific course that focuses on the skills necessary for residency, interpersonal communication, and interprofessional collaborative practice.

- Clerkship and postclerkship curriculum reform:

- With the opening of our regional campus in northern Virginia in 2021, we are embarking upon curriculum redesign.

- The size of our medical school class will not change.

- Guiding principles for this redesign include:

- Create core clinical learning experiences for learners to meet our program objectives and to develop competence for future practice

- Individualize learning to foster continued development and to prepare learners for the next stage of training

- Organize clinical learning experiences developmentally to ensure that learners are engaging in authentic roles in increasing complex environments as they demonstrate competence

- Align change with accreditation standards and emerging best practices in clinical education

- Sustain structures that support learners' sense of community and developing professional identity

- Continue to integrate clinical and basic science across all phases of the curriculum

- Development of a new learning management system (VMED)

\section{Assessment}

Our program objectives were initially developed using the AAMC Medical School Objectives Project, are aligned with the ACGME domains of competence, and map to the Physician Competency Reference Set.

See Supplemental Digital Appendix 2-Program Objectives and Assessment Methods—at http://links.lww.com/ACADMED/A855.

Recent additions to our assessment program include:

- Our EPA program

- Implementation of criterion-based standard setting for OSCEs

- Structured process for pre- and posttest review of multiplechoice question (MCQ) examinations used in the preclerkship curriculum
- Tagging of questions used in MCQ examinations in the preclerkship curriculum to learning objectives related to courses and threads (longitudinal thematic content, e.g., anatomy, ethics, patient safety)

- Redesign of summative student clinical evaluation forms using competency-based, behavioral anchors

\section{Pedagogy}

The courses within the preclerkship phase of our curriculum are delivered using a variety of pedagogies. The goal is that over $80 \%$ of learning activities are active, including casebased learning, weekly patient presentations/panels, teambased learning, in-class problem sets, and clinical anatomy and imaging labs. In FCM, students participate in smallgroup discussions and simulated exercises using high-fidelity simulation and standardized patients. Students also have a service learning requirement in their SIM course. The majority of teaching in the clerkship and postclerkship phases occurs in a clinical setting as students participate in patient care as members of an interprofessional team. Across all phases, students engage in self-directed learning activities.

\section{Clinical experiences}

Students complete the majority of their clinical education at UVA Health System. Students can also complete clinical rotations at our clinical affiliates: Salem VA Medical Center, INOVA Fairfax Medical Campus, and Bon Secours Health System. All students participate in 2 community-based required rotations during their clerkship year.

Students engage with patients in clinical settings beginning in the fall of their first year in FCM and PSP (longitudinal exposure).

Students have the opportunity to participate in electives at all of our affiliate sites, to arrange experiences at other LCMEaccredited institutions, or to participate in approved global health rotations.

Current challenges to implementing clinical experiences at sites associated with other health entities include establishing efficient processes for ensuring faculty certification, student credentialing, and creating affiliation agreements that meet each system's requirements.

\section{Curricular Governance}

The Curriculum Committee as established and codified in the School of Medicine bylaws and comprises elected and appointed members. It has the authority to set educational objectives; establish educational requirements; establish and approve content; ensure content integration, coordination, and coherence; allocate curriculum time; specify teaching methods; select and oversee course and clerkship directors; and evaluate educational outcomes.

See Figure 1 -Curriculum governance. 


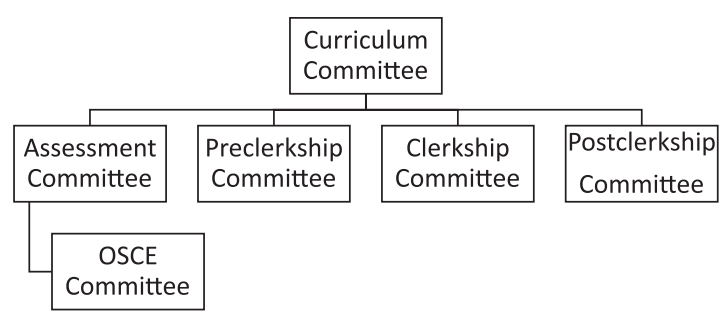

Figure 1 Curriculum governance.

\section{Education Staff}

The Office of Medical Education (OME) is the unit within the Office of Educational Affairs that provides administrative oversight and centralized support for undergraduate medical education. Members include:

- Decanal staff

- Faculty with expertise in student assessment and program evaluation

- Faculty administrative leaders for preclerkship courses: one who is also director of curriculum integration and development, and one who is the director of academic advancement

- A faculty director of anatomy and staff who support anatomy teaching

- Faculty who oversee FCM and PSP

- Faculty and staff who operate the clinical skills and medical simulation centers

- An instructional designer

- An educational technology unit that includes a director, instructional technologists, and staff who support systems for curriculum management and provide IT support for classrooms and instructional tools used in teaching

- Instructional support professionals

- Administrative staff

OME also provides financial support for clerkship coordinators who are members of clinical departmental administrative staff.

See Figure 2-Organizational chart.

OME also serves as an academic home within the SOM. Faculty with administrative roles in undergraduate medical education in addition to teaching faculty at clinical affiliate sites hold appointments on the instructional track within OME.

\section{Faculty Development and Support in Education}

Professional development for educators is organized by OME in collaboration with the Academy of Distinguished Educators (ADE) and the UVA SOM Office of Faculty Affairs and Faculty Development. Professional development sessions are offered through a series of highly interactive workshops, medical education grand rounds, and a monthly journal club. Faculty who participate in 10 workshops over a 2 -year period receive a Certificate of Commitment to Faculty Development in Medical Education that can be included in their teaching portfolio.

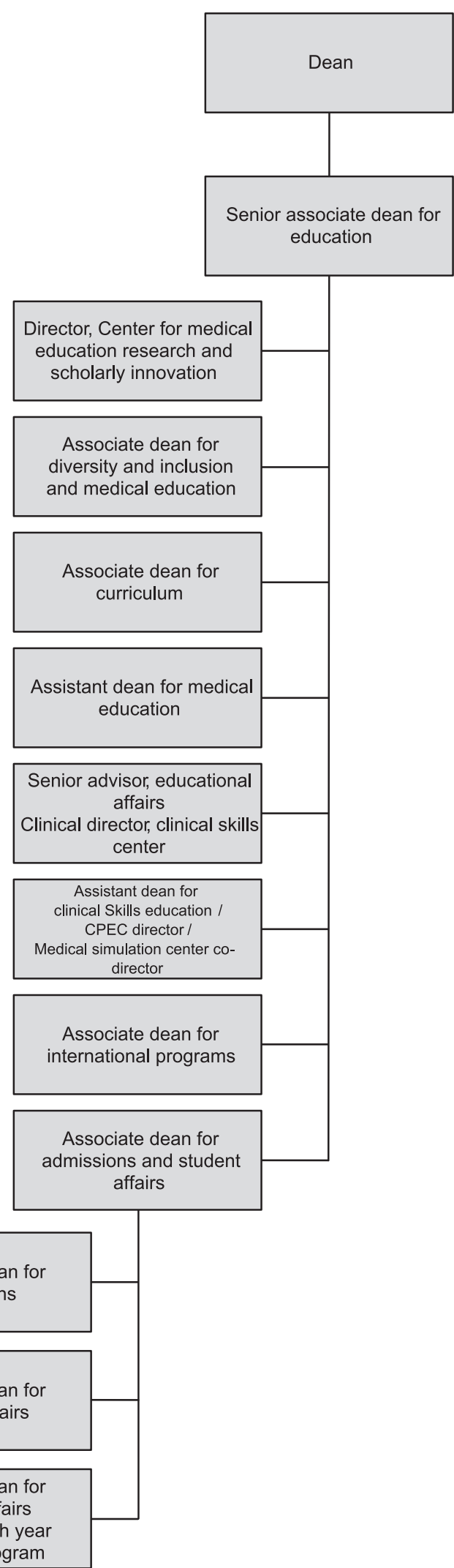

Figure 2 Organizational chart. 
Although faculty on all tracks contribute to each of the 3 missions of the SOM, faculty whose area of excellence is education are appointed to 1 of 3 tracks. Faculty on the clinician-educator track, a tenure eligible designation, spend at least $80 \%$ time devoted to patient care and/or medical education. The award of tenure is granted with evidence of excellence in both domains. There are also 2 tenure ineligible designations, clinical faculty and instructional faculty. Clinical faculty must demonstrate excellence in either patient care or education. Educational accomplishments are demonstrated through excellence in training, teaching, and advising learners or colleagues; election to honorary educational societies and receipt of teaching awards; and invited presentations and participation in educational professional development. Published scholarship is required for clinician-educators and for promotion to professor in the clinical and instructional faculty tracks. Scholarship includes peer-reviewed publications, textbooks or chapters, and dissemination of teaching materials. Instructional faculty spend the majority of their time in teaching or service. For this track, excellence in education can be demonstrated through activities done in support of the educational mission.

The ADE was founded in 2003 to recognize, reward, and promote excellence in education. Members of the medical school faculty are selected through an application process. In addition to funding to support medical education innovation and research, the ADE sponsors the annual Medical Education Week that includes an invited keynote speaker, a poster session, and oral presentations of educational projects done by learners and members of the UVA health science education community. The institution is currently restructuring the ADE to build an inclusive membership and serve learners and teachers across the biomedical community.

The SOM recently created the Center for Educational Research and Scholarly Innovation. This center will serve as an organizing unit and provide individual and programmatic support for members of the SOM community to apply a scholarly approach in their work as educators across the domains of teaching, curriculum development, learner assessment, advising/ mentoring, and educational leadership and administration. The center will support the development and dissemination of educational scholarship through professional development programming, coaching/mentoring, community building, and infrastructure support.

\section{Regional Medical Campuses}

In 2021, our first cohort of students (36) will begin their clerkship and postclerkship phases of the curriculum at the UVA SOM INOVA Campus in Fairfax, Virginia.

\section{Reference}

1 University of Virginia School of Medicine. Undergraduate medical education curriculum. Competencies required of the contemporary physician. https:// med.virginia.edu/ume-curriculum/curriculum/competencies-required-ofthe-contemporary-physician. Accessed March 16, 2020. 


\section{Virginia Commonwealth University School of Medicine}

Michael S. Ryan, MD, MEHP, Diane Biskobing, MD, Lelia Brinegar, EdD, Susan DiGiovanni, MD, and Christopher Woleben, MD

\section{Medical Education Program Highlights}

Students enrolled at Virginia Commonwealth University School of Medicine (VCU-SOM) are engaged in diverse learning opportunities in the classroom, patient care areas, and the larger community. In 2013, a major curriculum redesign was completed. A traditional 2-year preclinical curriculum was replaced with an 18-month integrated systems-based curriculum that emphasizes active learning opportunities. The clinical phase was expanded in duration and now includes career-exploratory electives as well as formative assessment and coaching programs developed to support the Core Entrustable Professional Activities (Core EPAs) for Entering Residency. Students have opportunities to participate in experiential learning opportunities based in programs such as the VCU Acute Care and System Strengthening in Low Resource Settings and the International/Inner City/Rural Preceptorship. And, for those exceptional students seeking an early entry to residency, the competency-based continuum allows students to graduate when deemed competent for residency. Dynamic faculty development initiatives such as the Teaching in Medicine Education (TiME) certificate program and monthly lunchtime learning sessions provide faculty and staff members with opportunities to continue their education in learning and teaching topics and techniques.

\section{Curriculum}

\section{Curriculum description}

The MD program curriculum is organized into a 3-semester basic science-oriented phase followed by a 24 -month clinically oriented phase.

See Supplemental Digital Appendix 1-Curriculum Schematicat http://links.lww.com/ACADMED/A896.

\section{Curriculum changes since 2010}

The VCU-SOM launched the $\mathrm{C}^{3}$ Curriculum (Competency Based, Centered on the needs of the learner, and Clinically Focused) in 2013. Additional modifications have been made on an annual basis following continuous evaluation of the program. Highlights curriculum include:

- Consolidated preclinical phase and extended clinical training

- Increased focus on active learning techniques

Acad Med. 2020;95:S538-S541.

doi: 10.1097/ACM.0000000000003389

Copyright (C) 2020 by the Association of American Medical Colleges

Supplemental digital content for this article is available at http://links.Iww.com/ ACADMED/A896.

Year school was established: 1838.

School URL: https://medschool.vcu.edu.
- Incorporation of curriculum in ultrasound, interprofessional education, quality, and safety

- Revision of core clerkships including reduction in the length of internal medicine, pediatrics, and psychiatry

- Creation of additional required clinical rotations in ambulatory medicine and a dedicated elective block in the third year

- Expansion of fourth-year requirements

- Development of interdepartmental acting internship experiences

- Adoption of Core EPAs and implementation across clinical rotations

- Development of required capstone course and specialtyspecific "boot camps" prior to graduation

\section{Assessment}

The VCU-SOM objectives were developed to fit the framework outlined by the ACGME core competencies. Within each primary objective there are several subobjectives.

See Table 1-Program Objectives and Assessment Methods.

The medical education objectives were based on the ACGME competency domains. In 2015, the subobjectives were modified to incorporate the Core EPAs.

- In the preclinical years, low-stakes summative assessments such as quizzes, team-based learning, and assignments were added to existing higher-stakes multiple-choice tests. Formative selfassessment questions were added to every course.

- In the clerkships, several additional modifications were made. Additional OSCEs were added to clerkships. A written clinical skills assessment was added to the pediatrics clerkship. Beginning in 2018, students were required to obtain formative workplace-based assessments for the Core EPAs. In 2018, the grading structure for the core clerkships and acting internships were revised to incorporate the RIME (reporter, interpreter, manager, educator) framework across clerkships.

- In the postclerkship phase, a formative clinical skills assessment was added to prepare students for the USMLE Step 2 Clinical Skills examination.

The VCU-SOM has begun piloting a competency-based graduation program. While this program is not specifically a 3-year program (students graduate when deemed "competent"), the duration is, in effect, 3 years.

\section{Pedagogy}

In the preclinical phase and clinical phases, a variety of teaching strategies are used. Active learning strategies are encouraged whenever appropriate. The following is a list of approaches used throughout the program: 


\section{Table 1}

\section{Program Objectives and Assessment Methods}

\section{Medical education program objective}

Professionalism: demonstrate the nature of professional and ethical behavior in the act of medical care including respect, responsibility, accountability, excellence, honor, integrity, altruism, leadership, cultural competency, compassion, maintenance of professional boundaries, and confidentiality

Patient engagement and communication: demonstrate the ability to engage and communicate with patients, their families, and professional associates, using interpersonal skills to build relationships for information gathering, guidance, education, support, and collaboration

\section{Application of scientific knowledge and method} discuss the biomedical, epidemiological, and social-behavioral aspects of clinical science and apply this knowledge to patient care

Patient care: provide patient care that is appropriate and effective for the treatment of health problems and the promotion of health

\section{Assessment methods}

- Peer evaluations in preclinical course

- Professionalism ratings in core clerkships

- Early concern notes, which may be filed by any individual at any point throughout the program

- Small-group leader ratings during Practice of Clinical Medicine course

- OSCEs administered Practice of Clinical Medicine course

- Summative workplace-based assessments completed across all clerkships

- Formative EPA-oriented workplace-based assessments throughout training

- OSCEs administered in several core clerkships

- Passage of Step 2 Clinical Skills examination

- Quizzes, assignments, and team-based learning activities during preclinical course

- Internally developed multiple-choice tests for each preclinical course

- NBME Clinical Science Subject Examinations during core clerkships

- Summative workplace-based assessments completed across all clerkships

- Passage of Step 1 and Step 2 Clinical Knowledge examinations

- Small-group leader ratings during Practice of Clinical Medicine course

- OSCEs administered Practice of Clinical Medicine course

- Summative workplace-based assessments completed across all clerkships

- Formative EPA-oriented workplace-based assessments throughout training

- OSCEs administered in several core clerkships

- Small-group leader ratings during Practice of Clinical Medicine course

- Summative assessment completed for Physician, Patient, and Society course

- Summative workplace-based assessments completed across all clerkships within the practical context of a patient's age, gender, personal values, family, health literacy, culture, religion, and social and economic circumstances, including consideration of relevant ethical, moral, and legal perspectives, patient advocacy, public health concerns, resources, and limitations of the health care system

Self-directed learning and self-assessment: assess and understand one's learning style, self-identify areas of strength and weakness, independently identify and evaluate resources to engage in lifelong learning, and critically appraise the evolving body of medical knowledge
- Summative assessment completed for Population Health and Evidence-based Medicine course

- Completion of individualized learning plans as part of EPA coaching program
- Case-based learning

- Clinical experience: ambulatory

- Clinical experience: inpatient

- Discussion: large group

- Discussion: small group

- Lecture

- Peer teaching

- Preceptorship

- Role play

- Self-directed learning

- Simulation

- Standardized patients

- Team-based learning

- Virtual patient

- Workshop

- Other: process-oriented guided inquiry learning

Since 2010, the VCU-SOM has established a goal of 50\% of class time devoted to active learning, accomplished through utilization of team-based learning, flipped classroom, standardized patients, and workshops. In addition, increased time has been allotted for self-directed learning. In the clinical phase of the curriculum, clerkships have expanded in the use of simulation, standardized patients, and team-based learning. Examples are provided below:

- Internal medicine: revised didactic curriculum to include team-based learning activities

- Obstetrics-gynecology: developed pelvic examination simulation

- Neurology: created simulation for lumbar puncture

- Pediatrics: developed standardized patient exercise to teach family-centered rounds and simulations around respiratory distress, lumbar puncture, and otoscopy

- Surgery: developed workshops to teach students suturing skills and nasogastric tube placement

\section{Clinical experiences}

VCU-SOM uses a variety of clinical sites for required educational experiences. 
- Ambulatory sites include primary care, subspecialty care, and surgical clinics at the VCU Health Medical Center, the Hunter Holmes McGuire VA Medical Center, and community-based settings throughout the state of Virginia.

- Inpatient sites are primarily based at the VCU Health and Hunter Holmes McGuire VA medical centers. In addition, a subset of medical students rotates through community hospital affiliates in the Richmond metropolitan area.

- The VCU health system (VCUHS) recently formed a partnership with Community Memorial Hospital in South Hill, Virginia. Plans are currently underdevelopment to initiate medical student rotations at this site.

- Practice of Clinical Medicine (PCM) course: This is an 18-month course that takes place throughout the preclinical phase of training. Students learn foundational skills of doctoring (e.g., history taking, physical examination) through a combination of didactics, small-group learning, and standardized patient exercises. Performance is assessed through multiple-choice-based examinations and OSCEs. Fourth-year students are provided an opportunity to function as coleaders in the small-group experience.

- Physician, Patient, and Society course: This course spans the entirety of the MD program. Its purpose is to provide students with humanistic, ethical, and legal skills required to practice medicine. A wide range of topics are presented and include career development, integrative/complementary medicine, palliative care, physician-patient relationship, spirituality, health disparities, physician bias, cultural competency, and application of ethics and law in medicine. Instruction includes didactics, patient panel activities, and experiential learning. Assessment is composed of multiple-choice tests, reflective writing assignments, and participation.

- Population Health and Evidence-Based Medicine course: This course spans the entirety of the MD program. In the preclinical component of the course, students learn foundational skills regarding social determinants of health, assessment of population-level health, methods for evaluating the health literature, and biostatistics. In the clinical phase, student apply evidence-based medicine skills to clinical situations encountered in their clerkships. Methods for instructions include didactics and small-group work. Assessment is conducted by participation and multiplechoice examinations.

- Patient Safety and Quality Improvement course: This interprofessional course spans the entirety of the MD program. Medical students work with learners from other health care professions to discuss principles of quality improvement and to apply those principles to the care of patients in the clinical environment. Instruction includes didactics, small-group learning, and simulation. Assessment is through participation, multiple-choice examinations, and standardized patient scenarios.

\section{Clinical experience first encounter}

Students are introduced to clinical medicine in the first week of the MD program in the context of the PCM course. In that setting, students participate in a simulation-based "boot camp" that includes standardized patient exercises. The first encounter with nonstandardized patients occurs in the third semester where students are paired with a preceptor for the PCM course. On a monthly basis, students spend at least 1 half day with the preceptor in his/her setting performing histories, physicals, and presenting patients.

\section{Required and elective community-based rotations}

- The family medicine clerkship uses community-based physicians to deliver the clinical experience in the clerkship. Students are paired with a family medicine physician throughout the state of Virginia for the duration of their clerkship.

- A subset of medical students completes portions of their obstetrics-gynecology and pediatrics clerkships at communitybased sites. The obstetrics-gynecology clerkship is offered at Riverside hospital, a community-based medical center in Hampton, Virginia. The inpatient component of pediatrics is offered at Chippenham hospital, a community-based facility within the Richmond metropolitan area.

\section{Challenges in designing and implementing clinical experiences for medical students}

VCUHS and the Hunter Holmes McGuire VA are outstanding major referral centers within the central Virginia region. While these sites are outstanding, there are several limitations. First, the high degree of specialization comes at some trade-off with respect to some lack of exposure to "bread and butter" clinical experiences. Second, while some specialties provide students with substantial patient volume, there are notable exceptions including pediatrics, obstetrics-gynecology, and to some degree psychiatry. The pediatrics volume will be improved with plans to complete the building of the first children's hospital in the region in 2023. In addition, VCUHS has a limited number of affiliated ambulatory sites, which creates some strain on providing ambulatory experiences for students within the area, particularly in primary care fields.

\section{Curricular Governance}

Central oversight for the curriculum is provided by the Curriculum Council, which has the authority to monitor, review, and revise the curriculum of the medical school and acts on the authority of the dean. Membership consists of voting and nonvoting members. Voting members include those elected by the larger faculty body, those appointed by nature of their positions (the deans for preclinical and clinical medical education), and students elected by the student body. With the exception of members with positions in the dean's office, all voting members serve a 4 -year term. Voting members for the Curriculum Council are:

- 1 chair

- 2 vice chairs

- 4 basic science faculty

- 7 clinical faculty

- 2 assistant deans

- 4 student representatives (1 per class) 


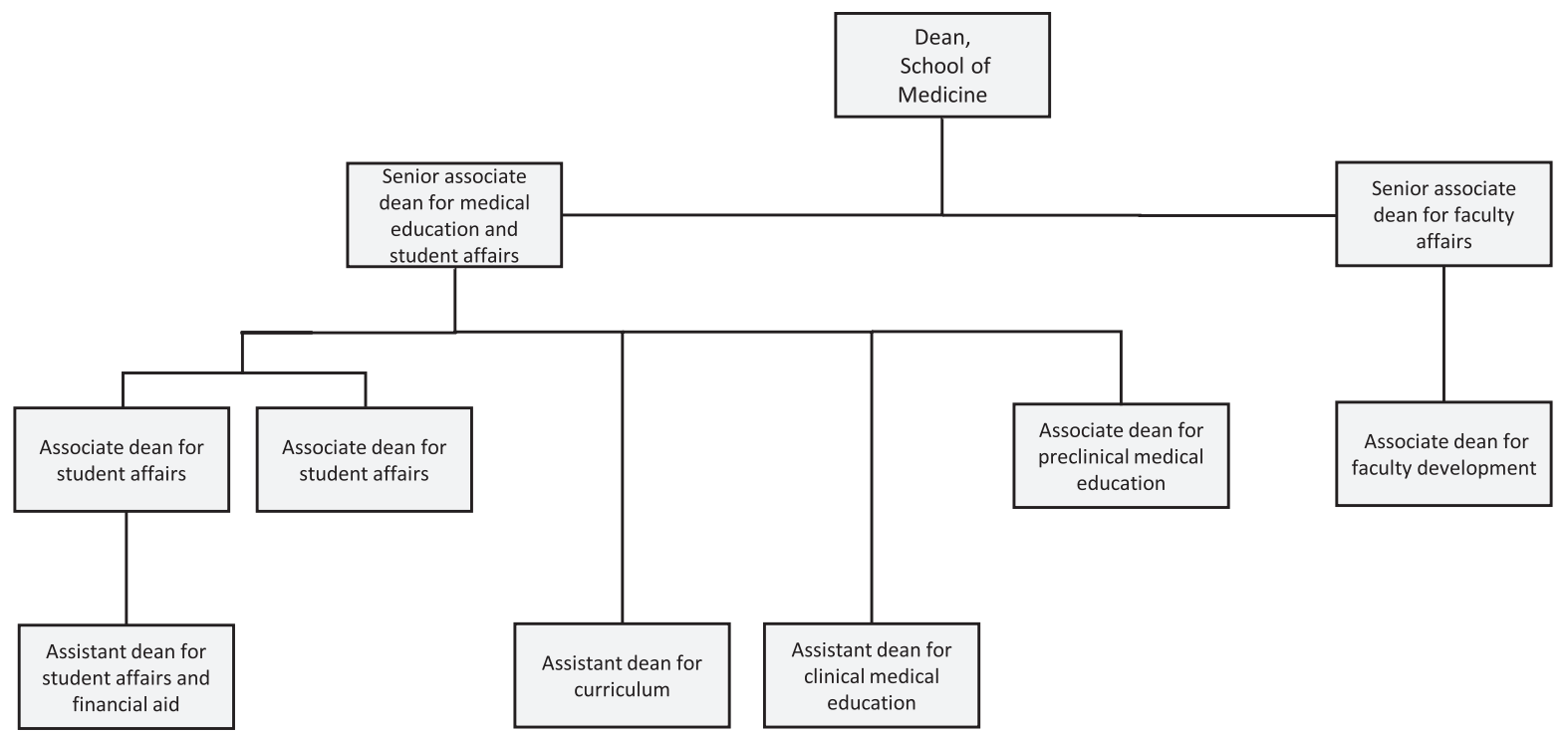

Figure 1 Organizational chart.

Curricular governance allows for departments to propose curricula, assessment strategies, budget for various initiatives (e.g., simulation, OSCEs), and leadership (e.g., course and clerkship directors). Final approval is determined by the Curriculum Council.

\section{Education Staff}

Administration of the medical education program is coordinated through several offices that work in parallel with direct reporting responsibilities to the dean. The Office of Curriculum and Student Affairs serves 3 functions relevant to the medical education program: student affairs, oversight of preclinical and clinical educational programs, and financial aid. The Office of Assessment, Evaluation, and Scholarship provides comprehensive program evaluation. The Curriculum Council provides overall oversight and participates in monitoring the program.

See Figure 1-Organizational chart.

The Office of Curriculum and Student Affairs is responsible for the undergraduate medical education program and student affairs only. Graduate medical education and continuing medical education are administered through separate offices with parallel reporting structures to the dean.

\section{Faculty Development and Support in Education}

\section{Professional development for faculty as educators}

The Office of Faculty Development supports the development of teaching faculty within the VCU-SOM. Multiple opportunities for faculty are provided, which include:
- TiME postgraduate certificate: 12 -credit certificate program coordinated through the VCU School of Education. Interested faculty are able to take coursework in adult learning, instructional design, educational scholarship, etc.

- iTeach website: a centralized resource for innovative teaching strategies. Includes best practice videos, featured faculty, audio/ video podcasts, original research articles regarding innovations, and a public forum for engagement

- Lunchtime Learning: 90-minute sessions offered live and recorded on a variety of core teaching topics

- 1-on-1 teaching consultation: provided by faculty and staff within the Office of Faculty Development to assist in creation of new learning technology, instructional methods, and assessment

\section{Role of teaching in promotion and tenure}

Promotion and tenure are driven by 3 components: service, scholarship, and teaching. Eligibility for promotion is determined by designations of "unsatisfactory," "satisfactory," "very good," or "excellent" ratings in each of the 3 categories. Faculty must demonstrate excellent performance in either scholarship or teaching for advancement unless the vast majority of their effort qualifies in the service domain. Teaching quality is measured holistically through a variety of categories including teaching activities, educational scholarship, educational leadership, and curriculum development.

\section{Regional Medical Campuses}

VCU-SOM maintained a partnership with INOVA Fairfax Hospital in Fairfax, Virginia, beginning in 2005. That partnership will be discontinued with the graduating class of 2021 . 


\section{University of Washington School of Medicine}

Suzanne M. Allen, MD, MPH, Michael J. Ryan, MD, Mark Whipple, MD, Kellie Engle, Lynne Robins, PhD, Matthew Cunningham, PhD, and Marjorie D. Wenrich, PhD, MPH

\section{Medical Education Program Highlights}

- The University of Washington School of Medicine (UWSOM) is a top-ranking scientific research institution and a leader in training primary care physicians. Since the 1970s, UWSOM has ranked among the top 8 medical schools in receipt of National Institutes of Health research support while maintaining a strong commitment to training rural physicians.

- Through its 48-year-old WWAMI (Washington, Wyoming, Alaska, Montana, and Idaho) Program, UWSOM provides medical education in 5 states. The preclinical curriculum is offered at 6 regional campuses affiliated with host state or private universities. Clinical rotations occur in diverse communities and practice types throughout WWAMI.

- UWSOM has a strong focus on clinical skills training and patient exposure. An integrated Foundations of Clinical Medicine (FCM) course begins at the start of medical school with a 2 - to 3 -week clinical immersion on the basic history and physical exam and continues for 15 months, combining a longitudinal outpatient clinic experience, clinical skills workshops, and hospital tutorials. The latter occurs in the context of sustained bedside teaching with a faculty mentor and small peer group through the Colleges program, a learning community offered on all campuses.

- Students have many options for sustained rural and regional primary care and community exposure, including the Rural/ Underserved Opportunities Program (4-week preclerkship rural clinical experience), WWAMI Rural Integrated Training Experience (22- to 24-week longitudinal integrated clerkship at a rural primary care teaching site), Targeted Rural Underserved Track (preclerkship-through-graduation immersion in a rural/ small town community), state/regional tracks, and other options.

\section{Curriculum}

\section{Curriculum description}

See Supplemental Digital Appendix 1-Curriculum Map-at http://links.lww.com/ACADMED/A944.

\section{Curriculum changes since 2010}

A new curriculum began in September 2015, with the most significant changes occurring in the foundations (basic science)

Acad Med. 2020;95:S542-S546.

doi: 10.1097/ACM.0000000000003455

Copyright (C) 2020 by the Association of American Medical Colleges

Supplemental digital content for this article is available at http://links.Iww.com/ ACADMED/A944.

Year school was established: 1945.

School URL: www.uwmedicine.org/school-of-medicine. phase. Changes resulted from a multiyear comprehensive curriculum review, needs assessment, and visioning process.

Foundations phase:

- Departmentally based courses were replaced with integrated basic science and organ system blocks with longitudinal integrated threads.

- The preclinical curriculum was shortened from 2 years to 15 months.

- Block courses feature increased clinical correlations.

- Active learning and independent study are increasingly emphasized.

- Clinical skills training has increased, including early meaningful clinical exposure in primary care clinics and clinical skills classroom training complemented by hospital tutorials focused on bedside learning with inpatients, a dedicated faculty mentor, and peer group.

- Overarching themes (health equity and diversity, ethics, professionalism, quality and safety, communication, population health, lifelong learning) are integrated through a longitudinal Ecology of Health and Medicine (EHM) course.

Patient case phase:

- Students begin clerkships 1 quarter earlier (spring of second year).

- Two 1-week intersessions were added for the EHM course.

Explore and focus phase:

- Students begin advanced clinical electives earlier (spring of third year), increasing time for career exploration.

- 2 required advanced patient care clerkships, either inpatient or outpatient (including a subinternship), were added.

- The Transition to Residency course was expanded, with an option to tailor preinternship training to specialty choice in some areas.

- A 1-week EHM intersession was added.

\section{Class size changes since 2010}

The class increased from 216 students in 2010 to 270 students in 2019. In the legacy curriculum, all students spent year 1 at their regional campus and year 2 in Seattle. In the new curriculum, all students remain on their regional campus for the 15-month foundations phase preclinical curriculum.

\section{Assessment}

For 2 years, curriculum leadership, including the Curriculum Committee and phase subcommittees, discussed a proposal to adopt the Physician Competency Reference Set with modifications. The Curriculum Committee approved the competencies and program objectives in September 2016. 
See Table 1-Program Objectives and Assessment Methods.

Student assessment changes since 2010 include:

- Preclinical phase: All summative assessments are standardized, delivered electronically across campuses, and given more frequently. Students receive formative feedback through in-class quizzes and immediate postexam written feedback. Narrative assessments are incorporated when consistent and meaningful teacher-student interactions occur.

- Clinical phases: Standardization of student evaluations has increased. All final exams have a minimum pass cut point; the passing level is based within each clerkship on national average data. A standard approach to grade determination and standard communication template are used.

\section{Pedagogy}

Foundations phase:

- Case-based learning: Clinical correlations in blocks

- Clinical experience, ambulatory: Yearlong twice monthly community-based Primary Care Practicum (PCP)

- Clinical experience, inpatient: Twice monthly hospital tutorials performing history, physical exam, oral case presentation, and write-up

- Discussion, large group (>12): Commonly used in courses

- Discussion, small group: All courses

- Laboratory: Anatomy lab across courses, pathology and microbiology labs

- Lecture: Lectures before small-group discussion

- Peer teaching: Within some small-group sessions

- Preceptorship: PCP twice monthly throughout most of foundations phase; optional additional preceptorships

- Self-directed learning/tutorial: 6-week Consolidation and Transition Phase modules and self-study in preparation for USMLE Step 1 examination and clerkships after completion of courses; independent investigative inquiry project completed during summer after first year

- Standardized/simulated patients: Frequent use during FCM course, formative and summative objective structured clinical examinations

- Video: In some blocks

- Workshop: Clinical skills workshops twice monthly throughout FCM

- Other: Group projects in some blocks

Patient care phase and explore and focus phase:

- Clinical experience, ambulatory: Clerkship experiences

- Laboratory: Required skills labs in emergency medicine clerkship

- Clinical experience: Clerkship experiences in inpatient settings

Pedagogical changes since 2010 include:

- Significant increase in active learning during foundations phase, with expectation of less daily lectures, frequent smallgroup, case-based discussion; less class time overall, with increased expectation of self-directed learning

- More frequent lower-stakes assessment, with feedback to measure and encourage growth

- Increased preclerkship clinical skills training through FCM
Table 1

Program Objectives and Assessment Methods

\begin{tabular}{|c|c|}
\hline Program objective & $\begin{array}{l}\text { Assessment } \\
\text { methods }\end{array}$ \\
\hline Patient care (8 objectives) & $\begin{array}{l}\text { Internal exams } \\
\text { Faculty ratings } \\
\text { OSCE } \\
\text { Mini-CEX }\end{array}$ \\
\hline Knowledge for practice (15 objectives) & $\begin{array}{l}\text { Internal exams } \\
\text { Lab exams } \\
\text { NBME subject exams } \\
\text { OSCE } \\
\text { Faculty ratings } \\
\text { Narrative assessment }\end{array}$ \\
\hline $\begin{array}{l}\text { Practice-based learning and improvement } \\
\text { (10 objectives) }\end{array}$ & $\begin{array}{l}\text { Faculty ratings } \\
\text { NBME subject exams } \\
\text { Written papers }\end{array}$ \\
\hline $\begin{array}{l}\text { Interprofessional and communication skills } \\
\text { (6 objectives) }\end{array}$ & $\begin{array}{l}\text { Internal exams } \\
\text { Faculty ratings } \\
\text { OSCE } \\
\text { Mini-CEX } \\
\text { Oral case presentations }\end{array}$ \\
\hline Professionalism (6 objectives) & $\begin{array}{l}\text { Internal exams } \\
\text { Faculty ratings } \\
\text { OSCE } \\
\text { Written papers }\end{array}$ \\
\hline Systems-based practice (4 objectives) & Written papers \\
\hline Interprofessional collaboration ( 3 objectives) & Faculty ratings \\
\hline $\begin{array}{l}\text { Personal and professional development } \\
\text { (8 objectives) }\end{array}$ & $\begin{array}{l}\text { Faculty ratings } \\
\text { OSCE } \\
\text { Written papers }\end{array}$ \\
\hline
\end{tabular}

\section{Clinical experiences}

- Foundations phase: PCP is in community practice settings. Hospital tutorials are in inpatient settings. Many students complete the optional Rural/Underserved Opportunities Program.

- Clinical phases: Most students participate in clerkships based on a "traditional" blocked clinical schedule in multiple locations throughout WWAMI. Practice sites vary from large-city hospital settings to remote rural clinics. Most required clerkships incorporate both inpatient and ambulatory settings for each student, but inpatient and ambulatory exposure mix varies by clerkship and clinical site. Each student must spend 8 weeks in 1 or more Seattle tertiary/quaternary care teaching institution.

- Patient care phase: Students can apply to participate in a special program with options including the longitudinal WWAMI Rural Integrated Training Experience, longitudinal integrated clerkship in Olympia, Washington, and city- or state-based programs allowing students to remain in one geographic location for most of their training. 


\section{Required longitudinal experiences}

In the foundations phase, the FCM course provides clinical skills workshops in laboratory/simulation/classroom settings, hospital tutorials, and the PCP in outpatient community practices. Six EHM intersessions are held across all 4 years.

\section{Clinical experience first encounter}

Students first encounter real patients during their first 2-3 weeks of medical school. This prepares them in taking a history and physical examination. Hospital tutorials start with the first course and the outpatient-based PCP starts in January of year 1 .

\section{Required and elective community-based rotations}

In addition to Seattle-based tertiary care training hospitals, UWSOM provides required and elective opportunities across WWAMI, including:

- Affiliated community hospitals

- Community health care centers (urban and rural)

- Private practices (urban and rural)

- International health clinics (local and abroad)

- Indian Health Service clinics

Students must meet core objectives and required clinical encounters for each required clerkship, whose leadership team tracks student success in meeting required clinical experiences.
Clinical assistant deans for each of 8 regions across WWAMI partner with UW clinical departments to identify and develop potential regional clerkships sites, provide faculty development, and perform in-person site visits.

\section{Challenges in designing and implementing clinical experiences for medical students}

Students participate in clerkships across a large geographic area consisting of more than one-quarter of the American land mass and 3 time zones. This makes identifying, developing, and monitoring widespread clerkship sites a challenge. Due to physicians' growing clinical demands, increasing numbers of allopathic and osteopathic medical schools, and expansion of regional campuses nationally, it is increasingly difficult to identify sustainable clerkship sites. However, UWSOM has been successful in achieving the number and quality of clerkship sites that sustain students' training needs.

\section{Curricular Governance}

\section{Decentralized curricular governance}

The following are managed at the department level:

- Full-time paid faculty appointment and promotion

- Courtesy faculty appointment and promotion

- Faculty effort for teaching

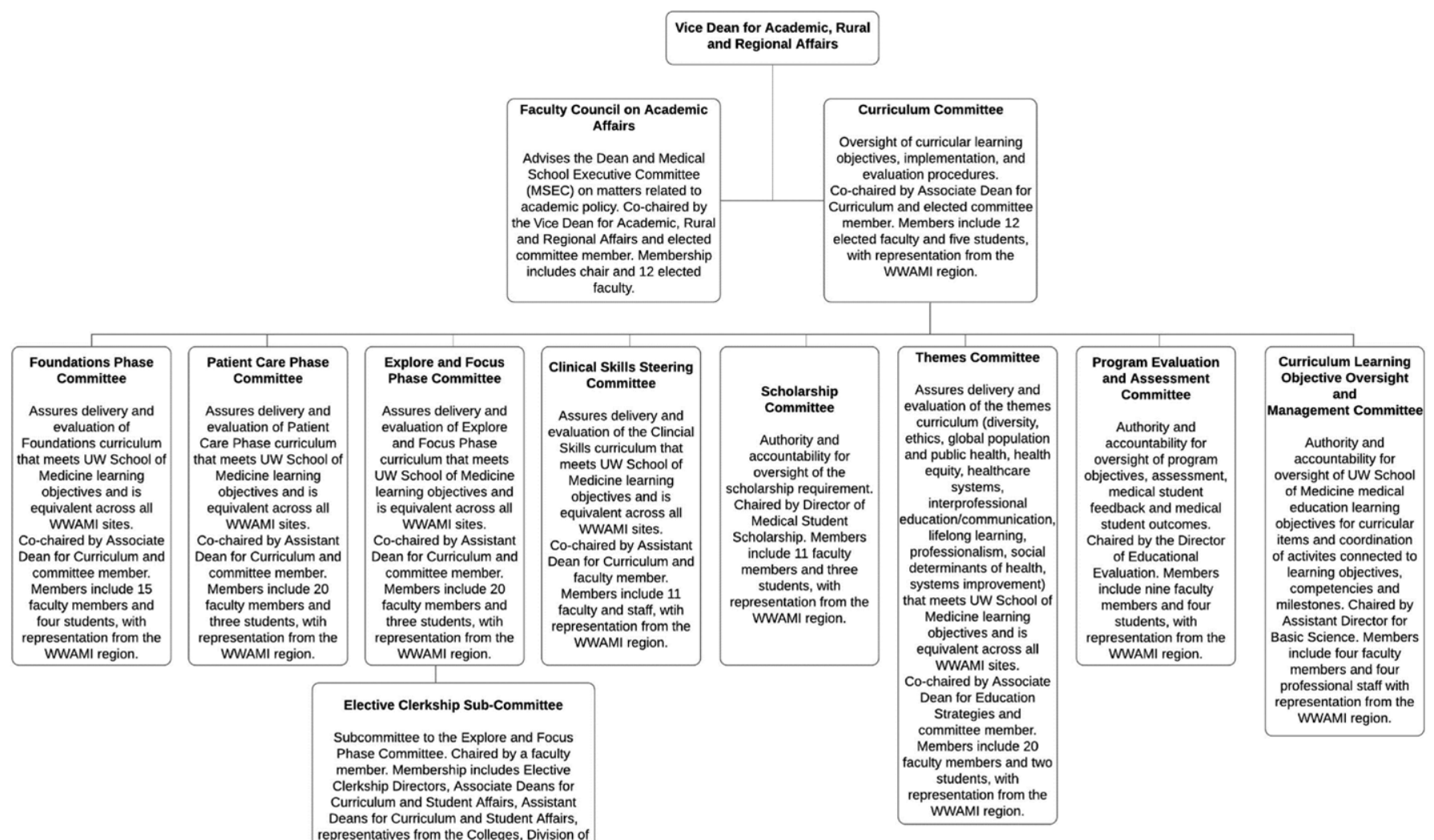

Figure 1 Curriculum governance structure. 
- Clinical clerkship training resources

- Clinical clerkship administration

See Figure 1-Curriculum governance structure.

\section{Education Staff}

The Office of Academic, Rural, and Regional Affairs (ARRA) is responsible for administrative and academic support for curricular planning, implementation, evaluation, and oversight and for developing and maintaining tools to support curriculum delivery, monitoring, and management. Within ARRA, orchestration of the curriculum is based in the Curriculum Office, led by the associate dean for curriculum and 3 assistant deans. The Curriculum Office works closely with:

- Curriculum Committee, which governs and oversees curriculum implementation and evaluation

- Educational Quality Improvement Strategic Planning Committee, which oversees ongoing monitoring, assessment, and implementation of the educational strategic plan

- Division of Medical Education and Evaluation, providing academic support for assessment/evaluation

\section{Medical education leadership}

The primary medical education staff and administrative faculty are responsible for all aspects of the UME program, from admissions through graduation. In addition to planning, overseeing, and administering the curriculum, ARRA is responsible for student affairs, medical school admissions, the Colleges program, educational quality improvement, and regional affairs.

See List 1-Dean-Level Positions.

The Division of Medical Education and Evaluation (MEE) within the Department of Biomedical Informatics and Medical Education has 8 regular and emeritus faculty who support student assessment, program evaluation, and educator professional development. Teaching faculty based outside MEE have primary appointments in their home department.

\section{Faculty Development and Support in Education}

Faculty development is conducted by several departments, units, and centers working in an integrated manner:

- Office of Faculty Development: This office, led by the associate dean for faculty development, offers workshops year-round to local and regional faculty on topics including clinical and teaching practice, research, mentoring, leadership skills, quality of life, wellness, and faculty promotion.

- MEE: Teaching Scholars is a longitudinal, cohort-based program for educators. Participants learn to develop, evaluate, enhance, and disseminate curricula using evidence-based practices and conduct and disseminate education research. MEE also presents skill-building workshops to equip faculty

\section{List 1}

\section{Dean-Level Positions}

Dean, School of Medicine

Vice dean for academic, rural, and regional affairs

Associate deans:

- Admissions

- Curriculum

- Eastern Washington

- Educational quality improvement

- Education strategies

- Faculty development

- Student affairs

Assistant deans

- Basic science curriculum

- Clinical curriculum

- Colleges

- Rural programs

- Student affairs—student development

- Student affairs—student support/Seattle foundations

Assistant clinical deans

- Alaska

- Central/Eastern Washington

- Idaho (2)

- Montana

- Spokane, Washington

- Western Washington

- Wyoming

Assistant foundations phase deans

- Alaska

- Idaho

- Montana

- Spokane, Washington

- Wyoming

participants with evidence-based teaching and assessment skills for classrooms and clinical settings.

- Center for Leadership and Innovation in Medical Education (CLIME): CLIME addresses the needs of local and regional faculty through an integrated continuum of medical, graduate, and lifelong education. Programs focus on building an educator community for networking and mentoring, educational scholarship, and teaching resources.

\section{Role of teaching in promotion and tenure}

Each department has developed criteria for appointment and promotion for specific faculty tracks and ranks. Regular-track faculty often have a blend of activities in teaching, research and scholarship, and clinical care (if applicable). In the clinician-teacher pathway, the most time is spent in patient care and clinical teaching responsibilities. Clinician-teachers are assessed on their contributions to teaching, patient care, 
and research/scholarship, which is often focused on education (e.g., development of curricula, educational materials and programs, and assessment tools). Faculty at regional campuses are governed by comparable faculty codes of their respective host universities.

While based in the UWSOM, CLIME, described above, welcomes faculty from other health professions. CLIME offerings are advertised through a membership listserv $(340+$ members $)$ and a School of Medicine faculty listserv.

\section{Regional Medical Campuses}

- All regional campuses have the same courses with identical duration, learning objectives, out-of-class resources, and assessments.

- Each course has a core team of faculty consisting of the course director(s) and leaders from all campuses, thread and theme directors, and a Seattle-based administrator. They meet yearround via distance technology to conduct course planning and implementation, student assessment, and course evaluation.

- Regional course leaders inform and orient faculty at their campus to core objectives, assessment methods, and grading. Faculty from all campuses participate in a formal review process for their course.

\section{Table 2}

\section{Regional Medical Campuses}

\begin{tabular}{llr} 
Regional campus name & Type & $\begin{array}{c}\text { Student } \\
\text { enrollment }\end{array}$ \\
University of Washington (Seattle) & Preclerkship & 100 \\
\hdashline Gonzaga University (Spokane) & Preclerkship & 60 \\
Üniversity of Wyoming (Laramie) & Preclerkship & 20 \\
\hdashline University of Alaska (Anchorage) & Preclerkship & 20 \\
\hdashline Montana State University (Bozeman) & Preclerkship & 30 \\
University of Idaho (Moscow) & Preclerkship & 40 \\
\hline
\end{tabular}

- A continuous quality improvement process occurs at the completion of each course. Student exam and overall grade performance are tracked across campuses. If inconsistencies are identified, block directors and campus leaders jointly discuss possible sources and implement corrective measures. Student course evaluations are examined by regional campus. If variability is detected, a root cause analysis is conducted, and discrepancies result in modifications through the formal change management process.

See Table 2-Regional Medical Campuses. 


\section{Washington State University Elson S. Floyd College of Medicine}

James Hupp, MD, DMD, JD, FACS

\section{Medical Education Program Highlights}

- Unique holistic admissions process with students selected based on their fit with the college's mission to prepare physicians with a passion to care for the underserved and take a leadership role in efforts to improve the health care system.

- Integrated, spiraling, case-based curriculum.

- College includes 4 regional community campuses of Washington State University where most of third- and fourthyear clinical education occurs; one-quarter of each class assigned to each regional campus.

- All students do a longitudinal integrated clerkship (LIC) for their third year.

- All students are enrolled in a 4-year leadership certificate program at the college.

- All students are required to conduct a scholarly project with a capstone deliverable in the fourth year.

- All students are required to participate in service learning and interprofessional learning activities during their 4 years.

\section{Curriculum}

\section{Curriculum description}

- First and second years: Foundational sciences in Spokane plus 6 weeks of clinical emersion at their regional campus.

- Clinical skills building across all 4 years, including extensive use of high-fidelity simulation and standardized and actual patient volunteers in the first 2 years. Regular use of OSCEs to assess learning.

- Completion of LIC at 1 of our 4 community-based campuses in Washington state for the third year.

- Fourth year includes required 4-week rotations in emergency medicine and underserved medicine, and a subinternship, along with 24 weeks of electives.

\section{Assessment}

- Students are assessed with formative exams every week for 2 weeks followed by high-stakes exam every third week.

- Workplace assessments are used extensively during clinical rotations.

- Academic and clinical coaches are used to monitor and guide student progress during the 4 years.

- Videotaped student encounters with standardized patients and OSCEs are used during the first 2 years.

- Specific learning objectives are required for every curricular learning session and are provided to guide student learning

Acad Med. 2020;95:S547.

doi: 10.1097/ACM.0000000000003318

Copyright (C) 2020 by the Association of American Medical Colleges

Year school was established: 2016.

School URL: https://medicine.wsu.edu. and design examination questions. Learning objectives are screened and edited by the curriculum associate dean.

- All students must pass/demonstrate all 13 AAMC Entrustable Professional Activities prior to graduation.

\section{Pedagogy}

- Most education during years 1 and 2 is through case-based and small-group learning.

- Some lecture sessions still occur, but faculty are highly encouraged and supported to use engaged teaching/learning strategies. Large-group session attendance is optional.

- Preceptors are used for all clinical learning, with some resident involvement, but primarily direct teaching by preceptors in all disciplines. A large percentage of clinical teaching occurs in the ambulatory setting.

- Standardized and actual patients are used for skills building.

- High-fidelity simulators are used to teach many types of clinical skills.

- Students are given ample opportunities for self-directed learning.

\section{Curricular Governance}

The college of medicine gives responsibility for curriculum design to a close collaboration between the associate deans for curriculum and assessment and the Curriculum Committee. The Curriculum Committee has several subcommittees that do detailed analysis of course evaluations and other data to help advise the Curriculum Committee on potential refinements and evolutionary changes to the curriculum. Students participate as members on all these committees, as well as on the Curriculum Committee. The faculty are responsible for serving on various subcommittees of the Curriculum Committee, as well as on the full committee. They are also charged with delivering the curriculum to the students.

The vice dean for student and faculty experience oversees the associate dean for faculty affairs and associate dean for curriculum, as well as the senior associate dean for admissions, student affairs and inclusion, allowing for a strong collaboration among these 3 offices, as well as with the associate dean for assessment and evaluation. Faculty development is coordinated with close guidance by chair of the major department in which faculty are appointed, as well as with the curriculum dean and the associate deans at each of the clinical campuses.

\section{Regional Medical Campuses}

The college has campuses in Everett, Spokane, Tri-Cities, and Vancouver, Washington. One-quarter of each class is assigned to each campus. The foundation sciences phase during years 1 and 2 is delivered at the Spokane campus, while all of the clinical learning takes place at a student's assigned campus, other than away rotations during the fourth year. 


\section{Marshall University Joan C. Edwards School of Medicine}

Nitin Puri, MD, PhD, and Bobby Miller, MD

\section{Medical Education Program Highlights}

Marshall University's Joan C. Edwards School of Medicine (JCESOM) is a state-supported, community-based medical school established in 1977. Our faculty and administration are committed to providing high-quality medical education and graduate training to meet the changing health care needs of our nation's citizens. We specialize in rural health issues as expressed in special efforts to recruit students from rural West Virginia and place graduates in clinical practice in rural areas. Additionally, because we believe that a diverse population offers a rich environment that leads to greater knowledge, understanding, acceptance, and mutual respect, we promote an inclusive environment by attracting, recruiting, and retaining individuals who represent varying backgrounds and perspectives.

JCESOM offers an integrated, competency-based MD curriculum with preclinical and clinical phases. Our core institutional competencies were adopted by the Curriculum Committee (CC) in 2012 and are based on the ACGME domains of competency:

- Professionalism

- Interpersonal and communication skills

- Patient care

- Practice-based learning and improvement

- Systems-based practice

- Medical knowledge

See Table 1—Core Competencies and Assessment Framework.

The preclinical phase of the curriculum is predominantly classroom- and clinical lab-based pedagogy centered around medical knowledge, patient care, and interpersonal and communication skills competencies. Students learn the normal structure and function of the body, and normal clinical exam in their first year. The second-year curriculum builds the concepts of diseases and therapeutics on the foundations of year 1 . Year 3 of the MD curriculum includes 6 required clinical rotations in 7 major clinical specialties, and year 4 is centered around career development and away rotations. Fourth-year requirements include emergency medicine, subinternship, and ICU.

Acad Med. 2020;95:S548-S551.

doi: 10.1097/ACM.0000000000003378

Copyright (C) 2020 by the Association of American Medical Colleges

Supplemental digital content for this article is available at http://links.Iww.com/ ACADMED/A882.

Year school established: 1977.

School URL: https://jcesom.marshall.edu.

\section{Curriculum}

Curriculum changes since 2010

Programmatic evaluation and continuous quality improvement are cornerstones of our curricular process. The MD curriculum at JCESOM was last modified in 2011 when we transitioned from a disciplines-based curriculum to the current organ systems-based $2 \times 2$ curriculum. In spite of the success rates of the current curriculum, the CC sought a major curricular redesign with the following objectives:

- Improved alignment of institutional competencies, course/ clerkship objectives, and individual assessments

- Cultivation of a student-driven learning environment with development of lifelong learning skills in our graduates

- True, systems-based, vertical integration of the preclerkship curricula with emphasis on active and self-directed learning pedagogies

- Adopt a model of programmatic assessment with a shift from assessment of learning to assessment for learning

\section{Table 1}

\section{Core Competencies and Assessment Framework}

\section{Institutional}

competency

Interpersonal and communication skills

Medical knowledge

Professionalism

Systems-based practice

learning

\section{Assessment framework}

Written, narrative feedback in the preclerkship and clerkship courses

OSCES

Peer-peer feedback in group activities

Direct observation in simulated or patientbased clinical settings

A combination of low-stakes and high-stakes assessments developed in-house

Customized or shelf NBME examinations Written, narrative feedback in the preclerkship and clerkship courses

OSCES

Peer-peer feedback in group activities Direct observation in the learning environment or simulated and patient-based clinical settings

Patient care OSCE

Narrative evaluation at midpoint and end of each clinical rotation

Practice-based $\quad$ Portfolio-based evaluation and feedback

Narrative feedback from faculty and residents in preclerkship and clerkship phase of the curriculum

Summer research and mentor feedback Narrative feedback from faculty and residents in preclerkship and clerkship phase of the curriculum 
- Curtail redundancies in the classroom-based curriculum and redistribute time to the clerkship phase for increased opportunities of clinical exposure and career electives

An extensive, collaborative effort of students and the faculty has led to the proposal and approval of the revised MD curriculum rolling out in July 2020.

See Supplemental Digital Appendix 1-Legacy Curriculum Map—at http://links.lww.com/ACADMED/A882.
See Supplemental Digital Appendix 2-Revised Curriculum Map—at http://links.lww.com/ACADMED/A882.

\section{Assessment}

See Figure 1-Programmatic assessment in the preclerkship phase.

\section{Pedagogy}

Pedagogies in the MD curriculum have evolved significantly over the past 10 years and continue to do so. Our classroom-based

\begin{tabular}{|c|c|c|c|c|c|}
\hline Week 1 & \multirow{2}{*}{$\begin{array}{c}\text { Low stakes } \\
\text { assessment - } \\
\text { quiz/TBLs/group } \\
\text { cases }\end{array}$} & Week 2 & \multirow{2}{*}{$\begin{array}{c}\text { Low stakes } \\
\text { assessment - } \\
\text { quiz/TBLs/group } \\
\text { cases }\end{array}$} & Week 3 & \multirow{2}{*}{$\begin{array}{c}\text { Medium stakes } \\
\text { assessment - } \\
\text { MK and CS }\end{array}$} \\
\hline $\begin{array}{l}\text { Learning } \\
\text { activities }\end{array}$ & & $\begin{array}{l}\text { Learning } \\
\text { activities }\end{array}$ & & $\begin{array}{l}\text { Learning } \\
\text { activities }\end{array}$ & \\
\hline \multicolumn{6}{|c|}{ Formative feedback } \\
\hline Week 4 & \multirow{2}{*}{$\begin{array}{c}\text { Low stakes } \\
\text { assessment - } \\
\text { quiz/TBLs/group } \\
\text { cases }\end{array}$} & Week 5 & \multirow{2}{*}{$\begin{array}{c}\text { Low stakes } \\
\text { assessment - } \\
\text { quiz/TBLs/group } \\
\text { cases }\end{array}$} & Week 6 & \multirow[b]{2}{*}{$\begin{array}{c}\text { Medium stakes } \\
\text { assessment - } \\
\text { MK and CS }\end{array}$} \\
\hline $\begin{array}{l}\text { Learning } \\
\text { activities }\end{array}$ & & $\begin{array}{l}\text { Learning } \\
\text { activities }\end{array}$ & & $\begin{array}{l}\text { Learning } \\
\text { activities }\end{array}$ & \\
\hline \multicolumn{6}{|c|}{ Formative feedback } \\
\hline
\end{tabular}

\section{Midpoint feedback}

\begin{tabular}{|c|c|c|c|c|c|}
\hline Week 7 & \multirow{2}{*}{$\begin{array}{c}\text { Low stakes } \\
\text { assessment - } \\
\text { quiz/TBLs/group } \\
\text { cases }\end{array}$} & Week 8 & \multirow{2}{*}{$\begin{array}{c}\text { Low stakes } \\
\text { assessment - } \\
\text { quiz/TBLs/group } \\
\text { cases }\end{array}$} & Week 9 & \multirow{2}{*}{$\begin{array}{c}\text { Medium stakes } \\
\text { assessment - } \\
\text { MK and CS }\end{array}$} \\
\hline $\begin{array}{l}\text { Learning } \\
\text { activities }\end{array}$ & & $\begin{array}{l}\text { Learning } \\
\text { activities }\end{array}$ & & $\begin{array}{l}\text { Learning } \\
\text { activities }\end{array}$ & \\
\hline \multicolumn{6}{|c|}{ Formative feedback } \\
\hline
\end{tabular}

\begin{tabular}{|c|c|c|c|c|c|}
\hline $\begin{array}{c}\text { Week } \\
10 \\
\end{array}$ & \multirow{2}{*}{$\begin{array}{c}\text { Low stakes } \\
\text { assessment - } \\
\text { quiz/TBLs/group } \\
\text { cases }\end{array}$} & $\begin{array}{c}\text { Week } \\
11\end{array}$ & \multirow{2}{*}{$\begin{array}{c}\text { Low stakes } \\
\text { assessment - } \\
\text { quiz/TBLs/group } \\
\text { cases }\end{array}$} & $\begin{array}{c}\text { Week } \\
12 \\
\end{array}$ & \multirow{2}{*}{$\begin{array}{c}\text { Medium stakes } \\
\text { assessment - } \\
\text { MK and CS }\end{array}$} \\
\hline $\begin{array}{l}\text { Learning } \\
\text { activities }\end{array}$ & & $\begin{array}{l}\text { Learning } \\
\text { activities }\end{array}$ & & $\begin{array}{l}\text { Learning } \\
\text { activities }\end{array}$ & \\
\hline \multicolumn{6}{|c|}{ Formative feedback } \\
\hline
\end{tabular}

\begin{tabular}{|c|c|c|}
\hline $\begin{array}{c}\text { Week } \\
13\end{array}$ & $\begin{array}{c}\text { Low stakes } \\
\text { assessment }- \\
\text { Learning } \\
\text { activities }\end{array}$ \\
& $\begin{array}{c}\text { Wuiz/TBLs/group } \\
\text { cases }\end{array}$ & Reviews and prep time 15 \\
\hline
\end{tabular}

High stakes assessment NBME/OSCES

Figure 1 Programmatic assessment in the preclerkship phase. 
pedagogy has shifted from predominantly lectures to active learning didactics, including case-based instruction, large- and small-group discussions, and peer-to-peer teaching. A significant portion of the curriculum is student-driven, independent, and self-directed learning. The clerkships use both ambulatory and inpatient settings to train our students.

The revised curriculum will use team-based learning and flipped classroom as central pedagogies in the classroom. Independent learning and self-directed learning are planned for out-of-class learning. Simulated clinical settings, patient and community panels, and peer-to-peer education will cover non-medicalknowledge competencies. The clerkship phase of the curriculum will continue to use ambulatory and inpatient settings to train students.

Several longitudinal themes are part of the MD curriculum at the JCESOM and are further expanded in the revised curriculum. These include clinical and translational sciences; medical ethics; radiology curriculum; evidence-based medicine; and environmental, nutritional, and social aspects of medicine.

\section{Clinical experiences}

The clerkship curriculum uses local and regional hospitals and variety of ambulatory care settings for student training. Students are first exposed to clinical experiences in the first year of the MD curriculum. First- and second-year students are matched with clinical mentors of their specialty of interest and are required to complete 12 hours of shadowing experience in each year.

See Table 2-Clinical Sites.

\section{Curricular Governance}

The CC, an elected body of basic sciences and clinical faculty and student representatives from each year of the MD curriculum, oversees and manages the MD curriculum.

The CC is responsible for a wide range of issues, including:

- Defining and adopting program objectives

\section{Table 2}

\section{Clinical Sites}

\begin{tabular}{|c|c|}
\hline Facility & Type of site \\
\hline Cabell Huntington Hospital & Inpatient \\
\hline St. Mary's Medical Center & Inpatient \\
\hline VAMC & Inpatient \\
\hline River Park & Inpatient \\
\hline Logan Regional Medical Center & Inpatient \\
\hline University hospital clinic & Ambulatory \\
\hline Community hospital clinics & Ambulatory \\
\hline Rural health centers & Ambulatory \\
\hline Private physician offices & Ambulatory \\
\hline Community health centers & Ambulatory \\
\hline
\end{tabular}

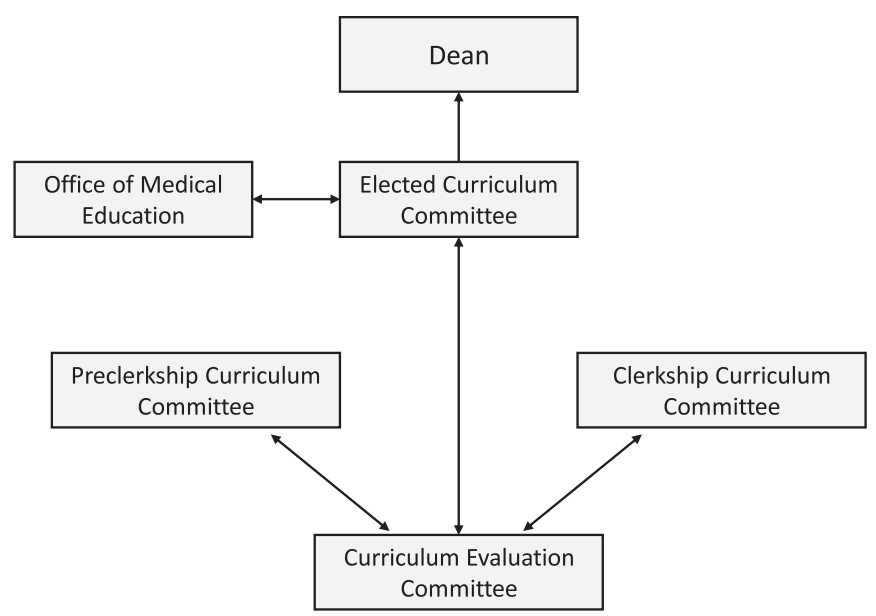

Figure 2 Curriculum committee.

- Defining minimum standards for graduation and graduation requirements

- Sequencing of the various segments of the curriculum, both within and across the academic periods of study

- Advising each course and clerkship on appropriate pedagogies and assessments best suited to meet the program objectives (institutional competencies)

- Ongoing evaluation of course and program effectiveness toward meeting the overall institutional outcomes

The CC is served by 3 subcommittees:

- The Preclerkship Curriculum Committee (PCC) oversees the organization and management of the preclerkship phase of the MD program, consisting of 10 integrated courses. The committee comprises all course directors, who are all voted on and approved by the committee itself. First- and secondyear student representatives sit on the committee. This committee meets monthly and minutes are posted online. All decisions of the PCC are debated and voted on by the CC.

- The Clerkship Curriculum Committee (CCC) oversees the organization and management of all required and elective clinical clerkships. The committee is made up of the clerkship directors and clerkship coordinators. The directors are all appointed members. Student representatives from year 3 and year 4 sit on this committee. Directors, coordinators, and students are voting members. The committee meets monthly and minutes are recorded. All decisions of the CCC are debated and voted on by the CC.

- The Curriculum Evaluation Committee (CEC) oversees programmatic evaluation and the adequacy of alignment of course and clerkship objectives with the institutional objectives. The CEC meets once a month to monitor the curriculum. This committee reviews the activities of all teaching units and determines whether or not the units are complying with the curricular goals and objectives. This subcommittee sends an annual questionnaire to block and clerkship directors who respond by describing the activities in their units of the curriculum. The subcommittee reviews these 


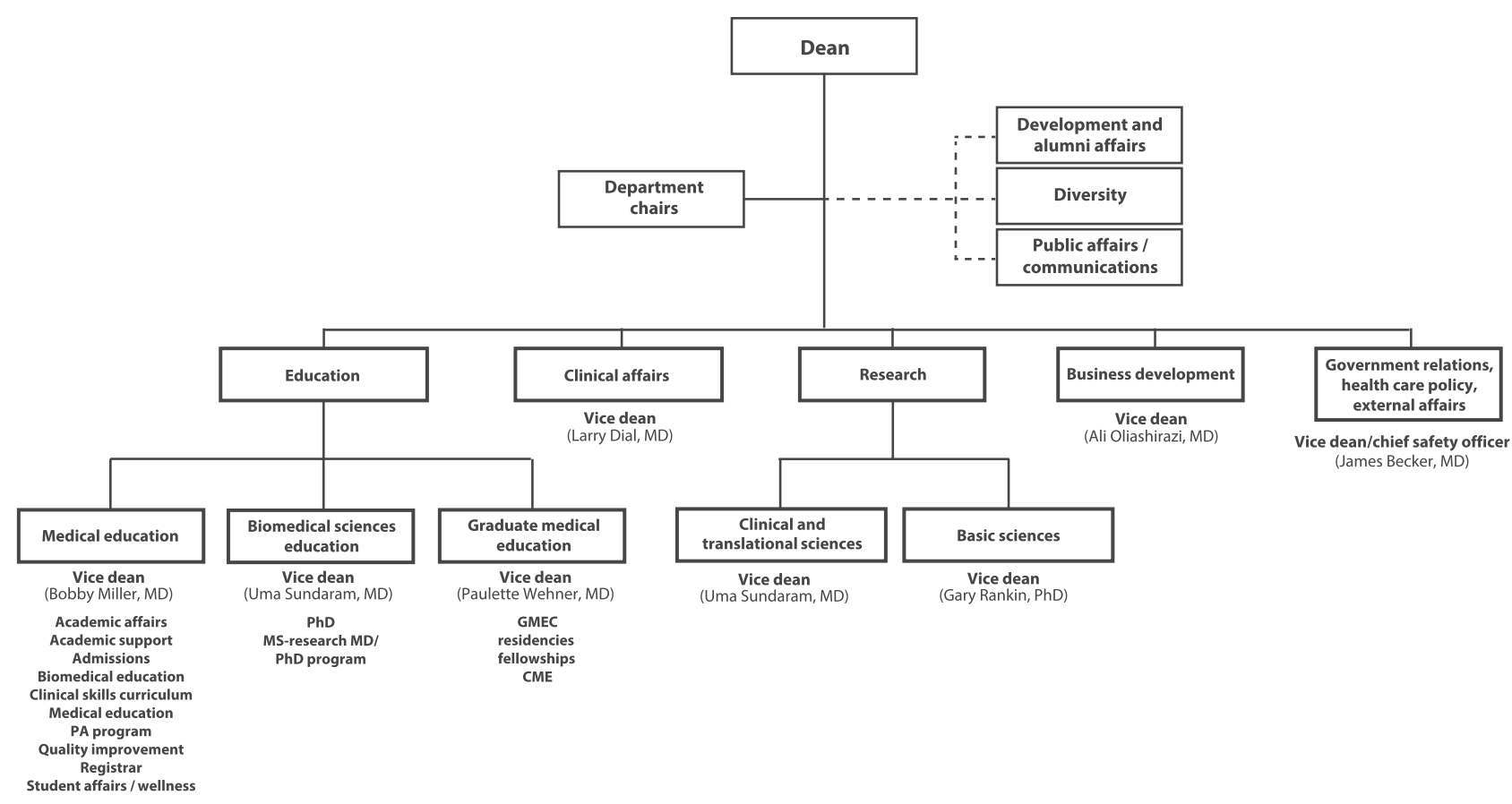

Support Staff

Figure $\mathbf{3}$ Organizational chart.

reports and makes recommendations that are reported directly to the CC. The CC discusses the report and recommendations and moves them along, intact or amended, to the block/ clerkship directors for appropriate amendments of their respective courses.

See Figure 2-Curriculum committee.

\section{Education Staff}

The Office of Medical Education (OME) supports the CC and all its subcommittees in the organization, delivery, and evaluation of the MD curriculum. The vice dean of medical education leads the OME and reports directly to the dean of JCESOM. The vice dean is supported by the associate dean of medical education and the assistant dean of academic affairs. The only decentralized component is budgets for teaching. Those are managed by the various departments. The OME is also responsible for providing administrative and academic support to the CC. The OME, with support from the chief information officer, develops and maintains tools for oversight of the curriculum including our learning management system and our assessment system(s). The OME oversees all aspects of the medical student experience including admissions, student affairs, medical education, and academic support services. The office also helps direct a combined BS-MD program and a master's program in biomedical sciences.

See Figure 3-Organizational chart.

\section{Faculty Development and Support in Education}

Faculty development is overseen by the associate dean for faculty advancement, who reports to supervisors outside the OME. Teaching and educational activities are a significant portion of the promotion and tenure process. These activities are reviewed with the faculty member by their department chair. We also have an academy called PACE (Promoting an Academic Community of Excellence). Its participants including medical school faculty, PA school faculty, and resident physicians. 


\title{
West Virginia University School of Medicine
}

\author{
Jason S. Hedrick, MA, Scott Cottrell, EdD, Karen Woodfork, PhD, and Norman D. Ferrari III, MD
}

\section{Medical Education Program Highlights}

West Virginia University School of Medicine (WVU SOM) has made major strides in the last decade in developing innovative educational experiences. An example is the implementation of 8 curricular threads that ensure deliberate vertical integration of content throughout the curriculum. Threads such as ultrasound have leveraged innovative technology to prepare learners for future practice. Diversity, inclusion, and health care disparities threads prepare graduates to treat patients from all backgrounds. The creation of the Mountaineer Accelerated Track To Enter Residency (MATTER) Track allows second-year students to apply for an accelerated curriculum that guarantees a residency position at 1 of 3 clinical campuses.

\section{Curriculum}

\section{Curriculum description}

See Supplemental Digital Appendix 1-Curriculum Map-at http://links.lww.com/ACADMED/A927.

\section{Curriculum changes since 2010}

The Curriculum Committee approved a new curriculum model with anticipated implementation in fall of 2020. The new curriculum seeks to:

- Expand content in emerging fields with increasing relevance for future practice and abridge content areas

- Rearrange content so normal physiology is integrated with organ system-based units

- Introduce content traditionally taught in the second year (microbiology/immunology, pathology, and pharmacology) to first-year foundations courses

- Use distance learning so students may engage in longer selective experiences between first and second years

- Realign clinical skills and basic sciences content between concurrent courses

- Reduce scheduled contact time to an average 18-20 hours/week

- Allow students to enter clerkships more expediently

\section{Medical education program objectives}

WVU SOM program objectives are based on the ACGME domains of competence. The SOM adopted the ACGME core competencies and program-level objectives (PLOs) in 2010.

Acad Med. 2020;95:S552-S555.

doi: 10.1097/ACM.0000000000003429

Copyright (C) 2020 by the Association of American Medical Colleges

Supplemental digital content for this article is available at http://links.Iww.com/ ACADMED/A927.

Year school was established: 1903.

School URL: https://medicine.hsc.wvu.edu.
The Curriculum Committee requires that all courses and clerkships develop learning objectives aligned with PLOs. This process ensures that courses and clerkships learning objectives are aligned with both institutional PLOs and attainment of core competencies. Each course and clerkship syllabus states learning objectives, their alignment with PLOs, and methods of evaluation. The committee reviewed and updated the PLOs in May 2014 and again in 2019. The most recent review was completed in conjunction with implementation of the new curricular model.

See Supplemental Digital Appendix 2-Program Objectives and Assessment Methods—at http://links.lww.com/ACADMED/A927.

\section{Assessment}

The Curriculum Committee referred to the MedBiquitous standardized vocabulary of assessment methods and encouraged course/clerkship directors to explore new ways to capture evidence of student learning and its development. We have made significant strides in furthering portfolio-based assessments. Academic advisors are leveraging this system to collect data and samples of students' academic and professional performance. Students submit annual reflections about their goals and career aspirations. Advisors couple these reflections with other data such as NBME examinations, narrative assessments, and performance-based assessments, to help students identify fourthyear electives needed to address any deficiencies in the core competencies and provide a better transition to GME.

\section{Parallel curriculum or tracks}

- The culinary and lifestyle medicine track aims to increase the number of physicians who have an understanding and appreciation of nutrition, food science and preparation, and lifestyle management. In the fourth year, students may sit for the national Culinary Medicine Certificate Exam.

- The global health track provides additional training for current students and also attracts students with an interest in global health. Graduating students are eligible to sit for the American Society of Tropical Medicine and Hygiene national exam.

- The rural health track begins the summer before program matriculation with a rural shadowing experience, followed by subsequent experiences during clerkships culminating with 2 months rotating in a rural medically underserved area of the state during the fourth year.

- The MATTER track provides students with a fast-track curriculum option to graduate earlier. The program trims off 1 semester and transitions students to select residencies at any of WVU's clinical campuses. MATTER students complete the curriculum in 137 weeks as opposed to 153 weeks, with no additional or modified learning outcomes. This track seeks to present a career option for students who are committed to a MATTER specialty, address student debt by minimizing 
costs of medical education without losing academic quality, efficiently transition medical students into a residency program, and attract talented students to become resident physicians serving the needs of our communities.

\section{Pedagogy}

The SOM uses a broad range of pedagogical approaches, including:

- Problem-based learning: This is a case-based small-group course using peer teaching and requiring students to develop learning objectives.

- Public health: Students perform a self-directed learning exercise that includes locating and assessing a research article, presenting it online, and engaging peers in their presentation.

- Physical Diagnosis and Clinical Integration: This course sequence offers innovative simulation and standardized patient encounters including a male urologic teaching assistant and a gynecologic teaching assistant experience, in which specially trained standardized patients teach students proper exam techniques. These experiences occur in the David and Jo Ann Shaw Center for Simulation Training and Education for Patient Safety, accredited by the Society for Simulation in Healthcare in all 4 areas, a designation held by fewer than 25 of the 700 accredited centers.

- The third-year clerkships offer robust simulation experiences. Surgery offers a lines and tubes experience to help students understand proper utilization of the many surgical implements they will encounter.

The Curriculum Committee has made an effort to reduce contact hours from about 22-24 contact hours a week to 20 hours per week, averaged across the semester. The reduction should further student wellness and enhance self-directed learning. Limiting the amount of contact time has challenged faculty to reconsider traditional pedagogical practices, for example, converting traditional 50-minute lectures to a series of 15- to 20-minute recorded didactics coupled with team-based learning, case-based large-group discussions, or simulations.

\section{Clinical experiences}

After completing the pre-Step 1 curriculum and taking the USMLE Step 1 examination, students transition to regional campuses. Approximately half of students remain in Morgantown, with the majority of their clinical experiences completed at WVU Hospitals in Morgantown. About 40\% of students transition to the Charleston campus and complete most of their required rotations at the Charleston Area Medical Center, composed of 3 hospitals and ancillary clinics. Both the Morgantown and Charleston campuses contain the state's only Level 1 trauma centers. Approximately 10\% of each class transitions to Eastern and spend the majority of required clinical experiences at 2 rural hospitals in the WVUMedicine system.

\section{Required longitudinal experiences}

Eastern campus students rotate through longitudinal experiences divided into 2 modules. One includes family medicine, pediatrics, and obstetrics-gynecology clerkships; the other includes internal medicine, psychiatry, neurology, and surgery.
The Eastern Division, in the eastern panhandle of the state, is associated with smaller, community-based hospitals. The integrated curriculum at Eastern allows for greater utilization of continuity clinic. Despite these differences, core content, learning outcomes, and required clerkship procedures and encounters remain the same across the 3 campuses, and students perform at an equal caliber to Morgantown and Charleston students on internal and national assessments.

\section{Clinical experience first encounter}

The SOM wastes no time integrating students into clinical experiences. Beginning in the first term, students in the Physical Diagnosis and Clinical Integration course complete 5 physician and 5 community shadowing experiences. A primary objective of the physician shadowing experience is to facilitate student comfortability in the clinical environment and around patients. Shadowing enables students to observe master clinicians and the communication skills they employ during encounters. Students can choose their community experiences from options including support groups, chaplaincy, community pharmacies, health departments, dental clinics, physical therapy, and other experiences.

\section{Required and elective community-based rotations}

- All third-year students complete 4 weeks of their family medicine clerkship with a community-based preceptor.

- All fourth-year students complete the rural community-based care rotation, which requires completion of 4 weeks at a community clinic or hospital in any discipline.

- Students complete a number of community-based shadowings early in their medical education.

- Two student organizations have been developed to serve locally underserved populations. The Multidisciplinary Un-Sheltered Homeless Relief Outreach of Morgantown and the Charleston Homeless and Street Medicine programs focus on outreach specific to local homeless populations.

- All students must complete 100 hours of community service/ service learning for graduation. Students embrace this expectation, often exceed the 100-hour requirement. The 2019 graduating class averaged more than 135 hours of service.

\section{Curricular Governance}

The Curriculum Committee is an autonomous decision-making body but relies on other organizational units for support. The Department of Medical Education provides administrative support for the committee including assessment resources such as experts in evaluation, curriculum mapping, and in general, educational expertise. The associate dean also interfaces with various groups to ensure potentially needed resources are taken into account and considered by individuals such as the associate dean for finance for things like the designation of new course or clerkship directors, expansion of threads, etc. Department leadership also serves as a representative and advocate for the MD curriculum at committees that address logistical issues such as management of academic space.

See Figure 1-Curricular governance. 


\section{Education Staff}

\section{Medical education leadership}

WVU SOM's Department of Medical Education provides administrative and academic support for the MD curriculum. The department's founding chair, Dr. Norman Ferrari, is also vice dean for education and academic affairs for the SOM. The department is composed of 3 divisions: CME, GME, and UME. A primary organizational function under the UME division is student services, under Dr. Scott Cottrell, associate dean for student services and curriculum. Student services provides various mechanisms and levels of support for students and delivery of the curriculum.

See Supplemental Digital Appendix 3-Organizational Chartat http://links.lww.com/ACADMED/A927.

\section{Department of Medical Education}

The Department of Medical Education serves a support role for UME, GME, and CME. Leaders of all 3 departmental divisions report directly to the vice dean. A primary function of UME is providing student services. An administrator with a background in social work directs the Office of Student Services, which helps ensure that wellness activities are closely aligned to support student needs.

Faculty of the Department of Medical Education includes educators with either primary or secondary appointments who have demonstrated commitment and expertise in medical education in areas such as curriculum design, teaching skills, medical education research, simulation, or assessment. It serves as an academic home for full-time faculty and community-based faculty physicians who contribute to the SOM's education mission.

\section{Faculty Development and Support in Education}

\section{Professional development for faculty as educators}

- Teaching Scholars Program: A 2-year longitudinal program for faculty members is offered by the Faculty Development Office.
- Summer Teaching Scholars Institute: The curriculum for the longitudinal Teaching Scholars Program is converted into a 5-day interdisciplinary emersion seminar.

\section{Role of teaching in promotion and tenure}

Quality of teaching is evaluated based upon student evaluations, peer evaluations, and evaluations by course directors. During promotion and tenure review, quantity and quality of teaching (and improvement) for the entire time at rank are considered. Outstanding faculty members are publicly recognized at the Dean's Awards for Excellence ceremony each spring. Additional programs celebrating teaching excellence and providing opportunities for developing teaching skills are operated by the Faculty Development Program, including the Academy of Excellence in Teaching and Learning, the Teaching Scholars Program, and the Summer Teaching Scholars Institute.

The WVU SOM Academy of Advisors mission is to "connect medical students with distinguished faculty who can offer advising, mentorship, and career development guidance." The academy is composed of SOM faculty across a number of disciplines from the 3 campuses, offering training sessions and regular meetings each semester. Guest speakers at Academy of Advisors meetings discuss topics relevant to advising, assessment, evaluation, teaching, and supervision of medical students.

\section{Regional Medical Campuses}

WVU SOM is composed of 3 total campuses: Morgantown, the main campus, which includes instruction of all 4 years of the curriculum; and 2 regional clinical campuses for the third- and fourth-year levels.

See Table 1-Regional Clinical Campuses.

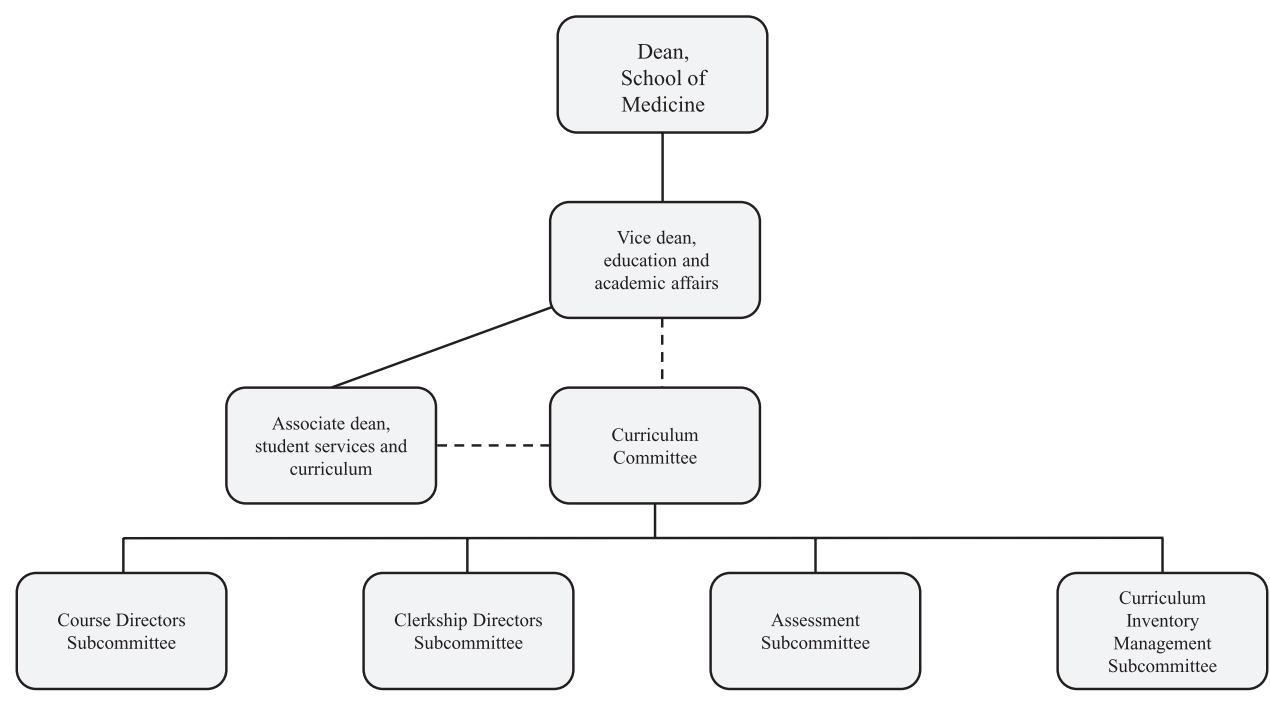

Figure 1 Curricular governance. 


\section{Table 1}

\section{Regional Clinical Campuses}

\begin{tabular}{|c|c|c|}
\hline Regional campus & Туре & $\begin{array}{l}\text { Student } \\
\text { enrollment }\end{array}$ \\
\hline $\begin{array}{l}\text { Eastern (Martinsburg, } \\
\text { West Virginia) }\end{array}$ & $\begin{array}{l}\text { Clinical campus } \\
\text { (years } 3 \text { and } 4 \text { ) }\end{array}$ & 12 per class \\
\hline $\begin{array}{l}\text { Charleston, West } \\
\text { Virginia }\end{array}$ & $\begin{array}{l}\text { Clinical campus } \\
\text { (years } 3 \text { and } 4 \text { ) }\end{array}$ & 40 per class \\
\hline
\end{tabular}

\section{Educational experiences across sites}

- All SOM required clerkships use a single online educational environment site, which presents a unified statement of the learning objectives and evaluation.

- Clerkship and rotation directors from the 3 campuses meet individually at least twice annually.

- The Assessment Subcommittee reviews evaluations from each clerkship and campus and designates remediation plans when any campus drift is identified.

- A single Committee on Academic and Professional Standards regularly reviews students' academic and professionalism performance for all campuses, which includes ensuring consistent and comparable grading methods and assessments. 


\section{Medical College of Wisconsin}

William J. Hueston, MD

\section{Medical Education Program Highlights}

The Medical College of Wisconsin (MCW) is one of the largest medical schools in the United States, enrolling an entering class of 249 students each year. The school's 3 campuses include the largest campus in Milwaukee where the School of Pharmacy, Master of Science in Anesthesia program, and Graduate School of Biomedical Sciences are located, and 2 regional campuses in Green Bay and Central Wisconsin (Wausau).

One of the unique features of the MCW structure is 2 community-based regional campuses that offer an accelerated medical school curriculum to all entering students so that they can complete their training in 133 weeks over 3 calendar years. Unlike other schools with accelerated tracks where students are guaranteed residency positions in selected specialties, students in the MCW accelerated tracks enter the Match in their third year and have competed successfully for residency spots, with an overall match rate of $98 \%$ for the first 3 cohorts who have graduated after 3 years. The school also offers students the opportunity to obtain combined degrees including an MD-PhD through the Medical Scientist Training Program, an MD-MS in 4 years, and an MD-MPH in 5 years.

A second unique feature of MCW is the Scholarly Pathways program, where students participate in 1 of 9 required "scholarly pathways" that allow learners to get in-depth knowledge and experience in a focused area. Students have a half day a week reserved for pathway activities, which include attending a large-group learning session monthly, self-directed learning through an individualized learning plan, and participation and completion of a scholarly project. Topic areas in Milwaukee for the program include bioethics and medical humanities, clinician-educator, clinical and translational research, global health, health systems management and policy, molecular and cellular research, quality improvement and patient safety, and urban and community health. Students at the regional campuses all participate in the pathway topic of physician in the community. As part of the Scholarly Pathways program, all students are required to complete and present their scholarly project before entering their final year of training.

Acad Med. 2020;95:S556-S558.

doi: 10.1097/ACM.0000000000003255

Copyright (C) 2020 by the Association of American Medical Colleges

Year school was established: 1893, as the Wisconsin College of Physicians and Surgeons School URL: https://www.mcw.edu.

\section{Curriculum}

\section{Curriculum description}

The MCW medical school curriculum is based on a traditional model of 2 years of foundational science followed by a year of clinical clerkships in 7 specialty areas and a final year of electives and required acting internships. The first 2 years also feature early clinical experiences that allow students to spend a half day every week with a clinician learning the basics of history taking and physical examination as well as the art of medicine.

Students in the regional campus accelerated programs begin their studies 6 weeks before students in Milwaukee, allowing them to complete their foundational clinical introduction course before starting foundational science classes. Students at these campuses engage in clinical clerkships over the summer between their first and second years, which allows time in the latter half of their third year for acting internships and electives. Students on these campuses can elect to extend to a fourth year of training; to date, $53 \%$ of students in the accelerated program have graduated after their third year.

\section{Curriculum changes since 2010}

As well as adding the 3-year regional campuses, the MCW curriculum has added several clinical experiences throughout the first 2 years of medical school and has integrated the foundational science teaching during the second year of medical school.

\section{Assessment \\ The MCW medical school global competencies are adapted from the AAMC's Physician Competencies Reference Set (2014), with some modifications to make these appropriate for medical students.}

MCW's student assessment strategies have been stable since 2010, although starting in 2019, students in the first 2 years of training are graded on a satisfactory/unsatisfactory scale rather than the previously used 5-tier grading system. Students in the first 2 years of medical school are assessed primarily with periodic multiple-choice examinations. Students in clinical rotations are assessed using a combination of clinical performance reviews by attending physicians and residents, scores on NBME subject examinations, or, in the case of family medicine and anesthesia, examinations developed by our own faculty and selected other factors such as reviews of history and physical examination report that vary according to the clerkship.

The MCW foundational sciences curriculum is primarily delivered through lectures and small-group anatomy laboratory sessions. All lectures are transmitted to the regional campuses in real time. Students may also access these lectures through livestreaming or at a later time through our digital capture system. 
The clinical clerkships all have 1 to 2 half days a week reserved for formal didactic instruction that supplements the clinical learning that students receive in the hospital or clinic setting.

In delivering the clinical curriculum, MCW partners with several regional medical centers in Milwaukee and across Wisconsin to offer students a rich clinical experience. In Milwaukee, the primary teaching sites for students are Froedtert Hospital and the Children's Hospital of Wisconsin, which share the Milwaukee Regional Medical Center campus with MCW along with the Zablocki Veteran's Administration Hospital. On the Green Bay campus, the primary clinical partners include the Bellin Health System, Hospital Sisters Health System, Prevea Health, ThedaCare, and the Ascension Health System, along with the Green Bay Veteran's Health Administration ambulatory site. The MCW-Central Wisconsin campus partners with a large number of medical groups spread across northern and central Wisconsin where students participate in the longitudinal integrated clerkship. These include hospitals and clinics affiliated with Aspirus Health, the Mayo Clinic, Gunderson Lutheran Health System, Hospital Sisters Health System, and the Oakleaf Surgical Center, among others.

MCW students on each of the campuses participate in a 9-month longitudinal rotation (clinical apprenticeship) early in the course of their training. This course pairs students with a physician for weekly 4 -hour sessions that introduce students to taking histories and performing physical examinations as well as the overall approach to patient care.

The MCW-Central Wisconsin campus also provides longitudinal experiences in the clinical years through a 12-month longitudinal integrated clerkship. Students begin their clerkship experience in the summer between their first and second years of medical school when they are placed at one of a number of sites across northern and central Wisconsin. Following a longitudinal specialty experience during the second year of medical school, students return to these sites in their third year to complete their clerkship training.

On the regional campuses, because students have an intensive clinical skills instruction course in the 6 weeks before students begin in Milwaukee, these students begin clinical experiences in early August at the same time they start their foundational science courses.

Like many medical schools, MCW continues to seek dedicated and talented community faculty members and clinical partners for our educational programs. These issues are faced by all 3 campuses where the pressures of clinical productivity and competition in the clinical arena have made volunteer teaching difficult for many community-based clinicians who are interested in contributing to the education of the next generation of physicians.

\section{Curricular Governance}

Governance and oversight of the MCW curriculum is provided by the Curriculum Evaluation Committee (CEC); slightly more than half of the members are elected through the MCW Faculty Council, with the remainder appointed by the dean of the medical school. The MCW student assembly also appoints 1 student from each class in Milwaukee and 1 student from each of the 2 regional campuses.

The CEC has 2 subcommittees, the M1-M2 Subcommittee and the M3-M4 Subcommittee. The subcommittees are primarily made up of faculty members teaching in the courses addressed by the subcommittees. Each subcommittee has student representatives enrolled in the academic years represented by the subcommittee.

\section{Education Staff}

Support for the medical school educational services is provided by a curriculum office with additional services contributed by the MCW Office of Academic Affairs. The curriculum office is overseen by the senior associate dean for medical education and staffed by the associate dean for curriculum, an assistant dean for basic science curriculum, and an assistant dean for clinical curriculum in Milwaukee. On the regional campuses, each campus is led by a campus dean and education is supported by an assistant dean for basic science curriculum and an assistant dean for clinical curriculum, who report to their campus dean. These teams are tasked with implementing the curriculum and provide direct oversight of the faculty.

The Office of Academic Affairs supports education for all MCW programs. In the Office of Academic Affairs, the Office of Educational Improvement includes teams that focus on support of educational information technology as well as instructional designers, interprofessional education support personnel, and a faculty development specialist. The Office of Assessment and Measurement provides analytics for examination support as well as medical educational research consultation. The Office of Academic Support and Enrichment provides student learning assessments, oversees student tutoring and peer-teaching programs, organizes national examination preparation sessions, and provides individualized student educational counseling. The Office of Academic Affairs also provides a career counselor who oversees a 4-year professional development curriculum, organizes mock-residency interviews, and provides individual student career counseling.

See Figure 1-Medical education leadership.

\section{Faculty Development and Support in Education}

Professional development for education is overseen by the Office of Educational Improvement. This office includes instructional designers who assist faculty in developing new course material and revamping previous material and in launching new courses. A faculty development staff individual coordinates monthly faculty development, which is also recorded and available to all faculty members through the faculty development website. The faculty development staff also maintain a "faculty toolbox" that provides educators with fundamental information regarding teaching and learning.

Additionally, all faculty members receive an evaluation dashboard annually that summarizes their teaching evaluations 


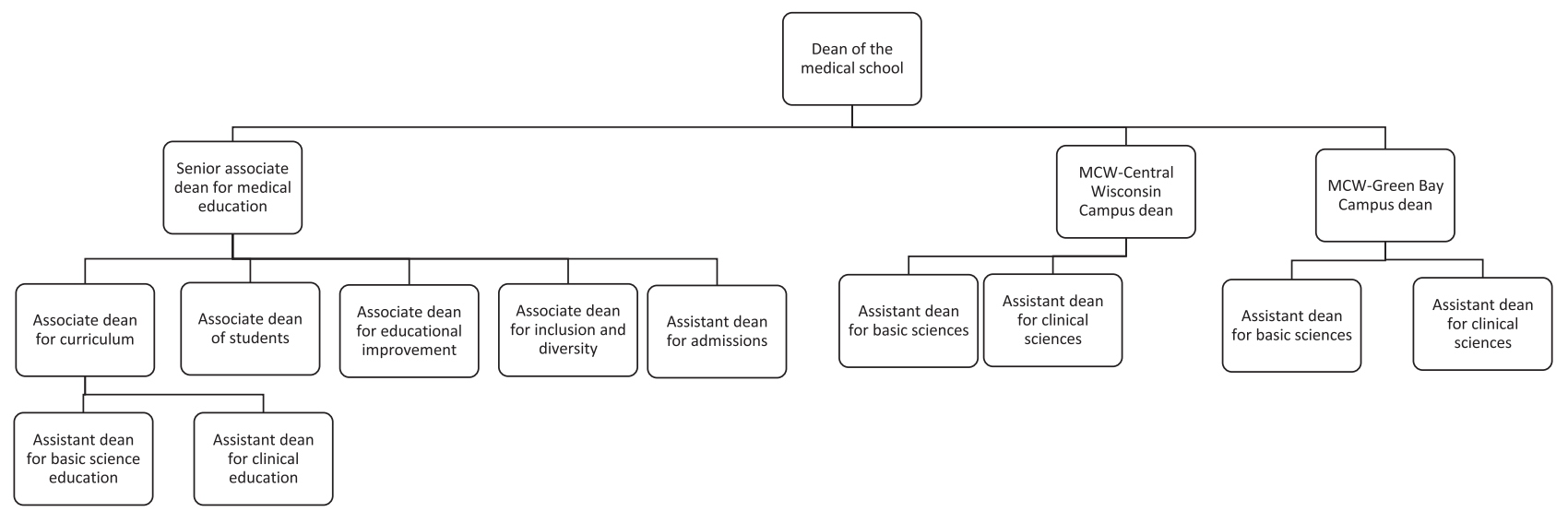

Figure 1 Medical education leadership.

from students. These evaluations are analyzed and any faculty member falling more than 1 standard deviation below the mean is provided with an expert review of their teaching conducted by a member of the Society of Teaching Scholars. Faculty members are also offered 1-on-1 coaching by a member of the society if they desire.

MCW has multiple faculty tracks. Educational contributions are considered for promotion on the clinician-educator/ educator pathway, traditional pathway, and the research pathway. For the latter 2 pathways, "education" is defined as contributing to medical student education or serving as a mentor for graduate students and postdoctoral fellows as well as contributing as a member of thesis or dissertation committees.

For tenure decisions, educational excellence is considered when it is a primary professional role for a faculty member and the faculty member can demonstrate superior teaching and

\section{Table 1}

Regional Medical Campuses

\begin{tabular}{|c|c|c|}
\hline $\begin{array}{l}\text { Regional } \\
\text { campus name }\end{array}$ & Type & $\begin{array}{l}\text { Student } \\
\text { enrollment }\end{array}$ \\
\hline MCW-Green Bay & $\begin{array}{l}\text { Offers all } 4 \text { years of } \\
\text { medical education with } \\
\text { all students starting in a } \\
\text { 3-year accelerated track }\end{array}$ & $\begin{array}{l}25 \text { students in each } \\
\text { entering class; total } \\
\text { enrollment in } 2019 \\
\text { was } 85\end{array}$ \\
\hline $\begin{array}{l}\text { MCW-Central } \\
\text { Wisconsin }\end{array}$ & $\begin{array}{l}\text { Offers all } 4 \text { years of } \\
\text { medical education with } \\
\text { all students starting in a } \\
\text { 3-year accelerated track }\end{array}$ & $\begin{array}{l}20 \text { students in each } \\
\text { entering class; total } \\
\text { enrollment in } 2019 \\
\text { was } 76\end{array}$ \\
\hline
\end{tabular}

scholarship in education compared with peers at MCW and other medical schools.

MCW has a medical school Society of Teaching Scholars that was established in the 1980s. The Society of Teaching Scholars provides support for teaching by providing teaching reviews as requested by the Office of Educational Improvement and offering coaching to new educators.

\section{Regional Medical Campuses}

See Table 1-Regional Medical Campuses.

\section{Educational experiences across sites}

Comparability of the educational experience across the 3 campuses is maintained by assuring that the objectives and assessment strategies are the same in all 3 sites. Students at the 2 regional campuses participate in the foundational sciences courses in the first 2 years using a distance education approach, which assures that the same material is taught in the same fashion among all the campuses. For clinical clerkships, MCW-Milwaukee and MCW-Green Bay have block rotations that are overseen by the same clerkship directors; MCW-Central Wisconsin employs a longitudinal clinical clerkship model that is based on the combined objectives of all 7 required clerkships at the other campuses.

\section{Initiatives in Progress}

The MCW medical school's major initiative is continued development of the accelerated 3-year campuses. To date, 2 classes have graduated from Green Bay and one class from Central Wisconsin, with residency match rates of $98 \%$ for those who finished in 3 years. 


\section{University of Wisconsin School of Medicine and Public Health}

Elaine M. Pelley, MD, Shobhina G. Chheda, MD, MPH, Christine S. Seibert, MD, Katharina S. Stewart, MD, William Aughenbaugh, MD, and Elizabeth M. Petty, MD

\section{Medical Education Program Highlights}

Since the last Snapshot in 2010, the University of Wisconsin School of Medicine and Public Health (UWSMPH) has implemented its most innovative curriculum change since the early 20th century. The ForWard curriculum, with its focus on competency-based education, self-directed learning, teamwork skills, and individual coaching, is delivered under new centralized institutional systems for governance and oversight, which facilitate continuous curricular improvements. This transformation has charted a new course for ensuring UWSMPH graduates are prepared to optimize the health of patients and populations for the twenty-first century and beyond.

Key features of the ForWard curriculum:

- 3-phase, 4-year, competency-based curricular model that horizontally, vertically, and developmentally integrates basic and clinical sciences with 10 longitudinal content threads

- Early clinical immersion in interprofessional teams starting in the first semester followed by ongoing interprofessional health learning

- Longitudinal coaching with each student to enhance mastery, based on program objectives

- Immersive parallel tracks in rural and urban health as well as $\mathrm{MPH}$ and PhD dual-degree programs available

- Statewide campus with 4 regional campus hubs in addition to central campus sites

\section{Curriculum}

\section{Curriculum description}

The ForWard curriculum, launched in 2016, fully integrates basic science, public health, and clinical science throughout medical students' education. ForWard is a 3-phase curriculum composed of integrated, multidisciplinary, thematic blocks within which all content threads are woven longitudinally:

- Phase 1 (3 semesters; 6 blocks): Core basic science content integrated with public health and clinical experiences

- Phase 2 (2 semesters; 4 blocks): Immersive clinical training with integrated public health and basic science content

- Phase 3 (3 semesters): Advanced specialty-specific experiences in clinical medicine, basic science, and public health

Acad Med. 2020;95:S559-S562.

doi: 10.1097/ACM.0000000000003394

Copyright (C) 2020 by the Association of American Medical Colleges

Supplemental digital content for this article is available at http://links.Iww.com/ ACADMED/A905.

Year school was established: 1907.

School URL: https://www.med.wisc.edu.
See Supplemental Digital Appendix 1-Curriculum

Description—at http://links.lww.com/ACADMED/A905.

\section{Curriculum changes since 2010}

The ForWard curriculum is the product of a multiyear curriculum transformation process that included input from hundreds of UWSMPH faculty, staff, and students. The school's graduation competencies (program objectives) were updated and revised with broad input. These competencies were used as the starting point for a formal backward design process to develop a horizontally and vertically integrated 3 -phase curriculum.

Each ForWard block was designed by a multidisciplinary faculty team. Before and during the design process, course and clerkship directors (integrated block leaders) met monthly for formal faculty development in educational best practices and to develop consensus on shared teaching and assessment toolboxes. This centrally coordinated process allows students to experience learning that is well-connected across all 4 years, with consistency in learning management systems, design, teaching modalities, and assessment methods across blocks. These educational leaders now compose the Curriculum Content Subcommittee, a standing subcommittee of the school's Educational Policy and Curriculum Committee, to ensure long-term curriculum coordination.

\section{Assessment}

The UWSMPH medical education program objectives are based on ACGME domains of competence. In the ForWard curriculum, phase-specific milestones and developmental benchmark scales were developed to ensure clear expectations for all students, at every level of their education, with respect to each of the 20 program objectives. To ensure specific and reliable outcome measures, students are evaluated via assessment rubrics based directly on the milestones and benchmark scales. Additional assessment tools (e.g., multiple-choice assessments, focused assignments) are linked to school competencies via course-level and learning activity-level objectives.

See Supplemental Digital Appendix 2-Graduation Competencies and Assessment Methods—at http://links.lww. com/ACADMED/A905.

\section{Parallel curriculum or tracks}

UWSMPH has 2 parallel tracks, the Wisconsin Academy for Rural Medicine (WARM) and the Training in Urban Medicine and Public Health (TRIUMPH) programs. WARM prepares students for rural medicine practice and to improve health in rural Wisconsin communities. TRIUMPH prepares students to become community-engaged physician leaders who will address health disparities in disadvantaged urban communities. After Phase 1 completion in Madison, students in these tracks relocate to a regional academic campus in either rural Wisconsin 
(WARM) or Milwaukee (TRIUMPH) to complete Phases 2 and 3. In addition, UWSMPH has formal MD-PhD and MD-MPH dual-degree programs.

\section{Pedagogy}

A teaching toolbox was developed for use by all blocks across the ForWard curriculum based on educational best practices.

In Phase $1, \leq 30 \%$ of instructional time is lecture based. Online learning modules, large-group (36 students in 6 groups/room) case-based learning, and small-group (8 students/room) selfdirected, problem-based learning each constitute $\sim 15 \%$ of instructional time. Laboratories, clinical skills with standardized patients, and clinical experiences (mostly ambulatory) each make up approximately $5 \%-10 \%$ of Phase 1 instructional time.

In Phase $2,80 \%$ of instructional time is spent in immersive clinical experiences, equally divided between inpatient and ambulatory settings. The remainder consists of structured discussions divided primarily between large-group and smallgroup case-based learning sessions that require preparatory readings/online activities and variably include simulation, standardized patients, and peer teaching.

In Phase 3, distribution of clinical versus didactic time varies, depending on the block (rotation). The inpatient acting internship and ambulatory acting internship are each $90 \%$ clinical activity and 10\% lecture- and case-based learning activities. Public health and basic science selectives may include synchronous and/or asynchronous teaching modalities, projects, presentations, discussions, clinical activities, and lab sessions. The internship preparatory course allows students to synthesize material with standardized patients, simulation, clinical skills, and group discussions.

The core curriculum, pedagogical approaches, and assessments for students in WARM and TRIUMPH are the same as that of the traditional MD program.

\section{Clinical experiences}

Students are paired with a physician preceptor at the beginning of Phase 1 with whom they complete a longitudinal clinical experience ( 15 sessions over 18 months) focused on practicing early clinical skills and contributing to an interprofessional team clinic-based quality improvement project.

Phase 2 consists of four 12-week integrated clinical blocks. In the acute care block (inpatient medicine, neurology, psychiatry, and emergency medicine), students have a consistent preceptor across all 12 weeks for case-based learning and bedside teaching. The chronic and preventive care block (ambulatory internal medicine, family medicine, neurology, and psychiatry/ behavioral health) also provides consistent ambulatory clinical teachers across the block. In the surgical and procedural care block (general surgery, surgical subspecialties, anesthesia, and other procedural subspecialties), students follow at least 1 patient longitudinally through anesthesia care, surgery, and postoperative care. The care across the life cycle block integrates pediatrics, obstetrics-gynecology, and geriatrics in various care settings, including a longitudinal clinic in pediatrics and in obstetrics-gynecology.

Phase 3 required experiences include a 4-week ambulatory acting internship in one of many statewide outpatient clinics, a 4-week inpatient acting internship, public health selectives (4 credits), basic science selective ( 4 credits), and an internship preparatory course ( 3 credits). Basic science and public health selectives offer a range of unique ways to engage in deep learning in areas related to students' field of future interest.

Required clinical experiences in Phases 2 and 3 occur at affiliated institutions throughout Wisconsin, including the local tertiary care, academic medical center's inpatient and ambulatory sites, as well as rural and urban community-based hospitals and ambulatory practices. These statewide campus opportunities help minimize any capacity issues created by having second-, third-, and fourth-year students concurrently doing clinical rotations.

\section{Curricular Governance}

The Educational Policy and Curriculum Committee is the primary curricular governing body and oversees 3 standing subcommittees that continuously monitor specific areas of the medical education program: Medical Student Subcommittee, Curriculum Content Subcommittee, and Assessment Subcommittee. Though not part of formal governance, the Health Equity Activation Team, composed of faculty and students, serves in a key advisory role to optimize diversity and inclusion throughout the curriculum.

Curricular governance is centralized, including all assessment and academic support, but departments and sites are responsible for recruiting and supporting teachers to assist in delivery of content, bedside teaching, and clinical assessment. The quantity of teaching provided by faculty and the number of learner-weeks are tracked centrally and used to allocate funding to departments and sites, respectively, to support teaching.

\section{Education Staff}

The Medical Education Office (MEO) is housed in the Office of Academic Affairs, which provides oversight to all health professions degree programs and graduate medical education as well as academic campuses. The associate dean for medical student education and services is responsible for the MD curriculum and related student programs through their leadership team, which includes the assistant dean for admissions, associate dean for student services, and associate dean for medical education.

Key faculty leaders within the MEO reporting to the associate dean of medical education include the 3 phase directors and the director of the longitudinal teacher/coach program. Each phase director oversees a team of faculty who direct required courses and clerkships in their respective phases. All faculty teaching roles, including faculty participants in the longitudinal teacher/ coach program, are financially supported through the MEO. The MEO also includes professional and support staff. The director 
of longitudinal curriculum and instruction oversees faculty leads (thread directors) in key content areas to ensure longitudinal integration. The director of assessment and analytics oversees the clinical teaching and assessment center, the standardized patient program, and staff to oversee testing. Additional staff support the implementation of all required courses under the leadership of their respective faculty leads. The MEO is an administrative office; teaching faculty have academic appointments within basic science or clinical departments.

See Figure 1-Institutional leadership structure.

\section{Faculty Development and Support in Education}

Numerous faculty development opportunities are available through various UW-Madison campus offices and UWSMPH. For example, Medical Education Day, held annually at UWSMPH, engages over 200 faculty, staff, fellows, and graduate students to explore innovations in medical education.

The associate dean for faculty affairs and development, in partnership with the Office of Continuing Professional Development in the Office of Academic Affairs, oversees professional development opportunities for faculty on all the
UWSMPH campuses, across all missions and departments, and across all faculty tracks. Together with the Schools of Pharmacy and Nursing, they established the interprofessional Clinical Teaching and Learning Community for faculty and academic staff, affiliated with both the UW-Madison Teaching Academy and the Interprofessional Continuing Education Partnership. The UW-Madison Teaching Academy is composed of faculty, instructional staff, and graduate students across the main university campus who have been recognized by peers for sustained teaching excellence. This academy works to promote, recognize, and support excellence in teaching and learning. The Interprofessional Continuing Education Partnership includes faculty and staff from the UW health professions schools and provides joint accreditation for faculty and staff development activities across health professions.

Teaching may be used as an area of excellence or significant accomplishment for academic promotion at UWSMPH. Candidates must demonstrate a record of achievement in education of the highest caliber. Examples of scholarly achievement include publication/presentation of educational scholarship, development/dissemination of a new curriculum, or creation of innovative teaching or assessment methods.

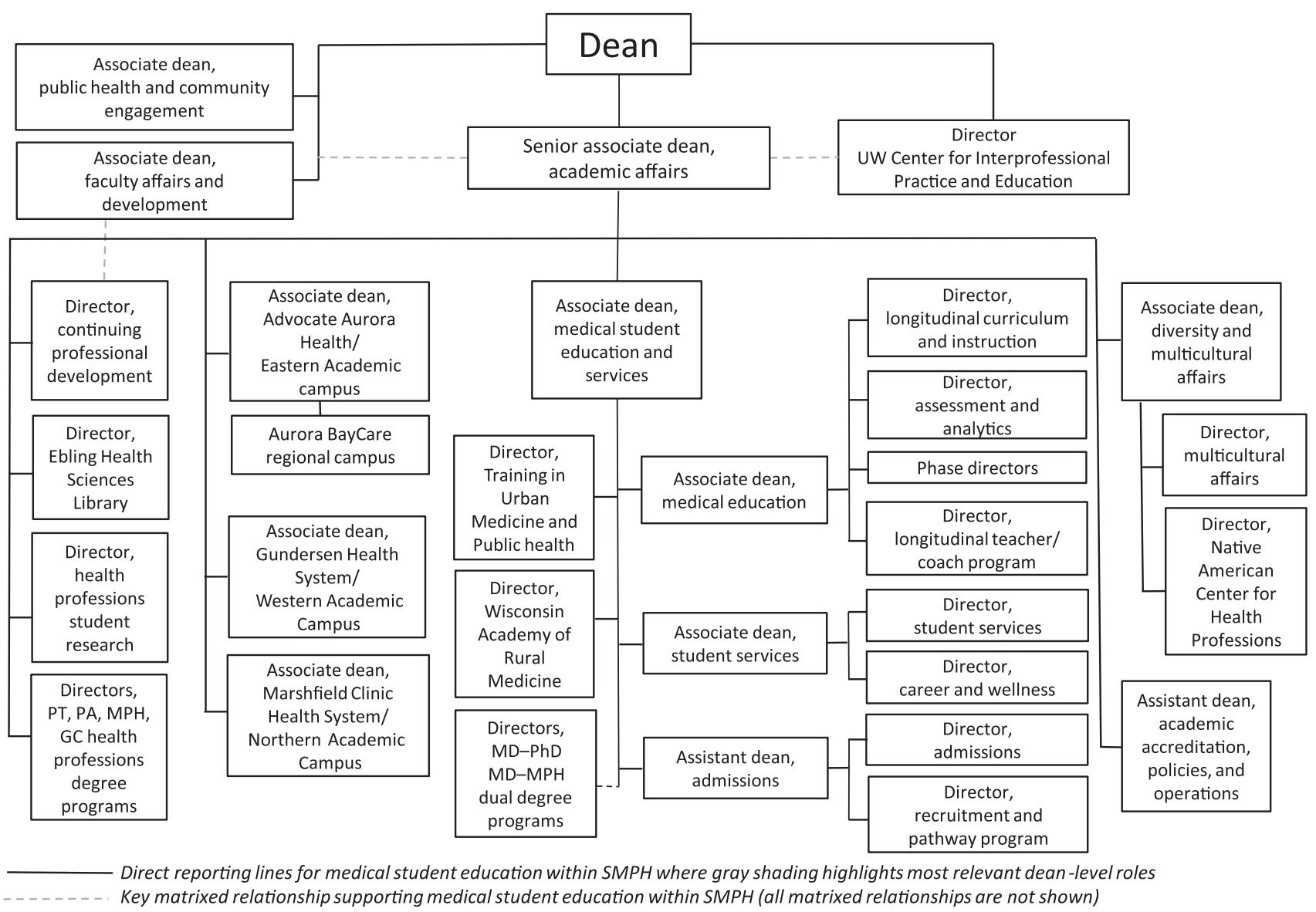

Figure 1 Institutional leadership structure. 


\section{Regional Medical Campuses}

UWSMPH has 4 regional campuses, including 3 designated academic campuses that support UME, GME, and significant research activities. The regional campuses offer Phase 2 and Phase 3 curriculum to all UME learners. WARM and TRIUMPH students are assigned to 1 of the regional campuses for Phases 2 and 3, and all other students are assigned at least 1 block at a regional campus. Maintenance of consistent educational experiences across sites is achieved via a novel CQI process termed "Going for Green," centered around a standardized checklist covering all important required clerkship elements (e.g., learning objectives, required clinical encounters/skills, curriculum delivery, development of faculty and residents as teachers/ assessors). Every site completes this checklist annually for each required clerkship block, providing details about how sites ensure completion of tasks. Completed checklists are reviewed centrally, discussed, and adjustments are made as needed to ensure any potential or identified inconsistencies are prevented or remedied.

\section{See Table 1-Regional Campuses.}

Robust communication and strong relationships are nurtured between central curriculum leadership and regional leadership, faculty, and clerkship coordinators through in-person meetings (including multi-annual site visits and distance conferencing) as well as by regular email and phone communication. Updates or changes are communicated via email, in conference calls,

\section{Table 1}

\section{Regional Campuses}

\begin{tabular}{|c|c|c|}
\hline $\begin{array}{l}\text { Regional campus } \\
\text { name }\end{array}$ & Tracks offered & $\begin{array}{l}\text { Student } \\
\text { enrollment } \\
\text { (average } \\
\text { number of } \\
\text { students } \\
\text { per year) }\end{array}$ \\
\hline \multirow{2}{*}{$\begin{array}{l}\text { Advocate Aurora Health } \\
\text { (Aurora BayCare)/ Eastern } \\
\text { Academic Campus, Green } \\
\text { Bay region }\end{array}$} & Traditional (rotation) & 5 \\
\hline & $\begin{array}{l}\text { WARM } \\
\text { (Phases } 2 \text { and 3) }\end{array}$ & 15 \\
\hline \multirow{2}{*}{$\begin{array}{l}\text { Advocate Aurora Health/ } \\
\text { Eastern Academic Campus, } \\
\text { Milwaukee region }\end{array}$} & Traditional (rotation) & 60 \\
\hline & $\begin{array}{l}\text { TRIUMPH } \\
\text { (Phases } 2 \text { and 3) }\end{array}$ & 40 \\
\hline \multirow{2}{*}{$\begin{array}{l}\text { Gundersen Health System/ } \\
\text { Western Academic Campus }\end{array}$} & Traditional (rotation) & 60 \\
\hline & $\begin{array}{l}\text { WARM } \\
\text { (Phases } 2 \text { and 3) }\end{array}$ & 25 \\
\hline \multirow{2}{*}{$\begin{array}{l}\text { Marshfield Clinic Health } \\
\text { System/ Northern } \\
\text { Academic Campus }\end{array}$} & Traditional (rotation) & 35 \\
\hline & $\begin{array}{l}\text { WARM } \\
\text { (Phases } 2 \text { and 3) }\end{array}$ & 25 \\
\hline
\end{tabular}

and during in-person meetings as needed. Regional faculty have access to clerkship curriculum, assessment methods, and requirements via the UWSMPH website and learning management system. 


\section{University of Alberta Faculty of Medicine and Dentistry}

Tracey Hillier, MD, Hollis Lai, PhD, Lyn Sonnenberg, MD, Melanie Lewis, MD, Helly (Rachel) Goez, MD, and Shirley Schipper, MD

\section{Medical Education Program Highlights}

The MD program of the University of Alberta is a 4-year postbaccalaureate program that provides medical training for 162 trainees each year to serve the needs of Albertans. Our program's mission is to prepare physicians to provide the highest quality health care to the people of Alberta and beyond, and to advance medical knowledge and its application through research. Program highlights include:

- The program has renewed its commitment to Indigenous peoples through various endeavors including its social accountability mandate, advances in admissions practices, and a major overhaul of its curriculum in Indigenous health.

- The Physicianship course delivers relevant and topical content such as global health initiatives, refugee health, and an innovative LGBTQ+ curriculum.

- The Patient Immersion Experience connects first- and secondyear students with volunteers living with chronic illness. This experience gives students the opportunity to learn from the experiences of their patient mentors.

- Heavy emphasis in training in the rural setting:

- Students in the GI block (year 2) can spend 4 weeks in a rural community through the Preclinical Networked Medical Education Program.

- The family medicine clerkship in the third year includes 4-week learning experiences in both rural and urban care settings.

- The integrated community clerkship places 20 third-year students into rural communities for a 38 -week longitudinal integrated clerkship.

- The fourth-year program places students in Grande Prairie for their clerkship experience in a rural center setting.

- The graduation questionnaire results show excellent outcomes in areas that are challenging for many medical education programs.

- The Office of Advocacy and Wellbeing is arm's length from the program and is highly supportive of students and student groups.

- There is a robust support system for faculty development that works effectively with faculty members to improve their teaching, academic, and scholarship skills.

- There are pioneering technological advancements to support the curriculum and delivery of the program.

Acad Med. 2020;95:S563-S565.

doi: 10.1097/ACM.0000000000003292

Copyright (C) 2020 by the Association of American Medical Colleges

Year school was established: 1936.

School URL: https://www.ualberta.ca/medicine.

\section{Curriculum}

Our curriculum and 4-year program-level objectives follow the CanMEDS framework. The MD Curriculum and Program Committee (MDCPC) is responsible for the overall design, management, and evaluation of a coherent and coordinated curriculum. A team of faculty members contributes to program operations based on their respective spheres of responsibilities. The program is divided into two 2-year phases_-preclerkship and clerkship - that all students are required to complete to meet programmatic degree requirements.

Students begin their education in the preclerkship phase, consisting of 11 systems-based courses along with complementary longitudinal courses, designed to provide contextual and clinical content. Each course presents material in a reasoned progression from basic scientific information to clinical application, building on foundational knowledge and skills, providing progressively increasing clinical exposure.

During the clerkship years, each student must maintain a record of the number and variety of their clinical experiences to ensure that the learning objectives of the clerkship have been met. Year 3 includes clinical rotations or an integrated community-based clerkship, a longitudinal Physicianship III/ Transitions course, and opportunities for elective courses. Year 4 is a senior clinical year, which includes clinical rotations, electives, and a longitudinal Physicianship IV/Transitions course. In addition, an intersession week was created between years 3 and 4 to enable the curriculum to adapt to and address important current social topics.

\section{Curriculum changes since 2010}

- 2012: MedSIS was introduced as a comprehensive education management system.

- 2012: A curriculum for Indigenous health was launched, with yearly additions and improvements.

- 2013: A comprehensive review of all aspects and curricular content was undertaken.

- 2013: Introduction of Physicianship, a comprehensive and longitudinal training program on clinically relevant content, was added. This course includes the start of the Patient Immersion Experience Program, academic service learning, physicianship discussion groups, and longitudinal clinical experience.

- 2013: The Neurosciences course was thoroughly revised by a dedicated curriculum working group. Subsequently, substantial changes were made, and a new Neurosciences and Organs of Special Senses course was developed and implemented.

- 2013: A new 4-week psychiatry systems block course was developed and implemented to address a critical curricular deficiency in the area of mental health. 
- 2015: Year 4 clinical rotation changes were made, as reformatting of year 4 clerkships was needed to target required skills for students to succeed in residency.

- 2016: Admissions review, a comprehensive review of the admissions process, was conducted to align our admissions process with program goals to improve equity and diversity.

- 2016: The Foundations block was introduced to provide a comprehensive training of skills and knowledge needed for the MD curriculum in year 1 .

- 2017: MedMAP, a mentorship program designed to provide structured, personalized feedback about a students' academic progress; identify academically struggling students at the earliest possible opportunity; and help students with access to support services as required, was introduced.

- 2018: A student wellness review group was established and reviewed current practice to recommend strategies to improve learning across the curriculum.

- 2019: Curriculum review is underway after strategic planning occurring in 2018.

In the medium term, the goal of the MD program is to improve student learning through adapting instruction methods and learning experiences to train independent learners with 21 stcentury skills. Increasing focus is being paid on novel modalities of instructions, student wellness, and team learning approaches. In the long term, the MD program aims to serve Albertans by producing excellent physicians able to adapt to changes in the profession, and contribute to the health of Albertans through scholarly work.

\section{Assessment}

In 2014, a custom assessment system was developed as a consolidated electronic resource. Since its deployment, the system has delivered quizzes, simulated patient cases, objective structured clinical examinations, peer assessments, team-based learning modules, problem-based learning, examinations, reflective essays, and clinical assessments. The customized system also allowed for the development of novel innovations that fit with our curriculum.

The clinical assessment processes were reviewed and revised in 2017. A single clinical assessment form is now administered across clerkships, and comments are solicited adaptively based on the need of the student by area.

Since 2013, all clerkships use the system to track required patient encounters and procedural skills using an electronic logging system. A new version of logging was introduced in 2019.

\section{Pedagogy}

Our MD program uses a variety of pedagogical approaches throughout the curriculum depending on the appropriateness of content, student knowledge, and availability. The preclerkship curriculum is delivered in a sequential, organ system-based approach using a hybrid of modalities including lectures, clerkships, discovery learning, small groups, teambased learning, virtual patients, anatomy labs, and other patient-involved experiences. Lectures are used to deliver prerequisite knowledge necessary to master the theoretical understanding in the field. Discovery learning exposes students to an independent, problem-solving learning environment. Clinical skill sessions and virtual patient cases prepare learners to develop and apply skills necessary for the profession, and professional development sessions enhance identity formation as a physician.

Clerkships are administered for year 3 and year 4 students to gain experiential knowledge and expose learners to a variety of work environments. Through a combination of experiencing the clinical setting and academic activities that include teambased learning, clinical decision-making activities, and case presentations, students are challenged to apply their knowledge and skills across a multitude of settings.

An increasing diversification of pedagogical approaches has been adopted and evaluated since 2010. Team-based learning has been adopted throughout the curriculum since 2011. Virtual patient cases have been adopted since 2014. Case-based learning activities have been in place since 2015 .

\section{Clinical experiences}

- To facilitate the clinical rotations during one academic year, approximately 1,100 practicing physicians along with 500 residents provide instruction from 100 unique locations (e.g., hospitals, sites, departments) to accommodate student learning.

- Clinical sites include hospitals, family medicine clinics, and health centers across Edmonton and Northern Alberta.

- Longitudinal clerkships offer an additional opportunity.

- Observership experience exists as part of Physicianship in year 1.

- The family medicine clerkship requires students to participate in a 4-week rural rotation in addition to a 4-week rotation in an urban clinic environment.

\section{Curricular Governance}

The MDCPC is duly constituted by the Faculty Council and has authority and responsibility for the MD program. All course and clerkship coordinators and chairs of committees who implement and deliver the curriculum operate under the authority of the MDCPC. Subcommittees coordinate specific aspects of the curriculum.

- The Preclerkship Subcommittee operates as a standing committee of the MDCPC with delegated responsibility for the preclerkship phase.

- The Clerkship Subcommittee operates as a standing committee of the MDCPC with delegated responsibility for the clerkship phase of the MD program.

- The Assessment Subcommittee operates as a standing committee of the MDCPC with delegated responsibility for monitoring and reviewing assessment processes across the MD curriculum.

- The Program Evaluation Subcommittee operates as a standing committee of the MDCPC with delegated responsibility for 
monitoring and reviewing teaching evaluations of the MD program.

- The Physicianship Thread Lead Subcommittee operates as a standing subcommittee of the MDCPC with delegated responsibility for the 4 Physicianship courses in the MD program.

All curricular governance related to the MD program is centralized and accountable at the Undergraduate Medical Education (UME) Office. Budget for faculty and delegation of teaching allocations are managed at the department level.

\section{Education Staff}

With a cadre of over 1,000 instructors and residents who teach students in various learning environments, there is also a team of assistant deans, directors, course coordinators, and thread leads who have responsibility for delivering and providing oversight of the program.

- The associate dean oversees all aspects of the MD program, including strategic planning, curriculum development, accreditation, collaboration with other team leaders within the MD program and across the faculty, and the day-to-day operations of the UME Office.

- Assistant dean, academic affairs is responsible for identifying students in academic difficulty and for arranging individualized assistance.

- Assistant dean, assessment oversees all aspects of student assessment for the MD program, including student assessments, assessment practices, standard setting, and the systems in place to run these aspects of the program.

- Assistant dean, program evaluation oversees all aspects of evaluation for the MD program, including teaching, course, and program evaluations, as well the systems in place to run these aspects of the program.

- Assistant dean, admissions leads all aspects of the admissions process, promotes diversity, chairs the admissions committee, and ensures that all admission policies and procedures are followed.

- Assistant dean, education quality and accreditation oversees the commitment of the Faculty of Medicine and Dentistry
(FoMD) to educational quality improvement, and the accreditation process for programs in the faculty, with emphasis on the MD program.

There are other offices of accountability that support MD program students. The associate dean, faculty development provides support to faculty development efforts. The associate dean, advocacy and well-being is responsible for developing resources (psychology, peer support), providing advocacy, and influencing an optimal health-promoting learning environment. The associate dean, academic technology supports the use of student technology and in teaching practices. The assistant dean, equity, diversity, and inclusion champions various related strategic initiatives. Other offices of accountability also support the efforts in rural, Indigenous, and global health initiatives.

\section{Faculty Development and Support in Education}

To facilitate and improve teaching practices of the program, various units within the FoMD provide consultation, collaboration, and support to help faculty members succeed in their academic roles. A comprehensive strategy has been carefully designed to meet the ongoing needs of faculty members.

- The FoMD offers peer-review opportunities and a mentoring program connecting senior academics with junior colleagues to support their scholarly and clinical work.

- The Teaching and Supervision Skills Program assists faculty with teaching and assessment.

- Faculty deliver workshops throughout the year and share skills, interests, and experience in teaching and supervision.

- The IDEAS Office offers membership for educators and provides formal opportunities to support faculty members on improving their teaching and learning.

- The Teaching Scholars Program of the IDEAS Office offers courses in teaching and education.

Teaching and education is an integral part of promotion and tenure decisions. The faculty evaluation committee guidelines outline specific expectations for teaching commitments according to the proportion of time allocated for teaching efforts. Creation of education content and products of education (including lectures and resources) are considered in annual reviews. 


\section{University of British Columbia Faculty of Medicine}

Roger Wong, MD, Sarah Brears, MD, Cheryl Holmes, MD, Amil Shah, MD, Paul Winwood, MB BS, Bruce Wright, MD, Kevin Eva, PhD, and the MD Undergraduate Program Team

\section{Medical Education Program Highlights}

- Publicly funded, 4-year program, admitting 288 learners annually; distributed across 4 locations in the province of British Columbia (BC) including 3 regional medical campuses: the Island Medical Program (Victoria), the Northern Medical Program (Prince George), and the Southern Medical Program (SMP) (Kelowna). The main medical campus is the Vancouver Fraser Medical Program (Vancouver and Fraser Valley). All graduates receive an MD from University of British Columbia (UBC).

- Social responsibility is central to the undergraduate curriculum as the Faculty of Medicine directs its education activities toward addressing the current and future needs of BC.

- Distributed medical education is fundamental to the Faculty of Medicine's structure and culture. Learners are exposed to a range of communities and patient populations and taught in underserved areas including remote and rural areas of BC. There are now distributed residency training programs in family medicine, internal medicine, psychiatry, pediatrics, and emergency medicine that complement the undergraduate training program.

- Admissions criteria are competitive, but holistic in nature, allowing learners from a wide range of backgrounds to succeed in the application process. Special streams exist for Indigenous, rural/remote, and $\mathrm{MD}-\mathrm{PhD}$ candidates.

\section{Curriculum}

\section{Curriculum description}

- Competency based and designed around the principles of horizontal and vertical (i.e., spiraled) integration. Integration occurs through a "body systems and themes" framework. More specifically, themes of medical sciences, diagnostic sciences, treatment, populations and diversity, care of patients, evidencebased medicine, and scholarship are integrated into relevant body system weeks across all courses.

- Systems and themes are revisited throughout all 4 years of the curriculum with increasing levels of difficulty (see https:// mdprogram.med.ubc.ca/about/curriculum).

- Year 1 and 2 content is anchored through 56 core case-based learning (CBL) cases, with related content explored in weekly clinical decision-making, clinical skills, and family medicine sessions.

Acad Med. 2020;95:S566-S569.

doi: 10.1097/ACM.0000000000003301

Copyright (C) 2020 by the Association of American Medical Colleges

Supplemental digital content for this article is available at http://links.Iww.com/ ACADMED/A834.

Year school was established: 1954.

School URL: https://www.med.ubc.ca.
- A Curriculum Integration Advisory Council, informed by theme leads, monitors curricular content for sequencing, gap identification, redundancies, and opportunities for further integration.

- Other key features include Flexible and Enhanced Learning (FLEX) courses, transitions courses (into medical education, into clinical education, and into postgraduate education and practice), and support for professional identity formation through small-group and reflective portfolio review sessions led by a coach.

\section{Curriculum changes since 2010}

- In August 2015, a renewed undergraduate curriculum was launched. Guiding principles included social responsibility and accountability competency-based and programmatic assessment, flexibility, scholarship, integration, continuity, and preparation for active participation in the current and future health care system.

- A significant innovation is the FLEX course. It is a required curricular component that spans all 4 years and emphasizes a broad understanding of scholarship, engagement, and social accountability. Students are prepared through a Foundations of Scholarship component and engaged in community and/ or research activities. Goals include fostering innovation, creativity, and critical thought as well as preparing graduates for roles as scholars, lifelong learners, and medical leaders.

- Indigenous health is a new theme (as part of Populations and Diversity) generated in collaboration with the central university's academic units including the UBC Centre for Indigenous Health. Preclinical students participate in a foundational Indigenous cultural safety learning experience covering topics of Indigenous perspectives of history, the legacy of colonialism in Canada, Indigenous peoples' health, and Canada's health care system. Web-based modules and face-to-face interdisciplinary workshops are used with lectures, orientation to local Indigenous communities, CBL, and smallgroup teaching sessions with Indigenous simulated patients and elders/educators.

- For ongoing quality improvement, the Curriculum Review Working Group formally reviews the curriculum in light of internal and external feedback to ensure the curriculum is accomplishing its intended outcomes.

- The SMP, based in the interior of BC, became the fourth undergraduate campus in 2011 and graduated its first class in 2015.

\section{Assessment}

See Supplemental Digital Appendix 1-Medical Education Program Objectives (Exit Competencies)—at http://links.lww. com/ACADMED/A834. 
- Exit competencies, developed during the curriculum renewal process, define the program's objectives. They were developed based on:

- Association of Faculties of Medicine of Canada (AFMC) (2010): The Future of Medical Education in Canada - College of Family Physicians of Canada's Undergraduate Education Committee (2009): CanMEDS-FM competency framework for undergraduate medical education from a family medicine perspective

- Royal College of Physician and Surgeons of Canada (2005): The CanMEDS Physician Competency framework

- A programmatic assessment approach was implemented with the renewed curriculum. It includes 4 assessment modalities, each designed to assess specific content and competencies: Written tests (multiple-choice question exams), portfolios, OSCEs, and workplace-based assessments. Progress tests are provided regularly for students to enable them to assess their progression. The goal is to provide longitudinal and competency-based assessment that enables regular feedback and opportunities for coaching.

- An MD-PhD program is jointly administered by the Faculty of Medicine and Faculty of Graduate and Postdoctoral Studies. Its purpose is to provide qualified students the opportunity to combine medical school with intensive scientific training for careers as clinician-scientists. Students usually complete the program in 7 years, with 3 years of full-time $\mathrm{PhD}$ training occurring between preclerkship and clerkship.

- The MD curriculum for all students is delivered using several pedagogical approaches. Most prevalent in terms of curricular hours are lecture, CBL, small-group discussion, independent learning, large-group discussion, ambulatory and inpatient clinical experience, laboratories, reflection, service learning, and research activity. In addition, approximately 3,600 hours of teaching is scheduled in the context of patient care.

- All learners' first encounter with clinical experiences occurs during clinical skills training and family medicine office placements, integrated throughout years 1 and 2. Both activities begin in the first term. Clinical skills involves exposure to a mix of volunteer patients, inpatients, and standardized patients.

- Clinical education takes place in urban, regional, and rural/remote settings in both academic and community centers across BC. Year 3 core clerkships and year 4 clinical electives are offered across the province at clinical academic campuses (larger, often highly specialized hospitals) and affiliated regional centers (medium-sized hospitals in urban or nonurban settings) that incorporate rotation-based and integrated community clerkships (ICCs).

- ICCs are currently delivered at 6 sites with 24 students in total, located in rural and remote communities. Year 2 students apply to ICCs for their third-year clerkships. Students are selected on the basis of merit and in accordance with regional quotas. For their entire clerkship year, ICC learners are assigned to a primary preceptor in family practice and work with patients whom they follow through various specialty clinics and other aspects of their care in the local community hospital.
- 24 weeks of clinical electives are offered in year 4, during which students can complete 2- or 4-week rotations in a variety of disciplines, in-province, out-of-province, and/or out-ofcountry.

\section{Curricular Governance}

- The Undergraduate Medical Education Committee (UGMEC) is responsible for design, management, integration, evaluation, and enhancement of the curriculum. Several subcommittees report to the UGMEC.

- Operational responsibility for the development, monitoring, and review of learning objectives is delegated to the years 1 \& 2 and years $3 \& 4$ Curriculum Subcommittees. These groups work across all sites.

- The Learning Environment Advisory Council is responsible for monitoring the learning environment, including mistreatment of students and promoting a culture of trust and respect among learners, faculty, and staff. It is advisory to the UGMEC, the regional associate deans, and the curriculum subcommittees.

See Figure 1-Curricular governance.

\section{Education Staff}

- The Curriculum Management Unit tracks objectives across the curriculum and maps them to link the granular, sessionlevel objectives with weekly objectives, course outcomes, yearly milestones, and exit competencies.

- The Educational Assessment Unit aligns objectives across assessment modalities to ensure what is taught is being assessed. They also monitor quality of assessment practices.

- The Evaluation Studies Unit conducts evaluations of courses and clinical rotations. They analyze and report to UGMEC on data arising from admissions, AFMC Graduation Questionnaires, learning environment surveys, Medical Council of Canada Qualifying Examinations, Canadian Residency Matching Services, and long-term outcomes.

- Student Affairs offices collaborate with UGMEC, but is purposefully separated from the undergraduate medical education structure. The Student Affairs Advisory Council advises the associate dean of medical student affairs, who reports directly to the executive associate dean of education, who is the chief academic officer of the Faculty of Medicine. Student Affairs offices, each with an assistant dean, exist at each regional campus. They are responsible for ensuring students have access to health services, personal counseling and well-being programs, and financial aid and debt management services. They also participate in electives advising and career counseling and can make referrals for academic advising and accommodations.

- The Centre for Health Education Scholarship (CHES) provides consultation regarding how modern understanding of educational practices can influence curriculum development. They also support faculty, staff, and trainees who wish to pursue educational scholarship of their own. 


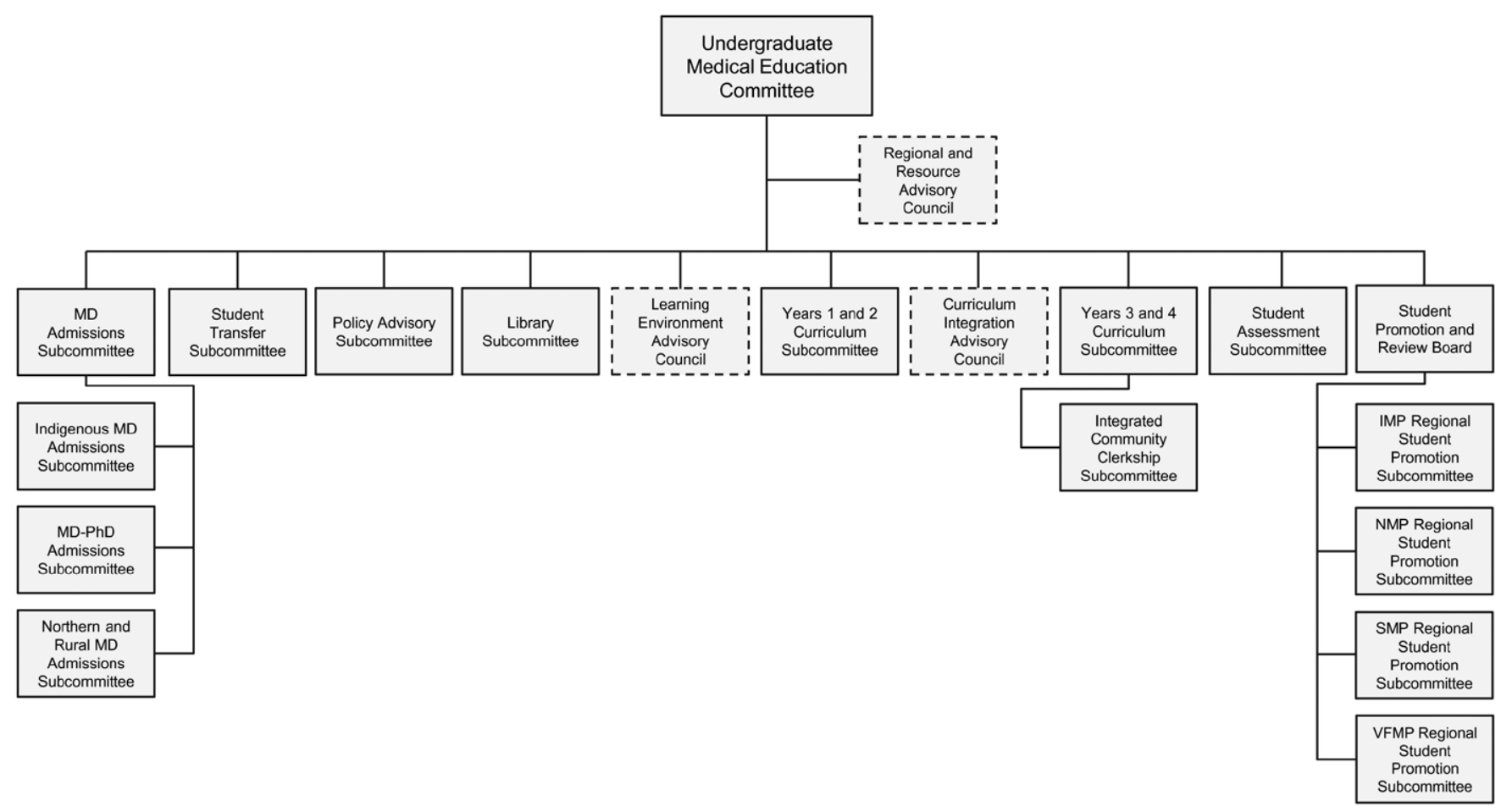

Figure 1 Curricular governance.

- The Faculty of Medicine's Education Office, led by the executive associate dean of education, provides strategic support to advance the education mandate of the Faculty of Medicine, overseeing 95 health professions programs.

See Supplemental Digital Appendix 2-Medical Education Leadership—at http://links.lww.com/ACADMED/A834.

\section{Faculty Development and Support in Education}

- The Faculty Development Office empowers teachers to be successful by developing a model for educational support that takes into account the needs of all teaching sites and teachers at all career stages. It maintains a distributed structure involving regional teams delivering local, central, and online offerings. In this way, faculty development offers a consistent core program while accommodating the unique needs of faculty in their geographical/departmental areas across regional sites.

- The faculty development team organizes and supports a collaborative, interprofessional network of teachers and clinician-educators and facilitates the development and renewal of foundational and advanced teaching and learning competencies.

- Full-time (academic) faculty and clinical faculty appointments are governed by the UBC Faculty Association Collective Agreement and follow the same policies and procedures for initial appointment, renewal, promotion, granting of tenure, remediation, and dismissal. Promotion decisions are based on performance in both teaching and scholarly activity/ educational leadership. Evidence is provided through teaching dossiers, examples of scholarly activity, and summative peer reviews of teaching.

- To support faculty and trainees interested in education scholarship, CHES was established to shape the theories and activities of learning in the health professions by creating new knowledge through research and informed innovation, building capacity through mentorship of individuals, and fostering a culture of collaboration and scholarly thinking in health professions education.

- CHES offers the Clinical Educator Fellowship Program aimed at training senior residents or junior faculty for roles as education leaders. It involves experiential teaching, a cohortbased academic program, and completion of a master's degree. CHES also delivers the Educators Leadership Program to support the development of multiprofessional health professions educators.

\section{Regional Medical Campuses}

- UBC has 3 geographically distributed campuses, each of which operate on a combined model (i.e., offering both preclerkship and clerkship training separate from the main campus).

- Regional associate deans are the principal academic officers for each campus. They are responsible for the delivery of undergraduate medical education as well as providing regional oversight of postgraduate residency and allied health professions programs. They are involved in all domains of activity at their geographically distributed campuses including research, faculty affairs, and engagement with partners (e.g., health authorities) and the community. 
- To help maintain a consistent educational experience across sites, the Faculty of Medicine has a stable, sustainable, and renewable technology platform that facilitates collaborative education, research, and administration. Further, the UGMEC and other committees include representatives from all distributed campuses and ensure that policies and procedures define common processes and expectations.

- Faculty leaders travel between regional campuses regularly, and a clear delineation is offered to faculty and students regarding what is context-specific versus core material. Students at all campuses receive the same materials, are directed to the same learning resources, and are assessed in the same way. Teaching faculty across campuses also receive the same materials that include the exit competencies, objectives, assessment criteria, and so forth.

- Program objectives and achievement markers are consistent across regional campuses with UGMEC monitoring written exam and clinical marks, OSCE results, graduation rates, residency match rates, Medical Council of Canada Qualifying Examination scores, and the AAMC Graduation Questionnaire, to ensure regional campus comparability.

See Table 1-Regional Medical Campuses.
Table 1

Regional Medical Campuses

\begin{tabular}{|c|c|c|c|}
\hline Program & Partners & Туре & Enrollment \\
\hline $\begin{array}{l}\text { Island Medical } \\
\text { Program }\end{array}$ & $\begin{array}{l}\text { University of } \\
\text { Victoria } \\
\text { Island Health }\end{array}$ & $\begin{array}{l}\text { Combined } \\
\text { academic and } \\
\text { clinical training }\end{array}$ & 128 \\
\hline $\begin{array}{l}\text { Northern Medical } \\
\text { Program }\end{array}$ & $\begin{array}{l}\text { University of } \\
\text { Northern British } \\
\text { Columbia } \\
\text { Fraser Health }\end{array}$ & $\begin{array}{l}\text { Combined } \\
\text { academic and } \\
\text { clinical training }\end{array}$ & 128 \\
\hline $\begin{array}{l}\text { Southern Medical } \\
\text { Program }\end{array}$ & $\begin{array}{l}\text { University of } \\
\text { British Columbia- } \\
\text { Okanagan } \\
\text { Interior Health }\end{array}$ & $\begin{array}{l}\text { Combined } \\
\text { academic and } \\
\text { clinical training }\end{array}$ & 128 \\
\hline
\end{tabular}

Acknowledgments: Submitted on behalf of the countless people who contributed to the educational program outlined in this document, the authors would like to extend a particular thank you to Lua Lynch for her assistance in aggregating the materials that enabled this snapshot's creation. 


\section{Memorial University of Newfoundland Faculty of Medicine}

Tanis Adey, MD, Carla Peddle, MA, and Margaret Steele, MD

\section{Medical Education Program Highlights}

The Faculty of Medicine (FOM) at Memorial University has recently celebrated 50 years since students first entered the classroom in 1969. The curriculum has seen many adaptations since its inception. Recent highlights include:

- Being the first Canadian medical school to implement Entrustable Professional Activities (EPAs) as the framework for learning and assessment in the final 2 years of clinical learning. EPAs are professional practice tasks or responsibilities that trainees must be able to perform without direct supervision at the time of graduation from the MD program. Each of the 13 adopted EPAs have been linked to CanMEDS ${ }^{1}$ competencies.

- The development of a Leadership in Medicine (LIM) Certificate option for learners of the MD program. In addition to the 8 physician competencies modules, learners wishing to pursue the LIM Certificate must complete an online capstone Leadership in Medicine module, which builds upon leadership concepts introduced in the MD program.

- A focus on rural medical education, requiring learners in the first 2 years of the MD program to complete, minimally, 2 rural clinical experiences. This allows learners to become familiar with the unique needs of rural populations of Newfoundland and Labrador. During the final 2 years, learners participate in an 8 -week rural family medicine rotation and a 4 -week rural selective in a core discipline.

- Memorial learners have hosted the Monte Carlo Charity Gala annually, since 1977, raising almost $\$ 2,000,000$ in support of local charities. This event embodies our philanthropic spirit and provides opportunities to make meaningful contributions to our communities.

\section{Curriculum}

\section{Curriculum description}

Our 4-year MD program is theme-based with both horizontal and vertical integration, whereby each phase builds on the knowledge acquired in the previous phase. Learners develop clinical skills, build on clinical knowledge, pursue a research project, and experience a variety of teaching and learning methods throughout the program. We strive to graduate wellrounded, generalist MDs.

Acad Med. 2020;95:S570-S574.

doi: 10.1097/ACM.0000000000003311

Copyright (C) 2020 by the Association of American Medical Colleges

Supplemental digital content for this article is available at http://links.Iww.com/ ACADMED/A841.

Year school was established: 1973.

School URL: https://www.med.mun.ca/medicine/home.aspx.
See Supplemental Digital Appendix 1-Curriculum

Description—at http://links.lww.com/ACADMED/A841.

\section{Curriculum changes since 2010}

Through the curriculum renewal process, we developed and implemented a spiral curriculum, which was introduced to firstyear medical learners in 2013. The spiral curriculum featured "stories" which were developed to allow learners to follow a set of fictional patients through the progression of health and disease across learning experiences.

A recent full review of the curriculum identified that it had not evolved into a purposeful spiral and the clinical skills sessions did not align with content delivered in learning sessions. From these recommendations, we began a reorganization to a themebased curriculum, whereby basic and clinical sciences are integrated into clinical encounters and clinical skills sessions are better aligned with the delivery of learning sessions. The theme-based curriculum was introduced for Phase 1 learners in 2019.

In 2013, we increased the incoming MD class size from 64 to 80 learners. In 2014, a new Medicine building was opened, complete with state-of-the-art classroom technology, seating accommodations for up to 120 learners in 2 designated lecture theatres, and many small learning rooms.

\section{Assessment \\ Our MD program objectives are linked directly to the CanMEDS competency framework. All session and course objectives covered in the curriculum have been mapped using an in-house software, CBlue.}

See Supplemental Digital Appendix 2-Medical Education Program Objectives - at http://links.lww.com/ACADMED/A841.

Since 2010, the MD program has implemented several changes in learner assessment, including increasing transparency through the development of assessment plans for each phase and course. Assessment plans detail formative and summative assessments, reassessment, and course success criteria.

Assessment plans are approved by the Undergraduate Medical Studies (UGMS) Committee prior to the commencement of the phase.

In 2013, the learning technology team introduced the use of QuestionMark, to create and deliver multiple-choice examinations and generate a cumulative question bank. Online multiple-choice examinations have now largely replaced paperbased assessments.

In 2015, we became the first MD program at a Canadian medical school to introduce EPAs as the primary learning and assessment tool during Phase 4, the final 2 clinical 
learning years. We transitioned away from discipline-specific medical expert examinations and adopted comprehensive clinical sciences examinations, which we deliver in the form of progress testing. Learners complete a baseline formative comprehensive exam prior to the commencement of Phase 4, 2 additional formative comprehensive exams during the Core Experiences course, and a summative comprehensive exam at the end of the course. In addition to the assessment of clinical and medical expert knowledge, the development of the core competency professionalism is assessed throughout the entire program.

\section{Parallel curriculum or tracks}

Our Core Experiences course has traditionally been made up of block-based, discipline-specific core experiences. In 2018, a longitudinal integrated clerkship (LIC) was introduced at one teaching hospital in parallel with the 50-week block-based Core Experiences course.

Learners in the final year of the MD program also have the opportunity to complete a 12 -week integrated selective in a rural setting. Traditional selective experiences are arranged in 2- to 4-week blocks to meet criteria for clinical diversification. We refer to this mini-LIC as Progression to Postgraduate, and it runs in parallel with the traditional 12 weeks of selective time.

\section{Pedagogy}

In addition to common pedagogical approaches, in 2013, the MD program implemented integrated learning sessions (ILSs) throughout the first 2 years of the MD program. ILS is a blended teaching approach, which helps learners integrate new medical expert knowledge into realistic clinical scenarios. Learners lead small-group discussions, followed by a facilitator-led large-group debrief and discussion. Learners gradually take on increasingly responsible roles in the large-group facilitation as they progress through the first and second years of the program. Faculty remain involved to provide oversight and input, as needed.

\section{Clinical experiences}

Learners in the MD program participate in rotations at several types of clinical sites, including:

- Hospital wards

- Medical specialist clinics, hospital and community based

- Family medicine clinics, rural and urban

- Community health clinics, rural and urban

Required and elective community-based rotations include:

- Phase 1 learners participate in weekly afternoon community placements, typically under the supervision of a family practitioner within an urban area.

- Phase 2 learners participate in a 2-week community visit at a rural site, during which $80 \%$ of the time is spent with community services and $20 \%$ of the time is spent under the supervision of a family practitioner.
- Phase 3 learners participate in a 2-week community visit, during which $80 \%$ of the time is spent under the supervision of a family practitioner and $20 \%$ of the time is spent with community services.

- During Phase 4, learners have many opportunities to participate in clinical learning experiences outside of the main teaching hospitals but are required to participate in an 8-week rural family medicine placement under the supervision of a family practitioner and a 4 -week rural placement in 1 of the 8 core disciplines.

- Electives and selectives may be completed at other Memorial affiliated sites, as well as other Canadian and international medical schools and teaching hospitals.

- Service learning opportunities are also available outside of the hospital setting.

The major challenges faced in designing and implementing clinical experiences for our medical learners are:

- Preceptor availability

- Housing accommodations at rural sites

- Inconsistent preceptor approaches to formative and summative feedback

\section{Curricular Governance}

The UGMS Committee, Curriculum Oversight Working Group (COWG), and Phase Management Teams are responsible for the planning, management, and monitoring of the MD curriculum. The COWG and the Phase Management Teams report to the UGMS Committee, which reports to Faculty Council and includes the curriculum lead; the four phase leads; representatives from the Student Assessment Subcommittee (SAS), Program Evaluation Subcommittee (PESC), and Informatics \& Technology Advisory Committee (iTac); and other related professionals.

The curriculum lead chairs the COWG, and the phase leads chair the respective management teams, all of which include representation from the instructional design team, as well as administrative personnel.

See Figure 1-Curricular governance.

\section{Education Staff}

The Undergraduate Medical Education (UGME) team coordinates the implementation of the MD curriculum. Program evaluation, learner assessment, and educational technologies are overseen by subcommittees of the UGMS (PESC, SAS, and iTac) and are partially administered by units outside of the educational office.

The UGME office is responsible for the delivery and assessment of the MD program and includes the faculty and administrative professionals who are responsible for the following elements of the MD program:

- Curriculum oversight

- Accreditation 


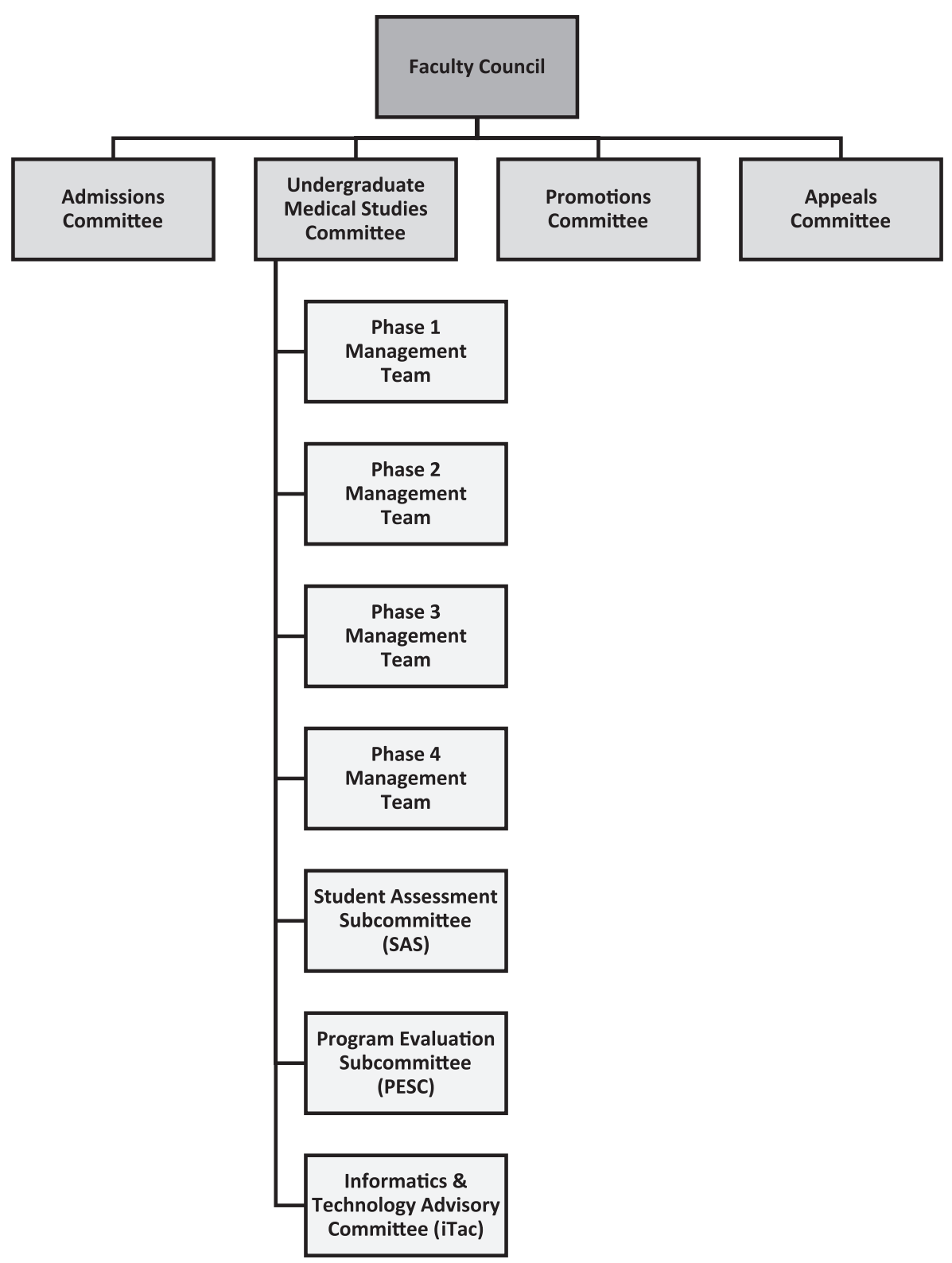

Figure 1. Curricular governance.

- Preclinical administration

- Clinical skills administration

- Core clinical administration

- Elective/selective administration

- Research administration

- ILS administration

- Assessment administration

Faculty and staff responsible for admissions, student affairs, and professional and educational development fall outside the auspices of the UGME office.
There are several faculty appointments with the UGME office:

- The associate dean, undergraduate medical education, in collaboration with the UGMS, has major responsibility for the education of our learners.

- The curriculum lead oversees the undergraduate medical education curriculum and seeks direction of the undergraduate content leads for the objectives relating to their specific discipline or division.

- The Phase 1,2, and 3 leads oversee faculty with designated responsibilities of assessment, research, ILSs, clinical skills, and coordinate matters relating to the respective phase. 
- The Phase 4 lead oversees faculty clerkship discipline coordinators, selectives coordinator, electives coordinator, course chairs, research lead, assessment lead, and coordinates matters relating to Phase 4 .

See Figure 2-Medical education leadership.

The UGME office administers the budget for curricular matters that cannot be met by appointed faculty. The UGME office pays the prorated salaries for nonfaculty MDs and non-MDs who play instructional or facilitative roles in the curriculum, as well as assessment invigilators and assessment markers.

\section{Faculty Development and Support in Education}

Applications for promotion and tenure must illustrate the candidate's excellence in scholarship of education, clinical excellence, excellence in scholarship of discovery, and excellence in leadership. Candidates must demonstrate meaningful contribution to the achievement of the FOM and Memorial's educational missions, through curriculum development, innovation in instructional delivery methods and evaluation, and leading major program development, among other educational contributions. The learner-completed evaluations of faculty teaching must attest to the faculty member's efficacy as an educator.

The Office of Professional and Educational Development (OPED) is an accredited provider of university continuing professional development for health care professionals and other faculty members at the FOM as well as the physicians in the province of Newfoundland and Labrador. OPED offers a breadth of professional development programming which is accredited by the Committee on Accreditation of Continuing Medical Education of the Association of Faculties of Medicine of Canada. The programs, offered in a variety of formats, are designed to address the needs of health care professionals who practice in both urban and rural communities throughout the provinces we serve, nationally and internationally.

\section{Distributed Medical Education}

At Memorial, we do not have regional medical campuses; however, approximately 10-14 learners participate in clinical learning opportunities such as community visits and the Core Experiences course in New Brunswick or Prince Edward Island each year.

All clinical learners must meet the same set of objectives irrespective of the clinical learning site. Each clinical discipline is overseen by one clerkship discipline coordinator, and in the case of the LIC, education is overseen by one site director. The site directors and clerkship discipline coordinators meet at the monthly Phase 4 Management Team meeting and discuss performance and assessment challenges, and unified approaches. All Phase 4 learners must meet success criteria for the phase. Learner performance issues are reviewed and addressed during the monthly Management Team meeting and as needed. Assessment profiles are presented at the Phase 4 Management Team meeting for learners who have not met the assessment criteria for the Core Experiences course at the 6-, 9-, and 12-month reviews. Remediation and reassessment are prescribed for learners, as required.

See Table 1-Distributed Medical Education Learners.

\section{Initiatives in Progress}

In conjunction with the LIC we currently offer in Moncton, New Brunswick, we intend to introduce another LIC site for our learners in Fredericton, New Brunswick, by August of 2020. We are also currently exploring opportunities for rural LIC sites in the province of Newfoundland and Labrador.

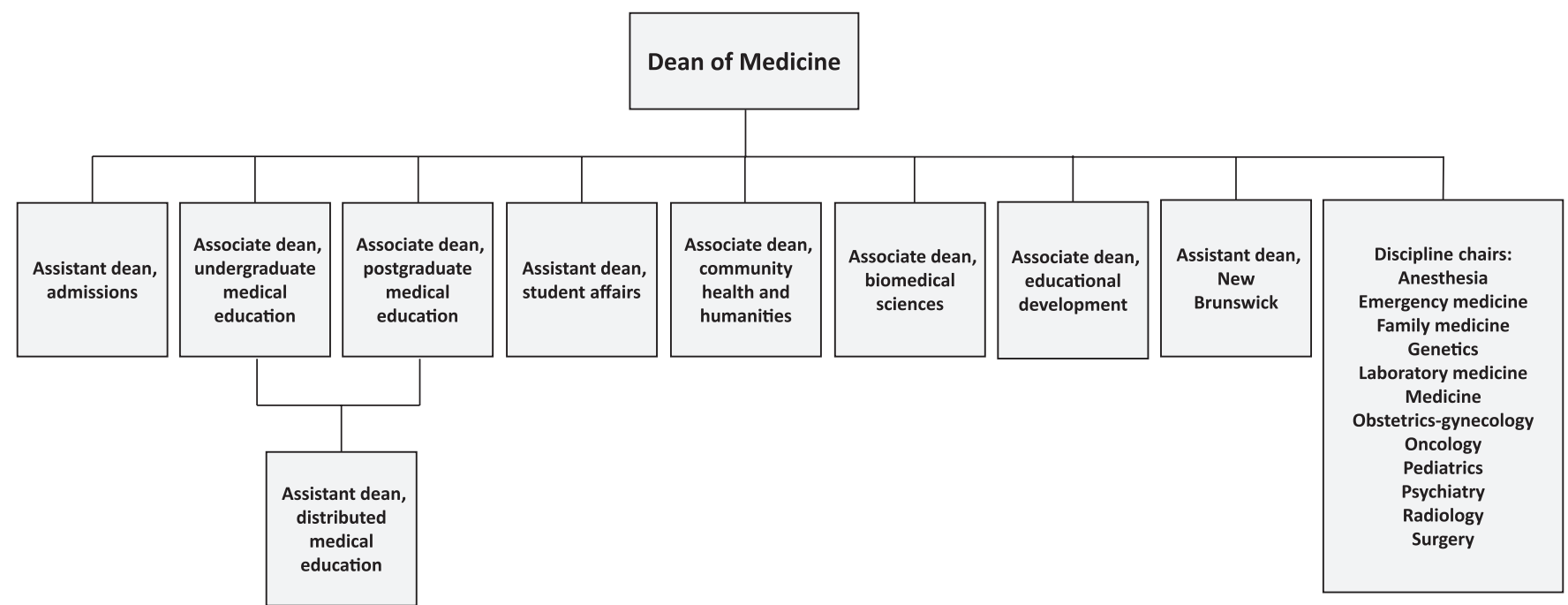

Figure 2. Medical education leadership. 


\section{Table 1}

\section{Distributed Medical Education Learners}

\begin{tabular}{llr} 
Location & Type & $\begin{array}{r}\text { Student } \\
\text { enrollment }\end{array}$ \\
$\begin{array}{l}\text { St. John's, Newfoundland } \\
\text { and Labrador }\end{array}$ & Preclinical learning \\
Newfoundland and & Clinical learning & 80 \\
Labrador & & $66-70$ \\
\hline New Brunswick & Clinical learning & \\
Prince Edward Island & Clinical learning & 10 \\
\hline
\end{tabular}

With the theme-based curriculum well underway for Phase 1 learners, we are looking forward to its full implementation by April of 2023.

Acknowledgments: The authors wish to thank Alison Haynes, Brian Kerr, and David Stokes for Memorial University's Doctor of Medicine Curriculum Infographic.

\section{Reference}

1 Frank JR, Snell L, Sherbino J, eds. CanMEDS 2015 Physician Competency Framework. Ottawa, Canada: Royal College of Physicians and Surgeons of Canada. 2015. 


\title{
Queen's University Faculty of Health Sciences
}

\author{
Leslie Flynn, MD, MEd, MMus, CCFP, FRCPC, and Denise Stockley, PhD
}

\section{Medicine Education Program Highlights}

In 2010, our primary focus within the School of Medicine was on undergraduate and postgraduate medical education. Fast forward 10 years and we now offer a complete 4-year undergraduate degree online in health sciences, an accelerated route to medical education, a combined MD with new graduate programs, and a graduate degree for health professionals. These programs provide a continuum of learning experiences from undergraduate students to clinical educators. A common thread for all programs was our move to a competency-based approach; taking an institutional approach to competency-based medical education (CBME) implementation has allowed for sharing resources and innovations across programs.

In the undergraduate sphere, to complement our long-standing UME program, we launched the Queen's University Accelerated Route to Medical School (QuARMS), which is a direct entry to the medical school program from high school, a first of its kind in Canada. We were also excited to launch our Bachelor of Health Sciences (BHSc) program, a completely online 4-year degree that provides prerequisites for a field in any of the health professions. Based on the success of this program, we launched an onsite format of the BHSc this past year.

At the graduate level, we launched 2 interdisciplinary graduate degrees in health professions education and in health care quality. To address the needs of internationally trained doctors, we created the Graduate Diploma in Medical Sciences and the Professional Master of Medical Sciences through a partnership between the Department of Biomedical and Molecular Sciences, post-undergraduate medical education, and UME. This program has been specifically designed to assist medical school graduates who have not yet matched to a postgraduate residency program in Canada achieve their goals.

We also created the Clinician Investigator program, which is an intensive, research-based postgraduate medical education program accredited by the Royal College of Physicians and Surgeons of Canada. It aims to develop clinician-scientist research leaders who will have the skill sets to achieve broadbased excellence not only in research but also as clinicians, supervisors/mentors, teachers, and academics. In addition, we launched a new combined opportunity wherein students can apply for the UME degree program and a master's or Doctorate

Acad Med. 2020;95:S575-S578.

doi: 10.1097/ACM.0000000000003489

Copyright (C) 2020 by the Association of American Medical Colleges

Supplemental digital content for this article is available at http://links.Iww.com/ ACADMED/A972.

Year school was established: 1854.

School URL: https://meds.queensu.ca.
(MD-MSc or MD-PhD), which they complete concurrently. This allows for unique opportunities for medical students to obtain advanced research and translational skills.

Our international reach has extended through teaching, research, and service, including the creation of international learning opportunities for both students and residents such as a family medicine rotation in the Falkland Islands. To further support our school, we have deliberately identified the need to place an emphasis on physician wellness. It has been embedded as a goal within our strategic framework for the School of Medicine.

Many of these programs are offered in our newly built, state-of-the-art School of Medicine Building, one of the premier teaching facilities in North America. It allows for the integration of modern teaching methods, technology, and interdisciplinary practices to provide a truly exceptional medical training experience. In the virtual realm, we have developed Elentra, which is an integrated teaching and learning and data management platform. This platform provides a seamless interaction between all aspects of our programs, including curriculum mapping and programmatic assessment, and has all the functionality of a robust learning management system. Elentra has matured into a consortium, where 22 international medical schools or other clinical sites are maintaining and growing the platform. In addition, our Faculty of Health Sciences is home to many different national and international research hubs, including the Canadian Frailty Network and the Canadian Institute for Military and Veteran Health Research.

Finally, in recognizing our responsibilities through the Truth and Reconciliation Commission calls to action we have revisited our responsibilities within the School of Medicine, including our admissions process, curricula, clerkships, support systems, and hiring processes, to be more inclusive for Indigenous peoples. We have also taken steps to redress historical inequities in our school. In 2018, the Queen's Senate revoked a 1918 motion that banned students of African descent in our School of Medicine. Although black students were admitted as of 1965, the motion continued to exist. The school has taken steps forward including personal letters of apologies to family members of those affected, curricular changes, outreach, and a Medicine Admission Award for Black Canadians.

\section{Curriculum}

\section{Curriculum description}

Since 2010, the provincial Ontario Universities Quality Assurance Council has monitored the quality of all university programs. At Queen's University we developed the Quality Assurance Process (QUQAP) to address this monitoring and have built institutional 
capacity through additional criteria of curriculum mapping, assessment, and academic integrity, among other unique features of our process. As such, all our programs are required to adhere to the accreditation standards and to the QUQAP, and all have curriculum maps that are vetted internally and externally.

See Supplemental Digital Appendix 1-Curriculum Map-at http://links.lww.com/ACADMED/A972.

\section{Curriculum changes since 2010}

There are significant changes within our curriculum since 2010 including CBME, Indigenous perspectives woven throughout our programs, an emphasis on physician wellness, and scholarship/ research. In addition, we launched the First Patient Program in 2016, which provides first-year UME students opportunities to interact with real patients, to learn empathy and skills they will carry with them throughout their academic and professional careers. Among other innovations, we have developed longitudinal clerkship rotations that allow our students opportunities to clerk in areas outside of our immediate area and in remote communities.

\section{Class size changes since 2010}

Our class size has not changed significantly since 2010, with our UME intake typically 100 students (currently 108 admissions); however, what has changed is that all students in the past were post-undergraduate degree students, now our numbers include 10 QuARMS students and a pathway for Indigenous students to our medical school. Further, at the request of the Canadian Armed Forces, the Queen's University UME program has completed arrangements with the Canadian Armed Forces to create up to 5 additional training positions in the Queen's Medical Doctorate Program. Canadian Forces staff, who are supported and funded by the Department of National Defence, are eligible to apply for these positions and offers of admission will be made to those who qualify.

\section{Assessment}

Our UME program adheres to the CanMEDs Framework, which identifies the 7 roles of a physician, and each of these are mapped to Entrustable Professional Activities (EPAs).

See Figure 1-Entrustable Professional Activities.

\section{Parallel curriculum or track}

Aside from the traditional route to our UME, we have 3 parallel curricula, all new since 2010.

\section{QuARMS:}

- Developed in 2013, QuARMS is Canada's only 2-year accelerated-entry premedical program, designed to reduce training time and the overall expense of medical training, as well as provide collaborative premedical experiences/training. Each year 10 students enter this program directly from high school and spend the first 2 years enrolled in an undergraduate degree program while also participating in an additional curriculum focusing on communication skills, critical thinking, the role of the physician, community service learning, and scientific foundations. They are then eligible to enter the first year of the MD curriculum.

MD-MSc and MD-PhD:

- The combined MD-PhD and MD-MSc programs provide benefits to both scholarship and to the professional development of physician-scientists by allowing better integration of clinical and research training experiences and providing better opportunities for fostering translational research. Our programs are in keeping with the strategic directions of both the Canadian Institutes of Health Research Strategic Plan and the government of Canada's Science and Technology Strategy, which emphasize the need for providing increased trans-sectorial and multidisciplinary training, building research excellence, translating knowledge into practical applications, and deepening the pool of highly skilled individuals.

\section{Curricular Governance}

As noted above, all academic programs at Queen's University are monitored through the QUQAP provincial quality assurance mandate. However, our Queen's Senate delegates to local programs the authority over all aspects of their educational programs. For example, within undergraduate medicine, the UME Curriculum Committee is delegated to have authority over all components of the MD educational program. The Curriculum Committee has both responsibility and authority regarding the design, content, implementation, and ongoing review of the program. This adheres to the CACMS/LCME Accreditation Standard 8: Curricular Management, Evaluation, and Enhancement. As well, the Curriculum Committee adheres to CACMS/LCME accreditation Standards 6: Competencies, Curricular Objectives, and Curricular Design; 7: Curricular Content; and 9: Teaching, Supervision, Assessment, and Student and Patient Safety. There are a number of subcommittees and individuals that report to the Curriculum Committee, including the Student Assessment Committee; the Teaching, Learning, and Integration Committee; and the Course and Faculty Review Committee.

The School of Medicine is situated within the Faculty of Health Sciences at Queen's University, which also includes the School of Nursing and the School of Rehabilitation Therapy.

See Figure 2-Organizational chart.

\section{Faculty Development and Support in Education}

The Faculty of Health Sciences includes the Office of Professional Development and Educational Scholarship (OPDES), an integration of continuing professional development, faculty development, education scholarship, and global health. OPDES conducts needs assessments and consultations, develops educational programs based on identified needs, develops online programs and courses, accredits and certifies programs on behalf 


\begin{tabular}{|c|c|c|c|c|c|c|c|}
\hline EPA & 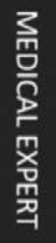 & $\begin{array}{l}\text { ᄋ } \\
\frac{8}{3} \\
\text { 윢 } \\
\frac{5}{3}\end{array}$ & 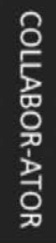 & $\begin{array}{l}\text { 罗 } \\
\text { 罖 }\end{array}$ & 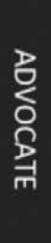 & 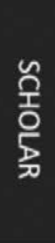 & 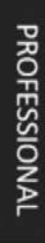 \\
\hline $\begin{array}{l}\text { EPA } 1 \text { - Obtain a history and perform a physical } \\
\text { examination adapted to the patient's clinical } \\
\text { situation } \\
H x \text { and } P x\end{array}$ & $x$ & $x$ & & & $x$ & & $x$ \\
\hline $\begin{array}{l}\text { EPA } 2 \text { - Formulate and justify a prioritized } \\
\text { differential diagnosis } \\
\text { Prioritized } D D x\end{array}$ & $x$ & & & & $x$ & $x$ & \\
\hline $\begin{array}{l}\text { EPA } 3 \text { - Formulate an initial plan of investigation } \\
\text { based on the diagnostic hypotheses } \\
\text { Investigation }\end{array}$ & $X$ & & & & & $x$ & \\
\hline $\begin{array}{l}\text { EPA } 4 \text { - Interpret and communicate results of } \\
\text { common diagnostic and screening tests } \\
\text { Diagnostic and screening tests }\end{array}$ & $x$ & $x$ & & & & $x$ & \\
\hline $\begin{array}{l}\text { EPA } 5 \text { - Formulate, communicate and implement } \\
\text { management plans } \\
\text { Management Plans }\end{array}$ & $X$ & $X$ & $x$ & & & $X$ & \\
\hline $\begin{array}{l}\text { EPA } 6 \text { - Present oral and written reports that } \\
\text { document a clinical encounter } \\
\text { Oral and written Reports }\end{array}$ & $X$ & $X$ & & & & & $X$ \\
\hline $\begin{array}{l}\text { EPA } 7 \text { - Provide and receive the handover in } \\
\text { transitions of care } \\
\text { Handover }\end{array}$ & $X$ & & $x$ & $x$ & & & \\
\hline $\begin{array}{l}\text { EPA } 8 \text { - Recognize a patient requiring urgent or } \\
\text { emergent care, provide initial management and } \\
\text { seek help } \\
\text { Urgent Care }\end{array}$ & $X$ & $x$ & $\mathbf{X}$ & & & & \\
\hline $\begin{array}{l}\text { EPA } 9 \text { - Communicate in difficult situations } \\
\text { Difficult Conversations }\end{array}$ & $x$ & $x$ & & & $x$ & & $x$ \\
\hline $\begin{array}{l}\text { EPA } 10-\text { Contribute to safety and quality in } \\
\text { patient care } \\
\text { Patient Safety/CQI }\end{array}$ & $X$ & & $\mathbf{X}$ & $X$ & & $X$ & \\
\hline $\begin{array}{l}\text { EPA } 11 \text { - Perform general procedures of a } \\
\text { physician } \\
\text { Procedural Skills }\end{array}$ & $X$ & $x$ & & & & & $x$ \\
\hline $\begin{array}{l}\text { EPA } 12 \\
\text { Educate patients on disease management, health } \\
\text { promotion and preventative medicine } \\
\text { Pt. Education/Promotion/Prevention }\end{array}$ & $X$ & $x$ & & $X$ & $x$ & & \\
\hline $\begin{array}{l}\text { EPA } 13 \\
\text { Design a research project } \\
\text { Research }\end{array}$ & & & $x$ & $x$ & & $\mathbf{X}$ & $\mathbf{X}$ \\
\hline
\end{tabular}

Figure 1 Entrustable Professional Activities.

of the Royal College of Physicians and Surgeons of Canada and the College of Family Physicians of Canada, and conducts educational research and program evaluations. OPDES offers programs for a diverse audience of health care professionals, has significant expertise and experience in the accreditation of learning activities for health professionals, and is a Committee on Accreditation of Continuing Medical Education accredited provider. The scope of this unit is to provide support and services to all 3 schools: Medicine, Nursing, and Rehabilitation Therapy.

Further, we have multiple noncredited and credited programs for faculty. An example of a noncredited program is our Fellows in
Educational Scholarship Program, which was designed to build individual expertise in educational scholarship and to create an identified cadre of faculty, graduate students, and residents who will advance the educational programs in the Faculty of Health Sciences through participation in educational scholarship. Individuals participating in this 2-year program focus on either a scholarly investigation of an educational issue or the development of an innovative educational program/curriculum. In 2020, we launched the Master of Health Professions Education program, a 2-year, part-time, interdisciplinary course/ competency-based program for clinical educators. All courses are 


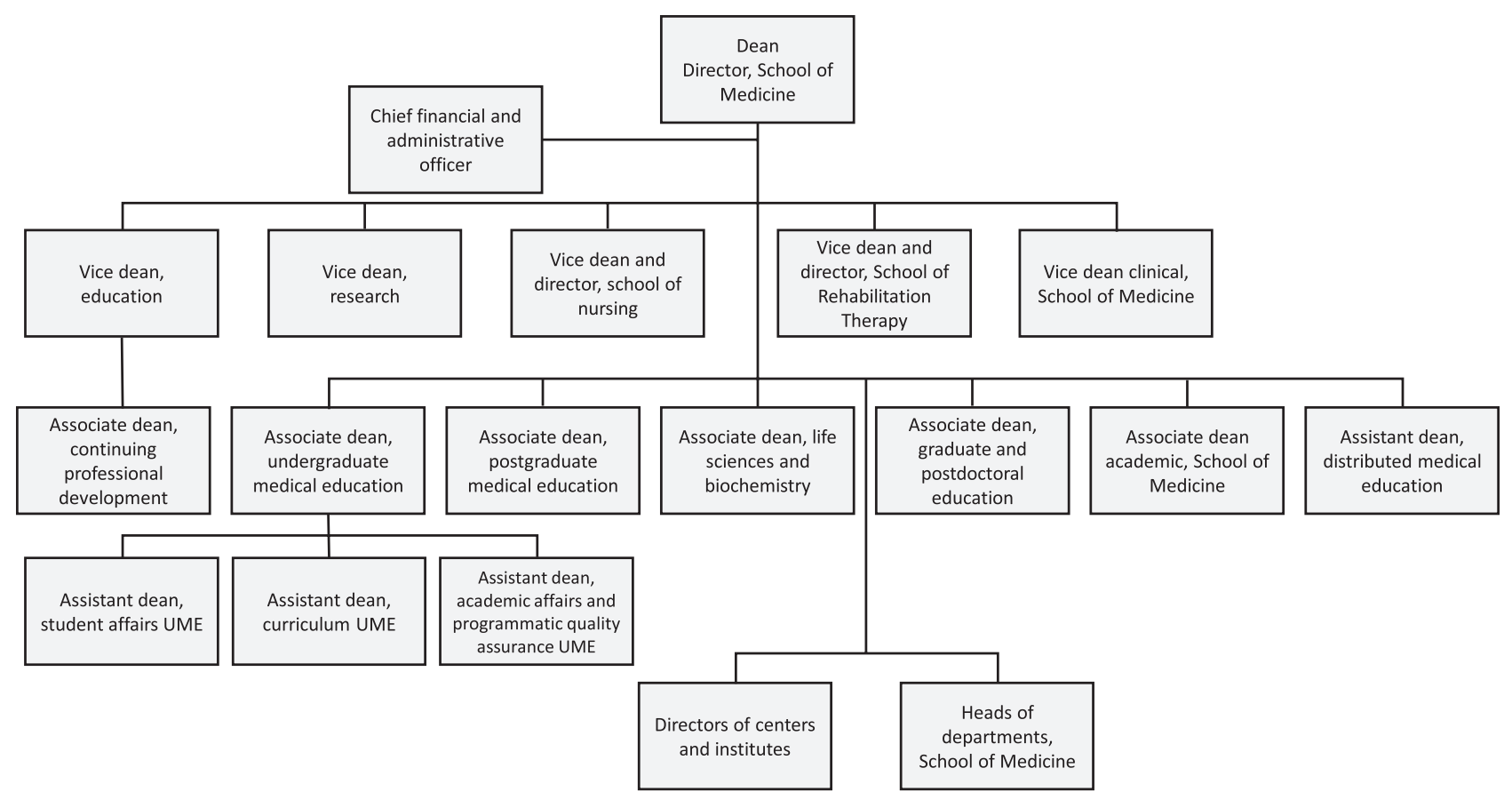

Figure 2 Organizational chart.

team-taught by a health professional and educational specialist and geared toward learners from all health professions.

\section{Role of teaching in promotion and tenure}

Our clinical faculty are promoted through the Queen's Senate, under the Statement on Promotion Policy for Geographically Full-Time and Adjunct-1 (non-Bargaining Unit) Appointees of the Faculty of Health Sciences.

There are 4 ranks: lecturer, assistant professor, associate professor, and professor. Promotion is based on teaching, research and scholarship, and administrative and professional service. The promotion package includes the curriculum vitae, teaching dossier, and other evidence. The following criteria are for each promotion category:

- Assistant: should be a good teacher and show evidence of the successful initiation of research and scholarly work. The appointee's participation in the operation of the department, the university, or contributions to the profession may be taken into consideration.
- Associate: should be a very good teacher, carrying a reasonable teaching load. The appointee's research and scholarly work should show high quality as judged by experts in the appointee's field of specialization. Contributions of the appointee to the operations of the department, the university, and the profession will be taken into account.

- Professor: must either combine distinguished scholarly work with very good teaching or continuing high-quality scholarly work with exceptional contributions in teaching. The appointee will have made a contribution to the successful operation of the department, the university, and the profession.

The ability to intersect theory and practice as fully integrated in all aspects of our School of Medicine highlights our intentionality to provide a multifaceted approach that is competency based. There have been significant strides in the past 10 years. Queen's University School of Medicine has undergone substantive shifts in admissions, curricular innovations, faculty support, and administrative processes. 


\section{University of Toronto Faculty of Medicine}

Paul Tonin, MEd, Stacey Bernstein, MD, Pier Bryden, MD, Kulamakan Kulasegaram, PhD, Marcus Law, MD, Maria Mylopoulos, PhD, Richard Pittini, MD, Glendon R. Tait, MD, and Patricia Houston, MD

\section{Medical Education Program Highlights}

The University of Toronto Faculty of Medicine was founded as a school of medicine in 1843. It is uniquely positioned as the only medical school operating in a greater Toronto area with a diverse population of more than 6 million people.

The MD program is delivered in partnership with $30+$ affiliated clinical teaching sites, including 9 fully affiliated hospitals and research institutes and 4 associate affiliated hospitals. Partnered with the Faculty of Medicine, they comprise the Toronto Academic Health Science Network (TAHSN), one of the largest biomedical research, education, and clinical care networks in North America.

A unique feature of the MD program is its academy system. The 4 academies-FitzGerald, Mississauga, Peters-Boyd, and Wightman-Berris - comprise clusters of affiliated hospitals. Medical students are assigned to an academy as part of the admissions process, with each academy providing students with a distinctive clinical community of learning for the program's duration.

With an annual entry class of 259 domestic students and up to 10 supernumerary international students, the MD program is the largest in Ontario and one of the largest in Canada. It has a large and highly competitive applicant pool and consistently high rate of acceptance of offers.

See Table 1-Student Application Characteristics, 2011-2019.

In alignment with our commitment to better reflect the society we serve, the MD program has established the Indigenous Student Application Program (first entry in 2012) and Black Student Application Program (first entry in 2018). The goals of these application pathways are to break down some of the barriers that might impede these students from applying to medical school and to increase their representation within the program. There is also a long-standing admission pathway for the MD-PhD program, the largest of its kind in Canada.

\section{Curriculum}

\section{Curriculum description}

The preclinical Foundations curriculum (years 1 and 2) is composed of 6 courses, 4 longitudinal components, and

Acad Med. 2020;95:S579-S582.

doi: 10.1097/ACM.0000000000003316

Copyright (C) 2020 by the Association of American Medical Colleges

Supplemental digital content for this article is available at http://links.Iww.com/ ACADMED/A843.

Year school was established: 1843.

School URL: https://medicine.utoronto.ca. longitudinal themes. The weekly curriculum is organized to include the equivalent of a full day of unscheduled time, providing students with time for self-study and with early exposure to the clinical environment.

See Supplemental Digital Appendix 1-Foundations Curriculum—at http://links.lww.com/ACADMED/A843.

Clerkship begins with the Transition to Clerkship course, which prepares students for a series of core clinical rotations that are the focus of year 3. Students reconvene from various clerkship rotations for centralized teaching (transition education days) throughout year 3. In year 4, students have the opportunity to pursue elective experiences and complete a Transition to Residency course.

See Supplemental Digital Appendix 2-Clerkship Curriculumat http://links.lww.com/ACADMED/A843.

Multiple curricular themes and a Portfolio course support longitudinal, integrated teaching in areas interwoven throughout all 4 years of the MD program. These longitudinal themes are grouped into 3 major categories related to priority population groups, specific CanMEDS roles, and specific content areas.

\section{Curriculum changes since 2010}

- The introduction of the Foundations curriculum over 2016-2017 (year 1) and 2017-2018 (year 2) is one of the most significant changes made to our delivery of medical education. Drawing on theories of expertise, particularly training for adaptive expertise, we implemented curricular strategies and instructional principles that align education activities with the goal of preparing learners to become adaptive physicians. Centered on 72 virtual patient cases, our spiral curriculum introduces learners to complexity and variation, which are necessary prerequisites for expertise development. Learners address this complexity through integrated instruction, productive struggle during guided discovery learning, and assessment that enhances guided learning. ${ }^{1,2}$

- Learning experiences focused on career planning, and wellness/ resiliency have been integrated into the core curriculum across all 4 years. This includes shadowing opportunities in Foundations, a longitudinal resiliency curriculum, and career planning learning sessions in both Foundations and year 3 transition education days.

- The clerkship structure underwent significant revisions over 2010-2011 and 2011-2012 to ensure that all students experience the core specialties and have opportunity to complete the same number of elective weeks before the residency ranking process. The opportunity to pursue a 2 -week home school elective in May/June of year 3 was introduced in 2019-2020. 
Table 1

Student Application Characteristics, 2011-2019

\begin{tabular}{|c|c|c|c|c|c|c|c|c|c|}
\hline Characteristic & Fall 2011 & Fall 2012 & Fall 2013 & Fall 2014 & Fall 2015 & Fall 2016 & Fall 2017 & Fall 2018 & Fall 2019 \\
\hline Applications & 2,956 & 3,052 & 3,153 & 3,463 & 3,488 & 3,121 & 3,167 & 3,262 & 3,555 \\
\hline Offers & 334 & 327 & 338 & 336 & 327 & 319 & 310 & 301 & 307 \\
\hline Yield (\%) & 78.4 & 79.2 & 76.6 & 77.1 & 79.5 & 81.2 & 84.2 & 84.2 & 87.3 \\
\hline Entering GPA & 3.88 & 3.90 & 3.92 & 3.94 & 3.96 & 3.95 & 3.95 & 3.96 & 3.96 \\
\hline
\end{tabular}

Includes data from all admission pathways.

- Two new courses (year 3, Transition to Clerkship, and year 4, Transition to Residency) to support students at key medical education transition points were introduced in 2010-2011.

- A new longitudinal Portfolio course designed to facilitate students' professional development through small-group discussions and guided reflections was introduced in 2010-2011. The initial iteration of this course spanned years 3 and 4 and expanded to years 1 and 2 concurrent with the introduction of Foundations.

\section{Assessment}

Over the 2017-2018 academic year, the MD program refreshed its overarching education goals to more clearly articulate the program's aspiration to prepare graduates who are:

- Clinically competent and prepared for life-long learning through the phases of their career

- Ethical decision-makers dedicated to acting in accordance with the highest standards of professionalism

- Adaptive in response to the needs of patients and communities from diverse and varied populations

- Engaged in integrated, team-based care wherein patient needs are addressed in an equitable, individualized, and holistic manner

- Reflective and able to act in the face of novelty, ambiguity, and complexity

- Resilient and mindful of their well-being and that of their colleagues

- Capable of and committed to evidence-informed practices and scholarship and a culture of continuous performance improvement

To support achievement of these goals, the MD program curriculum is governed by a competency framework adapted for an undergraduate medical education context from CanMEDS 2015. Our competency framework is also informed by Medical Council of Canada objectives and Association of Faculties of Medicine of Canada Entrustable Professional Activities for the transition from medical school to residency.

See Supplemental Digital Appendix 3-Medical Education Program Objectives—at http://links.lww.com/ACADMED/A843.

The Office of Assessment and Evaluation has expanded program evaluation measures beyond traditional measures, such as student satisfaction, to include analytics, meaningful qualitative data, and objective assessment data. These are collected and synthesized to evaluate specific courses and components as well as the program as a whole against the intended outcomes.

\section{Assessment changes since 2010}

- Concurrent with the development of Foundations, we adopted a programmatic assessment model that shifts the purpose of assessment from assessment of learning to assessment for learning. Foundations assessments include frequent nostakes, formative quizzes, as well as assessments to evaluate mastery every 2-3 weeks. An e-portfolio ("Learner Chart”) provides students with access to all of their assessment data. Foundations students meet regularly with an academic coach, with the focus on performance improvement, guided by personalized learning plans to address their challenges and strengths. ${ }^{1}$

- Our Foundations assessment model includes early identification of students who might need additional guidance as well as provision of resources and coaching to enhance self-regulated learning, study strategies, and forms of support tailored to their needs.

- The Foundations assessment items were (and continue to be) developed to test an integrated understanding of foundational and clinical knowledge. Evidence-informed practices in assessment writing and standard setting have been put into practice on an ongoing basis.

- Progress testing has been introduced to longitudinally track student and cohort performance against program objectives.

\section{Pedagogy}

- Independent learning: In Foundations, students are provided with readings, videos, e-learning modules, and other resources they are expected to engage with. Similar independent learning takes place in many core clinical rotations.

- Large-group lectures: Lectures are used in both Foundations and for centralized clerkship learning experiences. These lectures are video-conferenced between our St. George and Mississauga campuses. Some, but not all, of these lectures are recorded for future viewing.

- Small-group learning/discussions (tutorials, workshops, or seminars): In Foundations, tutorials support case-based learning as well as specific curriculum components and longitudinal experiences (e.g., health in the community, 
integrated clinical skills, portfolio, health science research). Clinical skills development is supported through skill-based and clinical decision-making workshops in both Foundations and core clinical rotations. Seminars provide an opportunity in both forums for discussion of longitudinal themes (e.g., ethics, interprofessional education, medical psychiatry, pharmacology) and rotation-specific content.

- Experiential learning: Interactions with standardized patients and real patients, community site visits, role play, and/ or simulations have been integrated into our Foundations curriculum.

- Anatomy labs: Scheduled throughout Foundations, students prepare for anatomy labs using digital anatomy apps and videos and attend in-person to explore dissections, prosections, and anatomical models.

- Clinical experiences (ambulatory and inpatient): Each clerkship rotation includes substantial time spent learning in the context of providing care to patients, often as part of a multidisciplinary team, in a variety of settings including ambulatory clinics, hospital wards, emergency departments, operating rooms, labor and delivery suites, and so forth. Simulations and standardized patients are also incorporated into our core rotations where appropriate.

\section{Changes in pedagogy since 2010}

To enable and support productive struggle, active learning, and cognitive integration, the curriculum evolved from its previous course-based organizational structure to a series of spiraled blocks that build upon each other conceptually, with each block comprised of a sequence of scaffolded cases with progressive introduction of complexity. This evolution involved a shift from lecture-heavy learning to case-based learning, with each case (virtual patient) acting as the core for the week's learning. These virtual patient cases enable students to contextualize their learning and integrate foundational knowledge within the structures of the curriculum. ${ }^{1}$

\section{Clinical experiences}

Clinical experiences are provided in our $30+$ affiliated clinical teaching sites.

In year 2, students are exposed to generalist care through the Family Medicine Longitudinal Experience. Each student is matched with a family physician with whom they spend 6 half days at a community-based clinical site.

In year 3, students complete a series of core clinical rotations of varying lengths. Core family and community medicine, internal medicine, and pediatrics rotations include significant ambulatory experiences.

All clinical teaching sites used by our family and community medicine rotation have identified one or more vulnerable populations as priorities for their practice in the local community. Students assigned to those sites interact with those vulnerable populations as part of required clinical learning activities.

In year 4 , all students are required to complete at least 1 of 3 selective rotations in a community setting.

\section{Curricular Governance}

The MD Program Curriculum Committee is the single, centralized governing body that has overall responsibility for the MD program curriculum. Curricular governance is supported by standing subcommittees that report to the Curriculum Committee.

See Figure 1-Curricular governance.

\section{Education Staff}

Oversight of the administrative, governance, and leadership structures that support the planning, implementation, delivery,

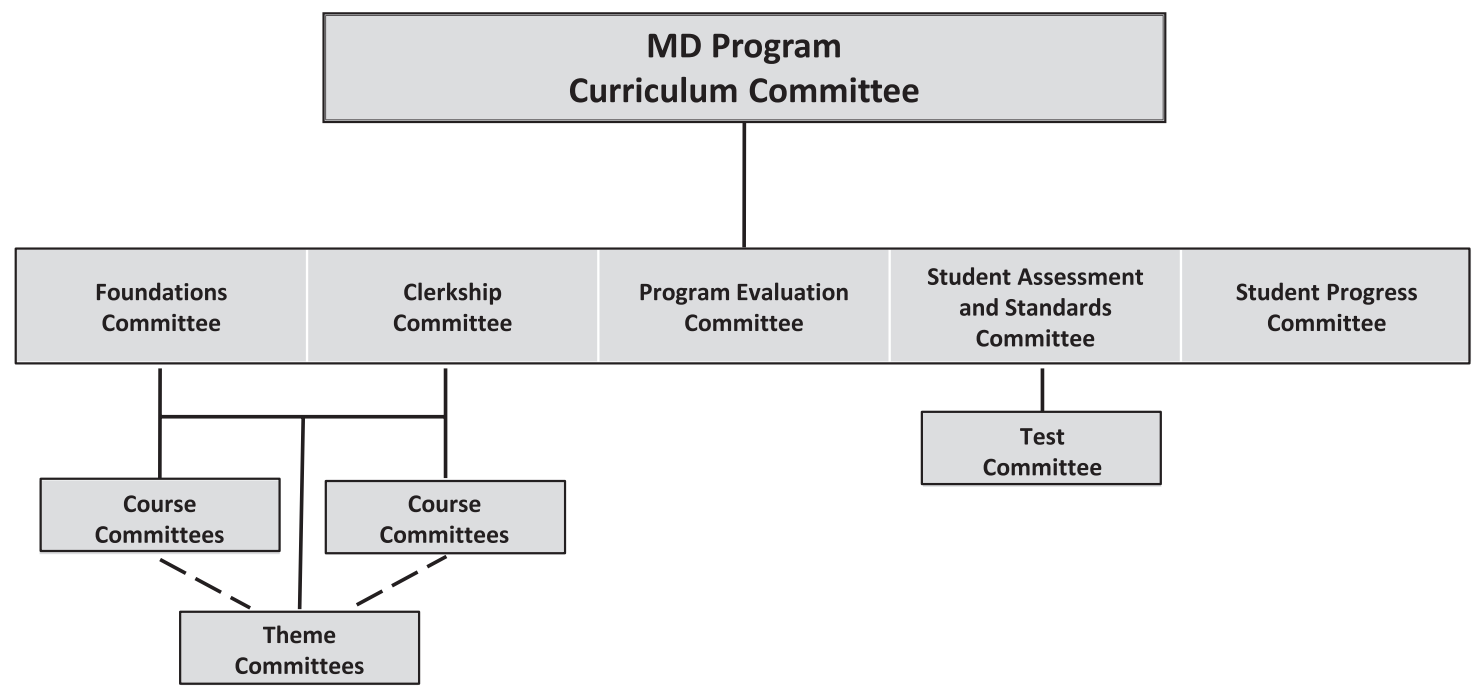

Figure 1 Curricular governance. 


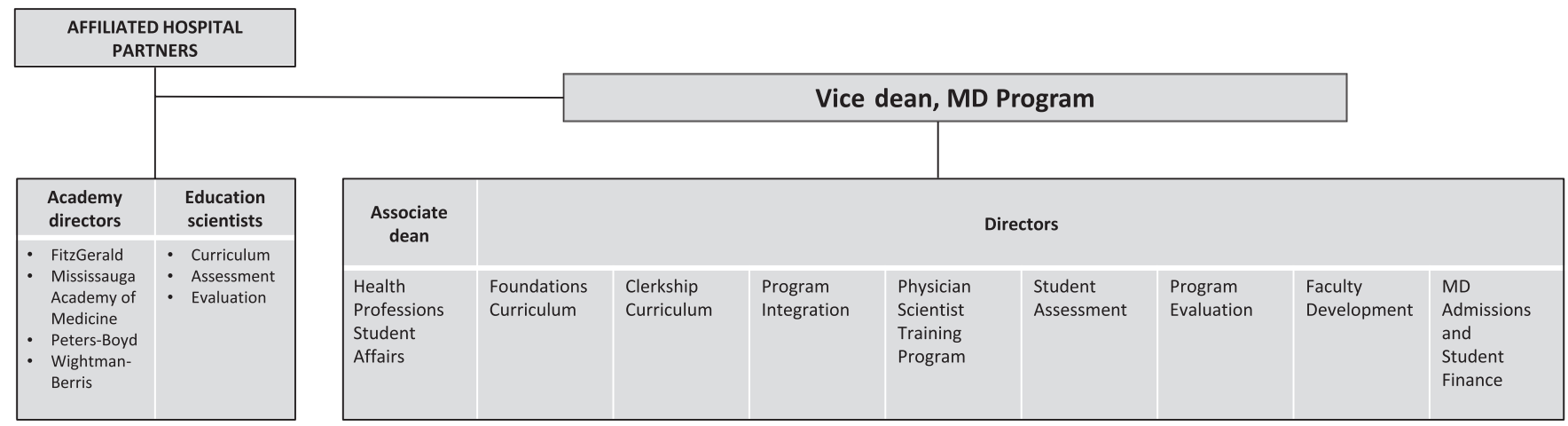

Figure 2 Medical education leadership.

and evaluation of the MD program curriculum is located within the Office of the Vice Dean. The following 6 units support MD program operations: Enrollment Services, Curriculum, Student Assessment and Program Evaluation, Integrated Physician Scientist Program, the Mississauga Academy of Medicine, and the Office of Health Professions Student Affairs.

See Figure 2-Medical education leadership.

\section{Faculty Development and Support in Education}

The MD program has a dedicated director of faculty development responsible for managing development and delivery of a wide range of faculty development events and resources designed specifically to support the various teaching roles across the curriculum. Faculty development is delivered at the clinical sites to make it as feasible and accessible as possible for distributed faculty.

\section{Regional Medical Campuses}

Formed in partnership with the Faculty of Medicine, the University of Toronto Mississauga, and Trillium Health Partners, the Mississauga Academy of Medicine (MAM) officially opened its doors in August 2011. In conjunction with the opening of MAM, the entering class size of the MD program increased by 9 seats in 2011 to our current entry target of 259 domestic students.

\section{Educational experiences across sites}

A consistent educational experience across sites is assured through the MD program's centralized and integrated curricular governance.

\section{Initiatives in Progress}

- Development of an integrated, longitudinal career advising and preparation curriculum that spans all 4 years focused on providing relevant and developmentally appropriate education and skills development.

- Shift to competency-based assessment in clerkship, specifically through the introduction of workplace-based assessments informed by programmatic assessment principles.

- Clerkship reorganization to provide students with greater scheduling flexibility (including to support wellness), enhanced opportunity for ambulatory clinics, integrated simulation opportunities, and longitudinal patient experiences.

- Revisions to our 2 transitions courses to more effectively support students at key transition points.

\section{References}

1 Kulasegaram K, Mylopoulos M, Tonin P, et al. The alignment imperative in curriculum renewal. Med Teach. 2018;40:443-448.

2 Kulasegaram, KM, Martimianakis MA, Mylopoulos M, Whitehead CR Woods NN. Cognition before curriculum. Acad Med. 2013;88:15781585 . 


\section{Western University Schulich School of Medicine \& Dentistry}

Gary Tithecott, MD, MBA, Kyle D. Massey, PhD, Teresa Van Deven, PhD, Shannon L. Venance, MD, PhD,

Fred Ross, Lawrence Jacobs, MD, and Jay Rosenfield, MD, MEd

\section{Medical Education Program Highlights}

- Our UME program mission is to develop generalist physicians as socially responsible leaders, collaborative decision makers, and lifelong adaptive learners.

- Our program uses a distributed model with core learning at London and Windsor campuses, and clinical learning delivered across affiliated community sites throughout Southwestern Ontario.

- Our model of curriculum delivery provides students with exposure to diverse patient care experiences, interprofessional and longitudinal learning, and the impact of social determinants of health in rural and urban communities.

- Using a competency-based medical education (CBME) structure, assessment activities measure student achievement of key and enabling competencies and Entrustable Professional Activities (EPAs).

- With our focus on competency rather than time, students are offered options for advanced or parallel learning aligned with future career goals.

- Experiential learning begins in year 1, including early primary care clinical care experiences, team-based research, service learning, and quality improvement (QI) projects.

- All students are assigned an academic coach to support individual academic success and cultivate learners' growth mindset.

- Program evaluation is carried out by program leaders in collaboration with a student evaluation team.

- Our admissions process is responsive to our commitments to diversity, social responsibility, and accountability, with practices in place to support increasing the number of Indigenous matriculants, those from lower socio-economic backgrounds, and those from rural locations.

- In response to the Truth \& Reconciliation Commission of Canada's Calls for Action, we have integrated Indigenous health and cultural values across all 4 years under guidance of our Indigenous Health Curriculum Task Force. The school is hiring an Indigenous leader in residence to guide these initiatives.

- Our curriculum integrates skill-based training in intercultural competency, human rights, and implicit bias to ensure graduates develop culturally safe health care practices.

Acad Med. 2020;95:S583-S587.

doi: 10.1097/ACM.0000000000003470

Copyright (C) 2020 by the Association of American Medical Colleges

Supplemental digital content for this article is available at http://links.Iww.com/ ACADMED/A960.

Year school was established: 1881.

School URL: https://www.schulich.uwo.ca.
- Student professional identity, professionalism, personal success, and wellness have been embedded in the longitudinal Professionalism, Career, and Wellness course.

- One day each week is set aside for independent learning and pursuit of clinical or scholarly activities of personal interest.

\section{Curriculum}

\section{Curriculum description}

- Our curriculum is composed of integrated semester courses covering core basic, clinical, and social sciences across organ systems and the life cycle.

- Particular emphasis is placed on embedding the social determinants of health and health care systems within a rich context of a patient/family-centeredness lens.

- Clinical decision-making and personal identity development are supported in clinical skills, experiential learning, and smallgroup learning throughout years 3 and 4 clerkships and clinical immersion.

- Following the introductory Foundations of Medicine course, the Principles of Medicine I, Principles of Medicine II, and Transition to Clerkship courses prepare students with knowledge, skills, attitudes, and behaviors necessary to begin clinical immersion.

- There is a 20-month immersion in clinical learning during years 3 and 4 beginning with the yearlong integrated clerkship course, followed by 16 weeks of electives. The clerkship learning experiences are delivered in London, Windsor, and across 60 sites in Southwestern Ontario.

- Critically, the curriculum prepares medical students at key transition points in the Transition to Clerkship (end of year 2 ) and Integration and Transition (end of year 4) courses. The complexity of assessment and management is elevated in these case-based active learning courses, with higher-order learning expectations for competencies and EPAs.

- The 4-year Professionalism, Career, and Wellness and Experiential Learning courses support achievement of the professional, leader, and scholar CanMEDS competencies.

- The curriculum is designed to graduate competent generalists prepared for the next stage of training, with particular preparation for competency-based postgraduate residency programs in Canada.

See Supplemental Digital Appendix 1-Curriculum Structureat http://links.lww.com/ACADMED/A960.

\section{Curriculum changes since 2010}

- Since 2010, we have implemented stepwise innovations in learning, assessment, outcomes, evaluation, and QI.

- In September 2019, we launched our CBME model, and are currently in a 3-year transition from our legacy systems-based curriculum, created in 2008. 
- Overall goals for this curriculum renewal include:

- Improving health care provision using an outcomes-based model

- Aligning with best practices of Canadian CBME

- Supporting and measuring students' demonstration of the competencies, knowledge, skills, and attitudes necessary to meet the needs of the diverse Canadian population we serve

- Preparing graduates for continual lifelong learning

- Our curricular objectives align with Canadian standards including: CanMEDS 2015; the Medical Council of Canada assessment blueprint; and Association of Faculties of Medicine of Canada (AFMC) EPAs.

\section{Assessment}

- Our competency framework is derived from the 2015 CanMEDS roles: medical expert, communicator, collaborator, leader, health advocate, scholar, and professional.

- Each role is defined by key and enabling competencies measured in stages across years 1-4 and assessed within the program before graduation.

- Each student also demonstrates achievement of all competencies by graduation using an electronic portfolio and dashboard.

- Achievement of competence in 12 defined clinical tasks is also assessed longitudinally with the AFMC EPA observed assessments across all 4 years using developmental markers (milestones).

- True to our CBME model, assessment for learning is carried out using a multifaceted system of frequent, formative, and low- and no-stakes assessments.

- Students are expected to monitor academic progress on formative assessments and in achieving key and enabling competencies monthly with their academic coach. Where appropriate, students develop learning plans to address their gaps or individual learning goals.

- We focus on effective and timely feedback to facilitate student developmental progression to reach GME entry competence.

See Table 1-Key Competencies and Assessment Methods.

\section{Parallel curriculum or tracks}

The Schulich Medicine MD program has legacy and evolving parallel tracks available to students:

- Legacy:

- MD-PhD program: For the past 20 years, cohorts of 3 students are admitted to this stream and complete their $\mathrm{PhD}$ with a Western University faculty in foundational and clinical sciences. Their $\mathrm{PhD}$ studies are pre-year 1 or after year 2 of the MD program.

- MD-MSc oral and maxillofacial surgery program: One student is admitted annually to this 6-year program. Candidates for admission must already possess a DDS or DMD degree. Acceptance includes admission into the oral and maxillofacial surgery, medical, and graduate studies programs.
- Planned:

- Western University certificates in special interest areas such as global health

- Joint master's degrees: MD-MBA, MD-MPH, and MD and other Western University master's programs

- Parallel certificates or master's degrees with University of Windsor for Windsor Campus students

- Expansion of MD-PhD offerings available across multiple faculties such as engineering and science

\section{Pedagogy}

- Teaching and learning methods support active engagement in clinically relevant sessions delivered in alignment with program outcomes.

- Exposure to clinical decision-making begins early in year 1 and continues across all courses.

- Years 1 and 2 courses use case-based learning, characterized by weekly authentic clinical cases that are revisited and built upon throughout the week using inquiry-based learning methods aligned to course and program outcomes.

- Faculty-led small groups build communication, leadership, advocacy, and collaboration skills such as listening, questioning, and responding, while strengthening critical thinking and problem solving.

- Experiential learning opportunities as required learning include research or QI projects, service learning, and longitudinal clinical exposure building professional, scholar, communicator, advocate, collaborator, with medical expert.

- Large-group sessions are interactive with required advance preparation. Educators promote active participation within the large-group context, using questions directed to deepen learning, group discussion, and using audienceresponse systems to poll students in real time to assess for understanding.

- Traditional cadaveric dissection anatomy is delivered with small-group educators and faculty/health professionals.

- Team-based projects and reflections are grounded in patient care and experiences.

- Service learning is mandatory and offered in partnership with local community organizations.

- Global and regional learning partner sites offer students optional additional clinical learning over year 1 , year 2, summer recess, and year 4 .

- Optional self-directed clinical and research learning is supported by the school in London, Windsor, and our distributed education region.

- Standardized patients are used with small-group sessions in years 1 and 2 to develop clinical skills, and simulation is employed for some clinical learning in year 3 and across other courses.

\section{Clinical experiences}

- All mandatory clinical experiences occur in clinical affiliates of the school or Canadian partners.

- Clinical learning before, during, and after clerkship is focused in affiliated London and Windsor hospitals, clinical health care affiliates, and community sites across Southwestern Ontario. 


\section{Table 1}

\section{Key Competencies and Assessment Methods}

Key competencies

\section{Medical expert (ME)}

- ME 1. Practice medicine within the scope of generalism as an undifferentiated generalist physician.

- ME 2. Perform a patient- and family-centered clinical assessment, formulate a diagnosis, create, and implement a management plan.

- ME 3. Plan and perform procedures and therapies for the purpose of patient management.

- ME 4. Formulate and implement plans for ongoing patient care and when appropriate seek timely consultation.

- ME 5. Actively contribute as a member of a team providing care, to the continuous improvement of health care quality and patient safety.

\section{Communicator (CM)}

- CM 1. Develop and recognize the essential skills of a communicator.

- CM 2. Develop a common understanding on issues, problems and plans with patients, families, colleagues, and other professionals to develop a shared plan of care.

- CM 3. Develop practices for documenting and sharing written and electronic information on the encounter to optimize clinical decision-making, patient safety, confidentiality, and privacy.

\section{Collaborator (CO)}

- CO 1. Work effectively and appropriately within an interprofessional health care team.

- $\mathrm{CO}$ 2. Contribute to a positive professional work and care environment.

\section{Assessment methods}

Formative and summative assessments

Projects

OSCES

Clinical assessments

Team submissions

\section{OSCE}

Clinical assessments

Small-group assessments

Peer assessments

Projects in teams

Simulation

Narrative reflections

Clinical assessments

Peer assessments

Projects

Small-group learning

Simulation

Service learning

Service learning

Small-group learning

Clerkship

Team projects in QI and year 4

Clinical assessments

Research proposal

Service learning

Clerkship clinical assessments

Narrative reflections

Small-group assessments

Team-based research and Q projects

Clinical assessments

Peer and faculty assessments in small-group and clinical assessments

Narrative reflections

Peer assessments

Specific course dedicated to this competency 
- Longitudinal clinical experiences in years 1 and 2, and our rural immersion week, occur in local family medicine clinics.

- QI projects and team-based chronic disease experiences are in clinical affiliates and community care sites.

- During years 1 and 2 and in summer recess, students are supported in undertaking clinical learning at local or regional sites.

- Students in year 4 are given 16 weeks of approved elective and selective clinical learning regionally, across Canada, or internationally.

\section{Curricular Governance}

- A centralized curriculum governance model is led by the Curriculum Committee, responsible for oversight of all program learning including development, management, and evaluation of the 4-year UME curriculum.

- The Competence Committee reviews learner assessment and makes recommendations on progression and achievement of competencies.

- QI processes and initiatives are guided and monitored by our Quality Committee and Accreditation Committee.

- All committees include representation (where appropriate) from program and school leadership, courses chairs, clinical and foundational science faculty, student affairs, staff, and junior and senior students from London and Windsor.
- The Curriculum Committee communicates with the faculty and the school through a monthly newsletter and reports to the Executive Committee of Schulich Council.

See Figure 1-Curricular governance structure.

\section{Education Staff}

- The UME Office, under the leadership of the associate dean, UME and UME manager, is responsible for the MD program.

- The UME Office is led by the manager and has 17 staff members who support students and teaching faculty in delivery of the MD program. Key positions include coordinators who are responsible for each of curriculum oversight, years 1 through 4 curriculum support, program evaluation, and educational technology.

- Our Windsor campus is led by the associate dean, Windsor, and a local manager with 16 administrative staff.

- Working in tandem with staff, UME has a number of faculty leads to provide direction and process oversight for assessment, QI, competency-based curriculum design, and population- and health-related topics.

See Figure 2-Organizational chart of decanal positions.

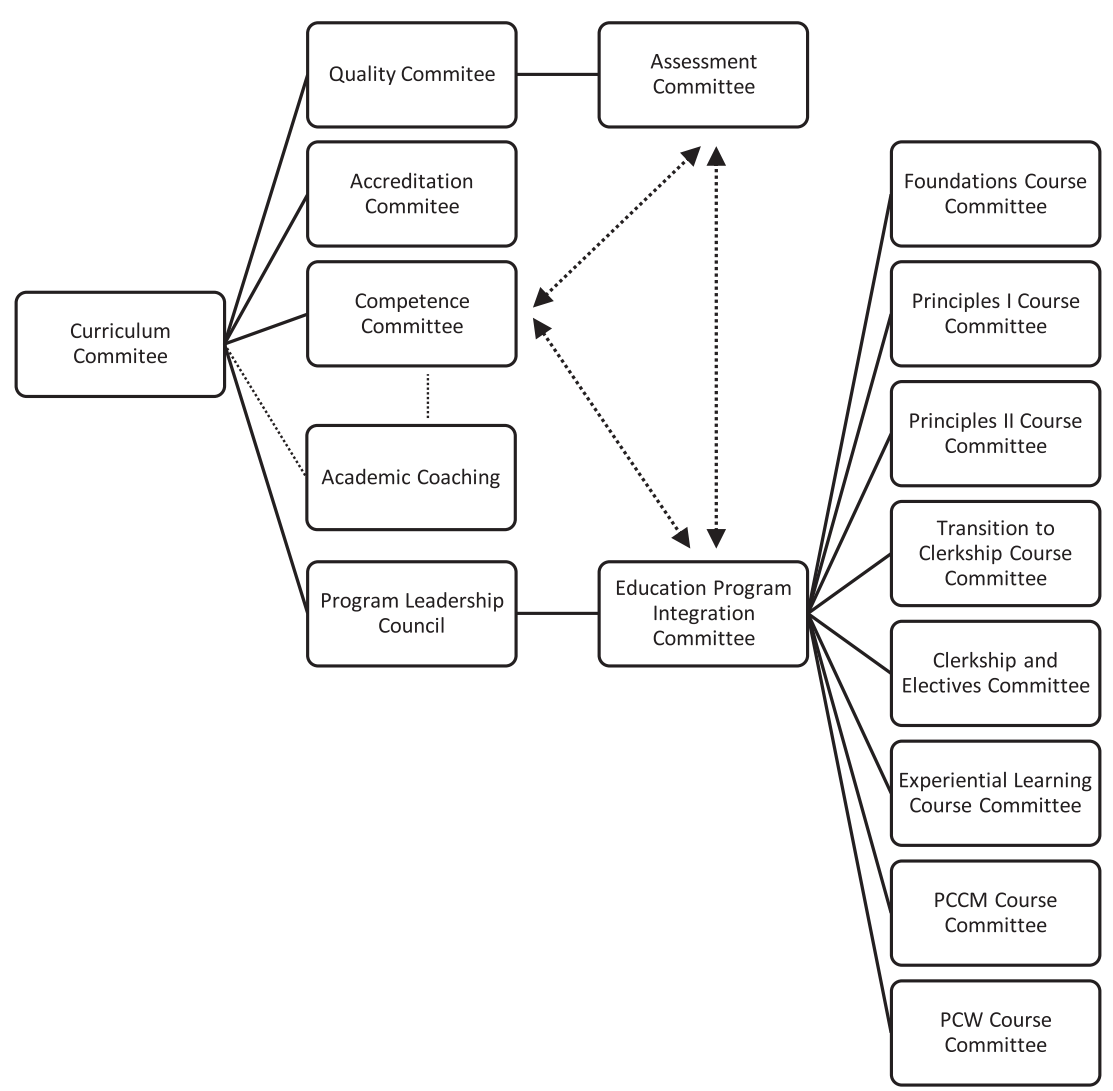

Figure 1 Curricular governance structure. 


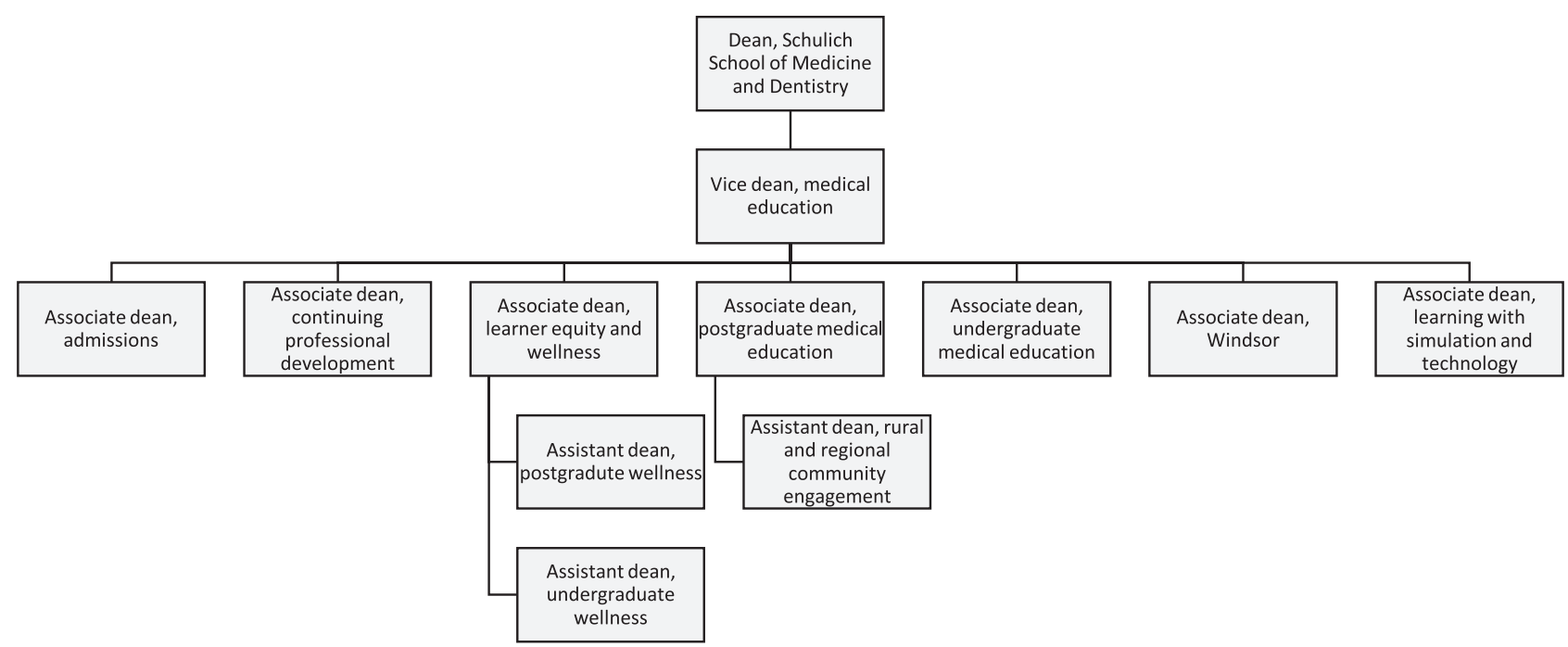

Figure 2 Organizational chart of decanal positions.

\section{Faculty Development and Support in Education}

- All Schulich Medicine faculty are supported with faculty development activities sponsored by the school and Western University to advance their competency as educators and leaders. These include in-person and distance learning workshops and seminars aimed at improving teaching effectiveness, curriculum development skills, academic coaching, assessment, and small-group facilitation.

- Residents with teaching responsibilities are supported in developing skills as evolving educators through the Postgraduate Medical Education Office annual bootcamp for resident teachers, an intensive 2-day program including sessions on cross-disciplinary teaching skills. Attendees are encouraged to teach others in a "train the trainer" process. Residents may enroll at no cost in courses offered by our Continuing Professional Development Office.

- Faculty, residents, and students may enroll in a Masters of Health Professions Education or other education master's and $\mathrm{PhD}$ programs through Western University's Centre for Education Research and Innovation, in partnership with the University of British Columbia and Maastricht University.

- The Centre for Teaching and Learning at Western University offers educators training, mentorship, research, and innovation opportunities through seminars, courses, certificate programs, and other resources.

- For promotion and tenure, Western University recognizes contributions to education innovation, teaching, and leadership as equivalent to research contributions.

\section{Regional Campus}

- The Windsor campus of Schulich Medicine has operated for over 10 years in collaboration with the University of Windsor, Windsor hospitals, clinics, and centers for patient care.

- The Windsor campus delivers the entire 4-year MD curriculum. Windsor has 38 students in each class of 171 first-year students. Resident presence in Windsor includes freestanding residency programs in family medicine and psychiatry, as well as resident rotations in pediatrics, medicine, emergency medicine, palliative care, obstetrics-gynecology, anesthesia, and some surgical disciplines.

- Educators, staff, and leaders from the Windsor campus are members of all, and cochair some, course and governance committees. Windsor personnel are engaged in curriculum innovation and ongoing improvement.

- Schulich Medicine partners with University of Windsor faculty members in the delivery of selected teaching and with respect to student research projects.

\section{Initiatives in Progress}

Our MD program is a part of an overall integrated medical education initiative, designed to better align the continuum of medical education (with a consistent CBME philosophy) and develop an integrated health and education network in Southwestern Ontario, supported by our distributed network and academy system. 


\title{
Northern Ontario School of Medicine
}

\author{
Brian M. Ross, PhD, MEd, and Catherine Cervin, MD, FCFP, MAEd
}

\section{Medical Education Program Highlights}

The Northern Ontario School of Medicine (NOSM) was established in 2005 as Canada's newest medical school. ${ }^{1}$ It is a not-for-profit corporation that acts as the Faculty of Medicine of 2 universities, Lakehead University and Laurentian University. ${ }^{1}$ The school was founded with a requirement to be socially accountable: that its activities should lead to an improvement in both the health and health care of those living in the region it serves.

Northern Ontarians experience generally poorer health than those living elsewhere in the province, due to the interplay of many factors including those related to geography and ethnicity. ${ }^{2}$ The region covers approximately 800,000 square kilometers, most of which is sparsely populated. Indeed, although some residents live in smaller cities of approximately 70,000-110,000 residents, many live in smaller urban, or in rural and often remote, communities. Many of the region's communities have experienced difficulties recruiting and retaining physicians and other health care professionals, thereby limiting access to health care services. The region is also home to Indigenous and Francophone persons who experience higher levels of health inequity, including higher rates of morbidity and mortality. ${ }^{3}$ Socially accountable medical education at NOSM has therefore focused on the areas of greatest need, these being Indigenous health, Francophone health, and rural health. The curriculum includes a unique collection of educational experiences that aim to equip students with the necessary knowledge, skills, and attitudes to meet the needs of those underserved populations. These include:

- During the first 2 years (preclerkship) of study, NOSM students take part in three 4 -week placements situated in rural communities across the region. In the first year, students study the historical, social, cultural, and geographic factors that determine the health of Indigenous peoples in Northern Ontario and take part in activities that prepare the student to deliver culturally safe care to their Indigenous patients. This begins with classroom-based sessions, some of which are taught by Indigenous persons including Elders. The year ends with a mandatory 4 -week cultural immersion experience during which students live and learn in an Indigenous community, following a curriculum designed by

Acad Med. 2020;95:S588-S591.

doi: 10.1097/ACM.0000000000003348

Copyright (C) 2020 by the Association of American Medical Colleges

Supplemental digital content for this article is available at http://links.Iww.com/ ACADMED/A871.

Year school was established: 2002.

School URL: https://www.nosm.ca. the community itself. In year 2, students spend time in a rural and/or remote community during 2-month-long community placements to learn about and develop an appreciation for rural practice. During these 4-week blocks, students are taught by one or more of NOSM's rural community-based faculty members. They also attend social and cultural events that aim to develop an understanding of what it is like to live in a small community as a physician.

- Once per week students take part in Community Learning Sessions (CLS) situated in a community setting related to health care. These could be the office of a physiotherapist or other health care professional as part of the school's interprofessionalism curriculum, or in a health or social services agency such as addiction services or children's mental health. CLS allows students to learn firsthand about the wider health care system.

- Clinical reasoning and decision-making, including the ability to generate differential diagnoses are key skills for medical students. A recent curricular innovation, Medicine in Practice (MIP), has first- and second-year NOSM students learn these skills directly from clinical faculty through a series of worked example cases. MIP also covers the realities of physician work, including ethical dilemmas, when and when not to order tests, and the influence of commercial interests on health care. Along with facilitated problem-based learning sessions, these innovative classroom activities assist students to gain confidence and ability in clinical work before entering their clerkship.

- In the program's third year, students complete a mandatory 8-month comprehensive community clerkship (CCC) (also known as a longitudinal integrated clerkship). This occurs outside of the 2 largest population centers in mainly primary care settings. The clerkship functions both as an introduction to small-community practice and as a means for students to become aware of available career opportunities.

\section{Curriculum}

\section{Curriculum description}

The MD is a 4-year distributed medical education program that supports each student to achieve the program outcomes (described below), which are aligned with the CanMEDS ${ }^{4}$ competency framework.

The program is split into 3 phases. Phase 1 encompasses years 1 and 2 and is a mainly classroom-based preclerkship that takes place at one of the school's 2 main campuses in Thunder Bay or Sudbury. This phase includes a cultural immersion, placements in remote and/or rural communities, and weekly communitybased learning experiences as described above.

Phase 1 consists of a 4-week introductory module followed by 10 modules ( 5 in each year) organized around the body systems: 
- Module 101: Introduction to medical studies

- Module 102: Gastrointestinal system

- Module 103: Cardiopulmonary system

- Module 104: Neuroscience 1 with a focus on neurology

- Module 105: Musculoskeletal system

- Module 106: Endocrine system

- Module 107: Reproductive system and genetics

- Module 108: Renal system

- Module 109: Hematology and Immunology

- Module 110: Neuroscience 2 with a focus on mental health

- Module 111: Integumentary system and integrative physiology

Phases 2 and 3 can be thought of as the equivalent to the traditional clerkship, providing clinical training supervised by a physician preceptor. Phase 2 , the CCC, takes place during year 3 in a mainly primary care setting at 1 of 15 sites ranging from small rural to small urban. During Phase 2, students learn the core clinical disciplines, not as distinct rotations, but longitudinally throughout the year.

Phase 3 in year 4 occurs in a tertiary care hospital, in Sudbury or Thunder Bay. It includes the traditional rotations of children's health, women's health, surgery, internal medicine, psychiatry, and emergency medicine, along with at least 14 weeks of electives.

The program uses an integrated teaching model containing 5 concurrent curricular elements, termed "themes." Each Theme Committee then designs and governs 4 year-long courses, 1 for each year of the program, making a total of 20 courses that all students must pass to obtain their degree. The 5 themes ${ }^{5}$ are:

- Theme 1: Northern and rural health

- Theme 2: Professional and personal aspects of health care

- Theme 3: Social and population health

- Theme 4: Foundations of medicine (the "sciences")

- Theme 5: Introduction to clinical medicine in Phase 1, clinical medicine and therapeutics in Phases 2 and 3

\section{Curriculum changes since 2010}

The curricular blueprint has not been fundamentally altered since 2010 although updates and enhancements have been implemented for quality improvement purposes, including curricula related to pharmacology and therapeutics, clinical reasoning, the assessment and teaching of professionalism, culinary medicine, advocacy, and research. The program has begun planning curriculum renewal, a process that will begin in earnest in the 2020-2021 academic year.

The class size was increased from 56 to 64 students beginning in the 2010-2011 academic year. To accommodate this change, additional community sites were added to the Phase 2 (year 3 ) clerkship, and two more 4-student streams to the Phase 3 (year 4) rotations.

\section{Assessment}

The medical education program objectives are organized by the program's 5 themes (described above), and were written to align with the CanMEDS framework.

See Supplemental Digital Appendix 1-Medical Education Program Outcomes-at http://links.lww.com/ACADMED/ A871.

The program's assessment structure requires that students obtain a passing grade in each of the 5 courses that constitute each year of study. Some new assessment exercises have been added including student reflective exercises about time spent in the weekly CLS, and in the rural and remote community placements in year 2. The program has also limited the term "remediation" to major course fails that require either the repeat of a course or rotation, or the addition of nonroutine educational activities to the student's program. Repeating a failed assessment is viewed as minor, is termed "reassessment," and is not recorded on the student's permanent record.

\section{Curricular Governance}

The academic governance of the program is unusual due to NOSM being the Faculty of Medicine of 2 universities (Lakehead and Laurentian Universities) and hence academic authority rests with 2 senates. Within the school curriculum, governance is situated in the Undergraduate Medical Education Committee and its Curriculum Committee Subcommittee, with the governance of each phase, theme, and courses, as well as different activities, being delegated to a series of subcommittees.

There is a single curriculum governance structure that applies to all the teaching sites that make up the program. The faculty, staff, and students participating in governance are drawn from across teaching, sites which helps ensure consistency and feasibility of the program's curriculum between locations. Participants attend meetings either in-person or by video- or teleconferencing.

See Supplemental Digital Appendix 2-Curriculum Governance Structure-at http://links.lww.com/ACADMED/A871.

\section{Education Staff}

The Office of Undergraduate Medical Education (UME) is led by the associate dean for UME, who is the head academic and administrative officer for the program. A number of academic staff report to the associate dean including the phase leads (the assistant dean for Phase 1, and the clerkship directors for phase 2 or 3 ), the chairs for themes 1 through 5 , the assistant dean for clinical education, the assistant dean for admissions, and the director of program evaluation and assessment. The senior director of UME administration reports to the associate dean for UME. The director is the most senior 
administrative position in UME and oversees the work of approximately 40 staff and managers.

NOSM is headed by the dean of medicine, who is also the president and CEO of the corporation to whom the vice dean academic and several associate deans report (a number of associate and assistant deans report to the vice dean academic who oversees education including UME, postgraduate education, learner affairs, and continuing education and professional development).

See Figure 1-Decanal organizational chart.

The Office of UME has responsibility for the 4-year MD program, and also for the admissions unit, which recruits and selects students for entry into UME.

Although NOSM does not have a Department of Medical Education, the Office of UME serves an equivalent function, appointing and supervising the faculty who serve in leadership positions within the program. NOSM also conducts medical education scholarship within the Medical Education Research Laboratory in the North, with which UME closely collaborates.
See Supplemental Digital Appendix 3-Academic and Corporate Governance Structure-at http://links.lww.com/ACADMED/A871.

\section{Faculty Development and Support in Education}

Faculty professional development at NOSM is led by the Office of Continuing Education and Professional Development (CEPD). CEPD delivers a program of development opportunities in person, by videoconference, and by means of prepared online learning modules or sessions. The CEPD unit also runs Northern Constellations, an annual faculty development conference open to all faculty, and has hosted Northern Lights, a leadership development event for physician faculty. The school's annual Northern Health Research Conference also provides faculty with the opportunity to develop their research and scholarship skills.

The policies governing appointment and promotion of all NOSM faculty foster teaching excellence as a key component of career progression. Faculty develop a teaching portfolio that contains their teaching evaluations (by peers and students), and this, along with recommendations from peers and those in leadership positions, is used in the promotions process.

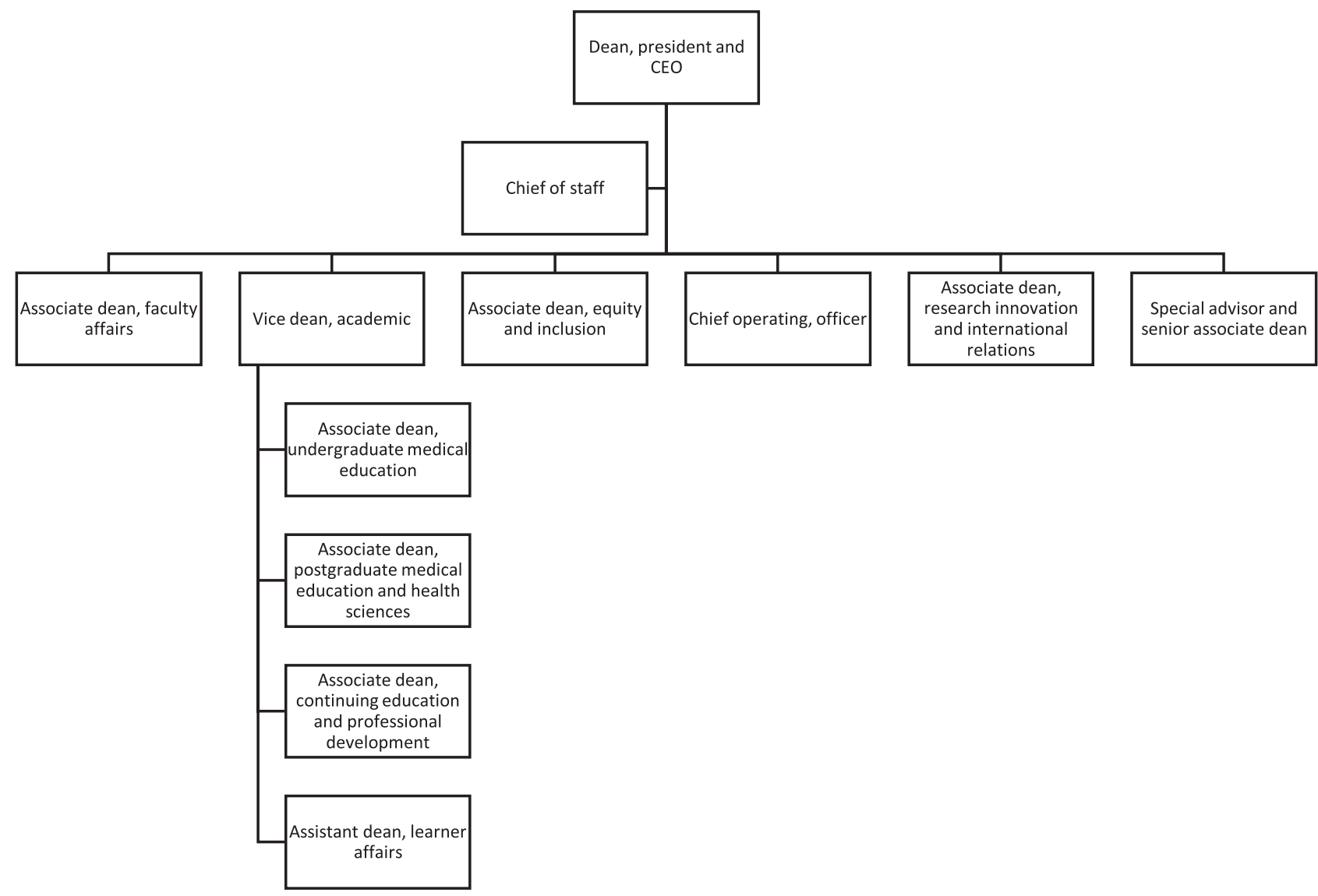

Figure 1 Decanal organizational chart. 


\section{Table 1}

\section{Regional Medical Campuses}

\begin{tabular}{|c|c|c|}
\hline $\begin{array}{l}\text { Regional } \\
\text { campus } \\
\text { name }\end{array}$ & Type & $\begin{array}{l}\text { Student } \\
\text { enrollment } \\
\text { in 2019-2020 }\end{array}$ \\
\hline Bracebridge & Comprehensive community clerkship 4 & \\
\hline Dryden & Comprehensive community clerkship 3 & 3 \\
\hline Fort Frances & Comprehensive community clerkship 2 & \\
\hline Hearst & Comprehensive community clerkship 2 & \\
\hline Huntsville & Comprehensive community clerkship 4 & \\
\hline Kapuska........... & Comprehensive community clerkship 4 & \\
\hline Kenora & Comprehensive community clerkship & \\
\hline Manitoulin Island & Comprehensive community clerkship 2 & \\
\hline Midland & Comprehensive community clerkship 4 & \\
\hline North Bay & Comprehensive community clerkship 9 & \\
\hline Parry Sound & Comprehensive community clerkship 4 & \\
\hline Sault Ste. Marie & Comprehensive community clerkship 8 & \\
\hline Sioux Lookout & Comprehensive community clerkship 4 & 4 \\
\hline $\begin{array}{l}\text { Temiskaming } \\
\text { Shores }\end{array}$ & Comprehensive community clerkship & 4 \\
\hline Timmins & Comprehensive community clerkship & 7 \\
\hline
\end{tabular}

\section{Regional Medical Campuses}

The Phase 2 CCC takes place at 15 regional sites.

See Table 1-Regional Medical Campuses.

\section{References}

1 Strasser R. Learning medicine in rural and remote community. In: Tesson G, Hudson G, Strasser R, Hunt D, eds. The Making of the Northern Ontario School of Medicine. A Case Study in the History of Medical Education. Montreal and Kingston, Canada: McGill-Queens University Press; 2009:33-46.

2 Statistics Canada. Table 13-10-0389-01. Life expectancy, at birth and at age 65 , by sex, three-year average, Canada, provinces, territories, health regions and peer groups. https://www150.statcan.gc.ca/t1/tbl1/en/ tv.action?pid=1310038901. Accessed March 22, 2020.

3 Young SW, Nishri ED, Candido E, Marrett LD. Health Reports. Colorectal cancer incidence in the aboriginal population of Ontario, 1998 to 2009. https://www150.statcan.agc.ca/n1/pub/82003-x/2015004/article/14157-eng.pdf. Published April 1, 2015. Accessed March 22, 2020.

4 Frank JR, Danoff D. The CanMEDS initiative: Implementing an outcomes-based framework of physician competencies. Med Teach. 2007;29:642-647.

5 Lakehead University. Undergraduate courses in the faculty of medicine. http://csdc.lakeheadu.ca/ /Catalog/ViewCatalog.aspx?pageid=viewcatalo g\&catalogid=26\&chapterid=7723. Accessed March 22, 2020. 


\section{McGill University Faculty of Medicine}

Beth-Ann Cummings, MD, MSc (HPE), Marion Koch, MD, Melanie Mondou, MD, MSc (HPE), Catherine Millar, MA, and Michelle Elizov, MD, MSc (HPE)

\section{Medical Education Program Highlights}

"Patient at heart, Science in hand" is at the core of the 4-year MD, CM program at McGill University. The Longitudinal Family Medicine Experience (LFME) immerses first-year medical students in early clinical exposure in the specialty of family medicine, while learning the basic and social sciences that underpin medicine. In year 2 of the program, students benefit from 6 months of the Transition to Clinical Practice (TCP) course, learning at the bedside without responsibility for patient care in preparation for taking an active role in caring for patients in clerkship. During clerkship, students complete their third-year rotations in 3 semiintegrated blocks that allow them to learn 2 disciplines hand in hand (e.g., medicine and surgery, pediatrics and obstetricsgynecology, family medicine and psychiatry). Throughout the 4-year program, groups of 6 students meet with a mentor (Osler Fellow) as part of the Physician Apprenticeship program.

\section{Curriculum}

\section{Curriculum description}

The mission of the MD, CM program is "to prepare students for careers as key members of the medical community, whether in clinical practice, in medical education, in research or as leaders of the health care system." Three longitudinal themes run throughout the curriculum: basic science, critical thinking, and knowledge translation (BSCTKT), emphasizing the basic sciences that underpin medical practice; social accountability, population health, and health advocacy (SAPHHA), emphasizing that medical practice is in service to society; and professional identity and practice, emphasizing professional identity formation and the dual role of the physician as professional and healer (i.e., physicianship). The MD, CM program includes 4 curricular components: Fundamentals of Medicine and Dentistry (FMD, first 1.5 years), TCP (second half of year 2), clerkship (years 3 and 4), and physicianship (longitudinal across all 4 years).

See Supplemental Digital Appendix 1-Curriculum Schema-at http://links.lww.com/ACADMED/A842.

\section{Curriculum changes since 2010}

The strategic planning process that concluded in 2010 led to the rollout of a revised MD, CM curriculum. The class of

Acad Med. 2020;95:S592-S595.

doi: 10.1097/ACM.0000000000003309

Copyright (C) 2020 by the Association of American Medical Colleges

Supplemental digital content for this article is available at http://links.Iww.com/ ACADMED/A842.

Year school was established: 1829.

School URL: mcgill.ca/medicine.
2017 was the first to graduate from the new curriculum. The curriculum features an increased focus on public health, social accountability, and primary care while maintaining traditional strengths in basic sciences and clinical training. The revised program also includes a longitudinal curriculum in pointof-care ultrasound, the Mindful Medical Practice course, and interprofessional education sessions with students from other professional programs in the Faculty of Medicine (nursing, physical therapy, occupational therapy, and speech language pathology). Aligned with the Future of Medical Education in Canada recommendations emphasizing the transitions between educational phases, the new curriculum includes the TCP (between FMD and clerkship), Transition to Clerkship (at the end of TCP), and Transition to Residency (at the end of clerkship). The assistant dean, student affairs, and the team at the Wellness Enhanced Lifelong Learning (WELL) Office (i.e., student affairs) oversee longitudinal curricula related to wellness and career advising that are integrated into the MD, CM program.

Since the implementation of the new curriculum, curricular changes have been on an annual basis in response to student and faculty feedback, student performance on licensing exams, and evolving societal problems. Upcoming changes already planned for 2020-2021 and beyond include the replacement of TCP Anesthesia in second year with an anesthesia clerkship in third year and enhancing teaching by family physicians during FMD. The MDCM Program Committee (curriculum committee) is also working on the integration of all 12 Association of Faculties of Medicine of Canada (AFMC) Entrustable Professional Activities (EPAs) into the curriculum, enhancing teaching related to the opioid crisis, and evolving the way human sexuality is taught in recognition of evolution in our understanding of gender and sexuality.

The size of the incoming class is increasing in 2020, coincident with the opening of our distributed medical campus in Gatineau, in the Outaouais Region of Quebec.

\section{Assessment}

The objectives of the MD, CM program follow the CanMEDS framework and incorporate the Medical Council of Canada list of clinical presentations, with the AFMC EPAs informing our assessment approach.

See Supplemental Digital Appendix 2-Medical Education Program Objectives and Assessment Methods-at http://links. lww.com/ACADMED/A842.

The assessment program relies on a variety of assessment methods and incorporates assessment that is purely formative, low-stakes summative, and high-stakes summative. Since the curricular renewal begun with the class of 2017, the MD, CM 
program uses progress tests in addition to other methods of assessment such as anatomy lab exams, final written examinations, written assignments, oral presentations, and objective structured clinical examinations. Some small-group sessions in FMD include peer assessment. In the clinical setting, the student assessment forms in TCP and clinical assessment forms in clerkship rate students across the CanMEDS competencies and emphasize the provision of formative feedback for ongoing development and summative feedback for Pass/Fail decisions. Beginning in 2020-2021, in addition to the existing progress tests used in clerkship, discipline-based clerkship exams are being reintroduced to favor discipline-based knowledge acquisition.

Teaching and learning methods include case-based learning in small groups of 12-18 students, large-group interactive sessions with up to 60 students, whole-class lectures, anatomy laboratory sessions with both prosection and dissection, simulation with standardized patients and with technical models, online virtual patient cases, online modules and podcasts, workshops and clinical experiences in ambulatory, ER, OR, birthing center, and inpatient settings. The curricular renewal was associated with an increase in interactive learning sessions, simulation, and online cases. Clerkship student integration into health care teams has been maintained in the revised curriculum, with students actively participating in the care of patients, including documenting consultations, admissions, progress notes, and discharge summaries and writing medical orders for revision and co-signature.

Clinical experiences occur in a variety of settings ranging from independent family doctor's offices, to group practices, community hospitals, and large tertiary and quaternary care academic health centers. Students have meaningful exposure to both acute and chronic presentations of common and serious diseases. All students participate in the LFME in outpatient family medicine in the first year of the program, and all students complete 4 weeks of regional family medicine outside of the major teaching hospitals in Montreal during the third year (clerkship).

\section{Curricular Governance}

The MDCM Program Committee is the curriculum committee for the program, with central oversight of the entire curriculum. The Program Committee is assisted by component subcommittees, theme subcommittees, the Program Evaluation and Curricular Outcomes subcommittee and the Wellness and Learning Environment subcommittee. While the curriculum is managed centrally, the clinical departments have an important role in clinical courses and input into the selection of course leadership for TCP and clerkship.

See Figure 1-Curriculum governance structure.

\section{Education Staff}

The Faculty of Medicine is in the process of being renamed the Faculty of Medicine and Health Sciences. The Faculty includes cross-cutting offices that serve all of the health sciences schools within its mandate, such as the Office of the ViceDean, Education, the Office of Accreditation and Education Quality Improvement, the WELL Office (student affairs office), and the Faculty Development Office. The Faculty is also in the process of establishing a formal School of Medicine, which will include undergraduate medical education (UME), postgraduate medical education (PGME), and continuing professional development (CPD). Currently, Medical Education Administration and Operations administratively includes UME, PGME, and educational systems. UME benefits from an administrative team of 28 people supporting the planning, implementation, and oversight of the curriculum, UME student records, interprofessional education, and distributed medical education. The UME Office works closely with the Medical Education Systems team (7 people, including 2 data analysts) for the development and maintenance of the tools to support curriculum delivery, monitoring, and management. An estimated 20 administrators will support the Outaouais campus opening in August 2020, including UME; student affairs and faculty development; and other dossiers such as PGME, finance, and academic affairs.

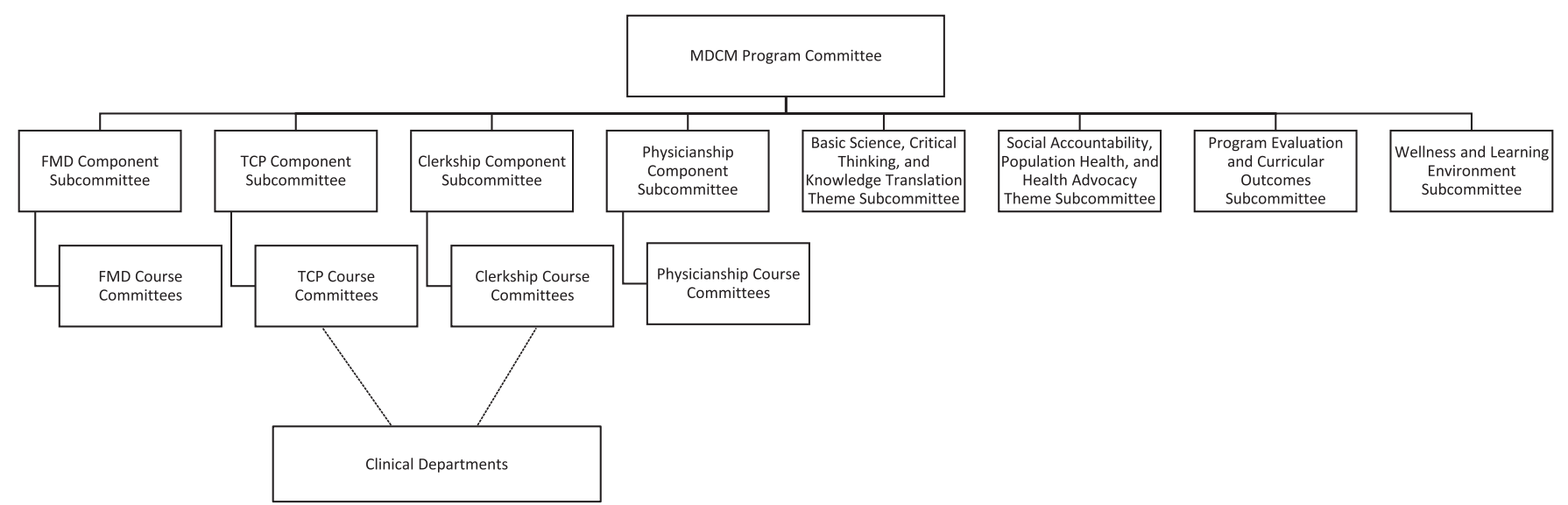

Figure 1 Curriculum governance structure. 
The associate dean, UME heads a 16-person academic team responsible for curriculum planning, implementation, and oversight:

- Assistant dean, UME

- Program director-Outaouais campus

- Chair, program evaluation, and curricular outcomes (PECO)

- Academic lead, curriculum design, and implementation

- Academic lead, assessment design, and implementation

- Two theme leaders (BSCTKT and SAPHHA)

- Eight component directors (4 at each campus: FMD, TCP, clerkship, physicianship)

See Figure 2-Organizational chart of deans.

The Institute of Health Sciences Education (IHSE; https://www. mcgill.ca/ihse) was established in 2019, building on the previous Centre for Medical Education in the Faculty of Medicine. The institute is a home for scholarship and innovation in health sciences education, and there are full-time $\mathrm{PhD}$ faculty as well as health care professionals with full-time or part-time appointments.

\section{Faculty Development and Support in Education}

The associate dean, faculty development (FacDev) works with the assistant dean, FacDev; assistant dean, FacDev-Outaouais campus; director of FacDev, UME; and others members of the FacDev Committee and the Faculty Development Office (https:// www.mcgill.ca/medicinefacdev) to offer activities and programs geared toward both new and experienced faculty. Programs range from short 15-minute targeted "capsules" for UME leaders at the beginning of committee meetings, to targeted workshops for clinical departments or the Outaouais campus based on needs identified by UME or PGME and to half day, full day, and multiday workshops open to all faculty in the Faculty of Medicine. FacDev is in the process of developing a longitudinal series of blended (online and face-to-face) modules to facilitate access to important activities.

Key topics relevant to UME offered by FacDev include:

- Orientation for new faculty
- Assessment in the clinical setting

- Assessment in the classroom setting

- Giving effective feedback

- Interactive lecturing skills

- Improving the learning environment

- Teaching culturally diverse learners

- Role modeling

- Assessing and teaching clinical reasoning

- The learner in "difficulty"

- Teaching when there's no time to teach

- Teaching in the ambulatory setting

- Small-group teaching skills

- Leadership development

FacDev has partnered with the Faculty's Academic Affairs Office (https://mcgill.ca/medicine-academic) and the University's Teaching and Learning Services (https://www.mcgill.ca/tls) to support faculty in academic promotion. This includes:

- Understanding how to maximally benefit from annual review/ performance dialogues

- Overview of promotions timelines and processes

- How teaching is recognized through the promotions process

- How to prepare the promotions dossier

Excellence in teaching and/or education activities is central to the promotions process. All faculty members are required to submit a teaching portfolio when applying for promotion. The teaching portfolio includes:

- Teaching approach: rationale for particular teaching methods; learning goals for students; how student learning is evaluated and why; how research and the "process of inquiry" is integrated into teaching; directions and plans for future development of teaching, in the light of teaching experiences

- Teaching responsibilities

- Evidence of teaching effectiveness

- Summary of activities undertaken to develop and enhance teaching: development and sharing of teaching innovations, materials, or strategies; advising and mentoring colleagues about teaching-related issues; mentoring; contributions

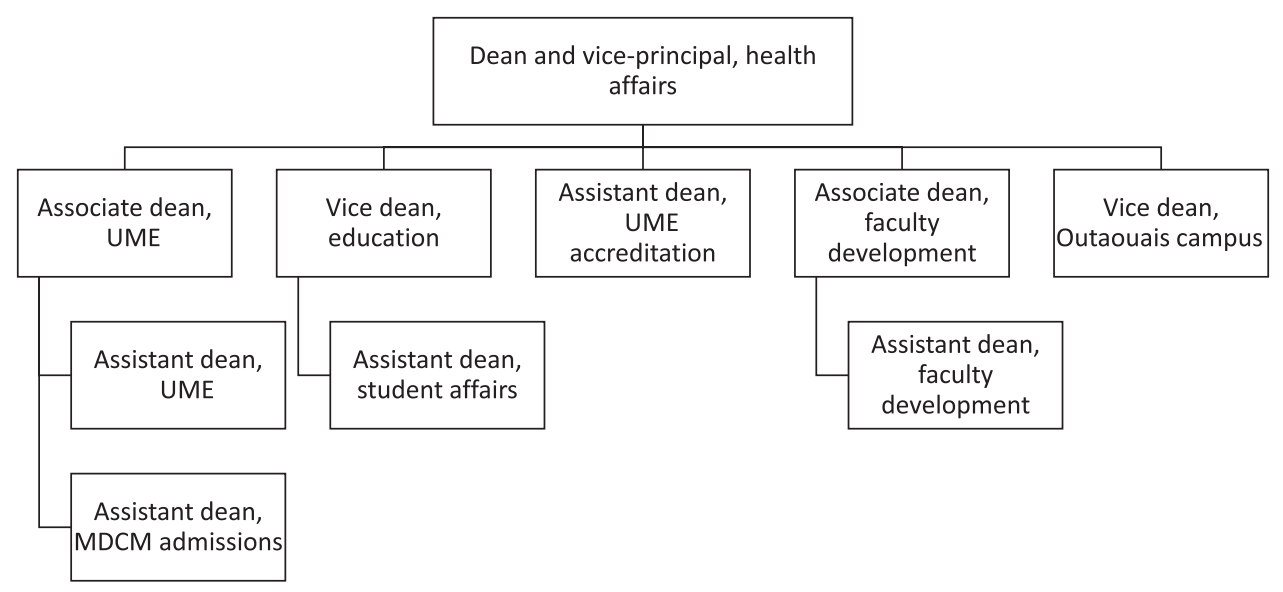

Figure 2 Organizational chart of deans. 
through teaching support units, teaching committees, associations; organizing or facilitating seminars, workshops, or conferences on teaching and learning; contributions to the development of policies on teaching and learning; teachingrelated publications

Educational scholarship is recognized and weighted on its own merits - the value of grants and impact factors of publications are rated compared with standards expected in the realm of educational scholarship, not clinical research. For faculty members in the clinician-teacher and clinician-educators tracks, teaching excellence and educational innovation, scholarship and research, and educational administration are weighted appropriately in the promotions process and these achievements can serve as the backbone for successful promotion. The Faculty Honour List for Educational Excellence also provides an opportunity for public recognition of excellence in teaching.

\section{Regional Medical Campuses}

The MD, CM program has an integrated clerkship in the city of Gatineau, in the Outaouais region of Quebec, which has been welcoming students for the core clerkship year since 2010 In August 2020, the Outaouais campus will officially open, welcoming 24 students into the first year of the medical program. The language of instruction at the Outaouais campus will be French, whereas the primary language of instruction at the main Montreal Campus is English. 


\section{Université de Montréal Faculty of Medicine}

Margaret Henri, MD, and Geneviève Grégoire, MD

\section{Medical Education Program Highlights}

About 1,300 students (including 200 at the Mauricie Regional Campus) are currently being trained in the undergraduate MD program of the Faculty of Medicine of Universite de Montréal, making it one of the largest in North America. The entire education is provided in French, as we serve a population whose mother tongue is French.

Social accountability of doctors as active members of society is an important value for our medical school, a value we wish to convey to students. Our faculty received the 2019 AMEE ASPIRE-to-Excellence Award in Social Accountability, underscoring this special feature. The beginning of year 1 is marked by a symposium where students are introduced to humanities and the concept of social accountability by speeches from local and international leaders who are involved with underserved populations. During clerkships, students take a 1 -week required rotation in social commitment and community health, with clinical exposure to vulnerable and marginalized populations, as refugee claimants, domestic abuse victims, sex trade workers, or prisoners.

Two bodies are prospectively involved in our curriculum: the Office of Clinical Ethics and Office for Patient Partnership and Collaboration. Faculty collaborators from both offices actively participate in the Program Committee.

Students benefit from a diverse and strong hospital network associated with the Université de Montréal, with several institutions of highly specialized care: the new Hospital University Center inaugurated in 2018, Sainte-Justine Mother and Child University Hospital Center, Montreal Heart Institute, and National Institute of Legal Psychiatry. Ten other integrated health centers and 17 family medicine groups located in Montreal and across Quebec constitute the University Integrated Health Network.

\section{Curriculum}

\section{Curriculum description}

See Supplemental Digital Appendix 1-Curriculum Structure and Sequencing - at http://links.lww.com/ACADMED/A961.

Acad Med. 2020;95:S596-S600.

doi: 10.1097/ACM.0000000000003472

Copyright (C) 2020 by the Association of American Medical Colleges

Supplemental digital content for this article is available at http://links.Iww.com/ ACADMED/A961.

Year school was established: 1843.

School URL: https://medecine.umontreal.ca/medical-school.

\section{Curriculum changes since 2010}

Major changes were implemented to clerkships in 2013:

- The surgery, pediatrics, internal medicine, obstetricsgynecology, and psychiatry rotations decreased from 8 to 6 weeks. Two of the original 8 weeks of the family medicine rotation were removed to create 2 new 1 -week rotations in palliative care and social commitment and community health.

- Elective rotations were increased from four 4-week rotations to twenty 2- or 4-week rotations, including three 2-week selectives in medicine, surgery, and pediatrics.

- Lectures taking place during clerkship and ethics workshops were combined into 7 "campus weeks" where students reunite at their respective central or regional campus.

- Simulation half days are now matched to associated rotations in internal medicine, obstetrics-gynecology, psychiatry, surgery, pediatrics, and emergency medicine.

- All end-of-rotation written assessments were eliminated, except for internal medicine. They were replaced by integrative progress tests taken throughout clerkship.

- To allow for more stability in students' rotation sites, 85 places are reserved for students wishing to remain in one institution during clerkship (centralized clerkship). For these students, 26-34 weeks of clerkship take place in a single institution. Students who are parents, elite sport students, and special accommodation students take precedence in these spots.

Other changes:

- 2017: Improved teaching in dermatology (module), allergy (simulation center), and ethics (preclinical workshops added)

- 2018: Curricular change in teaching pain management, with special focus on appropriate opioid usage

- 2018: Entrustable Professional Activities (EPAs) introduced in clerkship. In 2019, EPAs were formalized into a course; although EPAs remain formative assessments, students must reach specific EPA goals to pass

- 2019: Enhancement of the curriculum in First Nations' health and social responsibility

- 2019: Preparatory year curriculum was changed completely both at the Montreal Campus and at UQTR (Université du Québec à Trois-Rivières) for Mauricie students; courses were reformatted, sequencing was changed to avoid overlap, and an effort was made to standardize teaching and learning experiences at both universities

- 2019: Ultrasound skills added to several existing simulation scenarios at the simulation center

\footnotetext{
Assessment

Medical program objectives are based on the CanMEDS Framework.

See Table 1-Program Objectives and Assessment Methods.
} 
Table 1

Program Objectives and Assessment Methods

\begin{tabular}{|c|c|}
\hline $\begin{array}{l}\text { Program } \\
\text { objectives }\end{array}$ & Assessment methods \\
\hline Medical expertise & $\begin{array}{l}\text { - Written examinations (preclinical and clerkship) } \\
\text { - Clerkship competency-based evaluations } \\
\text { - Simulation-based assessments } \\
\text { - EPAs } \\
\text { - OSCE }\end{array}$ \\
\hline Collaboration & $\begin{array}{l}\text { - Preclinical years PBL and ICM-ICA tutor evaluations } \\
\text { - Simulation-based assessments } \\
\text { - Clerkship competency-based evaluations } \\
\text { - EPAs } \\
\text { - Evaluation of interprofessional collaboration }\end{array}$ \\
\hline Communication & $\begin{array}{l}\text { - Preclinical years PBL and ICM-ICA tutor and } \\
\text { - Assessment of feedback given to peer during TBL } \\
\text { - Simulation-based assessments } \\
\text { - Clerkship competency-based evaluations } \\
\text { - EPAs } \\
\text { - Oral presentations } \\
\text { - OSCE } \\
\text { - Evaluation of interprofessional collaboration }\end{array}$ \\
\hline Scholarship & $\begin{array}{l}\text { - Preclinical years PBL tutor and monitor } \\
\text { evaluations } \\
\text { - Clerkship competency-based evaluations } \\
\text { - EPAs } \\
\text { - Oral presentations }\end{array}$ \\
\hline $\begin{array}{l}\text { Leadership and } \\
\text { management }\end{array}$ & $\begin{array}{l}\text { - Simulation-based assessments } \\
\text { - EPAs }\end{array}$ \\
\hline Professionalism & $\begin{array}{l}\text { - Preclinical years PBL and ICM-ICA tutor and } \\
\text { - } \text { Assenitor evaluations } \\
\text { - Simulation-based assessments } \\
\text { - Clerkship competency-based evaluations } \\
\text { - EPAs } \\
\text { - OSCE } \\
\text { - Evaluation of interprofessional collaboration } \\
\text { - Ethics self-reflective essay }\end{array}$ \\
\hline Health advocacy & $\begin{array}{l}\text { - Clerkship competency-based evaluations } \\
\text { - EPAs } \\
\text { - OSCE }\end{array}$ \\
\hline
\end{tabular}

Preclinical years:

- Each course in years 1 and 2 has a midcourse low-stakes examination (2017). Previously, students had marks attributed completing the examination. With the use of EXAMSOFT software, students take examinations online and get the exact scoring of their performance, along with a retroaction based on success rate on learning objectives.
- The Nephrology and Urology course, in year 2, was transformed from problem-based learning (PBL) to teambased learning (TBL) (2011). Students are paired for mutual feedback about their participation within the team. Teachers assess the quality of feedback given by students.

- A formative progress test was added for second-year students (2016).

- Self-assessment of progression and learning strategies has been introduced to PBL (2015) and has been added in Introduction to Clinical Medicine and Initiation to the Clinical Approach courses in years 1 and 2 (2017).

Clerkship:

- "Learning opportunities," direct observation of clinical competencies as "disclosure of bad news," was introduced (2010). Direct observations were mapped into the Association of Faculties of Medicine of Canada EPAs (2017).

- Written examinations at the end of each clerkship rotation were removed (2013). Progress tests (PTs) in clerkship replaced them. Students perform 3 PTs in year 3 and 2 in year 4 . They get a personalized retroaction classified by learning objectives. Written examinations were reintroduced in medicine (2017) and in public health and public health and community medicine (2019).

- An end-of-clerkship OSCE was introduced (2017). If a student fails the OSCE, the remedial is in OSLER (Objective Structured Long Case Examination Record) examination. An end-of-clerkship written exam was added (2018). Both these assessments must be passed to earn the MD degree.

Preclinical and clerkship:

- Numeral marks were replaced by pass/fail scoring for all courses (2018).

\section{Parallel curriculum or track}

The University of Montreal does not offer a 3-year MD degree program.

\section{Pedagogy}

The following pedagogical approaches are used:

- Case-based learning

- Team-based learning

- Lectures

- Laboratory

- Role play/dramatization

- Simulation/standardized/simulated patients

- Clinical experience: ambulatory

- Clinical experience: inpatient

- Self-directed learning/tutorial

- Video/podcast

- Workshops

- Interfaculty workshops (involving pharmacy, nutrition, physiotherapy, kinesiology, nursing, dentistry, speech therapy, audiology, optometry, and social sciences students) 
Changes in pedagogical approaches since 2010:

- Workshops in clinical ethics, with one each practicing physician, resident, and patient collaborator

- Testimonial lectures from patient collaborators and First Nations patients

- Improvement in use of concept maps in PBL

- Podcasts in surgery

- Formative short-form PTs

- Preceptorship for clerk students who demonstrate clinical difficulties

- Clinical reasoning comprehensive assessment for students with difficulties during clerkship

Clinical experiences

Types of clinical site(s) required educational experiences:

- Tertiary and quaternary care hospitals

- Community hospitals

- Local community service centers

- Home visits

- Primary care physicians' offices and family medicine groups

\section{Required longitudinal experiences}

Our regional campus offers an integrated longitudinal clerkship. This approach aims at providing students with an authentic practice experience and at developing professional identity early on. This apprenticeship by iteration emphasizes self-reflective practice and collaborative work with other health care professionals, while students are exposed to ambulatory care. Students also benefit from individual and group mentorship.

\section{Clinical experience first encounter}

Learners have their first clinical encounters with patients in the first semester of year 1, during the Introduction to Clinical Medicine course, followed in year 2 by Introduction to Clinical Approach. They spend 1 half day every week learning history taking and physical examination as well as fundamentals of physicianship.

\section{Required and elective community-based rotations}

Required rotations:

- Family medicine

- Public health and community medicine

- Social commitment and community health

- Palliative care

Elective rotations:

- First Nation's health

- Remote area family medicine

- Addiction medicine

- Social pediatrics

- Primary care psychiatry and intellectual deficiency

- Day care internal medicine

- Social and legal medicine

\section{Challenges in designing and implementing clinical experiences} for medical students

The challenges we face are linked to large numbers of students needing placement. These numbers also imply faculty and faculty development, faculty training, and continuous pedagogical education, for ends of teaching and fair student assessment. The new implementation of EPAs illustrates this challenge as some teachers practice in remote centers, including regional family practice.

\section{Curricular Governance}

See Figure 1-Curricular governance.

\section{Decentralized curricular governance}

Curricular governance remains at the UME level. Budgets for teachers responsible for clerkship rotations are managed departmentally. However, the template for fair remuneration is decided by the UME director.

\section{Education Staff}

Academic and administrative support lies in the UME office. The academic direction counts 8 physicians in Montreal and 3 in Mauricie. There are 17 administrative management and office staff in Montreal and 3 in Mauricie.

The Program Committee is central for planning, implementing, evaluating, and overseeing the curriculum. Subcommittees that report to the Program Committee are:

- Clerkship Committee

- Pedagogical and Competency Apprenticeship Committee

- Clerkship Competencies Development Committee

- Committee of the Centre for Learning of Physician Abilities and Behaviors (simulation)

To help manage these tasks, a pedagogical assistant and faculty member are appointed to follow-up of development of competencies.

Regional Mauricie campus directors-the assistant dean, clerkship director, and preclinical director-are involved in decisions for planning and implementation. They share responsibilities of curricular oversight and maintenance of tools to support curriculum delivery and monitoring.

For the development and maintenance of tools supporting curriculum delivery, monitoring, and management, the Medical Studies Management Committee meets weekly in videoconference with Mauricie campus directors and administrative assistants of each campus. Decisions about curriculum delivery and application of academic rules for individual student situations are discussed by that committee.

The Evaluation Office has professional persons who collate results of written examinations, OSCE, and rotations assessments. They also prepare aggregated assessments of 


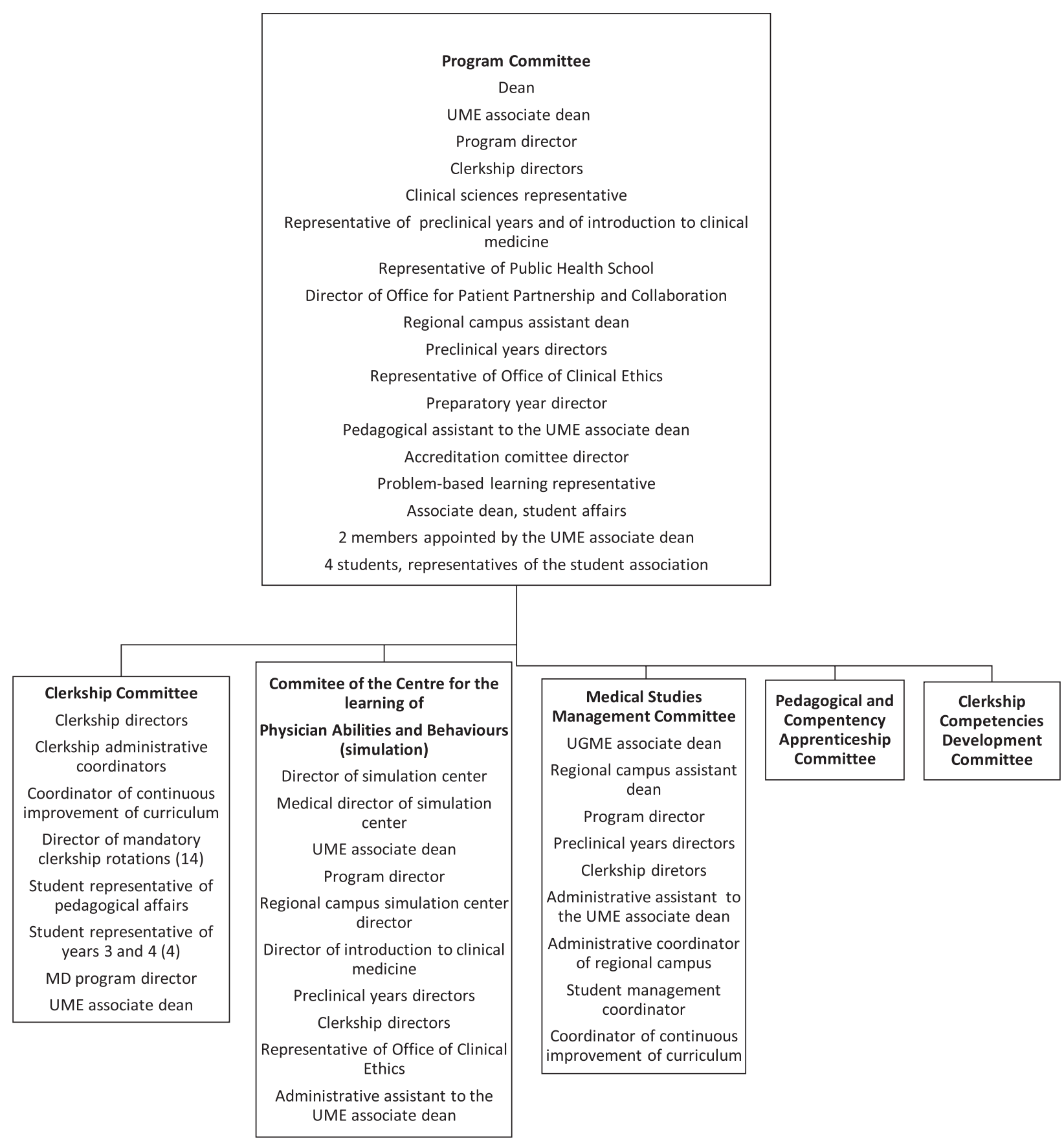

Figure 1 Curricular governance.

teachers, courses, and rotations, then diffuse these evaluations to the appropriate persons (students, direction, department heads, etc.). Teacher assessments are given only to individual teachers and their department head.

\section{Medical education leadership}

The associate deans who oversee UME are:

- UME associate dean and an assistant for the Mauricie campus
- Associate dean for faculty affairs and pedagogical and professional development, responsible for the Office of Medical Education (CPASS [Centre de pédagogie appliquée aux sciences de la santé])

- Associate dean for student life, responsible for students affairs (BAER)

- Executive associate dean, clinical sciences and network, responsible for the Evaluation Office and relationships with clinical teaching sites in the clinical network 


\section{Department of Medical Education}

The office is responsible for pre-UME and UME. Student Affairs is a distinct and confidential service.

\section{Faculty Development and Support in Education}

\section{Professional development for faculty as educators}

Professional development is under the associate dean for faculty affairs and pedagogical and professional development. The office is called CPASS.

\section{Role of teaching in promotion and tenure}

There are 4 aspects considered for promotion and tenure decisions: teaching, research, contribution to the institution, and academic visibility. Teaching and excellence in teaching include clinical teaching to clerks and residents in hospitals or clinics, lectures, and teaching in simulation or PBL, etc. Scholarship and research in medical education are considered in the research aspect. Academic administrative involvement is considered as contribution to the institution. Invited speaker activities and teaching awards are considered academic visibility.

CPASS provides continuous education for faculty members of the whole Faculté de Médecine. This includes pregraduate and postgraduate Medicine, Rehabilitation School, Speech Therapy and Audiology School, Nutrition School, and Kinesiology and Movement Science School.

CPASS has pedagogical leaders, educators from several disciplines. They are trained to teach faculty and residents and develop material for that end. Support from 2 educational technologists involved in development of interactive self-learning modules is provided to them.

\section{Regional Medical Campus}

There is one regional campus, in Trois-Rivières, in the Mauricie region. It hosts 44 students from years $1-4$. An integrated longitudinal clerkship experience is offered for 12 clerks.

\section{Educational experiences across sites}

All curricular aspects are coordinated at the direction level. Teachers or administrators of the Mauricie campus sit in every governance committee. The Medical Studies Management Committee meets weekly, using videoconference for the Mauricie directors. Administrative assistants for each campus are present.

All lectures from the Montreal campus are presented in videoconference in Mauricie. Sometimes lectures occur in Mauricie, transmitted by videoconference to Montreal. This helps students from both campuses understand videoconferencing best practices and behaviors, for example, asking questions at microphones so students from both campuses have identical learning experiences.

For PBL, Montreal and Trois-Rivières videoconference before every morning session, to ensure that teachers stress identical points and that their questions are answered.

Scenarios for the simulation center teaching are identical at both campuses.

Faculty development sessions are also given in Trois-Rivières. 


\section{College of Medicine, University of Saskatchewan}

Greg Malin, MD, PhD, Regina Gjevre, MD, MSc, FRCPC, Patricia Blakley, MD, PhD, FRCPC, and Kent Stobart, MD, MSc, FRCPC

\section{Medical Education Program Highlights}

The College of Medicine at the University of Saskatchewan offers an undergraduate 4-year MD degree. One hundred students matriculate each year. There are 2 main campuses and 3 distributed clinical training sites.

Of the 100 seats available each year, 10 are reserved for qualified, self-identified First Nations, Métis, and Inuit students and 6 for diversity and social-accountability applicants (based on family income and rural location).

Unique course offerings:

- Success in Medical School courses: Focused curricular time supports students to successfully transition into medicine (topics include professionalism, wellness, career advising, and study strategies).

- Clinical Integration courses: A series of preclerkship courses use a competency-based approach with focus on developing the clinical reasoning process through undifferentiated casebased problems.

- Medicine and Society courses: Patient- and family-centered care modules provide students with authentic patient perspectives on health care experiences. This course contains a longitudinal theme for both research/evidence-based medicine and arts and humanities experience. Finally, students engage in a community agency-based learning experience.

- Principles of Biomedical Sciences course: Integration of anatomy/ pathology and point of care ultrasound/medical imaging helps students understand and visualize structures and pathologies.

Unique student activities:

- Making the Links and Global Health Certificate: In this unique service-learning opportunity, students gain experience in traditionally underserved/ disadvantaged contexts, working in interprofessional teams, and cultural safety/health advocacy.

- MD-MBA dual degree: Students offered admission to the college can defer entry to complete an MBA.

Unique curricular oversight:

- The Curriculum Quality Review Subcommittee of the Curriculum Committee provides an internal peer-audit and course review to ensure alignment with program goals and objectives.

Acad Med. 2020;95:S601-S604.

doi: 10.1097/ACM.0000000000003486

Copyright (C) 2020 by the Association of American Medical Colleges

Supplemental digital content for this article is available at http://links.Iww.com/ ACADMED/A965.

Year school was established: 1953.

School URL: https://medicine.usask.ca.

\section{Curriculum}

The undergraduate medical education program consists of 2 years of preclerkship study and 2 years of clerkship. Horizontal and vertical integration of curricular themes are effected within this curricular structure.

See Supplemental Digital Appendix 1-Curriculum Overviewat http://links.lww.com/ACADMED/A965.

\section{Curriculum changes since 2010}

The medical school class size increased from 84 to 100 in AY 2011-2012 academic year. All students are based at the Saskatoon campus for year 1 . In year 2, a cohort of students (40\%) transfer to the Regina campus. This distributed model supports optimal access for clinical experience opportunities. In year 3 , there are 6 clerkship positions available at a regional site in Prince Albert and 4 positions available for students to engage in the rural Saskatchewan longitudinal integrated clerkship (SLIC), in Estevan and Meadow Lake, Saskatchewan.

In AY 2014-2015, the undergraduate medical program at University of Saskatchewan instituted the $2+2$ curriculum.

\section{Assessment}

At the University of Saskatchewan, the medical education program learning objectives and all subordinate course/module/ rotation/session-level objectives are aligned with the CanMEDS framework that identifies and describes the abilities physicians require to effectively meet the health care needs of the people they serve. A competent physician seamlessly integrates the competencies of all 7 CanMEDS roles.

See Supplemental Digital Appendix 2-Competencies, Program Objectives, and Outcome Measures_at http://links.lww.com/ ACADMED/A965.

Medical education program objectives are described in outcome-based terms that allow assessment of medical students' progress in developing competencies that the profession and the public expect of a physician (2020-2021 CACMS Standards 6.1).

The University of Saskatchewan implemented several changes around student assessment in the last several years. These changes include:

- Introduction of computer-based exams for examination administration

- Development of consistent standard-setting process for assessments that contribute $>20 \%$ to student grades

- Assessment mapping and monitoring

- Robust examination quality review processes

- Regular faculty development for exam construction 
- Processes for individual student performance feedback linked to specific learning objectives

- Rigorous OSCE station development and review processes

- Required narrative feedback to students from specific educational experiences

At present, the University of Saskatchewan has no plans to reduce our undergraduate medical program from the current duration of 4 years.

\section{Pedagogy}

University of Saskatchewan undergraduate medical program courses use a variety of pedagogical approaches to medical education. Each course has developed an individual strategy for employment of pedagogical methods. Approaches used in various courses include:

- Case-based learning

- Clinical experience: ambulatory

- Clinical experience: inpatient

- Discussion: large group (>12) (e.g., flipped classroom)

- Discussion: small group $(\leq 12)$

- Laboratory

- Lecture

- Peer teaching

- Preceptorship

- Problem-based learning

- Role play/dramatization

- Self-directed learning/tutorial

- Simulation

- Standardized/simulated patients

- Team-based learning

- Video/podcast

- Workshop/skills days

- Other: Experiential learning through service learning in the community

In the last decade, the medical school has increased diversity of teaching methodologies. We have introduced team-based learning within preclerkship and clerkship courses. Webinars have been used supporting teaching and learning for clerkship students in distributed geographic sites. Opportunities for clinically relevant exercises in application of learning have been expanded, with many faculty using a flipped classroom approach and assistive technologies to support active learning. There is increased emphasis on preclerkship experiential and service learning.

\section{Clinical experiences}

Hospital and outpatient clinic sites are primarily accessed for required educational experiences. These sites include urban major teaching hospitals and clinics through small rural community-based hospitals and clinics.

A subset of year 3 students participate in a 42-week longitudinal integrated clerkship in rural setting within Saskatchewan.

Students begin their patient clinical experiences and learn interviewing and physical examination skills in the first term of year 1.
Students complete a 1-week community hospital/clinic placement in the summer of year 1 . In year 2, students from both campuses participate in service-learning experiences engaging with different community agencies. Year 2 students have the option to engage in community clinical placements during the summer before clerkship. In year 3, students participating in the Core Clinical Rotations course rotate through a rural family medicine rotation in a rural/remote community. Students also have opportunities within some clinical rotations to have outpatient clinic experiences outside the teaching hospital in practitioner private offices. For students in the longitudinal integrated clerkship, their clinical experiences in year 3 are hosted within a community hospital and clinics. In year 4 , students have opportunities to select community-based rotations for a variety of disciplines including rural family medicine and indigenous health rotations.

Designing and implementing optimal experiences for medical students presents challenges in the real-world environment of clinical medicine. Competing needs with postgraduate programs have been somewhat of an obstacle for our undergraduate learners in some more specialized areas such as palliative care.

\section{Curricular Governance}

The preclerkship phase is managed from the centralized Office of Undergraduate Medical Education. The majority of clerkship administrative staff are employees of the undergraduate medical education office, but may be geographically located in clinical department offices. The undergraduate medical program provides funding for teaching, assessment, and academic support.

See Figure 1-Curriculum Committee.

\section{Education Staff}

Preclerkship:

- Preclerkship administrative coordinators work with faculty course/module leads to ensure effective planning, implementation, and day-to-day oversight of the curriculum.

- Year 1 and year 2 administrative assistants work with administrative coordinators to carry out planning, implementation, and day-to-day oversight of the curriculum. Additional administrative assistants support Regina campus for students in year 2 .

Clerkship:

- Two clerkship administrative coordinators (1 at each main campus) work with faculty course/rotation leads to ensure effective planning, implementation, and day-to-day oversight of the clerkship.

- Year 3 and year 4 administrative assistants work with administrative coordinators to carry out planning, implementation, and day-to-day oversight of the clerkship. Administrative assistants located at all campuses and distributed sites support learners and faculty. 


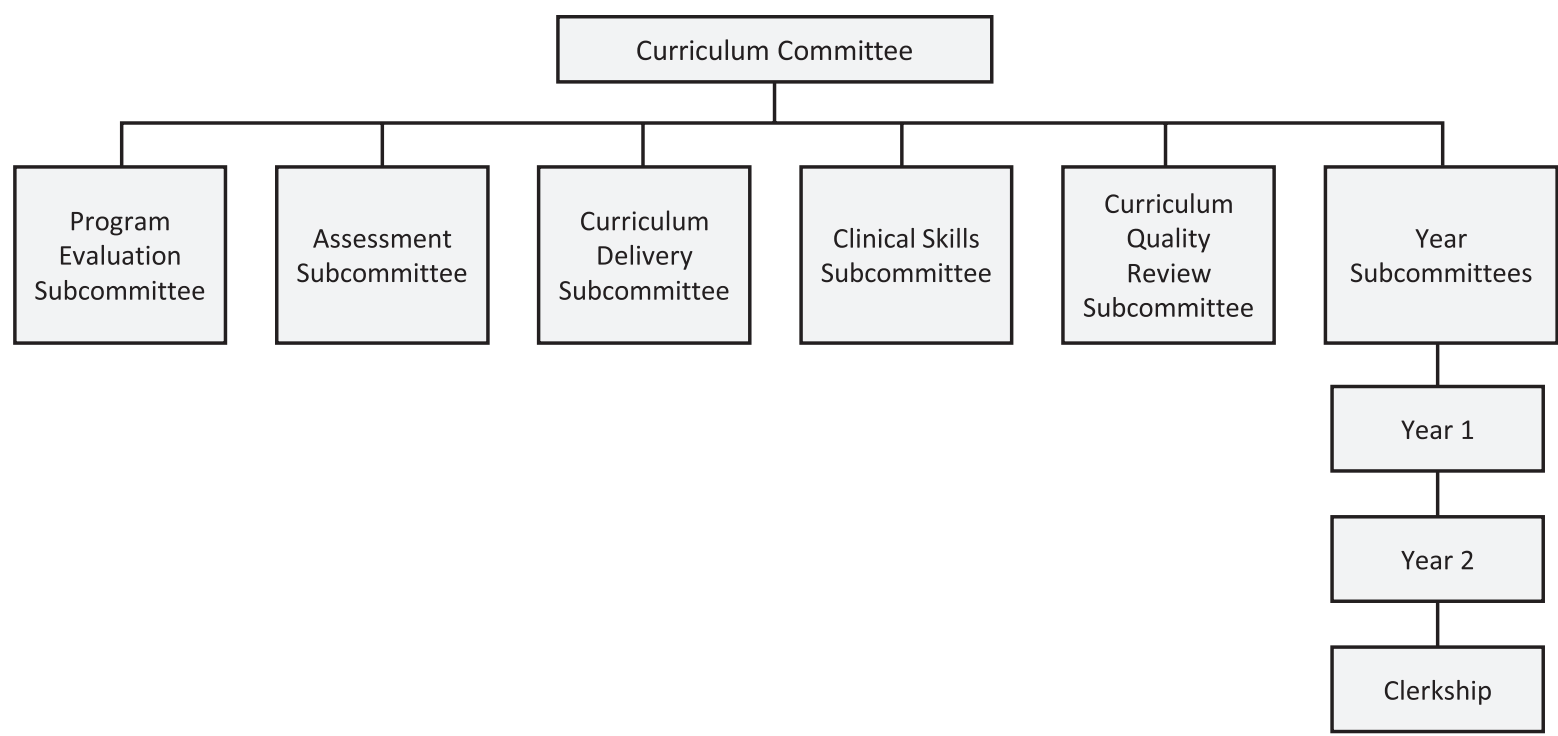

Figure 1 Curriculum Committee.

The College of Medicine has created 5 specialist positions for the major areas of undergraduate medical education program management:

- Curriculum specialist, who focuses on objective creation and alignment

- Assessment specialist, who focuses on methods of assessment, alignment with objectives, standard setting, and multiple choice question (MCQ) and OSCE development

- Indigenous curriculum specialist, with focus on curriculum integration of indigenous health and ways of knowing

- Program evaluation specialist, with focus on ensuring timely course and instructor evaluations, including loop closure, as well as longitudinal student performance outcome data

- Academic support specialist, with focus on identifying students in academic difficulty and coordinating academic support for students

The College of Medicine has a dedicated team of IT specialists to provide support for curriculum management, curriculum delivery (in-class assistive technology for active learning, asynchronous online learning technology, electronic exam software, recorded lectures), classroom videoconferencing for distributed large-group sessions, and computer troubleshooting and support.

See Figure 2-Organizational chart.

The undergraduate medical education program is responsible for the education delivery of the MD program. Student affairs reports directly to vice dean of education.

\section{Faculty Development and Support in Education}

The Office of Faculty Development provides a number of centrally located and developed professional development activities for faculty. Faculty development offers regularly scheduled monthly events (Teaching and Learning Tuesdays,
Webinar Wednesdays, medical education grand rounds, book club). They offer extensive website-based resources, peer-teaching mentorship, and both regularly scheduled and customized workshops and teaching sessions for faculty.

All faculty have expectations for demonstration of teaching ability and performance. We have specific evaluation criteria for faculty who have a focus in teaching and the scholarship of medical education, called practice of professional skills. This category evaluates for a scholarly approach to teaching, learning, and mentorship in the area of education. It also evaluates for excellence in teaching and engagement in translation and dissemination of effective and/or innovative teaching practices, as well as research and scholarship engagement, dissemination, translation, and leadership.

\section{Regional Medical Campuses}

A regional medical campus is a site distinct from the main campus where at least 1 student spends at least 6 consecutive months. The campus may offer the basic science portion of the curriculum, the clinical portion of the curriculum, or both. Excluded are affiliated clinical sites at a distance from the main campus where a given student would only take 1 or 2 clerkships.

See Table 1-Regional Medical Campuses.

The Prince Albert site has rotation site coordinators that work in tandem with the rotations site coordinators at the 2 main campus sites of Saskatoon and Regina to ensure consistency of curriculum delivery at the rotation level. All rotation coordinators have membership on, and report to, the Clerkship Subcommittee, where all curricular activities of clerkship are planned, implemented, evaluated, and monitored. The Clerkship Subcommittee then reports any recommendations for decision to the Curriculum Committee. 


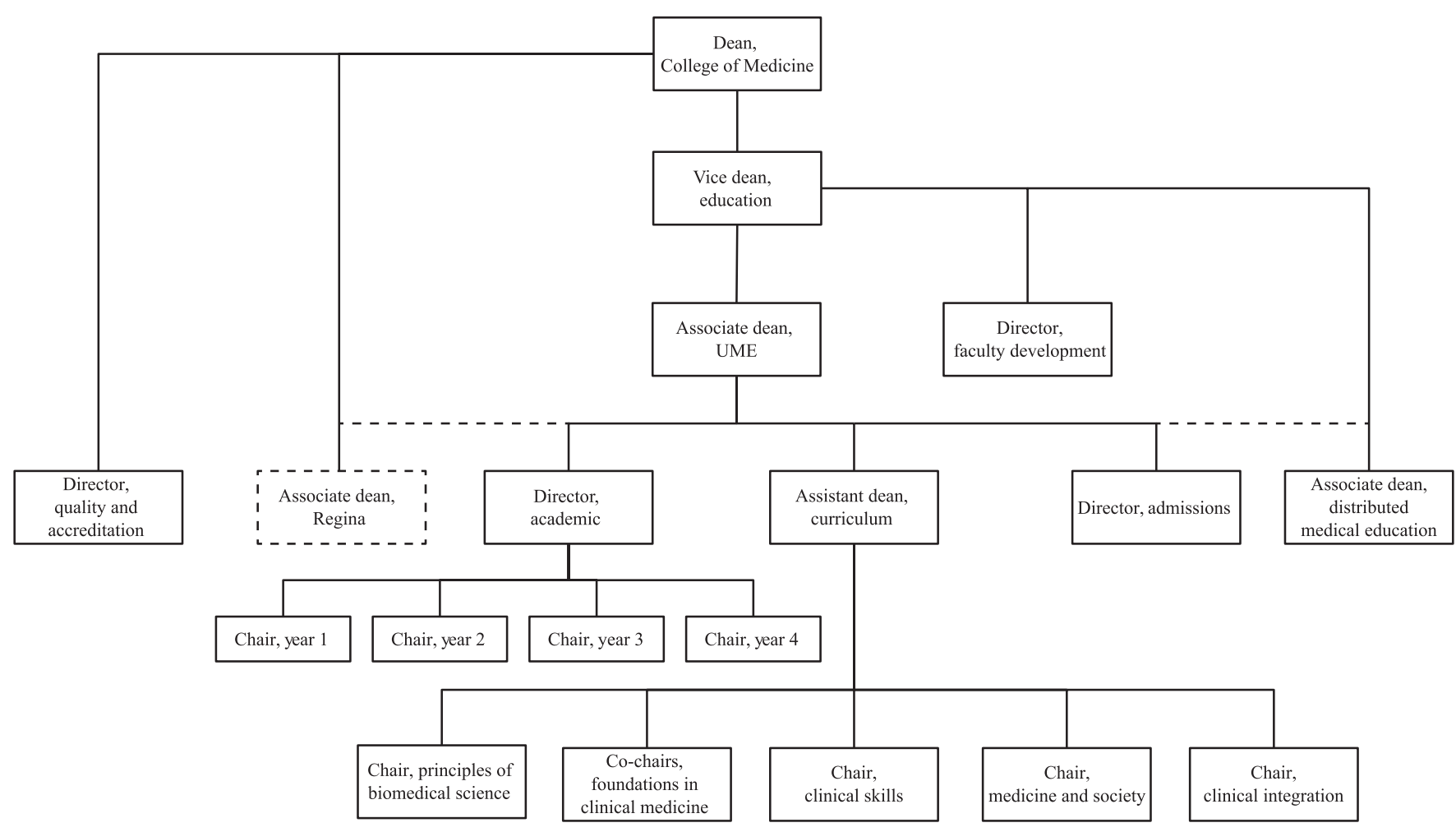

Figure 2 Organizational chart.

\section{Table 1}

Regional Medical Campuses

\begin{tabular}{|c|c|c|}
\hline Site & Туре & $\begin{array}{l}\text { Student } \\
\text { enrollment }\end{array}$ \\
\hline Prince Albert & Clerkship, block rotation based & 6 \\
\hline Estevan & Longitudinal integrated clerkship & 2 \\
\hline Meadow Lake & Longitudinal integrated clerkship & 2 \\
\hline
\end{tabular}

The 2 regional distributed clinical teaching sites for the SLIC have a committee that meets regularly to coordinate delivery of the SLIC. This committee includes representation from the year 3 cochairs, academic director, and assistant dean for curriculum. The SLIC lead also has membership on the Clerkship Subcommittee.

The Curriculum Quality Review Subcommittee, in reviewing the delivery and organization of the clerkship courses, may also identify opportunities or deficiencies in consistency across sites, which would be reported back to the regional site leads, SLIC and Prince Albert coordinators, and Curriculum Committee. 


\section{Selected Abbreviations Used in This \\ Supplement}

Jennifer M.G. McDonald

AAMC: Association of American Medical Colleges

ACGME: Accreditation Council for Graduate Medical Education

AHEC: area health education center

AI: artificial intelligence

AMA: American Medical Association

AMEE: Association for Medical

Education in Europe

APN: Advanced Practice Nurse

APRN: Advanced Practice Registered Nurse

AV: audiovisual

AY: academic year

CACMS: Committee on Accreditation of Canadian Medical Schools

CBL: case-based learning

CBME: competency-based medical education

CCC: clinical competency committee

CK: Clinical Knowledge (USMLE Step 1 $\mathrm{CK})$

CME: continuing medical education

CPD: continuing professional development

CQI: continuous quality improvement

CS: Clinical Skills (USMLE Step 1 CS)

CV: curriculum vitae
DACA: deferred action for childhood arrivals

DIO: designated institutional official

EBM: evidence-based medicine

ED: emergency department

EPA: Entrustable Professional Activity

EPO: educational program objective

FQHC: federally qualified health center

FTE: full-time equivalent

GME: graduate medical education

GQ: Graduation Questionnaire (AAMC)

HSS: health systems science

IAMSE: International Association of Medical Science Educators

IHI: Institute for Healthcare Improvement

IPE: interprofessional education

IT: information technology

IV: intravenous

KSAs: knowledge, skills, and attitudes; or knowledge, skills, and abilities

LCME: Liaison Committee on Medical Education

LIC: longitudinal integrated clerkship

MCQ: multiple-choice question

MSOP: Medical School Objectives Project

MSPE: Medical Student Performance

Evaluation
MSTP: Medical Scientist Training Program

NBME: National Board of Medical Examiners

NIH: National Institutes of Health

NRMP: National Resident Matching Program

OR: operating room

OSCE: objective structured clinical examination

PBL: problem-based learning

PCRS: Physician Competency Reference Set

PGY: postgraduate year

PRIME: Program in Medical Education

RVU: relative value unit

SP: standardized patient

TBL: team-based learning

TSP: Teaching Scholars Program

UME: undergraduate medical education

USMLE: United States Medical Licensing Examination

VA: Veterans Affairs

VR: virtual reality

VSAS: Visiting Student Application Service

VSLO: Visiting Student Learning Opportunities

WBA: workplace-based assessment 